\title{
ESTIMATION OF PEAK DISCHARGE QUANTILES FOR SELECTED ANNUAL EXCEEDANCE PROBABILITIES IN NORTHEASTERN ILLINOIS
}

\author{
Prepared By \\ Thomas M. Over \\ Riki J. Saito \\ Andrea G. Veilleux \\ Padraic S. O'Shea \\ Jennifer B. Sharpe \\ David T. Soong \\ Audrey L. Ishii
} U.S. Department of the Interior U.S. Geological Survey

Research Report No. FHWA-ICT-16-013

https://doi.org/10.36501/0197-9191/16-014

A report of the findings of

ICT-R27-144

Development and Implementation of Updated Urban Regional Flood Frequency Equations for Illinois

Originally published June 2016

Modified September 2017

Further modified April 2021 

TECHNICAL REPORT DOCUMENTATION PAGE

\section{Report No.}

FHWA-ICT-16-013

\section{Title and Subtitle}

Estimation of Peak Discharge Quantiles for Selected Annual Exceedance

Probabilities in Northeastern Illinois

2. Government Accession No.

\section{Recipient's Catalog No.}

\section{Report Date}

Originally published June 2016; Modified September 2017; Further modified April 2021

\section{Performing Organization Code}

\section{Author(s)}

Thomas M. Over, Riki J. Saito, Andrea G. Veilleux, Padraic S. O’Shea, Jennifer B. Sharpe, David T. Soong, and Audrey L. Ishii

\section{Performing Organization Name and Address}

U.S. Geological Survey

Illinois Water Science Center

405 N. Goodwin Ave.

Urbana, IL 61801

\section{Sponsoring Agency Name and Address}

Illinois Department of Transportation (SPR)

Bureau of Materials and Physical Research

126 East Ash Street

Springfield, IL 62704

\section{Supplementary Notes}

Conducted in cooperation with the U.S. Department of Transportation, Federal Highway Administration.

\section{Abstract}

This report provides two sets of equations for estimating peak discharge quantiles at annual exceedance probabilities (AEPs) of $0.50,0.20,0.10$, $0.04,0.02,0.01,0.005$, and 0.002 (recurrence intervals of $2,5,10,25,50,100,200$, and 500 years, respectively) for watersheds in Illinois based on annual maximum peak discharge data from 117 watersheds in and near northeastern Illinois. One set of equations was developed through a temporal analysis with a two-step least squares-quantile regression technique that measures the average effect of changes in the urbanization of the watersheds used in the study. The resulting equations can be used to adjust rural peak discharge quantiles for the effect of urbanization, and in this study the equations also were used to adjust the annual maximum peak discharges from the study watersheds to 2010 urbanization conditions.

The other set of equations was developed by a spatial analysis. This analysis used generalized least-squares regression to fit the peak discharge quantiles computed from the urbanization-adjusted annual maximum peak discharges from the study watersheds to drainage-basin characteristics. The peak discharge quantiles were computed by using the Expected Moments Algorithm following the removal of potentially influential low floods defined by a multiple Grubbs-Beck test. To improve the quantile estimates, regional skew coefficients were obtained from a newly developed regional skew model in which the skew increases with the urbanized land use fraction. The skew coefficient values for each streamgage were then computed as the variance-weighted average of at-site and regional skew coefficients. The drainage-basin characteristics used as explanatory variables in the spatial analysis include drainage area, the fraction of developed land, the fraction of land with poorly drained soils or likely water, and the basin slope estimated as the ratio of the basin relief to basin perimeter.

This report also provides: (1) examples to illustrate the use of the spatial and urbanization-adjustment equations for estimating peak discharge quantiles at ungaged sites and to improve flood-quantile estimates at and near a gaged site; (2) the urbanization-adjusted annual maximum peak discharges and peak discharge quantile estimates at streamgages from 181 watersheds including the 117 study watersheds and 64 additional watersheds in the study region that were originally considered for use in the study but later deemed to be redundant.

The urbanization-adjustment equations, spatial regression equations, and peak discharge quantile estimates developed in this study will be made available in the web-based application StreamStats, which provides automated regression-equation solutions for user-selected stream locations. Figures and tables comparing the observed and urbanization-adjusted peak discharge records by streamgage are provided at http://dx.doi.org/10.3133/sir20165050 for download.

\section{Key Words}

Floods, flood frequency, urbanization, peak discharge estimation, peak discharge, streamflow statistics, basin characteristics, regionalization, regression, Web application, StreamStats.

\section{Distribution Statement}

No restrictions. This document is available through the National Technical Information Service, Springfield, VA 22161.

\begin{tabular}{l|l}
$\begin{array}{l}\text { 19. Security Classif. (of this report) } \\
\text { Unclassified }\end{array}$ & $\begin{array}{l}\text { 20. Security Classif. (of this page) } \\
\text { Unclassified }\end{array}$ \\
\hline
\end{tabular}

21. No. of Pages $66+$ appendices
22. Price

N/A 





\section{Errata: FHWA-ICT-16-013}

\section{April 7, 2021}

Subsequent to original publication of FHWA-ICT-16-013 (June 2016) an error was found in the reported methodology. It was discovered that the so-called "weighted" skew coefficient values used in the computation of the at-site flood frequencies were not actually computed using the weighted skew, that is, as a weighted average of the station and regional skew values. Instead they were computed using the regional skew only.

The preferred skew coefficients to use for computing at-site flood frequencies are the weighted skews. As a result, a revised version of the report, which uses the weighted skew coefficient values to calculate the at-site flood frequencies was completed (April 2021).

In the process of carrying out the correction of the skew methodology, two other types of corrections were made: (1) the perception thresholds on crest-stage gages were revised according to clarifications on the appropriate methodology, and (2) an updated and corrected version of the regional regression software WREG was used to compute the regional regression equations.

The following revisions to FHWA-ICT-16-103 (modified September 2017) reflect what was intended in the original version of this report with regard to the at-site skew coefficients and indicate the ancillary updates.

\begin{tabular}{|c|c|c|c|}
\hline $\begin{array}{l}\text { Page number } \\
\text { in } 2017 \text { version }\end{array}$ & Location & Original text/image & Revised text/image \\
\hline \multicolumn{4}{|l|}{ Ancillary updates } \\
\hline $\begin{array}{l}\text { Title page and } \\
\text { report } \\
\text { documentation } \\
\text { page }\end{array}$ & & & Added Padraic S. O'Shea as an author \\
\hline 16 & $\begin{array}{l}\text { First } \\
\text { sentence of } \\
\text { second } \\
\text { paragraph }\end{array}$ & $\begin{array}{l}\text { "...56 were CSGs throughout their peak } \\
\text { discharge record, } 10 \text { were CSGs during } \\
\text { part of their peak discharge record, } \\
\text { and } 51 \text { had continuous record ..." }\end{array}$ & $\begin{array}{l}\text { “...57 were CSGs throughout their peak discharge } \\
\text { record, } 13 \text { were CSGs during part of their peak } \\
\text { discharge record, and } 47 \text { had continuous record ..." }\end{array}$ \\
\hline 17 & $\begin{array}{l}\text { First } \\
\text { paragraph of } \\
\text { section } 2.1 .4 \\
\end{array}$ & “...remove PILFs...” & “...censor PILFs...” \\
\hline 17 & $\begin{array}{l}\text { First } \\
\text { paragraph of } \\
\text { section } 2.1 .4 \\
\end{array}$ & “PeakFQ version 7.1" & $\begin{array}{l}\text { PeakFQ version 7.3, } \\
\text { https://water.usgs.gov/software/PeakFQ/ }\end{array}$ \\
\hline 29 & $\begin{array}{l}\text { First } \\
\text { paragraph } \\
\text { on page }\end{array}$ & $\begin{array}{l}\text { "...at an exceedance probability of } \\
0.002 "\end{array}$ & Added "(table 8)" following original text, \\
\hline 39 & $\begin{array}{l}\text { Second } \\
\text { paragraph } \\
\text { for Chapter } \\
4.2\end{array}$ & $\begin{array}{l}\text { “...the computer program WREG (Eng } \\
\text { et al. 2009), version 1.05, which was } \\
\text { downloaded from } \\
\text { http://water.usgs.gov/software/WREG. } \\
\text { WREG...." }\end{array}$ & $\begin{array}{l}\text { "the function WREG.GLS from the package WREG } \\
\text { (Farmer, 2017) in R version 3.6.3 (R Core Team, 2020). } \\
\text { WREG.GLS..." }\end{array}$ \\
\hline 39 & $\begin{array}{l}\text { Second } \\
\text { sentence of } \\
\text { second } \\
\text { paragraph of } \\
\text { Chapter } 4.2\end{array}$ & & Added "described in Eng and others (2009)" \\
\hline
\end{tabular}




\begin{tabular}{|c|c|c|c|}
\hline 62 & References & & $\begin{array}{l}\text { Added reference: } \\
\text { "Farmer, W.H., 2017, WREG: USGS WREG v. } 2.02 \text { R } \\
\text { package version 2.02. Accessed November 15, 2019, at } \\
\text { https://rdrr.io/github/USGS-R/WREG/." }\end{array}$ \\
\hline 62 & References & $\begin{array}{l}\text { Updated URL for Gesch and others, 2002: } \\
\text { "http://www.asprs.org/a/publications/pers } \\
\text { /2002journal/january" }\end{array}$ & $\begin{array}{l}\text { "https://www.asprs.org/wp- } \\
\text { content/uploads/pers/2002journal } \\
\text { /january/highlight.html" }\end{array}$ \\
\hline 64 & References & & $\begin{array}{l}\text { Added reference: } \\
\text { "R Core Team, 2020, R-A language and environment } \\
\text { for statistical computing. R Foundation for Statistical } \\
\text { Computing, Vienna, Austria accessed April 24, 2020, at } \\
\text { https://www.R-project.org/." }\end{array}$ \\
\hline 65 & References & $\begin{array}{l}\text { Updated URL for U.S. Army Corps of Engineers, } \\
\text { 2013: “[Updated version available at } \\
\text { http://nid.usace.army.mil/cm_apex/f?p=838:12.]" }\end{array}$ & $\begin{array}{l}\text { "[Updated version available at } \\
\text { https://nid.sec.usace.army.mil/ords/f?p=105:1::.:.:..]" }\end{array}$ \\
\hline 71 & $\begin{array}{l}\text { Second } \\
\text { paragraph } \\
\text { of 'Results } \\
\text { of Regional } \\
\text { Skew } \\
\text { Analysis in } \\
\text { Northeast } \\
\text { Illinois” } \\
\end{array}$ & $\begin{array}{l}\text { Updated URL for Theobald, 2015: "[Also available } \\
\text { at http://www.ecologyandsociety.org/vol10/ } \\
\text { iss1/art32/.]" }\end{array}$ & $\begin{array}{l}\text { "[Also available at } \\
\text { https://www.ecologyandsociety.org/vol10/iss1/art32/.]" }\end{array}$ \\
\hline $77-88$ & Table 1 & $\begin{array}{l}\text { Corrected 'Crest-stage gage (CSG)' value for the } \\
\text { following streamgages: } \\
\text { 05438250: “No", 05519500: “No", } \\
\text { 05528000: “No", 05542000: “No", } \\
\text { 05549000: “No", 05551030: “Partial” }\end{array}$ & $\begin{array}{l}\text { 05438250: “Partial”, 05519500: “Partial”, } \\
\text { 05528000: “Partial”, 05542000: “Partial”, } \\
\text { 05549000: “Partial”, 05551030: “Yes" }\end{array}$ \\
\hline \multicolumn{4}{|c|}{ Revisions related to skewness coefficients } \\
\hline $\begin{array}{l}\text { Documentation } \\
\text { page }\end{array}$ & $\begin{array}{l}\text { Second } \\
\text { paragraph, } \\
\text { second-to- } \\
\text { last } \\
\text { sentence } \\
\text { of Abstract }\end{array}$ & & $\begin{array}{l}\text { Added "The skew coefficient values for each streamgage } \\
\text { were then computed as the variance-weighted average } \\
\text { of at-site and regional skew coefficients." }\end{array}$ \\
\hline 10 & $\begin{array}{l}\text { Step } 3 \text { in } \\
\text { section } 2.1\end{array}$ & "for use in the frequency analysis" & $\begin{array}{l}\text { "that is weighted with the at-streamgage skewness } \\
\text { values in the frequency analysis" }\end{array}$ \\
\hline 17 & $\begin{array}{l}\text { Second } \\
\text { paragraph } \\
\text { of section } \\
2.1 .4\end{array}$ & $\begin{array}{l}\text { "For use in the frequency analysis, the skewness } \\
\text { values for each streamgage were taken as equal } \\
\text { to the regional skewness values developed as } \\
\text { described in the "Regional Skew Analysis" section } \\
\text { and appendix 1." }\end{array}$ & $\begin{array}{l}\text { "As part of the frequency analysis, the skewness values } \\
\text { for each streamgage were computed as a weighted } \\
\text { average AW of the station and regional skew values } \\
\text { following the method suggested in Bulletin 17B (U.S. } \\
\text { Interagency Advisory Com-mittee on Water Data, } \\
\text { 1982):" }\end{array}$ \\
\hline 17 & $\begin{array}{l}\text { Second } \\
\text { paragraph } \\
\text { of section } \\
2.1 .4\end{array}$ & & $\begin{array}{l}\text { Added equation } 4 \text { immediately following added text. } \\
\text { Updated all equation numbers that follow. }\end{array}$ \\
\hline 17 & $\begin{array}{l}\text { Last } \\
\text { sentence } \\
\text { of section } \\
2.1 .3\end{array}$ & $\begin{array}{l}\text { "The regional skewness estimate from equation 3, } \\
\text { without weighting with the at-site skewness, was } \\
\text { used to obtain at site peak discharge quantile } \\
\text { estimates as described in the Frequency Analysis } \\
\text { section." }\end{array}$ & $\begin{array}{l}\text { "The regional skewness estimate from equation } 3 \text { was } \\
\text { combined with the at-site skewness according to their } \\
\text { associated uncertainties to obtain a weighted skew } \\
\text { estimate for use in computing the at-site peak discharge } \\
\text { quantile estimates." }\end{array}$ \\
\hline
\end{tabular}




\begin{tabular}{|c|c|c|c|}
\hline 33 & $\begin{array}{l}\text { First } \\
\text { paragraph } \\
\text { of Chapter } \\
4\end{array}$ & $\begin{array}{l}\text { "...including application of the regional skew } \\
\text { model developed for this study" }\end{array}$ & "...including weighting with the regional skew model." \\
\hline 33 & $\begin{array}{l}\text { First } \\
\text { paragraph } \\
\text { of Chapter } \\
4.1, \\
\text { second } \\
\text { sentence }\end{array}$ & & $\begin{array}{l}\text { Added "For these exploratory regressions, a preliminary } \\
\text { set of peak-flow quantiles developed using regional } \\
\text { skew were used." }\end{array}$ \\
\hline 40 & $\begin{array}{l}\text { "where" } \\
\text { statements } \\
\text { following } \\
\text { equation } \\
11 \text { (was } \\
10 \text { ) }\end{array}$ & & $\begin{array}{l}\text { added "with weighted skew" to peak discharge quantile } \\
\text { definition }\end{array}$ \\
\hline 41 & Table 11 & & $\begin{array}{l}\text { All coefficients updated due to the use of 'weighted } \\
\text { skew' for at-site peak discharge quantile estimates. }\end{array}$ \\
\hline 42 & Table 12 & & $\begin{array}{l}\text { Pseudo } \mathrm{R}^{2} \text {, Average variance of prediction, Average } \\
\text { standard error of prediction, Model error variance } \mathrm{y}^{2} \text {, } \\
\text { Standard model error, and Standard model error WLS fit } \\
\text { values updated due to the use of 'weighted skew' for at- } \\
\text { site peak discharge quantile estimates. }\end{array}$ \\
\hline 46 & Figure 14 & & $\begin{array}{l}\text { Figure 14, graphs } A \& B \text {, updated based on the new } \\
\text { quantile estimates from using the weighted skew } \\
\text { estimate for at-site peak discharge quantile estimates. }\end{array}$ \\
\hline 49 & $\begin{array}{l}\text { Second } \\
\text { "where" } \\
\text { statement } \\
\text { following } \\
\text { equation } \\
17 \text { (was } \\
\text { equation } \\
16 \text { ) }\end{array}$ & “...by using EMA with regional skew as..." & “...by using EMA with weighted skew as...” \\
\hline 53 & $\begin{array}{l}\text { Equation } \\
24 \text { (was } \\
\text { equation } \\
23 \text { ) }\end{array}$ & & $\begin{array}{l}\text { Updated Q0.01 equation to reflect new exponent } \\
\text { values. Updated all mentions of output. }\end{array}$ \\
\hline 53 & $\begin{array}{l}\text { Example } 1 \\
\text { (section } \\
\text { 5.3.1) } \\
\end{array}$ & & $\begin{array}{l}\text { Updated values for } \gamma^{2} \text {, matrix }\left(X^{t} \Lambda^{-1} X\right)^{-1} \text {, and } \\
\text { subsequent calculations }\end{array}$ \\
\hline 55 & $\begin{array}{l}\text { Equation } \\
25-27 \\
\text { (was } 24- \\
26 \text { ) }\end{array}$ & & Updated equation values and outputs. \\
\hline 55 & $\begin{array}{l}\text { First } \\
\text { paragraph } \\
\text { of section } \\
5.3 .2\end{array}$ & “...streamgage 05527950 is $2,160 \mathrm{ft} 3 / \mathrm{s} . . . "$ & “...streamgage 05527950 is $2,050 \mathrm{ft} 3 / \mathrm{s} . . . "$ \\
\hline 55 & $\begin{array}{l}\text { Section } \\
5.3 .2\end{array}$ & & $\begin{array}{l}\text { Corrected all instances of }\left(\mathrm{Q}_{0.01}\right)_{\mathrm{u}, \mathrm{w}} \text { from } 1,830 \text { to } 1,740 \\
\mathrm{ft}^{3} / \mathrm{s}\end{array}$ \\
\hline 55 & $\begin{array}{l}\text { Example } 2 \\
\text { (section } \\
\text { 5.3.2) }\end{array}$ & & Updated equation and output with new values \\
\hline 56 & $\begin{array}{l}\text { Example } 3 \\
\text { (section } \\
\text { 5.3.3) }\end{array}$ & $\begin{array}{l}\text { "...the appropriate AEP } 0.01 \text { at-gage flood- } \\
\text { discharge quantile }\left(\mathrm{Q}_{0.01}\right)_{\mathrm{g}, \mathrm{w}} \text { is } 2,160 \mathrm{ft}^{3} / \mathrm{s}^{\prime}\end{array}$ & $\begin{array}{l}\text { "...the appropriate AEP } 0.01 \text { at-gage flood-discharge } \\
\text { quantile }\left(\mathrm{Q}_{0.01}\right)_{\mathrm{g}, \mathrm{w}} \text { is } 2,050 \mathrm{ft}^{3} / \mathrm{s}^{\prime}\end{array}$ \\
\hline
\end{tabular}




\begin{tabular}{|c|c|c|c|}
\hline 56 & $\begin{array}{l}\text { Example } 4 \\
\text { (section } \\
\text { 5.3.4) }\end{array}$ & & Updated $V_{u}$ and $S_{p u}$ calculation and results \\
\hline 57 & $\begin{array}{l}\text { Paragraph } \\
\text { following } \\
\text { equation } \\
31 \text { (was } \\
\text { 30) }\end{array}$ & & $\begin{array}{l}\text { Updated confidence intervals to reflect new equation } \\
\text { output }\end{array}$ \\
\hline 58 & $\begin{array}{l}\text { Example } 5 \\
\text { (section } \\
\text { 5.3.5) }\end{array}$ & & Updated $Q_{0.01}$ calculation and result \\
\hline 59 & $\begin{array}{l}\text { Third } \\
\text { paragraph }\end{array}$ & $\begin{array}{l}\text { "...following removal of potentially influential low } \\
\text { floods defined by..." }\end{array}$ & $\begin{array}{l}\text { "...following the censoring of potentially influential low } \\
\text { floods identified by..." }\end{array}$ \\
\hline 59 & $\begin{array}{l}\text { Third } \\
\text { paragraph }\end{array}$ & $\begin{array}{l}\text { "For the peak discharge quantile estimation, } \\
\text { regional skew coefficients were used in place of } \\
\text { the at-site skew." }\end{array}$ & $\begin{array}{l}\text { "To improve the skew estimates used for the peak } \\
\text { discharge quantile estimation, weighted skew } \\
\text { coefficients were computed as the variance-weighted } \\
\text { average of at-site and regional skew coefficients." } \\
\text { Reverted change made in update } 1 .\end{array}$ \\
\hline $87-132$ & Table 2 & $\begin{array}{l}\text { Updated the "Frequency analysis method", row } 3 \\
\text { for each site: } \\
\text { "At-site, adjusted for urbanization, EMA, regional } \\
\text { skew: (Qp)g,s" }\end{array}$ & $\begin{array}{l}\text { "At-site, adjusted for urbanization, EMA, weighted skew: } \\
\text { (Qp)g,s" }\end{array}$ \\
\hline $87-132$ & Table 2 & & $\begin{array}{l}\text { Of the five rows of discharge estimates for each site, the } \\
\text { first two rows (At-site, unadjusted and At-site, adjusted) } \\
\text { were updated for any sites which included crest-stage } \\
\text { gage years of operation. } \\
\text { The third rows were updated based on the use of } \\
\text { weighted skew, instead of regional skew. } \\
\text { The fourth rows were updated for all sites based on the } \\
\text { use of the updated WREG software. } \\
\text { The fifth rows are the weighted average of the third and } \\
\text { fourth rows and were also updated. }\end{array}$ \\
\hline $313-314$ & Table 13 & & $\begin{array}{l}\text { Updated }(X t \Lambda-1 X)-1 \text { matrices for all exceedance } \\
\text { probability quantiles due to the use of 'weighted skew' } \\
\text { for at-site peak discharge estimates. }\end{array}$ \\
\hline
\end{tabular}




\section{Errata: FHWA-ICT-16-013}

\section{September 30, 2017}

Subsequent to publication of FHWA-ICT-16-013 an error was found in the reported methodology. It was discovered that the so-called "weighted" skew coefficient values used in the computation of the at-site flood frequencies were not actually computed using the weighted skew, that is, as a weighted average of the station and regional skew values. Instead they were computed using the regional skew only.

The preferred skew coefficients to use for computing at-site flood frequencies are the weighted skews; however it was determined that the present results have only modest differences from the weighted skews. As a result, it was decided to correct the report to reflect the methodology actually implemented. An update of the analysis and report are planned which will use the weighted skew values and include additional years of record.

The following revisions to FHWA-ICT-16-103 reflect what was actually done in the analysis and correct a few typographical errors.

\begin{tabular}{|c|c|c|c|}
\hline $\begin{array}{l}\text { Page number in } \\
2016 \text { version }\end{array}$ & Location & Original text/image & Revised text/image \\
\hline \multicolumn{4}{|c|}{ Corrections of typographical errors } \\
\hline $\begin{array}{l}\text { Title page and } \\
\text { report } \\
\text { documentation } \\
\text { page }\end{array}$ & & & Middle initials added to authors' names. \\
\hline$x i$ & List of abbreviations & & Added definition of water year \\
\hline 1 & $\begin{array}{l}\text { At end of } 2^{\text {nd }} \\
\text { paragraph }\end{array}$ & $\begin{array}{l}\text { '...whereas for simulation models, } \\
\text { regional calibration is a "difficult, } \\
\text { unsolved problem" (Vogel 2006).' }\end{array}$ & $\begin{array}{l}\text { '...whereas significant challenges remain } \\
\text { for regional calibration of simulation } \\
\text { models (Vogel 2006).' }\end{array}$ \\
\hline 2 & $\begin{array}{l}\text { Last paragraph } \\
\text { beginning on page }\end{array}$ & $\begin{array}{l}\text { "...vegetation type, primarily alfisols, } \\
\text { which form under forest, nearer to Lake } \\
\text { Michigan and mollisols, which form } \\
\text { under grasslands, over..." }\end{array}$ & $\begin{array}{l}\text { "...vegetation type. Alfisols, which form } \\
\text { under forests, dominate nearer to Lake } \\
\text { Michigan, whereas mollisols, which form } \\
\text { under grasslands, cover..." }\end{array}$ \\
\hline 3 & $\begin{array}{l}\text { Next-to-last paragraph } \\
\text { on page }\end{array}$ & “(Figure 5)” & $\begin{array}{l}\text { "(see Streamgage Selection section } \\
\text { below)" }\end{array}$ \\
\hline 8 & $\begin{array}{l}\text { First paragraph of } \\
\text { section } 1.3\end{array}$ & & $\begin{array}{l}\text { Added a reference for the quote at the } \\
\text { end of the paragraph. }\end{array}$ \\
\hline 11 & Figure $4 \mathrm{x}$-axis & "Fraction of basins" & $\begin{array}{l}\text { "Urbanized fraction (Theobald, 2005, } \\
\text { classes 7-10) of basin at end of record } \\
\text { used in Soong and others (2004)" }\end{array}$ \\
\hline 11 & Figure 4 y-axis & $\begin{array}{l}\text { "Empirical distribution function of } \\
\text { fraction of Theobald (2005) classes 7-10 } \\
\text { at end of the streamgage record" }\end{array}$ & $\begin{array}{l}\text { "Fraction of basins having urbanized } \\
\text { fraction less than value on x axis" }\end{array}$ \\
\hline 11 & $\begin{array}{l}\text { First line of second } \\
\text { paragraph. }\end{array}$ & $\begin{array}{l}\text { "The list streamgages with at least } 10 \\
\text { years of record...." }\end{array}$ & $\begin{array}{l}\text { "The list of streamgages with at least } 10 \\
\text { years of record"... }\end{array}$ \\
\hline 14 & $\begin{array}{l}\text { Figure } 6 \text {, last line of } \\
\text { caption }\end{array}$ & "...greater than..." & "...fewer than..." \\
\hline 17 & $\begin{array}{l}\text { First paragraph of } \\
\text { section } 2.1 .4\end{array}$ & $\begin{array}{l}\text { "...described in the Temporal Analysis } \\
\text { and Adjustment section..." }\end{array}$ & $\begin{array}{l}\text { "...described in the Regional Temporal } \\
\text { Regression Analysis and Adjustment } \\
\text { section..." }\end{array}$ \\
\hline 20 & Chapter 3 title & $\begin{array}{l}\text { "REGIONAL TEMPORAL ANALYSIS AND } \\
\text { ADJUSTMENT" }\end{array}$ & $\begin{array}{l}\text { "REGIONAL TEMPORAL REGRESSION } \\
\text { ANALYSIS AND ADJUSTMENT" }\end{array}$ \\
\hline
\end{tabular}




\begin{tabular}{|c|c|c|c|}
\hline 20 & End of first paragraph & $\begin{array}{l}\text { "(see Table } 2 \text { for quantiles and Table } 6 \\
\text { for discharges)." }\end{array}$ & $\begin{array}{l}\text { "(see Table } 2 \text { for quantiles and the } \\
\text { Adjustment of Annual Maximum Peak } \\
\text { Discharges to Current (2010) Urban } \\
\text { Fractions section for discharges)." }\end{array}$ \\
\hline 22 & $\begin{array}{l}\text { First paragraph on } \\
\text { page }\end{array}$ & & $\begin{array}{l}\text { Added reference for National Inventory of } \\
\text { Dams }\end{array}$ \\
\hline 29 & Title of section 3.3 & $\begin{array}{l}\text { “ADJUSTMENT OF PEAK-FLOOD VALUES } \\
\text { TO CURRENT (2010) URBAN FRACTIONS" }\end{array}$ & $\begin{array}{l}\text { "ADJUSTMENT OF ANNUAL MAXIMUM } \\
\text { PEAK DISCHARGES TO CURRENT (2010) } \\
\text { URBAN FRACTIONS” }\end{array}$ \\
\hline 33 & $\begin{array}{l}\text { First paragraph of } \\
\text { Chapter } 4\end{array}$ & $\begin{array}{l}\text { "...as described in the Flood Frequency } \\
\text { section," }\end{array}$ & $\begin{array}{l}\text { "...as described in the Frequency Analysis } \\
\text { section," }\end{array}$ \\
\hline 33 & $\begin{array}{l}\text { "where" statements } \\
\text { following equation } 9 \\
\text { (was 8) }\end{array}$ & & Added missing units \\
\hline 42 & Footnote of table 12 & & $\begin{array}{l}\text { Added definition of } \mathrm{R}^{2} \text { ("coefficient of } \\
\text { determination"). }\end{array}$ \\
\hline 52 & First paragraph & $\begin{array}{l}\text { "...methods described in the } \\
\text { Applications of the Updated Illinois } \\
\text { Region } 2 \text { Spatial Regression Equations } \\
\text { section." }\end{array}$ & $\begin{array}{l}\text { "...methods described in the Applications } \\
\text { of the Spatial Regression Equations } \\
\text { section." }\end{array}$ \\
\hline 59 & Last sentence & $\begin{array}{l}\text { "...equations and quantile estimates will } \\
\text { be available..." }\end{array}$ & $\begin{array}{l}\text { "...equations and quantile estimates are } \\
\text { available..." }\end{array}$ \\
\hline 71 & Table 1-3 & & $\begin{array}{l}\text { Corrected several values in "Average } \\
\text { regional skew" column due to round-off } \\
\text { error. }\end{array}$ \\
\hline $87-132$ & $\begin{array}{l}\text { Table } 2 \text {, header of } \\
\text { next-to-last column }\end{array}$ & 0.01 & 0.005 \\
\hline $313-314$ & $\begin{array}{l}\text { Table 13, last header } \\
\text { entry on right and } \\
\text { entries in third column } \\
\text { from left }\end{array}$ & “DEM_P_1_0" & “DEM_1_0_P" \\
\hline \multicolumn{4}{|c|}{ Corrections related to skewness coefficients } \\
\hline NA & $\begin{array}{l}\text { Second paragraph of } \\
\text { abstract }\end{array}$ & “...generalized skew coefficients..." & “...regional skew coefficients...” \\
\hline ii & $\begin{array}{l}\text { Second paragraph of } \\
\text { Executive Summary }\end{array}$ & “...generalized skew coefficients...” & “...regional skew coefficients...” \\
\hline 10 & $\begin{array}{l}\text { First paragraph of } \\
\text { section } 2.1\end{array}$ & $\begin{array}{l}\text { "that is weighted with the at- } \\
\text { streamgage skewness values in the } \\
\text { frequency analysis" }\end{array}$ & "for use in the frequency analysis" \\
\hline 17 & $\begin{array}{l}\text { Last sentence of } \\
\text { section } 2.1 .3\end{array}$ & $\begin{array}{l}\text { "The skewness estimate from this } \\
\text { relation was combined with the at-site } \\
\text { skew according to their associated } \\
\text { uncertainties to obtain a weighted skew } \\
\text { estimate for use in obtaining the at-site } \\
\text { peak discharge quantile estimation..." }\end{array}$ & $\begin{array}{l}\text { "The regional skewness estimate from } \\
\text { equation } 3, \text { without weighting with the at- } \\
\text { site skewness, was used to obtain at-site } \\
\text { peak discharge quantile estimates..." }\end{array}$ \\
\hline $17-18$ & $\begin{array}{l}\text { Paragraph around } \\
\text { equation } 4\end{array}$ & Entire paragraph. & $\begin{array}{l}\text { "For use in the frequency analysis, the } \\
\text { skewness values for each streamgage } \\
\text { were taken as equal to the regional } \\
\text { skewness values developed as described } \\
\text { in the "regional Skew Analysis" section } \\
\text { and appendix } 1 .\end{array}$ \\
\hline 33 & $\begin{array}{l}\text { First paragraph of } \\
\text { section } 4\end{array}$ & $\begin{array}{l}\text { "...including weighting with the regional } \\
\text { skew model..." }\end{array}$ & $\begin{array}{l}\text { "...including application of the regional } \\
\text { skew model..." }\end{array}$ \\
\hline 33 & $\begin{array}{l}\text { First "where" } \\
\text { statement following } \\
\text { equation } 9 \text { (now } \\
\text { equation } 8 \text { ) }\end{array}$ & “...with weighted skew in cubic...” & “...with regional skew in cubic...” \\
\hline
\end{tabular}




\begin{tabular}{|c|c|c|c|}
\hline 49 & $\begin{array}{l}\text { Second "where" } \\
\text { statement following } \\
\text { equation } 17 \text { (now } \\
\text { equation 16) }\end{array}$ & $\begin{array}{l}\text { “...by using EMA with weighted skew } \\
\text { as..." }\end{array}$ & “...by using EMA with regional skew as...” \\
\hline 49 & $\begin{array}{l}\text { First paragraph after } \\
\text { "where" statements } \\
\text { following equation } 17 \\
\text { (now equation } 16 \text { ) }\end{array}$ & $\begin{array}{l}\text { "...in the rows labeled "EMA (weighted } \\
\text { skew"), "Regional regression," and } \\
\text { "Weighted EMA (weighted skew) and } \\
\text { regional regression," respectively." }\end{array}$ & $\begin{array}{l}\text { "...in the third, fourth, and fifth rows, } \\
\text { respectively, for each streamgage." }\end{array}$ \\
\hline 59 & $\begin{array}{l}\text { Third paragraph of } \\
\text { Summary }\end{array}$ & $\begin{array}{l}\text { "To improve the skew estimates used } \\
\text { for the peak discharge quantile } \\
\text { estimation, weighted skew coefficients } \\
\text { were computed as the variance- } \\
\text { weighted average of at-site and } \\
\text { generalized skew coefficients. The } \\
\text { generalized skew coefficients..." }\end{array}$ & $\begin{array}{l}\text { "For the peak discharge quantile } \\
\text { estimation, generalized skew coefficients } \\
\text { were used in place of the at-site skew. The } \\
\text { regional skew coefficients..." }\end{array}$ \\
\hline various & $\begin{array}{l}\text { equation numbers } 5 \\
\text { and larger }\end{array}$ & NA & $\begin{array}{l}\text { reduced by } 1 \text { because of deletion of } \\
\text { equation } 4\end{array}$ \\
\hline various & $\begin{array}{l}\text { references to } \\
\text { numbered equations } 5 \\
\text { and larger }\end{array}$ & NA & $\begin{array}{l}\text { reduced by } 1 \text { because of deletion of } \\
\text { equation } 4\end{array}$ \\
\hline table 2 & $\begin{array}{l}\text { row } 3 \text { of } 5 \text { for each } \\
\text { streamgage, } \\
\text { "Frequency analysis } \\
\text { method" column }\end{array}$ & $\begin{array}{l}\text { "At-site, adjusted for urbanization, EMA, } \\
\text { weighted skew: }\left(Q_{p}\right)_{g, s} \text { " }\end{array}$ & $\begin{array}{l}\text { "At-site, adjusted for urbanization, EMA, } \\
\text { regional skew: }\left(Q_{p}\right)_{g, s} \text { " }\end{array}$ \\
\hline
\end{tabular}





\section{ACKNOWLEDGMENT, DISCLAIMER, MANUFACTURERS' NAMES}

This publication is based on the results of ICT-R27-144, Development and Implementation of Updated Urban Regional Flood Frequency Equations for Illinois. ICT-R27-144 was conducted in cooperation with the Illinois Center for Transportation; the Illinois Department of Transportation; and the U.S. Department of Transportation, Federal Highway Administration.

Members of the Technical Review Panel were the following:

- Matt O' Connor, TRP Chair (Illinois Department of Transportation)

- Steve Ferguson (Illinois Department of Transportation)

- Dan Ghere (Federal Highway Administration)

- Rick Gosch (Illinois Department of Natural Resources)

- Perry Masouridis (Illinois Department of Transportation)

- Tzuoh-Ying Su (U.S. Army Corps of Engineers)

- Neil VanBebber (Illinois Department of Transportation)

- Rick Wojcik (Illinois Department of Transportation)

Marvin Harris and John Latour of the U.S. Geological Survey (USGS) Illinois Water Science Center, John Walker and Robert Waschbusch of the USGS Wisconsin Water Science Center, and Donald Arvin of the USGS Indiana Water Science Center provided key input on the quality of streamgage records used in this study. Allan Curtis (Midwestern Regional Climate Center) assisted with downloading and understanding the National Weather Service cooperative daily precipitation data used in this study.

The contents of this report reflect the view of the authors, who are responsible for the facts and the accuracy of the data presented herein. The contents do not necessarily reflect the official views or policies of the Illinois Center for Transportation, the Illinois Department of Transportation, or the Federal Highway Administration. This report does not constitute a standard, specification, or regulation.

Trademark or manufacturers' names appear in this report only because they are considered essential to the object of this document and do not constitute an endorsement of product by the Federal Highway Administration, the Illinois Department of Transportation, the Illinois Center for Transportation, or the U.S. Geological Survey, or other agencies of the U.S. Government. 


\section{EXECUTIVE SUMMARY}

This report presents the results of a study to improve the estimation of peak discharges for urbanized watersheds in northeastern Illinois (flood-frequency region 2). In order to maintain consistent relations for the entire region and to enable the transfer of the hydrologic effects of urbanization throughout the state for similarly developed watersheds, peak discharge estimations for rural watersheds in flood-frequency region 2 were also included in the study. The study included floodfrequency analysis at streamgages and regional regression analysis that resulted in techniques for estimating peak discharge quantiles at annual exceedance probabilities (AEPs) of 0.50, 0.20, 0.10, $0.04,0.02,0.01,0.005$, and 0.002 (recurrence intervals of 2, 5, 10, 25, 50, 100, 200, and 500 years, respectively) at ungaged sites throughout flood-frequency region 2. The equations were developed by a spatial analysis, applying generalized least-squares regression to flood-frequency and drainagebasin characteristics from 117 streamgages in and near Illinois flood-frequency region 2 . The drainage-basin characteristics used as explanatory variables in the spatial analysis included drainage area, the fraction of developed land, the fraction of land with poorly drained soils or likely water, and the basin slope estimated as the ratio of the basin relief to basin perimeter. The report also provides peak discharge quantile estimates at 181 streamgages: the 117 streamgages that were used to develop the spatial regression equations and 64 additional streamgages in the region that were originally considered for use in the study but later deemed to be redundant through an analysis that included watershed overlap areas and streamgage record lengths.

Peak discharge quantiles at the selected AEPs for streamgages were computed by using the Expected Moments Algorithm following the removal of potentially influential low floods defined by a multiple Grubbs-Beck test. To improve estimates of the peak discharge quantiles for given exceedance probabilities at the streamgages in the study region, regional skew coefficients were obtained from a newly developed regional skew model. The new regional skew model is an increasing function of the urbanized land use fraction.

The annual maximum discharge records of the streamgages used in the study were adjusted to 2010 urbanization conditions prior to computing the flood-frequency statistics. The adjustment is based on the temporal effect of urbanization estimated by a two-step least squares-quantile regression technique. The urbanization coefficients from the two-step temporal analysis also provide the basis for equations that can be used for adjusting rural peak discharge estimates for the effect of urbanization in other parts of Illinois. The adjusted annual maximum peak discharge records at the 181 streamgages considered for use in the study are also provided.

The accuracy of the spatial and temporal equations is described by several methods, including the model variance, standard error of prediction, and confidence intervals. The method for determining the $90 \%$ confidence interval for any estimated peak-flood discharge in flood-frequency region 2 is provided in the report, and will also be available in the USGS applications StreamStats and the National Streamflow Statistics program.

Examples are presented to illustrate the use of the spatial and urbanization-adjustment equations to estimate flood quantiles at ungaged sites and to improve peak discharge quantile estimates at and 
near a gaged site. The spatial regression equations, urbanization-adjustment equations, and peak discharge quantile data used in this study will be available in StreamStats. StreamStats is a web-based application providing automated regression-equation solutions for user-selected sites on streams. Figures and tables comparing the observed and urbanization-adjusted peak discharge records by streamgage are provided at http://dx.doi.org/10.3133/sir20165050 for download. 


\section{TABLE OF CONTENTS}

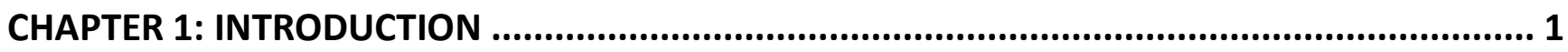

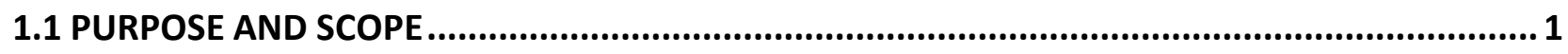

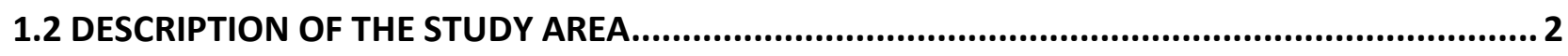

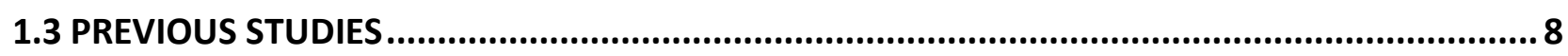

CHAPTER 2: DATA DEVELOPMENT ............................................................................ 10

2.1 PEAK DISCHARGE QUANTILES AT STREAMGAGES ...................................................... 10

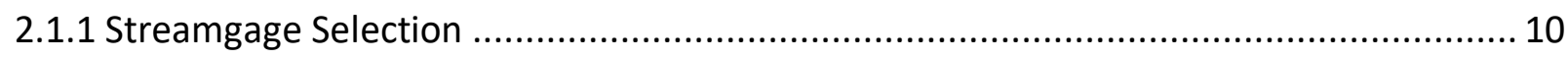

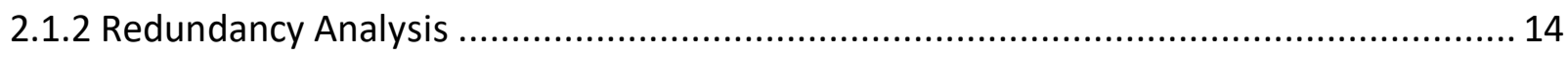

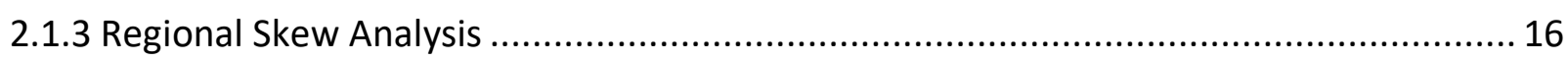

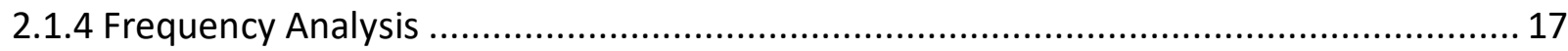

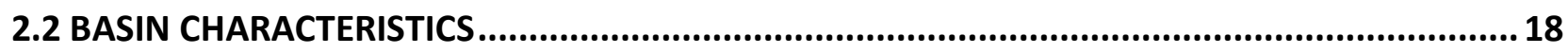

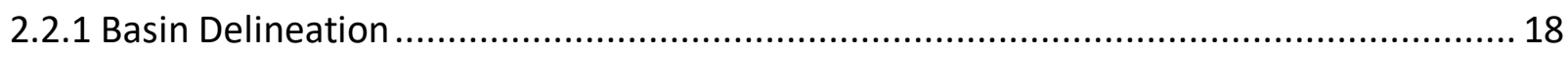

2.2.2 Spatially Averaged Basin Characteristics .................................................... 18

CHAPTER 3: REGIONAL TEMPORAL REGRESSION ANALYSIS AND ADJUSTMENT ............... 20

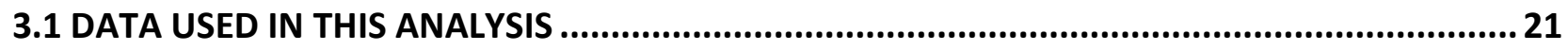

3.2 TEMPORAL ANALYSIS OF URBANIZATION EFFECT ....................................................... 23

3.3 ADJUSTMENT OF ANNUAL MAXIMUM PEAK DISCHARGES TO CURRENT (2010) URBAN

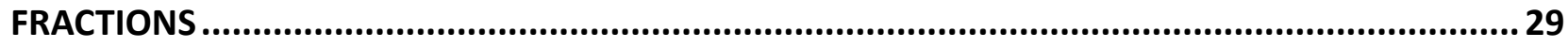

CHAPTER 4: REGIONAL SPATIAL REGRESSION ANALYSES ........................................... 33

4.1 SELECTION OF BASIN CHARACTERISTICS ........................................................................ 33

4.2 DEVELOPMENT OF FINAL SPATIAL REGRESSION EQUATIONS ........................................ 39

4.3 ACCURACY OF FINAL SPATIAL REGRESSION EQUATIONS ................................................. 41

CHAPTER 5: APPLICATIONS OF REGRESSION EQUATIONS................................................ 44

5.1 APPLICATIONS OF THE SPATIAL REGRESSION EQUATIONS ............................................ 48

5.1.1 Ungaged Locations Far from a Streamgage .................................................. 48

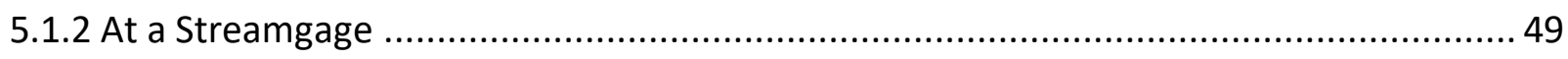

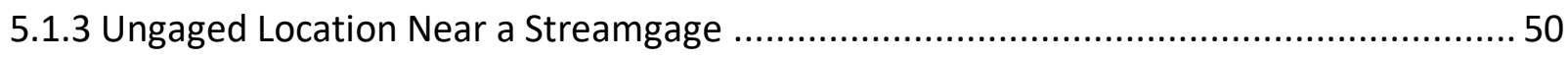

5.2 APPLICATIONS OF THE TEMPORAL URBANIZATION COEFFICIENTS ...................................51 
5.3.1 Example 1: Ungaged Location in the Study Region, Far from a Streamgage.................. 53

5.3.2 Example 2: Ungaged Location in the Study Region, Near a Streamgage ..................... 55

5.3.3 Example 3: At a Streamgage in the Study Region .............................................. 55

5.3.4 Example 4: At a Location Outside the Study Region ............................................ 56

5.3.5 Example 5: Adjustment for Effects of Future Urbanization...................................... 57

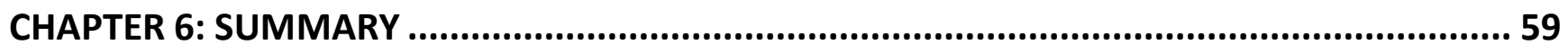

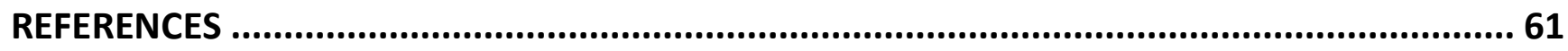

APPENDIX 1: NORTHEASTERN ILLINOIS REGIONAL SKEW ANALYSIS ............................. 67

APPLICATION OF B-WLS/B-GLS METHOD IN NORTHEASTERN ILLINOIS .................................67

RESULTS OF REGIONAL SKEW ANALYSIS IN NORTHEASTERN ILLINOIS................................... 70

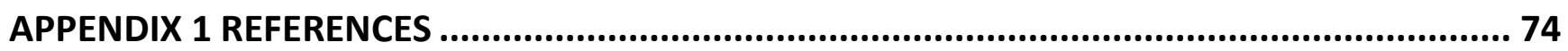




\section{LIST OF FIGURES}

Figure 1a. Map of entire study area and streamgages used in this study.....

Figure 1b. Map of study area and streamgages used in this study showing southern Lake, Cook, and DuPage counties in detail....

Figure 2. Plot of population growth in northeastern Illinois, 1850-2010 (from Karstensen et al. 2013).

Figure 3. Map of housing density by decade in northeastern Illinois (modified from Karstensen et al. 2013).

Figure 4. Empirical distribution functions of urbanized fraction of gaged basins in Illinois floodfrequency regions of Soong et al. (2004).

Figure 5. Properties of U.S. Geological Survey streamgage records used in this study in northeastern Illinois. (a) number of streamgages with annual maximum peak discharges each water year; (b) number of streamgages with annual maximum peak discharge records beginning each water year; (c) number of streamgages with peak discharge records ending each water year; (d) number of annual maximum peak discharges in each month of the year.

Figure 6. Properties of basins of U.S. Geological Survey streamgages used in this study in northeastern Illinois. (a) basin drainage areas, (b) urbanized basin fractions at first year of streamgage records, (c) urbanized basin fractions at last year of streamgage record, and (d) urbanized basin fractions in 2010, where urbanized is defined as Theobald (2005) housing density fewer than 10 acres per house plus commercial/industrial/transportation land use.

Figure 7. Locations of precipitation stations used in this study and their Thiessen polygons.

Figure 8. Segment intercepts as a function of drainage area for 181 streamgages used in this study in northeastern Illinois.

Figure 9. Urban fraction coefficients from temporal regression analysis of 117 streamgages in northeastern Illinois and adjacent states, as a function of exceedance probability.

Figure 10. Segment intercept-subtracted log-transformed annual maximum peak discharge from 181 streamgages in northeastern Illinois and adjacent states as a function of urban fraction, with quantile regression and least-squares regression line fits.

Figure 11. Changes in the mean, standard deviation, and skewness of the log-transformed annual maximum peak discharge records for 181 streamgages used in this study in northeastern Illinois, resulting from adjustment to 2010 urban fraction values.

Figure 12. Plots by annual exceedance probability of adjusted $R^{2}$ as a function of the regression coefficient $t$ ratio values for basin characteristic categories considered in this study in northeastern Illinois: (a) urbanization variables; (b) and (c) water and wetland variables; and (d) slope variables. When the absolute value of the $t$ ratio is greater than 2 , then the coefficient is significantly different 
from zero at about the $a=0.05$ significance level (Helsel and Hirsch 2002, p. 238). The variable names in the figure explanations are defined in Table 3; the strings preceding the variable names in the figure explanations indicate the transformation applied to the variable, where "lin" means "linear" (that is, no transformation), "sqrt" means a square-root transformation, and "log10" means a $\log _{10}$ transformation.

Figure 13. Comparison of square root of fraction of 2011 National Land Cover Dataset (NLCD, Jin et al. 2013) classes 22, 23, and 24 (NLCD_22_23_24 ${ }^{1 / 2}$ ) and 2010 Theobald urban fraction (Theobald 2005) for 117 basins used in this study in northeastern Illinois.

Figure 14. Ratios of peak discharge quantiles $Q_{p}(U) / Q_{p}\left(U_{0}\right)$ as a function of urban fraction $U$ for selected annual exceedance probabilities (AEPS) $p$ as implied by the two sets of urbanization coefficients obtained in this study in northeastern Illinois: (a) basin initially having no urbanization ( $U_{0}$ $=0)$; (b) basin initially $50 \%$ urbanized $\left(U_{0}=0.5\right)$.

Figure 1-1. Fitting of cross-correlation model of the log-annual maximum peak discharges in this study in northeastern Illinois: (a) relation between Fisher $Z$ transform of cross-correlation and distance; (b) relation between cross-correlation and distance.

Figure 1-2. Relations between the unbiased at-site skew and urbanization measure for 110 streamgages in northeastern Illinois regional skew study area. The lines represent a model based on a constant skew (CONSTANT) and a model with a linear relation between skew and the square root of fraction of National Land Cover Dataset classes 22, 23, and 24 (NLCD_22_23_24 ${ }^{1 / 2}$ ) urbanization measure (URBAN). The models were developed from B-WLS/B-GLS analyses. 


\section{LIST OF TABLES}

Table 1. Information regarding U.S. Geological Survey streamgages used in this study in northeastern Illinois and adjacent states.

Table 2. Estimated peak discharge quantiles for 181 streamgages in northeastern Illinois and adjacent states, at selected annual exceedance probabilities.

Table 3. Spatially averaged basin characteristics considered for developing spatial regression equations in this study in northeastern Illinois.

Table 4. Segment information for 181 U.S. Geological Survey streamgages used in this study, northeastern Illinois and adjacent states.

Table 5. Number of segments per streamgage record used in regression analysis of peak discharge for 117 streamgages, northeastern Illinois and adjacent states.

Table 6. Observed and urban-adjusted annual maximum peak discharges and associated urbanization and precipitation values at 181 streamgages in northeastern Illinois and adjacent states.

Table 7. Results of ordinary least-squares linear regression of 117 streamgages in northeastern Illinois and adjacent states to diagnose the temporal effects of urbanization and precipitation on annual maximum peak discharges in this study.

Table 8. Quantile regression coefficients from temporal analysis of 117 streamgages in northeastern Illinois and adjacent states, as a function of annual exceedance probability.

Table 9. Quantile regression coefficients of urban fraction from temporal analysis of 117 streamgages in northeastern Illinois and adjacent states, at selected annual exceedance probabilities.

Table 10. Correlation matrix and variance inflation factor (VIF) values of basin characteristics selected for use in spatial regression analysis of 117 streamgages in northeastern Illinois and adjacent states.

Table 11. Coefficients of the selected spatial regression equations in this study in northeastern Illinois.

Table 12. Measures of the average accuracy of the selected spatial regression equations in this study in northeastern Illinois.

Table 13. Components of variance of prediction for the selected spatial regression equations in northeastern Illinois.

Table 14. Ranges of basin characteristic values of streamgages used to fit selected spatial regression equations in this study in northeastern Illinois.

Table 15. Example peak discharge quantile computations in this study in northeastern Illinois.

Table 1-1. Skew statistics at U.S. Geological Survey streamgages used in the development of the regional skew model in this study in northeastern Illinois. 
Table 1-2. Regional skewness models and corresponding metrics in this study in northeastern Illinois.

Table 1-3. Average regional skew, variance of prediction, and equivalent record length for URBAN regional skew model for various values of NLCD_22_23_24, in this study in northeastern Illinois..... 71 Table 1-4. Pseudo analysis of variance (ANOVA) statistics for the northeastern Illinois URBAN regional

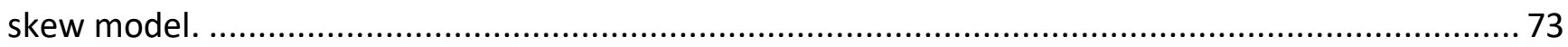




\section{CONVERSION FACTORS}

Inch/Pound to International System of Units

\begin{tabular}{|c|c|c|}
\hline Multiply & By & To obtain \\
\hline \multicolumn{3}{|l|}{ Length } \\
\hline inch (in.) & 2.54 & centimeter $(\mathrm{cm})$ \\
\hline inch (in.) & 25.4 & millimeter (mm) \\
\hline foot $(\mathrm{ft})$ & 0.3048 & meter $(\mathrm{m})$ \\
\hline mile (mi) & 1.609 & kilometer (km) \\
\hline \multicolumn{3}{|l|}{ Area } \\
\hline acre & 4,047 & square meter $\left(\mathrm{m}^{2}\right)$ \\
\hline acre & 0.4047 & hectare (ha) \\
\hline acre & 0.4047 & square hectometer $\left(\mathrm{hm}^{2}\right)$ \\
\hline acre & 0.004047 & square kilometer $\left(\mathrm{km}^{2}\right)$ \\
\hline square mile $\left(\mathrm{mi}^{2}\right)$ & 259.0 & hectare (ha) \\
\hline square mile $\left(\mathrm{mi}^{2}\right)$ & 2.590 & square kilometer $\left(\mathrm{km}^{2}\right)$ \\
\hline \multicolumn{3}{|l|}{ Volume } \\
\hline cubic foot $\left(\mathrm{ft}^{3}\right)$ & 28.32 & cubic decimeter $\left(\mathrm{dm}^{3}\right)$ \\
\hline cubic foot $\left(\mathrm{ft}^{3}\right)$ & 0.02832 & cubic meter $\left(\mathrm{m}^{3}\right)$ \\
\hline acre-foot (acre-ft) & 1,233 & cubic meter $\left(\mathrm{m}^{3}\right)$ \\
\hline acre-foot (acre-ft) & 0.001233 & cubic hectometer $\left(\mathrm{hm}^{3}\right)$ \\
\hline \multicolumn{3}{|l|}{ Flow rate } \\
\hline cubic foot per second $\left(\mathrm{ft}^{3} / \mathrm{s}\right)$ & 0.02832 & cubic meter per second $\left(\mathrm{m}^{3} / \mathrm{s}\right)$ \\
\hline
\end{tabular}

\section{DATUM}

Vertical coordinate information is referenced to the North American Vertical Datum of 1988 (NAVD 88).

Horizontal coordinate information is referenced to the North American Datum of 1927 (NAD 27) except as otherwise noted.

Elevation, as used in this report, refers to distance above the vertical datum. 


\section{ABBREVIATIONS}

\begin{tabular}{|c|c|}
\hline AEP & annual exceedance probability \\
\hline AFPD & annual flood-probability discharge \\
\hline AMS & annual maximum series \\
\hline BDF & basin development factor \\
\hline B-GLS & Bayesian generalized least squares \\
\hline B-WLS & Bayesian weighted least squares \\
\hline CCPN & Cook County Precipitation Network \\
\hline COOP & cooperative network \\
\hline CSG & crest-stage gage \\
\hline DEM & digital elevation model \\
\hline EMA & Expected Moments Algorithm \\
\hline Esri & Environmental Systems Research Institute, Inc. \\
\hline GIS & geographic information system \\
\hline GLS & generalized least squares \\
\hline HDB & hand-drawn boundary \\
\hline ICT & Illinois Center for Transportation \\
\hline IDOT & Illinois Department of Transportation \\
\hline MACS & MRCC Applied Climate System \\
\hline MRCC & Midwestern Regional Climate Center \\
\hline MSE & mean squared error \\
\hline MWRDGC & Metropolitan Water Reclamation District of Greater Chicago \\
\hline NED & National Elevation Dataset \\
\hline $\mathrm{NHD}$ & National Hydrography Dataset \\
\hline NLCD & National Land Cover Dataset \\
\hline NWI & National Wetlands Inventory \\
\hline NWIS & National Water Information System \\
\hline NWS & National Weather Service \\
\hline OLS & ordinary least squares \\
\hline OVB & omitted variable bias \\
\hline PDS & partial duration series \\
\hline
\end{tabular}




$\begin{array}{ll}\text { PeakFQ } & \text { Peak flow FreQuency program } \\ \text { PILF } & \text { potentially influential low flood } \\ \text { PRISM } & \text { Parameter-elevation Relationships on Independent Slopes Model } \\ \text { SE } & \text { standard error } \\ \text { SSURGO } & \text { State Soil Survey Geographic database } \\ \text { USGS } & \text { U.S. Geological Survey } \\ \text { VIF } & \text { variance inflation factor } \\ \text { WLS } & \text { weighted least squares } \\ \text { WREG } & \text { Weighted-Multiple-Linear Regression program } \\ \text { WY } & \begin{array}{l}\text { water year; a water year is the 12-month period October } 1 \text { through September } 30 \\ \text { designated by the calendar year in which it ends. }\end{array}\end{array}$




\section{CHAPTER 1: INTRODUCTION}

Estimates of the magnitude and frequency of floods, particularly in urban areas where there is an increased density of lives and property at risk, are a critical ingredient to floodplain management, emergency response planning, and infrastructure design tasks such as sizing of bridges and culverts. At the same time, urbanization, particularly the construction of impervious surfaces, changes the response of watersheds to precipitation by reducing infiltration and increasing flow velocities, which increases flood volumes and magnitudes of peaks (Konrad 2003).

Predictions of the effects of urbanization in ungaged basins or in basins where future urbanization is expected or planned are usually obtained by following one of two general approaches: (1) simulation with rainfall-runoff models driven by historical precipitation (either as design storms or by continuous simulation of long-term records), usually calibrated to observed streamflow data; and (2) regressions of peak discharge quantiles obtained from observed streamflow data at a collection of streamgages in and near the region of interest on relevant basin characteristics such as drainage area and fraction of urbanized area. The two approaches have different advantages and disadvantages (Rosbjerg et al. 2013); generally the simulation model approach is preferred for predicting the response of a basin to changes in the basin properties or climate characteristics, whereas for ungaged basins over a historical period, the regional regression approach may be more accurate (Hodgkins et al. 2007). Indeed, the regional regression-equation approach is a well-developed form of regional calibration of a watershed model, whereas significant challenges remain for regional calibration of simulation models (Vogel 2006).

Regional regression equations for urbanization-affected areas of northeast Illinois were first published by Allen and Bejcek (1979), who used data beginning around 1960 and ending in 1976. Since 1976 there have been many years of additional streamflow data, extensive growth of the urbanized area of northeast Illinois, and several historic floods (Juhl 2005). As a result, the U.S. Geological Survey (USGS) Illinois Water Science Center in cooperation with the Illinois Department of Transportation (IDOT) and the Federal Highway Administration (FHWA), through the Illinois Center for Transportation (ICT), developed a project to update the spatial regression equations of Allen and Bejcek (1979) for urbanization-affected streams in northeastern Illinois and for adjusting rural peak discharge quantile estimates for the effect of urbanization.

\subsection{PURPOSE AND SCOPE}

This report presents the methods and results of a study that applied a regional spatial regression approach to develop equations for estimating peak discharge quantiles with annual exceedance probabilities (AEPs) of 0.5, 0.2, 0.1, 0.04, 0.02, 0.01, 0.005, and 0.002 (often referred to as the 2-, 5-, 10-, 25-, 50-, 100-, 200-, and 500-year floods, respectively) for Illinois flood-frequency region 2 (previously defined by Soong et al. 2004). Flood-frequency region 2 consists of northeastern and parts of north-central Illinois and includes the greater Chicago region. This study updates the existing spatial regression equations (Soong et al. 2004; Allen and Bejcek 1979) for northeastern Illinois in the following ways: (1) it uses streamflow data through water year (WY) 2009, compared to through 1999 for Soong et al. (2004) and through 1976 for Allen and Bejcek (1979); (2) it tests and implements 
wetland-open water and slope variables replacing those of Soong et al. (2004); and (3) it uses the updated flood-frequency estimation methods and software, particularly in the estimation of at-site peak discharge quantiles by using multiple Grubbs-Beck potentially influential low flood (PILF) detection, the Expected Moments Algorithm (EMA) for handling censored and historical floods, and regional skew estimation by Bayesian generalized least squares.

The study described in this report also implements the temporal regression approach of Over et al. (2016) to the analysis and adjustment of annual maximum peak discharge records for the exceedance probability-dependent effect of urbanization. By this approach the observed peak discharge records are adjusted to current (2010) urbanization conditions before they are used in the spatial regression analysis, and a set of "temporal" urbanization coefficients are obtained that are applicable wherever hydrologic effects of urbanization are similar to those in northeastern Illinois. Because the historical urbanization data used in the temporal regression approach are available beginning 1940, the earliest streamflow data used in this study are from WY 1940.

There are three primary products from the study described in this report: (1) this report, published in both ICT and USGS versions, having the same content, and presenting the methods and results, including the two sets of regression equations for estimating peak discharge quantiles; (2) an implementation of the spatial and temporal regression equations in StreamStats (http://streamstats.usgs.gov); and (3) a collection of figures and tables comparing the observed and urbanization-adjusted peak discharges at http://dx.doi.org/10.1333/sir20165050.

\subsection{DESCRIPTION OF THE STUDY AREA}

The study area covers northeastern Illinois and portions of north-central Illinois, northwest Indiana, and southeast Wisconsin (Figures $1 \mathrm{a}$ and $1 \mathrm{~b}$ ). The physiography of the region is determined by recent glacial episodes. The last glacial episode was the Wisconsinan, which ended about 12,000 years ago and during which all except the western lobe of the study area in the valley of the Green River was covered in ice (Hansel and McKay 2010). The entire study region was covered with ice during the previous glacial episode, the Illinoian, which ended about 125,000 years ago. The glaciers of the Wisconsinan episode left behind various glacial features, the most prevalent of which are a series of moraines (Arnold et al. 1999). The physiographic regions of northeastern Illinois follow these glacial features, with the Chicago Lake Plain and the Wheaton Morainal Country making up the Great Lakes Section, and then, to the west and south, the Till Plains Section (Leighton et al. 1948), with corresponding regions in Indiana (Schneider 1966). As a result, the surficial geology in the study region consists of unconsolidated glacial drift of varying thickness up to 400 feet (Piskin and Bergstrom 1975). The character of the drift ranges from end moraines and till plains to outwash fans and lacustrine deposits (Hansel and McKay 2010).

Subsequent warmer and drier periods added loess to the surface, thinner toward Lake Michigan, so that soil parent materials combine the loess and the underlying drift (Fehrenbacher et al. 1984). Soil orders of the region vary with the original vegetation type. Alfisols, which form under forests, dominate nearer to Lake Michigan, whereas mollisols, which form under grasslands, cover most the 
rest of the region (Fehrenbacher et al. 1984). The permeability of the soils varies with the parent material from very low on clayey deposits to high on sandy outwash (Arnold et al. 1999).

The climate of the study region is classified as "Dfa" in the Köppen-Geiger system, which means humid continental with warm summers (Peel et al. 2007; Belda et al. 2014). This classification means winters are cold, summers are warm, and precipitation is common during the whole year. Based on 1971-2000 data, the average temperature at Chicago is about $50^{\circ}$ Fahrenheit (F), with average winter (DecemberFebruary) daily highs of about $33^{\circ} \mathrm{F}$ and lows of $17^{\circ} \mathrm{F}$ and average summer (June-August) daily highs of about $82^{\circ} \mathrm{F}$ and lows of $62^{\circ} \mathrm{F}$ (Changnon et al. 2004). Based on 1981-2010 Parameter-elevation Relationships on Independent Slopes Model (PRISM) data (http://www.prism.oregonstate.edu/normals, accessed April 15, 2014), precipitation for Chicago is about 35 inches per year, with a minimum monthly amount of about 2 inches in both January and February, increasing to about 4 inches per month during May-August before decreasing through the fall toward the winter minimum. About half the total precipitation is the result of thunderstorms, and 3 to 3.5 inches falls as snow, based on 1941-1995 data (Changnon et al. 2004).

In the flood climate classification of Hayden (1988), the study area lies at the border of the "TsuCpSs**" region to the north and the "TsuCpSe*" region to the south, where "Tsu" indicates barotropic (nonfrontal or convective) and unorganized (that is, without tropical cyclones) in the summer, "Cp" indicates that frontal storms are possible throughout the year, and "Ss**" indicates that winter snow cover is seasonal and exceeds 50 centimeters $(\mathrm{cm})$, so that there may be substantial spring snowmelt flooding, whereas "Se*" indicates seasonal, ephemeral snow cover for 10-50 days per year that may contribute to flooding during winter when rain falls on existing snow. The seasonality of precipitation in the study region and its flood classification explain the wide distribution of the timing of the annual maximum peak discharges used in this study (see Streamgage Selection section below), with the largest monthly fractions in March, April, and June, but also substantial fractions in February and May, with smaller fractions during July to September.

Nonurbanized land cover in the study area includes a substantial fraction of row crop agriculture but also more forest, grassland, and open water and wetlands than the surrounding regions, which are primarily agricultural (Illinois Department of Natural Resources 1996). Urbanized land use in the study area has been driven by population growth in metropolitan Chicago, which was dominated by that of the City of Chicago until 1940, when its population leveled off and population growth shifted to the suburbs (Figure 2). This suburban population growth was accompanied by even larger growth in developed land use; for example, from 1970 to 1990, the population grew by 4\%, but developed land use grew by $40 \%$, during which time more than 400 square miles $\left(\mathrm{mi}^{2}\right)$ of farmland that had been previously drained for agricultural use (Juhl 2005) were developed (Mariner 2005). This growth in developed land use is shown geographically in Figure 3. 


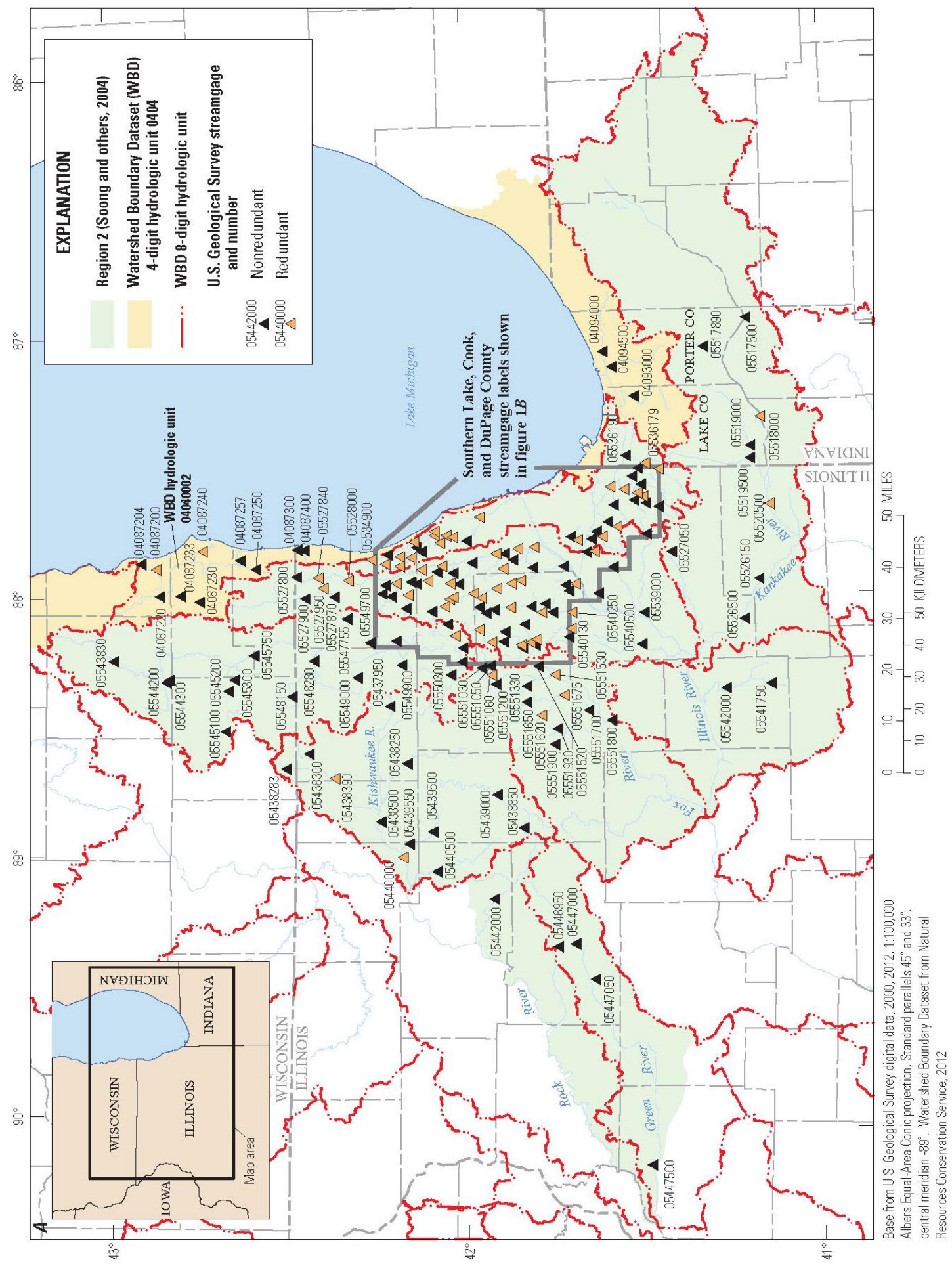

Figure 1a. Map of entire study area and streamgages used in this study. 


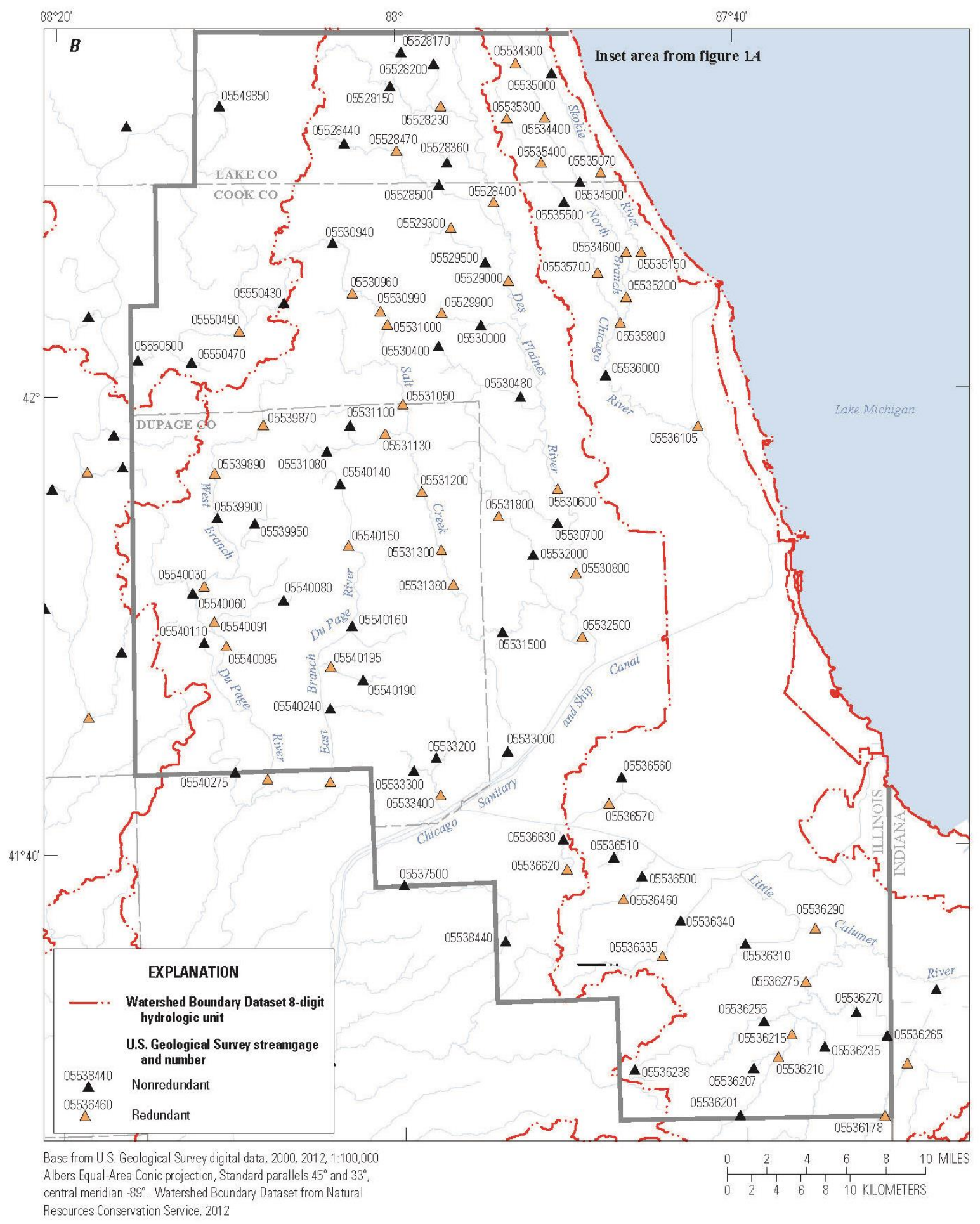

Figure 1b. Map of study area and streamgages used in this study showing southern Lake, Cook, and DuPage counties in detail. 


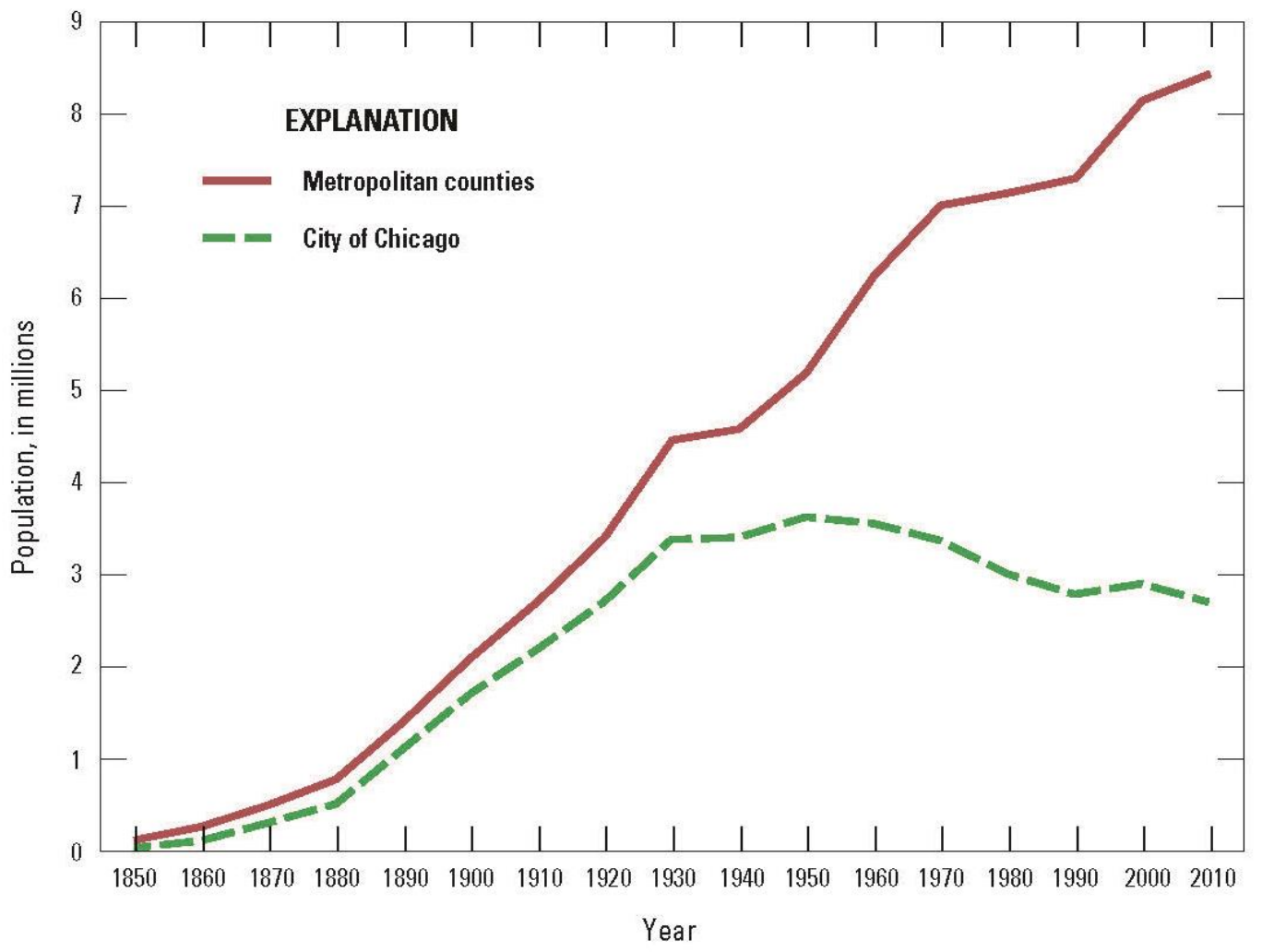

Figure 2. Plot of population growth in northeastern Illinois, 1850-2010 (from Karstensen et al. 2013). 


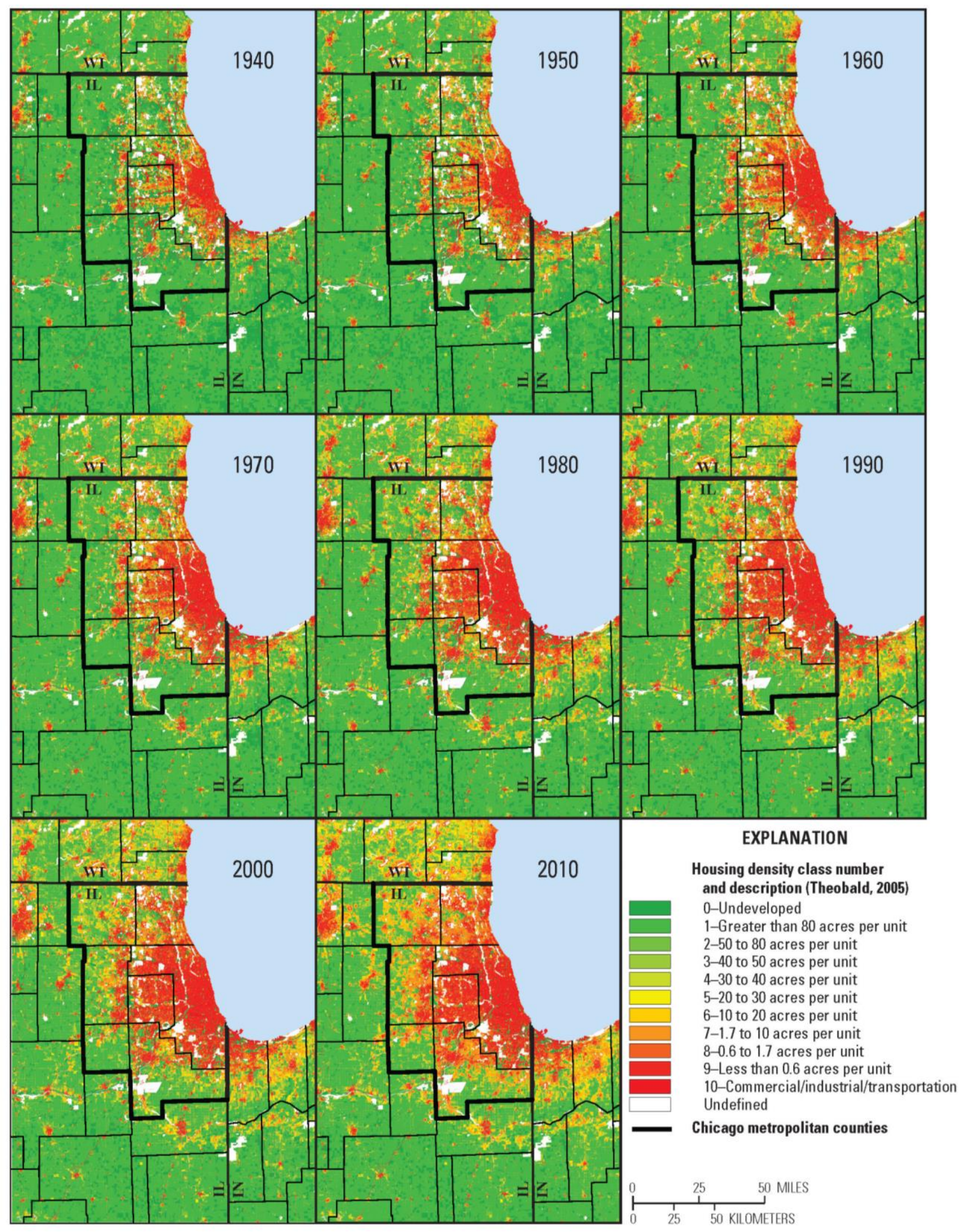

Figure 3. Map of housing density by decade in northeastern Illinois (modified from Karstensen et al. 2013). 
The hydrologic effects of this expansion of developed land in northeastern Illinois can be inferred from the series of historic floods that began in the 1950s and have extended to the present (2015) (Juhl 2005; Changnon 1999; Changnon and Westcott 2002a, 2002b; Angel and Changnon 2008; Changnon 2010, 2011; Fazio and Sharpe 2012; Villarini et al. 2013), though increases in precipitation have also been identified (Rougé and Cai 2014; Winters et al. 2015). These floods led to several policy and engineering responses (Juhl 2005). One such policy response was the introduction of ordinances requiring stormwater detention on new development, the first of which was promulgated in 1972 by the Metropolitan Water Reclamation District of Greater Chicago (MWRDGC). Another policy response was the passage of legislation by the State of Illinois allowing five suburban counties to plan and fund countywide stormwater management programs (Resource Coordination Policy Committee 1998). A third policy response was the planning and construction of channel improvements and flood-control reservoirs (Resource Coordination Policy Committee 1998).

\subsection{PREVIOUS STUDIES}

Allen and Bejcek (1979) developed regional spatial regression equations for peak discharge quantiles for the urbanized area of northeastern Illinois using data through WY 1976 as a function of impervious percentage, drainage area, and slope (all log-transformed). They used 103 streamgage records, including 70 crest-stage gages (CSGs), for many of which they developed stage-discharge ratings as part of the study. Drainage areas of the basins ranged from 0.07 to $630 \mathrm{mi}^{2}$ and impervious fractions from $1 \%$ to $38.7 \%$. A decreasing effect of urbanization (measured as an estimated fraction of impervious area) was determined with increasing flood magnitude (decreasing AEP), as is generally expected (Konrad 2003). The authors also suggest that the urbanization effect factor from their equations would be appropriate to use outside their study area elsewhere in Illinois and surrounding regions to adjust existing rural equations for use in urban areas, but that their results would not be appropriate for basins "completely served by underground drainage systems" or where "flood detention or retention reservoirs substantially affect the flood peaks" (Allen and Bejcek 1979, p.8).

Sauer et al. (1983) completed a nationwide spatial regression study of the effect of urbanization on flood frequency that used the results of Allen and Bejcek (1979). Sauer et al. used 199 streamgage records including 18 from Illinois, excluding streamgages with substantial manmade detention effects and those for which the developed fraction had increased by more than $50 \%$ during the period of record. They developed three sets of spatial regression equations. Two sets include seven explanatory variables and the remaining set has three. The two seven-variable equation sets have the same variables except one uses basin lag time (estimated from observed rainfall-runoff data, where available) in place of a measure of channel efficiency determined following the methods of Espey and Winslow (1974). All three sets of equations include as explanatory variables drainage area, the rural peak discharge quantile, and the basin development factor (BDF), which is a numerical index of the presence of channel improvements, curbs and gutters, and storm sewers, and is not easily computable from standard GIS databases. These three common explanatory variables constitute the variables of the three-variable equation set. Impervious area fraction is included in the two sevenvariable sets of equations, but it is not as significant as BDF. The coefficients of their equations also indicate a decreasing effect of urbanization for larger floods. 
More recently, Moglen and Shivers (2006) developed an iterative method of adjusting rural floodfrequency equations for the effects of time-varying urbanization, measured by one of two GIScomputable quantities - estimated impervious fraction and population density. Among their 78 study streamgages are 22 from Illinois, of which just one (Boneyard Creek at Urbana, USGS streamgage 03337000) is outside northeastern Illinois. One of the conclusions of the study listed as "Model Weaknesses" is that the apparent effects of urbanization are "relatively mild." In the use of GIScomputable quantities as the urbanization variable and directly incorporating time-varying urbanization, Moglen and Shivers' study is similar to the current study. Their method does not, however, produce an adjusted time series of annual maximum peak discharges for each streamgage; rather, it produces just a set of urbanization-adjusted peak discharge quantiles.

Other studies that have characterized the effect of urbanization on flooding in northeastern Illinois include Changnon and Demissie (1996), Changnon et al. (1996), Hejazi and Markus (2009), Villarini et al. (2013), and Rougé and Cai (2014).

Soong et al. (2004) divided the state of Illinois into seven hydrologic regions and developed regional regression equations for both annual maximum series (AMS) and partial duration series (PDS) peak discharges for rural basins in each region, using data through WY 1999. The basin characteristics used in the regression equations are drainage area, main channel slope, basin length, soil permeability, and percentage of open water and herbaceous wetland. For region 2, which is the focus area of the current study, they used drainage area, main channel slope, and percentage of open water and herbaceous wetland. The AMS regression equations from the study were later implemented in StreamStats (Ishii et al. 2010). 


\section{CHAPTER 2: DATA DEVELOPMENT}

Data development for this study consisted of the computation of two sets of data values: (1) observed peak discharge quantiles; that is, the statistical relation between flood magnitudes and frequencies based on observed floods at selected streamgages and (2) basin characteristics; that is, quantitative descriptors of characteristics of the basins draining to the locations of the streamgages. Regression equations for estimating the peak discharge quantiles at ungaged locations were then developed using the basin characteristics.

\subsection{PEAK DISCHARGE QUANTILES AT STREAMGAGES}

To develop equations for estimating peak discharge quantiles for ungaged stream locations, such quantiles were first computed at selected streamgages with at least 10 years of annual maximum peak discharges. This computation included four steps: (1) streamgage selection; (2) removal of redundant streamgages, where one streamgage of streamgage pairs whose basins are nested with substantial overlap are removed from the set of streamgages on which the regression analyses are done; (3) regional skew analysis, where a regional regression of at-streamgage skewness values is done to provide a regional estimate that is weighted with the at-streamgage skewness values in the frequency analysis; and (4) frequency analysis, where peak discharge quantiles at the selected AEPS are computed for each selected streamgage by fitting to the log-Pearson type III by a generalized method-of-moments method.

\subsubsection{Streamgage Selection}

The streamgages were chosen from the urbanized area of northeastern Illinois and nearby urbanized areas in northwestern Indiana and southeastern Wisconsin within hydrologic unit 0404, along with region 2 of Soong et al. (2004) (Figures 1a, 1b, and 3). In Indiana, the nearby urbanized area was selected as Lake and Porter counties, and in Wisconsin, as consisting of the 8-digit hydrologic unit 04040002 (Pike-Root) (Figures 1a and 1b). The decision to update only region 2 of Soong et al. (2004) as part of this study was in part made on the assumption that only this region includes substantial numbers of streamgages identified as rural drainage basins that contain substantial fractions of urbanized land use. This assumption is verified in Figure 4, which shows, for example, that $40 \%$ of gaged basins in region 2 used by Soong et al. (2004) had urbanized fractions greater than $20 \%$ as of the last year of record used by Soong et al. (2004), whereas only about $2 \%$ of gaged basins outside region 2 used by Soong et al. (2004) had such an urbanized fraction. 


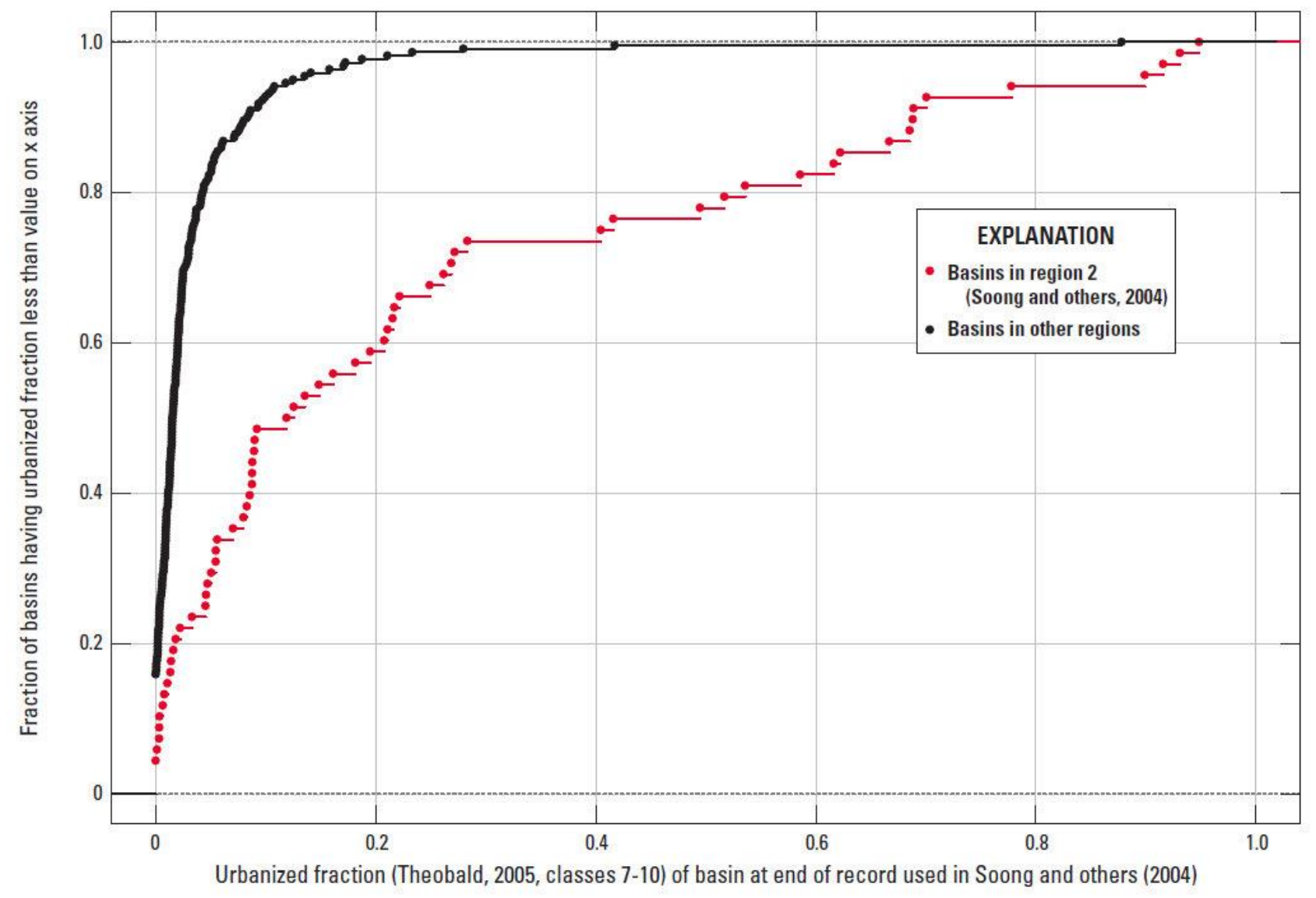

Figure 4. Empirical distribution functions of urbanized fraction of gaged basins in Illinois flood-frequency regions of Soong et al. (2004).

A minimum record-length criterion of 10 years of record between WYs 1940 and 2009 was applied when selecting stations. Water year 1940 was chosen as the starting point of the study period because that is the earliest year available in the housing density data of Theobald (2005) used to estimate historical urbanization in this study. Water year 2009 was chosen as the ending point of the study period for consistency with the analysis of Over et al. (2016).

The list of streamgages with at least 10 years of record in the study area was screened to eliminate those with potential measurement accuracy issues, using several criteria. One was to refer to the list of streamgages used in the most recent published rural flood-frequency studies (Soong et al. (2004) in Illinois, Rao (2006) in Indiana, and Walker and Krug (2003) in Wisconsin). John Walker (USGS, written commun. 2013) also provided a list of streamgages being used in an ongoing flood-frequency study in Wisconsin. If a streamgage had been used in one of those studies, it was retained for this study unless specific problems were identified by data section staff at the Illinois, Indiana, or Wisconsin USGS Water Science Centers.

The 181 selected streamgages and some properties of their records and drainage basins are listed in Table 1. Histograms of the annual maximum peak discharge record properties at the selected streamgages are shown in Figure 5 . These histograms show a maximum in streamgage records 
beginning around 1960 and ending around 1980, which were primarily CSGs on small (less than about $10 \mathrm{mi}^{2}$ ) drainage basins (Table 1). Histograms of the urban fractions of the streamgage drainage basins in Figure 6 show substantial urban fraction increases throughout the streamgage periods of record and further increases between the ends of the streamgage records and 2010. These urban fraction increases in urbanization underscore the nonstationary nature of the land use in the study basins and the need for adjustment to current (2010) conditions. Overall, drainage areas range from less than $0.01 \mathrm{mi}^{2}$ to more than $1000 \mathrm{mi}^{2}$, but the most are between 1 and $300 \mathrm{mi}^{2}$ (Figure 6).

Annual maximum peak discharge data for the selected stations were retrieved from the National Water Information System (U.S. Geological Survey 2013).

\section{Table 1. Information regarding U.S. Geological Survey streamgages} used in this study in northeastern Illinois and adjacent states.

[See Appendix 2.] 

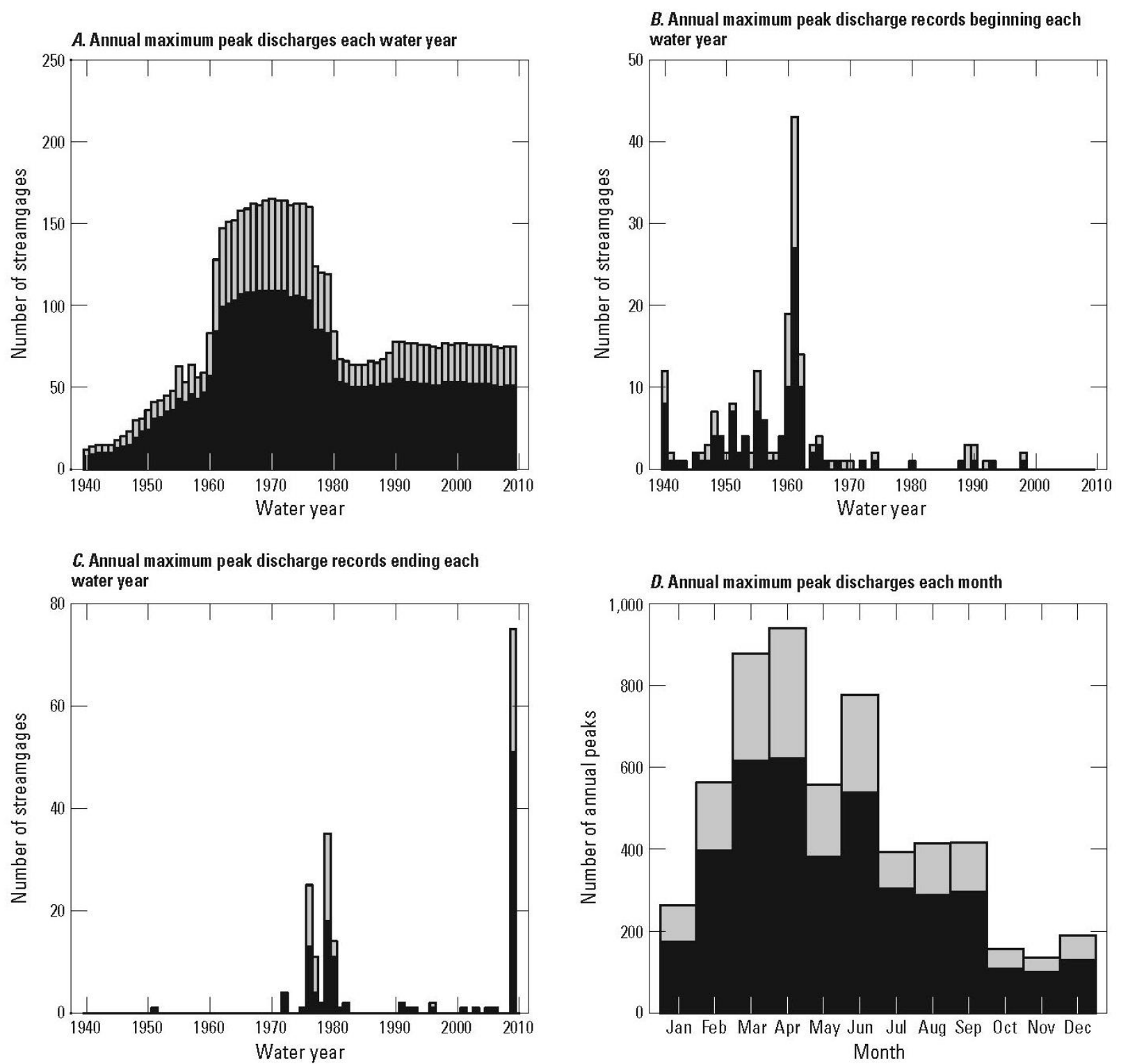

\section{EXPLANATION}

口 All streamgages

- Nonredundant streamgages

Figure 5. Properties of U.S. Geological Survey streamgage records used in this study in northeastern Illinois. (a) number of streamgages with annual maximum peak discharges each water year; (b) number of streamgages with annual maximum peak discharge records beginning each water year; (c) number of streamgages with peak discharge records ending each water year; (d) number of annual maximum peak discharges in each month of the year. 

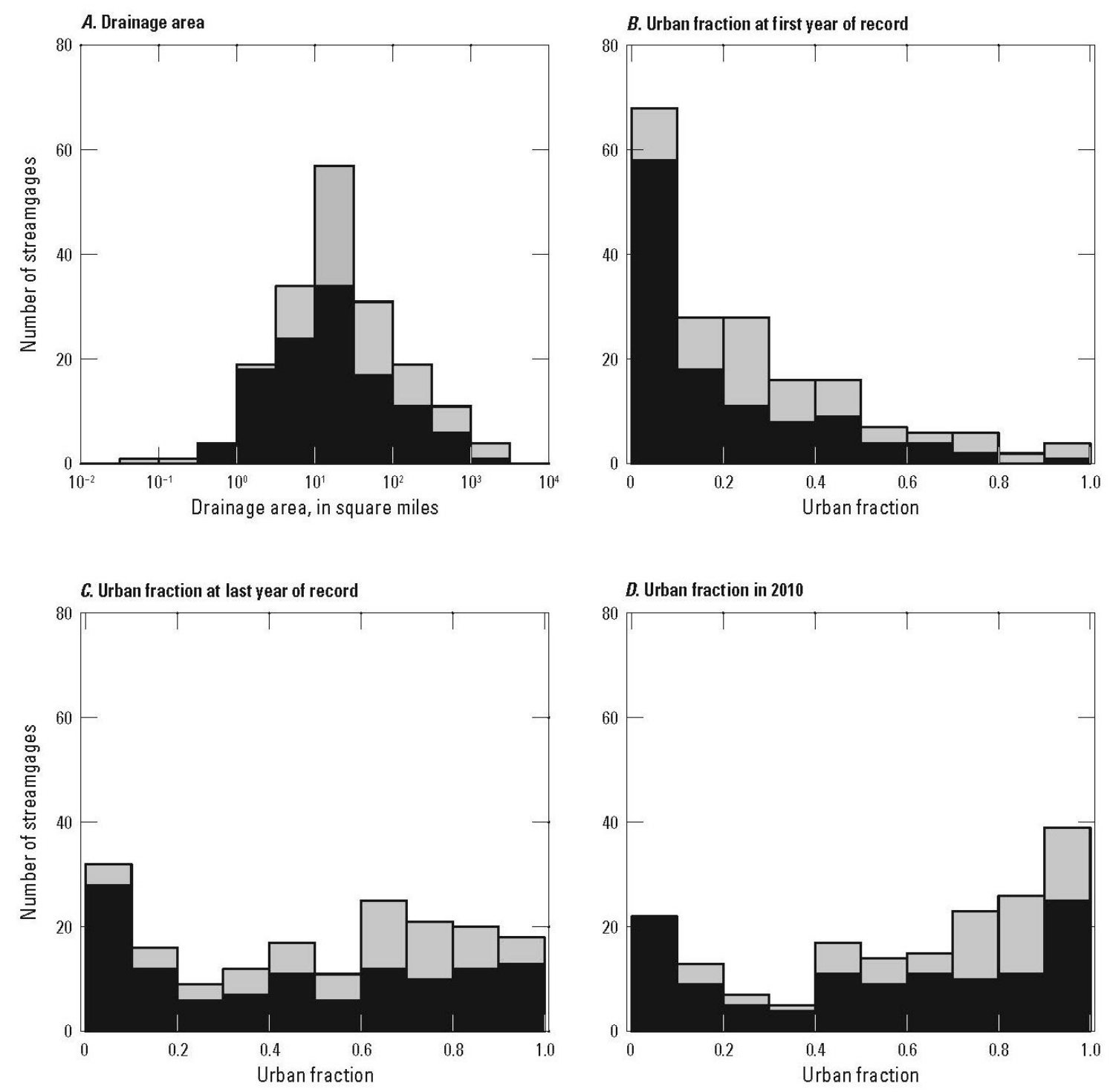

EXPLANATION

口 All streamgages

- Nonredundant streamgages

Figure 6. Properties of basins of U.S. Geological Survey streamgages used in this study in northeastern Illinois. (a) basin drainage areas, (b) urbanized basin fractions at first year of streamgage records, (c) urbanized basin fractions at last year of streamgage record, and (d) urbanized basin fractions in 2010, where urbanized is defined as Theobald (2005) housing density fewer than 10 acres per house plus commercial/industrial/transportation land use.

\subsubsection{Redundancy Analysis}

The generalized least-squares analysis that is used to fit the regression models for the peak discharge quantiles that form the basis of the final results of this study allows for cross-correlation among the observed quantiles that is induced by temporal factors such as the same storm hitting two nearby 
basins and causing the annual maximum peak discharge for both. Generalized least-squares regression, however, does not account for correlation in the model error that arises when two nested basins have an appreciable overlap in drainage area (Veilleux 2009). When the overlap is large enough, one of the basins is considered redundant for purposes of regional regression modeling (Veilleux 2009). To address this issue, a redundancy analysis was carried out to determine an optimal nonredundant subset of the original 181 stations.

Because any one gaged basin may be nested within multiple other gaged basins or have multiple other gaged basins nested within it, a way to decide in what order basins should be removed was needed. For this study, the decision was made by assigning a score to each gaged basin based on its record length and drainage-basin properties and its amount of overlap with other gaged basins, removing the basin with the worst (highest) score, followed by re-computation of the scores, removing the remaining basin with the worst recomputed score, and proceeding in this way until no basin remained with an overlap fraction larger than 0.205 , where the overlap fraction $D A R_{i j}$ for two nested basins $i$ and $j$ is defined as

$$
D A R_{i j}=\min \left[\frac{D A_{i}}{D A_{j}}, \frac{D A_{j}}{D A_{i}}\right],
$$

where $D A_{i}$ and $D A_{j}$ are the drainage areas of basins $i$ and $j$, respectively.

Originally a maximum overlap fraction of 0.2 as suggested by Veilleux (2009) was chosen, but it was determined that a few basins could be retained by increasing the value slightly to 0.205 . A script was written in $\mathrm{R}$ ( $\mathrm{R}$ Core Team 2014) to implement this algorithm.

The details of computation of the score are as follows: The score $S_{i}(t)$ for basin $i$ at iteration step $t$ is computed as the sum, $\sum s_{i j}(t)$, over all remaining basins $j$ having basins overlap with basin $i$, where $s_{i j}(t)$, the subscore of basin $i$ with overlapping basin $j$ at step $t$, is computed as

$$
s_{i j}(t)=D A R_{i j}\left[2-R L_{i} / \max (R L)\right]\left[d_{i}(t)+1\right],
$$

where

$D A R_{i j}$ is the overlap fraction between basins $i$ and $j$ (equation 1 ),

$R L_{i}$ is the record length of the streamgage at basin $i$, in years, $\max (R L)$ is the maximum record length in the dataset (70 years), and $d_{i}(t)$ is the probability density value of the drainage area of station $i$ at step $t$ scaled to lie between 0 and 1.

Based on their definitions, $D A R_{i j}$ lies between 0 and $1,2-R L_{i} / \max (R L)$ between 1 and 2 , and $d_{i}+1$ between 1 and 2 . The effect of each of the factors making up the subscore $s_{i j}$ on its value is as follows: 
(a) with more overlap of basin areas, $D A R_{i j}$ is larger, and so is $s_{i j}$; (b) with shorter record length $R L_{i}$, $2-R L_{i} / \max (R L)$ is larger and so is $s_{i j}$; and (c) when the drainage area of basin $i$ is at a larger density value (where there are many basins of similar drainage areas), $d_{i}$ is larger and so is $s_{i j}$; consequently, larger basin overlaps, shorter records, and larger drainage area overlaps raise the subscore and therefore the score, making the associated streamgage more likely to be removed.

The result of applying this algorithm was to remove 64 of the original 181 to leave 117 nonredundant streamgages, of which 57 were CSGs throughout their peak discharge record, 13 were CSGs during part of their peak discharge record, and 47 had continuous record throughout their peak discharge records. These 117 streamgages were used for all subsequent analyses. Streamgages that were used and streamgages that were considered redundant are indicated in the study area maps (Figures $1 \mathrm{a}$ and $1 \mathrm{~b}$ ), the histograms describing the basic properties of the streamgage records and their basins (Figures 5 and 6), and in the table listing the basic properties of the streamgage records and their basins (Table 1).

\subsubsection{Regional Skew Analysis}

Although the Expected Moments Algorithm (EMA) (Cohn et al. 1997, 2001) used in this study to obtain estimates of the peak discharge quantiles generalizes the method-of-moments method of Bulletin 17B (U.S. Interagency Advisory Committee on Water Data 1982), it still relies on estimates of the mean, variance, and skewness of the logarithms of the annual maximum peak discharge data at each streamgage. Of these moments, the skewness, because it involves raising the data values to the third power, is very sensitive to extreme values and thus has a large uncertainty when estimated from the data at a single site. Therefore, weighting at-site and regional skew estimates in inverse proportions to their uncertainties to obtain a weighted skew estimate was recommended in Bulletin 17B and continues to be recommended. Because the map in Bulletin 17B showing a grid of regional skew values has become outdated, newer and more refined maps have been created, such as the one in Soong et al. (2004) for Illinois. These maps, however, do not address the effects of urbanization. Furthermore, a new approach to the estimation of regional skew through Bayesian generalized leastsquares (GLS) analysis has been developed (Reis et al. 2005; Gruber et al. 2007; Gruber and Stedinger 2008; Veilleux 2011) that has shown the ability to reduce the uncertainty in regional skew estimates and allows the regional skew estimate to take into account basin characteristics where applicable.

Details of the new approach and its application in this study are given in Appendix 1 . The data analyzed was the annual maximum peak discharges at the nonredundant streamgages adjusted to 2010 urbanization conditions. The selected regional skew model is dependent on the urban fraction as measured by the NLCD_22_23_24 variable; that is, the sum of fractions of National Land Cover Database (NLCD) 2011 classes 22 (developed, low intensity), 23 (developed, medium intensity), and 24 (developed, high intensity), according to the linear relation:

$$
\hat{\gamma}_{R}=-0.39+0.97 N L C D_{-} 22_{-} 23 \_24^{1 / 2} \text {, }
$$

where $\hat{\gamma}_{R}$ is the regional skewness. 
Skewness increases substantially with urbanization from a value of -0.39 for a basin with no urbanization $\left(N L C D \_22 \_23 \_24=0\right)$ to $-0.39+0.97=0.58$ for a basin where the $N L C D \_22 \_23 \_24$ fraction is $100 \%$. The regional skewness estimate from equation 3 was combined with the at-site skewness according to their associated uncertainties to obtain a weighted skew estimate for use in computing the at-site peak discharge quantile estimates.

\subsubsection{Frequency Analysis}

The peak discharge quantiles with AEPs of $0.50,0.20,0.10,0.04,0.02,0.01,0.005$, and 0.002 for redundant and nonredundant streamgages (Table 2) were computed by applying the EMA approach (Cohn et al. 1997, 2001), along with the Multiple Grubbs-Beck test (Cohn et al. 2013) to detect and censor PILFs, to the annual maximum peak discharges adjusted to 2010 urbanization conditions as described in the Regional Temporal Regression Analysis and Adjustment section below. The EMA methodology generally follows guidelines provided in Bulletin 17B of the U.S. Interagency Advisory Committee on Water Data (1982) by using the log-Pearson Type III distribution fitted by the methodof-moments for estimating discharge frequency; however, the EMA methodology provides updated procedures for incorporating historical and censored peak discharges. Use of the EMA approach and the Multiple Grubbs-Beck test are as recommended by the Hydrologic Frequency Analysis Work Group (http://acwi.gov/hydrology/Frequency/HFAWG-B71B-Recommended-Rev-SOH-12June2013revised-final.pdf). Software developed by the USGS to analyze peak discharge data (PeakFQ version 7.3, https://water.usgs.gov/software/PeakFQ/) was used for these computations (Veilleux et al. 2013).

As part of the frequency analysis, the skewness values for each streamgage were computed as a weighted average $\widehat{W}$ of the station and regional skew values following the method suggested in Bulletin 17B (U.S. Interagency Advisory Committee on Water Data, 1982):

$$
\hat{\gamma}_{W}=\frac{A V P_{n e w} * \widehat{\gamma}_{S}+M S E\left[\widehat{\gamma}_{S}\right] * \widehat{\gamma}_{R}}{A V P_{n e w}+M S E\left[\widehat{\gamma}_{S}\right]},(4)
$$

where

$\hat{\gamma}_{W}$ is the weighted skewness,

$\hat{\gamma}_{R}$ is the regional skewness computed as described in the "Regional Skew Analysis" section and in appendix 1 ,

$\hat{\gamma}_{S}$ is the station skewness computed by using the EMA approach as implemented in PeakFQ for each streamgage,

$A V P_{\text {new }}=0.19$ is the average variance of prediction at a new site computed as described in appendix 1 , and

$\operatorname{MSE}\left[\hat{\gamma}_{S}\right]$ is the mean square error of the at-site skewness, which also is computed by using the EMA approach as implemented in PeakFQ. 


\section{Table 2. Estimated peak discharge quantiles for 181 streamgages in northeastern Illinois and adjacent states, at selected annual exceedance probabilities.}

[See Appendix 3.]

\subsection{BASIN CHARACTERISTICS}

The starting point in defining basin characteristics for regional statistical analyses is to delineate the basin boundaries. Following delineation, basin characteristics for this study were computed following two main approaches. The first was the traditional approach in projects of this type, using spatially averaged or basin-wide properties such as drainage area, fractions of various land uses and soil types, average soil properties, and physiographic properties such as basin slope. The second approach was to develop and analyze spatially distributed basin characteristics based on properties of estimated instantaneous unit hydrographs (for example, Grimaldi et al. 2010). The spatially distributed approach was proposed as a way to see if the effects of basin shape and the location, not just the amount, of different types of hydrologically relevant characteristics such as urbanized areas and open water and wetland on flood-frequency characteristics were significant and could improve floodfrequency prediction. Preliminary results with the spatially distributed approach were not promising and as a result it was dropped from further consideration. As this approach was not used in the final results, it is not discussed further in this report.

\subsubsection{Basin Delineation}

Most basins were delineated by using the online Illinois StreamStats application (http://streamstats.usgs.gov), which is based on Environmental Systems Research Institute's (Esri's) Arc Hydro Tools software (Maidment 2002). Illinois StreamStats uses the 1:100,000-scale National Hydrography Dataset (NHD) for hydrography and the 30-meter National Elevation Dataset (NED) resampled to a 10-meter resolution for elevation information for Illinois, Wisconsin, and Indiana. Seven Indiana basins were delineated using the Indiana StreamStats application, a similar application to Illinois StreamStats, because the results were slightly better in delineating the Kankakee River than the Illinois StreamStats results. Eight Wisconsin basins in the hydrologic unit 0404 (Lake Michigan) were provided by the USGS Wisconsin Water Science Center because the Illinois StreamStats does not cover that area and Wisconsin StreamStats did not exist at the time of the analysis. Resulting drainage areas from the Illinois StreamStats application were compared to the drainage areas of hand-drawn boundary (HDB) basin delineations, which are considered to be the most accurate delineation type. For those basins with discrepancies larger than about 10\%, it was decided that the HDB delineations were preferable. These basins are usually small urban basins with records that ended around 1980, where it is difficult to determine basin boundaries because of urban development and the related potential for later modifications to the hydrography and topography.

\subsubsection{Spatially Averaged Basin Characteristics}

A common challenge in projects of this type is to select among the many basin characteristics that are available in GIS databases. As this project is in part an update of the regional regression equations for region 2 from the rural flood-frequency study by Soong et al. (2004), which used spatially averaged basin characteristics, it was decided to simplify the process of the selection of 
basin characteristics by considering only the categories of characteristics used by Soong et al. (2004) in that region, which were drainage area, slope, and wetland-open water fraction, plus measures of urbanized area (Table 3).

The slope variable used in Soong et al. (2004) is main channel slope. The determination of the main channel requires sometimes lengthy computations in StreamStats and is in any case ambiguous, so alternative slope variables were considered for this study. These alternatives included the basin average of digital elevation model (DEM) slopes, the basin average of the State Soil Survey Geographic Database (SSURGO) soil slopes, and basin slope computed as maximum-minimum basin elevations divided by basin perimeter (Table 3 ).

The wetland-open water variable used in Soong et al. (2004) was the percentage of water and herbaceous wetland from the 2001 NLCD (Soong et al. 2004, p. 16). Additional open water and wetland variables derived from the 2011 NLCD, National Wetlands Inventory (NWI), and SSURGO were considered in this study (Table 3).

The urbanized area variable used for temporal analysis and adjustment of the annual maximum peak discharge records (Over et al. 2016) was derived from a year 2000 U.S. Census housing density database developed by David Theobald (Theobald 2005). For the spatial modeling of the peak discharge quantiles, it was preferred that the urbanization variable be something that is regularly updated; therefore, developed area fractions and impervious percentage from the 2011 NLCD were chosen, with the Theobald housing density urbanization estimates retained for comparison (Table 3).

\section{Table 3. Spatially averaged basin characteristics considered for developing spatial regression equations in this study in northeastern Illinois.}

[See Appendix 4.] 


\section{CHAPTER 3: REGIONAL TEMPORAL REGRESSION ANALYSIS AND ADJUSTMENT}

The purpose of the temporal analysis of the annual maximum peak discharges is to provide a means of adjusting the peak discharge records from the streamgages used in the study to their levels of urbanization at a common year (2010). This adjustment is needed to provide approximately stationary and contemporary streamgage records. The method of the analysis is to use regression to determine values of coefficients that characterize the regional average effect of urbanization on peak discharge quantiles as a function of exceedance probability in Illinois flood-frequency region 2 . The coefficients also are used to develop factors for adjusting estimates of peak discharge quantiles in other regions of Illinois for the effect of urbanization. The adjusted annual maximum discharges and associated peak discharge quantiles also are made available herein for general use (see Table 2 for quantiles and the Adjustment of Annual Maximum Peak Discharges to Current (2010) Urban Fractions section for discharges).

The effects of changes in urbanization in a streamgage record are potentially problematic in a regression analysis of peak discharge quantiles on basin characteristics for at least two reasons. One is that peak discharge records on basins with substantial changes in the fraction of urbanization over the streamgage period of record are difficult to use because no single value of urbanization fraction characterizes the entire record. The second is that historical urbanization information is needed to characterize the urban fraction of records that ended some time ago and urbanization of their basins continued after the records ended. Because both of these situations are fairly common in the study region (Figure 6), a method for adjustment of peak discharge records to their levels of urbanization at a common year was needed if such stations were going to be included in the study.

The methods used here for temporal analysis and adjustment were described by Over et al. (2016); a summary of the application of their method in this study follows. Over et al. use a two-step regression method applied to annual maximum peak discharge records as a function of contemporaneous urbanization and precipitation. In the first step, after breaking the peak discharge records into segments at the time of major abrupt changes in the basins, if any, ordinary leastsquares (OLS) regression is used to estimate an intercept for each segment. In the second step, after subtracting the segment intercepts to homogenize the data across all the segments, the homogenized data are analyzed with quantile regression, which provides regional urbanization and precipitation coefficients for a sequence of AEPs and a means of assigning AEPs to each flooddischarge observation. The urbanization coefficient for the AEP assigned to each peak discharge observation is then used to adjust the observation to the estimated 2010 urbanization level in the basin where it was observed. Adjustment for changes in precipitation was not implemented because, unlike changes in urbanization, changes in precipitation are highly uncertain and may reverse themselves in the future. 


\subsection{DATA USED IN THIS ANALYSIS}

In addition to the annual maximum peak discharge data from the nonredundant streamgages, three types of data were used for the temporal analysis and adjustment method applied in this study: (1) historical urbanization, (2) precipitation associated with each peak discharge, and (3) information on reservoirs and other structural flood-control measures such as channelization.

The historical urbanization data used, as in Over et al. (2016), were those of Theobald (2005), which are 1940-2010 decadal housing density data based on the 2000 U.S. Census for 10 categories of housing density (including undeveloped) plus nonhousing urbanization (commercial, industrial, and transportation) (Figure 3). Theobald's nonhousing urbanization estimates, which are constant year 2000 values, were adjusted in proportion to changes in housing density for each basin according to a technique presented in Over et al. to obtain 1940-2010 decadal estimates. Following Over et al., the decadal values of housing density classes 7-10 (Figure 3), which encompass areas with housing densities of no more than 10 acres per unit plus adjusted nonhousing urbanization, were linearly interpolated to annual values and used as the urbanization measure for the temporal analysis and adjustment in this study.

To account for effects of trends in precipitation alongside the effects of urbanization on peak discharges, precipitation values were estimated for each observed peak discharge used in this study. Because the density of daily precipitation gages is much higher than that of hourly gages, daily data were used. Three sets of daily precipitation data were used for the estimates: (1) National Weather Service (NWS) cooperative network (COOP) stations in the study area; (2) the Cook County Precipitation Network (CCPN) (Westcott 2015); and (3) precipitation data collected by the Argonne National Laboratory (http://www.atmos.anl.gov/ANLMET, accessed March 10, 2011). Data from COOP were downloaded from the Midwestern Regional Climate Center (MRCC) Applied Climate System (MACS) at https://mrcc.illinois.edu/CLIMATE/. Missing data during the study period (WYs 1940-2009) were filled by using a weighted average of values at neighboring gages, where weights were determined by an inverse-distance weighting technique (Over et al. 2016). Precipitation data were distributed spatially by using Thiessen polygons (Thiessen 1911) (Figure 7), and daily values for the basin upstream from each streamgage in the study were computed as area-weighted average of the values in each Thiessen polygon overlying the basin. An estimate of the precipitation associated with each annual maximum peak discharge was obtained by selecting the maximum daily precipitation during the period from 3 days before to 1 day after the date of the peak discharge. Precipitation data dated after the peak discharge were used because NWS cooperative precipitation measurements typically are made during the early morning of the date assigned to the data, and therefore include precipitation from the previous day. 


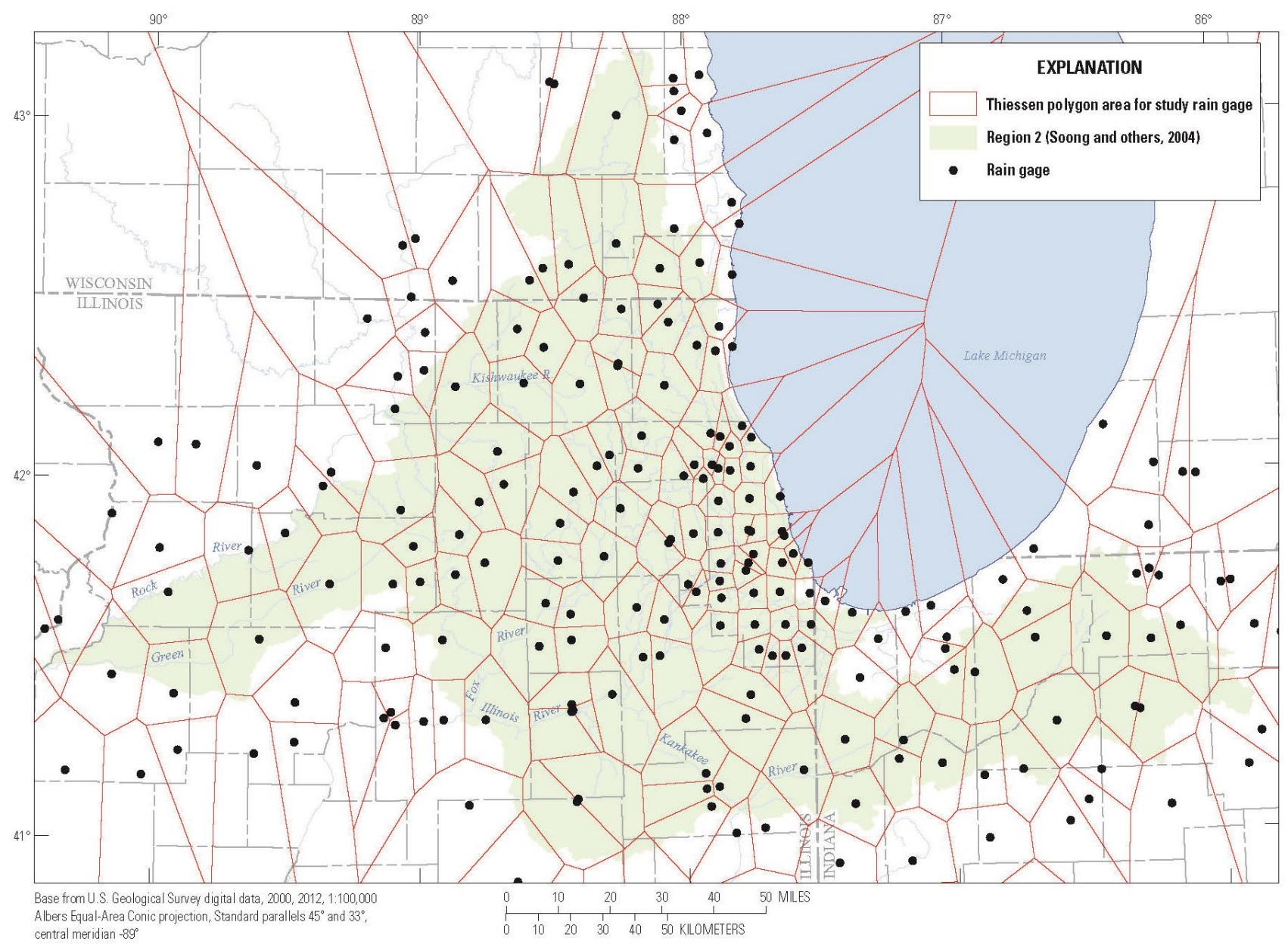

Figure 7. Locations of precipitation stations used in this study and their Thiessen polygons.

Information on reservoirs and other structural flood-control measures were obtained by accessing the 2013 National Inventory of Dams (U.S. Army Corps of Engineers, 2013), reviewing relevant reports, inquiring of various federal, state, county, and local agencies involved in flood control in northeastern Illinois, and searching historical imagery on Google Earth. By reviewing this information, reservoirs and other structural flood-control measures (for example, channelization) within the basins of the streamgages used in this study were determined. For the identified reservoirs, three particular quantities were obtained: (1) the year of construction, (2) the volumetric capacity, and (3) the basin area upstream of the reservoir or other flood-control measure.

The volumetric capacity and upstream area were used to estimate the magnitude of the hydrologic effect of the reservoir. When a reservoir controlling at least $10 \%$ of the streamgage drainage area and with a volumetric storage capacity divided by the streamgage drainage area of 0.40 inches or other substantial change in the effect of a basin's structural flood-control measures was deemed to have changed during the period of record of a streamgage used in the study, the year of the change was dropped from the temporal analysis and the remaining record was broken into segments at that year. After the segment $y$-intercept values were obtained by a preliminary run of the first, OLS, step of the temporal analysis procedure, if the intrastreamgage $y$-intercept values were anomalous (for example, 
showing a significant increase following the construction of a flood-control reservoir), further research was completed on the basin history. If no reason to explain the anomaly was found, the segment break where the anomaly occurred was dissolved and the record was re-connected. The final set of segments is presented in Table 4. According to Table 5, all but 18 of the 117 nonredundant streamgages have only 1 segment, and only 3 streamgage records were split into more than 2 segments. Peak discharges associated with segments shorter than 5 years, historic peak discharges, censored peak discharges, and peak discharges for which the date is not known also were dropped from the temporal analysis.

Table 4. Segment information for 181 U.S. Geological Survey streamgages used in this study, northeastern Illinois and adjacent states.

[See Appendix 5.]

Table 5. Number of segments per streamgage record used in regression analysis of peak discharge for 117 streamgages, northeastern Illinois and adjacent states.

\begin{tabular}{|c|c|c|c|c|c|}
\hline $\begin{array}{c}\text { Number of segments } \\
\text { per streamgage record }\end{array}$ & $\mathbf{> 0}$ & $\mathbf{> 1}$ & $\mathbf{> 2}$ & $\mathbf{> 3}$ & $\mathbf{> 4}$ \\
\hline Number of records & 117 & 18 & 3 & 2 & 0 \\
\hline
\end{tabular}

\subsection{TEMPORAL ANALYSIS OF URBANIZATION EFFECT}

The annual maximum peak discharges at the nonredundant streamgages during the study period, separated into segments as necessary based on the construction of structural flood-control measures with certain years of data dropped as described in the Data Used in This Analysis section, along with associated precipitation and urban fraction values, constituted the database for the temporal analysis of the effect of urbanization (Table 6). The results of this analysis were used to adjust the annual maximum peak discharges to their urban factions at a common year (2010) based on an analysis to determine the regional effect of changes in urban fraction on peak discharge magnitude.

In addition to the peak discharge values dropped from the temporal analysis because of segment breaks, certain coded peak discharge values were also dropped. Peak codes as they appear in the peak discharge data downloaded from NWIS are given in table 6, column titled "NWIS peak codes", and whether or not a particular peak discharge value was used in the temporal regression analysis to perform the urbanization adjustment is indicated in table 6, column titled "Discharge value used in adjustment regression". As documented by those two columns of table 6, peak discharge values with codes of $1,4,7,8$, or $B$ were dropped from the temporal regression analysis. These codes indicate that the published values are subject to censoring (codes 1, 4, 8), are historic peaks (code 7), or the date of the peak discharge event is not known (code B). These peak discharge values were nevertheless adjusted, and the adjusted values appear in table 6 . These values also were used in the spatial regression analysis, because the spatial analysis does not require knowing the date of the event and the EMA methodology is designed to handle censored and historic values, as discussed in the Frequency Analysis section. 


\section{Table 6. Observed and urban-adjusted annual maximum peak discharges and associated urbanization and precipitation values at 181 streamgages in northeastern Illinois and adjacent states.}

[See Appendix 6.]

The temporal analysis was carried out by the method described in Over et al. (2016), which follows a two-step linear regression technique suggested by Canay (2011), where the first step uses OLS regression to estimate intercepts for each segment of peak discharge data, and the second step applies quantile regression (Koenker 2005) to the peak discharge data with the segment intercept values subtracted. The segment intercepts are subtracted from the peak discharge observations to obtain segment-independent observations that are approximately homogeneous (that is, segmentindependent), to which quantile regression can be applied. Quantile regression is used in the second step because it provides an estimate of the coefficients of the regression model for different quantiles (exceedance probabilities). Because of this property, the disparate effect of urbanization at different exceedance probabilities (Espey and Winslow 1974 and references therein; Allen and Bejcek 1979; Konrad 2003 and references therein; Over et al. 2016 and references therein) can be estimated, and the adjustment of the peak discharges to their urban fractions at a common year (2010) can take this disparate effect into account.

The independent variables in the two-step regression analysis are urban fraction and precipitation. Precipitation is used in the regression analysis not so that the peak discharges can be adjusted to a present (2010) precipitation regime, as it was beyond the scope of this study to establish one, but so that by including precipitation, trends that happened to exist in the precipitation causing the observed peak discharges would not be falsely interpreted as the effect of changes in the urban fraction.

The following linear regression model was used for the least-squares regression analysis and was the first step of the method:

$$
y_{i}(t)=\hat{y}_{i}(t)+\varepsilon_{i}(t)=a_{i}+b_{1} P_{i}(t)+b_{2} U_{i}(t)+\varepsilon_{i}(t), i=1,2, \ldots, N,
$$

where

$y_{i}(t)=\log _{10} Q_{i}(t)$ is the base-10 logarithm of the annual maximum peak discharge at the $i$ th segment during year $t$,

$\hat{y}_{i}(t)$ is the fitted value of $y_{i}(t)$ according to the regression relation,

$a_{i}$ is the intercept of the $i$ th segment,

$P_{i}(t)$ and $U_{i}(t)$ are the precipitation and urban fraction, respectively, for the $i$ th segment during year $t$,

$b_{1}$ and $b_{2}$ are the regression coefficients for precipitation and urban fraction, respectively, and $\varepsilon_{i}(t)=y_{i}(t)-\hat{y}_{i}(t)$ is the regression error for the ith segment during year $t$. 
Notice there is one intercept for each segment, whereas there is only one value of each of the coefficients; therefore, they are estimated from the effect of precipitation and urbanization on all the segments combined, and are equivalent in value to an uncertainty-weighted mean of the coefficients of segment-by-segment least-squares regression analysis (Frees 2004, p. 32). Therefore, the segment intercepts contain all the information that distinguishes one segment from another; the effects of urbanization and precipitation, captured in their coefficients in equation 5, are assumed to be the same for all segments. The computation of the regression model was done in R (R Core Team 2014) using the plm function from the plm package (Croissant and Millo 2008).

The coefficients obtained by fitting the regression relation (equation 5) are given in Table 7, and the values of the intercepts for each segment are given with the other segment information in Table 4 and are plotted in Figure 8 . As can be seen from the linear relation with limited scatter in Figure 8, the segment intercept values are mostly but not completely explained by drainage area. According to the coefficients in Table 7, there is a small, positive effect of precipitation, which is highly significant because the magnitude of the coefficient is much larger than its standard error (SE). The urban fraction coefficient is also positive but larger and also highly significant. Because the regression relation is of the form $\log _{10} Q=a+b_{2} U$, the ratio of peak discharge for two different values of urban fraction $U_{1}$ and $U_{2}$ at the same site is given by $Q_{2} / Q_{1}=10^{b_{2}\left(U_{2}-U_{1}\right)}$; the value of the urban fraction coefficient $b_{2}$ indicates that for a $10 \%$ increase in urban fraction, the ratio of peak discharges is $Q_{2} / Q_{1}=10^{b_{2}(0.10)}=10^{0.5079 * 0.10}=1.124$, or about a $12 \%$ increase. Although the urbanization coefficient from this step of the analysis method is not used for adjustment of the peak discharges, the median value from the results of the next step is quite similar, so this coefficient gives an approximation of the average result.

Table 7. Results of ordinary least-squares linear regression of 117 streamgages in northeastern Illinois and adjacent states to diagnose the temporal effects of urbanization and precipitation on annual maximum peak discharges in this study.

\begin{tabular}{ccccc}
\hline $\begin{array}{c}\text { Precipitation } \\
\text { coefficient } \boldsymbol{b}_{\mathbf{1}}\end{array}$ & $\begin{array}{c}\text { Precipitation } \\
\text { coefficient SE }\end{array}$ & $\begin{array}{c}\text { Urban fraction } \\
\text { coefficient } \boldsymbol{b}_{\mathbf{2}}\end{array}$ & $\begin{array}{c}\text { Urban fraction } \\
\text { coefficient SE }\end{array}$ & $\begin{array}{c}\text { Weighted mean } \\
\text { dummy variable } \\
\text { (log units) }\end{array}$ \\
\hline 0.110 & 0.005 & 0.508 & 0.042 & $2.388^{\mathrm{a}}$ \\
\hline
\end{tabular}

aSee equation 6 for the computation of the weighted mean dummy variable value. [SE, standard error] 


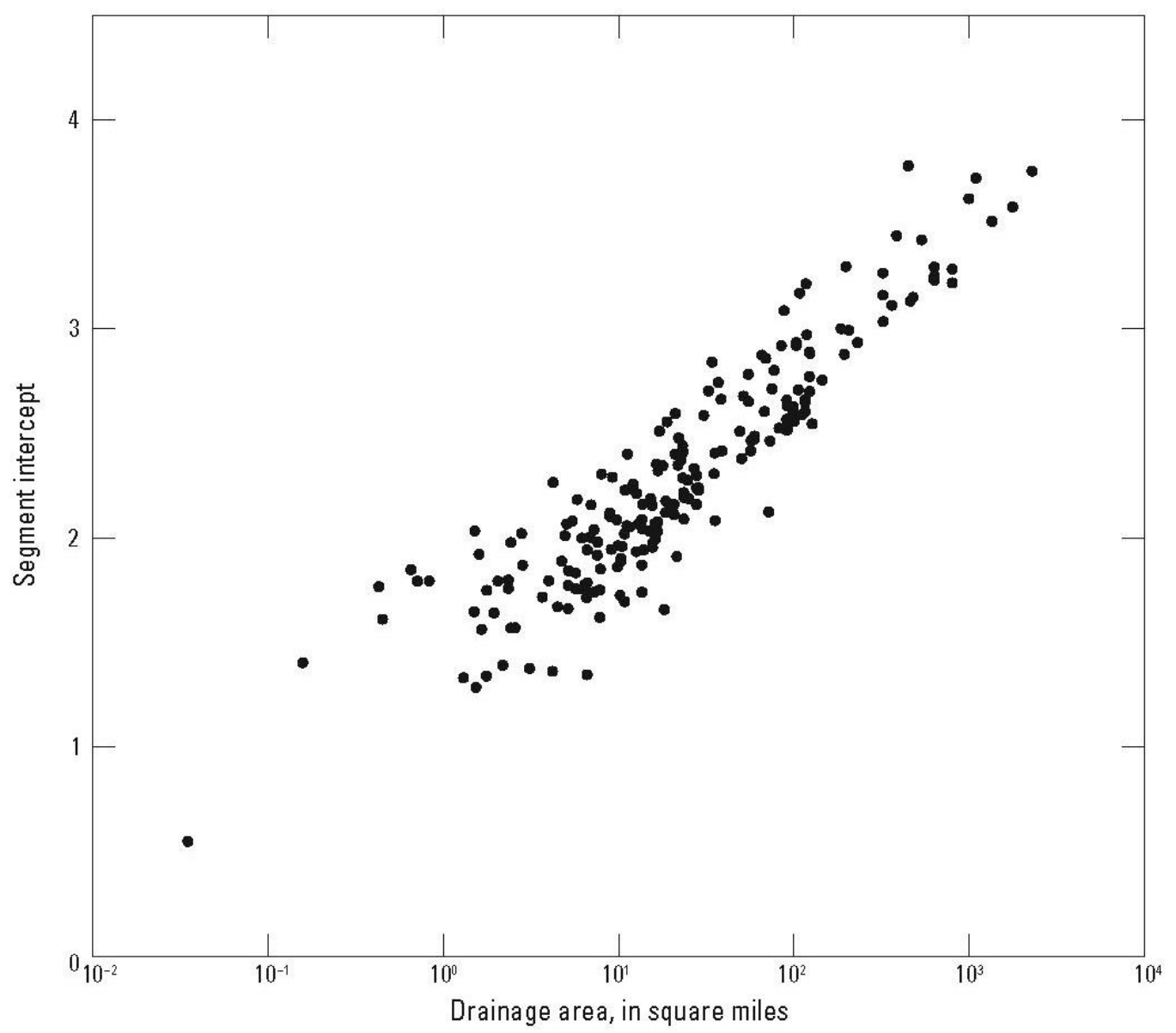

Figure 8. Segment intercepts as a function of drainage area for 181 streamgages used in this study in northeastern Illinois.

In the second, quantile regression-based, step of the method, the distinction between the segments is removed by subtracting the applicable segment intercept value from the dependent variable values for each segment; that is,

$$
y_{k}^{\prime}=y_{i}(t)+\bar{a}-a_{i}
$$

where $\bar{a}=\sum_{i} T_{i} a_{i} / \sum_{i} T_{i}$, where $T_{i}$ is the number of observations in the $i$ th segment, is the weighted mean of the segment intercepts obtained from the least-squares regression.

The independent variables are again the urban fractions and precipitation values associated with each peak discharge. 
The quantile regression model is as follows:

$$
y_{k}^{\prime}=\hat{y}_{k}^{\prime}(p)+\varepsilon_{k}^{\prime}(p)=a^{\prime}(p)+b_{1}^{\prime}(p) P_{k}+b_{2}^{\prime}(p) U_{k}+\varepsilon_{k}^{\prime}(p),
$$

where

$p$ is an element of a sequence of AEPs (Table 8),

$\hat{y}_{k}^{\prime}(p)$ is the fitted value of the quantile with $A E P=p$ of the $k$ th log-transformed peak discharge value $y_{k}^{\prime}$,

$a^{\prime}(p)$ is the intercept of the fitted linear relation between $\hat{y}_{k}^{\prime}(p)$ and the independent variables,

$P_{k}$ and $U_{k}$ are the precipitation and urban fraction, respectively, of $y_{k}^{\prime}$,

$b_{1}^{\prime}(p)$ and $b_{2}^{\prime}(p)$ are the quantile regression coefficients as a function of $p(A E P)$ for precipitation and urban fraction, respectively, and

$\varepsilon_{k}^{\prime}(p)=y_{k}^{\prime}-\hat{y}_{k}^{\prime}(p)$ is the regression error for the $k$ th observation at $A E P=p$.

This model was solved with the rq function of the quantreg package (Koenker 2013) of the $R$ language.

Two post-processing steps were applied to obtain the final quantile regression coefficients and their SEs. First, a bootstrap resampling approach suggested by Canay (2011) was applied to obtain SEs and unbiased mean coefficient values. Second, a continuous and monotonic version of the bootstrap mean urban fraction coefficients as a function of exceedance probability was obtained by fitting a seventh-order polynomial. The original and bootstrap mean and SE values for all the quantile regression coefficients, along with the fitted urban fraction coefficients, are given in Table 8 , and the three sets of urban fraction coefficients are plotted in Figure 9. A subset of the urbanization coefficients at the AEPs of the standard flood-frequency quantiles are given in Table 9. 


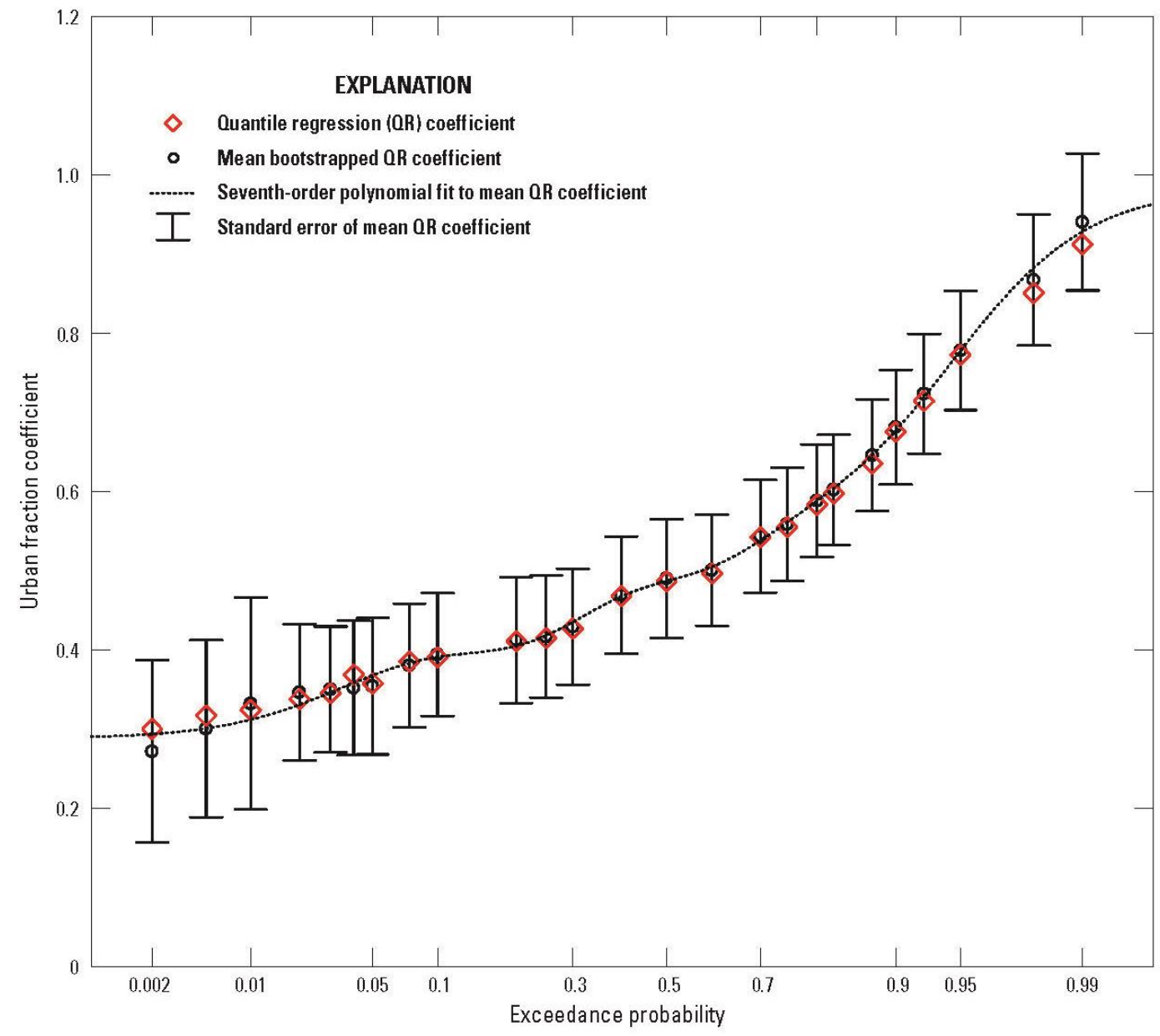

Figure 9. Urban fraction coefficients from temporal regression analysis of $\mathbf{1 1 7}$ streamgages in northeastern Illinois and adjacent states, as a function of exceedance probability.

Table 8. Quantile regression coefficients from temporal analysis of $\mathbf{1 1 7}$ streamgages in northeastern Illinois and adjacent states, as a function of annual exceedance probability. [See Appendix 7.] 
Table 9. Quantile regression coefficients of urban fraction from temporal analysis of 117 streamgages in northeastern Illinois and adjacent states, at selected annual exceedance probabilities.

\begin{tabular}{c|cccccccc}
\hline AEP & $\mathbf{0 . 5}$ & $\mathbf{0 . 2}$ & $\mathbf{0 . 1}$ & $\mathbf{0 . 0 4}$ & $\mathbf{0 . 0 2}$ & $\mathbf{0 . 0 1}$ & $\mathbf{0 . 0 0 5}$ & $\mathbf{0 . 0 0 2}$ \\
\hline $\begin{array}{c}\text { Coefficient } \\
\text { Standard } \\
\text { error }\end{array}$ & 0.487 & 0.405 & 0.391 & 0.359 & 0.331 & 0.312 & 0.301 & 0.294 \\
\hline
\end{tabular}

[AEP, annual exceedance probability]

The results in Tables 7-9 indicate that the median urban fraction and precipitation coefficients are quite similar to the values computed by the OLS regression carried out in the first step of the analysis, with values of about 0.5 and 0.1 . Results in these tables indicate that the precipitation coefficient varies only slightly with exceedance probability, whereas the urban fraction coefficient has a strong, mostly monotonic trend with exceedance probability, from a maximum value of 0.929 at an exceedance probability of 0.99 down to 0.294 at an exceedance probability of 0.002 (Table 8). The dependence of the urban fraction coefficient on exceedance probability gives direct empirical evidence that urbanization in the study area affects the magnitudes of the more common, large exceedance probability peak discharges more than it does the rarer, small exceedance probability peak discharges.

\subsection{ADJUSTMENT OF ANNUAL MAXIMUM PEAK DISCHARGES TO CURRENT (2010) URBAN FRACTIONS}

Following the application of the two-step method to obtain exceedance probability-dependent urbanization and precipitation coefficients, each peak discharge is assigned an exceedance probability by a procedure described in Over et al. (2016). This procedure was developed to adjust only for urbanization and therefore reduces the three-dimensional $\log _{10} Q, U$, and $P$ space in which the least-squares and quantile regressions were carried out to a two-dimensional $\log _{10} Q$ and $U$ space in which the quantile regression results are a set of lines in that space (Figure 10), where the diminishing effect of urbanization on the larger, rare floods can be seen graphically. 


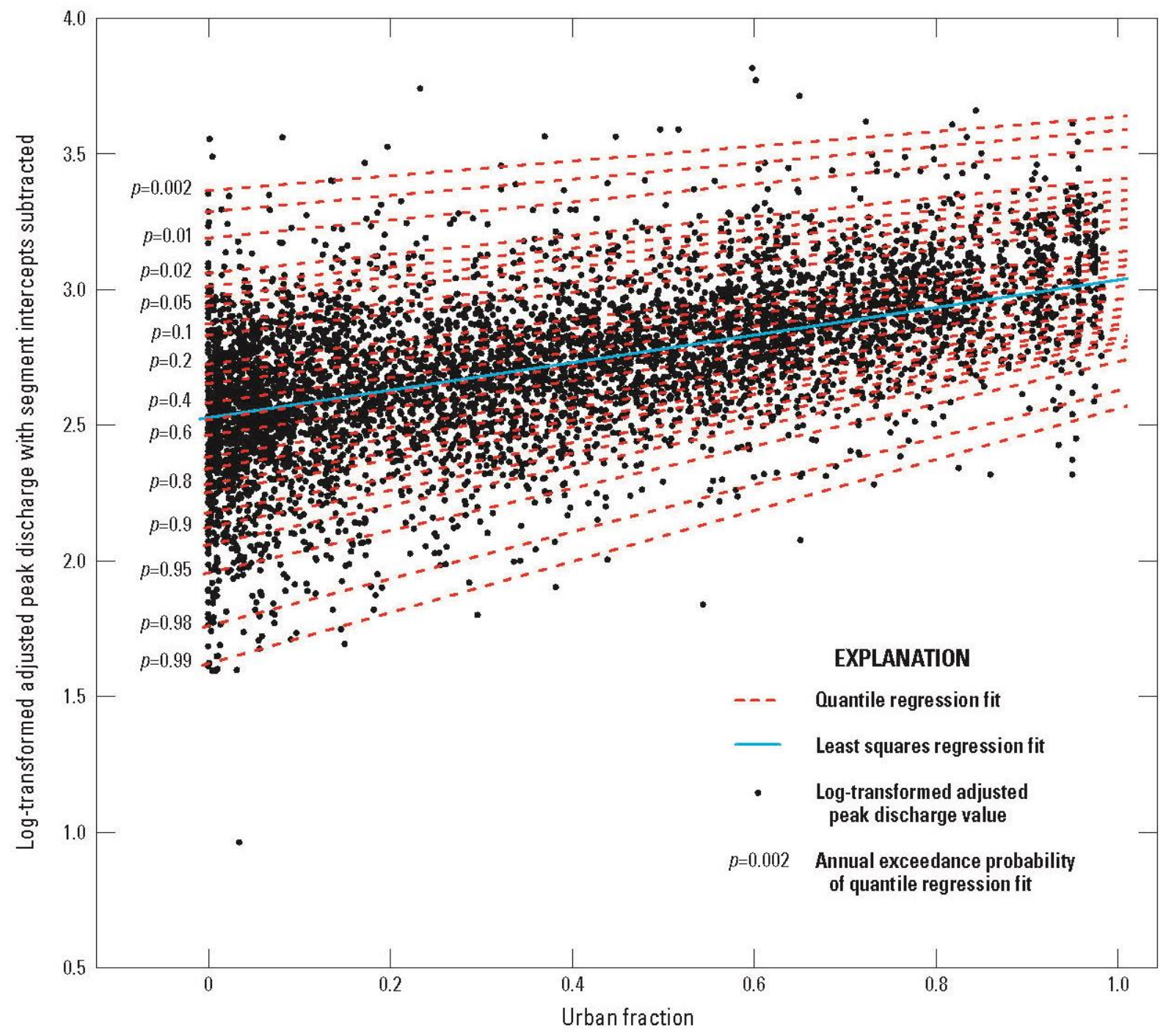

Figure 10. Segment intercept-subtracted log-transformed annual maximum peak discharge from 181 streamgages in northeastern Illinois and adjacent states as a function of urban fraction, with quantile regression and least-squares regression line fits.

Using the exceedance probability assigned to each peak discharge observation and the continuous urban fraction coefficient function $\bar{b}_{2}^{\prime \prime}(q)$, the annual maximum peak discharges were adjusted to the 2010 urban fraction obtained from the Theobald (2005) data by using the following equation applied to each streamgage record segment $i$ :

$$
y_{i}^{\prime \prime}(t)=a_{i}^{*}-a_{i}+y_{i}(t)+\bar{b}_{2}^{\prime \prime}(q)\left[U_{i}\left(t^{*}\right)-U_{i}(t)\right],
$$


where

$y_{i}^{\prime \prime}(t)$ and $y_{i}(t)$ are the base-10 logarithm of the adjusted and unadjusted, respectively, peak discharge value for year $t$ and segment $i$,

$a_{i}^{*}$ and $a_{i}$ are the segment intercept values for the most recent and current segments, respectively, of the streamgage record containing segment $i$,

$\bar{b}_{2}^{\prime \prime}(q)$ is the urban fraction coefficient value corresponding to the exceedance probability $q$ assigned to the peak discharge value $y_{i}(t)$, and

$U_{i}\left(t^{*}\right)$ and $U_{i}(t)$ are the urban fraction values for basin corresponding to segment $i$ during the year $t^{*}$ to which the peak discharges are being adjusted (here, 2010) and the year $t$ of the observation of the peak discharge $y_{i}(t)$, respectively.

Notice that if a streamgage record has only one segment, then $a_{i}^{*}=a_{i}$ and the quantity $a_{i}^{*}-a_{i}$ drops out.

For the peak discharges and censoring levels that were dropped from the temporal analysis, where the correct segment could be determined, the adjustment method allows those values also to be adjusted so that these data can be used for at-site frequency analysis. For peak discharge values between two segments, the adjustment was interpolated. As a result, only peak discharge values associated with segments shorter than five years were not adjusted and thus were dropped from the at-site frequency analysis.

The set of adjusted values $Q_{i}^{\prime \prime}(t)=10^{y_{i}^{\prime \prime}(t)}$ and the assigned exceedance probability values, $q$, on which the adjustment depends are given in Table 6 . The effects of the adjustment on mean, standard deviation, and skewness of the log-transformed peak discharges at each streamgage in the study are shown in Figure 11. The change in mean, which results from adjusting peaks with urban fractions less than the 2010 value upward, is almost always positive, and the change in standard deviation, which results from removal of urban fraction-induced trends in peak discharge in the observed data by the adjustment process and from the adjustment of smaller peak discharges more than larger ones, is almost always negative. The skewness increases more often than it decreases, also showing the effect of adjustment smaller peaks more than larger ones. The overall increase in skewness as peaks are adjusted to more urbanized conditions is also consistent with the increase in skewness with urban fraction determined in the spatial analysis, as discussed in the Regional Skew Analysis section and Appendix 1. 

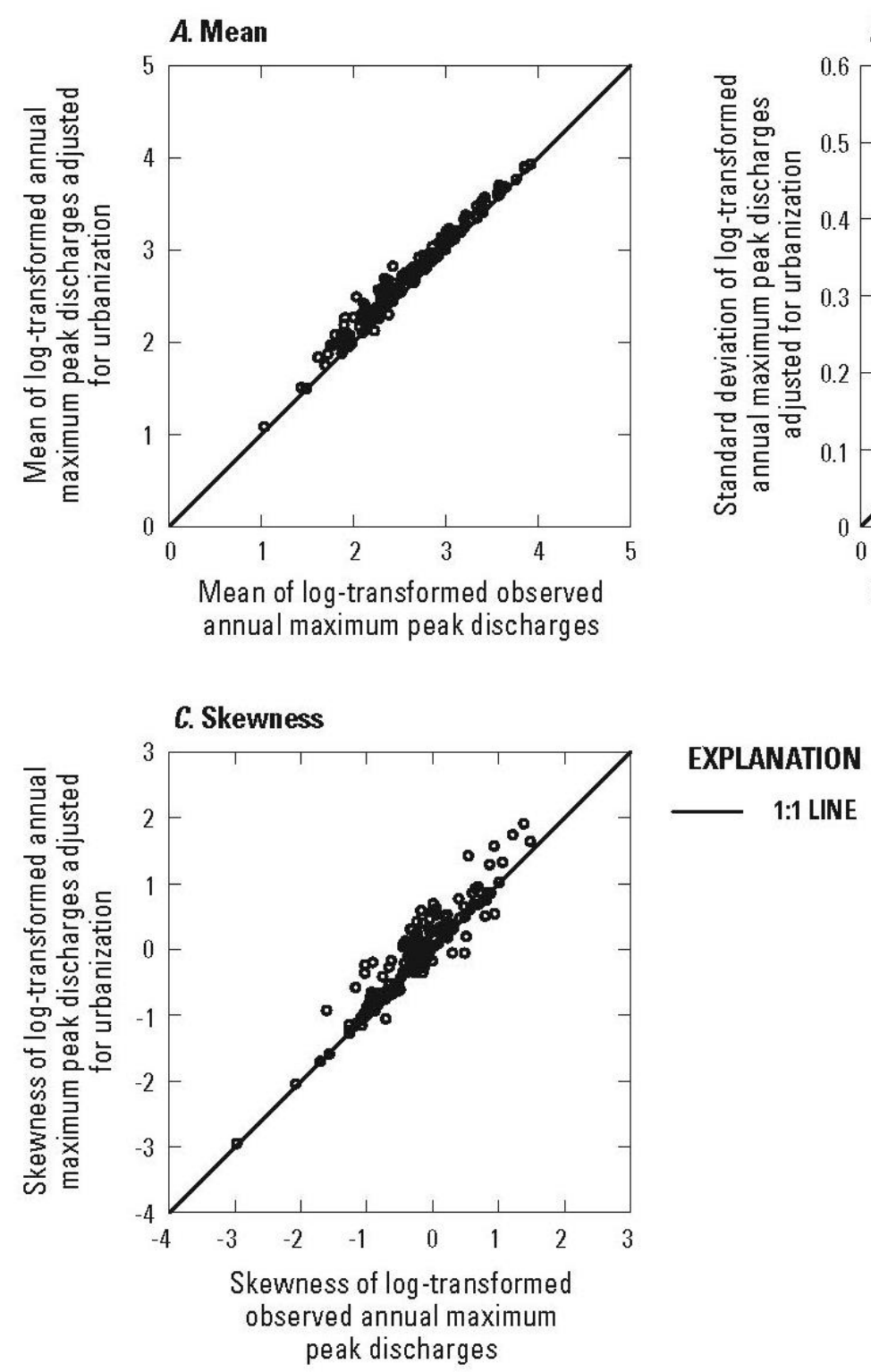

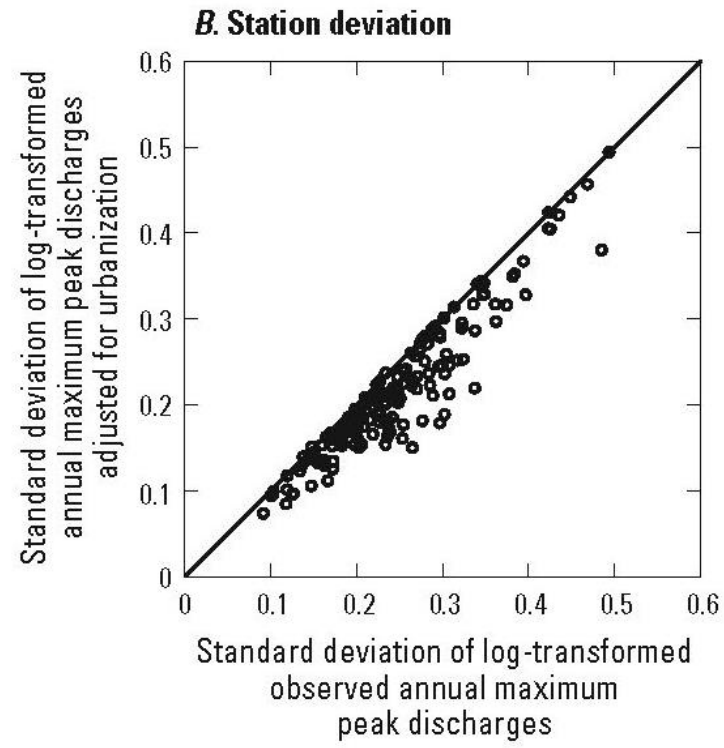

Figure 11. Changes in the mean, standard deviation, and skewness of the log-transformed annual maximum peak discharge records for 181 streamgages used in this study in northeastern Illinois, resulting from adjustment to 2010 urban fraction values. 


\section{CHAPTER 4: REGIONAL SPATIAL REGRESSION ANALYSES}

After the annual maximum peak discharges were adjusted to their levels of urbanization at a common year (2010) as described in the Regional Temporal Regression Analysis and Adjustment section, peak discharge quantiles for the selected AEPs were computed as described in the Frequency Analysis section, including weighting with the regional skew model. The computed quantiles constitute the dependent variable for the spatial regression analyses; the spatially averaged basin characteristics developed as described in the Spatially Averaged Basin Characteristics section constitute the independent variables.

\subsection{SELECTION OF BASIN CHARACTERISTICS}

The predictive capability of the basin characteristics in each category (Table 3) were investigated by fitting weighted least-squares (WLS) linear regression models with regression weights in proportion to record length for each AEP for all possible combinations of basin characteristics where at most one variable is selected from each category with $\log _{10}$-transformed drainage area always included. For these exploratory regressions, a preliminary set of peak-flow quantiles developed using regional skew were used. An R script (R Core Team 2014) was written to do the regression computations. The general form of the regression models fitted in this investigation was:

$$
\begin{gathered}
\log _{10} Q_{p}=\left(\beta_{0}\right)_{p}+\left(\beta_{A}\right)_{p} \log _{10} A+\left(\beta_{U}\right)_{p}\left(U+c_{2}\right)^{d_{2}}+\left(\beta_{W}\right)_{p}\left(W+c_{3}\right)^{d_{3}}+\left(\beta_{S}\right)_{p}\left(S+c_{4}\right)^{d_{4}}, \\
p=0.50,0.20,0.10,0.05,0.02,0.01,0.005,0.002
\end{gathered}
$$

where

$Q_{p}$ is the estimated peak discharge quantile with regional skew, in $\mathrm{ft}^{3} / \mathrm{s}$, with annual exceedance probability $p$,

$\left(\beta_{0}\right)_{p},\left(\beta_{A}\right)_{p},\left(\beta_{U}\right)_{p},\left(\beta_{W}\right)_{p}$, and $\left(\beta_{S}\right)_{p}$, are coefficients estimated by the WLS procedure,

$A$ is the drainage area, in square miles,

$U$ is an urbanization measure, a decimal fraction,

$W$ is a basin water and wetness measure, a decimal fraction,

$S$ is a basin slope measure, in feet per mile,

$d_{2}, d_{3}$, and $d_{4}$ are exponents used to transform the distribution of urbanization, water and wetness, and slope measures, respectively, to an approximately Gaussian shape ( $d_{i}=1,1 / 2,0$, where 1 implies no transformation, $1 / 2$ a square-root transformation, and 0 a $\log _{10}$-transformation were tested), and

$c_{2}, c_{3}$, and $c_{4}$ are constants added to the urbanization, water and wetness, and slope measures when a $\log _{10}$-transformation was used and the basin characteristic had zero values). 
The selected transformations are listed in Table 3.

Various plots of the regression results were made to facilitate examination of the results; the most useful plot was determined to be the adjusted coefficient of determination $\left(R^{2}\right)$ (Helsel and Hirsch 2002 , p. 313) as a function of the t ratio of the coefficients of variables of the urbanization, slope, and wetness categories (Figure 12), where the t ratio is the coefficient value divided by its standard error (Helsel and Hirsch 2002, p. 238). Such a plot shows which models provide the best fit (highest adjusted $R^{2}$ ) and the most significant coefficient values (largest magnitude $t$ statistics), and, by retaining the sign of the coefficient values, such plots show if the sign of the coefficient is physically reasonable. The variables whose corresponding regression model fits have the largest magnitude $t$ ratio values and highest adjusted $\mathrm{R}^{2}$, along with the sign of the coefficient being physically reasonable, were selected as candidate variables for the development of the final spatial regression equations.

Plots of this type for each of the categories (urbanization, slope, and water and wetland) are shown in Figure 12. The urbanization variable with the largest $t$ ratio values and highest adjusted $R^{2}$ values is usually the square root of NLCD_22_23_24, except for AEPs 0.5 and 0.2 where Theobald_Urban is of similar quality, as shown in Figure 12a. Both NLCD 22_23_24 and Theobald_Urban have positive coefficients, as is physically expected. Because only the NLCD-based urbanization variables are expected to be updated in the future, Theobald_Urban was eliminated from consideration for the spatial regression equations and NLCD_22_23_24 ${ }^{(1 / 2)}$ was chosen for all AEPs as the candidate urbanization variable for the final equations. Because there are too many water and wetland variables to distinguish on a single plot, the results for these are plotted on two sets of plots (Figures $12 b$ and $12 c)$. The variable with the highest adjusted $R^{2}$ values and largest magnitude $t$ ratio values varies from smaller to larger peak discharge quantiles, being NWI.emergent for the 0.5 annual exceedance probability (AEP) flood, NWI.total ${ }^{(1 / 2)}$ for 0.2 to 0.04 AEP floods, and DrainageClass $1 a^{(1 / 2)}$ for the larger floods ( 0.02 to $0.002 \mathrm{AEP}$ ), and all have physically expected negative coefficient values. Because NWI.total is almost as good as NWI.emergent for the 0.5 AEP flood, it was selected as the candidate variable for the smaller floods (AEPs of 0.5 to 0.04 ), and DrainageClass1a was selected for the larger floods (AEPs of 0.02 to 0.002 ). It can be seen in Figure12d that the slope variable with the highest adjusted $R^{2}$ values and largest magnitude $t$ statistic values for all AEPs (and physically expected positive coefficients) is $\log _{10}\left(D E M \_1 \_0 \_P\right)$, so $\log _{10}\left(D E M \_1 \_0 \_P\right)$ was chosen for all AEPs as the candidate urbanization variable for the final equations. 
A. Urbanization variables
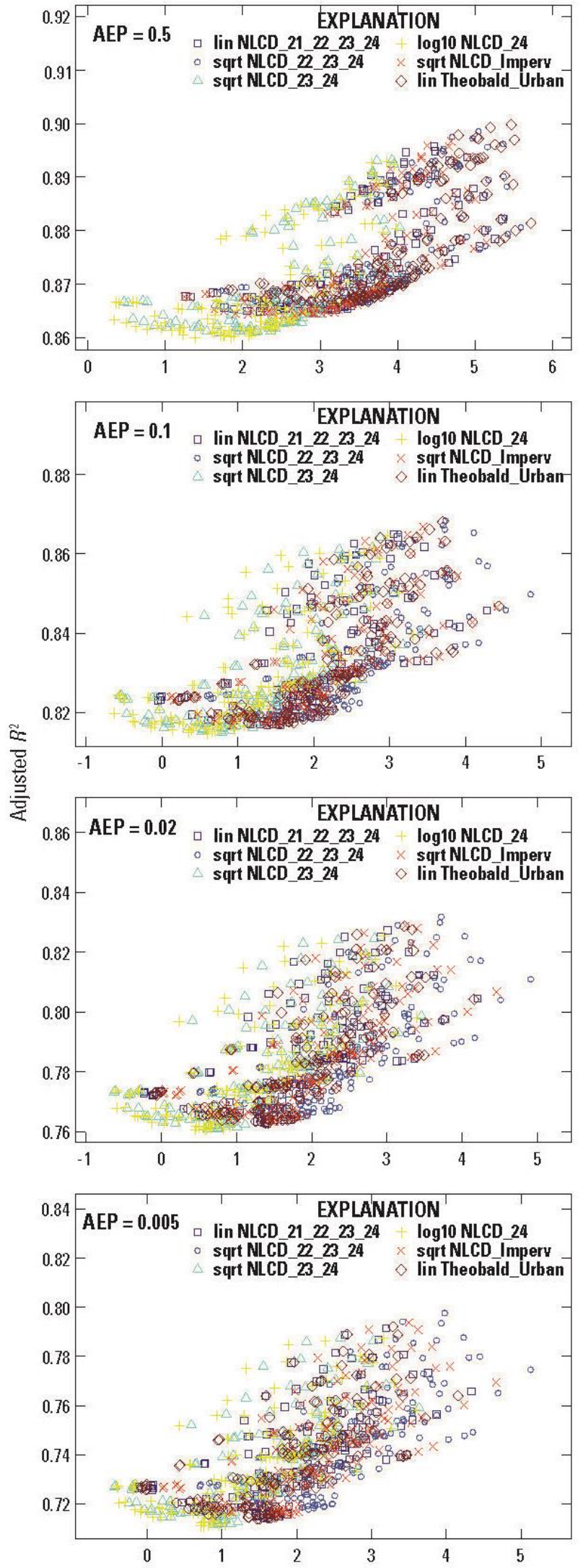
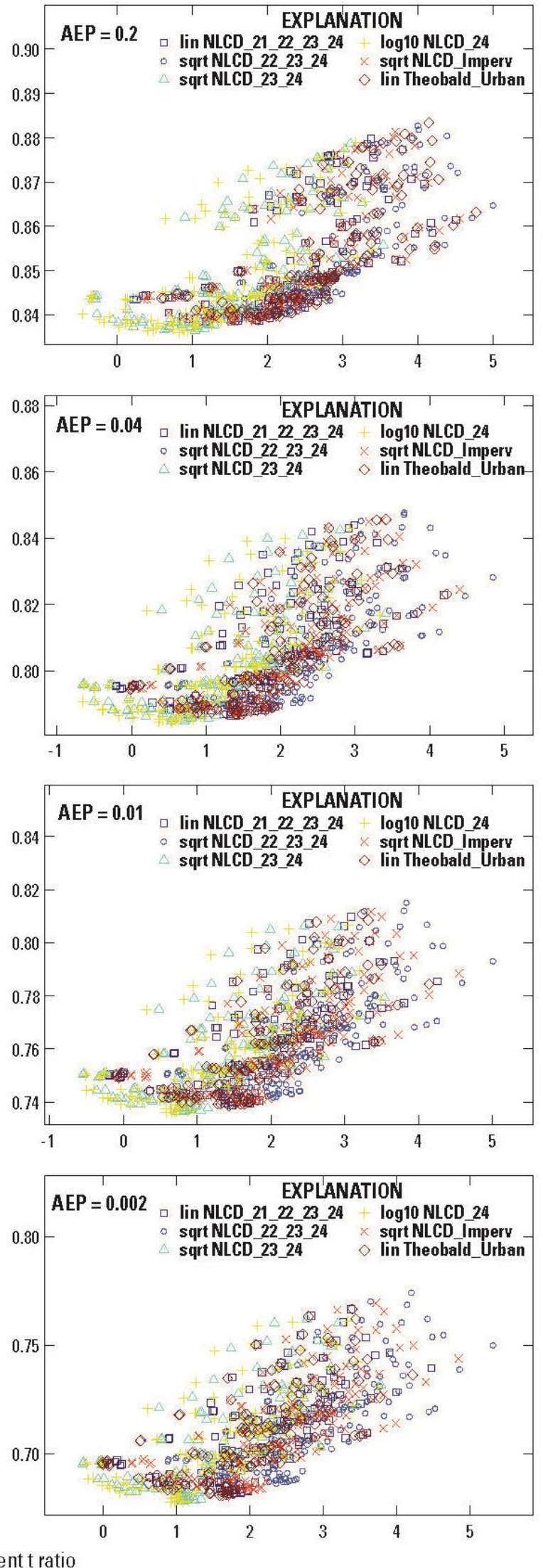


\section{$B$. Water and wetland variables}
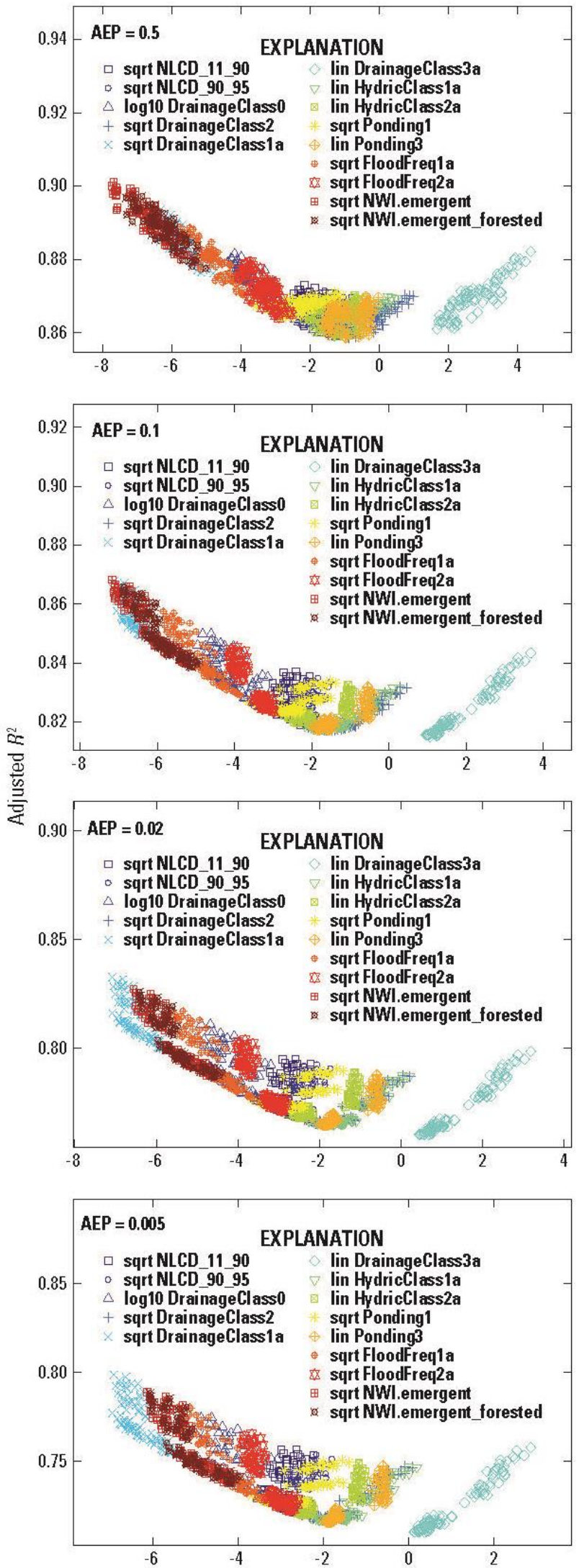
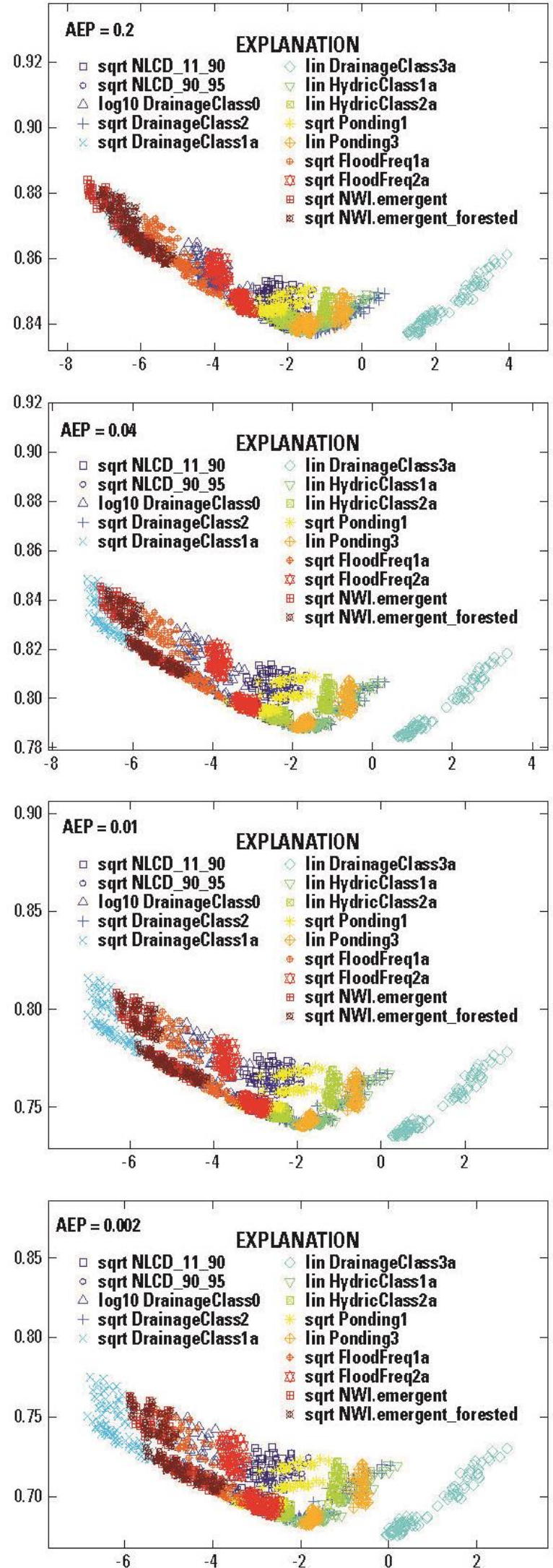

Coefficient t ratio 
C. Water and wetland variables
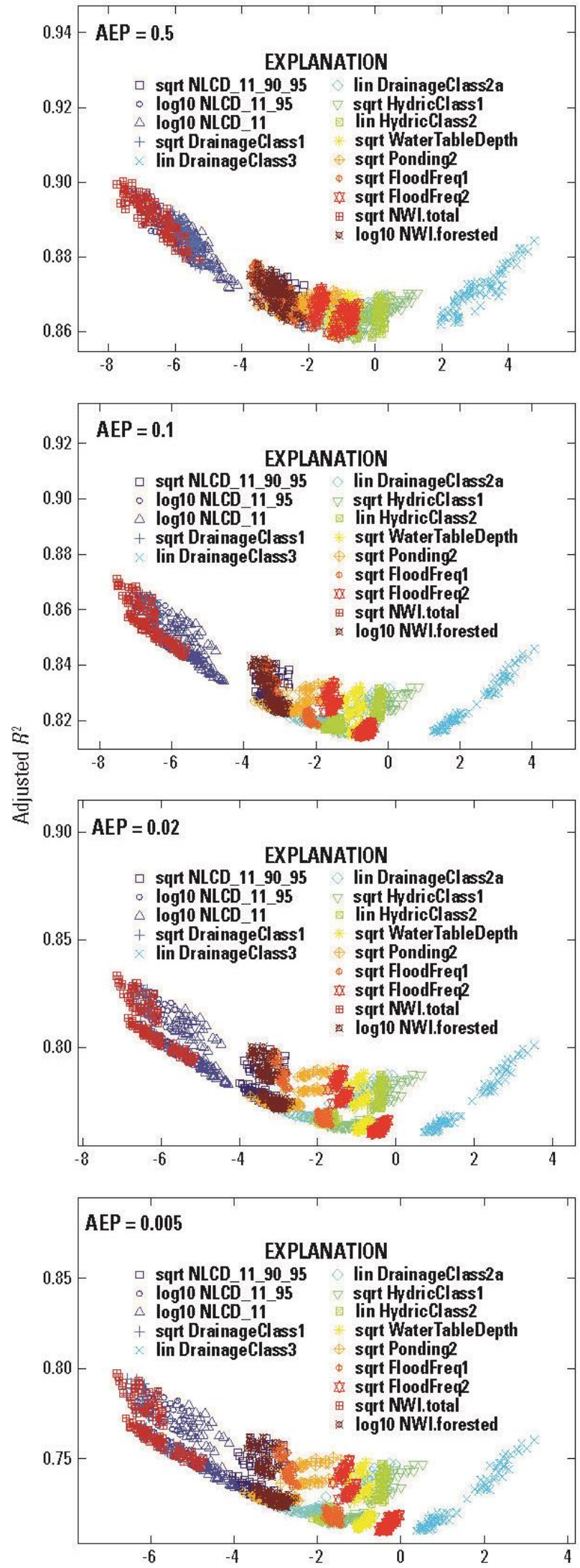
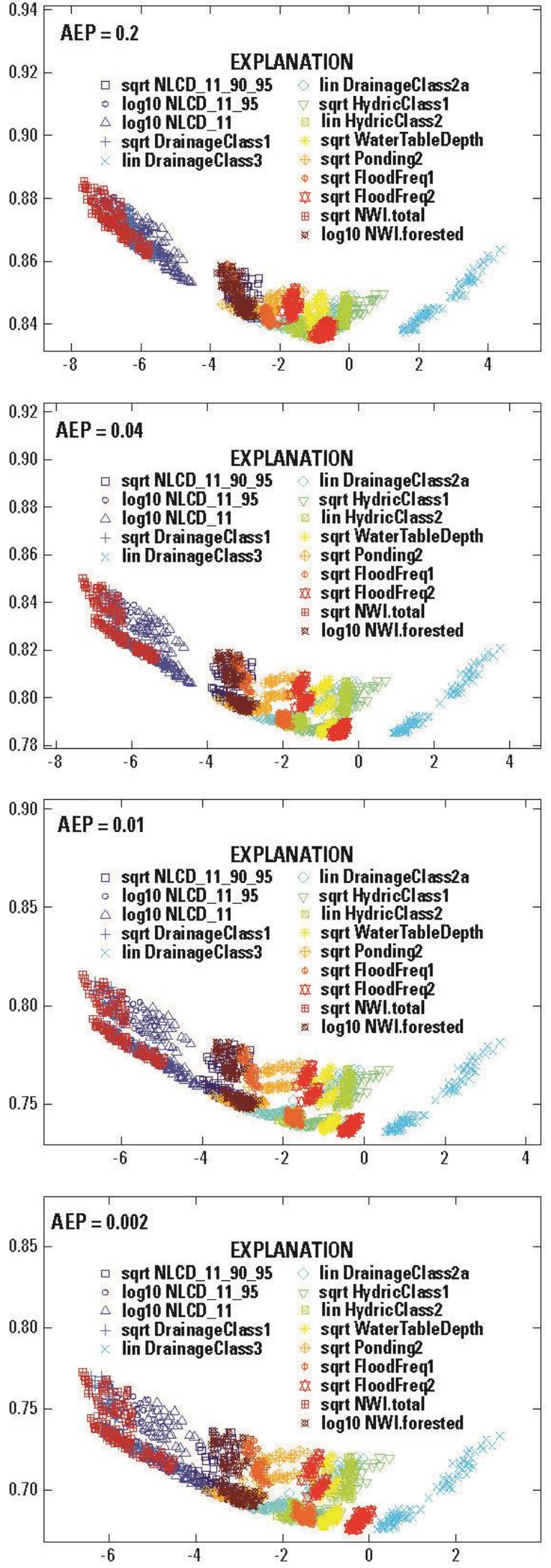

Coefficient t ratio 


\section{Slope variables}
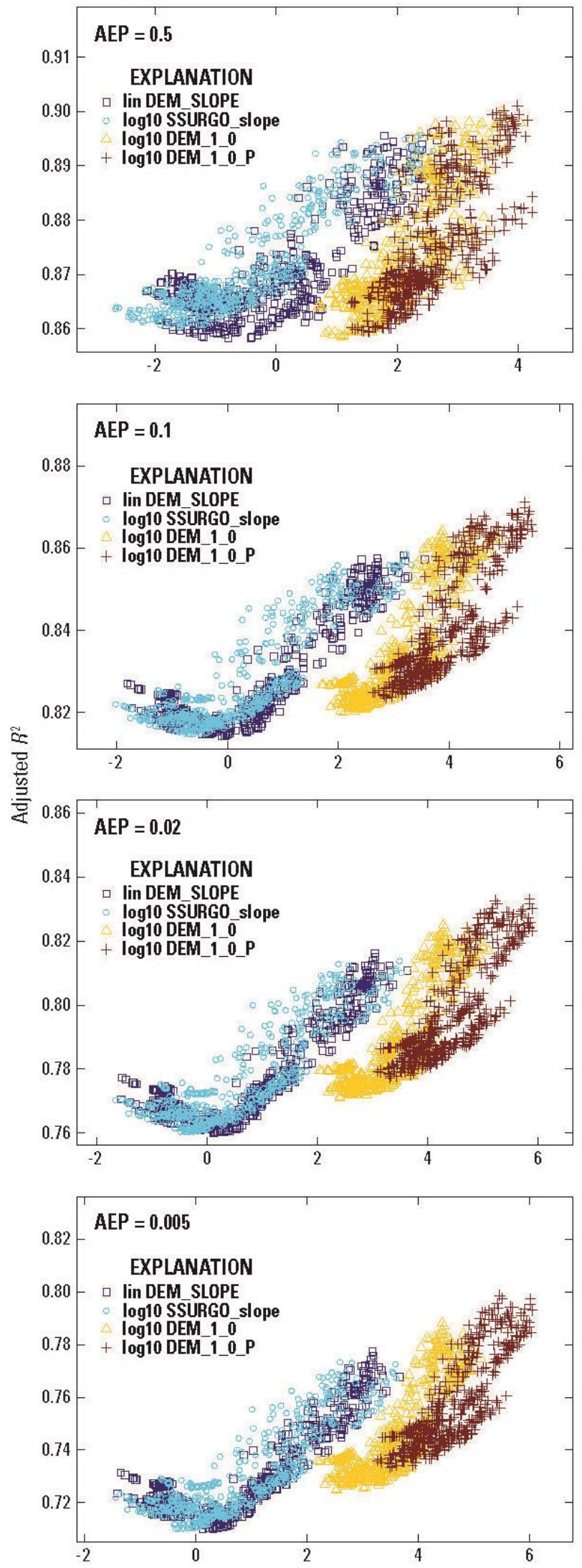
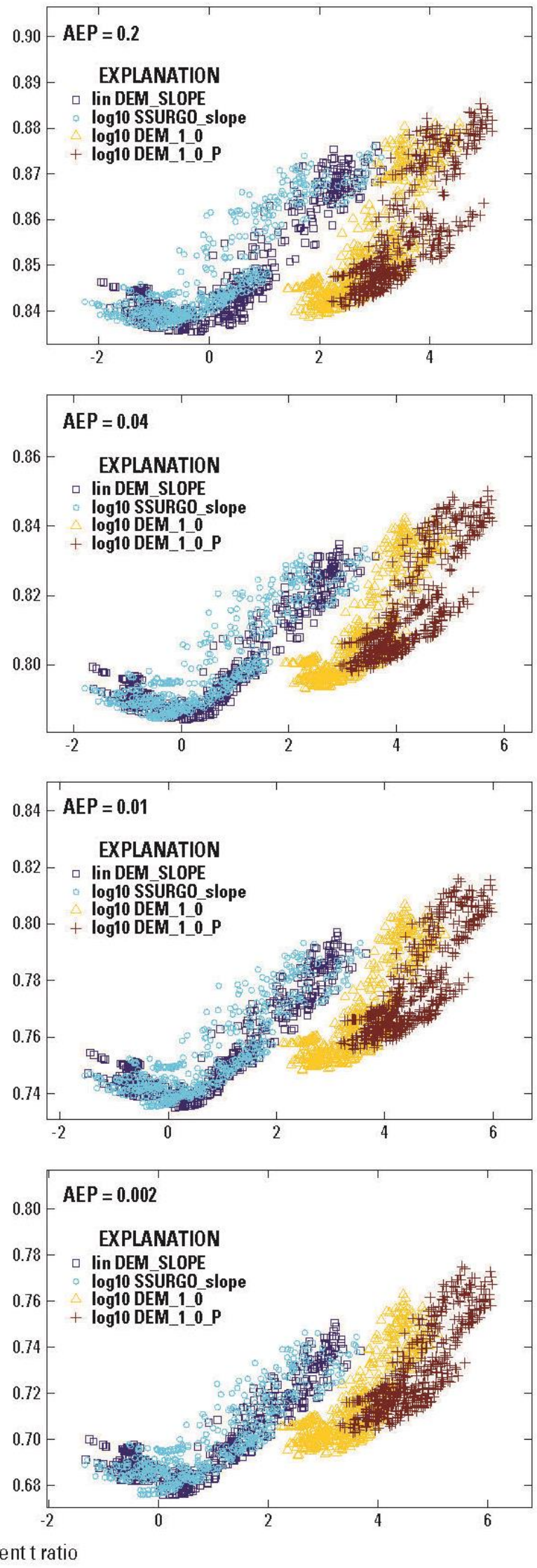
Figure 12. Plots by annual exceedance probability of adjusted $R^{2}$ as a function of the regression coefficient $t$ ratio values for basin characteristic categories considered in this study in northeastern Illinois: (a) urbanization variables; (b) and (c) water and wetland variables; and (d) slope variables. When the absolute value of the $t$ ratio is greater than 2 , then the coefficient is significantly different from zero at about the $a=0.05$ significance level (Helsel and Hirsch 2002, p. 238). The variable names in the figure explanations are defined in

Table 3; the strings preceding the variable names in the figure explanations indicate the transformation applied to the variable, where "lin" means "linear" (that is, no transformation),

"sqrt" means a square-root transformation, and "log10" means a $\log _{10}$ transformation.

\subsection{DEVELOPMENT OF FINAL SPATIAL REGRESSION EQUATIONS}

Following the selection of the most promising basin characteristics in each category by the WLS regression investigation, the final equations were computed with generalized least-squares (GLS) regression, which generalizes WLS regression by taking into account cross-correlations that arise among nearby streamgages with concurrent records. Stedinger and Tasker (1985) showed that GLS regression for regional spatial flood-frequency analysis provides more accurate coefficients and error estimates than OLS, with more modest improvement compared to WLS regression depending on the amount of cross-correlation among the streamgage records.

The GLS regression models were fitted with the function WREG.GLS from the package WREG (Farmer, 2017) in R version 3.6.3 (R Core Team, 2020). WREG.GLS implements a GLS approach to the estimation of peak discharge quantiles fit to a log-Pearson type III distribution using techniques described in Eng and others (2009) as developed by Stedinger and Tasker (1985), Tasker and Stedinger (1989), Griffis and Stedinger (2007), and Griffis and Stedinger (2009). The cross-correlations $\hat{\rho}_{i j}$ among streamgage records are estimated by selecting streamgage record pairs with a minimum number of concurrent years and fitting a function of the form:

$$
\hat{\rho}_{i j}=\theta^{d_{i j} /\left(\alpha d_{i j}+1\right)},
$$

where

$d_{i j}$ is the distance between streamgages $i$ and $j$, and

$\alpha$ and $\theta$ are dimensionless parameters fitted by a graphical method.

For this study the minimum number of concurrent years was taken as 45, and $\alpha$ and $\theta$ were taken as 0.005 and 0.975 , respectively.

When the selected water and wetland variables were used in the GLS regression analysis, it was found that use of DrainageClass1a gave a better fit than NWI.total from AEP 0.002 up to AEP 0.20 and had only a very small difference at AEP 0.50 , in the final equations, so DrainageClass1a is used for all AEPs in the final spatial regression equations. Values of the selected variables for each streamgage are given in Table 1 and their cross-correlations and variance inflation factors (VIFs) in Table 10. 
Table 10. Correlation matrix and variance inflation factor (VIF) values of basin characteristics selected for use in spatial regression analysis of 117 streamgages in northeastern Illinois and adjacent states.

\begin{tabular}{|c|c|c|c|c|}
\hline & $\log _{10}(D A)$ & $N L C D \_22 \_23 \_24^{1 / 2}$ & DrainageClass1a $a^{1 / 2}$ & $\log _{10}\left(D E M \_1 \_0 \_P\right)$ \\
\hline $\log _{10}(\mathrm{DA})$ & 1 & - & - & - \\
\hline$N L C D \_22 \_23 \_24^{1 / 2}$ & -0.156 & 1 & - & - \\
\hline${\text { DrainageClass } 1 a^{1 / 2}}$ & 0.288 & 0.221 & 1 & - \\
\hline $\log _{10}\left(D E M \_1 \_0 \_P\right)$ & -0.816 & 0.077 & -0.215 & 1 \\
\hline VIF & 3.4 & 1.12 & 1.19 & 3.02 \\
\hline
\end{tabular}

DA, drainage area, in mi²; NLCD_22_23_24, Sum of fractions of 2011 National Land Cover Dataset (NLCD) classes 22, 23, and 24; DrainageClass1a, sum of fractions of SSURGO fractions "very poorly drained" and "unknown (likely water)"; DEM_1_0_P, basin elevation range divided by basin perimeter in feet per mile; - , redundant value; VIF, variance inflation factor.

The general form of the selected equations follows in equation11:

$$
\begin{gathered}
\log _{10} Q_{p}=\left(b_{0}\right)_{p}+\left(b_{A}\right)_{p} \log _{10} A+\left(b_{U}\right)_{p} U^{1 / 2}+\left(b_{W}\right)_{p} W^{1 / 2}+\left(b_{S}\right)_{p} \log _{10} S, \\
p=0.50,0.20,0.10,0.05,0.02,0.01,0.005,0.002,
\end{gathered}
$$

where

$Q_{p}$ is the peak discharge quantile with weighted skew, in cubic feet per second, with annual exceedance

probability $p$,

$A$ is the drainage area, in square miles,

$U$ is the selected urbanization measure for the spatial equations NLCD_22_23_24 (a decimal fraction),

$W$ is the basin soil wetness measure DrainageClass1a (a decimal fraction),

$S$ is the basin slope measure $D E M \_1 \_0 \_P$ (basin elevation range divided by basin perimeter), in feet per mile, and

$\left(b_{0}\right)_{p},\left(b_{A}\right)_{p},\left(b_{U}\right)_{p},\left(b_{W}\right)_{p}$, and $\left(b_{S}\right)_{p}$ are coefficients estimated by using WREG (Table 11$)$.

After inverting the logarithmic transformation, the $Q_{p}$ prediction equation 12 is the result:

$$
\begin{gathered}
Q_{p}=10^{\left(b_{0}\right)_{p}} A^{\left(b_{A}\right)_{p}} 10^{\left(b_{U}\right)_{p} U^{1 / 2}} 10^{\left(b_{W}\right)_{p} W^{1 / 2}} S^{\left(b_{S}\right)_{p}}, \\
p=0.50,0.20,0.10,0.05,0.02,0.01,0.005,0.002,
\end{gathered}
$$


Table 11. Coefficients of the selected spatial regression equations in this study in northeastern Illinois.

\begin{tabular}{ccccccccccc}
\hline $\begin{array}{c}\text { Annual } \\
\text { exceedance } \\
\text { probability }\end{array}$ & $\boldsymbol{b}_{\boldsymbol{0}}$ & $\mathrm{SE}\left(\boldsymbol{b}_{\mathbf{0}}\right)$ & $\boldsymbol{b}_{\boldsymbol{A}}$ & $\mathrm{SE}\left(\boldsymbol{b}_{\boldsymbol{A}}\right)$ & $\boldsymbol{b}_{U}$ & $\mathrm{SE}\left(\boldsymbol{b}_{U}\right)$ & $\boldsymbol{b}_{\boldsymbol{w}}$ & $\mathrm{SE}\left(\boldsymbol{b}_{\boldsymbol{W}}\right)$ & $\boldsymbol{b}_{\boldsymbol{S}}$ & $\mathbf{S E}\left(\boldsymbol{b}_{\boldsymbol{S}}\right)$ \\
\hline 0.50 & 1.496 & 0.133 & 0.784 & 0.043 & 0.268 & 0.070 & -0.777 & 0.150 & 0.300 & 0.101 \\
0.20 & 1.691 & 0.137 & 0.777 & 0.045 & 0.212 & 0.074 & -0.878 & 0.156 & 0.371 & 0.105 \\
0.10 & 1.783 & 0.144 & 0.774 & 0.047 & 0.198 & 0.079 & -0.925 & 0.164 & 0.408 & 0.110 \\
0.04 & 1.873 & 0.156 & 0.772 & 0.051 & 0.195 & 0.088 & -0.972 & 0.178 & 0.447 & 0.119 \\
0.02 & 1.925 & 0.166 & 0.771 & 0.054 & 0.200 & 0.096 & -1.001 & 0.189 & 0.472 & 0.127 \\
0.01 & 1.968 & 0.176 & 0.771 & 0.057 & 0.207 & 0.103 & -1.025 & 0.201 & 0.494 & 0.135 \\
0.005 & 2.005 & 0.187 & 0.771 & 0.060 & 0.216 & 0.110 & -1.046 & 0.213 & 0.514 & 0.144 \\
0.002 & 2.045 & 0.200 & 0.773 & 0.065 & 0.230 & 0.119 & -1.071 & 0.227 & 0.539 & 0.155 \\
\hline
\end{tabular}

$b_{0}$, intercept; $b_{A}$, coefficient of $\log _{10}$ of drainage area in square miles; $b_{U}$, coefficient of square root of urbanization measure $N L C D \_22 \_23 \_24$ as a decimal fraction; $b_{W}$, coefficient of square root of basin wetness measure DrainageClass 1 a as a decimal fraction; $b_{S}$, coefficient of $\log _{10}$ of slope measure DEM_1_0_P as a percentage; SE, standard error.

\subsection{ACCURACY OF FINAL SPATIAL REGRESSION EQUATIONS}

Several measures of the average accuracy of the final GLS spatial regression equations are presented in Table 12, along with comparative values of the standard model error as a percentage from the corresponding WLS equations and the previous studies by Allen and Bejcek (1979) and Soong et al. (2004). All these measures are averages for all the streamgages in the analysis. The pseudo $\mathrm{R}^{2}$ measures the fraction of variability in the dependent variable that is explained by the regression model after removing the effect of the time-sampling error (Eng et al. 2009) and is analogous to the standard $\mathrm{R}^{2}$. The average variance of prediction includes both the model error and time-sampling error components and indicates the expected accuracy of a prediction at an ungaged location with basin characteristics that are near the centroid of the basin characteristics used in the GLS analysis. The standard model error indicates the accuracy of the model fit without the component in the average variance of prediction that indicates the additional error of making a prediction at an ungaged location; as a result it is always somewhat smaller than the average variance of prediction. All the accuracy measures decrease as the AEP decreases, as expected, because of the fewer data available for less frequent events. 
Table 12. Measures of the average accuracy of the selected spatial regression equations in this study in northeastern Illinois.

\begin{tabular}{|c|c|c|c|c|c|c|c|c|}
\hline $\begin{array}{c}\text { Annual } \\
\text { exceedance } \\
\text { probability }\end{array}$ & $\begin{array}{c}\text { Pseudo } \\
\mathbf{R}^{2} \\
\text { (percent) } \\
\end{array}$ & $\begin{array}{l}\text { Average } \\
\text { variance } \\
\text { of } \\
\text { prediction } \\
\text { (log units) }\end{array}$ & $\begin{array}{l}\text { Average } \\
\text { standard } \\
\text { error of } \\
\text { prediction } \\
\text { (percent) }\end{array}$ & $\begin{array}{c}\text { Model } \\
\text { error } \\
\text { variance } \\
V^{2} \text { (log } \\
\text { units) } \\
\end{array}$ & $\begin{array}{l}\text { Standard } \\
\text { model } \\
\text { error } \\
\text { (percent) }\end{array}$ & $\begin{array}{c}\text { Standard } \\
\text { model } \\
\text { error } \\
\text { (percent), } \\
\text { WLS fit }\end{array}$ & $\begin{array}{c}\text { Standard error } \\
\text { of estimate } \\
\text { (percent) from } \\
\text { Allen and } \\
\text { Bejcek (1979), } \\
\text { Table } 4 \\
\end{array}$ & $\begin{array}{l}\text { Standard model } \\
\text { error from } \\
\text { Soong et al. } \\
\text { (2004), table } 4 \\
\text { (applicable to } \\
\text { regions 2, 6, } \\
\text { and 7) } \\
\end{array}$ \\
\hline 0.50 & 87.5 & 0.0362 & 46.0 & 0.0341 & 44.5 & 45.2 & 36 & 39.1 \\
\hline 0.20 & 85.6 & 0.0384 & 47.5 & 0.0360 & 45.9 & 46.3 & 38 & 39.3 \\
\hline 0.10 & 83.9 & 0.0414 & 49.6 & 0.0387 & 47.7 & 48.6 & 40 & 40.6 \\
\hline 0.04 & 81.0 & 0.0473 & 53.4 & 0.0439 & 51.2 & 52.4 & 43 & 43.1 \\
\hline 0.02 & 78.6 & 0.0523 & 56.6 & 0.0485 & 54.2 & 55.8 & 45 & 45.2 \\
\hline 0.01 & 76.5 & 0.0571 & 59.5 & 0.0528 & 56.8 & 59.4 & 48 & 47.3 \\
\hline 0.005 & 74.2 & 0.0622 & 62.5 & 0.0573 & 59.6 & 63.2 & - & - \\
\hline 0.002 & 71.7 & 0.0681 & 66.0 & 0.0624 & 62.6 & 68.5 & 52 & 52.8 \\
\hline
\end{tabular}

$\mathrm{R}^{2}$, coefficient of determination; WLS, weighted least squares.

Techniques for computing accuracy estimates at individual locations (streamgages or ungaged locations) are also available, including the variance and standard error of prediction and confidence interval values. According to Hodge and Tasker (1995), the variance of prediction $V_{i}$ at an individual location based on a GLS regression analysis is:

$$
V_{i}=\gamma^{2}+x_{i}\left(X^{t} \Lambda^{-1} X\right)^{-1} x_{i}^{t}
$$

where

$\gamma^{2}$ is the model error variance (Table 12),

$x_{i}=\left[1, \log _{10} A_{i}, U_{\text {spat }, i}^{1 / 2}, W_{i}^{1 / 2}, \log _{10} S_{i}\right]$ is a row vector specifying the basin characteristics of the individual location augmented with a 1 ,

$X$ is a $(n \times p)$ matrix whose rows are the transformed basin characteristics for each streamgage used in the GLS model augmented by a 1 ( $n=117$ is the number of streamgages and $p=5$ is the number of basin characteristics plus 1 ),

$\Lambda^{-1}$ is the matrix inverse of $\Lambda$, the $(n \times n)$ covariance matrix used in the GLS regression analysis,

$X^{t}$ is the matrix transpose of $X$, and

$x_{i}^{t}$ is the matrix transpose of $x_{i}$.

The $\left(X^{t} \Lambda^{-1} X\right)^{-1}$ matrices for the selected AEPs are given in Table 13. 


\section{Table 13. Components of variance of prediction for the} selected spatial regression equations in northeastern Illinois.

[See Appendix 8.]

The variance of prediction $V_{i}$ can be converted to a standard error of prediction in log units by taking the square root; that is, $S_{i}=V_{i}^{1 / 2}$ and in percentage units by using the following formula (Eng et al. 2009):

$$
S_{p i}=100\left\{\exp \left[(\ln 10)^{2} V_{i}\right]-1\right\}^{1 / 2},
$$

The confidence intervals of the predicted discharge quantile $Q_{i}$ are computed as:

$$
\log _{10} Q_{i} \pm t_{\alpha / 2, n-p} S_{i}
$$

where $t_{\alpha / 2, n-p}$ is the critical value of the $t$ distribution at the alpha level $\alpha$ (for example, $\alpha=0.05$ for $90 \%$ confidence intervals) and $n-p$ degrees of freedom, where $n=117$ is the number of streamgages used in the spatial regressions and $p=5$ is the number of basin characteristics plus 1.

After inverting the logarithmic transformation, the interval is:

$$
\left\lfloor Q_{i} 10^{-t_{\alpha / 2, n-p} S_{i}}<Q_{i}<Q_{i} 10^{t_{\alpha / 2, n-p} S_{i}}\right\rfloor .
$$

In equation (16), $Q_{i}$ is the median prediction, and $Q_{i} 10^{t_{\alpha / 2, n-p} S_{i}}$ and $Q_{i} 10^{-t_{\alpha / 2, n-p} S_{i}}$ are the upper and lower $1-2 \alpha$ confidence limits, respectively. For example, for $\alpha=0.05$ (therefore, $90 \%$ confidence limits), according to the data and methods used in this study, the probability that the true value of the discharge quantile exceeds the upper limit $Q_{i} 10^{t_{\alpha / 2, n-p} S_{i}}$ is $\alpha=0.05$; likewise, the probability that the true value of the discharge quantile is less than the lower limit $Q_{i} 10^{-t_{\alpha / 2, n-p} S_{i}}$ is $\alpha=0.05$, so that the probability that the true value lies between the limits is $90 \%$. 


\section{CHAPTER 5: APPLICATIONS OF REGRESSION EQUATIONS}

There are two sets of regression equations presented in this report, the temporal (equation 7 and table 9) and the spatial (equation 12 and table 11). In both sets, the values of the urbanization coefficients are positive, as expected, and the urbanization coefficients from the temporal analysis decrease substantially as the peak discharge quantiles increase; that is, with decreasing AEP. The changes in the urbanization coefficients from the spatial regression equations as a function of AEP are more modest and have a minimum at $A E P=0.1$, with increases toward both smaller and larger AEP values; in fact, the largest urbanization coefficient occurs at $A E P=0.002$. The increase of the urbanization coefficients from the spatial analysis for decreasing AEPs beyond AEP $=0.1$ does not agree with the expectation that the effect of urbanization decreases with decreasing AEP and therefore is considered anomalous. Reasons for this anomaly and implications for applications of the spatial equations are discussed below.

Because the urbanization measures to which these coefficients apply are different but approximately commensurate (Figure 13), a direct comparison of their implications regarding the effect of urbanization can be considered as meaningful. Plots of the ratios of peak discharge quantiles for the different AEPs considered here are shown in Figure 14. These plots show that except for very small amounts of urbanization beginning with a completely rural basin, the larger coefficients from the temporal analysis imply larger effects of urbanization, especially for the larger AEPs (smaller floods). They also show the effect of the minimum value of the spatial coefficient occurring at $A E P=0.1$; for example, the largest effect of urbanization in the spatial curves is seen for AEP $=0.002$. 


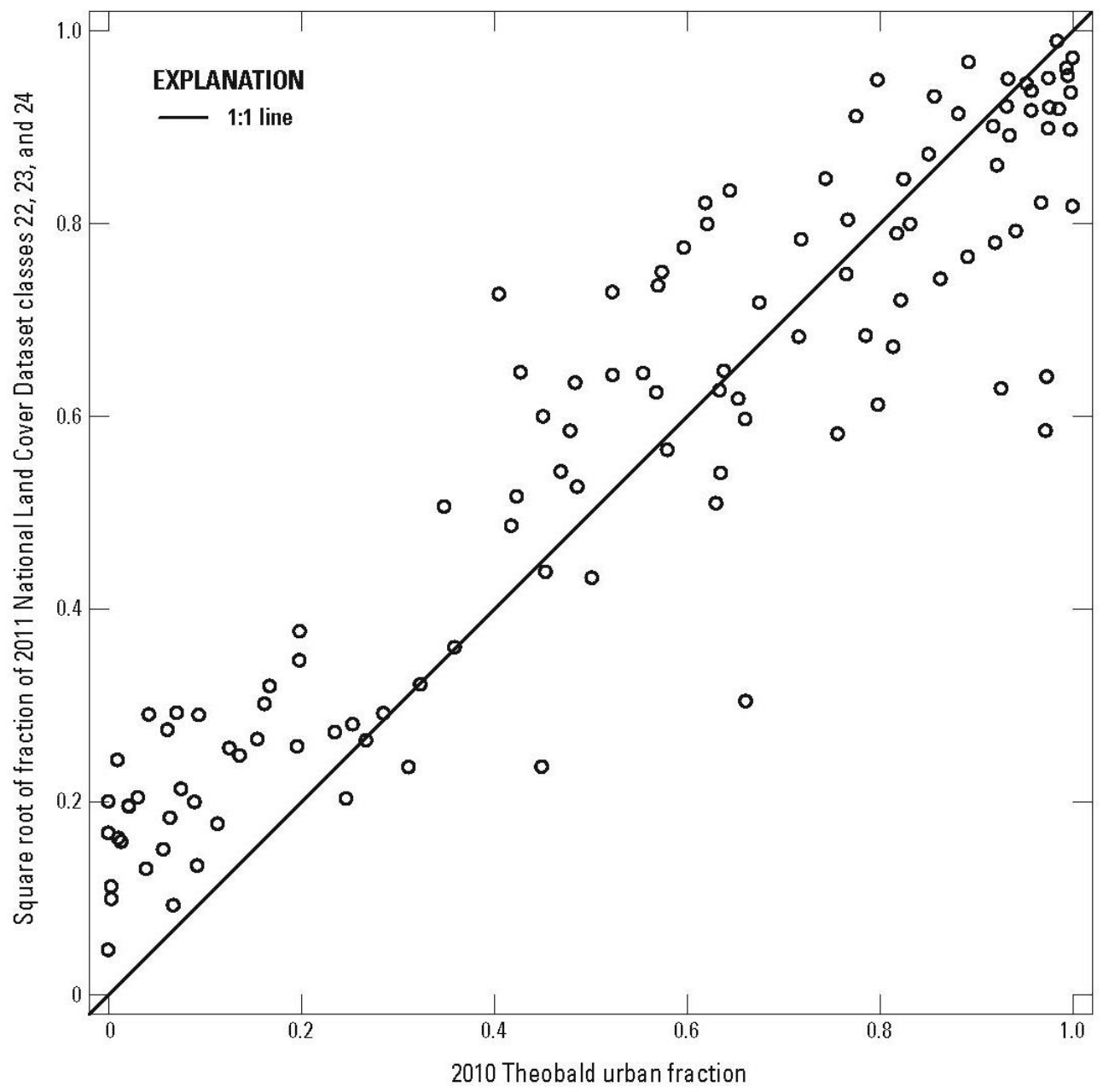

Figure 13. Comparison of square root of fraction of 2011 National Land Cover Dataset (NLCD, Jin et al. 2013) classes 22, 23, and 24 (NLCD_22_23_24 ${ }^{1 / 2}$ ) and 2010 Theobald urban fraction (Theobald 2005) for 117 basins used in this study in northeastern Illinois. 


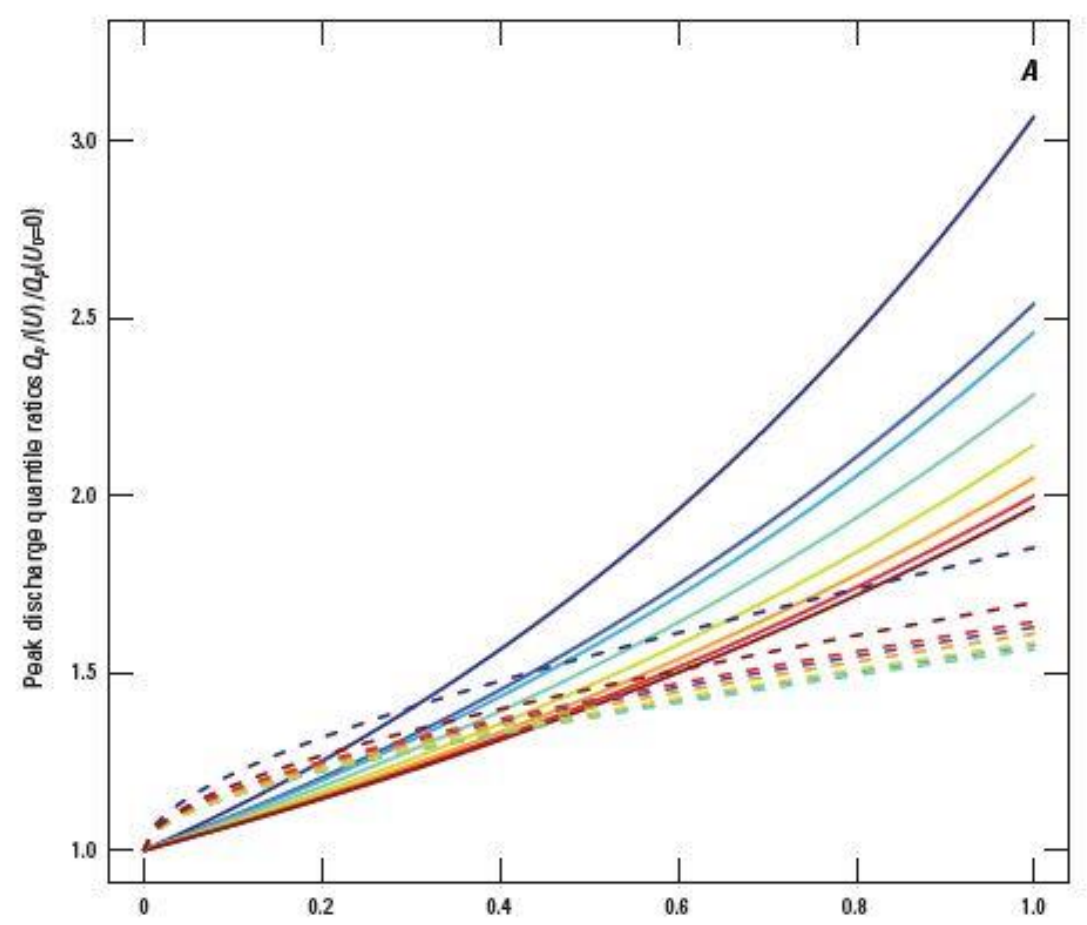

--- Spatial regression, $p=0.2$

- - Spatial regression, $p=0.1$

- Spatial regression, $p=0.04$

- Spatial regression, $p=0.02$

- Spatial regression, $p=0.01$

- - - Spatial regression, $p=0.005$

--- Spatial regression, $p=0.002$

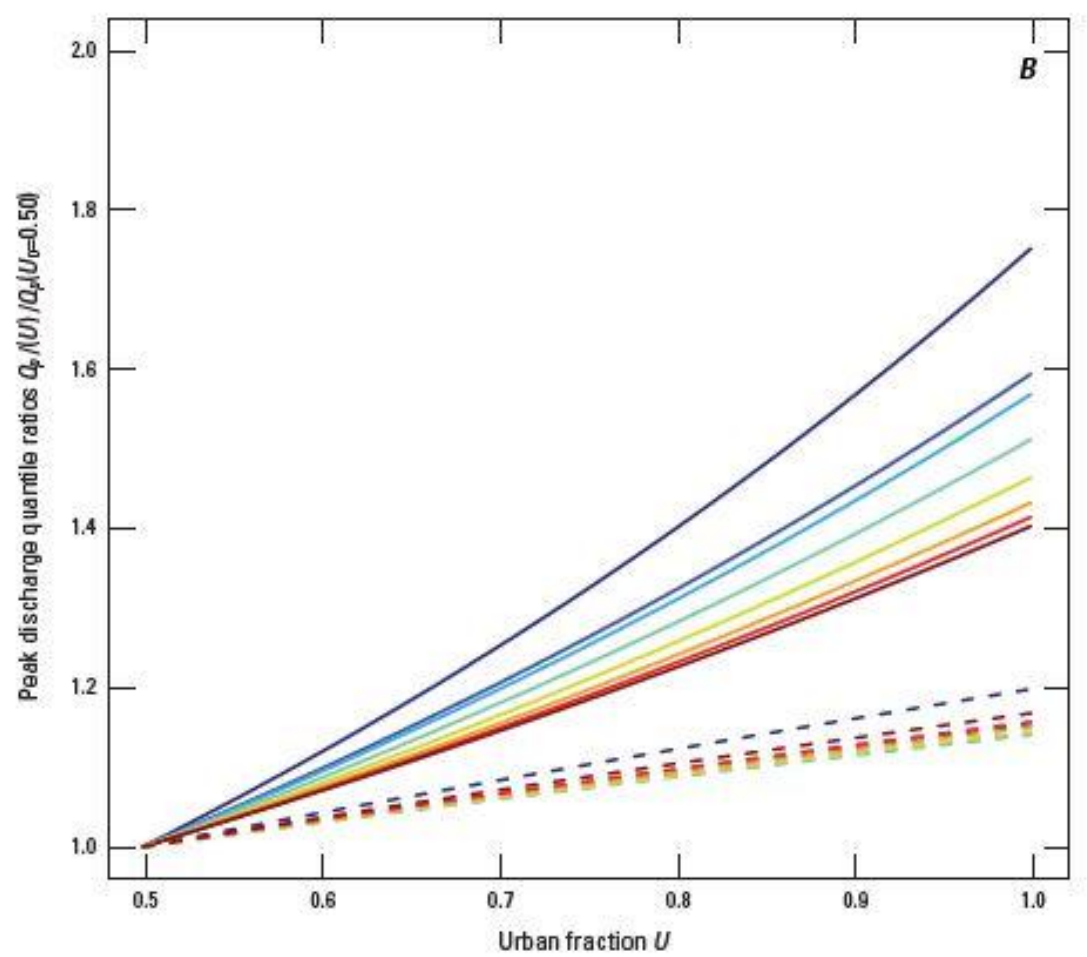

Figure 14. Ratios of peak discharge quantiles $Q_{p}(U) / Q_{p}\left(U_{0}\right)$ as a function of urban fraction $U$ for selected annual exceedance probabilities (AEPs) $p$ as implied by the two sets of urbanization coefficients obtained in this study in northeastern Illinois: (a) basin initially having no urbanization $\left(U_{0}=0\right)$; (b) basin initially $50 \%$ urbanized $\left(U_{0}=0.5\right)$. 
The urbanization coefficients in the temporal equations are estimated by a temporal analysis; that is, directly from the changes in the annual peak discharges as the basins urbanize. In the spatial equations, the urbanization coefficients are not computed from observed changes in the gaged basins, but indirectly, from the differences in flood-discharge quantiles among basins of different levels of urbanization. When applying flood-discharge regression equations, one is typically interested in the behavior at an ungaged location, and this spatial transfer of information is entirely appropriate. When it comes to urbanization, however, the primary interest is the temporal question: How did or how will the flood-discharge quantiles change when the basin is urbanized? Because they are based on a direct analysis of the results of this process, the temporal urbanization coefficients are more likely to reflect the effects of urbanization on a given basin.

Two general statistical issues, at least, that affect the values of regression coefficients in a multiple regression framework also need to be considered when the value of the coefficients is of interest, as in this study with respect to the urbanization coefficients. These issues are (1) omitted variable bias (OVB) and (2) measurement errors in the explanatory variables. The issue of OVB arises when an omitted variable is correlated with both the variable of interest and the dependent variable (Greene 1997). In this case, an omitted variable will cause a negative or positive bias in the coefficient of the variable of interest, though it does not bias the predictions of the equations. In the context of the present analysis, if there is some variable important in predicting the flood-discharge properties that is left out of the spatial or temporal analyses but is correlated with urbanization, the urbanization coefficient will not have its correct value. Measurement errors in an explanatory variable cause attenuation (reduction of the absolute value) of the variable, again without biasing the prediction of the equations (Fuller 1987).

It is possible that OVB is affecting either or both sets of coefficients, but because the urbanization coefficients from the spatial analysis are significantly smaller than those from the temporal analysis and measurement error always causes a reduction in positive coefficients, whereas OVB may cause an error in either direction, the first hypothesis that the results suggest is that the spatial urbanization coefficients are subject to a measurement error. In particular, if the relation between urbanization and its effects on peak discharges varies among basins because of different development practices related to stormwater and flood control, this variation would induce a measurement error relative to the hydrologically effective urbanization value. Such an error would, in addition, affect the spatial analyses more than the temporal ones because the temporal analyses depend only on the changes in time of the peak discharges with interbasin differences captured in the segment intercepts, whereas the spatial analyses depend directly on the values of the peak discharges.

Given this concern regarding the accuracy of the urbanization coefficients from the spatial models (which nevertheless does not bias the predictions) and the previous assertion that the basic question when it comes to the effect of urbanization is a temporal one, it seems clearly preferable to use temporal equations to predict the effect of urbanization where possible. It is not possible to use the temporal equations for adjustment of rural estimates in ungaged basins in region 2 because there are not enough truly rural basins to develop rural-only equations unaffected by urbanization (Figure 4). Therefore, to obtain flood-quantile estimates for ungaged basins in region 2 , the spatial regression 
equations should be used. But for situations where peak discharge quantile estimates are being adjusted for the effects of urbanization, the temporal equations should be used. Such situations include ungaged basins outside region 2, assuming urbanization has proceeded similarly as for the gaged basins analyzed in this study. Such situations may also include the prediction of future effects of urbanization for basins in region 2, again assuming urbanization will proceed as it did for the basins analyzed in this study. These situations are discussed in more detail in the Applications of the Temporal Urbanization Coefficients section.

\subsection{APPLICATIONS OF THE SPATIAL REGRESSION EQUATIONS}

The details of application of the updated Illinois region 2 spatial regression equations depend on the nearness of the location of interest to a streamgage. If the location of interest is far from a streamgage, the spatial regression equations are used. If the location is at a streamgage, a weighted average of the quantiles from regional equations and those from the streamgage record itself is used. If the location is near a streamgage, a weighted average of the quantiles from regional equations and an adjusted version of those from the streamgage record itself is used. The details of the different applications are described in this section.

\subsubsection{Ungaged Locations Far from a Streamgage}

For a general ungaged location in Illinois flood-frequency region 2, the final, real-space regression equation 12 should be used. The USGS StreamStats application (http://streamstats.usgs.gov) could be used to select the location of interest, delineate the basin, and compute the basin characteristics and the peak discharge quantiles.

It is important to realize that the regression equations represented by equations 12 apply only within the ranges of the basin characteristics used to fit the equations. These ranges are given in Table 14. StreamStats will not enforce this limitation, though it does provide a warning.

Table 14. Ranges of basin characteristic values of streamgages used to fit selected spatial regression equations in this study in northeastern Illinois.

\begin{tabular}{lccccc}
\hline Basin characteristic & StreamStats name & Units & Minimum & Maximum & Median \\
\hline Drainage area & DRNAREA & square miles & 0.078 & 1,351 & 13.6 \\
NLCD_22_23_24 & FLC11DVLMH & decimal fraction & 0.0022 & 0.979 & 0.391 \\
Theobald_Urban & URBTHE2010 & decimal fraction & 0.000 & 1.00 & 0.580 \\
DrainageClass1a & FSSURGDC78 & decimal fraction & 0.000 & 0.256 & 0.0511 \\
DEM_1_0_P & RELRELF & feet per mile & 0.821 & 37.2 & 4.79 \\
\hline
\end{tabular}

NLCD_22_23_24, fraction of 2011 National Land Cover Database (NLCD) classes 22, 23, and 24 (low, medium, and high intensity developed); Theobald_Urban, fraction of 2010 Theobald (2005) classes 7-10 (housing with no more than 10 acres per unit plus commercial/industrial/ transportation land use); DrainageClass1a, Soil Survey Geographic (SSURGO) Database fraction "very poorly drained" and "unknown (likely water)"; DEM_1_0_P, basin elevation range divided by basin perimeter. 


\subsubsection{At a Streamgage}

Because of the finite length of streamgage records, the regional equations can improve the accuracy of the peak discharge quantile estimates at streamgages by incorporating regional information. The procedure recommended by Cohn et al. 2012 (see also Tasker 1975) is to compute this peak discharge quantile from the weighted average of the regression-equation estimate and the result of the frequency analysis of the streamgage record, where the weights are the inverses of the variance of each of the discharge estimates. The weighted discharges are computed with the following equation:

$$
\log _{10}\left(Q_{p}\right)_{g, w}=\frac{\left(V_{p}\right)_{g, r} \log _{10}\left(Q_{p}\right)_{g, s}+\left(V_{p}\right)_{g, s} \log _{10}\left(Q_{p}\right)_{g, r}}{\left(V_{p}\right)_{g, r}+\left(V_{p}\right)_{g, s}},
$$

where

$\left(Q_{p}\right)_{g, w}$ is the weighted peak discharge quantile at the streamgage for an AEP of $p$,

$\left(Q_{p}\right)_{g, s}$ is the peak discharge quantile for an AEP of $p$ computed from the streamgage record by using EMA with weighted skew as described in the Frequency Analysis section,

$\left(Q_{p}\right)_{g, r}$ is the peak discharge quantile for the selected AEP obtained from the regression equations 12 applied at the streamgage,

$\left(V_{p}\right)_{g, r}$ is the variance of prediction of $\left(Q_{p}\right)_{g, r}$ for the an AEP of $p$ computed by using equation 13 , and

$\left(V_{p}\right)_{g, s}$ is the variance of prediction of $\left(Q_{p}\right)_{g, s}$ for the selected AEP computed by PeakFQ as part of the frequency analysis.

The values of $\left(Q_{p}\right)_{g, s},\left(Q_{p}\right)_{g, r}$, and $\left(Q_{p}\right)_{g, w}$ for both the redundant and nonredundant stations in this study are tabulated in Table 2, in the third, fourth, and fifth rows, respectively, for each streamgage. The variance of prediction for the weighted discharge $\left(Q_{p}\right)_{g, w}$ is given by:

$$
\left(V_{p}\right)_{g, w}=\left(V_{p}\right)_{g, s}\left(V_{p}\right)_{g, r} /\left\lfloor\left(V_{p}\right)_{g, s}+\left(V_{p}\right)_{g, r}\right\rfloor,
$$

(Tasker 1975). With this value of $\left(V_{p}\right)_{g, w}$, the standard error of prediction $\left(S_{p}\right)_{g, w}$ can be computed as $\left(S_{p}\right)_{g, w}=\left(V_{p}\right)_{g, w}^{1 / 2}$, and then equations 14-16 can be used to compute the standard error of prediction in percentage units and confidence intervals. 


\subsubsection{Ungaged Location Near a Streamgage}

If the ungaged location of interest is near to and on the same stream as a streamgage, the accuracy of the flood-discharge quantile estimate at the ungaged location can be improved if the estimate from the regional equation is combined with the estimate at the streamgage (Ries 2007). There are a few different methods in the literature for this adjustment; the method used here follows that of Soong et al. (2004) (as corrected August 10, 2010), which is the same as the method presented in the IDOT Drainage Manual (Drainage Manual Committee 2011). According to this method, the near-gage adjustment has an effect only if the ratio $A_{u} / A_{g}$ of the drainage area of the ungaged basin of interest $A_{u}$ to that of a gaged basin $A_{g}$ is between 0.5 and 1.5 (see equation 19); this constraint on the effect of the adjustment method defines being "near" a streamgage.

First define the adjustment weighting factor $w_{a}$, which is given by:

$$
w_{a}=\left\{\begin{array}{c}
2\left|\left(A_{u} / A_{g}\right)-1\right| \text { if } 0.5<\left(A_{u} / A_{g}\right)<1.5 \\
1 \text { otherwise },
\end{array}\right.
$$

where

$A_{u}$ is the drainage area at the ungaged location of interest, and

$A_{g}$ is the drainage area at the streamgage.

The near-gage adjustment equation can then be written as follows:

$$
\left(Q_{p}\right)_{u, w}=w_{a}\left(Q_{p}\right)_{u, r}+\left(1-w_{a}\right)\left(Q_{p}\right)_{g, w}\left(A_{u} / A_{g}\right),
$$

where

$\left(Q_{p}\right)_{u, w}$ is the gage-adjusted flood-discharge quantile estimate for an AEP of $p$ at the ungaged location of interest,

$\left(Q_{p}\right)_{u, r}$ is the flood-discharge quantile estimate for an AEP of $p$ at the ungaged location of interest from the spatial regression equations (equation 12),

$\left(Q_{p}\right)_{g, w}$ is the flood-discharge quantile estimate for an AEP of $p$ at the gage, weighted with the spatial regression equations (equation 17, Table 2), and

$w_{a}, A_{u}$, and $A_{g}$ are as defined for equation 19.

According to equations 19 and 20, the near-gage adjustment has no effect when $A_{u} / A_{g} \leq 0.5$ or $A_{u} / A_{g} \geq 1.5$ because $w_{a}=1$ (equation 19) and therefore (equation 20) the near-gage adjusted value $\left(Q_{p}\right)_{u, w}$ is identical to the value $\left(Q_{p}\right)_{u, r}$ from the regional regression equations. At a streamgage, 
$A_{u}=A_{g}$ and the near-gage adjusted value reduces to the weighted flood-discharge at the gage $\left(Q_{p}\right)_{g, w}$ because $w_{a}=0$. When $0.5<A_{u} / A_{g}<1.5$, then both $\left(Q_{p}\right)_{u, r}$ and $\left(Q_{p}\right)_{g, w}$ contribute to the value of $\left(Q_{p}\right)_{u, w}$ according to the values of the weights $w_{a}$ and $1-w_{a}$.

\subsection{APPLICATIONS OF THE TEMPORAL URBANIZATION COEFFICIENTS}

As discussed, there are two situations where the temporal urbanization coefficients may be appropriate for estimating the effects of urbanization, if it is the judgment of the analyst that the hydrologic effects of urbanization are or will be similar to those of the basins analyzed in this study: (1) to adjust the prediction of rural regression equations outside of Illinois flood-frequency region 2 for urbanization, and (2) to predict the future effects of urbanization for any basin in Illinois.

For either situation, the urbanization-adjusted flood-discharge quantile $Q_{p}(U)$ is given by:

$$
Q_{p}(U)=Q_{p}\left(U_{0}\right) 10^{\left(b_{U}\right)_{p}\left(U-U_{0}\right)},
$$

or, in log-transformed form:

$$
\log _{10} Q_{p}(U)=\log _{10} Q_{p}\left(U_{0}\right)+\left(b_{U}\right)_{p}\left(U-U_{0}\right),
$$

where

$Q_{p}(U)$ is the peak discharge quantile at the location of interest with AEP $p$ adjusted to correspond to Theobald (2005) urbanization fraction $U$,

$Q_{p}\left(U_{0}\right)$ is the peak discharge quantile with AEP $p$ at the initial urbanization fraction $U_{0}$, and $\left(b_{U}\right)_{p}$ is the temporal urbanization coefficient for AEP $p$ from Table 9.

The urbanization-adjustment factors $10^{\left(b_{U}\right)_{p}\left(U-U_{0}\right)}$ in equation 21 are the same ratios $Q_{p}(U) / Q_{p}\left(U_{0}\right)$ as are plotted with solid lines in Figure14a and b for particular values of $U_{0}(0.0$ and 0.5 , respectively). Because of the functional form, the urbanization effect as indicated by this relation depends only on the coefficient $\left(b_{U}\right)_{p}$ and the urban fraction increase $U-U_{0}$; the effect is estimated to be the same for equivalent increases of $U-U_{0}$ regardless of the value of the initial urban fraction $U_{0}$.

For an application outside region 2 using StreamStats, the initial urbanization fraction $U_{0}$ would be zero, and the unadjusted flood-discharge quantile $Q_{p}\left(U_{0}\right)$ would come from the rural regional flood- 
frequency equation where the basin of interest is found. For an application inside region 2 using StreamStats, the initial urbanization fraction $U_{0}$ might be positive, and the unadjusted flooddischarge quantile $Q_{p}\left(U_{0}\right)$ would come from the spatial regression equations for region 2 , such as the methods described in the Applications of the Spatial Regression Equations section. Whether inside or outside of region 2, the unadjusted flood-discharge quantile $Q_{p}\left(U_{0}\right)$ also could be a quantile estimate at or near a gage. The Theobald (2005) data for 2010 as adjusted has been installed in StreamStats so that fractions of urbanization appropriate for use with equations 21 and 22 can be computed within StreamStats.

The variance of prediction $V_{U}$ of the urbanization-adjusted flood-discharge quantile in log units, $\log _{10} Q_{p}(U)$, can be derived from equation 22 by computing the variances of both sides as:

$$
V_{U}=V_{U_{0}}+V_{b_{U}}\left(U-U_{0}\right)^{2}
$$

where

$V_{U_{0}}$ is the variance of prediction of the unadjusted flood-discharge in log units, $\log _{10} Q\left(U_{0}\right)$, and

$V_{b_{U}}$ is the variance of the urbanization coefficient $\left(b_{U}\right)_{p}$, which is the square of the standard error given in Table 9.

The standard error of prediction of $\log _{10} Q_{p}(U), S_{U}$, can be computed from $V_{U}$ as $S_{U}=V_{U}^{1 / 2}$, and then $S_{U}$ can be used in equations 14-16 to obtain a standard error in percentage units and confidence intervals.

Near-gage adjustment of peak discharge quantiles outside region 2 would be applicable only if the peak discharge quantiles at the streamgage arose from the same urbanization conditions as for the basin upstream from the site of interest.

\subsection{EXAMPLE COMPUTATIONS}

To provide further clarification on the use of the results presented in this report, five example computations are provided in this section. Basic information about the examples is given in Table 15. 
Table 15. Example peak discharge quantile computations in this study in northeastern Illinois.

\begin{tabular}{lcc}
\hline Number & \multicolumn{1}{c}{$\begin{array}{c}\text { Equation used } \\
\text { Description }\end{array}$} & $\begin{array}{c}\text { compute } \\
\text { do comarge values }\end{array}$ \\
\hline 1 & Ungaged location in study region, far from a streamgage & 12 \\
2 & Ungaged location in study region, near a streamgage & 20 \\
3 & At a streamgage in study region & $17^{\mathrm{b}}$ \\
4 & Locations outside study region & 21 \\
5 & Adjusting for future effects of urbanization & 21 \\
\hline aln this study, an ungaged location is considered to be near a streamgage if the ratio $A_{u} / A_{g}$ of the \\
drainage area at the ungaged location $A_{u}$ to the drainage area at the gage $A_{g}$ is between 0.5 and 1.5 \\
and they are on the same stream. \\
bValues were computed with equation 17 and are tabulated in Table 2.
\end{tabular}

\subsubsection{Example 1: Ungaged Location in the Study Region, Far from a Streamgage}

Consider a hypothetical ungaged basin with a drainage area $(A)$ of $50 \mathrm{mi}^{2}$, urbanized land use fractions (U) NLCD_22_23_24 of $20 \%$ and Theobald Urban of $40 \%$, water and wetland fraction $(W)$ DrainageClass1a of 15\%, and slope (S) DEM_1_0_P of 3.67 feet per mile. These values usually will be obtained by StreamStats after delineation of the basin. First, it can be seen that the basin characteristic values are well within their corresponding ranges (Table 14). If the basin is within region 2 , the peak discharge quantiles should be computed by using the spatial regression equations, defined by equations 11 or 12, with coefficient values given in Table 11. Taking the peak discharge quantile with $\mathrm{AEP}=0.01$ as an example, using equation 12 , this quantile is computed as:

$$
\begin{gathered}
\mathrm{Q}_{0.01}=10^{\left(b_{0}\right)_{0.01}} A^{\left(b_{A}\right)_{0.01}} 10^{\left(b_{U}\right)_{0.01} U^{1 / 2}} 10^{\left(b_{W}\right)_{0.01} W^{1 / 2}} S^{\left(b_{S}\right)_{0.01}} \\
\mathrm{Q}_{0.01}=10^{1.968} 50^{0.771} 10^{0.207 * 0.20^{1 / 2}} 10^{-1.025 * 0.15^{1 / 2}} 3.67^{0.494} \\
\mathrm{Q}_{0.01}=1790 \mathrm{ft}^{3} / \mathrm{s}
\end{gathered}
$$

where an NLCD_22_23_24 value of $20 \%$ is used for the urbanization measure $U$.

Notice the values of $W$ and $U$ enter the equation as decimal fractions, whereas $S$ enters in feet per mile.

The uncertainty of this estimate is computed by using equations 13-16. The variance $V_{i}$ of the estimate is computed from equation 13 :

$$
V_{i}=\gamma^{2}+x_{i}\left(X^{t} \Lambda^{-1} X\right)^{-1} x_{i}^{t}
$$

where

$$
\begin{aligned}
& \gamma^{2}=0.0528 \text { is the model error variance (Table 12), } \\
& x_{i}=\left[1, \log _{10} A_{i}, U_{i}^{1 / 2}, W_{i}^{1 / 2}, \log _{10} S_{i}\right] \\
& x_{i}=\left[1, \log _{10} 50,0.20^{1 / 2}, 0.15^{1 / 2}, \log _{10} 3.67\right]
\end{aligned}
$$




$$
x_{i}=[1,1.699,0.4472,0.3873,0.5647], \text { and }
$$

the matrix $\left(X^{t} \Lambda^{-1} X\right)^{-1}=0.001\left[\begin{array}{ccccc}31.003 & -8.3921 & -7.6988 & -4.5311 & -20.598 \\ -8.3921 & 3.2522 & 1.1394 & -2.6149 & 5.9206 \\ -7.6988 & 1.1394 & 10.542 & -1.2200 & 2.4035 \\ -4.5311 & -2.6149 & -1.2200 & 40.324 & -1.2044 \\ -20.598 & 5.9206 & 2.4035 & -1.2044 & 18.346\end{array}\right]$ (Table 13).

With these values, the second term of $V_{i}, x_{i}\left(X^{t} \Lambda^{-1} X\right)^{-1} x_{i}^{t}$, is computed as

$$
\begin{gathered}
x_{i}\left(X^{t} \Lambda^{-1} X\right)^{-1} x_{i}^{t}= \\
0.001[1,1.699,0.4472,0.3873,0.5647]\left[\begin{array}{ccccc}
31.003 & -8.3921 & -7.6988 & -4.5311 & -20.598 \\
-8.3921 & 3.2522 & 1.1394 & -2.6149 & 5.9206 \\
-7.6988 & 1.1394 & 10.542 & -1.2200 & 2.4035 \\
-4.5311 & -2.6149 & -1.2200 & 40.324 & -1.2044 \\
-20.598 & 5.9206 & 2.4035 & -1.2044 & 18.346
\end{array}\right]\left[\begin{array}{c}
1 \\
1.699 \\
0.4472 \\
0.3873 \\
0.5647
\end{array}\right] \\
=0.00214 .
\end{gathered}
$$

Therefore, $V_{i}=0.0528+0.00214=0.0549$.

Given the $V_{i}$ value, the standard error of prediction in log units is $S_{i}=V_{i}^{1 / 2}=0.236$, and from equation 14 , the standard error of prediction as a percentage is:

$$
\begin{aligned}
& S_{p i}=100\left\{\exp \left[(\ln 10)^{2} V_{i}\right]-1\right\}^{1 / 2} \\
& S_{p i}=100\left\{\exp \left[(\ln 10)^{2} 0.0549\right]-1\right\}^{1 / 2} \\
& S_{p i}=58.1 \%
\end{aligned}
$$

The confidence intervals are $\log _{10} Q_{i} \pm t_{\alpha / 2, n-p} S_{i}$ (equation 15), where $t_{\alpha / 2, n-p}$ is the critical value of the $t$ distribution at the alpha level $\alpha$ and $n-p$ degrees of freedom, where $n=117$ is the number streamgages used in the spatial regressions and $p=5$ is the number of basin characteristics plus 1. Here, for the $90 \%$ confidence intervals $t_{\alpha / 2, n-p}=t_{0.95,12}=1.659$, so the confidence intervals in log units are:

$$
\begin{gathered}
\log _{10} Q_{i} \pm t_{\alpha / 2, n-p} S_{i}=\log _{10}(1790) \pm 1.659 * 0.234 \\
\log _{10} Q_{i} \pm t_{\alpha / 2, n-p} S_{i}=[2.864,3.641]
\end{gathered}
$$

After inverting the logarithmic transformation, the $90 \%$ confidence intervals are: 


$$
\begin{gathered}
{\left[Q_{i} 10^{-t_{\alpha / 2, n-p} S_{i}}, Q_{i} 10^{t_{\alpha / 2, n-p} S_{i}}\right]=\left[1790 * 10^{-1.659 * 0.234}, 1790 * 10^{1.659 * 0.234}\right]} \\
{\left[Q_{i} 10^{-t_{\alpha / 2, n-p} S_{i}}, Q_{i} 10^{t_{\alpha / 2, n-p} S_{i}}\right]=10^{[2.864,3.641]}} \\
{\left[Q_{i} 10^{-t_{\alpha / 2, n-p} S_{i}}, Q_{i} 10^{t_{\alpha / 2, n-p} S_{i}}\right]=[730,4,380]}
\end{gathered}
$$

Summing up, the estimated 1\% AEP peak discharge quantile $Q_{0.01}$ for this hypothetical ungaged basin on a stream in region 2 far from a streamgage is $1,790 \mathrm{ft}^{3} / \mathrm{s}$ with a standard error of prediction of $58.1 \%$ and a $90 \%$ confidence interval of $[730,4,380] \mathrm{ft}^{3} / \mathrm{s}$.

\subsubsection{Example 2: Ungaged Location in the Study Region, Near a Streamgage}

Next assume that this hypothetical ungaged basin is located, still in region 2, upstream from USGS streamgage 05527950, Mill Creek at Old Mill Creek, Illinois, which has a drainage area of $59.87 \mathrm{mi}^{2}$ (Table 1). In this case the value of the ratio $A_{u} / A_{g}$ is $50 / 59.87=0.835$, and the adjustment weighting factor $w_{a}$ takes the value $2|50 / 59.87-1|=0.330$ (equation 19), so a near-gage adjustment (equation 20) is applicable (see discussion immediately following equation 20). From Table 2, the weighted AEP 0.01 at-gage flood-discharge quantile $\left(Q_{0.01}\right)_{g, w}$ at streamgage 05527950 is $2,050 \mathrm{ft}^{3} / \mathrm{s}$, and as previously computed the regional regression estimate at the ungaged site of interest $\left(Q_{0.01}\right)_{u, r}$ is $1,790 \mathrm{ft}^{3} / \mathrm{s}$. Therefore, the weighted estimate at the ungaged site of interest is:

$$
\begin{gathered}
\left(Q_{0.01}\right)_{u, w}=w_{a}\left(Q_{0.01}\right)_{u, r}+\left(1-w_{a}\right)\left(Q_{0.01}\right)_{g, w}\left(A_{u} / A_{g}\right) \\
\left(Q_{0.01}\right)_{u, w}=(0.330 * 1,790) \\
+(0.670 * 2,050 * 0.835) \\
\left(Q_{0.01}\right)_{u, w}=1,740 \mathrm{ft}^{3} / \mathrm{s}
\end{gathered}
$$

Summing up, the estimated 1\% AEP peak discharge quantile $Q_{0.01}$ for this hypothetical ungaged basin on a stream in region 2 near to streamgage $05527950\left(A_{u} / A_{g}=0.835\right)$ is $1,740 \mathrm{ft}^{3} / \mathrm{s}$, compared to $1,790 \mathrm{ft}^{3} / \mathrm{s}$ without the near-gage adjustment.

\subsubsection{Example 3: At a Streamgage in the Study Region}

To obtain an estimate at an applicable streamgage in the study region, no computations are needed. Although the method of computation is presented in the At a Streamgage section (equation 17), the results of the computations have been tabulated (Table 2). The particular value from Table 2 to be used is the same weighted at-gage flood-discharge quantile $\left(Q_{p}\right)_{g, w}$ used in the previous near-gage adjustment computation. Following that example, for USGS streamgage 05527950, Mill Creek at Old Mill Creek, Illinois, the appropriate AEP 0.01 at-gage flood-discharge quantile $\left(Q_{0.01}\right)_{g, w}$ is $2,050 \mathrm{ft}^{3} / \mathrm{s}$. 


\subsubsection{Example 4: At a Location Outside the Study Region}

If instead the hypothetical ungaged basin were outside region 2 , then the adjustment of a rural estimate for urbanization described in the Applications of the Temporal Urbanization Coefficients section would be applicable (equation 21). For example, if the AEP $=0.01$ quantile estimate from the rural regional regression equations from Soong et al. (2004) is $2,000 \mathrm{ft}^{3} / \mathrm{s}$, then the urbanizationadjusted $A E P=0.01$ quantile value at the location of interest is:

$$
Q_{0.01}(U)=Q_{0.01}\left(U_{0}\right) 10^{\left(b_{U}\right)_{0.01}\left(U-U_{0}\right)},
$$

where the urban fraction of interest, $U$, is, by assumption, $40 \%$ or 0.4 , and the current urban fraction, $U_{0}$, is 0 because the discharge quantile value being adjusted, $Q_{0.01}\left(U_{0}\right)$, is being estimated by using a rural regression equation.

Therefore,

$$
\begin{aligned}
& Q_{0.01}(U)=2,000 * 10^{0.312 *(0.40-0.0)} \\
& Q_{0.01}(U)=2,000 * 1.333 \\
& Q_{0.01}(U)=2,670 \mathrm{ft}^{3} / \mathrm{s},
\end{aligned}
$$

where $\left(b_{U}\right)_{0.01}=0.312$ is taken from Table 9.

The variance of prediction of this estimate in log units can be computed from equation 23 as follows:

$$
\begin{gathered}
V_{U}=V_{U_{0}}+V_{b_{U}}\left(U-U_{0}\right)^{2} \\
V_{U}=0.0549+0.134^{2}(0.4-0.0)^{2} \\
V_{U}=0.05777,
\end{gathered}
$$

where

$V_{U_{0}}$, the variance of prediction of the unadjusted flood-discharge in log units, $\log _{10} Q\left(U_{0}\right)$, is assumed, just for this example, to have the same value as $V_{i}$ which was computed previously for region 2 [in an actual computation it would be necessary to compute this value by the relevant method, such as the method given in Soong et al. (2004), which is currently (2016) implemented in StreamStats], and

$V_{b_{U}}$ is the square of the standard error of $\left(b_{U}\right)_{0.01}$, the value of which is given in Table 9.

Given this value of $V_{U}$, the standard error is $S_{U}=\left(V_{U}\right)^{1 / 2}=0.240$, and the standard error in percentage units is: 


$$
\begin{gathered}
S_{p U}=100\left\{\exp \left[(\ln 10)^{2} V_{U}\right]-1\right\}^{1 / 2} \\
S_{p U}=100\left\{\exp \left[(\ln 10)^{2} 0.05777\right]-1\right\}^{1 / 2} \\
S_{p U}=59.9 \% .
\end{gathered}
$$

Confidence intervals for this estimate can be computed by using equation 16 as already shown; the resulting $90 \%$ confidence interval in $\log _{10}$ units is [3.0269, 3.8247] and in cubic feet per second, $[1,060,6,680]$

Summing up, the estimated $1 \%$ AEP peak discharge quantile $Q_{0.01}$ for this hypothetical ungaged basin on a stream outside region 2 increases from 2,000 to $2,670 \mathrm{ft}^{3} / \mathrm{s}$ as a result of adjusting for the effects of urbanization increasing from $0 \%$ to $40 \%$. The estimated adjusted quantile has a standard error of prediction of $59.9 \%$ and a $90 \%$ confidence interval of $[1,060,6,680] \mathrm{ft}^{3} / \mathrm{s}$.

\subsubsection{Example 5: Adjustment for Effects of Future Urbanization}

Finally, assume the hypothetical basin is as originally assumed, with the same basin characteristics, but an estimate of the peak discharge quantile at "build-out" (100\% urbanization) is desired. If the future urbanization is expected to have similar hydrologic effects as the basins used in this study, the temporal urbanization coefficients would again be applicable, and the estimate of the AEP $=0.01$ peak discharge quantile would be obtained by equation 21 as follows:

$$
Q_{0.01}(U)=Q_{0.01}\left(U_{0}\right) 10^{\left(b_{U}\right)_{0.01}\left(U-U_{0}\right)},
$$

where the future urban fraction of interest, $U$, is taken to be $100 \%$ or 1.0 , and the current urban fraction, $U_{0}$, is $40 \%$ or 0.40 , according to the original assumptions on this hypothetical basin.

Therefore,

$$
\begin{aligned}
& Q_{0.01}(U)=1,790 * 10^{0.312 *(1.0-0.4)} \\
& Q_{0.01}(U)=1,790 * 1.539 \\
& Q_{0.01}(U)=2,750 \mathrm{ft}^{3} / \mathrm{s}
\end{aligned}
$$

where

$Q_{0.01}\left(U_{0}\right)=1,790$ is taken from the first example calculation (equation 24), and $\left(b_{U}\right)_{0.01}=0.312$ is taken from Table 9. 
The uncertainty of this estimate can be obtained from equation 23 as in the previous example; the resulting uncertainty $S_{p U}$ is $62.0 \%$, and the $90 \%$ confidence interval is $[1,070,7,090]$ in $\log _{10}$ units and $[3.0286,3.8507]$ in cubic feet per second.

Summing up, the estimated 1\% AEP peak discharge quantile $Q_{0.01}$ for this hypothetical ungaged basin increases from $1,790 \mathrm{ft}^{3} / \mathrm{s}$ to $2,750 \mathrm{ft}^{3} / \mathrm{s}$ as a result of adjusting for the effects of urbanization increasing from $40 \%$ to $100 \%$. The estimated adjusted quantile has a standard error of prediction of $62.0 \%$ and a $90 \%$ confidence interval of $[1,070,7,090] \mathrm{ft}^{3} / \mathrm{s}$.

A future increase in urbanization does not need to take the urban fraction to completed build-out; that is, $U=1.0$. Any value of $U$ greater than the current urban fraction $\left(U_{0}=0.40\right.$, in the example) could be used to estimate the effects of future increases in urbanization. 


\section{CHAPTER 6: SUMMARY}

This report provides two sets of equations for estimating peak discharge quantiles at annual exceedance probabilities (AEPs) of $0.50,0.20,0.10,0.04,0.02,0.01,0.005$, and 0.002 (recurrence intervals of $2,5,10,25,50,100,200$, and 500 years, respectively) for urbanized and urbanizing watersheds in Illinois based on analysis of 117 streamgage records in and near Illinois flood-frequency region 2. One set of equations was developed through a temporal analysis by using a two-step least squares-quantile regression technique that measures the average effect of changes in the urbanization of the gaged watersheds used in the study and were used to adjust the annual maximum peak discharge records to 2010 urbanization conditions. The other set of equations was developed through a spatial analysis using generalized least-squares (GLS) regression applied to quantiles estimated from the urbanization-adjusted records.

The effect of urbanization indicated by the two sets of equations is substantially different, with the temporal analysis indicating a larger effect, especially for larger AEPs (smaller recurrence intervals). This difference in urbanization effect was attributed to coefficient biases in the spatial analysis, and as a result, the urbanization coefficients from spatial equations are recommended for use only at ungaged locations in the study region. Other applications discussed in the report, which are estimating the effect of urbanization in regions of Illinois outside flood-frequency region 2 in combination with rural regression equations and estimating the effect of future urbanization anywhere in Illinois, use the results of the temporal analysis.

The peak discharge quantiles for the spatial analysis were computed by using the Expected Moments Algorithm following the censoring of potentially influential low floods identified by a multiple GrubbsBeck test. To improve the skew estimates used for the peak discharge quantile estimation, weighted skew coefficients were computed as the variance-weighted average of at-site and regional skew coefficients. The regional skew coefficients were obtained from a new regional skew model, which estimates skewness as an increasing linear function of the urbanized land use fraction, with values ranging from -0.39 to +0.58 .

Several urbanization, water and wetland, and slope variables, in addition to drainage area, were considered for use as basin characteristics in the spatial equations in a preliminary weighted leastsquares analysis. The combination of variables giving the best results-drainage area, the fraction of developed land, the fraction of land with poorly drained soils or likely water, and the basin slope estimated as the ratio of the basin relief to basin perimeter-were used in the GLS analysis to develop the final spatial regression equations.

In addition to this report, which details the development of the two sets of equations and provides guidance on their application, including numerical examples, the products of the study include the urbanization-adjusted annual maximum peak discharge records and peak discharge quantile estimates at 181 streamgages: the 117 streamgages used to develop the spatial regression equations and 64 additional streamgages in the region that originally were considered for use in the study but later deemed to be redundant. The equations and quantile estimates are available in the web- 
based application StreamStats, and the urbanization-adjusted peak discharge records are provided in a table as part of this report and as a collection of tables and plots by streamgage at http://dx.doi.org/10.3133/sir20165050. 


\section{REFERENCES}

Allen, H.E., Jr., and Bejcek, R.M., 1979, Effects of urbanization on the magnitude and frequency of floods in northeastern Illinois: U.S. Geological Survey Water-Resources Investigations Report 7936, $48 \mathrm{pp}$.

Angel, J.R., and Changnon. S.A., 2008, Record heavy rains in August 2007: Cause, magnitude, and impacts: Transactions of the Illinois State Academy of Science, v. 101, pp. 187-199.

Arnold, T.L., Sullivan, D.J., Harris, M.A., Fitzpatrick, F.A, Scudder, B.C., Ruhl, P.M., Hanchar, D.W., and Stewart, J.S., 1999, Environmental setting of the Upper Illinois River Basin and implications for water quality: U.S. Geological Survey Water-Resources Investigations Report 98-4268, 67 pp.

Belda, M., Holtanová, E., Halenka, T., and Kalvová, J., 2014, Climate classification revisited: From Köppen to Trewartha: Climate Research, v. 59, pp. 1-13.

Canay, I.A., 2011, A simple approach to quantile regression for panel data: The Econometrics Journal, v. 14 , pp. $368-386$.

Changnon, S.A., 1999, Record flood-producing rainstorms of 17-18 July 1996 in the Chicago metropolitan area, Part III: Impacts and responses to the flash flooding: Journal of Applied Meteorology, v. 38, pp. 273-280.

Changnon, S.A., 2010, Stormwater management for a record rainstorm at Chicago: Journal of Contemporary Water Research \& Education, no. 146, pp. 103-109.

Changnon, S.A., 2011, Major damages in northern Illinois from a record-setting rainstorm in 2010: Transactions of the Illinois State Academy of Science, v. 104, pp. 119-125.

Changnon, S.A., and Demissie, M., 1996, Detection of changes in streamflow and floods resulting from climate fluctuations and land use-drainage changes: Climatic Change, v. 32, pp. 411-421.

Changnon, S.A., and Westcott, N.E., 2002a, Heavy rainstorms in Chicago: Increasing frequency, altered impacts, and future implications: Journal of the American Water Resources Association, $v$. 28 , no. 5, pp. 1467-1475.

Changnon, S.A., and Westcott, N.E., 2002b, A record number of heavy rainstorms in Chicago in 2001: Transactions of the Illinois State Academy of Science, v. 95, no. 2, pp. 73-85.

Changnon, D., Fox, D., and Bork, S., 1996, Differences in warm-season, rainstorm-generated stormflows for northeastern Illinois urbanized basins: Water Resources Bulletin, v. 32, no. 6, pp. 1307-1317.

Changnon, S.A., Angel, J.R., Kunkel, K.E., and Lehman, C.M.B, 2004, Climate atlas of Illinois: Champaign, III., Illinois State Water Survey, 309 pp.

Cohn, T.A., Lane, W.L., and Baier, W.G., 1997, An algorithm for computing moments-based flood quantile estimates when historical flood information is available: Water Resources Research, $v$. 33, no. 9, pp. 2089-2096.

Cohn, T.A., Lane, W.L., and Stedinger, J.R., 2001, Confidence intervals for expected moments algorithm flood quantile estimates: Water Resources Research, v. 37, no. 6, pp. 1695-1706. 
Cohn, T.A., Berenbrock, C., Kiang, J.E., and Mason, R.R., Jr., 2012, Calculating weighted estimates of peak streamflow statistics: U.S. Geological Survey Fact Sheet 2012-3038, 4 pp. [Also available at http://pubs.usgs.gov/fs/2012/3038/.]

Cohn, T. A., J. F. England, C. E. Berenbrock, R. R. Mason, J. R. Stedinger, and J. R. Lamontagne (2013), A generalized Grubbs-Beck test statistic for detecting multiple potentially influential low outliers in flood series, Water Resources Research, v. 49, pp. 5047-5058, http://dx.doi.org:10.1002/wrcr.20392.

Cowardin, L.M., Carter, V., Golet, F.C., and LaRoe, E.T., 1979, Classification of wetlands and deepwater habitats of the United States: Washington, D.C., U.S. Department of the Interior, Fish and Wildlife Service, FWS/OBS-79/31, 131 pp. [Also available at http://www.fws.gov/wetlands/Documents/Classification-of-Wetlands-and-Deepwater-Habitatsof-the-United-States.pdf.]

Croissant, Y., and Millo, G., 2008, Panel data econometrics in R: The plm package: Journal of statistical software, v. 27 no. 2, 51 pp., accessed December 29, 2010, at http://www.jstatsoft.org/v27/i02/.

Drainage Manual Committee for IDOT Division of Highways, 2011, IDOT drainage manual: Springfield, III., Illinois Department of Transportation [variously paginated].

Eng, Ken, Chen, Yin-Yu, and Kiang, J.E., 2009, User's guide to the weighted-multiple-linear-regression program (WREG version 1.0): U.S. Geological Survey Techniques and Methods, book 4, chap. A8, $21 \mathrm{pp}$.

Espey, W.H., Jr., and Winslow, D.E., 1974, Urban flood frequency characteristics: Journal of the Hydraulics Division, Proceedings of the American Society of Civil Engineers, v. 100, no. HY2, pp. 279-293.

Farmer, W.H., 2017, WREG: USGS WREG v. 2.02 R package version 2.02. Accessed November 15, 2019, at https://rdrr.io/github/USGS-R/WREG/.

Fazio, D.J., and Sharpe, J.B., 2012, Flood of September 13-16, 2008, in northeastern Illinois: U.S. Geological Survey Data Series 726, 40 pp. [Also available at http://pubs.usgs.gov/ds/726/.]

Fehrenbacher, J.B., Alexander, J.D., Jansen, I.J., Darmody, R.G., Pope, R.A., Flock, M.A., Voss, E.E., Scott, J.W., Andrews, W.F., and Bushue, L.J., 1984, Soils of Illinois: University of Illinois, College of Agriculture, Agricultural Experiment Station, and U.S. Department of Agriculture, Soil Conservation Service, $85 \mathrm{pp}$.

Frees, E.W., 2004, Longitudinal and panel data: Analysis and applications in the social sciences: Cambridge, Cambridge University Press, 467 pp.

Fuller, W.A., 1987, Measurement error models: New York, John Wiley \& Sons, 440 pp.

Gesch, D.B., Oimoen, M.J., Greenlee, S.K., Nelson, C.A., Steuck, M., and Tyler, D.J., 2002, The National Elevation Dataset: Photogrammetric Engineering and Remote Sensing, v. 68, no. 1, pp. 5-11. [Also available at https://www.asprs.org/wpcontent/uploads/pers/2002journal/january/highlight.html.]

Greene, W.H., 1997, Econometric analysis $3^{\text {rd }}$ ed.: Upper Saddle River, NJ, Prentice Hall, 1075 pp. 
Grimaldi, S., Petroselli, A., Alonso, G., and Nardi, F., 2010, Flow time estimation with spatially variable hillslope velocity in ungauged basins: Advances in Water Resources, v. 33, pp. 1216-1223.

Griffis, V.W., and Stedinger, J.R., 2007, The use of GLS regression in regional hydrologic analyses: Journal of Hydrology, v. 344, pp. 82-95.

Griffis, V.W., and Stedinger, J.R., 2009, Log-Pearson type 3 distribution and its application in flood frequency analysis. III: sample skew and weighted skew estimators: Journal of Hydrologic Engineering, v. 14, no. 2, pp. 121-130.

Gruber, A.M., Reis, D.S., Jr., and Stedinger, J.R., 2007, Models of regional skew based on Bayesian GLS regression, Paper 40927-3285, in Kabbes, K.C., ed., Restoring our natural habitat-Proceedings of the 2007 World Environmental and Water Resources Congress-May 15-19, Tampa, Florida: Reston, Va., American Society of Civil Engineers [variously paginated].

Gruber, A.M., and Stedinger, J.R., 2008, Models of LP3 regional skew, data selection and Bayesian GLS regression, Paper 596, in Babcock, R.W., and Walton, Raymond, eds., World Environmental and Water Resources Congress 2008-Ahupua'a-Proceedings of the congress-May 12-16, 2008, Honolulu, Hawaii: Reston, Va., American Society of Civil Engineers [variously paginated].

Hansel, A.K., and McKay, E.D., III, 2010, Quaternary period, in Kolata, D.R., and Nimz, C.K., eds., Geology of Illinois: Champaign, Ill., Illinois State Geological Survey, pp. 216-247.

Hayden, B.P., 1988, Flood climates, in Baker, V.R., Kochel, R.C., and Patton, P.C., eds., Flood geomorphology: New York, John Wiley and Sons, pp. 13-26.

Helsel, D.R. and Hirsch, R.M., 2002, Statistical methods in water resources: U.S. Geological Survey Techniques of Water-Resources Investigations, book 4, chapter A3, $522 \mathrm{pp}$.

Hejazi, M.I., and Markus, M., 2009, Impacts of urbanization and climate variability on floods in northeastern Illinois: Journal of Hydrologic Engineering, v. 14, no. 6, pp. 606-616. [Also available at http://dx.doi.org/10.1061/(ASCE)HE.1943-5584.0000020.]

Hodge, S.A., and Tasker, G.D., 1995, Magnitude and frequency of floods in Arkansas: U.S. Geological Survey Water-Resources Investigations Report 95-4224 [variously paginated; see pp. 37-39].

Hodgkins, G.A., Hebson, Charles, Lombard, P.J., and Mann, Alexander, 2007, Comparison of peak-flow estimation methods for small drainage basins in Maine: U.S. Geological Survey Scientific Investigations Report 2007-5170, 32 pp.

Illinois Department of Natural Resources, 1996, Illinois land cover, an atlas: Springfield, Ill., Illinois Department of Natural Resources, 157 pp.

Ishii, A.L., Soong, D.T., and Sharpe, J.B., 2010, Implementation and evaluation of the Streamflow Statistics (StreamStats) web application for computing basin characteristics and flood peaks in Illinois: U.S. Geological Survey Scientific Investigations Report 2009-5197, 25 pp.

Jin, S., Yang, L., Danielson, P., Homer, C., Fry, J., and Xian, G. 2013. A comprehensive change detection method for updating the National Land Cover Database to circa 2011. Remote Sensing of Environment, v. 132, pp. 159-175.

Juhl, A.R., 2005, Flood control and drainage, in Reiff, J.L., Keating, A.D., and Grossman, J.R., The 
Electronic Encyclopedia of Chicago: Chicago, Chicago Historical Society, accessed August 4, 2015, at http://www.encyclopedia.chicagohistory.org/pages/460.html.

Karstensen, K.A., Shaver, D.K., Alexander, R.L, Over, T.M., and Soong, D., 2013, Land change in the Central Corn Belt Plains ecoregion and hydrologic consequences in developed areas-1939-2000: U.S. Geological Survey Open-File Report 2013-1157, 21 pp. [Also available at http://pubs.usgs.gov/of/2013/1157/.]

Koenker, R., 2005, Quantile regression: Cambridge, England, Cambridge University Press, 349 pp.

Koenker, R., 2013, quantreg: Quantile Regression: R package version 5.05, accessed November 6, 2014, at http://CRAN.R-project.org/package=quantreg.

Konrad, C.P., 2003, Effects of urban development on floods: U.S. Geological Survey Fact Sheet FS-07603, 4 pp.

Leighton, M.M., Ekblaw, G.E., and Horberg, L., 1948, Physiographic divisions of Illinois: The Journal of Geology, v. 56, no. 1, pp. 16-33.

Maidment, D.R., ed., 2002, Arc Hydro: GIS for water resources: Redlands, Cal., Esri, 220 pp.

Mariner, R.D., 2005, Land use, in Reiff, J.L., Keating, A.D., and Grossman, J.R., The Electronic Encyclopedia of Chicago: Chicago, Chicago Historical Society, accessed August 6, 2015, at http://www.encyclopedia.chicagohistory.org/pages/721.html.

Moglen, G.E., and Shivers, D.E., 2006, Methods for adjusting USGS rural regression peak discharges in an urban setting: U.S. Geological Survey Scientific Investigation Report 2006-5270, 65 pp.

Over, T.M., Saito, R.J., and Soong, D.T., 2016, Flood-peak magnitudes at selected stations in northeastern Illinois adjusted for changes in land-use conditions: U.S. Geological Survey Scientific Investigations Report 2016-5049, 33 p. [Also available at http://dx.doi.org.10.3133/sir20165049.]

Peel, M.C., Finlayson, B. L., and McMahon T.A., 2007, Updated world map of the Köppen-Geiger climate classification: Hydrology and Earth Systems Sciences, v. 11, pp. 1633-1644.

Piskin, K., and Bergstrom, R.E., 1975, Glacial drift in Illinois - thickness and character: Illinois State Geological Survey, Circular 490, 35 pp., 2 plates.

R Core Team, 2014, R: A language and environment for statistical computing: Vienna, Austria, R Foundation for Statistical Computing, accessed November 6, 2014, at http://www.R-project.org.

R Core Team, 2020, R-A language and environment for statistical computing. R Foundation for Statistical Computing, Vienna, Austria accessed April 24, 2020, at https://www.R-project.org/.

Rao, A.R., 2006, Flood frequency relationships for Indiana: West Lafayette, Ind., Joint Transportation Research Program, Purdue University, Final Report FHWA/IN/JTRP-2005/18, 140 pp.

Reis, D.S., Jr., Stedinger, J.R., and Martins, E.S., 2005, Bayesian generalized least squares regression with application to the log Pearson type 3 regional skew estimation: Water Resources Research, $v$. 41, no. 10, 14 pp. [Also available at http://dx.doi.org/10.1029/2004WR003445.]

Resource Coordination Policy Committee, 1998, Our community and flooding: A report of the status of floodwater management in the Chicago Metropolitan Area, 72 pp., accessed August 6, 2015, at 
https://www.dnr.illinois.gov/WaterResources/Documents/OurCommunityAndFlooding_Oct1998. pdf.

Ries III, K.G., 2007, The national streamflow statistics program: A computer program for estimating streamflow statistics for ungaged sites: U.S. Geological Survey Techniques and Methods 4-A6, $37 \mathrm{pp}$.

Rosbjerg, D. et al., 2013, Prediction of floods in ungauged basins, chap. 9 of Günter Blöschl, G., et al., eds., Runoff prediction in ungauged basins: Cambridge, Cambridge University Press, pp. 189-226, accessed August 4, 2015, at http://dx.doi.org/10.1017/СBO9781139235761.012.

Rougé. C. and Cai, X., 2014, Crossing-scale hydrological impacts of urbanization and climate variability in the greater Chicago area: Journal of Hydrology, v. 517, pp. 13-27. [Also available at http://dx.doi.org/10.1016/j.jhydrol.2014.05.005.]

Sauer, V.B., Thomas, W.O., Jr., Stricker, V.A., and Wilson, K.V., 1983 Flood characteristics of urban watersheds in the United States: U.S. Geological Survey Water-Supply Paper 2207, 63 pp.

Schneider, A.F., 1966, Physiography, in Lindsey, A.A., ed., Natural features of Indiana: Indianapolis, Indiana Academy of Science, pp. 40-56.

Soil Survey Staff, Natural Resources Conservation Service, United States Department of Agriculture, Web Soil Survey, 2013, website accessed October, 2013, at http://websoilsurvey.nrcs.usda.gov/.

Soong, D.T., Ishii, A.L., Sharpe, J.B., and Avery, C.F., 2004, Estimating flood-peak discharge magnitudes and frequencies for rural streams in Illinois: U.S. Geological Survey Scientific Investigations Report 2004-5103, 158 pp.

Stedinger, J.R., and Tasker, G.D., 1985, Regional hydrologic analysis, 1, ordinary, weighted, and generalized least squares compared: Water Resources Research, v. 21, no. 9, pp. 1421-1432.

Tasker, G.D., 1975, Combining estimates of low-flow characteristics of streams in Massachusetts and Rhode Island: Journal of Research of the U.S. Geological Survey, v. 3, no. 1, pp. 107-112.

Tasker, G.D., and Stedinger, J.R., 1989, An operational GLS model for hydrologic regression: Journal of Hydrology, v. 111, pp. 361-375.

Theobald, D., 2005, Landscape patterns of exurban growth in the USA from 1980 to 2020: Ecology and Society, v. 10, no. 1, article 32, 29 pp. [Also available at https://www.ecologyandsociety.org/vol10/iss1/art32/.]

Thiessen, A.H., 1911, Precipitation averages for large areas: Monthly Weather Review, v. 39, no. 7, pp. 1082-1089.

U.S. Army Corps of Engineers, 2013, National Inventory of Dams: U.S. Army Corps of Engineers, digital data, accessed August 16, 2013, at http://geo.usace.army.mil/pgis/f?p=397:12. [Updated version available at https://nid.sec.usace.army.mil/ords/f?p=105:1.]

U.S. Geological Survey, 2013, Peak streamflow for the Nation: National Water Information System: Web Interface, accessed December 2, 2013, at http://nwis.waterdata.usgs.gov/usa/nwis/peak.

U.S. Interagency Advisory Committee on Water Data, 1982, Guidelines for determining flood flow frequency-Bulletin \#17B of the Hydrology Subcommittee (revised and corrected): Reston, Va., 
U.S. Geological Survey, Office of Water Data Coordination, 28 pp. [plus appendixes].

Veilleux, A.G., 2009, Bayesian GLS regression for regionalization of hydrologic statistics, floods, and Bulletin 17 skew: Ithaca, N.Y., Cornell University, unpublished M.S. thesis.

Veilleux, A.G., 2011, Bayesian GLS regression, leverage and influence for regionalization of hydrologic statistics: Ithaca, N.Y., Cornell University, unpublished Ph.D. dissertation.

Veilleux, A.G., Cohn, T.A., Flynn, K.M., Mason, R.R., Jr., and Hummel, P.R., 2013, Estimating magnitude and frequency of floods using the PeakFQ 7.0 program: U.S. Geological Survey Fact Sheet 20133108, 2 pp. [Also available at http://dx.doi.org/10.3133/fs20133108.]

Villarini, G., Smith, J.A., Baeck, M.L., Smith, B.K., and Sturdevant-Rees, P., 2013, Hydrologic analyses of the July 17-18, 1996, flood in Chicago and the role of urbanization: Journal of Hydrologic Engineering, v. 18, pp. 250-259. [Also available at http://dx.doi.org:10.1061/(ASCE)HE.19435584.0000462.]

Vogel, R.M., 2006, Regional calibration of watershed models, chap. 3 of Singh, V.P., and Frevert, D.K., Watershed models: Boca Raton, Fla., CRC Press, pp. 47-71.

Walker, J.F., and Krug, W.R., 2003, Flood-frequency characteristics of Wisconsin streams: U.S. Geological Survey Water-Resources Investigations Report 03-4250, 37 pp.

Westcott, N.E., 2015, Continued operation of a 25-raingage network for collection, reduction, and analysis of precipitation data for Lake Michigan diversion accounting: Water year 2014: Contract Report 2015-01, Champaign, Illinois: Illinois State Water Survey, Prairie Research Institute, University of Illinois at Urbana-Champaign, $82 \mathrm{pp}$.

Winters, B.A., Angel, J., Ballerine, C., Byard, J., Flegel, A., Gambill, D., Jenkins, E., McConkey, S., Markus, M., Bender, B.A., and O'Toole, M.J., 2015, Report for the Urban Flooding Awareness Act: Illinois Department of Natural Resources, 89 pp., accessed August 9, 2015, at http://www.dnr.illinois.gov/WaterResources/ Pages/UrbanFloodAwareness.aspx. [Now available at https://www2.illinois.gov/dnr/waterresources/documents/Final_UFAA_Report.PDF.]

Wisconsin Department of Natural Resources, 1992, Wisconsin wetland inventory classification guide: Publication PUBL-WZ-WZ023, 3 pp. [Also available at http://dnr.wi.gov/topic/wetlands/ documents/WWI_Classification.pdf.]

Xian, G., Homer, C., Dewitz, J., Fry, J., Hossain, N., and Wickham, J., 2011, The change of impervious surface area between 2001 and 2006 in the conterminous United States: Photogrammetric Engineering and Remote Sensing, v. 77, no. 8, pp. 758-762. 


\section{APPENDIX 1: NORTHEASTERN ILLINOIS REGIONAL SKEW ANALYSIS}

For the log-transformation of annual peak discharges, Bulletin 17B (U.S. Interagency Advisory Committee on Water Data 1982) recommends using a weighted average of the station skew coefficient and a regional skew coefficient to improve estimates of annual flood-probability discharges (AFPDs). Bulletin 17B supplies a national map but also encourages hydrologists to develop more specific local relations. From the publication of the national map based on data through 1973 through the end of this study in 2009, some 36 years of additional information has accumulated and improved spatial estimation procedures have been developed (Stedinger and Griffis 2008). Furthermore, prior national and Illinois analyses of regional skew such as that in Soong et al. (2004) did not consider urbanization. For these reasons, for this study in northeastern Illinois a regression analysis was done to develop a regional skew model.

Reis et al. (2005), Gruber et al. (2007), and Gruber and Stedinger (2008) developed a Bayesian generalized least-squares (GLS) regression model for regional skewness analyses. The method provides a more reasonable description of the model error variance than either the generalized leastsquares method-of-moments or maximum likelihood point estimates (Veilleux 2011). However, because of complications introduced by the use of the Expected Moments Algorithm (EMA), with multiple Grubbs-Beck censoring of potentially influential low floods (Cohn et al. 2013) and large cross-correlations between annual peak discharges at pairs of streamgages, an alternate regression procedure was developed to provide stable and defensible results for regional skew regression (Veilleux et al. 2012; Veilleux 2011; Lamontagne et al. 2012). This alternate procedure is referred to as the Bayesian weighted least-squares/Bayesian generalized least-squares (B-WLS/B-GLS) regression framework (Veilleux et al. 2011; Veilleux 2011; Veilleux et al. 2012).

The B-WLS/B-GLS regression analysis uses an ordinary least-squares (OLS) analysis to fit an initial regional skewness model; the OLS model is then used to generate a regional skew-coefficient estimate for each streamgage. This regional estimate is the basis for computing the variance of each station skew-coefficient estimator used in the weighted least-squares (WLS) analysis. Then, B-WLS is used to generate estimators of the regional skew-coefficient model parameters. Finally, B-GLS is used to estimate the precision of those WLS parameter estimators, to estimate the model error variance and the precision of that variance estimator, and to compute various diagnostic statistics. The methodology for the regional skewness model is described in detail in Eash et al. (2013).

\section{APPLICATION OF B-WLS/B-GLS METHOD IN NORTHEASTERN ILLINOIS}

This regional skew study is based on the temporally adjusted annual peak discharge data from the 117 nonredundant streamgages in northeastern Illinois and the surrounding states that were included in the regional regression analyses described in the main report (Table 1). As stated in Bulletin 17B, the skew coefficient of the station is sensitive to extreme events, and more accurate estimates can be obtained from longer records. Thus, for regional skew studies it is preferred that each streamgage have a minimum of 30 to 35 years of record. However, because of the nature of the 
data available in northeastern Illinois, in particular at the urban stations, the minimum was reduced to 15 years to ensure that the entire study area was represented. Applying this minimum reduced the number of streamgages used in the regional skew study to 110 (Table 1-1).

Because the dataset includes censored data and historic information, the effective record length used to compute the precision of the skewness estimators is no longer simply the number of annual peak discharges at a streamgage. Instead, a more complex calculation is used to take into account the availability of historic information and censored values. Although historic information and censored peaks provide valuable information, they often provide less information than an equal number of years with systematically recorded peaks (Stedinger and Cohn 1986). The calculations made to compute the pseudo record length (PRL) are described in Eash et al. (2013). PRL equals the systematic record length if such a complete record is all that is available for a site.

The station logarithmic skew coefficient, $\hat{\gamma}_{S}$ (Table 1-1), and its mean square error, $M S E\left[\hat{\gamma}_{S}\right]$, were computed by using expected moments analysis (EMA) (Cohn et al. 1997; Griffis et al. 2004). The streamgage skewness estimates are ensured to be unbiased by using the correction factor developed by Tasker and Stedinger (1986) and used in Reis et al. (2005). In addition to the skew data, selected basin characteristics for each of the streamgages were available as explanatory variables for the regional skew study.

\section{Table 1-1. Skew statistics at U.S. Geological Survey streamgages used in the development of the regional skew model in this study in northeastern Illinois. [See Appendix 9.]}

A model for the cross-correlation of the logarithms of the annual maximum peak discharges between streamgage pairs is needed for the analysis because sample cross-correlations commonly are unavailable and are subject to large uncertainty, usually including unphysical negative values. Such a model for the northeastern Illinois study area was developed by using the 39 streamgages with at least 45 years of concurrent systematic peaks. A logit model, termed the Fisher $Z$ transformation (Kendall and Stuart, 1961), where $Z=\ln [1+r) /(1-r)]$, was used to transform the sample correlations $r_{i j}$ from their $(-1,+1)$ range to a $(-\infty,+\infty)$ range that is appropriate for least-squares fitting. Various models relating the transformed cross-correlations to various basin characteristics were considered. The adopted model relates the cross-correlations to the distance $D_{i j}$ between streamgage basin centroids according to the fitted equation $Z_{i j}=0.32+\exp \left[-0.40-0.042 D_{i j}\right\rfloor(0 a)$. The fitted relation between $Z$ and the distance between basin centroids together with sample data from the 567 streamgage pairs of data is shown in Figure 1-1a. The equivalent function relation between the cross-correlations and basin centroid distance, which is obtained by back-transforming from $Z$ to $r$ as $r=[\exp (2 Z)-1] /[\exp (2 Z)+1]$, is shown in Figure 1-1b. 

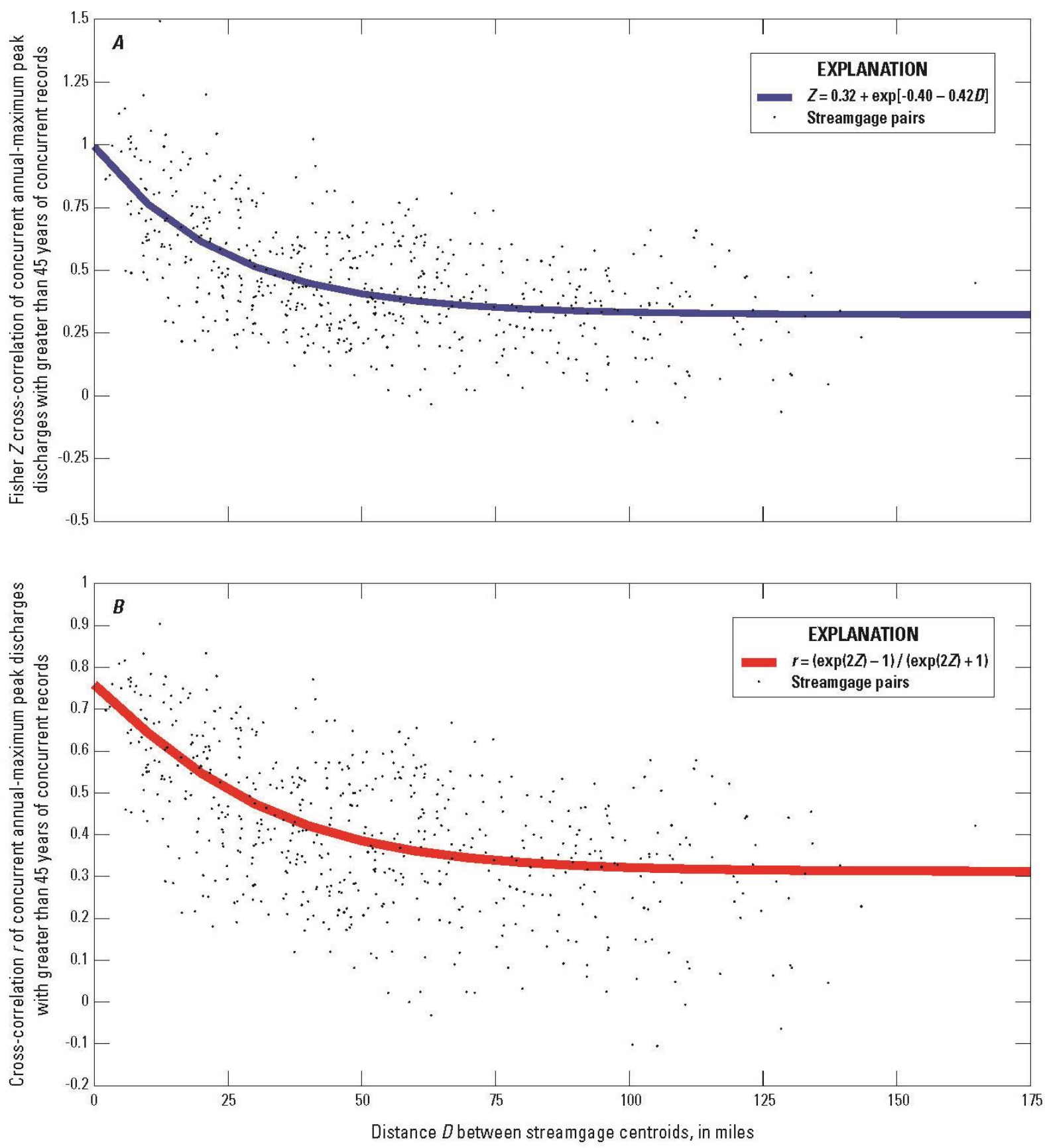

Figure 1-1. Fitting of cross-correlation model of the log-annual maximum peak discharges in this study in northeastern Illinois: (a) relation between Fisher $\mathbf{Z}$ transform of cross-correlation and distance; (b) relation between cross-correlation and distance. 


\section{RESULTS OF REGIONAL SKEW ANALYSIS IN NORTHEASTERN ILLINOIS}

The more promising basin characteristics from a preliminary analysis of the regional spatial regressions were selected for testing as explanatory variables in the regression analysis for regional skew. These selected basin characteristics included drainage area, the urbanization measure NLCD_22_23_24, the DEM-based slope variable DEM_slope, and several water and wetness variables: NWI.total, NWI.emergent, DrainageClass1, DrainageClass1a, and NLCD_11_95 (see Table 3 for definitions). Of these, only NLCD_22_23_24 was statistically significant in explaining the site-to-site variability in skewness, with a square-root transformation providing the better result as compared to untransformed values. Thus, the best model, as classified by having the smallest model error variance, $\sigma_{\delta}^{2}$, and largest pseudo $R_{\delta}^{2}$ is the model using NLCD_22_23_24 $4^{1 / 2}$, denoted URBAN. Results for both the URBAN model, as well as the constant regional model, denoted CONSTANT, for comparison are provided in Table 1-2.

Table 1-2. Regional skewness models and corresponding metrics in this study in northeastern Illinois.

\begin{tabular}{|c|c|c|c|c|c|c|}
\hline Model & $\beta_{1}$ & $\beta_{2}$ & $\sigma_{\delta}^{2}$ & ASEV & $\mathbf{A V P}_{\text {new }}$ & Pseudo $R_{\delta}^{2}$ \\
\hline & 0.16 & - & 0.19 & 0.022 & 0.21 & $0 \%$ \\
\hline CONSTANT: $\hat{\gamma}_{R}=\beta_{1}$ & $(0.15)$ & & $(0.05)$ & & & \\
\hline \multirow{3}{*}{$\begin{array}{c}\text { URBAN: } \\
\hat{\gamma}_{R}=\beta_{1}+\beta_{2}\left\lfloor\left(N L C D D_{-} 222_{-} 23_{-} 24\right)^{0.5}\right\rfloor\end{array}$} & -0.39 & 0.97 & 0.16 & 0.032 & 0.19 & $14 \%$ \\
\hline & $(0.19)$ & $(0.34)$ & $(0.05)$ & & & \\
\hline & & [0.4\%] & & & & \\
\hline
\end{tabular}

Standard deviations are in parentheses. Bayesian plausibility (as a percentage) is in square brackets. $\beta_{1}$, skew model intercept; $\beta_{2}$, skew model slope; $\sigma_{\delta}^{2}$, model error variance; ASEV, average sampling error variance; AVP $\mathrm{P}_{\text {new }}$, average variance of prediction for a new site; pseudo $R_{\delta}^{2}$ (\%), fraction of the variability in the true skews explained by each model in as a percentage (Gruber et al. 2007); $\hat{\gamma}_{R}$, estimated regional skewness; NLCD_22_23_24, fraction of the basin that has 2011 National Land Cover Database land cover classes 22, 23, and 24 (low, medium, and high intensity development, respectively).

Table 1-2 includes the pseudo $R_{\delta}^{2}$, which describes the estimated fraction of the variability in the true skewness from site-to-site explained by each model (Gruber et al. 2007; Parrett et al. 2011). A constant model does not explain any variability, so the pseudo $R_{\delta}^{2}$ for the CONSTANT model is equal to $0 \%$. The URBAN model has a pseudo $R_{\delta}^{2}$ of $14 \%$ indicating that inclusion of the NLCD_22_23_24 basin characteristic in the model explains $14 \%$ of the variability in the true skews. Although the URBAN model accounts for a relatively small portion of the total variation in skews, it is important to note that the focus of the study is on the effects of urbanization. A statistically significant dependence of skewness on the NLCD_22_23_24 characteristic, a measure of urbanization, suggests that not including it in the skewness model could negatively affect the final spatial regression equations. Also, the posterior mean of the model error variance, $\sigma_{\delta}^{2}$, for the URBAN model was 0.16 , 
which is smaller than that of the CONSTANT model $\left(\sigma_{\delta}^{2}=0.19\right)$. Thus, the URBAN model was chosen as the final regional skew model for the northeastern Illinois study area.

The average variance of prediction at a new site $\left(A V P_{n e w}\right)$ describes the precision of the regional skew. In Table 1-2, the URBAN model has the lower $A V P_{n e w}$, equal to 0.19. This $A V P_{n e w}$ is an average value computed by averaging the variance of prediction at a new site ( $\mathrm{VP}_{\text {new }}$ ) for all of the 110 streamgages in the analysis. Just as regional skew varies from streamgage to streamgage, depending on $N L C D \_22 \_23 \_24$, so too do the values of $V P_{\text {new. }}$. Values of the variance of prediction for the regional skew, $V P_{\text {new }}$, and effective record length (ERL) for the URBAN model for values of NLCD_22_23_24 between 0 and 1 are given in Table 1-3. Thus, the URBAN skew model for northeastern Illinois has $V P_{\text {new }}$ values ranging from 0.18 to 0.22 and effective record lengths ranging from 39 to 43 years, depending on NLCD_22_23_24. It is important to note that for the purposes of this study, the AVP new of 0.19 was used when weighting the at-site skew with the regional skew.

Table 1-3. Average regional skew, variance of prediction, and equivalent record length for URBAN regional skew model for various values of $N L C D \_22 \_23 \_24$, in this study in northeastern Illinois.

\begin{tabular}{cccc}
\hline $\begin{array}{c}\text { NLCD_22_23_24 } \\
\text { (fraction) }\end{array}$ & $\begin{array}{c}\text { Average regional } \\
\text { skew }\end{array}$ & VP $_{\text {new }}$ & ERL (years) \\
\hline 0.0 & -0.39 & 0.196 & 42 \\
0.1 & -0.29 & 0.180 & 43 \\
0.2 & -0.20 & 0.180 & 42 \\
0.3 & -0.10 & 0.182 & 41 \\
0.4 & 0.00 & 0.186 & 40 \\
0.5 & 0.10 & 0.191 & 39 \\
0.6 & 0.19 & 0.196 & 39 \\
0.7 & 0.29 & 0.203 & 39 \\
0.8 & 0.39 & 0.208 & 40 \\
0.9 & 0.48 & 0.215 & 40 \\
1.0 & 0.58 & 0.222 & 41 \\
\hline
\end{tabular}

$\mathrm{VP}_{\text {new }}$, variance of prediction; $E R L$, equivalent record length; $N L C D \_22 \_23 \_24$, the fraction of the basin that has 2011 National Land Cover Database land cover classes 22, 23, and 24 (low, medium, and high intensity development, respectively).

The URBAN model provides a reasonable fit for the northeastern Illinois regional skew data (Figure 12). As compared to the CONSTANT model, the URBAN model provides smaller values of regional skew for basins with less development (smaller NCLD_22_23_24 values) and larger values of regional skew for basins with more development (larger NCLD_22_23_24 values). The URBAN model indicates that skewness increases substantially with urbanization from a value of -0.39 for a basin with $N L C D \_22 \_23 \_24$ equal to 0 to +0.58 for a basin where the $N L C D \_22 \_23 \_24$ fraction is equal to one. 


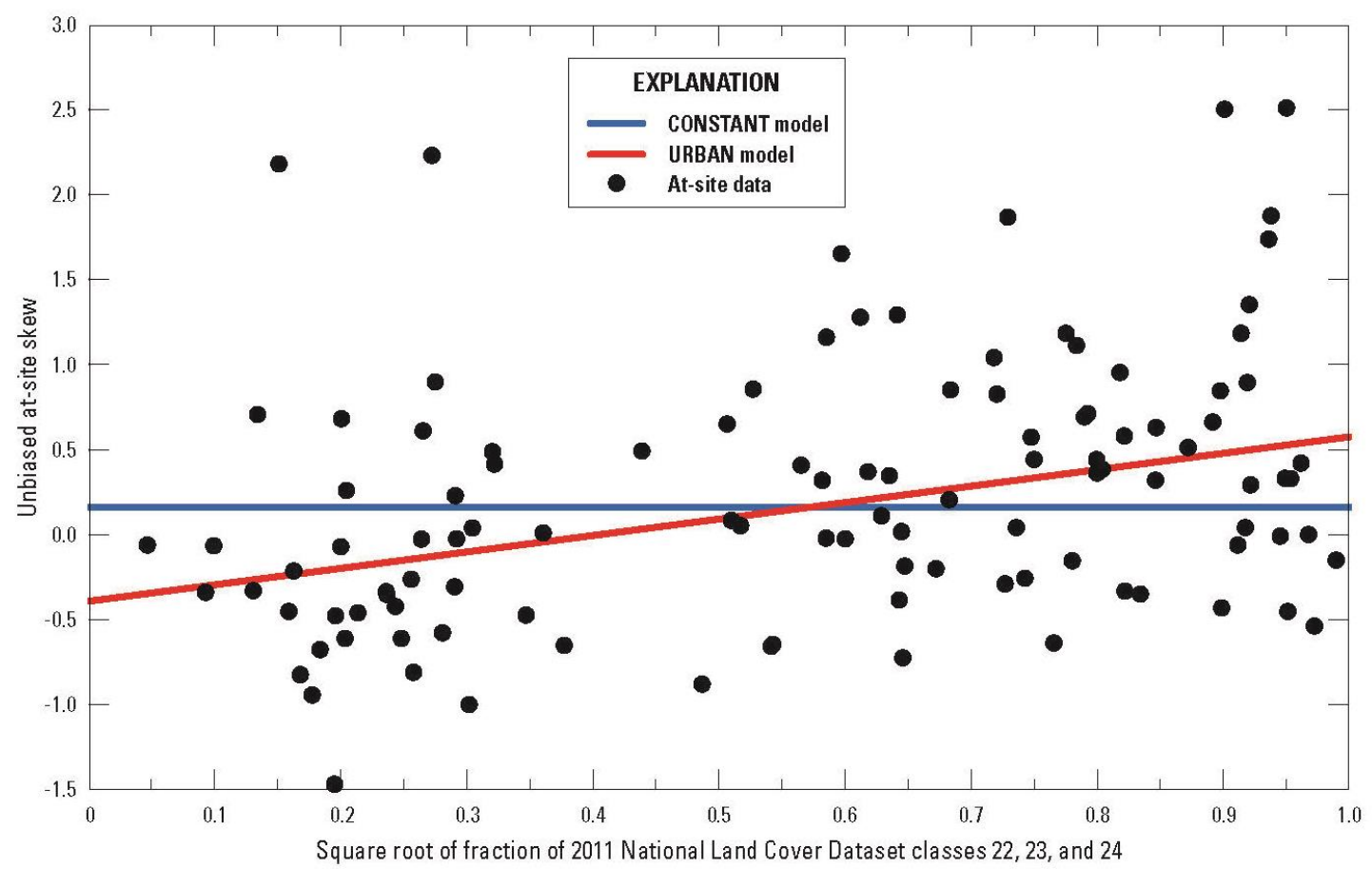

Figure 1-2. Relations between the unbiased at-site skew and urbanization measure for 110 streamgages in northeastern Illinois regional skew study area. The lines represent a model based on a constant skew (CONSTANT) and a model with a linear relation between skew and the square root of fraction of National Land Cover Dataset classes 22, 23, and 24 (NLCD_22_23_24 urbanization measure (URBAN). The models were developed from B-WLS/B-GLS analyses.

Pseudo analysis of variance (Pseudo ANOVA) statistics for the northeastern Illinois regional skew analysis were determined as additional diagnostics for the selected model (Table 1-4). Explanations of how the statistics were computed can be found in Eash et al. (2013). 
Table 1-4. Pseudo analysis of variance (ANOVA) statistics for the northeastern Illinois URBAN regional skew model.

\begin{tabular}{|c|c|c|c|c|}
\hline \multirow[b]{2}{*}{ Source } & \multicolumn{2}{|c|}{ Degrees of freedom } & \multicolumn{2}{|c|}{ Sum of squares } \\
\hline & Equations & URBAN model & Equations & URBAN model \\
\hline Model & $k$ & 1 & $n\left[\delta_{0}^{2}(0)-\delta_{0}^{2}(k)\right]$ & 2.9 \\
\hline Model Error & $n-k-1$ & 108 & $n\left[\delta_{\delta}^{2}(k)\right]$ & 18 \\
\hline Sampling Error & $n$ & 110 & $\sum_{i=1}^{n} \operatorname{Var}\left(\hat{\gamma}_{i}\right)$ & 37 \\
\hline Total & $2 n-1$ & 219 & $n\left[\delta_{\delta}^{2}(k)\right]+\sum_{i=1}^{n} \operatorname{Var}\left(\hat{\gamma}_{i}\right)$ & 58 \\
\hline EVR & & & & 2.1 \\
\hline MBV* & & & & 5.7 \\
\hline Pseudo- $R_{\delta}^{2}$ & & & & $14 \%$ \\
\hline
\end{tabular}

$k$, number of estimated regression parameters not including the constant; $n$, number of observations (streamgages) used in regression; $\delta_{0}^{2}(0)$, model error variance of a constant model; $\delta_{0}^{2}(k)$, model error variance of a model with $k$ regression parameters and a constant; $\operatorname{Var}\left(\hat{\gamma}_{i}\right)$, variance of the estimated sample skew at site $i$; EVR, error variance ratio; $\mathrm{MBV}^{*}$, misrepresentation of the beta variance; Pseudo $-R_{\delta}^{2}$, fraction of variability in the true skews explained by each model (Gruber et al. 2007). 


\section{APPENDIX 1 REFERENCES}

Cohn, T.A., Lane, W.L., and Baier, W.G., 1997, An algorithm for computing moments-based flood quantile estimates when historical flood information is available: Water Resources Research, v. 33, no. 9, pp. 2089-2096.

Cohn, T. A., J. F. England, C. E. Berenbrock, R. R. Mason, J. R. Stedinger, and J. R. Lamontagne (2013), A generalized Grubbs-Beck test statistic for detecting multiple potentially influential low outliers in flood series, Water Resources Research, v. 49, pp. 5047-5058. [Also available at http://dx.doi.org:10.1002/wrcr.20392.]

Eash, D.A., Barnes, K.K., and Veilleux, A.G., 2013, Methods for estimating annual exceedanceprobability discharges for streams in lowa, based on data through water year 2010: U.S. Geological Survey Scientific Investigations Report 2013-5086, 63 pp. [Also available at http://pubs.usgs.gov/ sir/2013/5086/.]

Griffis, V.W., Stedinger, J.R., and Cohn, T.A., 2004, LP3 quantile estimators with regional skew information and low outlier adjustments: Water Resources Research, v. 40, no. 7, [17 pp.]. [Also available at http://dx.doi.org/10.1029/2003WR002697.]

Griffis, V.W., 2006, Flood frequency analysis-Bulletin 17, regional information, and climate change: Ithaca, N.Y., Cornell University, unpublished Ph.D. dissertation.

Gruber, A.M., Reis, D.S., Jr., and Stedinger, J.R., 2007, Models of regional skew based on Bayesian GLS regression, Paper 40927-3285, in Kabbes, K.C., ed., Restoring our natural habitat-Proceedings of the 2007 World Environmental and Water Resources Congress-May 15-19, Tampa, Florida: Reston, Va., American Society of Civil Engineers [variously paginated]

Gruber, A.M., and Stedinger, J.R., 2008, Models of LP3 regional skew, data selection and Bayesian GLS regression, Paper 596, in Babcock, R.W., and Walton, Raymond, eds., World Environmental and Water Resources Congress 2008-Ahupua'a-Proceedings of the congress-May 12-16, 2008, Honolulu, Hawaii: Reston, Va., American Society of Civil Engineers [variously paginated].

Kendall, M.G., and Stuart, A., 1961, The advanced theory of statistics, v. 2: New York, Hafner Publishing Company, 676 p.

Lamontagne, J.R., Stedinger, J.R., Berenbrock, Charles, Veilleux, A.G., Ferris, J.C., and Knifong, D.L., 2012, Development of regional skews for selected flood durations for the Central Valley Region, California, based on data through water year 2008: U.S. Geological Survey Scientific Investigations Report 2012-5130, 60 pp.

Parrett, Charles, Veilleux, A.G., Stedinger, J.R., Barth, N.A., Knifong, D.L., and Ferris, J.C., 2011, Regional skew for California, and flood frequency for selected sites in the Sacramento-San Joaquin River Basin, based on data through water year 2006: U.S. Geological Survey Scientific Investigations Report 2010-5260, 94 pp.

Reis, D.S., Jr., Stedinger, J.R., and Martins, E.S., 2005, Bayesian generalized least squares regression with application to the log Pearson type 3 regional skew estimation: Water Resources Research, v. 41, no. 10, [14 pp.]. [Also available at http://dx.doi.org/10.1029/2004WR003445.] 
Soong, D.T., Ishii, A.L., Sharpe, J.B., and Avery, C.F., 2004, Estimating flood-peak discharge magnitudes and frequencies for rural streams in Illinois: U.S. Geological Survey Scientific Investigations Report 2004-5103, 158 pp.

Stedinger, J.R., and Cohn, T.A., 1986, Flood frequency analysis with historical and paleoflood information: Water Resources Research, v. 22, no. 5, pp. 785-793.

Stedinger, J.R., and Griffis, V.W., 2008, Flood frequency analysis in the United States - Time to update [editorial]: Journal of Hydrologic Engineering, v. 13, no. 4, pp. 199-204.

Tasker, G.D., and Stedinger, J.R., 1986, Regional skew with weighted LS regression: Journal of WaterResources Planning and Management, v. 112, no. 2, pp. 225-237.

U.S. Interagency Advisory Committee on Water Data, 1982, Guidelines for determining flood flow frequency-Bulletin \#17B of the Hydrology Subcommittee (revised and corrected): Reston, Va., U.S. Geological Survey, Office of Water Data Coordination, 28 pp. [plus appendixes].

Veilleux, A.G., 2011, Bayesian GLS regression, leverage and influence for regionalization of hydrologic statistics: Ithaca, N.Y., Cornell University, unpublished Ph.D. dissertation.

Veilleux, A.G., Stedinger, J.R., and Lamontagne, J.R., 2011, Bayesian WLS/GLS regression for regional skewness analysis for regions with large cross-correlations among flood flows, in Beighley, R.E., and Kilgore, M.W., eds., World Environmental and Water Resources Congress 2011-Bearing knowledge for sustainability-Proceedings of the 2011 World Environmental and Water Resources Congress, May 22-26, Palm Springs, Calif.: Reston, Va., American Society of Civil Engineers, pp. 3103-3112.

Veilleux, A.G., Stedinger, J.R., and Eash, D.A., 2012, Bayesian WLS/GLS regression for regional skewness analysis for regions with large crest stage gage networks, Paper 227, in Loucks, E.D., ed., World Environmental and Water Resources Congress 2012-Crossing boundaries-Proceedings of the 2012 congress, May 20-24, Albuquerque, N.M.: Reston, Va., American Society of Civil Engineers, pp. 2253-2263.

Veilleux, A.G., Cohn, T.A., Flynn, K.M., Mason, R.R., Jr., and Hummel, P.R., 2013, Estimating magnitude and frequency of floods using the PeakFQ 7.0 program: U.S. Geological Survey Fact Sheet 20133108, 2 pp. [Also available at http://dx.doi.org/10.3133/fs20133108.] 


\section{APPENDIX 2}

Table 1. U.S. Geological Survey streamgages used in this study in northeastern Illinois and adjacent states.

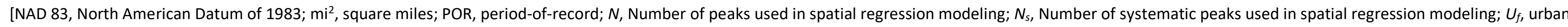

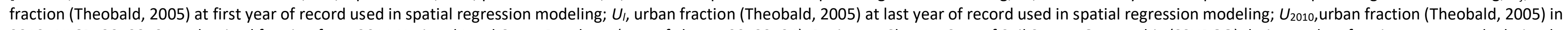

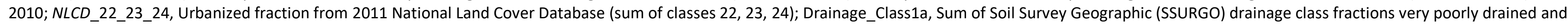

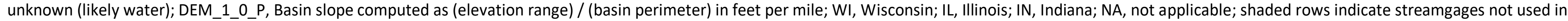
the regression analyses because they were deemed redundant but whose records were adjusted for urbanization in this study]

\begin{tabular}{|c|c|c|c|c|c|c|c|c|c|c|c|c|c|c|c|c|c|}
\hline $\begin{array}{l}\text { U.S. } \\
\text { Geological } \\
\text { Survey } \\
\text { Streamgage } \\
\text { number }\end{array}$ & Name & $\begin{array}{l}\text { Latitude } \\
\text { (NAD 83) }\end{array}$ & $\begin{array}{l}\text { Longitude } \\
\text { (NAD 83) }\end{array}$ & $\begin{array}{l}\text { Hydrologic } \\
\text { unit code }\end{array}$ & $\begin{array}{l}\text { Drainage } \\
\text { area }\left(\mathrm{mi}^{2}\right)\end{array}$ & $\begin{array}{l}\text { Used in } \\
\text { regression } \\
\text { analyses } \\
\text { (non- } \\
\text { redundant) }\end{array}$ & $\begin{array}{l}\text { Crest- } \\
\text { stage } \\
\text { gage } \\
\text { (CSG) }\end{array}$ & $\begin{array}{l}\text { Spatial } \\
\text { POR }\end{array}$ & $\begin{array}{l}\text { Temporal } \\
\text { POR }\end{array}$ & $N$ & $N_{s}$ & $u_{f}$ & $u_{l}$ & $U_{2010}$ & NLCD_22_23_24: & $\begin{array}{l}\text { Drainage } \\
\text { Class1a }\end{array}$ & $\begin{array}{l}\text { DEM_1_0_P: } \\
\text { Basin slope } \\
\text { computed as } \\
\text { (elevation } \\
\text { range) / (basin } \\
\text { perimeter), in } \\
\text { feet per mile }\end{array}$ \\
\hline 04087200 & $\begin{array}{l}\text { OAK CREEK NEAR SOUTH } \\
\text { MILWAUKEE, WI }\end{array}$ & 42.88279 & -87.89203 & 04040002 & 13.70 & No & Yes & $\begin{array}{l}1958- \\
2009\end{array}$ & $\begin{array}{l}1958- \\
2009\end{array}$ & 52 & 52 & 0.3066 & 0.7977 & 0.7977 & 0.5757 & 0.0234 & 6.9977 \\
\hline 04087204 & $\begin{array}{l}\text { OAK CREEK AT SOUTH MILWAUKEE, } \\
\text { WI }\end{array}$ & 42.925 & -87.870 & 04040002 & 24.93 & Yes & No & $\begin{array}{l}1964- \\
2009\end{array}$ & $\begin{array}{l}1964- \\
2009\end{array}$ & 46 & 45 & 0.4340 & 0.7655 & 0.7655 & 0.5588 & 0.0475 & 6.5998 \\
\hline 04087220 & ROOT RIVER NEAR FRANKLIN, WI & 42.87361 & -87.99583 & 04040002 & 49.28 & Yes & No & $\begin{array}{l}1964- \\
2009\end{array}$ & $\begin{array}{l}1960- \\
2009\end{array}$ & 47 & 45 & 0.5170 & 0.8200 & 0.8220 & 0.5190 & 0.0394 & 7.2925 \\
\hline 04087230 & $\begin{array}{l}\text { W BR ROOT R CANAL TRIB NEAR } \\
\text { NORTH CAPE, WI }\end{array}$ & 42.76224 & -88.01786 & 04040002 & 4.01 & Yes & Yes & $\begin{array}{l}1962- \\
1993\end{array}$ & $\begin{array}{l}1962- \\
1993\end{array}$ & 32 & 32 & 0.0143 & 0.0653 & 0.1134 & 0.0315 & 0.0699 & 12.8273 \\
\hline 04087233 & $\begin{array}{l}\text { ROOT RIVER CANAL NEAR } \\
\text { FRANKLIN, WI }\end{array}$ & 42.81556 & -87.99472 & 04040002 & 55.20 & Yes & No & $\begin{array}{l}1964- \\
2009\end{array}$ & $\begin{array}{l}1964- \\
2009\end{array}$ & 46 & 44 & 0.0333 & 0.1343 & 0.1363 & 0.0616 & 0.0702 & 4.1730 \\
\hline 04087240 & ROOT RIVER AT RACINE, WI & 42.75139 & -87.82361 & 04040002 & 186.59 & No & No & $\begin{array}{l}1964- \\
2009\end{array}$ & $\begin{array}{l}1964- \\
2009\end{array}$ & 46 & 46 & 0.2146 & 0.4069 & 0.4094 & 0.2213 & 0.0490 & 4.2149 \\
\hline 04087250 & PIKE CREEK NEAR KENOSHA, WI & 42.60335 & -87.89480 & 04040002 & 7.24 & Yes & Yes & $\begin{array}{l}1960- \\
2009\end{array}$ & $\begin{array}{l}1960- \\
2009\end{array}$ & 50 & 50 & 0.0740 & 0.4229 & 0.4276 & 0.4169 & 0.0623 & 6.3059 \\
\hline 04087257 & PIKE RIVER NEAR RACINE, WI & 42.64694 & -87.86056 & 04040002 & 38.57 & Yes & No & $\begin{array}{l}1972- \\
2009\end{array}$ & $\begin{array}{l}1972- \\
2009\end{array}$ & 38 & 38 & 0.2146 & 0.4648 & 0.4693 & 0.2945 & 0.0247 & 4.4086 \\
\hline 04087300 & $\begin{array}{l}\text { LAKE MICHIGAN TRIBUTARY AT } \\
\text { WINTHROP } \\
\text { HARBOR, IL }\end{array}$ & 42.48583 & -87.82222 & 04040002 & 1.50 & Yes & Yes & $\begin{array}{l}1956- \\
1972\end{array}$ & $\begin{array}{l}1956- \\
1972\end{array}$ & 17 & 17 & 0.1786 & 0.4046 & 0.9719 & 0.3425 & 0.0172 & 22.0903 \\
\hline 04087400 & KELLOGG RAVINE AT ZION, IL & 42.46724 & -87.82480 & 04040002 & 5.07 & Yes & Yes & $\begin{array}{l}1962- \\
1976 \\
\end{array}$ & $\begin{array}{l}1962- \\
1976\end{array}$ & 15 & 15 & 0.3060 & 0.4948 & 0.9733 & 0.4112 & 0.0174 & 19.2716 \\
\hline 04093000 & $\begin{array}{l}\text { DEEP RIVER AT LAKE GEORGE } \\
\text { OUTLET AT } \\
\text { HOBART, IN }\end{array}$ & 41.53615 & -87.25698 & 04040001 & 124.13 & Yes & No & $\begin{array}{l}1947- \\
2009\end{array}$ & $\begin{array}{l}1947- \\
2009\end{array}$ & 63 & 63 & 0.1180 & 0.4782 & 0.4791 & 0.3422 & 0.1565 & 2.7885 \\
\hline 04094000 & $\begin{array}{l}\text { LITTLE CALUMET RIVER AT PORTER, } \\
\text { IN }\end{array}$ & 41.62170 & -87.08698 & 04040001 & 66.01 & Yes & No & $\begin{array}{l}1945- \\
2009\end{array}$ & $\begin{array}{l}1945- \\
2009\end{array}$ & 65 & 65 & 0.0267 & 0.3155 & 0.3236 & 0.1036 & 0.1795 & 4.1226 \\
\hline 04094500 & SALT CREEK NEAR MCCOOL, IND. & 41.59670 & -87.14448 & 04040001 & 75.24 & Yes & No & $\begin{array}{l}1945- \\
1991\end{array}$ & $\begin{array}{l}1945- \\
1991\end{array}$ & 47 & 46 & 0.0733 & 0.4299 & 0.6303 & 0.2599 & 0.1802 & 4.1397 \\
\hline
\end{tabular}




\begin{tabular}{|c|c|c|c|c|c|c|c|c|c|c|c|c|c|c|c|c|c|}
\hline $\begin{array}{l}\text { U.S. } \\
\text { Geological } \\
\text { Survey } \\
\text { Streamgage } \\
\text { number }\end{array}$ & Name & $\begin{array}{l}\text { Latitude } \\
\text { (NAD 83) }\end{array}$ & $\begin{array}{l}\text { Longitude } \\
\text { (NAD 83) }\end{array}$ & $\begin{array}{l}\text { Hydrologic } \\
\text { unit code }\end{array}$ & $\begin{array}{l}\text { Drainage } \\
\text { area }\left(\mathrm{mi}^{2}\right)\end{array}$ & $\begin{array}{l}\text { Used in } \\
\text { regression } \\
\text { analyses } \\
\text { (non- } \\
\text { redundant) } \\
\end{array}$ & $\begin{array}{l}\text { Crest- } \\
\text { stage } \\
\text { gage } \\
\text { (CSG) } \\
\end{array}$ & \begin{tabular}{|l} 
Spatial \\
POR
\end{tabular} & $\begin{array}{l}\text { Temporal } \\
\text { POR }\end{array}$ & $N$ & $N_{s}$ & $u_{f}$ & $U_{t}$ & $U_{2010}$ & NLCD_22_23_24: & $\begin{array}{l}\text { Drainage } \\
\text { Class1a }\end{array}$ & $\begin{array}{l}\text { DEM_1_0_P: } \\
\text { Basin slope } \\
\text { computed as } \\
\text { (elevation } \\
\text { range) / (basin } \\
\text { perimeter), in } \\
\text { feet per mile }\end{array}$ \\
\hline 05437950 & $\begin{array}{l}\text { KISHWAUKEE RIVER NEAR } \\
\text { HUNTLEY, IL }\end{array}$ & 42.22975 & -88.42259 & 07090006 & 13.96 & Yes & Yes & $\begin{array}{l}1965- \\
1978\end{array}$ & $\begin{array}{l}1965- \\
1978\end{array}$ & 14 & 14 & 0.0768 & 0.1356 & 0.5015 & 0.1871 & 0.1852 & 4.2136 \\
\hline 05438250 & COON CREEK AT RILEY, IL & 42.18250 & -88.64139 & 07090006 & 85.12 & Yes & Partial & \begin{tabular}{|l}
$1962-$ \\
1991 \\
\end{tabular} & $\begin{array}{l}1962- \\
1991\end{array}$ & 30 & 30 & 0.0194 & 0.0471 & 0.1546 & 0.0704 & 0.0255 & 3.8994 \\
\hline 05438283 & $\begin{array}{l}\text { PISCASAW CREEK NEAR } \\
\text { WALWORTH, WI }\end{array}$ & 42.52167 & -88.66083 & 07090006 & 9.26 & Yes & No & $\begin{array}{l}1993- \\
2009\end{array}$ & $\begin{array}{l}1993- \\
2009\end{array}$ & 17 & 17 & 0.0319 & 0.0719 & 0.0755 & 0.0456 & 0.0066 & 7.9025 \\
\hline 05438300 & $\begin{array}{l}\text { LAWRENCE CREEK TRIBUTARY NEAR } \\
\text { HARVARD, IL }\end{array}$ & 42.45835 & -88.60288 & 07090006 & 0.81 & Yes & Yes & \begin{tabular}{|l}
$1961-$ \\
1980
\end{tabular} & $\begin{array}{l}1961- \\
1980\end{array}$ & 20 & 19 & 0.0095 & 0.0095 & 0.0095 & 0.0593 & 0.0196 & 37.2431 \\
\hline 05438390 & $\begin{array}{l}\text { PISCASAW CREEK BELOW MOKELER } \\
\text { CREEK NR } \\
\text { CAPRON, IL }\end{array}$ & 42.38557 & -88.69704 & 07090006 & 88.15 & No & Yes & $\begin{array}{l}1970- \\
1979 \\
\end{array}$ & $\begin{array}{l}1970- \\
1979\end{array}$ & 10 & 10 & 0.0427 & 0.0504 & 0.1304 & 0.0699 & 0.0302 & 5.4835 \\
\hline 05438500 & $\begin{array}{l}\text { KISHWAUKEE RIVER AT BELVIDERE, } \\
\text { IL }\end{array}$ & 42.25613 & -88.86316 & 07090006 & 536.74 & Yes & No & $\begin{array}{l}1940- \\
2009\end{array}$ & $\begin{array}{l}1940- \\
2009\end{array}$ & 70 & 70 & 0.0209 & 0.1545 & 0.1622 & 0.0910 & 0.0441 & 2.1308 \\
\hline 05438850 & $\begin{array}{l}\text { MIDDLE BR OF S B KISHWAUKEE } \\
\text { RIVER NEAR } \\
\text { MALTA, IL }\end{array}$ & 41.85558 & -88.88620 & 07090006 & 1.52 & Yes & Yes & \begin{tabular}{|l}
$1956-$ \\
1980 \\
\end{tabular} & $\begin{array}{l}1956- \\
1980\end{array}$ & 25 & 24 & 0.0000 & 0.0000 & 0.0000 & 0.0282 & 0.0370 & 13.1427 \\
\hline 05439000 & $\begin{array}{l}\text { SOUTH BRANCH KISHWAUKEE } \\
\text { RIVER AT } \\
\text { DEKALB, IL } \\
\end{array}$ & 41.93111 & -88.75972 & 07090006 & 77.41 & Yes & No & $\begin{array}{l}1980- \\
2009\end{array}$ & $\begin{array}{l}1980- \\
2009\end{array}$ & 30 & 30 & 0.0470 & 0.0609 & 0.0615 & 0.0756 & 0.0170 & 2.2440 \\
\hline 05439500 & $\begin{array}{l}\text { SOUTH BRANCH KISHWAUKEE } \\
\text { RIVER NR } \\
\text { FAIRDALE IL }\end{array}$ & 42.11058 & -88.90065 & 07090006 & 387.01 & Yes & No & $\begin{array}{l}1940- \\
2009 \\
\end{array}$ & $\begin{array}{l}1940- \\
2009\end{array}$ & 70 & 70 & 0.0245 & 0.0916 & 0.0939 & 0.0843 & 0.0149 & 1.9568 \\
\hline 05439550 & $\begin{array}{l}\text { SOUTH BRANCH KISHWAUKEE } \\
\text { RIVER TRIB NR } \\
\text { IRENE, IL } \\
\end{array}$ & 42.17725 & -88.94732 & 07090006 & 1.78 & Yes & Yes & $\begin{array}{l}1959- \\
1976\end{array}$ & $\begin{array}{l}1959- \\
1976\end{array}$ & 18 & 18 & 0.0044 & 0.0222 & 0.0675 & 0.0086 & 0.0010 & 15.7822 \\
\hline 05440000 & $\begin{array}{l}\text { KISHWAUKEE RIVER NEAR } \\
\text { PERRYVILLE, IL }\end{array}$ & 42.19444 & -88.99889 & 07090006 & 1098.23 & No & No & $\begin{array}{l}1940- \\
2009 \\
\end{array}$ & $\begin{array}{l}1940- \\
2009\end{array}$ & 70 & 70 & 0.0228 & 0.1385 & 0.1437 & 0.0949 & 0.0311 & 1.7133 \\
\hline 05440500 & $\begin{array}{l}\text { KILLBUCK CREEK NEAR MONROE } \\
\text { CENTER, IL }\end{array}$ & 42.09889 & -89.05194 & 07090006 & 117.64 & Yes & Partial & $\begin{array}{l}1940- \\
1980 \\
\end{array}$ & $\begin{array}{l}1940- \\
1980\end{array}$ & 41 & 41 & 0.0078 & 0.0161 & 0.0216 & 0.0381 & 0.0067 & 2.7263 \\
\hline 05442000 & KYTE RIVER NEAR FLAGG CENTER, IL & 41.93750 & -89.15639 & 07090005 & 118.78 & Yes & No & $\begin{array}{l}1940- \\
1951\end{array}$ & $\begin{array}{l}1940- \\
1951\end{array}$ & 12 & 12 & 0.0256 & 0.0330 & 0.0710 & 0.0856 & 0.0085 & 2.3363 \\
\hline 05446950 & $\begin{array}{l}\text { GREEN RIVER TRIBUTARY NEAR } \\
\text { AMBOY, IL }\end{array}$ & 41.75837 & -89.33621 & 07090007 & 0.66 & Yes & Yes & \begin{tabular}{|l|}
$1961-$ \\
1976 \\
\end{tabular} & $\begin{array}{l}1961- \\
1976 \\
\end{array}$ & 16 & 16 & 0.0000 & 0.0000 & 0.0000 & 0.0403 & 0.0000 & 16.7906 \\
\hline 05447000 & GREEN RIVER AT AMBOY, IL & 41.70976 & -89.32454 & 07090007 & 198.69 & Yes & Partial & \begin{tabular}{|l}
$1940-$ \\
1982 \\
\end{tabular} & $\begin{array}{l}1940- \\
1982\end{array}$ & 42 & 41 & 0.0052 & 0.0078 & 0.0105 & 0.0265 & 0.0086 & 2.6497 \\
\hline 05447050 & $\begin{array}{l}\text { GREEN RIVER TRIBUTARY NEAR } \\
\text { OHIO, IL }\end{array}$ & 41.65365 & -89.45871 & 07090007 & 4.96 & Yes & Yes & $\begin{array}{l}1959- \\
1972 \\
\end{array}$ & $\begin{array}{l}1959- \\
1972 \\
\end{array}$ & 14 & 14 & 0.0031 & 0.0031 & 0.0031 & 0.0126 & 0.0274 & 9.6476 \\
\hline 05447500 & GREEN RIVER NEAR GENESEO, IL & 41.48892 & -90.15762 & 07090007 & 999.57 & Yes & No & $\begin{array}{l}1940- \\
2009\end{array}$ & $\begin{array}{l}1940- \\
2009\end{array}$ & 70 & 70 & 0.0077 & 0.0135 & 0.0135 & 0.0252 & 0.0399 & 1.1626 \\
\hline
\end{tabular}




\begin{tabular}{|c|c|c|c|c|c|c|c|c|c|c|c|c|c|c|c|c|c|}
\hline $\begin{array}{l}\text { U.S. } \\
\text { Geological } \\
\text { Survey } \\
\text { Streamgage } \\
\text { number }\end{array}$ & Name & $\begin{array}{l}\text { Latitude } \\
\text { (NAD 83) }\end{array}$ & $\begin{array}{l}\text { Longitude } \\
\text { (NAD 83) }\end{array}$ & $\begin{array}{l}\text { Hydrologic } \\
\text { unit code }\end{array}$ & $\begin{array}{l}\text { Drainage } \\
\text { area }\left(\mathrm{mi}^{2}\right)\end{array}$ & \begin{tabular}{|l} 
Used in \\
regression \\
analyses \\
(non- \\
redundant)
\end{tabular} & $\begin{array}{l}\text { Crest- } \\
\text { stage } \\
\text { gage } \\
\text { (CSG) }\end{array}$ & \begin{tabular}{|l} 
Spatial \\
POR
\end{tabular} & $\begin{array}{l}\text { Temporal } \\
\text { POR }\end{array}$ & $N$ & $N_{s}$ & $u_{f}$ & $u_{1}$ & $U_{2010}$ & NLCD_22_23_24: & $\begin{array}{l}\text { Drainage_ } \\
\text { Class1a }\end{array}$ & $\begin{array}{l}\text { DEM_1_0_P: } \\
\text { Basin slope } \\
\text { computed as } \\
\text { (elevation } \\
\text { range) / (basin } \\
\text { perimeter), in } \\
\text { feet per mile }\end{array}$ \\
\hline 05517500 & $\begin{array}{l}\text { KANKAKEE RIVER AT DUNNS } \\
\text { BRIDGE, IN }\end{array}$ & 41.22004 & -86.96836 & 07120001 & 1351.30 & Yes & No & $\begin{array}{l}1949- \\
2009\end{array}$ & $\begin{array}{l}1949- \\
2009\end{array}$ & 61 & 61 & 0.0238 & 0.0888 & 0.0894 & 0.0400 & 0.1721 & 0.8207 \\
\hline 05517890 & COBB DITCH NEAR KOUTS, IND. & 41.33865 & -87.07503 & 07120001 & 30.62 & Yes & No & $\begin{array}{l}1968- \\
2003 \\
\end{array}$ & $\begin{array}{l}1968- \\
2003\end{array}$ & 36 & 35 & 0.0216 & 0.1891 & 0.2466 & 0.0414 & 0.2562 & 4.2023 \\
\hline 05518000 & KANKAKEE RIVER AT SHELBY, IN & 41.18281 & -87.34031 & 07120001 & 1777.67 & No & No & $\begin{array}{l}1940- \\
2009\end{array}$ & $\begin{array}{l}1940- \\
2009\end{array}$ & 70 & 70 & 0.0164 & 0.1000 & 0.1012 & 0.0436 & 0.1859 & 0.6956 \\
\hline 05519000 & $\begin{array}{l}\text { SINGLETON DITCH AT SCHNEIDER, } \\
\text { IN }\end{array}$ & 41.21226 & -87.44837 & 07120001 & 122.84 & Yes & No & $\begin{array}{l}1951- \\
2001\end{array}$ & $\begin{array}{l}1949- \\
2001\end{array}$ & 53 & 50 & 0.0442 & 0.1604 & 0.1676 & 0.1026 & 0.1564 & 2.0038 \\
\hline 05519500 & $\begin{array}{l}\text { WEST CREEK NEAR SCHNEIDER, } \\
\text { IND. }\end{array}$ & 41.21365 & -87.49642 & 07120001 & 55.05 & Yes & Partial & $\begin{array}{l}1949- \\
1972\end{array}$ & $\begin{array}{l}1949- \\
1972\end{array}$ & 23 & 21 & 0.0312 & 0.0879 & 0.1986 & 0.1422 & 0.1478 & 2.1296 \\
\hline 05520500 & KANKAKEE RIVER AT MOMENCE, IL & 41.16003 & -87.66865 & 07120001 & 2296.66 & No & No & $\begin{array}{l}1940- \\
2009\end{array}$ & $\begin{array}{l}1940- \\
2009\end{array}$ & 70 & 70 & 0.0162 & 0.0991 & 0.1001 & 0.0501 & 0.1636 & 0.6007 \\
\hline 05526150 & $\begin{array}{l}\text { KANKAKEE RIVER TRIBUTARY NEAR } \\
\text { BOURBONNAIS, IL } \\
\end{array}$ & 41.19167 & -87.94972 & 07120001 & 0.16 & Yes & Yes & $\begin{array}{l}1956- \\
1980\end{array}$ & $\begin{array}{l}1956- \\
1980\end{array}$ & 25 & 24 & 0.0000 & 0.0000 & 0.0000 & 0.0022 & 0.0000 & 24.1357 \\
\hline 05526500 & $\begin{array}{l}\begin{array}{l}\text { TERRY CREEK NEAR CUSTER PARK, } \\
\text { IL }\end{array} \\
\end{array}$ & 41.23333 & -88.09833 & 07120001 & 13.00 & Yes & No & $\begin{array}{l}1950- \\
1975\end{array}$ & $\begin{array}{l}1950- \\
1975\end{array}$ & 26 & 26 & 0.0003 & 0.0034 & 0.0570 & 0.0228 & 0.0182 & 3.5253 \\
\hline 05527050 & $\begin{array}{l}\text { PRAIRIE CREEK NEAR FRANKFORT, } \\
\text { IL }\end{array}$ & 41.43670 & -87.84505 & 07120001 & 0.83 & Yes & Yes & $\begin{array}{l}1956- \\
1972\end{array}$ & $\begin{array}{l}1956- \\
1972\end{array}$ & 17 & 17 & 0.0046 & 0.0065 & 0.2350 & 0.0742 & 0.0006 & 11.5575 \\
\hline 05527800 & DES PLAINES RIVER AT RUSSELL, IL & 42.48919 & -87.92647 & 07120004 & 123.65 & Yes & Partial & $\begin{array}{l}1960- \\
2009\end{array}$ & $\begin{array}{l}1960- \\
2009\end{array}$ & 50 & 50 & 0.0499 & 0.1950 & 0.1982 & 0.1204 & 0.0878 & 2.4754 \\
\hline 05527840 & $\begin{array}{l}\text { DES PLAINES RIVER AT } \\
\text { WADSWORTH, IL }\end{array}$ & 42.42919 & -87.93035 & 07120004 & 145.52 & No & Yes & $\begin{array}{l}1962- \\
1976\end{array}$ & $\begin{array}{l}1962- \\
1976\end{array}$ & 15 & 15 & 0.0544 & 0.0921 & 0.2309 & 0.1255 & 0.0860 & 2.2110 \\
\hline 05527870 & MILL CREEK AT WEDGES CORNER, IL & 42.38335 & -88.00425 & 07120004 & 18.33 & Yes & Yes & $\begin{array}{l}1962- \\
1976\end{array}$ & $\begin{array}{l}1960- \\
1976\end{array}$ & 16 & 14 & 0.2181 & 0.3048 & 0.5683 & 0.3905 & 0.2273 & 3.5393 \\
\hline 05527900 & $\begin{array}{l}\text { NORTH MILL CREEK AT HICKORY } \\
\text { CORNERS, IL }\end{array}$ & 42.46583 & -88.00917 & 07120004 & 20.79 & Yes & Yes & $\begin{array}{l}1962- \\
1976\end{array}$ & $\begin{array}{l}1960- \\
2009\end{array}$ & 18 & 15 & 0.0428 & 0.2578 & 0.2674 & 0.0697 & 0.1902 & 4.7889 \\
\hline 05527950 & MILL CREEK AT OLD MILL CREEK, IL & 42.41528 & -87.96917 & 07120004 & 59.87 & No & Partial & $\begin{array}{l}1962- \\
2009\end{array}$ & $\begin{array}{l}1960- \\
2009\end{array}$ & 36 & 35 & 0.1317 & 0.4428 & 0.4565 & 0.2385 & 0.1744 & 3.0358 \\
\hline 05528000 & $\begin{array}{l}\text { DES PLAINES RIVER NEAR GURNEE, } \\
\text { IL }\end{array}$ & 42.34389 & -87.94111 & 07120004 & 232.00 & No & Partial & $\begin{array}{l}1946- \\
2009\end{array}$ & $\begin{array}{l}1946- \\
2009\end{array}$ & 63 & 62 & 0.0429 & 0.3374 & 0.3465 & 0.1885 & 0.1051 & 1.7730 \\
\hline 05528150 & $\begin{array}{l}\begin{array}{l}\text { INDIAN CREEK AT DIAMOND LAKE, } \\
\text { IL }\end{array} \\
\end{array}$ & 42.22419 & -88.00480 & 07120004 & 10.92 & Yes & Yes & $\begin{array}{l}1960- \\
1976\end{array}$ & $\begin{array}{l}1960- \\
1976\end{array}$ & 17 & 17 & 0.1469 & 0.2169 & 0.9258 & 0.3955 & 0.0973 & 9.6960 \\
\hline 05528170 & $\begin{array}{l}\text { DIAMOND LAKE DRAIN AT } \\
\text { MUNDELEIN, IL }\end{array}$ & 42.24891 & -87.99369 & 07120004 & 2.58 & Yes & Yes & $\begin{array}{l}1961- \\
1976\end{array}$ & $\begin{array}{l}1961- \\
1976\end{array}$ & 16 & 16 & 0.4165 & 0.4548 & 0.7162 & 0.4660 & 0.1927 & 11.3353 \\
\hline 05528200 & $\begin{array}{l}\text { HAWTHORN DRAINAGE DITCH } \\
\text { NEAR } \\
\text { MUNDELEIN, IL }\end{array}$ & 42.24030 & -87.96174 & 07120004 & 5.91 & Yes & Yes & $\begin{array}{l}1963- \\
1976\end{array}$ & $\begin{array}{l}1961- \\
1976\end{array}$ & 16 & 14 & 0.4517 & 0.6639 & 0.9217 & 0.7402 & 0.0779 & 10.9833 \\
\hline 05528230 & INDIAN CREEK AT PRAIRIE VIEW, IL & 42.20944 & -87.95500 & 07120004 & 35.51 & No & Partial & $\begin{array}{l}1963- \\
1996\end{array}$ & $\begin{array}{l}1960- \\
1996\end{array}$ & 21 & 18 & 0.2174 & 0.6281 & 0.9130 & 0.5174 & 0.0844 & 6.8325 \\
\hline
\end{tabular}




\begin{tabular}{|c|c|c|c|c|c|c|c|c|c|c|c|c|c|c|c|c|c|}
\hline $\begin{array}{l}\text { U.S. } \\
\text { Geological } \\
\text { Survey } \\
\text { Streamgage } \\
\text { number }\end{array}$ & Name & $\begin{array}{l}\text { Latitude } \\
\text { (NAD 83) }\end{array}$ & $\begin{array}{l}\text { Longitude } \\
\text { (NAD 83) }\end{array}$ & $\begin{array}{l}\text { Hydrologic } \\
\text { unit code }\end{array}$ & $\begin{array}{l}\text { Drainage } \\
\text { area }\left(\mathrm{mi}^{2}\right)\end{array}$ & \begin{tabular}{|l} 
Used in \\
regression \\
analyses \\
(non- \\
redundant)
\end{tabular} & \begin{tabular}{|l|} 
Crest- \\
stage \\
gage \\
(CSG) \\
\end{tabular} & $\begin{array}{l}\text { Spatial } \\
\text { POR }\end{array}$ & $\begin{array}{l}\text { Temporal } \\
\text { POR }\end{array}$ & $\boldsymbol{N}$ & $N_{s}$ & $u_{f}$ & $u_{1}$ & $U_{2010}$ & NLCD_22_23_24: & $\begin{array}{l}\begin{array}{l}\text { Drainage_ } \\
\text { Class1a }\end{array} \\
\end{array}$ & $\begin{array}{l}\text { DEM_1_0_P: } \\
\text { Basin slope } \\
\text { computed as } \\
\text { (elevation } \\
\text { range) / (basin } \\
\text { perimeter), in } \\
\text { feet per mile }\end{array}$ \\
\hline 05528360 & APTAKISIC CREEK AT APTAKISIC, IL & 42.16808 & -87.94952 & 07120004 & 2.83 & Yes & Yes & $\begin{array}{l}1961- \\
1976\end{array}$ & $\begin{array}{l}1961- \\
1976\end{array}$ & 16 & 16 & 0.0621 & 0.2616 & 1.0000 & 0.6690 & 0.0664 & 4.7725 \\
\hline 05528400 & $\begin{array}{l}\text { DES PLAINES RIVER AT WHEELING, } \\
\text { IL }\end{array}$ & 42.13919 & -87.90396 & 07120004 & 325.15 & No & Yes & $\begin{array}{l}1962- \\
1977\end{array}$ & $\begin{array}{l}1962- \\
1977\end{array}$ & 11 & 11 & 0.1360 & 0.2174 & 0.4823 & 0.2705 & 0.0952 & 1.7224 \\
\hline 05528440 & $\begin{array}{l}\text { BUFFALO CREEK NEAR LAKE } \\
\text { ZURICH, IL }\end{array}$ & 42.18280 & -88.05063 & 07120004 & 1.66 & Yes & Yes & $\begin{array}{l}1961- \\
1976\end{array}$ & $\begin{array}{l}1961- \\
1976\end{array}$ & 16 & 16 & 0.3332 & 0.6854 & 0.9974 & 0.8055 & 0.0746 & 15.7818 \\
\hline 05528470 & $\begin{array}{l}\text { BUFFALO CREEK AT LONG GROVE, } \\
\text { IL }\end{array}$ & 42.17725 & -87.99924 & 07120004 & 8.96 & No & Yes & $\begin{array}{l}1961- \\
1976\end{array}$ & $\begin{array}{l}1961- \\
1976\end{array}$ & 16 & 15 & 0.1920 & 0.5169 & 0.9862 & 0.4627 & 0.1001 & 6.8250 \\
\hline 05528500 & $\begin{array}{l}\text { BUFFALO CREEK NEAR WHEELING, } \\
\text { IL }\end{array}$ & 42.15194 & -87.95778 & 07120004 & 19.64 & Yes & No & $\begin{array}{l}1953- \\
2009\end{array}$ & $\begin{array}{l}1953- \\
2009\end{array}$ & 57 & 56 & 0.0952 & 0.8807 & 0.8914 & 0.5859 & 0.0835 & 6.5236 \\
\hline 05529000 & $\begin{array}{l}\text { DES PLAINES RIVER NEAR DES } \\
\text { PLAINES, IL }\end{array}$ & 42.08167 & -87.89056 & 07120004 & 364.30 & No & No & $\begin{array}{l}1941- \\
2009\end{array}$ & $\begin{array}{l}1941- \\
2009\end{array}$ & 69 & 69 & 0.0423 & 0.5052 & 0.5166 & 0.3087 & 0.0921 & 1.5720 \\
\hline 05529300 & $\begin{array}{l}\text { MC DONALD CREEK NEAR } \\
\text { WHEELING, IL }\end{array}$ & 42.12058 & -87.94646 & 07120004 & 4.50 & No & Yes & $\begin{array}{l}1961- \\
1979\end{array}$ & $\begin{array}{l}1955- \\
1979\end{array}$ & 21 & 19 & 0.3820 & 0.9468 & 0.9535 & 0.8819 & 0.0206 & 6.0650 \\
\hline 05529500 & $\begin{array}{l}\text { MC DONALD CREEK NEAR } \\
\text { MOUNT PROSPECT, IL }\end{array}$ & 42.09530 & -87.91285 & 07120004 & 7.83 & Yes & No & $\begin{array}{l}1953- \\
2009\end{array}$ & $\begin{array}{l}1953- \\
2009\end{array}$ & 57 & 56 & 0.3807 & 0.9571 & 0.9571 & 0.8413 & 0.0218 & 4.8367 \\
\hline 05529900 & $\begin{array}{l}\text { WELLER CREEK AT MOUNT } \\
\text { PROSPECT, IL }\end{array}$ & 42.05892 & -87.95646 & 07120004 & 8.96 & No & Yes & $\begin{array}{l}1961- \\
1977\end{array}$ & $\begin{array}{l}1961- \\
1979\end{array}$ & 18 & 16 & 0.9256 & 0.9617 & 0.9693 & 0.9005 & 0.0047 & 4.7531 \\
\hline 05530000 & WELLER CREEK AT DES PLAINES, IL & 42.04947 & -87.91812 & 07120004 & 12.73 & Yes & No & $\begin{array}{l}1951- \\
2009\end{array}$ & $\begin{array}{l}1951- \\
2009\end{array}$ & 59 & 59 & 0.6469 & 0.9751 & 0.9751 & 0.9043 & 0.0038 & 3.9615 \\
\hline 05530400 & $\begin{array}{l}\text { HIGGINS CREEK NEAR MOUNT } \\
\text { PROSPECT, IL }\end{array}$ & 42.03447 & -87.95979 & 07120004 & 2.05 & Yes & Yes & $\begin{array}{l}1961- \\
1979\end{array}$ & $\begin{array}{l}1961- \\
1979\end{array}$ & 19 & 19 & 0.2441 & 0.5259 & 0.6447 & 0.6959 & 0.0671 & 6.5743 \\
\hline 05530480 & $\begin{array}{l}\text { WILLOW CREEK AT ORCHARD } \\
\text { PLACE, IL }\end{array}$ & 41.99697 & -87.87979 & 07120004 & 17.92 & Yes & Yes & $\begin{array}{l}1961- \\
1979\end{array}$ & $\begin{array}{l}1955- \\
1979\end{array}$ & 21 & 19 & 0.1563 & 0.6599 & 0.7978 & 0.9006 & 0.0215 & 2.9481 \\
\hline 05530600 & $\begin{array}{l}\text { DES PLAINES RIVER AT RIVER } \\
\text { GROVE, IL }\end{array}$ & 41.92948 & -87.84451 & 07120004 & 463.01 & No & Yes & $\begin{array}{l}1960- \\
1977\end{array}$ & $\begin{array}{l}1960- \\
1977\end{array}$ & 18 & 18 & 0.2504 & 0.3769 & 0.5862 & 0.4259 & 0.0756 & 1.3236 \\
\hline 05530700 & SILVER CREEK AT MELROSE PARK, IL & 41.90475 & -87.84506 & 07120004 & 11.15 & Yes & Yes & $\begin{array}{l}1961- \\
1980\end{array}$ & $\begin{array}{l}1955- \\
1980\end{array}$ & 21 & 20 & 0.6507 & 0.8474 & 0.8922 & 0.9361 & 0.0107 & 4.1101 \\
\hline 05530800 & $\begin{array}{l}\text { DES PLAINES RIVER AT FOREST } \\
\text { PARK, IL }\end{array}$ & 41.86809 & -87.82756 & 07120004 & 481.08 & No & Yes & $\begin{array}{l}1954- \\
1976\end{array}$ & $\begin{array}{l}1954- \\
1976\end{array}$ & 23 & 23 & 0.2172 & 0.3919 & 0.5979 & 0.4445 & 0.0730 & 1.2716 \\
\hline 05530940 & SALT CREEK AT PALATINE, IL & 42.11058 & -88.06313 & 07120004 & 5.71 & Yes & Yes & $\begin{array}{l}1961- \\
1980\end{array}$ & $\begin{array}{l}1961- \\
1980\end{array}$ & 19 & 19 & 0.3773 & 0.8531 & 0.9413 & 0.6277 & 0.1429 & 8.7956 \\
\hline 05530960 & SALT CREEK NEAR PALATINE, IL & 42.07392 & -88.04396 & 07120004 & 15.83 & No & Yes & $\begin{array}{l}1961- \\
1979 \\
\end{array}$ & $\begin{array}{l}1961- \\
1979\end{array}$ & 19 & 19 & 0.3344 & 0.7149 & 0.7891 & 0.6453 & 0.1169 & 5.6670 \\
\hline 05530990 & $\begin{array}{l}\text { SALT CREEK AT ROLLING } \\
\text { MEADOWS, IL }\end{array}$ & 42.06056 & -88.01667 & 07120004 & 27.11 & No & No & $\begin{array}{l}1974- \\
2009\end{array}$ & $\begin{array}{l}1974- \\
2009\end{array}$ & 36 & 36 & 0.7261 & 0.8420 & 0.8420 & 0.7367 & 0.0920 & 5.1206 \\
\hline 05531000 & $\begin{array}{l}\text { SALT CREEK NEAR ARLINGTON } \\
\text { HEIGHTS, IL } \\
\end{array}$ & 42.05083 & -88.01000 & 07120004 & 28.62 & No & Partial & $\begin{array}{l}1951- \\
1976 \\
\end{array}$ & $\begin{array}{l}1951- \\
1976 \\
\end{array}$ & 26 & 26 & 0.2428 & 0.7567 & 0.8465 & 0.7459 & 0.0875 & 4.6420 \\
\hline 05531050 & SALT CREEK NEAR WOOD DALE, IL & 41.99281 & -87.99562 & 07120004 & 50.40 & No & Yes & $\begin{array}{l}1961- \\
1976\end{array}$ & $\begin{array}{l}1955- \\
1979\end{array}$ & 22 & 16 & 0.2631 & 0.7281 & 0.7841 & 0.7183 & 0.0885 & 3.9700 \\
\hline
\end{tabular}




\begin{tabular}{|c|c|c|c|c|c|c|c|c|c|c|c|c|c|c|c|c|c|}
\hline $\begin{array}{l}\text { U.S. } \\
\text { Geological } \\
\text { Survey } \\
\text { Streamgage } \\
\text { number }\end{array}$ & Name & $\begin{array}{l}\text { Latitude } \\
\text { (NAD 83) }\end{array}$ & $\begin{array}{l}\text { Longitude } \\
\text { (NAD 83) }\end{array}$ & $\begin{array}{l}\text { Hydrologic } \\
\text { unit code }\end{array}$ & $\begin{array}{l}\text { Drainage } \\
\text { area }\left(\mathrm{mi}^{2}\right)\end{array}$ & $\begin{array}{l}\text { Used in } \\
\text { regression } \\
\text { analyses } \\
\text { (non- } \\
\text { redundant) } \\
\end{array}$ & $\begin{array}{l}\text { Crest- } \\
\text { stage } \\
\text { gage } \\
\text { (CSG) } \\
\end{array}$ & \begin{tabular}{|l} 
Spatial \\
POR
\end{tabular} & $\begin{array}{l}\text { Temporal } \\
\text { POR }\end{array}$ & $N$ & $N_{s}$ & $u_{f}$ & $u_{1}$ & $U_{2010}$ & NLCD_22_23_24: & $\begin{array}{l}\text { Drainage } \\
\text { Class1a }\end{array}$ & $\begin{array}{l}\text { DEM_1_0_P: } \\
\text { Basin slope } \\
\text { computed as } \\
\text { (elevation } \\
\text { range) / (basin } \\
\text { perimeter), in } \\
\text { feet per mile }\end{array}$ \\
\hline 05531080 & $\begin{array}{l}\text { SPRING BROOK AT } \\
\text { BLOOMINGDALE, IL }\end{array}$ & 41.95864 & -88.07063 & 07120004 & 6.17 & Yes & Yes & \begin{tabular}{|l}
$1961-$ \\
1979
\end{tabular} & $\begin{array}{l}1961- \\
1979\end{array}$ & 19 & 17 & 0.4205 & 0.7656 & 0.9178 & 0.8118 & 0.0511 & 5.6661 \\
\hline 05531100 & MEACHAM CREEK AT MEDINAH, IL & 41.97753 & -88.04785 & 07120004 & 4.20 & Yes & Yes & \begin{tabular}{|l}
$1956-$ \\
1979 \\
\end{tabular} & $\begin{array}{l}1956- \\
1979\end{array}$ & 19 & 19 & 0.4144 & 0.8867 & 0.9761 & 0.8473 & 0.0703 & 8.4784 \\
\hline 05531130 & $\begin{array}{l}\text { SPRING BROOK AT WALNUT AVE AT } \\
\text { ITASCA, IL }\end{array}$ & 41.97114 & -88.01312 & 07120004 & 15.04 & No & Yes & \begin{tabular}{|l|}
$1961-$ \\
1979 \\
\end{tabular} & $\begin{array}{l}1961- \\
1979 \\
\end{array}$ & 18 & 15 & 0.4560 & 0.7312 & 0.9211 & 0.7634 & 0.0540 & 5.0997 \\
\hline 05531200 & SALT CREEK AT ADDISON, IL & 41.92892 & -87.97784 & 07120004 & 82.56 & No & Yes & $\begin{array}{l}1960- \\
1976 \\
\end{array}$ & $\begin{array}{l}1948- \\
1976\end{array}$ & 21 & 17 & 0.1529 & 0.6894 & 0.8200 & 0.7409 & 0.0758 & 2.7069 \\
\hline 05531300 & SALT CREEK AT ELMHURST, IL & 41.88614 & -87.95923 & 07120004 & 91.25 & No & Partial & $\begin{array}{l}1960- \\
2009 \\
\end{array}$ & $\begin{array}{l}1948- \\
2009\end{array}$ & 46 & 33 & 0.2102 & 0.8308 & 0.8332 & 0.7585 & 0.0709 & 2.6206 \\
\hline 05531380 & SALT CREEK AT OAK BROOK, IL & 41.86086 & -87.94784 & 07120004 & 100.85 & No & Yes & $\begin{array}{l}1960- \\
1976 \\
\end{array}$ & $\begin{array}{l}1948- \\
1976\end{array}$ & 21 & 17 & 0.2468 & 0.7432 & 0.8475 & 0.7687 & 0.0659 & 2.3553 \\
\hline 05531500 & $\begin{array}{l}\text { SALT CREEK AT WESTERN SPRINGS, } \\
\text { IL }\end{array}$ & 41.82583 & -87.90028 & 07120004 & 116.25 & Yes & No & $\begin{array}{l}1946- \\
2009 \\
\end{array}$ & $\begin{array}{l}1946- \\
2009\end{array}$ & 64 & 57 & 0.2219 & 0.8474 & 0.8508 & 0.7602 & 0.0660 & 2.3509 \\
\hline 05531800 & ADDISON CREEK AT NORTHLAKE, IL & 41.91031 & -87.90284 & 07120004 & 6.89 & No & Yes & $\begin{array}{l}1961- \\
1976\end{array}$ & $\begin{array}{l}1961- \\
1980\end{array}$ & 19 & 15 & 0.6265 & 0.8094 & 0.9117 & 0.7853 & 0.0222 & 4.3848 \\
\hline 05532000 & ADDISON CREEK AT BELLWOOD, IL & 41.88170 & -87.86923 & 07120004 & 16.19 & Yes & No & \begin{tabular}{|l}
$1951-$ \\
2009
\end{tabular} & $\begin{array}{l}1951- \\
2009\end{array}$ & 59 & 58 & 0.6007 & 0.9487 & 0.9523 & 0.8931 & 0.0118 & 3.4123 \\
\hline 05532500 & DES PLAINES RIVER AT RIVERSIDE, IL & 41.82167 & -87.82194 & 07120004 & 635.65 & No & Partial & $\begin{array}{l}1940- \\
2009\end{array}$ & $\begin{array}{l}1940- \\
2009\end{array}$ & 70 & 68 & 0.1452 & 0.6509 & 0.6582 & 0.5265 & 0.0680 & 1.2028 \\
\hline 05533000 & $\begin{array}{l}\text { FLAG CREEK NEAR WILLOW } \\
\text { SPRINGS, IL }\end{array}$ & 41.73889 & -87.89639 & 07120004 & 16.82 & Yes & No & $\begin{array}{l}1951- \\
2009\end{array}$ & $\begin{array}{l}1951- \\
2009\end{array}$ & 59 & 59 & 0.4949 & 0.9299 & 0.9318 & 0.8492 & 0.0395 & 6.1458 \\
\hline 05533200 & $\begin{array}{l}\text { SAWMILL CREEK TRIBUTARY NEAR } \\
\text { TIEDTVILLE, IL }\end{array}$ & 41.73475 & -87.96673 & 07120004 & 2.35 & Yes & Yes & \begin{tabular}{|l}
$1961-$ \\
1979 \\
\end{tabular} & $\begin{array}{l}1961- \\
1979\end{array}$ & 18 & 17 & 0.5995 & 0.9485 & 0.9889 & 0.9090 & 0.0051 & 12.3731 \\
\hline 05533300 & $\begin{array}{l}\text { WARDS CREEK NEAR WOODRIDGE, } \\
\text { IL }\end{array}$ & 41.72559 & -87.98867 & 07120004 & 3.11 & Yes & Yes & \begin{tabular}{|l|}
$1962-$ \\
1976 \\
\end{tabular} & $\begin{array}{l}1962- \\
1976\end{array}$ & 15 & 15 & 0.3059 & 0.7006 & 0.9676 & 0.6753 & 0.0735 & 7.9840 \\
\hline 05533400 & SAWMILL CREEK NEAR LEMONT, IL & 41.70778 & -87.96278 & 07120004 & 12.16 & No & Partial & $\begin{array}{l}1961- \\
2009\end{array}$ & $\begin{array}{l}1961- \\
2009\end{array}$ & 43 & 40 & 0.3362 & 0.7845 & 0.7903 & 0.6504 & 0.0477 & 6.3108 \\
\hline 05534300 & $\begin{array}{l}\text { NORTH BRANCH CHICAGO RIVER AT } \\
\text { LAKE FOREST, IL }\end{array}$ & 42.24002 & -87.88063 & 07120003 & 11.18 & No & Yes & $\begin{array}{l}1961- \\
1976 \\
\end{array}$ & $\begin{array}{l}1961- \\
1976\end{array}$ & 16 & 16 & 0.0697 & 0.1907 & 0.7346 & 0.4606 & 0.0415 & 2.8830 \\
\hline 05534400 & $\begin{array}{l}\text { NORTH BRANCH CHICAGO RIVER AT } \\
\text { BANNOCKBURN, IL }\end{array}$ & 42.20002 & -87.85285 & 07120003 & 15.61 & No & Yes & $\begin{array}{l}1960- \\
1976 \\
\end{array}$ & $\begin{array}{l}1960- \\
1976\end{array}$ & 17 & 17 & 0.1129 & 0.2687 & 0.7748 & 0.4276 & 0.0386 & 2.5479 \\
\hline 05534500 & $\begin{array}{l}\text { NORTH BRANCH CHICAGO RIVER AT } \\
\text { DEERFIELD, IL }\end{array}$ & 42.15278 & -87.81861 & 07120003 & 19.70 & Yes & No & \begin{tabular}{|l}
$1953-$ \\
2009
\end{tabular} & $\begin{array}{l}1953- \\
2009\end{array}$ & 57 & 56 & 0.1443 & 0.8000 & 0.8139 & 0.4517 & 0.0323 & 2.0827 \\
\hline 05534600 & $\begin{array}{l}\text { NORTH BRANCH CHICAGO RIVER AT } \\
\text { NORTHFIELD, IL }\end{array}$ & 42.10142 & -87.77395 & 07120003 & 23.70 & No & Yes & $\begin{array}{l}1960- \\
1980 \\
\end{array}$ & $\begin{array}{l}1960- \\
1980\end{array}$ & 20 & 19 & 0.3166 & 0.5076 & 0.7970 & 0.4686 & 0.0278 & 2.1406 \\
\hline 05534900 & SKOKIE RIVER AT LAKE BLUFF, IL & 42.27947 & -87.86285 & 07120003 & 7.87 & No & Yes & $\begin{array}{l}1962- \\
1976 \\
\end{array}$ & $\begin{array}{l}1962- \\
1976 \\
\end{array}$ & 15 & 15 & 0.3758 & 0.6002 & 0.8271 & 0.6611 & 0.0630 & 3.5010 \\
\hline 05535000 & SKOKIE RIVER AT LAKE FOREST, IL & 42.23250 & -87.84528 & 07120003 & 12.63 & Yes & No & \begin{tabular}{|l}
$1952-$ \\
2009
\end{tabular} & $\begin{array}{l}1952- \\
2009\end{array}$ & 58 & 58 & 0.2110 & 0.8497 & 0.8629 & 0.5515 & 0.0443 & 2.8227 \\
\hline
\end{tabular}




\begin{tabular}{|c|c|c|c|c|c|c|c|c|c|c|c|c|c|c|c|c|c|}
\hline $\begin{array}{l}\text { U.S. } \\
\text { Geological } \\
\text { Survey } \\
\text { Streamgage } \\
\text { number }\end{array}$ & Name & $\begin{array}{l}\text { Latitude } \\
\text { (NAD 83) }\end{array}$ & $\begin{array}{l}\text { Longitude } \\
\text { (NAD 83) }\end{array}$ & $\begin{array}{l}\text { Hydrologic } \\
\text { unit code }\end{array}$ & $\begin{array}{l}\text { Drainage } \\
\text { area }\left(\mathrm{mi}^{2}\right)\end{array}$ & $\begin{array}{l}\text { Used in } \\
\text { regression } \\
\text { analyses } \\
\text { (non- } \\
\text { redundant) }\end{array}$ & $\begin{array}{l}\text { Crest- } \\
\text { stage } \\
\text { gage } \\
\text { (CSG) }\end{array}$ & $\begin{array}{l}\text { Spatial } \\
\text { POR }\end{array}$ & $\begin{array}{l}\text { Temporal } \\
\text { POR }\end{array}$ & $N$ & $N_{s}$ & $u_{f}$ & $u_{t}$ & $U_{2010}$ & NLCD_22_23_24: & $\begin{array}{l}\text { Drainage } \\
\text { Class1a }\end{array}$ & $\begin{array}{l}\text { DEM_1_0_P: } \\
\text { Basin slope } \\
\text { computed as } \\
\text { (elevation } \\
\text { range) / (basin } \\
\text { perimeter), in } \\
\text { feet per mile }\end{array}$ \\
\hline 05535070 & $\begin{array}{l}\text { SKOKIE RIVER NEAR HIGHLAND } \\
\text { PARK, IL }\end{array}$ & 42.15972 & -87.79806 & 07120003 & 20.75 & No & No & \begin{tabular}{|l|}
$1967-$ \\
2009
\end{tabular} & $\begin{array}{l}1967- \\
2009\end{array}$ & 43 & 43 & 0.5727 & 0.8953 & 0.9076 & 0.5495 & 0.0385 & 2.4357 \\
\hline 05535150 & SKOKIE RIVER AT NORTHFIELD, IL & 42.10142 & -87.75923 & 07120003 & 27.93 & No & Yes & \begin{tabular}{|l|}
$1960-$ \\
1979
\end{tabular} & $\begin{array}{l}1960- \\
1979\end{array}$ & 19 & 18 & 0.4710 & 0.6553 & 0.8333 & 0.5591 & 0.0479 & 2.0473 \\
\hline 05535200 & $\begin{array}{l}\text { NORTH BRANCH CHICAGO RIVER AT } \\
\text { GLENVIEW, IL }\end{array}$ & 42.06892 & -87.77451 & 07120003 & 57.17 & No & Yes & \begin{tabular}{|l|}
$1960-$ \\
1969 \\
\end{tabular} & $\begin{array}{l}1960- \\
1977\end{array}$ & 18 & 10 & 0.4420 & 0.6004 & 0.8159 & 0.5321 & 0.0357 & 1.8011 \\
\hline 05535300 & $\begin{array}{l}\text { WF OF NB CHICAGO RIVER AT } \\
\text { BANNOCKBURN, IL }\end{array}$ & 42.20002 & -87.89007 & 07120003 & 2.80 & No & Yes & \begin{tabular}{|l|}
$1961-$ \\
1976
\end{tabular} & $\begin{array}{l}1961- \\
1976\end{array}$ & 16 & 16 & 0.2424 & 0.4784 & 0.9025 & 0.3678 & 0.0185 & 4.2389 \\
\hline 05535400 & $\begin{array}{l}\text { WF OF NB CHICAGO RIVER AT } \\
\text { DEERFIELD, IL }\end{array}$ & 42.16725 & -87.85673 & 07120003 & 6.99 & No & Yes & $\begin{array}{l}1961- \\
1976\end{array}$ & $\begin{array}{l}1961- \\
1976\end{array}$ & 16 & 16 & 0.4225 & 0.6556 & 0.9354 & 0.4453 & 0.0277 & 2.9551 \\
\hline 05535500 & $\begin{array}{l}\text { WF OF NB CHICAGO RIVER AT } \\
\text { NORTHBROOK IL }\end{array}$ & 42.13833 & -87.83472 & 07120003 & 11.64 & Yes & No & \begin{tabular}{|l|}
$1953-$ \\
2009 \\
\end{tabular} & $\begin{array}{l}1953- \\
2009\end{array}$ & 57 & 54 & 0.2507 & 0.9105 & 0.9198 & 0.6085 & 0.0236 & 2.8439 \\
\hline 05535700 & $\begin{array}{l}\text { WF OF NB CHICAGO RIVER AT } \\
\text { GLENVIEW, IL }\end{array}$ & 42.08697 & -87.80229 & 07120003 & 21.89 & No & Yes & $\begin{array}{l}1960- \\
1977\end{array}$ & $\begin{array}{l}1960- \\
1977\end{array}$ & 18 & 18 & 0.4549 & 0.7159 & 0.8565 & 0.7235 & 0.0224 & 2.3616 \\
\hline 05535800 & $\begin{array}{l}\text { NORTH BRANCH CHICAGO RIVER AT } \\
\text { MORTON GROVE, IL }\end{array}$ & 42.05003 & -87.78062 & 07120003 & 92.03 & No & Yes & $\begin{array}{l}1960- \\
1978\end{array}$ & $\begin{array}{l}1960- \\
1979\end{array}$ & 20 & 15 & 0.4996 & 0.6772 & 0.8297 & 0.6202 & 0.0281 & 1.7657 \\
\hline 05536000 & $\begin{array}{l}\text { NORTH BRANCH CHICAGO RIVER AT } \\
\text { NILES, IL }\end{array}$ & 42.01222 & -87.79583 & 07120003 & 99.34 & Yes & No & $\begin{array}{l}1951- \\
2009\end{array}$ & $\begin{array}{l}1951- \\
2009\end{array}$ & 59 & 53 & 0.3514 & 0.8249 & 0.8315 & 0.6393 & 0.0264 & 1.6934 \\
\hline 05536105 & $\begin{array}{l}\text { NB CHICAGO RIVER AT ALBANY } \\
\text { AVENUE AT CHICAGO, IL }\end{array}$ & 41.97417 & -87.70583 & 07120003 & 112.17 & No & No & $\begin{array}{l}1990- \\
2009\end{array}$ & $\begin{array}{l}1990- \\
2009\end{array}$ & 19 & 19 & 0.7463 & 0.8286 & 0.8344 & 0.6651 & 0.0241 & 1.5828 \\
\hline 05536178 & PLUM CREEK NEAR DYER, IN & 41.47004 & -87.53254 & 07120003 & 34.26 & No & Yes & $\begin{array}{l}1966- \\
1977\end{array}$ & $\begin{array}{l}1955- \\
1977\end{array}$ & 13 & 12 & 0.0278 & 0.1189 & 0.4707 & 0.1309 & 0.0402 & 3.4243 \\
\hline 05536179 & HART DITCH AT DYER, IN & 41.50781 & -87.51004 & 07120003 & 37.16 & No & No & $\begin{array}{l}1990- \\
2009\end{array}$ & $\begin{array}{l}1990- \\
2009\end{array}$ & 20 & 20 & 0.2181 & 0.4575 & 0.4753 & 0.1614 & 0.0669 & 3.0727 \\
\hline 05536190 & HART DITCH AT MUNSTER, IN & 41.56115 & -87.48060 & 07120003 & 69.28 & Yes & No & $\begin{array}{l}1943- \\
2009\end{array}$ & $\begin{array}{l}1943- \\
2009\end{array}$ & 67 & 63 & 0.1241 & 0.6290 & 0.6385 & 0.4190 & 0.1227 & 2.3585 \\
\hline 05536201 & THORN CREEK AT PARK FOREST, IL & 41.47198 & -87.67393 & 07120003 & 5.82 & Yes & Yes & \begin{tabular}{|l|}
$1962-$ \\
1978 \\
\end{tabular} & $\begin{array}{l}1955- \\
1978\end{array}$ & 19 & 16 & 0.1982 & 0.3387 & 0.5798 & 0.3195 & 0.0205 & 7.0569 \\
\hline 05536207 & $\begin{array}{l}\text { THORN CREEK TRIBUTARY AT } \\
\text { CHICAGO HEIGHTS, IL }\end{array}$ & 41.50615 & -87.66004 & 07120003 & 3.93 & Yes & Yes & $\begin{array}{l}1962- \\
1977\end{array}$ & $\begin{array}{l}1962- \\
1977\end{array}$ & 16 & 16 & 0.8305 & 0.8509 & 0.8815 & 0.8355 & 0.1221 & 27.8415 \\
\hline 05536210 & $\begin{array}{l}\text { THORN CREEK NEAR CHICAGO } \\
\text { HEIGHTS, IL }\end{array}$ & 41.51389 & -87.63583 & 07120003 & 17.14 & No & No & $\begin{array}{l}1965- \\
1979\end{array}$ & $\begin{array}{l}1965- \\
1979\end{array}$ & 15 & 15 & 0.5225 & 0.5788 & 0.6843 & 0.5535 & 0.0443 & 5.6029 \\
\hline 05536215 & THORN CREEK AT GLENWOOD, IL & 41.53031 & -87.62227 & 07120003 & 25.03 & No & No & $\begin{array}{l}1950- \\
2009 \\
\end{array}$ & $\begin{array}{l}1950- \\
2009\end{array}$ & 60 & 60 & 0.3966 & 0.6942 & 0.7010 & 0.6298 & 0.0308 & 4.7866 \\
\hline 05536235 & $\begin{array}{l}\text { DEER CREEK NEAR CHICAGO } \\
\text { HEIGHTS, IL }\end{array}$ & 41.52087 & -87.59032 & 07120003 & 23.36 & Yes & No & $\begin{array}{l}1948- \\
2005 \\
\end{array}$ & $\begin{array}{l}1948- \\
2009\end{array}$ & 62 & 58 & 0.1420 & 0.6305 & 0.6534 & 0.3822 & 0.0269 & 4.1747 \\
\hline 05536238 & $\begin{array}{l}\text { BUTTERFIELD CREEK NEAR LINCOLN } \\
\text { ESTATES, IL }\end{array}$ & 41.50615 & -87.77616 & 07120003 & 1.76 & Yes & Yes & \begin{tabular}{|l|}
$1961-$ \\
1979
\end{tabular} & $\begin{array}{l}1961- \\
1979\end{array}$ & 19 & 18 & 0.0702 & 0.2289 & 0.4052 & 0.5282 & 0.0498 & 6.7099 \\
\hline 05536255 & $\begin{array}{l}\text { BUTTERFIELD CREEK AT } \\
\text { FLOSSMOOR, IL }\end{array}$ & 41.54003 & -87.64921 & 07120003 & 23.21 & Yes & No & $\begin{array}{l}1948- \\
2009\end{array}$ & $\begin{array}{l}1948- \\
2009\end{array}$ & 62 & 61 & 0.1243 & 0.6182 & 0.6193 & 0.6745 & 0.0487 & 4.4021 \\
\hline
\end{tabular}




\begin{tabular}{|c|c|c|c|c|c|c|c|c|c|c|c|c|c|c|c|c|c|}
\hline $\begin{array}{l}\text { U.S. } \\
\text { Geological } \\
\text { Survey } \\
\text { Streamgage } \\
\text { number }\end{array}$ & Name & $\begin{array}{l}\text { Latitude } \\
\text { (NAD 83) }\end{array}$ & $\begin{array}{l}\text { Longitude } \\
\text { (NAD 83) }\end{array}$ & $\begin{array}{l}\text { Hydrologic } \\
\text { unit code }\end{array}$ & $\begin{array}{l}\text { Drainage } \\
\text { area }\left(\mathrm{mi}^{2}\right)\end{array}$ & $\begin{array}{l}\text { Used in } \\
\text { regression } \\
\text { analyses } \\
\text { (non- } \\
\text { redundant) }\end{array}$ & $\begin{array}{l}\text { Crest- } \\
\text { stage } \\
\text { gage } \\
\text { (CSG) }\end{array}$ & $\begin{array}{l}\text { Spatial } \\
\text { POR }\end{array}$ & $\begin{array}{l}\text { Temporal } \\
\text { POR }\end{array}$ & $N$ & $N_{s}$ & $u_{f}$ & $u_{t}$ & $U_{2010}$ & NLCD_22_23_24: & $\begin{array}{l}\text { Drainage } \\
\text { Class1a }\end{array}$ & $\begin{array}{l}\text { DEM_1_0_P: } \\
\text { Basin slope } \\
\text { computed as } \\
\text { (elevation } \\
\text { range) / (basin } \\
\text { perimeter), in } \\
\text { feet per mile }\end{array}$ \\
\hline 05536265 & LANSING DITCH NEAR LANSING, IL & 41.52837 & -87.52921 & 07120003 & 8.02 & Yes & No & \begin{tabular}{|l|}
$1948-$ \\
2009 \\
\end{tabular} & $\begin{array}{l}1948- \\
2009\end{array}$ & 62 & 61 & 0.0402 & 0.5124 & 0.5231 & 0.4134 & 0.0356 & 5.5774 \\
\hline 05536270 & NORTH CREEK NEAR LANSING, IL & 41.54556 & -87.55889 & 07120003 & 16.83 & Yes & No & \begin{tabular}{|l|}
$1948-$ \\
1979 \\
\end{tabular} & $\begin{array}{l}1948- \\
1979\end{array}$ & 32 & 32 & 0.1246 & 0.4413 & 0.5702 & 0.5412 & 0.1215 & 3.9938 \\
\hline 05536275 & THORN CREEK AT THORNTON, IL & 41.56837 & -87.60782 & 07120003 & 103.58 & No & No & $\begin{array}{l}1947- \\
2006\end{array}$ & $\begin{array}{l}1947- \\
2009\end{array}$ & 63 & 59 & 0.1852 & 0.5953 & 0.6031 & 0.5434 & 0.0441 & 2.7702 \\
\hline 05536290 & $\begin{array}{l}\text { LITTLE CALUMET RIVER AT SOUTH } \\
\text { HOLLAND, IL }\end{array}$ & 41.60698 & -87.59782 & 07120003 & 206.58 & No & No & $\begin{array}{l}1947- \\
2009\end{array}$ & $\begin{array}{l}1947- \\
2009\end{array}$ & 63 & 63 & 0.2214 & 0.6311 & 0.6382 & 0.5308 & 0.0861 & 1.8084 \\
\hline 05536310 & $\begin{array}{l}\text { CALUMET UNION DRAINAGE CANAL } \\
\text { NEAR MARKHAM, IL }\end{array}$ & 41.59670 & -87.66644 & 07120003 & 12.64 & Yes & Yes & \begin{tabular}{|l}
$1961-$ \\
1974
\end{tabular} & $\begin{array}{l}1955- \\
1976\end{array}$ & 18 & 14 & 0.5770 & 0.8209 & 0.8568 & 0.8684 & 0.0149 & 4.8727 \\
\hline 05536335 & $\begin{array}{l}\text { MIDLOTHIAN CREEK NEAR TINLEY } \\
\text { PARK, IL }\end{array}$ & 41.58837 & -87.74783 & 07120003 & 9.98 & No & Yes & $\begin{array}{l}1954- \\
1979\end{array}$ & $\begin{array}{l}1954- \\
1979\end{array}$ & 26 & 26 & 0.2697 & 0.7689 & 0.8621 & 0.9026 & 0.0737 & 4.4944 \\
\hline 05536340 & $\begin{array}{l}\text { MIDLOTHIAN CREEK AT OAK } \\
\text { FOREST, IL }\end{array}$ & 41.61420 & -87.72949 & 07120003 & 13.62 & Yes & No & $\begin{array}{l}1951- \\
2009\end{array}$ & $\begin{array}{l}1951- \\
2009\end{array}$ & 59 & 57 & 0.2094 & 0.7754 & 0.7755 & 0.8306 & 0.0798 & 4.0485 \\
\hline 05536460 & TINLEY CREEK NEAR OAK FOREST, IL & 41.63031 & -87.78477 & 07120003 & 8.00 & No & Yes & $\begin{array}{l}1961- \\
1979\end{array}$ & $\begin{array}{l}1961- \\
1979\end{array}$ & 19 & 18 & 0.2100 & 0.6544 & 0.7231 & 0.7102 & 0.0554 & 6.3055 \\
\hline 05536500 & TINLEY CREEK NEAR PALOS PARK, IL & 41.64670 & -87.76644 & 07120003 & 11.27 & Yes & No & $\begin{array}{l}1951- \\
2009\end{array}$ & $\begin{array}{l}1951- \\
2009\end{array}$ & 58 & 58 & 0.0462 & 0.6212 & 0.6212 & 0.6392 & 0.0514 & 5.4185 \\
\hline 05536510 & $\begin{array}{l}\text { NAVAJO CREEK AT PALOS } \\
\text { HEIGHTS, IL }\end{array}$ & 41.66087 & -87.79394 & 07120003 & 1.60 & Yes & Yes & $\begin{array}{l}1961- \\
1979\end{array}$ & $\begin{array}{l}1961- \\
1979\end{array}$ & 19 & 19 & 0.6310 & 0.7187 & 0.7440 & 0.7167 & 0.0160 & 13.3884 \\
\hline 05536560 & $\begin{array}{l}\text { MELVINA DITCH NEAR OAK } \\
\text { LAWN, IL }\end{array}$ & 41.71920 & -87.78533 & 07120003 & 5.54 & Yes & Yes & $\begin{array}{l}1962- \\
1980\end{array}$ & $\begin{array}{l}1962- \\
1980\end{array}$ & 19 & 16 & 0.9716 & 0.9840 & 0.9840 & 0.9787 & 0.0000 & 2.3942 \\
\hline 05536570 & STONY CREEK (WEST) AT WORTH, IL & 41.70003 & -87.79783 & 07120003 & 17.96 & No & Yes & $\begin{array}{l}1962- \\
1970 \\
\end{array}$ & $\begin{array}{l}1962- \\
1976\end{array}$ & 15 & 9 & 0.9502 & 0.9641 & 0.9652 & 0.9725 & 0.0085 & 1.7950 \\
\hline 05536620 & MILL CREEK NEAR PALOS PARK, IL & 41.65253 & -87.83978 & 07120003 & 5.69 & No & Yes & $\begin{array}{l}1961- \\
1977\end{array}$ & $\begin{array}{l}1961- \\
1977\end{array}$ & 17 & 17 & 0.2933 & 0.4812 & 0.5845 & 0.6473 & 0.1764 & 5.2094 \\
\hline 05536630 & MILL CREEK AT PALOS PARK, IL & 41.67420 & -87.84311 & 07120003 & 10.52 & Yes & Yes & $\begin{array}{l}1961- \\
1979\end{array}$ & $\begin{array}{l}1955- \\
1979\end{array}$ & 20 & 19 & 0.2327 & 0.5030 & 0.5969 & 0.6007 & 0.1362 & 7.1825 \\
\hline 05537500 & LONG RUN NEAR LEMONT, IL & 41.64253 & -87.99923 & 07120004 & 21.11 & Yes & No & \begin{tabular}{|l}
$1951-$ \\
2009
\end{tabular} & $\begin{array}{l}1951- \\
2009\end{array}$ & 59 & 58 & 0.0399 & 0.7768 & 0.7855 & 0.4674 & 0.0694 & 4.5653 \\
\hline 05538440 & $\begin{array}{l}\text { SPRING CREEK NEAR ORLAND } \\
\text { PARK, IL }\end{array}$ & 41.60059 & -87.90061 & 07120004 & 1.54 & Yes & Yes & $\begin{array}{l}1961- \\
1977\end{array}$ & $\begin{array}{l}1955- \\
1977\end{array}$ & 18 & 17 & 0.1916 & 0.4732 & 0.8177 & 0.6238 & 0.2496 & 7.0869 \\
\hline 05539000 & HICKORY CREEK AT JOLIET, IL & 41.51503 & -88.07339 & 07120004 & 108.21 & Yes & No & $\begin{array}{l}1945- \\
2008 \\
\end{array}$ & $\begin{array}{l}1942- \\
2009\end{array}$ & 66 & 64 & 0.0789 & 0.6572 & 0.6752 & 0.5157 & 0.0264 & 2.7395 \\
\hline 05539870 & $\begin{array}{l}\text { WEST BRANCH DU PAGE RIVER AT } \\
\text { ONTARIOVILLEE, IL }\end{array}$ & 41.97836 & -88.13313 & 07120004 & 10.10 & No & Yes & $\begin{array}{l}1961- \\
1976\end{array}$ & $\begin{array}{l}1961- \\
1979\end{array}$ & 19 & 16 & 0.3871 & 0.8953 & 0.9458 & 0.8979 & 0.0928 & 3.3021 \\
\hline 05539890 & $\begin{array}{l}\text { WEST BRANCH DU PAGE RIVER } \\
\text { NEAR WAYNE, IL }\end{array}$ & 41.94364 & -88.18091 & 07120004 & 23.34 & No & Yes & $\begin{array}{l}1961- \\
1976\end{array}$ & $\begin{array}{l}1955- \\
1979\end{array}$ & 20 & 16 & 0.1904 & 0.6889 & 0.8311 & 0.7341 & 0.1060 & 5.1838 \\
\hline 05539900 & $\begin{array}{l}\text { WEST BRANCH DU PAGE RIVER } \\
\text { NEAR WEST CHICAGO, IL }\end{array}$ & 41.91086 & -88.17896 & 07120004 & 27.95 & Yes & No & $\begin{array}{l}1961- \\
2009\end{array}$ & $\begin{array}{l}1961- \\
2009\end{array}$ & 49 & 48 & 0.2582 & 0.8185 & 0.8249 & 0.7160 & 0.1009 & 5.6098 \\
\hline
\end{tabular}




\begin{tabular}{|c|c|c|c|c|c|c|c|c|c|c|c|c|c|c|c|c|c|}
\hline $\begin{array}{l}\text { U.S. } \\
\text { Geological } \\
\text { Survey } \\
\text { Streamgage } \\
\text { number }\end{array}$ & Name & $\begin{array}{l}\text { Latitude } \\
\text { (NAD 83) }\end{array}$ & $\begin{array}{l}\text { Longitude } \\
\text { (NAD 83) }\end{array}$ & $\begin{array}{l}\text { Hydrologic } \\
\text { unit code }\end{array}$ & $\begin{array}{l}\text { Drainage } \\
\text { area }\left(\mathrm{mi}^{2}\right)\end{array}$ & $\begin{array}{l}\text { Used in } \\
\text { regression } \\
\text { analyses } \\
\text { (non- } \\
\text { redundant) }\end{array}$ & $\begin{array}{l}\text { Crest- } \\
\text { stage } \\
\text { gage } \\
\text { (CSG) }\end{array}$ & $\begin{array}{l}\text { Spatial } \\
\text { POR }\end{array}$ & $\begin{array}{l}\text { Temporal } \\
\text { POR }\end{array}$ & $N$ & $N_{s}$ & $u_{f}$ & $u_{t}$ & $U_{2010}$ & NLCD_22_23_24: & $\begin{array}{l}\text { Drainage } \\
\text { Class1a }\end{array}$ & $\begin{array}{l}\text { DEM_1_0_P: } \\
\text { Basin slope } \\
\text { computed as } \\
\text { (elevation } \\
\text { range) / (basin } \\
\text { perimeter), in } \\
\text { feet per mile }\end{array}$ \\
\hline 05539950 & KLEIN CREEK AT CAROL STREAM, IL & 41.90670 & -88.14229 & 07120004 & 9.11 & Yes & Yes & $\begin{array}{l}1961- \\
1979\end{array}$ & $\begin{array}{l}1961- \\
1979\end{array}$ & 19 & 19 & 0.1967 & 0.6882 & 0.9331 & 0.9027 & 0.0716 & 8.6120 \\
\hline 05540030 & $\begin{array}{l}\text { WEST BRANCH DU PAGE RIVER AT } \\
\text { WEST CHICAGO, IL }\end{array}$ & 41.86142 & -88.19257 & 07120004 & 59.77 & No & Yes & $\begin{array}{l}1961- \\
1979\end{array}$ & $\begin{array}{l}1955- \\
1979\end{array}$ & 20 & 19 & 0.2321 & 0.6669 & 0.8465 & 0.7333 & 0.0807 & 4.1513 \\
\hline 05540060 & KRESS CREEK AT WEST CHICAGO, IL & 41.85639 & -88.20389 & 07120004 & 18.63 & Yes & Partial & $\begin{array}{l}1961- \\
2009\end{array}$ & $\begin{array}{l}1961- \\
2009\end{array}$ & 44 & 42 & 0.1894 & 0.5098 & 0.5229 & 0.5314 & 0.0667 & 3.7049 \\
\hline 05540080 & SPRING BROOK AT WHEATON, IL & 41.85058 & -88.11479 & 07120004 & 2.07 & Yes & Yes & $\begin{array}{l}1961- \\
1979\end{array}$ & $\begin{array}{l}1961- \\
1979\end{array}$ & 19 & 19 & 0.7411 & 0.9212 & 0.9949 & 0.8760 & 0.0425 & 6.4418 \\
\hline 05540091 & $\begin{array}{l}\text { SPRING BK AT FOREST PRESERVE NR } \\
\text { WARRENVILLE, IL }\end{array}$ & 41.83528 & -88.18278 & 07120004 & 6.83 & No & No & $\begin{array}{l}1992- \\
2009\end{array}$ & $\begin{array}{l}1992- \\
2009\end{array}$ & 18 & 18 & 0.7288 & 0.9033 & 0.9211 & 0.6509 & 0.0535 & 4.8716 \\
\hline 05540095 & $\begin{array}{l}\text { WEST BRANCH DU PAGE RIVER } \\
\text { NEAR WARRENVILLE, IL }\end{array}$ & 41.81750 & -88.17139 & 07120004 & 91.45 & No & No & $\begin{array}{l}1969- \\
2009\end{array}$ & $\begin{array}{l}1969- \\
2009\end{array}$ & 41 & 41 & 0.4112 & 0.7666 & 0.7766 & 0.6621 & 0.0763 & 3.4273 \\
\hline 05540110 & FERRY CREEK AT WARRENVILLE, IL & 41.82031 & -88.19313 & 07120004 & 3.69 & Yes & Yes & $\begin{array}{l}1961- \\
1979\end{array}$ & $\begin{array}{l}1961- \\
1979\end{array}$ & 19 & 19 & 0.1311 & 0.2398 & 0.4865 & 0.2778 & 0.0843 & 3.8298 \\
\hline 05540130 & $\begin{array}{l}\text { WEST BRANCH DU PAGE RIVER } \\
\text { NEAR NAPERVILLE, IL }\end{array}$ & 41.72056 & -88.13194 & 07120004 & 123.27 & No & No & $\begin{array}{l}1989- \\
2009\end{array}$ & $\begin{array}{l}1989- \\
2009\end{array}$ & 21 & 21 & 0.6589 & 0.7868 & 0.7965 & 0.6807 & 0.0678 & 3.0496 \\
\hline 05540140 & $\begin{array}{l}\text { EAST BRANCH DU PAGE RIVER } \\
\text { NEAR BLOOMINGDALE, IL }\end{array}$ & 41.93503 & -88.05812 & 07120004 & 2.42 & Yes & Yes & $\begin{array}{l}1961- \\
1979\end{array}$ & $\begin{array}{l}1961- \\
1979\end{array}$ & 19 & 19 & 0.3124 & 0.9225 & 0.9845 & 0.9245 & 0.0422 & 10.3462 \\
\hline 05540150 & $\begin{array}{l}\text { EAST BR DU PAGE RIVER AT GLEN } \\
\text { ELLYN, IL }\end{array}$ & 41.89003 & -88.05035 & 07120004 & 13.68 & No & Yes & $\begin{array}{l}1961- \\
1980\end{array}$ & $\begin{array}{l}1961- \\
1980\end{array}$ & 18 & 18 & 0.5464 & 0.8416 & 0.9141 & 0.7923 & 0.0468 & 4.6220 \\
\hline 05540160 & $\begin{array}{l}\text { EAST BRANCH DU PAGE RIVER } \\
\text { NEAR DOWNERS GROVE, IL }\end{array}$ & 41.83167 & -88.04778 & 07120004 & 25.21 & Yes & Partial & $\begin{array}{l}1961- \\
2009\end{array}$ & $\begin{array}{l}1955- \\
2009\end{array}$ & 36 & 35 & 0.4797 & 0.9291 & 0.9347 & 0.7946 & 0.0567 & 3.6796 \\
\hline 05540190 & ST. JOSEPH CREEK AT BELMONT, IL & 41.79197 & -88.03756 & 07120004 & 8.82 & Yes & Yes & $\begin{array}{l}1961- \\
1977\end{array}$ & $\begin{array}{l}1961- \\
1977\end{array}$ & 17 & 17 & 0.8906 & 0.9339 & 0.9683 & 0.8075 & 0.0219 & 4.6491 \\
\hline 05540195 & $\begin{array}{l}\text { ST. JOSEPH CREEK AT ROUTE } 34 \text { AT } \\
\text { LISLE, IL }\end{array}$ & 41.80194 & -88.06889 & 07120004 & 11.10 & No & No & $\begin{array}{l}1989- \\
2009\end{array}$ & $\begin{array}{l}1989- \\
2009\end{array}$ & 21 & 21 & 0.9195 & 0.9561 & 0.9601 & 0.8024 & 0.0272 & 5.0182 \\
\hline 05540240 & PRENTISS CREEK NEAR LISLE, IL & 41.77142 & -88.06979 & 07120004 & 6.57 & Yes & Yes & $\begin{array}{l}1961- \\
1980\end{array}$ & $\begin{array}{l}1961- \\
1980\end{array}$ & 20 & 20 & 0.5572 & 0.9316 & 0.9807 & 0.8442 & 0.0207 & 6.8289 \\
\hline 05540250 & $\begin{array}{l}\text { EAST BRANCH DU PAGE RIVER AT } \\
\text { BOLINGBROOK, IL }\end{array}$ & 41.71806 & -88.07056 & 07120004 & 73.34 & No & No & $\begin{array}{l}1992- \\
2009\end{array}$ & $\begin{array}{l}1989- \\
2009\end{array}$ & 21 & 18 & 0.7886 & 0.8658 & 0.8729 & 0.7146 & 0.0469 & 2.2427 \\
\hline 05540275 & $\begin{array}{l}\text { SPRING BROOK AT 87TH STREET } \\
\text { NEAR NAPERVILLE, IL }\end{array}$ & 41.72583 & -88.16361 & 07120004 & 9.89 & Yes & No & $\begin{array}{l}1988- \\
2009\end{array}$ & $\begin{array}{l}1988- \\
2009\end{array}$ & 22 & 22 & 0.4807 & 0.7114 & 0.7186 & 0.6140 & 0.0220 & 3.1703 \\
\hline 05540500 & DU PAGE RIVER AT SHOREWOOD, IL & 41.52225 & -88.19256 & 07120004 & 323.19 & Yes & No & $\begin{array}{l}1941- \\
2009 \\
\end{array}$ & $\begin{array}{l}1941- \\
2009\end{array}$ & 69 & 68 & 0.1054 & 0.7540 & 0.7671 & 0.6462 & 0.0519 & 2.3473 \\
\hline 05541750 & $\begin{array}{l}\text { MAZON RIVER TRIBUTARY NEAR } \\
\text { GARDNER, IL }\end{array}$ & 41.16003 & -88.34312 & 07120005 & 4.74 & Yes & Yes & $\begin{array}{l}1959- \\
1980\end{array}$ & $\begin{array}{l}1959- \\
1980\end{array}$ & 22 & 22 & 0.0033 & 0.0033 & 0.0033 & 0.0099 & 0.0013 & 2.2336 \\
\hline 05542000 & MAZON RIVER NEAR COAL CITY, IL & 41.28614 & -88.35979 & 07120005 & 451.80 & Yes & Partial & $\begin{array}{l}1940- \\
2009\end{array}$ & $\begin{array}{l}1940- \\
2009 \\
\end{array}$ & 69 & 69 & 0.0094 & 0.0208 & 0.0211 & 0.0384 & 0.0189 & 1.8277 \\
\hline 05543830 & FOX RIVER AT WAUKESHA, WI & 43.00472 & -88.24361 & 07120006 & 127.74 & Yes & No & $\begin{array}{l}1963- \\
2009\end{array}$ & $\begin{array}{l}1960- \\
2009\end{array}$ & 48 & 47 & 0.2656 & 0.7412 & 0.7565 & 0.3386 & 0.1717 & 3.5814 \\
\hline
\end{tabular}




\begin{tabular}{|c|c|c|c|c|c|c|c|c|c|c|c|c|c|c|c|c|c|}
\hline $\begin{array}{l}\text { U.S. } \\
\text { Geological } \\
\text { Survey } \\
\text { Streamgage } \\
\text { number }\end{array}$ & Name & $\begin{array}{l}\text { Latitude } \\
\text { (NAD 83) }\end{array}$ & $\begin{array}{l}\text { Longitude } \\
\text { (NAD 83) }\end{array}$ & $\begin{array}{l}\text { Hydrologic } \\
\text { unit code }\end{array}$ & $\begin{array}{l}\text { Drainage } \\
\text { area }\left(\mathrm{mi}^{2}\right)\end{array}$ & $\begin{array}{l}\text { Used in } \\
\text { regression } \\
\text { analyses } \\
\text { (non- } \\
\text { redundant) }\end{array}$ & $\begin{array}{l}\text { Crest- } \\
\text { stage } \\
\text { gage } \\
\text { (CSG) }\end{array}$ & $\begin{array}{l}\text { Spatial } \\
\text { POR }\end{array}$ & $\begin{array}{l}\text { Temporal } \\
\text { POR }\end{array}$ & $N$ & $N_{s}$ & $u_{f}$ & $u_{t}$ & $U_{2010}$ & NLCD_22_23_24: & $\begin{array}{l}\text { Drainage } \\
\text { Class1a }\end{array}$ & $\begin{array}{l}\text { DEM_1_0_P: } \\
\text { Basin slope } \\
\text { computed as } \\
\text { (elevation } \\
\text { range) / (basin } \\
\text { perimeter), in } \\
\text { feet per mile }\end{array}$ \\
\hline 05544200 & $\begin{array}{l}\text { MUKWONAGO RIVER AT } \\
\text { MUKWONAGO, WI }\end{array}$ & 42.85667 & -88.32778 & 07120006 & 72.09 & Yes & No & $\begin{array}{l}1974- \\
2009\end{array}$ & $\begin{array}{l}1974- \\
2009\end{array}$ & 34 & 34 & 0.0876 & 0.3057 & 0.3115 & 0.0558 & 0.1036 & 4.4455 \\
\hline 05544300 & $\begin{array}{l}\text { MUKWONAGO RIVER TRIBUTARY } \\
\text { NEAR MUKWONAGO, WI }\end{array}$ & 42.84946 & -88.31732 & 07120006 & 1.31 & Yes & Yes & $\begin{array}{l}1960- \\
1981\end{array}$ & $\begin{array}{l}1960- \\
1981\end{array}$ & 21 & 19 & 0.0534 & 0.1119 & 0.1958 & 0.0664 & 0.0973 & 14.3308 \\
\hline 05545100 & SUGAR CREEK AT ELKHORN, WI & 42.69078 & -88.51644 & 07120006 & 6.19 & Yes & Yes & $\begin{array}{l}1962- \\
2009\end{array}$ & $\begin{array}{l}1962- \\
2009\end{array}$ & 48 & 48 & 0.0214 & 0.0418 & 0.0422 & 0.0845 & 0.0000 & 3.3575 \\
\hline 05545200 & $\begin{array}{l}\text { WHITE RIVER TRIBUTARY NEAR } \\
\text { BURLINGTON, WI }\end{array}$ & 42.68363 & -88.36148 & 07120006 & 2.36 & Yes & Yes & $\begin{array}{l}1960- \\
2009\end{array}$ & $\begin{array}{l}1958- \\
2009\end{array}$ & 51 & 49 & 0.0049 & 0.0389 & 0.0393 & 0.0171 & 0.0012 & 16.0636 \\
\hline 05545300 & $\begin{array}{l}\text { WHITE RIVER NEAR } \\
\text { BURLINGTON, WI }\end{array}$ & 42.66585 & -88.31759 & 07120006 & 106.37 & Yes & Partial & \begin{tabular}{|l|}
$1960-$ \\
1982
\end{tabular} & $\begin{array}{l}1959- \\
1982\end{array}$ & 24 & 23 & 0.1305 & 0.1772 & 0.2855 & 0.0852 & 0.0765 & 4.0675 \\
\hline 05545750 & $\begin{array}{l}\text { FOX RIVER NEAR NEW } \\
\text { MUNSTER, WI }\end{array}$ & 42.61085 & -88.22592 & 07120006 & 804.31 & Yes & Partial & \begin{tabular}{|l|}
$1940-$ \\
2009 \\
\end{tabular} & $\begin{array}{l}1940- \\
2009\end{array}$ & 70 & 69 & 0.0505 & 0.3523 & 0.3593 & 0.1300 & 0.1459 & 1.5424 \\
\hline 05547755 & SQUAW CREEK AT ROUND LAKE, IL & 42.34972 & -88.08833 & 07120006 & 16.37 & Yes & No & $\begin{array}{l}1990- \\
2005\end{array}$ & $\begin{array}{l}1990- \\
2005\end{array}$ & 16 & 16 & 0.0661 & 0.3061 & 0.4180 & 0.2367 & 0.1953 & 4.0509 \\
\hline 05548150 & $\begin{array}{l}\text { NORTH BRANCH NIPPERSINK CREEK } \\
\text { NEAR GENOA CITY, WI }\end{array}$ & 42.50418 & -88.38371 & 07120006 & 13.75 & Yes & Yes & $\begin{array}{l}1962- \\
2009\end{array}$ & $\begin{array}{l}1962- \\
2009\end{array}$ & 48 & 44 & 0.0335 & 0.0623 & 0.0641 & 0.0337 & 0.1177 & 6.2355 \\
\hline 05548280 & $\begin{array}{l}\text { NIPPERSINK CREEK NEAR SPRING } \\
\text { GROVE, IL }\end{array}$ & 42.44333 & -88.24750 & 07120006 & 195.14 & Yes & No & $\begin{array}{l}1967- \\
2009 \\
\end{array}$ & $\begin{array}{l}1966- \\
2009\end{array}$ & 43 & 42 & 0.0840 & 0.2435 & 0.2534 & 0.0787 & 0.0880 & 3.4186 \\
\hline 05549000 & BOONE CREEK NEAR MC HENRY, IL & 42.32056 & -88.31278 & 07120006 & 15.65 & Yes & Partial & $\begin{array}{l}1949- \\
1992\end{array}$ & $\begin{array}{l}1949- \\
1992\end{array}$ & 44 & 42 & 0.0007 & 0.1255 & 0.4496 & 0.0560 & 0.1473 & 7.3301 \\
\hline 05549700 & MUTTON CREEK AT ISLAND LAKE, IL & 42.28475 & -88.17980 & 07120006 & 10.24 & Yes & Yes & $\begin{array}{l}1962- \\
1976\end{array}$ & $\begin{array}{l}1960- \\
1976\end{array}$ & 16 & 15 & 0.0211 & 0.0453 & 0.4535 & 0.1925 & 0.2117 & 5.1296 \\
\hline 05549850 & $\begin{array}{l}\text { FLINT CREEK NEAR FOX RIVER } \\
\text { GROVE, IL }\end{array}$ & 42.21111 & -88.17361 & 07120006 & 35.57 & Yes & Partial & \begin{tabular}{|l|}
$1962-$ \\
1996
\end{tabular} & $\begin{array}{l}1960- \\
1996\end{array}$ & 23 & 22 & 0.2389 & 0.6163 & 0.7983 & 0.3746 & 0.1660 & 3.8487 \\
\hline 05549900 & $\begin{array}{l}\text { FOX RIVER TRIBUTARY NEAR } \\
\text { CARY, IL }\end{array}$ & 42.19669 & -88.26508 & 07120006 & 0.08 & Yes & Yes & $\begin{array}{l}1956- \\
1979\end{array}$ & $\begin{array}{l}1956- \\
1979\end{array}$ & 23 & 20 & 0.7700 & 0.9500 & 1.0000 & 0.9450 & 0.0000 & 28.4190 \\
\hline 05550300 & TYLER CREEK AT ELGIN, IL & 42.05833 & -88.30389 & 07120006 & 39.04 & Yes & Partial & $\begin{array}{l}1962- \\
2009\end{array}$ & $\begin{array}{l}1962- \\
2009\end{array}$ & 29 & 29 & 0.0684 & 0.3346 & 0.3483 & 0.2566 & 0.0600 & 6.3855 \\
\hline 05550430 & $\begin{array}{l}\text { EAST BRANCH POPLAR CREEK NEAR } \\
\text { PALATINE, IL }\end{array}$ & 42.06725 & -88.11146 & 07120006 & 2.57 & Yes & Yes & \begin{tabular}{|l|}
$1961-$ \\
1977
\end{tabular} & $\begin{array}{l}1961- \\
1977\end{array}$ & 17 & 17 & 0.1777 & 0.4210 & 0.4842 & 0.4032 & 0.1614 & 7.1875 \\
\hline 05550450 & $\begin{array}{l}\text { POPLAR CREEK NEAR } \\
\text { ONTARIOVILLE, IL }\end{array}$ & 42.04669 & -88.15563 & 07120006 & 16.70 & No & Yes & $\begin{array}{l}1961- \\
1977\end{array}$ & $\begin{array}{l}1961- \\
1977\end{array}$ & 17 & 16 & 0.1328 & 0.3878 & 0.5715 & 0.5244 & 0.1620 & 3.8626 \\
\hline 05550470 & $\begin{array}{l}\text { POPLAR CREEK TRIBUTARY NEAR } \\
\text { BARTLETT, IL }\end{array}$ & 42.02447 & -88.20285 & 07120006 & 5.15 & Yes & Yes & $\begin{array}{l}1961- \\
1979\end{array}$ & $\begin{array}{l}1961- \\
1979\end{array}$ & 19 & 17 & 0.3894 & 0.8991 & 0.9573 & 0.8794 & 0.1020 & 5.8190 \\
\hline 05550500 & POPLAR CREEK AT ELGIN, IL & 42.02611 & -88.25556 & 07120006 & 35.09 & Yes & No & $\begin{array}{l}1952- \\
2009\end{array}$ & $\begin{array}{l}1952- \\
2009\end{array}$ & 58 & 58 & 0.0753 & 0.5742 & 0.5742 & 0.5621 & 0.1179 & 3.7406 \\
\hline 05551030 & $\begin{array}{l}\text { BREWSTER CREEK AT VALLEY } \\
\text { VIEW, IL }\end{array}$ & 41.97194 & -88.27972 & 07120007 & 15.28 & Yes & Yes & $\begin{array}{l}1962- \\
1979\end{array}$ & $\begin{array}{l}1962- \\
2006\end{array}$ & 21 & 17 & 0.0701 & 0.5198 & 0.5549 & 0.4157 & 0.1129 & 5.9096 \\
\hline 05551050 & NORTON CREEK NEAR WAYNE, IL & 41.94836 & -88.27146 & 07120007 & 7.37 & Yes & Yes & $\begin{array}{l}1962- \\
1979\end{array}$ & $\begin{array}{l}1957- \\
1979\end{array}$ & 19 & 18 & 0.0815 & 0.1780 & 0.6605 & 0.3565 & 0.0988 & 3.9399 \\
\hline
\end{tabular}




\begin{tabular}{|c|c|c|c|c|c|c|c|c|c|c|c|c|c|c|c|c|c|}
\hline $\begin{array}{l}\text { U.S. } \\
\text { Geological } \\
\text { Survey } \\
\text { Streamgage } \\
\text { number }\end{array}$ & Name & $\begin{array}{l}\text { Latitude } \\
\text { (NAD 83) }\end{array}$ & $\begin{array}{l}\text { Longitude } \\
\text { (NAD 83) }\end{array}$ & $\begin{array}{l}\text { Hydrologic } \\
\text { unit code }\end{array}$ & $\begin{array}{l}\text { Drainage } \\
\text { area }\left(\mathrm{mi}^{2}\right)\end{array}$ & $\begin{array}{l}\text { Used in } \\
\text { regression } \\
\text { analyses } \\
\text { (non- } \\
\text { redundant) }\end{array}$ & $\begin{array}{l}\text { Crest- } \\
\text { stage } \\
\text { gage } \\
\text { (CSG) }\end{array}$ & $\begin{array}{l}\text { Spatial } \\
\text { POR }\end{array}$ & $\begin{array}{l}\text { Temporal } \\
\text { POR }\end{array}$ & $N$ & $N_{s}$ & $u_{f}$ & $U_{1}$ & $U_{2010}$ & NLCD_22_23_24: & $\begin{array}{l}\text { Drainage_ } \\
\text { Class1a }\end{array}$ & $\begin{array}{l}\text { DEM_1_0_P: } \\
\text { Basin slope } \\
\text { computed as } \\
\text { (elevation } \\
\text { range) / (basin } \\
\text { perimeter), in } \\
\text { feet per mile }\end{array}$ \\
\hline 05551060 & $\begin{array}{l}\text { NORTON CREEK NEAR } \\
\text { ST. CHARLES, IL }\end{array}$ & 41.94503 & -88.30619 & 07120007 & 11.53 & No & Yes & $\begin{array}{l}1962- \\
1979\end{array}$ & $\begin{array}{l}1957- \\
1979\end{array}$ & 19 & 18 & 0.1079 & 0.2621 & 0.7822 & 0.3743 & 0.0680 & 4.6437 \\
\hline 05551200 & $\begin{array}{l}\text { FERSON CREEK NEAR } \\
\text { ST. CHARLES, IL }\end{array}$ & 41.93278 & -88.34083 & 07120007 & 51.80 & Yes & No & $\begin{array}{l}1961- \\
2009\end{array}$ & $\begin{array}{l}1961- \\
2009\end{array}$ & 49 & 49 & 0.0421 & 0.6143 & 0.6351 & 0.2929 & 0.0443 & 5.7631 \\
\hline 05551330 & MILL CREEK NEAR BATAVIA, IL & 41.84583 & -88.34917 & 07120007 & 28.78 & Yes & No & $\begin{array}{l}1998- \\
2009\end{array}$ & $\begin{array}{l}1998- \\
2009\end{array}$ & 12 & 12 & 0.4268 & 0.6160 & 0.6339 & 0.3931 & 0.0237 & 7.7810 \\
\hline 05551520 & $\begin{array}{l}\text { INDIAN CREEK NEAR NORTH } \\
\text { AURORA, IL }\end{array}$ & 41.81392 & -88.27396 & 07120007 & 5.19 & Yes & Yes & $\begin{array}{l}1961- \\
1979\end{array}$ & $\begin{array}{l}1961- \\
1979\end{array}$ & 19 & 19 & 0.0912 & 0.1483 & 0.4510 & 0.3599 & 0.0552 & 4.2888 \\
\hline 05551530 & INDIAN CREEK AT AURORA, IL & 41.76642 & -88.30674 & 07120007 & 16.52 & No & Yes & $\begin{array}{l}1961- \\
1979\end{array}$ & $\begin{array}{l}1961- \\
1979\end{array}$ & 19 & 19 & 0.2226 & 0.3635 & 0.7498 & 0.6095 & 0.0394 & 5.3852 \\
\hline 05551620 & $\begin{array}{l}\text { BLACKBERRY CREEK NEAR } \\
\text { KANEVILLE, IL } \\
\end{array}$ & 41.80475 & -88.45952 & 07120007 & 22.09 & No & Yes & $\begin{array}{l}1961- \\
1979\end{array}$ & $\begin{array}{l}1961- \\
1979\end{array}$ & 17 & 17 & 0.0412 & 0.0855 & 0.2827 & 0.1268 & 0.0372 & 8.0919 \\
\hline 05551650 & LAKE RUN AT BALD MOUND, IL & 41.84611 & -88.40889 & 07120007 & 1.95 & Yes & Yes & $\begin{array}{l}1961- \\
1976\end{array}$ & $\begin{array}{l}1961- \\
1976\end{array}$ & 16 & 15 & 0.0000 & 0.0012 & 0.0923 & 0.0180 & 0.0047 & 18.6516 \\
\hline 05551675 & $\begin{array}{l}\text { BLACKBERRY CREEK NEAR } \\
\text { MONTGOMERY, IL }\end{array}$ & 41.74083 & -88.38333 & 07120007 & 56.88 & No & No & $\begin{array}{l}1998- \\
2009\end{array}$ & $\begin{array}{l}1998- \\
2009\end{array}$ & 12 & 12 & 0.2191 & 0.4006 & 0.4194 & 0.2563 & 0.0588 & 6.0869 \\
\hline 05551700 & $\begin{array}{l}\text { BLACKBERRY CREEK NEAR } \\
\text { YORKVILLE, IL } \\
\end{array}$ & 41.67167 & -88.44139 & 07120007 & 68.19 & Yes & No & $\begin{array}{l}1961- \\
2009\end{array}$ & $\begin{array}{l}1961- \\
2009\end{array}$ & 49 & 49 & 0.0694 & 0.4035 & 0.4237 & 0.2674 & 0.0570 & 5.0357 \\
\hline 05551800 & $\begin{array}{l}\text { FOX RIVER TRIBUTARY NO } 2 \text { NEAR } \\
\text { FOX, IL }\end{array}$ & 41.60781 & -88.47868 & 07120007 & 0.45 & Yes & Yes & $\begin{array}{l}1961- \\
1980\end{array}$ & $\begin{array}{l}1961- \\
1980\end{array}$ & 19 & 19 & 0.1297 & 0.1949 & 0.6610 & 0.0928 & 0.0000 & 30.6422 \\
\hline 05551900 & $\begin{array}{l}\text { EAST BRANCH BIG ROCK CREEK } \\
\text { NEAR BIG ROCK, IL }\end{array}$ & 41.76781 & -88.56980 & 07120007 & 32.72 & Yes & Yes & $\begin{array}{l}1965- \\
1979\end{array}$ & $\begin{array}{l}1965- \\
1979\end{array}$ & 15 & 15 & 0.0040 & 0.0141 & 0.0307 & 0.0419 & 0.0167 & 4.8644 \\
\hline 05551930 & WELCH CREEK NEAR BIG ROCK, IL & 41.76003 & -88.51063 & 07120007 & 22.75 & Yes & Yes & $\begin{array}{l}1965- \\
1980\end{array}$ & $\begin{array}{l}1965- \\
1980\end{array}$ & 16 & 16 & 0.0442 & 0.0546 & 0.1257 & 0.0655 & 0.0103 & 5.4502 \\
\hline
\end{tabular}




\section{APPENDIX 3}

Table 2. Estimated peak discharge quantiles for 181 streamgages in northeastern Illinois and adjacent states, at selected annual exceedance probabilities.

[USGS, U.S. Geological Survey; EMA, Expected Probability Adjustment; fts/s, cubic feet per second; NA, not available; highlighting in column “Used in regression analyses (non-redundant)" indicates streamgages not used in the regression analyses because they were deemed redundant]

\begin{tabular}{|c|c|c|c|c|c|c|c|c|c|c|c|}
\hline \multirow[b]{2}{*}{$\begin{array}{c}\text { USGS } \\
\text { streamgage } \\
\text { number }\end{array}$} & \multirow[b]{2}{*}{ Streamgage name } & \multirow[b]{2}{*}{$\begin{array}{l}\text { Used in regression } \\
\text { analyses (non- } \\
\text { redundant) }\end{array}$} & \multirow[b]{2}{*}{ Frequency analysis method } & \multicolumn{8}{|c|}{ Discharge for given annual exceedance probability, $\mathrm{ft}^{3} / \mathrm{s}$} \\
\hline & & & & 0.50 & 0.20 & 0.10 & 0.04 & 0.02 & 0.01 & 0.005 & 0.002 \\
\hline \multirow{5}{*}{04087200} & \multirow{5}{*}{ OAK CREEK NEAR SOUTH MILWAUKEE, WI } & \multirow{5}{*}{ No } & $\begin{array}{l}\text { At-site, unadjusted for urbanization, EMA, } \\
\text { at-site skew }\end{array}$ & 334 & 564 & 727 & 940 & 1,100 & 1,260 & 1,420 & 1,640 \\
\hline & & & $\begin{array}{l}\text { At-site, adjusted for urbanization, EMA, at- } \\
\text { site skew }\end{array}$ & 399 & 625 & 802 & 1,060 & 1,280 & 1,510 & 1,780 & 2,170 \\
\hline & & & $\begin{array}{l}\text { At-site, adjusted for urbanization, EMA, } \\
\text { wighted skew: }\left(Q_{p}\right), g, 5\end{array}$ & 399 & 625 & 803 & 1,060 & 1,280 & 1,520 & 1,790 & 2,190 \\
\hline & & & Regional regression: $\left(Q_{p}\right)_{g, r}$ & 531 & 820 & 1,040 & 1,340 & 1,580 & 1,830 & 2,090 & 2,450 \\
\hline & & & 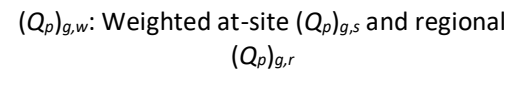 & 402 & 631 & 814 & 1,080 & 1,310 & 1,560 & 1,830 & 2,240 \\
\hline \multirow{5}{*}{04087204} & \multirow{5}{*}{ OAK CREEK AT SOUTH MILWAUKEE, WI } & \multirow{5}{*}{ Yes } & $\begin{array}{l}\text { At-site, unadjusted for urbanization, EMA, } \\
\text { at-site skew }\end{array}$ & 597 & 852 & 1,060 & 1,370 & 1,640 & 1,940 & 2,290 & 2,820 \\
\hline & & & $\begin{array}{l}\text { At-site, adjusted for urbanization, EMA, at- } \\
\text { site skew }\end{array}$ & 697 & 965 & 1,170 & 1,450 & 1,680 & 1,920 & 2,190 & 2,580 \\
\hline & & & $\begin{array}{l}\text { At-site, adjusted for urbanization, EMA, } \\
\text { weighted skew: }\left(Q_{p}\right) \text { g,s }\end{array}$ & 700 & 968 & 1,160 & 1,430 & 1,650 & 1,880 & 2,140 & 2,490 \\
\hline & & & Regional regression: $\left(Q_{p}\right)_{g, r}$ & 737 & 1,110 & 1,400 & 1,780 & 2,090 & 2,410 & 2,740 & 3,200 \\
\hline & & & $\begin{array}{l}\left(Q_{p}\right)_{g, w:} \text { Weighted at-site }\left(Q_{p}\right)_{Q, s} \text { and regional } \\
\qquad\left(Q_{p}\right)_{Q, r}\end{array}$ & 701 & 971 & 1,170 & 1,450 & 1,680 & 1,920 & 2,190 & 2,570 \\
\hline \multirow{5}{*}{04087220} & \multirow{5}{*}{ ROOT RIVER NEAR FRANKLIN, WI } & \multirow{5}{*}{ Yes } & $\begin{array}{l}\text { At-site, unadjusted for urbanization, EMA, } \\
\text { at-site skew }\end{array}$ & 968 & 1,720 & 2,440 & 3,670 & 4,880 & 6,400 & 8,300 & 11,600 \\
\hline & & & $\begin{array}{l}\text { At-site, adjusted for urbanization, EMA, at- } \\
\text { site skew }\end{array}$ & 1,090 & 1,940 & 2,730 & 4,090 & 5,420 & 7,090 & 9,150 & 12,700 \\
\hline & & & $\begin{array}{l}\text { At-site, adjusted for urbanization, EMA, } \\
\text { weighted skew: }\left(Q_{p}\right) g_{g, s}\end{array}$ & 1,120 & 1,960 & 2,720 & 3,960 & 5,110 & 6,500 & 8,170 & 10,900 \\
\hline & & & Regional regression: $\left(Q_{p}\right) g_{, r}$ & 1,320 & 2,020 & 2,540 & 3,250 & 3,820 & 4,420 & 5,040 & 5,910 \\
\hline & & & $\begin{array}{l}\left(Q_{p}\right)_{g, w} \text { : Weighted at-site }\left(Q_{p}\right)_{Q, s} \text { and regional } \\
\left(Q_{p} q_{g, r}\right.\end{array}$ & 1,120 & 1,960 & 2,700 & 3,850 & 4,870 & 6,000 & 7,250 & 9,080 \\
\hline
\end{tabular}




\begin{tabular}{|c|c|c|c|c|c|c|c|c|c|c|c|}
\hline \multirow{2}{*}{$\begin{array}{c}\text { USGS } \\
\text { streamgage } \\
\text { number }\end{array}$} & \multirow[b]{2}{*}{ Streamgage name } & \multirow{2}{*}{$\begin{array}{l}\text { Used in regression } \\
\text { analyses (non- } \\
\text { redundant) }\end{array}$} & \multirow[b]{2}{*}{ Frequency analysis method } & \multicolumn{8}{|c|}{ Discharge for given annual exceedance probability, $\mathrm{ft}^{3} / \mathrm{s}$} \\
\hline & & & & 0.50 & 0.20 & 0.10 & 0.04 & 0.02 & 0.01 & 0.005 & 0.002 \\
\hline \multirow{5}{*}{04087230} & \multirow{5}{*}{$\begin{array}{l}\text { W BR ROOT R CANAL TRIB NEAR NORTH CAPE, } \\
\text { WI }\end{array}$} & \multirow{5}{*}{ Yes } & $\begin{array}{l}\text { At-site, unadjusted for urbanization, EMA, } \\
\text { at-site skew }\end{array}$ & 99 & 137 & 157 & 178 & 190 & 201 & 210 & 220 \\
\hline & & & $\begin{array}{l}\text { At-site, adjusted for urbanization, EMA, at- } \\
\text { site skew }\end{array}$ & 107 & 146 & 166 & 188 & 201 & 212 & 222 & 233 \\
\hline & & & $\begin{array}{l}\text { At-site, adjusted for urbanization, EMA, } \\
\left.\text { weighted skew: ( } 0_{0}\right)_{g, s}\end{array}$ & 105 & 144 & 168 & 196 & 216 & 234 & 251 & 273 \\
\hline & & & Regional regression: $(Q p)_{a, r}$ & 139 & 238 & 311 & 408 & 483 & 558 & 633 & 734 \\
\hline & & & 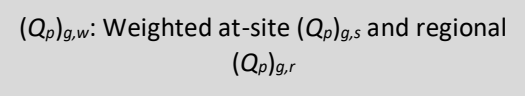 & 105 & 146 & 171 & 202 & 224 & 245 & 266 & 294 \\
\hline \multirow{5}{*}{04087233} & \multirow{5}{*}{ ROOT RIVER CANAL NEAR FRANKLIN, WI } & \multirow{5}{*}{ Yes } & $\begin{array}{l}\text { At-site, unadjusted for urbanization, EMA, } \\
\text { at-site skew }\end{array}$ & 793 & 1,080 & 1,260 & 1,460 & 1,610 & 1,740 & 1,870 & 2,030 \\
\hline & & & $\begin{array}{l}\text { At-site, adjusted for urbanization, EMA, at- } \\
\text { site skew }\end{array}$ & 841 & 1,130 & 1,300 & 1,490 & 1,610 & 1,720 & 1,820 & 1,940 \\
\hline & & & $\begin{array}{l}\text { At-site, adjusted for urbanization, EMA, } \\
\text { weighted skew: }\left(Q_{p}\right)_{g, s}\end{array}$ & 831 & 1,130 & 1,310 & 1,530 & 1,670 & 1,810 & 1,940 & 2,110 \\
\hline & & & Regional regression: $\left(Q_{p}\right)_{a, r}$ & 810 & 1,240 & 1,550 & 1,930 & 2,210 & 2,500 & 2,780 & 3,160 \\
\hline & & & $\begin{array}{l}\left(Q_{\rho}\right)_{Q, w}: \text { Weighted at-site } \\
\qquad\left(Q_{\rho}\right)_{\rho, g, s} \text { and regional }\end{array}$ & 831 & 1,130 & 1,320 & 1,540 & 1,690 & 1,830 & 1,980 & 2,160 \\
\hline \multirow{5}{*}{04087240} & \multirow{5}{*}{ ROOT RIVER AT RACINE, WI } & \multirow{5}{*}{ No } & $\begin{array}{l}\text { At-site, unadjusted for urbanization, EMA, } \\
\text { at-site skew }\end{array}$ & 1,860 & 2,810 & 3,530 & 4,570 & 5,430 & 6,380 & 7,410 & 8,940 \\
\hline & & & $\begin{array}{l}\text { At-site, adjusted for urbanization, EMA, at- } \\
\text { site skew }\end{array}$ & 2,070 & 3,080 & 3,830 & 4,860 & 5,690 & 6,580 & 7,540 & 8,900 \\
\hline & & & $\begin{array}{l}\text { At-site, adjusted for urbanization, EMA, } \\
\text { weighted skew: ( }\left(Q_{p}\right)_{g, s}\end{array}$ & 2,080 & 3,090 & 3,820 & 4,820 & 5,610 & 6,450 & 7,330 & 8,590 \\
\hline & & & Regional regression: $(Q p)_{g, r}$ & 2,620 & 3,910 & 4,830 & 6,040 & 6,960 & 7,910 & 8,870 & 10,200 \\
\hline & & & 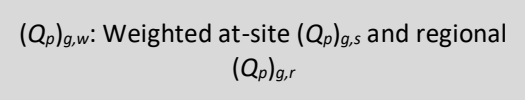 & 2,090 & 3,110 & 3,860 & 4,880 & 5,700 & 6,570 & 7,480 & 8,780 \\
\hline \multirow{5}{*}{04087250} & \multirow{5}{*}{ PIIKE CREEK NEAR KENOSHA, WI } & \multirow{5}{*}{ Yes } & $\begin{array}{l}\text { At-site, unadjusted for urbanization, EMA, } \\
\text { at-site skew }\end{array}$ & 101 & 156 & 185 & 212 & 228 & 241 & 251 & 261 \\
\hline & & & $\begin{array}{l}\text { At-site, adjusted for urbanization, EMA, at- } \\
\text { site skew }\end{array}$ & 125 & 183 & 217 & 256 & 281 & 303 & 324 & 348 \\
\hline & & & $\begin{array}{l}\text { At-site, adjusted for urbanization, EMA, } \\
\left.\text { weighted skew: (Q. } Q_{0}\right)_{g, s}\end{array}$ & 121 & 183 & 224 & 275 & 312 & 349 & 385 & 432 \\
\hline & & & Regional regression: $\left(Q_{p}\right)_{g, r}$ & 245 & 375 & 470 & 599 & 699 & 802 & 908 & 1,050 \\
\hline & & & $\begin{array}{c}\left(Q_{\rho}\right)_{g, w}: \text { Weighted at-site } \\
\left(Q_{\rho} Q_{g, r}\right)_{g, s} \text { and regional }\end{array}$ & 124 & 187 & 229 & 284 & 326 & 368 & 412 & 472 \\
\hline
\end{tabular}




\begin{tabular}{|c|c|c|c|c|c|c|c|c|c|c|c|}
\hline \multirow{2}{*}{$\begin{array}{c}\text { USGS } \\
\text { streamgage } \\
\text { number }\end{array}$} & \multirow[b]{2}{*}{ Streamgage name } & \multirow{2}{*}{$\begin{array}{l}\text { Used in regression } \\
\text { analyses (non- } \\
\text { redundant) }\end{array}$} & \multirow[b]{2}{*}{ Frequency analysis method } & \multicolumn{8}{|c|}{ Discharge for given annual exceedance probability, $\mathrm{ft}^{3} / \mathrm{s}$} \\
\hline & & & & 0.50 & 0.20 & 0.10 & 0.04 & 0.02 & 0.01 & 0.005 & 0.002 \\
\hline \multirow{5}{*}{04087257} & \multirow{5}{*}{ PIKE RIVER NEAR RACINE, WI } & \multirow{5}{*}{ Yes } & $\begin{array}{l}\text { At-site, unadjusted for urbanization, EMA, } \\
\text { at-site skew }\end{array}$ & 969 & 1,330 & 1,560 & 1,840 & 2,040 & 2,230 & 2,410 & 2,650 \\
\hline & & & $\begin{array}{l}\text { At-site, adjusted for urbanization, EMA, at- } \\
\text { site skew }\end{array}$ & 1,140 & 1,510 & 1,710 & 1,930 & 2,070 & 2,200 & 2,320 & 2,450 \\
\hline & & & $\begin{array}{l}\text { At-site, adjusted for urbanization, EMA, } \\
\text { weighted skew: }\left(Q_{p}\right)_{g, s}\end{array}$ & 1,120 & 1,490 & 1,720 & 2,000 & 2,210 & 2,410 & 2,600 & 2,860 \\
\hline & & & Regional regression: $(Q p)_{g, r}$ & 904 & 1,380 & 1,720 & 2,180 & 2,530 & 2,890 & 3,260 & 3,760 \\
\hline & & & 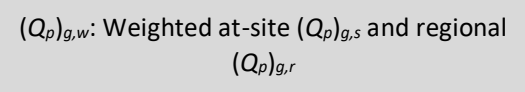 & 1,120 & 1,490 & 1,720 & 2,010 & 2,220 & 2,430 & 2,630 & 2,910 \\
\hline \multirow{5}{*}{04087300} & \multirow{5}{*}{$\begin{array}{l}\text { LAKE MICHIGAN TRIBUTARY AT WINTHROP } \\
\text { HARBOR, IL }\end{array}$} & \multirow{5}{*}{ Yes } & $\begin{array}{l}\text { At-site, unadjusted for urbanization, EMA, } \\
\text { at-site skew }\end{array}$ & 75 & 143 & 208 & 321 & 433 & 575 & 752 & 1,060 \\
\hline & & & $\begin{array}{l}\text { At-site, adjusted for urbanization, EMA, at- } \\
\text { site skew }\end{array}$ & 170 & 266 & 351 & 487 & 614 & 765 & 946 & 1,240 \\
\hline & & & $\begin{array}{l}\text { At-site, adjusted for urbanization, EMA, } \\
\left.\text { weighted skew: (o } a_{0}\right)_{g, s}\end{array}$ & 177 & 273 & 347 & 454 & 544 & 642 & 752 & 914 \\
\hline & & & Regional regression: $\left(Q_{p}\right)_{g, r}$ & 124 & 217 & 291 & 395 & 480 & 570 & 664 & 796 \\
\hline & & & $\begin{array}{l}\left(Q_{\rho}\right)_{Q, w}: \text { Weighted at-site } \\
\qquad\left(Q_{\rho}\right)_{g, s} \text { and regional }\end{array}$ & 173 & 267 & 339 & 444 & 531 & 626 & 729 & 878 \\
\hline \multirow{5}{*}{04087400} & \multirow{5}{*}{ KELLOGG RAVINE AT ZION, IL } & \multirow{5}{*}{ Yes } & $\begin{array}{l}\text { At-site, unadjusted for urbanization, EMA, } \\
\text { at-site skew }\end{array}$ & 218 & 398 & 568 & 859 & 1,140 & 1,500 & 1,940 & 2,690 \\
\hline & & & $\begin{array}{l}\text { At-site, adjusted for urbanization, EMA, at- } \\
\text { site skew }\end{array}$ & 436 & 680 & 898 & 1,250 & 1,580 & 1,980 & 2,460 & 3,250 \\
\hline & & & $\begin{array}{l}\text { At-site, adjusted for urbanization, EMA, } \\
\text { weighted skew: }\left(Q_{p}\right)_{g, s}\end{array}$ & 455 & 698 & 887 & 1,160 & 1,390 & 1,640 & 1,930 & 2,350 \\
\hline & & & Regional regression: $(Q p)_{g, r}$ & 319 & 544 & 722 & 974 & 1,180 & 1,400 & 1,620 & 1,950 \\
\hline & & & 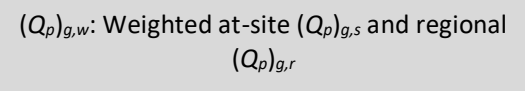 & 443 & 679 & 862 & 1,120 & 1,340 & 1,580 & 1,840 & 2,220 \\
\hline \multirow{5}{*}{04093000} & \multirow{5}{*}{$\begin{array}{l}\text { DEEP RIVER AT LAKE GEORGE OUTLET AT } \\
\text { HOBART, IN }\end{array}$} & \multirow{5}{*}{ Yes } & $\begin{array}{l}\text { At-site, unadjusted for urbanization, EMA, } \\
\text { at-site skew }\end{array}$ & 1,660 & 2,580 & 3,220 & 4,050 & 4,670 & 5,300 & 5,930 & 6,780 \\
\hline & & & $\begin{array}{l}\text { At-site, adjusted for urbanization, EMA, at- } \\
\text { site skew }\end{array}$ & 1,920 & 2,890 & 3,570 & 4,470 & 5,170 & 5,890 & 6,630 & 7,660 \\
\hline & & & $\begin{array}{l}\text { At-site, adjusted for urbanization, EMA, } \\
\text { weighted skew: (Q) } Q_{0} g_{g, s}\end{array}$ & 1,910 & 2,880 & 3,580 & 4,520 & 5,250 & 6,020 & 6,820 & 7,940 \\
\hline & & & Regional regression: $\left(Q_{p}\right)_{g, r}$ & 1,320 & 1,820 & 2,170 & 2,610 & 2,950 & 3,300 & 3,650 & 4,110 \\
\hline & & & $\begin{array}{l}\left(Q_{\rho}\right)_{g, w}: \text { Weighted at-site }\left(Q_{p}\right)_{g, s} \text { and regional } \\
\left(Q_{p} g_{g, r}\right.\end{array}$ & 1,900 & 2,850 & 3,520 & 4,410 & 5,080 & 5,770 & 6,470 & 7,400 \\
\hline
\end{tabular}




\begin{tabular}{|c|c|c|c|c|c|c|c|c|c|c|c|}
\hline \multirow{2}{*}{$\begin{array}{c}\text { USGS } \\
\text { streamgage } \\
\text { number }\end{array}$} & \multirow[b]{2}{*}{ Streamgage name } & \multirow{2}{*}{$\begin{array}{l}\text { Used in regression } \\
\text { analyses (non- } \\
\text { redundant) }\end{array}$} & \multirow[b]{2}{*}{ Frequency analysis method } & \multicolumn{8}{|c|}{ Discharge for given annual exceedance probability, $\mathrm{ft}^{3} / \mathrm{s}$} \\
\hline & & & & 0.50 & 0.20 & 0.10 & 0.04 & 0.02 & 0.01 & 0.005 & 0.002 \\
\hline \multirow{5}{*}{04094000} & \multirow{5}{*}{ LITTLE CALUMET RIVER AT PORTER, IN } & \multirow{5}{*}{ Yes } & $\begin{array}{l}\text { At-site, unadjusted for urbanization, EMA, } \\
\text { at-site skew }\end{array}$ & 1,150 & 1,880 & 2,450 & 3,290 & 4,010 & 4,800 & 5,680 & 7,000 \\
\hline & & & $\begin{array}{l}\text { At-site, adjusted for urbanization, EMA, at- } \\
\text { site skew }\end{array}$ & 1,440 & 2,240 & 2,880 & 3,820 & 4,630 & 5,520 & 6,520 & 8,020 \\
\hline & & & $\begin{array}{l}\text { At-site, adjusted for urbanization, EMA, } \\
\text { weighted skew: (Q }\left(Q_{p}\right)_{g, s}\end{array}$ & 1,460 & 2,260 & 2,860 & 3,730 & 4,440 & 5,210 & 6,040 & 7,260 \\
\hline & & & Regional regression: $(Q p)_{a, r}$ & 732 & 1,070 & 1,300 & 1,600 & 1,810 & 2,030 & 2,250 & 2,530 \\
\hline & & & $\begin{array}{c}\left(Q_{p}\right)_{Q, w} \text { : Weighted at-site }\left(Q_{\rho}\right)_{Q s, \text { and regional }} \\
\left(Q_{\rho}\right)_{Q, r}\end{array}$ & 1,430 & 2,210 & 2,780 & 3,560 & 4,170 & 4,790 & 5,440 & 6,310 \\
\hline \multirow{5}{*}{04094500} & \multirow{5}{*}{ SALT CREEK NEAR MCCOOL, IND. } & \multirow{5}{*}{ Yes } & $\begin{array}{l}\text { At-site, unadjusted for urbanization, EMA, } \\
\text { at-site skew }\end{array}$ & 1,030 & 1,650 & 2,110 & 2,720 & 3,200 & 3,700 & 4,220 & 4,950 \\
\hline & & & $\begin{array}{l}\text { At-site, adjusted for urbanization, EMA, at- } \\
\text { site skew }\end{array}$ & 1,580 & 2,340 & 2,890 & 3,630 & 4,210 & 4,810 & 5,440 & 6,330 \\
\hline & & & $\begin{array}{l}\text { At-site, adjusted for urbanization, EMA, } \\
\text { weighted skew: (Q }\left(Q_{p}\right)_{g, s}\end{array}$ & 1,580 & 2,340 & 2,900 & 3,640 & 4,220 & 4,830 & 5,470 & 6,370 \\
\hline & & & Regional regression: $\left(Q_{p}\right)_{g, r}$ & 910 & 1,300 & 1,570 & 1,920 & 2,190 & 2,460 & 2,730 & 3,090 \\
\hline & & & $\begin{array}{c}\left(Q_{\rho}\right)_{g, w:} \text { : Weighted at-site } \\
\qquad\left(Q_{\rho \rho}\right)_{g, s} \text { and regional } \\
\left(Q_{\rho}\right)_{g, r}\end{array}$ & 1,550 & 2,300 & 2,820 & 3,510 & 4,030 & 4,560 & 5,100 & 5,820 \\
\hline \multirow{5}{*}{05437950} & \multirow{5}{*}{ KISHWAUKEE RIVER NEAR HUNTLEY, IL } & \multirow{5}{*}{ Yes } & $\begin{array}{l}\text { At-site, unadjusted for urbanization, EMA, } \\
\text { at-site skew }\end{array}$ & 129 & 158 & 174 & 190 & 200 & 209 & 217 & 226 \\
\hline & & & $\begin{array}{l}\text { At-site, adjusted for urbanization, EMA, at- } \\
\text { site skew }\end{array}$ & 203 & 242 & 261 & 280 & 292 & 302 & 310 & 320 \\
\hline & & & $\begin{array}{l}\text { At-site, adjusted for urbanization, EMA, } \\
\left.\text { weighted skew: ( } Q_{p}\right)_{g, s}\end{array}$ & 200 & 241 & 265 & 292 & 310 & 326 & 342 & 361 \\
\hline & & & Regional regression: $(Q p)_{g, r}$ & 230 & 336 & 409 & 503 & 573 & 642 & 712 & 804 \\
\hline & & & $\begin{array}{c}\left(Q_{p}\right)_{g, w} \text { : Weighted at-site } \\
\left(Q_{\rho}\right)_{Q, s} \text { and regional } \\
\left(Q_{\rho}\right)_{g, r}\end{array}$ & 201 & 243 & 268 & 296 & 316 & 335 & 354 & 378 \\
\hline \multirow{5}{*}{05438250} & \multirow{5}{*}{ COON CREEK AT RILEY, IL } & \multirow{5}{*}{ Yes } & $\begin{array}{l}\text { At-site, unadjusted for urbanization, EMA, } \\
\text { at-site skew }\end{array}$ & 1,220 & 1,940 & 2,540 & 3,460 & 4,260 & 5,190 & 6,260 & 7,920 \\
\hline & & & $\begin{array}{l}\text { At-site, adjusted for urbanization, EMA, at- } \\
\text { site skew }\end{array}$ & 1,390 & 2,180 & 2,830 & 3,810 & 4,670 & 5,650 & 6,760 & 8,480 \\
\hline & & & 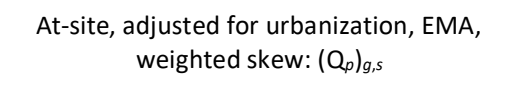 & 1,430 & 2,230 & 2,820 & 3,640 & 4,300 & 5,010 & 5,760 & 6,830 \\
\hline & & & Regional regression: $\left(Q_{p}\right)_{g, r}$ & 1,360 & 2,120 & 2,650 & 3,330 & 3,850 & 4,360 & 4,880 & 5,560 \\
\hline & & & $\begin{array}{l}\left(Q_{\rho}\right)_{g, w} \text { : Weighted at-site }\left(Q_{\rho}\right)_{g, \text { s a d r regional }} \\
\left(Q_{\rho} g_{g, r}\right.\end{array}$ & 1,420 & 2,220 & 2,810 & 3,610 & 4,250 & 4,910 & 5,600 & 6,550 \\
\hline
\end{tabular}




\begin{tabular}{|c|c|c|c|c|c|c|c|c|c|c|c|}
\hline \multirow{2}{*}{$\begin{array}{c}\text { USGS } \\
\text { streamgage } \\
\text { number }\end{array}$} & \multirow[b]{2}{*}{ Streamgage name } & \multirow{2}{*}{$\begin{array}{c}\text { Used in regression } \\
\text { analyses (non-- } \\
\text { redundant) }\end{array}$} & \multirow[b]{2}{*}{ Frequency analysis method } & \multicolumn{8}{|c|}{ Discharge for given annual exceedance probability, $\mathrm{ft}^{3} / \mathrm{s}$} \\
\hline & & & & 0.50 & 0.20 & 0.10 & 0.04 & 0.02 & 0.01 & 0.005 & 0.002 \\
\hline \multirow{5}{*}{05438283} & \multirow{5}{*}{ PISCASAW CREEK NEAR WALWORTH, WI } & \multirow{5}{*}{ Yes } & $\begin{array}{l}\text { At-site, unadjusted for urbanization, EMA, } \\
\text { at-site skew }\end{array}$ & 353 & 458 & 519 & 589 & 636 & 680 & 722 & 773 \\
\hline & & & $\begin{array}{l}\text { At-site, adjusted for urbanization, EMA, at- } \\
\text { site skew }\end{array}$ & 362 & 472 & 536 & 610 & 660 & 707 & 751 & 806 \\
\hline & & & 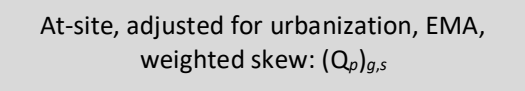 & 361 & 470 & 537 & 615 & 670 & 722 & 773 & 837 \\
\hline & & & Regional regression: $(Q p)_{a, r}$ & 329 & 561 & 733 & 961 & 1,140 & 1,310 & 1,490 & 1,730 \\
\hline & & & 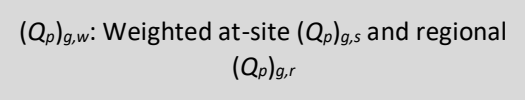 & 360 & 473 & 544 & 631 & 693 & 755 & 817 & 899 \\
\hline \multirow{5}{*}{05438300} & \multirow{5}{*}{ LAWRENCE CREEK TRIBUTARY NEAR HARVARD, IL } & \multirow{5}{*}{ Yes } & $\begin{array}{l}\text { At-site, unadjusted for urbanization, EMA, } \\
\text { at-site skew }\end{array}$ & 76 & 132 & 171 & 223 & 263 & 304 & 344 & 399 \\
\hline & & & $\begin{array}{l}\text { At-site, adjusted for urbanization, EMA, at- } \\
\text { site skew }\end{array}$ & 76 & 132 & 171 & 223 & 263 & 304 & 344 & 399 \\
\hline & & & $\begin{array}{l}\text { At-site, adjusted for urbanization, EMA, } \\
\text { weighted skew: ( }\left(Q_{p}\right) \text { g,s }\end{array}$ & 75 & 131 & 172 & 229 & 274 & 320 & 368 & 435 \\
\hline & & & Regional regression: $\left(Q_{p}\right)_{g, r}$ & 71 & 136 & 188 & 262 & 321 & 382 & 447 & 536 \\
\hline & & & $\begin{array}{l}\left(Q_{p \rho}\right)_{y, w} \text { Weighted at-site }\left(Q_{0}\right)_{g, s} \text { and regional } \\
\left(Q_{p}\right)_{g, r}\end{array}$ & 75 & 132 & 174 & 234 & 282 & 332 & 385 & 460 \\
\hline \multirow{5}{*}{05438390} & \multirow{5}{*}{$\begin{array}{l}\text { PISCASAW CREEK BELOW MOKELER CREEK NR } \\
\text { CAPRON, IL }\end{array}$} & \multirow{5}{*}{ No } & $\begin{array}{l}\text { At-site, unadjusted for urbanization, EMA, } \\
\text { at-site skew }\end{array}$ & 1,690 & 2,490 & 3,110 & 4,010 & 4,770 & 5,620 & 6,570 & 7,980 \\
\hline & & & $\begin{array}{l}\text { At-site, adjusted for urbanization, EMA, at- } \\
\text { site skew }\end{array}$ & 1,860 & 2,710 & 3,370 & 4,320 & 5,120 & 6,000 & 6,990 & 8,460 \\
\hline & & & $\begin{array}{l}\text { At-site, adjusted for urbanization, EMA, } \\
\text { weighted skew: (Q Q Q }) \text { g,s }\end{array}$ & 1,920 & 2,760 & 3,340 & 4,090 & 4,660 & 5,250 & 5,840 & 6,660 \\
\hline & & & Regional regression: $(Q p)_{g, r}$ & 1,510 & 2,400 & 3,040 & 3,860 & 4,490 & 5,130 & 5,770 & 6,630 \\
\hline & & & $\begin{array}{c}\left(Q_{0}\right)_{a, w} \text { : Weighted at-site }\left(Q_{0}\right)_{a, s} \text { and regional } \\
\left(Q_{p}\right)_{Q, r}\end{array}$ & 1,880 & 2,720 & 3,300 & 4,050 & 4,630 & 5,220 & 5,830 & 6,650 \\
\hline \multirow{5}{*}{05438500} & \multirow{5}{*}{ KISHWAUKEE RIVER AT BELVIDERE, IL } & \multirow{5}{*}{ Yes } & $\begin{array}{l}\text { At-site, unadjusted for urbanization, EMA, } \\
\text { at-site skew }\end{array}$ & 4,060 & 7,010 & 8,650 & 10,300 & 11,300 & 12,000 & 12,600 & 13,300 \\
\hline & & & $\begin{array}{l}\text { At-site, adjusted for urbanization, EMA, at- } \\
\text { site skew }\end{array}$ & 4,570 & 7,650 & 9,460 & 11,400 & 12,600 & 13,700 & 14,600 & 15,600 \\
\hline & & & $\begin{array}{l}\text { At-site, adjusted for urbanization, EMA, } \\
\text { weighted skew: ( }\left(_{0}\right)_{g, s}\end{array}$ & 4,460 & 7,390 & 9,460 & 12,200 & 14,200 & 16,300 & 18,400 & 21,200 \\
\hline & & & Regional regression: $\left(Q_{p}\right)_{g, r}$ & 4,490 & 6,510 & 7,870 & 9,570 & 10,800 & 12,100 & 13,300 & 15,000 \\
\hline & & & $\begin{array}{l}\left(Q_{\rho}\right)_{g, w}: \text { Weighted at-site } \\
\qquad\left(Q_{\rho}\right)_{\rho, g, s} \text { and regional }\end{array}$ & 4,460 & 7,360 & 9,390 & 12,000 & 14,000 & 16,000 & 17,900 & 20,500 \\
\hline
\end{tabular}




\begin{tabular}{|c|c|c|c|c|c|c|c|c|c|c|c|}
\hline \multirow{2}{*}{$\begin{array}{c}\text { USGS } \\
\text { streamgage } \\
\text { number }\end{array}$} & \multirow[b]{2}{*}{ Streamgage name } & \multirow{2}{*}{$\begin{array}{l}\text { Used in regression } \\
\text { analyses (non- } \\
\text { redundant) }\end{array}$} & \multirow[b]{2}{*}{ Frequency analysis method } & \multicolumn{8}{|c|}{ Discharge for given annual exceedance probability, $\mathrm{ft}^{3} / \mathrm{s}$} \\
\hline & & & & 0.50 & 0.20 & 0.10 & 0.04 & 0.02 & 0.01 & 0.005 & 0.002 \\
\hline \multirow{5}{*}{05438850} & \multirow{5}{*}{$\begin{array}{c}\text { MIDDLE BR OF S B KISHWAUKEE RIVER NEAR } \\
\text { MALTA, IL }\end{array}$} & \multirow{5}{*}{ Yes } & $\begin{array}{l}\text { At-site, unadjusted for urbanization, EMA, } \\
\text { at-site skew }\end{array}$ & 142 & 239 & 301 & 374 & 424 & 470 & 512 & 564 \\
\hline & & & $\begin{array}{l}\text { At-site, adjusted for urbanization, EMA, at- } \\
\text { site skew }\end{array}$ & 142 & 239 & 301 & 374 & 424 & 470 & 512 & 564 \\
\hline & & & $\begin{array}{l}\text { At-site, adjusted for urbanization, EMA, } \\
\left.\text { weighted skew: }\left(Q_{0}\right)_{g}\right)_{s, s}\end{array}$ & 138 & 236 & 306 & 396 & 465 & 534 & 603 & 695 \\
\hline & & & Regional regression: $(Q p)_{g, r}$ & 74 & 130 & 172 & 228 & 271 & 315 & 359 & 417 \\
\hline & & & $\begin{array}{l}\left(Q_{p}\right)_{g, w}: \text { Weighted at-site } \\
\left(Q_{\rho} Q_{a, r}\right)_{g, s} \text { and regional }\end{array}$ & 131 & 224 & 288 & 370 & 430 & 489 & 547 & 622 \\
\hline \multirow{5}{*}{05439000} & \multirow{5}{*}{$\begin{array}{l}\text { SOUTH BRANCH KISHWAUKEE RIVER AT DEKALB, } \\
\text { IL }\end{array}$} & \multirow{5}{*}{ Yes } & $\begin{array}{l}\text { At-site, unadjusted for urbanization, EMA, } \\
\text { at-site skew }\end{array}$ & 1,030 & 1,600 & 2,100 & 2,860 & 3,560 & 4,380 & 5,340 & 6,880 \\
\hline & & & $\begin{array}{l}\text { At-site, adjusted for urbanization, EMA, at- } \\
\text { site skew }\end{array}$ & 1,040 & 1,620 & 2,110 & 2,880 & 3,580 & 4,400 & 5,370 & 6,910 \\
\hline & & & $\begin{array}{l}\text { At-site, adjusted for urbanization, EMA, } \\
\left.\text { wighted skew: (Q } Q_{0}\right)_{g, s}\end{array}$ & 1,080 & 1,660 & 2,100 & 2,710 & 3,210 & 3,750 & 4,330 & 5,170 \\
\hline & & & Regional regression: $\left(Q_{\rho}\right)_{g, r}$ & 1,130 & 1,710 & 2,100 & 2,600 & 2,960 & 3,320 & 3,680 & 4,150 \\
\hline & & & $\begin{array}{l}\left(Q_{p}\right)_{y, w}: \text { Weighted at-site }\left(Q_{p}\right)_{g, s} \text { and regional } \\
\left(Q_{\rho} g_{g, r}\right.\end{array}$ & 1,080 & 1,660 & 2,100 & 2,700 & 3,180 & 3,680 & 4,210 & 4,940 \\
\hline \multirow{5}{*}{05439500} & \multirow{5}{*}{$\begin{array}{l}\text { SOUTH BRANCH KISHWAUKEE RIVER NR } \\
\text { FAIRDALE IL }\end{array}$} & \multirow{5}{*}{ Yes } & $\begin{array}{l}\text { At-site, unadjusted for urbanization, EMA, } \\
\text { at-site skew }\end{array}$ & 4,000 & 6,820 & 8,850 & 11,600 & 13,600 & 15,700 & 17,900 & 20,800 \\
\hline & & & $\begin{array}{l}\text { At-site, adjusted for urbanization, EMA, at- } \\
\text { site skew }\end{array}$ & 4,220 & 7,090 & 9,150 & 11,900 & 13,900 & 16,000 & 18,200 & 21,100 \\
\hline & & & $\begin{array}{l}\text { At-site, adjusted for urbanization, EMA, } \\
\text { weighted skew: (Q }\left(Q_{p}\right)_{g, s}\end{array}$ & 4,190 & 7,080 & 9,200 & 12,000 & 14,200 & 16,500 & 18,800 & 22,000 \\
\hline & & & Regional regression: $(Q p)_{g, r}$ & 3,940 & 5,810 & 7,080 & 8,680 & 9,850 & 11,000 & 12,200 & 13,800 \\
\hline & & & 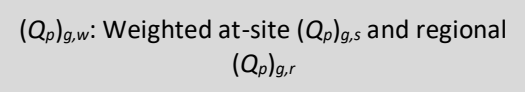 & 4,180 & 7,040 & 9,100 & 11,800 & 13,900 & 16,000 & 18,000 & 20,800 \\
\hline \multirow{5}{*}{05439550} & \multirow{5}{*}{$\begin{array}{l}\text { SOUTH BRANCH KISHWAUKEE RIVER TRIB NR } \\
\text { IRENE, IL }\end{array}$} & \multirow{5}{*}{ Yes } & $\begin{array}{l}\text { At-site, unadjusted for urbanization, EMA, } \\
\text { at-site skew }\end{array}$ & 80 & 190 & 292 & 451 & 590 & 747 & 922 & 1,180 \\
\hline & & & $\begin{array}{l}\text { At-site, adjusted for urbanization, EMA, at- } \\
\text { site skew }\end{array}$ & 85 & 199 & 302 & 465 & 607 & 767 & 945 & 1,210 \\
\hline & & & $\begin{array}{l}\text { At-site, adjusted for urbanization, EMA, } \\
\left.\text { weighted skew: (Q. } Q_{0}\right)_{g, s}\end{array}$ & 85 & 199 & 301 & 460 & 598 & 751 & 920 & 1,170 \\
\hline & & & Regional regression: $\left(Q_{p}\right)_{g, r}$ & 113 & 210 & 285 & 388 & 468 & 549 & 632 & 743 \\
\hline & & & $\begin{array}{l}\left(Q_{p}\right)_{g, w}: \text { Weighted at-site }\left(Q_{p}\right)_{g, s} \text { and regional } \\
\left(Q_{p} g_{g, r}\right.\end{array}$ & 91 & 202 & 297 & 435 & 547 & 663 & 782 & 942 \\
\hline
\end{tabular}




\begin{tabular}{|c|c|c|c|c|c|c|c|c|c|c|c|}
\hline \multirow{2}{*}{$\begin{array}{c}\text { USGS } \\
\text { streamgage } \\
\text { number }\end{array}$} & \multirow[b]{2}{*}{ Streamgage name } & \multirow{2}{*}{$\begin{array}{l}\text { Used in regression } \\
\text { analyses (non- } \\
\text { redundant) }\end{array}$} & \multirow[b]{2}{*}{ Frequency analysis method } & \multicolumn{8}{|c|}{ Discharge for given annual exceedance probability, $\mathrm{ft}^{3} / \mathrm{s}$} \\
\hline & & & & 0.50 & 0.20 & 0.10 & 0.04 & 0.02 & 0.01 & 0.005 & 0.002 \\
\hline \multirow{5}{*}{05440000} & \multirow{5}{*}{ KIISHWAUKEE RIVER NEAR PERRYVILLE, IL } & \multirow{5}{*}{ No } & $\begin{array}{l}\text { At-site, unadjusted for urbanization, EMA, } \\
\text { at-site skew }\end{array}$ & 8,450 & 12,600 & 15,300 & 18,600 & 20,900 & 23,100 & 25,200 & 27,900 \\
\hline & & & $\begin{array}{l}\text { At-site, adjusted for urbanization, EMA, at- } \\
\text { site skew }\end{array}$ & 9,190 & 13,700 & 16,500 & 19,900 & 22,200 & 24,300 & 26,400 & 29,000 \\
\hline & & & $\begin{array}{l}\text { At-site, adjusted for urbanization, EMA, } \\
\text { weighted skew: ( }\left(_{0}\right)_{g, s}\end{array}$ & 9,180 & 13,600 & 16,500 & 20,100 & 22,900 & 25,600 & 28,300 & 31,800 \\
\hline & & & Regional regression: $(Q p)_{g, r}$ & 7,860 & 11,200 & 13,500 & 16,300 & 18,400 & 20,500 & 22,500 & 25,300 \\
\hline & & & $\begin{array}{c}\left(Q_{\rho \rho}\right)_{y, w} \text { : Weighted at-site }\left(Q_{\rho}\right)_{Q, s} \text { and regional } \\
\left(Q_{\rho}\right)_{Q, r}\end{array}$ & 9,140 & 13,500 & 16,400 & 20,000 & 22,600 & 25,300 & 27,900 & 31,300 \\
\hline \multirow{5}{*}{05440500} & \multirow{5}{*}{ KILLBUCK CREEK NEAR MONROE CENTER, IL } & \multirow{5}{*}{ Yes } & $\begin{array}{l}\text { At-site, unadjusted for urbanization, EMA, } \\
\text { at-site skew }\end{array}$ & 2,680 & 4,270 & 5,060 & 5,780 & 6,160 & 6,440 & 6,650 & 6,850 \\
\hline & & & $\begin{array}{l}\text { At-site, adjusted for urbanization, EMA, at- } \\
\text { site skew }\end{array}$ & 2,710 & 4,310 & 5,100 & 5,830 & 6,210 & 6,490 & 6,700 & 6,900 \\
\hline & & & $\begin{array}{l}\text { At-site, adjusted for urbanization, EMA, } \\
\text { weighted skew: (Q⿱中⿰㇀丶㇀ })_{g, s}\end{array}$ & 2,570 & 4,140 & 5,200 & 6,550 & 7,540 & 8,520 & 9,490 & 10,800 \\
\hline & & & Regional regression: $\left(Q_{p}\right)_{Q, r}$ & 1,730 & 2,700 & 3,360 & 4,210 & 4,830 & 5,450 & 6,060 & 6,880 \\
\hline & & & $\begin{array}{c}\left(Q_{\rho \rho}\right)_{y, w} \text { Weighted at-site }\left(Q_{p}\right)_{Q, s} \text { and regional } \\
\left(Q_{\rho}\right)_{Q, r}\end{array}$ & 2,520 & 4,050 & 5,070 & 6,340 & 7,260 & 8,160 & 9,020 & 10,100 \\
\hline \multirow{5}{*}{05442000} & \multirow{5}{*}{ KYTE RIVER NEAR FLAGG CENTER, IL } & \multirow{5}{*}{ Yes } & $\begin{array}{l}\text { At-site, unadjusted for urbanization, EMA, } \\
\text { at-site skew }\end{array}$ & 1,220 & 1,720 & 2,090 & 2,580 & 2,980 & 3,400 & 3,850 & 4,500 \\
\hline & & & $\begin{array}{l}\text { At-site, adjusted for urbanization, EMA, at- } \\
\text { site skew }\end{array}$ & 1,280 & 1,800 & 2,170 & 2,670 & 3,080 & 3,510 & 3,960 & 4,610 \\
\hline & & & $\begin{array}{l}\text { At-site, adjusted for urbanization, EMA, } \\
\text { weighted skew: ( (a) olg,s }\end{array}$ & 1,310 & 1,810 & 2,140 & 2,570 & 2,900 & 3,220 & 3,560 & 4,010 \\
\hline & & & Regional regression: $(Q p)_{a, r}$ & 1,740 & 2,640 & 3,250 & 4,040 & 4,620 & 5,200 & 5,770 & 6,540 \\
\hline & & & 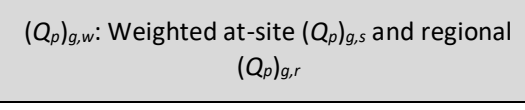 & 1,330 & 1,860 & 2,230 & 2,710 & 3,080 & 3,470 & 3,870 & 4,410 \\
\hline \multirow{5}{*}{05446950} & \multirow{5}{*}{ GREEN RIVER TRIBUTARY NEAR AMBOY, IL } & \multirow{5}{*}{ Yes } & $\begin{array}{l}\text { At-site, unadjusted for urbanization, EMA, } \\
\text { at-site skew }\end{array}$ & 81 & 194 & 320 & 566 & 836 & 1,200 & 1,700 & 2,630 \\
\hline & & & $\begin{array}{l}\text { At-site, adjusted for urbanization, EMA, at- } \\
\text { site skew }\end{array}$ & 81 & 194 & 320 & 566 & 836 & 1,200 & 1,700 & 2,630 \\
\hline & & & $\begin{array}{l}\text { At-site, adjusted for urbanization, EMA, } \\
\text { weighted skew: }\left(Q_{p}\right)_{g, s}\end{array}$ & 89 & 200 & 306 & 478 & 636 & 822 & 1,040 & 1,380 \\
\hline & & & Regional regression: $\left(Q_{p}\right)_{g, r}$ & 59 & 111 & 152 & 208 & 252 & 298 & 344 & 407 \\
\hline & & & $\begin{array}{l}\left(Q_{\rho}\right)_{a, w} \text { : Weighted at-site } \\
\left.\qquad\left(Q_{\rho}\right)_{Q, r}\right)_{a, s} \text { and regional }\end{array}$ & 80 & 170 & 242 & 344 & 424 & 504 & 587 & 693 \\
\hline
\end{tabular}




\begin{tabular}{|c|c|c|c|c|c|c|c|c|c|c|c|}
\hline \multirow{2}{*}{$\begin{array}{c}\text { USGS } \\
\text { streamgage } \\
\text { number }\end{array}$} & \multirow[b]{2}{*}{ Streamgage name } & \multirow{2}{*}{$\begin{array}{l}\text { Used in regression } \\
\text { analyses (non- } \\
\text { redundant) }\end{array}$} & \multirow[b]{2}{*}{ Frequency analysis method } & \multicolumn{8}{|c|}{ Discharge for given annual exceedance probability, $\mathrm{ft}^{3} / \mathrm{s}$} \\
\hline & & & & 0.50 & 0.20 & 0.10 & 0.04 & 0.02 & 0.01 & 0.005 & 0.002 \\
\hline \multirow{5}{*}{05447000} & \multirow{5}{*}{ GREEN RIVER AT AMBOY, IL } & \multirow{5}{*}{ Yes } & $\begin{array}{l}\text { At-site, unadjusted for urbanization, EMA, } \\
\text { at-site skew }\end{array}$ & 2,840 & 4,160 & 5,040 & 6,150 & 6,970 & 7,780 & 8,600 & 9,670 \\
\hline & & & $\begin{array}{l}\text { At-site, adjusted for urbanization, EMA, at- } \\
\text { site skew }\end{array}$ & 2,850 & 4,180 & 5,060 & 6,170 & 6,990 & 7,800 & 8,610 & 9,690 \\
\hline & & & $\begin{array}{l}\text { At-site, adjusted for urbanization, EMA, } \\
\text { weighted skew: }\left(Q_{p}\right)_{g, s}\end{array}$ & 2,860 & 4,180 & 5,060 & 6,150 & 6,960 & 7,750 & 8,540 & 9,570 \\
\hline & & & Regional regression: $(Q p)_{a, r}$ & 2,490 & 3,860 & 4,800 & 5,990 & 6,860 & 7,720 & 8,580 & 9,710 \\
\hline & & & $\begin{array}{c}\left(Q_{\rho \rho}\right)_{y, w} \text { Weighted at-site }\left(Q_{p}\right)_{Q, s} \text { and regional } \\
\left(Q_{\rho}\right)_{Q, r}\end{array}$ & 2,850 & 4,170 & 5,050 & 6,150 & 6,950 & 7,750 & 8,540 & 9,590 \\
\hline \multirow{5}{*}{05447050} & \multirow{5}{*}{ GREEN RIVER TRIBUTARY NEAR OHIO, IL } & \multirow{5}{*}{ Yes } & $\begin{array}{l}\text { At-site, unadjusted for urbanization, EMA, } \\
\text { at-site skew }\end{array}$ & 138 & 232 & 307 & 418 & 512 & 616 & 732 & 905 \\
\hline & & & $\begin{array}{l}\text { At-site, adjusted for urbanization, EMA, at- } \\
\text { site skew }\end{array}$ & 138 & 232 & 307 & 418 & 512 & 616 & 732 & 905 \\
\hline & & & $\begin{array}{l}\text { At-site, adjusted for urbanization, EMA, } \\
\text { weighted skew: (Q }\left(Q_{p}\right)_{g, s}\end{array}$ & 143 & 236 & 304 & 395 & 467 & 541 & 618 & 724 \\
\hline & & & Regional regression: $\left(Q_{p}\right)_{g, r}$ & 173 & 299 & 391 & 513 & 606 & 699 & 792 & 915 \\
\hline & & & $\begin{array}{c}\left(Q_{\rho \rho}\right)_{y, w} \text { : Weighted at-site }\left(Q_{p}\right)_{Q, s} \text { and regional } \\
\left(Q_{\rho}\right)_{Q, r}\end{array}$ & 146 & 243 & 316 & 416 & 495 & 576 & 661 & 779 \\
\hline \multirow{5}{*}{05447500} & \multirow{5}{*}{ GREEN RIVER NEAR GENESEO, IL } & \multirow{5}{*}{ Yes } & $\begin{array}{l}\text { At-site, unadjusted for urbanization, EMA, } \\
\text { at-site skew }\end{array}$ & 6,580 & 8,680 & 9,850 & 11,100 & 12,000 & 12,700 & 13,400 & 14,200 \\
\hline & & & $\begin{array}{l}\text { At-site, adjusted for urbanization, EMA, at- } \\
\text { site skew }\end{array}$ & 6,650 & 8,660 & 9,820 & 11,100 & 12,000 & 12,800 & 13,500 & 14,400 \\
\hline & & & $\begin{array}{l}\text { At-site, adjusted for urbanization, EMA, } \\
\text { weighted skew: }\left(Q_{0}\right)_{g, s}\end{array}$ & 6,650 & 8,630 & 9,800 & 11,200 & 12,100 & 13,000 & 13,800 & 14,900 \\
\hline & & & Regional regression: $(Q p)_{g, r}$ & 5,690 & 8,030 & 9,520 & 11,300 & 12,600 & 13,900 & 15,100 & 16,600 \\
\hline & & & $\begin{array}{l}\left(Q_{\rho p}\right)_{y, w} \text { : Weighted at-site }\left(Q_{p}\right)_{Q, s} \text { and regional } \\
\left(Q_{0}\right)_{p, r}\end{array}$ & 6,640 & 8,620 & 9,800 & 11,200 & 12,100 & 13,000 & 13,900 & 14,900 \\
\hline \multirow{5}{*}{05517500} & \multirow{5}{*}{ KANKAKEE RIVER AT DUNNS BRIDGE, IN } & \multirow{5}{*}{ Yes } & $\begin{array}{l}\text { At-site, unadjusted for urbanization, EMA, } \\
\text { at-site skew }\end{array}$ & 3,740 & 4,500 & 4,940 & 5,450 & 5,800 & 6,140 & 6,460 & 6,870 \\
\hline & & & $\begin{array}{l}\text { At-site, adjusted for urbanization, EMA, at- } \\
\text { site skew }\end{array}$ & 3,900 & 4,650 & 5,090 & 5,600 & 5,950 & 6,280 & 6,600 & 7,010 \\
\hline & & & 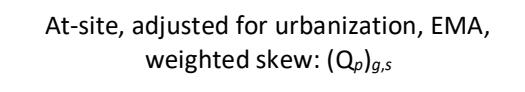 & 3,910 & 4,650 & 5,080 & 5,580 & 5,920 & 6,240 & 6,540 & 6,930 \\
\hline & & & Regional regression: $\left(Q_{p}\right)_{g, r}$ & 4,530 & 5,890 & 6,720 & 7,690 & 8,370 & 9,030 & 9,660 & 10,500 \\
\hline & & & $\begin{array}{l}\left(Q_{\rho}\right)_{g, w} \text { : Weighted at-site }\left(Q_{p}\right)_{g_{s, s}} \text { and regional } \\
\left(Q_{p}\right)_{Q, r}\end{array}$ & 3,910 & 4,660 & 5,090 & 5,590 & 5,940 & 6,270 & 6,580 & 6,980 \\
\hline
\end{tabular}




\begin{tabular}{|c|c|c|c|c|c|c|c|c|c|c|c|}
\hline \multirow{2}{*}{$\begin{array}{c}\text { USGS } \\
\text { streamgage } \\
\text { number }\end{array}$} & \multirow[b]{2}{*}{ Streamgage name } & \multirow{2}{*}{$\begin{array}{l}\text { Used in regression } \\
\text { analyses (non- } \\
\text { redundant) }\end{array}$} & \multirow[b]{2}{*}{ Frequency analysis method } & \multicolumn{8}{|c|}{ Discharge for given annual exceedance probability, $\mathrm{ft}^{3} / \mathrm{s}$} \\
\hline & & & & 0.50 & 0.20 & 0.10 & 0.04 & 0.02 & 0.01 & 0.005 & 0.002 \\
\hline \multirow{5}{*}{05517890} & \multirow{5}{*}{ COBB DITCH NEAR KOUTS, IND. } & \multirow{5}{*}{ Yes } & $\begin{array}{l}\text { At-site, unadjusted for urbanization, EMA, } \\
\text { at-site skew }\end{array}$ & 615 & 866 & 988 & 1,100 & 1,160 & 1,210 & 1,250 & 1,290 \\
\hline & & & $\begin{array}{l}\text { At-site, adjusted for urbanization, EMA, at- } \\
\text { site skew }\end{array}$ & 708 & 982 & 1,140 & 1,320 & 1,440 & 1,550 & 1,650 & 1,780 \\
\hline & & & $\begin{array}{l}\text { At-site, adjusted for urbanization, EMA, } \\
\left.\text { weighted skew: ( } Q_{p}\right)_{g, s}\end{array}$ & 700 & 981 & 1,150 & 1,360 & 1,500 & 1,640 & 1,760 & 1,930 \\
\hline & & & Regional regression: $(Q p)_{g, r}$ & 323 & 474 & 575 & 701 & 793 & 882 & 969 & 1,080 \\
\hline & & & $\begin{array}{l}\left(Q_{\rho}\right)_{a, w} \text { : Weighted at-site }\left(Q_{e}\right)_{Q, s} \text { and regional } \\
\qquad\left(Q_{\rho}\right)_{Q, r}\end{array}$ & 686 & 964 & 1,130 & 1,330 & 1,460 & 1,580 & 1,700 & 1,840 \\
\hline \multirow{5}{*}{05518000} & \multirow{5}{*}{ KANKAKEE RIVER AT SHELBY, IN } & \multirow{5}{*}{ No } & $\begin{array}{l}\text { At-site, unadjusted for urbanization, EMA, } \\
\text { at-site skew }\end{array}$ & 4,590 & 5,450 & 5,920 & 6,440 & 6,790 & 7,100 & 7,390 & 7,750 \\
\hline & & & $\begin{array}{l}\text { At-site, adjusted for urbanization, EMA, at- } \\
\text { site skew }\end{array}$ & 4,860 & 5,700 & 6,170 & 6,690 & 7,030 & 7,350 & 7,650 & 8,020 \\
\hline & & & $\begin{array}{l}\text { At-site, adjusted for urbanization, EMA, } \\
\left.\text { weighted skew: ( } Q_{\rho}\right)_{g, s}\end{array}$ & 4,860 & 5,700 & 6,170 & 6,690 & 7,050 & 7,370 & 7,670 & 8,050 \\
\hline & & & Regional regression: $\left(Q_{p}\right)_{g, r}$ & 5,220 & 6,670 & 7,530 & 8,550 & 9,250 & 9,930 & 10,600 & 11,400 \\
\hline & & & $\begin{array}{l}\left(Q_{\rho \rho}\right)_{y, w} \text { : Weighted at-site }\left(Q_{p}\right)_{Q, s} \text { and regional } \\
\left(Q_{p}\right)_{g, r}\end{array}$ & 4,860 & 5,700 & 6,170 & 6,700 & 7,060 & 7,390 & 7,700 & 8,090 \\
\hline \multirow{5}{*}{05519000} & \multirow{5}{*}{ SINGLETON DITCH AT SCHNEIDER, IN } & \multirow{5}{*}{ Yes } & $\begin{array}{l}\text { At-site, unadjusted for urbanization, EMA, } \\
\text { at-site skew }\end{array}$ & 1,240 & 1,790 & 2,150 & 2,580 & 2,900 & 3,200 & 3,500 & 3,890 \\
\hline & & & $\begin{array}{l}\text { At-site, adjusted for urbanization, EMA, at- } \\
\text { site skew }\end{array}$ & 1,330 & 1,820 & 2,190 & 2,680 & 3,080 & 3,510 & 3,970 & 4,630 \\
\hline & & & $\begin{array}{l}\text { At-site, adjusted for urbanization, EMA, } \\
\left.\text { weighted skew: ( } Q_{p}\right)_{g, s}\end{array}$ & 1,340 & 1,840 & 2,180 & 2,630 & 2,970 & 3,320 & 3,690 & 4,190 \\
\hline & & & Regional regression: $(Q p)_{g, r}$ & 1,010 & 1,400 & 1,660 & 1,990 & 2,220 & 2,450 & 2,680 & 2,970 \\
\hline & & & $\begin{array}{c}\left(Q_{\rho}\right)_{y, w} \text { : Weighted at-site }\left(Q_{\rho}\right)_{Q, s} \text { and regional } \\
\left(Q_{\rho}\right)_{Q, r}\end{array}$ & 1,340 & 1,830 & 2,170 & 2,600 & 2,930 & 3,270 & 3,610 & 4,070 \\
\hline \multirow{5}{*}{05519500} & \multirow{5}{*}{ WEST CREEK NEAR SCHNEIDER, IND. } & \multirow{5}{*}{ Yes } & $\begin{array}{l}\text { At-site, unadjusted for urbanization, EMA, } \\
\text { at-site skew }\end{array}$ & 952 & 1,380 & 1,640 & 1,950 & 2,160 & 2,360 & 2,550 & 2,790 \\
\hline & & & $\begin{array}{l}\text { At-site, adjusted for urbanization, EMA, at- } \\
\text { site skew }\end{array}$ & 1,110 & 1,570 & 1,840 & 2,160 & 2,370 & 2,560 & 2,740 & 2,950 \\
\hline & & & $\begin{array}{l}\text { At-site, adjusted for urbanization, EMA, } \\
\left.\text { weighted skew: (Q } Q_{p}\right)_{g, s}\end{array}$ & 1,090 & 1,560 & 1,870 & 2,250 & 2,540 & 2,820 & 3,090 & 3,460 \\
\hline & & & Regional regression: $\left(Q_{p}\right)_{g, r}$ & 578 & 809 & 963 & 1,160 & 1,300 & 1,430 & 1,570 & 1,750 \\
\hline & & & $\begin{array}{l}\left(Q_{\rho}\right)_{g, w}: \text { Weighted at-site }\left(Q_{p}\right)_{g_{s, s}} \text { and regional } \\
\left(Q_{p}\right)_{g, r}\end{array}$ & 1,060 & 1,510 & 1,800 & 2,140 & 2,390 & 2,630 & 2,860 & 3,150 \\
\hline
\end{tabular}




\begin{tabular}{|c|c|c|c|c|c|c|c|c|c|c|c|}
\hline \multirow{2}{*}{$\begin{array}{c}\text { USGS } \\
\text { streamgage } \\
\text { number }\end{array}$} & \multirow{2}{*}{ Streamgage name } & \multirow{2}{*}{$\begin{array}{l}\text { Used in regression } \\
\text { analyses (non- } \\
\text { redundant) }\end{array}$} & \multirow{2}{*}{ Frequency analysis method } & \multicolumn{8}{|c|}{ Discharge for given annual exceedance probability, $\mathrm{ft}^{3} / \mathrm{s}$} \\
\hline & & & & 0.50 & 0.20 & 0.10 & 0.04 & 0.02 & 0.01 & 0.005 & 0.002 \\
\hline \multirow{5}{*}{05520500} & \multirow{5}{*}{ KANKAKEE RIVER AT MOMENCE, IL } & \multirow{5}{*}{ No } & $\begin{array}{l}\text { At-site, unadjusted for urbanization, EMA, } \\
\text { at-site skew }\end{array}$ & 7,300 & 9,390 & 10,700 & 12,300 & 13,400 & 14,500 & 15,600 & 17,000 \\
\hline & & & $\begin{array}{l}\text { At-site, adjusted for urbanization, EMA, at- } \\
\text { site skew }\end{array}$ & 7,740 & 9,800 & 11,100 & 12,600 & 13,600 & 14,600 & 15,600 & 16,900 \\
\hline & & & $\begin{array}{l}\text { At-site, adjusted for urbanization, EMA, } \\
\text { weighted skew: }\left(Q_{\rho}\right)_{\text {g,s }}\end{array}$ & 7,750 & 9,810 & 11,000 & 12,500 & 13,600 & 14,600 & 15,500 & 16,800 \\
\hline & & & Regional regression: $(Q p)_{g, r}$ & 6,470 & 8,190 & 9,220 & 10,400 & 11,300 & 12,100 & 12,900 & 13,900 \\
\hline & & & 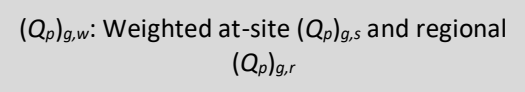 & 7,740 & 9,790 & 11,000 & 12,500 & 13,500 & 14,500 & 15,500 & 16,700 \\
\hline \multirow{5}{*}{05526150} & \multirow{5}{*}{$\begin{array}{l}\text { KANKAKEE RIVER TRIBUTARY NEAR } \\
\text { BOURBONNAIS, IL }\end{array}$} & \multirow{5}{*}{ Yes } & $\begin{array}{l}\text { At-site, unadjusted for urbanization, EMA, } \\
\text { at-site skew }\end{array}$ & 31 & 82 & 137 & 235 & 332 & 452 & 599 & 841 \\
\hline & & & $\begin{array}{l}\text { At-site, adjusted for urbanization, EMA, at- } \\
\text { site skew }\end{array}$ & 31 & 82 & 137 & 235 & 332 & 452 & 599 & 841 \\
\hline & & & 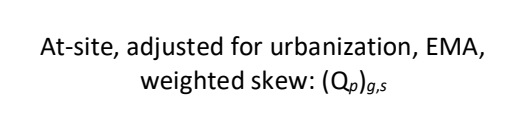 & 32 & 83 & 134 & 218 & 296 & 386 & 489 & 647 \\
\hline & & & Regional regression: $\left(Q_{\rho}\right)_{Q, r}$ & 20 & 39 & 55 & 76 & 94 & 111 & 129 & 153 \\
\hline & & & $\begin{array}{c}\left(Q_{\rho} \rho\right)_{\text {Qww }}: \text { Weighted at-site } \\
\left(Q_{\rho}\right)_{g, s} \text { and regional } \\
\left(S_{\rho} g_{g, r}\right.\end{array}$ & 29 & 70 & 106 & 158 & 199 & 239 & 279 & 329 \\
\hline \multirow{5}{*}{05526500} & \multirow{5}{*}{ TERRY CREEK NEAR CUSTER PARK, IL } & \multirow{5}{*}{ Yes } & $\begin{array}{l}\text { At-site, unadjusted for urbanization, EMA, } \\
\text { at-site skew }\end{array}$ & 135 & 259 & 415 & 762 & 1,200 & 1,880 & 2,950 & 5,310 \\
\hline & & & $\begin{array}{l}\text { At-site, adjusted for urbanization, EMA, at- } \\
\text { site skew }\end{array}$ & 144 & 273 & 435 & 794 & 1,250 & 1,950 & 3,040 & 5,450 \\
\hline & & & $\begin{array}{l}\text { At-site, adjusted for urbanization, EMA, } \\
\text { weighted skew: (Zopos,s }\end{array}$ & 168 & 311 & 434 & 626 & 797 & 994 & 1,220 & 1,570 \\
\hline & & & Regional regression: $(Q p)_{a r}$ & 294 & 471 & 594 & 750 & 865 & 978 & 1,090 & 1,230 \\
\hline & & & 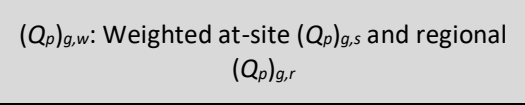 & 178 & 328 & 456 & 650 & 813 & 989 & 1,180 & 1,440 \\
\hline \multirow{5}{*}{05527050} & \multirow{5}{*}{ PRAIRIE CREEK NEAR FRANKFORT, IL } & \multirow{5}{*}{ Yes } & $\begin{array}{l}\text { At-site, unadjusted for urbanization, EMA, } \\
\text { at-site skew }\end{array}$ & 76 & 151 & 242 & 441 & 686 & 1,060 & 1,630 & 2,850 \\
\hline & & & $\begin{array}{l}\text { At-site, adjusted for urbanization, EMA, at- } \\
\text { site skew }\end{array}$ & 99 & 186 & 293 & 522 & 802 & 1,230 & 1,870 & 3,240 \\
\hline & & & $\begin{array}{l}\text { At-site, adjusted for urbanization, EMA, } \\
\text { weighted skew: ( }\left(_{0}\right)_{g, s}\end{array}$ & 113 & 210 & 293 & 423 & 539 & 673 & 826 & 1,060 \\
\hline & & & Regional regression: $\left(Q_{p}\right) g_{r}$ & 64 & 115 & 154 & 207 & 249 & 291 & 334 & 392 \\
\hline & & & 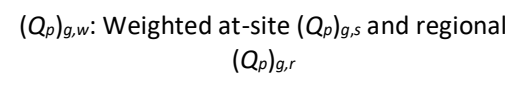 & 105 & 189 & 255 & 350 & 426 & 505 & 588 & 698 \\
\hline
\end{tabular}




\begin{tabular}{|c|c|c|c|c|c|c|c|c|c|c|c|}
\hline \multirow{2}{*}{$\begin{array}{c}\text { USGS } \\
\text { streamgage } \\
\text { number }\end{array}$} & \multirow[b]{2}{*}{ Streamgage name } & \multirow{2}{*}{$\begin{array}{l}\text { Used in regression } \\
\text { analyses (non- } \\
\text { redundant) }\end{array}$} & \multirow[b]{2}{*}{ Frequency analysis method } & \multicolumn{8}{|c|}{ Discharge for given annual exceedance probability, $\mathrm{ft}^{3} / \mathrm{s}$} \\
\hline & & & & 0.50 & 0.20 & 0.10 & 0.04 & 0.02 & 0.01 & 0.005 & 0.002 \\
\hline \multirow{5}{*}{05527800} & \multirow{5}{*}{ DES PLAINES RIVER AT RUSSELL, IL } & \multirow{5}{*}{ Yes } & $\begin{array}{l}\text { At-site, unadjusted for urbanization, EMA, } \\
\text { at-site skew }\end{array}$ & 762 & 1,420 & 1,910 & 2,540 & 3,020 & 3,490 & 3,960 & 4,580 \\
\hline & & & $\begin{array}{l}\text { At-site, adjusted for urbanization, EMA, at- } \\
\text { site skew }\end{array}$ & 838 & 1,520 & 2,010 & 2,660 & 3,150 & 3,640 & 4,130 & 4,780 \\
\hline & & & $\begin{array}{l}\text { At-site, adjusted for urbanization, EMA, } \\
\text { weighted skew: }\left(Q_{p}\right)_{g, s}\end{array}$ & 822 & 1,510 & 2,040 & 2,780 & 3,360 & 3,970 & 4,600 & 5,480 \\
\hline & & & Regional regression: $(Q p)_{a, r}$ & 1,310 & 1,890 & 2,280 & 2,770 & 3,140 & 3,500 & 3,860 & 4,330 \\
\hline & & & $\begin{array}{c}\left(Q_{\rho \rho}\right)_{y, w} \text { Weighted at-site }\left(Q_{p}\right)_{Q, s} \text { and regional } \\
\left(Q_{\rho}\right)_{Q, r}\end{array}$ & 847 & 1,540 & 2,060 & 2,780 & 3,330 & 3,900 & 4,470 & 5,230 \\
\hline \multirow{5}{*}{05527840} & \multirow{5}{*}{ DES PLAINES RIVER AT WADSWORTH, IL } & \multirow{5}{*}{ No } & $\begin{array}{l}\text { At-site, unadjusted for urbanization, EMA, } \\
\text { at-site skew }\end{array}$ & 952 & 1,510 & 1,840 & 2,210 & 2,440 & 2,640 & 2,820 & 3,030 \\
\hline & & & $\begin{array}{l}\text { At-site, adjusted for urbanization, EMA, at- } \\
\text { site skew }\end{array}$ & 1,130 & 1,750 & 2,100 & 2,480 & 2,710 & 2,900 & 3,070 & 3,260 \\
\hline & & & $\begin{array}{l}\text { At-site, adjusted for urbanization, EMA, } \\
\text { weighted skew: ( }\left(_{0}\right)_{g, s}\end{array}$ & 1,080 & 1,700 & 2,140 & 2,710 & 3,140 & 3,590 & 4,040 & 4,650 \\
\hline & & & Regional regression: $\left(Q_{0}\right)_{g, r}$ & 1,450 & 2,080 & 2,490 & 3,020 & 3,410 & 3,790 & 4,170 & 4,670 \\
\hline & & & $\begin{array}{c}\left(Q_{\rho \rho}\right)_{y, w} \text { Weighted at-site }\left(Q_{p}\right)_{Q, s} \text { and regional } \\
\left(Q_{p}\right)_{Q, r}\end{array}$ & 1,120 & 1,740 & 2,180 & 2,760 & 3,190 & 3,630 & 4,070 & 4,650 \\
\hline \multirow{5}{*}{05527870} & \multirow{5}{*}{ MILL CREEK AT WEDGES CORNER, IL } & \multirow{5}{*}{ Yes } & $\begin{array}{l}\text { At-site, unadjusted for urbanization, EMA, } \\
\text { at-site skew }\end{array}$ & 81 & 140 & 188 & 257 & 315 & 379 & 450 & 553 \\
\hline & & & $\begin{array}{l}\text { At-site, adjusted for urbanization, EMA, at- } \\
\text { site skew }\end{array}$ & 112 & 183 & 241 & 328 & 405 & 491 & 589 & 738 \\
\hline & & & $\begin{array}{l}\text { At-site, adjusted for urbanization, EMA, } \\
\text { weighted skew: }\left(Q_{p}\right)_{\text {g,s }}\end{array}$ & 114 & 184 & 240 & 323 & 392 & 469 & 555 & 683 \\
\hline & & & Regional regression: $\left(\left.Q p\right|_{g r}\right.$ & 281 & 389 & 465 & 564 & 639 & 714 & 790 & 892 \\
\hline & & & $\begin{array}{c}\left(Q_{\rho}\right)_{g, w} \text { : Weighted at-site }\left(Q_{\rho}\right)_{Q, s} \text { and regional } \\
\left(Q_{\rho}\right)_{Q, r}\end{array}$ & 125 & 203 & 268 & 362 & 441 & 526 & 618 & 748 \\
\hline \multirow{5}{*}{05527900} & \multirow{5}{*}{ NORTH MILL CREEK AT HICKORY CORNERS, IL } & \multirow{5}{*}{ Yes } & $\begin{array}{l}\text { At-site, unadjusted for urbanization, EMA, } \\
\text { at-site skew }\end{array}$ & 203 & 309 & 383 & 481 & 556 & 633 & 712 & 820 \\
\hline & & & $\begin{array}{l}\text { At-site, adjusted for urbanization, EMA, at- } \\
\text { site skew }\end{array}$ & 256 & 378 & 462 & 573 & 659 & 746 & 836 & 959 \\
\hline & & & $\begin{array}{l}\text { At-site, adjusted for urbanization, EMA, } \\
\text { weighted skew: (Qop) })_{g, s}\end{array}$ & 257 & 378 & 461 & 568 & 648 & 730 & 812 & 923 \\
\hline & & & Regional regression: $\left(Q_{p}\right)_{a, r}$ & 292 & 437 & 537 & 662 & 755 & 847 & 937 & 1,060 \\
\hline & & & $\begin{array}{c}\left(Q_{\rho \rho}\right)_{y, w} \text { : Weighted at-site }\left(Q_{p}\right)_{Q, s} \text { and regional } \\
\left(Q_{p}\right)_{Q, r}\end{array}$ & 259 & 383 & 468 & 578 & 662 & 746 & 832 & 948 \\
\hline
\end{tabular}




\begin{tabular}{|c|c|c|c|c|c|c|c|c|c|c|c|}
\hline \multirow{2}{*}{$\begin{array}{c}\text { USGS } \\
\text { streamgage } \\
\text { number }\end{array}$} & \multirow[b]{2}{*}{ Streamgage name } & \multirow{2}{*}{$\begin{array}{l}\text { Used in regression } \\
\text { analyses (non- } \\
\text { redundant) }\end{array}$} & \multirow[b]{2}{*}{ Frequency analysis method } & \multicolumn{8}{|c|}{ Discharge for given annual exceedance probability, $\mathrm{ft}^{3} / \mathrm{s}$} \\
\hline & & & & 0.50 & 0.20 & 0.10 & 0.04 & 0.02 & 0.01 & 0.005 & 0.002 \\
\hline \multirow{5}{*}{05527950} & \multirow{5}{*}{ MILL CREEK AT OLD MILL CREEK, IL } & \multirow{5}{*}{ No } & $\begin{array}{l}\text { At-site, unadjusted for urbanization, EMA, } \\
\text { at-site skew }\end{array}$ & 629 & 952 & 1,160 & 1,400 & 1,570 & 1,720 & 1,880 & 2,060 \\
\hline & & & $\begin{array}{l}\text { At-site, adjusted for urbanization, EMA, at- } \\
\text { site skew }\end{array}$ & 781 & 1,120 & 1,320 & 1,560 & 1,710 & 1,850 & 1,980 & 2,140 \\
\hline & & & $\begin{array}{l}\text { At-site, adjusted for urbanization, EMA, } \\
\left.\text { weighted skew: ( } 0_{0}\right)_{g, s}\end{array}$ & 762 & 1,110 & 1,340 & 1,640 & 1,850 & 2,070 & 2,290 & 2,580 \\
\hline & & & Regional regression: $(Q p)_{g, r}$ & 693 & 972 & 1,160 & 1,410 & 1,590 & 1,780 & 1,960 & 2,210 \\
\hline & & & 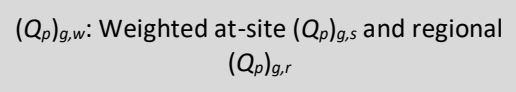 & 760 & 1,100 & 1,330 & 1,620 & 1,840 & 2,050 & 2,260 & 2,540 \\
\hline \multirow{5}{*}{05528000} & \multirow{5}{*}{ DES PLAINES RIVER NEAR GURNEE, IL } & \multirow{5}{*}{ No } & $\begin{array}{l}\text { At-site, unadjusted for urbanization, EMA, } \\
\text { at-site skew }\end{array}$ & 1,340 & 2,050 & 2,510 & 3,080 & 3,480 & 3,870 & 4,250 & 4,730 \\
\hline & & & $\begin{array}{l}\text { At-site, adjusted for urbanization, EMA, at- } \\
\text { site skew }\end{array}$ & 1,650 & 2,450 & 2,960 & 3,580 & 4,020 & 4,430 & 4,840 & 5,350 \\
\hline & & & $\begin{array}{l}\text { At-site, adjusted for urbanization, EMA, } \\
\text { weighted skew: ( (opog,s }\end{array}$ & 1,620 & 2,430 & 2,970 & 3,670 & 4,200 & 4,720 & 5,260 & 5,970 \\
\hline & & & Regional regression: $\left(Q_{p}\right)_{g, r}$ & 1,950 & 2,680 & 3,180 & 3,790 & 4,250 & 4,700 & 5,150 & 5,750 \\
\hline & & & $\begin{array}{c}\left(Q_{\rho}\right)_{g, w}: \text { Weighted at-site } \\
\left(Q_{\rho}\right)_{g, s} \text { and regional } \\
\left(S_{\rho} g_{g, r}\right.\end{array}$ & 1,630 & 2,430 & 2,980 & 3,680 & 4,200 & 4,720 & 5,250 & 5,950 \\
\hline \multirow{5}{*}{05528150} & \multirow{5}{*}{ INDIAN CREEK AT DIAMOND LAKE, IL } & \multirow{5}{*}{ Yes } & $\begin{array}{l}\text { At-site, unadjusted for urbanization, EMA, } \\
\text { at-site skew }\end{array}$ & 284 & 522 & 696 & 924 & 1,100 & 1,270 & 1,440 & 1,670 \\
\hline & & & $\begin{array}{l}\text { At-site, adjusted for urbanization, EMA, at- } \\
\text { site skew }\end{array}$ & 655 & 1,010 & 1,260 & 1,620 & 1,900 & 2,200 & 2,520 & 2,970 \\
\hline & & & $\begin{array}{l}\text { At-site, adjusted for urbanization, EMA, } \\
\left.\text { weighted skew: ( } Q_{p}\right)_{g, s}\end{array}$ & 650 & 1,000 & 1,270 & 1,640 & 1,950 & 2,280 & 2,630 & 3,140 \\
\hline & & & Regional regression: $(Q p)_{a, r}$ & 340 & 529 & 669 & 860 & 1,010 & 1,170 & 1,330 & 1,550 \\
\hline & & & 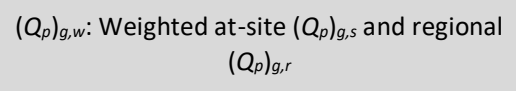 & 618 & 942 & 1,170 & 1,490 & 1,730 & 1,980 & 2,240 & 2,590 \\
\hline \multirow{5}{*}{05528170} & \multirow{5}{*}{ DIAMOND LAKE DRAIN AT MUNDELEIN, IL } & \multirow{5}{*}{ Yes } & $\begin{array}{l}\text { At-site, unadjusted for urbanization, EMA, } \\
\text { at-site skew }\end{array}$ & 53 & 75 & 91 & 111 & 126 & 142 & 158 & 180 \\
\hline & & & $\begin{array}{l}\text { At-site, adjusted for urbanization, EMA, at- } \\
\text { site skew }\end{array}$ & 73 & 99 & 117 & 141 & 159 & 177 & 196 & 222 \\
\hline & & & $\begin{array}{l}\text { At-site, adjusted for urbanization, EMA, } \\
\text { weighted skew: }\left(Q_{0}\right)_{g, s}\end{array}$ & 72 & 99 & 118 & 142 & 162 & 181 & 202 & 231 \\
\hline & & & Regional regression: $\left(Q_{p}\right)_{g, r}$ & 95 & 145 & 182 & 233 & 273 & 315 & 357 & 415 \\
\hline & & & $\begin{array}{l}\left(Q_{\rho}\right)_{g, w}: \text { Weighted at-site }\left(Q_{\rho}\right)_{g, s} \text { and regional } \\
\left(\rho_{\rho} g_{g, r}\right.\end{array}$ & 73 & 101 & 121 & 149 & 171 & 194 & 219 & 255 \\
\hline
\end{tabular}




\begin{tabular}{|c|c|c|c|c|c|c|c|c|c|c|c|}
\hline \multirow{2}{*}{$\begin{array}{c}\text { USGS } \\
\text { streamgage } \\
\text { number }\end{array}$} & \multirow[b]{2}{*}{ Streamgage name } & \multirow{2}{*}{$\begin{array}{l}\text { Used in regression } \\
\text { analyses (non- } \\
\text { redundant) }\end{array}$} & \multirow[b]{2}{*}{ Frequency analysis method } & \multicolumn{8}{|c|}{ Discharge for given annual exceedance probability, $\mathrm{ft}^{3} / \mathrm{s}$} \\
\hline & & & & 0.50 & 0.20 & 0.10 & 0.04 & 0.02 & 0.01 & 0.005 & 0.002 \\
\hline \multirow{5}{*}{05528200} & \multirow{5}{*}{$\begin{array}{l}\text { HAWTHORN DRAINAGE DITCH NEAR } \\
\text { MUNDELEN, IL }\end{array}$} & \multirow{5}{*}{ Yes } & $\begin{array}{l}\text { At-site, unadjusted for urbanization, EMA, } \\
\text { at-site skew }\end{array}$ & 212 & 331 & 424 & 557 & 669 & 791 & 925 & 1,120 \\
\hline & & & $\begin{array}{l}\text { At-site, adjusted for urbanization, EMA, at- } \\
\text { site skew }\end{array}$ & 329 & 483 & 597 & 756 & 884 & 1,020 & 1,170 & 1,380 \\
\hline & & & 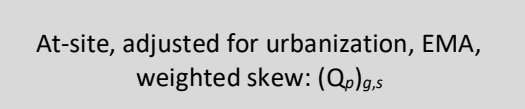 & 326 & 481 & 600 & 768 & 909 & 1,060 & 1,230 & 1,480 \\
\hline & & & Regional regression: $(Q p)_{a, r}$ & 267 & 411 & 521 & 676 & 800 & 932 & 1,070 & 1,260 \\
\hline & & & $\begin{array}{c}\left(Q_{p}\right)_{Q, w} \text { : Weighted at-site }\left(Q_{\rho}\right)_{Q s, \text { and regional }} \\
\left(Q_{\rho}\right)_{Q, r}\end{array}$ & 322 & 474 & 589 & 752 & 887 & 1,030 & 1,190 & 1,410 \\
\hline \multirow{5}{*}{05528230} & \multirow{5}{*}{ INDIAN CREEK AT PRAIRIE VIEW, IL } & \multirow{5}{*}{ No } & $\begin{array}{l}\text { At-site, unadjusted for urbanization, EMA, } \\
\text { at-site skew }\end{array}$ & 514 & 841 & 1,080 & 1,390 & 1,630 & 1,870 & 2,120 & 2,460 \\
\hline & & & $\begin{array}{l}\text { At-site, adjusted for urbanization, EMA, at- } \\
\text { site skew }\end{array}$ & 916 & 1,310 & 1,600 & 1,980 & 2,280 & 2,610 & 2,940 & 3,420 \\
\hline & & & $\begin{array}{l}\text { At-site, adjusted for urbanization, EMA, } \\
\text { weighted skew: (Q }\left(Q_{p}\right)_{g, s}\end{array}$ & 913 & 1,310 & 1,600 & 2,000 & 2,320 & 2,660 & 3,020 & 3,540 \\
\hline & & & Regional regression: $\left(Q_{p}\right)_{g, r}$ & 849 & 1,270 & 1,580 & 2,000 & 2,330 & 2,670 & 3,030 & 3,530 \\
\hline & & & $\begin{array}{c}\left(Q_{\rho}\right)_{g, w:} \text { : Weighted at-site } \\
\qquad\left(Q_{\rho \rho}\right)_{g, s} \text { and regional } \\
\left(Q_{\rho}\right)_{g, r}\end{array}$ & 909 & 1,310 & 1,600 & 2,000 & 2,320 & 2,660 & 3,020 & 3,540 \\
\hline \multirow{5}{*}{05528360} & \multirow{5}{*}{ APTAKIIIC CREEK AT APTAKISIC, IL } & \multirow{5}{*}{ Yes } & $\begin{array}{l}\text { At-site, unadjusted for urbanization, EMA, } \\
\text { at-site skew }\end{array}$ & 107 & 191 & 258 & 357 & 440 & 531 & 631 & 778 \\
\hline & & & $\begin{array}{l}\text { At-site, adjusted for urbanization, EMA, at- } \\
\text { site skew }\end{array}$ & 294 & 426 & 533 & 691 & 827 & 981 & 1,160 & 1,420 \\
\hline & & & $\begin{array}{l}\text { At-site, adjusted for urbanization, EMA, } \\
\left.\text { weighted skew: ( } Q_{p}\right)_{g, s}\end{array}$ & 298 & 430 & 530 & 674 & 793 & 923 & 1,070 & 1,280 \\
\hline & & & Regional regression: $(Q p)_{g, r}$ & 118 & 174 & 216 & 272 & 316 & 361 & 407 & 470 \\
\hline & & & $\begin{array}{c}\left(Q_{p}\right)_{g, w} \text { : Weighted at-site } \\
\left(Q_{\rho}\right)_{Q, s} \text { and regional } \\
\left(Q_{\rho}\right)_{g, r}\end{array}$ & 283 & 399 & 482 & 593 & 680 & 770 & 862 & 988 \\
\hline \multirow{5}{*}{05528400} & \multirow{5}{*}{ DES PLAIINES RIVER AT WHEELING, LL } & \multirow{5}{*}{ No } & $\begin{array}{l}\text { At-site, unadjusted for urbanization, EMA, } \\
\text { at-site skew }\end{array}$ & 1,890 & 2,600 & 2,940 & 3,260 & 3,440 & 3,570 & 3,680 & 3,780 \\
\hline & & & $\begin{array}{l}\text { At-site, adjusted for urbanization, EMA, at- } \\
\text { site skew }\end{array}$ & 2,570 & 3,530 & 4,000 & 4,450 & 4,710 & 4,910 & 5,080 & 5,250 \\
\hline & & & $\begin{array}{l}\text { At-site, adjusted for urbanization, EMA, } \\
\left.\text { weighted skew: ( } Q_{p}\right)_{g, s}\end{array}$ & 2,420 & 3,530 & 4,250 & 5,150 & 5,800 & 6,440 & 7,080 & 7,910 \\
\hline & & & Regional regression: $\left(Q_{p}\right)_{g, r}$ & 2,730 & 3,720 & 4,380 & 5,230 & 5,860 & 6,500 & 7,140 & 8,000 \\
\hline & & & $\begin{array}{l}\left(Q_{\rho}\right)_{g, w} \text { : Weighted at-site }\left(Q_{\rho}\right)_{g, \text { s a d r regional }} \\
\left(Q_{\rho} g_{g, r}\right.\end{array}$ & 2,450 & 3,540 & 4,260 & 5,160 & 5,810 & 6,450 & 7,090 & 7,930 \\
\hline
\end{tabular}




\begin{tabular}{|c|c|c|c|c|c|c|c|c|c|c|c|}
\hline \multirow{2}{*}{$\begin{array}{c}\text { USGS } \\
\text { streamgage } \\
\text { number }\end{array}$} & \multirow[b]{2}{*}{ Streamgage name } & \multirow{2}{*}{$\begin{array}{l}\text { Used in regression } \\
\text { analyses (non- } \\
\text { redundant) }\end{array}$} & \multirow[b]{2}{*}{ Frequency analysis method } & \multicolumn{8}{|c|}{ Discharge for given annual exceedance probability, $\mathrm{ft}^{3} / \mathrm{s}$} \\
\hline & & & & 0.50 & 0.20 & 0.10 & 0.04 & 0.02 & 0.01 & 0.005 & 0.002 \\
\hline \multirow{5}{*}{05528440} & \multirow{5}{*}{ BUFFALO CREEK NEAR LAKE ZURICH, IL } & \multirow{5}{*}{ Yes } & $\begin{array}{l}\text { At-site, unadjusted for urbanization, EMA, } \\
\text { at-site skew }\end{array}$ & 79 & 130 & 168 & 224 & 269 & 317 & 370 & 445 \\
\hline & & & $\begin{array}{l}\text { At-site, adjusted for urbanization, EMA, at- } \\
\text { site skew }\end{array}$ & 147 & 205 & 250 & 314 & 367 & 425 & 489 & 584 \\
\hline & & & $\begin{array}{l}\text { At-site, adjusted for urbanization, EMA, } \\
\left.\text { weighted skew: }\left(Q_{0}\right)_{g}\right)_{s, s}\end{array}$ & 148 & 206 & 250 & 310 & 360 & 414 & 473 & 559 \\
\hline & & & Regional regression: $(Q p)_{g, r}$ & 114 & 180 & 233 & 307 & 368 & 432 & 500 & 595 \\
\hline & & & 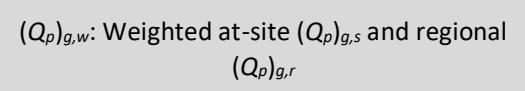 & 146 & 204 & 248 & 310 & 361 & 417 & 478 & 566 \\
\hline \multirow{5}{*}{05528470} & \multirow{5}{*}{ BUFFALO CREEK AT LONG GROVE, IL } & \multirow{5}{*}{ No } & $\begin{array}{l}\text { At-site, unadjusted for urbanization, EMA, } \\
\text { at-site skew }\end{array}$ & 245 & 380 & 443 & 497 & 525 & 545 & 559 & 571 \\
\hline & & & $\begin{array}{l}\text { At-site, adjusted for urbanization, EMA, at- } \\
\text { site skew }\end{array}$ & 499 & 665 & 757 & 858 & 923 & 981 & 1,030 & 1,100 \\
\hline & & & $\begin{array}{l}\text { At-site, adjusted for urbanization, EMA, } \\
\left.\text { weighted skew: ( } Q_{\rho}\right)_{g, s}\end{array}$ & 483 & 656 & 771 & 916 & 1,020 & 1,130 & 1,240 & 1,390 \\
\hline & & & Regional regression: $\left(Q_{p}\right)_{g, r}$ & 269 & 404 & 504 & 639 & 745 & 853 & 965 & 1,120 \\
\hline & & & $\begin{array}{l}\left(Q_{p}\right)_{y, w}: \text { Weighted at-site } \\
\left(Q_{\rho} Q_{g, r}\right.\end{array}$ & 470 & 639 & 749 & 888 & 993 & 1,100 & 1,200 & 1,350 \\
\hline \multirow{5}{*}{05528500} & \multirow{5}{*}{ BUFFALO CREEK NEAR WHEELING, IL } & \multirow[t]{5}{*}{ Yes } & $\begin{array}{l}\text { At-site, unadjusted for urbanization, EMA, } \\
\text { at-site skew }\end{array}$ & 407 & 585 & 698 & 833 & 929 & 1,020 & 1,110 & 1,220 \\
\hline & & & $\begin{array}{l}\text { At-site, adjusted for urbanization, EMA, at- } \\
\text { site skew }\end{array}$ & 492 & 671 & 772 & 884 & 957 & 1,020 & 1,080 & 1,150 \\
\hline & & & $\begin{array}{l}\text { At-site, adjusted for urbanization, EMA, } \\
\left.\text { weighted skew: (Q } Q_{p}\right)_{g, s}\end{array}$ & 480 & 669 & 790 & 938 & 1,040 & 1,150 & 1,250 & 1,380 \\
\hline & & & Regional regression: $(Q p)_{a, r}$ & 543 & 806 & 1,000 & 1,270 & 1,480 & 1,700 & 1,930 & 2,240 \\
\hline & & & $\begin{array}{l}\left(Q_{\rho}\right)_{g, w}: \text { Weighted at-site }\left(Q_{\rho}\right)_{g, s} \text { and regional } \\
\left(Q_{\rho}\right)_{g, r}\end{array}$ & 481 & 671 & 793 & 945 & 1,060 & 1,170 & 1,280 & 1,430 \\
\hline \multirow{5}{*}{05529000} & \multirow{5}{*}{ DES PLAINES RIVER NEAR DES PLAINES, IL } & \multirow{5}{*}{ No } & $\begin{array}{l}\text { At-site, unadjusted for urbanization, EMA, } \\
\text { at-site skew }\end{array}$ & 2,320 & 3,130 & 3,650 & 4,280 & 4,740 & 5,180 & 5,620 & 6,190 \\
\hline & & & $\begin{array}{l}\text { At-site, adjusted for urbanization, EMA, at- } \\
\text { site skew }\end{array}$ & 3,100 & 4,140 & 4,740 & 5,400 & 5,850 & 6,260 & 6,630 & 7,090 \\
\hline & & & 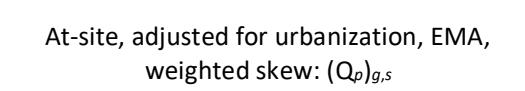 & 3,060 & 4,130 & 4,790 & 5,580 & 6,140 & 6,670 & 7,190 & 7,860 \\
\hline & & & Regional regression: $\left(Q_{p}\right)_{q, r}$ & 2,990 & 4,030 & 4,740 & 5,630 & 6,300 & 6,980 & 7,660 & 8,580 \\
\hline & & & $\begin{array}{l}\left(Q_{\rho}\right)_{a, w} \text { : Weighted at-site } \\
\left.\qquad\left(Q_{\rho}\right)_{Q, r}\right)_{a, s} \text { and regional }\end{array}$ & 3,060 & 4,130 & 4,790 & 5,580 & 6,140 & 6,680 & 7,210 & 7,890 \\
\hline
\end{tabular}




\begin{tabular}{|c|c|c|c|c|c|c|c|c|c|c|c|}
\hline \multirow{2}{*}{$\begin{array}{c}\text { USGS } \\
\text { streamgage } \\
\text { number }\end{array}$} & \multirow[b]{2}{*}{ Streamgage name } & \multirow{2}{*}{$\begin{array}{l}\text { Used in regression } \\
\text { analyses (non- } \\
\text { redundant) }\end{array}$} & \multirow[b]{2}{*}{ Frequency analysis method } & \multicolumn{8}{|c|}{ Discharge for given annual exceedance probability, $\mathrm{ft}^{3} / \mathrm{s}$} \\
\hline & & & & 0.50 & 0.20 & 0.10 & 0.04 & 0.02 & 0.01 & 0.005 & 0.002 \\
\hline \multirow{5}{*}{05529300} & \multirow{5}{*}{ MC DONALD CREEK NEAR WHEELING, IL } & \multirow{5}{*}{ No } & $\begin{array}{l}\text { At-site, unadjusted for urbanization, EMA, } \\
\text { at-site skew }\end{array}$ & 181 & 323 & 427 & 566 & 674 & 782 & 892 & 1,040 \\
\hline & & & $\begin{array}{l}\text { At-site, adjusted for urbanization, EMA, at- } \\
\text { site skew }\end{array}$ & 211 & 371 & 501 & 693 & 857 & 1,040 & 1,240 & 1,540 \\
\hline & & & $\begin{array}{l}\text { At-site, adjusted for urbanization, EMA, } \\
\text { weighted skew: }\left(Q_{p}\right)_{g, s}\end{array}$ & 204 & 365 & 506 & 729 & 932 & 1,170 & 1,450 & 1,890 \\
\hline & & & Regional regression: $(Q p)_{g, r}$ & 242 & 365 & 459 & 590 & 695 & 805 & 922 & 1,080 \\
\hline & & & $\begin{array}{c}\left(Q_{\rho \rho}\right)_{y, w} \text { : Weighted at-site }\left(Q_{\rho}\right)_{Q, s} \text { and regional } \\
\left(Q_{\rho}\right)_{Q, r}\end{array}$ & 208 & 365 & 498 & 697 & 866 & 1,050 & 1,250 & 1,530 \\
\hline \multirow{5}{*}{05529500} & \multirow{5}{*}{ MC DONALD CREEK NEAR MOUNT PROSPECT, IL } & \multirow{5}{*}{ Yes } & $\begin{array}{l}\text { At-site, unadjusted for urbanization, EMA, } \\
\text { at-site skew }\end{array}$ & 198 & 342 & 450 & 600 & 720 & 846 & 979 & 1,160 \\
\hline & & & $\begin{array}{l}\text { At-site, adjusted for urbanization, EMA, at- } \\
\text { site skew }\end{array}$ & 178 & 288 & 370 & 485 & 578 & 677 & 782 & 934 \\
\hline & & & $\begin{array}{l}\text { At-site, adjusted for urbanization, EMA, } \\
\text { weighted skew: }\left(Q_{p}\right)_{g, s}\end{array}$ & 176 & 286 & 373 & 499 & 604 & 720 & 848 & 1,040 \\
\hline & & & Regional regression: $\left(Q_{p}\right)_{g, r}$ & 341 & 506 & 630 & 801 & 938 & 1,080 & 1,230 & 1,440 \\
\hline & & & $\begin{array}{c}\left(Q_{\rho \rho}\right)_{y, w} \text { Weighted at-site }\left(Q_{p}\right)_{Q, s} \text { and regional } \\
\left(Q_{\rho}\right)_{Q, r}\end{array}$ & 180 & 293 & 384 & 516 & 629 & 755 & 892 & 1,090 \\
\hline \multirow{5}{*}{05529900} & \multirow{5}{*}{ WELLER CREEK AT MOUNT PROSPECT, IL } & \multirow{5}{*}{ No } & $\begin{array}{l}\text { At-site, unadjusted for urbanization, EMA, } \\
\text { at-site skew }\end{array}$ & 492 & 814 & 1,040 & 1,350 & 1,580 & 1,810 & 2,050 & 2,370 \\
\hline & & & $\begin{array}{l}\text { At-site, adjusted for urbanization, EMA, at- } \\
\text { site skew }\end{array}$ & 504 & 824 & 1,060 & 1,360 & 1,600 & 1,840 & 2,090 & 2,430 \\
\hline & & & $\begin{array}{l}\text { At-site, adjusted for urbanization, EMA, } \\
\left.\text { weighted skew: ( } Q_{p}\right)_{g, s}\end{array}$ & 482 & 808 & 1,080 & 1,480 & 1,840 & 2,240 & 2,700 & 3,410 \\
\hline & & & Regional regression: $(Q p)_{g, r}$ & 443 & 665 & 833 & 1,070 & 1,260 & 1,450 & 1,660 & 1,950 \\
\hline & & & $\begin{array}{c}\left(Q_{\rho}\right)_{Q, w}, \text { Weighted at-site } \\
\left.\left(Q_{p}\right)_{Q, r}\right)_{Q, s} \text { and regional }\end{array}$ & 478 & 786 & 1,030 & 1,380 & 1,670 & 1,980 & 2,310 & 2,770 \\
\hline \multirow{5}{*}{05530000} & \multirow{5}{*}{ WELLER CREEK AT DES PLAINES, IL } & \multirow{5}{*}{ Yes } & $\begin{array}{l}\text { At-site, unadjusted for urbanization, EMA, } \\
\text { at-site skew }\end{array}$ & 815 & 1,060 & 1,210 & 1,410 & 1,550 & 1,680 & 1,820 & 2,010 \\
\hline & & & $\begin{array}{l}\text { At-site, adjusted for urbanization, EMA, at- } \\
\text { site skew }\end{array}$ & 771 & 1,070 & 1,260 & 1,470 & 1,610 & 1,750 & 1,880 & 2,040 \\
\hline & & & $\begin{array}{l}\text { At-site, adjusted for urbanization, EMA, } \\
\text { weighted skew: }\left(Q_{p}\right)_{g, s}\end{array}$ & 752 & 1,070 & 1,280 & 1,550 & 1,750 & 1,960 & 2,160 & 2,440 \\
\hline & & & Regional regression: $\left(Q_{p}\right)_{g, r}$ & 560 & 829 & 1,030 & 1,310 & 1,540 & 1,770 & 2,020 & 2,360 \\
\hline & & & $\begin{array}{l}\left(Q_{\rho}\right)_{g, w}: \text { Weighted at-site }\left(Q_{p}\right)_{g, s} \text { and regional } \\
\left(Q_{p}\right)_{Q, r}\end{array}$ & 748 & 1,060 & 1,270 & 1,540 & 1,740 & 1,950 & 2,150 & 2,430 \\
\hline
\end{tabular}




\begin{tabular}{|c|c|c|c|c|c|c|c|c|c|c|c|}
\hline \multirow{2}{*}{$\begin{array}{c}\text { USGS } \\
\text { streamgage } \\
\text { number }\end{array}$} & \multirow[b]{2}{*}{ Streamgage name } & \multirow{2}{*}{$\begin{array}{l}\text { Used in regression } \\
\text { analyses (non- } \\
\text { redundant) }\end{array}$} & \multirow[b]{2}{*}{ Frequency analysis method } & \multicolumn{8}{|c|}{ Discharge for given annual exceedance probability, $\mathrm{ft}^{3} / \mathrm{s}$} \\
\hline & & & & 0.50 & 0.20 & 0.10 & 0.04 & 0.02 & 0.01 & 0.005 & 0.002 \\
\hline \multirow{5}{*}{05530400} & \multirow{5}{*}{ HIGGINS CREEK NEAR MOUNT PROSPECT, IL } & \multirow{5}{*}{ Yes } & $\begin{array}{l}\text { At-site, unadjusted for urbanization, EMA, } \\
\text { at-site skew }\end{array}$ & 134 & 250 & 340 & 464 & 563 & 666 & 773 & 921 \\
\hline & & & $\begin{array}{l}\text { At-site, adjusted for urbanization, EMA, at- } \\
\text { site skew }\end{array}$ & 178 & 309 & 405 & 535 & 636 & 739 & 845 & 990 \\
\hline & & & $\begin{array}{l}\text { At-site, adjusted for urbanization, EMA, } \\
\left.\text { weighted skew: ( } Q_{p}\right)_{g, s}\end{array}$ & 170 & 305 & 418 & 590 & 741 & 910 & 1,100 & 1,390 \\
\hline & & & Regional regression: $(Q p)_{a, r}$ & 102 & 154 & 193 & 246 & 288 & 332 & 377 & 439 \\
\hline & & & $\begin{array}{c}\left(Q_{\rho \rho}\right)_{y, w} \text { Weighted at-site }\left(Q_{p}\right)_{Q, s} \text { and regional } \\
\left(Q_{\rho}\right)_{Q, r}\end{array}$ & 160 & 276 & 364 & 484 & 579 & 675 & 773 & 904 \\
\hline \multirow{5}{*}{05530480} & \multirow{5}{*}{ WILLOW CREEK AT ORCHARD PLACE, IL } & \multirow{5}{*}{ Yes } & $\begin{array}{l}\text { At-site, unadjusted for urbanization, EMA, } \\
\text { at-site skew }\end{array}$ & 547 & 1,000 & 1,360 & 1,880 & 2,300 & 2,760 & 3,240 & 3,940 \\
\hline & & & $\begin{array}{l}\text { At-site, adjusted for urbanization, EMA, at- } \\
\text { site skew }\end{array}$ & 823 & 1,340 & 1,760 & 2,370 & 2,890 & 3,470 & 4,120 & 5,080 \\
\hline & & & $\begin{array}{l}\text { At-site, adjusted for urbanization, EMA, } \\
\text { weighted skew: (Q }\left(Q_{p}\right)_{g, s}\end{array}$ & 808 & 1,330 & 1,770 & 2,440 & 3,040 & 3,730 & 4,520 & 5,740 \\
\hline & & & Regional regression: $\left(Q_{p}\right)_{g, r}$ & 575 & 816 & 993 & 1,240 & 1,430 & 1,630 & 1,840 & 2,130 \\
\hline & & & $\begin{array}{c}\left(Q_{\rho \rho}\right)_{y, w} \text { Weighted at-site }\left(Q_{p}\right)_{Q, s} \text { and regional } \\
\left(Q_{p}\right)_{Q, r}\end{array}$ & 788 & 1,270 & 1,640 & 2,180 & 2,610 & 3,060 & 3,530 & 4,170 \\
\hline \multirow{5}{*}{05530600} & \multirow{5}{*}{ DES PLAINES RIVER AT RIVER GROVE, IL } & \multirow{5}{*}{ No } & $\begin{array}{l}\text { At-site, unadjusted for urbanization, EMA, } \\
\text { at-site skew }\end{array}$ & 2,750 & 3,380 & 3,670 & 3,930 & 4,080 & 4,190 & 4,280 & 4,380 \\
\hline & & & $\begin{array}{l}\text { At-site, adjusted for urbanization, EMA, at- } \\
\text { site skew }\end{array}$ & 3,620 & 4,430 & 4,800 & 5,150 & 5,340 & 5,500 & 5,630 & 5,760 \\
\hline & & & $\begin{array}{l}\text { At-site, adjusted for urbanization, EMA, } \\
\text { weighted skew: ( }\left(Q_{0}\right)_{g, s}\end{array}$ & 3,530 & 4,340 & 4,830 & 5,420 & 5,850 & 6,260 & 6,670 & 7,200 \\
\hline & & & Regional regression: $(Q p)_{a, r}$ & 3,840 & 5,060 & 5,900 & 6,990 & 7,810 & 8,640 & 9,490 & $10,60 \mathrm{c}$ \\
\hline & & & $\begin{array}{c}\left(Q_{\rho \rho}\right)_{y, w} \text { : Weighted at-site }\left(Q_{p}\right)_{Q, s} \text { and regional } \\
\left(Q_{\rho}\right)_{Q, r}\end{array}$ & 3,540 & 4,350 & 4,860 & 5,480 & 5,930 & 6,370 & 6,810 & 7,390 \\
\hline \multirow{5}{*}{05530700} & \multirow{5}{*}{ SILVER CREEK AT MELROSE PARK, IL } & \multirow{5}{*}{ Yes } & $\begin{array}{l}\text { At-site, unadjusted for urbanization, EMA, } \\
\text { at-site skew }\end{array}$ & 437 & 545 & 610 & 687 & 741 & 792 & 841 & 905 \\
\hline & & & $\begin{array}{l}\text { At-site, adjusted for urbanization, EMA, at- } \\
\text { site skew }\end{array}$ & 479 & 597 & 670 & 756 & 818 & 877 & 935 & 1,010 \\
\hline & & & $\begin{array}{l}\text { At-site, adjusted for urbanization, EMA, } \\
\text { weighted skew: }\left(Q_{p}\right)_{g, s}\end{array}$ & 471 & 592 & 673 & 777 & 856 & 936 & 1,020 & 1,130 \\
\hline & & & Regional regression: $\left(Q_{p}\right)_{g, r}$ & 479 & 703 & 872 & 1,110 & 1,290 & 1,490 & 1,700 & 1,980 \\
\hline & & & $\begin{array}{c}\left(Q_{\rho}\right)_{Q, w}, \text { Weighted at-site } \\
\left.\left(Q_{p}\right)_{Q, r} Q_{p}\right)_{g, s} \text { and regional }\end{array}$ & 471 & 595 & 678 & 788 & 873 & 961 & 1,050 & 1,180 \\
\hline
\end{tabular}




\begin{tabular}{|c|c|c|c|c|c|c|c|c|c|c|c|}
\hline \multirow{2}{*}{$\begin{array}{c}\text { USGS } \\
\text { streamgage } \\
\text { number }\end{array}$} & \multirow[b]{2}{*}{ Streamgage name } & \multirow{2}{*}{$\begin{array}{l}\text { Used in regression } \\
\text { analyses (non- } \\
\text { redundant) }\end{array}$} & \multirow[b]{2}{*}{ Frequency analysis method } & \multicolumn{8}{|c|}{ Discharge for given annual exceedance probability, $\mathrm{ft}^{3} / \mathrm{s}$} \\
\hline & & & & 0.50 & 0.20 & 0.10 & 0.04 & 0.02 & 0.01 & 0.005 & 0.002 \\
\hline \multirow{5}{*}{05530800} & \multirow{5}{*}{ DES PLAINES RIVER AT FOREST PARK, IL } & \multirow{5}{*}{ No } & $\begin{array}{l}\text { At-site, unadjusted for urbanization, EMA, } \\
\text { at-site skew }\end{array}$ & 2,980 & 3,680 & 3,970 & 4,230 & 4,360 & 4,450 & 4,520 & 4,590 \\
\hline & & & $\begin{array}{l}\text { At-site, adjusted for urbanization, EMA, at- } \\
\text { site skew }\end{array}$ & 3,830 & 4,820 & 5,330 & 5,870 & 6,200 & 6,490 & 6,740 & 7,030 \\
\hline & & & $\begin{array}{l}\text { At-site, adjusted for urbanization, EMA, } \\
\text { weighted skew: }\left(Q_{0}\right)_{g, s}\end{array}$ & 3,730 & 4,800 & 5,450 & 6,220 & 6,760 & 7,280 & 7,770 & 8,410 \\
\hline & & & Regional regression: $(Q p)_{g, r}$ & 3,970 & 5,230 & 6,080 & 7,190 & 8,030 & 8,890 & 9,750 & 10,900 \\
\hline & & & $\begin{array}{c}\left(Q_{\rho \rho}\right)_{y, w} \text { : Weighted at-site }\left(Q_{\rho}\right)_{Q, s} \text { and regional } \\
\left(Q_{\rho}\right)_{Q, r}\end{array}$ & 3,740 & 4,810 & 5,470 & 6,250 & 6,810 & 7,350 & 7,880 & 8,570 \\
\hline \multirow{5}{*}{05530940} & \multirow{5}{*}{ SALT CREEK AT PALATINE, IL } & \multirow{5}{*}{ Yes } & $\begin{array}{l}\text { At-site, unadjusted for urbanization, EMA, } \\
\text { at-site skew }\end{array}$ & 191 & 281 & 357 & 476 & 583 & 709 & 857 & 1,090 \\
\hline & & & $\begin{array}{l}\text { At-site, adjusted for urbanization, EMA, at- } \\
\text { site skew }\end{array}$ & 281 & 399 & 488 & 617 & 724 & 841 & 969 & 1,160 \\
\hline & & & $\begin{array}{l}\text { At-site, adjusted for urbanization, EMA, } \\
\text { weighted skew: }\left(Q_{p}\right)_{g, s}\end{array}$ & 283 & 400 & 487 & 609 & 709 & 816 & 932 & 1,100 \\
\hline & & & Regional regression: $\left(Q_{p}\right)_{g, r}$ & 195 & 292 & 364 & 463 & 542 & 623 & 708 & 825 \\
\hline & & & $\begin{array}{c}\left(Q_{\rho}\right)_{g, w} \text { : Weighted at-site }\left(Q_{p}\right)_{g, s} \text { and regional } \\
\left(Q_{\rho}\right)_{g, r}\end{array}$ & 278 & 392 & 476 & 591 & 684 & 783 & 887 & 1,040 \\
\hline \multirow{5}{*}{05530960} & \multirow{5}{*}{ SALT CREEK NEAR PALATINE, IL } & \multirow{5}{*}{ No } & $\begin{array}{l}\text { At-site, unadjusted for urbanization, EMA, } \\
\text { at-site skew }\end{array}$ & 295 & 464 & 590 & 765 & 906 & 1,060 & 1,220 & 1,440 \\
\hline & & & $\begin{array}{l}\text { At-site, adjusted for urbanization, EMA, at- } \\
\text { site skew }\end{array}$ & 376 & 546 & 679 & 871 & 1,030 & 1,210 & 1,410 & 1,710 \\
\hline & & & $\begin{array}{l}\text { At-site, adjusted for urbanization, EMA, } \\
\left.\text { weighted skew: ( } Q_{p}\right)_{g, s}\end{array}$ & 378 & 548 & 678 & 861 & 1,010 & 1,180 & 1,360 & 1,620 \\
\hline & & & Regional regression: $(Q p)_{a, r}$ & 409 & 592 & 728 & 911 & 1,060 & 1,200 & 1,360 & 1,570 \\
\hline & & & 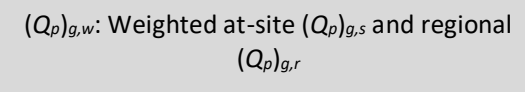 & 380 & 551 & 682 & 867 & 1,020 & 1,180 & 1,360 & 1,610 \\
\hline \multirow{5}{*}{05530990} & \multirow{5}{*}{ SALT CREEK AT ROLLING MEADOWS, IL } & \multirow{5}{*}{ No } & $\begin{array}{l}\text { At-site, unadjusted for urbanization, EMA, } \\
\text { at-site skew }\end{array}$ & 819 & 1,200 & 1,480 & 1,870 & 2,190 & 2,530 & 2,890 & 3,420 \\
\hline & & & $\begin{array}{l}\text { At-site, adjusted for urbanization, EMA, at- } \\
\text { site skew }\end{array}$ & 841 & 1,210 & 1,490 & 1,870 & 2,170 & 2,500 & 2,840 & 3,340 \\
\hline & & & $\begin{array}{l}\text { At-site, adjusted for urbanization, EMA, } \\
\text { weighted skew: }\left(Q_{p}\right)_{g, s}\end{array}$ & 836 & 1,210 & 1,490 & 1,880 & 2,200 & 2,550 & 2,930 & 3,470 \\
\hline & & & Regional regression: $\left(Q_{p}\right)_{g, r}$ & 671 & 962 & 1,180 & 1,470 & 1,710 & 1,950 & 2,210 & 2,560 \\
\hline & & & $\begin{array}{l}(Q p)_{g, w} \text { : Weighted at-site }\left(Q_{p}\right)_{g, s} \text { and regional } \\
\left(Q_{e}\right)_{g_{1}, r}\end{array}$ & 831 & 1,200 & 1,470 & 1,850 & 2,150 & 2,480 & 2,820 & 3,300 \\
\hline
\end{tabular}




\begin{tabular}{|c|c|c|c|c|c|c|c|c|c|c|c|}
\hline \multirow{2}{*}{$\begin{array}{c}\text { USGS } \\
\text { streamgage } \\
\text { number }\end{array}$} & \multirow[b]{2}{*}{ Streamgage name } & \multirow{2}{*}{$\begin{array}{l}\text { Used in regression } \\
\text { analyses (non- } \\
\text { redundant) }\end{array}$} & \multirow[b]{2}{*}{ Frequency analysis method } & \multicolumn{8}{|c|}{ Discharge for given annual exceedance probability, $\mathrm{ft}^{3} / \mathrm{s}$} \\
\hline & & & & 0.50 & 0.20 & 0.10 & 0.04 & 0.02 & 0.01 & 0.005 & 0.002 \\
\hline \multirow{5}{*}{05531000} & \multirow{5}{*}{ SALT CREEK NEAR ARLINGTON HEIGHTS, IL } & \multirow{5}{*}{ No } & $\begin{array}{l}\text { At-site, unadjusted for urbanization, EMA, } \\
\text { at-site skew }\end{array}$ & 452 & 700 & 866 & 1,080 & 1,230 & 1,380 & 1,530 & 1,720 \\
\hline & & & $\begin{array}{l}\text { At-site, adjusted for urbanization, EMA, at- } \\
\text { site skew }\end{array}$ & 645 & 896 & 1,060 & 1,260 & 1,420 & 1,560 & 1,710 & 1,910 \\
\hline & & & $\begin{array}{l}\text { At-site, adjusted for urbanization, EMA, } \\
\text { weighted skew: ( }\left(_{0}\right)_{g, s}\end{array}$ & 633 & 891 & 1,070 & 1,310 & 1,500 & 1,700 & 1,900 & 2,190 \\
\hline & & & Regional regression: $(Q p)_{g, r}$ & 691 & 985 & 1,200 & 1,500 & 1,730 & 1,980 & 2,230 & 2,580 \\
\hline & & & $\begin{array}{l}\left(Q_{\rho p}\right)_{g, w} \text { Weighted at-site }\left(Q_{p}\right)_{g, s} \text { and regional } \\
\left(Q_{0}\right)_{p, r}\end{array}$ & 635 & 895 & 1,080 & 1,330 & 1,520 & 1,730 & 1,940 & 2,250 \\
\hline \multirow{5}{*}{05531050} & \multirow{5}{*}{ SALT CREEK NEAR WOOD DALE, IL } & \multirow{5}{*}{ No } & $\begin{array}{l}\text { At-site, unadjusted for urbanization, EMA, } \\
\text { at-site skew }\end{array}$ & NA & NA & NA & NA & NA & NA & NA & NA \\
\hline & & & $\begin{array}{l}\text { At-site, adjusted for urbanization, EMA, at- } \\
\text { site skew }\end{array}$ & 747 & 1,010 & 1,210 & 1,490 & 1,730 & 1,980 & 2,260 & 2,680 \\
\hline & & & $\begin{array}{l}\text { At-site, adjusted for urbanization, EMA, } \\
\text { wighted skew: (Qoplg,s, }\end{array}$ & 755 & 1,020 & 1,210 & 1,470 & 1,680 & 1,910 & 2,150 & 2,490 \\
\hline & & & Regional regression: $\left(Q_{p}\right)_{g, r}$ & 1,010 & 1,430 & 1,730 & 2,140 & 2,460 & 2,800 & 3,150 & 3,630 \\
\hline & & & $\begin{array}{c}\left(Q_{\rho \rho}\right)_{y, w} \text { Weighted at-site }\left(Q_{p}\right)_{Q, s} \text { and regional } \\
\left(Q_{\rho}\right)_{Q, r}\end{array}$ & 762 & 1,030 & 1,240 & 1,520 & 1,750 & 2,000 & 2,260 & 2,650 \\
\hline \multirow{5}{*}{05531080} & \multirow{5}{*}{ SPRING BROOK AT BLOOMINGDALE, IL } & \multirow{5}{*}{ Yes } & $\begin{array}{l}\text { At-site, unadjusted for urbanization, EMA, } \\
\text { at-site skew }\end{array}$ & 135 & 218 & 299 & 440 & 581 & 762 & 993 & 1,400 \\
\hline & & & $\begin{array}{l}\text { At-site, adjusted for urbanization, EMA, at- } \\
\text { site skew }\end{array}$ & 197 & 287 & 378 & 545 & 717 & 942 & 1,240 & 1,770 \\
\hline & & & $\begin{array}{l}\text { At-site, adjusted for urbanization, EMA, } \\
\text { weighted skew: (Q }\left(Q_{p}\right)_{g, s}\end{array}$ & 207 & 307 & 388 & 510 & 617 & 738 & 877 & 1,090 \\
\hline & & & Regional regression: $(Q p)_{g, r}$ & 255 & 377 & 469 & 596 & 697 & 802 & 912 & 1,060 \\
\hline & & & $\begin{array}{c}\left(Q_{\rho \rho}\right)_{y, w} \text { Weighted at-site }\left(Q_{\rho}\right)_{g, s} \text { and regional } \\
\left(Q_{\rho}\right)_{Q, r}\end{array}$ & 209 & 312 & 397 & 523 & 632 & 753 & 886 & 1,080 \\
\hline \multirow{5}{*}{05531100} & \multirow{5}{*}{ MEACHAM CREEK AT MEDINAH, IL } & \multirow{5}{*}{ Yes } & $\begin{array}{l}\text { At-site, unadjusted for urbanization, EMA, } \\
\text { at-site skew }\end{array}$ & 57 & 92 & 125 & 181 & 235 & 302 & 386 & 530 \\
\hline & & & $\begin{array}{l}\text { At-site, adjusted for urbanization, EMA, at- } \\
\text { site skew }\end{array}$ & 86 & 129 & 166 & 226 & 281 & 347 & 426 & 555 \\
\hline & & & $\begin{array}{l}\text { At-site, adjusted for urbanization, EMA, } \\
\text { weighted skew: (Q }\left(Q_{p}\right)_{g, s}\end{array}$ & 89 & 131 & 165 & 216 & 259 & 309 & 364 & 449 \\
\hline & & & Regional regression: $\left(Q_{p}\right)_{g, r}$ & 201 & 304 & 382 & 491 & 578 & 671 & 768 & 902 \\
\hline & & & $\begin{array}{c}(Q p)_{g, w} \text { : Weighted at-site }\left(Q_{\rho}\right)_{g_{s, s}} \text { and regional } \\
\left(Q_{p}\right)_{g, r}\end{array}$ & 92 & 141 & 181 & 244 & 300 & 364 & 436 & 547 \\
\hline
\end{tabular}




\begin{tabular}{|c|c|c|c|c|c|c|c|c|c|c|c|}
\hline \multirow{2}{*}{$\begin{array}{c}\text { USGS } \\
\text { streamgage } \\
\text { number }\end{array}$} & \multirow[b]{2}{*}{ Streamgage name } & \multirow{2}{*}{$\begin{array}{l}\text { Used in regression } \\
\text { analyses (non- } \\
\text { redundant) }\end{array}$} & \multirow[b]{2}{*}{ Frequency analysis method } & \multicolumn{8}{|c|}{ Discharge for given annual exceedance probability, $\mathrm{ft}^{3} / \mathrm{s}$} \\
\hline & & & & 0.50 & 0.20 & 0.10 & 0.04 & 0.02 & 0.01 & 0.005 & 0.002 \\
\hline \multirow{5}{*}{05531130} & \multirow{5}{*}{ SPRING BROOK AT WALNUT AVE AT ITASCA, IL } & \multirow{5}{*}{ No } & $\begin{array}{l}\text { At-site, unadjusted for urbanization, EMA, } \\
\text { at-site skew }\end{array}$ & 231 & 341 & 437 & 592 & 735 & 905 & 1,110 & 1,440 \\
\hline & & & $\begin{array}{l}\text { At-site, adjusted for urbanization, EMA, at- } \\
\text { site skew }\end{array}$ & 348 & 467 & 577 & 758 & 928 & 1,140 & 1,380 & 1,800 \\
\hline & & & $\begin{array}{l}\text { At-site, adjusted for urbanization, EMA, } \\
\text { weighted skew: }\left(Q_{0}\right)_{g, s}\end{array}$ & 361 & 490 & 585 & 718 & 825 & 941 & 1,070 & 1,250 \\
\hline & & & Regional regression: $(Q p)_{g, r}$ & 484 & 707 & 873 & 1,100 & 1,280 & 1,470 & 1,670 & 1,940 \\
\hline & & & $\begin{array}{c}\left(Q_{p}\right)_{g, w} \text { : Weighted at-site }\left(Q_{\rho}\right)_{g, s} \text { and regional } \\
\left(Q_{\rho} g_{g, r}\right.\end{array}$ & 365 & 499 & 603 & 750 & 872 & 1,010 & 1,150 & 1,360 \\
\hline \multirow{5}{*}{05531200} & \multirow{5}{*}{ SALT CREEK AT ADDISON, IL } & \multirow{5}{*}{ No } & $\begin{array}{l}\text { At-site, unadjusted for urbanization, EMA, } \\
\text { at-site skew }\end{array}$ & 744 & 1,020 & 1,240 & 1,560 & 1,830 & 2,130 & 2,480 & 2,990 \\
\hline & & & $\begin{array}{l}\text { At-site, adjusted for urbanization, EMA, at- } \\
\text { site skew }\end{array}$ & 1,110 & 1,480 & 1,740 & 2,100 & 2,380 & 2,670 & 2,990 & 3,430 \\
\hline & & & $\begin{array}{l}\text { At-site, adjusted for urbanization, EMA, } \\
\text { weighted skew: (apog,s, }\end{array}$ & 1,110 & 1,480 & 1,740 & 2,090 & 2,370 & 2,670 & 2,980 & 3,420 \\
\hline & & & Regional regression: $\left(Q_{p}\right)_{g, r}$ & 1,400 & 1,910 & 2,290 & 2,790 & 3,190 & 3,590 & 4,020 & 4,600 \\
\hline & & & $\begin{array}{c}\left(Q_{p}\right)_{g, w}: \text { Weighted at-site }\left(Q_{\rho}\right)_{g, s} \text { and regional } \\
\left(Q_{\rho}\right)_{g, r}\end{array}$ & 1,120 & 1,490 & 1,760 & 2,130 & 2,420 & 2,740 & 3,070 & 3,550 \\
\hline \multirow{5}{*}{05531300} & \multirow{5}{*}{ SALT CREEK AT ELMHURST, IL } & \multirow{5}{*}{ No } & $\begin{array}{l}\text { At-site, unadjusted for urbanization, EMA, } \\
\text { at-site skew }\end{array}$ & 1,030 & 1,360 & 1,570 & 1,820 & 2,010 & 2,190 & 2,380 & 2,610 \\
\hline & & & $\begin{array}{l}\text { At-site, adjusted for urbanization, EMA, at- } \\
\text { site skew }\end{array}$ & 1,170 & 1,500 & 1,700 & 1,950 & 2,130 & 2,300 & 2,470 & 2,700 \\
\hline & & & $\begin{array}{l}\text { At-site, adjusted for urbanization, EMA, } \\
\left.\text { weighted skew: ( } Q_{p}\right)_{g, s}\end{array}$ & 1,160 & 1,490 & 1,710 & 1,990 & 2,190 & 2,400 & 2,610 & 2,900 \\
\hline & & & Regional regression: $(Q p)_{a, r}$ & 1,530 & 2,090 & 2,500 & 3,050 & 3,480 & 3,920 & 4,390 & 5,020 \\
\hline & & & $\begin{array}{c}\left(Q_{p}\right)_{g, w} \text { : Weighted at-site } \\
\qquad\left(Q_{\rho}\right)_{g, \text { s and regional }} \\
\left(Q_{\rho}\right)_{g, r}\end{array}$ & 1,160 & 1,500 & 1,720 & 2,000 & 2,220 & 2,440 & 2,670 & 2,980 \\
\hline \multirow{5}{*}{05531380} & \multirow{5}{*}{ SALT CREEK AT OAK BROOK, IL } & \multirow{5}{*}{ No } & $\begin{array}{l}\text { At-site, unadjusted for urbanization, EMA, } \\
\text { at-site skew }\end{array}$ & 952 & 1,250 & 1,430 & 1,660 & 1,820 & 1,980 & 2,140 & 2,350 \\
\hline & & & $\begin{array}{l}\text { At-site, adjusted for urbanization, EMA, at- } \\
\text { site skew }\end{array}$ & 1,280 & 1,670 & 1,940 & 2,300 & 2,580 & 2,860 & 3,160 & 3,570 \\
\hline & & & $\begin{array}{l}\text { At-site, adjusted for urbanization, EMA, } \\
\text { weighted skew: }\left(Q_{p}\right)_{g, s}\end{array}$ & 1,270 & 1,670 & 1,940 & 2,310 & 2,600 & 2,900 & 3,220 & 3,660 \\
\hline & & & Regional regression: $\left(Q_{p}\right)_{g, r}$ & 1,640 & 2,220 & 2,640 & 3,210 & 3,660 & 4,120 & 4,600 & 5,260 \\
\hline & & & $\begin{array}{l}\left(Q_{p}\right)_{g, w} \text { : Weighted at-site }\left(Q_{\rho}\right)_{g, \text { s and regional }} \\
\left(Q_{\rho} \rho_{g, r}\right.\end{array}$ & 1,280 & 1,680 & 1,970 & 2,350 & 2,660 & 2,990 & 3,330 & 3,820 \\
\hline
\end{tabular}




\begin{tabular}{|c|c|c|c|c|c|c|c|c|c|c|c|}
\hline \multirow{2}{*}{$\begin{array}{c}\text { USGS } \\
\text { streamgage } \\
\text { number }\end{array}$} & \multirow[b]{2}{*}{ Streamgage name } & \multirow{2}{*}{$\begin{array}{l}\text { Used in regression } \\
\text { analyses (non- } \\
\text { redundant) }\end{array}$} & \multirow[b]{2}{*}{ Frequency analysis method } & \multicolumn{8}{|c|}{ Discharge for given annual exceedance probability, $\mathrm{ft}^{3} / \mathrm{s}$} \\
\hline & & & & 0.50 & 0.20 & 0.10 & 0.04 & 0.02 & 0.01 & 0.005 & 0.002 \\
\hline \multirow{5}{*}{05531500} & \multirow{5}{*}{ SALT CREEK AT WESTERN SPRINGS, IL } & \multirow{5}{*}{ Yes } & $\begin{array}{l}\text { At-site, unadjusted for urbanization, EMA, } \\
\text { at-site skew }\end{array}$ & 1,290 & 1,770 & 2,080 & 2,480 & 2,770 & 3,060 & 3,350 & 3,740 \\
\hline & & & $\begin{array}{l}\text { At-site, adjusted for urbanization, EMA, at- } \\
\text { site skew }\end{array}$ & 1,430 & 1,860 & 2,160 & 2,570 & 2,890 & 3,220 & 3,580 & 4,070 \\
\hline & & & $\begin{array}{l}\text { At-site, adjusted for urbanization, EMA, } \\
\text { weighted skew: }\left(Q_{p}\right)_{g, s}\end{array}$ & 1,430 & 1,860 & 2,160 & 2,570 & 2,890 & 3,220 & 3,570 & 4,060 \\
\hline & & & Regional regression: $(Q p)_{g, r}$ & 1,820 & 2,470 & 2,940 & 3,570 & 4,070 & 4,580 & 5,120 & 5,850 \\
\hline & & & $\begin{array}{l}\left(Q_{p}\right)_{g, w}: \text { Weighted at-site }\left(Q_{\rho}\right)_{g_{s} \text { a and regional }} \\
\left(Q_{p}\right)_{Q, r}\end{array}$ & 1,430 & 1,870 & 2,180 & 2,590 & 2,920 & 3,270 & 3,640 & 4,170 \\
\hline \multirow{5}{*}{05531800} & \multirow{5}{*}{ ADDISON CREEK AT NORTHLAKE, IL } & \multirow{5}{*}{ No } & $\begin{array}{l}\text { At-site, unadjusted for urbanization, EMA, } \\
\text { at-site skew }\end{array}$ & 337 & 380 & 407 & 442 & 467 & 493 & 518 & 552 \\
\hline & & & $\begin{array}{l}\text { At-site, adjusted for urbanization, EMA, at- } \\
\text { site skew }\end{array}$ & 418 & 470 & 502 & 539 & 566 & 591 & 616 & 648 \\
\hline & & & 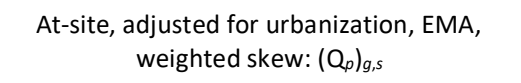 & 417 & 469 & 502 & 542 & 570 & 598 & 626 & 663 \\
\hline & & & Regional regression: $\left(Q_{p}\right)_{g, r}$ & 293 & 434 & 539 & 683 & 798 & 917 & 1,040 & 1,210 \\
\hline & & & $\begin{array}{c}\left(Q_{\rho}^{\rho}\right)_{Q, w}: \text { Weighted at-site }\left(Q_{\rho}\right)_{g, s} \text { and regional } \\
\left(Q_{\rho}\right)_{g, r}\end{array}$ & 416 & 469 & 503 & 544 & 574 & 605 & 636 & 677 \\
\hline \multirow{5}{*}{05532000} & \multirow{5}{*}{ ADDISON CREEK AT BELLWOOD, IL } & \multirow{5}{*}{ Yes } & $\begin{array}{l}\text { At-site, unadjusted for urbanization, EMA, } \\
\text { at-site skew }\end{array}$ & 473 & 644 & 748 & 870 & 956 & 1,040 & 1,110 & 1,210 \\
\hline & & & $\begin{array}{l}\text { At-site, adjusted for urbanization, EMA, at- } \\
\text { site skew }\end{array}$ & 448 & 597 & 694 & 815 & 904 & 992 & 1,080 & 1,200 \\
\hline & & & $\begin{array}{l}\text { At-site, adjusted for urbanization, EMA, } \\
\text { weighted skew: (Q }\left(Q_{p}\right)_{g, s}\end{array}$ & 443 & 595 & 698 & 831 & 933 & 1,040 & 1,140 & 1,290 \\
\hline & & & Regional regression: $(Q p)_{g, r}$ & 593 & 858 & 1,060 & 1,330 & 1,550 & 1,770 & 2,010 & 2,340 \\
\hline & & & $\begin{array}{c}\left(Q_{\rho}\right)_{g, w}: \text { Weighted at-site }\left(Q_{\rho}\right)_{g, s} \text { and regional } \\
\left(S_{\rho}\right)_{g, r}\end{array}$ & 445 & 598 & 704 & 842 & 949 & 1,060 & 1,180 & 1,340 \\
\hline \multirow{5}{*}{05532500} & \multirow{5}{*}{ DES PLAINES RIVER AT RIVERSIDE, IL } & \multirow{5}{*}{ No } & $\begin{array}{l}\text { At-site, unadjusted for urbanization, EMA, } \\
\text { at-site skew }\end{array}$ & 4,190 & 5,630 & 6,490 & 7,480 & 8,150 & 8,780 & 9,380 & 10,100 \\
\hline & & & $\begin{array}{l}\text { At-site, adjusted for urbanization, EMA, at- } \\
\text { site skew }\end{array}$ & 4,890 & 6,320 & 7,200 & 8,260 & 9,010 & 9,730 & 10,400 & 11,400 \\
\hline & & & $\begin{array}{l}\text { At-site, adjusted for urbanization, EMA, } \\
\left.\text { weighted skew: ( } Q_{0}\right)_{g, s}\end{array}$ & 4,850 & 6,310 & 7,240 & 8,380 & 9,210 & 10,000 & 10,800 & 11,900 \\
\hline & & & Regional regression: $\left(Q_{p}\right)_{g, r}$ & 5,130 & 6,670 & 7,730 & 9,120 & 10,200 & 11,300 & 12,400 & 13,900 \\
\hline & & & $\begin{array}{c}\left(Q_{\rho}\right)_{g, w} \text { : Weighted at-site }\left(Q_{\rho}\right)_{g, s} \text { and regional } \\
\left(\rho_{\rho}\right)_{g, r}\end{array}$ & 4,860 & 6,310 & 7,240 & 8,390 & 9,230 & 10,100 & 10,900 & 12,000 \\
\hline
\end{tabular}




\begin{tabular}{|c|c|c|c|c|c|c|c|c|c|c|c|}
\hline \multirow{2}{*}{$\begin{array}{c}\text { USGS } \\
\text { streamgage } \\
\text { number }\end{array}$} & \multirow[b]{2}{*}{ Streamgage name } & \multirow{2}{*}{$\begin{array}{l}\text { Used in regression } \\
\text { analyses (non- } \\
\text { redundant) }\end{array}$} & \multirow[b]{2}{*}{ Frequency analysis method } & \multicolumn{8}{|c|}{ Discharge for given annual exceedance probability, $\mathrm{ft}^{3} / \mathrm{s}$} \\
\hline & & & & 0.50 & 0.20 & 0.10 & 0.04 & 0.02 & 0.01 & 0.005 & 0.002 \\
\hline \multirow{5}{*}{05533000} & \multirow{5}{*}{ FLAG CREEK NEAR WILLOW SPRINGS, IL } & \multirow{5}{*}{ Yes } & $\begin{array}{l}\text { At-site, unadjusted for urbanization, EMA, } \\
\text { at-site skew }\end{array}$ & 799 & 1,270 & 1,620 & 2,100 & 2,470 & 2,860 & 3,280 & 3,860 \\
\hline & & & $\begin{array}{l}\text { At-site, adjusted for urbanization, EMA, at- } \\
\text { site skew }\end{array}$ & 882 & 1,360 & 1,720 & 2,250 & 2,680 & 3,150 & 3,660 & 4,410 \\
\hline & & & $\begin{array}{l}\text { At-site, adjusted for urbanization, EMA, } \\
\text { weighted skew: (Q }\left(Q_{p}\right)_{g, s}\end{array}$ & 876 & 1,360 & 1,730 & 2,280 & 2,740 & 3,240 & 3,810 & 4,650 \\
\hline & & & Regional regression: $(Q p)_{a, r}$ & 611 & 906 & 1,130 & 1,440 & 1,690 & 1,950 & 2,230 & 2,610 \\
\hline & & & $\begin{array}{c}\left(Q_{\rho \rho}\right)_{y, w} \text { Weighted at-site }\left(Q_{p}\right)_{Q, s} \text { and regional } \\
\left(Q_{\rho}\right)_{Q, r}\end{array}$ & 868 & 1,340 & 1,700 & 2,210 & 2,630 & 3,080 & 3,560 & 4,250 \\
\hline \multirow{5}{*}{05533200} & \multirow{5}{*}{ SAWMILL CREEK TRIBUTARY NEAR TIEDTVILLE, IL } & \multirow{5}{*}{ Yes } & $\begin{array}{l}\text { At-site, unadjusted for urbanization, EMA, } \\
\text { at-site skew }\end{array}$ & 230 & 281 & 307 & 333 & 350 & 363 & 375 & 389 \\
\hline & & & $\begin{array}{l}\text { At-site, adjusted for urbanization, EMA, at- } \\
\text { site skew }\end{array}$ & 292 & 314 & 326 & 340 & 350 & 359 & 368 & 380 \\
\hline & & & $\begin{array}{l}\text { At-site, adjusted for urbanization, EMA, } \\
\text { weighted skew: (Q }\left(Q_{p}\right)_{g, s}\end{array}$ & 292 & 313 & 326 & 341 & 352 & 362 & 372 & 385 \\
\hline & & & Regional regression: $\left(Q_{p}\right)_{g, r}$ & 206 & 334 & 436 & 581 & 701 & 829 & 965 & 1,160 \\
\hline & & & $\begin{array}{l}\left(Q_{\rho \rho}\right)_{y, w} \text { Weighted at-site }\left(Q_{\rho}\right)_{g, s} \text { and regional } \\
\left(Q_{\rho}\right)_{Q, r}\end{array}$ & 292 & 313 & 326 & 342 & 353 & 364 & 376 & 390 \\
\hline \multirow{5}{*}{05533300} & \multirow{5}{*}{ WARDS CREEK NEAR WOODRIDGE, IL } & \multirow{5}{*}{ Yes } & $\begin{array}{l}\text { At-site, unadjusted for urbanization, EMA, } \\
\text { at-site skew }\end{array}$ & 80 & 113 & 134 & 159 & 176 & 193 & 208 & 228 \\
\hline & & & $\begin{array}{l}\text { At-site, adjusted for urbanization, EMA, at- } \\
\text { site skew }\end{array}$ & 119 & 158 & 182 & 211 & 231 & 250 & 268 & 292 \\
\hline & & & $\begin{array}{l}\text { At-site, adjusted for urbanization, EMA, } \\
\left.\text { weighted skew: ( } Q_{p}\right)_{g, s}\end{array}$ & 116 & 157 & 185 & 222 & 250 & 279 & 309 & 350 \\
\hline & & & Regional regression: $(Q p)_{g, r}$ & 145 & 221 & 279 & 358 & 421 & 486 & 554 & 648 \\
\hline & & & $\begin{array}{l}\left(Q_{\rho p}\right)_{y, w} \text { : Weighted at-site }\left(Q_{p}\right)_{Q, s} \text { and regional } \\
\left(Q_{0}\right)_{p, r}\end{array}$ & 117 & 160 & 191 & 232 & 265 & 299 & 336 & 388 \\
\hline \multirow{5}{*}{05533400} & \multirow{5}{*}{ SAWMILL CREEK NEAR LEMONT, IL } & \multirow{5}{*}{ No } & $\begin{array}{l}\text { At-site, unadjusted for urbanization, EMA, } \\
\text { at-site skew }\end{array}$ & 639 & 1,030 & 1,340 & 1,820 & 2,240 & 2,710 & 3,250 & 4,070 \\
\hline & & & $\begin{array}{l}\text { At-site, adjusted for urbanization, EMA, at- } \\
\text { site skew }\end{array}$ & 734 & 1,180 & 1,520 & 1,970 & 2,330 & 2,710 & 3,110 & 3,670 \\
\hline & & & 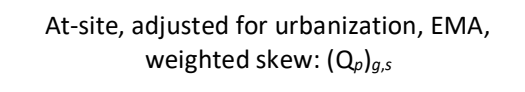 & 722 & 1,170 & 1,520 & 2,030 & 2,450 & 2,920 & 3,420 & 4,170 \\
\hline & & & Regional regression: $\left(Q_{p}\right)_{g, r}$ & 430 & 646 & 808 & 1,030 & 1,210 & 1,390 & 1,580 & 1,850 \\
\hline & & & $\begin{array}{l}(Q p)_{g, w} \text { : Weighted at-site }\left(Q_{\rho}\right)_{g, s} \text { and regional } \\
\left(Q_{\rho}\right)_{Q, r}\end{array}$ & 707 & 1,140 & 1,460 & 1,910 & 2,260 & 2,630 & 3,010 & 3,540 \\
\hline
\end{tabular}




\begin{tabular}{|c|c|c|c|c|c|c|c|c|c|c|c|}
\hline \multirow{2}{*}{$\begin{array}{c}\text { USGS } \\
\text { streamgage } \\
\text { number }\end{array}$} & \multirow[b]{2}{*}{ Streamgage name } & \multirow{2}{*}{$\begin{array}{l}\text { Used in regression } \\
\text { analyses (non- } \\
\text { redundant) }\end{array}$} & \multirow[b]{2}{*}{ Frequency analysis method } & \multicolumn{8}{|c|}{ Discharge for given annual exceedance probability, $\mathrm{ft}^{3} / \mathrm{s}$} \\
\hline & & & & 0.50 & 0.20 & 0.10 & 0.04 & 0.02 & 0.01 & 0.005 & 0.002 \\
\hline \multirow{5}{*}{05534300} & \multirow{5}{*}{$\begin{array}{l}\text { NORTH BRANCH CHIIAGG RIVER AT LAKE } \\
\text { FOREST, IL }\end{array}$} & \multirow{5}{*}{ No } & $\begin{array}{l}\text { At-site, unadjusted for urbanization, EMA, } \\
\text { at-site skew }\end{array}$ & 193 & 262 & 301 & 344 & 373 & 399 & 422 & 450 \\
\hline & & & $\begin{array}{l}\text { At-site, adjusted for urbanization, EMA, at- } \\
\text { site skew }\end{array}$ & 378 & 469 & 518 & 568 & 600 & 628 & 652 & 681 \\
\hline & & & $\begin{array}{l}\text { At-site, adjusted for urbanization, EMA, } \\
\left.\text { weighted skew: ( } Q_{0}\right)_{s, 5}\end{array}$ & 368 & 468 & 529 & 602 & 654 & 704 & 753 & 816 \\
\hline & & & Regional regression: $(Q p)_{g, r}$ & 302 & 438 & 535 & 663 & 762 & 862 & 963 & 1,100 \\
\hline & & & $\begin{array}{c}\left(Q_{p}\right)_{g, w}: \text { Weighted at-site }\left(Q_{\rho}\right)_{g, s} \text { and regional } \\
\left(Q_{\rho} g_{g, r}\right.\end{array}$ & 366 & 467 & 529 & 605 & 660 & 713 & 767 & 838 \\
\hline \multirow{5}{*}{05534400} & \multirow{5}{*}{$\begin{array}{l}\text { NORTH BRANCH CHICAGO RIVER AT } \\
\text { BANNOCKBURN, IL }\end{array}$} & \multirow{5}{*}{ No } & $\begin{array}{l}\text { At-site, unadjusted for urbanization, EMA, } \\
\text { at-site skew }\end{array}$ & 270 & 315 & 340 & 366 & 383 & 398 & 412 & 429 \\
\hline & & & $\begin{array}{l}\text { At-site, adjusted for urbanization, EMA, at- } \\
\text { site skew }\end{array}$ & 503 & 561 & 595 & 634 & 660 & 685 & 708 & 738 \\
\hline & & & $\begin{array}{l}\text { At-site, adjusted for urbanization, EMA, } \\
\text { weighted skew: (( }\left(_{p}\right)_{g, s}\end{array}$ & 503 & 560 & 595 & 635 & 663 & 690 & 715 & 749 \\
\hline & & & Regional regression: $\left(Q_{\rho}\right)_{g, r}$ & 377 & 544 & 661 & 816 & 935 & 1,050 & 1,180 & 1,340 \\
\hline & & & $\begin{array}{c}\left(Q_{p}\right)_{g, w}: \text { Weighted at-site }\left(Q_{\rho}\right)_{g, s} \text { and regional } \\
\left(Q_{\rho}\right)_{g, r}\end{array}$ & 501 & 560 & 595 & 637 & 667 & 696 & 724 & 761 \\
\hline \multirow{5}{*}{05534500} & \multirow{5}{*}{$\begin{array}{l}\text { NORTH BRANCH CHICAGO RIVER AT DEERFIELD, } \\
\text { IL }\end{array}$} & \multirow{5}{*}{ Yes } & $\begin{array}{l}\text { At-site, unadjusted for urbanization, EMA, } \\
\text { at-site skew }\end{array}$ & 361 & 538 & 652 & 792 & 892 & 989 & 1,080 & 1,200 \\
\hline & & & $\begin{array}{l}\text { At-site, adjusted for urbanization, EMA, at- } \\
\text { site skew }\end{array}$ & 529 & 704 & 812 & 942 & 1,040 & 1,120 & 1,210 & 1,320 \\
\hline & & & $\begin{array}{l}\text { At-site, adjusted for urbanization, EMA, } \\
\text { weighted skew: }\left(Q_{p}\right)_{g, s}\end{array}$ & 524 & 702 & 817 & 961 & 1,070 & 1,170 & 1,280 & 1,420 \\
\hline & & & Regional regression: $(Q p)_{g, r}$ & 443 & 631 & 762 & 934 & 1,070 & 1,200 & 1,330 & 1,510 \\
\hline & & & $\begin{array}{l}\left(Q_{p}\right)_{g, w} \text { : Weighted at-site }\left(Q_{\rho}\right)_{g, s} \text { and regional } \\
\qquad\left(Q_{\rho}\right)_{g, r}\end{array}$ & 523 & 700 & 816 & 960 & 1,070 & 1,170 & 1,280 & 1,420 \\
\hline \multirow{5}{*}{05534600} & \multirow{5}{*}{$\begin{array}{l}\text { NORTH BRANCH CHICAGO RIVER AT } \\
\text { NORTHFIELD, IL }\end{array}$} & \multirow{5}{*}{ No } & $\begin{array}{l}\text { At-site, unadjusted for urbanization, EMA, } \\
\text { at-site skew }\end{array}$ & 346 & 434 & 481 & 533 & 567 & 597 & 625 & 658 \\
\hline & & & $\begin{array}{l}\text { At-site, adjusted for urbanization, EMA, at- } \\
\text { site skew }\end{array}$ & 537 & 634 & 684 & 735 & 766 & 793 & 817 & 844 \\
\hline & & & $\begin{array}{l}\text { At-site, adjusted for urbanization, EMA, } \\
\text { weighted skew: }\left(\mathrm{Q}_{\mathrm{Q}}\right)_{g, s}\end{array}$ & 527 & 633 & 695 & 767 & 816 & 863 & 908 & 964 \\
\hline & & & Regional regression: $\left(Q_{p}\right)_{g, r}$ & 533 & 760 & 919 & 1,130 & 1,290 & 1,450 & 1,620 & 1,840 \\
\hline & & & $\begin{array}{c}(Q p)_{g, w}: \text { Weighted at-site }\left(Q_{\rho}\right)_{g, s} \text { and regional } \\
\left(Q_{\rho} \rho g_{g, r}\right.\end{array}$ & 527 & 635 & 699 & 774 & 827 & 878 & 928 & 993 \\
\hline
\end{tabular}




\begin{tabular}{|c|c|c|c|c|c|c|c|c|c|c|c|}
\hline \multirow{2}{*}{$\begin{array}{c}\text { USGS } \\
\text { streamgage } \\
\text { number }\end{array}$} & \multirow{2}{*}{ Streamgage name } & \multirow{2}{*}{$\begin{array}{l}\text { Used in regression } \\
\text { analyses (non-- } \\
\text { redundant) }\end{array}$} & \multirow[b]{2}{*}{ Frequency analysis method } & \multicolumn{8}{|c|}{ Discharge for given annual exceedance probability, $\mathrm{ft}^{3} / \mathrm{s}$} \\
\hline & & & & 0.50 & 0.20 & 0.10 & 0.04 & 0.02 & 0.01 & 0.005 & 0.002 \\
\hline \multirow{5}{*}{05534900} & \multirow{5}{*}{ SKOKIE RIVER AT LAKE BLUFF, IL } & \multirow{5}{*}{ No } & $\begin{array}{l}\text { At-site, unadjusted for urbanization, EMA, } \\
\text { at-site skew }\end{array}$ & 205 & 314 & 384 & 468 & 528 & 585 & 640 & 710 \\
\hline & & & $\begin{array}{l}\text { At-site, adjusted for urbanization, EMA, at- } \\
\text { site skew }\end{array}$ & 287 & 401 & 472 & 555 & 614 & 670 & 723 & 791 \\
\hline & & & $\begin{array}{l}\text { At-site, adjusted for urbanization, EMA, } \\
\text { weighted skew: ( }\left(_{0}\right)_{g, s}\end{array}$ & 278 & 398 & 483 & 596 & 685 & 777 & 873 & 1,010 \\
\hline & & & Regional regression: $(Q p)_{g, r}$ & 243 & 348 & 424 & 528 & 608 & 690 & 775 & 890 \\
\hline & & & $\begin{array}{l}\left(Q_{p \rho}\right)_{y, w} \text { Weighted at-site }\left(Q_{p}\right)_{g, s} \text { and regional } \\
\left(Q_{0}\right)_{p, r}\end{array}$ & 276 & 394 & 477 & 587 & 673 & 762 & 854 & 981 \\
\hline \multirow{5}{*}{05535000} & \multirow{5}{*}{ SKOKIE RIVER AT LAKE FOREST, IL } & \multirow{5}{*}{ Yes } & $\begin{array}{l}\text { At-site, unadjusted for urbanization, EMA, } \\
\text { at-site skew }\end{array}$ & 254 & 363 & 428 & 504 & 556 & 605 & 650 & 707 \\
\hline & & & $\begin{array}{l}\text { At-site, adjusted for urbanization, EMA, at- } \\
\text { site skew }\end{array}$ & 348 & 455 & 520 & 596 & 649 & 700 & 748 & 810 \\
\hline & & & $\begin{array}{l}\text { At-site, adjusted for urbanization, EMA, } \\
\text { weighted skew: ( (a) o gl,s }\end{array}$ & 344 & 453 & 523 & 610 & 674 & 737 & 800 & 884 \\
\hline & & & Regional regression: $\left(Q_{p}\right)_{Q, r}$ & 339 & 486 & 592 & 732 & 841 & 951 & 1,060 & 1,220 \\
\hline & & & $\begin{array}{c}\left(Q_{\rho \rho}\right)_{y, w} \text { Weighted at-site }\left(Q_{p}\right)_{Q, s} \text { and regional } \\
\left(Q_{\rho}\right)_{Q, r}\end{array}$ & 344 & 453 & 524 & 612 & 678 & 744 & 810 & 900 \\
\hline \multirow{5}{*}{05535070} & \multirow{5}{*}{ SKOKIE RIVER NEAR HIGHLAND PARK, IL } & \multirow{5}{*}{ No } & $\begin{array}{l}\text { At-site, unadjusted for urbanization, EMA, } \\
\text { at-site skew }\end{array}$ & 501 & 712 & 856 & 1,040 & 1,180 & 1,320 & 1,460 & 1,650 \\
\hline & & & $\begin{array}{l}\text { At-site, adjusted for urbanization, EMA, at- } \\
\text { site skew }\end{array}$ & 611 & 820 & 956 & 1,130 & 1,250 & 1,380 & 1,500 & 1,670 \\
\hline & & & $\begin{array}{l}\text { At-site, adjusted for urbanization, EMA, } \\
\text { weighted skew: (Oaplgs, }\end{array}$ & 606 & 818 & 961 & 1,140 & 1,280 & 1,420 & 1,570 & 1,770 \\
\hline & & & Regional regression: $(Q p)_{a r}$ & 491 & 696 & 843 & 1,040 & 1,190 & 1,340 & 1,500 & 1,710 \\
\hline & & & $\begin{array}{c}\left(Q_{\rho \rho}\right)_{y, w} \text { Weighted at-site }\left(Q_{p}\right)_{Q, s} \text { and regional } \\
\left(Q_{p}\right)_{Q, r}\end{array}$ & 604 & 815 & 957 & 1,140 & 1,280 & 1,420 & 1,560 & 1,760 \\
\hline \multirow{5}{*}{05535150} & \multirow{5}{*}{ SKOKIE RIVER AT NORTHFIELD, IL } & \multirow{5}{*}{ No } & $\begin{array}{l}\text { At-site, unadjusted for urbanization, EMA, } \\
\text { at-site skew }\end{array}$ & 374 & 448 & 492 & 544 & 580 & 615 & 650 & 694 \\
\hline & & & $\begin{array}{l}\text { At-site, adjusted for urbanization, EMA, at- } \\
\text { site skew }\end{array}$ & 494 & 572 & 619 & 674 & 714 & 752 & 789 & 838 \\
\hline & & & $\begin{array}{l}\text { At-site, adjusted for urbanization, EMA, } \\
\text { weighted skew: ( }\left(\alpha_{0}\right)_{g, s}\end{array}$ & 493 & 571 & 620 & 678 & 721 & 762 & 803 & 856 \\
\hline & & & Regional regression: $\left(Q_{p}\right)_{g, r}$ & 567 & 788 & 944 & 1,150 & 1,310 & 1,470 & 1,630 & 1,850 \\
\hline & & & $\begin{array}{c}\left(Q_{\rho \rho}\right)_{y, w} \text { : Weighted at-site }\left(Q_{\rho}\right)_{Q, s} \text { and regional } \\
\left(Q_{p}\right)_{Q, r}\end{array}$ & 494 & 573 & 623 & 686 & 731 & 777 & 823 & 885 \\
\hline
\end{tabular}




\begin{tabular}{|c|c|c|c|c|c|c|c|c|c|c|c|}
\hline \multirow{2}{*}{$\begin{array}{c}\text { USGS } \\
\text { streamgage } \\
\text { number }\end{array}$} & \multirow[b]{2}{*}{ Streamgage name } & \multirow{2}{*}{$\begin{array}{l}\text { Used in regression } \\
\text { analyses (non- } \\
\text { redundant) }\end{array}$} & \multirow[b]{2}{*}{ Frequency analysis method } & \multicolumn{8}{|c|}{ Discharge for given annual exceedance probability, $\mathrm{ft}^{3} / \mathrm{s}$} \\
\hline & & & & 0.50 & 0.20 & 0.10 & 0.04 & 0.02 & 0.01 & 0.005 & 0.002 \\
\hline \multirow{5}{*}{05535200} & \multirow{5}{*}{$\begin{array}{l}\text { NORTH BRANCH CHICAGO RIVER AT GLENVIEW, } \\
\text { IL }\end{array}$} & \multirow{5}{*}{ No } & $\begin{array}{l}\text { At-site, unadjusted for urbanization, EMA, } \\
\text { at-site skew }\end{array}$ & 636 & 806 & 926 & 1,090 & 1,210 & 1,340 & 1,480 & 1,680 \\
\hline & & & $\begin{array}{l}\text { At-site, adjusted for urbanization, EMA, at- } \\
\text { site skew }\end{array}$ & 932 & 1,140 & 1,270 & 1,440 & 1,560 & 1,690 & 1,820 & 1,990 \\
\hline & & & $\begin{array}{l}\text { At-site, adjusted for urbanization, EMA, } \\
\text { weighted skew: }\left(Q_{p}\right)_{g, s}\end{array}$ & 933 & 1,140 & 1,270 & 1,430 & 1,550 & 1,680 & 1,800 & 1,970 \\
\hline & & & Regional regression: $(Q p)_{g, r}$ & 998 & 1,380 & 1,650 & 2,000 & 2,280 & 2,550 & 2,830 & 3,210 \\
\hline & & & $\begin{array}{c}\left(Q_{\rho \rho}\right)_{y, w} \text { : Weighted at-site }\left(Q_{p}\right)_{Q, s} \text { and regional } \\
\left(Q_{\rho}\right)_{Q, r}\end{array}$ & 935 & 1,140 & 1,280 & 1,460 & 1,600 & 1,740 & 1,880 & 2,080 \\
\hline \multirow{5}{*}{05535300} & \multirow{5}{*}{ WF OF NB CHICAGO RIVER AT BANNOCKBURN, IL } & \multirow{5}{*}{ No } & $\begin{array}{l}\text { At-site, unadjusted for urbanization, EMA, } \\
\text { at-site skew }\end{array}$ & 208 & 285 & 334 & 394 & 438 & 481 & 523 & 578 \\
\hline & & & $\begin{array}{l}\text { At-site, adjusted for urbanization, EMA, at- } \\
\text { site skew }\end{array}$ & 380 & 473 & 531 & 602 & 654 & 704 & 754 & 819 \\
\hline & & & $\begin{array}{l}\text { At-site, adjusted for urbanization, EMA, } \\
\text { weighted skew: ( (opog,s }\end{array}$ & 379 & 472 & 532 & 606 & 660 & 714 & 768 & 839 \\
\hline & & & Regional regression: $\left(Q_{p}\right)_{g, r}$ & 123 & 191 & 240 & 305 & 355 & 406 & 459 & 528 \\
\hline & & & $\begin{array}{c}(Q p)_{g, w} \text { : Weighted at-site }\left(Q_{p}\right)_{g, s} \text { and regional } \\
\left(Q_{p}\right)_{g, r}\end{array}$ & 370 & 461 & 518 & 587 & 638 & 688 & 738 & 805 \\
\hline \multirow{5}{*}{05535400} & \multirow{5}{*}{ WF OF NB CHICAGO RIVER AT DEERFIELD, IL } & \multirow{5}{*}{ No } & $\begin{array}{l}\text { At-site, unadjusted for urbanization, EMA, } \\
\text { at-site skew }\end{array}$ & 362 & 453 & 506 & 568 & 611 & 652 & 690 & 739 \\
\hline & & & $\begin{array}{l}\text { At-site, adjusted for urbanization, EMA, at- } \\
\text { site skew }\end{array}$ & 555 & 655 & 715 & 787 & 837 & 885 & 932 & 993 \\
\hline & & & $\begin{array}{l}\text { At-site, adjusted for urbanization, EMA, } \\
\left.\text { weighted skew: (Q } Q_{p}\right)_{g, 5}\end{array}$ & 553 & 654 & 717 & 793 & 847 & 900 & 953 & 1,020 \\
\hline & & & Regional regression: $(Q p)_{a, r}$ & 223 & 329 & 404 & 505 & 582 & 659 & 739 & 844 \\
\hline & & & $\begin{array}{l}\left(Q_{\rho p}\right)_{y, w} \text { Weighted at-site }\left(Q_{p}\right)_{g, s} \text { and regional } \\
\left(Q_{0}\right)_{g, r}\end{array}$ & 546 & 646 & 708 & 782 & 837 & 889 & 942 & 1,010 \\
\hline \multirow{5}{*}{05535500} & \multirow{5}{*}{ WF OF NB CHICAGO RIVER AT NORTHBROOK IL } & \multirow{5}{*}{ Yes } & $\begin{array}{l}\text { At-site, unadjusted for urbanization, EMA, } \\
\text { at-site skew }\end{array}$ & 487 & 673 & 794 & 943 & 1,050 & 1,160 & 1,260 & 1,400 \\
\hline & & & $\begin{array}{l}\text { At-site, adjusted for urbanization, EMA, at- } \\
\text { site skew }\end{array}$ & 470 & 619 & 711 & 822 & 901 & 977 & 1,050 & 1,150 \\
\hline & & & $\begin{array}{l}\text { At-site, adjusted for urbanization, EMA, } \\
\text { weighted skew: }\left(Q_{p}\right)_{g, s}\end{array}$ & 465 & 616 & 715 & 839 & 931 & 1,020 & 1,120 & 1,240 \\
\hline & & & Regional regression: $\left(Q_{p}\right)_{g, r}$ & 361 & 523 & 640 & 796 & 918 & 1,040 & 1,170 & 1,340 \\
\hline & & & $\begin{array}{l}\left(Q_{\rho}\right)_{g, w}: \text { Weighted at-site }\left(Q_{p}\right)_{g, s} \text { and regional } \\
\left(Q_{p}\right)_{Q, r}\end{array}$ & 464 & 615 & 714 & 838 & 931 & 1,020 & 1,120 & 1,250 \\
\hline
\end{tabular}




\begin{tabular}{|c|c|c|c|c|c|c|c|c|c|c|c|}
\hline \multirow{2}{*}{$\begin{array}{c}\text { USGS } \\
\text { streamgage } \\
\text { number }\end{array}$} & \multirow[b]{2}{*}{ Streamgage name } & \multirow{2}{*}{$\begin{array}{l}\text { Used in regression } \\
\text { analyses (non- } \\
\text { redundant) }\end{array}$} & \multirow[b]{2}{*}{ Frequency analysis method } & \multicolumn{8}{|c|}{ Discharge for given annual exceedance probability, $\mathrm{ft}^{3} / \mathrm{s}$} \\
\hline & & & & 0.50 & 0.20 & 0.10 & 0.04 & 0.02 & 0.01 & 0.005 & 0.002 \\
\hline \multirow{5}{*}{05535700} & \multirow{5}{*}{ WF OF NB CHICAGO RIVER AT GLENVIEW, II } & \multirow{5}{*}{ No } & $\begin{array}{l}\text { At-site, unadjusted for urbanization, EMA, } \\
\text { at-site skew }\end{array}$ & 628 & 856 & 986 & 1,130 & 1,220 & 1,310 & 1,390 & 1,480 \\
\hline & & & $\begin{array}{l}\text { At-site, adjusted for urbanization, EMA, at- } \\
\text { site skew }\end{array}$ & 832 & 1,070 & 1,210 & 1,370 & 1,480 & 1,580 & 1,670 & 1,790 \\
\hline & & & $\begin{array}{l}\text { At-site, adjusted for urbanization, EMA, } \\
\left.\text { weighted skew: ( } 0_{0}\right)_{g, s}\end{array}$ & 813 & 1,060 & 1,220 & 1,430 & 1,590 & 1,750 & 1,920 & 2,140 \\
\hline & & & Regional regression: $(Q p)_{g, r}$ & 589 & 831 & 1,010 & 1,240 & 1,430 & 1,620 & 1,810 & 2,080 \\
\hline & & & $\begin{array}{c}\left(Q_{\rho \rho}\right)_{y, w} \text { : Weighted at-site }\left(Q_{\rho}\right)_{Q, s} \text { and regional } \\
\left(Q_{\rho}\right)_{Q, r}\end{array}$ & 806 & 1,050 & 1,210 & 1,420 & 1,580 & 1,740 & 1,900 & 2,130 \\
\hline \multirow{5}{*}{05535800} & \multirow{5}{*}{$\begin{array}{l}\text { NORTH BRANCH CHICAGO RIVER AT MORTON } \\
\text { GROVE, IL }\end{array}$} & \multirow{5}{*}{ No } & $\begin{array}{l}\text { At-site, unadjusted for urbanization, EMA, } \\
\text { at-site skew }\end{array}$ & 1,010 & 1,340 & 1,570 & 1,860 & 2,080 & 2,320 & 2,550 & 2,880 \\
\hline & & & $\begin{array}{l}\text { At-site, adjusted for urbanization, EMA, at- } \\
\text { site skew }\end{array}$ & 1,120 & 1,440 & 1,650 & 1,910 & 2,110 & 2,310 & 2,510 & 2,780 \\
\hline & & & $\begin{array}{l}\text { At-site, adjusted for urbanization, EMA, } \\
\text { weighted skew: }\left(Q_{p}\right)_{g, s}\end{array}$ & 1,120 & 1,440 & 1,650 & 1,930 & 2,140 & 2,360 & 2,590 & 2,900 \\
\hline & & & Regional regression: $\left(Q_{p}\right)_{g, r}$ & 1,550 & 2,130 & 2,540 & 3,090 & 3,510 & 3,940 & 4,380 & 4,990 \\
\hline & & & $\begin{array}{c}(Q p)_{Q, w} \text { : Weighted at-site } \\
\left.\qquad\left(Q_{p}\right)_{Q, r}\right)_{g, s} \text { and regional }\end{array}$ & 1,130 & 1,450 & 1,680 & 1,980 & 2,220 & 2,460 & 2,720 & 3,090 \\
\hline \multirow{5}{*}{05536000} & \multirow{5}{*}{ NORTH BRANCH CHICAGO RIVER AT NLLES, IL } & \multirow{5}{*}{ Yes } & $\begin{array}{l}\text { At-site, unadjusted for urbanization, EMA, } \\
\text { at-site skew }\end{array}$ & 1,220 & 1,660 & 1,960 & 2,350 & 2,660 & 2,960 & 3,280 & 3,730 \\
\hline & & & $\begin{array}{l}\text { At-site, adjusted for urbanization, EMA, at- } \\
\text { site skew }\end{array}$ & 1,450 & 1,890 & 2,200 & 2,600 & 2,910 & 3,230 & 3,570 & 4,030 \\
\hline & & & $\begin{array}{l}\text { At-site, adjusted for urbanization, EMA, } \\
\left.\text { weighted skew: ( } Q_{p}\right)_{g, s}\end{array}$ & 1,440 & 1,890 & 2,200 & 2,610 & 2,920 & 3,250 & 3,590 & 4,060 \\
\hline & & & Regional regression: $(Q p)_{g, r}$ & 1,650 & 2,260 & 2,690 & 3,270 & 3,710 & 4,170 & 4,640 & 5,270 \\
\hline & & & $\begin{array}{c}\left(Q_{\rho \rho}\right)_{y, w} \text { Weighted at-site }\left(Q_{\rho}\right)_{Q, s} \text { and regional } \\
\left(Q_{\rho}\right)_{Q, r}\end{array}$ & 1,450 & 1,900 & 2,210 & 2,620 & 2,950 & 3,280 & 3,640 & 4,130 \\
\hline \multirow{5}{*}{05536105} & \multirow{5}{*}{$\begin{array}{l}\text { NB CHICAGO RIVER AT ALBANY AVENUE AT } \\
\text { CHICAGO, AL }\end{array}$} & \multirow{5}{*}{ No } & $\begin{array}{l}\text { At-site, unadjusted for urbanization, EMA, } \\
\text { at-site skew }\end{array}$ & 1,400 & 1,960 & 2,410 & 3,070 & 3,640 & 4,290 & 5,020 & 6,130 \\
\hline & & & $\begin{array}{l}\text { At-site, adjusted for urbanization, EMA, at- } \\
\text { site skew }\end{array}$ & 1,490 & 2,060 & 2,500 & 3,140 & 3,680 & 4,280 & 4,940 & 5,950 \\
\hline & & & $\begin{array}{l}\text { At-site, adjusted for urbanization, EMA, } \\
\text { weighted skew: }\left(Q_{p}\right)_{g, s}\end{array}$ & 1,510 & 2,070 & 2,490 & 3,070 & 3,540 & 4,050 & 4,600 & 5,400 \\
\hline & & & Regional regression: $\left(Q_{p}\right)_{q, r}$ & 1,820 & 2,480 & 2,950 & 3,570 & 4,050 & 4,540 & 5,040 & 5,730 \\
\hline & & & 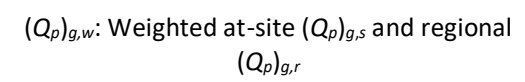 & 1,520 & 2,090 & 2,520 & 3,110 & 3,600 & 4,110 & 4,670 & 5,460 \\
\hline
\end{tabular}




\begin{tabular}{|c|c|c|c|c|c|c|c|c|c|c|c|}
\hline \multirow{2}{*}{$\begin{array}{c}\text { USGS } \\
\text { streamgage } \\
\text { number }\end{array}$} & \multirow[b]{2}{*}{ Streamgage name } & \multirow{2}{*}{$\begin{array}{l}\text { Used in regression } \\
\text { analyses (non- } \\
\text { redundant) }\end{array}$} & \multirow[b]{2}{*}{ Frequency analysis method } & \multicolumn{8}{|c|}{ Discharge for given annual exceedance probability, $\mathrm{ft}^{3} / \mathrm{s}$} \\
\hline & & & & 0.50 & 0.20 & 0.10 & 0.04 & 0.02 & 0.01 & 0.005 & 0.002 \\
\hline \multirow{5}{*}{05536178} & \multirow{5}{*}{ PLUM CREEK NEAR DYER, IN } & \multirow{5}{*}{ No } & $\begin{array}{l}\text { At-site, unadjusted for urbanization, EMA, } \\
\text { at-site skew }\end{array}$ & 1,170 & 1,660 & 1,990 & 2,430 & 2,760 & 3,100 & 3,440 & 3,920 \\
\hline & & & $\begin{array}{l}\text { At-site, adjusted for urbanization, EMA, at- } \\
\text { site skew }\end{array}$ & 1,780 & 2,430 & 2,880 & 3,460 & 3,910 & 4,370 & 4,850 & 5,510 \\
\hline & & & $\begin{array}{l}\text { At-site, adjusted for urbanization, EMA, } \\
\text { weighted skew: }\left(Q_{p}\right) q_{g},\end{array}$ & 1,790 & 2,450 & 2,890 & 3,440 & 3,850 & 4,260 & 4,680 & 5,240 \\
\hline & & & Regional regression: $(Q p)_{g, r}$ & 632 & 961 & 1,190 & 1,480 & 1,710 & 1,930 & 2,150 & 2,440 \\
\hline & & & $\begin{array}{c}\left(Q_{p}\right)_{g, w} \text { : Weighted at-site }\left(Q_{\rho}\right)_{g, s} \text { and regional } \\
\left(Q_{\rho} g_{g, r}\right.\end{array}$ & 1,690 & 2,320 & 2,710 & 3,190 & 3,540 & 3,890 & 4,230 & 4,670 \\
\hline \multirow{5}{*}{05536179} & \multirow{5}{*}{ HART DITCH AT DYER, IN } & \multirow{5}{*}{ No } & $\begin{array}{l}\text { At-site, unadjusted for urbanization, EMA, } \\
\text { at-site skew }\end{array}$ & 1,210 & 1,850 & 2,330 & 3,010 & 3,560 & 4,160 & 4,810 & 5,750 \\
\hline & & & $\begin{array}{l}\text { At-site, adjusted for urbanization, EMA, at- } \\
\text { site skew }\end{array}$ & 1,450 & 2,130 & 2,620 & 3,290 & 3,820 & 4,380 & 4,980 & 5,820 \\
\hline & & & 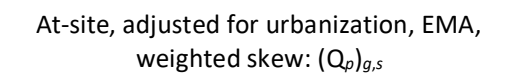 & 1,460 & 2,130 & 2,610 & 3,240 & 3,730 & 4,240 & 4,770 & 5,510 \\
\hline & & & Regional regression: $\left(Q_{p}\right)_{g, r}$ & 602 & 891 & 1,090 & 1,350 & 1,540 & 1,730 & 1,920 & 2,170 \\
\hline & & & $\begin{array}{c}\left(Q_{p}\right)_{g, w}: \text { Weighted at-site }\left(Q_{\rho}\right)_{g, s} \text { and regional } \\
\left(Q_{\rho}\right)_{g, r}\end{array}$ & 1,390 & 2,010 & 2,430 & 2,950 & 3,340 & 3,720 & 4,100 & 4,590 \\
\hline \multirow{5}{*}{05536190} & \multirow{5}{*}{ HART DITCH AT MUNSTER, IN } & \multirow{5}{*}{ Yes } & $\begin{array}{l}\text { At-site, unadjusted for urbanization, EMA, } \\
\text { at-site skew }\end{array}$ & 1,640 & 2,350 & 2,800 & 3,340 & 3,730 & 4,090 & 4,450 & 4,900 \\
\hline & & & $\begin{array}{l}\text { At-site, adjusted for urbanization, EMA, at- } \\
\text { site skew }\end{array}$ & 2,160 & 2,880 & 3,340 & 3,880 & 4,260 & 4,640 & 5,000 & 5,480 \\
\hline & & & $\begin{array}{l}\text { At-site, adjusted for urbanization, EMA, } \\
\text { weighted skew: (Q }\left(Q_{p}\right)_{g, s}\end{array}$ & 2,140 & 2,880 & 3,350 & 3,940 & 4,370 & 4,800 & 5,220 & 5,790 \\
\hline & & & Regional regression: $(Q p)_{a, r}$ & 896 & 1,230 & 1,460 & 1,760 & 1,990 & 2,220 & 2,450 & 2,770 \\
\hline & & & $\begin{array}{c}\left(Q_{p}\right)_{Q, w} \text { : Weighted at-site }\left(Q_{\rho}\right)_{Q s, \text { and regional }} \\
\left(Q_{\rho}\right)_{Q, r}\end{array}$ & 2,120 & 2,850 & 3,310 & 3,870 & 4,270 & 4,660 & 5,050 & 5,550 \\
\hline \multirow{5}{*}{05536201} & \multirow{5}{*}{ THORN CREEK AT PARK FOREST, IL } & \multirow{5}{*}{ Yes } & $\begin{array}{l}\text { At-site, unadjusted for urbanization, EMA, } \\
\text { at-site skew }\end{array}$ & 294 & 598 & 864 & 1,280 & 1,640 & 2,050 & 2,520 & 3,220 \\
\hline & & & $\begin{array}{l}\text { At-site, adjusted for urbanization, EMA, at- } \\
\text { site skew }\end{array}$ & 404 & 759 & 1,080 & 1,600 & 2,070 & 2,640 & 3,310 & 4,380 \\
\hline & & & $\begin{array}{l}\text { At-site, adjusted for urbanization, EMA, } \\
\text { weighted skew: (Q }\left(Q_{p}\right)_{g, s}\end{array}$ & 408 & 765 & 1,080 & 1,570 & 2,010 & 2,520 & 3,120 & 4,040 \\
\hline & & & Regional regression: $\left(Q_{p}\right)_{g, r}$ & 246 & 393 & 503 & 651 & 768 & 887 & 1,010 & 1,180 \\
\hline & & & $\begin{array}{c}\left(Q_{\rho}\right)_{Q, w} \text { : Weighted at-site }\left(Q_{\rho}\right)_{g, s} \text { and regional } \\
\left(Q_{\rho}\right)_{g, r}\end{array}$ & 382 & 692 & 938 & 1,280 & 1,550 & 1,820 & 2,100 & 2,460 \\
\hline
\end{tabular}




\begin{tabular}{|c|c|c|c|c|c|c|c|c|c|c|c|}
\hline \multirow{2}{*}{$\begin{array}{c}\text { USGS } \\
\text { streamgage } \\
\text { number }\end{array}$} & \multirow[b]{2}{*}{ Streamgage name } & \multirow{2}{*}{$\begin{array}{l}\text { Used in regression } \\
\text { analyses (non- } \\
\text { redundant) }\end{array}$} & \multirow[b]{2}{*}{ Frequency analysis method } & \multicolumn{8}{|c|}{ Discharge for given annual exceedance probability, $\mathrm{ft}^{3} / \mathrm{s}$} \\
\hline & & & & 0.50 & 0.20 & 0.10 & 0.04 & 0.02 & 0.01 & 0.005 & 0.002 \\
\hline \multirow{5}{*}{05536207} & \multirow{5}{*}{$\begin{array}{l}\text { THORN CREEK TRIBUTARY AT CHICAGO HEIGHTS, } \\
\text { IL }\end{array}$} & \multirow{5}{*}{ Yes } & $\begin{array}{l}\text { At-site, unadjusted for urbanization, EMA, } \\
\text { at-site skew }\end{array}$ & 247 & 431 & 607 & 909 & 1,210 & 1,580 & 2,060 & 2,870 \\
\hline & & & $\begin{array}{l}\text { At-site, adjusted for urbanization, EMA, at- } \\
\text { site skew }\end{array}$ & 257 & 444 & 623 & 931 & 1,240 & 1,620 & 2,100 & 2,930 \\
\hline & & & 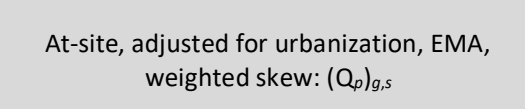 & 263 & 451 & 619 & 892 & 1,150 & 1,450 & 1,820 & 2,420 \\
\hline & & & Regional regression: $(Q p)_{a, r}$ & 234 & 377 & 491 & 655 & 791 & 937 & 1,090 & 1,320 \\
\hline & & & $\begin{array}{c}\left(Q_{\rho \rho}\right)_{y, w} \text { : Weighted at-site }\left(Q_{p}\right)_{Q, s} \text { and regional } \\
\left(Q_{\rho}\right)_{Q, r}\end{array}$ & 260 & 439 & 591 & 824 & 1,030 & 1,250 & 1,510 & 1,870 \\
\hline \multirow{5}{*}{05536210} & \multirow{5}{*}{ THORN CREEK NEAR CHICAGO HEIGHTS, IL } & \multirow{5}{*}{ No } & $\begin{array}{l}\text { At-site, unadjusted for urbanization, EMA, } \\
\text { at-site skew }\end{array}$ & 968 & 1,420 & 1,750 & 2,210 & 2,590 & 2,990 & 3,420 & 4,040 \\
\hline & & & $\begin{array}{l}\text { At-site, adjusted for urbanization, EMA, at- } \\
\text { site skew }\end{array}$ & 1,100 & 1,590 & 1,950 & 2,460 & 2,880 & 3,320 & 3,810 & 4,520 \\
\hline & & & $\begin{array}{l}\text { At-site, adjusted for urbanization, EMA, } \\
\text { weighted skew: }\left(Q_{p}\right)_{g, s}\end{array}$ & 1,110 & 1,590 & 1,950 & 2,450 & 2,850 & 3,290 & 3,760 & 4,440 \\
\hline & & & Regional regression: $\left(Q_{p}\right)_{g, r}$ & 529 & 795 & 992 & 1,260 & 1,470 & 1,690 & 1,920 & 2,230 \\
\hline & & & $\begin{array}{c}(Q p)_{g, w} \text { : Weighted at-site }\left(Q_{\rho}\right)_{g, s} \text { and regional } \\
\left(Q_{\rho}\right)_{g, r}\end{array}$ & 1,060 & 1,500 & 1,810 & 2,230 & 2,560 & 2,890 & 3,250 & 3,730 \\
\hline \multirow{5}{*}{05536215} & \multirow{5}{*}{ THORN CREEK AT GLENWOOD, IL } & \multirow{5}{*}{ No } & $\begin{array}{l}\text { At-site, unadjusted for urbanization, EMA, } \\
\text { at-site skew }\end{array}$ & 1,120 & 1,640 & 2,020 & 2,530 & 2,950 & 3,390 & 3,860 & 4,540 \\
\hline & & & $\begin{array}{l}\text { At-site, adjusted for urbanization, EMA, at- } \\
\text { site skew }\end{array}$ & 1,260 & 1,780 & 2,160 & 2,680 & 3,090 & 3,530 & 3,990 & 4,650 \\
\hline & & & 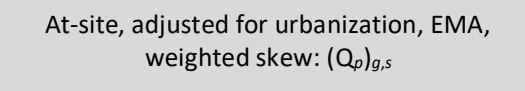 & 1,260 & 1,780 & 2,160 & 2,690 & 3,110 & 3,560 & 4,040 & 4,730 \\
\hline & & & Regional regression: $(Q p)_{g, r}$ & 746 & 1,110 & 1,370 & 1,740 & 2,030 & 2,330 & 2,640 & 3,070 \\
\hline & & & $\begin{array}{c}\left(Q_{\rho \rho g}\right)_{y, w} \text { Weighted at-site }\left(Q_{\rho}\right)_{g, s} \text { and regional } \\
\left(Q_{\rho}\right)_{Q, r}\end{array}$ & 1,250 & 1,760 & 2,140 & 2,640 & 3,040 & 3,460 & 3,900 & 4,520 \\
\hline \multirow{5}{*}{05536235} & \multirow{5}{*}{ DEER CREEK NEAR CHICAGO HEIGHTS, IL } & \multirow{5}{*}{ Yes } & $\begin{array}{l}\text { At-site, unadjusted for urbanization, EMA, } \\
\text { at-site skew }\end{array}$ & 566 & 770 & 914 & 1,110 & 1,260 & 1,420 & 1,580 & 1,810 \\
\hline & & & $\begin{array}{l}\text { At-site, adjusted for urbanization, EMA, at- } \\
\text { site skew }\end{array}$ & 778 & 989 & 1,130 & 1,320 & 1,460 & 1,600 & 1,740 & 1,940 \\
\hline & & & $\begin{array}{l}\text { At-site, adjusted for urbanization, EMA, } \\
\left.\text { weighted skew: ( } Q_{0}\right)_{g, s}\end{array}$ & 780 & 991 & 1,130 & 1,310 & 1,450 & 1,580 & 1,720 & 1,910 \\
\hline & & & Regional regression: $\left(Q_{p}\right)_{g, r}$ & 621 & 937 & 1,170 & 1,470 & 1,710 & 1,950 & 2,200 & 2,530 \\
\hline & & & $\begin{array}{l}\left(Q_{\rho \rho g}\right)_{y, w} \text { Weighted at-site }\left(Q_{\rho}\right)_{g, s} \text { and regional } \\
\left(Q_{\rho}\right)_{g, r}\end{array}$ & 778 & 990 & 1,130 & 1,310 & 1,450 & 1,590 & 1,740 & 1,940 \\
\hline
\end{tabular}




\begin{tabular}{|c|c|c|c|c|c|c|c|c|c|c|c|}
\hline \multirow{2}{*}{$\begin{array}{c}\text { USGS } \\
\text { streamgage } \\
\text { number }\end{array}$} & \multirow[b]{2}{*}{ Streamgage name } & \multirow{2}{*}{$\begin{array}{l}\text { Used in regression } \\
\text { analyses (non- } \\
\text { redundant) }\end{array}$} & \multirow[b]{2}{*}{ Frequency analysis method } & \multicolumn{8}{|c|}{ Discharge for given annual exceedance probability, $\mathrm{ft}^{3} / \mathrm{s}$} \\
\hline & & & & 0.50 & 0.20 & 0.10 & 0.04 & 0.02 & 0.01 & 0.005 & 0.002 \\
\hline \multirow{5}{*}{05536238} & \multirow{5}{*}{ BUTTERFFELD CREEK NEAR LINCOLN ESTATES, IL } & \multirow{5}{*}{ Yes } & $\begin{array}{l}\text { At-site, unadjusted for urbanization, EMA, } \\
\text { at-site skew }\end{array}$ & 140 & 270 & 370 & 507 & 616 & 728 & 844 & 1,000 \\
\hline & & & $\begin{array}{l}\text { At-site, adjusted for urbanization, EMA, at- } \\
\text { site skew }\end{array}$ & 194 & 341 & 452 & 604 & 724 & 849 & 979 & 1,160 \\
\hline & & & $\begin{array}{l}\text { At-site, adjusted for urbanization, EMA, } \\
\text { weighted skew: ( }\left(_{p}\right)_{g, s}\end{array}$ & 187 & 336 & 462 & 651 & 817 & 1,000 & 1,220 & 1,540 \\
\hline & & & Regional regression: $(Q p)_{a, r}$ & 91 & 140 & 177 & 227 & 267 & 307 & 349 & 406 \\
\hline & & & $\begin{array}{c}\left(Q_{p}\right)_{Q, w} \text { : Weighted at-site }\left(Q_{\rho}\right)_{g, s} \text { and regional } \\
\left(Q_{\rho}\right)_{g, r}\end{array}$ & 171 & 295 & 387 & 511 & 607 & 701 & 798 & 922 \\
\hline \multirow{5}{*}{05536255} & \multirow{5}{*}{ BUTTERFIELD CREEK AT FLOSSMOOR, IL } & \multirow{5}{*}{ Yes } & $\begin{array}{l}\text { At-site, unadjusted for urbanization, EMA, } \\
\text { at-site skew }\end{array}$ & 717 & 1,200 & 1,580 & 2,150 & 2,640 & 3,190 & 3,800 & 4,710 \\
\hline & & & $\begin{array}{l}\text { At-site, adjusted for urbanization, EMA, at- } \\
\text { site skew }\end{array}$ & 840 & 1,330 & 1,740 & 2,370 & 2,920 & 3,560 & 4,290 & 5,420 \\
\hline & & & $\begin{array}{l}\text { At-site, adjusted for urbanization, EMA, } \\
\text { weighted skew: ( }\left(Q_{p}\right)_{g, s}\end{array}$ & 844 & 1,340 & 1,740 & 2,350 & 2,890 & 3,500 & 4,190 & 5,260 \\
\hline & & & Regional regression: $\left(Q_{0}\right)_{g, r}$ & 643 & 936 & 1,150 & 1,450 & 1,680 & 1,920 & 2,170 & 2,510 \\
\hline & & & $\begin{array}{c}\left(Q_{p}\right)_{Q, w} \text { : Weighted at-site }\left(Q_{\rho}\right)_{g, s} \text { and regional } \\
\left(Q_{\rho}\right)_{g, r}\end{array}$ & 838 & 1,320 & 1,700 & 2,270 & 2,740 & 3,250 & 3,790 & 4,580 \\
\hline \multirow{5}{*}{05536265} & \multirow{5}{*}{ LANSING DITCH NEAR LANSING, IL } & \multirow{5}{*}{ Yes } & $\begin{array}{l}\text { At-site, unadjusted for urbanization, EMA, } \\
\text { at-site skew }\end{array}$ & 171 & 250 & 302 & 369 & 418 & 468 & 517 & 583 \\
\hline & & & $\begin{array}{l}\text { At-site, adjusted for urbanization, EMA, at- } \\
\text { site skew }\end{array}$ & 136 & 194 & 230 & 274 & 304 & 333 & 361 & 397 \\
\hline & & & $\begin{array}{l}\text { At-site, adjusted for urbanization, EMA, } \\
\text { weighted skew: }\left(Q_{p}\right)_{\text {g,s }}\end{array}$ & 134 & 194 & 233 & 283 & 320 & 358 & 395 & 444 \\
\hline & & & Regional regression: $(Q p)_{g, r}$ & 285 & 438 & 550 & 702 & 820 & 942 & 1,070 & 1,240 \\
\hline & & & $\begin{array}{c}\left(Q_{\rho}\right)_{g, w} \text { : Weighted at-site }\left(Q_{\rho}\right)_{g, s} \text { and regional } \\
\left(Q_{\rho} g_{g, r}\right.\end{array}$ & 136 & 197 & 238 & 292 & 333 & 375 & 419 & 479 \\
\hline \multirow{5}{*}{05536270} & \multirow{5}{*}{ NORTH CREEK NEAR LANSING, IL } & \multirow{5}{*}{ Yes } & $\begin{array}{l}\text { At-site, unadjusted for urbanization, EMA, } \\
\text { at-site skew }\end{array}$ & 354 & 512 & 614 & 737 & 826 & 912 & 997 & 1,110 \\
\hline & & & $\begin{array}{l}\text { At-site, adjusted for urbanization, EMA, at- } \\
\text { site skew }\end{array}$ & 479 & 699 & 853 & 1,060 & 1,210 & 1,380 & 1,540 & 1,770 \\
\hline & & & $\begin{array}{l}\text { At-site, adjusted for urbanization, EMA, } \\
\text { weighted skew: (Qop) })_{g, s}\end{array}$ & 474 & 697 & 858 & 1,080 & 1,250 & 1,440 & 1,630 & 1,910 \\
\hline & & & Regional regression: $\left(Q_{p}\right)_{a, r}$ & 366 & 521 & 632 & 780 & 895 & 1,010 & 1,130 & 1,300 \\
\hline & & & $\begin{array}{c}\left(Q_{p}\right)_{g, w}: \text { Weighted at-site } \\
\qquad\left(Q_{\rho}\right)_{g, s,} \text { and regional }\end{array}$ & 470 & 688 & 844 & 1,050 & 1,210 & 1,380 & 1,550 & 1,790 \\
\hline
\end{tabular}




\begin{tabular}{|c|c|c|c|c|c|c|c|c|c|c|c|}
\hline \multirow{2}{*}{$\begin{array}{c}\text { USGS } \\
\text { streamgage } \\
\text { number }\end{array}$} & \multirow[b]{2}{*}{ Streamgage name } & \multirow{2}{*}{$\begin{array}{l}\text { Used in regression } \\
\text { analyses (non- } \\
\text { redundant) }\end{array}$} & \multirow[b]{2}{*}{ Frequency analysis method } & \multicolumn{8}{|c|}{ Discharge for given annual exceedance probability, $\mathrm{ft}^{3} / \mathrm{s}$} \\
\hline & & & & 0.50 & 0.20 & 0.10 & 0.04 & 0.02 & 0.01 & 0.005 & 0.002 \\
\hline \multirow{5}{*}{05536275} & \multirow{5}{*}{ THORN CREEK AT THORNTON, IL } & \multirow{5}{*}{ No } & $\begin{array}{l}\text { At-site, unadjusted for urbanization, EMA, } \\
\text { at-site skew }\end{array}$ & 2,160 & 3,210 & 3,940 & 4,890 & 5,630 & 6,380 & 7,140 & 8,190 \\
\hline & & & $\begin{array}{l}\text { At-site, adjusted for urbanization, EMA, at- } \\
\text { site skew }\end{array}$ & 2,460 & 3,560 & 4,330 & 5,350 & 6,150 & 6,980 & 7,830 & 9,020 \\
\hline & & & $\begin{array}{l}\text { At-site, adjusted for urbanization, EMA, } \\
\left.\text { weighted skew: ( } 0_{0}\right)_{g, s}\end{array}$ & 2,440 & 3,550 & 4,340 & 5,420 & 6,260 & 7,150 & 8,090 & 9,410 \\
\hline & & & Regional regression: $(Q p)_{g, r}$ & 1,750 & 2,470 & 2,990 & 3,680 & 4,210 & 4,760 & 5,330 & 6,110 \\
\hline & & & $\begin{array}{c}\left(Q_{\rho \rho}\right)_{y, w} \text { : Weighted at-site }\left(Q_{\rho}\right)_{Q, s} \text { and regional } \\
\left(Q_{\rho}\right)_{Q, r}\end{array}$ & 2,430 & 3,520 & 4,290 & 5,320 & 6,130 & 6,960 & 7,820 & 9,000 \\
\hline \multirow{5}{*}{05536290} & \multirow{5}{*}{ LITTLE CALUMET RIVER AT SOUTH HOLLAND, IL } & \multirow{5}{*}{ No } & $\begin{array}{l}\text { At-site, unadjusted for urbanization, EMA, } \\
\text { at-site skew }\end{array}$ & 2,520 & 3,300 & 3,780 & 4,330 & 4,710 & 5,070 & 5,410 & 5,840 \\
\hline & & & $\begin{array}{l}\text { At-site, adjusted for urbanization, EMA, at- } \\
\text { site skew }\end{array}$ & 2,960 & 3,920 & 4,560 & 5,380 & 5,990 & 6,620 & 7,250 & 8,110 \\
\hline & & & $\begin{array}{l}\text { At-site, adjusted for urbanization, EMA, } \\
\text { weighted skew: ( (opog,s }\end{array}$ & 2,950 & 3,920 & 4,570 & 5,410 & 6,050 & 6,710 & 7,380 & 8,300 \\
\hline & & & Regional regression: $\left(Q_{p}\right)_{g, r}$ & 2,270 & 3,040 & 3,570 & 4,280 & 4,820 & 5,370 & 5,940 & 6,710 \\
\hline & & & 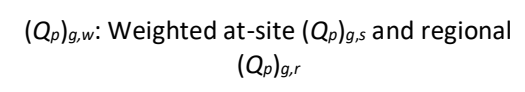 & 2,940 & 3,900 & 4,550 & 5,380 & 6,010 & 6,650 & 7,300 & 8,190 \\
\hline \multirow{5}{*}{05536310} & \multirow{5}{*}{$\begin{array}{l}\text { CALUMET UNION DRAINAGE CANAL NEAR } \\
\text { MARKHAM, IL }\end{array}$} & \multirow{5}{*}{ Yes } & $\begin{array}{l}\text { At-site, unadjusted for urbanization, EMA, } \\
\text { at-site skew }\end{array}$ & 321 & 411 & 458 & 504 & 533 & 557 & 578 & 601 \\
\hline & & & $\begin{array}{l}\text { At-site, adjusted for urbanization, EMA, at- } \\
\text { site skew }\end{array}$ & 364 & 468 & 524 & 586 & 625 & 660 & 692 & 730 \\
\hline & & & $\begin{array}{l}\text { At-site, adjusted for urbanization, EMA, } \\
\text { weighted skew: }\left(Q_{0}\right) g, s\end{array}$ & 351 & 461 & 534 & 627 & 696 & 765 & 834 & 929 \\
\hline & & & Regional regression: $(Q p)_{g, r}$ & 526 & 781 & 974 & 1,240 & 1,460 & 1,680 & 1,920 & 2,240 \\
\hline & & & $\begin{array}{c}\left(Q_{\rho \rho}\right)_{y, w} \text { Weighted at-site }\left(Q_{\rho}\right)_{Q, s} \text { and regional } \\
\left(Q_{\rho}\right)_{Q, r}\end{array}$ & 356 & 471 & 549 & 653 & 733 & 817 & 904 & 1,030 \\
\hline \multirow{5}{*}{05536335} & \multirow{5}{*}{ MIDLOTHIAN CREEK NEAR TINLEY PARK, IL } & \multirow{5}{*}{ No } & $\begin{array}{l}\text { At-site, unadjusted for urbanization, EMA, } \\
\text { at-site skew }\end{array}$ & 218 & 308 & 383 & 496 & 597 & 713 & 846 & 1,060 \\
\hline & & & $\begin{array}{l}\text { At-site, adjusted for urbanization, EMA, at- } \\
\text { site skew }\end{array}$ & 323 & 450 & 547 & 685 & 800 & 925 & 1,060 & 1,270 \\
\hline & & & $\begin{array}{l}\text { At-site, adjusted for urbanization, EMA, } \\
\left.\text { weighted skew: ( } Q_{0}\right)_{g, s}\end{array}$ & 324 & 451 & 547 & 683 & 796 & 918 & 1,050 & 1,250 \\
\hline & & & Regional regression: $\left(Q_{p}\right)_{q, r}$ & 330 & 471 & 575 & 720 & 834 & 954 & 1,080 & 1,250 \\
\hline & & & $\begin{array}{l}(Q p)_{g, w:} \text { : Weighted at-site }\left(Q_{0}\right)_{g, s} \text { and regional } \\
\left(Q_{0}\right)_{q_{1}}\end{array}$ & 324 & 452 & 549 & 686 & 800 & 923 & 1,060 & 1,250 \\
\hline
\end{tabular}




\begin{tabular}{|c|c|c|c|c|c|c|c|c|c|c|c|}
\hline \multirow{2}{*}{$\begin{array}{c}\text { USGS } \\
\text { streamgage } \\
\text { number }\end{array}$} & \multirow[b]{2}{*}{ Streamgage name } & \multirow{2}{*}{$\begin{array}{l}\text { Used in regression } \\
\text { analyses (non- } \\
\text { redundant) }\end{array}$} & \multirow[b]{2}{*}{ Frequency analysis method } & \multicolumn{8}{|c|}{ Discharge for given annual exceedance probability, $\mathrm{ft}^{3} / \mathrm{s}$} \\
\hline & & & & 0.50 & 0.20 & 0.10 & 0.04 & 0.02 & 0.01 & 0.005 & 0.002 \\
\hline \multirow{5}{*}{05536340} & \multirow{5}{*}{ MIDLOTHIAN CREEK AT OAK FOREST, IL } & \multirow{5}{*}{ Yes } & $\begin{array}{l}\text { At-site, unadjusted for urbanization, EMA, } \\
\text { at-site skew }\end{array}$ & 237 & 320 & 378 & 454 & 514 & 576 & 641 & 732 \\
\hline & & & $\begin{array}{l}\text { At-site, adjusted for urbanization, EMA, at- } \\
\text { site skew }\end{array}$ & 198 & 265 & 307 & 360 & 398 & 436 & 474 & 524 \\
\hline & & & $\begin{array}{l}\text { At-site, adjusted for urbanization, EMA, } \\
\text { weighted skew: }\left(Q_{p}\right)_{g, s}\end{array}$ & 196 & 264 & 309 & 368 & 412 & 457 & 502 & 564 \\
\hline & & & Regional regression: $(Q p)_{a, r}$ & 391 & 553 & 673 & 837 & 966 & 1,100 & 1,240 & 1,430 \\
\hline & & & $\begin{array}{c}\left(Q_{\rho p}\right)_{y, w} \text { : Weighted at-site }\left(Q_{p}\right)_{Q, s} \text { and regional } \\
\left(\left.Q_{p}\right|_{Q, r}\right.\end{array}$ & 198 & 267 & 314 & 376 & 424 & 474 & 526 & 599 \\
\hline \multirow{5}{*}{05536460} & \multirow{5}{*}{ TINLEY CREEK NEAR OAK FOREST, IL } & \multirow{5}{*}{ No } & $\begin{array}{l}\text { At-site, unadjusted for urbanization, EMA, } \\
\text { at-site skew }\end{array}$ & 432 & 667 & 796 & 928 & 1,010 & 1,070 & 1,130 & 1,190 \\
\hline & & & $\begin{array}{l}\text { At-site, adjusted for urbanization, EMA, at- } \\
\text { site skew }\end{array}$ & 595 & 879 & 1,060 & 1,260 & 1,400 & 1,540 & 1,660 & 1,820 \\
\hline & & & $\begin{array}{l}\text { At-site, adjusted for urbanization, EMA, } \\
\text { weighted skew: ( }\left(_{0}\right)_{g, s}\end{array}$ & 569 & 864 & 1,080 & 1,380 & 1,620 & 1,870 & 2,130 & 2,510 \\
\hline & & & Regional regression: $\left(Q_{0}\right)_{g, r}$ & 307 & 458 & 572 & 729 & 854 & 983 & 1,120 & 1,310 \\
\hline & & & $\begin{array}{c}\left(Q_{\rho \rho}\right)_{y, w} \text { Weighted at-site }\left(Q_{p}\right)_{Q, s} \text { and regional } \\
\left(Q_{p}\right)_{Q, r}\end{array}$ & 546 & 820 & 1,010 & 1,270 & 1,470 & 1,670 & 1,870 & 2,150 \\
\hline \multirow{5}{*}{05536500} & \multirow{5}{*}{ TINLEY CREEK NEAR PALOS PARK, IL } & \multirow{5}{*}{ Yes } & $\begin{array}{l}\text { At-site, unadjusted for urbanization, EMA, } \\
\text { at-site skew }\end{array}$ & 609 & 974 & 1,260 & 1,660 & 1,990 & 2,350 & 2,740 & 3,310 \\
\hline & & & $\begin{array}{l}\text { At-site, adjusted for urbanization, EMA, at- } \\
\text { site skew }\end{array}$ & 712 & 1,100 & 1,420 & 1,870 & 2,260 & 2,690 & 3,180 & 3,910 \\
\hline & & & $\begin{array}{l}\text { At-site, adjusted for urbanization, EMA, } \\
\text { weighted skew: }\left(Q_{p}\right)_{\text {g,s }}\end{array}$ & 712 & 1,100 & 1,420 & 1,870 & 2,260 & 2,690 & 3,170 & 3,900 \\
\hline & & & Regional regression: $\left(\left.Q p\right|_{g r}\right.$ & 379 & 564 & 701 & 888 & 1,040 & 1,190 & 1,350 & 1,570 \\
\hline & & & $\begin{array}{c}\left(Q_{\rho}\right)_{g, w} \text { : Weighted at-site }\left(Q_{\rho}\right)_{Q, s} \text { and regional } \\
\left(Q_{\rho}\right)_{Q, r}\end{array}$ & 701 & 1,080 & 1,370 & 1,770 & 2,110 & 2,460 & 2,830 & 3,340 \\
\hline \multirow{5}{*}{05536510} & \multirow{5}{*}{ NAVAJO CREEK AT PALOS HEIGHTS, IL } & \multirow{5}{*}{ Yes } & $\begin{array}{l}\text { At-site, unadjusted for urbanization, EMA, } \\
\text { at-site skew }\end{array}$ & 230 & 310 & 368 & 446 & 508 & 574 & 644 & 743 \\
\hline & & & $\begin{array}{l}\text { At-site, adjusted for urbanization, EMA, at- } \\
\text { site skew }\end{array}$ & 254 & 336 & 395 & 474 & 538 & 604 & 674 & 775 \\
\hline & & & $\begin{array}{l}\text { At-site, adjusted for urbanization, EMA, } \\
\text { weighted skew: (Qop) })_{g, s}\end{array}$ & 254 & 336 & 395 & 473 & 535 & 600 & 668 & 765 \\
\hline & & & Regional regression: $\left(Q_{p}\right)_{a, r}$ & 133 & 217 & 283 & 377 & 454 & 535 & 621 & 741 \\
\hline & & & $\begin{array}{c}(Q p)_{Q, w} \text { : Weighted at-site } \\
\left.\qquad\left(Q_{p}\right)_{Q, r}\right)_{g, s} \text { and regional }\end{array}$ & 250 & 330 & 388 & 465 & 527 & 592 & 662 & 762 \\
\hline
\end{tabular}




\begin{tabular}{|c|c|c|c|c|c|c|c|c|c|c|c|}
\hline \multirow{2}{*}{$\begin{array}{c}\text { USGS } \\
\text { streamgage } \\
\text { number }\end{array}$} & \multirow[b]{2}{*}{ Streamgage name } & \multirow{2}{*}{$\begin{array}{l}\text { Used in regression } \\
\text { analyses (non- } \\
\text { redundant) }\end{array}$} & \multirow[b]{2}{*}{ Frequency analysis method } & \multicolumn{8}{|c|}{ Discharge for given annual exceedance probability, $\mathrm{ft}^{3} / \mathrm{s}$} \\
\hline & & & & 0.50 & 0.20 & 0.10 & 0.04 & 0.02 & 0.01 & 0.005 & 0.002 \\
\hline \multirow{5}{*}{05536560} & \multirow{5}{*}{ MELVINA DITCH NEAR OAK LAWN, IL } & \multirow{5}{*}{ Yes } & $\begin{array}{l}\text { At-site, unadjusted for urbanization, EMA, } \\
\text { at-site skew }\end{array}$ & 139 & 234 & 303 & 395 & 467 & 540 & 616 & 720 \\
\hline & & & $\begin{array}{l}\text { At-site, adjusted for urbanization, EMA, at- } \\
\text { site skew }\end{array}$ & 241 & 341 & 406 & 488 & 549 & 609 & 670 & 750 \\
\hline & & & $\begin{array}{l}\text { At-site, adjusted for urbanization, EMA, } \\
\text { weighted skew: }\left(Q_{p}\right)_{g, s}\end{array}$ & 234 & 337 & 413 & 518 & 602 & 692 & 788 & 927 \\
\hline & & & Regional regression: $(Q p)_{g, r}$ & 287 & 416 & 512 & 644 & 749 & 858 & 971 & 1,130 \\
\hline & & & $\begin{array}{c}\left(Q_{p}\right)_{Q, w} \text { : Weighted at-site }\left(Q_{\rho}\right)_{Q s, \text { and regional }} \\
\left(Q_{\rho}\right)_{Q, r}\end{array}$ & 237 & 342 & 420 & 530 & 619 & 714 & 816 & 963 \\
\hline \multirow{5}{*}{05536570} & \multirow{5}{*}{ STONY CREEK (WEST) AT WORTH, IL } & \multirow{5}{*}{ No } & $\begin{array}{l}\text { At-site, unadjusted for urbanization, EMA, } \\
\text { at-site skew }\end{array}$ & 406 & 622 & 732 & 838 & 897 & 944 & 980 & 1,020 \\
\hline & & & $\begin{array}{l}\text { At-site, adjusted for urbanization, EMA, at- } \\
\text { site skew }\end{array}$ & 410 & 625 & 736 & 842 & 902 & 949 & 986 & 1,020 \\
\hline & & & $\begin{array}{l}\text { At-site, adjusted for urbanization, EMA, } \\
\text { weighted skew: }\left(Q_{p}\right)_{g, s}\end{array}$ & 364 & 623 & 826 & 1,110 & 1,350 & 1,610 & 1,890 & 2,290 \\
\hline & & & Regional regression: $\left(Q_{p}\right)_{g, r}$ & 560 & 773 & 929 & 1,140 & 1,310 & 1,480 & 1,660 & 1,900 \\
\hline & & & $\begin{array}{c}\left(Q_{\rho}\right)_{g, w:} \text { : Weighted at-site } \\
\qquad\left(Q_{\rho \rho}\right)_{g, s} \text { and regional } \\
\left(Q_{\rho}\right)_{g, r}\end{array}$ & 395 & 654 & 851 & 1,120 & 1,340 & 1,560 & 1,790 & 2,110 \\
\hline \multirow{5}{*}{05536620} & \multirow{5}{*}{ MILL CREEK NEAR PALOS PARK, IL } & \multirow{5}{*}{ No } & $\begin{array}{l}\text { At-site, unadjusted for urbanization, EMA, } \\
\text { at-site skew }\end{array}$ & 120 & 184 & 228 & 283 & 324 & 364 & 404 & 458 \\
\hline & & & $\begin{array}{l}\text { At-site, adjusted for urbanization, EMA, at- } \\
\text { site skew }\end{array}$ & 148 & 223 & 275 & 342 & 394 & 447 & 501 & 574 \\
\hline & & & $\begin{array}{l}\text { At-site, adjusted for urbanization, EMA, } \\
\text { weighted skew: }\left(Q_{p}\right) \text { g,s }\end{array}$ & 144 & 221 & 279 & 360 & 427 & 499 & 577 & 690 \\
\hline & & & Regional regression: $(Q p)_{g, r}$ & 156 & 222 & 270 & 335 & 385 & 437 & 490 & 563 \\
\hline & & & 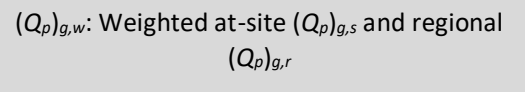 & 145 & 221 & 278 & 356 & 419 & 486 & 556 & 653 \\
\hline \multirow{5}{*}{05536630} & \multirow{5}{*}{ MILL CREEK AT PALOS PARK, IL } & \multirow{5}{*}{ Yes } & $\begin{array}{l}\text { At-site, unadjusted for urbanization, EMA, } \\
\text { at-site skew }\end{array}$ & 197 & 442 & 712 & 1,240 & 1,820 & 2,610 & 3,690 & 5,710 \\
\hline & & & $\begin{array}{l}\text { At-site, adjusted for urbanization, EMA, at- } \\
\text { site skew }\end{array}$ & 239 & 512 & 822 & 1,440 & 2,150 & 3,140 & 4,540 & 7,280 \\
\hline & & & $\begin{array}{l}\text { At-site, adjusted for urbanization, EMA, } \\
\text { weighted skew: }\left(\mathrm{Q}_{\rho}\right)_{g, s}\end{array}$ & 253 & 533 & 821 & 1,340 & 1,880 & 2,570 & 3,460 & 5,030 \\
\hline & & & Regional regression: $\left(Q_{p}\right)_{g, r}$ & 299 & 440 & 544 & 686 & 799 & 915 & 1,040 & 1,200 \\
\hline & & & $\begin{array}{c}\left(Q_{\rho}\right)_{g, w} \text { : Weighted at-site }\left(Q_{\rho \rho g}\right)_{g, \text { and regional }} \\
\qquad\left(Q_{\rho}\right)_{g, r}\end{array}$ & 260 & 510 & 730 & 1,060 & 1,330 & 1,610 & 1,910 & 2,290 \\
\hline
\end{tabular}




\begin{tabular}{|c|c|c|c|c|c|c|c|c|c|c|c|}
\hline \multirow{2}{*}{$\begin{array}{c}\text { USGS } \\
\text { streamgage } \\
\text { number }\end{array}$} & \multirow[b]{2}{*}{ Streamgage name } & \multirow{2}{*}{$\begin{array}{l}\text { Used in regression } \\
\text { analyses (non- } \\
\text { redundant) }\end{array}$} & \multirow[b]{2}{*}{ Frequency analysis method } & \multicolumn{8}{|c|}{ Discharge for given annual exceedance probability, $\mathrm{ft}^{3} / \mathrm{s}$} \\
\hline & & & & 0.50 & 0.20 & 0.10 & 0.04 & 0.02 & 0.01 & 0.005 & 0.002 \\
\hline \multirow{5}{*}{05537500} & \multirow{5}{*}{ LONG RUN NEAR LEMONT, IL } & \multirow{5}{*}{ Yes } & $\begin{array}{l}\text { At-site, unadjusted for urbanization, EMA, } \\
\text { at-site skew }\end{array}$ & 624 & 1,150 & 1,620 & 2,400 & 3,120 & 3,980 & 5,010 & 6,670 \\
\hline & & & $\begin{array}{l}\text { At-site, adjusted for urbanization, EMA, at- } \\
\text { site skew }\end{array}$ & 840 & 1,400 & 1,920 & 2,760 & 3,570 & 4,550 & 5,750 & 7,740 \\
\hline & & & $\begin{array}{l}\text { At-site, adjusted for urbanization, EMA, } \\
\text { weighted skew: }\left(Q_{p}\right)_{g, s}\end{array}$ & 857 & 1,420 & 1,900 & 2,670 & 3,370 & 4,190 & 5,150 & 6,680 \\
\hline & & & Regional regression: $(Q p)_{g, r}$ & 514 & 756 & 932 & 1,170 & 1,350 & 1,540 & 1,730 & 1,990 \\
\hline & & & $\begin{array}{l}\left(Q_{p}\right)_{g, w}: \text { Weighted at-site }\left(Q_{p}\right)_{g, s} \text { and regional } \\
\left(Q_{p} g_{g, r}\right.\end{array}$ & 843 & 1,380 & 1,810 & 2,460 & 2,990 & 3,550 & 4,160 & 4,990 \\
\hline \multirow{5}{*}{05538440} & \multirow{5}{*}{ SPRING CREEK NEAR ORLAND PARK, IL } & \multirow{5}{*}{ Yes } & $\begin{array}{l}\text { At-site, unadjusted for urbanization, EMA, } \\
\text { at-site skew }\end{array}$ & 41 & 61 & 75 & 93 & 107 & 122 & 136 & 156 \\
\hline & & & $\begin{array}{l}\text { At-site, adjusted for urbanization, EMA, at- } \\
\text { site skew }\end{array}$ & 66 & 94 & 115 & 145 & 170 & 198 & 228 & 272 \\
\hline & & & $\begin{array}{l}\text { At-site, adjusted for urbanization, EMA, } \\
\text { weighted skew: }\left(Q_{p}\right)_{g, s}\end{array}$ & 66 & 94 & 115 & 144 & 167 & 193 & 220 & 260 \\
\hline & & & Regional regression: $\left(Q_{p}\right)_{g, r}$ & 53 & 76 & 93 & 116 & 134 & 153 & 172 & 197 \\
\hline & & & $\begin{array}{l}(Q p)_{g, w}: \text { Weighted at-site }\left(Q_{p}\right)_{g, s} \text { and regional } \\
\qquad\left(Q_{\rho} g_{g, r}\right.\end{array}$ & 66 & 93 & 113 & 140 & 163 & 186 & 211 & 247 \\
\hline \multirow{5}{*}{05539000} & \multirow{5}{*}{ HICKORY CREEK AT JOLLT, IL } & \multirow{5}{*}{ Yes } & $\begin{array}{l}\text { At-site, unadjusted for urbanization, EMA, } \\
\text { at-site skew }\end{array}$ & 2,960 & 5,120 & 7,040 & 10,100 & 13,000 & 16,400 & 20,500 & 27,100 \\
\hline & & & $\begin{array}{l}\text { At-site, adjusted for urbanization, EMA, at- } \\
\text { site skew }\end{array}$ & 4,350 & 7,070 & 9,580 & 13,800 & 17,900 & 22,900 & 29,100 & 39,600 \\
\hline & & & $\begin{array}{l}\text { At-site, adjusted for urbanization, EMA, } \\
\text { weighted skew: }\left(Q_{p}\right)_{g, s}\end{array}$ & 4,470 & 7,190 & 9,550 & 13,300 & 16,600 & 20,600 & 25,300 & 32,900 \\
\hline & & & Regional regression: $(Q p)_{g, r}$ & 1,940 & 2,780 & 3,370 & 4,170 & 4,790 & 5,430 & 6,090 & 6,990 \\
\hline & & & 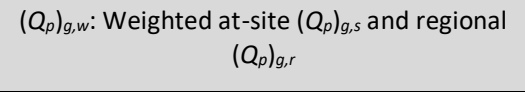 & 4,380 & 6,940 & 9,010 & 12,000 & 14,400 & 17,000 & 19,700 & 23,300 \\
\hline \multirow{5}{*}{05539870} & \multirow{5}{*}{$\begin{array}{l}\text { WEST BRANCH DI PAGE RIVER AT } \\
\text { ONTARIOVILLE, IL }\end{array}$} & \multirow{5}{*}{ No } & $\begin{array}{l}\text { At-site, unadjusted for urbanization, EMA, } \\
\text { at-site skew }\end{array}$ & 310 & 490 & 598 & 720 & 801 & 873 & 937 & 1,010 \\
\hline & & & $\begin{array}{l}\text { At-site, adjusted for urbanization, EMA, at- } \\
\text { site skew }\end{array}$ & 425 & 578 & 670 & 774 & 845 & 911 & 973 & 1,050 \\
\hline & & & $\begin{array}{l}\text { At-site, adjusted for urbanization, EMA, } \\
\text { weighted skew: }\left(Q_{0}\right) g, s\end{array}$ & 408 & 573 & 689 & 842 & 961 & 1,080 & 1,210 & 1,390 \\
\hline & & & Regional regression: $\left(Q_{p}\right)_{g, r}$ & 286 & 396 & 476 & 587 & 674 & 763 & 856 & 983 \\
\hline & & & $\begin{array}{l}\left(Q_{\rho}\right)_{g, w}: \text { Weighted at-site }\left(Q_{p}\right)_{g, s} \text { and regional } \\
\left(Q_{p} g_{g, r}\right.\end{array}$ & 401 & 559 & 668 & 811 & 921 & 1,030 & 1,150 & 1,310 \\
\hline
\end{tabular}




\begin{tabular}{|c|c|c|c|c|c|c|c|c|c|c|c|}
\hline \multirow{2}{*}{$\begin{array}{c}\text { USGS } \\
\text { streamgage } \\
\text { number }\end{array}$} & \multirow[b]{2}{*}{ Streamgage name } & \multirow{2}{*}{$\begin{array}{l}\text { Used in regression } \\
\text { analyses (non- } \\
\text { redundant) }\end{array}$} & \multirow[b]{2}{*}{ Frequency analysis method } & \multicolumn{8}{|c|}{ Discharge for given annual exceedance probability, $\mathrm{ft}^{3} / \mathrm{s}$} \\
\hline & & & & 0.50 & 0.20 & 0.10 & 0.04 & 0.02 & 0.01 & 0.005 & 0.002 \\
\hline \multirow{5}{*}{05539890} & \multirow{5}{*}{ WEST BRANCH DU PAGE RIVER NEAR WAYNE, IL } & \multirow{5}{*}{ No } & $\begin{array}{l}\text { At-site, unadjusted for urbanization, EMA, } \\
\text { at-site skew }\end{array}$ & 453 & 745 & 981 & 1,330 & 1,640 & 1,980 & 2,360 & 2,940 \\
\hline & & & $\begin{array}{l}\text { At-site, adjusted for urbanization, EMA, at- } \\
\text { site skew }\end{array}$ & 649 & 998 & 1,330 & 1,890 & 2,440 & 3,140 & 4,000 & 5,500 \\
\hline & & & $\begin{array}{l}\text { At-site, adjusted for urbanization, EMA, } \\
\left.\text { weighted skew: ( } Q_{0}\right)_{g, s}\end{array}$ & 684 & 1,040 & 1,340 & 1,780 & 2,170 & 2,610 & 3,110 & 3,890 \\
\hline & & & Regional regression: $(Q p)_{g, r}$ & 575 & 822 & 1,010 & 1,250 & 1,450 & 1,660 & 1,870 & 2,170 \\
\hline & & & $\begin{array}{c}\left(Q_{p}\right)_{g, w}: \text { Weighted at-site }\left(Q_{e}\right)_{g, s} \text { and regional } \\
\left(Q_{p}\right)_{g, r}\end{array}$ & 676 & 1,020 & 1,290 & 1,680 & 2,000 & 2,340 & 2,710 & 3,230 \\
\hline \multirow{5}{*}{05539900} & \multirow{5}{*}{$\begin{array}{l}\text { WEST BRANCH DU PAGE RIVER NEAR WEST } \\
\text { CHICAGO, IL }\end{array}$} & \multirow{5}{*}{ Yes } & $\begin{array}{l}\text { At-site, unadjusted for urbanization, EMA, } \\
\text { at-site skew }\end{array}$ & 547 & 795 & 956 & 1,150 & 1,300 & 1,440 & 1,570 & 1,750 \\
\hline & & & $\begin{array}{l}\text { At-site, adjusted for urbanization, EMA, at- } \\
\text { site skew }\end{array}$ & 631 & 861 & 1,020 & 1,240 & 1,400 & 1,580 & 1,760 & 2,020 \\
\hline & & & $\begin{array}{l}\text { At-site, adjusted for urbanization, EMA, } \\
\text { weighted skew: ( }\left(_{0}\right)_{9, s}\end{array}$ & 629 & 860 & 1,020 & 1,240 & 1,420 & 1,600 & 1,800 & 2,080 \\
\hline & & & Regional regression: $\left(Q_{p}\right)_{g, r}$ & 683 & 984 & 1,210 & 1,510 & 1,760 & 2,010 & 2,270 & 2,640 \\
\hline & & & $\begin{array}{c}\left(Q_{\rho}^{\rho}\right)_{Q, w}: \text { Weighted at-site }\left(Q_{\rho}\right)_{g, s} \text { and regional } \\
\left(Q_{\rho}\right)_{g, r}\end{array}$ & 630 & 862 & 1,030 & 1,260 & 1,440 & 1,630 & 1,830 & 2,120 \\
\hline \multirow{5}{*}{05539950} & \multirow{5}{*}{ KLEIN CREEK AT CAROL STREAM, IL } & \multirow{5}{*}{ Yes } & $\begin{array}{l}\text { At-site, unadjusted for urbanization, EMA, } \\
\text { at-site skew }\end{array}$ & 168 & 283 & 402 & 623 & 859 & 1,180 & 1,600 & 2,390 \\
\hline & & & $\begin{array}{l}\text { At-site, adjusted for urbanization, EMA, at- } \\
\text { site skew }\end{array}$ & 307 & 455 & 609 & 892 & 1,190 & 1,580 & 2,110 & 3,080 \\
\hline & & & $\begin{array}{l}\text { At-site, adjusted for urbanization, EMA, } \\
\text { weighted skew: ( }\left(_{0}\right)_{1, s}\end{array}$ & 332 & 488 & 615 & 804 & 970 & 1,160 & 1,370 & 1,700 \\
\hline & & & Regional regression: $(Q p)_{a, r}$ & 376 & 562 & 705 & 905 & 1,070 & 1,240 & 1,420 & 1,670 \\
\hline & & & $\begin{array}{c}\left(Q_{\rho}\right)_{g, w}: \text { Weighted at-site }\left(Q_{\rho}\right)_{g, s} \text { and regional } \\
\left(S_{\rho}\right)_{g, r}\end{array}$ & 334 & 494 & 624 & 819 & 987 & 1,170 & 1,380 & 1,690 \\
\hline \multirow{5}{*}{05540030} & \multirow{5}{*}{$\begin{array}{l}\text { WEST BRANCH DU PAGE RIVER AT WEST } \\
\text { CHICAGO, IL }\end{array}$} & \multirow{5}{*}{ No } & $\begin{array}{l}\text { At-site, unadjusted for urbanization, EMA, } \\
\text { at-site skew }\end{array}$ & 720 & 1,020 & 1,200 & 1,430 & 1,590 & 1,740 & 1,900 & 2,090 \\
\hline & & & $\begin{array}{l}\text { At-site, adjusted for urbanization, EMA, at- } \\
\text { site skew }\end{array}$ & 1,070 & 1,430 & 1,700 & 2,060 & 2,350 & 2,660 & 3,000 & 3,490 \\
\hline & & & 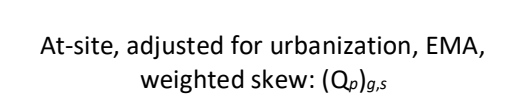 & 1,080 & 1,440 & 1,700 & 2,050 & 2,330 & 2,630 & 2,950 & 3,400 \\
\hline & & & Regional regression: $\left(Q_{p}\right)_{q, r}$ & 1,210 & 1,710 & 2,080 & 2,580 & 2,970 & 3,380 & 3,810 & 4,410 \\
\hline & & & $\begin{array}{l}\left(Q_{\rho}\right)_{g, w}: \text { Weighted at-site }\left(Q_{\rho}\right)_{g, s} \text { and regional } \\
\qquad\left(Q_{p}\right)_{g, r}\end{array}$ & 1,080 & 1,450 & 1,710 & 2,080 & 2,380 & 2,700 & 3,040 & 3,530 \\
\hline
\end{tabular}




\begin{tabular}{|c|c|c|c|c|c|c|c|c|c|c|c|}
\hline \multirow{2}{*}{$\begin{array}{c}\text { USGS } \\
\text { streamgage } \\
\text { number }\end{array}$} & \multirow[b]{2}{*}{ Streamgage name } & \multirow{2}{*}{$\begin{array}{l}\text { Used in regression } \\
\text { analyses (non- } \\
\text { redundant) }\end{array}$} & \multirow[b]{2}{*}{ Frequency analysis method } & \multicolumn{8}{|c|}{ Discharge for given annual exceedance probability, $\mathrm{ft}^{3} / \mathrm{s}$} \\
\hline & & & & 0.50 & 0.20 & 0.10 & 0.04 & 0.02 & 0.01 & 0.005 & 0.002 \\
\hline \multirow{5}{*}{05540060} & \multirow{5}{*}{ KRESS CREEK AT WEST CHICAGO, IL } & \multirow{5}{*}{ Yes } & $\begin{array}{l}\text { At-site, unadjusted for urbanization, EMA, } \\
\text { at-site skew }\end{array}$ & 257 & 446 & 653 & 1,060 & 1,500 & 2,120 & 2,990 & 4,670 \\
\hline & & & $\begin{array}{l}\text { At-site, adjusted for urbanization, EMA, at- } \\
\text { site skew }\end{array}$ & 293 & 491 & 708 & 1,130 & 1,600 & 2,260 & 3,170 & 4,950 \\
\hline & & & $\begin{array}{l}\text { At-site, adjusted for urbanization, EMA, } \\
\text { weighted skew: (Q }\left(Q_{p}\right)_{g, s}\end{array}$ & 319 & 527 & 710 & 1,000 & 1,280 & 1,600 & 1,990 & 2,610 \\
\hline & & & Regional regression: $(Q p)_{a, r}$ & 454 & 656 & 802 & 996 & 1,150 & 1,300 & 1,460 & 1,680 \\
\hline & & & 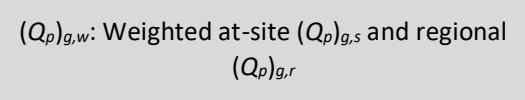 & 323 & 534 & 718 & 1,000 & 1,250 & 1,530 & 1,840 & 2,290 \\
\hline \multirow{5}{*}{05540080} & \multirow{5}{*}{ SPRING BROOK AT WHEATON, IL } & \multirow{5}{*}{ Yes } & $\begin{array}{l}\text { At-site, unadjusted for urbanization, EMA, } \\
\text { at-site skew }\end{array}$ & 143 & 213 & 274 & 373 & 465 & 575 & 706 & 922 \\
\hline & & & $\begin{array}{l}\text { At-site, adjusted for urbanization, EMA, at- } \\
\text { site skew }\end{array}$ & 171 & 245 & 312 & 422 & 525 & 650 & 801 & 1,050 \\
\hline & & & $\begin{array}{l}\text { At-site, adjusted for urbanization, EMA, } \\
\text { weighted skew: ( }\left(Q_{p}\right)_{g, s}\end{array}$ & 177 & 252 & 312 & 400 & 476 & 561 & 658 & 804 \\
\hline & & & Regional regression: $\left(Q_{0}\right)_{g, r}$ & 119 & 180 & 225 & 289 & 340 & 393 & 448 & 525 \\
\hline & & & 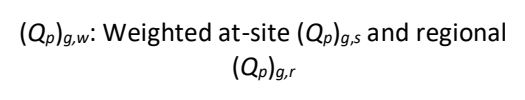 & 174 & 246 & 302 & 383 & 450 & 524 & 603 & 718 \\
\hline \multirow{5}{*}{05540091} & \multirow{5}{*}{$\begin{array}{l}\text { SPRING BK AT FOREST PRESERVE NR } \\
\text { WARRENVILE, IL }\end{array}$} & \multirow{5}{*}{ No } & $\begin{array}{l}\text { At-site, unadjusted for urbanization, EMA, } \\
\text { at-site skew }\end{array}$ & 241 & 323 & 376 & 442 & 491 & 538 & 586 & 648 \\
\hline & & & $\begin{array}{l}\text { At-site, adjusted for urbanization, EMA, at- } \\
\text { site skew }\end{array}$ & 276 & 360 & 414 & 481 & 530 & 578 & 626 & 689 \\
\hline & & & $\begin{array}{l}\text { At-site, adjusted for urbanization, EMA, } \\
\text { weighted skew: ( }\left(Q_{p}\right) \text { g,s }\end{array}$ & 272 & 359 & 417 & 493 & 550 & 609 & 670 & 753 \\
\hline & & & Regional regression: $(Q p)_{g, r}$ & 247 & 365 & 452 & 571 & 664 & 761 & 861 & 997 \\
\hline & & & 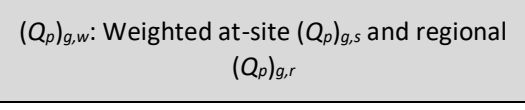 & 271 & 359 & 419 & 498 & 559 & 622 & 689 & 781 \\
\hline \multirow{5}{*}{05540095} & \multirow{5}{*}{$\begin{array}{l}\text { WEST BRANCH DU PAGE RIVER NEAR } \\
\text { WARRENVILE, IL }\end{array}$} & \multirow{5}{*}{ No } & $\begin{array}{l}\text { At-site, unadjusted for urbanization, EMA, } \\
\text { at-site skew }\end{array}$ & 1,320 & 2,020 & 2,560 & 3,340 & 3,990 & 4,700 & 5,490 & 6,660 \\
\hline & & & $\begin{array}{l}\text { At-site, adjusted for urbanization, EMA, at- } \\
\text { site skew }\end{array}$ & 1,570 & 2,280 & 2,820 & 3,590 & 4,220 & 4,910 & 5,670 & 6,780 \\
\hline & & & $\begin{array}{l}\text { At-site, adjusted for urbanization, EMA, } \\
\text { weighted skew: (Q. }\left(Q_{p}\right)_{g, s}\end{array}$ & 1,570 & 2,280 & 2,820 & 3,580 & 4,210 & 4,900 & 5,650 & 6,750 \\
\hline & & & Regional regression: $\left(Q_{p}\right)_{g, r}$ & 1,580 & 2,200 & 2,660 & 3,280 & 3,760 & 4,260 & 4,780 & 5,500 \\
\hline & & & $\begin{array}{l}\left(Q_{\rho}\right)_{y, w}: \text { Weighted at-site } \\
\qquad\left(Q_{p}\right)_{g, s} \text { and regional }\end{array}$ & 1,570 & 2,280 & 2,810 & 3,560 & 4,170 & 4,830 & 5,530 & 6,530 \\
\hline
\end{tabular}




\begin{tabular}{|c|c|c|c|c|c|c|c|c|c|c|c|}
\hline \multirow{2}{*}{$\begin{array}{c}\text { USGS } \\
\text { streamgage } \\
\text { number }\end{array}$} & \multirow[b]{2}{*}{ Streamgage name } & \multirow{2}{*}{$\begin{array}{l}\text { Used in regression } \\
\text { analyses (non- } \\
\text { redundant) }\end{array}$} & \multirow[b]{2}{*}{ Frequency analysis method } & \multicolumn{8}{|c|}{ Discharge for given annual exceedance probability, $\mathrm{ft}^{3} / \mathrm{s}$} \\
\hline & & & & 0.50 & 0.20 & 0.10 & 0.04 & 0.02 & 0.01 & 0.005 & 0.002 \\
\hline \multirow{5}{*}{05540110} & \multirow{5}{*}{ FERRY CREEK AT WARRENVILLE, IL } & \multirow{5}{*}{ Yes } & $\begin{array}{l}\text { At-site, unadjusted for urbanization, EMA, } \\
\text { at-site skew }\end{array}$ & 84 & 126 & 160 & 210 & 252 & 300 & 353 & 433 \\
\hline & & & $\begin{array}{l}\text { At-site, adjusted for urbanization, EMA, at- } \\
\text { site skew }\end{array}$ & 118 & 169 & 209 & 269 & 319 & 376 & 439 & 535 \\
\hline & & & $\begin{array}{l}\text { At-site, adjusted for urbanization, EMA, } \\
\text { weighted skew: (Q }\left(Q_{p}\right)_{g, s}\end{array}$ & 120 & 171 & 207 & 257 & 297 & 339 & 383 & 446 \\
\hline & & & Regional regression: $(Q p)_{a, r}$ & 107 & 160 & 198 & 246 & 283 & 320 & 357 & 405 \\
\hline & & & $\begin{array}{c}\left(Q_{\rho \rho}\right)_{y, w} \text { Weighted at-site }\left(Q_{p}\right)_{Q, s} \text { and regional } \\
\left(Q_{\rho}\right)_{Q, r}\end{array}$ & 120 & 170 & 207 & 256 & 295 & 336 & 379 & 438 \\
\hline \multirow{5}{*}{05540130} & \multirow{5}{*}{$\begin{array}{l}\text { WEST BRANCH DU PAGE RIVER NEAR } \\
\text { NAPERVILLE, IL }\end{array}$} & \multirow{5}{*}{ No } & $\begin{array}{l}\text { At-site, unadjusted for urbanization, EMA, } \\
\text { at-site skew }\end{array}$ & 2,060 & 3,060 & 3,860 & 5,070 & 6,110 & 7,280 & 8,620 & 10,700 \\
\hline & & & $\begin{array}{l}\text { At-site, adjusted for urbanization, EMA, at- } \\
\text { site skew }\end{array}$ & 2,250 & 3,310 & 4,160 & 5,440 & 6,550 & 7,810 & 9,240 & 11,400 \\
\hline & & & $\begin{array}{l}\text { At-site, adjusted for urbanization, EMA, } \\
\text { weighted skew: (Q (Q) } g_{s,}\end{array}$ & 2,280 & 3,330 & 4,150 & 5,340 & 6,340 & 7,440 & 8,670 & 10,500 \\
\hline & & & Regional regression: $\left(Q_{p}\right)_{g, r}$ & 1,990 & 2,760 & 3,330 & 4,080 & 4,670 & 5,290 & 5,930 & 6,810 \\
\hline & & & $\begin{array}{l}\left(Q_{\rho \rho}\right)_{y, w} \text { Weighted at-site }\left(Q_{\rho}\right)_{g, s} \text { and regional } \\
\left(Q_{\rho}\right)_{Q, r}\end{array}$ & 2,260 & 3,290 & 4,070 & 5,160 & 6,040 & 6,990 & 7,990 & 9,410 \\
\hline \multirow{5}{*}{05540140} & \multirow{5}{*}{$\begin{array}{l}\text { EAST BRANCH DU PAGE RIVER NEAR } \\
\text { BLOOMINGDALE, IL }\end{array}$} & \multirow{5}{*}{ Yes } & $\begin{array}{l}\text { At-site, unadjusted for urbanization, EMA, } \\
\text { at-site skew }\end{array}$ & 58 & 102 & 134 & 176 & 209 & 243 & 277 & 323 \\
\hline & & & $\begin{array}{l}\text { At-site, adjusted for urbanization, EMA, at- } \\
\text { site skew }\end{array}$ & 89 & 130 & 161 & 204 & 240 & 277 & 318 & 378 \\
\hline & & & $\begin{array}{l}\text { At-site, adjusted for urbanization, EMA, } \\
\left.\text { weighted skew: }\left(Q_{0}\right)\right)_{a, s}\end{array}$ & 88 & 130 & 162 & 208 & 247 & 290 & 338 & 408 \\
\hline & & & Regional regression: $(Q p)_{g, r}$ & 158 & 245 & 312 & 408 & 485 & 567 & 654 & 776 \\
\hline & & & $\begin{array}{l}\left(Q_{\rho p}\right)_{y, w} \text { : Weighted at-site }\left(Q_{p}\right)_{Q, s} \text { and regional } \\
\left(Q_{0}\right)_{p, r}\end{array}$ & 91 & 136 & 173 & 228 & 276 & 329 & 389 & 480 \\
\hline \multirow{5}{*}{05540150} & \multirow{5}{*}{ EAST BR DU PAGE RIVER AT GLEN ELLYN, IL } & \multirow{5}{*}{ No } & $\begin{array}{l}\text { At-site, unadjusted for urbanization, EMA, } \\
\text { at-site skew }\end{array}$ & 240 & 398 & 511 & 661 & 776 & 894 & 1,010 & 1,180 \\
\hline & & & $\begin{array}{l}\text { At-site, adjusted for urbanization, EMA, at- } \\
\text { site skew }\end{array}$ & 292 & 454 & 584 & 777 & 943 & 1,130 & 1,340 & 1,650 \\
\hline & & & $\begin{array}{l}\text { At-site, adjusted for urbanization, EMA, } \\
\left.\text { weighted skew: ( } Q_{p}\right)_{g, s}\end{array}$ & 291 & 454 & 585 & 782 & 951 & 1,140 & 1,360 & 1,690 \\
\hline & & & Regional regression: $\left(Q_{p}\right)_{g, r}$ & 453 & 659 & 813 & 1,020 & 1,190 & 1,360 & 1,550 & 1,800 \\
\hline & & & $\begin{array}{c}\left(Q_{\rho \rho}\right)_{y, w} \text { : Weighted at-site }\left(Q_{p}\right)_{Q, s} \text { and regional } \\
\left(Q_{p}\right)_{Q, r}\end{array}$ & 300 & 472 & 612 & 821 & 998 & 1,190 & 1,410 & 1,720 \\
\hline
\end{tabular}




\begin{tabular}{|c|c|c|c|c|c|c|c|c|c|c|c|}
\hline \multirow{2}{*}{$\begin{array}{c}\text { USGS } \\
\text { streamgage } \\
\text { number }\end{array}$} & \multirow[b]{2}{*}{ Streamgage name } & \multirow{2}{*}{$\begin{array}{l}\text { Used in regression } \\
\text { analyses (non- } \\
\text { redundant) }\end{array}$} & \multirow[b]{2}{*}{ Frequency analysis method } & \multicolumn{8}{|c|}{ Discharge for given annual exceedance probability, $\mathrm{ft}^{3} / \mathrm{s}$} \\
\hline & & & & 0.50 & 0.20 & 0.10 & 0.04 & 0.02 & 0.01 & 0.005 & 0.002 \\
\hline \multirow{5}{*}{05540160} & \multirow{5}{*}{$\begin{array}{l}\text { EAST BRANCH DU PAGE RIVER NEAR DOWNERS } \\
\text { GROVE, IL }\end{array}$} & \multirow{5}{*}{ Yes } & $\begin{array}{l}\text { At-site, unadjusted for urbanization, EMA, } \\
\text { at-site skew }\end{array}$ & 609 & 895 & 1,090 & 1,360 & 1,560 & 1,760 & 1,970 & 2,260 \\
\hline & & & $\begin{array}{l}\text { At-site, adjusted for urbanization, EMA, at- } \\
\text { site skew }\end{array}$ & 669 & 981 & 1,220 & 1,580 & 1,870 & 2,200 & 2,570 & 3,120 \\
\hline & & & 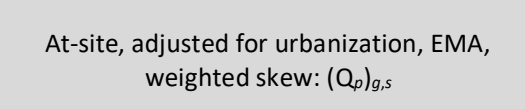 & 670 & 983 & 1,220 & 1,570 & 1,870 & 2,190 & 2,550 & 3,090 \\
\hline & & & Regional regression: $(Q p)_{a, r}$ & 658 & 933 & 1,140 & 1,410 & 1,630 & 1,860 & 2,090 & 2,420 \\
\hline & & & $\begin{array}{c}\left(Q_{\rho \rho}\right)_{y, w} \text { Weighted at-site }\left(Q_{p}\right)_{Q, s} \text { and regional } \\
\left(Q_{\rho}\right)_{Q, r}\end{array}$ & 670 & 981 & 1,220 & 1,560 & 1,850 & 2,150 & 2,480 & 2,960 \\
\hline \multirow{5}{*}{05540190} & \multirow{5}{*}{ ST. JOSEPH CREEK AT BELMONT, IL } & \multirow{5}{*}{ Yes } & $\begin{array}{l}\text { At-site, unadjusted for urbanization, EMA, } \\
\text { at-site skew }\end{array}$ & 345 & 517 & 627 & 759 & 853 & 942 & 1,030 & 1,140 \\
\hline & & & $\begin{array}{l}\text { At-site, adjusted for urbanization, EMA, at- } \\
\text { site skew }\end{array}$ & 363 & 539 & 653 & 794 & 895 & 993 & 1,090 & 1,210 \\
\hline & & & $\begin{array}{l}\text { At-site, adjusted for urbanization, EMA, } \\
\text { weighted skew: (Q }\left(Q_{p}\right)_{g, s}\end{array}$ & 348 & 533 & 672 & 866 & 1,020 & 1,190 & 1,380 & 1,640 \\
\hline & & & Regional regression: $\left(Q_{p}\right)_{g, r}$ & 366 & 542 & 674 & 855 & 1,000 & 1,150 & 1,310 & 1,530 \\
\hline & & & $\begin{array}{l}(Q p)_{g, w} \text { : Weighted at-site }\left(Q_{\rho}\right)_{g, s} \text { and regional } \\
\left(Q_{\rho}\right)_{g, r}\end{array}$ & 349 & 534 & 672 & 865 & 1,020 & 1,190 & 1,360 & 1,610 \\
\hline \multirow{5}{*}{05540195} & \multirow{5}{*}{ ST. JOSEPH CREEK AT ROUTE 34 AT LISLE, IL } & \multirow{5}{*}{ No } & $\begin{array}{l}\text { At-site, unadjusted for urbanization, EMA, } \\
\text { at-site skew }\end{array}$ & 580 & 808 & 972 & 1,190 & 1,370 & 1,550 & 1,750 & 2,020 \\
\hline & & & $\begin{array}{l}\text { At-site, adjusted for urbanization, EMA, at- } \\
\text { site skew }\end{array}$ & 599 & 830 & 996 & 1,220 & 1,390 & 1,580 & 1,770 & 2,050 \\
\hline & & & 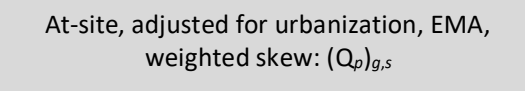 & 596 & 828 & 998 & 1,230 & 1,420 & 1,620 & 1,840 & 2,150 \\
\hline & & & Regional regression: $(Q p)_{g, r}$ & 434 & 643 & 800 & 1,020 & 1,190 & 1,370 & 1,560 & 1,820 \\
\hline & & & $\begin{array}{c}\left(Q_{\rho \rho}\right)_{y, w} \text { Weighted at-site }\left(Q_{\rho}\right)_{g, s} \text { and regional } \\
\left(Q_{\rho}\right)_{Q, r}\end{array}$ & 589 & 817 & 983 & 1,210 & 1,390 & 1,590 & 1,790 & 2,080 \\
\hline \multirow{5}{*}{05540240} & \multirow{5}{*}{ PRENTISS CREEK NEAR LISLE, IL } & \multirow{5}{*}{ Yes } & $\begin{array}{l}\text { At-site, unadjusted for urbanization, EMA, } \\
\text { at-site skew }\end{array}$ & 181 & 296 & 396 & 558 & 708 & 888 & 1,100 & 1,450 \\
\hline & & & $\begin{array}{l}\text { At-site, adjusted for urbanization, EMA, at- } \\
\text { site skew }\end{array}$ & 225 & 368 & 494 & 697 & 884 & 1,110 & 1,380 & 1,810 \\
\hline & & & $\begin{array}{l}\text { At-site, adjusted for urbanization, EMA, } \\
\left.\text { weighted skew: ( } Q_{0}\right)_{g, s}\end{array}$ & 228 & 370 & 492 & 682 & 853 & 1,050 & 1,280 & 1,650 \\
\hline & & & Regional regression: $\left(Q_{p}\right)_{g, r}$ & 332 & 506 & 639 & 824 & 974 & 1,130 & 1,300 & 1,530 \\
\hline & & & $\begin{array}{l}\left(Q_{\rho \rho g}\right)_{y, w} \text { Weighted at-site }\left(Q_{\rho}\right)_{g, s} \text { and regional } \\
\left(Q_{\rho}\right)_{g, r}\end{array}$ & 234 & 384 & 512 & 709 & 881 & 1,070 & 1,290 & 1,610 \\
\hline
\end{tabular}




\begin{tabular}{|c|c|c|c|c|c|c|c|c|c|c|c|}
\hline \multirow{2}{*}{$\begin{array}{l}\text { USGS } \\
\text { streamgage } \\
\text { number }\end{array}$} & \multirow[b]{2}{*}{ Streamgage name } & \multirow{2}{*}{$\begin{array}{l}\text { Used in regression } \\
\text { analyses (non- } \\
\text { redundant) }\end{array}$} & \multirow[b]{2}{*}{ Frequency analysis method } & \multicolumn{8}{|c|}{ Discharge for given annual exceedance probability, $\mathrm{ft}^{3} / \mathrm{s}$} \\
\hline & & & & 0.50 & 0.20 & 0.10 & 0.04 & 0.02 & 0.01 & 0.005 & 0.002 \\
\hline \multirow{5}{*}{05540250} & \multirow{5}{*}{$\begin{array}{l}\text { EAST BRANCH DU PAGE RIVER AT BOLINGBROOK, } \\
\text { IL }\end{array}$} & \multirow{5}{*}{ No } & $\begin{array}{l}\text { At-site, unadjusted for urbanization, EMA, } \\
\text { at-site skew }\end{array}$ & 1,160 & 1,820 & 2,380 & 3,240 & 4,020 & 4,920 & 5,960 & 7,610 \\
\hline & & & $\begin{array}{l}\text { At-site, adjusted for urbanization, EMA, at- } \\
\text { site skew }\end{array}$ & 1,230 & 1,900 & 2,470 & 3,350 & 4,130 & 5,030 & 6,080 & 7,730 \\
\hline & & & $\begin{array}{l}\text { At-site, adjusted for urbanization, EMA, } \\
\text { weighted skew: }\left(Q_{0}\right)_{g, s}\end{array}$ & 1,240 & 1,920 & 2,460 & 3,280 & 3,990 & 4,790 & 5,700 & 7,100 \\
\hline & & & Regional regression: $(Q p)_{g, r}$ & 1,320 & 1,820 & 2,170 & 2,650 & 3,020 & 3,410 & 3,800 & 4,350 \\
\hline & & & $\begin{array}{c}\left(Q_{\rho \rho}\right)_{y, w} \text { : Weighted at-site }\left(Q_{p}\right)_{Q, s} \text { and regional } \\
\left(Q_{\rho}\right)_{Q, r}\end{array}$ & 1,250 & 1,910 & 2,420 & 3,160 & 3,760 & 4,400 & 5,090 & 6,050 \\
\hline \multirow{5}{*}{05540275} & \multirow{5}{*}{$\begin{array}{c}\text { SPRING BROOK AT 87TH STREET NEAR } \\
\text { NAPERVILLE, IL }\end{array}$} & \multirow{5}{*}{ Yes } & $\begin{array}{l}\text { At-site, unadjusted for urbanization, EMA, } \\
\text { at-site skew }\end{array}$ & 204 & 421 & 652 & 1,080 & 1,550 & 2,160 & 2,990 & 4,500 \\
\hline & & & $\begin{array}{l}\text { At-site, adjusted for urbanization, EMA, at- } \\
\text { site skew }\end{array}$ & 226 & 452 & 694 & 1,150 & 1,650 & 2,320 & 3,240 & 4,940 \\
\hline & & & $\begin{array}{l}\text { At-site, adjusted for urbanization, EMA, } \\
\text { weighted skew: }\left(Q_{0}\right)_{g, s}\end{array}$ & 235 & 463 & 687 & 1,080 & 1,480 & 1,980 & 2,610 & 3,710 \\
\hline & & & Regional regression: $\left(Q_{p}\right)_{g, r}$ & 332 & 485 & 597 & 747 & 865 & 985 & 1,110 & 1,280 \\
\hline & & & $\begin{array}{c}\left(Q_{\rho \rho}\right)_{y, w} \text { : Weighted at-site } \\
\left(Q_{p}\right)_{Q, r}\left(Q_{\rho}\right)_{Q, s} \text { and regional }\end{array}$ & 246 & 467 & 664 & 963 & 1,210 & 1,480 & 1,760 & 2,130 \\
\hline \multirow{5}{*}{05540500} & \multirow{5}{*}{ DU PAGE RIVER AT SHOREWOOD, IL } & \multirow{5}{*}{ Yes } & $\begin{array}{l}\text { At-site, unadjusted for urbanization, EMA, } \\
\text { at-site skew }\end{array}$ & 3,750 & 5,950 & 7,650 & 10,100 & 12,100 & 14,300 & 16,700 & 20,200 \\
\hline & & & $\begin{array}{l}\text { At-site, adjusted for urbanization, EMA, at- } \\
\text { site skew }\end{array}$ & 4,890 & 7,330 & 9,200 & 11,900 & 14,100 & 16,500 & 19,200 & 23,100 \\
\hline & & & $\begin{array}{l}\text { At-site, adjusted for urbanization, EMA, } \\
\text { weighted skew: ( } Q_{p} g_{g, s}\end{array}$ & 4,880 & 7,330 & 9,210 & 11,900 & 14,100 & 16,600 & 19,300 & 23,200 \\
\hline & & & Regional regression: $(Q p)_{g, r}$ & 4,100 & 5,610 & 6,690 & 8,140 & 9,270 & 10,400 & 11,700 & 13,300 \\
\hline & & & $\begin{array}{c}\left(Q_{\rho \rho}\right)_{y, w} \text { : Weighted at-site }\left(Q_{\rho}\right)_{Q, s} \text { and regional } \\
\left(Q_{\rho}\right)_{Q, r}\end{array}$ & 4,870 & 7,280 & 9,110 & 11,700 & 13,800 & 16,000 & 18,300 & 21,700 \\
\hline \multirow{5}{*}{05541750} & \multirow{5}{*}{ MAZON RIVER TRIBUTARY NEAR GARDNER, IL } & \multirow{5}{*}{ Yes } & $\begin{array}{l}\text { At-site, unadjusted for urbanization, EMA, } \\
\text { at-site skew }\end{array}$ & 113 & 139 & 155 & 174 & 187 & 200 & 212 & 228 \\
\hline & & & $\begin{array}{l}\text { At-site, adjusted for urbanization, EMA, at- } \\
\text { site skew }\end{array}$ & 113 & 139 & 155 & 174 & 187 & 200 & 212 & 228 \\
\hline & & & $\begin{array}{l}\text { At-site, adjusted for urbanization, EMA, } \\
\left.\text { weighted skew: ( } Q_{0}\right)_{g, s}\end{array}$ & 113 & 140 & 155 & 173 & 185 & 196 & 206 & 220 \\
\hline & & & Regional regression: $\left(Q_{p}\right)_{g, r}$ & 135 & 217 & 273 & 343 & 394 & 443 & 490 & 550 \\
\hline & & & 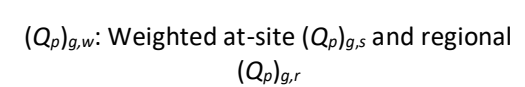 & 114 & 141 & 157 & 176 & 190 & 202 & 215 & 230 \\
\hline
\end{tabular}




\begin{tabular}{|c|c|c|c|c|c|c|c|c|c|c|c|}
\hline \multirow{2}{*}{$\begin{array}{c}\text { USGS } \\
\text { streamgage } \\
\text { number }\end{array}$} & \multirow[b]{2}{*}{ Streamgage name } & \multirow{2}{*}{$\begin{array}{l}\text { Used in regression } \\
\text { analyses (non- } \\
\text { redundant) }\end{array}$} & \multirow[b]{2}{*}{ Frequency analysis method } & \multicolumn{8}{|c|}{ Discharge for given annual exceedance probability, $\mathrm{ft}^{3} / \mathrm{s}$} \\
\hline & & & & 0.50 & 0.20 & 0.10 & 0.04 & 0.02 & 0.01 & 0.005 & 0.002 \\
\hline \multirow{5}{*}{05542000} & \multirow{5}{*}{ MAZON RIVER NEAR COAL CITY, IL } & \multirow{5}{*}{ Yes } & $\begin{array}{l}\text { At-site, unadjusted for urbanization, EMA, } \\
\text { at-site skew }\end{array}$ & 9,580 & 13,900 & 16,600 & 19,800 & 22,000 & 24,000 & 26,000 & 28,400 \\
\hline & & & $\begin{array}{l}\text { At-site, adjusted for urbanization, EMA, at- } \\
\text { site skew }\end{array}$ & 9,650 & 14,000 & 16,700 & 19,900 & 22,100 & 24,200 & 26,100 & 28,600 \\
\hline & & & $\begin{array}{l}\text { At-site, adjusted for urbanization, EMA, } \\
\left.\text { weighted skew: }\left(Q_{0}\right)_{g}\right)_{s, s}\end{array}$ & 9,600 & 13,900 & 16,700 & 20,100 & 22,600 & 25,000 & 27,300 & 30,300 \\
\hline & & & Regional regression: $(Q p)_{g, r}$ & 4,000 & 5,920 & 7,190 & 8,780 & 9,930 & 11,100 & 12,200 & 13,700 \\
\hline & & & 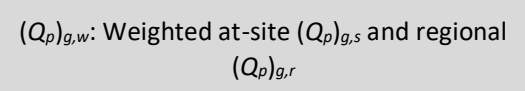 & 9,440 & 13,700 & 16,400 & 19,700 & 22,000 & 24,200 & 26,300 & 28,900 \\
\hline \multirow{5}{*}{05543830} & \multirow{5}{*}{ FOX RIVER AT WAUKESHA, WI } & \multirow{5}{*}{ Yes } & $\begin{array}{l}\text { At-site, unadjusted for urbanization, EMA, } \\
\text { at-site skew }\end{array}$ & 929 & 1,370 & 1,680 & 2,110 & 2,440 & 2,800 & 3,170 & 3,690 \\
\hline & & & $\begin{array}{l}\text { At-site, adjusted for urbanization, EMA, at- } \\
\text { site skew }\end{array}$ & 1,230 & 1,740 & 2,110 & 2,610 & 3,010 & 3,430 & 3,880 & 4,520 \\
\hline & & & $\begin{array}{l}\text { At-site, adjusted for urbanization, EMA, } \\
\text { weighted skew: }\left(Q_{0}\right)_{g, s}\end{array}$ & 1,230 & 1,740 & 2,100 & 2,600 & 2,980 & 3,390 & 3,820 & 4,420 \\
\hline & & & Regional regression: $\left(Q_{\rho}\right)_{g, r}$ & 1,410 & 1,960 & 2,350 & 2,860 & 3,250 & 3,640 & 4,050 & 4,590 \\
\hline & & & $\begin{array}{l}\left(Q_{p}\right)_{y, w}: \text { Weighted at-site } \\
\left(Q_{\rho} Q_{g, r}\right.\end{array}$ & 1,240 & 1,750 & 2,110 & 2,610 & 3,000 & 3,410 & 3,840 & 4,440 \\
\hline \multirow{5}{*}{05544200} & \multirow{5}{*}{ MUKWONAGO RIVER AT MUKWONAGO, WI } & \multirow{5}{*}{ Yes } & $\begin{array}{l}\text { At-site, unadjusted for urbanization, EMA, } \\
\text { at-site skew }\end{array}$ & 219 & 265 & 291 & 321 & 342 & 361 & 379 & 402 \\
\hline & & & $\begin{array}{l}\text { At-site, adjusted for urbanization, EMA, at- } \\
\text { site skew }\end{array}$ & 251 & 299 & 326 & 355 & 375 & 393 & 410 & 430 \\
\hline & & & $\begin{array}{l}\text { At-site, adjusted for urbanization, EMA, } \\
\text { wighted skew: }\left(Q_{0}\right)_{g, s}\end{array}$ & 251 & 299 & 326 & 357 & 378 & 397 & 415 & 437 \\
\hline & & & Regional regression: $(Q p)_{g, r}$ & 913 & 1,390 & 1,720 & 2,130 & 2,440 & 2,750 & 3,060 & 3,470 \\
\hline & & & 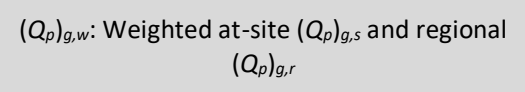 & 254 & 303 & 332 & 366 & 389 & 411 & 433 & 462 \\
\hline \multirow{5}{*}{05544300} & \multirow{5}{*}{$\begin{array}{l}\text { MUKWONAGO RIVER TRIBUTARY NEAR } \\
\text { MUKWONAGO, WI }\end{array}$} & \multirow{5}{*}{ Yes } & $\begin{array}{l}\text { At-site, unadjusted for urbanization, EMA, } \\
\text { at-site skew }\end{array}$ & 32 & 50 & 62 & 76 & 85 & 94 & 102 & 112 \\
\hline & & & $\begin{array}{l}\text { At-site, adjusted for urbanization, EMA, at- } \\
\text { site skew }\end{array}$ & 36 & 56 & 68 & 82 & 92 & 100 & 108 & 117 \\
\hline & & & $\begin{array}{l}\text { At-site, adjusted for urbanization, EMA, } \\
\text { weighted skew: }\left(Q_{0}\right) g, s\end{array}$ & 36 & 55 & 68 & 85 & 97 & 110 & 122 & 139 \\
\hline & & & Regional regression: $\left(Q_{p}\right)_{g, r}$ & 58 & 98 & 128 & 169 & 200 & 231 & 263 & 305 \\
\hline & & & $\begin{array}{l}\left(Q_{p}\right)_{g, w}: \text { Weighted at-site }\left(Q_{p}\right)_{g, s} \text { and regional } \\
\left(Q_{p} g_{g, r}\right.\end{array}$ & 37 & 57 & 72 & 92 & 108 & 124 & 141 & 164 \\
\hline
\end{tabular}




\begin{tabular}{|c|c|c|c|c|c|c|c|c|c|c|c|}
\hline \multirow{2}{*}{$\begin{array}{c}\text { USGS } \\
\text { streamgage } \\
\text { number }\end{array}$} & \multirow[b]{2}{*}{ Streamgage name } & \multirow{2}{*}{$\begin{array}{c}\text { Used in regression } \\
\text { analyses (non-- } \\
\text { redundant) }\end{array}$} & \multirow[b]{2}{*}{ Frequency analysis method } & \multicolumn{8}{|c|}{ Discharge for given annual exceedance probability, $\mathrm{ft}^{3} / \mathrm{s}$} \\
\hline & & & & 0.50 & 0.20 & 0.10 & 0.04 & 0.02 & 0.01 & 0.005 & 0.002 \\
\hline \multirow{5}{*}{05545100} & \multirow{5}{*}{ SUGAR CREEK AT ELKHORN, WI } & \multirow{5}{*}{ Yes } & $\begin{array}{l}\text { At-site, unadjusted for urbanization, EMA, } \\
\text { at-site skew }\end{array}$ & 134 & 231 & 311 & 431 & 534 & 651 & 782 & 979 \\
\hline & & & $\begin{array}{l}\text { At-site, adjusted for urbanization, EMA, at- } \\
\text { site skew }\end{array}$ & 136 & 234 & 314 & 434 & 539 & 656 & 787 & 986 \\
\hline & & & $\begin{array}{l}\text { At-site, adjusted for urbanization, EMA, } \\
\text { weighted skew: }\left(Q_{p}\right)_{g, s}\end{array}$ & 138 & 235 & 312 & 423 & 516 & 617 & 729 & 892 \\
\hline & & & Regional regression: $(Q p)_{a, r}$ & 225 & 366 & 466 & 597 & 695 & 792 & 890 & 1,020 \\
\hline & & & $\begin{array}{c}\left(Q_{\rho \rho}\right)_{y, w} \text { Weighted at-site }\left(Q_{p}\right)_{Q, s} \text { and regional } \\
\left(Q_{\rho}\right)_{Q, r}\end{array}$ & 141 & 240 & 320 & 436 & 533 & 638 & 752 & 915 \\
\hline \multirow{5}{*}{05545200} & \multirow{5}{*}{ WHITE RIVER TRIBUTARY NEAR BURLINGTON, WI } & \multirow{5}{*}{ Yes } & $\begin{array}{l}\text { At-site, unadjusted for urbanization, EMA, } \\
\text { at-site skew }\end{array}$ & 95 & 166 & 218 & 288 & 343 & 399 & 457 & 536 \\
\hline & & & $\begin{array}{l}\text { At-site, adjusted for urbanization, EMA, at- } \\
\text { site skew }\end{array}$ & 96 & 168 & 220 & 291 & 345 & 400 & 457 & 534 \\
\hline & & & $\begin{array}{l}\text { At-site, adjusted for urbanization, EMA, } \\
\text { weighted skew: ( }\left(Q_{p}\right)_{g, s}\end{array}$ & 96 & 168 & 221 & 292 & 347 & 403 & 461 & 540 \\
\hline & & & Regional regression: $\left(Q_{0}\right)_{g, r}$ & 144 & 266 & 362 & 492 & 594 & 698 & 804 & 947 \\
\hline & & & $\begin{array}{c}\left(Q_{\rho \rho}\right)_{y, w} \text { Weighted at-site }\left(Q_{p}\right)_{Q, s} \text { and regional } \\
\left(Q_{p}\right)_{Q, r}\end{array}$ & 98 & 172 & 227 & 303 & 363 & 428 & 495 & 589 \\
\hline \multirow{5}{*}{05545300} & \multirow{5}{*}{ WHITE RIVER NEAR BURLINGTON, WI } & \multirow{5}{*}{ Yes } & $\begin{array}{l}\text { At-site, unadjusted for urbanization, EMA, } \\
\text { at-site skew }\end{array}$ & 797 & 1,300 & 1,670 & 2,180 & 2,580 & 3,010 & 3,460 & 4,080 \\
\hline & & & $\begin{array}{l}\text { At-site, adjusted for urbanization, EMA, at- } \\
\text { site skew }\end{array}$ & 929 & 1,480 & 1,880 & 2,440 & 2,870 & 3,340 & 3,820 & 4,500 \\
\hline & & & $\begin{array}{l}\text { At-site, adjusted for urbanization, EMA, } \\
\text { weighted skew: }\left(Q_{p}\right)_{\text {g,s }}\end{array}$ & 934 & 1,480 & 1,880 & 2,410 & 2,830 & 3,270 & 3,720 & 4,350 \\
\hline & & & Regional regression: $\left(\left.Q p\right|_{g r}\right.$ & 1,350 & 2,050 & 2,530 & 3,140 & 3,600 & 4,060 & 4,520 & 5,140 \\
\hline & & & $\begin{array}{c}\left(Q_{\rho}\right)_{g, w} \text { : Weighted at-site }\left(Q_{\rho}\right)_{Q, s} \text { and regional } \\
\left(Q_{\rho}\right)_{Q, r}\end{array}$ & 957 & 1,520 & 1,930 & 2,490 & 2,930 & 3,380 & 3,860 & 4,510 \\
\hline \multirow{5}{*}{05545750} & \multirow{5}{*}{ FOX RIVER NEAR NEW MUNSTER, WI } & \multirow{5}{*}{ Yes } & $\begin{array}{l}\text { At-site, unadjusted for urbanization, EMA, } \\
\text { at-site skew }\end{array}$ & 2,760 & 3,960 & 4,780 & 5,830 & 6,630 & 7,440 & 8,260 & 9,370 \\
\hline & & & $\begin{array}{l}\text { At-site, adjusted for urbanization, EMA, at- } \\
\text { site skew }\end{array}$ & 3,140 & 4,380 & 5,200 & 6,260 & 7,050 & 7,850 & 8,660 & 9,760 \\
\hline & & & $\begin{array}{l}\text { At-site, adjusted for urbanization, EMA, } \\
\text { weighted skew: (Qop) })_{g, s}\end{array}$ & 3,150 & 4,380 & 5,200 & 6,240 & 7,030 & 7,820 & 8,620 & 9,690 \\
\hline & & & Regional regression: $\left(Q_{p}\right)_{a, r}$ & 4,270 & 5,750 & 6,720 & 7,910 & 8,780 & 9,640 & 10,500 & 11,600 \\
\hline & & & $\begin{array}{c}\left(Q_{\rho \rho}\right)_{y, w} \text { : Weighted at-site }\left(Q_{p}\right)_{Q, s} \text { and regional } \\
\left(Q_{p}\right)_{Q, r}\end{array}$ & 3,160 & 4,400 & 5,220 & 6,280 & 7,080 & 7,890 & 8,710 & 9,810 \\
\hline
\end{tabular}




\begin{tabular}{|c|c|c|c|c|c|c|c|c|c|c|c|}
\hline \multirow{2}{*}{$\begin{array}{c}\text { USGS } \\
\text { streamgage } \\
\text { number }\end{array}$} & \multirow[b]{2}{*}{ Streamgage name } & \multirow{2}{*}{$\begin{array}{l}\text { Used in regression } \\
\text { analyses (non- } \\
\text { redundant) }\end{array}$} & \multirow[b]{2}{*}{ Frequency analysis method } & \multicolumn{8}{|c|}{ Discharge for given annual exceedance probability, $\mathrm{ft}^{3} / \mathrm{s}$} \\
\hline & & & & 0.50 & 0.20 & 0.10 & 0.04 & 0.02 & 0.01 & 0.005 & 0.002 \\
\hline \multirow{5}{*}{05547755} & \multirow{5}{*}{ SQUAW CREEK AT ROUND LAKE, IL } & \multirow{5}{*}{ Yes } & $\begin{array}{l}\text { At-site, unadjusted for urbanization, EMA, } \\
\text { at-site skew }\end{array}$ & 184 & 250 & 288 & 330 & 358 & 384 & 408 & 436 \\
\hline & & & $\begin{array}{l}\text { At-site, adjusted for urbanization, EMA, at- } \\
\text { site skew }\end{array}$ & 244 & 325 & 369 & 416 & 447 & 474 & 497 & 525 \\
\hline & & & $\begin{array}{l}\text { At-site, adjusted for urbanization, EMA, } \\
\text { weighted skew: }\left(Q_{\rho}\right)_{g, s}\end{array}$ & 237 & 324 & 379 & 446 & 495 & 542 & 589 & 650 \\
\hline & & & Regional regression: $(Q p)_{g, r}$ & 261 & 376 & 455 & 558 & 635 & 711 & 788 & 890 \\
\hline & & & 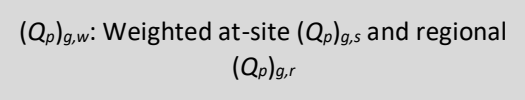 & 238 & 326 & 383 & 454 & 506 & 557 & 609 & 678 \\
\hline \multirow{5}{*}{05548150} & \multirow{5}{*}{$\begin{array}{l}\text { NORTH BRANCH NIPPERSINK CREEK NEAR } \\
\text { GENOA CITY, WI }\end{array}$} & \multirow{5}{*}{ Yes } & $\begin{array}{l}\text { At-site, unadjusted for urbanization, EMA, } \\
\text { at-site skew }\end{array}$ & 191 & 303 & 373 & 455 & 512 & 564 & 613 & 673 \\
\hline & & & $\begin{array}{l}\text { At-site, adjusted for urbanization, EMA, at- } \\
\text { site skew }\end{array}$ & 196 & 309 & 379 & 461 & 517 & 569 & 617 & 675 \\
\hline & & & $\begin{array}{l}\text { At-site, adjusted for urbanization, EMA, } \\
\left.\text { weighted skew: (o } 0_{0}\right)_{s, 5}\end{array}$ & 193 & 307 & 383 & 479 & 550 & 618 & 686 & 774 \\
\hline & & & Regional regression: $\left(Q_{p}\right)_{g, r}$ & 257 & 406 & 510 & 644 & 743 & 841 & 939 & 1,070 \\
\hline & & & 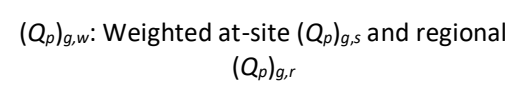 & 195 & 310 & 388 & 487 & 561 & 634 & 706 & 802 \\
\hline \multirow{5}{*}{05548280} & \multirow{5}{*}{ NIPPERSINK CREEK NEAR SPRING GROVE, IL } & \multirow{5}{*}{ Yes } & $\begin{array}{l}\text { At-site, unadjusted for urbanization, EMA, } \\
\text { at-site skew }\end{array}$ & 1,250 & 1,990 & 2,440 & 2,950 & 3,290 & 3,610 & 3,890 & 4,230 \\
\hline & & & $\begin{array}{l}\text { At-site, adjusted for urbanization, EMA, at- } \\
\text { site skew }\end{array}$ & 1,420 & 2,200 & 2,700 & 3,300 & 3,710 & 4,110 & 4,480 & 4,940 \\
\hline & & & $\begin{array}{l}\text { At-site, adjusted for urbanization, EMA, } \\
\text { weighted skew: ( }\left(_{0}\right)_{g, s}\end{array}$ & 1,390 & 2,190 & 2,740 & 3,430 & 3,940 & 4,450 & 4,960 & 5,630 \\
\hline & & & Regional regression: $(Q p)_{g, r}$ & 1,980 & 2,940 & 3,590 & 4,420 & 5,030 & 5,650 & 6,260 & 7,070 \\
\hline & & & $\begin{array}{c}\left(Q_{\rho}\right)_{\text {g,w: }} \text { : Weighted at-site }\left(Q_{\rho}\right)_{g, s} \text { and regional } \\
\left(Q_{\rho \rho \rho} g_{y,}\right.\end{array}$ & 1,410 & 2,220 & 2,770 & 3,480 & 4,010 & 4,540 & 5,080 & 5,790 \\
\hline \multirow{5}{*}{05549000} & \multirow{5}{*}{ BOONE CREEK NEAR MC HENRY, IL } & \multirow{5}{*}{ Yes } & $\begin{array}{l}\text { At-site, unadjusted for urbanization, EMA, } \\
\text { at-site skew }\end{array}$ & 129 & 202 & 246 & 296 & 329 & 358 & 385 & 416 \\
\hline & & & $\begin{array}{l}\text { At-site, adjusted for urbanization, EMA, at- } \\
\text { site skew }\end{array}$ & 203 & 300 & 362 & 439 & 494 & 547 & 599 & 667 \\
\hline & & & 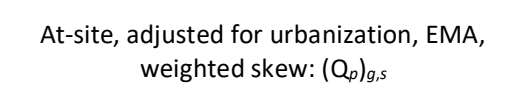 & 202 & 300 & 364 & 444 & 503 & 560 & 618 & 693 \\
\hline & & & Regional regression: $\left(Q_{\rho}\right)_{g, r}$ & 287 & 450 & 566 & 715 & 827 & 938 & 1,050 & 1,200 \\
\hline & & & 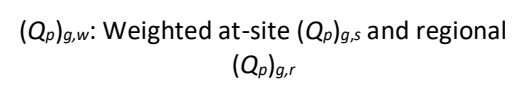 & 204 & 303 & 369 & 454 & 517 & 581 & 645 & 732 \\
\hline
\end{tabular}




\begin{tabular}{|c|c|c|c|c|c|c|c|c|c|c|c|}
\hline \multirow{2}{*}{$\begin{array}{c}\text { USGS } \\
\text { streamgage } \\
\text { number }\end{array}$} & \multirow[b]{2}{*}{ Streamgage name } & \multirow{2}{*}{$\begin{array}{l}\text { Used in regression } \\
\text { analyses (non- } \\
\text { redundant) }\end{array}$} & \multirow[b]{2}{*}{ Frequency analysis method } & \multicolumn{8}{|c|}{ Discharge for given annual exceedance probability, $\mathrm{ft}^{3} / \mathrm{s}$} \\
\hline & & & & 0.50 & 0.20 & 0.10 & 0.04 & 0.02 & 0.01 & 0.005 & 0.002 \\
\hline \multirow{5}{*}{05549700} & \multirow{5}{*}{ MUTTON CREEK AT ISLAND LAKE, IL } & \multirow{5}{*}{ Yes } & $\begin{array}{l}\text { At-site, unadjusted for urbanization, EMA, } \\
\text { at-site skew }\end{array}$ & 76 & 159 & 238 & 371 & 498 & 651 & 835 & 1,140 \\
\hline & & & $\begin{array}{l}\text { At-site, adjusted for urbanization, EMA, at- } \\
\text { site skew }\end{array}$ & 122 & 231 & 329 & 491 & 642 & 823 & 1,040 & 1,390 \\
\hline & & & $\begin{array}{l}\text { At-site, adjusted for urbanization, EMA, } \\
\text { weighted skew: }\left(Q_{p}\right)_{g, s}\end{array}$ & 126 & 234 & 325 & 466 & 590 & 732 & 892 & 1,140 \\
\hline & & & Regional regression: $(Q p)_{g, r}$ & 182 & 268 & 328 & 405 & 463 & 521 & 579 & 656 \\
\hline & & & 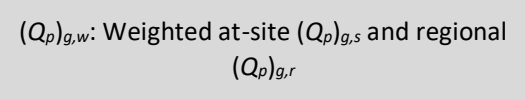 & 133 & 240 & 326 & 449 & 548 & 650 & 756 & 898 \\
\hline \multirow{5}{*}{05549850} & \multirow{5}{*}{ FLLNT CREEK NEAR FOX RIVER GROVE, IL } & \multirow{5}{*}{ Yes } & $\begin{array}{l}\text { At-site, unadjusted for urbanization, EMA, } \\
\text { at-site skew }\end{array}$ & 271 & 386 & 472 & 595 & 696 & 806 & 927 & 1,100 \\
\hline & & & $\begin{array}{l}\text { At-site, adjusted for urbanization, EMA, at- } \\
\text { site skew }\end{array}$ & 420 & 535 & 624 & 752 & 860 & 979 & 1,110 & 1,300 \\
\hline & & & $\begin{array}{l}\text { At-site, adjusted for urbanization, EMA, } \\
\text { weighted skew: ( }\left(z_{p}\right)_{g, s}\end{array}$ & 430 & 544 & 623 & 725 & 804 & 886 & 970 & 1,090 \\
\hline & & & Regional regression: $\left(Q_{p}\right)_{g, r}$ & 543 & 768 & 927 & 1,130 & 1,290 & 1,450 & 1,620 & 1,840 \\
\hline & & & 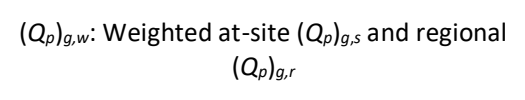 & 432 & 549 & 631 & 741 & 827 & 918 & 1,010 & 1,150 \\
\hline \multirow{5}{*}{05549900} & \multirow{5}{*}{ FOX RIVER TRIBUTARY NEAR CARY, IL } & \multirow{5}{*}{ Yes } & $\begin{array}{l}\text { At-site, unadjusted for urbanization, EMA, } \\
\text { at-site skew }\end{array}$ & 11 & 26 & 40 & 60 & 77 & 96 & 115 & 142 \\
\hline & & & $\begin{array}{l}\text { At-site, adjusted for urbanization, EMA, at- } \\
\text { site skew }\end{array}$ & 12 & 28 & 42 & 62 & 77 & 94 & 111 & 134 \\
\hline & & & $\begin{array}{l}\text { At-site, adjusted for urbanization, EMA, } \\
\text { weighted skew: ( }\left(Q_{p}\right)_{g, s}\end{array}$ & 11 & 27 & 43 & 74 & 106 & 146 & 198 & 287 \\
\hline & & & Regional regression: $(Q p)_{g, r}$ & 21 & 38 & 51 & 72 & 89 & 108 & 128 & 157 \\
\hline & & & $\begin{array}{c}\left(Q_{\rho}\right)_{\text {g,w: }} \text { : Weighted at-site }\left(Q_{\rho}\right)_{g, s} \text { and regional } \\
\left(Q_{\rho \rho \rho} g_{y,}\right.\end{array}$ & 12 & 29 & 46 & 73 & 99 & 128 & 160 & 208 \\
\hline \multirow{5}{*}{05550300} & \multirow{5}{*}{ TYLER CREEK AT ELGIN, IL } & \multirow{5}{*}{ Yes } & $\begin{array}{l}\text { At-site, unadjusted for urbanization, EMA, } \\
\text { at-site skew }\end{array}$ & 436 & 738 & 1,000 & 1,430 & 1,830 & 2,310 & 2,870 & 3,790 \\
\hline & & & $\begin{array}{l}\text { At-site, adjusted for urbanization, EMA, at- } \\
\text { site skew }\end{array}$ & 542 & 844 & 1,090 & 1,470 & 1,810 & 2,190 & 2,630 & 3,320 \\
\hline & & & 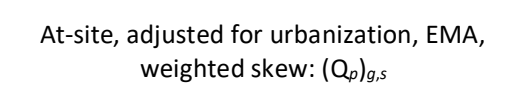 & 554 & 855 & 1,090 & 1,420 & 1,700 & 2,000 & 2,330 & 2,820 \\
\hline & & & Regional regression: $\left(Q_{\rho}\right)_{g, r}$ & 852 & 1,310 & 1,650 & 2,100 & 2,440 & 2,800 & 3,160 & 3,660 \\
\hline & & & $\begin{array}{l}\left(Q_{p}\right)_{g, w}: \text { Weighted at-site }\left(Q_{p}\right)_{g, s} \text { and regional } \\
\left(Q_{p} g_{g, r}\right.\end{array}$ & 565 & 878 & 1,120 & 1,480 & 1,780 & 2,120 & 2,480 & 3,000 \\
\hline
\end{tabular}




\begin{tabular}{|c|c|c|c|c|c|c|c|c|c|c|c|}
\hline \multirow{2}{*}{$\begin{array}{c}\text { USGS } \\
\text { streamgage } \\
\text { number }\end{array}$} & \multirow[b]{2}{*}{ Streamgage name } & \multirow{2}{*}{$\begin{array}{l}\text { Used in regression } \\
\text { analyses (non- } \\
\text { redundant) }\end{array}$} & \multirow[b]{2}{*}{ Frequency analysis method } & \multicolumn{8}{|c|}{ Discharge for given annual exceedance probability, $\mathrm{ft}^{3} / \mathrm{s}$} \\
\hline & & & & 0.50 & 0.20 & 0.10 & 0.04 & 0.02 & 0.01 & 0.005 & 0.002 \\
\hline \multirow{5}{*}{05550430} & \multirow{5}{*}{ EAST BRANCH POPLAR CREEK NEAR PALATINE, IL } & \multirow{5}{*}{ Yes } & $\begin{array}{l}\text { At-site, unadjusted for urbanization, EMA, } \\
\text { at-site skew }\end{array}$ & 80 & 122 & 157 & 212 & 262 & 320 & 388 & 496 \\
\hline & & & $\begin{array}{l}\text { At-site, adjusted for urbanization, EMA, at- } \\
\text { site skew }\end{array}$ & 99 & 148 & 185 & 236 & 279 & 324 & 374 & 445 \\
\hline & & & $\begin{array}{l}\text { At-site, adjusted for urbanization, EMA, } \\
\text { weighted skew: }\left(Q_{p}\right)_{g, s}\end{array}$ & 99 & 148 & 185 & 236 & 278 & 322 & 370 & 440 \\
\hline & & & Regional regression: $(Q p)_{a, r}$ & 86 & 129 & 160 & 202 & 234 & 267 & 301 & 346 \\
\hline & & & $\begin{array}{c}\left(Q_{\rho \rho}\right)_{y, w} \text { Weighted at-site }\left(Q_{p}\right)_{Q, s} \text { and regional } \\
\left(Q_{\rho}\right)_{Q, r}\end{array}$ & 98 & 146 & 182 & 231 & 270 & 311 & 354 & 414 \\
\hline \multirow{5}{*}{05550450} & \multirow{5}{*}{ POPLAR CREEK NEAR ONTARIOVILLE, IL } & \multirow{5}{*}{ No } & $\begin{array}{l}\text { At-site, unadjusted for urbanization, EMA, } \\
\text { at-site skew }\end{array}$ & 207 & 289 & 344 & 414 & 467 & 520 & 574 & 647 \\
\hline & & & $\begin{array}{l}\text { At-site, adjusted for urbanization, EMA, at- } \\
\text { site skew }\end{array}$ & 285 & 380 & 439 & 509 & 559 & 608 & 655 & 716 \\
\hline & & & $\begin{array}{l}\text { At-site, adjusted for urbanization, EMA, } \\
\text { weighted skew: ( }\left(_{p}\right)_{g, s}\end{array}$ & 280 & 378 & 444 & 529 & 593 & 659 & 726 & 818 \\
\hline & & & Regional regression: $\left(Q_{0}\right)_{g, r}$ & 325 & 456 & 550 & 674 & 770 & 867 & 967 & 1,100 \\
\hline & & & $\begin{array}{c}\left(Q_{\rho}\right)_{Q, w:} \text { : Weighted at-site }\left(Q_{\rho}\right)_{g, s} \text { and regional } \\
\left(Q_{\rho}\right)_{g, r}\end{array}$ & 282 & 381 & 450 & 539 & 608 & 679 & 752 & 854 \\
\hline \multirow{5}{*}{05550470} & \multirow{5}{*}{ POPLAR CREEK TRIBUTARY NEAR BARTLETT, IL } & \multirow{5}{*}{ Yes } & $\begin{array}{l}\text { At-site, unadjusted for urbanization, EMA, } \\
\text { at-site skew }\end{array}$ & 157 & 247 & 321 & 434 & 534 & 648 & 780 & 983 \\
\hline & & & $\begin{array}{l}\text { At-site, adjusted for urbanization, EMA, at- } \\
\text { site skew }\end{array}$ & 170 & 242 & 309 & 418 & 522 & 649 & 804 & 1,060 \\
\hline & & & $\begin{array}{l}\text { At-site, adjusted for urbanization, EMA, } \\
\text { weighted skew: }\left(Q_{p}\right)_{\text {g,s }}\end{array}$ & 177 & 250 & 308 & 394 & 466 & 548 & 639 & 778 \\
\hline & & & Regional regression: $\left(\left.Q p\right|_{g r}\right.$ & 193 & 279 & 344 & 433 & 504 & 578 & 655 & 761 \\
\hline & & & $\begin{array}{c}\left(Q_{\rho}\right)_{g, w} \text { : Weighted at-site }\left(Q_{\rho}\right)_{Q, s} \text { and regional } \\
\left(Q_{\rho}\right)_{Q, r}\end{array}$ & 178 & 252 & 312 & 398 & 472 & 553 & 643 & 774 \\
\hline \multirow{5}{*}{05550500} & \multirow{5}{*}{ POPLAR CREEK AT ELGIN, IL } & \multirow{5}{*}{ Yes } & $\begin{array}{l}\text { At-site, unadjusted for urbanization, EMA, } \\
\text { at-site skew }\end{array}$ & 457 & 706 & 884 & 1,120 & 1,300 & 1,500 & 1,690 & 1,960 \\
\hline & & & $\begin{array}{l}\text { At-site, adjusted for urbanization, EMA, at- } \\
\text { site skew }\end{array}$ & 546 & 766 & 927 & 1,150 & 1,330 & 1,520 & 1,730 & 2,030 \\
\hline & & & $\begin{array}{l}\text { At-site, adjusted for urbanization, EMA, } \\
\text { weighted skew: (Qop) })_{g, s}\end{array}$ & 547 & 766 & 927 & 1,150 & 1,320 & 1,520 & 1,720 & 2,010 \\
\hline & & & Regional regression: $\left(Q_{p}\right)_{a, r}$ & 651 & 916 & 1,110 & 1,360 & 1,560 & 1,760 & 1,970 & 2,250 \\
\hline & & & 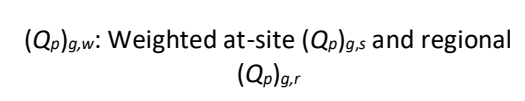 & 548 & 769 & 932 & 1,160 & 1,340 & 1,530 & 1,740 & 2,030 \\
\hline
\end{tabular}




\begin{tabular}{|c|c|c|c|c|c|c|c|c|c|c|c|}
\hline \multirow{2}{*}{$\begin{array}{c}\text { USGS } \\
\text { streamgage } \\
\text { number }\end{array}$} & \multirow[b]{2}{*}{ Streamgage name } & \multirow{2}{*}{$\begin{array}{l}\text { Used in regression } \\
\text { analyses (non- } \\
\text { redundant) }\end{array}$} & \multirow[b]{2}{*}{ Frequency analysis method } & \multicolumn{8}{|c|}{ Discharge for given annual exceedance probability, $\mathrm{ft}^{3} / \mathrm{s}$} \\
\hline & & & & 0.50 & 0.20 & 0.10 & 0.04 & 0.02 & 0.01 & 0.005 & 0.002 \\
\hline \multirow{5}{*}{05551030} & \multirow{5}{*}{ BREWSTER CREEK AT VALLEY VIEW, IL } & \multirow{5}{*}{ Yes } & $\begin{array}{l}\text { At-site, unadjusted for urbanization, EMA, } \\
\text { at-site skew }\end{array}$ & 185 & 350 & 491 & 707 & 897 & 1,110 & 1,360 & 1,730 \\
\hline & & & $\begin{array}{l}\text { At-site, adjusted for urbanization, EMA, at- } \\
\text { site skew }\end{array}$ & 373 & 594 & 758 & 983 & 1,160 & 1,350 & 1,560 & 1,840 \\
\hline & & & $\begin{array}{l}\text { At-site, adjusted for urbanization, EMA, } \\
\text { weighted skew: (Q }\left(Q_{p}\right)_{g, s}\end{array}$ & 368 & 591 & 764 & 1,010 & 1,220 & 1,440 & 1,680 & 2,030 \\
\hline & & & Regional regression: $(Q p)_{a, r}$ & 369 & 548 & 678 & 852 & 987 & 1,130 & 1,270 & 1,460 \\
\hline & & & 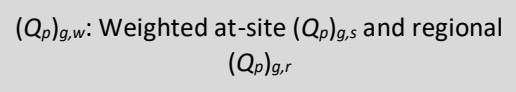 & 368 & 586 & 751 & 979 & 1,160 & 1,350 & 1,560 & 1,830 \\
\hline \multirow{5}{*}{05551050} & \multirow{5}{*}{ NORTON CREEK NEAR WAYNE, IL } & \multirow{5}{*}{ Yes } & $\begin{array}{l}\text { At-site, unadjusted for urbanization, EMA, } \\
\text { at-site skew }\end{array}$ & 88 & 200 & 330 & 598 & 908 & 1,350 & 1,980 & 3,230 \\
\hline & & & $\begin{array}{l}\text { At-site, adjusted for urbanization, EMA, at- } \\
\text { site skew }\end{array}$ & 158 & 313 & 492 & 860 & 1,290 & 1,910 & 2,800 & 4,600 \\
\hline & & & $\begin{array}{l}\text { At-site, adjusted for urbanization, EMA, } \\
\text { weighted skew: (Q }\left(Q_{p}\right)_{g, s}\end{array}$ & 175 & 339 & 494 & 754 & 1,000 & 1,310 & 1,680 & 2,310 \\
\hline & & & Regional regression: $\left(Q_{p}\right)_{g, r}$ & 186 & 273 & 335 & 416 & 478 & 540 & 603 & 687 \\
\hline & & & $\begin{array}{l}\left(Q_{\rho}\right)_{g, w:}: \text { Weighted at-site }\left(Q_{\rho}\right)_{g, s} \text { and regional } \\
\left(Q_{\rho}\right)_{g, r}\end{array}$ & 177 & 325 & 449 & 629 & 775 & 925 & 1,080 & 1,280 \\
\hline \multirow{5}{*}{05551060} & \multirow{5}{*}{ NORTON CREEK NEAR ST. CHARLES, IL } & \multirow{5}{*}{ No } & $\begin{array}{l}\text { At-site, unadjusted for urbanization, EMA, } \\
\text { at-site skew }\end{array}$ & 109 & 202 & 300 & 482 & 678 & 941 & 1,290 & 1,950 \\
\hline & & & $\begin{array}{l}\text { At-site, adjusted for urbanization, EMA, at- } \\
\text { site skew }\end{array}$ & 218 & 351 & 495 & 770 & 1,070 & 1,480 & 2,050 & 3,140 \\
\hline & & & 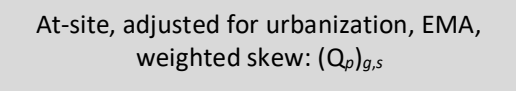 & 240 & 383 & 500 & 678 & 834 & 1,010 & 1,220 & 1,530 \\
\hline & & & Regional regression: $(Q p)_{g, r}$ & 309 & 462 & 572 & 718 & 831 & 945 & 1,060 & 1,220 \\
\hline & & & $\begin{array}{l}\left(Q_{\rho}\right)_{g, w:} \text { : Weighted at-site }\left(Q_{\rho}\right)_{g, s} \text { and regional } \\
\qquad\left(Q_{\rho}\right)_{g, r}\end{array}$ & 244 & 390 & 509 & 685 & 833 & 996 & 1,170 & 1,420 \\
\hline \multirow{5}{*}{05551200} & \multirow{5}{*}{ FERSON CREEK NEAR ST. CHARLES, IL } & \multirow{5}{*}{ Yes } & $\begin{array}{l}\text { At-site, unadjusted for urbanization, EMA, } \\
\text { at-site skew }\end{array}$ & 959 & 1,610 & 2,040 & 2,560 & 2,910 & 3,250 & 3,570 & 3,960 \\
\hline & & & $\begin{array}{l}\text { At-site, adjusted for urbanization, EMA, at- } \\
\text { site skew }\end{array}$ & 1,440 & 2,200 & 2,680 & 3,230 & 3,610 & 3,960 & 4,280 & 4,670 \\
\hline & & & $\begin{array}{l}\text { At-site, adjusted for urbanization, EMA, } \\
\left.\text { weighted skew: ( } Q_{0}\right)_{g, s}\end{array}$ & 1,390 & 2,200 & 2,750 & 3,460 & 3,990 & 4,520 & 5,050 & 5,760 \\
\hline & & & Regional regression: $\left(Q_{p}\right)_{g, r}$ & 1,120 & 1,720 & 2,150 & 2,730 & 3,180 & 3,650 & 4,120 & 4,770 \\
\hline & & & $\begin{array}{l}\left(Q_{\rho}\right)_{g, w}: \text { Weighted at-site }\left(Q_{\rho}\right)_{g, s} \text { and regional } \\
\qquad\left(\rho_{\rho} g_{g, r}\right.\end{array}$ & 1,380 & 2,180 & 2,720 & 3,420 & 3,930 & 4,440 & 4,950 & 5,630 \\
\hline
\end{tabular}




\begin{tabular}{|c|c|c|c|c|c|c|c|c|c|c|c|}
\hline \multirow{2}{*}{$\begin{array}{c}\text { USGS } \\
\text { streamgage } \\
\text { number }\end{array}$} & \multirow[b]{2}{*}{ Streamgage name } & \multirow{2}{*}{$\begin{array}{l}\text { Used in regression } \\
\text { analyses (non- } \\
\text { redundant) }\end{array}$} & \multirow[b]{2}{*}{ Frequency analysis method } & \multicolumn{8}{|c|}{ Discharge for given annual exceedance probability, $\mathrm{ft}^{3} / \mathrm{s}$} \\
\hline & & & & 0.50 & 0.20 & 0.10 & 0.04 & 0.02 & 0.01 & 0.005 & 0.002 \\
\hline \multirow{5}{*}{05551330} & \multirow{5}{*}{ MILL CREEK NEAR BATAVIA, IL } & \multirow{5}{*}{ Yes } & $\begin{array}{l}\text { At-site, unadjusted for urbanization, EMA, } \\
\text { at-site skew }\end{array}$ & 410 & 1,000 & 1,740 & 3,370 & 5,360 & 8,350 & 12,800 & 22,200 \\
\hline & & & $\begin{array}{l}\text { At-site, adjusted for urbanization, EMA, at- } \\
\text { site skew }\end{array}$ & 474 & 1,110 & 1,880 & 3,520 & 5,460 & 8,300 & 12,400 & 20,800 \\
\hline & & & $\begin{array}{l}\text { At-site, adjusted for urbanization, EMA, } \\
\text { weighted skew: (Q }\left(Q_{p}\right)_{g, s}\end{array}$ & 508 & 1,160 & 1,850 & 3,120 & 4,460 & 6,200 & 8,460 & 12,500 \\
\hline & & & Regional regression: $(Q p)_{a, r}$ & 903 & 1,420 & 1,810 & 2,340 & 2,760 & 3,200 & 3,660 & 4,290 \\
\hline & & & 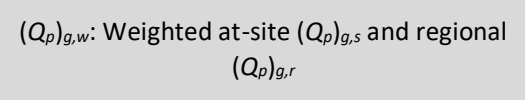 & 598 & 1,250 & 1,830 & 2,700 & 3,420 & 4,190 & 5,000 & 6,100 \\
\hline \multirow{5}{*}{05551520} & \multirow{5}{*}{ INDIAN CREEK NEAR NORTH AURORA, IL } & \multirow{5}{*}{ Yes } & $\begin{array}{l}\text { At-site, unadjusted for urbanization, EMA, } \\
\text { at-site skew }\end{array}$ & 130 & 216 & 280 & 367 & 436 & 510 & 587 & 695 \\
\hline & & & $\begin{array}{l}\text { At-site, adjusted for urbanization, EMA, at- } \\
\text { site skew }\end{array}$ & 184 & 288 & 362 & 463 & 542 & 625 & 712 & 833 \\
\hline & & & $\begin{array}{l}\text { At-site, adjusted for urbanization, EMA, } \\
\text { weighted skew: ( }\left(Q_{p}\right)_{g, s}\end{array}$ & 182 & 286 & 365 & 474 & 563 & 659 & 761 & 908 \\
\hline & & & Regional regression: $\left(Q_{0}\right)_{g, r}$ & 168 & 253 & 314 & 394 & 457 & 519 & 583 & 668 \\
\hline & & & 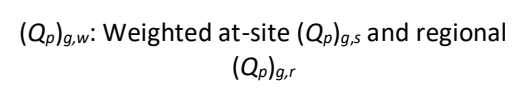 & 181 & 283 & 358 & 462 & 543 & 628 & 716 & 837 \\
\hline \multirow{5}{*}{05551530} & \multirow{5}{*}{ INDIAN CREEK AT AURORA, IL } & \multirow{5}{*}{ No } & $\begin{array}{l}\text { At-site, unadjusted for urbanization, EMA, } \\
\text { at-site skew }\end{array}$ & 530 & 659 & 727 & 798 & 842 & 880 & 914 & 953 \\
\hline & & & $\begin{array}{l}\text { At-site, adjusted for urbanization, EMA, at- } \\
\text { site skew }\end{array}$ & 848 & 998 & 1,070 & 1,150 & 1,190 & 1,230 & 1,260 & 1,300 \\
\hline & & & 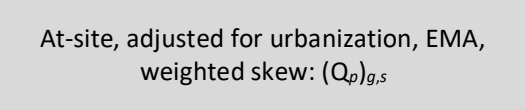 & 829 & 996 & 1,090 & 1,210 & 1,290 & 1,360 & 1,430 & 1,520 \\
\hline & & & Regional regression: $(Q p)_{g, r}$ & 531 & 794 & 989 & 1,260 & 1,470 & 1,680 & 1,910 & 2,230 \\
\hline & & & 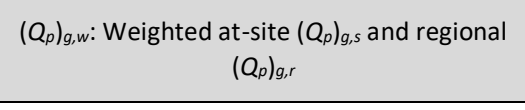 & 824 & 992 & 1,090 & 1,210 & 1,290 & 1,370 & 1,450 & 1,550 \\
\hline \multirow{5}{*}{05551620} & \multirow{5}{*}{ BLACKBERRY CREEK NEAR KANEVILLE, IL } & \multirow{5}{*}{ No } & $\begin{array}{l}\text { At-site, unadjusted for urbanization, EMA, } \\
\text { at-site skew }\end{array}$ & 423 & 529 & 593 & 667 & 719 & 768 & 816 & 877 \\
\hline & & & $\begin{array}{l}\text { At-site, adjusted for urbanization, EMA, at- } \\
\text { site skew }\end{array}$ & 546 & 669 & 741 & 824 & 881 & 934 & 986 & 1,050 \\
\hline & & & $\begin{array}{l}\text { At-site, adjusted for urbanization, EMA, } \\
\text { weighted skew: (Oe o ols, }\end{array}$ & 544 & 668 & 742 & 830 & 890 & 948 & 1,000 & 1,080 \\
\hline & & & Regional regression: $\left(Q_{p}\right)_{g, r}$ & 586 & 952 & 1,220 & 1,580 & 1,850 & 2,130 & 2,420 & 2,810 \\
\hline & & & $\begin{array}{l}\left(Q_{\rho}\right)_{y, w}: \text { Weighted at-site } \\
\qquad\left(Q_{p}\right)_{g, s} \text { and regional }\end{array}$ & 545 & 674 & 752 & 849 & 918 & 986 & 1,050 & 1,140 \\
\hline
\end{tabular}




\begin{tabular}{|c|c|c|c|c|c|c|c|c|c|c|c|}
\hline \multirow{2}{*}{$\begin{array}{c}\text { USGS } \\
\text { streamgage } \\
\text { number }\end{array}$} & \multirow[b]{2}{*}{ Streamgage name } & \multirow{2}{*}{$\begin{array}{l}\text { Used in regression } \\
\text { analyses (non- } \\
\text { redundant) }\end{array}$} & \multirow[b]{2}{*}{ Frequency analysis method } & \multicolumn{8}{|c|}{ Discharge for given annual exceedance probability, $\mathrm{ft}^{3} / \mathrm{s}$} \\
\hline & & & & 0.50 & 0.20 & 0.10 & 0.04 & 0.02 & 0.01 & 0.005 & 0.002 \\
\hline \multirow{5}{*}{05551650} & \multirow{5}{*}{ LAKE RUN AT BALD MOUND, IL } & \multirow{5}{*}{ Yes } & $\begin{array}{l}\text { At-site, unadjusted for urbanization, EMA, } \\
\text { at-site skew }\end{array}$ & 45 & 112 & 191 & 348 & 524 & 767 & 1,100 & 1,730 \\
\hline & & & $\begin{array}{l}\text { At-site, adjusted for urbanization, EMA, at- } \\
\text { site skew }\end{array}$ & 50 & 123 & 205 & 369 & 551 & 802 & 1,140 & 1,790 \\
\hline & & & $\begin{array}{l}\text { At-site, adjusted for urbanization, EMA, } \\
\text { weighted skew: }\left(Q_{p}\right)_{g, s}\end{array}$ & 55 & 129 & 199 & 314 & 419 & 543 & 686 & 908 \\
\hline & & & Regional regression: $(Q p)_{a, r}$ & 122 & 227 & 308 & 420 & 508 & 598 & 690 & 814 \\
\hline & & & $\begin{array}{c}\left(Q_{\rho p}\right)_{y, w} \text { : Weighted at-site }\left(Q_{p}\right)_{Q, s} \text { and regional } \\
\left(\left.Q_{p}\right|_{Q, r}\right.\end{array}$ & 68 & 151 & 229 & 351 & 455 & 567 & 688 & 856 \\
\hline \multirow{5}{*}{05551675} & \multirow{5}{*}{ BLACKBERRY CREEK NEAR MONTGOMERY, IL } & \multirow{5}{*}{ No } & $\begin{array}{l}\text { At-site, unadjusted for urbanization, EMA, } \\
\text { at-site skew }\end{array}$ & 520 & 914 & 1,220 & 1,640 & 1,980 & 2,340 & 2,730 & 3,280 \\
\hline & & & $\begin{array}{l}\text { At-site, adjusted for urbanization, EMA, at- } \\
\text { site skew }\end{array}$ & 606 & 1,020 & 1,320 & 1,710 & 2,010 & 2,320 & 2,630 & 3,050 \\
\hline & & & $\begin{array}{l}\text { At-site, adjusted for urbanization, EMA, } \\
\text { weighted skew: ( }\left(_{0}\right)_{g, s}\end{array}$ & 589 & 1,020 & 1,350 & 1,820 & 2,210 & 2,630 & 3,080 & 3,740 \\
\hline & & & Regional regression: $\left(Q_{0}\right)_{g, r}$ & 1,130 & 1,740 & 2,180 & 2,760 & 3,210 & 3,670 & 4,150 & 4,800 \\
\hline & & & $\begin{array}{c}\left(Q_{\rho \rho}\right)_{y, w} \text { Weighted at-site }\left(Q_{p}\right)_{Q, s} \text { and regional } \\
\left(Q_{p}\right)_{Q, r}\end{array}$ & 655 & 1,130 & 1,500 & 2,040 & 2,480 & 2,950 & 3,440 & 4,140 \\
\hline \multirow{5}{*}{05551700} & \multirow{5}{*}{ BLACKBERRY CREEK NEAR YORKVILLE, IL } & \multirow{5}{*}{ Yes } & $\begin{array}{l}\text { At-site, unadjusted for urbanization, EMA, } \\
\text { at-site skew }\end{array}$ & 666 & 1,240 & 1,730 & 2,450 & 3,080 & 3,780 & 4,550 & 5,710 \\
\hline & & & $\begin{array}{l}\text { At-site, adjusted for urbanization, EMA, at- } \\
\text { site skew }\end{array}$ & 889 & 1,560 & 2,100 & 2,900 & 3,560 & 4,300 & 5,100 & 6,300 \\
\hline & & & $\begin{array}{l}\text { At-site, adjusted for urbanization, EMA, } \\
\text { weighted skew: }\left(Q_{p}\right)_{\text {g,s }}\end{array}$ & 887 & 1,560 & 2,110 & 2,910 & 3,590 & 4,350 & 5,180 & 6,420 \\
\hline & & & Regional regression: $\left(\left.Q p\right|_{g r}\right.$ & 1,250 & 1,890 & 2,350 & 2,950 & 3,420 & 3,900 & 4,390 & 5,060 \\
\hline & & & $\begin{array}{c}\left(Q_{\rho}\right)_{g, w} \text { : Weighted at-site }\left(Q_{\rho}\right)_{Q, s} \text { and regional } \\
\left(Q_{\rho}\right)_{Q, r}\end{array}$ & 903 & 1,580 & 2,120 & 2,920 & 3,570 & 4,280 & 5,030 & 6,100 \\
\hline \multirow{5}{*}{05551800} & \multirow{5}{*}{ FOX RIVER TRIBUTARY NO 2 NEAR FOX, IL } & \multirow{5}{*}{ Yes } & $\begin{array}{l}\text { At-site, unadjusted for urbanization, EMA, } \\
\text { at-site skew }\end{array}$ & 65 & 164 & 262 & 429 & 586 & 775 & 997 & 1,350 \\
\hline & & & $\begin{array}{l}\text { At-site, adjusted for urbanization, EMA, at- } \\
\text { site skew }\end{array}$ & 118 & 248 & 366 & 556 & 729 & 930 & 1,160 & 1,530 \\
\hline & & & $\begin{array}{l}\text { At-site, adjusted for urbanization, EMA, } \\
\text { weighted skew: (Qop) })_{g, s}\end{array}$ & 120 & 249 & 364 & 542 & 701 & 882 & 1,090 & 1,400 \\
\hline & & & Regional regression: $\left(Q_{p}\right)_{g, r}$ & 56 & 109 & 151 & 213 & 262 & 314 & 369 & 443 \\
\hline & & & $\begin{array}{c}\left(Q_{\rho \rho}\right)_{y, w} \text { : Weighted at-site }\left(Q_{p}\right)_{Q, s} \text { and regional } \\
\left(Q_{p}\right)_{Q, r}\end{array}$ & 105 & 210 & 294 & 410 & 503 & 598 & 694 & 823 \\
\hline
\end{tabular}




\begin{tabular}{|c|c|c|c|c|c|c|c|c|c|c|c|}
\hline \multirow{2}{*}{$\begin{array}{c}\text { USGS } \\
\text { streamgage } \\
\text { number }\end{array}$} & \multirow[b]{2}{*}{ Streamgage name } & \multirow{2}{*}{$\begin{array}{l}\text { Used in regression } \\
\text { analyses (non- } \\
\text { redundant) }\end{array}$} & \multirow[b]{2}{*}{ Frequency analysis method } & \multicolumn{8}{|c|}{ Discharge for given annual exceedance probability, $\mathrm{ft}^{3} / \mathrm{s}$} \\
\hline & & & & 0.50 & 0.20 & 0.10 & 0.04 & 0.02 & 0.01 & 0.005 & 0.002 \\
\hline \multirow{5}{*}{05551900} & \multirow{5}{*}{$\begin{array}{l}\text { EAST BRANCH BIG ROCK CREEK NEAR BIG ROCK, } \\
\text { IL }\end{array}$} & \multirow{5}{*}{ Yes } & $\begin{array}{l}\text { At-site, unadjusted for urbanization, EMA, } \\
\text { at-site skew }\end{array}$ & 620 & 941 & 1,180 & 1,510 & 1,780 & 2,060 & 2,370 & 2,810 \\
\hline & & & $\begin{array}{l}\text { At-site, adjusted for urbanization, EMA, at- } \\
\text { site skew }\end{array}$ & 637 & 963 & 1,210 & 1,540 & 1,810 & 2,100 & 2,420 & 2,860 \\
\hline & & & $\begin{array}{l}\text { At-site, adjusted for urbanization, EMA, } \\
\text { weighted skew: (o o o g,s }\end{array}$ & 650 & 969 & 1,190 & 1,480 & 1,700 & 1,920 & 2,150 & 2,460 \\
\hline & & & Regional regression: $(Q p)_{Q, r}$ & 699 & 1,130 & 1,440 & 1,830 & 2,130 & 2,430 & 2,730 & 3,120 \\
\hline & & & $\begin{array}{c}\left(Q_{\rho}\right)_{g, w}: \text { Weighted at-site }\left(Q_{\rho}\right)_{g, s} \text { and regional } \\
\left(Q_{\rho}\right)_{g, r}\end{array}$ & 654 & 983 & 1,210 & 1,520 & 1,760 & 2,000 & 2,250 & 2,600 \\
\hline \multirow{5}{*}{05551930} & \multirow{5}{*}{ WELCH CREEK NEAR BIG ROCK, IL } & \multirow{5}{*}{ Yes } & $\begin{array}{l}\text { At-site, unadjusted for urbanization, EMA, } \\
\text { at-site skew }\end{array}$ & 316 & 459 & 552 & 669 & 755 & 840 & 924 & 1,040 \\
\hline & & & $\begin{array}{l}\text { At-site, adjusted for urbanization, EMA, at- } \\
\text { site skew }\end{array}$ & 346 & 496 & 594 & 716 & 805 & 893 & 980 & 1,090 \\
\hline & & & $\begin{array}{l}\text { At-site, adjusted for urbanization, EMA, } \\
\text { weighted skew: }\left(Q_{p}\right)_{g, s}\end{array}$ & 345 & 496 & 595 & 720 & 812 & 903 & 993 & 1,110 \\
\hline & & & Regional regression: $\left(Q_{p}\right)_{g, r}$ & 590 & 963 & 1,230 & 1,590 & 1,850 & 2,120 & 2,400 & 2,760 \\
\hline & & & $\begin{array}{c}\left(Q_{p}\right)_{g, w}: \text { Weighted at-site }\left(Q_{\rho}\right)_{g, s} \text { and regional } \\
\left(Q_{p}\right)_{g, r}\end{array}$ & 357 & 519 & 632 & 780 & 895 & 1,010 & 1,140 & 1,310 \\
\hline
\end{tabular}




\section{APPENDIX 4}

Table 3. Spatially averaged basin characteristics considered for developing spatial regression equations in this study in northeastern Illinois.

[NLCD, National Land Cover Database; SSURGO, Soil Survey Geographic; NED, National Elevation Dataset; NWI, National Wetlands Inventory; WWI, Wisconsin Wetlands Inventory]

\begin{tabular}{|c|c|c|c|c|c|c|}
\hline Category & Variable name & Variable description & Units & $\begin{array}{l}\text { Selected } \\
\text { transformation }\end{array}$ & Data source & Reference \\
\hline \multicolumn{7}{|c|}{ Drainage Area } \\
\hline & Drainage Area & Drainage area of basin upstream from streamgage. & $\begin{array}{l}\text { square } \\
\text { miles }\end{array}$ & $\log _{10}$ & $\begin{array}{l}\text { As the area of the streamgage drainage basins } \\
\text { delineated as described in the report. }\end{array}$ & \\
\hline \multicolumn{7}{|c|}{ Urbanization } \\
\hline & NLCD_21_22_23_24 & $\begin{array}{l}\text { Sum of fractions of NLCD } 2011 \text { classes } 21 \text { (developed, open } \\
\text { space), } 22 \text { (developed, low intensity), } 23 \text { (developed, } \\
\text { medium intensity), } 24 \text { (developed, high intensity) }\end{array}$ & $\begin{array}{l}\text { decimal } \\
\text { fraction }\end{array}$ & none & NLCD 2011 (http://www.mrlc.gov/nlcd2011.php) & Jin et al. (2013) \\
\hline & NLCD_22_23_24 & $\begin{array}{l}\text { Sum of fractions of NLCD } 2011 \text { classes } 22 \text { (developed, low } \\
\text { intensity), } 23 \text { (developed, medium intensity), } 24 \\
\text { (developed, high intensity) }\end{array}$ & $\begin{array}{l}\text { decimal } \\
\text { fraction }\end{array}$ & $x^{(1 / 2)}$ & NLCD 2011 (http://www.mrlc.gov/nlcd2011.php) & Jin et al. (2013) \\
\hline & NLCD_23_24 & $\begin{array}{l}\text { Sum of fractions of NLCD } 2011 \text { classes } 23 \text { (developed, } \\
\text { medium intensity), } 24 \text { (developed, high intensity) }\end{array}$ & $\begin{array}{l}\text { decimal } \\
\text { fraction }\end{array}$ & $x^{(1 / 2)}$ & NLCD 2011 (http://www.mrlc.gov/nlcd2011.php) & Jin et al. (2013) \\
\hline & NLCD_24 & Fraction of NLCD 2011 class 24 (developed, high intensity) & $\begin{array}{l}\text { decimal } \\
\text { fraction }\end{array}$ & $\log _{10}(X+0.001)$ & NLCD 2011 (http://www.mrlc.gov/nlcd2011.php) & Jin et al. (2013) \\
\hline & NCLD_Imperv & mean NLCD 2011 percent developed imperviousness & percent & $x^{(1 / 2)}$ & NLCD 2011 (http://www.mrlc.gov/nlcd2011.php) & Xian et al. (2011) \\
\hline & Theobald_Urban & $\begin{array}{l}\text { Sum of fractions of Theobald housing density classes } 7 \\
\text { (urban/exurban: } 1.7-10 \text { acres per lot), } 8 \text { (urban/exurban } \\
0.6-1.7 \text { acres per lot), } 9 \text { (urban/exurban, less than } 0.6 \\
\text { acres per lot), and } 10 \text { (commercial/industrial/ } \\
\text { transportation) }\end{array}$ & $\begin{array}{l}\text { decimal } \\
\text { fraction }\end{array}$ & none & $\begin{array}{l}\text { file bhcs_fote20080612.zip downloaded from } \\
\text { www.nrel.colostate.edu/ftp/theobald on 5/12/2009; } \\
\text { now at } \\
\text { https://pubs.er.usgs.gov/publication/sir20165049 }\end{array}$ & Theobald (2005) \\
\hline
\end{tabular}




\begin{tabular}{|c|c|c|c|c|c|c|}
\hline Category & Variable name & Variable description & Units & $\begin{array}{l}\text { Selected } \\
\text { transformation }\end{array}$ & Data source & Reference \\
\hline \multicolumn{7}{|c|}{ Water and wetlands } \\
\hline & NLCD_11 & Fraction of NLCD 2011 class 11 (open water) & $\begin{array}{l}\text { decimal } \\
\text { fraction }\end{array}$ & $\log _{10}(X+0.0001)$ & NLCD 2011 (http://www.mrlc.gov/nlcd2011.php) & Jin et al. (2013) \\
\hline & NLCD_11_90 & $\begin{array}{l}\text { Sum of fractions of NLCD } 2011 \text { classes } 11 \text { (open water) and } \\
90 \text { (woody wetlands) }\end{array}$ & $\begin{array}{l}\text { decimal } \\
\text { fraction }\end{array}$ & $x^{(1 / 2)}$ & NLCD 2011 (http://www.mrlc.gov/nlcd2011.php) & Jin et al. (2013) \\
\hline & NLCD_11_95 & $\begin{array}{l}\text { Sum of fractions of NLCD } 2011 \text { classes } 11 \text { (open water) and } \\
95 \text { (emergent herbaceous wetlands) }\end{array}$ & $\begin{array}{l}\text { decimal } \\
\text { fraction }\end{array}$ & $\log _{10}(X+0.0001)$ & NLCD 2011 (http://www.mrlc.gov/nlcd2011.php) & Jin et al. (2013) \\
\hline & NLCD_11_90_95 & $\begin{array}{l}\text { Sum of fractions of NLCD } 2011 \text { classes } 11 \text { (open water), } 90 \\
\text { (woody wetlands), and } 95 \text { (emergent herbaceous wetlands) }\end{array}$ & $\begin{array}{l}\text { decimal } \\
\text { fraction }\end{array}$ & $x^{(1 / 2)}$ & NLCD 2011 (http://www.mrlc.gov/nlcd2011.php) & Jin et al. (2013) \\
\hline & NLCD_90_95 & $\begin{array}{l}\text { Sum of fractions of NLCD } 2011 \text { classes } 90 \text { (woody wetlands) } \\
\text { and } 95 \text { (emergent herbaceous wetlands) }\end{array}$ & $\begin{array}{l}\text { decimal } \\
\text { fraction }\end{array}$ & $x^{(1 / 2)}$ & NLCD 2011 (http://www.mrlc.gov/nlcd2011.php) & Jin et al. (2013) \\
\hline & NWI.total & $\begin{array}{l}\text { Sum of fractions of emergent wetland, farmed wetland, } \\
\text { filled/drained wetland, forested wetland, non-wetland, } \\
\text { open-water wetland, shallow lake, and undefined. }\end{array}$ & $\begin{array}{l}\text { decimal } \\
\text { fraction }\end{array}$ & $x^{(1 / 2)}$ & $\begin{array}{l}\text { NWI: http://www.fws.gov/wetlands/); WWI: } \\
\text { http://dnr.wi.gov/topic/wetlands/inventory.html }\end{array}$ & $\begin{array}{l}\text { NWI: Cowardin et al. (1979); WWI: Wisconsin Department of } \\
\text { Natural Resources( 1992) }\end{array}$ \\
\hline & NWI.emergent & Fraction of NWI/WWI emergent wetlands & $\begin{array}{l}\text { decimal } \\
\text { fraction }\end{array}$ & $x^{(1 / 2)}$ & $\begin{array}{l}\text { NWI: http://www.fws.gov/wetlands//; wWI: } \\
\text { http://dnr.wi.gov/topic/wetlands/inventory.html }\end{array}$ & $\begin{array}{l}\text { NWI: Cowardin et al. (1979); WWI: Wisconsin Department of } \\
\text { Natural Resources (1992) }\end{array}$ \\
\hline & NWI.forested & Fraction of NWI/WWI forested wetlands & $\begin{array}{l}\text { decimal } \\
\text { fraction }\end{array}$ & $\log _{10}(X+0.001)$ & $\begin{array}{l}\text { NWI: http://www.fws.gov/wetlands//; wWI: } \\
\text { http://dnr.wi.gov/topic/wetlands/inventory.html }\end{array}$ & $\begin{array}{l}\text { NWI: Cowardin et al. (1979); WWI: Wisconsin Department of } \\
\text { Natural Resources (1992) }\end{array}$ \\
\hline & NWI.emergent_forested & $\begin{array}{l}\text { Sum of fractions of NWI/WWI emergent and forested } \\
\text { wetlands }\end{array}$ & $\begin{array}{l}\text { decimal } \\
\text { fraction }\end{array}$ & $x^{(1 / 2)}$ & $\begin{array}{l}\text { NWI: http://www.fws.gov/wetlands/); wWI: } \\
\text { http://dnr.wi.gov/topic/wetlands/inventory.html }\end{array}$ & $\begin{array}{l}\text { NWI: Cowardin et al. (1979); WWI: Wisconsin Department of } \\
\text { Natural Resources (1992) }\end{array}$ \\
\hline & DrainageClasso & Fraction "unknown (likely water)" & $\begin{array}{l}\text { decimal } \\
\text { fraction }\end{array}$ & $x^{(1 / 2)}$ & $\begin{array}{l}\text { SSURGO Database; table: muaggatt; column: drclassdcd } \\
\text { (Drainage Class - Dominant Condition) }\end{array}$ & Soil Survey Staff (2013) \\
\hline & DrainageClass1 & Fraction "very poorly drained" & $\begin{array}{l}\text { decimal } \\
\text { fraction }\end{array}$ & $x^{(1 / 2)}$ & $\begin{array}{l}\text { SSURGO Database; table: muaggatt; column: drclassdcd } \\
\text { (Drainage Class - Dominant Condition) }\end{array}$ & Soil Survey Staff (2013) \\
\hline
\end{tabular}




\begin{tabular}{|c|c|c|c|c|c|c|}
\hline Category & Variable name & Variable description & Units & $\begin{array}{l}\text { Selected } \\
\text { transformation }\end{array}$ & Data source & Reference \\
\hline & DrainageClass2 & Sum of fractions "poorly" and "very poorly drained" & $\begin{array}{l}\text { decimal } \\
\text { fraction }\end{array}$ & $x^{(1 / 2)}$ & $\begin{array}{l}\text { SSURGO Database; table: muaggatt; column: drclassdcd } \\
\text { (Drainage Class - Dominant Condition) }\end{array}$ & Soil Survey Staff (2013) \\
\hline & DrainageClass3 & $\begin{array}{l}\text { Sum of fractions "somewhat poorly", "poorly", and "very } \\
\text { poorly drained" }\end{array}$ & $\begin{array}{l}\text { decimal } \\
\text { fraction }\end{array}$ & none & $\begin{array}{l}\text { SSURGO Database; table: muaggatt; column: drclassdcd } \\
\text { (Drainage Class - Dominant Condition) }\end{array}$ & Soil Survey Staff (2013) \\
\hline & DrainageClass1a & $\begin{array}{l}\text { Sum of fractions "very poorly drained" and "unknown (likely } \\
\text { water)" }\end{array}$ & $\begin{array}{l}\text { decimal } \\
\text { fraction }\end{array}$ & $x^{(1 / 2)}$ & $\begin{array}{l}\text { SSURGO Database; table: muaggatt; column: drclassdcd } \\
\text { (Drainage Class - Dominant Condition) }\end{array}$ & Soil Survey Staff (2013) \\
\hline & DrainageClass2a & $\begin{array}{l}\text { Sum of fractions "poorly" and "very poorly drained" and } \\
\text { "unknown (likely water)" }\end{array}$ & $\begin{array}{l}\text { decimal } \\
\text { fraction }\end{array}$ & none & $\begin{array}{l}\text { SSURGO Database; table: muaggatt; column: drclassdcd } \\
\text { (Drainage Class - Dominant Condition) }\end{array}$ & Soil Survey Staff (2013) \\
\hline & DrainageClass3a & $\begin{array}{l}\text { Sum of fractions "somewhat poorly", "poorly", and "very } \\
\text { poorly drained" and "unknown (likely water") }\end{array}$ & $\begin{array}{l}\text { decimal } \\
\text { fraction }\end{array}$ & none & $\begin{array}{l}\text { SSURGO Database; table: muaggatt; column: drclassdcd } \\
\text { (Drainage Class - Dominant Condition) }\end{array}$ & Soil Survey Staff (2013) \\
\hline & HydricClass1 & Fraction "all hydric" soils & $\begin{array}{l}\text { decimal } \\
\text { fraction }\end{array}$ & $x^{(1 / 2)}$ & $\begin{array}{l}\text { SSURGO Database; table: muaggatt; column: hydclprs } \\
\text { (Hydric Classication - Presence) }\end{array}$ & Soil Survey Staff (2013) \\
\hline & HydricClass1a & Sum of fractions "all hydric" soils and "likely water" & $\begin{array}{l}\text { decimal } \\
\text { fraction }\end{array}$ & none & $\begin{array}{l}\text { SSURGO Database; table: muaggatt; column: hydclprs } \\
\text { (Hydric Classication - Presence) }\end{array}$ & Soil Survey Staff (2013) \\
\hline & HydricClass2 & Sum of fractions "all hydric" and "partially hydric" soils & $\begin{array}{l}\text { decimal } \\
\text { fraction }\end{array}$ & none & $\begin{array}{l}\text { SSURGO Database; table: muaggatt; column: hydclprs } \\
\text { (Hydric Classication - Presence) }\end{array}$ & Soil Survey Staff (2013) \\
\hline & HydricClass2a & $\begin{array}{l}\text { Sum of fractions "all hydric" and "partially hydric" soils and } \\
\text { "likely water" }\end{array}$ & $\begin{array}{l}\text { decimal } \\
\text { fraction }\end{array}$ & none & $\begin{array}{l}\text { SSURGO Database; table: muaggatt; column: hydclprs } \\
\text { (Hydric Classication - Presence) }\end{array}$ & Soil Survey Staff (2013) \\
\hline & WaterTableDepth & Mean water table depth & $\mathrm{cm}$ & $x^{(1 / 2)}$ & $\begin{array}{l}\text { SSURGO Database; table: muaggatt; column: } \\
\text { wtdepannmin (Water Table Depth - Annual - Minimum) }\end{array}$ & Soil Survey Staff (2013) \\
\hline & Ponding1 & Fraction $75-100 \%$ ponding & $\begin{array}{l}\text { decimal } \\
\text { fraction }\end{array}$ & $x^{(1 / 2)}$ & $\begin{array}{l}\text { SSURGO Database; table: muaggatt; column: } \\
\text { wtdepannmin (Water Table Depth - Annual - Minimum) }\end{array}$ & Soil Survey Staff (2013) \\
\hline
\end{tabular}




\begin{tabular}{|c|c|c|c|c|c|c|}
\hline Category & Variable name & Variable description & Units & $\begin{array}{l}\text { Selected } \\
\text { transformation }\end{array}$ & Data source & Reference \\
\hline & Ponding2 & Sum of fractions $50-74 \%$ and $75-100 \%$ ponding & $\begin{array}{l}\text { decimal } \\
\text { fraction }\end{array}$ & $x^{(1 / 2)}$ & $\begin{array}{l}\text { SSURGO Database; table: muaggatt; column: } \\
\text { wtdepannmin (Water Table Depth - Annual - Minimum) }\end{array}$ & Soil Survey Staff (2013) \\
\hline & Ponding3 & Sum of fractions $15-49 \%, 50-74 \%$, and $75-100 \%$ ponding & $\begin{array}{l}\text { decimal } \\
\text { fraction }\end{array}$ & none & $\begin{array}{l}\text { SSURGO Database; table: muaggatt; column: } \\
\text { wtdepannmin (Water Table Depth - Annual - Minimum) }\end{array}$ & Soil Survey Staff (2013) \\
\hline & FloodFreq1 & Sum of fractions frequent and very frequent flooding & $\begin{array}{l}\text { decimal } \\
\text { fraction }\end{array}$ & $x^{(1 / 2)}$ & $\begin{array}{l}\text { SSURGO Database; table: muaggatt; column: flodfreqcd } \\
\text { (Flooding Frequency - Dominant Condition) }\end{array}$ & Soil Survey Staff (2013) \\
\hline & FloodFreq1a & $\begin{array}{l}\text { Sum of fractions frequent and very frequent flooding and } \\
\text { likely water }\end{array}$ & $\begin{array}{l}\text { decimal } \\
\text { fraction }\end{array}$ & $x^{(1 / 2)}$ & $\begin{array}{l}\text { SSURGO Database; table: muaggatt; column: flodfreqcd } \\
\text { (Flooding Frequency - Dominant Condition) }\end{array}$ & Soil Survey Staff (2013) \\
\hline & FloodFreq2 & $\begin{array}{l}\text { Sum of fractions occasional, frequent, and very frequent } \\
\text { flooding }\end{array}$ & $\begin{array}{l}\text { decimal } \\
\text { fraction }\end{array}$ & $x^{(1 / 2)}$ & $\begin{array}{l}\text { SSURGO Database; table: muaggatt; column: flodfreqcd } \\
\text { (Flooding Frequency - Dominant Condition) }\end{array}$ & Soil Survey Staff (2013) \\
\hline & FloodFreq2a & $\begin{array}{l}\text { Sum of fractions occasional, frequent, and very frequent } \\
\text { flooding and likely water }\end{array}$ & $\begin{array}{l}\text { decimal } \\
\text { fraction }\end{array}$ & $x^{(1 / 2)}$ & $\begin{array}{l}\text { SSURGO Database; table: muaggatt; column: flodfreqcd } \\
\text { (Flooding Frequency - Dominant Condition) }\end{array}$ & Soil Survey Staff (2013) \\
\hline
\end{tabular}




\begin{tabular}{|c|c|c|c|c|c|c|}
\hline Category & Variable name & Variable description & Units & $\begin{array}{l}\text { Selected } \\
\text { transformation }\end{array}$ & Data source & Reference \\
\hline \multicolumn{7}{|l|}{ Slope } \\
\hline & DEM_slope & $\begin{array}{l}\text { Average of slope grid derived from the NED by using the } \\
\text { ArcHydro Tools Terrain Preprocessing Slope tool. }\end{array}$ & percent & none & $\begin{array}{l}\text { 1/3 arc-second National Elevation Dataset (NED), U.S. } \\
\text { Geological Survey, 2013. Downloaded from } \\
\text { http://nationalmap.usgs.gov/viewer.html, Feb. 12, } \\
\text { 2014. }\end{array}$ & Gesch et al. (2002) \\
\hline & SSURGO_slope & Area-weighted average SSURGO soil slopes & percent & $\log _{10}(X)$ & $\begin{array}{l}\text { SSURGO Database; table: muaggatt; column: } \\
\text { slopegraddcp (Slope Gradient - Dominant Component) }\end{array}$ & Soil Survey Staff (2013) \\
\hline & DEM_X_Y (DEM_1_0) & $\begin{array}{l}\text { Differences of elevation quantiles at probabilities } Y=1 \text { (the } \\
\text { maximum), } 0.999,0.99,0.90,0.75 \text { and their } \\
\text { complementary values } X=0 \text { (the minimum), } 0.001,0.01 \text {, } \\
0.10 \text {, and } 0.25 \text {, respectively. }\end{array}$ & feet & $\log _{10}(X)$ & $\begin{array}{l}\text { 1/3 arc-second National Elevation Dataset (NED), U.S. } \\
\text { Geological Survey, 2013. Downloaded from } \\
\text { http://nationalmap.usgs.gov/viewer.html, Feb. 12, } \\
2014 .\end{array}$ & Gesch et al. (2002) \\
\hline & $\begin{array}{l}\text { DEM_X_Y P } \\
\text { (DEM_1_O_P) }\end{array}$ & $\begin{array}{l}\text { Differences of elevation quantiles at probabilities } Y=1 \text { (the } \\
\text { maximum), } 0.999,0.99,0.90,0.75 \text { and their } \\
\text { complementary values } X=0 \text { (the minimum), } 0.001,0.01 \text {, } \\
0.10 \text {, and } 0.25 \text {, respectively, divided by basin perimeter. }\end{array}$ & $\begin{array}{l}\text { feet per } \\
\text { mile }\end{array}$ & $\log _{10}(X)$ & $\begin{array}{l}\text { 1/3 arc-second National Elevation Dataset (NED), U.S. } \\
\text { Geological Survey, 2013. Downloaded from } \\
\text { http://nationalmap.usgs.gov/viewer.html, Feb. 12, } \\
\text { 2014. }\end{array}$ & Gesch et al. (2002) \\
\hline
\end{tabular}




\section{APPENDIX 5}

Table 4. Segment information for U.S. Geological Survey streamgages used in this study, northeastern Illinois and adjacent states.

[NA, not available; shaded rows indicate streamgages not used in the regression analyses because they were deemed redundant but whose records were adjusted for urbanization in this study]

\begin{tabular}{|c|c|c|c|c|c|c|c|c|}
\hline $\begin{array}{l}\text { U.S. } \\
\text { Geological } \\
\text { Survey } \\
\text { streamgage } \\
\text { number }\end{array}$ & $\begin{array}{l}\begin{array}{l}\text { Segment } \\
\text { number }\end{array} \\
\end{array}$ & $\begin{array}{l}\text { Used in regression } \\
\text { analyses (non- } \\
\text { redundant) }\end{array}$ & $\begin{array}{l}\text { Beginning } \\
\text { water } \\
\text { year }\end{array}$ & $\begin{array}{l}\text { Ending } \\
\text { water } \\
\text { year }\end{array}$ & $\begin{array}{l}\text { Segment-average } \\
\text { maximum dam } \\
\text { storage, acre-feet }\end{array}$ & $\begin{array}{l}\text { Segment intercept } \\
\text { estimate, } \\
\log _{10}\left(\mathrm{ft}^{3} / \mathrm{s}\right)\end{array}$ & $\begin{array}{l}\text { Standard error of } \\
\text { segment intercept } \\
\text { effect estimate, } \\
\log _{10}\left(\mathrm{ft}^{3} / \mathrm{s}\right)\end{array}$ & $\begin{array}{l}\text { Intra-streamgage } \\
\text { segment intercept } \\
\text { differences, } \\
\log _{10}\left(\mathrm{ft}^{3} / \mathrm{s}\right)\end{array}$ \\
\hline 04087200 & 1 & No & 1958 & 2009 & 0 & 2.042 & 0.039 & NA \\
\hline 04087204 & 1 & Yes & 1964 & 2009 & 0 & 2.275 & 0.042 & NA \\
\hline 04087220 & 1 & Yes & 1964 & 2009 & 0 & 2.509 & 0.044 & NA \\
\hline 04087230 & 1 & Yes & 1962 & 1993 & 0 & 1.795 & 0.042 & NA \\
\hline 04087233 & 1 & Yes & 1964 & 2009 & 115 & 2.651 & 0.036 & NA \\
\hline 04087240 & 1 & No & 1964 & 2009 & 465 & 3.000 & 0.037 & NA \\
\hline 04087250 & 1 & Yes & 1960 & 2009 & 0 & 1.739 & 0.034 & NA \\
\hline 04087257 & 1 & Yes & 1972 & 2009 & 0 & 2.662 & 0.040 & NA \\
\hline 04087300 & 1 & Yes & 1956 & 1972 & 0 & 1.646 & 0.058 & NA \\
\hline 04087400 & 1 & Yes & 1962 & 1976 & 0 & 2.065 & 0.062 & NA \\
\hline 04093000 & 1 & Yes & 1947 & 2009 & 4390 & 2.882 & 0.032 & NA \\
\hline 04094000 & 1 & Yes & 1945 & 2009 & 447 & 2.873 & 0.030 & NA \\
\hline 04094500 & 1 & Yes & 1945 & 1991 & 1120 & 2.712 & 0.036 & NA \\
\hline 05437950 & 1 & Yes & 1965 & 1978 & 0 & 1.942 & 0.063 & NA \\
\hline 05438250 & 1 & Yes & 1962 & 1991 & 0 & 2.918 & 0.043 & NA \\
\hline 05438283 & 1 & Yes & 1993 & 2009 & 0 & 2.290 & 0.057 & NA \\
\hline 05438300 & 1 & Yes & 1961 & 1980 & 0 & 1.792 & 0.054 & NA \\
\hline 05438390 & 1 & No & 1970 & 1979 & 0 & 3.086 & 0.074 & NA \\
\hline 05438500 & 1 & Yes & 1940 & 2009 & 939 & 3.425 & 0.028 & NA \\
\hline 05438850 & 1 & Yes & 1956 & 1980 & 0 & 2.033 & 0.048 & NA \\
\hline 05439000 & 1 & Yes & 1980 & 2009 & 0 & 2.800 & 0.043 & NA \\
\hline 05439500 & 1 & Yes & 1940 & 2009 & 1130 & 3.445 & 0.028 & NA \\
\hline 05439550 & 1 & Yes & 1959 & 1976 & 0 & 1.749 & 0.056 & NA \\
\hline 05440000 & 1 & No & 1940 & 2009 & 5740 & 3.720 & 0.028 & NA \\
\hline 05440500 & 1 & Yes & 1940 & 1980 & 0 & 3.214 & 0.037 & NA \\
\hline 05442000 & 1 & Yes & 1940 & 1951 & 440 & 2.970 & 0.068 & NA \\
\hline 05446950 & 1 & Yes & 1961 & 1976 & 0 & 1.848 & 0.059 & NA \\
\hline 05447000 & 1 & Yes & 1940 & 1982 & 31.8 & 3.297 & 0.037 & NA \\
\hline 05447050 & 1 & Yes & 1959 & 1972 & 0 & 2.009 & 0.063 & NA \\
\hline 05447500 & 1 & Yes & 1940 & 2009 & 1440 & 3.621 & 0.029 & NA \\
\hline
\end{tabular}




\begin{tabular}{|c|c|c|c|c|c|c|c|c|}
\hline $\begin{array}{l}\text { U.S. } \\
\text { Geological } \\
\text { Survey } \\
\text { streamgage } \\
\text { number }\end{array}$ & $\begin{array}{l}\text { Segment } \\
\text { number }\end{array}$ & $\begin{array}{l}\text { Used in regression } \\
\text { analyses (non- } \\
\text { redundant) }\end{array}$ & $\begin{array}{l}\text { Beginning } \\
\text { water } \\
\text { year }\end{array}$ & $\begin{array}{l}\text { Ending } \\
\text { water } \\
\text { year }\end{array}$ & $\begin{array}{l}\text { Segment-average } \\
\text { maximum dam } \\
\text { storage, acre-feet }\end{array}$ & $\begin{array}{l}\text { Segment intercept } \\
\text { estimate, } \\
\log _{10}\left(\mathrm{ft}^{3} / \mathrm{s}\right)\end{array}$ & \begin{tabular}{|l} 
Standard error of \\
segment intercept \\
effect estimate, \\
$\log _{10}\left(\mathrm{ft}^{3} / \mathrm{s}\right)$ \\
\end{tabular} & $\begin{array}{l}\text { Intra-streamgage } \\
\text { segment intercept } \\
\text { differences, } \\
\log _{10}\left(\mathrm{ft}^{3} / \mathrm{s}\right)\end{array}$ \\
\hline 05517500 & 1 & Yes & 1949 & 2009 & 12700 & 3.513 & 0.030 & NA \\
\hline 05517890 & 1 & Yes & 1968 & 2003 & 29.1 & 2.585 & 0.040 & NA \\
\hline 05518000 & 1 & No & 1940 & 2009 & 12100 & 3.582 & 0.028 & NA \\
\hline 05519000 & 1 & Yes & 1951 & 2001 & 6260 & 2.888 & 0.034 & NA \\
\hline 05519500 & 1 & Yes & 1949 & 1972 & 0 & 2.782 & 0.052 & $\mathrm{NA}$ \\
\hline 05520500 & 1 & No & 1940 & 2009 & 18200 & 3.753 & 0.028 & NA \\
\hline 05526150 & 1 & Yes & 1956 & 1980 & 0 & 1.403 & 0.048 & NA \\
\hline 05526500 & 1 & Yes & 1950 & 1975 & 0 & 2.066 & 0.046 & NA \\
\hline 05527050 & 1 & Yes & 1956 & 1972 & 0 & 1.794 & 0.057 & NA \\
\hline 05527800 & 1 & Yes & 1960 & 2009 & 684 & 2.699 & 0.034 & $\mathrm{NA}$ \\
\hline 05527840 & 1 & No & 1962 & 1976 & 300 & 2.754 & 0.061 & NA \\
\hline 05527870 & 1 & Yes & 1962 & 1976 & 0 & 1.657 & 0.064 & NA \\
\hline 05527900 & 1 & Yes & 1962 & 1976 & 1200 & 2.111 & 0.061 & $\mathrm{NA}$ \\
\hline 05527950 & 1 & No & 1962 & 2009 & 1960 & 2.486 & 0.041 & NA \\
\hline 05528000 & 1 & No & 1946 & 2009 & 2410 & 2.933 & 0.030 & NA \\
\hline 05528150 & 1 & Yes & 1960 & 1976 & 2070 & 2.227 & 0.057 & NA \\
\hline 05528170 & 1 & Yes & 1961 & 1976 & 0 & 1.391 & 0.061 & NA \\
\hline 05528200 & 1 & Yes & 1963 & 1976 & 1400 & 1.941 & 0.066 & NA \\
\hline 05528230 & 1 & No & 1963 & 1996 & 3490 & 2.405 & 0.057 & $\mathrm{NA}$ \\
\hline 05528360 & 1 & Yes & 1961 & 1976 & 0 & 1.869 & 0.059 & NA \\
\hline 05528400 & 1 & No & 1962 & 1977 & 7930 & 3.033 & 0.071 & $\mathrm{NA}$ \\
\hline 05528440 & 1 & Yes & 1961 & 1976 & 0 & 1.562 & 0.061 & $\mathrm{NA}$ \\
\hline 05528470 & 1 & No & 1961 & 1976 & 0 & 2.103 & 0.062 & NA \\
\hline 05528500 & 1 & Yes & 1953 & 1982 & 0 & 2.216 & 0.045 & -0.126 \\
\hline 05528500 & 2 & Yes & 1984 & 2009 & 605 & 2.090 & 0.054 & NA \\
\hline 05529000 & 1 & No & 1941 & 2009 & 7930 & 3.112 & 0.030 & NA \\
\hline 05529300 & 1 & No & 1961 & 1979 & 0 & 1.669 & 0.061 & NA \\
\hline 05529500 & 1 & Yes & 1953 & 1989 & 0 & 1.751 & 0.048 & -0.132 \\
\hline 05529500 & 2 & Yes & 1991 & 2009 & 540 & 1.619 & 0.064 & NA \\
\hline 05529900 & 1 & No & 1961 & 1977 & 53.8 & 2.120 & 0.068 & NA \\
\hline 05530000 & 1 & Yes & 1951 & 2009 & 251 & 2.212 & 0.045 & NA \\
\hline 05530400 & 1 & Yes & 1961 & 1979 & 0 & 1.792 & 0.056 & NA \\
\hline 05530480 & 1 & Yes & 1961 & 1979 & 0 & 2.344 & 0.056 & NA \\
\hline 05530600 & 1 & No & 1960 & 1977 & 7790 & 3.132 & 0.057 & NA \\
\hline 05530700 & 1 & Yes & 1961 & 1980 & 0 & 2.080 & 0.060 & NA \\
\hline 05530800 & 1 & No & 1954 & 1976 & 7400 & 3.150 & 0.050 & NA \\
\hline 05530940 & 1 & Yes & 1961 & 1980 & 0 & 1.832 & 0.058 & NA \\
\hline
\end{tabular}




\begin{tabular}{|c|c|c|c|c|c|c|c|c|}
\hline $\begin{array}{l}\text { U.S. } \\
\text { Geological } \\
\text { Survey } \\
\text { streamgage } \\
\text { number }\end{array}$ & $\begin{array}{l}\begin{array}{l}\text { Segment } \\
\text { number }\end{array} \\
\end{array}$ & $\begin{array}{l}\text { Used in regression } \\
\text { analyses (non- } \\
\text { redundant) }\end{array}$ & $\begin{array}{l}\text { Beginning } \\
\text { water } \\
\text { year }\end{array}$ & \begin{tabular}{|l} 
Ending \\
water \\
year
\end{tabular} & $\begin{array}{l}\text { Segment-average } \\
\text { maximum dam } \\
\text { storage, acre-feet }\end{array}$ & $\begin{array}{l}\text { Segment intercept } \\
\text { estimate, } \\
\log _{10}\left(\mathrm{ft}^{3} / \mathrm{s}\right)\end{array}$ & $\begin{array}{l}\text { Standard error of } \\
\text { segment intercept } \\
\text { effect estimate, } \\
\log _{10}\left(\mathrm{ft}^{3} / \mathrm{s}\right) \\
\end{array}$ & $\begin{array}{l}\text { Intra-streamgage } \\
\text { segment intercept } \\
\text { differences, } \\
\log _{10}\left(\mathrm{ft}^{3} / \mathrm{s}\right)\end{array}$ \\
\hline 05530960 & 1 & No & 1961 & 1979 & 0 & 2.042 & 0.057 & NA \\
\hline 05530990 & 1 & No & 1974 & 2009 & 1570 & 2.332 & 0.049 & NA \\
\hline 05531000 & 1 & No & 1951 & 1976 & 0 & 2.239 & 0.050 & NA \\
\hline 05531050 & 1 & No & 1961 & 1976 & 0 & 2.378 & 0.062 & NA \\
\hline 05531080 & 1 & Yes & 1961 & 1979 & 0 & 1.754 & 0.061 & NA \\
\hline 05531100 & 1 & Yes & 1956 & 1979 & 0 & 1.361 & 0.058 & NA \\
\hline 05531130 & 1 & No & 1961 & 1979 & 359 & 2.031 & 0.064 & NA \\
\hline 05531200 & 1 & No & 1960 & 1976 & 462 & 2.524 & 0.060 & NA \\
\hline 05531300 & 1 & No & 1960 & 1976 & 462 & 2.567 & 0.061 & -0.052 \\
\hline 05531300 & 2 & No & 1989 & 1997 & 7720 & 2.519 & 0.083 & -0.004 \\
\hline 05531300 & 3 & No & 2003 & 2009 & 18300 & 2.515 & 0.094 & NA \\
\hline 05531380 & 1 & No & 1960 & 1976 & 462 & 2.556 & 0.061 & NA \\
\hline 05531500 & 1 & Yes & 1946 & 1976 & 518 & 2.649 & 0.046 & -0.045 \\
\hline 05531500 & 2 & Yes & 1978 & 1985 & 5190 & 2.661 & 0.088 & -0.057 \\
\hline 05531500 & 3 & Yes & 1987 & 1997 & 7630 & 2.655 & 0.076 & -0.051 \\
\hline 05531500 & 4 & Yes & 2003 & 2009 & 18400 & 2.604 & 0.094 & NA \\
\hline 05531800 & 1 & No & 1961 & 1976 & 0 & 2.002 & 0.066 & NA \\
\hline 05532000 & 1 & Yes & 1951 & 1991 & 311 & 2.068 & 0.048 & -0.077 \\
\hline 05532000 & 2 & Yes & 1993 & 2009 & 1370 & 1.992 & 0.066 & $\mathrm{NA}$ \\
\hline 05532500 & 1 & No & 1940 & 1976 & 7490 & 3.294 & 0.040 & -0.062 \\
\hline 05532500 & 2 & No & 1978 & 1997 & 17800 & 3.251 & 0.056 & -0.019 \\
\hline 05532500 & 3 & No & 1999 & 2009 & 32400 & 3.232 & 0.074 & NA \\
\hline 05533000 & 1 & Yes & 1951 & 2009 & 0 & 2.320 & 0.043 & NA \\
\hline 05533200 & 1 & Yes & 1961 & 1979 & 0 & 1.757 & 0.064 & NA \\
\hline 05533300 & 1 & Yes & 1962 & 1976 & 0 & 1.375 & 0.064 & NA \\
\hline 05533400 & 1 & No & 1961 & 2009 & 0 & 2.256 & 0.044 & NA \\
\hline 05534300 & 1 & No & 1961 & 1976 & 0 & 2.058 & 0.059 & NA \\
\hline 05534400 & 1 & No & 1960 & 1976 & 0 & 2.154 & 0.057 & NA \\
\hline 05534500 & 1 & Yes & 1953 & 1991 & 0 & 2.148 & 0.040 & 0.013 \\
\hline 05534500 & 2 & Yes & 1993 & 2009 & 500 & 2.160 & 0.062 & NA \\
\hline 05534600 & 1 & No & 1960 & 1972 & 0 & 2.193 & 0.067 & 0.005 \\
\hline 05534600 & 2 & No & 1974 & 1980 & 600 & 2.198 & 0.097 & NA \\
\hline 05534900 & 1 & No & 1962 & 1976 & 0 & 1.850 & 0.064 & NA \\
\hline 05535000 & 1 & Yes & 1952 & 2009 & 0 & 1.934 & 0.037 & NA \\
\hline 05535070 & 1 & No & 1967 & 2009 & 0 & 2.159 & 0.044 & NA \\
\hline 05535150 & 1 & No & 1960 & 1969 & 0 & 2.160 & 0.077 & -0.003 \\
\hline 05535150 & 2 & No & 1971 & 1979 & 961 & 2.158 & 0.086 & NA \\
\hline
\end{tabular}




\begin{tabular}{|c|c|c|c|c|c|c|c|c|}
\hline $\begin{array}{l}\text { U.S. } \\
\text { Geological } \\
\text { Survey } \\
\text { streamgage } \\
\text { number }\end{array}$ & $\begin{array}{l}\text { Segment } \\
\text { number }\end{array}$ & $\begin{array}{l}\text { Used in regression } \\
\text { analyses (non- } \\
\text { redundant) }\end{array}$ & $\begin{array}{l}\text { Beginning } \\
\text { water } \\
\text { year }\end{array}$ & $\begin{array}{l}\text { Ending } \\
\text { water } \\
\text { year }\end{array}$ & $\begin{array}{l}\text { Segment-average } \\
\text { maximum dam } \\
\text { storage, acre-feet }\end{array}$ & $\begin{array}{l}\text { Segment intercept } \\
\text { estimate, } \\
\log _{10}\left(\mathrm{ft}^{3} / \mathrm{s}\right)\end{array}$ & $\begin{array}{l}\text { Standard error of } \\
\text { segment intercept } \\
\text { effect estimate, } \\
\log _{10}\left(\mathrm{ft}^{3} / \mathrm{s}\right)\end{array}$ & $\begin{array}{l}\text { Intra-streamgage } \\
\text { segment intercept } \\
\text { differences, } \\
\log _{10}\left(\mathrm{ft}^{3} / \mathrm{s}\right)\end{array}$ \\
\hline 05535200 & 1 & No & 1960 & 1969 & 0 & 2.464 & 0.076 & NA \\
\hline 05535300 & 1 & No & 1961 & 1976 & 0 & 2.020 & 0.060 & NA \\
\hline 05535400 & 1 & No & 1961 & 1976 & 0 & 2.157 & 0.062 & NA \\
\hline 05535500 & 1 & Yes & 1953 & 1989 & 0 & 2.233 & 0.044 & -0.182 \\
\hline 05535500 & 2 & Yes & 1993 & 2009 & 1100 & 2.051 & 0.065 & $\mathrm{NA}$ \\
\hline 05535700 & 1 & No & 1960 & 1977 & 0 & 2.347 & 0.059 & $\mathrm{NA}$ \\
\hline 05535800 & 1 & No & 1960 & 1969 & 0 & 2.630 & 0.077 & -0.113 \\
\hline 05535800 & 2 & No & 1974 & 1978 & 1560 & 2.517 & 0.108 & NA \\
\hline 05536000 & 1 & Yes & 1951 & 1969 & 0 & 2.627 & 0.057 & -0.032 \\
\hline 05536000 & 2 & Yes & 1974 & 1978 & 1560 & 2.585 & 0.108 & 0.010 \\
\hline 05536000 & 3 & Yes & 1980 & 1991 & 3110 & 2.581 & 0.073 & 0.014 \\
\hline 05536000 & 4 & Yes & 1993 & 2009 & 4920 & 2.595 & 0.064 & NA \\
\hline 05536105 & 1 & No & 1990 & 2009 & 4810 & 2.590 & 0.061 & NA \\
\hline 05536178 & 1 & No & 1966 & 1977 & 0 & 2.840 & 0.068 & NA \\
\hline 05536179 & 1 & No & 1990 & 2009 & 0 & 2.742 & 0.054 & NA \\
\hline 05536190 & 1 & Yes & 1943 & 2009 & 0 & 2.858 & 0.033 & NA \\
\hline 05536201 & 1 & Yes & 1962 & 1978 & 0 & 2.183 & 0.060 & NA \\
\hline 05536207 & 1 & Yes & 1962 & 1977 & 0 & 1.766 & 0.066 & NA \\
\hline 05536210 & 1 & No & 1965 & 1979 & 376 & 2.510 & 0.064 & $\mathrm{NA}$ \\
\hline 05536215 & 1 & No & 1950 & 2009 & 376 & 2.555 & 0.037 & NA \\
\hline 05536235 & 1 & Yes & 1948 & 2005 & 600 & 2.410 & 0.033 & $\mathrm{NA}$ \\
\hline 05536238 & 1 & Yes & 1961 & 1979 & 0 & 1.977 & 0.056 & $\mathrm{NA}$ \\
\hline 05536255 & 1 & Yes & 1948 & 1968 & 0 & 2.409 & 0.053 & 0.031 \\
\hline 05536255 & 2 & Yes & 1970 & 2009 & 584 & 2.440 & 0.043 & NA \\
\hline 05536265 & 1 & Yes & 1948 & 1987 & 0 & 2.018 & 0.038 & -0.324 \\
\hline 05536265 & 2 & Yes & 1989 & 2009 & 1090 & 1.695 & 0.054 & NA \\
\hline 05536270 & 1 & Yes & 1948 & 1979 & 0 & 2.264 & 0.043 & NA \\
\hline 05536275 & 1 & No & 1947 & 1987 & 1240 & 2.934 & 0.039 & -0.014 \\
\hline 05536275 & 2 & No & 1989 & 2006 & 2670 & 2.920 & 0.059 & $\mathrm{NA}$ \\
\hline 05536290 & 1 & No & 1947 & 2009 & 1750 & 2.992 & 0.034 & NA \\
\hline 05536310 & 1 & Yes & 1961 & 1974 & 0 & 1.979 & 0.068 & NA \\
\hline 05536335 & 1 & No & 1954 & 1979 & 0 & 1.962 & 0.050 & NA \\
\hline 05536340 & 1 & Yes & 1951 & 1973 & 0 & 2.087 & 0.051 & -0.347 \\
\hline 05536340 & 2 & Yes & 1975 & 1987 & 950 & 1.870 & 0.070 & -0.129 \\
\hline 05536340 & 3 & Yes & 1989 & 2009 & 1570 & 1.740 & 0.059 & NA \\
\hline 05536460 & 1 & No & 1961 & 1979 & 0 & 2.304 & 0.057 & NA \\
\hline 05536500 & 1 & Yes & 1951 & 2009 & 0 & 2.400 & 0.035 & NA \\
\hline
\end{tabular}




\begin{tabular}{|c|c|c|c|c|c|c|c|c|}
\hline \begin{tabular}{l|} 
U.S. \\
Geological \\
Survey \\
streamgage \\
number
\end{tabular} & $\begin{array}{l}\text { Segment } \\
\text { number }\end{array}$ & $\begin{array}{l}\text { Used in regression } \\
\text { analyses (non- } \\
\text { redundant) }\end{array}$ & $\begin{array}{l}\text { Beginning } \\
\text { water } \\
\text { year }\end{array}$ & $\begin{array}{l}\text { Ending } \\
\text { water } \\
\text { year }\end{array}$ & $\begin{array}{l}\text { Segment-average } \\
\text { maximum dam } \\
\text { storage, acre-feet }\end{array}$ & $\begin{array}{l}\text { Segment intercept } \\
\text { estimate, } \\
\log _{10}\left(\mathrm{ft}^{3} / \mathrm{s}\right)\end{array}$ & $\begin{array}{l}\text { Standard error of } \\
\text { segment intercept } \\
\text { effect estimate, } \\
\log _{10}\left(\mathrm{ft}^{3} / \mathrm{s}\right)\end{array}$ & $\begin{array}{l}\text { Intra-streamgage } \\
\text { segment intercept } \\
\text { differences, } \\
\log _{10}\left(\mathrm{ft}^{3} / \mathrm{s}\right)\end{array}$ \\
\hline 05536510 & 1 & Yes & 1961 & 1979 & 0 & 1.921 & 0.059 & $\mathrm{NA}$ \\
\hline 05536560 & 1 & Yes & 1962 & 1970 & 0 & 1.345 & 0.086 & 0.440 \\
\hline 05536560 & 2 & Yes & 1974 & 1980 & 165 & 1.785 & 0.095 & NA \\
\hline 05536570 & 1 & No & 1962 & 1970 & 2.67 & 1.910 & 0.085 & NA \\
\hline 05536620 & 1 & No & 1961 & 1977 & 0 & 1.756 & 0.059 & NA \\
\hline 05536630 & 1 & Yes & 1961 & 1979 & 143 & 1.959 & 0.056 & NA \\
\hline 05537500 & 1 & Yes & 1951 & 1963 & 0 & 2.595 & 0.065 & -0.195 \\
\hline 05537500 & 2 & Yes & 1965 & 2009 & 859 & 2.400 & 0.040 & NA \\
\hline 05538440 & 1 & Yes & 1961 & 1977 & 0 & 1.284 & 0.059 & $\mathrm{NA}$ \\
\hline 05539000 & 1 & Yes & 1945 & 2008 & 1020 & 3.170 & 0.032 & NA \\
\hline 05539870 & 1 & No & 1961 & 1976 & 0 & 2.038 & 0.063 & $\mathrm{NA}$ \\
\hline 05539890 & 1 & No & 1961 & 1976 & 0 & 2.285 & 0.061 & NA \\
\hline 05539900 & 1 & Yes & 1961 & 1976 & 0 & 2.298 & 0.061 & -0.061 \\
\hline 05539900 & 2 & Yes & 1978 & 2009 & 230 & 2.237 & 0.049 & $\mathrm{NA}$ \\
\hline 05539950 & 1 & Yes & 1961 & 1979 & 0 & 1.945 & 0.056 & NA \\
\hline 05540030 & 1 & No & 1961 & 1979 & 594 & 2.473 & 0.057 & NA \\
\hline 05540060 & 1 & Yes & 1961 & 1991 & 0 & 2.176 & 0.047 & -0.055 \\
\hline 05540060 & 2 & Yes & 1994 & 2009 & 540 & 2.121 & 0.061 & NA \\
\hline 05540080 & 1 & Yes & 1961 & 1979 & 0 & 1.569 & 0.062 & $\mathrm{NA}$ \\
\hline 05540091 & 1 & No & 1992 & 2009 & 0 & 1.735 & 0.062 & $\mathrm{NA}$ \\
\hline 05540095 & 1 & No & 1969 & 2009 & 1040 & 2.659 & 0.043 & $\mathrm{NA}$ \\
\hline 05540110 & 1 & Yes & 1961 & 1979 & 711 & 1.716 & 0.054 & $\mathrm{NA}$ \\
\hline 05540130 & 1 & No & 1989 & 2009 & 9560 & 2.770 & 0.057 & NA \\
\hline 05540140 & 1 & Yes & 1961 & 1979 & 0 & 1.339 & 0.058 & NA \\
\hline 05540150 & 1 & No & 1961 & 1980 & 0 & 1.886 & 0.061 & NA \\
\hline 05540160 & 1 & Yes & 1961 & 2009 & 478 & 2.187 & 0.050 & $\mathrm{NA}$ \\
\hline 05540190 & 1 & Yes & 1961 & 1977 & 0 & 1.917 & 0.066 & NA \\
\hline 05540195 & 1 & No & 1989 & 2009 & 0 & 2.086 & 0.061 & NA \\
\hline 05540240 & 1 & Yes & 1961 & 1980 & 0 & 1.714 & 0.060 & NA \\
\hline 05540250 & 1 & No & 1992 & 2009 & 2190 & 2.463 & 0.063 & NA \\
\hline 05540275 & 1 & Yes & 1988 & 2009 & 117 & 1.860 & 0.055 & NA \\
\hline 05540500 & 1 & Yes & 1941 & 1971 & 2030 & 3.265 & 0.043 & -0.106 \\
\hline 05540500 & 2 & Yes & 1973 & 2009 & 11600 & 3.160 & 0.044 & NA \\
\hline 05541750 & 1 & Yes & 1959 & 1980 & 0 & 1.888 & 0.050 & $\mathrm{NA}$ \\
\hline 05542000 & 1 & Yes & 1940 & 2009 & 35000 & 3.778 & 0.029 & NA \\
\hline 05543830 & 1 & Yes & 1963 & 2009 & 37200 & 2.545 & 0.039 & $\mathrm{NA}$ \\
\hline 05544200 & 1 & Yes & 1974 & 2009 & 11500 & 2.124 & 0.041 & NA \\
\hline
\end{tabular}




\begin{tabular}{|c|c|c|c|c|c|c|c|c|}
\hline $\begin{array}{l}\text { U.S. } \\
\text { Geological } \\
\text { Survey } \\
\text { streamgage } \\
\text { number } \\
\end{array}$ & $\begin{array}{l}\begin{array}{l}\text { Segment } \\
\text { number }\end{array} \\
\end{array}$ & $\begin{array}{l}\text { Used in regression } \\
\text { analyses (non- } \\
\text { redundant) }\end{array}$ & $\begin{array}{l}\text { Beginning } \\
\text { water } \\
\text { year }\end{array}$ & \begin{tabular}{|l} 
Ending \\
water \\
year \\
\end{tabular} & $\begin{array}{l}\text { Segment-average } \\
\text { maximum dam } \\
\text { storage, acre-feet }\end{array}$ & $\begin{array}{l}\text { Segment intercept } \\
\text { estimate, } \\
\log _{10}\left(\mathrm{ft}^{3} / \mathrm{s}\right) \\
\end{array}$ & $\begin{array}{l}\text { Standard error of } \\
\text { segment intercept } \\
\text { effect estimate, } \\
\log _{10}\left(\mathrm{ft}^{3} / \mathrm{s}\right) \\
\end{array}$ & $\begin{array}{l}\text { Intra-streamgage } \\
\text { segment intercept } \\
\text { differences, } \\
\log _{10}\left(\mathrm{ft}^{3} / \mathrm{s}\right) \\
\end{array}$ \\
\hline 05544300 & 1 & Yes & 1960 & 1981 & 0 & 1.330 & 0.054 & NA \\
\hline 05545100 & 1 & Yes & 1962 & 2009 & 0 & 1.998 & 0.034 & NA \\
\hline 05545200 & 1 & Yes & 1960 & 2009 & 0 & 1.798 & 0.034 & NA \\
\hline 05545300 & 1 & Yes & 1960 & 1982 & 40100 & 2.708 & 0.049 & NA \\
\hline 05545750 & 1 & Yes & 1940 & 1976 & 109000 & 3.284 & 0.039 & -0.066 \\
\hline 05545750 & 2 & Yes & 1978 & 2009 & 122000 & 3.218 & 0.043 & NA \\
\hline 05547755 & 1 & Yes & 1990 & 2005 & 62 & 2.046 & 0.059 & NA \\
\hline 05548150 & 1 & Yes & 1962 & 2009 & 0 & 2.160 & 0.036 & NA \\
\hline 05548280 & 1 & Yes & 1967 & 2009 & 10300 & 2.877 & 0.037 & NA \\
\hline 05549000 & 1 & Yes & 1949 & 1964 & 0 & 1.954 & 0.059 & 0.020 \\
\hline 05549000 & 2 & Yes & 1966 & 1992 & 400 & 1.974 & 0.046 & NA \\
\hline 05549700 & 1 & Yes & 1962 & 1976 & 0 & 1.724 & 0.061 & NA \\
\hline 05549850 & 1 & Yes & 1962 & 1996 & 282 & 2.082 & 0.052 & NA \\
\hline 05549900 & 1 & Yes & 1956 & 1979 & 0 & 0.548 & 0.062 & NA \\
\hline 05550300 & 1 & Yes & 1962 & 2009 & 97.9 & 2.415 & 0.044 & NA \\
\hline 05550430 & 1 & Yes & 1961 & 1977 & 633 & 1.569 & 0.058 & NA \\
\hline 05550450 & 1 & No & 1961 & 1968 & 633 & 2.075 & 0.083 & -0.046 \\
\hline 05550450 & 2 & No & 1970 & 1977 & 1150 & 2.030 & 0.084 & NA \\
\hline 05550470 & 1 & Yes & 1961 & 1972 & 0 & 1.772 & 0.071 & -0.111 \\
\hline 05550470 & 2 & Yes & 1975 & 1979 & 154 & 1.661 & 0.110 & NA \\
\hline 05550500 & 1 & Yes & 1952 & 2009 & 1170 & 2.306 & 0.034 & NA \\
\hline 05551030 & 1 & Yes & 1962 & 1979 & 0 & 2.187 & 0.057 & NA \\
\hline 05551050 & 1 & Yes & 1962 & 1979 & 0 & 1.777 & 0.056 & NA \\
\hline 05551060 & 1 & No & 1962 & 1979 & 0 & 1.901 & 0.056 & NA \\
\hline 05551200 & 1 & Yes & 1961 & 2009 & 297 & 2.677 & 0.035 & NA \\
\hline 05551330 & 1 & Yes & 1998 & 2009 & 302 & 2.226 & 0.070 & NA \\
\hline 05551520 & 1 & Yes & 1961 & 1979 & 0 & 1.843 & 0.055 & NA \\
\hline 05551530 & 1 & No & 1961 & 1979 & 0 & 2.352 & 0.055 & NA \\
\hline 05551620 & 1 & No & 1961 & 1979 & 0 & 2.477 & 0.057 & NA \\
\hline 05551650 & 1 & Yes & 1961 & 1976 & 0 & 1.641 & 0.061 & NA \\
\hline 05551675 & 1 & No & 1998 & 2009 & 532 & 2.415 & 0.069 & NA \\
\hline 05551700 & 1 & Yes & 1961 & 2009 & 331 & 2.603 & 0.034 & NA \\
\hline 05551800 & 1 & Yes & 1961 & 1980 & 0 & 1.610 & 0.054 & NA \\
\hline 05551900 & 1 & Yes & 1965 & 1979 & 0 & 2.702 & 0.061 & NA \\
\hline 05551930 & 1 & Yes & 1965 & 1980 & 0 & 2.372 & 0.059 & NA \\
\hline
\end{tabular}




\section{APPENDIX 6}

Table 6. Observed and urban-adjusted annual maximum peak discharges and associated urbanization

and precipitation values at $\mathbf{1 8 1}$ streamgages in northeastern Illinois and adjacent states.

[NWIS, National Water Information System; $\mathrm{ft}^{3} / \mathrm{s}$, cubic feet per second, --, no data; NA, not available]

[NWIS peak codes: 1 , Discharge is a maximum daily average; 2 , Discharge is an estimate; 4 , Discharge less than indicated value, which is the minimum recordable discharge at this site; 5 , Discharge affected to an unknown degree by regulation or diversion; 6 , Discharge affected by regulation or diversion; 7, Discharge is an historic peak; 8, Discharge actually greater than indicated value, 9 , Discharge due to snowmelt,

hurricane, ice-jam or debris dam breakup; B, Month or day of occurrence is unknown or not exact; C, All or part of the record affected by urbanization, mining, agricultural changes, channelization, or other; D, Base discharge changed during this year; $E$, Only maximum peak available for this year.]

\begin{tabular}{|c|c|c|c|c|c|c|c|c|c|c|c|}
\hline $\begin{array}{c}\text { U.S. } \\
\text { Geological } \\
\text { Survey } \\
\text { streamgage } \\
\text { number }\end{array}$ & $\begin{array}{c}\text { Segment } \\
\text { number }\end{array}$ & $\begin{array}{l}\text { Streamgage used in } \\
\text { regression analyses } \\
\text { (non-redundant) }\end{array}$ & $\begin{array}{c}\text { Discharge value } \\
\text { used in } \\
\text { adjustment } \\
\text { regression }\end{array}$ & $\begin{array}{l}\text { Water } \\
\text { year }\end{array}$ & $\begin{array}{c}\text { Observed annual } \\
\text { maximum peak } \\
\text { discharge }\left(\mathrm{ft}^{3} / \mathrm{s}\right)\end{array}$ & $\begin{array}{l}\text { NWIS peak } \\
\text { code }\end{array}$ & $\begin{array}{c}\text { Observed annual } \\
\text { maximum peak } \\
\text { discharge with } \\
\text { segment intercept } \\
\text { value subtracted } \\
\left(\mathrm{ft}^{3} / \mathrm{s}\right) \\
\end{array}$ & $\begin{array}{l}\text { Observed } \\
\text { precipitation } \\
\text { (inches) }\end{array}$ & Urban fraction & $\begin{array}{l}\text { Exceedance } \\
\text { probability }\end{array}$ & $\begin{array}{c}\text { Urban-adjusted } \\
\text { annual maximum } \\
\text { peak discharge } \\
\left(\mathrm{ft}^{3} / \mathrm{s}\right)\end{array}$ \\
\hline 04087200 & 1 & No & No & 1958 & 57 & -- & 127 & 0.937 & 0.307 & 0.966 & 146 \\
\hline 04087200 & 1 & No & No & 1959 & 170 & -- & 378 & 0.708 & 0.322 & 0.672 & 303 \\
\hline 04087200 & 1 & No & No & 1960 & 1100 & -- & 2443 & 1.54 & 0.338 & 0.00502 & 1510 \\
\hline 04087200 & 1 & No & No & 1961 & 85 & -- & 189 & 2.11 & 0.35 & 0.936 & 183 \\
\hline 04087200 & 1 & No & No & 1962 & 185 & -- & 411 & 0.000352 & 0.362 & 0.648 & 312 \\
\hline 04087200 & 1 & No & No & 1963 & 75 & -- & 167 & 0.0369 & 0.374 & 0.955 & 163 \\
\hline 04087200 & 1 & No & No & 1964 & 130 & -- & 289 & 2.38 & 0.386 & 0.846 & 234 \\
\hline 04087200 & 1 & No & No & 1965 & 165 & -- & 366 & 1.1 & 0.398 & 0.748 & 277 \\
\hline 04087200 & 1 & No & No & 1966 & 235 & -- & 522 & 0.713 & 0.41 & 0.507 & 363 \\
\hline 04087200 & 1 & No & No & 1967 & 165 & -- & 366 & 1.95 & 0.422 & 0.764 & 270 \\
\hline 04087200 & 1 & No & No & 1968 & 255 & -- & 566 & 1.93 & 0.434 & 0.463 & 382 \\
\hline 04087200 & 1 & No & No & 1969 & 630 & - & 1399 & 2.16 & 0.446 & 0.0363 & 839 \\
\hline 04087200 & 1 & No & No & 1970 & 180 & -- & 400 & 1.16 & 0.458 & 0.742 & 279 \\
\hline 04087200 & 1 & No & No & 1971 & 210 & -- & 466 & 0.698 & 0.471 & 0.651 & 311 \\
\hline 04087200 & 1 & No & No & 1972 & 740 & -- & 1643 & 1.69 & 0.483 & 0.0227 & 944 \\
\hline 04087200 & 1 & No & No & 1973 & 260 & -- & 577 & 2.71 & 0.496 & 0.504 & 365 \\
\hline 04087200 & 1 & No & No & 1974 & 255 & -- & 566 & 0.76 & 0.509 & 0.533 & 354 \\
\hline 04087200 & 1 & No & No & 1975 & 195 & -- & 433 & 1.65 & 0.522 & 0.743 & 278 \\
\hline 04087200 & 1 & No & No & 1976 & 580 & -- & 1288 & 2.17 & 0.534 & 0.0595 & 728 \\
\hline 04087200 & 1 & No & No & 1977 & 145 & -- & 322 & 1.81 & 0.547 & 0.892 & 213 \\
\hline 04087200 & 1 & No & No & 1978 & 600 & -- & 1332 & 1.67 & 0.56 & 0.0569 & 736 \\
\hline 04087200 & 1 & No & No & 1979 & 600 & -- & 1332 & 0.899 & 0.573 & 0.0592 & 729 \\
\hline 04087200 & 1 & No & No & 1980 & 240 & -- & 533 & 1.7 & 0.585 & 0.655 & 310 \\
\hline 04087200 & 1 & No & No & 1981 & 265 & -- & 588 & 0.95 & 0.591 & 0.58 & 336 \\
\hline 04087200 & 1 & No & No & 1982 & 390 & -- & 866 & 0.922 & 0.597 & 0.251 & 473 \\
\hline
\end{tabular}




\begin{tabular}{|c|c|c|c|c|c|c|c|c|c|c|c|}
\hline $\begin{array}{l}\text { U.S. } \\
\text { Geological } \\
\text { Survey } \\
\text { streamgage } \\
\text { number }\end{array}$ & $\begin{array}{l}\text { Segment } \\
\text { number }\end{array}$ & $\begin{array}{l}\text { Streamgage used in } \\
\text { regression analyses } \\
\text { (non-redundant) }\end{array}$ & $\begin{array}{c}\text { Discharge value } \\
\text { used in } \\
\text { adjustment } \\
\text { regression }\end{array}$ & $\begin{array}{l}\text { Water } \\
\text { year }\end{array}$ & $\begin{array}{l}\text { Observed annual } \\
\text { maximum peak } \\
\text { discharge }\left(\mathrm{ft}^{3} / \mathrm{s}\right)\end{array}$ & $\begin{array}{l}\text { NWIS peak } \\
\text { code }\end{array}$ & $\begin{array}{l}\text { Observed annual } \\
\text { maximum peak } \\
\text { discharge with } \\
\text { segment intercept } \\
\text { value subtracted } \\
\left(\mathrm{ft}^{3} / \mathrm{s}\right)\end{array}$ & $\begin{array}{l}\text { Observed } \\
\text { precipitation } \\
\text { (inches) }\end{array}$ & Urban fraction & $\begin{array}{l}\text { Exceedance } \\
\text { probability }\end{array}$ & $\begin{array}{c}\text { Urban-adjusted } \\
\text { annual maximum } \\
\text { peak discharge } \\
\left(\mathrm{ft}^{3} / \mathrm{s}\right)\end{array}$ \\
\hline 04087200 & 1 & No & No & 1983 & 570 & -- & 1266 & 2.12 & 0.603 & 0.0761 & 677 \\
\hline 04087200 & 1 & No & No & 1984 & 270 & -- & 600 & 1.44 & 0.609 & 0.581 & 336 \\
\hline 04087200 & 1 & No & No & 1985 & 225 & -- & 500 & 0.166 & 0.615 & 0.728 & 284 \\
\hline 04087200 & 1 & No & No & 1986 & 410 & -- & 910 & 0.012 & 0.621 & 0.233 & 485 \\
\hline 04087200 & 1 & No & No & 1987 & 280 & -- & 622 & 1.38 & 0.627 & 0.567 & 341 \\
\hline 04087200 & 1 & No & No & 1988 & 380 & -- & 844 & 0.18 & 0.632 & 0.298 & 448 \\
\hline 04087200 & 1 & No & No & 1989 & 295 & -- & 655 & 0.583 & 0.638 & 0.534 & 354 \\
\hline 04087200 & 1 & No & No & 1990 & 520 & -- & 1155 & 1.77 & 0.644 & 0.125 & 598 \\
\hline 04087200 & 1 & No & No & 1991 & 315 & -- & 700 & 0.787 & 0.659 & 0.498 & 368 \\
\hline 04087200 & 1 & No & No & 1992 & 370 & -- & 822 & 2.13 & 0.675 & 0.365 & 421 \\
\hline 04087200 & 1 & No & No & 1993 & 660 & -- & 1466 & 1.89 & 0.69 & 0.0599 & 724 \\
\hline 04087200 & 1 & No & No & 1994 & 350 & 2 & 777 & 0.878 & 0.706 & 0.449 & 387 \\
\hline 04087200 & 1 & No & No & 1995 & 340 & -- & 755 & 2.38 & 0.721 & 0.492 & 370 \\
\hline 04087200 & 1 & No & No & 1996 & 440 & -- & 977 & 2.02 & 0.736 & 0.262 & 467 \\
\hline 04087200 & 1 & No & No & 1997 & 560 & 2 & 1244 & 3.85 & 0.752 & 0.136 & 584 \\
\hline 04087200 & 1 & No & No & 1998 & 300 & 2 & 666 & 0.644 & 0.767 & 0.651 & 311 \\
\hline 04087200 & 1 & No & No & 1999 & 910 & -- & 2021 & 1.48 & 0.782 & 0.0258 & 921 \\
\hline 04087200 & 1 & No & No & 2000 & 1360 & -- & 3020 & 3.89 & 0.798 & 0.00826 & 1360 \\
\hline 04087200 & 1 & No & No & 2001 & 450 & -- & 999 & 1.14 & 0.798 & 0.293 & 450 \\
\hline 04087200 & 1 & No & No & 2002 & 247 & -- & 549 & 1.12 & 0.798 & 0.817 & 247 \\
\hline 04087200 & 1 & No & No & 2003 & 144 & -- & 320 & 0.668 & 0.798 & 0.967 & 144 \\
\hline 04087200 & 1 & No & No & 2004 & 446 & -- & 990 & 1.4 & 0.798 & 0.301 & 446 \\
\hline 04087200 & 1 & No & No & 2005 & 446 & -- & 990 & 2.04 & 0.798 & 0.301 & 446 \\
\hline 04087200 & 1 & No & No & 2006 & 628 & -- & 1395 & 0.969 & 0.798 & 0.103 & 628 \\
\hline 04087200 & 1 & No & No & 2007 & 511 & -- & 1135 & 1.53 & 0.798 & 0.2 & 511 \\
\hline 04087200 & 1 & No & No & 2008 & 628 & -- & 1395 & 4.38 & 0.798 & 0.103 & 628 \\
\hline 04087200 & 1 & No & No & 2009 & 420 & -- & 933 & 2.32 & 0.798 & 0.368 & 420 \\
\hline 04087204 & 1 & Yes & Yes & 1964 & 398 & -- & 517 & 2.4 & 0.434 & 0.538 & 580 \\
\hline 04087204 & 1 & Yes & Yes & 1965 & 480 & -- & 624 & 1.13 & 0.446 & 0.395 & 676 \\
\hline 04087204 & 1 & Yes & Yes & 1966 & 526 & -- & 683 & 0.73 & 0.458 & 0.33 & 721 \\
\hline 04087204 & 1 & Yes & Yes & 1967 & 612 & -- & 795 & 1.98 & 0.47 & 0.227 & 811 \\
\hline 04087204 & 1 & Yes & Yes & 1968 & 606 & -- & 787 & 1.97 & 0.481 & 0.241 & 796 \\
\hline 04087204 & 1 & Yes & Yes & 1969 & 704 & -- & 915 & 2.24 & 0.493 & 0.167 & 905 \\
\hline 04087204 & 1 & Yes & Yes & 1970 & 399 & -- & 518 & 1.14 & 0.505 & 0.603 & 541 \\
\hline 04087204 & 1 & Yes & Yes & 1971 & 441 & -- & 573 & 0.717 & 0.516 & 0.53 & 585 \\
\hline
\end{tabular}




\begin{tabular}{|c|c|c|c|c|c|c|c|c|c|c|c|}
\hline $\begin{array}{c}\text { U.S. } \\
\text { Geological } \\
\text { Survey } \\
\text { streamgage } \\
\text { number }\end{array}$ & $\begin{array}{l}\text { Segment } \\
\text { number }\end{array}$ & $\begin{array}{l}\text { Streamgage used in } \\
\text { regression analyses } \\
\text { (non-redundant) }\end{array}$ & $\begin{array}{l}\text { Discharge value } \\
\text { used in } \\
\text { adjustment } \\
\text { regression }\end{array}$ & $\begin{array}{l}\text { Water } \\
\text { year }\end{array}$ & $\begin{array}{l}\text { Observed annual } \\
\text { maximum peak } \\
\text { discharge }\left(\mathrm{ft}^{3} / \mathrm{s}\right)\end{array}$ & $\begin{array}{l}\text { NWIS peak } \\
\text { code }\end{array}$ & $\begin{array}{l}\text { Observed annual } \\
\text { maximum peak } \\
\text { discharge with } \\
\text { segment intercept } \\
\text { value subtracted } \\
\left(\mathrm{ft}^{3} / \mathrm{s}\right)\end{array}$ & $\begin{array}{l}\text { Observed } \\
\text { precipitation } \\
\text { (inches) }\end{array}$ & Urban fraction & $\begin{array}{l}\text { Exceedance } \\
\text { probability }\end{array}$ & $\begin{array}{c}\text { Urban-adjusted } \\
\text { annual maximum } \\
\text { peak discharge } \\
\left(\mathrm{ft}^{3} / \mathrm{s}\right)\end{array}$ \\
\hline 04087204 & 1 & Yes & Yes & 1972 & 916 & -- & 1190 & 1.71 & 0.527 & 0.0746 & 1130 \\
\hline 04087204 & 1 & Yes & Yes & 1973 & 774 & -- & 1005 & 2.74 & 0.537 & 0.143 & 954 \\
\hline 04087204 & 1 & Yes & Yes & 1974 & 839 & -- & 1090 & 0.769 & 0.548 & 0.111 & 1020 \\
\hline 04087204 & 1 & Yes & Yes & 1975 & 581 & -- & 755 & 1.68 & 0.559 & 0.333 & 719 \\
\hline 04087204 & 1 & Yes & Yes & 1976 & 935 & -- & 1215 & 2.23 & 0.57 & 0.0799 & 1110 \\
\hline 04087204 & 1 & Yes & Yes & 1977 & 378 & -- & 491 & 1.84 & 0.58 & 0.712 & 477 \\
\hline 04087204 & 1 & Yes & Yes & 1978 & 1020 & -- & 1325 & 1.7 & 0.591 & 0.0637 & 1190 \\
\hline 04087204 & 1 & Yes & Yes & 1979 & 660 & -- & 857 & 1.48 & 0.602 & 0.262 & 774 \\
\hline 04087204 & 1 & Yes & Yes & 1980 & 541 & -- & 703 & 1.77 & 0.613 & 0.448 & 640 \\
\hline 04087204 & 1 & Yes & Yes & 1981 & 472 & -- & 613 & 1.5 & 0.616 & 0.569 & 560 \\
\hline 04087204 & 1 & Yes & Yes & 1982 & 492 & -- & 639 & 1.2 & 0.62 & 0.538 & 580 \\
\hline 04087204 & 1 & Yes & Yes & 1983 & 670 & -- & 870 & 2.01 & 0.624 & 0.267 & 769 \\
\hline 04087204 & 1 & Yes & Yes & 1984 & 450 & -- & 585 & 1.49 & 0.628 & 0.621 & 529 \\
\hline 04087204 & 1 & Yes & Yes & 1985 & 403 & -- & 524 & 0.723 & 0.632 & 0.712 & 477 \\
\hline 04087204 & 1 & Yes & Yes & 1986 & 1140 & -- & 1481 & 6.46 & 0.635 & 0.0485 & 1270 \\
\hline 04087204 & 1 & Yes & Yes & 1987 & 457 & -- & 594 & 1.63 & 0.639 & 0.619 & 530 \\
\hline 04087204 & 1 & Yes & Yes & 1988 & 568 & -- & 738 & 1.47 & 0.643 & 0.434 & 650 \\
\hline 04087204 & 1 & Yes & Yes & 1989 & 422 & -- & 548 & 1.26 & 0.647 & 0.693 & 489 \\
\hline 04087204 & 1 & Yes & Yes & 1990 & 817 & -- & 1061 & 2.38 & 0.65 & 0.166 & 908 \\
\hline 04087204 & 1 & Yes & Yes & 1991 & 451 & -- & 586 & 0.925 & 0.662 & 0.653 & 511 \\
\hline 04087204 & 1 & Yes & Yes & 1992 & 508 & -- & 660 & 2.22 & 0.673 & 0.562 & 565 \\
\hline 04087204 & 1 & Yes & Yes & 1993 & 887 & -- & 1152 & 1.96 & 0.685 & 0.143 & 955 \\
\hline 04087204 & 1 & Yes & No & 1994 & 600 & 1,8 & 779 & 0.893 & 0.696 & 0.438 & 647 \\
\hline 04087204 & 1 & Yes & Yes & 1995 & 438 & -- & 569 & 0.71 & 0.708 & 0.721 & 471 \\
\hline 04087204 & 1 & Yes & Yes & 1996 & 573 & -- & 744 & 2.07 & 0.719 & 0.503 & 603 \\
\hline 04087204 & 1 & Yes & Yes & 1997 & 1110 & -- & 1442 & 4.02 & 0.731 & 0.0716 & 1140 \\
\hline 04087204 & 1 & Yes & Yes & 1998 & 542 & -- & 704 & 0.669 & 0.742 & 0.575 & 557 \\
\hline 04087204 & 1 & Yes & Yes & 1999 & 1060 & -- & 1377 & 1.5 & 0.754 & 0.0926 & 1070 \\
\hline 04087204 & 1 & Yes & Yes & 2000 & 1120 & -- & 1455 & 4.13 & 0.766 & 0.0773 & 1120 \\
\hline 04087204 & 1 & Yes & Yes & 2001 & 798 & -- & 1037 & 1.17 & 0.766 & 0.239 & 798 \\
\hline 04087204 & 1 & Yes & Yes & 2002 & 511 & -- & 664 & 1.15 & 0.766 & 0.653 & 511 \\
\hline 04087204 & 1 & Yes & Yes & 2003 & 267 & -- & 347 & 0.651 & 0.766 & 0.953 & 267 \\
\hline 04087204 & 1 & Yes & Yes & 2004 & 732 & -- & 951 & 1.46 & 0.766 & 0.311 & 732 \\
\hline 04087204 & 1 & Yes & Yes & 2005 & 611 & -- & 794 & 2.16 & 0.766 & 0.492 & 611 \\
\hline 04087204 & 1 & Yes & Yes & 2006 & 772 & -- & 1003 & 1.03 & 0.766 & 0.264 & 772 \\
\hline
\end{tabular}




\begin{tabular}{|c|c|c|c|c|c|c|c|c|c|c|c|}
\hline $\begin{array}{c}\text { U.S. } \\
\text { Geological } \\
\text { Survey } \\
\text { streamgage } \\
\text { number } \\
\end{array}$ & $\begin{array}{c}\text { Segment } \\
\text { number }\end{array}$ & $\begin{array}{l}\text { Streamgage used in } \\
\text { regression analyses } \\
\text { (non-redundant) }\end{array}$ & $\begin{array}{c}\text { Discharge value } \\
\text { used in } \\
\text { adjustment } \\
\text { regression }\end{array}$ & $\begin{array}{c}\text { Water } \\
\text { year }\end{array}$ & $\begin{array}{c}\text { Observed annual } \\
\text { maximum peak } \\
\text { discharge }\left(\mathrm{ft}^{3} / \mathrm{s}\right)\end{array}$ & $\begin{array}{c}\text { NWIS peak } \\
\text { code }\end{array}$ & $\begin{array}{l}\text { Observed annual } \\
\text { maximum peak } \\
\text { discharge with } \\
\text { segment intercept } \\
\text { value subtracted } \\
\left(\mathrm{ft}^{3} / \mathrm{s}\right)\end{array}$ & $\begin{array}{l}\text { Observed } \\
\text { precipitation } \\
\text { (inches) }\end{array}$ & Urban fraction & $\begin{array}{l}\text { Exceedance } \\
\text { probability }\end{array}$ & $\begin{array}{c}\text { Urban-adjusted } \\
\text { annual maximum } \\
\text { peak discharge } \\
\left(\mathrm{ft}^{3} / \mathrm{s}\right)\end{array}$ \\
\hline 04087204 & 1 & Yes & Yes & 2007 & 590 & -- & 766 & 1.52 & 0.766 & 0.523 & 590 \\
\hline 04087204 & 1 & Yes & Yes & 2008 & 2370 & -- & 3079 & 4.63 & 0.766 & 0.00697 & 2370 \\
\hline 04087204 & 1 & Yes & Yes & 2009 & 774 & -- & 1005 & 2.29 & 0.766 & 0.262 & 774 \\
\hline 04087220 & 1 & Yes & No & 1960 & 5130 & 7 & 3889 & 0.892 & 0.517 & 0 & 6290 \\
\hline 04087220 & 1 & Yes & Yes & 1964 & 792 & -- & 600 & 2.11 & 0.555 & 0.528 & 1070 \\
\hline 04087220 & 1 & Yes & Yes & 1965 & 1600 & -- & 1213 & 0.713 & 0.565 & 0.079 & 2010 \\
\hline 04087220 & 1 & Yes & Yes & 1966 & 1300 & -- & 986 & 0.483 & 0.575 & 0.168 & 1630 \\
\hline 04087220 & 1 & Yes & Yes & 1967 & 808 & -- & 613 & 1.48 & 0.584 & 0.539 & 1060 \\
\hline 04087220 & 1 & Yes & Yes & 1968 & 910 & -- & 690 & 1.23 & 0.594 & 0.446 & 1170 \\
\hline 04087220 & 1 & Yes & Yes & 1969 & 2650 & -- & 2009 & 0.976 & 0.603 & 0.0173 & 3120 \\
\hline 04087220 & 1 & Yes & Yes & 1970 & 1220 & -- & 925 & 1.48 & 0.613 & 0.218 & 1490 \\
\hline 04087220 & 1 & Yes & Yes & 1971 & 1020 & -- & 773 & 0.207 & 0.621 & 0.37 & 1260 \\
\hline 04087220 & 1 & Yes & Yes & 1972 & 2270 & -- & 1721 & 1.47 & 0.63 & 0.0294 & 2640 \\
\hline 04087220 & 1 & Yes & Yes & 1973 & 3700 & -- & 2805 & 2.29 & 0.638 & 0.007 & 4210 \\
\hline 04087220 & 1 & Yes & Yes & 1974 & 1680 & -- & 1274 & 0.644 & 0.647 & 0.0865 & 1960 \\
\hline 04087220 & 1 & Yes & Yes & 1975 & 1420 & -- & 1077 & 1.21 & 0.655 & 0.162 & 1660 \\
\hline 04087220 & 1 & Yes & Yes & 1976 & 2160 & -- & 1638 & 1.21 & 0.663 & 0.0386 & 2460 \\
\hline 04087220 & 1 & Yes & Yes & 1977 & 534 & -- & 405 & 1.28 & 0.672 & 0.878 & 669 \\
\hline 04087220 & 1 & Yes & Yes & 1978 & 980 & -- & 743 & 1.24 & 0.68 & 0.465 & 1150 \\
\hline 04087220 & 1 & Yes & Yes & 1979 & 1300 & -- & 986 & 0.272 & 0.689 & 0.224 & 1480 \\
\hline 04087220 & 1 & Yes & Yes & 1980 & 860 & -- & 652 & 0.668 & 0.697 & 0.596 & 995 \\
\hline 04087220 & 1 & Yes & Yes & 1981 & 474 & -- & 359 & 0.576 & 0.702 & 0.93 & 579 \\
\hline 04087220 & 1 & Yes & Yes & 1982 & 556 & -- & 422 & 0.184 & 0.708 & 0.884 & 661 \\
\hline 04087220 & 1 & Yes & Yes & 1983 & 1080 & -- & 819 & 1.55 & 0.714 & 0.41 & 1210 \\
\hline 04087220 & 1 & Yes & Yes & 1984 & 550 & 2 & 417 & 0.41 & 0.719 & 0.894 & 645 \\
\hline 04087220 & 1 & Yes & Yes & 1985 & 698 & -- & 529 & 0.478 & 0.725 & 0.781 & 794 \\
\hline 04087220 & 1 & Yes & Yes & 1986 & 708 & -- & 537 & 0.345 & 0.73 & 0.776 & 800 \\
\hline 04087220 & 1 & Yes & Yes & 1987 & 579 & -- & 439 & 1.36 & 0.736 & 0.884 & 660 \\
\hline 04087220 & 1 & Yes & Yes & 1988 & 530 & 9 & 402 & 0.535 & 0.741 & 0.919 & 605 \\
\hline 04087220 & 1 & Yes & Yes & 1989 & 442 & -- & 335 & 1.23 & 0.747 & 0.953 & 507 \\
\hline 04087220 & 1 & Yes & Yes & 1990 & 880 & -- & 667 & 1.16 & 0.752 & 0.634 & 956 \\
\hline 04087220 & 1 & Yes & Yes & 1991 & 587 & -- & 445 & 0.861 & 0.757 & 0.892 & 648 \\
\hline 04087220 & 1 & Yes & Yes & 1992 & 641 & -- & 486 & 0.757 & 0.762 & 0.855 & 699 \\
\hline 04087220 & 1 & Yes & Yes & 1993 & 1180 & -- & 895 & 0.893 & 0.767 & 0.378 & 1250 \\
\hline 04087220 & 1 & Yes & No & 1994 & 600 & 1,8 & 455 & 0.653 & 0.772 & 0.892 & 648 \\
\hline
\end{tabular}




\begin{tabular}{|c|c|c|c|c|c|c|c|c|c|c|c|}
\hline $\begin{array}{l}\text { U.S. } \\
\text { Geological } \\
\text { Survey } \\
\text { streamgage } \\
\text { number }\end{array}$ & $\begin{array}{l}\text { Segment } \\
\text { number }\end{array}$ & $\begin{array}{l}\text { Streamgage used in } \\
\text { regression analyses } \\
\text { (non-redundant) }\end{array}$ & $\begin{array}{c}\text { Discharge value } \\
\text { used in } \\
\text { adjustment } \\
\text { regression }\end{array}$ & $\begin{array}{l}\text { Water } \\
\text { year }\end{array}$ & $\begin{array}{c}\text { Observed annual } \\
\text { maximum peak } \\
\text { discharge }\left(\mathrm{ft}^{3} / \mathrm{s}\right)\end{array}$ & $\begin{array}{l}\text { NWIS peak } \\
\text { code }\end{array}$ & $\begin{array}{l}\text { Observed annual } \\
\text { maximum peak } \\
\text { discharge with } \\
\text { segment intercept } \\
\text { value subtracted } \\
\left(\mathrm{ft}^{3} / \mathrm{s}\right)\end{array}$ & $\begin{array}{l}\text { Observed } \\
\text { precipitation } \\
\text { (inches) }\end{array}$ & Urban fraction & $\begin{array}{l}\text { Exceedance } \\
\text { probability }\end{array}$ & $\begin{array}{c}\text { Urban-adjusted } \\
\text { annual maximum } \\
\text { peak discharge } \\
\left(\mathrm{ft}^{3} / \mathrm{s}\right)\end{array}$ \\
\hline 04087220 & 1 & Yes & Yes & 1995 & 430 & -- & 326 & 0.799 & 0.777 & 0.961 & 468 \\
\hline 04087220 & 1 & Yes & Yes & 1996 & 1020 & -- & 773 & 1.17 & 0.782 & 0.532 & 1070 \\
\hline 04087220 & 1 & Yes & Yes & 1997 & 1750 & -- & 1327 & 1.24 & 0.787 & 0.121 & 1810 \\
\hline 04087220 & 1 & Yes & Yes & 1998 & 558 & -- & 423 & 1.09 & 0.792 & 0.927 & 587 \\
\hline 04087220 & 1 & Yes & Yes & 1999 & 2050 & -- & 1554 & 1.32 & 0.797 & 0.0673 & 2100 \\
\hline 04087220 & 1 & Yes & Yes & 2000 & 2420 & -- & 1835 & 4.33 & 0.802 & 0.0384 & 2460 \\
\hline 04087220 & 1 & Yes & Yes & 2001 & 917 & -- & 695 & 1.98 & 0.804 & 0.653 & 937 \\
\hline 04087220 & 1 & Yes & Yes & 2002 & 780 & -- & 591 & 1.35 & 0.806 & 0.779 & 797 \\
\hline 04087220 & 1 & Yes & Yes & 2003 & 449 & -- & 340 & 0.921 & 0.808 & 0.963 & 461 \\
\hline 04087220 & 1 & Yes & Yes & 2004 & 1190 & -- & 902 & 2.15 & 0.81 & 0.417 & 1210 \\
\hline 04087220 & 1 & Yes & Yes & 2005 & 618 & -- & 469 & 0.431 & 0.812 & 0.905 & 628 \\
\hline 04087220 & 1 & Yes & Yes & 2006 & 1160 & -- & 879 & 1.18 & 0.814 & 0.446 & 1170 \\
\hline 04087220 & 1 & Yes & Yes & 2007 & 1070 & -- & 811 & 1.72 & 0.816 & 0.524 & 1080 \\
\hline 04087220 & 1 & Yes & Yes & 2008 & 5350 & -- & 4056 & 4.82 & 0.818 & 0 & 5360 \\
\hline 04087220 & 1 & Yes & Yes & 2009 & 2260 & -- & 1713 & 2.88 & 0.82 & 0.0491 & 2260 \\
\hline 04087230 & 1 & Yes & Yes & 1962 & 108 & -- & 424 & 0.02 & 0.0143 & 0.346 & 120 \\
\hline 04087230 & 1 & Yes & Yes & 1963 & 30 & -- & 118 & 0.05 & 0.0172 & 0.925 & 35.2 \\
\hline 04087230 & 1 & Yes & Yes & 1964 & 70 & -- & 275 & 4 & 0.02 & 0.644 & 78.3 \\
\hline 04087230 & 1 & Yes & Yes & 1965 & 47 & -- & 184 & 0.13 & 0.0229 & 0.825 & 53.3 \\
\hline 04087230 & 1 & Yes & Yes & 1966 & 150 & -- & 588 & 0.83 & 0.0257 & 0.168 & 163 \\
\hline 04087230 & 1 & Yes & Yes & 1967 & 65 & -- & 255 & 2.03 & 0.0286 & 0.691 & 72.2 \\
\hline 04087230 & 1 & Yes & Yes & 1968 & 60 & -- & 235 & 1.25 & 0.0315 & 0.734 & 66.6 \\
\hline 04087230 & 1 & Yes & Yes & 1969 & 107 & -- & 420 & 2.3 & 0.0343 & 0.365 & 116 \\
\hline 04087230 & 1 & Yes & Yes & 1970 & 98 & -- & 384 & 0.51 & 0.0372 & 0.425 & 106 \\
\hline 04087230 & 1 & Yes & Yes & 1971 & 100 & -- & 392 & 0.95 & 0.0386 & 0.412 & 108 \\
\hline 04087230 & 1 & Yes & Yes & 1972 & 85 & -- & 333 & 1.66 & 0.04 & 0.53 & 92.4 \\
\hline 04087230 & 1 & Yes & Yes & 1973 & 48 & -- & 188 & 1.15 & 0.0415 & 0.827 & 53.1 \\
\hline 04087230 & 1 & Yes & Yes & 1974 & 105 & -- & 412 & 0.55 & 0.0429 & 0.383 & 113 \\
\hline 04087230 & 1 & Yes & Yes & 1975 & 32 & -- & 126 & 0.02 & 0.0443 & 0.922 & 35.9 \\
\hline 04087230 & 1 & Yes & Yes & 1976 & 90 & -- & 353 & 1.23 & 0.0458 & 0.492 & 97.1 \\
\hline 04087230 & 1 & Yes & Yes & 1977 & 25 & -- & 98 & 0.62 & 0.0472 & 0.95 & 28.2 \\
\hline 04087230 & 1 & Yes & Yes & 1978 & 82 & -- & 322 & 1.91 & 0.0486 & 0.565 & 88.3 \\
\hline 04087230 & 1 & Yes & Yes & 1979 & 72 & -- & 282 & 0.49 & 0.05 & 0.648 & 77.7 \\
\hline 04087230 & 1 & Yes & Yes & 1980 & 100 & -- & 392 & 2.7 & 0.0515 & 0.422 & 107 \\
\hline 04087230 & 1 & Yes & Yes & 1981 & 80 & -- & 314 & 0.69 & 0.0519 & 0.587 & 85.9 \\
\hline
\end{tabular}




\begin{tabular}{|c|c|c|c|c|c|c|c|c|c|c|c|}
\hline $\begin{array}{c}\text { U.S. } \\
\text { Geological } \\
\text { Survey } \\
\text { streamgage } \\
\text { number } \\
\end{array}$ & $\begin{array}{c}\text { Segment } \\
\text { number }\end{array}$ & $\begin{array}{l}\text { Streamgage used in } \\
\text { regression analyses } \\
\text { (non-redundant) }\end{array}$ & $\begin{array}{c}\text { Discharge value } \\
\text { used in } \\
\text { adjustment } \\
\text { regression }\end{array}$ & $\begin{array}{c}\text { Water } \\
\text { year }\end{array}$ & $\begin{array}{c}\text { Observed annual } \\
\text { maximum peak } \\
\text { discharge }\left(\mathrm{ft}^{3} / \mathrm{s}\right)\end{array}$ & $\begin{array}{c}\text { NWIS peak } \\
\text { code }\end{array}$ & $\begin{array}{l}\text { Observed annual } \\
\text { maximum peak } \\
\text { discharge with } \\
\text { segment intercept } \\
\text { value subtracted } \\
\left(\mathrm{ft}^{3} / \mathrm{s}\right)\end{array}$ & $\begin{array}{l}\text { Observed } \\
\text { precipitation } \\
\text { (inches) }\end{array}$ & Urban fraction & $\begin{array}{l}\text { Exceedance } \\
\text { probability }\end{array}$ & $\begin{array}{c}\text { Urban-adjusted } \\
\text { annual maximum } \\
\text { peak discharge } \\
\left(\mathrm{ft}^{3} / \mathrm{s}\right)\end{array}$ \\
\hline 04087230 & 1 & Yes & Yes & 1982 & 96 & -- & 377 & 0.6 & 0.0522 & 0.451 & 103 \\
\hline 04087230 & 1 & Yes & Yes & 1983 & 155 & -- & 608 & 1.15 & 0.0526 & 0.165 & 164 \\
\hline 04087230 & 1 & Yes & Yes & 1984 & 140 & -- & 549 & 1.29 & 0.053 & 0.21 & 148 \\
\hline 04087230 & 1 & Yes & Yes & 1985 & 128 & -- & 502 & 0.78 & 0.0534 & 0.265 & 136 \\
\hline 04087230 & 1 & Yes & Yes & 1986 & 168 & -- & 659 & 1.08 & 0.0538 & 0.133 & 177 \\
\hline 04087230 & 1 & Yes & Yes & 1987 & 182 & -- & 714 & 2.35 & 0.0541 & 0.0999 & 192 \\
\hline 04087230 & 1 & Yes & Yes & 1988 & 152 & -- & 596 & 0.02 & 0.0545 & 0.174 & 161 \\
\hline 04087230 & 1 & Yes & Yes & 1989 & 142 & -- & 557 & 4.33 & 0.0549 & 0.203 & 150 \\
\hline 04087230 & 1 & Yes & Yes & 1990 & 132 & -- & 518 & 1.52 & 0.0553 & 0.247 & 140 \\
\hline 04087230 & 1 & Yes & Yes & 1991 & 123 & -- & 482 & 1.27 & 0.0586 & 0.293 & 130 \\
\hline 04087230 & 1 & Yes & Yes & 1992 & 95 & -- & 373 & 0.95 & 0.062 & 0.466 & 101 \\
\hline 04087230 & 1 & Yes & Yes & 1993 & 148 & -- & 581 & 2.05 & 0.0653 & 0.189 & 155 \\
\hline 04087233 & 1 & Yes & Yes & 1964 & 309 & -- & 169 & 3.97 & 0.0333 & 0.856 & 359 \\
\hline 04087233 & 1 & Yes & No & 1965 & 500 & 1,8 & 273 & 0.556 & 0.0363 & 0.658 & 564 \\
\hline 04087233 & 1 & Yes & Yes & 1966 & 774 & -- & 422 & 0.823 & 0.0394 & 0.365 & 857 \\
\hline 04087233 & 1 & Yes & Yes & 1967 & 574 & -- & 313 & 2.02 & 0.0424 & 0.58 & 640 \\
\hline 04087233 & 1 & Yes & Yes & 1968 & 470 & -- & 256 & 1.25 & 0.0454 & 0.699 & 526 \\
\hline 04087233 & 1 & Yes & Yes & 1969 & 461 & -- & 252 & 2.27 & 0.0484 & 0.711 & 515 \\
\hline 04087233 & 1 & Yes & Yes & 1970 & 696 & -- & 380 & 2.01 & 0.0514 & 0.445 & 764 \\
\hline 04087233 & 1 & Yes & Yes & 1971 & 760 & -- & 415 & 0.0303 & 0.0544 & 0.386 & 829 \\
\hline 04087233 & 1 & Yes & Yes & 1972 & 704 & -- & 384 & 1.59 & 0.0575 & 0.441 & 768 \\
\hline 04087233 & 1 & Yes & Yes & 1973 & 740 & -- & 404 & 0.36 & 0.0605 & 0.408 & 803 \\
\hline 04087233 & 1 & Yes & Yes & 1974 & 1440 & D & 786 & 0.551 & 0.0635 & 0.0769 & 1540 \\
\hline 04087233 & 1 & Yes & Yes & 1975 & 482 & -- & 263 & 0.000498 & 0.0666 & 0.7 & 526 \\
\hline 04087233 & 1 & Yes & Yes & 1976 & 1200 & -- & 655 & 1.23 & 0.0696 & 0.141 & 1280 \\
\hline 04087233 & 1 & Yes & Yes & 1977 & 108 & -- & 59 & 0.621 & 0.0726 & 0.984 & 123 \\
\hline 04087233 & 1 & Yes & Yes & 1978 & 990 & -- & 540 & 5.29 & 0.0757 & 0.233 & 1050 \\
\hline 04087233 & 1 & Yes & Yes & 1979 & 1060 & -- & 578 & 0.251 & 0.0787 & 0.196 & 1120 \\
\hline 04087233 & 1 & Yes & Yes & 1980 & 437 & -- & 238 & 2.66 & 0.0817 & 0.758 & 469 \\
\hline 04087233 & 1 & Yes & Yes & 1981 & 673 & -- & 367 & 2.3 & 0.0823 & 0.493 & 715 \\
\hline 04087233 & 1 & Yes & Yes & 1982 & 552 & -- & 301 & 2.31 & 0.0828 & 0.634 & 588 \\
\hline 04087233 & 1 & Yes & Yes & 1983 & 1140 & -- & 622 & 1.16 & 0.0833 & 0.168 & 1200 \\
\hline 04087233 & 1 & Yes & Yes & 1984 & 583 & -- & 318 & 1.06 & 0.0839 & 0.604 & 620 \\
\hline 04087233 & 1 & Yes & Yes & 1985 & 942 & -- & 514 & 0.451 & 0.0844 & 0.269 & 991 \\
\hline 04087233 & 1 & Yes & Yes & 1986 & 1120 & -- & 611 & 0.159 & 0.085 & 0.175 & 1170 \\
\hline
\end{tabular}




\begin{tabular}{|c|c|c|c|c|c|c|c|c|c|c|c|}
\hline $\begin{array}{c}\text { U.S. } \\
\text { Geological } \\
\text { Survey } \\
\text { streamgage } \\
\text { number } \\
\end{array}$ & $\begin{array}{c}\text { Segment } \\
\text { number }\end{array}$ & $\begin{array}{l}\text { Streamgage used in } \\
\text { regression analyses } \\
\text { (non-redundant) }\end{array}$ & $\begin{array}{c}\text { Discharge value } \\
\text { used in } \\
\text { adjustment } \\
\text { regression }\end{array}$ & $\begin{array}{c}\text { Water } \\
\text { year }\end{array}$ & $\begin{array}{c}\text { Observed annual } \\
\text { maximum peak } \\
\text { discharge }\left(\mathrm{ft}^{3} / \mathrm{s}\right)\end{array}$ & $\begin{array}{c}\text { NWIS peak } \\
\text { code }\end{array}$ & $\begin{array}{c}\text { Observed annual } \\
\text { maximum peak } \\
\text { discharge with } \\
\text { segment intercept } \\
\text { value subtracted } \\
\left(\mathrm{ft}^{3} / \mathrm{s}\right)\end{array}$ & $\begin{array}{c}\text { Observed } \\
\text { precipitation } \\
\text { (inches) }\end{array}$ & Urban fraction & $\begin{array}{l}\text { Exceedance } \\
\text { probability }\end{array}$ & $\begin{array}{c}\text { Urban-adjusted } \\
\text { annual maximum } \\
\text { peak discharge } \\
\left(\mathrm{ft}^{3} / \mathrm{s}\right)\end{array}$ \\
\hline 04087233 & 1 & Yes & Yes & 1987 & 901 & -- & 492 & 1.19 & 0.0855 & 0.298 & 948 \\
\hline 04087233 & 1 & Yes & Yes & 1988 & 788 & -- & 430 & 1.51 & 0.086 & 0.384 & 831 \\
\hline 04087233 & 1 & Yes & Yes & 1989 & 760 & -- & 415 & 4.28 & 0.0866 & 0.409 & 802 \\
\hline 04087233 & 1 & Yes & Yes & 1990 & 1080 & -- & 589 & 1.51 & 0.0871 & 0.191 & 1130 \\
\hline 04087233 & 1 & Yes & Yes & 1991 & 1050 & -- & 573 & 1.26 & 0.0901 & 0.206 & 1100 \\
\hline 04087233 & 1 & Yes & Yes & 1992 & 635 & -- & 347 & 0.939 & 0.0931 & 0.547 & 667 \\
\hline 04087233 & 1 & Yes & Yes & 1993 & 1260 & -- & 688 & 2.03 & 0.096 & 0.131 & 1310 \\
\hline 04087233 & 1 & Yes & No & 1994 & 1000 & 1,8 & 546 & 1.47 & 0.099 & 0.241 & 1040 \\
\hline 04087233 & 1 & Yes & Yes & 1995 & 750 & -- & 409 & 1.98 & 0.102 & 0.431 & 779 \\
\hline 04087233 & 1 & Yes & Yes & 1996 & 721 & -- & 393 & 2.37 & 0.105 & 0.462 & 746 \\
\hline 04087233 & 1 & Yes & Yes & 1997 & 704 & -- & 384 & 1.23 & 0.108 & 0.481 & 727 \\
\hline 04087233 & 1 & Yes & Yes & 1998 & 659 & -- & 360 & 1.19 & 0.111 & 0.534 & 678 \\
\hline 04087233 & 1 & Yes & Yes & 1999 & 830 & -- & 453 & 1.59 & 0.114 & 0.37 & 850 \\
\hline 04087233 & 1 & Yes & Yes & 2000 & 1300 & $-{ }_{-1}$ & 709 & 1.48 & 0.117 & 0.126 & 1320 \\
\hline 04087233 & 1 & Yes & Yes & 2001 & 1100 & 9 & 600 & 1.83 & 0.119 & 0.196 & 1120 \\
\hline 04087233 & 1 & Yes & Yes & 2002 & 768 & -- & 419 & 1.97 & 0.121 & 0.429 & 781 \\
\hline 04087233 & 1 & Yes & Yes & 2003 & 356 & -- & 194 & 0.723 & 0.123 & 0.852 & 363 \\
\hline 04087233 & 1 & Yes & Yes & 2004 & 1430 & -- & 780 & 2.82 & 0.125 & 0.0932 & 1440 \\
\hline 04087233 & 1 & Yes & Yes & 2005 & 599 & -- & 327 & 0.551 & 0.127 & 0.617 & 606 \\
\hline 04087233 & 1 & Yes & Yes & 2006 & 792 & -- & 432 & 2.02 & 0.129 & 0.412 & 799 \\
\hline 04087233 & 1 & Yes & Yes & 2007 & 1280 & -- & 698 & 3.89 & 0.13 & 0.138 & 1290 \\
\hline 04087233 & 1 & Yes & Yes & 2008 & 1560 & -- & 851 & 2.53 & 0.132 & 0.0729 & 1570 \\
\hline 04087233 & 1 & Yes & Yes & 2009 & 988 & -- & 539 & 2.2 & 0.134 & 0.27 & 990 \\
\hline 04087240 & 1 & No & No & 1964 & 997 & -- & 244 & 2.71 & 0.215 & 0.82 & 1310 \\
\hline 04087240 & 1 & No & No & 1965 & 1610 & -- & 394 & 0.634 & 0.221 & 0.562 & 2000 \\
\hline 04087240 & 1 & No & No & 1966 & 2500 & -- & 611 & 0.532 & 0.227 & 0.247 & 2980 \\
\hline 04087240 & 1 & No & No & 1967 & 2250 & -- & 550 & 1.65 & 0.234 & 0.322 & 2690 \\
\hline 04087240 & 1 & No & No & 1968 & 1470 & -- & 359 & 0.455 & 0.24 & 0.64 & 1800 \\
\hline 04087240 & 1 & No & No & 1969 & 2150 & -- & 526 & 1.27 & 0.246 & 0.363 & 2550 \\
\hline 04087240 & 1 & No & No & 1970 & 1280 & -- & 313 & 1.65 & 0.252 & 0.732 & 1560 \\
\hline 04087240 & 1 & No & No & 1971 & 1560 & -- & 381 & 0.0569 & 0.257 & 0.616 & 1860 \\
\hline 04087240 & 1 & No & No & 1972 & 2740 & -- & 670 & 1.45 & 0.262 & 0.211 & 3150 \\
\hline 04087240 & 1 & No & No & 1973 & 3790 & -- & 927 & 1.78 & 0.267 & 0.081 & 4300 \\
\hline 04087240 & 1 & No & No & 1974 & 4500 & -- & 1100 & 0.595 & 0.272 & 0.0487 & 5050 \\
\hline 04087240 & 1 & No & No & 1975 & 1930 & -- & 472 & 0.0185 & 0.277 & 0.469 & 2240 \\
\hline
\end{tabular}




\begin{tabular}{|c|c|c|c|c|c|c|c|c|c|c|c|}
\hline $\begin{array}{l}\text { U.S. } \\
\text { Geological } \\
\text { Survey } \\
\text { streamgage } \\
\text { number }\end{array}$ & $\begin{array}{l}\text { Segment } \\
\text { number }\end{array}$ & $\begin{array}{l}\text { Streamgage used in } \\
\text { regression analyses } \\
\text { (non-redundant) }\end{array}$ & $\begin{array}{c}\text { Discharge value } \\
\text { used in } \\
\text { adjustment } \\
\text { regression }\end{array}$ & $\begin{array}{l}\text { Water } \\
\text { year }\end{array}$ & $\begin{array}{c}\text { Observed annual } \\
\text { maximum peak } \\
\text { discharge }\left(\mathrm{ft}^{3} / \mathrm{s}\right)\end{array}$ & $\begin{array}{l}\text { NWIS peak } \\
\text { code }\end{array}$ & $\begin{array}{l}\text { Observed annual } \\
\text { maximum peak } \\
\text { discharge with } \\
\text { segment intercept } \\
\text { value subtracted } \\
\left(\mathrm{ft}^{3} / \mathrm{s}\right)\end{array}$ & $\begin{array}{l}\text { Observed } \\
\text { precipitation } \\
\text { (inches) }\end{array}$ & Urban fraction & $\begin{array}{l}\text { Exceedance } \\
\text { probability }\end{array}$ & $\begin{array}{c}\text { Urban-adjusted } \\
\text { annual maximum } \\
\text { peak discharge } \\
\left(\mathrm{ft}^{3} / \mathrm{s}\right)\end{array}$ \\
\hline 04087240 & 1 & No & No & 1976 & 2930 & -- & 716 & 1.11 & 0.282 & 0.187 & 3300 \\
\hline 04087240 & 1 & No & No & 1977 & 340 & -- & 83 & 0.646 & 0.287 & 0.988 & 440 \\
\hline 04087240 & 1 & No & No & 1978 & 1490 & -- & 364 & 1.47 & 0.292 & 0.672 & 1720 \\
\hline 04087240 & 1 & No & No & 1979 & 3460 & -- & 846 & 0.268 & 0.297 & 0.122 & 3830 \\
\hline 04087240 & 1 & No & No & 1980 & 1000 & -- & 244 & 1.05 & 0.302 & 0.862 & 1170 \\
\hline 04087240 & 1 & No & No & 1981 & 1320 & -- & 323 & 1.2 & 0.304 & 0.752 & 1510 \\
\hline 04087240 & 1 & No & No & 1982 & 1940 & -- & 474 & 0.409 & 0.307 & 0.491 & 2180 \\
\hline 04087240 & 1 & No & No & 1983 & 3480 & -- & 851 & 1.4 & 0.309 & 0.124 & 3810 \\
\hline 04087240 & 1 & No & No & 1984 & 1580 & -- & 386 & 0.518 & 0.312 & 0.649 & 1780 \\
\hline 04087240 & 1 & No & No & 1985 & 1810 & -- & 442 & 0.471 & 0.314 & 0.553 & 2020 \\
\hline 04087240 & 1 & No & No & 1986 & 2140 & -- & 523 & 0.204 & 0.317 & 0.423 & 2370 \\
\hline 04087240 & 1 & No & No & 1987 & 1450 & -- & 354 & 0.863 & 0.319 & 0.71 & 1620 \\
\hline 04087240 & 1 & No & No & 1988 & 2090 & -- & 511 & 0.122 & 0.322 & 0.446 & 2300 \\
\hline 04087240 & 1 & No & No & 1989 & 847 & -- & 207 & 0.408 & 0.324 & 0.914 & 971 \\
\hline 04087240 & 1 & No & No & 1990 & 2530 & -- & 618 & 1.25 & 0.327 & 0.305 & 2750 \\
\hline 04087240 & 1 & No & No & 1991 & 2000 & -- & 489 & 1.01 & 0.333 & 0.49 & 2180 \\
\hline 04087240 & 1 & No & No & 1992 & 1220 & -- & 298 & 0.742 & 0.338 & 0.808 & 1340 \\
\hline 04087240 & 1 & No & No & 1993 & 3010 & -- & 736 & 1.27 & 0.344 & 0.201 & 3200 \\
\hline 04087240 & 1 & No & No & 1994 & 1940 & -- & 474 & 0.947 & 0.35 & 0.53 & 2080 \\
\hline 04087240 & 1 & No & No & 1995 & 1240 & -- & 303 & 1.29 & 0.356 & 0.811 & 1330 \\
\hline 04087240 & 1 & No & No & 1996 & 1900 & -- & 464 & 0.986 & 0.361 & 0.558 & 2010 \\
\hline 04087240 & 1 & No & No & 1997 & 1800 & -- & 440 & 1.13 & 0.367 & 0.606 & 1890 \\
\hline 04087240 & 1 & No & No & 1998 & 1370 & -- & 335 & 0.828 & 0.373 & 0.776 & 1440 \\
\hline 04087240 & 1 & No & No & 1999 & 2690 & -- & 658 & 1.1 & 0.379 & 0.297 & 2770 \\
\hline 04087240 & 1 & No & No & 2000 & 2550 & -- & 623 & 1.81 & 0.384 & 0.343 & 2620 \\
\hline 04087240 & 1 & No & No & 2001 & 2270 & -- & 555 & 1.16 & 0.387 & 0.437 & 2330 \\
\hline 04087240 & 1 & No & No & 2002 & 1400 & -- & 342 & 0.915 & 0.389 & 0.776 & 1440 \\
\hline 04087240 & 1 & No & No & 2003 & 789 & -- & 193 & 0.523 & 0.392 & 0.943 & 814 \\
\hline 04087240 & 1 & No & No & 2004 & 2940 & -- & 719 & 1.86 & 0.394 & 0.246 & 2980 \\
\hline 04087240 & 1 & No & No & 2005 & 1330 & -- & 325 & 0.344 & 0.397 & 0.805 & 1350 \\
\hline 04087240 & 1 & No & No & 2006 & 2050 & -- & 501 & 1.01 & 0.399 & 0.531 & 2070 \\
\hline 04087240 & 1 & No & No & 2007 & 2350 & -- & 574 & 1.04 & 0.402 & 0.422 & 2370 \\
\hline 04087240 & 1 & No & No & 2008 & 8050 & -- & 1968 & 3.07 & 0.404 & 0.0125 & 8080 \\
\hline 04087240 & 1 & No & No & 2009 & 2140 & -- & 523 & 1.7 & 0.407 & 0.503 & 2150 \\
\hline 04087250 & 1 & Yes & Yes & 1960 & 170 & -- & 758 & 0.67 & 0.074 & 0.0889 & 233 \\
\hline
\end{tabular}




\begin{tabular}{|c|c|c|c|c|c|c|c|c|c|c|c|}
\hline $\begin{array}{l}\text { U.S. } \\
\text { Geological } \\
\text { Survey } \\
\text { streamgage } \\
\text { number }\end{array}$ & $\begin{array}{l}\text { Segment } \\
\text { number }\end{array}$ & $\begin{array}{l}\text { Streamgage used in } \\
\text { regression analyses } \\
\text { (non-redundant) }\end{array}$ & $\begin{array}{c}\text { Discharge value } \\
\text { used in } \\
\text { adjustment } \\
\text { regression }\end{array}$ & $\begin{array}{l}\text { Water } \\
\text { year }\end{array}$ & $\begin{array}{l}\text { Observed annual } \\
\text { maximum peak } \\
\text { discharge }\left(\mathrm{ft}^{3} / \mathrm{s}\right)\end{array}$ & $\begin{array}{l}\text { NWIS peak } \\
\text { code }\end{array}$ & $\begin{array}{l}\text { Observed annual } \\
\text { maximum peak } \\
\text { discharge with } \\
\text { segment intercept } \\
\text { value subtracted } \\
\left(\mathrm{ft}^{3} / \mathrm{s}\right)\end{array}$ & $\begin{array}{l}\text { Observed } \\
\text { precipitation } \\
\text { (inches) }\end{array}$ & Urban fraction & $\begin{array}{l}\text { Exceedance } \\
\text { probability }\end{array}$ & $\begin{array}{c}\text { Urban-adjusted } \\
\text { annual maximum } \\
\text { peak discharge } \\
\left(\mathrm{ft}^{3} / \mathrm{s}\right)\end{array}$ \\
\hline 04087250 & 1 & Yes & Yes & 1961 & 65 & -- & 290 & 0.411 & 0.0764 & 0.652 & 99 \\
\hline 04087250 & 1 & Yes & Yes & 1962 & 85 & -- & 379 & 0.00336 & 0.0787 & 0.468 & 125 \\
\hline 04087250 & 1 & Yes & Yes & 1963 & 43 & -- & 192 & 1.54 & 0.081 & 0.838 & 70.2 \\
\hline 04087250 & 1 & Yes & Yes & 1964 & 20 & -- & 89 & 1.89 & 0.0834 & 0.961 & 38.1 \\
\hline 04087250 & 1 & Yes & Yes & 1965 & 55 & -- & 245 & 0.101 & 0.0857 & 0.749 & 85.5 \\
\hline 04087250 & 1 & Yes & Yes & 1966 & 210 & -- & 936 & 1.2 & 0.0881 & 0.0501 & 280 \\
\hline 04087250 & 1 & Yes & Yes & 1967 & 42 & -- & 187 & 1.36 & 0.0904 & 0.849 & 68.1 \\
\hline 04087250 & 1 & Yes & Yes & 1968 & 40 & -- & 178 & 1.34 & 0.0928 & 0.865 & 65.4 \\
\hline 04087250 & 1 & Yes & Yes & 1969 & 85 & -- & 379 & 1.05 & 0.0951 & 0.481 & 123 \\
\hline 04087250 & 1 & Yes & Yes & 1970 & 75 & -- & 334 & 1.52 & 0.0974 & 0.579 & 110 \\
\hline 04087250 & 1 & Yes & Yes & 1971 & 85 & -- & 379 & 0.497 & 0.101 & 0.486 & 122 \\
\hline 04087250 & 1 & Yes & Yes & 1972 & 110 & -- & 490 & 1.1 & 0.104 & 0.312 & 153 \\
\hline 04087250 & 1 & Yes & Yes & 1973 & 90 & -- & 401 & 1.55 & 0.108 & 0.45 & 128 \\
\hline 04087250 & 1 & Yes & Yes & 1974 & 95 & -- & 423 & 0.536 & 0.111 & 0.413 & 134 \\
\hline 04087250 & 1 & Yes & Yes & 1975 & 40 & -- & 178 & 0.855 & 0.114 & 0.875 & 63.8 \\
\hline 04087250 & 1 & Yes & Yes & 1976 & 180 & -- & 802 & 1.2 & 0.118 & 0.0841 & 237 \\
\hline 04087250 & 1 & Yes & Yes & 1977 & 30 & -- & 134 & 0.93 & 0.121 & 0.93 & 50.3 \\
\hline 04087250 & 1 & Yes & Yes & 1978 & 220 & -- & 981 & 0.949 & 0.124 & 0.048 & 284 \\
\hline 04087250 & 1 & Yes & Yes & 1979 & 145 & -- & 646 & 0.319 & 0.128 & 0.169 & 191 \\
\hline 04087250 & 1 & Yes & Yes & 1980 & 50 & -- & 223 & 0.601 & 0.131 & 0.811 & 75.1 \\
\hline 04087250 & 1 & Yes & Yes & 1981 & 40 & -- & 178 & 0.749 & 0.137 & 0.882 & 62 \\
\hline 04087250 & 1 & Yes & Yes & 1982 & 120 & -- & 535 & 0.112 & 0.143 & 0.28 & 159 \\
\hline 04087250 & 1 & Yes & Yes & 1983 & 140 & -- & 624 & 1.44 & 0.149 & 0.192 & 181 \\
\hline 04087250 & 1 & Yes & Yes & 1984 & 65 & -- & 290 & 0.474 & 0.155 & 0.707 & 91.3 \\
\hline 04087250 & 1 & Yes & Yes & 1985 & 100 & -- & 446 & 0.715 & 0.162 & 0.416 & 133 \\
\hline 04087250 & 1 & Yes & Yes & 1986 & 170 & -- & 758 & 0.869 & 0.168 & 0.118 & 215 \\
\hline 04087250 & 1 & Yes & Yes & 1987 & 85 & -- & 379 & 0.794 & 0.174 & 0.549 & 114 \\
\hline 04087250 & 1 & Yes & Yes & 1988 & 90 & -- & 401 & 0.00985 & 0.18 & 0.51 & 119 \\
\hline 04087250 & 1 & Yes & Yes & 1989 & 20 & -- & 89 & 0.619 & 0.186 & 0.975 & 32.3 \\
\hline 04087250 & 1 & Yes & Yes & 1990 & 100 & -- & 446 & 1.24 & 0.192 & 0.441 & 129 \\
\hline 04087250 & 1 & Yes & Yes & 1991 & 100 & -- & 446 & 0.985 & 0.211 & 0.457 & 127 \\
\hline 04087250 & 1 & Yes & Yes & 1992 & 60 & -- & 267 & 0.702 & 0.23 & 0.791 & 78.2 \\
\hline 04087250 & 1 & Yes & Yes & 1993 & 140 & -- & 624 & 0.84 & 0.249 & 0.247 & 166 \\
\hline 04087250 & 1 & Yes & Yes & 1994 & 100 & 2 & 446 & 0.647 & 0.268 & 0.505 & 120 \\
\hline 04087250 & 1 & Yes & Yes & 1995 & 128 & -- & 571 & 0.996 & 0.287 & 0.334 & 148 \\
\hline
\end{tabular}




\begin{tabular}{|c|c|c|c|c|c|c|c|c|c|c|c|}
\hline $\begin{array}{c}\text { U.S. } \\
\text { Geological } \\
\text { Survey } \\
\text { streamgage } \\
\text { number }\end{array}$ & $\begin{array}{l}\text { Segment } \\
\text { number }\end{array}$ & $\begin{array}{l}\text { Streamgage used in } \\
\text { regression analyses } \\
\text { (non-redundant) }\end{array}$ & $\begin{array}{l}\text { Discharge value } \\
\text { used in } \\
\text { adjustment } \\
\text { regression }\end{array}$ & $\begin{array}{l}\text { Water } \\
\text { year }\end{array}$ & $\begin{array}{l}\text { Observed annual } \\
\text { maximum peak } \\
\text { discharge }\left(\mathrm{ft}^{3} / \mathrm{s}\right)\end{array}$ & $\begin{array}{l}\text { NWIS peak } \\
\text { code }\end{array}$ & $\begin{array}{l}\text { Observed annual } \\
\text { maximum peak } \\
\text { discharge with } \\
\text { segment intercept } \\
\text { value subtracted } \\
\left(\mathrm{ft}^{3} / \mathrm{s}\right)\end{array}$ & $\begin{array}{l}\text { Observed } \\
\text { precipitation } \\
\text { (inches) }\end{array}$ & Urban fraction & $\begin{array}{l}\text { Exceedance } \\
\text { probability }\end{array}$ & $\begin{array}{c}\text { Urban-adjusted } \\
\text { annual maximum } \\
\text { peak discharge } \\
\left(\mathrm{ft}^{3} / \mathrm{s}\right)\end{array}$ \\
\hline 04087250 & 1 & Yes & Yes & 1996 & 174 & -- & 776 & 0.795 & 0.306 & 0.162 & 195 \\
\hline 04087250 & 1 & Yes & Yes & 1997 & 129 & -- & 575 & 1.73 & 0.325 & 0.357 & 144 \\
\hline 04087250 & 1 & Yes & Yes & 1998 & 121 & -- & 539 & 1.37 & 0.343 & 0.422 & 133 \\
\hline 04087250 & 1 & Yes & Yes & 1999 & 174 & -- & 776 & 1.14 & 0.362 & 0.185 & 185 \\
\hline 04087250 & 1 & Yes & Yes & 2000 & 235 & -- & 1047 & 3.84 & 0.381 & 0.0747 & 245 \\
\hline 04087250 & 1 & Yes & Yes & 2001 & 118 & -- & 526 & 0.62 & 0.386 & 0.479 & 124 \\
\hline 04087250 & 1 & Yes & Yes & 2002 & 111 & -- & 495 & 2 & 0.391 & 0.533 & 116 \\
\hline 04087250 & 1 & Yes & Yes & 2003 & 33 & -- & 147 & 0.33 & 0.395 & 0.968 & 35.1 \\
\hline 04087250 & 1 & Yes & Yes & 2004 & 127 & 2 & 566 & 1.99 & 0.4 & 0.433 & 131 \\
\hline 04087250 & 1 & Yes & Yes & 2005 & 78 & -- & 348 & 0.56 & 0.404 & 0.778 & 80.4 \\
\hline 04087250 & 1 & Yes & Yes & 2006 & 104 & -- & 464 & 1.05 & 0.409 & 0.603 & 106 \\
\hline 04087250 & 1 & Yes & Yes & 2007 & 190 & -- & 847 & 2.73 & 0.414 & 0.168 & 192 \\
\hline 04087250 & 1 & Yes & Yes & 2008 & 156 & -- & 695 & 2.31 & 0.418 & 0.285 & 157 \\
\hline 04087250 & 1 & Yes & Yes & 2009 & 179 & -- & 798 & 0.95 & 0.423 & 0.198 & 180 \\
\hline 04087257 & 1 & Yes & Yes & 1972 & 1200 & -- & 639 & 1.27 & 0.215 & 0.212 & 1520 \\
\hline 04087257 & 1 & Yes & Yes & 1973 & 824 & -- & 439 & 1.55 & 0.221 & 0.477 & 1090 \\
\hline 04087257 & 1 & Yes & Yes & 1974 & 1240 & -- & 661 & 0.554 & 0.228 & 0.2 & 1550 \\
\hline 04087257 & 1 & Yes & Yes & 1975 & 862 & -- & 459 & 1.04 & 0.235 & 0.454 & 1120 \\
\hline 04087257 & 1 & Yes & Yes & 1976 & 1480 & -- & 789 & 1.34 & 0.242 & 0.13 & 1820 \\
\hline 04087257 & 1 & Yes & Yes & 1977 & 200 & -- & 107 & 0.983 & 0.249 & 0.971 & 307 \\
\hline 04087257 & 1 & Yes & Yes & 1978 & 1340 & -- & 714 & 1.25 & 0.256 & 0.178 & 1630 \\
\hline 04087257 & 1 & Yes & Yes & 1979 & 1100 & -- & 586 & 1.24 & 0.263 & 0.298 & 1350 \\
\hline 04087257 & 1 & Yes & Yes & 1980 & 443 & -- & 236 & 0.789 & 0.27 & 0.858 & 592 \\
\hline 04087257 & 1 & Yes & Yes & 1981 & 820 & -- & 437 & 0.846 & 0.274 & 0.527 & 1020 \\
\hline 04087257 & 1 & Yes & Yes & 1982 & 674 & -- & 359 & 1.75 & 0.278 & 0.67 & 850 \\
\hline 04087257 & 1 & Yes & Yes & 1983 & 959 & -- & 511 & 1.39 & 0.282 & 0.412 & 1170 \\
\hline 04087257 & 1 & Yes & Yes & 1984 & 695 & -- & 370 & 0.505 & 0.286 & 0.656 & 866 \\
\hline 04087257 & 1 & Yes & Yes & 1985 & 588 & -- & 313 & 1.02 & 0.29 & 0.757 & 742 \\
\hline 04087257 & 1 & Yes & Yes & 1986 & 1090 & -- & 581 & 0.163 & 0.294 & 0.327 & 1300 \\
\hline 04087257 & 1 & Yes & Yes & 1987 & 836 & -- & 445 & 0.765 & 0.298 & 0.533 & 1020 \\
\hline 04087257 & 1 & Yes & Yes & 1988 & 915 & -- & 488 & 0.93 & 0.302 & 0.465 & 1100 \\
\hline 04087257 & 1 & Yes & Yes & 1989 & 601 & -- & 320 & 1.71 & 0.306 & 0.756 & 743 \\
\hline 04087257 & 1 & Yes & Yes & 1990 & 1040 & -- & 554 & 1.23 & 0.31 & 0.374 & 1230 \\
\hline 04087257 & 1 & Yes & Yes & 1991 & 801 & -- & 427 & 0.974 & 0.321 & 0.589 & 951 \\
\hline 04087257 & 1 & Yes & Yes & 1992 & 791 & -- & 421 & 0.714 & 0.333 & 0.608 & 928 \\
\hline
\end{tabular}




\begin{tabular}{|c|c|c|c|c|c|c|c|c|c|c|c|}
\hline $\begin{array}{c}\text { U.S. } \\
\text { Geological } \\
\text { Survey } \\
\text { streamgage } \\
\text { number }\end{array}$ & $\begin{array}{c}\text { Segment } \\
\text { number }\end{array}$ & $\begin{array}{l}\text { Streamgage used in } \\
\text { regression analyses } \\
\text { (non-redundant) }\end{array}$ & $\begin{array}{c}\text { Discharge value } \\
\text { used in } \\
\text { adjustment } \\
\text { regression }\end{array}$ & $\begin{array}{l}\text { Water } \\
\text { year }\end{array}$ & $\begin{array}{c}\text { Observed annual } \\
\text { maximum peak } \\
\text { discharge }\left(\mathrm{ft}^{3} / \mathrm{s}\right)\end{array}$ & $\begin{array}{l}\text { NWIS peak } \\
\text { code }\end{array}$ & $\begin{array}{c}\text { Observed annual } \\
\text { maximum peak } \\
\text { discharge with } \\
\text { segment intercept } \\
\text { value subtracted } \\
\left(\mathrm{ft}^{3} / \mathrm{s}\right)\end{array}$ & $\begin{array}{c}\text { Observed } \\
\text { precipitation } \\
\text { (inches) }\end{array}$ & Urban fraction & $\begin{array}{l}\text { Exceedance } \\
\text { probability }\end{array}$ & $\begin{array}{c}\text { Urban-adjusted } \\
\text { annual maximum } \\
\text { peak discharge } \\
\left(\mathrm{ft}^{3} / \mathrm{s}\right)\end{array}$ \\
\hline 04087257 & 1 & Yes & Yes & 1993 & 1140 & -- & 607 & 0.947 & 0.344 & 0.331 & 1300 \\
\hline 04087257 & 1 & Yes & Yes & 1994 & 940 & 9 & 501 & 0.705 & 0.356 & 0.491 & 1070 \\
\hline 04087257 & 1 & Yes & Yes & 1995 & 878 & -- & 468 & 1.11 & 0.367 & 0.557 & 987 \\
\hline 04087257 & 1 & Yes & Yes & 1996 & 1360 & -- & 725 & 0.912 & 0.378 & 0.231 & 1480 \\
\hline 04087257 & 1 & Yes & Yes & 1997 & 944 & -- & 503 & 1.71 & 0.39 & 0.519 & 1030 \\
\hline 04087257 & 1 & Yes & Yes & 1998 & 959 & -- & 511 & 1.26 & 0.401 & 0.517 & 1040 \\
\hline 04087257 & 1 & Yes & Yes & 1999 & 1230 & -- & 655 & 1.3 & 0.412 & 0.326 & 1300 \\
\hline 04087257 & 1 & Yes & Yes & 2000 & 1580 & -- & 842 & 2.8 & 0.424 & 0.175 & 1650 \\
\hline 04087257 & 1 & Yes & Yes & 2001 & 1360 & -- & 725 & 1.37 & 0.428 & 0.263 & 1420 \\
\hline 04087257 & 1 & Yes & Yes & 2002 & 1010 & -- & 538 & 1.85 & 0.433 & 0.504 & 1050 \\
\hline 04087257 & 1 & Yes & Yes & 2003 & 321 & -- & 171 & 0.443 & 0.437 & 0.962 & 341 \\
\hline 04087257 & 1 & Yes & Yes & 2004 & 1650 & -- & 879 & 1.83 & 0.442 & 0.163 & 1690 \\
\hline 04087257 & 1 & Yes & Yes & 2005 & 614 & -- & 327 & 0.518 & 0.447 & 0.831 & 634 \\
\hline 04087257 & 1 & Yes & Yes & 2006 & 555 & -- & 296 & 1.01 & 0.451 & 0.873 & 570 \\
\hline 04087257 & 1 & Yes & Yes & 2007 & 1720 & -- & 916 & 3.08 & 0.456 & 0.151 & 1740 \\
\hline 04087257 & 1 & Yes & Yes & 2008 & 1960 & -- & 1044 & 2.26 & 0.46 & 0.0964 & 1980 \\
\hline 04087257 & 1 & Yes & Yes & 2009 & 1860 & -- & 991 & 0.842 & 0.465 & 0.12 & 1870 \\
\hline 04087300 & 1 & Yes & Yes & 1956 & 78 & -- & 431 & 0.0783 & 0.179 & 0.454 & 187 \\
\hline 04087300 & 1 & Yes & Yes & 1957 & 51 & -- & 282 & 0.661 & 0.195 & 0.749 & 139 \\
\hline 04087300 & 1 & Yes & Yes & 1958 & 55 & -- & 304 & 0.00428 & 0.212 & 0.719 & 143 \\
\hline 04087300 & 1 & Yes & Yes & 1959 & 76 & -- & 420 & 1.29 & 0.228 & 0.516 & 176 \\
\hline 04087300 & 1 & Yes & Yes & 1960 & 145 & -- & 802 & 0.93 & 0.245 & 0.124 & 281 \\
\hline 04087300 & 1 & Yes & Yes & 1961 & 42 & -- & 232 & 0.877 & 0.257 & 0.857 & 118 \\
\hline 04087300 & 1 & Yes & Yes & 1962 & 94 & -- & 520 & 0.263 & 0.27 & 0.389 & 199 \\
\hline 04087300 & 1 & Yes & Yes & 1963 & 30 & -- & 166 & 2.39 & 0.282 & 0.937 & 98.3 \\
\hline 04087300 & 1 & Yes & Yes & 1964 & 33 & -- & 182 & 2.01 & 0.295 & 0.928 & 103 \\
\hline 04087300 & 1 & Yes & Yes & 1965 & 80 & -- & 442 & 0.498 & 0.307 & 0.547 & 171 \\
\hline 04087300 & 1 & Yes & Yes & 1966 & 95 & -- & 525 & 0.867 & 0.32 & 0.422 & 193 \\
\hline 04087300 & 1 & Yes & Yes & 1967 & 51 & -- & 282 & 0.675 & 0.332 & 0.827 & 124 \\
\hline 04087300 & 1 & Yes & Yes & 1968 & 39 & -- & 216 & 1.33 & 0.345 & 0.913 & 107 \\
\hline 04087300 & 1 & Yes & Yes & 1969 & 355 & -- & 1963 & 1.63 & 0.357 & 0.0113 & 554 \\
\hline 04087300 & 1 & Yes & Yes & 1970 & 145 & -- & 802 & 1.25 & 0.37 & 0.174 & 253 \\
\hline 04087300 & 1 & Yes & Yes & 1971 & 134 & -- & 741 & 1.23 & 0.387 & 0.222 & 233 \\
\hline 04087300 & 1 & Yes & Yes & 1972 & 282 & -- & 1560 & 1.67 & 0.405 & 0.0218 & 436 \\
\hline 04087400 & 1 & Yes & Yes & 1962 & 121 & $\begin{array}{ll}- \\
\end{array}$ & 254 & 0.628 & 0.306 & 0.85 & 315 \\
\hline
\end{tabular}




\begin{tabular}{|c|c|c|c|c|c|c|c|c|c|c|c|}
\hline $\begin{array}{c}\text { U.S. } \\
\text { Geological } \\
\text { Survey } \\
\text { streamgage } \\
\text { number } \\
\end{array}$ & $\begin{array}{c}\text { Segment } \\
\text { number }\end{array}$ & $\begin{array}{l}\text { Streamgage used in } \\
\text { regression analyses } \\
\text { (non-redundant) }\end{array}$ & $\begin{array}{c}\text { Discharge value } \\
\text { used in } \\
\text { adjustment } \\
\text { regression }\end{array}$ & $\begin{array}{c}\text { Water } \\
\text { year }\end{array}$ & $\begin{array}{c}\text { Observed annual } \\
\text { maximum peak } \\
\text { discharge }\left(\mathrm{ft}^{3} / \mathrm{s}\right)\end{array}$ & $\begin{array}{c}\text { NWIS peak } \\
\text { code }\end{array}$ & $\begin{array}{l}\text { Observed annual } \\
\text { maximum peak } \\
\text { discharge with } \\
\text { segment intercept } \\
\text { value subtracted } \\
\left(\mathrm{ft}^{3} / \mathrm{s}\right)\end{array}$ & $\begin{array}{l}\text { Observed } \\
\text { precipitation } \\
\text { (inches) }\end{array}$ & Urban fraction & $\begin{array}{l}\text { Exceedance } \\
\text { probability }\end{array}$ & $\begin{array}{c}\text { Urban-adjusted } \\
\text { annual maximum } \\
\text { peak discharge } \\
\left(\mathrm{ft}^{3} / \mathrm{s}\right)\end{array}$ \\
\hline 04087400 & 1 & Yes & Yes & 1963 & 125 & -- & 263 & 0.36 & 0.319 & 0.845 & 318 \\
\hline 04087400 & 1 & Yes & Yes & 1964 & 122 & -- & 257 & 1.83 & 0.332 & 0.861 & 310 \\
\hline 04087400 & 1 & Yes & Yes & 1965 & 206 & -- & 433 & 0.555 & 0.346 & 0.599 & 428 \\
\hline 04087400 & 1 & Yes & Yes & 1966 & 288 & -- & 606 & 0.468 & 0.359 & 0.345 & 545 \\
\hline 04087400 & 1 & Yes & Yes & 1967 & 265 & -- & 557 & 1.29 & 0.372 & 0.42 & 510 \\
\hline 04087400 & 1 & Yes & Yes & 1968 & 119 & -- & 250 & 0.991 & 0.385 & 0.892 & 294 \\
\hline 04087400 & 1 & Yes & Yes & 1969 & 937 & -- & 1971 & 1.08 & 0.398 & 0.0122 & 1430 \\
\hline 04087400 & 1 & Yes & Yes & 1970 & 224 & -- & 471 & 1.04 & 0.411 & 0.593 & 430 \\
\hline 04087400 & 1 & Yes & Yes & 1971 & 388 & -- & 816 & 0.428 & 0.425 & 0.189 & 646 \\
\hline 04087400 & 1 & Yes & Yes & 1972 & 601 & -- & 1264 & 1.16 & 0.439 & 0.0483 & 943 \\
\hline 04087400 & 1 & Yes & Yes & 1973 & 114 & -- & 240 & 1.41 & 0.453 & 0.927 & 272 \\
\hline 04087400 & 1 & Yes & Yes & 1974 & 285 & -- & 599 & 1.07 & 0.467 & 0.446 & 497 \\
\hline 04087400 & 1 & Yes & Yes & 1975 & 136 & -- & 286 & 0.319 & 0.481 & 0.897 & 292 \\
\hline 04087400 & 1 & Yes & Yes & 1976 & 471 & -- & 991 & 1.26 & 0.495 & 0.132 & 729 \\
\hline 04093000 & 1 & Yes & Yes & 1947 & 2410 & -- & 773 & 2.51 & 0.118 & 0.0942 & 3330 \\
\hline 04093000 & 1 & Yes & Yes & 1948 & 2740 & -- & 879 & 1.48 & 0.124 & 0.0662 & 3730 \\
\hline 04093000 & 1 & Yes & Yes & 1949 & 620 & -- & 199 & 0.975 & 0.131 & 0.849 & 1020 \\
\hline 04093000 & 1 & Yes & Yes & 1950 & 2390 & -- & 766 & 1.47 & 0.137 & 0.102 & 3250 \\
\hline 04093000 & 1 & Yes & Yes & 1951 & 1440 & -- & 462 & 1.91 & 0.145 & 0.379 & 2050 \\
\hline 04093000 & 1 & Yes & Yes & 1952 & 1340 & -- & 430 & 1.78 & 0.154 & 0.437 & 1910 \\
\hline 04093000 & 1 & Yes & Yes & 1953 & 912 & -- & 292 & 0.47 & 0.162 & 0.707 & 1350 \\
\hline 04093000 & 1 & Yes & Yes & 1954 & 1440 & -- & 462 & 1.79 & 0.17 & 0.397 & 2010 \\
\hline 04093000 & 1 & Yes & Yes & 1955 & 3880 & -- & 1244 & 3.65 & 0.178 & 0.0258 & 4910 \\
\hline 04093000 & 1 & Yes & Yes & 1956 & 1320 & -- & 423 & 1.03 & 0.186 & 0.475 & 1830 \\
\hline 04093000 & 1 & Yes & Yes & 1957 & 1650 & -- & 529 & 3.16 & 0.195 & 0.322 & 2210 \\
\hline 04093000 & 1 & Yes & Yes & 1958 & 720 & -- & 231 & 1.17 & 0.203 & 0.834 & 1060 \\
\hline 04093000 & 1 & Yes & Yes & 1959 & 1970 & -- & 632 & 2.15 & 0.211 & 0.217 & 2540 \\
\hline 04093000 & 1 & Yes & Yes & 1960 & 1260 & -- & 404 & 1.22 & 0.219 & 0.539 & 1690 \\
\hline 04093000 & 1 & Yes & Yes & 1961 & 1200 & -- & 385 & 1.73 & 0.227 & 0.585 & 1610 \\
\hline 04093000 & 1 & Yes & Yes & 1962 & 900 & -- & 289 & 0.31 & 0.234 & 0.761 & 1240 \\
\hline 04093000 & 1 & Yes & Yes & 1963 & 560 & 2 & 180 & 0.45 & 0.242 & 0.916 & 823 \\
\hline 04093000 & 1 & Yes & Yes & 1964 & 393 & -- & 126 & 0.588 & 0.249 & 0.959 & 602 \\
\hline 04093000 & 1 & Yes & Yes & 1965 & 1080 & -- & 346 & 0.958 & 0.257 & 0.676 & 1420 \\
\hline 04093000 & 1 & Yes & Yes & 1966 & 2400 & -- & 770 & 2.13 & 0.264 & 0.149 & 2920 \\
\hline 04093000 & 1 & Yes & Yes & 1967 & 1120 & -- & 359 & 1 & 0.272 & 0.665 & 1440 \\
\hline
\end{tabular}




\begin{tabular}{|c|c|c|c|c|c|c|c|c|c|c|c|}
\hline $\begin{array}{c}\text { U.S. } \\
\text { Geological } \\
\text { Survey } \\
\text { streamgage } \\
\text { number } \\
\end{array}$ & $\begin{array}{c}\text { Segment } \\
\text { number }\end{array}$ & $\begin{array}{l}\text { Streamgage used in } \\
\text { regression analyses } \\
\text { (non-redundant) }\end{array}$ & $\begin{array}{c}\text { Discharge value } \\
\text { used in } \\
\text { adjustment } \\
\text { regression }\end{array}$ & $\begin{array}{c}\text { Water } \\
\text { year }\end{array}$ & $\begin{array}{c}\text { Observed annual } \\
\text { maximum peak } \\
\text { discharge }\left(\mathrm{ft}^{3} / \mathrm{s}\right)\end{array}$ & $\begin{array}{c}\text { NWIS peak } \\
\text { code }\end{array}$ & $\begin{array}{c}\text { Observed annual } \\
\text { maximum peak } \\
\text { discharge with } \\
\text { segment intercept } \\
\text { value subtracted } \\
\left(\mathrm{ft}^{3} / \mathrm{s}\right)\end{array}$ & $\begin{array}{l}\text { Observed } \\
\text { precipitation } \\
\text { (inches) }\end{array}$ & Urban fraction & $\begin{array}{l}\text { Exceedance } \\
\text { probability }\end{array}$ & $\begin{array}{c}\text { Urban-adjusted } \\
\text { annual maximum } \\
\text { peak discharge } \\
\left(\mathrm{ft}^{3} / \mathrm{s}\right)\end{array}$ \\
\hline 04093000 & 1 & Yes & Yes & 1968 & 1990 & -- & 638 & 0.949 & 0.279 & 0.251 & 2410 \\
\hline 04093000 & 1 & Yes & Yes & 1969 & 1050 & -- & 337 & 0.593 & 0.287 & 0.716 & 1340 \\
\hline 04093000 & 1 & Yes & Yes & 1970 & 2900 & 2 & 930 & 1.61 & 0.294 & 0.0867 & 3420 \\
\hline 04093000 & 1 & Yes & Yes & 1971 & 742 & -- & 238 & 0.495 & 0.302 & 0.872 & 964 \\
\hline 04093000 & 1 & Yes & Yes & 1972 & 973 & -- & 312 & 0.745 & 0.311 & 0.771 & 1210 \\
\hline 04093000 & 1 & Yes & Yes & 1973 & 1840 & -- & 590 & 0.831 & 0.319 & 0.334 & 2170 \\
\hline 04093000 & 1 & Yes & Yes & 1974 & 1100 & -- & 353 & 0.72 & 0.327 & 0.719 & 1330 \\
\hline 04093000 & 1 & Yes & Yes & 1975 & 1900 & -- & 609 & 1.38 & 0.336 & 0.323 & 2200 \\
\hline 04093000 & 1 & Yes & Yes & 1976 & 2020 & -- & 648 & 0.978 & 0.344 & 0.284 & 2310 \\
\hline 04093000 & 1 & Yes & Yes & 1977 & 575 & -- & 184 & 1.13 & 0.352 & 0.939 & 716 \\
\hline 04093000 & 1 & Yes & Yes & 1978 & 1320 & -- & 423 & 0.88 & 0.361 & 0.627 & 1520 \\
\hline 04093000 & 1 & Yes & Yes & 1979 & 2540 & -- & 814 & 0.561 & 0.369 & 0.167 & 2810 \\
\hline 04093000 & 1 & Yes & Yes & 1980 & 1060 & -- & 340 & 0.283 & 0.377 & 0.772 & 1210 \\
\hline 04093000 & 1 & Yes & Yes & 1981 & 4000 & -- & 1283 & 2.29 & 0.381 & 0.0405 & 4340 \\
\hline 04093000 & 1 & Yes & Yes & 1982 & 2600 & -- & 834 & 0.458 & 0.385 & 0.163 & 2840 \\
\hline 04093000 & 1 & Yes & Yes & 1983 & 3710 & -- & 1190 & 2.81 & 0.388 & 0.0512 & 4010 \\
\hline 04093000 & 1 & Yes & Yes & 1984 & 1090 & -- & 349 & 0.968 & 0.392 & 0.768 & 1220 \\
\hline 04093000 & 1 & Yes & Yes & 1985 & 2870 & -- & 920 & 1.13 & 0.396 & 0.124 & 3100 \\
\hline 04093000 & 1 & Yes & Yes & 1986 & 2780 & -- & 891 & 1.41 & 0.399 & 0.14 & 2990 \\
\hline 04093000 & 1 & Yes & Yes & 1987 & 1360 & -- & 436 & 1.41 & 0.403 & 0.641 & 1490 \\
\hline 04093000 & 1 & Yes & Yes & 1988 & 944 & -- & 303 & 0.861 & 0.407 & 0.839 & 1050 \\
\hline 04093000 & 1 & Yes & Yes & 1989 & 3530 & -- & 1132 & 2.66 & 0.41 & 0.0645 & 3750 \\
\hline 04093000 & 1 & Yes & Yes & 1990 & 2450 & -- & 786 & 2.04 & 0.414 & 0.201 & 2600 \\
\hline 04093000 & 1 & Yes & Yes & 1991 & 4230 & -- & 1356 & 2.32 & 0.419 & 0.0375 & 4440 \\
\hline 04093000 & 1 & Yes & Yes & 1992 & 919 & -- & 295 & 0.532 & 0.425 & 0.859 & 994 \\
\hline 04093000 & 1 & Yes & Yes & 1993 & 2070 & -- & 664 & 2.09 & 0.431 & 0.331 & 2180 \\
\hline 04093000 & 1 & Yes & Yes & 1994 & 1630 & -- & 523 & 2.68 & 0.436 & 0.531 & 1710 \\
\hline 04093000 & 1 & Yes & Yes & 1995 & 1230 & -- & 394 & 0.883 & 0.442 & 0.738 & 1290 \\
\hline 04093000 & 1 & Yes & Yes & 1996 & 2080 & -- & 667 & 4.01 & 0.447 & 0.341 & 2150 \\
\hline 04093000 & 1 & Yes & Yes & 1997 & 2060 & -- & 660 & 1.82 & 0.453 & 0.354 & 2120 \\
\hline 04093000 & 1 & Yes & Yes & 1998 & 1850 & -- & 593 & 0.552 & 0.459 & 0.447 & 1890 \\
\hline 04093000 & 1 & Yes & Yes & 1999 & 1690 & -- & 542 & 0.709 & 0.464 & 0.527 & 1720 \\
\hline 04093000 & 1 & Yes & Yes & 2000 & 1260 & -- & 404 & 1.29 & 0.47 & 0.745 & 1280 \\
\hline 04093000 & 1 & Yes & Yes & 2001 & 1460 & -- & 468 & 0.48 & 0.471 & 0.648 & 1470 \\
\hline 04093000 & 1 & Yes & Yes & 2002 & 2310 & -- & 741 & 1.71 & 0.472 & 0.277 & 2330 \\
\hline
\end{tabular}




\begin{tabular}{|c|c|c|c|c|c|c|c|c|c|c|c|}
\hline $\begin{array}{c}\text { U.S. } \\
\text { Geological } \\
\text { Survey } \\
\text { streamgage } \\
\text { number }\end{array}$ & $\begin{array}{l}\text { Segment } \\
\text { number }\end{array}$ & $\begin{array}{l}\text { Streamgage used in } \\
\text { regression analyses } \\
\text { (non-redundant) }\end{array}$ & $\begin{array}{c}\text { Discharge value } \\
\text { used in } \\
\text { adjustment } \\
\text { regression }\end{array}$ & $\begin{array}{c}\text { Water } \\
\text { year }\end{array}$ & $\begin{array}{c}\text { Observed annual } \\
\text { maximum peak } \\
\text { discharge }\left(\mathrm{ft}^{3} / \mathrm{s}\right)\end{array}$ & $\begin{array}{l}\text { NWIS peak } \\
\text { code }\end{array}$ & $\begin{array}{c}\text { Observed annual } \\
\text { maximum peak } \\
\text { discharge with } \\
\text { segment intercept } \\
\text { value subtracted } \\
\left(\mathrm{ft}^{3} / \mathrm{s}\right)\end{array}$ & $\begin{array}{c}\text { Observed } \\
\text { precipitation } \\
\text { (inches) }\end{array}$ & Urban fraction & $\begin{array}{l}\text { Exceedance } \\
\text { probability }\end{array}$ & $\begin{array}{c}\text { Urban-adjusted } \\
\text { annual maximum } \\
\text { peak discharge } \\
\left(\mathrm{ft}^{3} / \mathrm{s}\right)\end{array}$ \\
\hline 04093000 & 1 & Yes & Yes & 2003 & 1700 & -- & 545 & 2.43 & 0.473 & 0.53 & 1710 \\
\hline 04093000 & 1 & Yes & Yes & 2004 & 1160 & -- & 372 & 1.2 & 0.474 & 0.79 & 1170 \\
\hline 04093000 & 1 & Yes & Yes & 2005 & 2210 & -- & 709 & 1.17 & 0.474 & 0.313 & 2220 \\
\hline 04093000 & 1 & Yes & Yes & 2006 & 2240 & -- & 718 & 1.34 & 0.475 & 0.303 & 2250 \\
\hline 04093000 & 1 & Yes & Yes & 2007 & 3390 & -- & 1087 & 2.71 & 0.476 & 0.0886 & 3400 \\
\hline 04093000 & 1 & Yes & Yes & 2008 & 5280 & -- & 1693 & 3.72 & 0.477 & 0.0198 & 5290 \\
\hline 04093000 & 1 & Yes & Yes & 2009 & 2060 & -- & 660 & 1.17 & 0.478 & 0.375 & 2060 \\
\hline 04094000 & 1 & Yes & Yes & 1945 & 2440 & -- & 798 & 1.04 & 0.0267 & 0.0682 & 3160 \\
\hline 04094000 & 1 & Yes & Yes & 1946 & 715 & -- & 234 & 1.22 & 0.0275 & 0.734 & 1040 \\
\hline 04094000 & 1 & Yes & Yes & 1947 & 2140 & -- & 700 & 2.05 & 0.0282 & 0.0988 & 2790 \\
\hline 04094000 & 1 & Yes & Yes & 1948 & 1960 & -- & 641 & 1.59 & 0.029 & 0.134 & 2560 \\
\hline 04094000 & 1 & Yes & Yes & 1949 & 690 & -- & 226 & 0.869 & 0.0298 & 0.753 & 1010 \\
\hline 04094000 & 1 & Yes & Yes & 1950 & 1720 & -- & 563 & 1.38 & 0.0305 & 0.188 & 2260 \\
\hline 04094000 & 1 & Yes & Yes & 1951 & 1360 & -- & 445 & 1.96 & 0.0323 & 0.327 & 1830 \\
\hline 04094000 & 1 & Yes & Yes & 1952 & 1060 & -- & 347 & 0.821 & 0.0341 & 0.495 & 1470 \\
\hline 04094000 & 1 & Yes & Yes & 1953 & 521 & -- & 170 & 0.731 & 0.036 & 0.854 & 789 \\
\hline 04094000 & 1 & Yes & Yes & 1954 & 1170 & -- & 383 & 1.25 & 0.0378 & 0.428 & 1600 \\
\hline 04094000 & 1 & Yes & Yes & 1955 & 3110 & -- & 1018 & 3.44 & 0.0396 & 0.035 & 3920 \\
\hline 04094000 & 1 & Yes & Yes & 1956 & 1370 & -- & 448 & 1.75 & 0.0414 & 0.328 & 1830 \\
\hline 04094000 & 1 & Yes & Yes & 1957 & 848 & -- & 277 & 0.651 & 0.0432 & 0.654 & 1190 \\
\hline 04094000 & 1 & Yes & Yes & 1958 & 490 & -- & 160 & 0.318 & 0.045 & 0.876 & 742 \\
\hline 04094000 & 1 & Yes & Yes & 1959 & 1420 & -- & 465 & 1.56 & 0.0468 & 0.309 & 1880 \\
\hline 04094000 & 1 & Yes & Yes & 1960 & 1120 & -- & 366 & 1 & 0.0486 & 0.468 & 1520 \\
\hline 04094000 & 1 & Yes & Yes & 1961 & 1020 & -- & 334 & 1.12 & 0.0508 & 0.539 & 1390 \\
\hline 04094000 & 1 & Yes & Yes & 1962 & 710 & -- & 232 & 0.256 & 0.0529 & 0.753 & 1010 \\
\hline 04094000 & 1 & Yes & Yes & 1963 & 460 & -- & 151 & 1.21 & 0.0551 & 0.892 & 695 \\
\hline 04094000 & 1 & Yes & Yes & 1964 & 282 & -- & 92 & 0.607 & 0.0572 & 0.956 & 459 \\
\hline 04094000 & 1 & Yes & Yes & 1965 & 680 & -- & 223 & 0.806 & 0.0593 & 0.774 & 964 \\
\hline 04094000 & 1 & Yes & Yes & 1966 & 1990 & -- & 651 & 1.78 & 0.0615 & 0.14 & 2530 \\
\hline 04094000 & 1 & Yes & Yes & 1967 & 890 & -- & 291 & 1.06 & 0.0636 & 0.64 & 1210 \\
\hline 04094000 & 1 & Yes & Yes & 1968 & 1290 & -- & 422 & 1.15 & 0.0658 & 0.383 & 1700 \\
\hline 04094000 & 1 & Yes & Yes & 1969 & 616 & -- & 202 & 0.72 & 0.0679 & 0.816 & 876 \\
\hline 04094000 & 1 & Yes & Yes & 1970 & 965 & -- & 316 & 1.37 & 0.0701 & 0.598 & 1300 \\
\hline 04094000 & 1 & Yes & Yes & 1971 & 708 & -- & 232 & 0.57 & 0.075 & 0.766 & 981 \\
\hline 04094000 & 1 & Yes & Yes & 1972 & 1300 & -- & 425 & 1.68 & 0.08 & 0.387 & 1690 \\
\hline
\end{tabular}




\begin{tabular}{|c|c|c|c|c|c|c|c|c|c|c|c|}
\hline $\begin{array}{c}\text { U.S. } \\
\text { Geological } \\
\text { Survey } \\
\text { streamgage } \\
\text { number }\end{array}$ & $\begin{array}{l}\text { Segment } \\
\text { number }\end{array}$ & $\begin{array}{l}\text { Streamgage used in } \\
\text { regression analyses } \\
\text { (non-redundant) }\end{array}$ & $\begin{array}{l}\text { Discharge value } \\
\text { used in } \\
\text { adjustment } \\
\text { regression }\end{array}$ & $\begin{array}{l}\text { Water } \\
\text { year }\end{array}$ & $\begin{array}{l}\text { Observed annual } \\
\text { maximum peak } \\
\text { discharge }\left(\mathrm{ft}^{3} / \mathrm{s}\right)\end{array}$ & $\begin{array}{l}\text { NWIS peak } \\
\text { code }\end{array}$ & $\begin{array}{l}\text { Observed annual } \\
\text { maximum peak } \\
\text { discharge with } \\
\text { segment intercept } \\
\text { value subtracted } \\
\left(\mathrm{ft}^{3} / \mathrm{s}\right)\end{array}$ & $\begin{array}{l}\text { Observed } \\
\text { precipitation } \\
\text { (inches) }\end{array}$ & Urban fraction & $\begin{array}{l}\text { Exceedance } \\
\text { probability }\end{array}$ & $\begin{array}{c}\text { Urban-adjusted } \\
\text { annual maximum } \\
\text { peak discharge } \\
\left(\mathrm{ft}^{3} / \mathrm{s}\right)\end{array}$ \\
\hline 04094000 & 1 & Yes & Yes & 1973 & 1400 & -- & 458 & 1.33 & 0.085 & 0.343 & 1790 \\
\hline 04094000 & 1 & Yes & Yes & 1974 & 752 & -- & 246 & 0.767 & 0.09 & 0.749 & 1020 \\
\hline 04094000 & 1 & Yes & Yes & 1975 & 1080 & -- & 353 & 1.34 & 0.095 & 0.533 & 1400 \\
\hline 04094000 & 1 & Yes & Yes & 1976 & 1250 & -- & 409 & 0.953 & 0.0999 & 0.43 & 1600 \\
\hline 04094000 & 1 & Yes & Yes & 1977 & 453 & -- & 148 & 0.773 & 0.105 & 0.91 & 642 \\
\hline 04094000 & 1 & Yes & Yes & 1978 & 986 & -- & 323 & 0.369 & 0.11 & 0.613 & 1270 \\
\hline 04094000 & 1 & Yes & Yes & 1979 & 1680 & -- & 550 & 0.821 & 0.115 & 0.246 & 2050 \\
\hline 04094000 & 1 & Yes & Yes & 1980 & 707 & -- & 231 & 0.361 & 0.12 & 0.79 & 929 \\
\hline 04094000 & 1 & Yes & Yes & 1981 & 2410 & -- & 789 & 2.06 & 0.123 & 0.0901 & 2880 \\
\hline 04094000 & 1 & Yes & Yes & 1982 & 2030 & -- & 664 & 0.341 & 0.127 & 0.157 & 2430 \\
\hline 04094000 & 1 & Yes & Yes & 1983 & 1300 & -- & 425 & 0.714 & 0.13 & 0.425 & 1600 \\
\hline 04094000 & 1 & Yes & Yes & 1984 & 1000 & -- & 327 & 0.621 & 0.134 & 0.621 & 1250 \\
\hline 04094000 & 1 & Yes & Yes & 1985 & 1840 & -- & 602 & 0.833 & 0.137 & 0.203 & 2190 \\
\hline 04094000 & 1 & Yes & Yes & 1986 & 1800 & -- & 589 & 1.13 & 0.141 & 0.219 & 2140 \\
\hline 04094000 & 1 & Yes & Yes & 1987 & 900 & -- & 294 & 1.34 & 0.145 & 0.691 & 1120 \\
\hline 04094000 & 1 & Yes & Yes & 1988 & 883 & -- & 289 & 0.775 & 0.148 & 0.704 & 1100 \\
\hline 04094000 & 1 & Yes & Yes & 1989 & 2270 & -- & 743 & 1.82 & 0.152 & 0.12 & 2650 \\
\hline 04094000 & 1 & Yes & Yes & 1990 & 3220 & -- & 1054 & 2.8 & 0.155 & 0.0421 & 3700 \\
\hline 04094000 & 1 & Yes & Yes & 1991 & 3880 & -- & 1270 & 1.79 & 0.164 & 0.0229 & 4390 \\
\hline 04094000 & 1 & Yes & Yes & 1992 & 631 & $\mathrm{E}$ & 206 & 1.54 & 0.173 & 0.856 & 785 \\
\hline 04094000 & 1 & Yes & Yes & 1993 & 2220 & -- & 726 & 1.9 & 0.181 & 0.141 & 2530 \\
\hline 04094000 & 1 & Yes & Yes & 1994 & 1120 & -- & 366 & 1.97 & 0.19 & 0.59 & 1310 \\
\hline 04094000 & 1 & Yes & Yes & 1995 & 773 & -- & 253 & 0.855 & 0.199 & 0.797 & 915 \\
\hline 04094000 & 1 & Yes & Yes & 1996 & 3400 & -- & 1113 & 1.73 & 0.208 & 0.0407 & 3740 \\
\hline 04094000 & 1 & Yes & Yes & 1997 & 1300 & -- & 425 & 1.6 & 0.217 & 0.496 & 1470 \\
\hline 04094000 & 1 & Yes & Yes & 1998 & 928 & -- & 304 & 0.625 & 0.225 & 0.73 & 1050 \\
\hline 04094000 & 1 & Yes & Yes & 1999 & 896 & -- & 293 & 0.922 & 0.234 & 0.754 & 1010 \\
\hline 04094000 & 1 & Yes & Yes & 2000 & 1310 & -- & 429 & 0.965 & 0.243 & 0.514 & 1430 \\
\hline 04094000 & 1 & Yes & Yes & 2001 & 1200 & -- & 393 & 0.556 & 0.251 & 0.591 & 1310 \\
\hline 04094000 & 1 & Yes & Yes & 2002 & 1130 & -- & 370 & 1.22 & 0.259 & 0.637 & 1220 \\
\hline 04094000 & 1 & Yes & Yes & 2003 & 793 & -- & 259 & 0.927 & 0.267 & 0.824 & 858 \\
\hline 04094000 & 1 & Yes & Yes & 2004 & 740 & -- & 242 & 1.42 & 0.275 & 0.852 & 793 \\
\hline 04094000 & 1 & Yes & Yes & 2005 & 1690 & -- & 553 & 1.21 & 0.283 & 0.355 & 1760 \\
\hline 04094000 & 1 & Yes & Yes & 2006 & 881 & -- & 288 & 1.15 & 0.291 & 0.795 & 920 \\
\hline 04094000 & 1 & Yes & Yes & 2007 & 1370 & -- & 448 & 2.36 & 0.299 & 0.529 & 1410 \\
\hline
\end{tabular}




\begin{tabular}{|c|c|c|c|c|c|c|c|c|c|c|c|}
\hline $\begin{array}{c}\text { U.S. } \\
\text { Geological } \\
\text { Survey } \\
\text { streamgage } \\
\text { number }\end{array}$ & $\begin{array}{l}\text { Segment } \\
\text { number }\end{array}$ & $\begin{array}{l}\text { Streamgage used in } \\
\text { regression analyses } \\
\text { (non-redundant) }\end{array}$ & $\begin{array}{l}\text { Discharge value } \\
\text { used in } \\
\text { adjustment } \\
\text { regression }\end{array}$ & $\begin{array}{c}\text { Water } \\
\text { year }\end{array}$ & $\begin{array}{l}\text { Observed annual } \\
\text { maximum peak } \\
\text { discharge }\left(\mathrm{ft}^{3} / \mathrm{s}\right)\end{array}$ & $\begin{array}{l}\text { NWIS peak } \\
\text { code }\end{array}$ & $\begin{array}{l}\text { Observed annual } \\
\text { maximum peak } \\
\text { discharge with } \\
\text { segment intercept } \\
\text { value subtracted } \\
\left(\mathrm{ft}^{3} / \mathrm{s}\right)\end{array}$ & $\begin{array}{l}\text { Observed } \\
\text { precipitation } \\
\text { (inches) }\end{array}$ & Urban fraction & $\begin{array}{l}\text { Exceedance } \\
\text { probability }\end{array}$ & $\begin{array}{c}\text { Urban-adjusted } \\
\text { annual maximum } \\
\text { peak discharge } \\
\left(\mathrm{ft}^{3} / \mathrm{s}\right)\end{array}$ \\
\hline 04094000 & 1 & Yes & Yes & 2008 & 5320 & -- & 1741 & 3.76 & 0.307 & 0.0141 & 5380 \\
\hline 04094000 & 1 & Yes & Yes & 2009 & 1620 & -- & 530 & 0.632 & 0.316 & 0.412 & 1630 \\
\hline 04094500 & 1 & Yes & Yes & 1945 & 990 & -- & 470 & 1.52 & 0.0733 & 0.319 & 1750 \\
\hline 04094500 & 1 & Yes & Yes & 1946 & 1280 & -- & 608 & 1.98 & 0.0762 & 0.174 & 2140 \\
\hline 04094500 & 1 & Yes & Yes & 1947 & 1580 & -- & 750 & 1.94 & 0.0792 & 0.0928 & 2590 \\
\hline 04094500 & 1 & Yes & Yes & 1948 & 1910 & -- & 907 & 2.3 & 0.0822 & 0.0547 & 3050 \\
\hline 04094500 & 1 & Yes & Yes & 1949 & 525 & -- & 249 & 0.761 & 0.0851 & 0.74 & 1060 \\
\hline 04094500 & 1 & Yes & Yes & 1950 & 1700 & -- & 807 & 1.68 & 0.0881 & 0.0756 & 2740 \\
\hline 04094500 & 1 & Yes & Yes & 1951 & 970 & -- & 460 & 2.39 & 0.0939 & 0.346 & 1690 \\
\hline 04094500 & 1 & Yes & Yes & 1952 & 912 & -- & 433 & 0.642 & 0.0998 & 0.39 & 1610 \\
\hline 04094500 & 1 & Yes & Yes & 1953 & 454 & -- & 216 & 0.469 & 0.106 & 0.81 & 931 \\
\hline 04094500 & 1 & Yes & Yes & 1954 & 910 & -- & 432 & 1.72 & 0.111 & 0.399 & 1590 \\
\hline 04094500 & 1 & Yes & Yes & 1955 & 3180 & -- & 1510 & 4.64 & 0.117 & 0.0139 & 4640 \\
\hline 04094500 & 1 & Yes & Yes & 1956 & 1280 & -- & 608 & 2.2 & 0.123 & 0.193 & 2050 \\
\hline 04094500 & 1 & Yes & Yes & 1957 & 725 & -- & 344 & 0.821 & 0.129 & 0.584 & 1290 \\
\hline 04094500 & 1 & Yes & Yes & 1958 & 456 & -- & 216 & 1.3 & 0.135 & 0.824 & 908 \\
\hline 04094500 & 1 & Yes & Yes & 1959 & 1200 & -- & 570 & 2.21 & 0.141 & 0.239 & 1920 \\
\hline 04094500 & 1 & Yes & Yes & 1960 & 980 & 2 & 465 & 0.826 & 0.146 & 0.375 & 1640 \\
\hline 04094500 & 1 & Yes & Yes & 1961 & 780 & -- & 370 & 1.32 & 0.153 & 0.548 & 1340 \\
\hline 04094500 & 1 & Yes & Yes & 1962 & 578 & -- & 274 & 0.333 & 0.159 & 0.739 & 1060 \\
\hline 04094500 & 1 & Yes & Yes & 1963 & 290 & -- & 138 & 1.26 & 0.166 & 0.936 & 642 \\
\hline 04094500 & 1 & Yes & Yes & 1964 & 290 & -- & 138 & 0.665 & 0.172 & 0.937 & 637 \\
\hline 04094500 & 1 & Yes & Yes & 1965 & 700 & -- & 332 & 1.04 & 0.178 & 0.643 & 1200 \\
\hline 04094500 & 1 & Yes & Yes & 1966 & 1610 & -- & 764 & 2.41 & 0.185 & 0.121 & 2410 \\
\hline 04094500 & 1 & Yes & Yes & 1967 & 805 & -- & 382 & 1.23 & 0.191 & 0.558 & 1330 \\
\hline 04094500 & 1 & Yes & Yes & 1968 & 1780 & -- & 845 & 1.53 & 0.197 & 0.0893 & 2620 \\
\hline 04094500 & 1 & Yes & Yes & 1969 & 578 & -- & 274 & 0.583 & 0.204 & 0.765 & 1010 \\
\hline 04094500 & 1 & Yes & Yes & 1970 & 900 & -- & 427 & 1.9 & 0.21 & 0.488 & 1440 \\
\hline 04094500 & 1 & Yes & No & 1971 & 780 & 1,8 & 370 & 0.538 & 0.225 & 0.611 & 1250 \\
\hline 04094500 & 1 & Yes & Yes & 1972 & 894 & -- & 424 & 2.67 & 0.24 & 0.519 & 1390 \\
\hline 04094500 & 1 & Yes & Yes & 1973 & 1490 & -- & 707 & 1.67 & 0.254 & 0.181 & 2110 \\
\hline 04094500 & 1 & Yes & Yes & 1974 & 989 & -- & 470 & 0.874 & 0.269 & 0.466 & 1480 \\
\hline 04094500 & 1 & Yes & Yes & 1975 & 1240 & -- & 589 & 1.75 & 0.284 & 0.309 & 1760 \\
\hline 04094500 & 1 & Yes & Yes & 1976 & 1590 & -- & 755 & 1.06 & 0.298 & 0.171 & 2160 \\
\hline 04094500 & 1 & Yes & Yes & 1977 & 398 & -- & 189 & 1.12 & 0.313 & 0.928 & 676 \\
\hline
\end{tabular}




\begin{tabular}{|c|c|c|c|c|c|c|c|c|c|c|c|}
\hline $\begin{array}{l}\text { U.S. } \\
\text { Geological } \\
\text { Survey } \\
\text { streamgage } \\
\text { number }\end{array}$ & $\begin{array}{l}\text { Segment } \\
\text { number }\end{array}$ & $\begin{array}{l}\text { Streamgage used in } \\
\text { regression analyses } \\
\text { (non-redundant) }\end{array}$ & $\begin{array}{c}\text { Discharge value } \\
\text { used in } \\
\text { adjustment } \\
\text { regression }\end{array}$ & $\begin{array}{l}\text { Water } \\
\text { year }\end{array}$ & $\begin{array}{l}\text { Observed annual } \\
\text { maximum peak } \\
\text { discharge }\left(\mathrm{ft}^{3} / \mathrm{s}\right)\end{array}$ & $\begin{array}{l}\text { NWIS peak } \\
\text { code }\end{array}$ & $\begin{array}{l}\text { Observed annual } \\
\text { maximum peak } \\
\text { discharge with } \\
\text { segment intercept } \\
\text { value subtracted } \\
\left(\mathrm{ft}^{3} / \mathrm{s}\right)\end{array}$ & $\begin{array}{l}\text { Observed } \\
\text { precipitation } \\
\text { (inches) }\end{array}$ & Urban fraction & $\begin{array}{l}\text { Exceedance } \\
\text { probability }\end{array}$ & $\begin{array}{c}\text { Urban-adjusted } \\
\text { annual maximum } \\
\text { peak discharge } \\
\left(\mathrm{ft}^{3} / \mathrm{s}\right)\end{array}$ \\
\hline 04094500 & 1 & Yes & Yes & 1978 & 606 & -- & 288 & 0.233 & 0.328 & 0.817 & 920 \\
\hline 04094500 & 1 & Yes & Yes & 1979 & 1600 & -- & 760 & 0.836 & 0.342 & 0.186 & 2090 \\
\hline 04094500 & 1 & Yes & Yes & 1980 & 716 & -- & 340 & 0.495 & 0.357 & 0.759 & 1020 \\
\hline 04094500 & 1 & Yes & Yes & 1981 & 2950 & -- & 1400 & 3.82 & 0.364 & 0.0286 & 3640 \\
\hline 04094500 & 1 & Yes & Yes & 1982 & 1200 & 2 & 570 & 0.452 & 0.37 & 0.402 & 1590 \\
\hline 04094500 & 1 & Yes & Yes & 1983 & 3120 & -- & 1481 & 4.78 & 0.377 & 0.0244 & 3800 \\
\hline 04094500 & 1 & Yes & Yes & 1984 & 692 & -- & 329 & 1.51 & 0.383 & 0.791 & 965 \\
\hline 04094500 & 1 & Yes & Yes & 1985 & 1500 & -- & 712 & 1.31 & 0.39 & 0.249 & 1890 \\
\hline 04094500 & 1 & Yes & Yes & 1986 & 1370 & -- & 650 & 2.03 & 0.396 & 0.319 & 1740 \\
\hline 04094500 & 1 & Yes & Yes & 1987 & 681 & -- & 323 & 1.39 & 0.403 & 0.811 & 930 \\
\hline 04094500 & 1 & Yes & Yes & 1988 & 815 & -- & 387 & 1.24 & 0.409 & 0.725 & 1080 \\
\hline 04094500 & 1 & Yes & Yes & 1989 & 2000 & -- & 949 & 2.97 & 0.416 & 0.119 & 2430 \\
\hline 04094500 & 1 & Yes & Yes & 1990 & 1630 & -- & 774 & 3.63 & 0.422 & 0.216 & 1980 \\
\hline 04094500 & 1 & Yes & Yes & 1991 & 2320 & -- & 1101 & 2.86 & 0.43 & 0.0732 & 2770 \\
\hline 05437950 & 1 & Yes & Yes & 1965 & 105 & -- & 293 & 0.0605 & 0.0768 & 0.645 & 174 \\
\hline 05437950 & 1 & Yes & Yes & 1966 & 150 & -- & 419 & 0.512 & 0.0796 & 0.397 & 236 \\
\hline 05437950 & 1 & Yes & Yes & 1967 & 154 & -- & 430 & 0.92 & 0.0824 & 0.382 & 241 \\
\hline 05437950 & 1 & Yes & Yes & 1968 & 89 & -- & 248 & 1.3 & 0.0853 & 0.741 & 152 \\
\hline 05437950 & 1 & Yes & Yes & 1969 & 125 & -- & 349 & 1.37 & 0.0881 & 0.537 & 200 \\
\hline 05437950 & 1 & Yes & Yes & 1970 & 124 & -- & 346 & 1.56 & 0.0909 & 0.546 & 198 \\
\hline 05437950 & 1 & Yes & Yes & 1971 & 139 & -- & 388 & 0.427 & 0.0965 & 0.465 & 218 \\
\hline 05437950 & 1 & Yes & Yes & 1972 & 192 & -- & 536 & 0.696 & 0.102 & 0.254 & 283 \\
\hline 05437950 & 1 & Yes & Yes & 1973 & 136 & -- & 380 & 1.56 & 0.108 & 0.49 & 211 \\
\hline 05437950 & 1 & Yes & Yes & 1974 & 120 & -- & 335 & 1.14 & 0.113 & 0.591 & 188 \\
\hline 05437950 & 1 & Yes & Yes & 1975 & 91 & -- & 254 & 0.0334 & 0.119 & 0.752 & 149 \\
\hline 05437950 & 1 & Yes & Yes & 1976 & 172 & -- & 480 & 1.3 & 0.124 & 0.339 & 254 \\
\hline 05437950 & 1 & Yes & Yes & 1977 & 70 & -- & 195 & 0.821 & 0.13 & 0.854 & 120 \\
\hline 05437950 & 1 & Yes & Yes & 1978 & 144 & -- & 402 & 1.46 & 0.136 & 0.471 & 216 \\
\hline 05438250 & 1 & Yes & Yes & 1962 & 1150 & -- & 339 & 0.361 & 0.0194 & 0.499 & 1340 \\
\hline 05438250 & 1 & Yes & Yes & 1963 & 220 & 2, E & 65 & 0.247 & 0.0197 & 0.975 & 287 \\
\hline 05438250 & 1 & Yes & Yes & 1964 & 220 & $\mathrm{E}$ & 65 & 0.817 & 0.02 & 0.975 & 287 \\
\hline 05438250 & 1 & Yes & Yes & 1965 & 985 & -- & 291 & 0.105 & 0.0203 & 0.613 & 1150 \\
\hline 05438250 & 1 & Yes & Yes & 1966 & 1390 & -- & 410 & 0.642 & 0.0206 & 0.371 & 1600 \\
\hline 05438250 & 1 & Yes & Yes & 1967 & 1250 & -- & 369 & 1.89 & 0.0209 & 0.441 & 1450 \\
\hline 05438250 & 1 & Yes & Yes & 1968 & 1220 & $E$ & 360 & 1.81 & 0.0211 & 0.459 & 1410 \\
\hline
\end{tabular}




\begin{tabular}{|c|c|c|c|c|c|c|c|c|c|c|c|}
\hline $\begin{array}{l}\text { U.S. } \\
\text { Geological } \\
\text { Survey } \\
\text { streamgage } \\
\text { number }\end{array}$ & $\begin{array}{l}\text { Segment } \\
\text { number }\end{array}$ & $\begin{array}{l}\text { Streamgage used in } \\
\text { regression analyses } \\
\text { (non-redundant) }\end{array}$ & $\begin{array}{c}\text { Discharge value } \\
\text { used in } \\
\text { adjustment } \\
\text { regression }\end{array}$ & $\begin{array}{l}\text { Water } \\
\text { year }\end{array}$ & $\begin{array}{l}\text { Observed annual } \\
\text { maximum peak } \\
\text { discharge }\left(\mathrm{ft}^{3} / \mathrm{s}\right)\end{array}$ & $\begin{array}{l}\text { NWIS peak } \\
\text { code }\end{array}$ & $\begin{array}{l}\text { Observed annual } \\
\text { maximum peak } \\
\text { discharge with } \\
\text { segment intercept } \\
\text { value subtracted } \\
\left(\mathrm{ft}^{3} / \mathrm{s}\right)\end{array}$ & $\begin{array}{l}\text { Observed } \\
\text { precipitation } \\
\text { (inches) }\end{array}$ & Urban fraction & $\begin{array}{l}\text { Exceedance } \\
\text { probability }\end{array}$ & $\begin{array}{c}\text { Urban-adjusted } \\
\text { annual maximum } \\
\text { peak discharge } \\
\left(\mathrm{ft}^{3} / \mathrm{s}\right)\end{array}$ \\
\hline 05438250 & 1 & Yes & Yes & 1969 & 1670 & -- & 493 & 0.127 & 0.0214 & 0.258 & 1900 \\
\hline 05438250 & 1 & Yes & Yes & 1970 & 1940 & -- & 572 & 1.97 & 0.0217 & 0.178 & 2190 \\
\hline 05438250 & 1 & Yes & Yes & 1971 & 1670 & -- & 493 & 0.381 & 0.0231 & 0.259 & 1900 \\
\hline 05438250 & 1 & Yes & Yes & 1972 & 1770 & -- & 522 & 1.05 & 0.0245 & 0.224 & 2000 \\
\hline 05438250 & 1 & Yes & Yes & 1973 & 3020 & -- & 891 & 1.51 & 0.0258 & 0.0499 & 3370 \\
\hline 05438250 & 1 & Yes & Yes & 1974 & 1150 & -- & 339 & 0.594 & 0.0272 & 0.506 & 1330 \\
\hline 05438250 & 1 & Yes & Yes & 1975 & 797 & -- & 235 & 0.794 & 0.0286 & 0.732 & 936 \\
\hline 05438250 & 1 & Yes & Yes & 1976 & 1480 & -- & 437 & 1.28 & 0.03 & 0.338 & 1680 \\
\hline 05438250 & 1 & Yes & Yes & 1977 & 134 & -- & 40 & 1.26 & 0.0314 & 1 & 177 \\
\hline 05438250 & 1 & Yes & Yes & 1978 & 5090 & -- & 1502 & 2.26 & 0.0327 & 0.0119 & 5560 \\
\hline 05438250 & 1 & Yes & Yes & 1979 & 2140 & -- & 631 & 0.289 & 0.0341 & 0.143 & 2390 \\
\hline 05438250 & 1 & Yes & Yes & 1980 & 811 & -- & 239 & 0.388 & 0.0355 & 0.728 & 943 \\
\hline 05438250 & 1 & Yes & Yes & 1981 & 479 & $E$ & 141 & 1.89 & 0.0364 & 0.899 & 576 \\
\hline 05438250 & 1 & Yes & Yes & 1982 & 1360 & -- & 401 & 0.34 & 0.0373 & 0.395 & 1540 \\
\hline 05438250 & 1 & Yes & Yes & 1983 & 2520 & -- & 744 & 1.87 & 0.0382 & 0.0855 & 2800 \\
\hline 05438250 & 1 & Yes & Yes & 1984 & 738 & -- & 218 & 0.766 & 0.0391 & 0.772 & 859 \\
\hline 05438250 & 1 & Yes & Yes & 1985 & 2020 & -- & 596 & 0.532 & 0.0401 & 0.168 & 2250 \\
\hline 05438250 & 1 & Yes & Yes & 1986 & 1490 & -- & 440 & 1.09 & 0.041 & 0.34 & 1680 \\
\hline 05438250 & 1 & Yes & Yes & 1987 & 2110 & -- & 623 & 2.26 & 0.0419 & 0.151 & 2340 \\
\hline 05438250 & 1 & Yes & Yes & 1988 & 779 & -- & 230 & 1 & 0.0428 & 0.753 & 900 \\
\hline 05438250 & 1 & Yes & Yes & 1989 & 850 & -- & 251 & 1.18 & 0.0437 & 0.709 & 976 \\
\hline 05438250 & 1 & Yes & Yes & 1990 & 1130 & -- & 333 & 1.59 & 0.0446 & 0.534 & 1280 \\
\hline 05438250 & 1 & Yes & Yes & 1991 & 970 & -- & 286 & 1.23 & 0.0471 & 0.639 & 1100 \\
\hline 05438283 & 1 & Yes & Yes & 1993 & 322 & -- & 404 & 1.28 & 0.0319 & 0.387 & 337 \\
\hline 05438283 & 1 & Yes & Yes & 1994 & 371 & -- & 466 & 0.733 & 0.033 & 0.299 & 387 \\
\hline 05438283 & 1 & Yes & Yes & 1995 & 7.3 & -- & 9.2 & 0.994 & 0.034 & 1 & 8.0 \\
\hline 05438283 & 1 & Yes & Yes & 1996 & 544 & -- & 683 & 0.894 & 0.035 & 0.111 & 564 \\
\hline 05438283 & 1 & Yes & Yes & 1997 & 484 & -- & 608 & 1.8 & 0.0361 & 0.159 & 502 \\
\hline 05438283 & 1 & Yes & Yes & 1998 & 433 & -- & 544 & 0.817 & 0.0371 & 0.207 & 449 \\
\hline 05438283 & 1 & Yes & Yes & 1999 & 571 & -- & 717 & 1.21 & 0.0382 & 0.0951 & 590 \\
\hline 05438283 & 1 & Yes & Yes & 2000 & 478 & -- & 600 & 0.977 & 0.0392 & 0.165 & 494 \\
\hline 05438283 & 1 & Yes & Yes & 2001 & 283 & -- & 355 & 0.84 & 0.0428 & 0.485 & 294 \\
\hline 05438283 & 1 & Yes & Yes & 2002 & 90 & -- & 113 & 1.48 & 0.0465 & 0.935 & 94.6 \\
\hline 05438283 & 1 & Yes & Yes & 2003 & 152 & -- & 191 & 0.913 & 0.0501 & 0.826 & 157 \\
\hline 05438283 & 1 & Yes & Yes & 2004 & 345 & -- & 433 & 1.11 & 0.0537 & 0.358 & 353 \\
\hline
\end{tabular}




\begin{tabular}{|c|c|c|c|c|c|c|c|c|c|c|c|}
\hline $\begin{array}{l}\text { U.S. } \\
\text { Geological } \\
\text { Survey } \\
\text { streamgage } \\
\text { number }\end{array}$ & $\begin{array}{c}\text { Segment } \\
\text { number }\end{array}$ & $\begin{array}{l}\text { Streamgage used in } \\
\text { regression analyses } \\
\text { (non-redundant) }\end{array}$ & $\begin{array}{c}\text { Discharge value } \\
\text { used in } \\
\text { adjustment } \\
\text { regression }\end{array}$ & $\begin{array}{l}\text { Water } \\
\text { year }\end{array}$ & $\begin{array}{c}\text { Observed annual } \\
\text { maximum peak } \\
\text { discharge }\left(\mathrm{ft}^{3} / \mathrm{s}\right)\end{array}$ & $\begin{array}{l}\text { NWIS peak } \\
\text { code }\end{array}$ & $\begin{array}{l}\text { Observed annual } \\
\text { maximum peak } \\
\text { discharge with } \\
\text { segment intercept } \\
\text { value subtracted } \\
\left(\mathrm{ft}^{3} / \mathrm{s}\right)\end{array}$ & $\begin{array}{l}\text { Observed } \\
\text { precipitation } \\
\text { (inches) }\end{array}$ & Urban fraction & $\begin{array}{l}\text { Exceedance } \\
\text { probability }\end{array}$ & $\begin{array}{c}\text { Urban-adjusted } \\
\text { annual maximum } \\
\text { peak discharge } \\
\left(\mathrm{ft}^{3} / \mathrm{s}\right)\end{array}$ \\
\hline 05438283 & 1 & Yes & Yes & 2005 & 332 & -- & 417 & 0.485 & 0.0574 & 0.385 & 338 \\
\hline 05438283 & 1 & Yes & Yes & 2006 & 259 & -- & 325 & 1.02 & 0.061 & 0.568 & 263 \\
\hline 05438283 & 1 & Yes & Yes & 2007 & 330 & -- & 414 & 1.36 & 0.0646 & 0.394 & 334 \\
\hline 05438283 & 1 & Yes & Yes & 2008 & 397 & -- & 498 & 0.894 & 0.0683 & 0.279 & 400 \\
\hline 05438283 & 1 & Yes & Yes & 2009 & 397 & -- & 498 & 0.338 & 0.0719 & 0.281 & 398 \\
\hline 05438300 & 1 & Yes & Yes & 1961 & 45 & -- & 177 & 0.758 & 0.00952 & 0.831 & 45 \\
\hline 05438300 & 1 & Yes & Yes & 1962 & 70 & -- & 276 & 1.28 & 0.00952 & 0.634 & 70 \\
\hline 05438300 & 1 & Yes & Yes & 1963 & 39 & -- & 154 & 1.34 & 0.00952 & 0.872 & 39 \\
\hline 05438300 & 1 & Yes & No & 1964 & 26 & 4, B & 103 & 0.0318 & 0.00952 & 0.938 & 26 \\
\hline 05438300 & 1 & Yes & Yes & 1965 & 48 & -- & 189 & 0.787 & 0.00952 & 0.809 & 48 \\
\hline 05438300 & 1 & Yes & Yes & 1966 & 85 & -- & 335 & 1.46 & 0.00952 & 0.5 & 85 \\
\hline 05438300 & 1 & Yes & Yes & 1967 & 92 & -- & 363 & 1.41 & 0.00952 & 0.444 & 92 \\
\hline 05438300 & 1 & Yes & Yes & 1968 & 35 & -- & 138 & 0.744 & 0.00952 & 0.895 & 35 \\
\hline 05438300 & 1 & Yes & Yes & 1969 & 159 & -- & 627 & 0.779 & 0.00952 & 0.136 & 159 \\
\hline 05438300 & 1 & Yes & Yes & 1970 & 139 & -- & 548 & 0.971 & 0.00952 & 0.191 & 139 \\
\hline 05438300 & 1 & Yes & Yes & 1971 & 103 & -- & 406 & 0.467 & 0.00952 & 0.369 & 103 \\
\hline 05438300 & 1 & Yes & Yes & 1972 & 180 & -- & 710 & 0.56 & 0.00952 & 0.0908 & 180 \\
\hline 05438300 & 1 & Yes & Yes & 1973 & 144 & -- & 568 & 0.622 & 0.00952 & 0.176 & 144 \\
\hline 05438300 & 1 & Yes & Yes & 1974 & 63 & -- & 248 & 1.07 & 0.00952 & 0.692 & 63 \\
\hline 05438300 & 1 & Yes & Yes & 1975 & 55 & -- & 217 & 0.0318 & 0.00952 & 0.758 & 55 \\
\hline 05438300 & 1 & Yes & Yes & 1976 & 155 & -- & 611 & 1.03 & 0.00952 & 0.146 & 155 \\
\hline 05438300 & 1 & Yes & Yes & 1977 & 35 & -- & 138 & 0.727 & 0.00952 & 0.895 & 35 \\
\hline 05438300 & 1 & Yes & Yes & 1978 & 31 & -- & 122 & 1.45 & 0.00952 & 0.917 & 31 \\
\hline 05438300 & 1 & Yes & Yes & 1979 & 123 & -- & 485 & 1.57 & 0.00952 & 0.26 & 123 \\
\hline 05438300 & 1 & Yes & Yes & 1980 & 175 & -- & 690 & 0.545 & 0.00952 & 0.0981 & 175 \\
\hline 05438390 & 1 & No & No & 1970 & 1750 & -- & 351 & 0.988 & 0.0427 & 0.493 & 1930 \\
\hline 05438390 & 1 & No & No & 1971 & 2750 & -- & 552 & 0.477 & 0.0436 & 0.202 & 2980 \\
\hline 05438390 & 1 & No & No & 1972 & 2020 & -- & 405 & 1.2 & 0.0444 & 0.394 & 2210 \\
\hline 05438390 & 1 & No & No & 1973 & 4000 & -- & 803 & 1.8 & 0.0453 & 0.07 & 4310 \\
\hline 05438390 & 1 & No & No & 1974 & 1760 & -- & 353 & 0.587 & 0.0461 & 0.492 & 1930 \\
\hline 05438390 & 1 & No & No & 1975 & 1530 & -- & 307 & 0.0316 & 0.047 & 0.599 & 1690 \\
\hline 05438390 & 1 & No & No & 1976 & 2020 & -- & 405 & 1.22 & 0.0479 & 0.396 & 2210 \\
\hline 05438390 & 1 & No & No & 1977 & 370 & -- & 74 & 0.791 & 0.0487 & 0.969 & 433 \\
\hline 05438390 & 1 & No & No & 1978 & 1380 & -- & 277 & 1.4 & 0.0496 & 0.659 & 1520 \\
\hline 05438390 & 1 & No & No & 1979 & 1060 & -- & 213 & 0.302 & 0.0504 & 0.787 & 1180 \\
\hline
\end{tabular}




\begin{tabular}{|c|c|c|c|c|c|c|c|c|c|c|c|}
\hline $\begin{array}{c}\text { U.S. } \\
\text { Geological } \\
\text { Survey } \\
\text { streamgage } \\
\text { number } \\
\end{array}$ & $\begin{array}{l}\text { Segment } \\
\text { number }\end{array}$ & $\begin{array}{l}\text { Streamgage used in } \\
\text { regression analyses } \\
\text { (non-redundant) }\end{array}$ & $\begin{array}{c}\text { Discharge value } \\
\text { used in } \\
\text { adjustment } \\
\text { regression }\end{array}$ & $\begin{array}{c}\text { Water } \\
\text { year }\end{array}$ & $\begin{array}{c}\text { Observed annual } \\
\text { maximum peak } \\
\text { discharge }\left(\mathrm{ft}^{3} / \mathrm{s}\right)\end{array}$ & $\begin{array}{c}\text { NWIS peak } \\
\text { code }\end{array}$ & $\begin{array}{c}\text { Observed annual } \\
\text { maximum peak } \\
\text { discharge with } \\
\text { segment intercept } \\
\text { value subtracted } \\
\left(\mathrm{ft}^{3} / \mathrm{s}\right)\end{array}$ & $\begin{array}{c}\text { Observed } \\
\text { precipitation } \\
\text { (inches) }\end{array}$ & Urban fraction & $\begin{array}{l}\text { Exceedance } \\
\text { probability }\end{array}$ & $\begin{array}{c}\text { Urban-adjusted } \\
\text { annual maximum } \\
\text { peak discharge } \\
\left(\mathrm{ft}^{3} / \mathrm{s}\right)\end{array}$ \\
\hline 05438500 & 1 & Yes & Yes & 1940 & 1330 & -- & 122 & 0.653 & 0.0209 & 0.92 & 1680 \\
\hline 05438500 & 1 & Yes & Yes & 1941 & 2240 & -- & 206 & 0.0391 & 0.0212 & 0.784 & 2700 \\
\hline 05438500 & 1 & Yes & Yes & 1942 & 3230 & -- & 297 & 0.789 & 0.0216 & 0.602 & 3810 \\
\hline 05438500 & 1 & Yes & Yes & 1943 & 10300 & -- & 947 & 1.18 & 0.0219 & 0.042 & 11600 \\
\hline 05438500 & 1 & Yes & Yes & 1944 & 9200 & -- & 845 & 1.33 & 0.0222 & 0.0581 & 10400 \\
\hline 05438500 & 1 & Yes & Yes & 1945 & 1850 & -- & 170 & 0.428 & 0.0225 & 0.849 & 2260 \\
\hline 05438500 & 1 & Yes & Yes & 1946 & 9830 & -- & 903 & 0.625 & 0.0229 & 0.0479 & 11100 \\
\hline 05438500 & 1 & Yes & Yes & 1947 & 2170 & -- & 199 & 1.51 & 0.0232 & 0.797 & 2620 \\
\hline 05438500 & 1 & Yes & Yes & 1948 & 8000 & -- & 735 & 1.88 & 0.0235 & 0.085 & 9050 \\
\hline 05438500 & 1 & Yes & Yes & 1949 & 5750 & -- & 528 & 0.349 & 0.0238 & 0.217 & 6550 \\
\hline 05438500 & 1 & Yes & Yes & 1950 & 5240 & -- & 482 & 1.35 & 0.0242 & 0.273 & 6000 \\
\hline 05438500 & 1 & Yes & Yes & 1951 & 6410 & -- & 589 & 0.188 & 0.0247 & 0.167 & 7280 \\
\hline 05438500 & 1 & Yes & Yes & 1952 & 6690 & -- & 615 & 0.569 & 0.0253 & 0.15 & 7580 \\
\hline 05438500 & 1 & Yes & Yes & 1953 & 1850 & $E$ & 170 & 1.46 & 0.0258 & 0.85 & 2250 \\
\hline 05438500 & 1 & Yes & Yes & 1954 & 3290 & -- & 302 & 1.55 & 0.0264 & 0.593 & 3850 \\
\hline 05438500 & 1 & Yes & Yes & 1955 & 2140 & $\mathrm{E}$ & 197 & 0.284 & 0.027 & 0.804 & 2570 \\
\hline 05438500 & 1 & Yes & Yes & 1956 & 935 & $\mathrm{E}$ & 86 & 1.15 & 0.0275 & 0.957 & 1200 \\
\hline 05438500 & 1 & Yes & Yes & 1957 & 1070 & $\mathrm{E}$ & 98 & 0.916 & 0.0281 & 0.946 & 1360 \\
\hline 05438500 & 1 & Yes & Yes & 1958 & 1270 & $\mathrm{E}$ & 117 & 0.193 & 0.0286 & 0.928 & 1590 \\
\hline 05438500 & 1 & Yes & Yes & 1959 & 3330 & -- & 306 & 0.02 & 0.0292 & 0.586 & 3880 \\
\hline 05438500 & 1 & Yes & Yes & 1960 & 8230 & -- & 756 & 0.664 & 0.0298 & 0.0791 & 9250 \\
\hline 05438500 & 1 & Yes & Yes & 1961 & 1420 & $E$ & 131 & 0.797 & 0.0305 & 0.911 & 1750 \\
\hline 05438500 & 1 & Yes & Yes & 1962 & 4600 & -- & 423 & 0.312 & 0.0312 & 0.359 & 5280 \\
\hline 05438500 & 1 & Yes & Yes & 1963 & 955 & $E$ & 88 & 0.152 & 0.0319 & 0.956 & 1210 \\
\hline 05438500 & 1 & Yes & Yes & 1964 & 1090 & $E$ & 100 & 0.729 & 0.0326 & 0.945 & 1370 \\
\hline 05438500 & 1 & Yes & Yes & 1965 & 3300 & 2 & 303 & 0.167 & 0.0333 & 0.597 & 3830 \\
\hline 05438500 & 1 & Yes & Yes & 1966 & 5450 & $E$ & 501 & 0.649 & 0.034 & 0.255 & 6170 \\
\hline 05438500 & 1 & Yes & Yes & 1967 & 3550 & $E$ & 326 & 1.93 & 0.0347 & 0.542 & 4100 \\
\hline 05438500 & 1 & Yes & Yes & 1968 & 2150 & $E$ & 198 & 1.64 & 0.0354 & 0.807 & 2560 \\
\hline 05438500 & 1 & Yes & Yes & 1969 & 3640 & -- & 335 & 1.74 & 0.0361 & 0.524 & 4200 \\
\hline 05438500 & 1 & Yes & Yes & 1970 & 2790 & -- & 256 & 1.89 & 0.0369 & 0.693 & 3260 \\
\hline 05438500 & 1 & Yes & Yes & 1971 & 8350 & $\mathrm{E}$ & 767 & 0.412 & 0.0383 & 0.0773 & 9320 \\
\hline 05438500 & 1 & Yes & Yes & 1972 & 6700 & -- & 616 & 0.941 & 0.0396 & 0.155 & 7500 \\
\hline 05438500 & 1 & Yes & Yes & 1973 & 6270 & -- & 576 & 1.6 & 0.041 & 0.183 & 7020 \\
\hline 05438500 & 1 & Yes & Yes & 1974 & 6620 & -- & 608 & 1.11 & 0.0424 & 0.161 & 7390 \\
\hline
\end{tabular}




\begin{tabular}{|c|c|c|c|c|c|c|c|c|c|c|c|}
\hline $\begin{array}{c}\text { U.S. } \\
\text { Geological } \\
\text { Survey } \\
\text { streamgage } \\
\text { number } \\
\end{array}$ & $\begin{array}{l}\text { Segment } \\
\text { number }\end{array}$ & $\begin{array}{l}\text { Streamgage used in } \\
\text { regression analyses } \\
\text { (non-redundant) }\end{array}$ & $\begin{array}{c}\text { Discharge value } \\
\text { used in } \\
\text { adjustment } \\
\text { regression }\end{array}$ & $\begin{array}{c}\text { Water } \\
\text { year }\end{array}$ & $\begin{array}{c}\text { Observed annual } \\
\text { maximum peak } \\
\text { discharge }\left(\mathrm{ft}^{3} / \mathrm{s}\right)\end{array}$ & $\begin{array}{c}\text { NWIS peak } \\
\text { code }\end{array}$ & $\begin{array}{c}\text { Observed annual } \\
\text { maximum peak } \\
\text { discharge with } \\
\text { segment intercept } \\
\text { value subtracted } \\
\left(\mathrm{ft}^{3} / \mathrm{s}\right)\end{array}$ & $\begin{array}{c}\text { Observed } \\
\text { precipitation } \\
\text { (inches) }\end{array}$ & Urban fraction & $\begin{array}{l}\text { Exceedance } \\
\text { probability }\end{array}$ & $\begin{array}{c}\text { Urban-adjusted } \\
\text { annual maximum } \\
\text { peak discharge } \\
\left(\mathrm{ft}^{3} / \mathrm{s}\right)\end{array}$ \\
\hline 05438500 & 1 & Yes & Yes & 1975 & 3840 & $E$ & 353 & 0.0233 & 0.0438 & 0.491 & 4380 \\
\hline 05438500 & 1 & Yes & Yes & 1976 & 6130 & -- & 563 & 1.32 & 0.0452 & 0.194 & 6840 \\
\hline 05438500 & 1 & Yes & Yes & 1977 & 1980 & $E$ & 182 & 0.527 & 0.0466 & 0.839 & 2330 \\
\hline 05438500 & 1 & Yes & Yes & 1978 & 8340 & -- & 766 & 2.16 & 0.048 & 0.0799 & 9230 \\
\hline 05438500 & 1 & Yes & Yes & 1979 & 9040 & -- & 831 & 0.282 & 0.0494 & 0.0648 & 9970 \\
\hline 05438500 & 1 & Yes & Yes & 1980 & 2090 & $E$ & 192 & 0.692 & 0.0508 & 0.825 & 2440 \\
\hline 05438500 & 1 & Yes & Yes & 1981 & 1480 & $E$ & 136 & 1.8 & 0.0519 & 0.91 & 1770 \\
\hline 05438500 & 1 & Yes & Yes & 1982 & 4670 & -- & 429 & 0.336 & 0.053 & 0.363 & 5240 \\
\hline 05438500 & 1 & Yes & Yes & 1983 & 6470 & -- & 595 & 1.74 & 0.054 & 0.175 & 7150 \\
\hline 05438500 & 1 & Yes & Yes & 1984 & 5070 & $E$ & 466 & 0.726 & 0.0551 & 0.313 & 5650 \\
\hline 05438500 & 1 & Yes & Yes & 1985 & 3900 & -- & 358 & 0.5 & 0.0562 & 0.49 & 4390 \\
\hline 05438500 & 1 & Yes & Yes & 1986 & 4660 & -- & 428 & 0.121 & 0.0573 & 0.368 & 5210 \\
\hline 05438500 & 1 & Yes & Yes & 1987 & 4590 & -- & 422 & 2.17 & 0.0584 & 0.378 & 5120 \\
\hline 05438500 & 1 & Yes & Yes & 1988 & 3960 & -- & 364 & 0.192 & 0.0595 & 0.481 & 4440 \\
\hline 05438500 & 1 & Yes & Yes & 1989 & 1830 & $\mathrm{E}$ & 168 & 2.06 & 0.0605 & 0.868 & 2130 \\
\hline 05438500 & 1 & Yes & Yes & 1990 & 2580 & -- & 237 & 0.439 & 0.0616 & 0.75 & 2940 \\
\hline 05438500 & 1 & Yes & Yes & 1991 & 4240 & $\mathrm{E}$ & 390 & 1.23 & 0.064 & 0.436 & 4720 \\
\hline 05438500 & 1 & Yes & Yes & 1992 & 1710 & $\mathrm{E}$ & 157 & 1.11 & 0.0663 & 0.887 & 1980 \\
\hline 05438500 & 1 & Yes & Yes & 1993 & 7870 & -- & 723 & 2.06 & 0.0687 & 0.0999 & 8560 \\
\hline 05438500 & 1 & Yes & Yes & 1994 & 11900 & -- & 1094 & 1.18 & 0.0711 & 0.0296 & 12800 \\
\hline 05438500 & 1 & Yes & Yes & 1995 & 2360 & $\mathrm{E}$ & 217 & 1.48 & 0.0734 & 0.791 & 2660 \\
\hline 05438500 & 1 & Yes & Yes & 1996 & 5860 & -- & 539 & 1.61 & 0.0758 & 0.235 & 6360 \\
\hline 05438500 & 1 & Yes & Yes & 1997 & 7620 & -- & 700 & 1.69 & 0.0781 & 0.117 & 8220 \\
\hline 05438500 & 1 & Yes & Yes & 1998 & 3570 & C & 328 & 1.69 & 0.0805 & 0.578 & 3920 \\
\hline 05438500 & 1 & Yes & Yes & 1999 & 11400 & $C$ & 1048 & 2.01 & 0.0828 & 0.0357 & 12200 \\
\hline 05438500 & 1 & Yes & Yes & 2000 & 5730 & $C$ & 527 & 2.17 & 0.0852 & 0.255 & 6170 \\
\hline 05438500 & 1 & Yes & Yes & 2001 & 4040 & $C$ & 371 & 0.658 & 0.0929 & 0.494 & 4370 \\
\hline 05438500 & 1 & Yes & Yes & 2002 & 5040 & $C$ & 463 & 2.29 & 0.101 & 0.346 & 5370 \\
\hline 05438500 & 1 & Yes & Yes & 2003 & 896 & $\mathrm{C}, \mathrm{E}$ & 82 & 1.73 & 0.108 & 0.97 & 995 \\
\hline 05438500 & 1 & Yes & Yes & 2004 & 3960 & $C$ & 364 & 1.66 & 0.116 & 0.529 & 4170 \\
\hline 05438500 & 1 & Yes & Yes & 2005 & 2610 & $C, E$ & 240 & 0.716 & 0.124 & 0.778 & 2750 \\
\hline 05438500 & 1 & Yes & Yes & 2006 & 1290 & $C, E$ & 119 & 1.6 & 0.131 & 0.946 & 1360 \\
\hline 05438500 & 1 & Yes & Yes & 2007 & 7420 & $C$ & 682 & 1.62 & 0.139 & 0.151 & 7580 \\
\hline 05438500 & 1 & Yes & Yes & 2008 & 4810 & C & 442 & 0.596 & 0.147 & 0.41 & 4890 \\
\hline 05438500 & 1 & Yes & Yes & 2009 & 4510 & C & 414 & 1.02 & 0.154 & 0.464 & 4550 \\
\hline
\end{tabular}




\begin{tabular}{|c|c|c|c|c|c|c|c|c|c|c|c|}
\hline $\begin{array}{l}\text { U.S. } \\
\text { Geological } \\
\text { Survey } \\
\text { streamgage } \\
\text { number }\end{array}$ & $\begin{array}{c}\text { Segment } \\
\text { number }\end{array}$ & $\begin{array}{l}\text { Streamgage used in } \\
\text { regression analyses } \\
\text { (non-redundant) }\end{array}$ & $\begin{array}{c}\text { Discharge value } \\
\text { used in } \\
\text { adjustment } \\
\text { regression }\end{array}$ & $\begin{array}{l}\text { Water } \\
\text { year }\end{array}$ & $\begin{array}{c}\text { Observed annual } \\
\text { maximum peak } \\
\text { discharge }\left(\mathrm{ft}^{3} / \mathrm{s}\right)\end{array}$ & $\begin{array}{l}\text { NWIS peak } \\
\text { code }\end{array}$ & $\begin{array}{l}\text { Observed annual } \\
\text { maximum peak } \\
\text { discharge with } \\
\text { segment intercept } \\
\text { value subtracted } \\
\left(\mathrm{ft}^{3} / \mathrm{s}\right)\end{array}$ & $\begin{array}{l}\text { Observed } \\
\text { precipitation } \\
\text { (inches) }\end{array}$ & Urban fraction & $\begin{array}{l}\text { Exceedance } \\
\text { probability }\end{array}$ & $\begin{array}{c}\text { Urban-adjusted } \\
\text { annual maximum } \\
\text { peak discharge } \\
\left(\mathrm{ft}^{3} / \mathrm{s}\right)\end{array}$ \\
\hline 05438850 & 1 & Yes & Yes & 1956 & 18 & -- & 41 & 0.929 & 0 & 1 & 18 \\
\hline 05438850 & 1 & Yes & Yes & 1957 & 200 & -- & 454 & 0.241 & 0 & 0.295 & 200 \\
\hline 05438850 & 1 & Yes & Yes & 1958 & 310 & -- & 703 & 0.963 & 0 & 0.0911 & 310 \\
\hline 05438850 & 1 & Yes & Yes & 1959 & 393 & -- & 892 & 1.38 & 0 & 0.0472 & 393 \\
\hline 05438850 & 1 & Yes & Yes & 1960 & 257 & -- & 583 & 1.23 & 0 & 0.162 & 257 \\
\hline 05438850 & 1 & Yes & Yes & 1961 & 31 & -- & 70 & 0.761 & 0 & 0.967 & 31 \\
\hline 05438850 & 1 & Yes & Yes & 1962 & 100 & -- & 227 & 0.796 & 0 & 0.732 & 100 \\
\hline 05438850 & 1 & Yes & Yes & 1963 & 92 & -- & 209 & 0.422 & 0 & 0.768 & 92 \\
\hline 05438850 & 1 & Yes & Yes & 1964 & 59 & -- & 134 & 0.861 & 0 & 0.899 & 59 \\
\hline 05438850 & 1 & Yes & Yes & 1965 & 231 & -- & 524 & 0.85 & 0 & 0.208 & 231 \\
\hline 05438850 & 1 & Yes & Yes & 1966 & 85 & -- & 193 & 0.537 & 0 & 0.798 & 85 \\
\hline 05438850 & 1 & Yes & Yes & 1967 & 72 & -- & 163 & 0.0108 & 0 & 0.851 & 72 \\
\hline 05438850 & 1 & Yes & No & 1968 & 28 & $4, B$ & 64 & 0.00236 & 0 & 0.973 & 28 \\
\hline 05438850 & 1 & Yes & Yes & 1969 & 101 & -- & 229 & 1.39 & 0 & 0.727 & 101 \\
\hline 05438850 & 1 & Yes & Yes & 1970 & 150 & -- & 340 & 1.59 & 0 & 0.482 & 150 \\
\hline 05438850 & 1 & Yes & Yes & 1971 & 96 & -- & 218 & 0.236 & 0 & 0.752 & 96 \\
\hline 05438850 & 1 & Yes & Yes & 1972 & 228 & -- & 517 & 1.32 & 0 & 0.216 & 228 \\
\hline 05438850 & 1 & Yes & Yes & 1973 & 185 & -- & 420 & 0.408 & 0 & 0.343 & 185 \\
\hline 05438850 & 1 & Yes & Yes & 1974 & 233 & -- & 529 & 1.5 & 0 & 0.203 & 233 \\
\hline 05438850 & 1 & Yes & Yes & 1975 & 274 & -- & 622 & 0.893 & 0 & 0.136 & 274 \\
\hline 05438850 & 1 & Yes & Yes & 1976 & 131 & -- & 297 & 0.00236 & 0 & 0.583 & 131 \\
\hline 05438850 & 1 & Yes & Yes & 1977 & 112 & -- & 254 & 0.757 & 0 & 0.673 & 112 \\
\hline 05438850 & 1 & Yes & Yes & 1978 & 234 & -- & 531 & 1.11 & 0 & 0.2 & 234 \\
\hline 05438850 & 1 & Yes & Yes & 1979 & 136 & -- & 309 & 1.61 & 0 & 0.555 & 136 \\
\hline 05438850 & 1 & Yes & Yes & 1980 & 219 & -- & 497 & 0.731 & 0 & 0.24 & 219 \\
\hline 05439000 & 1 & Yes & Yes & 1980 & 1510 & -- & 585 & 1.08 & 0.047 & 0.179 & 1530 \\
\hline 05439000 & 1 & Yes & Yes & 1981 & 1050 & -- & 407 & 2.17 & 0.0474 & 0.393 & 1070 \\
\hline 05439000 & 1 & Yes & Yes & 1982 & 718 & -- & 278 & 3.38 & 0.0477 & 0.655 & 730 \\
\hline 05439000 & 1 & Yes & Yes & 1983 & 3500 & -- & 1356 & 1.91 & 0.0481 & 0.0157 & 3540 \\
\hline 05439000 & 1 & Yes & Yes & 1984 & 787 & -- & 305 & 0.692 & 0.0484 & 0.604 & 799 \\
\hline 05439000 & 1 & Yes & Yes & 1985 & 1130 & -- & 438 & 1.35 & 0.0488 & 0.348 & 1150 \\
\hline 05439000 & 1 & Yes & Yes & 1986 & 910 & -- & 353 & 1.23 & 0.0491 & 0.495 & 923 \\
\hline 05439000 & 1 & Yes & Yes & 1987 & 1600 & -- & 620 & 2.13 & 0.0495 & 0.156 & 1620 \\
\hline 05439000 & 1 & Yes & Yes & 1988 & 694 & -- & 269 & 0.27 & 0.0498 & 0.675 & 704 \\
\hline 05439000 & 1 & Yes & Yes & 1989 & 697 & -- & 270 & 1.24 & 0.0502 & 0.673 & 707 \\
\hline
\end{tabular}




\begin{tabular}{|c|c|c|c|c|c|c|c|c|c|c|c|}
\hline $\begin{array}{c}\text { U.S. } \\
\text { Geological } \\
\text { Survey } \\
\text { streamgage } \\
\text { number } \\
\end{array}$ & $\begin{array}{c}\text { Segment } \\
\text { number }\end{array}$ & $\begin{array}{l}\text { Streamgage used in } \\
\text { regression analyses } \\
\text { (non-redundant) }\end{array}$ & $\begin{array}{c}\text { Discharge value } \\
\text { used in } \\
\text { adjustment } \\
\text { regression }\end{array}$ & $\begin{array}{c}\text { Water } \\
\text { year }\end{array}$ & $\begin{array}{c}\text { Observed annual } \\
\text { maximum peak } \\
\text { discharge }\left(\mathrm{ft}^{3} / \mathrm{s}\right)\end{array}$ & $\begin{array}{c}\text { NWIS peak } \\
\text { code }\end{array}$ & $\begin{array}{c}\text { Observed annual } \\
\text { maximum peak } \\
\text { discharge with } \\
\text { segment intercept } \\
\text { value subtracted } \\
\left(\mathrm{ft}^{3} / \mathrm{s}\right)\end{array}$ & $\begin{array}{c}\text { Observed } \\
\text { precipitation } \\
\text { (inches) }\end{array}$ & Urban fraction & $\begin{array}{l}\text { Exceedance } \\
\text { probability }\end{array}$ & $\begin{array}{c}\text { Urban-adjusted } \\
\text { annual maximum } \\
\text { peak discharge } \\
\left(\mathrm{ft}^{3} / \mathrm{s}\right)\end{array}$ \\
\hline 05439000 & 1 & Yes & Yes & 1990 & 671 & -- & 260 & 0.6 & 0.0505 & 0.695 & 680 \\
\hline 05439000 & 1 & Yes & Yes & 1991 & 1170 & -- & 453 & 0.708 & 0.051 & 0.327 & 1180 \\
\hline 05439000 & 1 & Yes & Yes & 1992 & 323 & $\mathrm{E}$ & 125 & 0.655 & 0.0514 & 0.925 & 328 \\
\hline 05439000 & 1 & Yes & Yes & 1993 & 1310 & -- & 508 & 1.02 & 0.0519 & 0.257 & 1320 \\
\hline 05439000 & 1 & Yes & Yes & 1994 & 1260 & -- & 488 & 0.917 & 0.0524 & 0.282 & 1270 \\
\hline 05439000 & 1 & Yes & Yes & 1995 & 806 & C & 312 & 1.08 & 0.0528 & 0.591 & 814 \\
\hline 05439000 & 1 & Yes & Yes & 1996 & 2280 & C, D & 884 & 4.56 & 0.0533 & 0.055 & 2300 \\
\hline 05439000 & 1 & Yes & Yes & 1997 & 2170 & C & 841 & 2.24 & 0.0538 & 0.0634 & 2180 \\
\hline 05439000 & 1 & Yes & Yes & 1998 & 750 & C & 291 & 0.984 & 0.0542 & 0.635 & 756 \\
\hline 05439000 & 1 & Yes & Yes & 1999 & 1080 & C & 419 & 1.08 & 0.0547 & 0.38 & 1090 \\
\hline 05439000 & 1 & Yes & Yes & 2000 & 818 & $\mathrm{C}, \mathrm{E}$ & 317 & 1.02 & 0.0552 & 0.582 & 824 \\
\hline 05439000 & 1 & Yes & Yes & 2001 & 1080 & C & 419 & 0.703 & 0.0558 & 0.381 & 1090 \\
\hline 05439000 & 1 & Yes & Yes & 2002 & 1090 & C & 422 & 1.76 & 0.0564 & 0.376 & 1100 \\
\hline 05439000 & 1 & Yes & Yes & 2003 & 760 & $\mathrm{C}, \mathrm{E}$ & 295 & 0.92 & 0.0571 & 0.629 & 764 \\
\hline 05439000 & 1 & Yes & Yes & 2004 & 1270 & C & 492 & 1.54 & 0.0577 & 0.28 & 1270 \\
\hline 05439000 & 1 & Yes & Yes & 2005 & 273 & $C$ & 106 & 0.458 & 0.0583 & 0.944 & 275 \\
\hline 05439000 & 1 & Yes & Yes & 2006 & 136 & $\mathrm{C}, \mathrm{E}$ & 53 & 0.743 & 0.059 & 0.987 & 137 \\
\hline 05439000 & 1 & Yes & Yes & 2007 & 3020 & C & 1170 & 3.86 & 0.0596 & 0.0227 & 3020 \\
\hline 05439000 & 1 & Yes & Yes & 2008 & 2030 & C & 787 & 5.44 & 0.0602 & 0.0759 & 2030 \\
\hline 05439000 & 1 & Yes & Yes & 2009 & 1370 & C & 531 & 1.54 & 0.0609 & 0.235 & 1370 \\
\hline 05439500 & 1 & Yes & Yes & 1940 & 1350 & -- & 119 & 0.00345 & 0.0245 & 0.926 & 1520 \\
\hline 05439500 & 1 & Yes & Yes & 1941 & 2380 & -- & 209 & 0.0426 & 0.0249 & 0.78 & 2610 \\
\hline 05439500 & 1 & Yes & Yes & 1942 & 4530 & -- & 398 & 0.693 & 0.0252 & 0.392 & 4880 \\
\hline 05439500 & 1 & Yes & Yes & 1943 & 5680 & -- & 499 & 0.709 & 0.0255 & 0.252 & 6070 \\
\hline 05439500 & 1 & Yes & Yes & 1944 & 5850 & -- & 514 & 1.17 & 0.0259 & 0.235 & 6240 \\
\hline 05439500 & 1 & Yes & Yes & 1945 & 2510 & -- & 220 & 1.52 & 0.0262 & 0.76 & 2740 \\
\hline 05439500 & 1 & Yes & Yes & 1946 & 6840 & -- & 601 & 0.569 & 0.0265 & 0.16 & 7280 \\
\hline 05439500 & 1 & Yes & Yes & 1947 & 2210 & -- & 194 & 0.68 & 0.0269 & 0.809 & 2420 \\
\hline 05439500 & 1 & Yes & Yes & 1948 & 5680 & -- & 499 & 1.76 & 0.0272 & 0.253 & 6060 \\
\hline 05439500 & 1 & Yes & Yes & 1949 & 6000 & 2 & 527 & 0.299 & 0.0275 & 0.221 & 6390 \\
\hline 05439500 & 1 & Yes & Yes & 1950 & 4530 & -- & 398 & 1.45 & 0.0279 & 0.394 & 4860 \\
\hline 05439500 & 1 & Yes & Yes & 1951 & 6670 & -- & 586 & 2.43 & 0.0288 & 0.171 & 7080 \\
\hline 05439500 & 1 & Yes & Yes & 1952 & 4690 & -- & 412 & 0.486 & 0.0298 & 0.374 & 5020 \\
\hline 05439500 & 1 & Yes & Yes & 1953 & 1290 & $\mathrm{E}$ & 113 & 0.735 & 0.0307 & 0.932 & 1440 \\
\hline 05439500 & 1 & Yes & Yes & 1954 & 2340 & $E$ & 206 & 1.09 & 0.0317 & 0.79 & 2540 \\
\hline
\end{tabular}




\begin{tabular}{|c|c|c|c|c|c|c|c|c|c|c|c|}
\hline $\begin{array}{c}\text { U.S. } \\
\text { Geological } \\
\text { Survey } \\
\text { streamgage } \\
\text { number }\end{array}$ & $\begin{array}{l}\text { Segment } \\
\text { number }\end{array}$ & $\begin{array}{l}\text { Streamgage used in } \\
\text { regression analyses } \\
\text { (non-redundant) }\end{array}$ & $\begin{array}{l}\text { Discharge value } \\
\text { used in } \\
\text { adjustment } \\
\text { regression }\end{array}$ & $\begin{array}{l}\text { Water } \\
\text { year }\end{array}$ & $\begin{array}{c}\text { Observed annual } \\
\text { maximum peak } \\
\text { discharge }\left(\mathrm{ft}^{3} / \mathrm{s}\right)\end{array}$ & $\begin{array}{l}\text { NWIS peak } \\
\text { code }\end{array}$ & $\begin{array}{l}\text { Observed annual } \\
\text { maximum peak } \\
\text { discharge with } \\
\text { segment intercept } \\
\text { value subtracted } \\
\left(\mathrm{ft}^{3} / \mathrm{s}\right)\end{array}$ & $\begin{array}{l}\text { Observed } \\
\text { precipitation } \\
\text { (inches) }\end{array}$ & Urban fraction & $\begin{array}{l}\text { Exceedance } \\
\text { probability }\end{array}$ & $\begin{array}{c}\text { Urban-adjusted } \\
\text { annual maximum } \\
\text { peak discharge } \\
\left(\mathrm{ft}^{3} / \mathrm{s}\right)\end{array}$ \\
\hline 05439500 & 1 & Yes & Yes & 1955 & 5910 & -- & 519 & 3.47 & 0.0326 & 0.233 & 6270 \\
\hline 05439500 & 1 & Yes & Yes & 1956 & 1740 & $\mathrm{E}$ & 153 & 0.709 & 0.0335 & 0.882 & 1910 \\
\hline 05439500 & 1 & Yes & Yes & 1957 & 2000 & $\mathrm{E}$ & 176 & 0.566 & 0.0345 & 0.844 & 2180 \\
\hline 05439500 & 1 & Yes & Yes & 1958 & 4290 & -- & 377 & 1.83 & 0.0354 & 0.438 & 4570 \\
\hline 05439500 & 1 & Yes & Yes & 1959 & 2900 & 2 & 255 & 0.459 & 0.0364 & 0.696 & 3110 \\
\hline 05439500 & 1 & Yes & Yes & 1960 & 5240 & -- & 460 & 0.576 & 0.0373 & 0.309 & 5550 \\
\hline 05439500 & 1 & Yes & Yes & 1961 & 1010 & $\mathrm{E}$ & 89 & 1.29 & 0.0382 & 0.956 & 1120 \\
\hline 05439500 & 1 & Yes & Yes & 1962 & 4760 & -- & 418 & 0.319 & 0.0392 & 0.371 & 5040 \\
\hline 05439500 & 1 & Yes & Yes & 1963 & 1510 & $\mathrm{E}$ & 133 & 0.872 & 0.0401 & 0.911 & 1650 \\
\hline 05439500 & 1 & Yes & Yes & 1964 & 1090 & $\mathrm{E}$ & 96 & 0.734 & 0.041 & 0.951 & 1200 \\
\hline 05439500 & 1 & Yes & Yes & 1965 & 4000 & 2 & 351 & 0.112 & 0.0419 & 0.492 & 4240 \\
\hline 05439500 & 1 & Yes & Yes & 1966 & 5060 & -- & 445 & 0.519 & 0.0428 & 0.335 & 5330 \\
\hline 05439500 & 1 & Yes & Yes & 1967 & 2850 & -- & 250 & 0.998 & 0.0438 & 0.71 & 3030 \\
\hline 05439500 & 1 & Yes & Yes & 1968 & 4460 & $E$ & 392 & 1.51 & 0.0447 & 0.417 & 4700 \\
\hline 05439500 & 1 & Yes & Yes & 1969 & 5030 & -- & 442 & 0.126 & 0.0456 & 0.34 & 5290 \\
\hline 05439500 & 1 & Yes & Yes & 1970 & 3910 & -- & 343 & 1.93 & 0.0465 & 0.513 & 4120 \\
\hline 05439500 & 1 & Yes & Yes & 1971 & 3930 & -- & 345 & 0.492 & 0.0474 & 0.51 & 4140 \\
\hline 05439500 & 1 & Yes & Yes & 1972 & 6020 & -- & 529 & 1.65 & 0.0484 & 0.231 & 6290 \\
\hline 05439500 & 1 & Yes & Yes & 1973 & 8460 & -- & 743 & 1.7 & 0.0493 & 0.0882 & 8800 \\
\hline 05439500 & 1 & Yes & Yes & 1974 & 6780 & -- & 596 & 1.37 & 0.0502 & 0.173 & 7060 \\
\hline 05439500 & 1 & Yes & Yes & 1975 & 3740 & -- & 329 & 0.0251 & 0.0511 & 0.551 & 3930 \\
\hline 05439500 & 1 & Yes & Yes & 1976 & 6690 & -- & 588 & 1.13 & 0.052 & 0.179 & 6950 \\
\hline 05439500 & 1 & Yes & Yes & 1977 & 799 & -- & 70 & 1.42 & 0.0529 & 0.974 & 866 \\
\hline 05439500 & 1 & Yes & Yes & 1978 & 5470 & -- & 481 & 1.18 & 0.0538 & 0.293 & 5690 \\
\hline 05439500 & 1 & Yes & Yes & 1979 & 7600 & -- & 668 & 0.343 & 0.0547 & 0.128 & 7880 \\
\hline 05439500 & 1 & Yes & Yes & 1980 & 3390 & -- & 298 & 0.454 & 0.0556 & 0.622 & 3550 \\
\hline 05439500 & 1 & Yes & Yes & 1981 & 2840 & -- & 249 & 2.08 & 0.0561 & 0.72 & 2980 \\
\hline 05439500 & 1 & Yes & Yes & 1982 & 3460 & -- & 304 & 0.27 & 0.0565 & 0.611 & 3610 \\
\hline 05439500 & 1 & Yes & Yes & 1983 & 7220 & -- & 634 & 1.73 & 0.057 & 0.149 & 7470 \\
\hline 05439500 & 1 & Yes & Yes & 1984 & 4400 & -- & 387 & 0.67 & 0.0574 & 0.437 & 4580 \\
\hline 05439500 & 1 & Yes & Yes & 1985 & 5200 & -- & 457 & 1.31 & 0.0578 & 0.327 & 5400 \\
\hline 05439500 & 1 & Yes & Yes & 1986 & 3120 & -- & 274 & 1.13 & 0.0583 & 0.671 & 3260 \\
\hline 05439500 & 1 & Yes & Yes & 1987 & 5300 & -- & 466 & 2.95 & 0.0587 & 0.316 & 5490 \\
\hline 05439500 & 1 & Yes & Yes & 1988 & 3680 & -- & 323 & 0.26 & 0.0592 & 0.571 & 3830 \\
\hline 05439500 & 1 & Yes & Yes & 1989 & 5370 & -- & 472 & 1.42 & 0.0596 & 0.308 & 5560 \\
\hline
\end{tabular}




\begin{tabular}{|c|c|c|c|c|c|c|c|c|c|c|c|}
\hline $\begin{array}{l}\text { U.S. } \\
\text { Geological } \\
\text { Survey } \\
\text { streamgage } \\
\text { number }\end{array}$ & $\begin{array}{l}\text { Segment } \\
\text { number }\end{array}$ & $\begin{array}{l}\text { Streamgage used in } \\
\text { regression analyses } \\
\text { (non-redundant) }\end{array}$ & $\begin{array}{l}\text { Discharge value } \\
\text { used in } \\
\text { adjustment } \\
\text { regression }\end{array}$ & $\begin{array}{l}\text { Water } \\
\text { year }\end{array}$ & $\begin{array}{c}\text { Observed annual } \\
\text { maximum peak } \\
\text { discharge }\left(\mathrm{ft}^{3} / \mathrm{s}\right)\end{array}$ & $\begin{array}{l}\text { NWIS peak } \\
\text { code }\end{array}$ & $\begin{array}{l}\text { Observed annual } \\
\text { maximum peak } \\
\text { discharge with } \\
\text { segment intercept } \\
\text { value subtracted } \\
\left(\mathrm{ft}^{3} / \mathrm{s}\right)\end{array}$ & $\begin{array}{l}\text { Observed } \\
\text { precipitation } \\
\text { (inches) }\end{array}$ & Urban fraction & $\begin{array}{l}\text { Exceedance } \\
\text { probability }\end{array}$ & $\begin{array}{c}\text { Urban-adjusted } \\
\text { annual maximum } \\
\text { peak discharge } \\
\left(\mathrm{ft}^{3} / \mathrm{s}\right)\end{array}$ \\
\hline 05439500 & 1 & Yes & Yes & 1990 & 2880 & -- & 253 & 0.55 & 0.06 & 0.715 & 3010 \\
\hline 05439500 & 1 & Yes & Yes & 1991 & 3070 & -- & 270 & 0.74 & 0.0612 & 0.682 & 3200 \\
\hline 05439500 & 1 & Yes & Yes & 1992 & 1250 & $E$ & 110 & 0.631 & 0.0623 & 0.941 & 1320 \\
\hline 05439500 & 1 & Yes & Yes & 1993 & 8190 & -- & 719 & 1.7 & 0.0634 & 0.1 & 8420 \\
\hline 05439500 & 1 & Yes & Yes & 1994 & 8790 & -- & 772 & 0.839 & 0.0646 & 0.0818 & 9020 \\
\hline 05439500 & 1 & Yes & Yes & 1995 & 2610 & -- & 229 & 0.898 & 0.0657 & 0.765 & 2710 \\
\hline 05439500 & 1 & Yes & Yes & 1996 & 25400 & -- & 2231 & 4.01 & 0.0669 & 0.00336 & 25900 \\
\hline 05439500 & 1 & Yes & Yes & 1997 & 9940 & -- & 873 & 2.17 & 0.068 & 0.0591 & 10200 \\
\hline 05439500 & 1 & Yes & Yes & 1998 & 3160 & -- & 278 & 0.794 & 0.0691 & 0.671 & 3260 \\
\hline 05439500 & 1 & Yes & Yes & 1999 & 12200 & -- & 1072 & 1.1 & 0.0703 & 0.0318 & 12400 \\
\hline 05439500 & 1 & Yes & Yes & 2000 & 2650 & -- & 233 & 0.874 & 0.0714 & 0.762 & 2730 \\
\hline 05439500 & 1 & Yes & Yes & 2001 & 3900 & -- & 343 & 0.707 & 0.0737 & 0.539 & 3990 \\
\hline 05439500 & 1 & Yes & Yes & 2002 & 11000 & -- & 966 & 2.26 & 0.0759 & 0.0449 & 11200 \\
\hline 05439500 & 1 & Yes & Yes & 2003 & 1820 & $E$ & 160 & 0.712 & 0.0782 & 0.887 & 1860 \\
\hline 05439500 & 1 & Yes & Yes & 2004 & 5110 & -- & 449 & 1.38 & 0.0804 & 0.353 & 5180 \\
\hline 05439500 & 1 & Yes & Yes & 2005 & 1700 & -- & 149 & 0.607 & 0.0826 & 0.902 & 1730 \\
\hline 05439500 & 1 & Yes & Yes & 2006 & 882 & $\mathrm{E}$ & 77 & 1.18 & 0.0849 & 0.971 & 898 \\
\hline 05439500 & 1 & Yes & Yes & 2007 & 7980 & -- & 701 & 2.54 & 0.0871 & 0.12 & 8030 \\
\hline 05439500 & 1 & Yes & Yes & 2008 & 4850 & -- & 426 & 0.294 & 0.0894 & 0.393 & 4870 \\
\hline 05439500 & 1 & Yes & Yes & 2009 & 5010 & -- & 440 & 0.832 & 0.0916 & 0.373 & 5020 \\
\hline 05439550 & 1 & Yes & Yes & 1959 & 19 & -- & 83 & 0.99 & 0.00436 & 0.956 & 21.3 \\
\hline 05439550 & 1 & Yes & Yes & 1960 & 154 & -- & 672 & 0.517 & 0.00436 & 0.106 & 163 \\
\hline 05439550 & 1 & Yes & Yes & 1961 & 16 & -- & 70 & 0.97 & 0.00523 & 0.968 & 18 \\
\hline 05439550 & 1 & Yes & Yes & 1962 & 71 & -- & 310 & 1.31 & 0.0061 & 0.557 & 76.2 \\
\hline 05439550 & 1 & Yes & Yes & 1963 & 43 & -- & 188 & 1.54 & 0.00697 & 0.811 & 46.7 \\
\hline 05439550 & 1 & Yes & Yes & 1964 & 9 & -- & 39 & 0.827 & 0.00784 & 1 & 10.3 \\
\hline 05439550 & 1 & Yes & Yes & 1965 & 67 & -- & 292 & 0.865 & 0.00871 & 0.602 & 71.8 \\
\hline 05439550 & 1 & Yes & Yes & 1966 & 23 & -- & 100 & 1.2 & 0.00959 & 0.941 & 25.4 \\
\hline 05439550 & 1 & Yes & Yes & 1967 & 56 & -- & 244 & 1.24 & 0.0105 & 0.701 & 60.1 \\
\hline 05439550 & 1 & Yes & Yes & 1968 & 73 & -- & 318 & 1.26 & 0.0113 & 0.54 & 77.8 \\
\hline 05439550 & 1 & Yes & Yes & 1969 & 89 & -- & 388 & 1.5 & 0.0122 & 0.399 & 94.5 \\
\hline 05439550 & 1 & Yes & Yes & 1970 & 152 & -- & 663 & 1.55 & 0.0131 & 0.115 & 160 \\
\hline 05439550 & 1 & Yes & Yes & 1971 & 452 & -- & 1972 & 0.34 & 0.0146 & 0.00485 & 469 \\
\hline 05439550 & 1 & Yes & Yes & 1972 & 219 & -- & 955 & 1.18 & 0.0161 & 0.0403 & 228 \\
\hline 05439550 & 1 & Yes & Yes & 1973 & 278 & -- & 1213 & 1.92 & 0.0176 & 0.0187 & 289 \\
\hline
\end{tabular}




\begin{tabular}{|c|c|c|c|c|c|c|c|c|c|c|c|}
\hline $\begin{array}{l}\text { U.S. } \\
\text { Geological } \\
\text { Survey } \\
\text { streamgage } \\
\text { number }\end{array}$ & $\begin{array}{l}\text { Segment } \\
\text { number }\end{array}$ & $\begin{array}{l}\text { Streamgage used in } \\
\text { regression analyses } \\
\text { (non-redundant) }\end{array}$ & $\begin{array}{l}\text { Discharge value } \\
\text { used in } \\
\text { adjustment } \\
\text { regression }\end{array}$ & $\begin{array}{l}\text { Water } \\
\text { year }\end{array}$ & $\begin{array}{c}\text { Observed annual } \\
\text { maximum peak } \\
\text { discharge }\left(\mathrm{ft}^{3} / \mathrm{s}\right)\end{array}$ & $\begin{array}{l}\text { NWIS peak } \\
\text { code }\end{array}$ & $\begin{array}{l}\text { Observed annual } \\
\text { maximum peak } \\
\text { discharge with } \\
\text { segment intercept } \\
\text { value subtracted } \\
\left(\mathrm{ft}^{3} / \mathrm{s}\right)\end{array}$ & $\begin{array}{l}\text { Observed } \\
\text { precipitation } \\
\text { (inches) }\end{array}$ & Urban fraction & $\begin{array}{l}\text { Exceedance } \\
\text { probability }\end{array}$ & $\begin{array}{c}\text { Urban-adjusted } \\
\text { annual maximum } \\
\text { peak discharge } \\
\left(\mathrm{ft}^{3} / \mathrm{s}\right)\end{array}$ \\
\hline 05439550 & 1 & Yes & Yes & 1974 & 314 & -- & 1370 & 1.17 & 0.0192 & 0.0146 & 325 \\
\hline 05439550 & 1 & Yes & Yes & 1975 & 97 & -- & 423 & 0.893 & 0.0207 & 0.351 & 102 \\
\hline 05439550 & 1 & Yes & Yes & 1976 & 74 & -- & 323 & 1.28 & 0.0222 & 0.539 & 77.9 \\
\hline 05440000 & 1 & No & No & 1940 & 3240 & -- & 151 & 0.665 & 0.0228 & 0.881 & 3890 \\
\hline 05440000 & 1 & No & No & 1941 & 4220 & -- & 197 & 0.0363 & 0.0231 & 0.802 & 4970 \\
\hline 05440000 & 1 & No & No & 1942 & 5840 & -- & 272 & 0.65 & 0.0235 & 0.651 & 6740 \\
\hline 05440000 & 1 & No & No & 1943 & 12600 & -- & 587 & 1.02 & 0.0239 & 0.168 & 14100 \\
\hline 05440000 & 1 & No & No & 1944 & 14800 & -- & 690 & 1.25 & 0.0242 & 0.103 & 16500 \\
\hline 05440000 & 1 & No & No & 1945 & 4360 & -- & 203 & 0.471 & 0.0246 & 0.791 & 5120 \\
\hline 05440000 & 1 & No & No & 1946 & 16400 & -- & 765 & 0.624 & 0.025 & 0.0752 & 18200 \\
\hline 05440000 & 1 & No & No & 1947 & 3990 & -- & 186 & 1.4 & 0.0253 & 0.824 & 4700 \\
\hline 05440000 & 1 & No & No & 1948 & 15200 & -- & 709 & 1.84 & 0.0257 & 0.0951 & 16900 \\
\hline 05440000 & 1 & No & No & 1949 & 9500 & 2 & 443 & 0.317 & 0.026 & 0.326 & 10700 \\
\hline 05440000 & 1 & No & No & 1950 & 8670 & -- & 404 & 1.35 & 0.0264 & 0.383 & 9820 \\
\hline 05440000 & 1 & No & No & 1951 & 11900 & -- & 555 & 0.206 & 0.0273 & 0.193 & 13300 \\
\hline 05440000 & 1 & No & No & 1952 & 10900 & -- & 508 & 0.538 & 0.0282 & 0.243 & 12200 \\
\hline 05440000 & 1 & No & No & 1953 & 2620 & $\mathrm{E}$ & 122 & 1.13 & 0.029 & 0.922 & 3160 \\
\hline 05440000 & 1 & No & No & 1954 & 4680 & -- & 218 & 1.46 & 0.0299 & 0.766 & 5430 \\
\hline 05440000 & 1 & No & No & 1955 & 8190 & -- & 382 & 3.11 & 0.0308 & 0.425 & 9260 \\
\hline 05440000 & 1 & No & No & 1956 & 2340 & -- & 109 & 0.643 & 0.0317 & 0.936 & 2830 \\
\hline 05440000 & 1 & No & No & 1957 & 2360 & $\mathrm{E}$ & 110 & 0.508 & 0.0325 & 0.935 & 2850 \\
\hline 05440000 & 1 & No & No & 1958 & 4650 & -- & 217 & 1.45 & 0.0334 & 0.77 & 5380 \\
\hline 05440000 & 1 & No & No & 1959 & 5890 & -- & 275 & 0.023 & 0.0343 & 0.653 & 6720 \\
\hline 05440000 & 1 & No & No & 1960 & 14100 & -- & 657 & 0.611 & 0.0352 & 0.127 & 15600 \\
\hline 05440000 & 1 & No & No & 1961 & 2690 & $\mathrm{E}$ & 125 & 0.763 & 0.0363 & 0.92 & 3210 \\
\hline 05440000 & 1 & No & No & 1962 & 10500 & -- & 490 & 0.319 & 0.0373 & 0.271 & 11700 \\
\hline 05440000 & 1 & No & No & 1963 & 2020 & E & 94 & 0.215 & 0.0384 & 0.952 & 2440 \\
\hline 05440000 & 1 & No & No & 1964 & 2080 & $\mathrm{E}$ & 97 & 0.716 & 0.0395 & 0.95 & 2510 \\
\hline 05440000 & 1 & No & No & 1965 & 8000 & 2 & 373 & 0.143 & 0.0406 & 0.449 & 8960 \\
\hline 05440000 & 1 & No & No & 1966 & 11300 & -- & 527 & 0.593 & 0.0416 & 0.229 & 12500 \\
\hline 05440000 & 1 & No & No & 1967 & 4550 & -- & 212 & 1.51 & 0.0427 & 0.784 & 5210 \\
\hline 05440000 & 1 & No & No & 1968 & 5940 & $\mathrm{E}$ & 277 & 1.55 & 0.0438 & 0.655 & 6700 \\
\hline 05440000 & 1 & No & No & 1969 & 11100 & -- & 517 & 0.113 & 0.0449 & 0.242 & 12200 \\
\hline 05440000 & 1 & No & No & 1970 & 5870 & -- & 274 & 1.85 & 0.0459 & 0.663 & 6610 \\
\hline 05440000 & 1 & No & No & 1971 & 14200 & -- & 662 & 0.462 & 0.0475 & 0.128 & 15500 \\
\hline
\end{tabular}




\begin{tabular}{|c|c|c|c|c|c|c|c|c|c|c|c|}
\hline $\begin{array}{c}\text { U.S. } \\
\text { Geological } \\
\text { Survey } \\
\text { streamgage } \\
\text { number }\end{array}$ & $\begin{array}{l}\text { Segment } \\
\text { number }\end{array}$ & $\begin{array}{l}\text { Streamgage used in } \\
\text { regression analyses } \\
\text { (non-redundant) }\end{array}$ & $\begin{array}{l}\text { Discharge value } \\
\text { used in } \\
\text { adjustment } \\
\text { regression }\end{array}$ & $\begin{array}{l}\text { Water } \\
\text { year }\end{array}$ & $\begin{array}{l}\text { Observed annual } \\
\text { maximum peak } \\
\text { discharge }\left(\mathrm{ft}^{3} / \mathrm{s}\right)\end{array}$ & $\begin{array}{l}\text { NWIS peak } \\
\text { code }\end{array}$ & $\begin{array}{l}\text { Observed annual } \\
\text { maximum peak } \\
\text { discharge with } \\
\text { segment intercept } \\
\text { value subtracted } \\
\left(\mathrm{ft}^{3} / \mathrm{s}\right)\end{array}$ & $\begin{array}{l}\text { Observed } \\
\text { precipitation } \\
\text { (inches) }\end{array}$ & Urban fraction & $\begin{array}{l}\text { Exceedance } \\
\text { probability }\end{array}$ & $\begin{array}{c}\text { Urban-adjusted } \\
\text { annual maximum } \\
\text { peak discharge } \\
\left(\mathrm{ft}^{3} / \mathrm{s}\right)\end{array}$ \\
\hline 05440000 & 1 & No & No & 1972 & 9080 & -- & 423 & 1.58 & 0.049 & 0.369 & 10000 \\
\hline 05440000 & 1 & No & No & 1973 & 14800 & -- & 690 & 1.78 & 0.0505 & 0.112 & 16100 \\
\hline 05440000 & 1 & No & No & 1974 & 13000 & -- & 606 & 1.18 & 0.052 & 0.166 & 14100 \\
\hline 05440000 & 1 & No & No & 1975 & 8060 & -- & 376 & 0.0239 & 0.0535 & 0.454 & 8900 \\
\hline 05440000 & 1 & No & No & 1976 & 9520 & -- & 444 & 1.26 & 0.055 & 0.344 & 10400 \\
\hline 05440000 & 1 & No & No & 1977 & 1340 & $\mathrm{E}$ & 62 & 0.485 & 0.0565 & 0.981 & 1600 \\
\hline 05440000 & 1 & No & No & 1978 & 13000 & -- & 606 & 1.8 & 0.0581 & 0.169 & 14100 \\
\hline 05440000 & 1 & No & No & 1979 & 16700 & -- & 779 & 0.305 & 0.0596 & 0.0784 & 18000 \\
\hline 05440000 & 1 & No & No & 1980 & 5040 & -- & 235 & 0.374 & 0.0611 & 0.753 & 5610 \\
\hline 05440000 & 1 & No & No & 1981 & 3760 & $E$ & 175 & 1.97 & 0.0621 & 0.857 & 4230 \\
\hline 05440000 & 1 & No & No & 1982 & 7990 & -- & 372 & 0.296 & 0.0631 & 0.468 & 8740 \\
\hline 05440000 & 1 & No & No & 1983 & 11300 & -- & 527 & 1.62 & 0.0642 & 0.242 & 12200 \\
\hline 05440000 & 1 & No & No & 1984 & 8820 & -- & 411 & 0.683 & 0.0652 & 0.399 & 9600 \\
\hline 05440000 & 1 & No & No & 1985 & 7970 & -- & 372 & 1.27 & 0.0662 & 0.472 & 8690 \\
\hline 05440000 & 1 & No & No & 1986 & 7890 & -- & 368 & 0.135 & 0.0672 & 0.48 & 8590 \\
\hline 05440000 & 1 & No & No & 1987 & 8720 & -- & 407 & 2.47 & 0.0683 & 0.409 & 9460 \\
\hline 05440000 & 1 & No & No & 1988 & 7580 & -- & 353 & 0.221 & 0.0693 & 0.511 & 8240 \\
\hline 05440000 & 1 & No & No & 1989 & 5570 & -- & 260 & 1.03 & 0.0703 & 0.709 & 6100 \\
\hline 05440000 & 1 & No & No & 1990 & 5240 & -- & 244 & 0.932 & 0.0713 & 0.741 & 5750 \\
\hline 05440000 & 1 & No & No & 1991 & 6170 & -- & 288 & 1.07 & 0.0734 & 0.654 & 6710 \\
\hline 05440000 & 1 & No & No & 1992 & 2710 & $\mathrm{E}$ & 126 & 0.626 & 0.0755 & 0.928 & 3040 \\
\hline 05440000 & 1 & No & No & 1993 & 11400 & -- & 531 & 0.814 & 0.0775 & 0.244 & 12100 \\
\hline 05440000 & 1 & No & No & 1994 & 17100 & -- & 797 & 0.997 & 0.0796 & 0.0768 & 18100 \\
\hline 05440000 & 1 & No & No & 1995 & 4260 & -- & 199 & 1.23 & 0.0817 & 0.828 & 4650 \\
\hline 05440000 & 1 & No & No & 1996 & 24200 & -- & 1128 & 2.88 & 0.0838 & 0.0278 & 25400 \\
\hline 05440000 & 1 & No & No & 1997 & 17400 & -- & 811 & 1.85 & 0.0858 & 0.0742 & 18300 \\
\hline 05440000 & 1 & No & No & 1998 & 5480 & -- & 255 & 0.922 & 0.0879 & 0.729 & 5880 \\
\hline 05440000 & 1 & No & No & 1999 & 16300 & -- & 760 & 1.46 & 0.09 & 0.092 & 17100 \\
\hline 05440000 & 1 & No & No & 2000 & 11300 & -- & 527 & 1.67 & 0.092 & 0.259 & 11900 \\
\hline 05440000 & 1 & No & No & 2001 & 8810 & -- & 411 & 0.683 & 0.0972 & 0.424 & 9270 \\
\hline 05440000 & 1 & No & No & 2002 & 15600 & -- & 727 & 2.43 & 0.102 & 0.11 & 16200 \\
\hline 05440000 & 1 & No & No & 2003 & 2360 & $\mathrm{E}$ & 110 & 0.368 & 0.108 & 0.95 & 2520 \\
\hline 05440000 & 1 & No & No & 2004 & 8420 & -- & 393 & 1.44 & 0.113 & 0.47 & 8710 \\
\hline 05440000 & 1 & No & No & 2005 & 4630 & -- & 216 & 0.653 & 0.118 & 0.816 & 4800 \\
\hline 05440000 & 1 & No & No & 2006 & 2970 & E & 138 & 0.798 & 0.123 & 0.927 & 3070 \\
\hline
\end{tabular}




\begin{tabular}{|c|c|c|c|c|c|c|c|c|c|c|c|}
\hline $\begin{array}{l}\text { U.S. } \\
\text { Geological } \\
\text { Survey } \\
\text { streamgage } \\
\text { number }\end{array}$ & $\begin{array}{l}\text { Segment } \\
\text { number }\end{array}$ & $\begin{array}{l}\text { Streamgage used in } \\
\text { regression analyses } \\
\text { (non-redundant) }\end{array}$ & $\begin{array}{c}\text { Discharge value } \\
\text { used in } \\
\text { adjustment } \\
\text { regression }\end{array}$ & $\begin{array}{l}\text { Water } \\
\text { year }\end{array}$ & $\begin{array}{l}\text { Observed annual } \\
\text { maximum peak } \\
\text { discharge }\left(\mathrm{ft}^{3} / \mathrm{s}\right)\end{array}$ & $\begin{array}{l}\text { NWIS peak } \\
\text { code }\end{array}$ & $\begin{array}{l}\text { Observed annual } \\
\text { maximum peak } \\
\text { discharge with } \\
\text { segment intercept } \\
\text { value subtracted } \\
\left(\mathrm{ft}^{3} / \mathrm{s}\right)\end{array}$ & $\begin{array}{l}\text { Observed } \\
\text { precipitation } \\
\text { (inches) }\end{array}$ & Urban fraction & $\begin{array}{l}\text { Exceedance } \\
\text { probability }\end{array}$ & $\begin{array}{c}\text { Urban-adjusted } \\
\text { annual maximum } \\
\text { peak discharge } \\
\left(\mathrm{ft}^{3} / \mathrm{s}\right)\end{array}$ \\
\hline 05440000 & 1 & No & No & 2007 & 14000 & -- & 653 & 1.51 & 0.128 & 0.165 & 14200 \\
\hline 05440000 & 1 & No & No & 2008 & 10300 & -- & 480 & 0.465 & 0.133 & 0.345 & 10400 \\
\hline 05440000 & 1 & No & No & 2009 & 14400 & -- & 671 & 1.02 & 0.139 & 0.157 & 14500 \\
\hline 05440500 & 1 & Yes & Yes & 1940 & 640 & 2 & 96 & 0.687 & 0.00779 & 0.945 & 656 \\
\hline 05440500 & 1 & Yes & Yes & 1941 & 2360 & -- & 353 & 0.583 & 0.00782 & 0.463 & 2400 \\
\hline 05440500 & 1 & Yes & Yes & 1942 & 1100 & -- & 164 & 0.615 & 0.00786 & 0.852 & 1120 \\
\hline 05440500 & 1 & Yes & Yes & 1943 & 3450 & -- & 516 & 1.08 & 0.00789 & 0.222 & 3490 \\
\hline 05440500 & 1 & Yes & Yes & 1944 & 2370 & -- & 354 & 1.6 & 0.00792 & 0.46 & 2410 \\
\hline 05440500 & 1 & Yes & Yes & 1945 & 2520 & -- & 377 & 1.26 & 0.00795 & 0.417 & 2560 \\
\hline 05440500 & 1 & Yes & Yes & 1946 & 4750 & -- & 710 & 0.654 & 0.00799 & 0.0905 & 4810 \\
\hline 05440500 & 1 & Yes & Yes & 1947 & 795 & -- & 119 & 1.36 & 0.00802 & 0.921 & 813 \\
\hline 05440500 & 1 & Yes & Yes & 1948 & 4390 & -- & 656 & 1.81 & 0.00805 & 0.117 & 4440 \\
\hline 05440500 & 1 & Yes & Yes & 1949 & 2620 & -- & 392 & 0.289 & 0.00809 & 0.391 & 2660 \\
\hline 05440500 & 1 & Yes & Yes & 1950 & 2820 & -- & 421 & 0.0146 & 0.00812 & 0.346 & 2860 \\
\hline 05440500 & 1 & Yes & Yes & 1951 & 6100 & D & 912 & 3.34 & 0.00831 & 0.0453 & 6170 \\
\hline 05440500 & 1 & Yes & Yes & 1952 & 4030 & -- & 602 & 2.57 & 0.0085 & 0.152 & 4080 \\
\hline 05440500 & 1 & Yes & Yes & 1953 & 770 & $\mathrm{E}$ & 115 & 0.342 & 0.00869 & 0.926 & 787 \\
\hline 05440500 & 1 & Yes & Yes & 1954 & 620 & $\mathrm{E}$ & 93 & 1.74 & 0.00888 & 0.949 & 634 \\
\hline 05440500 & 1 & Yes & Yes & 1955 & 6100 & -- & 912 & 4.73 & 0.00907 & 0.0454 & 6160 \\
\hline 05440500 & 1 & Yes & Yes & 1956 & 3050 & -- & 456 & 0.667 & 0.00925 & 0.298 & 3090 \\
\hline 05440500 & 1 & Yes & Yes & 1957 & 1450 & -- & 217 & 0.352 & 0.00944 & 0.759 & 1470 \\
\hline 05440500 & 1 & Yes & Yes & 1958 & 2490 & -- & 372 & 1.71 & 0.00963 & 0.427 & 2520 \\
\hline 05440500 & 1 & Yes & Yes & 1959 & 3610 & 2 & 540 & 0.352 & 0.00982 & 0.197 & 3650 \\
\hline 05440500 & 1 & Yes & Yes & 1960 & 2630 & -- & 393 & 0.705 & 0.01 & 0.39 & 2660 \\
\hline 05440500 & 1 & Yes & Yes & 1961 & 267 & $\mathrm{E}$ & 40 & 0.999 & 0.0102 & 1 & 274 \\
\hline 05440500 & 1 & Yes & Yes & 1962 & 3370 & -- & 504 & 0.471 & 0.0104 & 0.238 & 3410 \\
\hline 05440500 & 1 & Yes & Yes & 1963 & 978 & $\mathrm{E}$ & 146 & 0.147 & 0.0105 & 0.884 & 994 \\
\hline 05440500 & 1 & Yes & Yes & 1964 & 298 & $\mathrm{E}$ & 45 & 1.09 & 0.0107 & 0.989 & 305 \\
\hline 05440500 & 1 & Yes & Yes & 1965 & 1800 & 2 & 269 & 0.0448 & 0.0109 & 0.649 & 1820 \\
\hline 05440500 & 1 & Yes & Yes & 1966 & 4040 & $\mathrm{E}$ & 604 & 0.699 & 0.011 & 0.152 & 4080 \\
\hline 05440500 & 1 & Yes & Yes & 1967 & 612 & $\mathrm{E}$ & 91 & 1.11 & 0.0112 & 0.951 & 623 \\
\hline 05440500 & 1 & Yes & Yes & 1968 & 1650 & $\mathrm{E}$ & 247 & 1.14 & 0.0114 & 0.697 & 1670 \\
\hline 05440500 & 1 & Yes & Yes & 1969 & 3550 & -- & 531 & 0.152 & 0.0116 & 0.207 & 3580 \\
\hline 05440500 & 1 & Yes & Yes & 1970 & 1870 & -- & 279 & 1.86 & 0.0117 & 0.629 & 1890 \\
\hline 05440500 & 1 & Yes & Yes & 1971 & 3720 & -- & 556 & 0.465 & 0.0122 & 0.186 & 3750 \\
\hline
\end{tabular}




\begin{tabular}{|c|c|c|c|c|c|c|c|c|c|c|c|}
\hline $\begin{array}{c}\text { U.S. } \\
\text { Geological } \\
\text { Survey } \\
\text { streamgage } \\
\text { number } \\
\end{array}$ & $\begin{array}{l}\text { Segment } \\
\text { number }\end{array}$ & $\begin{array}{l}\text { Streamgage used in } \\
\text { regression analyses } \\
\text { (non-redundant) }\end{array}$ & $\begin{array}{c}\text { Discharge value } \\
\text { used in } \\
\text { adjustment } \\
\text { regression }\end{array}$ & $\begin{array}{c}\text { Water } \\
\text { year }\end{array}$ & $\begin{array}{c}\text { Observed annual } \\
\text { maximum peak } \\
\text { discharge }\left(\mathrm{ft}^{3} / \mathrm{s}\right)\end{array}$ & $\begin{array}{c}\text { NWIS peak } \\
\text { code }\end{array}$ & $\begin{array}{c}\text { Observed annual } \\
\text { maximum peak } \\
\text { discharge with } \\
\text { segment intercept } \\
\text { value subtracted } \\
\left(\mathrm{ft}^{3} / \mathrm{s}\right)\end{array}$ & $\begin{array}{c}\text { Observed } \\
\text { precipitation } \\
\text { (inches) }\end{array}$ & Urban fraction & $\begin{array}{l}\text { Exceedance } \\
\text { probability }\end{array}$ & $\begin{array}{c}\text { Urban-adjusted } \\
\text { annual maximum } \\
\text { peak discharge } \\
\left(\mathrm{ft}^{3} / \mathrm{s}\right)\end{array}$ \\
\hline 05440500 & 1 & Yes & Yes & 1972 & 4700 & -- & 702 & 1.56 & 0.0126 & 0.0943 & 4740 \\
\hline 05440500 & 1 & Yes & Yes & 1973 & 5570 & -- & 832 & 2.3 & 0.013 & 0.0594 & 5610 \\
\hline 05440500 & 1 & Yes & Yes & 1974 & 5400 & -- & 807 & 1.74 & 0.0135 & 0.0646 & 5440 \\
\hline 05440500 & 1 & Yes & Yes & 1975 & 3200 & -- & 478 & 0.00847 & 0.0139 & 0.272 & 3220 \\
\hline 05440500 & 1 & Yes & Yes & 1976 & 2670 & -- & 399 & 1.43 & 0.0143 & 0.383 & 2690 \\
\hline 05440500 & 1 & Yes & Yes & 1977 & 1330 & -- & 199 & 0.691 & 0.0148 & 0.794 & 1340 \\
\hline 05440500 & 1 & Yes & Yes & 1978 & 3190 & -- & 477 & 1.19 & 0.0152 & 0.274 & 3210 \\
\hline 05440500 & 1 & Yes & Yes & 1979 & 3900 & -- & 583 & 0.402 & 0.0156 & 0.168 & 3920 \\
\hline 05440500 & 1 & Yes & Yes & 1980 & 2830 & -- & 423 & 1.12 & 0.016 & 0.349 & 2850 \\
\hline 05442000 & 1 & Yes & Yes & 1940 & 800 & 2 & 209 & 0.796 & 0.0256 & 0.78 & 850 \\
\hline 05442000 & 1 & Yes & Yes & 1941 & 1100 & -- & 288 & 0.755 & 0.0263 & 0.622 & 1160 \\
\hline 05442000 & 1 & Yes & Yes & 1942 & 692 & -- & 181 & 1.31 & 0.027 & 0.832 & 736 \\
\hline 05442000 & 1 & Yes & Yes & 1943 & 1380 & -- & 361 & 1.12 & 0.0277 & 0.461 & 1450 \\
\hline 05442000 & 1 & Yes & Yes & 1944 & 1280 & -- & 335 & 1.72 & 0.0284 & 0.516 & 1340 \\
\hline 05442000 & 1 & Yes & Yes & 1945 & 889 & -- & 233 & 1.98 & 0.0291 & 0.738 & 938 \\
\hline 05442000 & 1 & Yes & Yes & 1946 & 2030 & -- & 532 & 0.652 & 0.0298 & 0.217 & 2110 \\
\hline 05442000 & 1 & Yes & Yes & 1947 & 889 & -- & 233 & 1.28 & 0.0305 & 0.739 & 936 \\
\hline 05442000 & 1 & Yes & Yes & 1948 & 1480 & -- & 388 & 1.83 & 0.0311 & 0.414 & 1550 \\
\hline 05442000 & 1 & Yes & Yes & 1949 & 1600 & -- & 419 & 0.00166 & 0.0318 & 0.365 & 1670 \\
\hline 05442000 & 1 & Yes & Yes & 1950 & 1330 & -- & 348 & 0.143 & 0.0325 & 0.491 & 1390 \\
\hline 05442000 & 1 & Yes & Yes & 1951 & 2630 & D & 689 & 0.575 & 0.033 & 0.107 & 2720 \\
\hline 05446950 & 1 & Yes & Yes & 1961 & 33 & -- & 115 & 0.59 & 0 & 0.925 & 33 \\
\hline 05446950 & 1 & Yes & Yes & 1962 & 31 & -- & 108 & 1.03 & 0 & 0.932 & 31 \\
\hline 05446950 & 1 & Yes & Yes & 1963 & 113 & -- & 392 & 0.44 & 0 & 0.384 & 113 \\
\hline 05446950 & 1 & Yes & Yes & 1964 & 34 & -- & 118 & 1.09 & 0 & 0.92 & 34 \\
\hline 05446950 & 1 & Yes & Yes & 1965 & 47 & -- & 163 & 0.863 & 0 & 0.851 & 47 \\
\hline 05446950 & 1 & Yes & Yes & 1966 & 36 & -- & 125 & 1.47 & 0 & 0.91 & 36 \\
\hline 05446950 & 1 & Yes & Yes & 1967 & 493 & -- & 1712 & 0.808 & 0 & 0.00774 & 493 \\
\hline 05446950 & 1 & Yes & Yes & 1968 & 37 & -- & 129 & 0.284 & 0 & 0.906 & 37 \\
\hline 05446950 & 1 & Yes & Yes & 1969 & 39 & -- & 135 & 0.166 & 0 & 0.896 & 39 \\
\hline 05446950 & 1 & Yes & Yes & 1970 & 348 & -- & 1209 & 1.53 & 0 & 0.0183 & 348 \\
\hline 05446950 & 1 & Yes & Yes & 1971 & 135 & -- & 469 & 0.335 & 0 & 0.275 & 135 \\
\hline 05446950 & 1 & Yes & Yes & 1972 & 280 & -- & 972 & 1.17 & 0 & 0.0366 & 280 \\
\hline 05446950 & 1 & Yes & Yes & 1973 & 244 & -- & 847 & 1.69 & 0 & 0.0547 & 244 \\
\hline 05446950 & 1 & Yes & Yes & 1974 & 227 & -- & 788 & 1.8 & 0 & 0.0665 & 227 \\
\hline
\end{tabular}




\begin{tabular}{|c|c|c|c|c|c|c|c|c|c|c|c|}
\hline $\begin{array}{c}\text { U.S. } \\
\text { Geological } \\
\text { Survey } \\
\text { streamgage } \\
\text { number }\end{array}$ & $\begin{array}{l}\text { Segment } \\
\text { number }\end{array}$ & $\begin{array}{l}\text { Streamgage used in } \\
\text { regression analyses } \\
\text { (non-redundant) }\end{array}$ & $\begin{array}{l}\text { Discharge value } \\
\text { used in } \\
\text { adjustment } \\
\text { regression }\end{array}$ & $\begin{array}{l}\text { Water } \\
\text { year }\end{array}$ & $\begin{array}{c}\text { Observed annual } \\
\text { maximum peak } \\
\text { discharge }\left(\mathrm{ft}^{3} / \mathrm{s}\right)\end{array}$ & $\begin{array}{l}\text { NWIS peak } \\
\text { code }\end{array}$ & $\begin{array}{l}\text { Observed annual } \\
\text { maximum peak } \\
\text { discharge with } \\
\text { segment intercept } \\
\text { value subtracted } \\
\left(\mathrm{ft}^{3} / \mathrm{s}\right)\end{array}$ & $\begin{array}{l}\text { Observed } \\
\text { precipitation } \\
\text { (inches) }\end{array}$ & Urban fraction & $\begin{array}{l}\text { Exceedance } \\
\text { probability }\end{array}$ & $\begin{array}{c}\text { Urban-adjusted } \\
\text { annual maximum } \\
\text { peak discharge } \\
\left(\mathrm{ft}^{3} / \mathrm{s}\right)\end{array}$ \\
\hline 05446950 & 1 & Yes & Yes & 1975 & 73 & -- & 254 & 0.854 & 0 & 0.674 & 73 \\
\hline 05446950 & 1 & Yes & Yes & 1976 & 51 & -- & 177 & 0.00212 & 0 & 0.828 & 51 \\
\hline 05447000 & 1 & Yes & Yes & 1940 & 2030 & -- & 250 & 0.532 & 0.0052 & 0.685 & 2040 \\
\hline 05447000 & 1 & Yes & Yes & 1941 & 2490 & -- & 307 & 0.577 & 0.00522 & 0.563 & 2510 \\
\hline 05447000 & 1 & Yes & Yes & 1942 & 2230 & -- & 275 & 1.61 & 0.00525 & 0.634 & 2240 \\
\hline 05447000 & 1 & Yes & Yes & 1943 & 2680 & -- & 330 & 0.678 & 0.00527 & 0.507 & 2700 \\
\hline 05447000 & 1 & Yes & Yes & 1944 & 2820 & -- & 348 & 1.38 & 0.00529 & 0.471 & 2840 \\
\hline 05447000 & 1 & Yes & Yes & 1945 & 2100 & -- & 259 & 1.02 & 0.00531 & 0.666 & 2110 \\
\hline 05447000 & 1 & Yes & Yes & 1946 & 3960 & -- & 488 & 0.634 & 0.00533 & 0.254 & 3980 \\
\hline 05447000 & 1 & Yes & Yes & 1947 & 2020 & -- & 249 & 0.966 & 0.00535 & 0.688 & 2030 \\
\hline 05447000 & 1 & Yes & Yes & 1948 & 3030 & -- & 374 & 1.83 & 0.00537 & 0.421 & 3050 \\
\hline 05447000 & 1 & Yes & Yes & 1949 & 2890 & -- & 356 & 0.17 & 0.00539 & 0.454 & 2910 \\
\hline 05447000 & 1 & Yes & Yes & 1950 & 2890 & -- & 356 & 1.39 & 0.00542 & 0.454 & 2910 \\
\hline 05447000 & 1 & Yes & Yes & 1951 & 2770 & -- & 342 & 0.35 & 0.00545 & 0.483 & 2790 \\
\hline 05447000 & 1 & Yes & Yes & 1952 & 2560 & -- & 316 & 0.487 & 0.00547 & 0.542 & 2570 \\
\hline 05447000 & 1 & Yes & Yes & 1953 & 615 & -- & 76 & 0.89 & 0.0055 & 0.962 & 621 \\
\hline 05447000 & 1 & Yes & Yes & 1954 & 1350 & -- & 166 & 1.58 & 0.00553 & 0.848 & 1360 \\
\hline 05447000 & 1 & Yes & Yes & 1955 & 6120 & -- & 755 & 3.2 & 0.00556 & 0.0745 & 6150 \\
\hline 05447000 & 1 & Yes & Yes & 1956 & 1790 & -- & 221 & 0.478 & 0.00559 & 0.75 & 1800 \\
\hline 05447000 & 1 & Yes & Yes & 1957 & 593 & -- & 73 & 0.417 & 0.00562 & 0.965 & 598 \\
\hline 05447000 & 1 & Yes & Yes & 1958 & 1910 & -- & 235 & 1.31 & 0.00565 & 0.717 & 1920 \\
\hline 05447000 & 1 & Yes & Yes & 1959 & 3740 & -- & 461 & 1.71 & 0.00568 & 0.289 & 3760 \\
\hline 05447000 & 1 & Yes & Yes & 1960 & 4240 & -- & 523 & 0.526 & 0.00571 & 0.213 & 4260 \\
\hline 05447000 & 1 & Yes & Yes & 1961 & 480 & -- & 59 & 2.41 & 0.00573 & 0.979 & 485 \\
\hline 05447000 & 1 & Yes & Yes & 1962 & 3280 & -- & 404 & 0.344 & 0.00575 & 0.37 & 3300 \\
\hline 05447000 & 1 & Yes & Yes & 1963 & 2220 & -- & 274 & 0.398 & 0.00577 & 0.636 & 2230 \\
\hline 05447000 & 1 & Yes & Yes & 1964 & 1030 & -- & 127 & 1.12 & 0.00579 & 0.909 & 1040 \\
\hline 05447000 & 1 & Yes & Yes & 1965 & 3290 & -- & 406 & 0.9 & 0.00581 & 0.368 & 3310 \\
\hline 05447000 & 1 & Yes & Yes & 1966 & 4600 & -- & 567 & 1.85 & 0.00583 & 0.176 & 4620 \\
\hline 05447000 & 1 & Yes & Yes & 1967 & 2530 & -- & 312 & 1.35 & 0.00585 & 0.551 & 2540 \\
\hline 05447000 & 1 & Yes & No & 1968 & 502 & 4, B & 62 & 0.00387 & 0.00588 & 0.976 & 507 \\
\hline 05447000 & 1 & Yes & Yes & 1969 & 4110 & -- & 507 & 0.184 & 0.0059 & 0.232 & 4130 \\
\hline 05447000 & 1 & Yes & Yes & 1970 & 4130 & -- & 509 & 1.67 & 0.00592 & 0.229 & 4150 \\
\hline 05447000 & 1 & Yes & Yes & 1971 & 3600 & -- & 444 & 0.374 & 0.00609 & 0.312 & 3620 \\
\hline 05447000 & 1 & Yes & Yes & 1972 & 4260 & -- & 525 & 1.45 & 0.00626 & 0.21 & 4280 \\
\hline
\end{tabular}




\begin{tabular}{|c|c|c|c|c|c|c|c|c|c|c|c|}
\hline $\begin{array}{l}\text { U.S. } \\
\text { Geological } \\
\text { Survey } \\
\text { streamgage } \\
\text { number }\end{array}$ & $\begin{array}{l}\text { Segment } \\
\text { number }\end{array}$ & $\begin{array}{l}\text { Streamgage used in } \\
\text { regression analyses } \\
\text { (non-redundant) }\end{array}$ & $\begin{array}{c}\text { Discharge value } \\
\text { used in } \\
\text { adjustment } \\
\text { regression }\end{array}$ & $\begin{array}{l}\text { Water } \\
\text { year }\end{array}$ & $\begin{array}{c}\text { Observed annual } \\
\text { maximum peak } \\
\text { discharge }\left(\mathrm{ft}^{3} / \mathrm{s}\right)\end{array}$ & $\begin{array}{l}\text { NWIS peak } \\
\text { code }\end{array}$ & $\begin{array}{l}\text { Observed annual } \\
\text { maximum peak } \\
\text { discharge with } \\
\text { segment intercept } \\
\text { value subtracted } \\
\left(\mathrm{ft}^{3} / \mathrm{s}\right)\end{array}$ & $\begin{array}{l}\text { Observed } \\
\text { precipitation } \\
\text { (inches) }\end{array}$ & Urban fraction & $\begin{array}{l}\text { Exceedance } \\
\text { probability }\end{array}$ & $\begin{array}{c}\text { Urban-adjusted } \\
\text { annual maximum } \\
\text { peak discharge } \\
\left(\mathrm{ft}^{3} / \mathrm{s}\right)\end{array}$ \\
\hline 05447000 & 1 & Yes & Yes & 1973 & 4460 & -- & 550 & 1.4 & 0.00643 & 0.188 & 4480 \\
\hline 05447000 & 1 & Yes & Yes & 1974 & 5010 & -- & 618 & 1.87 & 0.00659 & 0.141 & 5030 \\
\hline 05447000 & 1 & Yes & Yes & 1975 & 2740 & -- & 338 & 0.00387 & 0.00676 & 0.492 & 2750 \\
\hline 05447000 & 1 & Yes & Yes & 1976 & 2410 & -- & 297 & 1.27 & 0.00693 & 0.589 & 2420 \\
\hline 05447000 & 1 & Yes & Yes & 1978 & 4740 & -- & 584 & 2 & 0.00727 & 0.164 & 4750 \\
\hline 05447000 & 1 & Yes & Yes & 1979 & 5750 & -- & 709 & 0.323 & 0.00744 & 0.0907 & 5770 \\
\hline 05447000 & 1 & Yes & Yes & 1980 & 950 & 2 & 117 & 0.321 & 0.00761 & 0.923 & 955 \\
\hline 05447000 & 1 & Yes & Yes & 1981 & 7600 & -- & 937 & 1.91 & 0.00772 & 0.0418 & 7620 \\
\hline 05447000 & 1 & Yes & Yes & 1982 & 3530 & -- & 435 & 1.59 & 0.00782 & 0.326 & 3540 \\
\hline 05447050 & 1 & Yes & Yes & 1959 & 148 & -- & 354 & 0.738 & 0.00312 & 0.456 & 148 \\
\hline 05447050 & 1 & Yes & Yes & 1960 & 135 & -- & 323 & 1.22 & 0.00312 & 0.522 & 135 \\
\hline 05447050 & 1 & Yes & Yes & 1961 & 23 & -- & 55 & 2.26 & 0.00312 & 0.982 & 23 \\
\hline 05447050 & 1 & Yes & Yes & 1962 & 121 & -- & 290 & 1.02 & 0.00312 & 0.604 & 121 \\
\hline 05447050 & 1 & Yes & Yes & 1963 & 61 & -- & 146 & 0.467 & 0.00312 & 0.882 & 61 \\
\hline 05447050 & 1 & Yes & Yes & 1964 & 150 & -- & 359 & 1.09 & 0.00312 & 0.447 & 150 \\
\hline 05447050 & 1 & Yes & Yes & 1965 & 120 & -- & 287 & 0.807 & 0.00312 & 0.608 & 120 \\
\hline 05447050 & 1 & Yes & Yes & 1966 & 228 & -- & 546 & 1.47 & 0.00312 & 0.19 & 228 \\
\hline 05447050 & 1 & Yes & Yes & 1967 & 115 & -- & 275 & 0.573 & 0.00312 & 0.632 & 115 \\
\hline 05447050 & 1 & Yes & Yes & 1968 & 90 & -- & 215 & 1.52 & 0.00312 & 0.758 & 90 \\
\hline 05447050 & 1 & Yes & Yes & 1969 & 431 & -- & 1032 & 1.39 & 0.00312 & 0.0299 & 431 \\
\hline 05447050 & 1 & Yes & Yes & 1970 & 115 & -- & 275 & 1.67 & 0.00312 & 0.632 & 115 \\
\hline 05447050 & 1 & Yes & Yes & 1971 & 278 & -- & 665 & 0.388 & 0.00312 & 0.11 & 278 \\
\hline 05447050 & 1 & Yes & Yes & 1972 & 296 & -- & 709 & 0.136 & 0.00312 & 0.0899 & 296 \\
\hline 05447500 & 1 & Yes & Yes & 1940 & 3750 & -- & 219 & 0.531 & 0.0077 & 0.753 & 3780 \\
\hline 05447500 & 1 & Yes & Yes & 1941 & 4350 & -- & 254 & 1.3 & 0.00779 & 0.678 & 4380 \\
\hline 05447500 & 1 & Yes & Yes & 1942 & 5240 & -- & 306 & 1.08 & 0.00789 & 0.567 & 5270 \\
\hline 05447500 & 1 & Yes & Yes & 1943 & 5890 & -- & 344 & 1.71 & 0.00799 & 0.48 & 5930 \\
\hline 05447500 & 1 & Yes & Yes & 1944 & 6730 & -- & 393 & 1.55 & 0.00808 & 0.388 & 6770 \\
\hline 05447500 & 1 & Yes & Yes & 1945 & 4930 & -- & 288 & 1.53 & 0.00818 & 0.61 & 4960 \\
\hline 05447500 & 1 & Yes & Yes & 1946 & 8050 & -- & 471 & 0.704 & 0.00828 & 0.278 & 8090 \\
\hline 05447500 & 1 & Yes & Yes & 1947 & 6400 & -- & 374 & 2.23 & 0.00837 & 0.422 & 6440 \\
\hline 05447500 & 1 & Yes & Yes & 1948 & 6670 & -- & 390 & 1.19 & 0.00847 & 0.394 & 6710 \\
\hline 05447500 & 1 & Yes & Yes & 1949 & 6310 & -- & 369 & 0.0608 & 0.00857 & 0.432 & 6340 \\
\hline 05447500 & 1 & Yes & Yes & 1950 & 5590 & -- & 327 & 1.69 & 0.00866 & 0.518 & 5620 \\
\hline 05447500 & 1 & Yes & Yes & 1951 & 6940 & -- & 406 & 2.12 & 0.00875 & 0.369 & 6970 \\
\hline
\end{tabular}




\begin{tabular}{|c|c|c|c|c|c|c|c|c|c|c|c|}
\hline $\begin{array}{c}\text { U.S. } \\
\text { Geological } \\
\text { Survey } \\
\text { streamgage } \\
\text { number }\end{array}$ & $\begin{array}{l}\text { Segment } \\
\text { number }\end{array}$ & $\begin{array}{l}\text { Streamgage used in } \\
\text { regression analyses } \\
\text { (non-redundant) }\end{array}$ & $\begin{array}{l}\text { Discharge value } \\
\text { used in } \\
\text { adjustment } \\
\text { regression }\end{array}$ & $\begin{array}{l}\text { Water } \\
\text { year }\end{array}$ & $\begin{array}{c}\text { Observed annual } \\
\text { maximum peak } \\
\text { discharge }\left(\mathrm{ft}^{3} / \mathrm{s}\right)\end{array}$ & $\begin{array}{l}\text { NWIS peak } \\
\text { code }\end{array}$ & $\begin{array}{l}\text { Observed annual } \\
\text { maximum peak } \\
\text { discharge with } \\
\text { segment intercept } \\
\text { value subtracted } \\
\left(\mathrm{ft}^{3} / \mathrm{s}\right)\end{array}$ & $\begin{array}{l}\text { Observed } \\
\text { precipitation } \\
\text { (inches) }\end{array}$ & Urban fraction & $\begin{array}{l}\text { Exceedance } \\
\text { probability }\end{array}$ & $\begin{array}{c}\text { Urban-adjusted } \\
\text { annual maximum } \\
\text { peak discharge } \\
\left(\mathrm{ft}^{3} / \mathrm{s}\right)\end{array}$ \\
\hline 05447500 & 1 & Yes & Yes & 1952 & 5230 & -- & 306 & 0.587 & 0.00884 & 0.569 & 5260 \\
\hline 05447500 & 1 & Yes & Yes & 1953 & 3970 & -- & 232 & 1.52 & 0.00894 & 0.726 & 3990 \\
\hline 05447500 & 1 & Yes & Yes & 1954 & 2630 & -- & 154 & 1.89 & 0.00903 & 0.872 & 2650 \\
\hline 05447500 & 1 & Yes & Yes & 1955 & 8900 & -- & 520 & 3.1 & 0.00912 & 0.218 & 8940 \\
\hline 05447500 & 1 & Yes & Yes & 1956 & 3790 & -- & 222 & 0.489 & 0.00921 & 0.75 & 3810 \\
\hline 05447500 & 1 & Yes & Yes & 1957 & 1340 & -- & 78 & 0.656 & 0.0093 & 0.961 & 1350 \\
\hline 05447500 & 1 & Yes & Yes & 1958 & 3320 & -- & 194 & 1.15 & 0.00939 & 0.801 & 3340 \\
\hline 05447500 & 1 & Yes & Yes & 1959 & 7100 & -- & 415 & 0.373 & 0.00948 & 0.356 & 7130 \\
\hline 05447500 & 1 & Yes & Yes & 1960 & 8500 & -- & 497 & 0.383 & 0.00957 & 0.246 & 8530 \\
\hline 05447500 & 1 & Yes & Yes & 1961 & 4690 & -- & 274 & 2.43 & 0.00967 & 0.638 & 4710 \\
\hline 05447500 & 1 & Yes & Yes & 1962 & 7100 & -- & 415 & 0.324 & 0.00978 & 0.356 & 7130 \\
\hline 05447500 & 1 & Yes & Yes & 1963 & 3600 & 2 & 210 & 0.539 & 0.00988 & 0.77 & 3620 \\
\hline 05447500 & 1 & Yes & Yes & 1964 & 2380 & -- & 139 & 1.47 & 0.00998 & 0.894 & 2390 \\
\hline 05447500 & 1 & Yes & Yes & 1965 & 5910 & -- & 346 & 1.06 & 0.0101 & 0.479 & 5930 \\
\hline 05447500 & 1 & Yes & Yes & 1966 & 5240 & -- & 306 & 1.54 & 0.0102 & 0.569 & 5260 \\
\hline 05447500 & 1 & Yes & Yes & 1967 & 4500 & -- & 263 & 0.824 & 0.0103 & 0.661 & 4520 \\
\hline 05447500 & 1 & Yes & Yes & 1968 & 1690 & -- & 99 & 0.689 & 0.0104 & 0.942 & 1700 \\
\hline 05447500 & 1 & Yes & Yes & 1969 & 8000 & 2 & 468 & 0.292 & 0.0105 & 0.283 & 8020 \\
\hline 05447500 & 1 & Yes & Yes & 1970 & 9820 & -- & 574 & 1.81 & 0.0106 & 0.172 & 9850 \\
\hline 05447500 & 1 & Yes & Yes & 1971 & 9000 & 2 & 526 & 0.525 & 0.0107 & 0.212 & 9020 \\
\hline 05447500 & 1 & Yes & Yes & 1972 & 7600 & -- & 444 & 1.38 & 0.0109 & 0.315 & 7620 \\
\hline 05447500 & 1 & Yes & Yes & 1973 & 10900 & -- & 637 & 1.91 & 0.011 & 0.13 & 10900 \\
\hline 05447500 & 1 & Yes & Yes & 1974 & 12100 & -- & 707 & 1.65 & 0.0112 & 0.0921 & 12100 \\
\hline 05447500 & 1 & Yes & Yes & 1975 & 6160 & -- & 360 & 0.00186 & 0.0113 & 0.451 & 6170 \\
\hline 05447500 & 1 & Yes & Yes & 1976 & 6740 & -- & 394 & 1.31 & 0.0114 & 0.389 & 6750 \\
\hline 05447500 & 1 & Yes & Yes & 1977 & 2300 & -- & 134 & 1.04 & 0.0116 & 0.901 & 2310 \\
\hline 05447500 & 1 & Yes & Yes & 1978 & 8100 & -- & 474 & 1.57 & 0.0117 & 0.276 & 8110 \\
\hline 05447500 & 1 & Yes & Yes & 1979 & 12000 & -- & 702 & 0.372 & 0.0119 & 0.0945 & 12000 \\
\hline 05447500 & 1 & Yes & Yes & 1980 & 2500 & -- & 146 & 0.921 & 0.012 & 0.884 & 2510 \\
\hline 05447500 & 1 & Yes & Yes & 1981 & 7870 & -- & 460 & 2.19 & 0.0121 & 0.294 & 7880 \\
\hline 05447500 & 1 & Yes & Yes & 1982 & 7910 & -- & 462 & 1.2 & 0.0121 & 0.291 & 7920 \\
\hline 05447500 & 1 & Yes & Yes & 1983 & 7870 & -- & 460 & 1.36 & 0.0122 & 0.294 & 7880 \\
\hline 05447500 & 1 & Yes & Yes & 1984 & 4450 & -- & 260 & 0.678 & 0.0122 & 0.668 & 4460 \\
\hline 05447500 & 1 & Yes & Yes & 1985 & 8060 & -- & 471 & 1.07 & 0.0123 & 0.28 & 8070 \\
\hline 05447500 & 1 & Yes & Yes & 1986 & 8560 & -- & 500 & 1.51 & 0.0123 & 0.243 & 8570 \\
\hline
\end{tabular}




\begin{tabular}{|c|c|c|c|c|c|c|c|c|c|c|c|}
\hline $\begin{array}{c}\text { U.S. } \\
\text { Geological } \\
\text { Survey } \\
\text { streamgage } \\
\text { number } \\
\end{array}$ & $\begin{array}{c}\text { Segment } \\
\text { number }\end{array}$ & $\begin{array}{l}\text { Streamgage used in } \\
\text { regression analyses } \\
\text { (non-redundant) }\end{array}$ & $\begin{array}{c}\text { Discharge value } \\
\text { used in } \\
\text { adjustment } \\
\text { regression }\end{array}$ & $\begin{array}{c}\text { Water } \\
\text { year }\end{array}$ & $\begin{array}{c}\text { Observed annual } \\
\text { maximum peak } \\
\text { discharge }\left(\mathrm{ft}^{3} / \mathrm{s}\right)\end{array}$ & $\begin{array}{c}\text { NWIS peak } \\
\text { code }\end{array}$ & $\begin{array}{c}\text { Observed annual } \\
\text { maximum peak } \\
\text { discharge with } \\
\text { segment intercept } \\
\text { value subtracted } \\
\left(\mathrm{ft}^{3} / \mathrm{s}\right)\end{array}$ & $\begin{array}{c}\text { Observed } \\
\text { precipitation } \\
\text { (inches) }\end{array}$ & Urban fraction & $\begin{array}{l}\text { Exceedance } \\
\text { probability }\end{array}$ & $\begin{array}{c}\text { Urban-adjusted } \\
\text { annual maximum } \\
\text { peak discharge } \\
\left(\mathrm{ft}^{3} / \mathrm{s}\right)\end{array}$ \\
\hline 05447500 & 1 & Yes & Yes & 1987 & 10100 & -- & 591 & 2.1 & 0.0124 & 0.162 & 10100 \\
\hline 05447500 & 1 & Yes & Yes & 1988 & 6430 & -- & 376 & 0.625 & 0.0124 & 0.422 & 6440 \\
\hline 05447500 & 1 & Yes & Yes & 1989 & 3380 & -- & 198 & 1.17 & 0.0125 & 0.795 & 3380 \\
\hline 05447500 & 1 & Yes & Yes & 1990 & 10100 & -- & 591 & 2.05 & 0.0126 & 0.162 & 10100 \\
\hline 05447500 & 1 & Yes & Yes & 1991 & 6710 & -- & 392 & 0.848 & 0.0126 & 0.393 & 6720 \\
\hline 05447500 & 1 & Yes & Yes & 1992 & 5550 & -- & 324 & 1.97 & 0.0127 & 0.527 & 5550 \\
\hline 05447500 & 1 & Yes & Yes & 1993 & 9600 & -- & 561 & 1.89 & 0.0128 & 0.183 & 9610 \\
\hline 05447500 & 1 & Yes & Yes & 1994 & 7370 & -- & 431 & 0.775 & 0.0129 & 0.335 & 7370 \\
\hline 05447500 & 1 & Yes & Yes & 1995 & 7050 & -- & 412 & 1.34 & 0.013 & 0.362 & 7050 \\
\hline 05447500 & 1 & Yes & Yes & 1996 & 8180 & -- & 478 & 0.98 & 0.0131 & 0.271 & 8180 \\
\hline 05447500 & 1 & Yes & Yes & 1997 & 10300 & -- & 602 & 2.23 & 0.0132 & 0.154 & 10300 \\
\hline 05447500 & 1 & Yes & Yes & 1998 & 4800 & -- & 281 & 0.926 & 0.0133 & 0.628 & 4800 \\
\hline 05447500 & 1 & Yes & Yes & 1999 & 10300 & -- & 602 & 2.49 & 0.0133 & 0.154 & 10300 \\
\hline 05447500 & 1 & Yes & Yes & 2000 & 4480 & -- & 262 & 0.788 & 0.0134 & 0.665 & 4480 \\
\hline 05447500 & 1 & Yes & Yes & 2001 & 6520 & -- & 381 & 0.744 & 0.0134 & 0.413 & 6520 \\
\hline 05447500 & 1 & Yes & Yes & 2002 & 9520 & -- & 557 & 2.22 & 0.0134 & 0.186 & 9520 \\
\hline 05447500 & 1 & Yes & Yes & 2003 & 5340 & -- & 312 & 1.08 & 0.0135 & 0.557 & 5340 \\
\hline 05447500 & 1 & Yes & Yes & 2004 & 5840 & -- & 341 & 1.14 & 0.0135 & 0.49 & 5840 \\
\hline 05447500 & 1 & Yes & Yes & 2005 & 2490 & $\mathrm{E}$ & 146 & 0.562 & 0.0135 & 0.886 & 2490 \\
\hline 05447500 & 1 & Yes & Yes & 2006 & 996 & $\mathrm{E}$ & 58 & 0.783 & 0.0135 & 0.98 & 996 \\
\hline 05447500 & 1 & Yes & Yes & 2007 & 7610 & -- & 445 & 0.519 & 0.0135 & 0.316 & 7610 \\
\hline 05447500 & 1 & Yes & Yes & 2008 & 9110 & -- & 533 & 3.89 & 0.0135 & 0.206 & 9110 \\
\hline 05447500 & 1 & Yes & Yes & 2009 & 9930 & -- & 581 & 0.765 & 0.0135 & 0.169 & 9930 \\
\hline 05517500 & 1 & Yes & Yes & 1949 & 2980 & -- & 224 & 0.195 & 0.0238 & 0.754 & 3240 \\
\hline 05517500 & 1 & Yes & Yes & 1950 & 5200 & -- & 390 & 0.499 & 0.0244 & 0.404 & 5580 \\
\hline 05517500 & 1 & Yes & Yes & 1951 & 3230 & -- & 242 & 0.0326 & 0.0254 & 0.715 & 3500 \\
\hline 05517500 & 1 & Yes & Yes & 1952 & 3380 & -- & 254 & 0.178 & 0.0264 & 0.692 & 3650 \\
\hline 05517500 & 1 & Yes & Yes & 1953 & 2190 & -- & 164 & 0.159 & 0.0273 & 0.861 & 2400 \\
\hline 05517500 & 1 & Yes & Yes & 1954 & 3100 & -- & 233 & 0.402 & 0.0283 & 0.738 & 3350 \\
\hline 05517500 & 1 & Yes & Yes & 1955 & 5300 & -- & 398 & 0.00239 & 0.0293 & 0.395 & 5650 \\
\hline 05517500 & 1 & Yes & Yes & 1956 & 3800 & -- & 285 & 0.18 & 0.0303 & 0.63 & 4080 \\
\hline 05517500 & 1 & Yes & Yes & 1957 & 3100 & -- & 233 & 0.633 & 0.0312 & 0.74 & 3340 \\
\hline 05517500 & 1 & Yes & Yes & 1958 & 2730 & -- & 205 & 0.361 & 0.0322 & 0.792 & 2950 \\
\hline 05517500 & 1 & Yes & Yes & 1959 & 3850 & -- & 289 & 0.0818 & 0.0332 & 0.624 & 4110 \\
\hline 05517500 & 1 & Yes & Yes & 1960 & 3660 & -- & 275 & 0.0277 & 0.0341 & 0.653 & 3910 \\
\hline
\end{tabular}




\begin{tabular}{|c|c|c|c|c|c|c|c|c|c|c|c|}
\hline $\begin{array}{c}\text { U.S. } \\
\text { Geological } \\
\text { Survey } \\
\text { streamgage } \\
\text { number } \\
\end{array}$ & $\begin{array}{l}\text { Segment } \\
\text { number }\end{array}$ & $\begin{array}{l}\text { Streamgage used in } \\
\text { regression analyses } \\
\text { (non-redundant) }\end{array}$ & $\begin{array}{c}\text { Discharge value } \\
\text { used in } \\
\text { adjustment } \\
\text { regression }\end{array}$ & $\begin{array}{c}\text { Water } \\
\text { year }\end{array}$ & $\begin{array}{c}\text { Observed annual } \\
\text { maximum peak } \\
\text { discharge }\left(\mathrm{ft}^{3} / \mathrm{s}\right)\end{array}$ & $\begin{array}{c}\text { NWIS peak } \\
\text { code }\end{array}$ & $\begin{array}{c}\text { Observed annual } \\
\text { maximum peak } \\
\text { discharge with } \\
\text { segment intercept } \\
\text { value subtracted } \\
\left(\mathrm{ft}^{3} / \mathrm{s}\right)\end{array}$ & $\begin{array}{c}\text { Observed } \\
\text { precipitation } \\
\text { (inches) }\end{array}$ & Urban fraction & $\begin{array}{l}\text { Exceedance } \\
\text { probability }\end{array}$ & $\begin{array}{c}\text { Urban-adjusted } \\
\text { annual maximum } \\
\text { peak discharge } \\
\left(\mathrm{ft}^{3} / \mathrm{s}\right)\end{array}$ \\
\hline 05517500 & 1 & Yes & Yes & 1961 & 3950 & -- & 296 & 0.192 & 0.0349 & 0.611 & 4210 \\
\hline 05517500 & 1 & Yes & Yes & 1962 & 3900 & -- & 293 & 0.0101 & 0.0357 & 0.619 & 4150 \\
\hline 05517500 & 1 & Yes & Yes & 1963 & 2910 & -- & 218 & 0.259 & 0.0365 & 0.769 & 3120 \\
\hline 05517500 & 1 & Yes & Yes & 1964 & 1700 & -- & 128 & 0.288 & 0.0373 & 0.917 & 1850 \\
\hline 05517500 & 1 & Yes & Yes & 1965 & 3000 & -- & 225 & 0.0389 & 0.0382 & 0.758 & 3210 \\
\hline 05517500 & 1 & Yes & Yes & 1966 & 3360 & -- & 252 & 0.059 & 0.039 & 0.703 & 3580 \\
\hline 05517500 & 1 & Yes & Yes & 1967 & 3610 & -- & 271 & 0.0817 & 0.0398 & 0.664 & 3830 \\
\hline 05517500 & 1 & Yes & Yes & 1968 & 4120 & -- & 309 & 0.0457 & 0.0406 & 0.588 & 4360 \\
\hline 05517500 & 1 & Yes & Yes & 1969 & 3800 & -- & 285 & 0.224 & 0.0414 & 0.637 & 4020 \\
\hline 05517500 & 1 & Yes & Yes & 1970 & 3450 & -- & 259 & 0.0942 & 0.0422 & 0.691 & 3660 \\
\hline 05517500 & 1 & Yes & Yes & 1971 & 2590 & -- & 194 & 0.38 & 0.0437 & 0.817 & 2760 \\
\hline 05517500 & 1 & Yes & Yes & 1972 & 3140 & -- & 236 & 0.344 & 0.0452 & 0.742 & 3320 \\
\hline 05517500 & 1 & Yes & Yes & 1973 & 3720 & -- & 279 & 0.439 & 0.0467 & 0.652 & 3920 \\
\hline 05517500 & 1 & Yes & Yes & 1974 & 3530 & -- & 265 & 0.121 & 0.0482 & 0.683 & 3710 \\
\hline 05517500 & 1 & Yes & Yes & 1975 & 3510 & -- & 263 & 0.301 & 0.0497 & 0.687 & 3690 \\
\hline 05517500 & 1 & Yes & Yes & 1976 & 4310 & -- & 323 & 0.741 & 0.0512 & 0.563 & 4500 \\
\hline 05517500 & 1 & Yes & Yes & 1977 & 3670 & -- & 275 & 0.484 & 0.0527 & 0.664 & 3840 \\
\hline 05517500 & 1 & Yes & Yes & 1978 & 4540 & -- & 341 & 0.296 & 0.0542 & 0.526 & 4720 \\
\hline 05517500 & 1 & Yes & Yes & 1979 & 4550 & -- & 341 & 0.149 & 0.0557 & 0.525 & 4730 \\
\hline 05517500 & 1 & Yes & Yes & 1980 & 3170 & -- & 238 & 0.119 & 0.0572 & 0.745 & 3300 \\
\hline 05517500 & 1 & Yes & Yes & 1981 & 4890 & -- & 367 & 0.205 & 0.0581 & 0.474 & 5060 \\
\hline 05517500 & 1 & Yes & Yes & 1982 & 5870 & -- & 441 & 0.54 & 0.059 & 0.351 & 6060 \\
\hline 05517500 & 1 & Yes & Yes & 1983 & 4480 & -- & 336 & 0.205 & 0.0599 & 0.541 & 4630 \\
\hline 05517500 & 1 & Yes & Yes & 1984 & 3320 & -- & 249 & 0.251 & 0.0608 & 0.724 & 3440 \\
\hline 05517500 & 1 & Yes & Yes & 1985 & 5370 & -- & 403 & 0.485 & 0.0617 & 0.41 & 5530 \\
\hline 05517500 & 1 & Yes & Yes & 1986 & 3190 & -- & 239 & 0.223 & 0.0626 & 0.745 & 3300 \\
\hline 05517500 & 1 & Yes & Yes & 1987 & 3530 & -- & 265 & 0.169 & 0.0636 & 0.693 & 3640 \\
\hline 05517500 & 1 & Yes & Yes & 1988 & 3390 & -- & 254 & 0.0698 & 0.0645 & 0.715 & 3500 \\
\hline 05517500 & 1 & Yes & Yes & 1989 & 3450 & -- & 259 & 0.0599 & 0.0654 & 0.707 & 3560 \\
\hline 05517500 & 1 & Yes & Yes & 1990 & 4250 & -- & 319 & 0.391 & 0.0663 & 0.587 & 4370 \\
\hline 05517500 & 1 & Yes & Yes & 1991 & 4930 & -- & 370 & 0.179 & 0.068 & 0.476 & 5050 \\
\hline 05517500 & 1 & Yes & Yes & 1992 & 2270 & -- & 170 & 0.134 & 0.0696 & 0.869 & 2340 \\
\hline 05517500 & 1 & Yes & Yes & 1993 & 4820 & -- & 362 & 0.411 & 0.0713 & 0.495 & 4920 \\
\hline 05517500 & 1 & Yes & Yes & 1994 & 4310 & -- & 323 & 0.0611 & 0.073 & 0.582 & 4390 \\
\hline 05517500 & 1 & Yes & Yes & 1995 & 3550 & -- & 266 & 0.103 & 0.0747 & 0.698 & 3620 \\
\hline
\end{tabular}




\begin{tabular}{|c|c|c|c|c|c|c|c|c|c|c|c|}
\hline $\begin{array}{l}\text { U.S. } \\
\text { Geological } \\
\text { Survey } \\
\text { streamgage } \\
\text { number }\end{array}$ & $\begin{array}{l}\text { Segment } \\
\text { number }\end{array}$ & $\begin{array}{l}\text { Streamgage used in } \\
\text { regression analyses } \\
\text { (non-redundant) }\end{array}$ & $\begin{array}{c}\text { Discharge value } \\
\text { used in } \\
\text { adjustment } \\
\text { regression }\end{array}$ & $\begin{array}{l}\text { Water } \\
\text { year }\end{array}$ & $\begin{array}{c}\text { Observed annual } \\
\text { maximum peak } \\
\text { discharge }\left(\mathrm{ft}^{3} / \mathrm{s}\right)\end{array}$ & $\begin{array}{l}\text { NWIS peak } \\
\text { code }\end{array}$ & $\begin{array}{l}\text { Observed annual } \\
\text { maximum peak } \\
\text { discharge with } \\
\text { segment intercept } \\
\text { value subtracted } \\
\left(\mathrm{ft}^{3} / \mathrm{s}\right)\end{array}$ & $\begin{array}{l}\text { Observed } \\
\text { precipitation } \\
\text { (inches) }\end{array}$ & Urban fraction & $\begin{array}{l}\text { Exceedance } \\
\text { probability }\end{array}$ & $\begin{array}{c}\text { Urban-adjusted } \\
\text { annual maximum } \\
\text { peak discharge } \\
\left(\mathrm{ft}^{3} / \mathrm{s}\right)\end{array}$ \\
\hline 05517500 & 1 & Yes & Yes & 1996 & 4650 & -- & 349 & 0.0794 & 0.0764 & 0.527 & 4720 \\
\hline 05517500 & 1 & Yes & Yes & 1997 & 4500 & -- & 338 & 0.375 & 0.0781 & 0.554 & 4560 \\
\hline 05517500 & 1 & Yes & Yes & 1998 & 4360 & -- & 327 & 0.266 & 0.0797 & 0.579 & 4410 \\
\hline 05517500 & 1 & Yes & Yes & 1999 & 4140 & -- & 311 & 0.196 & 0.0814 & 0.615 & 4180 \\
\hline 05517500 & 1 & Yes & Yes & 2000 & 3310 & -- & 248 & 0.815 & 0.0831 & 0.74 & 3340 \\
\hline 05517500 & 1 & Yes & Yes & 2001 & 3920 & -- & 294 & 1.53 & 0.0837 & 0.648 & 3950 \\
\hline 05517500 & 1 & Yes & Yes & 2002 & 4180 & -- & 314 & 0.362 & 0.0844 & 0.612 & 4200 \\
\hline 05517500 & 1 & Yes & Yes & 2003 & 2950 & -- & 221 & 0.336 & 0.085 & 0.789 & 2970 \\
\hline 05517500 & 1 & Yes & Yes & 2004 & 2960 & -- & 222 & 0.037 & 0.0856 & 0.788 & 2980 \\
\hline 05517500 & 1 & Yes & Yes & 2005 & 4520 & -- & 339 & 0.0176 & 0.0863 & 0.557 & 4540 \\
\hline 05517500 & 1 & Yes & Yes & 2006 & 2980 & -- & 224 & 0.106 & 0.0869 & 0.786 & 2990 \\
\hline 05517500 & 1 & Yes & Yes & 2007 & 4170 & -- & 313 & 0.28 & 0.0875 & 0.615 & 4180 \\
\hline 05517500 & 1 & Yes & Yes & 2008 & 5420 & -- & 407 & 0.0747 & 0.0881 & 0.424 & 5430 \\
\hline 05517500 & 1 & Yes & Yes & 2009 & 5510 & -- & 414 & 0.102 & 0.0888 & 0.413 & 5510 \\
\hline 05517890 & 1 & Yes & Yes & 1968 & 194 & -- & 123 & 1.86 & 0.0216 & 0.919 & 280 \\
\hline 05517890 & 1 & Yes & Yes & 1969 & 300 & 2 & 191 & 0.507 & 0.0224 & 0.813 & 408 \\
\hline 05517890 & 1 & Yes & Yes & 1970 & 444 & -- & 282 & 0.645 & 0.0233 & 0.631 & 578 \\
\hline 05517890 & 1 & Yes & No & 1971 & 290 & 1,8 & 184 & 0.528 & 0.027 & 0.827 & 394 \\
\hline 05517890 & 1 & Yes & Yes & 1972 & 466 & -- & 296 & 0.742 & 0.0307 & 0.609 & 600 \\
\hline 05517890 & 1 & Yes & Yes & 1973 & 744 & -- & 473 & 1.4 & 0.0344 & 0.291 & 919 \\
\hline 05517890 & 1 & Yes & Yes & 1974 & 347 & -- & 220 & 0.9 & 0.0382 & 0.766 & 456 \\
\hline 05517890 & 1 & Yes & Yes & 1975 & 576 & -- & 366 & 1.38 & 0.0419 & 0.464 & 723 \\
\hline 05517890 & 1 & Yes & Yes & 1976 & 777 & -- & 494 & 1.02 & 0.0456 & 0.271 & 946 \\
\hline 05517890 & 1 & Yes & Yes & 1977 & 220 & -- & 140 & 0.791 & 0.0493 & 0.905 & 300 \\
\hline 05517890 & 1 & Yes & Yes & 1978 & 498 & -- & 316 & 0.35 & 0.053 & 0.582 & 623 \\
\hline 05517890 & 1 & Yes & Yes & 1979 & 650 & -- & 413 & 0.837 & 0.0567 & 0.39 & 796 \\
\hline 05517890 & 1 & Yes & Yes & 1980 & 252 & -- & 160 & 0.339 & 0.0604 & 0.881 & 333 \\
\hline 05517890 & 1 & Yes & Yes & 1981 & 761 & -- & 483 & 2.34 & 0.0622 & 0.294 & 915 \\
\hline 05517890 & 1 & Yes & Yes & 1982 & 751 & -- & 477 & 0.354 & 0.0639 & 0.303 & 902 \\
\hline 05517890 & 1 & Yes & Yes & 1983 & 763 & -- & 485 & 2.73 & 0.0657 & 0.295 & 914 \\
\hline 05517890 & 1 & Yes & Yes & 1984 & 531 & -- & 337 & 0.91 & 0.0674 & 0.545 & 651 \\
\hline 05517890 & 1 & Yes & Yes & 1985 & 795 & -- & 505 & 0.765 & 0.0692 & 0.271 & 946 \\
\hline 05517890 & 1 & Yes & Yes & 1986 & 1070 & -- & 680 & 1.33 & 0.0709 & 0.126 & 1260 \\
\hline 05517890 & 1 & Yes & Yes & 1987 & 661 & -- & 420 & 1.03 & 0.0727 & 0.391 & 796 \\
\hline 05517890 & 1 & Yes & Yes & 1988 & 424 & -- & 269 & 0.855 & 0.0744 & 0.692 & 524 \\
\hline
\end{tabular}




\begin{tabular}{|c|c|c|c|c|c|c|c|c|c|c|c|}
\hline $\begin{array}{l}\text { U.S. } \\
\text { Geological } \\
\text { Survey } \\
\text { streamgage } \\
\text { number }\end{array}$ & $\begin{array}{l}\text { Segment } \\
\text { number }\end{array}$ & $\begin{array}{l}\text { Streamgage used in } \\
\text { regression analyses } \\
\text { (non-redundant) }\end{array}$ & $\begin{array}{c}\text { Discharge value } \\
\text { used in } \\
\text { adjustment } \\
\text { regression }\end{array}$ & $\begin{array}{l}\text { Water } \\
\text { year }\end{array}$ & $\begin{array}{l}\text { Observed annual } \\
\text { maximum peak } \\
\text { discharge }\left(\mathrm{ft}^{3} / \mathrm{s}\right)\end{array}$ & $\begin{array}{l}\text { NWIS peak } \\
\text { code }\end{array}$ & $\begin{array}{l}\text { Observed annual } \\
\text { maximum peak } \\
\text { discharge with } \\
\text { segment intercept } \\
\text { value subtracted } \\
\left(\mathrm{ft}^{3} / \mathrm{s}\right)\end{array}$ & $\begin{array}{l}\text { Observed } \\
\text { precipitation } \\
\text { (inches) }\end{array}$ & Urban fraction & $\begin{array}{l}\text { Exceedance } \\
\text { probability }\end{array}$ & $\begin{array}{c}\text { Urban-adjusted } \\
\text { annual maximum } \\
\text { peak discharge } \\
\left(\mathrm{ft}^{3} / \mathrm{s}\right)\end{array}$ \\
\hline 05517890 & 1 & Yes & Yes & 1989 & 916 & -- & 582 & 1.98 & 0.0762 & 0.192 & 1070 \\
\hline 05517890 & 1 & Yes & Yes & 1990 & 1070 & -- & 680 & 2.03 & 0.0779 & 0.129 & 1250 \\
\hline 05517890 & 1 & Yes & Yes & 1991 & 1160 & -- & 737 & 2.13 & 0.0866 & 0.0993 & 1340 \\
\hline 05517890 & 1 & Yes & Yes & 1992 & 236 & $\mathrm{E}$ & 150 & 0.677 & 0.0952 & 0.905 & 300 \\
\hline 05517890 & 1 & Yes & Yes & 1993 & 779 & -- & 495 & 1.18 & 0.104 & 0.305 & 899 \\
\hline 05517890 & 1 & Yes & Yes & 1994 & 481 & -- & 306 & 1.32 & 0.113 & 0.646 & 565 \\
\hline 05517890 & 1 & Yes & Yes & 1995 & 426 & -- & 271 & 0.866 & 0.121 & 0.721 & 499 \\
\hline 05517890 & 1 & Yes & Yes & 1996 & 991 & -- & 630 & 3.53 & 0.13 & 0.181 & 1100 \\
\hline 05517890 & 1 & Yes & Yes & 1997 & 1030 & -- & 654 & 1.04 & 0.139 & 0.168 & 1140 \\
\hline 05517890 & 1 & Yes & Yes & 1998 & 593 & -- & 377 & 0.705 & 0.147 & 0.53 & 664 \\
\hline 05517890 & 1 & Yes & Yes & 1999 & 643 & -- & 408 & 0.846 & 0.156 & 0.476 & 711 \\
\hline 05517890 & 1 & Yes & Yes & 2000 & 700 & -- & 445 & 1.37 & 0.165 & 0.42 & 765 \\
\hline 05517890 & 1 & Yes & Yes & 2001 & 548 & -- & 348 & 0.866 & 0.173 & 0.611 & 598 \\
\hline 05517890 & 1 & Yes & Yes & 2002 & 585 & -- & 372 & 1.23 & 0.181 & 0.571 & 631 \\
\hline 05517890 & 1 & Yes & Yes & 2003 & 808 & -- & 513 & 1.3 & 0.189 & 0.339 & 857 \\
\hline 05518000 & 1 & No & No & 1940 & 3600 & -- & 231 & 0.447 & 0.0164 & 0.735 & 4010 \\
\hline 05518000 & 1 & No & No & 1941 & 2150 & -- & 138 & 0.27 & 0.017 & 0.898 & 2450 \\
\hline 05518000 & 1 & No & No & 1942 & 4260 & -- & 273 & 0.161 & 0.0176 & 0.646 & 4710 \\
\hline 05518000 & 1 & No & No & 1943 & 6350 & -- & 407 & 0.533 & 0.0182 & 0.374 & 6930 \\
\hline 05518000 & 1 & No & No & 1944 & 4820 & -- & 309 & 0.685 & 0.0188 & 0.571 & 5300 \\
\hline 05518000 & 1 & No & No & 1945 & 3550 & -- & 227 & 0.0551 & 0.0194 & 0.744 & 3940 \\
\hline 05518000 & 1 & No & No & 1946 & 3200 & -- & 205 & 0.68 & 0.02 & 0.785 & 3570 \\
\hline 05518000 & 1 & No & No & 1947 & 3970 & -- & 254 & 0.592 & 0.0206 & 0.687 & 4380 \\
\hline 05518000 & 1 & No & No & 1948 & 4420 & -- & 283 & 0.31 & 0.0212 & 0.628 & 4860 \\
\hline 05518000 & 1 & No & No & 1949 & 3500 & -- & 224 & 0.214 & 0.0218 & 0.752 & 3880 \\
\hline 05518000 & 1 & No & No & 1950 & 5910 & -- & 378 & 0.0193 & 0.0224 & 0.425 & 6440 \\
\hline 05518000 & 1 & No & No & 1951 & 3890 & -- & 249 & 0.341 & 0.0233 & 0.7 & 4280 \\
\hline 05518000 & 1 & No & No & 1952 & 4200 & -- & 269 & 0.923 & 0.0242 & 0.658 & 4610 \\
\hline 05518000 & 1 & No & No & 1953 & 2650 & -- & 170 & 0.179 & 0.0251 & 0.851 & 2960 \\
\hline 05518000 & 1 & No & No & 1954 & 3620 & -- & 232 & 0.379 & 0.026 & 0.738 & 3990 \\
\hline 05518000 & 1 & No & No & 1955 & 5040 & -- & 323 & 0.0565 & 0.0269 & 0.544 & 5480 \\
\hline 05518000 & 1 & No & No & 1956 & 4540 & -- & 291 & 0.702 & 0.0278 & 0.618 & 4950 \\
\hline 05518000 & 1 & No & No & 1957 & 4240 & -- & 271 & 0.762 & 0.0287 & 0.656 & 4630 \\
\hline 05518000 & 1 & No & No & 1958 & 3480 & -- & 223 & 1.23 & 0.0296 & 0.758 & 3820 \\
\hline 05518000 & 1 & No & No & 1959 & 5100 & -- & 327 & 0.0816 & 0.0305 & 0.538 & 5530 \\
\hline
\end{tabular}




\begin{tabular}{|c|c|c|c|c|c|c|c|c|c|c|c|}
\hline $\begin{array}{c}\text { U.S. } \\
\text { Geological } \\
\text { Survey } \\
\text { streamgage } \\
\text { number }\end{array}$ & $\begin{array}{l}\text { Segment } \\
\text { number }\end{array}$ & $\begin{array}{l}\text { Streamgage used in } \\
\text { regression analyses } \\
\text { (non-redundant) }\end{array}$ & $\begin{array}{l}\text { Discharge value } \\
\text { used in } \\
\text { adjustment } \\
\text { regression }\end{array}$ & $\begin{array}{c}\text { Water } \\
\text { year }\end{array}$ & $\begin{array}{l}\text { Observed annual } \\
\text { maximum peak } \\
\text { discharge }\left(\mathrm{ft}^{3} / \mathrm{s}\right)\end{array}$ & $\begin{array}{l}\text { NWIS peak } \\
\text { code }\end{array}$ & $\begin{array}{l}\text { Observed annual } \\
\text { maximum peak } \\
\text { discharge with } \\
\text { segment intercept } \\
\text { value subtracted } \\
\left(\mathrm{ft}^{3} / \mathrm{s}\right)\end{array}$ & $\begin{array}{l}\text { Observed } \\
\text { precipitation } \\
\text { (inches) }\end{array}$ & Urban fraction & $\begin{array}{l}\text { Exceedance } \\
\text { probability }\end{array}$ & $\begin{array}{c}\text { Urban-adjusted } \\
\text { annual maximum } \\
\text { peak discharge } \\
\left(\mathrm{ft}^{3} / \mathrm{s}\right)\end{array}$ \\
\hline 05518000 & 1 & No & No & 1960 & 4160 & -- & 266 & 0.0252 & 0.0314 & 0.668 & 4530 \\
\hline 05518000 & 1 & No & No & 1961 & 4640 & -- & 297 & 0.179 & 0.0322 & 0.608 & 5030 \\
\hline 05518000 & 1 & No & No & 1962 & 4840 & -- & 310 & 0.439 & 0.033 & 0.58 & 5240 \\
\hline 05518000 & 1 & No & No & 1963 & 3410 & -- & 218 & 0.139 & 0.0338 & 0.768 & 3730 \\
\hline 05518000 & 1 & No & No & 1964 & 2200 & 2 & 141 & 0.286 & 0.0345 & 0.899 & 2440 \\
\hline 05518000 & 1 & No & No & 1965 & 3760 & -- & 241 & 0.741 & 0.0353 & 0.725 & 4090 \\
\hline 05518000 & 1 & No & No & 1966 & 4270 & -- & 273 & 0.257 & 0.0361 & 0.657 & 4620 \\
\hline 05518000 & 1 & No & No & 1967 & 4770 & -- & 305 & 0.431 & 0.0369 & 0.595 & 5140 \\
\hline 05518000 & 1 & No & No & 1968 & 5020 & -- & 321 & 0.0527 & 0.0376 & 0.556 & 5400 \\
\hline 05518000 & 1 & No & No & 1969 & 4770 & -- & 305 & 0.238 & 0.0384 & 0.596 & 5130 \\
\hline 05518000 & 1 & No & No & 1970 & 4030 & -- & 258 & 1.57 & 0.0392 & 0.691 & 4350 \\
\hline 05518000 & 1 & No & No & 1971 & 3590 & -- & 230 & 0.398 & 0.0411 & 0.752 & 3880 \\
\hline 05518000 & 1 & No & No & 1972 & 4220 & -- & 270 & 0.37 & 0.0429 & 0.668 & 4530 \\
\hline 05518000 & 1 & No & No & 1973 & 5060 & -- & 324 & 0.382 & 0.0448 & 0.556 & 5400 \\
\hline 05518000 & 1 & No & No & 1974 & 4700 & -- & 301 & 0.865 & 0.0467 & 0.611 & 5010 \\
\hline 05518000 & 1 & No & No & 1975 & 4720 & -- & 302 & 0.525 & 0.0486 & 0.609 & 5020 \\
\hline 05518000 & 1 & No & No & 1976 & 5200 & -- & 333 & 0.823 & 0.0504 & 0.54 & 5510 \\
\hline 05518000 & 1 & No & No & 1977 & 4250 & -- & 272 & 0.47 & 0.0523 & 0.671 & 4510 \\
\hline 05518000 & 1 & No & No & 1978 & 5490 & -- & 352 & 0.97 & 0.0542 & 0.502 & 5790 \\
\hline 05518000 & 1 & No & No & 1979 & 5420 & -- & 347 & 0.834 & 0.056 & 0.513 & 5700 \\
\hline 05518000 & 1 & No & No & 1980 & 4030 & -- & 258 & 0.134 & 0.0579 & 0.704 & 4250 \\
\hline 05518000 & 1 & No & No & 1981 & 6200 & -- & 397 & 0.192 & 0.0589 & 0.419 & 6490 \\
\hline 05518000 & 1 & No & No & 1982 & 7650 & -- & 490 & 0.225 & 0.06 & 0.285 & 7970 \\
\hline 05518000 & 1 & No & No & 1983 & 5120 & -- & 328 & 0.414 & 0.061 & 0.561 & 5360 \\
\hline 05518000 & 1 & No & No & 1984 & 4290 & -- & 275 & 0.226 & 0.0621 & 0.672 & 4500 \\
\hline 05518000 & 1 & No & No & 1985 & 6340 & -- & 406 & 0.0167 & 0.0631 & 0.406 & 6610 \\
\hline 05518000 & 1 & No & No & 1986 & 4700 & -- & 301 & 0.51 & 0.0642 & 0.622 & 4910 \\
\hline 05518000 & 1 & No & No & 1987 & 4450 & -- & 285 & 0.203 & 0.0652 & 0.654 & 4650 \\
\hline 05518000 & 1 & No & No & 1988 & 4300 & -- & 275 & 0.381 & 0.0662 & 0.674 & 4490 \\
\hline 05518000 & 1 & No & No & 1989 & 4830 & -- & 309 & 0.726 & 0.0673 & 0.609 & 5030 \\
\hline 05518000 & 1 & No & No & 1990 & 5150 & -- & 330 & 0.44 & 0.0683 & 0.563 & 5350 \\
\hline 05518000 & 1 & No & No & 1991 & 5660 & -- & 362 & 0.229 & 0.0705 & 0.493 & 5860 \\
\hline 05518000 & 1 & No & No & 1992 & 2820 & -- & 181 & 0.385 & 0.0726 & 0.852 & 2940 \\
\hline 05518000 & 1 & No & No & 1993 & 5580 & -- & 357 & 0.346 & 0.0748 & 0.507 & 5750 \\
\hline 05518000 & 1 & No & No & 1994 & 5480 & -- & 351 & 0.143 & 0.0769 & 0.523 & 5630 \\
\hline
\end{tabular}




\begin{tabular}{|c|c|c|c|c|c|c|c|c|c|c|c|}
\hline $\begin{array}{l}\text { U.S. } \\
\text { Geological } \\
\text { Survey } \\
\text { streamgage } \\
\text { number }\end{array}$ & $\begin{array}{c}\text { Segment } \\
\text { number }\end{array}$ & $\begin{array}{l}\text { Streamgage used in } \\
\text { regression analyses } \\
\text { (non-redundant) }\end{array}$ & $\begin{array}{c}\text { Discharge value } \\
\text { used in } \\
\text { adjustment } \\
\text { regression }\end{array}$ & $\begin{array}{l}\text { Water } \\
\text { year }\end{array}$ & $\begin{array}{c}\text { Observed annual } \\
\text { maximum peak } \\
\text { discharge }\left(\mathrm{ft}^{3} / \mathrm{s}\right)\end{array}$ & $\begin{array}{l}\text { NWIS peak } \\
\text { code }\end{array}$ & $\begin{array}{l}\text { Observed annual } \\
\text { maximum peak } \\
\text { discharge with } \\
\text { segment intercept } \\
\text { value subtracted } \\
\left(\mathrm{ft}^{3} / \mathrm{s}\right)\end{array}$ & $\begin{array}{l}\text { Observed } \\
\text { precipitation } \\
\text { (inches) }\end{array}$ & Urban fraction & $\begin{array}{l}\text { Exceedance } \\
\text { probability }\end{array}$ & $\begin{array}{c}\text { Urban-adjusted } \\
\text { annual maximum } \\
\text { peak discharge } \\
\left(\mathrm{ft}^{3} / \mathrm{s}\right)\end{array}$ \\
\hline 05518000 & 1 & No & No & 1995 & 4430 & -- & 284 & 0.197 & 0.0791 & 0.666 & 4550 \\
\hline 05518000 & 1 & No & No & 1996 & 5480 & -- & 351 & 0.242 & 0.0812 & 0.527 & 5600 \\
\hline 05518000 & 1 & No & No & 1997 & 5850 & -- & 375 & 0.334 & 0.0834 & 0.48 & 5970 \\
\hline 05518000 & 1 & No & No & 1998 & 5220 & -- & 334 & 0.351 & 0.0855 & 0.568 & 5310 \\
\hline 05518000 & 1 & No & No & 1999 & 5160 & -- & 330 & 0.215 & 0.0877 & 0.579 & 5240 \\
\hline 05518000 & 1 & No & No & 2000 & 4480 & -- & 287 & 0.624 & 0.0898 & 0.667 & 4540 \\
\hline 05518000 & 1 & No & No & 2001 & 4600 & -- & 295 & 1.28 & 0.091 & 0.652 & 4660 \\
\hline 05518000 & 1 & No & No & 2002 & 5110 & -- & 327 & 0.282 & 0.0921 & 0.59 & 5160 \\
\hline 05518000 & 1 & No & No & 2003 & 4510 & -- & 289 & 0.532 & 0.0932 & 0.665 & 4550 \\
\hline 05518000 & 1 & No & No & 2004 & 3570 & -- & 229 & 0.027 & 0.0944 & 0.781 & 3600 \\
\hline 05518000 & 1 & No & No & 2005 & 5570 & -- & 357 & 0.411 & 0.0955 & 0.527 & 5610 \\
\hline 05518000 & 1 & No & No & 2006 & 3500 & -- & 224 & 0.0985 & 0.0966 & 0.79 & 3520 \\
\hline 05518000 & 1 & No & No & 2007 & 5500 & -- & 352 & 0.399 & 0.0978 & 0.539 & 5520 \\
\hline 05518000 & 1 & No & No & 2008 & 6230 & -- & 399 & 0.0294 & 0.0989 & 0.447 & 6250 \\
\hline 05518000 & 1 & No & No & 2009 & 6460 & -- & 414 & 0.455 & 0.1 & 0.422 & 6470 \\
\hline 05519000 & 1 & Yes & No & 1949 & 550 & 1,8 & 174 & 0.668 & 0.0442 & 0.851 & 657 \\
\hline 05519000 & 1 & Yes & No & 1950 & 1100 & 1,8 & 348 & 0.606 & 0.0453 & 0.502 & 1260 \\
\hline 05519000 & 1 & Yes & Yes & 1951 & 841 & -- & 266 & 0.345 & 0.047 & 0.68 & 975 \\
\hline 05519000 & 1 & Yes & Yes & 1952 & 1010 & -- & 319 & 1.2 & 0.0487 & 0.571 & 1160 \\
\hline 05519000 & 1 & Yes & Yes & 1953 & 812 & -- & 257 & 0.538 & 0.0504 & 0.702 & 939 \\
\hline 05519000 & 1 & Yes & Yes & 1954 & 810 & -- & 256 & 1.66 & 0.052 & 0.704 & 935 \\
\hline 05519000 & 1 & Yes & Yes & 1955 & 953 & -- & 301 & 2.81 & 0.0537 & 0.614 & 1090 \\
\hline 05519000 & 1 & Yes & Yes & 1956 & 1000 & -- & 316 & 0.42 & 0.0554 & 0.584 & 1140 \\
\hline 05519000 & 1 & Yes & Yes & 1957 & 1110 & -- & 351 & 0.623 & 0.0571 & 0.506 & 1260 \\
\hline 05519000 & 1 & Yes & Yes & 1958 & 799 & -- & 253 & 1.31 & 0.0588 & 0.715 & 916 \\
\hline 05519000 & 1 & Yes & Yes & 1959 & 1120 & -- & 354 & 0.082 & 0.0604 & 0.502 & 1260 \\
\hline 05519000 & 1 & Yes & Yes & 1960 & 1100 & -- & 348 & 1.05 & 0.0621 & 0.517 & 1240 \\
\hline 05519000 & 1 & Yes & Yes & 1961 & 1050 & -- & 332 & 0.656 & 0.0648 & 0.555 & 1180 \\
\hline 05519000 & 1 & Yes & Yes & 1962 & 1020 & -- & 323 & 0.448 & 0.0675 & 0.58 & 1140 \\
\hline 05519000 & 1 & Yes & No & 1963 & 1000 & 1,8 & 316 & 0.517 & 0.0702 & 0.597 & 1120 \\
\hline 05519000 & 1 & Yes & Yes & 1964 & 200 & -- & 63 & 0.93 & 0.0729 & 0.982 & 243 \\
\hline 05519000 & 1 & Yes & Yes & 1965 & 978 & -- & 309 & 1.24 & 0.0756 & 0.614 & 1090 \\
\hline 05519000 & 1 & Yes & Yes & 1966 & 1100 & -- & 348 & 2.62 & 0.0783 & 0.531 & 1220 \\
\hline 05519000 & 1 & Yes & Yes & 1967 & 1030 & -- & 326 & 1.2 & 0.081 & 0.584 & 1140 \\
\hline 05519000 & 1 & Yes & Yes & 1968 & 1140 & -- & 361 & 1.69 & 0.0837 & 0.508 & 1250 \\
\hline
\end{tabular}




\begin{tabular}{|c|c|c|c|c|c|c|c|c|c|c|c|}
\hline $\begin{array}{l}\text { U.S. } \\
\text { Geological } \\
\text { Survey } \\
\text { streamgage } \\
\text { number }\end{array}$ & $\begin{array}{c}\text { Segment } \\
\text { number }\end{array}$ & $\begin{array}{l}\text { Streamgage used in } \\
\text { regression analyses } \\
\text { (non-redundant) }\end{array}$ & $\begin{array}{c}\text { Discharge value } \\
\text { used in } \\
\text { adjustment } \\
\text { regression }\end{array}$ & $\begin{array}{l}\text { Water } \\
\text { year }\end{array}$ & $\begin{array}{c}\text { Observed annual } \\
\text { maximum peak } \\
\text { discharge }\left(\mathrm{ft}^{3} / \mathrm{s}\right)\end{array}$ & $\begin{array}{l}\text { NWIS peak } \\
\text { code }\end{array}$ & $\begin{array}{l}\text { Observed annual } \\
\text { maximum peak } \\
\text { discharge with } \\
\text { segment intercept } \\
\text { value subtracted } \\
\left(\mathrm{ft}^{3} / \mathrm{s}\right)\end{array}$ & $\begin{array}{l}\text { Observed } \\
\text { precipitation } \\
\text { (inches) }\end{array}$ & Urban fraction & $\begin{array}{l}\text { Exceedance } \\
\text { probability }\end{array}$ & $\begin{array}{c}\text { Urban-adjusted } \\
\text { annual maximum } \\
\text { peak discharge } \\
\left(\mathrm{ft}^{3} / \mathrm{s}\right)\end{array}$ \\
\hline 05519000 & 1 & Yes & Yes & 1969 & 952 & -- & 301 & 0.506 & 0.0863 & 0.637 & 1050 \\
\hline 05519000 & 1 & Yes & Yes & 1970 & 1160 & -- & 367 & 1.93 & 0.089 & 0.499 & 1270 \\
\hline 05519000 & 1 & Yes & Yes & 1971 & 615 & -- & 194 & 0.764 & 0.0914 & 0.838 & 685 \\
\hline 05519000 & 1 & Yes & Yes & 1972 & 969 & -- & 306 & 3.36 & 0.0938 & 0.632 & 1060 \\
\hline 05519000 & 1 & Yes & Yes & 1973 & 1230 & -- & 389 & 1.61 & 0.0962 & 0.463 & 1330 \\
\hline 05519000 & 1 & Yes & Yes & 1974 & 1380 & -- & 436 & 1.7 & 0.0986 & 0.384 & 1490 \\
\hline 05519000 & 1 & Yes & Yes & 1975 & 1350 & -- & 427 & 0.625 & 0.101 & 0.4 & 1450 \\
\hline 05519000 & 1 & Yes & Yes & 1976 & 3550 & -- & 1123 & 1.33 & 0.103 & 0.0296 & 3740 \\
\hline 05519000 & 1 & Yes & Yes & 1977 & 370 & 2 & 117 & 0.2 & 0.106 & 0.942 & 412 \\
\hline 05519000 & 1 & Yes & Yes & 1978 & 1630 & -- & 515 & 0.766 & 0.108 & 0.282 & 1730 \\
\hline 05519000 & 1 & Yes & Yes & 1979 & 1700 & 2 & 538 & 1.03 & 0.11 & 0.257 & 1800 \\
\hline 05519000 & 1 & Yes & Yes & 1980 & 525 & -- & 166 & 1.48 & 0.113 & 0.89 & 571 \\
\hline 05519000 & 1 & Yes & Yes & 1981 & 2000 & -- & 632 & 1.7 & 0.114 & 0.173 & 2100 \\
\hline 05519000 & 1 & Yes & Yes & 1982 & 1910 & -- & 604 & 0.556 & 0.115 & 0.192 & 2010 \\
\hline 05519000 & 1 & Yes & Yes & 1983 & 2150 & -- & 680 & 1.2 & 0.116 & 0.143 & 2250 \\
\hline 05519000 & 1 & Yes & Yes & 1984 & 1350 & -- & 427 & 1.43 & 0.117 & 0.412 & 1430 \\
\hline 05519000 & 1 & Yes & Yes & 1985 & 2120 & -- & 670 & 1.22 & 0.118 & 0.15 & 2220 \\
\hline 05519000 & 1 & Yes & Yes & 1986 & 2070 & -- & 655 & 1.46 & 0.12 & 0.16 & 2160 \\
\hline 05519000 & 1 & Yes & Yes & 1987 & 1300 & -- & 411 & 1.4 & 0.121 & 0.442 & 1370 \\
\hline 05519000 & 1 & Yes & Yes & 1988 & 947 & -- & 299 & 0.743 & 0.122 & 0.664 & 1000 \\
\hline 05519000 & 1 & Yes & Yes & 1989 & 2260 & -- & 715 & 2.48 & 0.123 & 0.125 & 2350 \\
\hline 05519000 & 1 & Yes & Yes & 1990 & 1300 & -- & 411 & 1.18 & 0.124 & 0.445 & 1360 \\
\hline 05519000 & 1 & Yes & Yes & 1991 & 2460 & -- & 778 & 3.39 & 0.128 & 0.0947 & 2550 \\
\hline 05519000 & 1 & Yes & Yes & 1992 & 547 & $E$ & 173 & 0.529 & 0.131 & 0.887 & 578 \\
\hline 05519000 & 1 & Yes & Yes & 1993 & 1940 & -- & 614 & 1.39 & 0.135 & 0.193 & 2000 \\
\hline 05519000 & 1 & Yes & Yes & 1994 & 1750 & -- & 553 & 1.73 & 0.138 & 0.256 & 1800 \\
\hline 05519000 & 1 & Yes & Yes & 1995 & 1340 & -- & 424 & 1.24 & 0.142 & 0.437 & 1380 \\
\hline 05519000 & 1 & Yes & Yes & 1996 & 1870 & -- & 591 & 3.78 & 0.145 & 0.219 & 1910 \\
\hline 05519000 & 1 & Yes & Yes & 1997 & 2270 & -- & 718 & 1.91 & 0.149 & 0.133 & 2310 \\
\hline 05519000 & 1 & Yes & Yes & 1998 & 1950 & -- & 617 & 0.586 & 0.152 & 0.198 & 1980 \\
\hline 05519000 & 1 & Yes & Yes & 1999 & 1590 & -- & 503 & 1.35 & 0.156 & 0.33 & 1610 \\
\hline 05519000 & 1 & Yes & Yes & 2000 & 1900 & -- & 601 & 2.2 & 0.16 & 0.217 & 1910 \\
\hline 05519000 & 1 & Yes & Yes & 2001 & 1400 & -- & 443 & 0.564 & 0.16 & 0.42 & 1410 \\
\hline 05519500 & 1 & Yes & Yes & 1949 & 504 & -- & 203 & 0.685 & 0.0312 & 0.794 & 631 \\
\hline 05519500 & 1 & Yes & Yes & 1950 & 1050 & -- & 424 & 1.52 & 0.0335 & 0.359 & 1250 \\
\hline
\end{tabular}




\begin{tabular}{|c|c|c|c|c|c|c|c|c|c|c|c|}
\hline $\begin{array}{l}\text { U.S. } \\
\text { Geological } \\
\text { Survey } \\
\text { streamgage } \\
\text { number }\end{array}$ & $\begin{array}{c}\text { Segment } \\
\text { number }\end{array}$ & $\begin{array}{l}\text { Streamgage used in } \\
\text { regression analyses } \\
\text { (non-redundant) }\end{array}$ & $\begin{array}{c}\text { Discharge value } \\
\text { used in } \\
\text { adjustment } \\
\text { regression }\end{array}$ & $\begin{array}{l}\text { Water } \\
\text { year }\end{array}$ & $\begin{array}{c}\text { Observed annual } \\
\text { maximum peak } \\
\text { discharge }\left(\mathrm{ft}^{3} / \mathrm{s}\right)\end{array}$ & $\begin{array}{l}\text { NWIS peak } \\
\text { code }\end{array}$ & $\begin{array}{l}\text { Observed annual } \\
\text { maximum peak } \\
\text { discharge with } \\
\text { segment intercept } \\
\text { value subtracted } \\
\left(\mathrm{ft}^{3} / \mathrm{s}\right)\end{array}$ & $\begin{array}{l}\text { Observed } \\
\text { precipitation } \\
\text { (inches) }\end{array}$ & Urban fraction & $\begin{array}{l}\text { Exceedance } \\
\text { probability }\end{array}$ & $\begin{array}{c}\text { Urban-adjusted } \\
\text { annual maximum } \\
\text { peak discharge } \\
\left(\mathrm{ft}^{3} / \mathrm{s}\right)\end{array}$ \\
\hline 05519500 & 1 & Yes & Yes & 1951 & 738 & -- & 298 & 0.342 & 0.0364 & 0.61 & 892 \\
\hline 05519500 & 1 & Yes & Yes & 1952 & 584 & -- & 236 & 0.918 & 0.0394 & 0.738 & 716 \\
\hline 05519500 & 1 & Yes & Yes & 1954 & 1000 & -- & 404 & 1.64 & 0.0453 & 0.397 & 1180 \\
\hline 05519500 & 1 & Yes & Yes & 1955 & 1840 & -- & 743 & 2.89 & 0.0482 & 0.0882 & 2100 \\
\hline 05519500 & 1 & Yes & Yes & 1956 & 710 & -- & 287 & 0.426 & 0.0512 & 0.641 & 846 \\
\hline 05519500 & 1 & Yes & Yes & 1957 & 1250 & -- & 505 & 2.52 & 0.0541 & 0.263 & 1440 \\
\hline 05519500 & 1 & Yes & Yes & 1958 & 794 & -- & 320 & 1.2 & 0.0571 & 0.575 & 935 \\
\hline 05519500 & 1 & Yes & Yes & 1959 & 1200 & -- & 484 & 1.24 & 0.06 & 0.292 & 1380 \\
\hline 05519500 & 1 & Yes & Yes & 1960 & 940 & -- & 379 & 1.03 & 0.063 & 0.454 & 1090 \\
\hline 05519500 & 1 & Yes & Yes & 1961 & 880 & -- & 355 & 0.894 & 0.0648 & 0.503 & 1020 \\
\hline 05519500 & 1 & Yes & Yes & 1962 & 950 & -- & 383 & 0.253 & 0.0666 & 0.45 & 1100 \\
\hline 05519500 & 1 & Yes & No & 1963 & 360 & 1,8 & 145 & 0.508 & 0.0684 & 0.903 & 442 \\
\hline 05519500 & 1 & Yes & Yes & 1964 & 159 & -- & 64 & 0.943 & 0.0701 & 0.981 & 207 \\
\hline 05519500 & 1 & Yes & Yes & 1965 & 1000 & -- & 404 & 1.23 & 0.0719 & 0.417 & 1150 \\
\hline 05519500 & 1 & Yes & Yes & 1966 & 1510 & -- & 609 & 2.67 & 0.0737 & 0.172 & 1690 \\
\hline 05519500 & 1 & Yes & Yes & 1967 & 1480 & -- & 597 & 1.2 & 0.0755 & 0.181 & 1660 \\
\hline 05519500 & 1 & Yes & Yes & 1968 & 1760 & -- & 710 & 3.61 & 0.0773 & 0.111 & 1960 \\
\hline 05519500 & 1 & Yes & Yes & 1969 & 750 & 2 & 303 & 0.514 & 0.0791 & 0.629 & 864 \\
\hline 05519500 & 1 & Yes & Yes & 1970 & 1460 & -- & 589 & 1.95 & 0.0809 & 0.189 & 1630 \\
\hline 05519500 & 1 & Yes & No & 1971 & 500 & 1,8 & 202 & 0.765 & 0.0844 & 0.824 & 586 \\
\hline 05519500 & 1 & Yes & Yes & 1972 & 1670 & -- & 674 & 3.62 & 0.0879 & 0.136 & 1850 \\
\hline 05520500 & 1 & No & No & 1940 & 4860 & -- & 210 & 0.0464 & 0.0162 & 0.774 & 5430 \\
\hline 05520500 & 1 & No & No & 1941 & 2910 & -- & 126 & 0.773 & 0.0168 & 0.914 & 3330 \\
\hline 05520500 & 1 & No & No & 1942 & 6310 & -- & 272 & 0.154 & 0.0174 & 0.647 & 6970 \\
\hline 05520500 & 1 & No & No & 1943 & 8450 & -- & 365 & 0.609 & 0.018 & 0.447 & 9250 \\
\hline 05520500 & 1 & No & No & 1944 & 6950 & -- & 300 & 0.82 & 0.0186 & 0.592 & 7640 \\
\hline 05520500 & 1 & No & No & 1945 & 4940 & -- & 213 & 0.504 & 0.0193 & 0.77 & 5490 \\
\hline 05520500 & 1 & No & No & 1946 & 5460 & -- & 236 & 0.283 & 0.0199 & 0.726 & 6040 \\
\hline 05520500 & 1 & No & No & 1947 & 6260 & -- & 270 & 0.587 & 0.0205 & 0.653 & 6890 \\
\hline 05520500 & 1 & No & No & 1948 & 6260 & -- & 270 & 0.345 & 0.0211 & 0.653 & 6880 \\
\hline 05520500 & 1 & No & No & 1949 & 5060 & -- & 218 & 0.215 & 0.0217 & 0.762 & 5610 \\
\hline 05520500 & 1 & No & No & 1950 & 10100 & -- & 436 & 1.01 & 0.0223 & 0.334 & 10900 \\
\hline 05520500 & 1 & No & No & 1951 & 8400 & 2 & 363 & 0.317 & 0.0232 & 0.455 & 9140 \\
\hline 05520500 & 1 & No & No & 1952 & 6960 & -- & 300 & 1.23 & 0.0242 & 0.596 & 7600 \\
\hline 05520500 & 1 & No & No & 1953 & 4560 & -- & 197 & 0.436 & 0.0251 & 0.803 & 5050 \\
\hline
\end{tabular}




\begin{tabular}{|c|c|c|c|c|c|c|c|c|c|c|c|}
\hline $\begin{array}{c}\text { U.S. } \\
\text { Geological } \\
\text { Survey } \\
\text { streamgage } \\
\text { number }\end{array}$ & $\begin{array}{l}\text { Segment } \\
\text { number }\end{array}$ & $\begin{array}{l}\text { Streamgage used in } \\
\text { regression analyses } \\
\text { (non-redundant) }\end{array}$ & $\begin{array}{l}\text { Discharge value } \\
\text { used in } \\
\text { adjustment } \\
\text { regression }\end{array}$ & $\begin{array}{l}\text { Water } \\
\text { year }\end{array}$ & $\begin{array}{c}\text { Observed annual } \\
\text { maximum peak } \\
\text { discharge }\left(\mathrm{ft}^{3} / \mathrm{s}\right)\end{array}$ & $\begin{array}{l}\text { NWIS peak } \\
\text { code }\end{array}$ & $\begin{array}{l}\text { Observed annual } \\
\text { maximum peak } \\
\text { discharge with } \\
\text { segment intercept } \\
\text { value subtracted } \\
\left(\mathrm{ft}^{3} / \mathrm{s}\right)\end{array}$ & $\begin{array}{l}\text { Observed } \\
\text { precipitation } \\
\text { (inches) }\end{array}$ & Urban fraction & $\begin{array}{l}\text { Exceedance } \\
\text { probability }\end{array}$ & $\begin{array}{c}\text { Urban-adjusted } \\
\text { annual maximum } \\
\text { peak discharge } \\
\left(\mathrm{ft}^{3} / \mathrm{s}\right)\end{array}$ \\
\hline 05520500 & 1 & No & No & 1954 & 4960 & -- & 214 & 1.7 & 0.0261 & 0.772 & 5470 \\
\hline 05520500 & 1 & No & No & 1955 & 5880 & -- & 254 & 0.809 & 0.027 & 0.692 & 6430 \\
\hline 05520500 & 1 & No & No & 1956 & 5460 & -- & 236 & 0.683 & 0.028 & 0.731 & 5980 \\
\hline 05520500 & 1 & No & No & 1957 & 6960 & -- & 300 & 1.96 & 0.0289 & 0.6 & 7560 \\
\hline 05520500 & 1 & No & No & 1958 & 5670 & -- & 245 & 1.26 & 0.0299 & 0.713 & 6190 \\
\hline 05520500 & 1 & No & No & 1959 & 7100 & 2 & 306 & 0.0729 & 0.0308 & 0.587 & 7690 \\
\hline 05520500 & 1 & No & No & 1960 & 6520 & -- & 281 & 1.12 & 0.0318 & 0.638 & 7070 \\
\hline 05520500 & 1 & No & No & 1961 & 6740 & -- & 291 & 1.13 & 0.0326 & 0.62 & 7300 \\
\hline 05520500 & 1 & No & No & 1962 & 7180 & -- & 310 & 0.446 & 0.0335 & 0.58 & 7750 \\
\hline 05520500 & 1 & No & No & 1963 & 6500 & 2 & 281 & 0.219 & 0.0344 & 0.641 & 7030 \\
\hline 05520500 & 1 & No & No & 1964 & 2410 & -- & 104 & 0.0981 & 0.0352 & 0.942 & 2700 \\
\hline 05520500 & 1 & No & No & 1965 & 5670 & -- & 245 & 0.911 & 0.0361 & 0.717 & 6150 \\
\hline 05520500 & 1 & No & No & 1966 & 7310 & -- & 316 & 1.25 & 0.037 & 0.57 & 7860 \\
\hline 05520500 & 1 & No & No & 1967 & 7540 & -- & 325 & 1.06 & 0.0378 & 0.547 & 8090 \\
\hline 05520500 & 1 & No & No & 1968 & 8240 & -- & 356 & 1.18 & 0.0387 & 0.481 & 8820 \\
\hline 05520500 & 1 & No & No & 1969 & 6300 & 2 & 272 & 0.254 & 0.0396 & 0.662 & 6780 \\
\hline 05520500 & 1 & No & No & 1970 & 9480 & -- & 409 & 1.64 & 0.0404 & 0.385 & 10100 \\
\hline 05520500 & 1 & No & No & 1971 & 5200 & 2 & 224 & 0.218 & 0.0422 & 0.761 & 5610 \\
\hline 05520500 & 1 & No & No & 1972 & 6490 & -- & 280 & 1.14 & 0.044 & 0.649 & 6940 \\
\hline 05520500 & 1 & No & No & 1973 & 8380 & -- & 362 & 0.998 & 0.0458 & 0.475 & 8900 \\
\hline 05520500 & 1 & No & No & 1974 & 10000 & -- & 432 & 0.905 & 0.0476 & 0.356 & 10600 \\
\hline 05520500 & 1 & No & No & 1975 & 8440 & -- & 364 & 0.765 & 0.0494 & 0.473 & 8930 \\
\hline 05520500 & 1 & No & No & 1976 & 11900 & -- & 514 & 0.903 & 0.0512 & 0.25 & 12500 \\
\hline 05520500 & 1 & No & No & 1977 & 4780 & -- & 206 & 0.467 & 0.053 & 0.8 & 5100 \\
\hline 05520500 & 1 & No & No & 1978 & 7760 & -- & 335 & 0.931 & 0.0548 & 0.539 & 8170 \\
\hline 05520500 & 1 & No & No & 1979 & 16000 & 2 & 691 & 0.867 & 0.0566 & 0.114 & 16600 \\
\hline 05520500 & 1 & No & No & 1980 & 4800 & -- & 207 & 0.147 & 0.0584 & 0.801 & 5080 \\
\hline 05520500 & 1 & No & No & 1981 & 9760 & -- & 421 & 0.946 & 0.0594 & 0.379 & 10200 \\
\hline 05520500 & 1 & No & No & 1982 & 11000 & -- & 475 & 0.573 & 0.0604 & 0.304 & 11400 \\
\hline 05520500 & 1 & No & No & 1983 & 9840 & -- & 425 & 0.341 & 0.0614 & 0.376 & 10300 \\
\hline 05520500 & 1 & No & No & 1984 & 7200 & 2 & 311 & 0.216 & 0.0623 & 0.603 & 7520 \\
\hline 05520500 & 1 & No & No & 1985 & 9450 & -- & 408 & 0.612 & 0.0633 & 0.403 & 9830 \\
\hline 05520500 & 1 & No & No & 1986 & 9080 & -- & 392 & 0.609 & 0.0643 & 0.432 & 9440 \\
\hline 05520500 & 1 & No & No & 1987 & 5780 & -- & 250 & 1.03 & 0.0653 & 0.726 & 6040 \\
\hline 05520500 & 1 & No & No & 1988 & 6400 & -- & 276 & 0.456 & 0.0663 & 0.672 & 6670 \\
\hline
\end{tabular}




\begin{tabular}{|c|c|c|c|c|c|c|c|c|c|c|c|}
\hline $\begin{array}{l}\text { U.S. } \\
\text { Geological } \\
\text { Survey } \\
\text { streamgage } \\
\text { number }\end{array}$ & $\begin{array}{l}\text { Segment } \\
\text { number }\end{array}$ & $\begin{array}{l}\text { Streamgage used in } \\
\text { regression analyses } \\
\text { (non-redundant) }\end{array}$ & $\begin{array}{c}\text { Discharge value } \\
\text { used in } \\
\text { adjustment } \\
\text { regression }\end{array}$ & $\begin{array}{l}\text { Water } \\
\text { year }\end{array}$ & $\begin{array}{c}\text { Observed annual } \\
\text { maximum peak } \\
\text { discharge }\left(\mathrm{ft}^{3} / \mathrm{s}\right)\end{array}$ & $\begin{array}{l}\text { NWIS peak } \\
\text { code }\end{array}$ & $\begin{array}{l}\text { Observed annual } \\
\text { maximum peak } \\
\text { discharge with } \\
\text { segment intercept } \\
\text { value subtracted } \\
\left(\mathrm{ft}^{3} / \mathrm{s}\right)\end{array}$ & $\begin{array}{l}\text { Observed } \\
\text { precipitation } \\
\text { (inches) }\end{array}$ & Urban fraction & $\begin{array}{l}\text { Exceedance } \\
\text { probability }\end{array}$ & $\begin{array}{c}\text { Urban-adjusted } \\
\text { annual maximum } \\
\text { peak discharge } \\
\left(\mathrm{ft}^{3} / \mathrm{s}\right)\end{array}$ \\
\hline 05520500 & 1 & No & No & 1989 & 8080 & -- & 349 & 0.981 & 0.0673 & 0.519 & 8380 \\
\hline 05520500 & 1 & No & No & 1990 & 7050 & -- & 304 & 0.396 & 0.0683 & 0.618 & 7320 \\
\hline 05520500 & 1 & No & No & 1991 & 10600 & -- & 458 & 0.899 & 0.0705 & 0.334 & 10900 \\
\hline 05520500 & 1 & No & No & 1992 & 4620 & -- & 199 & 0.0647 & 0.0726 & 0.822 & 4800 \\
\hline 05520500 & 1 & No & No & 1993 & 9160 & -- & 395 & 0.711 & 0.0747 & 0.434 & 9420 \\
\hline 05520500 & 1 & No & No & 1994 & 8050 & -- & 347 & 0.879 & 0.0769 & 0.531 & 8260 \\
\hline 05520500 & 1 & No & No & 1995 & 7470 & -- & 322 & 0.597 & 0.079 & 0.59 & 7660 \\
\hline 05520500 & 1 & No & No & 1996 & 10000 & -- & 432 & 0.592 & 0.0811 & 0.379 & 10200 \\
\hline 05520500 & 1 & No & No & 1997 & 10100 & -- & 436 & 0.453 & 0.0833 & 0.374 & 10300 \\
\hline 05520500 & 1 & No & No & 1998 & 9990 & -- & 431 & 0.683 & 0.0854 & 0.382 & 10100 \\
\hline 05520500 & 1 & No & No & 1999 & 8510 & -- & 367 & 0.437 & 0.0876 & 0.497 & 8630 \\
\hline 05520500 & 1 & No & No & 2000 & 6380 & -- & 275 & 0.919 & 0.0897 & 0.69 & 6460 \\
\hline 05520500 & 1 & No & No & 2001 & 8310 & -- & 359 & 0.622 & 0.0907 & 0.518 & 8400 \\
\hline 05520500 & 1 & No & No & 2002 & 10300 & -- & 445 & 0.746 & 0.0918 & 0.367 & 10400 \\
\hline 05520500 & 1 & No & No & 2003 & 7110 & -- & 307 & 0.846 & 0.0928 & 0.63 & 7170 \\
\hline 05520500 & 1 & No & No & 2004 & 7250 & -- & 313 & 0.837 & 0.0939 & 0.62 & 7300 \\
\hline 05520500 & 1 & No & No & 2005 & 10100 & -- & 436 & 0.566 & 0.0949 & 0.382 & 10200 \\
\hline 05520500 & 1 & No & No & 2006 & 3780 & -- & 163 & 0.395 & 0.096 & 0.888 & 3800 \\
\hline 05520500 & 1 & No & No & 2007 & 9720 & -- & 420 & 0.513 & 0.097 & 0.409 & 9750 \\
\hline 05520500 & 1 & No & No & 2008 & 11800 & -- & 509 & 1.19 & 0.098 & 0.283 & 11800 \\
\hline 05520500 & 1 & No & No & 2009 & 12000 & -- & 518 & 0.833 & 0.0991 & 0.273 & 12000 \\
\hline 05526150 & 1 & Yes & Yes & 1956 & 44 & -- & 426 & 0.438 & 0 & 0.334 & 44 \\
\hline 05526150 & 1 & Yes & Yes & 1957 & 233 & -- & 2254 & 3.66 & 0 & 0.00246 & 233 \\
\hline 05526150 & 1 & Yes & Yes & 1958 & 153 & -- & 1480 & 1.2 & 0 & 0.0115 & 153 \\
\hline 05526150 & 1 & Yes & Yes & 1959 & 92 & -- & 890 & 1.28 & 0 & 0.0474 & 92 \\
\hline 05526150 & 1 & Yes & Yes & 1960 & 32 & -- & 310 & 0.959 & 0 & 0.552 & 32 \\
\hline 05526150 & 1 & Yes & Yes & 1961 & 13 & -- & 126 & 1.82 & 0 & 0.909 & 13 \\
\hline 05526150 & 1 & Yes & Yes & 1962 & 10 & -- & 97 & 0.291 & 0 & 0.943 & 10 \\
\hline 05526150 & 1 & Yes & Yes & 1963 & 62 & -- & 600 & 0.56 & 0 & 0.151 & 62 \\
\hline 05526150 & 1 & Yes & Yes & 1964 & 5 & -- & 48 & 1.04 & 0 & 0.985 & 5 \\
\hline 05526150 & 1 & Yes & Yes & 1965 & 46 & -- & 445 & 0.981 & 0 & 0.307 & 46 \\
\hline 05526150 & 1 & Yes & Yes & 1966 & 23 & -- & 223 & 1.49 & 0 & 0.742 & 23 \\
\hline 05526150 & 1 & Yes & Yes & 1967 & 65 & -- & 629 & 0.72 & 0 & 0.131 & 65 \\
\hline 05526150 & 1 & Yes & Yes & 1968 & 39 & -- & 377 & 2.15 & 0 & 0.409 & 39 \\
\hline 05526150 & 1 & Yes & Yes & 1969 & 6 & -- & 58 & 1.25 & 0 & 0.979 & 6 \\
\hline
\end{tabular}




\begin{tabular}{|c|c|c|c|c|c|c|c|c|c|c|c|}
\hline $\begin{array}{l}\text { U.S. } \\
\text { Geological } \\
\text { Survey } \\
\text { streamgage } \\
\text { number }\end{array}$ & $\begin{array}{l}\text { Segment } \\
\text { number }\end{array}$ & $\begin{array}{l}\text { Streamgage used in } \\
\text { regression analyses } \\
\text { (non-redundant) }\end{array}$ & $\begin{array}{c}\text { Discharge value } \\
\text { used in } \\
\text { adjustment } \\
\text { regression }\end{array}$ & $\begin{array}{l}\text { Water } \\
\text { year }\end{array}$ & $\begin{array}{c}\text { Observed annual } \\
\text { maximum peak } \\
\text { discharge }\left(\mathrm{ft}^{3} / \mathrm{s}\right)\end{array}$ & $\begin{array}{l}\text { NWIS peak } \\
\text { code }\end{array}$ & $\begin{array}{l}\text { Observed annual } \\
\text { maximum peak } \\
\text { discharge with } \\
\text { segment intercept } \\
\text { value subtracted } \\
\left(\mathrm{ft}^{3} / \mathrm{s}\right)\end{array}$ & $\begin{array}{l}\text { Observed } \\
\text { precipitation } \\
\text { (inches) }\end{array}$ & Urban fraction & $\begin{array}{l}\text { Exceedance } \\
\text { probability }\end{array}$ & $\begin{array}{c}\text { Urban-adjusted } \\
\text { annual maximum } \\
\text { peak discharge } \\
\left(\mathrm{ft}^{3} / \mathrm{s}\right)\end{array}$ \\
\hline 05526150 & 1 & Yes & Yes & 1970 & 200 & -- & 1935 & 1.88 & 0 & 0.005 & 200 \\
\hline 05526150 & 1 & Yes & Yes & 1971 & 13 & -- & 126 & 0.293 & 0 & 0.909 & 13 \\
\hline 05526150 & 1 & Yes & Yes & 1972 & 22 & -- & 213 & 0.893 & 0 & 0.761 & 22 \\
\hline 05526150 & 1 & Yes & Yes & 1973 & 19 & -- & 184 & 1.59 & 0 & 0.815 & 19 \\
\hline 05526150 & 1 & Yes & Yes & 1974 & 8 & -- & 77 & 0.65 & 0 & 0.96 & 8 \\
\hline 05526150 & 1 & Yes & Yes & 1975 & 22 & -- & 213 & 0.569 & 0 & 0.761 & 22 \\
\hline 05526150 & 1 & Yes & Yes & 1976 & 17 & -- & 164 & 0.72 & 0 & 0.849 & 17 \\
\hline 05526150 & 1 & Yes & No & 1977 & 5 & 4 & 48 & 0.303 & 0 & 0.985 & 5 \\
\hline 05526150 & 1 & Yes & Yes & 1978 & 32 & -- & 310 & 0.845 & 0 & 0.552 & 32 \\
\hline 05526150 & 1 & Yes & Yes & 1979 & 129 & -- & 1248 & 0.329 & 0 & 0.0173 & 129 \\
\hline 05526150 & 1 & Yes & Yes & 1980 & 105 & -- & 1016 & 1.42 & 0 & 0.0314 & 105 \\
\hline 05526500 & 1 & Yes & Yes & 1950 & 162 & -- & 340 & 1.11 & 0.000297 & 0.483 & 173 \\
\hline 05526500 & 1 & Yes & Yes & 1951 & 152 & -- & 319 & 0.407 & 0.000327 & 0.53 & 162 \\
\hline 05526500 & 1 & Yes & Yes & 1952 & 125 & -- & 262 & 1.24 & 0.000356 & 0.656 & 134 \\
\hline 05526500 & 1 & Yes & Yes & 1953 & 150 & -- & 315 & 1.2 & 0.000386 & 0.54 & 160 \\
\hline 05526500 & 1 & Yes & Yes & 1954 & 112 & -- & 235 & 1.87 & 0.000416 & 0.715 & 120 \\
\hline 05526500 & 1 & Yes & Yes & 1955 & 71 & -- & 149 & 2.25 & 0.000446 & 0.877 & 77.3 \\
\hline 05526500 & 1 & Yes & Yes & 1956 & 154 & -- & 323 & 0.436 & 0.000475 & 0.52 & 164 \\
\hline 05526500 & 1 & Yes & Yes & 1957 & 545 & -- & 1144 & 3.64 & 0.000505 & 0.0206 & 569 \\
\hline 05526500 & 1 & Yes & Yes & 1958 & 285 & -- & 598 & 1.2 & 0.000535 & 0.152 & 300 \\
\hline 05526500 & 1 & Yes & Yes & 1959 & 140 & 2 & 294 & 0.883 & 0.000564 & 0.592 & 149 \\
\hline 05526500 & 1 & Yes & Yes & 1960 & 155 & -- & 325 & 0.974 & 0.000594 & 0.515 & 165 \\
\hline 05526500 & 1 & Yes & Yes & 1961 & 101 & -- & 212 & 1.18 & 0.000683 & 0.763 & 109 \\
\hline 05526500 & 1 & Yes & Yes & 1962 & 81 & -- & 170 & 0.268 & 0.000772 & 0.84 & 87.7 \\
\hline 05526500 & 1 & Yes & Yes & 1963 & 110 & 2 & 231 & 0.22 & 0.000861 & 0.724 & 118 \\
\hline 05526500 & 1 & Yes & Yes & 1964 & 20 & -- & 42 & 1.18 & 0.00095 & 0.99 & 22.5 \\
\hline 05526500 & 1 & Yes & Yes & 1965 & 90 & -- & 189 & 1.04 & 0.00104 & 0.806 & 97.1 \\
\hline 05526500 & 1 & Yes & Yes & 1966 & 162 & -- & 340 & 1.48 & 0.00113 & 0.483 & 172 \\
\hline 05526500 & 1 & Yes & Yes & 1967 & 140 & -- & 294 & 0.529 & 0.00122 & 0.593 & 149 \\
\hline 05526500 & 1 & Yes & Yes & 1968 & 230 & -- & 483 & 2.14 & 0.00131 & 0.258 & 243 \\
\hline 05526500 & 1 & Yes & Yes & 1969 & 93 & -- & 195 & 0.435 & 0.0014 & 0.794 & 100 \\
\hline 05526500 & 1 & Yes & Yes & 1970 & 1710 & -- & 3589 & 1.87 & 0.00148 & 0 & 1770 \\
\hline 05526500 & 1 & Yes & Yes & 1971 & 97 & -- & 204 & 0.371 & 0.00187 & 0.779 & 104 \\
\hline 05526500 & 1 & Yes & Yes & 1972 & 132 & -- & 277 & 0.888 & 0.00226 & 0.628 & 141 \\
\hline 05526500 & 1 & Yes & Yes & 1973 & 269 & -- & 565 & 0.822 & 0.00264 & 0.176 & 283 \\
\hline
\end{tabular}




\begin{tabular}{|c|c|c|c|c|c|c|c|c|c|c|c|}
\hline $\begin{array}{l}\text { U.S. } \\
\text { Geological } \\
\text { Survey } \\
\text { streamgage } \\
\text { number }\end{array}$ & $\begin{array}{l}\text { Segment } \\
\text { number }\end{array}$ & $\begin{array}{l}\text { Streamgage used in } \\
\text { regression analyses } \\
\text { (non-redundant) }\end{array}$ & $\begin{array}{c}\text { Discharge value } \\
\text { used in } \\
\text { adjustment } \\
\text { regression }\end{array}$ & $\begin{array}{l}\text { Water } \\
\text { year }\end{array}$ & $\begin{array}{c}\text { Observed annual } \\
\text { maximum peak } \\
\text { discharge }\left(\mathrm{ft}^{3} / \mathrm{s}\right)\end{array}$ & $\begin{array}{l}\text { NWIS peak } \\
\text { code }\end{array}$ & $\begin{array}{l}\text { Observed annual } \\
\text { maximum peak } \\
\text { discharge with } \\
\text { segment intercept } \\
\text { value subtracted } \\
\left(\mathrm{ft}^{3} / \mathrm{s}\right)\end{array}$ & $\begin{array}{l}\text { Observed } \\
\text { precipitation } \\
\text { (inches) }\end{array}$ & Urban fraction & $\begin{array}{l}\text { Exceedance } \\
\text { probability }\end{array}$ & $\begin{array}{c}\text { Urban-adjusted } \\
\text { annual maximum } \\
\text { peak discharge } \\
\left(\mathrm{ft}^{3} / \mathrm{s}\right)\end{array}$ \\
\hline 05526500 & 1 & Yes & Yes & 1974 & 469 & -- & 984 & 0.61 & 0.00303 & 0.0355 & 490 \\
\hline 05526500 & 1 & Yes & Yes & 1975 & 237 & -- & 497 & 1.43 & 0.00342 & 0.242 & 250 \\
\hline 05527050 & 1 & Yes & Yes & 1956 & 171 & -- & 672 & 0.634 & 0.00461 & 0.106 & 211 \\
\hline 05527050 & 1 & Yes & Yes & 1957 & 786 & -- & 3087 & 3.14 & 0.00461 & 0 & 917 \\
\hline 05527050 & 1 & Yes & Yes & 1958 & 63 & -- & 247 & 0.606 & 0.00461 & 0.691 & 83.7 \\
\hline 05527050 & 1 & Yes & Yes & 1959 & 100 & 2 & 393 & 1.36 & 0.00461 & 0.387 & 128 \\
\hline 05527050 & 1 & Yes & Yes & 1960 & 55 & -- & 216 & 0.412 & 0.00461 & 0.757 & 74.2 \\
\hline 05527050 & 1 & Yes & Yes & 1961 & 140 & -- & 550 & 1.46 & 0.00461 & 0.188 & 173 \\
\hline 05527050 & 1 & Yes & Yes & 1962 & 44 & -- & 173 & 0.302 & 0.00461 & 0.837 & 60.9 \\
\hline 05527050 & 1 & Yes & Yes & 1963 & 65 & 2 & 255 & 1.44 & 0.00461 & 0.674 & 86 \\
\hline 05527050 & 1 & Yes & Yes & 1964 & 10 & 2 & 39 & 1.02 & 0.00461 & 1 & 16.8 \\
\hline 05527050 & 1 & Yes & Yes & 1965 & 80 & -- & 314 & 0.979 & 0.00461 & 0.545 & 104 \\
\hline 05527050 & 1 & Yes & Yes & 1966 & 92 & -- & 361 & 1.58 & 0.00461 & 0.443 & 118 \\
\hline 05527050 & 1 & Yes & Yes & 1967 & 64 & -- & 251 & 1.18 & 0.00461 & 0.682 & 84.9 \\
\hline 05527050 & 1 & Yes & Yes & 1968 & 96 & -- & 377 & 1.68 & 0.00461 & 0.413 & 123 \\
\hline 05527050 & 1 & Yes & Yes & 1969 & 105 & -- & 412 & 0.713 & 0.00461 & 0.357 & 134 \\
\hline 05527050 & 1 & Yes & Yes & 1970 & 105 & -- & 412 & 1.72 & 0.00461 & 0.357 & 134 \\
\hline 05527050 & 1 & Yes & Yes & 1971 & 35 & -- & 137 & 0.289 & 0.00553 & 0.895 & 49.9 \\
\hline 05527050 & 1 & Yes & Yes & 1972 & 170 & -- & 668 & 1.56 & 0.00645 & 0.109 & 209 \\
\hline 05527800 & 1 & Yes & Yes & 1960 & 1320 & -- & 645 & 0.776 & 0.0499 & 0.14 & 1510 \\
\hline 05527800 & 1 & Yes & Yes & 1961 & 425 & 2 & 208 & 0.273 & 0.0518 & 0.797 & 518 \\
\hline 05527800 & 1 & Yes & Yes & 1962 & 820 & -- & 401 & 0.281 & 0.0536 & 0.408 & 959 \\
\hline 05527800 & 1 & Yes & Yes & 1963 & 104 & -- & 51 & 1.57 & 0.0554 & 0.988 & 141 \\
\hline 05527800 & 1 & Yes & Yes & 1964 & 157 & -- & 77 & 0.692 & 0.0573 & 0.968 & 206 \\
\hline 05527800 & 1 & Yes & Yes & 1965 & 600 & -- & 293 & 0.527 & 0.0591 & 0.633 & 708 \\
\hline 05527800 & 1 & Yes & Yes & 1966 & 740 & -- & 362 & 0.536 & 0.0609 & 0.487 & 863 \\
\hline 05527800 & 1 & Yes & Yes & 1967 & 720 & -- & 352 & 1.52 & 0.0628 & 0.509 & 838 \\
\hline 05527800 & 1 & Yes & Yes & 1968 & 235 & -- & 115 & 0.349 & 0.0646 & 0.936 & 296 \\
\hline 05527800 & 1 & Yes & Yes & 1969 & 273 & -- & 133 & 0.88 & 0.0664 & 0.918 & 338 \\
\hline 05527800 & 1 & Yes & Yes & 1970 & 508 & -- & 248 & 1.63 & 0.0683 & 0.731 & 599 \\
\hline 05527800 & 1 & Yes & Yes & 1971 & 898 & -- & 439 & 0.276 & 0.0715 & 0.362 & 1030 \\
\hline 05527800 & 1 & Yes & Yes & 1972 & 816 & -- & 399 & 1.28 & 0.0748 & 0.428 & 934 \\
\hline 05527800 & 1 & Yes & Yes & 1973 & 940 & -- & 459 & 1.47 & 0.078 & 0.337 & 1060 \\
\hline 05527800 & 1 & Yes & Yes & 1974 & 1690 & -- & 826 & 0.53 & 0.0812 & 0.0704 & 1870 \\
\hline 05527800 & 1 & Yes & Yes & 1975 & 407 & -- & 199 & 0.327 & 0.0845 & 0.828 & 477 \\
\hline
\end{tabular}




\begin{tabular}{|c|c|c|c|c|c|c|c|c|c|c|c|}
\hline $\begin{array}{l}\text { U.S. } \\
\text { Geological } \\
\text { Survey } \\
\text { streamgage } \\
\text { number }\end{array}$ & $\begin{array}{l}\text { Segment } \\
\text { number }\end{array}$ & $\begin{array}{l}\text { Streamgage used in } \\
\text { regression analyses } \\
\text { (non-redundant) }\end{array}$ & $\begin{array}{c}\text { Discharge value } \\
\text { used in } \\
\text { adjustment } \\
\text { regression }\end{array}$ & $\begin{array}{l}\text { Water } \\
\text { year }\end{array}$ & $\begin{array}{c}\text { Observed annual } \\
\text { maximum peak } \\
\text { discharge }\left(\mathrm{ft}^{3} / \mathrm{s}\right)\end{array}$ & $\begin{array}{l}\text { NWIS peak } \\
\text { code }\end{array}$ & $\begin{array}{l}\text { Observed annual } \\
\text { maximum peak } \\
\text { discharge with } \\
\text { segment intercept } \\
\text { value subtracted } \\
\left(\mathrm{ft}^{3} / \mathrm{s}\right)\end{array}$ & $\begin{array}{l}\text { Observed } \\
\text { precipitation } \\
\text { (inches) }\end{array}$ & Urban fraction & $\begin{array}{l}\text { Exceedance } \\
\text { probability }\end{array}$ & $\begin{array}{c}\text { Urban-adjusted } \\
\text { annual maximum } \\
\text { peak discharge } \\
\left(\mathrm{ft}^{3} / \mathrm{s}\right)\end{array}$ \\
\hline 05527800 & 1 & Yes & Yes & 1976 & 1990 & -- & 973 & 1.21 & 0.0877 & 0.0453 & 2180 \\
\hline 05527800 & 1 & Yes & Yes & 1977 & 105 & -- & 51 & 0.732 & 0.0909 & 0.99 & 132 \\
\hline 05527800 & 1 & Yes & Yes & 1978 & 1380 & -- & 674 & 1.68 & 0.0942 & 0.138 & 1520 \\
\hline 05527800 & 1 & Yes & Yes & 1979 & 2120 & -- & 1036 & 0.286 & 0.0974 & 0.0385 & 2300 \\
\hline 05527800 & 1 & Yes & Yes & 1980 & 484 & -- & 237 & 0.773 & 0.101 & 0.771 & 550 \\
\hline 05527800 & 1 & Yes & Yes & 1981 & 378 & -- & 185 & 0.195 & 0.103 & 0.859 & 434 \\
\hline 05527800 & 1 & Yes & Yes & 1982 & 873 & -- & 427 & 0.427 & 0.104 & 0.403 & 966 \\
\hline 05527800 & 1 & Yes & Yes & 1983 & 1630 & -- & 797 & 1.4 & 0.106 & 0.0833 & 1770 \\
\hline 05527800 & 1 & Yes & Yes & 1984 & 597 & -- & 292 & 0.203 & 0.108 & 0.67 & 666 \\
\hline 05527800 & 1 & Yes & Yes & 1985 & 950 & -- & 464 & 1.03 & 0.11 & 0.351 & 1040 \\
\hline 05527800 & 1 & Yes & Yes & 1986 & 1640 & -- & 802 & 1.19 & 0.112 & 0.083 & 1770 \\
\hline 05527800 & 1 & Yes & Yes & 1987 & 435 & -- & 213 & 0.786 & 0.114 & 0.82 & 489 \\
\hline 05527800 & 1 & Yes & Yes & 1988 & 442 & -- & 216 & 0.948 & 0.116 & 0.815 & 495 \\
\hline 05527800 & 1 & Yes & Yes & 1989 & 155 & -- & 76 & 0.617 & 0.118 & 0.977 & 182 \\
\hline 05527800 & 1 & Yes & Yes & 1990 & 852 & -- & 416 & 0.248 & 0.12 & 0.433 & 928 \\
\hline 05527800 & 1 & Yes & Yes & 1991 & 587 & -- & 287 & 1.09 & 0.124 & 0.691 & 643 \\
\hline 05527800 & 1 & Yes & Yes & 1992 & 428 & -- & 209 & 0.36 & 0.129 & 0.832 & 472 \\
\hline 05527800 & 1 & Yes & Yes & 1993 & 1750 & -- & 855 & 1.06 & 0.134 & 0.0722 & 1850 \\
\hline 05527800 & 1 & Yes & Yes & 1994 & 870 & 2 & 425 & 0.815 & 0.138 & 0.432 & 929 \\
\hline 05527800 & 1 & Yes & Yes & 1995 & 805 & -- & 393 & 1.19 & 0.143 & 0.493 & 856 \\
\hline 05527800 & 1 & Yes & Yes & 1996 & 1200 & -- & 586 & 1.02 & 0.147 & 0.225 & 1260 \\
\hline 05527800 & 1 & Yes & Yes & 1997 & 853 & -- & 417 & 1.44 & 0.152 & 0.458 & 898 \\
\hline 05527800 & 1 & Yes & Yes & 1998 & 794 & -- & 388 & 0.966 & 0.157 & 0.515 & 832 \\
\hline 05527800 & 1 & Yes & Yes & 1999 & 1250 & -- & 611 & 1.11 & 0.161 & 0.208 & 1290 \\
\hline 05527800 & 1 & Yes & Yes & 2000 & 2130 & -- & 1041 & 2.38 & 0.166 & 0.0448 & 2190 \\
\hline 05527800 & 1 & Yes & Yes & 2001 & 501 & -- & 245 & 0.141 & 0.169 & 0.794 & 521 \\
\hline 05527800 & 1 & Yes & Yes & 2002 & 527 & -- & 258 & 0.0144 & 0.172 & 0.775 & 545 \\
\hline 05527800 & 1 & Yes & Yes & 2003 & 241 & -- & 118 & 0.395 & 0.176 & 0.954 & 251 \\
\hline 05527800 & 1 & Yes & Yes & 2004 & 3500 & -- & 1711 & 1.71 & 0.179 & 0.0113 & 3550 \\
\hline 05527800 & 1 & Yes & Yes & 2005 & 672 & -- & 328 & 0.289 & 0.182 & 0.653 & 685 \\
\hline 05527800 & 1 & Yes & Yes & 2006 & 509 & -- & 249 & 0.754 & 0.185 & 0.797 & 518 \\
\hline 05527800 & 1 & Yes & Yes & 2007 & 1610 & -- & 787 & 1.11 & 0.189 & 0.11 & 1620 \\
\hline 05527800 & 1 & Yes & Yes & 2008 & 1910 & -- & 933 & 1.9 & 0.192 & 0.0657 & 1920 \\
\hline 05527800 & 1 & Yes & Yes & 2009 & 2470 & -- & 1207 & 2.02 & 0.195 & 0.0297 & 2480 \\
\hline 05527840 & 1 & No & No & 1962 & 1370 & -- & 591 & 0.153 & 0.0544 & 0.178 & 1610 \\
\hline
\end{tabular}




\begin{tabular}{|c|c|c|c|c|c|c|c|c|c|c|c|}
\hline $\begin{array}{c}\text { U.S. } \\
\text { Geological } \\
\text { Survey } \\
\text { streamgage } \\
\text { number } \\
\end{array}$ & $\begin{array}{l}\text { Segment } \\
\text { number }\end{array}$ & $\begin{array}{l}\text { Streamgage used in } \\
\text { regression analyses } \\
\text { (non-redundant) }\end{array}$ & $\begin{array}{c}\text { Discharge value } \\
\text { used in } \\
\text { adjustment } \\
\text { regression }\end{array}$ & $\begin{array}{c}\text { Water } \\
\text { year }\end{array}$ & $\begin{array}{c}\text { Observed annual } \\
\text { maximum peak } \\
\text { discharge }\left(\mathrm{ft}^{3} / \mathrm{s}\right)\end{array}$ & $\begin{array}{c}\text { NWIS peak } \\
\text { code }\end{array}$ & $\begin{array}{c}\text { Observed annual } \\
\text { maximum peak } \\
\text { discharge with } \\
\text { segment intercept } \\
\text { value subtracted } \\
\left(\mathrm{ft}^{3} / \mathrm{s}\right)\end{array}$ & $\begin{array}{c}\text { Observed } \\
\text { precipitation } \\
\text { (inches) }\end{array}$ & Urban fraction & $\begin{array}{l}\text { Exceedance } \\
\text { probability }\end{array}$ & $\begin{array}{c}\text { Urban-adjusted } \\
\text { annual maximum } \\
\text { peak discharge } \\
\left(\mathrm{ft}^{3} / \mathrm{s}\right)\end{array}$ \\
\hline 05527840 & 1 & No & No & 1963 & 110 & -- & 47 & 1.53 & 0.0565 & 0.99 & 160 \\
\hline 05527840 & 1 & No & No & 1964 & 173 & -- & 75 & 0.695 & 0.0586 & 0.97 & 242 \\
\hline 05527840 & 1 & No & No & 1965 & 940 & -- & 405 & 0.532 & 0.0607 & 0.405 & 1130 \\
\hline 05527840 & 1 & No & No & 1966 & 1190 & -- & 513 & 0.526 & 0.0628 & 0.257 & 1400 \\
\hline 05527840 & 1 & No & No & 1967 & 1040 & -- & 448 & 1.48 & 0.0648 & 0.343 & 1240 \\
\hline 05527840 & 1 & No & No & 1968 & 240 & -- & 103 & 0.473 & 0.0669 & 0.948 & 322 \\
\hline 05527840 & 1 & No & No & 1969 & 795 & -- & 343 & 1.21 & 0.069 & 0.534 & 955 \\
\hline 05527840 & 1 & No & No & 1970 & 630 & -- & 272 & 1.62 & 0.0711 & 0.685 & 766 \\
\hline 05527840 & 1 & No & No & 1971 & 1470 & -- & 634 & 0.271 & 0.0746 & 0.156 & 1700 \\
\hline 05527840 & 1 & No & No & 1972 & 1320 & -- & 569 & 0.584 & 0.0781 & 0.203 & 1520 \\
\hline 05527840 & 1 & No & No & 1973 & 1180 & -- & 509 & 1.46 & 0.0816 & 0.274 & 1370 \\
\hline 05527840 & 1 & No & No & 1974 & 1730 & -- & 746 & 0.523 & 0.0851 & 0.0957 & 1970 \\
\hline 05527840 & 1 & No & No & 1975 & 535 & -- & 231 & 0.307 & 0.0886 & 0.775 & 646 \\
\hline 05527840 & 1 & No & No & 1976 & 2170 & -- & 936 & 1.22 & 0.0921 & 0.0507 & 2440 \\
\hline 05527870 & 1 & Yes & No & 1960 & 326 & 7 & 1757 & 0.548 & 0.218 & 0.0114 & 420 \\
\hline 05527870 & 1 & Yes & Yes & 1962 & 118 & -- & 636 & 0.251 & 0.229 & 0.223 & 163 \\
\hline 05527870 & 1 & Yes & Yes & 1963 & 32 & -- & 172 & 1.54 & 0.234 & 0.921 & 55.4 \\
\hline 05527870 & 1 & Yes & No & 1964 & 39 & $4, B$ & 210 & 0.251 & 0.239 & 0.88 & 64 \\
\hline 05527870 & 1 & Yes & Yes & 1965 & 93 & -- & 501 & 0.769 & 0.244 & 0.396 & 132 \\
\hline 05527870 & 1 & Yes & Yes & 1966 & 44 & -- & 237 & 0.482 & 0.25 & 0.847 & 69.4 \\
\hline 05527870 & 1 & Yes & Yes & 1967 & 69 & -- & 372 & 1.32 & 0.255 & 0.63 & 100 \\
\hline 05527870 & 1 & Yes & Yes & 1968 & 31 & -- & 167 & 0.544 & 0.26 & 0.932 & 52.2 \\
\hline 05527870 & 1 & Yes & Yes & 1969 & 92 & -- & 496 & 1.39 & 0.266 & 0.421 & 128 \\
\hline 05527870 & 1 & Yes & Yes & 1970 & 95 & -- & 512 & 1.61 & 0.271 & 0.401 & 131 \\
\hline 05527870 & 1 & Yes & Yes & 1971 & 163 & -- & 878 & 0.454 & 0.276 & 0.0984 & 212 \\
\hline 05527870 & 1 & Yes & Yes & 1972 & 76 & -- & 410 & 0.468 & 0.282 & 0.586 & 106 \\
\hline 05527870 & 1 & Yes & Yes & 1973 & 85 & -- & 458 & 1.42 & 0.288 & 0.501 & 116 \\
\hline 05527870 & 1 & Yes & Yes & 1974 & 138 & -- & 744 & 1.14 & 0.293 & 0.175 & 178 \\
\hline 05527870 & 1 & Yes & Yes & 1975 & 74 & -- & 399 & 0.841 & 0.299 & 0.619 & 102 \\
\hline 05527870 & 1 & Yes & Yes & 1976 & 125 & -- & 674 & 1.35 & 0.305 & 0.233 & 161 \\
\hline 05527900 & 1 & Yes & No & 1960 & 510 & 7 & 966 & 0.689 & 0.0428 & 0.0417 & 614 \\
\hline 05527900 & 1 & Yes & Yes & 1962 & 203 & -- & 384 & 0.207 & 0.0439 & 0.43 & 259 \\
\hline 05527900 & 1 & Yes & Yes & 1963 & 50 & -- & 95 & 2.02 & 0.0444 & 0.952 & 74.8 \\
\hline 05527900 & 1 & Yes & Yes & 1964 & 142 & -- & 269 & 1.44 & 0.045 & 0.672 & 186 \\
\hline 05527900 & 1 & Yes & Yes & 1965 & 236 & -- & 447 & 0.555 & 0.0455 & 0.333 & 297 \\
\hline
\end{tabular}




\begin{tabular}{|c|c|c|c|c|c|c|c|c|c|c|c|}
\hline $\begin{array}{c}\text { U.S. } \\
\text { Geological } \\
\text { Survey } \\
\text { streamgage } \\
\text { number } \\
\end{array}$ & $\begin{array}{c}\text { Segment } \\
\text { number }\end{array}$ & $\begin{array}{l}\text { Streamgage used in } \\
\text { regression analyses } \\
\text { (non-redundant) }\end{array}$ & $\begin{array}{c}\text { Discharge value } \\
\text { used in } \\
\text { adjustment } \\
\text { regression } \\
\end{array}$ & $\begin{array}{l}\text { Water } \\
\text { year }\end{array}$ & $\begin{array}{c}\text { Observed annual } \\
\text { maximum peak } \\
\text { discharge }\left(\mathrm{ft}^{3} / \mathrm{s}\right)\end{array}$ & $\begin{array}{c}\text { NWIS peak } \\
\text { code }\end{array}$ & $\begin{array}{c}\text { Observed annual } \\
\text { maximum peak } \\
\text { discharge with } \\
\text { segment intercept } \\
\text { value subtracted } \\
\left(\mathrm{ft}^{3} / \mathrm{s}\right) \\
\end{array}$ & $\begin{array}{c}\text { Observed } \\
\text { precipitation } \\
\text { (inches) }\end{array}$ & Urban fraction & $\begin{array}{l}\text { Exceedance } \\
\text { probability }\end{array}$ & $\begin{array}{c}\text { Urban-adjusted } \\
\text { annual maximum } \\
\text { peak discharge } \\
\left(\mathrm{ft}^{3} / \mathrm{s}\right)\end{array}$ \\
\hline 05527900 & 1 & Yes & Yes & 1966 & 231 & -- & 437 & 0.516 & 0.046 & 0.347 & 291 \\
\hline 05527900 & 1 & Yes & Yes & 1967 & 181 & -- & 343 & 1.55 & 0.0466 & 0.515 & 232 \\
\hline 05527900 & 1 & Yes & Yes & 1968 & 95 & -- & 180 & 0.478 & 0.0471 & 0.843 & 130 \\
\hline 05527900 & 1 & Yes & Yes & 1969 & 306 & -- & 579 & 1.13 & 0.0476 & 0.183 & 375 \\
\hline 05527900 & 1 & Yes & Yes & 1970 & 217 & -- & 411 & 2.43 & 0.0482 & 0.388 & 274 \\
\hline 05527900 & 1 & Yes & Yes & 1971 & 212 & -- & 401 & 0.697 & 0.0534 & 0.407 & 267 \\
\hline 05527900 & 1 & Yes & Yes & 1972 & 166 & -- & 314 & 0.532 & 0.0587 & 0.592 & 211 \\
\hline 05527900 & 1 & Yes & Yes & 1973 & 180 & -- & 341 & 1.24 & 0.064 & 0.534 & 227 \\
\hline 05527900 & 1 & Yes & Yes & 1974 & 401 & -- & 759 & 1.58 & 0.0692 & 0.0873 & 479 \\
\hline 05527900 & 1 & Yes & Yes & 1975 & 120 & 2 & 227 & 1.04 & 0.0745 & 0.773 & 155 \\
\hline 05527900 & 1 & Yes & Yes & 1976 & 357 & -- & 676 & 2.01 & 0.0798 & 0.132 & 424 \\
\hline 05527900 & 0 & Yes & No & 2008 & 183 & -- & NA & 0.699 & 0.248 & NA & NA \\
\hline 05527900 & 0 & Yes & No & 2009 & 283 & -- & NA & 1.29 & 0.258 & NA & NA \\
\hline 05527950 & 1 & No & No & 1960 & 778 & 7 & 621 & 0.606 & 0.132 & 0.187 & 1050 \\
\hline 05527950 & 1 & No & No & 1962 & 439 & -- & 350 & 0.234 & 0.141 & 0.581 & 632 \\
\hline 05527950 & 1 & No & No & 1963 & 70 & 2 & 56 & 1.42 & 0.146 & 1 & 141 \\
\hline 05527950 & 1 & No & No & 1964 & 120 & $2, B$ & 96 & 1.16 & 0.151 & 0.965 & 215 \\
\hline 05527950 & 1 & No & No & 1965 & 423 & -- & 338 & 0.558 & 0.156 & 0.618 & 602 \\
\hline 05527950 & 1 & No & No & 1966 & 500 & 2 & 399 & 0.495 & 0.161 & 0.497 & 697 \\
\hline 05527950 & 1 & No & No & 1967 & 395 & -- & 315 & 1.41 & 0.165 & 0.665 & 562 \\
\hline 05527950 & 1 & No & No & 1968 & 146 & 2 & 117 & 0.518 & 0.17 & 0.954 & 246 \\
\hline 05527950 & 1 & No & No & 1969 & 720 & -- & 575 & 1.46 & 0.175 & 0.254 & 945 \\
\hline 05527950 & 1 & No & No & 1970 & 744 & -- & 594 & 1.92 & 0.18 & 0.236 & 969 \\
\hline 05527950 & 1 & No & No & 1971 & 583 & -- & 465 & 0.548 & 0.185 & 0.403 & 781 \\
\hline 05527950 & 1 & No & No & 1972 & 921 & -- & 735 & 0.89 & 0.189 & 0.139 & 1180 \\
\hline 05527950 & 1 & No & No & 1973 & 510 & -- & 407 & 1.36 & 0.194 & 0.511 & 685 \\
\hline 05527950 & 1 & No & No & 1974 & 840 & -- & 671 & 1.3 & 0.199 & 0.182 & 1070 \\
\hline 05527950 & 1 & No & No & 1975 & 319 & -- & 255 & 0.919 & 0.204 & 0.797 & 449 \\
\hline 05527950 & 1 & No & No & 1976 & 1050 & -- & 838 & 1.59 & 0.209 & 0.0943 & 1310 \\
\hline 05527950 & 1 & No & No & 1990 & 486 & -- & 388 & 0.651 & 0.257 & 0.605 & 614 \\
\hline 05527950 & 1 & No & No & 1991 & 931 & -- & 743 & 1.58 & 0.263 & 0.163 & 1110 \\
\hline 05527950 & 1 & No & No & 1992 & 336 & $\mathrm{E}$ & 268 & 0.969 & 0.269 & 0.812 & 434 \\
\hline 05527950 & 1 & No & No & 1993 & 1090 & -- & 870 & 2.58 & 0.275 & 0.101 & 1280 \\
\hline 05527950 & 1 & No & No & 1994 & 988 & -- & 789 & 0.604 & 0.282 & 0.146 & 1160 \\
\hline 05527950 & 1 & No & No & 1995 & 696 & -- & 556 & 1.37 & 0.288 & 0.355 & 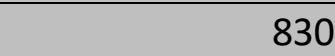 \\
\hline
\end{tabular}




\begin{tabular}{|c|c|c|c|c|c|c|c|c|c|c|c|}
\hline $\begin{array}{l}\text { U.S. } \\
\text { Geological } \\
\text { Survey } \\
\text { streamgage } \\
\text { number }\end{array}$ & $\begin{array}{l}\text { Segment } \\
\text { number }\end{array}$ & $\begin{array}{l}\text { Streamgage used in } \\
\text { regression analyses } \\
\text { (non-redundant) }\end{array}$ & $\begin{array}{c}\text { Discharge value } \\
\text { used in } \\
\text { adjustment } \\
\text { regression }\end{array}$ & $\begin{array}{l}\text { Water } \\
\text { year }\end{array}$ & $\begin{array}{l}\text { Observed annual } \\
\text { maximum peak } \\
\text { discharge }\left(\mathrm{ft}^{3} / \mathrm{s}\right)\end{array}$ & $\begin{array}{l}\text { NWIS peak } \\
\text { code }\end{array}$ & $\begin{array}{l}\text { Observed annual } \\
\text { maximum peak } \\
\text { discharge with } \\
\text { segment intercept } \\
\text { value subtracted } \\
\left(\mathrm{ft}^{3} / \mathrm{s}\right)\end{array}$ & $\begin{array}{l}\text { Observed } \\
\text { precipitation } \\
\text { (inches) }\end{array}$ & Urban fraction & $\begin{array}{l}\text { Exceedance } \\
\text { probability }\end{array}$ & $\begin{array}{c}\text { Urban-adjusted } \\
\text { annual maximum } \\
\text { peak discharge } \\
\left(\mathrm{ft}^{3} / \mathrm{s}\right)\end{array}$ \\
\hline 05527950 & 1 & No & No & 1996 & 1020 & -- & 814 & 1.32 & 0.294 & 0.137 & 1180 \\
\hline 05527950 & 1 & No & No & 1997 & 1000 & -- & 798 & 1.82 & 0.3 & 0.148 & 1150 \\
\hline 05527950 & 1 & No & No & 1998 & 609 & -- & 486 & 0.689 & 0.307 & 0.472 & 719 \\
\hline 05527950 & 1 & No & No & 1999 & 1160 & -- & 926 & 1.34 & 0.313 & 0.0927 & 1320 \\
\hline 05527950 & 1 & No & No & 2000 & 1060 & -- & 846 & 1.22 & 0.319 & 0.13 & 1200 \\
\hline 05527950 & 1 & No & No & 2001 & 405 & $\mathrm{E}$ & 323 & 0.535 & 0.333 & 0.768 & 476 \\
\hline 05527950 & 1 & No & No & 2002 & 581 & -- & 464 & 1.26 & 0.347 & 0.545 & 658 \\
\hline 05527950 & 1 & No & No & 2003 & 138 & $\mathrm{E}$ & 110 & 0.679 & 0.36 & 0.983 & 168 \\
\hline 05527950 & 1 & No & No & 2004 & 831 & -- & 663 & 1.47 & 0.374 & 0.288 & 902 \\
\hline 05527950 & 1 & No & No & 2005 & 436 & -- & 348 & 0.617 & 0.388 & 0.767 & 477 \\
\hline 05527950 & 1 & No & No & 2006 & 465 & -- & 371 & 1.2 & 0.402 & 0.744 & 499 \\
\hline 05527950 & 1 & No & No & 2007 & 770 & -- & 615 & 1.57 & 0.415 & 0.38 & 804 \\
\hline 05527950 & 1 & No & No & 2008 & 1070 & -- & 854 & 1.33 & 0.429 & 0.171 & 1100 \\
\hline 05527950 & 1 & No & No & 2009 & 1730 & -- & 1381 & 1.27 & 0.443 & 0.0376 & 1750 \\
\hline 05528000 & 1 & No & No & 1946 & 1580 & -- & 451 & 0.683 & 0.0429 & 0.326 & 2160 \\
\hline 05528000 & 1 & No & No & 1947 & 931 & -- & 266 & 1.18 & 0.0451 & 0.679 & 1350 \\
\hline 05528000 & 1 & No & No & 1948 & 2620 & -- & 747 & 1.8 & 0.0474 & 0.0863 & 3420 \\
\hline 05528000 & 1 & No & No & 1949 & 1300 & 2 & 371 & 0.315 & 0.0496 & 0.46 & 1810 \\
\hline 05528000 & 1 & No & No & 1950 & 1780 & -- & 508 & 1.5 & 0.0519 & 0.257 & 2370 \\
\hline 05528000 & 1 & No & No & 1951 & 1600 & -- & 456 & 0.162 & 0.0554 & 0.326 & 2160 \\
\hline 05528000 & 1 & No & No & 1952 & 1830 & -- & 522 & 0.95 & 0.0589 & 0.244 & 2410 \\
\hline 05528000 & 1 & No & No & 1953 & 391 & -- & 112 & 0.399 & 0.0625 & 0.939 & 639 \\
\hline 05528000 & 1 & No & No & 1954 & 960 & -- & 274 & 1.44 & 0.066 & 0.676 & 1350 \\
\hline 05528000 & 1 & No & No & 1955 & 1100 & -- & 314 & 0.1 & 0.0695 & 0.602 & 1520 \\
\hline 05528000 & 1 & No & No & 1956 & 420 & -- & 120 & 1.01 & 0.0731 & 0.933 & 669 \\
\hline 05528000 & 1 & No & No & 1957 & 745 & -- & 213 & 0.725 & 0.0766 & 0.801 & 1070 \\
\hline 05528000 & 1 & No & No & 1958 & 845 & -- & 241 & 0.168 & 0.0801 & 0.753 & 1190 \\
\hline 05528000 & 1 & No & No & 1960 & 3070 & -- & 876 & 0.0787 & 0.0872 & 0.0613 & 3840 \\
\hline 05528000 & 1 & No & No & 1961 & 960 & -- & 274 & 0.425 & 0.0904 & 0.694 & 1320 \\
\hline 05528000 & 1 & No & No & 1962 & 2140 & -- & 610 & 0.161 & 0.0936 & 0.179 & 2700 \\
\hline 05528000 & 1 & No & No & 1963 & 190 & -- & 54 & 1.48 & 0.0969 & 0.988 & 322 \\
\hline 05528000 & 1 & No & No & 1964 & 700 & 4, B & 200 & 0.0168 & 0.1 & 0.834 & 990 \\
\hline 05528000 & 1 & No & No & 1965 & 1520 & -- & 434 & 0.541 & 0.103 & 0.391 & 1970 \\
\hline 05528000 & 1 & No & No & 1966 & 1580 & -- & 451 & 0.515 & 0.107 & 0.368 & 2040 \\
\hline 05528000 & 1 & No & No & 1967 & 1520 & -- & 434 & 1.44 & 0.11 & 0.396 & 1960 \\
\hline
\end{tabular}




\begin{tabular}{|c|c|c|c|c|c|c|c|c|c|c|c|}
\hline $\begin{array}{l}\text { U.S. } \\
\text { Geological } \\
\text { Survey } \\
\text { streamgage } \\
\text { number }\end{array}$ & $\begin{array}{l}\text { Segment } \\
\text { number }\end{array}$ & $\begin{array}{l}\text { Streamgage used in } \\
\text { regression analyses } \\
\text { (non-redundant) }\end{array}$ & $\begin{array}{c}\text { Discharge value } \\
\text { used in } \\
\text { adjustment } \\
\text { regression }\end{array}$ & $\begin{array}{l}\text { Water } \\
\text { year }\end{array}$ & $\begin{array}{l}\text { Observed annual } \\
\text { maximum peak } \\
\text { discharge }\left(\mathrm{ft}^{3} / \mathrm{s}\right)\end{array}$ & $\begin{array}{l}\text { NWIS peak } \\
\text { code }\end{array}$ & $\begin{array}{l}\text { Observed annual } \\
\text { maximum peak } \\
\text { discharge with } \\
\text { segment intercept } \\
\text { value subtracted } \\
\left(\mathrm{ft}^{3} / \mathrm{s}\right)\end{array}$ & $\begin{array}{l}\text { Observed } \\
\text { precipitation } \\
\text { (inches) }\end{array}$ & Urban fraction & $\begin{array}{l}\text { Exceedance } \\
\text { probability }\end{array}$ & $\begin{array}{c}\text { Urban-adjusted } \\
\text { annual maximum } \\
\text { peak discharge } \\
\left(\mathrm{ft}^{3} / \mathrm{s}\right)\end{array}$ \\
\hline 05528000 & 1 & No & No & 1968 & 394 & -- & 112 & 0.488 & 0.113 & 0.948 & 598 \\
\hline 05528000 & 1 & No & No & 1969 & 980 & -- & 280 & 1.32 & 0.116 & 0.7 & 1300 \\
\hline 05528000 & 1 & No & No & 1970 & 1160 & -- & 331 & 1.7 & 0.119 & 0.605 & 1510 \\
\hline 05528000 & 1 & No & No & 1971 & 1610 & 2 & 459 & 0.242 & 0.124 & 0.368 & 2040 \\
\hline 05528000 & 1 & No & No & 1972 & 1750 & -- & 499 & 0.595 & 0.128 & 0.316 & 2180 \\
\hline 05528000 & 1 & No & No & 1973 & 1500 & -- & 428 & 1.41 & 0.133 & 0.423 & 1890 \\
\hline 05528000 & 1 & No & No & 1974 & 2140 & -- & 610 & 0.48 & 0.137 & 0.196 & 2600 \\
\hline 05528000 & 1 & No & No & 1975 & 661 & -- & 189 & 0.899 & 0.141 & 0.87 & 895 \\
\hline 05528000 & 1 & No & No & 1976 & 2590 & -- & 739 & 1.34 & 0.146 & 0.12 & 3110 \\
\hline 05528000 & 1 & No & No & 1977 & 173 & -- & 49 & 0.816 & 0.15 & 1 & 269 \\
\hline 05528000 & 1 & No & No & 1978 & 1700 & -- & 485 & 0.259 & 0.154 & 0.353 & 2080 \\
\hline 05528000 & 1 & No & No & 1979 & 2860 & -- & 816 & 0.295 & 0.159 & 0.0894 & 3380 \\
\hline 05528000 & 1 & No & No & 1980 & 955 & -- & 272 & 0.307 & 0.163 & 0.745 & 1210 \\
\hline 05528000 & 1 & No & No & 1981 & 651 & -- & 186 & 0.172 & 0.166 & 0.883 & 855 \\
\hline 05528000 & 1 & No & No & 1982 & 1590 & -- & 454 & 0.496 & 0.169 & 0.409 & 1930 \\
\hline 05528000 & 1 & No & No & 1983 & 2370 & -- & 676 & 0.512 & 0.172 & 0.168 & 2780 \\
\hline 05528000 & 1 & No & No & 1984 & 1050 & -- & 300 & 0.2 & 0.176 & 0.703 & 1300 \\
\hline 05528000 & 1 & No & No & 1985 & 1800 & -- & 513 & 1.05 & 0.179 & 0.331 & 2140 \\
\hline 05528000 & 1 & No & No & 1986 & 3530 & -- & 1007 & 1.13 & 0.182 & 0.0508 & 4060 \\
\hline 05528000 & 1 & No & No & 1987 & 1260 & -- & 359 & 0.0168 & 0.185 & 0.601 & 1520 \\
\hline 05528000 & 1 & No & No & 1988 & 1390 & -- & 397 & 0.173 & 0.188 & 0.526 & 1660 \\
\hline 05528000 & 1 & No & No & 1989 & 279 & -- & 80 & 0.443 & 0.191 & 0.982 & 384 \\
\hline 05528000 & 1 & No & No & 1990 & 1400 & -- & 399 & 0.287 & 0.194 & 0.526 & 1660 \\
\hline 05528000 & 1 & No & No & 1991 & 1260 & -- & 359 & 0.256 & 0.2 & 0.611 & 1500 \\
\hline 05528000 & 1 & No & No & 1992 & 820 & -- & 234 & 0.0696 & 0.207 & 0.831 & 998 \\
\hline 05528000 & 1 & No & No & 1993 & 2370 & -- & 676 & 0.898 & 0.213 & 0.184 & 2680 \\
\hline 05528000 & 1 & No & No & 1994 & 1370 & -- & 391 & 0.768 & 0.219 & 0.565 & 1590 \\
\hline 05528000 & 1 & No & No & 1995 & 1220 & C & 348 & 0.106 & 0.225 & 0.649 & 1410 \\
\hline 05528000 & 1 & No & No & 1996 & 1640 & C & 468 & 0.963 & 0.231 & 0.437 & 1860 \\
\hline 05528000 & 1 & No & No & 1997 & 1320 & C & 377 & 1.66 & 0.237 & 0.609 & 1500 \\
\hline 05528000 & 1 & No & No & 1998 & 1180 & C & 337 & 0.732 & 0.243 & 0.683 & 1340 \\
\hline 05528000 & 1 & No & No & 1999 & 1470 & C & 419 & 1.14 & 0.249 & 0.537 & 1640 \\
\hline 05528000 & 1 & No & No & 2000 & 2690 & $C, E$ & 767 & 0.925 & 0.255 & 0.147 & 2920 \\
\hline 05528000 & 1 & No & No & 2001 & 1140 & C & 325 & 0.122 & 0.264 & 0.719 & 1260 \\
\hline 05528000 & 1 & No & No & 2002 & 989 & C & 282 & 0.881 & 0.273 & 0.794 & 1090 \\
\hline
\end{tabular}




\begin{tabular}{|c|c|c|c|c|c|c|c|c|c|c|c|}
\hline $\begin{array}{l}\text { U.S. } \\
\text { Geological } \\
\text { Survey } \\
\text { streamgage } \\
\text { number }\end{array}$ & $\begin{array}{l}\text { Segment } \\
\text { number }\end{array}$ & $\begin{array}{l}\text { Streamgage used in } \\
\text { regression analyses } \\
\text { (non-redundant) }\end{array}$ & $\begin{array}{c}\text { Discharge value } \\
\text { used in } \\
\text { adjustment } \\
\text { regression }\end{array}$ & $\begin{array}{l}\text { Water } \\
\text { year }\end{array}$ & $\begin{array}{c}\text { Observed annual } \\
\text { maximum peak } \\
\text { discharge }\left(\mathrm{ft}^{3} / \mathrm{s}\right)\end{array}$ & $\begin{array}{l}\text { NWIS peak } \\
\text { code }\end{array}$ & $\begin{array}{l}\text { Observed annual } \\
\text { maximum peak } \\
\text { discharge with } \\
\text { segment intercept } \\
\text { value subtracted } \\
\left(\mathrm{ft}^{3} / \mathrm{s}\right)\end{array}$ & $\begin{array}{l}\text { Observed } \\
\text { precipitation } \\
\text { (inches) }\end{array}$ & Urban fraction & $\begin{array}{l}\text { Exceedance } \\
\text { probability }\end{array}$ & $\begin{array}{c}\text { Urban-adjusted } \\
\text { annual maximum } \\
\text { peak discharge } \\
\left(\mathrm{ft}^{3} / \mathrm{s}\right)\end{array}$ \\
\hline 05528000 & 1 & No & No & 2003 & 516 & $C, E$ & 147 & 0.483 & 0.283 & 0.951 & 579 \\
\hline 05528000 & 1 & No & No & 2004 & 3890 & C & 1110 & 1.6 & 0.292 & 0.0496 & 4070 \\
\hline 05528000 & 1 & No & No & 2005 & 1090 & C & 311 & 0.284 & 0.301 & 0.766 & 1160 \\
\hline 05528000 & 1 & No & No & 2006 & 838 & C & 239 & 0.0897 & 0.31 & 0.874 & 885 \\
\hline 05528000 & 1 & No & No & 2007 & 2390 & C & 682 & 0.807 & 0.319 & 0.234 & 2450 \\
\hline 05528000 & 1 & No & No & 2008 & 1900 & C & 542 & 0.888 & 0.328 & 0.405 & 1940 \\
\hline 05528000 & 1 & No & No & 2009 & 2120 & C & 605 & 1.92 & 0.337 & 0.329 & 2140 \\
\hline 05528150 & 1 & Yes & Yes & 1960 & 1150 & -- & 1668 & 0.5 & 0.147 & 0.0113 & 2020 \\
\hline 05528150 & 1 & Yes & Yes & 1961 & 111 & -- & 161 & 1.22 & 0.15 & 0.908 & 381 \\
\hline 05528150 & 1 & Yes & Yes & 1962 & 466 & -- & 676 & 0.261 & 0.152 & 0.16 & 949 \\
\hline 05528150 & 1 & Yes & Yes & 1963 & 67 & 2 & 97 & 1.26 & 0.155 & 0.965 & 290 \\
\hline 05528150 & 1 & Yes & Yes & 1964 & 80 & -- & 116 & 1.73 & 0.158 & 0.952 & 321 \\
\hline 05528150 & 1 & Yes & Yes & 1965 & 306 & -- & 444 & 0.565 & 0.16 & 0.418 & 703 \\
\hline 05528150 & 1 & Yes & Yes & 1966 & 280 & -- & 406 & 0.476 & 0.163 & 0.486 & 656 \\
\hline 05528150 & 1 & Yes & Yes & 1967 & 284 & -- & 412 & 0.924 & 0.166 & 0.478 & 662 \\
\hline 05528150 & 1 & Yes & Yes & 1968 & 80 & -- & 116 & 1.25 & 0.168 & 0.954 & 317 \\
\hline 05528150 & 1 & Yes & Yes & 1969 & 406 & -- & 589 & 1.34 & 0.171 & 0.237 & 835 \\
\hline 05528150 & 1 & Yes & Yes & 1970 & 590 & -- & 856 & 1.51 & 0.174 & 0.0801 & 1150 \\
\hline 05528150 & 1 & Yes & Yes & 1971 & 375 & -- & 544 & 0.415 & 0.181 & 0.294 & 789 \\
\hline 05528150 & 1 & Yes & Yes & 1972 & 417 & -- & 605 & 1.2 & 0.188 & 0.23 & 841 \\
\hline 05528150 & 1 & Yes & Yes & 1973 & 386 & -- & 560 & 1.44 & 0.195 & 0.284 & 796 \\
\hline 05528150 & 1 & Yes & Yes & 1974 & 306 & -- & 444 & 1.11 & 0.202 & 0.453 & 679 \\
\hline 05528150 & 1 & Yes & Yes & 1975 & 174 & -- & 252 & 1.12 & 0.21 & 0.804 & 461 \\
\hline 05528150 & 1 & Yes & Yes & 1976 & 403 & -- & 584 & 1.26 & 0.217 & 0.27 & 807 \\
\hline 05528170 & 1 & Yes & Yes & 1961 & 64 & -- & 637 & 1.21 & 0.416 & 0.353 & 87.5 \\
\hline 05528170 & 1 & Yes & Yes & 1962 & 56 & -- & 557 & 0.261 & 0.419 & 0.463 & 77.8 \\
\hline 05528170 & 1 & Yes & Yes & 1963 & 35 & -- & 348 & 1.26 & 0.421 & 0.788 & 52 \\
\hline 05528170 & 1 & Yes & Yes & 1964 & 67 & -- & 666 & 0.685 & 0.423 & 0.322 & 90.3 \\
\hline 05528170 & 1 & Yes & Yes & 1965 & 36 & -- & 358 & 0.565 & 0.426 & 0.777 & 52.9 \\
\hline 05528170 & 1 & Yes & Yes & 1966 & 78 & -- & 776 & 1.16 & 0.428 & 0.218 & 102 \\
\hline 05528170 & 1 & Yes & Yes & 1967 & 49 & -- & 487 & 0.922 & 0.43 & 0.583 & 68.2 \\
\hline 05528170 & 1 & Yes & Yes & 1968 & 40 & -- & 398 & 1.25 & 0.433 & 0.726 & 57.3 \\
\hline 05528170 & 1 & Yes & Yes & 1969 & 23 & -- & 229 & 1.34 & 0.435 & 0.93 & 36.9 \\
\hline 05528170 & 1 & Yes & Yes & 1970 & 36 & -- & 358 & 1.51 & 0.437 & 0.785 & 52.2 \\
\hline 05528170 & 1 & Yes & Yes & 1971 & 110 & -- & 1094 & 0.415 & 0.44 & 0.077 & 140 \\
\hline
\end{tabular}




\begin{tabular}{|c|c|c|c|c|c|c|c|c|c|c|c|}
\hline $\begin{array}{l}\text { U.S. } \\
\text { Geological } \\
\text { Survey } \\
\text { streamgage } \\
\text { number }\end{array}$ & $\begin{array}{l}\text { Segment } \\
\text { number }\end{array}$ & $\begin{array}{l}\text { Streamgage used in } \\
\text { regression analyses } \\
\text { (non-redundant) }\end{array}$ & $\begin{array}{c}\text { Discharge value } \\
\text { used in } \\
\text { adjustment } \\
\text { regression }\end{array}$ & $\begin{array}{l}\text { Water } \\
\text { year }\end{array}$ & $\begin{array}{l}\text { Observed annual } \\
\text { maximum peak } \\
\text { discharge }\left(\mathrm{ft}^{3} / \mathrm{s}\right)\end{array}$ & $\begin{array}{l}\text { NWIS peak } \\
\text { code }\end{array}$ & $\begin{array}{l}\text { Observed annual } \\
\text { maximum peak } \\
\text { discharge with } \\
\text { segment intercept } \\
\text { value subtracted } \\
\left(\mathrm{ft}^{3} / \mathrm{s}\right)\end{array}$ & $\begin{array}{l}\text { Observed } \\
\text { precipitation } \\
\text { (inches) }\end{array}$ & Urban fraction & $\begin{array}{l}\text { Exceedance } \\
\text { probability }\end{array}$ & $\begin{array}{c}\text { Urban-adjusted } \\
\text { annual maximum } \\
\text { peak discharge } \\
\left(\mathrm{ft}^{3} / \mathrm{s}\right)\end{array}$ \\
\hline 05528170 & 1 & Yes & Yes & 1972 & 68 & -- & 676 & 1.2 & 0.443 & 0.326 & 89.9 \\
\hline 05528170 & 1 & Yes & Yes & 1973 & 56 & -- & 557 & 1.43 & 0.446 & 0.488 & 75.7 \\
\hline 05528170 & 1 & Yes & Yes & 1974 & 54 & -- & 537 & 1.11 & 0.449 & 0.52 & 73 \\
\hline 05528170 & 1 & Yes & Yes & 1975 & 107 & -- & 1064 & 0.819 & 0.452 & 0.0884 & 136 \\
\hline 05528170 & 1 & Yes & Yes & 1976 & 41 & -- & 408 & 1.26 & 0.455 & 0.728 & 57.1 \\
\hline 05528200 & 0 & Yes & No & 1961 & 206 & -- & NA & 1.97 & 0.452 & NA & NA \\
\hline 05528200 & 0 & Yes & No & 1962 & 125 & -- & NA & 0.258 & 0.46 & NA & NA \\
\hline 05528200 & 1 & Yes & Yes & 1963 & 106 & -- & 297 & 1.26 & 0.468 & 0.88 & 209 \\
\hline 05528200 & 1 & Yes & Yes & 1964 & 148 & -- & 414 & 1.76 & 0.477 & 0.735 & 261 \\
\hline 05528200 & 1 & Yes & Yes & 1965 & 197 & -- & 551 & 0.563 & 0.485 & 0.532 & 323 \\
\hline 05528200 & 1 & Yes & Yes & 1966 & 230 & -- & 644 & 0.476 & 0.493 & 0.411 & 366 \\
\hline 05528200 & 1 & Yes & Yes & 1967 & 273 & -- & 764 & 1.28 & 0.502 & 0.276 & 413 \\
\hline 05528200 & 1 & Yes & Yes & 1968 & 277 & -- & 775 & 1.2 & 0.51 & 0.271 & 415 \\
\hline 05528200 & 1 & Yes & Yes & 1969 & 416 & -- & 1164 & 1.36 & 0.518 & 0.0789 & 595 \\
\hline 05528200 & 1 & Yes & Yes & 1970 & 543 & -- & 1520 & 1.52 & 0.527 & 0.0342 & 748 \\
\hline 05528200 & 1 & Yes & Yes & 1971 & 214 & -- & 599 & 0.417 & 0.55 & 0.524 & 326 \\
\hline 05528200 & 1 & Yes & Yes & 1972 & 433 & -- & 1212 & 1.2 & 0.572 & 0.0813 & 590 \\
\hline 05528200 & 1 & Yes & Yes & 1973 & 146 & -- & 409 & 1.42 & 0.595 & 0.825 & 230 \\
\hline 05528200 & 1 & Yes & Yes & 1974 & 152 & -- & 425 & 0.641 & 0.618 & 0.821 & 231 \\
\hline 05528200 & 1 & Yes & Yes & 1975 & 206 & 2 & 577 & 0.784 & 0.641 & 0.645 & 288 \\
\hline 05528200 & 1 & Yes & Yes & 1976 & 100 & -- & 280 & 1.27 & 0.664 & 0.956 & 160 \\
\hline 05528230 & 0 & No & No & 1960 & 1410 & -- & NA & 0.494 & 0.217 & NA & NA \\
\hline 05528230 & 0 & No & No & 1961 & 393 & -- & NA & 1.22 & 0.224 & NA & NA \\
\hline 05528230 & 1 & No & No & 1963 & 162 & -- & 156 & 1.25 & 0.236 & 0.935 & 515 \\
\hline 05528230 & 1 & No & No & 1964 & 222 & -- & 213 & 1.74 & 0.243 & 0.878 & 605 \\
\hline 05528230 & 1 & No & No & 1965 & 656 & -- & 631 & 0.561 & 0.249 & 0.24 & 1240 \\
\hline 05528230 & 1 & No & No & 1967 & 945 & -- & 908 & 0.924 & 0.262 & 0.0851 & 1690 \\
\hline 05528230 & 1 & No & No & 1968 & 354 & -- & 340 & 1.23 & 0.268 & 0.696 & 786 \\
\hline 05528230 & 1 & No & No & 1969 & 625 & -- & 601 & 1.34 & 0.274 & 0.288 & 1180 \\
\hline 05528230 & 1 & No & No & 1970 & 852 & -- & 819 & 1.5 & 0.281 & 0.129 & 1520 \\
\hline 05528230 & 1 & No & No & 1971 & 364 & $4 B$ & 350 & 0.249 & 0.293 & 0.699 & 784 \\
\hline 05528230 & 1 & No & No & 1973 & 617 & -- & 593 & 1.43 & 0.319 & 0.33 & 1140 \\
\hline 05528230 & 1 & No & No & 1974 & 526 & -- & 506 & 0.62 & 0.332 & 0.463 & 1000 \\
\hline 05528230 & 1 & No & No & 1975 & 235 & -- & 226 & 0.823 & 0.345 & 0.902 & 573 \\
\hline 05528230 & 1 & No & No & 1976 & 537 & -- & 516 & 1.24 & 0.358 & 0.469 & 994 \\
\hline
\end{tabular}




\begin{tabular}{|c|c|c|c|c|c|c|c|c|c|c|c|}
\hline $\begin{array}{c}\text { U.S. } \\
\text { Geological } \\
\text { Survey } \\
\text { streamgage } \\
\text { number }\end{array}$ & $\begin{array}{l}\text { Segment } \\
\text { number }\end{array}$ & $\begin{array}{l}\text { Streamgage used in } \\
\text { regression analyses } \\
\text { (non-redundant) }\end{array}$ & $\begin{array}{c}\text { Discharge value } \\
\text { used in } \\
\text { adjustment } \\
\text { regression }\end{array}$ & $\begin{array}{c}\text { Water } \\
\text { year }\end{array}$ & $\begin{array}{c}\text { Observed annual } \\
\text { maximum peak } \\
\text { discharge }\left(\mathrm{ft}^{3} / \mathrm{s}\right)\end{array}$ & $\begin{array}{c}\text { NWIS peak } \\
\text { code }\end{array}$ & $\begin{array}{c}\text { Observed annual } \\
\text { maximum peak } \\
\text { discharge with } \\
\text { segment intercept } \\
\text { value subtracted } \\
\left(\mathrm{ft}^{3} / \mathrm{s}\right) \\
\end{array}$ & $\begin{array}{c}\text { Observed } \\
\text { precipitation } \\
\text { (inches) }\end{array}$ & Urban fraction & $\begin{array}{l}\text { Exceedance } \\
\text { probability }\end{array}$ & $\begin{array}{c}\text { Urban-adjusted } \\
\text { annual maximum } \\
\text { peak discharge } \\
\left(\mathrm{ft}^{3} / \mathrm{s}\right)\end{array}$ \\
\hline 05528230 & 1 & No & No & 1990 & 586 & -- & 563 & 0.535 & 0.578 & 0.604 & 866 \\
\hline 05528230 & 1 & No & No & 1991 & 646 & -- & 621 & 0.861 & 0.586 & 0.529 & 935 \\
\hline 05528230 & 1 & No & No & 1992 & 268 & $E$ & 258 & 0.599 & 0.595 & 0.952 & 476 \\
\hline 05528230 & 1 & No & No & 1993 & 576 & -- & 554 & 0.872 & 0.603 & 0.641 & 833 \\
\hline 05528230 & 1 & No & No & 1994 & 815 & $C, E$ & 784 & 0.624 & 0.611 & 0.348 & 1120 \\
\hline 05528230 & 1 & No & No & 1995 & 623 & $C$ & 599 & 1.05 & 0.62 & 0.593 & 876 \\
\hline 05528230 & 1 & No & No & 1996 & 1800 & C & 1730 & 0.249 & 0.628 & 0.0287 & 2260 \\
\hline 05528360 & 1 & Yes & Yes & 1961 & 34 & -- & 112 & 1.38 & 0.0621 & 0.938 & 172 \\
\hline 05528360 & 1 & Yes & Yes & 1962 & 100 & 2 & 331 & 0.249 & 0.0643 & 0.558 & 292 \\
\hline 05528360 & 1 & Yes & Yes & 1963 & 38 & -- & 126 & 1.13 & 0.0665 & 0.927 & 180 \\
\hline 05528360 & 1 & Yes & Yes & 1964 & 47 & -- & 155 & 1.77 & 0.0687 & 0.89 & 195 \\
\hline 05528360 & 1 & Yes & Yes & 1965 & 78 & 2 & 258 & 0.0906 & 0.0708 & 0.713 & 250 \\
\hline 05528360 & 1 & Yes & Yes & 1966 & 79 & 2 & 261 & 0.416 & 0.073 & 0.708 & 251 \\
\hline 05528360 & 1 & Yes & Yes & 1967 & 147 & -- & 486 & 1.47 & 0.0752 & 0.299 & 371 \\
\hline 05528360 & 1 & Yes & Yes & 1968 & 62 & -- & 205 & 1.14 & 0.0774 & 0.814 & 221 \\
\hline 05528360 & 1 & Yes & Yes & 1969 & 123 & 2 & 407 & 1.28 & 0.0796 & 0.418 & 334 \\
\hline 05528360 & 1 & Yes & Yes & 1970 & 300 & 2 & 992 & 1.33 & 0.0817 & 0.0423 & 643 \\
\hline 05528360 & 1 & Yes & Yes & 1971 & 157 & 2 & 519 & 0.385 & 0.112 & 0.28 & 377 \\
\hline 05528360 & 1 & Yes & Yes & 1972 & 390 & -- & 1289 & 0.692 & 0.142 & 0.02 & 750 \\
\hline 05528360 & 1 & Yes & Yes & 1973 & 173 & -- & 572 & 1.38 & 0.172 & 0.255 & 386 \\
\hline 05528360 & 1 & Yes & Yes & 1974 & 126 & 2 & 416 & 0.523 & 0.202 & 0.5 & 308 \\
\hline 05528360 & 1 & Yes & Yes & 1975 & 114 & -- & 377 & 0.951 & 0.232 & 0.605 & 280 \\
\hline 05528360 & 1 & Yes & Yes & 1976 & 166 & -- & 549 & 0.974 & 0.262 & 0.344 & 357 \\
\hline 05528400 & 1 & No & No & 1962 & 2300 & -- & 521 & 0.00304 & 0.136 & 0.293 & 3250 \\
\hline 05528400 & 1 & No & No & 1963 & 660 & -- & 149 & 1.41 & 0.14 & 0.919 & 1150 \\
\hline 05528400 & 1 & No & No & 1967 & 1730 & -- & 392 & 0.774 & 0.158 & 0.509 & 2490 \\
\hline 05528400 & 1 & No & No & 1969 & 1940 & -- & 439 & 1.36 & 0.166 & 0.431 & 2740 \\
\hline 05528400 & 1 & No & No & 1970 & 3240 & -- & 734 & 1.66 & 0.171 & 0.133 & 4310 \\
\hline 05528400 & 1 & No & No & 1972 & 1870 & -- & 423 & 1.07 & 0.184 & 0.472 & 2600 \\
\hline 05528400 & 1 & No & No & 1973 & 2450 & -- & 555 & 1.39 & 0.191 & 0.287 & 3270 \\
\hline 05528400 & 1 & No & No & 1974 & 2100 & -- & 475 & 0.285 & 0.197 & 0.397 & 2850 \\
\hline 05528400 & 1 & No & No & 1975 & 1160 & -- & 263 & 0.873 & 0.204 & 0.784 & 1680 \\
\hline 05528400 & 1 & No & No & 1976 & 2400 & -- & 543 & 0.0211 & 0.211 & 0.314 & 3160 \\
\hline 05528400 & 1 & No & No & 1977 & 610 & -- & 138 & 0.825 & 0.217 & 0.946 & 975 \\
\hline 05528440 & 1 & Yes & Yes & 1961 & 44 & 2 & 295 & 1.21 & 0.333 & 0.809 & 109 \\
\hline
\end{tabular}




\begin{tabular}{|c|c|c|c|c|c|c|c|c|c|c|c|}
\hline $\begin{array}{c}\text { U.S. } \\
\text { Geological } \\
\text { Survey } \\
\text { streamgage } \\
\text { number } \\
\end{array}$ & $\begin{array}{c}\text { Segment } \\
\text { number }\end{array}$ & $\begin{array}{l}\text { Streamgage used in } \\
\text { regression analyses } \\
\text { (non-redundant) }\end{array}$ & $\begin{array}{c}\text { Discharge value } \\
\text { used in } \\
\text { adjustment } \\
\text { regression } \\
\end{array}$ & $\begin{array}{c}\text { Water } \\
\text { year }\end{array}$ & $\begin{array}{c}\text { Observed annual } \\
\text { maximum peak } \\
\text { discharge }\left(\mathrm{ft}^{3} / \mathrm{s}\right)\end{array}$ & $\begin{array}{c}\text { NWIS peak } \\
\text { code }\end{array}$ & $\begin{array}{c}\text { Observed annual } \\
\text { maximum peak } \\
\text { discharge with } \\
\text { segment intercept } \\
\text { value subtracted } \\
\left(\mathrm{ft}^{3} / \mathrm{s}\right)\end{array}$ & $\begin{array}{c}\text { Observed } \\
\text { precipitation } \\
\text { (inches) }\end{array}$ & Urban fraction & $\begin{array}{l}\text { Exceedance } \\
\text { probability }\end{array}$ & $\begin{array}{c}\text { Urban-adjusted } \\
\text { annual maximum } \\
\text { peak discharge } \\
\left(\mathrm{ft}^{3} / \mathrm{s}\right) \\
\end{array}$ \\
\hline 05528440 & 1 & Yes & Yes & 1962 & 53 & 2 & 355 & 0.261 & 0.342 & 0.724 & 122 \\
\hline 05528440 & 1 & Yes & Yes & 1963 & 34 & -- & 228 & 1.26 & 0.35 & 0.902 & 93.9 \\
\hline 05528440 & 1 & Yes & Yes & 1964 & 30 & -- & 201 & 1.73 & 0.358 & 0.929 & 87.8 \\
\hline 05528440 & 1 & Yes & Yes & 1965 & 72 & -- & 483 & 0.0937 & 0.367 & 0.531 & 147 \\
\hline 05528440 & 1 & Yes & Yes & 1966 & 64 & -- & 429 & 0.475 & 0.375 & 0.629 & 134 \\
\hline 05528440 & 1 & Yes & Yes & 1967 & 64 & -- & 429 & 0.923 & 0.384 & 0.636 & 133 \\
\hline 05528440 & 1 & Yes & Yes & 1968 & 52 & 2 & 349 & 1.25 & 0.392 & 0.769 & 115 \\
\hline 05528440 & 1 & Yes & Yes & 1969 & 76 & -- & 510 & 1.34 & 0.4 & 0.518 & 149 \\
\hline 05528440 & 1 & Yes & Yes & 1970 & 193 & -- & 1294 & 1.51 & 0.409 & 0.0422 & 315 \\
\hline 05528440 & 1 & Yes & Yes & 1971 & 83 & -- & 557 & 0.415 & 0.455 & 0.496 & 152 \\
\hline 05528440 & 1 & Yes & Yes & 1972 & 203 & -- & 1361 & 1.2 & 0.501 & 0.0453 & 308 \\
\hline 05528440 & 1 & Yes & Yes & 1973 & 121 & -- & 811 & 1.43 & 0.547 & 0.264 & 188 \\
\hline 05528440 & 1 & Yes & Yes & 1974 & 125 & -- & 838 & 1.11 & 0.593 & 0.273 & 186 \\
\hline 05528440 & 1 & Yes & Yes & 1975 & 155 & -- & 1039 & 0.82 & 0.639 & 0.171 & 216 \\
\hline 05528440 & 1 & Yes & Yes & 1976 & 118 & -- & 791 & 1.26 & 0.685 & 0.413 & 165 \\
\hline 05528470 & 1 & No & No & 1961 & 102 & -- & 197 & 1.22 & 0.192 & 0.879 & 335 \\
\hline 05528470 & 1 & No & No & 1962 & 204 & -- & 394 & 0.262 & 0.207 & 0.548 & 496 \\
\hline 05528470 & 1 & No & No & 1963 & 55 & $4, B$ & 106 & 0.0872 & 0.222 & 0.967 & 239 \\
\hline 05528470 & 1 & No & No & 1964 & 118 & 2 & 228 & 0.672 & 0.237 & 0.854 & 348 \\
\hline 05528470 & 1 & No & No & 1965 & 152 & -- & 293 & 0.0872 & 0.252 & 0.764 & 397 \\
\hline 05528470 & 1 & No & No & 1966 & 204 & -- & 394 & 0.483 & 0.267 & 0.603 & 472 \\
\hline 05528470 & 1 & No & No & 1967 & 340 & -- & 656 & 1.28 & 0.282 & 0.235 & 666 \\
\hline 05528470 & 1 & No & No & 1968 & 126 & -- & 243 & 1.48 & 0.297 & 0.861 & 344 \\
\hline 05528470 & 1 & No & No & 1969 & 221 & -- & 427 & 1.43 & 0.312 & 0.581 & 481 \\
\hline 05528470 & 1 & No & No & 1970 & 403 & -- & 778 & 1.62 & 0.327 & 0.17 & 740 \\
\hline 05528470 & 1 & No & No & 1971 & 227 & -- & 438 & 0.413 & 0.359 & 0.602 & 472 \\
\hline 05528470 & 1 & No & No & 1972 & 539 & -- & 1041 & 1.24 & 0.39 & 0.0788 & 914 \\
\hline 05528470 & 1 & No & No & 1973 & 387 & -- & 747 & 1.38 & 0.422 & 0.238 & 664 \\
\hline 05528470 & 1 & No & No & 1974 & 264 & -- & 510 & 0.664 & 0.454 & 0.568 & 487 \\
\hline 05528470 & 1 & No & No & 1975 & 296 & -- & 571 & 0.901 & 0.485 & 0.503 & 519 \\
\hline 05528470 & 1 & No & No & 1976 & 426 & -- & 822 & 1.31 & 0.517 & 0.235 & 667 \\
\hline 05528500 & 1 & Yes & Yes & 1953 & 55 & $\mathrm{E}$ & 82 & 0.598 & 0.0952 & 0.969 & 192 \\
\hline 05528500 & 1 & Yes & Yes & 1954 & 268 & -- & 399 & 1.36 & 0.107 & 0.454 & 476 \\
\hline 05528500 & 1 & Yes & Yes & 1955 & 161 & -- & 240 & 2.92 & 0.118 & 0.775 & 335 \\
\hline 05528500 & 1 & Yes & Yes & 1956 & 110 & $E$ & 164 & 1.18 & 0.13 & 0.899 & 270 \\
\hline
\end{tabular}




\begin{tabular}{|c|c|c|c|c|c|c|c|c|c|c|c|}
\hline $\begin{array}{l}\text { U.S. } \\
\text { Geological } \\
\text { Survey } \\
\text { streamgage } \\
\text { number }\end{array}$ & $\begin{array}{l}\text { Segment } \\
\text { number }\end{array}$ & $\begin{array}{l}\text { Streamgage used in } \\
\text { regression analyses } \\
\text { (non-redundant) }\end{array}$ & $\begin{array}{l}\text { Discharge value } \\
\text { used in } \\
\text { adjustment } \\
\text { regression }\end{array}$ & $\begin{array}{l}\text { Water } \\
\text { year }\end{array}$ & $\begin{array}{l}\text { Observed annual } \\
\text { maximum peak } \\
\text { discharge }\left(\mathrm{ft}^{3} / \mathrm{s}\right)\end{array}$ & $\begin{array}{l}\text { NWIS peak } \\
\text { code }\end{array}$ & $\begin{array}{l}\text { Observed annual } \\
\text { maximum peak } \\
\text { discharge with } \\
\text { segment intercept } \\
\text { value subtracted } \\
\left(\mathrm{ft}^{3} / \mathrm{s}\right)\end{array}$ & $\begin{array}{l}\text { Observed } \\
\text { precipitation } \\
\text { (inches) }\end{array}$ & Urban fraction & $\begin{array}{l}\text { Exceedance } \\
\text { probability }\end{array}$ & $\begin{array}{c}\text { Urban-adjusted } \\
\text { annual maximum } \\
\text { peak discharge } \\
\left(\mathrm{ft}^{3} / \mathrm{s}\right)\end{array}$ \\
\hline 05528500 & 1 & Yes & Yes & 1957 & 430 & -- & 640 & 1.87 & 0.141 & 0.179 & 644 \\
\hline 05528500 & 1 & Yes & Yes & 1958 & 130 & $\mathrm{E}$ & 193 & 0.209 & 0.153 & 0.867 & 288 \\
\hline 05528500 & 1 & Yes & Yes & 1959 & 320 & 2 & 476 & 0.392 & 0.165 & 0.372 & 516 \\
\hline 05528500 & 1 & Yes & Yes & 1960 & 457 & -- & 680 & 0.456 & 0.176 & 0.167 & 660 \\
\hline 05528500 & 1 & Yes & Yes & 1961 & 370 & -- & 551 & 1.3 & 0.197 & 0.296 & 554 \\
\hline 05528500 & 1 & Yes & Yes & 1962 & 318 & -- & 473 & 0.254 & 0.218 & 0.417 & 494 \\
\hline 05528500 & 1 & Yes & Yes & 1963 & 76 & $E$ & 113 & 1.24 & 0.239 & 0.965 & 197 \\
\hline 05528500 & 1 & Yes & Yes & 1964 & 264 & -- & 393 & 0.877 & 0.26 & 0.599 & 412 \\
\hline 05528500 & 1 & Yes & Yes & 1965 & 274 & -- & 408 & 0.089 & 0.281 & 0.588 & 416 \\
\hline 05528500 & 1 & Yes & Yes & 1966 & 343 & -- & 510 & 0.455 & 0.302 & 0.43 & 488 \\
\hline 05528500 & 1 & Yes & Yes & 1967 & 758 & -- & 1128 & 1.35 & 0.323 & 0.0511 & 918 \\
\hline 05528500 & 1 & Yes & Yes & 1968 & 617 & -- & 918 & 1.33 & 0.344 & 0.105 & 756 \\
\hline 05528500 & 1 & Yes & Yes & 1969 & 335 & -- & 498 & 1.37 & 0.365 & 0.503 & 453 \\
\hline 05528500 & 1 & Yes & Yes & 1970 & 600 & $\mathrm{D}$ & 893 & 1.5 & 0.386 & 0.134 & 712 \\
\hline 05528500 & 1 & Yes & Yes & 1971 & 350 & $\mathrm{E}$ & 521 & 0.402 & 0.414 & 0.513 & 448 \\
\hline 05528500 & 1 & Yes & Yes & 1972 & 802 & -- & 1193 & 1.16 & 0.442 & 0.0593 & 884 \\
\hline 05528500 & 1 & Yes & Yes & 1973 & 454 & -- & 675 & 1.38 & 0.47 & 0.349 & 527 \\
\hline 05528500 & 1 & Yes & Yes & 1974 & 424 & -- & 631 & 0.605 & 0.498 & 0.432 & 487 \\
\hline 05528500 & 1 & Yes & Yes & 1975 & 388 & -- & 577 & 0.918 & 0.526 & 0.533 & 439 \\
\hline 05528500 & 1 & Yes & Yes & 1976 & 458 & -- & 681 & 1.17 & 0.554 & 0.419 & 494 \\
\hline 05528500 & 1 & Yes & Yes & 1977 & 116 & $\mathrm{E}$ & 173 & 0.814 & 0.582 & 0.983 & 164 \\
\hline 05528500 & 1 & Yes & Yes & 1978 & 372 & -- & 553 & 1.32 & 0.61 & 0.648 & 389 \\
\hline 05528500 & 1 & Yes & Yes & 1979 & 409 & -- & 609 & 1.01 & 0.638 & 0.598 & 411 \\
\hline 05528500 & 1 & Yes & Yes & 1980 & 571 & -- & 850 & 0.675 & 0.666 & 0.324 & 537 \\
\hline 05528500 & 1 & Yes & Yes & 1981 & 597 & -- & 888 & 1.14 & 0.675 & 0.291 & 554 \\
\hline 05528500 & 1 & Yes & Yes & 1982 & 887 & -- & 1320 & 1.87 & 0.684 & 0.0857 & 798 \\
\hline 05528500 & 0 & Yes & No & 1983 & 610 & -- & 1050 & 1.6 & 0.692 & 0.189 & 635 \\
\hline 05528500 & 2 & Yes & Yes & 1984 & 382 & -- & 760 & 1.2 & 0.701 & 0.465 & 472 \\
\hline 05528500 & 2 & Yes & Yes & 1985 & 455 & $-{ }_{-1}$ & 905 & 1.24 & 0.709 & 0.303 & 547 \\
\hline 05528500 & 2 & Yes & Yes & 1986 & 596 & \begin{tabular}{l|}
- \\
\end{tabular} & 1186 & 0.74 & 0.718 & 0.144 & 699 \\
\hline 05528500 & 2 & Yes & Yes & 1987 & 717 & -- & 1427 & 2.44 & 0.726 & 0.0731 & 829 \\
\hline 05528500 & 2 & Yes & Yes & 1988 & 308 & -- & 613 & 0.434 & 0.735 & 0.691 & 374 \\
\hline 05528500 & 2 & Yes & Yes & 1989 & 352 & -- & 700 & 1.37 & 0.743 & 0.58 & 418 \\
\hline 05528500 & 2 & Yes & Yes & 1990 & 613 & -- & 1220 & 1.56 & 0.752 & 0.146 & 697 \\
\hline 05528500 & 2 & Yes & Yes & 1991 & 374 & -- & 744 & 1.76 & 0.755 & 0.539 & 437 \\
\hline
\end{tabular}




\begin{tabular}{|c|c|c|c|c|c|c|c|c|c|c|c|}
\hline $\begin{array}{l}\text { U.S. } \\
\text { Geological } \\
\text { Survey } \\
\text { streamgage } \\
\text { number }\end{array}$ & $\begin{array}{l}\text { Segment } \\
\text { number }\end{array}$ & $\begin{array}{l}\text { Streamgage used in } \\
\text { regression analyses } \\
\text { (non-redundant) }\end{array}$ & $\begin{array}{c}\text { Discharge value } \\
\text { used in } \\
\text { adjustment } \\
\text { regression }\end{array}$ & $\begin{array}{l}\text { Water } \\
\text { year }\end{array}$ & $\begin{array}{l}\text { Observed annual } \\
\text { maximum peak } \\
\text { discharge }\left(\mathrm{ft}^{3} / \mathrm{s}\right)\end{array}$ & $\begin{array}{l}\text { NWIS peak } \\
\text { code }\end{array}$ & $\begin{array}{l}\text { Observed annual } \\
\text { maximum peak } \\
\text { discharge with } \\
\text { segment intercept } \\
\text { value subtracted } \\
\left(\mathrm{ft}^{3} / \mathrm{s}\right)\end{array}$ & $\begin{array}{l}\text { Observed } \\
\text { precipitation } \\
\text { (inches) }\end{array}$ & Urban fraction & $\begin{array}{l}\text { Exceedance } \\
\text { probability }\end{array}$ & $\begin{array}{c}\text { Urban-adjusted } \\
\text { annual maximum } \\
\text { peak discharge } \\
\left(\mathrm{ft}^{3} / \mathrm{s}\right)\end{array}$ \\
\hline 05528500 & 2 & Yes & Yes & 1992 & 213 & $E$ & 424 & 0.551 & 0.758 & 0.91 & 264 \\
\hline 05528500 & 2 & Yes & Yes & 1993 & 287 & $C, E$ & 571 & 0.929 & 0.761 & 0.763 & 340 \\
\hline 05528500 & 2 & Yes & Yes & 1994 & 437 & C & 869 & 1.41 & 0.765 & 0.405 & 501 \\
\hline 05528500 & 2 & Yes & Yes & 1995 & 337 & C & 671 & 1.03 & 0.768 & 0.646 & 391 \\
\hline 05528500 & 2 & Yes & Yes & 1996 & 670 & C & 1333 & 0.286 & 0.771 & 0.112 & 747 \\
\hline 05528500 & 2 & Yes & Yes & 1997 & 541 & $C, E$ & 1076 & 2.25 & 0.774 & 0.22 & 604 \\
\hline 05528500 & 2 & Yes & Yes & 1998 & 272 & $C, E$ & 541 & 0.777 & 0.778 & 0.81 & 318 \\
\hline 05528500 & 2 & Yes & Yes & 1999 & 621 & C & 1236 & 1.05 & 0.781 & 0.152 & 687 \\
\hline 05528500 & 2 & Yes & Yes & 2000 & 272 & C & 541 & 1.74 & 0.784 & 0.814 & 315 \\
\hline 05528500 & 2 & Yes & Yes & 2001 & 680 & C & 1353 & 2.45 & 0.795 & 0.116 & 742 \\
\hline 05528500 & 2 & Yes & Yes & 2002 & 742 & C & 1476 & 1.52 & 0.805 & 0.0845 & 801 \\
\hline 05528500 & 2 & Yes & Yes & 2003 & 193 & $C, E$ & 384 & 1.19 & 0.816 & 0.952 & 221 \\
\hline 05528500 & 2 & Yes & Yes & 2004 & 495 & C & 985 & 1.23 & 0.827 & 0.341 & 529 \\
\hline 05528500 & 2 & Yes & Yes & 2005 & 247 & $C, E$ & 491 & 0.574 & 0.838 & 0.903 & 269 \\
\hline 05528500 & 2 & Yes & Yes & 2006 & 338 & C & 673 & 1.26 & 0.848 & 0.728 & 357 \\
\hline 05528500 & 2 & Yes & Yes & 2007 & 830 & C & 1651 & 1.67 & 0.859 & 0.0655 & 854 \\
\hline 05528500 & 2 & Yes & Yes & 2008 & 664 & C & 1321 & 3.3 & 0.87 & 0.16 & 677 \\
\hline 05528500 & 2 & Yes & Yes & 2009 & 444 & C & 883 & 0.596 & 0.881 & 0.513 & 449 \\
\hline 05529000 & 1 & No & No & 1941 & 1430 & -- & 270 & 0.19 & 0.0423 & 0.668 & 2540 \\
\hline 05529000 & 1 & No & No & 1942 & 2060 & -- & 389 & 0.439 & 0.0453 & 0.422 & 3440 \\
\hline 05529000 & 1 & No & No & 1943 & 2470 & -- & 467 & 0.102 & 0.0484 & 0.307 & 3960 \\
\hline 05529000 & 1 & No & No & 1944 & 2470 & -- & 467 & 1.2 & 0.0514 & 0.309 & 3950 \\
\hline 05529000 & 1 & No & No & 1945 & 1830 & -- & 346 & 1.32 & 0.0545 & 0.515 & 3080 \\
\hline 05529000 & 1 & No & No & 1946 & 2510 & -- & 474 & 0.706 & 0.0576 & 0.303 & 3980 \\
\hline 05529000 & 1 & No & No & 1947 & 1920 & -- & 363 & 1.2 & 0.0606 & 0.484 & 3190 \\
\hline 05529000 & 1 & No & No & 1948 & 3490 & -- & 660 & 1.8 & 0.0637 & 0.136 & 5280 \\
\hline 05529000 & 1 & No & No & 1949 & 1700 & -- & 321 & 0.31 & 0.0667 & 0.582 & 2860 \\
\hline 05529000 & 1 & No & No & 1950 & 4040 & -- & 763 & 1.46 & 0.0698 & 0.0859 & 6020 \\
\hline 05529000 & 1 & No & No & 1951 & 2410 & -- & 455 & 0.133 & 0.077 & 0.341 & 3800 \\
\hline 05529000 & 1 & No & No & 1952 & 2690 & -- & 508 & 1.29 & 0.0842 & 0.276 & 4120 \\
\hline 05529000 & 1 & No & No & 1953 & 669 & $\mathrm{E}$ & 126 & 0.565 & 0.0914 & 0.931 & 1370 \\
\hline 05529000 & 1 & No & No & 1954 & 1770 & -- & 334 & 1.33 & 0.0986 & 0.579 & 2870 \\
\hline 05529000 & 1 & No & No & 1955 & 1800 & -- & 340 & 2.4 & 0.106 & 0.573 & 2890 \\
\hline 05529000 & 1 & No & No & 1956 & 880 & $\mathrm{E}$ & 166 & 1.23 & 0.113 & 0.89 & 1630 \\
\hline 05529000 & 1 & No & No & 1957 & 2610 & -- & 493 & 1.31 & 0.12 & 0.318 & 3910 \\
\hline
\end{tabular}




\begin{tabular}{|c|c|c|c|c|c|c|c|c|c|c|c|}
\hline $\begin{array}{l}\text { U.S. } \\
\text { Geological } \\
\text { Survey } \\
\text { streamgage } \\
\text { number }\end{array}$ & $\begin{array}{l}\text { Segment } \\
\text { number }\end{array}$ & $\begin{array}{l}\text { Streamgage used in } \\
\text { regression analyses } \\
\text { (non-redundant) }\end{array}$ & $\begin{array}{c}\text { Discharge value } \\
\text { used in } \\
\text { adjustment } \\
\text { regression }\end{array}$ & $\begin{array}{l}\text { Water } \\
\text { year }\end{array}$ & $\begin{array}{l}\text { Observed annual } \\
\text { maximum peak } \\
\text { discharge }\left(\mathrm{ft}^{3} / \mathrm{s}\right)\end{array}$ & $\begin{array}{l}\text { NWIS peak } \\
\text { code }\end{array}$ & $\begin{array}{l}\text { Observed annual } \\
\text { maximum peak } \\
\text { discharge with } \\
\text { segment intercept } \\
\text { value subtracted } \\
\left(\mathrm{ft}^{3} / \mathrm{s}\right)\end{array}$ & $\begin{array}{l}\text { Observed } \\
\text { precipitation } \\
\text { (inches) }\end{array}$ & Urban fraction & $\begin{array}{l}\text { Exceedance } \\
\text { probability }\end{array}$ & $\begin{array}{c}\text { Urban-adjusted } \\
\text { annual maximum } \\
\text { peak discharge } \\
\left(\mathrm{ft}^{3} / \mathrm{s}\right)\end{array}$ \\
\hline 05529000 & 1 & No & No & 1958 & 1220 & -- & 231 & 0.146 & 0.128 & 0.796 & 2060 \\
\hline 05529000 & 1 & No & No & 1959 & 1190 & $\mathrm{E}$ & 225 & 1.27 & 0.135 & 0.809 & 2010 \\
\hline 05529000 & 1 & No & No & 1960 & 4670 & -- & 883 & 0.604 & 0.142 & 0.068 & 6480 \\
\hline 05529000 & 1 & No & No & 1961 & 1150 & -- & 217 & 0.425 & 0.148 & 0.828 & 1920 \\
\hline 05529000 & 1 & No & No & 1962 & 2820 & $\mathrm{E}$ & 533 & 0.00314 & 0.154 & 0.29 & 4040 \\
\hline 05529000 & 1 & No & No & 1963 & 732 & $\mathrm{E}$ & 138 & 1.4 & 0.161 & 0.934 & 1340 \\
\hline 05529000 & 1 & No & No & 1964 & 840 & $\mathrm{E}$ & 159 & 0.715 & 0.167 & 0.916 & 1480 \\
\hline 05529000 & 1 & No & No & 1965 & 2160 & -- & 408 & 0.541 & 0.173 & 0.491 & 3170 \\
\hline 05529000 & 1 & No & No & 1966 & 1920 & -- & 363 & 0.496 & 0.179 & 0.588 & 2840 \\
\hline 05529000 & 1 & No & No & 1967 & 2820 & -- & 533 & 1.41 & 0.185 & 0.31 & 3940 \\
\hline 05529000 & 1 & No & No & 1968 & 1730 & $E$ & 327 & 1.01 & 0.192 & 0.663 & 2560 \\
\hline 05529000 & 1 & No & No & 1969 & 2430 & -- & 459 & 1.37 & 0.198 & 0.423 & 3440 \\
\hline 05529000 & 1 & No & No & 1970 & 3620 & -- & 684 & 1.65 & 0.204 & 0.175 & 4830 \\
\hline 05529000 & 1 & No & No & 1971 & 2440 & -- & 461 & 0.0771 & 0.212 & 0.432 & 3400 \\
\hline 05529000 & 1 & No & No & 1972 & 2710 & -- & 512 & 1.03 & 0.22 & 0.363 & 3700 \\
\hline 05529000 & 1 & No & No & 1973 & 2900 & -- & 548 & 1.39 & 0.229 & 0.321 & 3890 \\
\hline 05529000 & 1 & No & No & 1974 & 2480 & -- & 469 & 0.652 & 0.237 & 0.44 & 3370 \\
\hline 05529000 & 1 & No & No & 1975 & 1810 & -- & 342 & 0.848 & 0.245 & 0.675 & 2520 \\
\hline 05529000 & 1 & No & No & 1976 & 2600 & -- & 491 & 1.31 & 0.253 & 0.418 & 3460 \\
\hline 05529000 & 1 & No & No & 1977 & 514 & $\mathrm{E}$ & 97 & 0.825 & 0.261 & 0.98 & 863 \\
\hline 05529000 & 1 & No & No & 1978 & 1960 & -- & 370 & 1.28 & 0.27 & 0.643 & 2630 \\
\hline 05529000 & 1 & No & No & 1979 & 3430 & -- & 648 & 0.3 & 0.278 & 0.24 & 4310 \\
\hline 05529000 & 1 & No & No & 1980 & 1440 & -- & 272 & 0.294 & 0.286 & 0.816 & 1980 \\
\hline 05529000 & 1 & No & No & 1981 & 1700 & -- & 321 & 1.15 & 0.292 & 0.746 & 2270 \\
\hline 05529000 & 1 & No & No & 1982 & 3920 & -- & 741 & 1.9 & 0.298 & 0.179 & 4800 \\
\hline 05529000 & 1 & No & No & 1983 & 3900 & -- & 737 & 1.53 & 0.304 & 0.183 & 4750 \\
\hline 05529000 & 1 & No & No & 1984 & 1830 & $\mathrm{E}$ & 346 & 0.5 & 0.31 & 0.717 & 2370 \\
\hline 05529000 & 1 & No & No & 1985 & 3490 & -- & 660 & 1.08 & 0.316 & 0.252 & 4240 \\
\hline 05529000 & 1 & No & No & 1986 & 2890 & -- & 546 & 0.597 & 0.321 & 0.394 & 3560 \\
\hline 05529000 & 1 & No & No & 1987 & 4900 & -- & 926 & 0.804 & 0.327 & 0.0964 & 5810 \\
\hline 05529000 & 1 & No & No & 1988 & 1810 & -- & 342 & 0.567 & 0.333 & 0.74 & 2290 \\
\hline 05529000 & 1 & No & No & 1989 & 1930 & $\mathrm{E}$ & 365 & 1.06 & 0.339 & 0.708 & 2410 \\
\hline 05529000 & 1 & No & No & 1990 & 2430 & -- & 459 & 1.57 & 0.345 & 0.552 & 2960 \\
\hline 05529000 & 1 & No & No & 1991 & 2170 & -- & 410 & 0.88 & 0.351 & 0.641 & 2640 \\
\hline 05529000 & 1 & No & No & 1992 & 1220 & $D, E$ & 231 & 0.784 & 0.357 & 0.902 & 1570 \\
\hline
\end{tabular}




\begin{tabular}{|c|c|c|c|c|c|c|c|c|c|c|c|}
\hline $\begin{array}{c}\text { U.S. } \\
\text { Geological } \\
\text { Survey } \\
\text { streamgage } \\
\text { number } \\
\end{array}$ & $\begin{array}{c}\text { Segment } \\
\text { number }\end{array}$ & $\begin{array}{l}\text { Streamgage used in } \\
\text { regression analyses } \\
\text { (non-redundant) }\end{array}$ & $\begin{array}{c}\text { Discharge value } \\
\text { used in } \\
\text { adjustment } \\
\text { regression } \\
\end{array}$ & $\begin{array}{l}\text { Water } \\
\text { year }\end{array}$ & $\begin{array}{c}\text { Observed annual } \\
\text { maximum peak } \\
\text { discharge }\left(\mathrm{ft}^{3} / \mathrm{s}\right)\end{array}$ & $\begin{array}{c}\text { NWIS peak } \\
\text { code }\end{array}$ & $\begin{array}{c}\text { Observed annual } \\
\text { maximum peak } \\
\text { discharge with } \\
\text { segment intercept } \\
\text { value subtracted } \\
\left(\mathrm{ft}^{3} / \mathrm{s}\right) \\
\end{array}$ & $\begin{array}{c}\text { Observed } \\
\text { precipitation } \\
\text { (inches) }\end{array}$ & Urban fraction & $\begin{array}{l}\text { Exceedance } \\
\text { probability }\end{array}$ & $\begin{array}{c}\text { Urban-adjusted } \\
\text { annual maximum } \\
\text { peak discharge } \\
\left(\mathrm{ft}^{3} / \mathrm{s}\right) \\
\end{array}$ \\
\hline 05529000 & 1 & No & No & 1993 & 2660 & C & 503 & 0.92 & 0.362 & 0.494 & 3160 \\
\hline 05529000 & 1 & No & No & 1994 & 2700 & C & 510 & 0.742 & 0.368 & 0.488 & 3190 \\
\hline 05529000 & 1 & No & No & 1995 & 2470 & C & 467 & 1.17 & 0.374 & 0.565 & 2910 \\
\hline 05529000 & 1 & No & No & 1996 & 3850 & C & 728 & 0.975 & 0.38 & 0.229 & 4390 \\
\hline 05529000 & 1 & No & No & 1997 & 3540 & C & 669 & 1.89 & 0.385 & 0.29 & 4030 \\
\hline 05529000 & 1 & No & No & 1998 & 1860 & C & 351 & 0.59 & 0.391 & 0.764 & 2190 \\
\hline 05529000 & 1 & No & No & 1999 & 3420 & C & 646 & 1.16 & 0.397 & 0.325 & 3870 \\
\hline 05529000 & 1 & No & No & 2000 & 2460 & C & 465 & 0.0862 & 0.403 & 0.596 & 2810 \\
\hline 05529000 & 1 & No & No & 2001 & 2090 & C & 395 & 1.83 & 0.414 & 0.716 & 2380 \\
\hline 05529000 & 1 & No & No & 2002 & 2970 & C & 561 & 1.35 & 0.425 & 0.462 & 3290 \\
\hline 05529000 & 1 & No & No & 2003 & 1630 & $C, E$ & 308 & 0.602 & 0.437 & 0.849 & 1830 \\
\hline 05529000 & 1 & No & No & 2004 & 3760 & C & 711 & 0.0874 & 0.448 & 0.291 & 4030 \\
\hline 05529000 & 1 & No & No & 2005 & 1860 & $C, E$ & 351 & 0.464 & 0.46 & 0.808 & 2010 \\
\hline 05529000 & 1 & No & No & 2006 & 2030 & C & 384 & 0.95 & 0.471 & 0.773 & 2160 \\
\hline 05529000 & 1 & No & No & 2007 & 3780 & C & 714 & 1.04 & 0.482 & 0.313 & 3910 \\
\hline 05529000 & 1 & No & No & 2008 & 3010 & C & 569 & 2.48 & 0.494 & 0.515 & 3090 \\
\hline 05529000 & 1 & No & No & 2009 & 3190 & C & 603 & 0.665 & 0.505 & 0.477 & 3230 \\
\hline 05529300 & 1 & No & No & 1955 & 372 & 7 & 1948 & 3.45 & 0.382 & 0.0122 & 565 \\
\hline 05529300 & 1 & No & No & 1957 & 698 & 7 & 3655 & 2.7 & 0.448 & 0 & 978 \\
\hline 05529300 & 1 & No & No & 1961 & 138 & -- & 723 & 1.54 & 0.581 & 0.393 & 206 \\
\hline 05529300 & 1 & No & No & 1962 & 80 & -- & 419 & 0.225 & 0.616 & 0.826 & 128 \\
\hline 05529300 & 1 & No & No & 1963 & 39 & -- & 204 & 1.13 & 0.651 & 0.982 & 72.5 \\
\hline 05529300 & 1 & No & No & 1964 & 76 & -- & 398 & 1.78 & 0.685 & 0.892 & 115 \\
\hline 05529300 & 1 & No & No & 1965 & 76 & -- & 398 & 0.5 & 0.72 & 0.911 & 110 \\
\hline 05529300 & 1 & No & No & 1966 & 125 & -- & 655 & 1.14 & 0.755 & 0.654 & 159 \\
\hline 05529300 & 1 & No & No & 1967 & 389 & -- & 2037 & 1.48 & 0.79 & 0.0256 & 442 \\
\hline 05529300 & 1 & No & No & 1968 & 184 & -- & 964 & 1.18 & 0.824 & 0.363 & 211 \\
\hline 05529300 & 1 & No & No & 1969 & 181 & -- & 948 & 1.29 & 0.859 & 0.421 & 201 \\
\hline 05529300 & 1 & No & No & 1970 & 326 & -- & 1707 & 1.32 & 0.894 & 0.0646 & 343 \\
\hline 05529300 & 1 & No & No & 1971 & 169 & -- & 885 & 0.382 & 0.9 & 0.531 & 180 \\
\hline 05529300 & 1 & No & No & 1972 & 485 & -- & 2540 & 1.06 & 0.906 & 0.0174 & 503 \\
\hline 05529300 & 1 & No & No & 1973 & 221 & -- & 1157 & 0.781 & 0.912 & 0.262 & 230 \\
\hline 05529300 & 1 & No & No & 1974 & 244 & -- & 1278 & 1.04 & 0.917 & 0.197 & 252 \\
\hline 05529300 & 1 & No & No & 1975 & 271 & -- & 1419 & 0.963 & 0.923 & 0.149 & 279 \\
\hline 05529300 & 1 & No & No & 1976 & 257 & -- & 1346 & 0.961 & 0.929 & 0.178 & 263 \\
\hline
\end{tabular}




\begin{tabular}{|c|c|c|c|c|c|c|c|c|c|c|c|}
\hline $\begin{array}{l}\text { U.S. } \\
\text { Geological } \\
\text { Survey } \\
\text { streamgage } \\
\text { number }\end{array}$ & $\begin{array}{l}\text { Segment } \\
\text { number }\end{array}$ & $\begin{array}{l}\text { Streamgage used in } \\
\text { regression analyses } \\
\text { (non-redundant) }\end{array}$ & $\begin{array}{c}\text { Discharge value } \\
\text { used in } \\
\text { adjustment } \\
\text { regression }\end{array}$ & $\begin{array}{l}\text { Water } \\
\text { year }\end{array}$ & $\begin{array}{l}\text { Observed annual } \\
\text { maximum peak } \\
\text { discharge }\left(\mathrm{ft}^{3} / \mathrm{s}\right)\end{array}$ & $\begin{array}{l}\text { NWIS peak } \\
\text { code }\end{array}$ & $\begin{array}{l}\text { Observed annual } \\
\text { maximum peak } \\
\text { discharge with } \\
\text { segment intercept } \\
\text { value subtracted } \\
\left(\mathrm{ft}^{3} / \mathrm{s}\right)\end{array}$ & $\begin{array}{l}\text { Observed } \\
\text { precipitation } \\
\text { (inches) }\end{array}$ & Urban fraction & $\begin{array}{l}\text { Exceedance } \\
\text { probability }\end{array}$ & $\begin{array}{c}\text { Urban-adjusted } \\
\text { annual maximum } \\
\text { peak discharge } \\
\left(\mathrm{ft}^{3} / \mathrm{s}\right)\end{array}$ \\
\hline 05529300 & 1 & No & No & 1977 & 51 & -- & 267 & 1.2 & 0.935 & 1 & 53.2 \\
\hline 05529300 & 1 & No & No & 1978 & 178 & -- & 932 & 1.48 & 0.941 & 0.526 & 181 \\
\hline 05529300 & 1 & No & No & 1979 & 222 & -- & 1163 & 0.896 & 0.947 & 0.289 & 223 \\
\hline 05529500 & 1 & Yes & Yes & 1953 & 27 & $\mathrm{E}$ & 117 & 0.694 & 0.381 & 0.982 & 65 \\
\hline 05529500 & 1 & Yes & Yes & 1954 & 103 & -- & 447 & 1.5 & 0.421 & 0.638 & 144 \\
\hline 05529500 & 1 & Yes & Yes & 1955 & 146 & -- & 634 & 3.33 & 0.46 & 0.394 & 184 \\
\hline 05529500 & 1 & Yes & Yes & 1956 & 45 & $\mathrm{E}$ & 195 & 1.24 & 0.5 & 0.961 & 78 \\
\hline 05529500 & 1 & Yes & Yes & 1957 & 430 & -- & 1868 & 2.42 & 0.54 & 0.0181 & 435 \\
\hline 05529500 & 1 & Yes & Yes & 1958 & 42 & $E$ & 182 & 0.216 & 0.58 & 0.98 & 66.8 \\
\hline 05529500 & 1 & Yes & Yes & 1959 & 76 & $E$ & 330 & 0.371 & 0.62 & 0.918 & 97.2 \\
\hline 05529500 & 1 & Yes & Yes & 1960 & 261 & -- & 1134 & 0.384 & 0.659 & 0.14 & 253 \\
\hline 05529500 & 1 & Yes & Yes & 1961 & 194 & -- & 843 & 1.49 & 0.684 & 0.35 & 190 \\
\hline 05529500 & 1 & Yes & Yes & 1962 & 154 & -- & 669 & 0.233 & 0.708 & 0.585 & 152 \\
\hline 05529500 & 1 & Yes & Yes & 1963 & 44 & $E$ & 191 & 1.13 & 0.732 & 1 & 54 \\
\hline 05529500 & 1 & Yes & Yes & 1964 & 74 & -- & 321 & 0.783 & 0.756 & 0.959 & 79.3 \\
\hline 05529500 & 1 & Yes & Yes & 1965 & 93 & -- & 404 & 0.501 & 0.78 & 0.932 & 92.6 \\
\hline 05529500 & 1 & Yes & Yes & 1966 & 175 & -- & 760 & 1.32 & 0.804 & 0.57 & 154 \\
\hline 05529500 & 1 & Yes & Yes & 1967 & 618 & D & 2684 & 1.48 & 0.829 & 0.0131 & 501 \\
\hline 05529500 & 1 & Yes & Yes & 1968 & 134 & -- & 582 & 1.17 & 0.853 & 0.827 & 114 \\
\hline 05529500 & 1 & Yes & Yes & 1969 & 239 & -- & 1038 & 1.29 & 0.877 & 0.339 & 192 \\
\hline 05529500 & 1 & Yes & Yes & 1970 & 434 & D & 1885 & 1.32 & 0.901 & 0.0453 & 336 \\
\hline 05529500 & 1 & Yes & Yes & 1971 & 260 & 2, E & 1129 & 0.383 & 0.906 & 0.28 & 202 \\
\hline 05529500 & 1 & Yes & Yes & 1972 & 664 & -- & 2884 & 0.683 & 0.911 & 0.0128 & 507 \\
\hline 05529500 & 1 & Yes & Yes & 1973 & 396 & -- & 1720 & 1.38 & 0.916 & 0.0677 & 303 \\
\hline 05529500 & 1 & Yes & Yes & 1974 & 291 & -- & 1264 & 0.52 & 0.921 & 0.206 & 222 \\
\hline 05529500 & 1 & Yes & Yes & 1975 & 276 & -- & 1199 & 0.96 & 0.927 & 0.245 & 210 \\
\hline 05529500 & 1 & Yes & Yes & 1976 & 305 & D & 1325 & 0.965 & 0.932 & 0.186 & 231 \\
\hline 05529500 & 1 & Yes & Yes & 1977 & 86 & $\mathrm{E}$ & 374 & 0.725 & 0.937 & 0.98 & 66.2 \\
\hline 05529500 & 1 & Yes & Yes & 1978 & 204 & -- & 886 & 1.19 & 0.942 & 0.574 & 153 \\
\hline 05529500 & 1 & Yes & Yes & 1979 & 246 & -- & 1068 & 0.898 & 0.947 & 0.396 & 184 \\
\hline 05529500 & 1 & Yes & Yes & 1980 & 144 & $\mathrm{E}$ & 625 & 0.745 & 0.952 & 0.869 & 107 \\
\hline 05529500 & 1 & Yes & Yes & 1981 & 247 & $\mathrm{E}$ & 1073 & 0.927 & 0.953 & 0.398 & 183 \\
\hline 05529500 & 1 & Yes & Yes & 1982 & 484 & -- & 2102 & 1.93 & 0.953 & 0.0367 & 358 \\
\hline 05529500 & 1 & Yes & Yes & 1983 & 548 & -- & 2380 & 1.51 & 0.954 & 0.0233 & 406 \\
\hline 05529500 & 1 & Yes & Yes & 1984 & 148 & $E$ & 643 & 0.677 & 0.954 & 0.853 & 110 \\
\hline
\end{tabular}




\begin{tabular}{|c|c|c|c|c|c|c|c|c|c|c|c|}
\hline $\begin{array}{c}\text { U.S. } \\
\text { Geological } \\
\text { Survey } \\
\text { streamgage } \\
\text { number }\end{array}$ & $\begin{array}{l}\text { Segment } \\
\text { number }\end{array}$ & $\begin{array}{l}\text { Streamgage used in } \\
\text { regression analyses } \\
\text { (non-redundant) }\end{array}$ & $\begin{array}{c}\text { Discharge value } \\
\text { used in } \\
\text { adjustment } \\
\text { regression }\end{array}$ & $\begin{array}{c}\text { Water } \\
\text { year }\end{array}$ & $\begin{array}{c}\text { Observed annual } \\
\text { maximum peak } \\
\text { discharge }\left(\mathrm{ft}^{3} / \mathrm{s}\right)\end{array}$ & $\begin{array}{c}\text { NWIS peak } \\
\text { code }\end{array}$ & $\begin{array}{c}\text { Observed annual } \\
\text { maximum peak } \\
\text { discharge with } \\
\text { segment intercept } \\
\text { value subtracted } \\
\left(\mathrm{ft}^{3} / \mathrm{s}\right)\end{array}$ & $\begin{array}{c}\text { Observed } \\
\text { precipitation } \\
\text { (inches) }\end{array}$ & Urban fraction & $\begin{array}{l}\text { Exceedance } \\
\text { probability }\end{array}$ & $\begin{array}{c}\text { Urban-adjusted } \\
\text { annual maximum } \\
\text { peak discharge } \\
\left(\mathrm{ft}^{3} / \mathrm{s}\right)\end{array}$ \\
\hline 05529500 & 1 & Yes & Yes & 1985 & 346 & $E$ & 1503 & 1.25 & 0.955 & 0.136 & 256 \\
\hline 05529500 & 1 & Yes & Yes & 1986 & 403 & -- & 1750 & 0.786 & 0.955 & 0.0718 & 298 \\
\hline 05529500 & 1 & Yes & Yes & 1987 & 806 & -- & 3501 & 2.8 & 0.956 & 0.00726 & 596 \\
\hline 05529500 & 1 & Yes & Yes & 1988 & 146 & $E$ & 634 & 0.738 & 0.956 & 0.864 & 108 \\
\hline 05529500 & 1 & Yes & Yes & 1989 & 232 & $E$ & 1008 & 1.53 & 0.957 & 0.468 & 171 \\
\hline 05529500 & 0 & Yes & No & 1990 & 208 & $E$ & 1051 & 1.67 & 0.957 & 0.424 & 179 \\
\hline 05529500 & 2 & Yes & Yes & 1991 & 121 & $E$ & 712 & 2.01 & 0.957 & 0.79 & 121 \\
\hline 05529500 & 2 & Yes & Yes & 1992 & 115 & $\mathrm{C}, \mathrm{E}$ & 677 & 1.81 & 0.957 & 0.824 & 115 \\
\hline 05529500 & 2 & Yes & Yes & 1993 & 160 & $C, E$ & 941 & 1.14 & 0.957 & 0.534 & 160 \\
\hline 05529500 & 2 & Yes & Yes & 1994 & 180 & $C, E$ & 1059 & 1.41 & 0.957 & 0.417 & 180 \\
\hline 05529500 & 2 & Yes & Yes & 1995 & 202 & $C, E$ & 1188 & 0.913 & 0.957 & 0.278 & 202 \\
\hline 05529500 & 2 & Yes & Yes & 1996 & 194 & C & 1141 & 0.925 & 0.957 & 0.322 & 194 \\
\hline 05529500 & 2 & Yes & Yes & 1997 & 322 & $C, E$ & 1894 & 2.38 & 0.957 & 0.0513 & 322 \\
\hline 05529500 & 2 & Yes & Yes & 1998 & 111 & $C, E$ & 653 & 1.62 & 0.957 & 0.846 & 111 \\
\hline 05529500 & 2 & Yes & Yes & 1999 & 286 & C & 1683 & 1.42 & 0.957 & 0.0859 & 286 \\
\hline 05529500 & 2 & Yes & Yes & 2000 & 153 & $C, E$ & 900 & 1.92 & 0.957 & 0.576 & 153 \\
\hline 05529500 & 2 & Yes & Yes & 2001 & 206 & $\mathrm{C}, \mathrm{E}$ & 1212 & 2.75 & 0.957 & 0.26 & 206 \\
\hline 05529500 & 2 & Yes & Yes & 2002 & 345 & C & 2030 & 0.31 & 0.957 & 0.0416 & 345 \\
\hline 05529500 & 2 & Yes & Yes & 2003 & 135 & $C, E$ & 794 & 1.6 & 0.957 & 0.707 & 135 \\
\hline 05529500 & 2 & Yes & Yes & 2004 & 171 & $C, E$ & 1006 & 0.12 & 0.957 & 0.47 & 171 \\
\hline 05529500 & 2 & Yes & Yes & 2005 & 141 & C & 830 & 0.58 & 0.957 & 0.662 & 141 \\
\hline 05529500 & 2 & Yes & Yes & 2006 & 90 & $\mathrm{C}, \mathrm{E}$ & 530 & 0.508 & 0.957 & 0.939 & 90 \\
\hline 05529500 & 2 & Yes & Yes & 2007 & 220 & $C$ & 1294 & 1.9 & 0.957 & 0.213 & 220 \\
\hline 05529500 & 2 & Yes & Yes & 2008 & 474 & $C, E$ & 2789 & 4.25 & 0.957 & 0.0154 & 474 \\
\hline 05529500 & 2 & Yes & Yes & 2009 & 252 & $C$ & 1483 & 1.04 & 0.957 & 0.143 & 252 \\
\hline 05529900 & 1 & No & No & 1961 & 444 & -- & 824 & 0.936 & 0.926 & 0.627 & 468 \\
\hline 05529900 & 1 & No & No & 1962 & 210 & $4, B$ & 390 & 0.372 & 0.929 & 0.973 & 227 \\
\hline 05529900 & 1 & No & No & 1963 & 195 & -- & 362 & 1.55 & 0.932 & 0.981 & 210 \\
\hline 05529900 & 1 & No & No & 1964 & 235 & 2 & 436 & 0.455 & 0.935 & 0.962 & 250 \\
\hline 05529900 & 1 & No & No & 1965 & 291 & -- & 540 & 0.685 & 0.939 & 0.928 & 306 \\
\hline 05529900 & 1 & No & No & 1966 & 821 & -- & 1524 & 1.15 & 0.942 & 0.123 & 842 \\
\hline 05529900 & 1 & No & No & 1967 & 1160 & -- & 2153 & 1.54 & 0.945 & 0.0329 & 1180 \\
\hline 05529900 & 1 & No & No & 1968 & 627 & -- & 1164 & 1.3 & 0.949 & 0.29 & 640 \\
\hline 05529900 & 1 & No & No & 1969 & 418 & -- & 776 & 0.835 & 0.952 & 0.72 & 427 \\
\hline 05529900 & 1 & No & No & 1970 & 919 & -- & 1706 & 1.26 & 0.955 & 0.0801 & 931 \\
\hline
\end{tabular}




\begin{tabular}{|c|c|c|c|c|c|c|c|c|c|c|c|}
\hline $\begin{array}{l}\text { U.S. } \\
\text { Geological } \\
\text { Survey } \\
\text { streamgage } \\
\text { number }\end{array}$ & $\begin{array}{l}\text { Segment } \\
\text { number }\end{array}$ & $\begin{array}{l}\text { Streamgage used in } \\
\text { regression analyses } \\
\text { (non-redundant) }\end{array}$ & $\begin{array}{l}\text { Discharge value } \\
\text { used in } \\
\text { adjustment } \\
\text { regression }\end{array}$ & $\begin{array}{l}\text { Water } \\
\text { year }\end{array}$ & $\begin{array}{l}\text { Observed annual } \\
\text { maximum peak } \\
\text { discharge }\left(\mathrm{ft}^{3} / \mathrm{s}\right)\end{array}$ & $\begin{array}{l}\text { NWIS peak } \\
\text { code }\end{array}$ & $\begin{array}{l}\text { Observed annual } \\
\text { maximum peak } \\
\text { discharge with } \\
\text { segment intercept } \\
\text { value subtracted } \\
\left(\mathrm{ft}^{3} / \mathrm{s}\right)\end{array}$ & $\begin{array}{l}\text { Observed } \\
\text { precipitation } \\
\text { (inches) }\end{array}$ & Urban fraction & $\begin{array}{l}\text { Exceedance } \\
\text { probability }\end{array}$ & $\begin{array}{c}\text { Urban-adjusted } \\
\text { annual maximum } \\
\text { peak discharge } \\
\left(\mathrm{ft}^{3} / \mathrm{s}\right)\end{array}$ \\
\hline 05529900 & 1 & No & No & 1971 & 680 & 2 & 1262 & 0.372 & 0.956 & 0.229 & 689 \\
\hline 05529900 & 1 & No & No & 1972 & 1190 & -- & 2209 & 1.07 & 0.957 & 0.0309 & 1200 \\
\hline 05529900 & 1 & No & No & 1973 & 840 & 2 & 1559 & 0.809 & 0.957 & 0.119 & 849 \\
\hline 05529900 & 1 & No & No & 1974 & 444 & -- & 824 & 1.02 & 0.958 & 0.67 & 450 \\
\hline 05529900 & 1 & No & No & 1975 & 571 & -- & 1060 & 1.01 & 0.959 & 0.418 & 578 \\
\hline 05529900 & 1 & No & No & 1976 & 392 & -- & 728 & 0.829 & 0.959 & 0.776 & 397 \\
\hline 05529900 & 1 & No & No & 1977 & 266 & -- & 494 & 1.27 & 0.96 & 0.953 & 270 \\
\hline 05529900 & 0 & No & No & 1979 & 287 & -- & NA & 0.877 & 0.962 & NA & NA \\
\hline 05530000 & 1 & Yes & Yes & 1951 & 245 & -- & 368 & 0.588 & 0.647 & 0.899 & 409 \\
\hline 05530000 & 1 & Yes & Yes & 1952 & 328 & -- & 493 & 1.53 & 0.676 & 0.785 & 489 \\
\hline 05530000 & 1 & Yes & Yes & 1953 & 169 & $\mathrm{E}$ & 254 & 1.51 & 0.706 & 0.973 & 287 \\
\hline 05530000 & 1 & Yes & Yes & 1954 & 461 & -- & 692 & 3.11 & 0.735 & 0.582 & 608 \\
\hline 05530000 & 1 & Yes & Yes & 1955 & 535 & -- & 804 & 4.74 & 0.764 & 0.479 & 676 \\
\hline 05530000 & 1 & Yes & Yes & 1956 & 245 & -- & 368 & 1.56 & 0.794 & 0.952 & 340 \\
\hline 05530000 & 1 & Yes & Yes & 1957 & 668 & -- & 1003 & 5.8 & 0.823 & 0.315 & 779 \\
\hline 05530000 & 1 & Yes & Yes & 1958 & 384 & -- & 577 & 1.59 & 0.853 & 0.832 & 456 \\
\hline 05530000 & 1 & Yes & Yes & 1959 & 376 & $\mathrm{E}$ & 565 & 1.87 & 0.882 & 0.868 & 431 \\
\hline 05530000 & 1 & Yes & Yes & 1960 & 562 & -- & 844 & 1.89 & 0.911 & 0.586 & 605 \\
\hline 05530000 & 1 & Yes & Yes & 1961 & 596 & -- & 895 & 2.81 & 0.917 & 0.538 & 637 \\
\hline 05530000 & 1 & Yes & Yes & 1962 & 394 & -- & 592 & 2.64 & 0.922 & 0.876 & 426 \\
\hline 05530000 & 1 & Yes & Yes & 1963 & 478 & D & 718 & 1.55 & 0.927 & 0.754 & 509 \\
\hline 05530000 & 1 & Yes & Yes & 1964 & 432 & -- & 649 & 0.455 & 0.933 & 0.828 & 458 \\
\hline 05530000 & 1 & Yes & Yes & 1965 & 336 & -- & 505 & 0.236 & 0.938 & 0.942 & 358 \\
\hline 05530000 & 1 & Yes & Yes & 1966 & 1530 & D & 2298 & 1.15 & 0.943 & 0.0259 & 1570 \\
\hline 05530000 & 1 & Yes & Yes & 1967 & 1590 & -- & 2388 & 1.54 & 0.949 & 0.0226 & 1620 \\
\hline 05530000 & 1 & Yes & Yes & 1968 & 1140 & -- & 1712 & 1.3 & 0.954 & 0.0784 & 1160 \\
\hline 05530000 & 1 & Yes & Yes & 1969 & 897 & -- & 1347 & 1.3 & 0.959 & 0.191 & 910 \\
\hline 05530000 & 1 & Yes & Yes & 1970 & 1060 & -- & 1592 & 1.25 & 0.965 & 0.112 & 1070 \\
\hline 05530000 & 1 & Yes & Yes & 1971 & 1000 & 2 & 1502 & 0.371 & 0.965 & 0.141 & 1010 \\
\hline 05530000 & 1 & Yes & Yes & 1972 & 1280 & -- & 1923 & 1.06 & 0.966 & 0.0497 & 1290 \\
\hline 05530000 & 1 & Yes & Yes & 1973 & 1190 & -- & 1787 & 1.35 & 0.966 & 0.0688 & 1200 \\
\hline 05530000 & 1 & Yes & Yes & 1974 & 673 & D & 1011 & 0.848 & 0.967 & 0.476 & 679 \\
\hline 05530000 & 1 & Yes & Yes & 1975 & 1040 & -- & 1562 & 1.04 & 0.967 & 0.123 & 1050 \\
\hline 05530000 & 1 & Yes & Yes & 1976 & 887 & -- & 1332 & 0.904 & 0.968 & 0.2 & 893 \\
\hline 05530000 & 1 & Yes & Yes & 1977 & 515 & $\mathrm{E}$ & 774 & 1.27 & 0.969 & 0.738 & 519 \\
\hline
\end{tabular}




\begin{tabular}{|c|c|c|c|c|c|c|c|c|c|c|c|}
\hline $\begin{array}{l}\text { U.S. } \\
\text { Geological } \\
\text { Survey } \\
\text { streamgage } \\
\text { number }\end{array}$ & $\begin{array}{l}\text { Segment } \\
\text { number }\end{array}$ & $\begin{array}{l}\text { Streamgage used in } \\
\text { regression analyses } \\
\text { (non-redundant) }\end{array}$ & $\begin{array}{l}\text { Discharge value } \\
\text { used in } \\
\text { adjustment } \\
\text { regression }\end{array}$ & $\begin{array}{l}\text { Water } \\
\text { year }\end{array}$ & $\begin{array}{l}\text { Observed annual } \\
\text { maximum peak } \\
\text { discharge }\left(\mathrm{ft}^{3} / \mathrm{s}\right)\end{array}$ & $\begin{array}{l}\text { NWIS peak } \\
\text { code }\end{array}$ & $\begin{array}{l}\text { Observed annual } \\
\text { maximum peak } \\
\text { discharge with } \\
\text { segment intercept } \\
\text { value subtracted } \\
\left(\mathrm{ft}^{3} / \mathrm{s}\right)\end{array}$ & $\begin{array}{l}\text { Observed } \\
\text { precipitation } \\
\text { (inches) }\end{array}$ & Urban fraction & $\begin{array}{l}\text { Exceedance } \\
\text { probability }\end{array}$ & $\begin{array}{c}\text { Urban-adjusted } \\
\text { annual maximum } \\
\text { peak discharge } \\
\left(\mathrm{ft}^{3} / \mathrm{s}\right)\end{array}$ \\
\hline 05530000 & 1 & Yes & Yes & 1978 & 883 & -- & 1326 & 1.54 & 0.969 & 0.204 & 888 \\
\hline 05530000 & 1 & Yes & Yes & 1979 & 919 & -- & 1380 & 0.873 & 0.97 & 0.184 & 924 \\
\hline 05530000 & 1 & Yes & Yes & 1980 & 658 & -- & 988 & 0.626 & 0.97 & 0.503 & 662 \\
\hline 05530000 & 1 & Yes & Yes & 1981 & 896 & -- & 1346 & 0.905 & 0.971 & 0.197 & 900 \\
\hline 05530000 & 1 & Yes & Yes & 1982 & 966 & -- & 1451 & 0.286 & 0.971 & 0.16 & 969 \\
\hline 05530000 & 1 & Yes & Yes & 1983 & 792 & -- & 1190 & 1.54 & 0.972 & 0.291 & 795 \\
\hline 05530000 & 1 & Yes & Yes & 1984 & 812 & $\mathrm{E}$ & 1220 & 1.32 & 0.972 & 0.268 & 814 \\
\hline 05530000 & 1 & Yes & Yes & 1985 & 913 & -- & 1371 & 1.06 & 0.973 & 0.188 & 915 \\
\hline 05530000 & 1 & Yes & Yes & 1986 & 962 & -- & 1445 & 0.818 & 0.973 & 0.163 & 964 \\
\hline 05530000 & 1 & Yes & Yes & 1987 & 1490 & -- & 2238 & 2.88 & 0.974 & 0.031 & 1490 \\
\hline 05530000 & 1 & Yes & Yes & 1988 & 610 & $E$ & 916 & 0.381 & 0.974 & 0.577 & 611 \\
\hline 05530000 & 1 & Yes & Yes & 1989 & 1170 & -- & 1757 & 1.51 & 0.975 & 0.0754 & 1170 \\
\hline 05530000 & 1 & Yes & Yes & 1990 & 1190 & -- & 1787 & 1.55 & 0.975 & 0.0708 & 1190 \\
\hline 05530000 & 1 & Yes & Yes & 1991 & 831 & -- & 1248 & 2.05 & 0.975 & 0.249 & 831 \\
\hline 05530000 & 1 & Yes & Yes & 1992 & 911 & $\mathrm{E}$ & 1368 & 1.83 & 0.975 & 0.191 & 911 \\
\hline 05530000 & 1 & Yes & Yes & 1993 & 962 & C & 1445 & 1.1 & 0.975 & 0.164 & 962 \\
\hline 05530000 & 1 & Yes & Yes & 1994 & 1150 & C & 1727 & 1.42 & 0.975 & 0.0821 & 1150 \\
\hline 05530000 & 1 & Yes & Yes & 1995 & 586 & $C, E$ & 880 & 0.934 & 0.975 & 0.619 & 586 \\
\hline 05530000 & 1 & Yes & Yes & 1996 & 820 & C & 1232 & 0.985 & 0.975 & 0.261 & 820 \\
\hline 05530000 & 1 & Yes & Yes & 1997 & 1040 & C & 1562 & 2.41 & 0.975 & 0.126 & 1040 \\
\hline 05530000 & 1 & Yes & Yes & 1998 & 484 & $C, E$ & 727 & 1.4 & 0.975 & 0.792 & 484 \\
\hline 05530000 & 1 & Yes & Yes & 1999 & 792 & C & 1190 & 1.32 & 0.975 & 0.294 & 792 \\
\hline 05530000 & 1 & Yes & Yes & 2000 & 531 & $C, E$ & 798 & 1.45 & 0.975 & 0.721 & 531 \\
\hline 05530000 & 1 & Yes & Yes & 2001 & 890 & C & 1337 & 1.46 & 0.975 & 0.203 & 890 \\
\hline 05530000 & 1 & Yes & Yes & 2002 & 1070 & C & 1607 & 1.4 & 0.975 & 0.112 & 1070 \\
\hline 05530000 & 1 & Yes & Yes & 2003 & 619 & C & 930 & 1.14 & 0.975 & 0.565 & 619 \\
\hline 05530000 & 1 & Yes & Yes & 2004 & 768 & C & 1154 & 0.586 & 0.975 & 0.333 & 768 \\
\hline 05530000 & 1 & Yes & Yes & 2005 & 414 & C & 622 & 0.754 & 0.975 & 0.893 & 414 \\
\hline 05530000 & 1 & Yes & Yes & 2006 & 292 & $C, E$ & 439 & 0.811 & 0.975 & 0.97 & 292 \\
\hline 05530000 & 1 & Yes & Yes & 2007 & 927 & C & 1392 & 1.5 & 0.975 & 0.182 & 927 \\
\hline 05530000 & 1 & Yes & Yes & 2008 & 1310 & C & 1968 & 3.62 & 0.975 & 0.0476 & 1310 \\
\hline 05530000 & 1 & Yes & Yes & 2009 & 818 & C & 1229 & 1.42 & 0.975 & 0.264 & 818 \\
\hline 05530400 & 1 & Yes & Yes & 1961 & 63 & -- & 249 & 2.2 & 0.244 & 0.828 & 110 \\
\hline 05530400 & 1 & Yes & Yes & 1962 & 50 & 2 & 197 & 0.13 & 0.261 & 0.903 & 91.4 \\
\hline 05530400 & 1 & Yes & Yes & 1963 & 95 & -- & 375 & 0.353 & 0.279 & 0.643 & 147 \\
\hline
\end{tabular}




\begin{tabular}{|c|c|c|c|c|c|c|c|c|c|c|c|}
\hline $\begin{array}{c}\text { U.S. } \\
\text { Geological } \\
\text { Survey } \\
\text { streamgage } \\
\text { number } \\
\end{array}$ & $\begin{array}{c}\text { Segment } \\
\text { number }\end{array}$ & $\begin{array}{l}\text { Streamgage used in } \\
\text { regression analyses } \\
\text { (non-redundant) }\end{array}$ & $\begin{array}{c}\text { Discharge value } \\
\text { used in } \\
\text { adjustment } \\
\text { regression }\end{array}$ & $\begin{array}{c}\text { Water } \\
\text { year }\end{array}$ & $\begin{array}{c}\text { Observed annual } \\
\text { maximum peak } \\
\text { discharge }\left(\mathrm{ft}^{3} / \mathrm{s}\right)\end{array}$ & $\begin{array}{c}\text { NWIS peak } \\
\text { code }\end{array}$ & $\begin{array}{l}\text { Observed annual } \\
\text { maximum peak } \\
\text { discharge with } \\
\text { segment intercept } \\
\text { value subtracted } \\
\left(\mathrm{ft}^{3} / \mathrm{s}\right)\end{array}$ & $\begin{array}{l}\text { Observed } \\
\text { precipitation } \\
\text { (inches) }\end{array}$ & Urban fraction & $\begin{array}{l}\text { Exceedance } \\
\text { probability }\end{array}$ & $\begin{array}{c}\text { Urban-adjusted } \\
\text { annual maximum } \\
\text { peak discharge } \\
\left(\mathrm{ft}^{3} / \mathrm{s}\right)\end{array}$ \\
\hline 05530400 & 1 & Yes & Yes & 1964 & 16 & -- & 63 & 0.806 & 0.296 & 1 & 35.1 \\
\hline 05530400 & 1 & Yes & Yes & 1965 & 38 & -- & 150 & 0.149 & 0.313 & 0.954 & 69.6 \\
\hline 05530400 & 1 & Yes & Yes & 1966 & 158 & -- & 623 & 1.42 & 0.33 & 0.301 & 217 \\
\hline 05530400 & 1 & Yes & Yes & 1967 & 382 & -- & 1507 & 1.54 & 0.347 & 0.0207 & 480 \\
\hline 05530400 & 1 & Yes & Yes & 1968 & 156 & -- & 615 & 1.3 & 0.365 & 0.337 & 208 \\
\hline 05530400 & 1 & Yes & Yes & 1969 & 195 & -- & 769 & 1.3 & 0.382 & 0.197 & 249 \\
\hline 05530400 & 1 & Yes & Yes & 1970 & 343 & 2 & 1353 & 1.25 & 0.399 & 0.0357 & 419 \\
\hline 05530400 & 1 & Yes & Yes & 1971 & 133 & 2 & 525 & 0.622 & 0.413 & 0.506 & 173 \\
\hline 05530400 & 1 & Yes & Yes & 1972 & 463 & -- & 1826 & 1.06 & 0.427 & 0.0157 & 545 \\
\hline 05530400 & 1 & Yes & Yes & 1973 & 324 & -- & 1278 & 0.809 & 0.441 & 0.0471 & 384 \\
\hline 05530400 & 1 & Yes & Yes & 1974 & 87 & -- & 343 & 0.495 & 0.455 & 0.816 & 113 \\
\hline 05530400 & 1 & Yes & Yes & 1975 & 184 & -- & 726 & 1.04 & 0.469 & 0.291 & 219 \\
\hline 05530400 & 1 & Yes & Yes & 1976 & 116 & -- & 458 & 0.905 & 0.484 & 0.676 & 141 \\
\hline 05530400 & 1 & Yes & Yes & 1977 & 114 & -- & 450 & 1.12 & 0.498 & 0.702 & 137 \\
\hline 05530400 & 1 & Yes & Yes & 1978 & 145 & -- & 572 & 1.54 & 0.512 & 0.527 & 169 \\
\hline 05530400 & 1 & Yes & Yes & 1979 & 115 & -- & 454 & 0.875 & 0.526 & 0.718 & 134 \\
\hline 05530480 & 1 & Yes & No & 1955 & 782 & 7 & 865 & 3.79 & 0.156 & 0.0736 & 1380 \\
\hline 05530480 & 1 & Yes & No & 1957 & 1200 & 7 & 1328 & 3.38 & 0.186 & 0.0205 & 1920 \\
\hline 05530480 & 1 & Yes & Yes & 1961 & 322 & -- & 356 & 2.03 & 0.254 & 0.656 & 619 \\
\hline 05530480 & 1 & Yes & Yes & 1962 & 310 & -- & 343 & 0.18 & 0.277 & 0.698 & 591 \\
\hline 05530480 & 1 & Yes & Yes & 1963 & 126 & -- & 139 & 1.6 & 0.299 & 0.958 & 317 \\
\hline 05530480 & 1 & Yes & Yes & 1964 & 210 & -- & 232 & 1.14 & 0.322 & 0.886 & 433 \\
\hline 05530480 & 1 & Yes & Yes & 1965 & 184 & -- & 204 & 0.185 & 0.345 & 0.925 & 390 \\
\hline 05530480 & 1 & Yes & Yes & 1966 & 692 & -- & 766 & 2.17 & 0.368 & 0.193 & 1030 \\
\hline 05530480 & 1 & Yes & Yes & 1967 & 2100 & -- & 2324 & 2.46 & 0.391 & 0.00725 & 2800 \\
\hline 05530480 & 1 & Yes & Yes & 1968 & 340 & -- & 376 & 2.56 & 0.413 & 0.745 & 558 \\
\hline 05530480 & 1 & Yes & Yes & 1969 & 494 & -- & 547 & 1.42 & 0.436 & 0.494 & 740 \\
\hline 05530480 & 1 & Yes & Yes & 1970 & 845 & -- & 935 & 1.26 & 0.459 & 0.143 & 1150 \\
\hline 05530480 & 1 & Yes & Yes & 1971 & 366 & -- & 405 & 1.62 & 0.481 & 0.752 & 551 \\
\hline 05530480 & 1 & Yes & Yes & 1972 & 1350 & -- & 1494 & 1.83 & 0.504 & 0.034 & 1710 \\
\hline 05530480 & 1 & Yes & Yes & 1973 & 718 & -- & 794 & 0.899 & 0.526 & 0.265 & 936 \\
\hline 05530480 & 1 & Yes & Yes & 1974 & 494 & -- & 547 & 0.791 & 0.548 & 0.601 & 661 \\
\hline 05530480 & 1 & Yes & Yes & 1975 & 1070 & 2 & 1184 & 1.56 & 0.571 & 0.0883 & 1310 \\
\hline 05530480 & 1 & Yes & Yes & 1976 & 754 & -- & 834 & 1.23 & 0.593 & 0.277 & 922 \\
\hline 05530480 & 1 & Yes & Yes & 1977 & 426 & -- & 471 & 1.21 & 0.615 & 0.764 & 541 \\
\hline
\end{tabular}




\begin{tabular}{|c|c|c|c|c|c|c|c|c|c|c|c|}
\hline $\begin{array}{c}\text { U.S. } \\
\text { Geological } \\
\text { Survey } \\
\text { streamgage } \\
\text { number }\end{array}$ & $\begin{array}{c}\text { Segment } \\
\text { number }\end{array}$ & $\begin{array}{l}\text { Streamgage used in } \\
\text { regression analyses } \\
\text { (non-redundant) }\end{array}$ & $\begin{array}{c}\text { Discharge value } \\
\text { used in } \\
\text { adjustment } \\
\text { regression }\end{array}$ & $\begin{array}{c}\text { Water } \\
\text { year }\end{array}$ & $\begin{array}{c}\text { Observed annual } \\
\text { maximum peak } \\
\text { discharge }\left(\mathrm{ft}^{3} / \mathrm{s}\right)\end{array}$ & $\begin{array}{c}\text { NWIS peak } \\
\text { code }\end{array}$ & $\begin{array}{l}\text { Observed annual } \\
\text { maximum peak } \\
\text { discharge with } \\
\text { segment intercept } \\
\text { value subtracted } \\
\left(\mathrm{ft}^{3} / \mathrm{s}\right)\end{array}$ & $\begin{array}{c}\text { Observed } \\
\text { precipitation } \\
\text { (inches) }\end{array}$ & Urban fraction & $\begin{array}{l}\text { Exceedance } \\
\text { probability }\end{array}$ & $\begin{array}{c}\text { Urban-adjusted } \\
\text { annual maximum } \\
\text { peak discharge } \\
\left(\mathrm{ft}^{3} / \mathrm{s}\right)\end{array}$ \\
\hline 05530480 & 1 & Yes & Yes & 1978 & 1680 & -- & 1859 & 2.3 & 0.638 & 0.0228 & 1900 \\
\hline 05530480 & 1 & Yes & Yes & 1979 & 1010 & -- & 1118 & 1.36 & 0.66 & 0.147 & 1150 \\
\hline 05530600 & 1 & No & No & 1960 & 3620 & -- & 653 & 0.542 & 0.25 & 0.219 & 4970 \\
\hline 05530600 & 1 & No & No & 1961 & 1280 & -- & 231 & 1.33 & 0.258 & 0.859 & 2060 \\
\hline 05530600 & 1 & No & No & 1962 & 2850 & -- & 514 & 0.003 & 0.265 & 0.393 & $402 C$ \\
\hline 05530600 & 1 & No & No & 1963 & 1550 & -- & 280 & 1.31 & 0.273 & 0.797 & 2370 \\
\hline 05530600 & 1 & No & No & 1964 & 1540 & -- & 278 & 0.758 & 0.281 & 0.804 & 2330 \\
\hline 05530600 & 1 & No & No & 1965 & 2100 & -- & 379 & 0.512 & 0.288 & 0.643 & 3000 \\
\hline 05530600 & 1 & No & No & 1966 & 2800 & -- & 505 & 1.17 & 0.296 & 0.432 & 3850 \\
\hline 05530600 & 1 & No & No & 1967 & 3620 & -- & 653 & 1.5 & 0.303 & 0.251 & 4760 \\
\hline 05530600 & 1 & No & No & 1968 & 2050 & -- & 370 & 0.988 & 0.311 & 0.676 & 2870 \\
\hline 05530600 & 1 & No & No & 1969 & 2880 & -- & 520 & 1.36 & 0.318 & 0.429 & 3860 \\
\hline 05530600 & 1 & No & No & 1970 & 3600 & -- & 650 & 1.54 & 0.326 & 0.269 & $464 C$ \\
\hline 05530600 & 1 & No & No & 1971 & 2400 & -- & 433 & 0.0848 & 0.333 & 0.588 & 3220 \\
\hline 05530600 & 1 & No & No & 1972 & 3600 & -- & 650 & 1.09 & 0.341 & 0.279 & 4590 \\
\hline 05530600 & 1 & No & No & 1973 & 3100 & -- & 560 & 1.37 & 0.348 & 0.397 & 4000 \\
\hline 05530600 & 1 & No & No & 1974 & 2880 & -- & 520 & 0.603 & 0.355 & 0.461 & $372 C$ \\
\hline 05530600 & 1 & No & No & 1975 & 2890 & -- & 522 & 0.79 & 0.362 & 0.465 & 3700 \\
\hline 05530600 & 1 & No & No & 1976 & 3300 & -- & 596 & 1.18 & 0.37 & 0.366 & 4150 \\
\hline 05530600 & 1 & No & No & 1977 & 1290 & -- & 233 & 1.07 & 0.377 & 0.907 & $180 C$ \\
\hline 05530700 & 1 & Yes & No & 1955 & 547 & 7 & 1113 & 3.35 & 0.651 & 0.145 & 682 \\
\hline 05530700 & 1 & Yes & Yes & 1961 & 589 & -- & 1198 & 2 & 0.776 & 0.164 & 656 \\
\hline 05530700 & 1 & Yes & Yes & 1962 & 262 & -- & 533 & 1.98 & 0.781 & 0.821 & $30 \epsilon$ \\
\hline 05530700 & 1 & Yes & Yes & 1963 & 309 & -- & 628 & 1.59 & 0.787 & 0.721 & 353 \\
\hline 05530700 & 1 & Yes & Yes & 1964 & 524 & -- & 1066 & 1.9 & 0.792 & 0.238 & $57 \epsilon$ \\
\hline 05530700 & 1 & Yes & Yes & 1965 & 586 & -- & 1192 & 0.495 & 0.798 & 0.177 & 639 \\
\hline 05530700 & 1 & Yes & Yes & 1966 & 356 & -- & 724 & 1.89 & 0.804 & 0.613 & 395 \\
\hline 05530700 & 1 & Yes & Yes & 1967 & 372 & -- & 757 & 2.07 & 0.809 & 0.579 & $40 \mathrm{~s}$ \\
\hline 05530700 & 1 & Yes & Yes & 1968 & 600 & 2 & 1220 & 2.09 & 0.815 & 0.173 & 644 \\
\hline 05530700 & 1 & Yes & Yes & 1969 & 560 & -- & 1139 & 0.807 & 0.82 & 0.211 & 595 \\
\hline 05530700 & 1 & Yes & Yes & 1970 & 317 & -- & 645 & 1.04 & 0.826 & 0.738 & 345 \\
\hline 05530700 & 1 & Yes & Yes & 1971 & 378 & -- & 769 & 1.2 & 0.828 & 0.584 & 407 \\
\hline 05530700 & 1 & Yes & Yes & 1972 & 752 & -- & 1530 & 1.29 & 0.83 & 0.0797 & 795 \\
\hline 05530700 & 1 & Yes & Yes & 1973 & 459 & -- & 934 & 0.482 & 0.832 & 0.408 & 490 \\
\hline 05530700 & 1 & Yes & Yes & 1974 & 347 & -- & 706 & 0.756 & 0.834 & 0.674 & 372 \\
\hline
\end{tabular}




\begin{tabular}{|c|c|c|c|c|c|c|c|c|c|c|c|}
\hline $\begin{array}{l}\text { U.S. } \\
\text { Geological } \\
\text { Survey } \\
\text { streamgage } \\
\text { number }\end{array}$ & $\begin{array}{c}\text { Segment } \\
\text { number }\end{array}$ & $\begin{array}{l}\text { Streamgage used in } \\
\text { regression analyses } \\
\text { (non-redundant) }\end{array}$ & $\begin{array}{c}\text { Discharge value } \\
\text { used in } \\
\text { adjustment } \\
\text { regression }\end{array}$ & $\begin{array}{l}\text { Water } \\
\text { year }\end{array}$ & $\begin{array}{c}\text { Observed annual } \\
\text { maximum peak } \\
\text { discharge }\left(\mathrm{ft}^{3} / \mathrm{s}\right)\end{array}$ & $\begin{array}{l}\text { NWIS peak } \\
\text { code }\end{array}$ & $\begin{array}{l}\text { Observed annual } \\
\text { maximum peak } \\
\text { discharge with } \\
\text { segment intercept } \\
\text { value subtracted } \\
\left(\mathrm{ft}^{3} / \mathrm{s}\right)\end{array}$ & $\begin{array}{l}\text { Observed } \\
\text { precipitation } \\
\text { (inches) }\end{array}$ & Urban fraction & $\begin{array}{l}\text { Exceedance } \\
\text { probability }\end{array}$ & $\begin{array}{c}\text { Urban-adjusted } \\
\text { annual maximum } \\
\text { peak discharge } \\
\left(\mathrm{ft}^{3} / \mathrm{s}\right)\end{array}$ \\
\hline 05530700 & 1 & Yes & Yes & 1975 & 499 & -- & 1015 & 1.39 & 0.837 & 0.317 & 528 \\
\hline 05530700 & 1 & Yes & Yes & 1976 & 308 & -- & 626 & 1.69 & 0.839 & 0.769 & 330 \\
\hline 05530700 & 1 & Yes & Yes & 1977 & 434 & -- & 883 & 0.349 & 0.841 & 0.471 & 459 \\
\hline 05530700 & 1 & Yes & Yes & 1978 & 496 & -- & 1009 & 1.9 & 0.843 & 0.332 & 522 \\
\hline 05530700 & 1 & Yes & Yes & 1979 & 511 & -- & 1039 & 1.14 & 0.845 & 0.3 & 536 \\
\hline 05530700 & 1 & Yes & Yes & 1980 & 450 & -- & 915 & 0.676 & 0.847 & 0.443 & 473 \\
\hline 05530800 & 1 & No & No & 1954 & 3020 & -- & 523 & 1.43 & 0.217 & 0.345 & 4480 \\
\hline 05530800 & 1 & No & No & 1955 & 3800 & -- & 658 & 2.71 & 0.227 & 0.201 & 5370 \\
\hline 05530800 & 1 & No & No & 1956 & 1700 & -- & 295 & 1.25 & 0.238 & 0.754 & 2710 \\
\hline 05530800 & 1 & No & No & 1957 & 4080 & -- & 707 & 1.61 & 0.248 & 0.179 & 5640 \\
\hline 05530800 & 1 & No & No & 1958 & 1320 & -- & 229 & 0.185 & 0.258 & 0.863 & 2170 \\
\hline 05530800 & 1 & No & No & 1959 & 1200 & -- & 208 & 1.19 & 0.268 & 0.894 & 1990 \\
\hline 05530800 & 1 & No & No & 1960 & 3800 & -- & 658 & 0.535 & 0.278 & 0.231 & 5150 \\
\hline 05530800 & 1 & No & No & 1961 & 2330 & -- & 404 & 1.33 & 0.286 & 0.601 & 3350 \\
\hline 05530800 & 1 & No & No & 1962 & 3050 & -- & 528 & 0.249 & 0.293 & 0.395 & 4230 \\
\hline 05530800 & 1 & No & No & 1963 & 1520 & -- & 263 & 1.31 & 0.3 & 0.835 & 2310 \\
\hline 05530800 & 1 & No & No & 1964 & 1550 & -- & 269 & 0.759 & 0.307 & 0.832 & 2330 \\
\hline 05530800 & 1 & No & No & 1965 & 2180 & -- & 378 & 0.512 & 0.315 & 0.666 & 3070 \\
\hline 05530800 & 1 & No & No & 1966 & 3280 & -- & 568 & 1.18 & 0.322 & 0.364 & 4390 \\
\hline 05530800 & 1 & No & No & 1967 & 3600 & -- & 624 & 1.5 & 0.329 & 0.3 & 4710 \\
\hline 05530800 & 1 & No & No & 1968 & 2230 & -- & 386 & 0.997 & 0.336 & 0.668 & 3060 \\
\hline 05530800 & 1 & No & No & 1969 & 3200 & -- & 554 & 1.35 & 0.343 & 0.4 & 4210 \\
\hline 05530800 & 1 & No & No & 1970 & 3870 & -- & 671 & 1.52 & 0.351 & 0.264 & 4930 \\
\hline 05530800 & 1 & No & No & 1971 & 2780 & -- & 482 & 0.0865 & 0.357 & 0.524 & 3650 \\
\hline 05530800 & 1 & No & No & 1972 & 2620 & -- & 454 & 1.09 & 0.364 & 0.579 & 3430 \\
\hline 05530800 & 1 & No & No & 1973 & 3200 & -- & 554 & 1.37 & 0.371 & 0.424 & 4100 \\
\hline 05530800 & 1 & No & No & 1974 & 3340 & -- & 579 & 0.599 & 0.378 & 0.396 & 4230 \\
\hline 05530800 & 1 & No & No & 1975 & 3610 & -- & 625 & 0.784 & 0.385 & 0.341 & 4500 \\
\hline 05530800 & 1 & No & No & 1976 & 3850 & -- & 667 & 1.17 & 0.392 & 0.296 & 4730 \\
\hline 05530940 & 1 & Yes & Yes & 1961 & 215 & -- & 774 & 1.27 & 0.377 & 0.192 & 363 \\
\hline 05530940 & 1 & Yes & Yes & 1962 & 177 & -- & 637 & 0.269 & 0.403 & 0.341 & 309 \\
\hline 05530940 & 1 & Yes & Yes & 1963 & 171 & -- & 615 & 2.01 & 0.428 & 0.39 & 296 \\
\hline 05530940 & 1 & Yes & Yes & 1964 & 106 & -- & 381 & 0.901 & 0.454 & 0.764 & 201 \\
\hline 05530940 & 1 & Yes & Yes & 1965 & 151 & -- & 543 & 0.01 & 0.479 & 0.539 & 255 \\
\hline 05530940 & 1 & Yes & Yes & 1966 & 173 & -- & 623 & 1.23 & 0.505 & 0.449 & 280 \\
\hline
\end{tabular}




\begin{tabular}{|c|c|c|c|c|c|c|c|c|c|c|c|}
\hline $\begin{array}{l}\text { U.S. } \\
\text { Geological } \\
\text { Survey } \\
\text { streamgage } \\
\text { number }\end{array}$ & $\begin{array}{l}\text { Segment } \\
\text { number }\end{array}$ & $\begin{array}{l}\text { Streamgage used in } \\
\text { regression analyses } \\
\text { (non-redundant) }\end{array}$ & $\begin{array}{c}\text { Discharge value } \\
\text { used in } \\
\text { adjustment } \\
\text { regression }\end{array}$ & $\begin{array}{l}\text { Water } \\
\text { year }\end{array}$ & $\begin{array}{c}\text { Observed annual } \\
\text { maximum peak } \\
\text { discharge }\left(\mathrm{ft}^{3} / \mathrm{s}\right)\end{array}$ & $\begin{array}{l}\text { NWIS peak } \\
\text { code }\end{array}$ & $\begin{array}{l}\text { Observed annual } \\
\text { maximum peak } \\
\text { discharge with } \\
\text { segment intercept } \\
\text { value subtracted } \\
\left(\mathrm{ft}^{3} / \mathrm{s}\right)\end{array}$ & $\begin{array}{l}\text { Observed } \\
\text { precipitation } \\
\text { (inches) }\end{array}$ & Urban fraction & $\begin{array}{l}\text { Exceedance } \\
\text { probability }\end{array}$ & $\begin{array}{c}\text { Urban-adjusted } \\
\text { annual maximum } \\
\text { peak discharge } \\
\left(\mathrm{ft}^{3} / \mathrm{s}\right)\end{array}$ \\
\hline 05530940 & 1 & Yes & Yes & 1967 & 482 & -- & 1734 & 1.23 & 0.53 & 0.0212 & 661 \\
\hline 05530940 & 1 & Yes & Yes & 1968 & 180 & -- & 648 & 4.15 & 0.555 & 0.463 & 276 \\
\hline 05530940 & 1 & Yes & Yes & 1969 & 184 & -- & 662 & 2.46 & 0.581 & 0.469 & 274 \\
\hline 05530940 & 1 & Yes & Yes & 1970 & 275 & -- & 990 & 2.87 & 0.606 & 0.179 & 375 \\
\hline 05530940 & 1 & Yes & Yes & 1971 & 183 & -- & 658 & 0.386 & 0.631 & 0.523 & 260 \\
\hline 05530940 & 1 & Yes & Yes & 1972 & 513 & -- & 1846 & 1.75 & 0.656 & 0.0248 & 641 \\
\hline 05530940 & 1 & Yes & Yes & 1973 & 356 & -- & 1281 & 2.2 & 0.68 & 0.0946 & 450 \\
\hline 05530940 & 1 & Yes & Yes & 1974 & 210 & -- & 756 & 1.13 & 0.705 & 0.475 & 273 \\
\hline 05530940 & 1 & Yes & Yes & 1975 & 205 & -- & 738 & 1.85 & 0.73 & 0.521 & 260 \\
\hline 05530940 & 1 & Yes & Yes & 1976 & 148 & -- & 533 & 1.89 & 0.754 & 0.801 & 191 \\
\hline 05530940 & 1 & Yes & Yes & 1978 & 249 & -- & 896 & 2.2 & 0.804 & 0.417 & 289 \\
\hline 05530940 & 1 & Yes & Yes & 1979 & 148 & -- & 533 & 0.38 & 0.828 & 0.857 & 174 \\
\hline 05530940 & 1 & Yes & Yes & 1980 & 122 & -- & 439 & 0.951 & 0.853 & 0.94 & 142 \\
\hline 05530960 & 1 & No & No & 1961 & 213 & -- & 473 & 1.35 & 0.334 & 0.518 & 356 \\
\hline 05530960 & 1 & No & No & 1962 & 215 & -- & 477 & 0.26 & 0.362 & 0.536 & 349 \\
\hline 05530960 & 1 & No & No & 1963 & 123 & -- & 273 & 1.75 & 0.389 & 0.868 & 222 \\
\hline 05530960 & 1 & No & No & 1964 & 112 & -- & 249 & 0.739 & 0.417 & 0.907 & 202 \\
\hline 05530960 & 1 & No & No & 1965 & 210 & -- & 466 & 0.56 & 0.444 & 0.628 & 316 \\
\hline 05530960 & 1 & No & No & 1966 & 367 & -- & 815 & 1.22 & 0.472 & 0.213 & 495 \\
\hline 05530960 & 1 & No & No & 1967 & 744 & -- & 1652 & 1.29 & 0.499 & 0.0234 & 932 \\
\hline 05530960 & 1 & No & No & 1968 & 240 & -- & 533 & 3.28 & 0.526 & 0.601 & 326 \\
\hline 05530960 & 1 & No & No & 1969 & 180 & -- & 400 & 2.11 & 0.554 & 0.808 & 248 \\
\hline 05530960 & 1 & No & No & 1970 & 371 & -- & 824 & 2.39 & 0.581 & 0.278 & 455 \\
\hline 05530960 & 1 & No & No & 1971 & 232 & 2 & 515 & 0.38 & 0.596 & 0.692 & 294 \\
\hline 05530960 & 1 & No & No & 1972 & 609 & -- & 1352 & 1.58 & 0.611 & 0.063 & 711 \\
\hline 05530960 & 1 & No & No & 1973 & 526 & -- & 1168 & 1.82 & 0.626 & 0.112 & 610 \\
\hline 05530960 & 1 & No & No & 1974 & 264 & -- & 586 & 0.947 & 0.641 & 0.631 & 315 \\
\hline 05530960 & 1 & No & No & 1975 & 314 & -- & 697 & 1.59 & 0.656 & 0.497 & 365 \\
\hline 05530960 & 1 & No & No & 1976 & 417 & -- & 926 & 1.61 & 0.67 & 0.254 & 468 \\
\hline 05530960 & 1 & No & No & 1977 & 244 & -- & 542 & 0.703 & 0.685 & 0.734 & 279 \\
\hline 05530960 & 1 & No & No & 1978 & 697 & -- & 1547 & 1.9 & 0.7 & 0.0497 & 751 \\
\hline 05530960 & 1 & No & No & 1979 & 366 & -- & 813 & 0.504 & 0.715 & 0.418 & 397 \\
\hline 05530990 & 1 & No & No & 1974 & 540 & -- & 615 & 1.03 & 0.726 & 0.679 & 622 \\
\hline 05530990 & 1 & No & No & 1975 & 910 & -- & 1036 & 0.797 & 0.738 & 0.222 & 1000 \\
\hline 05530990 & 1 & No & No & 1976 & 731 & D & 832 & 1.39 & 0.749 & 0.431 & 809 \\
\hline
\end{tabular}




\begin{tabular}{|c|c|c|c|c|c|c|c|c|c|c|c|}
\hline $\begin{array}{c}\text { U.S. } \\
\text { Geological } \\
\text { Survey } \\
\text { streamgage } \\
\text { number } \\
\end{array}$ & $\begin{array}{c}\text { Segment } \\
\text { number }\end{array}$ & $\begin{array}{l}\text { Streamgage used in } \\
\text { regression analyses } \\
\text { (non-redundant) }\end{array}$ & $\begin{array}{c}\text { Discharge value } \\
\text { used in } \\
\text { adjustment } \\
\text { regression }\end{array}$ & $\begin{array}{c}\text { Water } \\
\text { year }\end{array}$ & $\begin{array}{c}\text { Observed annual } \\
\text { maximum peak } \\
\text { discharge }\left(\mathrm{ft}^{3} / \mathrm{s}\right)\end{array}$ & $\begin{array}{c}\text { NWIS peak } \\
\text { code }\end{array}$ & $\begin{array}{c}\text { Observed annual } \\
\text { maximum peak } \\
\text { discharge with } \\
\text { segment intercept } \\
\text { value subtracted } \\
\left(\mathrm{ft}^{3} / \mathrm{s}\right)\end{array}$ & $\begin{array}{l}\text { Observed } \\
\text { precipitation } \\
\text { (inches) }\end{array}$ & Urban fraction & $\begin{array}{l}\text { Exceedance } \\
\text { probability }\end{array}$ & $\begin{array}{c}\text { Urban-adjusted } \\
\text { annual maximum } \\
\text { peak discharge } \\
\left(\mathrm{ft}^{3} / \mathrm{s}\right)\end{array}$ \\
\hline 05530990 & 1 & No & No & 1977 & 213 & $E$ & 242 & 1 & 0.761 & 0.984 & 252 \\
\hline 05530990 & 1 & No & No & 1978 & 642 & -- & 731 & 1.67 & 0.773 & 0.573 & 695 \\
\hline 05530990 & 1 & No & No & 1979 & 755 & -- & 859 & 0.585 & 0.784 & 0.437 & 804 \\
\hline 05530990 & 1 & No & No & 1980 & 422 & -- & 480 & 0.909 & 0.796 & 0.885 & 452 \\
\hline 05530990 & 1 & No & No & 1981 & 575 & -- & 654 & 1.66 & 0.8 & 0.704 & 606 \\
\hline 05530990 & 1 & No & No & 1982 & 609 & -- & 693 & 0.533 & 0.804 & 0.656 & 638 \\
\hline 05530990 & 1 & No & No & 1983 & 1060 & -- & 1207 & 1.77 & 0.808 & 0.175 & 1090 \\
\hline 05530990 & 1 & No & No & 1984 & 548 & -- & 624 & 0.703 & 0.812 & 0.749 & 570 \\
\hline 05530990 & 1 & No & No & 1985 & 763 & -- & 868 & 1.57 & 0.816 & 0.46 & 785 \\
\hline 05530990 & 1 & No & No & 1986 & 887 & -- & 1010 & 0.837 & 0.82 & 0.304 & 907 \\
\hline 05530990 & 1 & No & No & 1987 & 1650 & -- & 1878 & 2.17 & 0.824 & 0.0377 & 1670 \\
\hline 05530990 & 1 & No & No & 1988 & 535 & -- & 609 & 0.211 & 0.828 & 0.778 & 545 \\
\hline 05530990 & 1 & No & No & 1989 & 1110 & -- & 1263 & 2.25 & 0.832 & 0.164 & 1120 \\
\hline 05530990 & 1 & No & No & 1990 & 1010 & -- & 1150 & 2.02 & 0.836 & 0.215 & 1020 \\
\hline 05530990 & 1 & No & No & 1991 & 673 & -- & 766 & 1.84 & 0.836 & 0.596 & 677 \\
\hline 05530990 & 1 & No & No & 1992 & 444 & $\mathrm{E}$ & 505 & 1.58 & 0.837 & 0.89 & 447 \\
\hline 05530990 & 1 & No & No & 1993 & 1080 & C, D & 1229 & 0.999 & 0.838 & 0.18 & 1080 \\
\hline 05530990 & 1 & No & No & 1994 & 1200 & C & 1366 & 1.9 & 0.838 & 0.13 & 1200 \\
\hline 05530990 & 1 & No & No & 1995 & 827 & C & 941 & 1.84 & 0.839 & 0.406 & 830 \\
\hline 05530990 & 1 & No & No & 1996 & 796 & C & 906 & 1.46 & 0.84 & 0.444 & 798 \\
\hline 05530990 & 1 & No & No & 1997 & 1450 & C & 1650 & 2.75 & 0.84 & 0.0617 & 1450 \\
\hline 05530990 & 1 & No & No & 1998 & 668 & $C, E$ & 760 & 1.42 & 0.841 & 0.608 & 669 \\
\hline 05530990 & 1 & No & No & 1999 & 1140 & C & 1298 & 1.72 & 0.841 & 0.156 & 1140 \\
\hline 05530990 & 1 & No & No & 2000 & 837 & C & 953 & 2.41 & 0.842 & 0.398 & 837 \\
\hline 05530990 & 1 & No & No & 2001 & 1370 & C & 1559 & 2.44 & 0.842 & 0.0765 & 1370 \\
\hline 05530990 & 1 & No & No & 2002 & 1450 & $C$ & 1650 & 2.3 & 0.842 & 0.0621 & 1450 \\
\hline 05530990 & 1 & No & No & 2003 & 811 & C & 923 & 1.6 & 0.842 & 0.429 & 811 \\
\hline 05530990 & 1 & No & No & 2004 & 854 & $C$ & 972 & 1.19 & 0.842 & 0.374 & 854 \\
\hline 05530990 & 1 & No & No & 2005 & 505 & $C, E$ & 575 & 0.745 & 0.842 & 0.826 & 505 \\
\hline 05530990 & 1 & No & No & 2006 & 521 & $C$ & 593 & 1.34 & 0.842 & 0.808 & 521 \\
\hline 05530990 & 1 & No & No & 2007 & 1400 & C & 1594 & 2.05 & 0.842 & 0.0707 & 1400 \\
\hline 05530990 & 1 & No & No & 2008 & 2510 & C & 2857 & 3.53 & 0.842 & 0.0112 & 2510 \\
\hline 05530990 & 1 & No & No & 2009 & 1470 & C & 1673 & 0.92 & 0.842 & 0.0587 & 1470 \\
\hline 05531000 & 1 & No & No & 1951 & 350 & 2 & 493 & 0.421 & 0.243 & 0.406 & 671 \\
\hline 05531000 & 1 & No & No & 1952 & 430 & -- & 606 & 0.862 & 0.264 & 0.276 & 763 \\
\hline
\end{tabular}




\begin{tabular}{|c|c|c|c|c|c|c|c|c|c|c|c|}
\hline $\begin{array}{l}\text { U.S. } \\
\text { Geological } \\
\text { Survey } \\
\text { streamgage } \\
\text { number }\end{array}$ & $\begin{array}{l}\text { Segment } \\
\text { number }\end{array}$ & $\begin{array}{l}\text { Streamgage used in } \\
\text { regression analyses } \\
\text { (non-redundant) }\end{array}$ & $\begin{array}{c}\text { Discharge value } \\
\text { used in } \\
\text { adjustment } \\
\text { regression }\end{array}$ & $\begin{array}{l}\text { Water } \\
\text { year }\end{array}$ & $\begin{array}{c}\text { Observed annual } \\
\text { maximum peak } \\
\text { discharge }\left(\mathrm{ft}^{3} / \mathrm{s}\right)\end{array}$ & $\begin{array}{l}\text { NWIS peak } \\
\text { code }\end{array}$ & $\begin{array}{l}\text { Observed annual } \\
\text { maximum peak } \\
\text { discharge with } \\
\text { segment intercept } \\
\text { value subtracted } \\
\left(\mathrm{ft}^{3} / \mathrm{s}\right)\end{array}$ & $\begin{array}{l}\text { Observed } \\
\text { precipitation } \\
\text { (inches) }\end{array}$ & Urban fraction & $\begin{array}{l}\text { Exceedance } \\
\text { probability }\end{array}$ & $\begin{array}{c}\text { Urban-adjusted } \\
\text { annual maximum } \\
\text { peak discharge } \\
\left(\mathrm{ft}^{3} / \mathrm{s}\right)\end{array}$ \\
\hline 05531000 & 1 & No & No & 1953 & 139 & -- & 196 & 0.759 & 0.285 & 0.912 & 342 \\
\hline 05531000 & 1 & No & No & 1954 & 540 & -- & 761 & 1.95 & 0.306 & 0.171 & 889 \\
\hline 05531000 & 1 & No & No & 1955 & 516 & -- & 727 & 3.64 & 0.327 & 0.199 & 838 \\
\hline 05531000 & 1 & No & No & 1956 & 188 & -- & 265 & 1.3 & 0.348 & 0.857 & 387 \\
\hline 05531000 & 1 & No & No & 1957 & 572 & -- & 806 & 3.45 & 0.369 & 0.171 & 889 \\
\hline 05531000 & 1 & No & No & 1958 & 200 & -- & 282 & 0.769 & 0.39 & 0.857 & 388 \\
\hline 05531000 & 1 & No & No & 1959 & 350 & 2 & 493 & 0.402 & 0.411 & 0.555 & 576 \\
\hline 05531000 & 1 & No & No & 1960 & 721 & -- & 1016 & 0.394 & 0.432 & 0.0971 & 1050 \\
\hline 05531000 & 1 & No & No & 1961 & 551 & -- & 776 & 1.62 & 0.458 & 0.235 & 798 \\
\hline 05531000 & 1 & No & No & 1962 & 385 & 2 & 543 & 0.219 & 0.484 & 0.545 & 582 \\
\hline 05531000 & 1 & No & No & 1963 & 200 & -- & 282 & 1.52 & 0.509 & 0.912 & 344 \\
\hline 05531000 & 1 & No & No & 1964 & 210 & -- & 296 & 0.616 & 0.535 & 0.91 & 346 \\
\hline 05531000 & 1 & No & No & 1965 & 294 & -- & 414 & 0.534 & 0.56 & 0.795 & 432 \\
\hline 05531000 & 1 & No & No & 1966 & 410 & -- & 578 & 0.476 & 0.586 & 0.59 & 555 \\
\hline 05531000 & 1 & No & No & 1967 & 1060 & -- & 1494 & 1.38 & 0.611 & 0.0449 & 1290 \\
\hline 05531000 & 1 & No & No & 1968 & 396 & -- & 558 & 2.52 & 0.637 & 0.668 & 511 \\
\hline 05531000 & 1 & No & No & 1969 & 388 & -- & 547 & 1.82 & 0.662 & 0.709 & 488 \\
\hline 05531000 & 1 & No & No & 1970 & 731 & -- & 1030 & 1.99 & 0.688 & 0.196 & 847 \\
\hline 05531000 & 1 & No & No & 1971 & 427 & -- & 602 & 0.377 & 0.699 & 0.669 & 510 \\
\hline 05531000 & 1 & No & No & 1972 & 1060 & 2 & 1494 & 1.4 & 0.711 & 0.0595 & 1190 \\
\hline 05531000 & 1 & No & No & 1973 & 712 & -- & 1003 & 1.48 & 0.722 & 0.233 & 801 \\
\hline 05531000 & 1 & No & No & 1974 & 550 & 2 & 775 & 1.03 & 0.734 & 0.481 & 624 \\
\hline 05531000 & 1 & No & No & 1975 & 927 & 2 & 1306 & 0.81 & 0.745 & 0.111 & 1020 \\
\hline 05531000 & 1 & No & No & 1976 & 745 & 2 & 1050 & 1.36 & 0.757 & 0.225 & 811 \\
\hline 05531050 & 1 & No & No & 1955 & 610 & 7 & 624 & 3.53 & 0.263 & 0.255 & 1010 \\
\hline 05531050 & 1 & No & No & 1957 & 287 & 7 & 294 & 0.0405 & 0.305 & 0.795 & 547 \\
\hline 05531050 & 1 & No & No & 1960 & 583 & 7 & 597 & 0.386 & 0.368 & 0.364 & 903 \\
\hline 05531050 & 1 & No & No & 1961 & 465 & -- & 476 & 1.56 & 0.393 & 0.567 & 729 \\
\hline 05531050 & 1 & No & No & 1962 & 565 & -- & 578 & 0.232 & 0.417 & 0.43 & 844 \\
\hline 05531050 & 1 & No & No & 1963 & 305 & -- & 312 & 1.36 & 0.441 & 0.846 & 498 \\
\hline 05531050 & 1 & No & No & 1964 & 307 & -- & 314 & 0.71 & 0.465 & 0.857 & 487 \\
\hline 05531050 & 1 & No & No & 1965 & 456 & -- & 467 & 0.132 & 0.49 & 0.667 & 652 \\
\hline 05531050 & 1 & No & No & 1966 & 575 & -- & 588 & 1.22 & 0.514 & 0.505 & 779 \\
\hline 05531050 & 1 & No & No & 1967 & 1160 & -- & 1187 & 1.41 & 0.538 & 0.0783 & 1440 \\
\hline 05531050 & 1 & No & No & 1968 & 363 & -- & 371 & 1.98 & 0.562 & 0.846 & 498 \\
\hline
\end{tabular}




\begin{tabular}{|c|c|c|c|c|c|c|c|c|c|c|c|}
\hline $\begin{array}{l}\text { U.S. } \\
\text { Geological } \\
\text { Survey } \\
\text { streamgage } \\
\text { number }\end{array}$ & $\begin{array}{l}\text { Segment } \\
\text { number }\end{array}$ & $\begin{array}{l}\text { Streamgage used in } \\
\text { regression analyses } \\
\text { (non-redundant) }\end{array}$ & $\begin{array}{c}\text { Discharge value } \\
\text { used in } \\
\text { adjustment } \\
\text { regression }\end{array}$ & $\begin{array}{c}\text { Water } \\
\text { year }\end{array}$ & $\begin{array}{c}\text { Observed annual } \\
\text { maximum peak } \\
\text { discharge }\left(\mathrm{ft}^{3} / \mathrm{s}\right)\end{array}$ & $\begin{array}{l}\text { NWIS peak } \\
\text { code }\end{array}$ & $\begin{array}{l}\text { Observed annual } \\
\text { maximum peak } \\
\text { discharge with } \\
\text { segment intercept } \\
\text { value subtracted } \\
\left(\mathrm{ft}^{3} / \mathrm{s}\right)\end{array}$ & $\begin{array}{l}\text { Observed } \\
\text { precipitation } \\
\text { (inches) }\end{array}$ & Urban fraction & $\begin{array}{l}\text { Exceedance } \\
\text { probability }\end{array}$ & $\begin{array}{c}\text { Urban-adjusted } \\
\text { annual maximum } \\
\text { peak discharge } \\
\left(\mathrm{ft}^{3} / \mathrm{s}\right)\end{array}$ \\
\hline 05531050 & 1 & No & No & 1969 & 508 & -- & 520 & 1.59 & 0.587 & 0.676 & 646 \\
\hline 05531050 & 1 & No & No & 1970 & 654 & -- & 669 & 1.69 & 0.611 & 0.489 & 794 \\
\hline 05531050 & 1 & No & No & 1971 & 714 & -- & 731 & 0.372 & 0.624 & 0.424 & 850 \\
\hline 05531050 & 1 & No & No & 1972 & 1510 & -- & 1545 & 1.3 & 0.637 & 0.0432 & 1710 \\
\hline 05531050 & 1 & No & No & 1973 & 619 & -- & 633 & 1.24 & 0.65 & 0.575 & 722 \\
\hline 05531050 & 1 & No & No & 1974 & 648 & -- & 663 & 1.04 & 0.663 & 0.548 & 744 \\
\hline 05531050 & 1 & No & No & 1975 & 848 & -- & 868 & 0.894 & 0.676 & 0.313 & 946 \\
\hline 05531050 & 1 & No & No & 1976 & 1110 & -- & 1136 & 1.19 & 0.689 & 0.152 & 1210 \\
\hline 05531050 & 0 & No & No & 1977 & 365 & -- & NA & 0.95 & 0.702 & NA & NA \\
\hline 05531050 & 0 & No & No & 1978 & 1180 & -- & NA & 1.46 & 0.715 & NA & NA \\
\hline 05531050 & 0 & No & No & 1979 & 1740 & -- & NA & 0.676 & 0.728 & NA & NA \\
\hline 05531080 & 1 & Yes & Yes & 1961 & 176 & -- & 758 & 1.44 & 0.42 & 0.228 & 282 \\
\hline 05531080 & 1 & Yes & Yes & 1962 & 146 & -- & 629 & 0.254 & 0.436 & 0.379 & 244 \\
\hline 05531080 & 1 & Yes & Yes & 1963 & 173 & -- & 745 & 1.16 & 0.451 & 0.259 & 272 \\
\hline 05531080 & 1 & Yes & No & 1964 & 70 & 4, B & 302 & 0.254 & 0.467 & 0.874 & 137 \\
\hline 05531080 & 1 & Yes & No & 1965 & 70 & 4, B & 302 & 0.254 & 0.482 & 0.881 & 135 \\
\hline 05531080 & 1 & Yes & Yes & 1966 & 98 & -- & 422 & 1.33 & 0.498 & 0.74 & 168 \\
\hline 05531080 & 1 & Yes & Yes & 1967 & 98 & -- & 422 & 1.41 & 0.513 & 0.752 & 166 \\
\hline 05531080 & 1 & Yes & Yes & 1968 & 90 & -- & 388 & 1.31 & 0.529 & 0.806 & 153 \\
\hline 05531080 & 1 & Yes & Yes & 1969 & 16 & -- & 69 & 1.31 & 0.544 & 1 & 37.2 \\
\hline 05531080 & 1 & Yes & Yes & 1970 & 126 & -- & 543 & 1.32 & 0.56 & 0.616 & 192 \\
\hline 05531080 & 1 & Yes & Yes & 1971 & 114 & -- & 491 & 0.365 & 0.582 & 0.713 & 174 \\
\hline 05531080 & 1 & Yes & Yes & 1972 & 645 & -- & 2778 & 1.2 & 0.605 & 0.00661 & 803 \\
\hline 05531080 & 1 & Yes & Yes & 1973 & 193 & -- & 831 & 0.445 & 0.628 & 0.308 & 259 \\
\hline 05531080 & 1 & Yes & Yes & 1974 & 263 & -- & 1133 & 0.535 & 0.651 & 0.137 & 335 \\
\hline 05531080 & 1 & Yes & Yes & 1975 & 256 & -- & 1103 & 0.98 & 0.674 & 0.159 & 320 \\
\hline 05531080 & 1 & Yes & Yes & 1976 & 165 & -- & 711 & 0.988 & 0.697 & 0.521 & 212 \\
\hline 05531080 & 1 & Yes & Yes & 1977 & 181 & -- & 780 & 0.738 & 0.72 & 0.461 & 225 \\
\hline 05531080 & 1 & Yes & Yes & 1978 & 158 & -- & 681 & 1.24 & 0.743 & 0.605 & 194 \\
\hline 05531080 & 1 & Yes & Yes & 1979 & 180 & -- & 775 & 1.25 & 0.766 & 0.513 & 214 \\
\hline 05531100 & 1 & Yes & Yes & 1956 & 73 & -- & 777 & 1.18 & 0.414 & 0.208 & 124 \\
\hline 05531100 & 1 & Yes & Yes & 1957 & 51 & -- & 543 & 0.967 & 0.452 & 0.514 & 92 \\
\hline 05531100 & 1 & Yes & Yes & 1958 & 38 & -- & 405 & 1.41 & 0.49 & 0.758 & 71.6 \\
\hline 05531100 & 1 & Yes & Yes & 1959 & 54 & -- & 575 & 0.692 & 0.528 & 0.538 & 89.9 \\
\hline 05531100 & 1 & Yes & Yes & 1960 & 87 & -- & 926 & 0.445 & 0.565 & 0.192 & 128 \\
\hline
\end{tabular}




\begin{tabular}{|c|c|c|c|c|c|c|c|c|c|c|c|}
\hline $\begin{array}{l}\text { U.S. } \\
\text { Geological } \\
\text { Survey } \\
\text { streamgage } \\
\text { number }\end{array}$ & $\begin{array}{l}\text { Segment } \\
\text { number }\end{array}$ & $\begin{array}{l}\text { Streamgage used in } \\
\text { regression analyses } \\
\text { (non-redundant) }\end{array}$ & $\begin{array}{c}\text { Discharge value } \\
\text { used in } \\
\text { adjustment } \\
\text { regression }\end{array}$ & $\begin{array}{l}\text { Water } \\
\text { year }\end{array}$ & $\begin{array}{c}\text { Observed annual } \\
\text { maximum peak } \\
\text { discharge }\left(\mathrm{ft}^{3} / \mathrm{s}\right)\end{array}$ & $\begin{array}{l}\text { NWIS peak } \\
\text { code }\end{array}$ & $\begin{array}{l}\text { Observed annual } \\
\text { maximum peak } \\
\text { discharge with } \\
\text { segment intercept } \\
\text { value subtracted } \\
\left(\mathrm{ft}^{3} / \mathrm{s}\right)\end{array}$ & $\begin{array}{l}\text { Observed } \\
\text { precipitation } \\
\text { (inches) }\end{array}$ & Urban fraction & $\begin{array}{l}\text { Exceedance } \\
\text { probability }\end{array}$ & $\begin{array}{c}\text { Urban-adjusted } \\
\text { annual maximum } \\
\text { peak discharge } \\
\left(\mathrm{ft}^{3} / \mathrm{s}\right)\end{array}$ \\
\hline 05531100 & 1 & Yes & Yes & 1961 & 77 & -- & 820 & 1.47 & 0.578 & 0.279 & 114 \\
\hline 05531100 & 1 & Yes & Yes & 1962 & 67 & -- & 713 & 0.311 & 0.591 & 0.413 & 102 \\
\hline 05531100 & 1 & Yes & Yes & 1963 & 35 & -- & 373 & 1.14 & 0.603 & 0.87 & 60.8 \\
\hline 05531100 & 1 & Yes & Yes & 1964 & 33 & -- & 351 & 0.779 & 0.616 & 0.898 & 57.8 \\
\hline 05531100 & 1 & Yes & Yes & 1965 & 55 & -- & 586 & 0.505 & 0.628 & 0.62 & 82.8 \\
\hline 05531100 & 1 & Yes & Yes & 1966 & 98 & -- & 1043 & 1.37 & 0.641 & 0.17 & 133 \\
\hline 05531100 & 1 & Yes & Yes & 1967 & 119 & -- & 1267 & 1.46 & 0.654 & 0.0904 & 159 \\
\hline 05531100 & 1 & Yes & Yes & 1968 & 40 & -- & 426 & 1.24 & 0.666 & 0.852 & 62.5 \\
\hline 05531100 & 1 & Yes & Yes & 1969 & 64 & -- & 681 & 1.29 & 0.679 & 0.54 & 89.7 \\
\hline 05531100 & 1 & Yes & Yes & 1970 & 32 & -- & 341 & 1.32 & 0.692 & 0.935 & 52.1 \\
\hline 05531100 & 1 & Yes & Yes & 1971 & 39 & 2 & 415 & 0.364 & 0.713 & 0.892 & 58.4 \\
\hline 05531100 & 1 & Yes & Yes & 1972 & 256 & 2 & 2725 & 1.16 & 0.735 & 0.00992 & 304 \\
\hline 05531100 & 1 & Yes & Yes & 1978 & 56 & -- & 596 & 1.2 & 0.865 & 0.823 & 65.3 \\
\hline 05531100 & 1 & Yes & Yes & 1979 & 96 & -- & 1022 & 0.781 & 0.887 & 0.371 & 106 \\
\hline 05531130 & 1 & No & No & 1961 & 208 & -- & 474 & 1.45 & 0.456 & 0.627 & 360 \\
\hline 05531130 & 1 & No & No & 1962 & 196 & -- & 446 & 0.251 & 0.468 & 0.681 & 341 \\
\hline 05531130 & 1 & No & No & 1963 & 171 & 4 & 390 & 1.15 & 0.481 & 0.772 & 306 \\
\hline 05531130 & 1 & No & No & 1964 & 171 & 4, B & 390 & 0.144 & 0.493 & 0.78 & 302 \\
\hline 05531130 & 1 & No & No & 1965 & 191 & -- & 435 & 0.144 & 0.505 & 0.728 & 324 \\
\hline 05531130 & 1 & No & No & 1966 & 365 & -- & 831 & 1.35 & 0.518 & 0.228 & 535 \\
\hline 05531130 & 1 & No & No & 1967 & 330 & -- & 752 & 1.44 & 0.53 & 0.311 & 490 \\
\hline 05531130 & 1 & No & No & 1968 & 213 & -- & 485 & 1.27 & 0.543 & 0.687 & 339 \\
\hline 05531130 & 1 & No & No & 1969 & 258 & -- & 588 & 1.3 & 0.555 & 0.546 & 391 \\
\hline 05531130 & 1 & No & No & 1970 & 148 & -- & 337 & 1.29 & 0.567 & 0.887 & 254 \\
\hline 05531130 & 1 & No & No & 1971 & 171 & 4, B & 390 & 0.144 & 0.586 & 0.84 & 275 \\
\hline 05531130 & 1 & No & No & 1972 & 770 & -- & 1754 & 1.18 & 0.604 & 0.0257 & 987 \\
\hline 05531130 & 1 & No & No & 1973 & 256 & -- & 583 & 0.805 & 0.622 & 0.617 & 364 \\
\hline 05531130 & 1 & No & No & 1974 & 274 & -- & 624 & 0.522 & 0.64 & 0.578 & 379 \\
\hline 05531130 & 1 & No & No & 1975 & 297 & -- & 677 & 1 & 0.658 & 0.526 & 400 \\
\hline 05531130 & 1 & No & No & 1976 & 312 & -- & 711 & 0.964 & 0.677 & 0.501 & 410 \\
\hline 05531130 & 1 & No & No & 1978 & 239 & -- & 544 & 1.22 & 0.713 & 0.754 & 313 \\
\hline 05531130 & 1 & No & No & 1979 & 404 & -- & 920 & 0.784 & 0.731 & 0.309 & 489 \\
\hline 05531200 & 1 & No & No & 1948 & 1060 & 7 & 776 & 1.89 & 0.153 & 0.102 & 1930 \\
\hline 05531200 & 1 & No & No & 1950 & 910 & 7 & 666 & 1.23 & 0.171 & 0.173 & 1660 \\
\hline 05531200 & 1 & No & No & 1955 & 1150 & 7 & 842 & 3.46 & 0.283 & 0.118 & 1870 \\
\hline
\end{tabular}




\begin{tabular}{|c|c|c|c|c|c|c|c|c|c|c|c|}
\hline $\begin{array}{l}\text { U.S. } \\
\text { Geological } \\
\text { Survey } \\
\text { streamgage } \\
\text { number }\end{array}$ & $\begin{array}{c}\text { Segment } \\
\text { number }\end{array}$ & $\begin{array}{l}\text { Streamgage used in } \\
\text { regression analyses } \\
\text { (non-redundant) }\end{array}$ & $\begin{array}{c}\text { Discharge value } \\
\text { used in } \\
\text { adjustment } \\
\text { regression }\end{array}$ & $\begin{array}{l}\text { Water } \\
\text { year }\end{array}$ & $\begin{array}{c}\text { Observed annual } \\
\text { maximum peak } \\
\text { discharge }\left(\mathrm{ft}^{3} / \mathrm{s}\right)\end{array}$ & $\begin{array}{l}\text { NWIS peak } \\
\text { code }\end{array}$ & $\begin{array}{l}\text { Observed annual } \\
\text { maximum peak } \\
\text { discharge with } \\
\text { segment intercept } \\
\text { value subtracted } \\
\left(\mathrm{ft}^{3} / \mathrm{s}\right)\end{array}$ & $\begin{array}{l}\text { Observed } \\
\text { precipitation } \\
\text { (inches) }\end{array}$ & Urban fraction & $\begin{array}{l}\text { Exceedance } \\
\text { probability }\end{array}$ & $\begin{array}{c}\text { Urban-adjusted } \\
\text { annual maximum } \\
\text { peak discharge } \\
\left(\mathrm{ft}^{3} / \mathrm{s}\right)\end{array}$ \\
\hline 05531200 & 1 & No & No & 1957 & 776 & 7 & 568 & 2.83 & 0.328 & 0.369 & 1300 \\
\hline 05531200 & 1 & No & No & 1960 & 984 & -- & 720 & 0.381 & 0.395 & 0.245 & 1480 \\
\hline 05531200 & 1 & No & No & 1961 & 755 & -- & 553 & 1.52 & 0.416 & 0.467 & 1180 \\
\hline 05531200 & 1 & No & No & 1962 & 838 & -- & 613 & 0.241 & 0.437 & 0.4 & 1260 \\
\hline 05531200 & 1 & No & No & 1963 & 531 & -- & 389 & 1.28 & 0.459 & 0.758 & 850 \\
\hline 05531200 & 1 & No & No & 1964 & 528 & -- & 387 & 0.74 & 0.48 & 0.775 & 828 \\
\hline 05531200 & 1 & No & No & 1965 & 613 & -- & 449 & 0.514 & 0.501 & 0.705 & 912 \\
\hline 05531200 & 1 & No & No & 1966 & 1070 & -- & 783 & 1.29 & 0.522 & 0.272 & 1430 \\
\hline 05531200 & 1 & No & No & 1967 & 1260 & -- & 922 & 1.43 & 0.543 & 0.184 & 1630 \\
\hline 05531200 & 1 & No & No & 1968 & 609 & -- & 446 & 1.69 & 0.564 & 0.758 & 850 \\
\hline 05531200 & 1 & No & No & 1969 & 876 & -- & 641 & 1.48 & 0.585 & 0.501 & 1140 \\
\hline 05531200 & 1 & No & No & 1970 & 570 & -- & 417 & 1.34 & 0.607 & 0.822 & 766 \\
\hline 05531200 & 1 & No & No & 1971 & 768 & -- & 562 & 0.368 & 0.62 & 0.646 & 975 \\
\hline 05531200 & 1 & No & No & 1972 & 2110 & -- & 1545 & 1.25 & 0.634 & 0.043 & 2460 \\
\hline 05531200 & 1 & No & No & 1973 & 531 & -- & 389 & 1.24 & 0.648 & 0.88 & 688 \\
\hline 05531200 & 1 & No & No & 1974 & 1050 & -- & 769 & 1.04 & 0.662 & 0.416 & 1250 \\
\hline 05531200 & 1 & No & No & 1975 & 989 & -- & 724 & 0.949 & 0.676 & 0.484 & 1160 \\
\hline 05531200 & 1 & No & No & 1976 & 1150 & -- & 842 & 1.09 & 0.689 & 0.356 & 1320 \\
\hline 05531300 & 1 & No & No & 1948 & 1130 & 7 & 749 & 1.9 & 0.21 & 0.139 & 1770 \\
\hline 05531300 & 1 & No & No & 1950 & 1160 & 7 & 769 & 1.23 & 0.23 & 0.136 & 1790 \\
\hline 05531300 & 1 & No & No & 1955 & 1380 & 7 & 915 & 3.45 & 0.34 & 0.105 & 1910 \\
\hline 05531300 & 1 & No & No & 1957 & 895 & 7 & 594 & 2.78 & 0.384 & 0.381 & 1280 \\
\hline 05531300 & 1 & No & No & 1960 & 1260 & -- & 836 & 0.377 & 0.45 & 0.189 & 1600 \\
\hline 05531300 & 1 & No & No & 1961 & 688 & -- & 456 & 2 & 0.47 & 0.666 & 948 \\
\hline 05531300 & 1 & No & No & 1962 & 1090 & -- & 723 & 0.246 & 0.489 & 0.309 & 1370 \\
\hline 05531300 & 1 & No & No & 1963 & 608 & -- & 403 & 1.27 & 0.509 & 0.773 & 828 \\
\hline 05531300 & 1 & No & No & 1964 & 614 & -- & 407 & 0.745 & 0.528 & 0.781 & 818 \\
\hline 05531300 & 1 & No & No & 1965 & 688 & -- & 456 & 0.515 & 0.548 & 0.732 & 878 \\
\hline 05531300 & 1 & No & No & 1966 & 1400 & -- & 928 & 1.29 & 0.568 & 0.192 & 1590 \\
\hline 05531300 & 1 & No & No & 1967 & 1480 & -- & 982 & 1.43 & 0.587 & 0.175 & 1650 \\
\hline 05531300 & 1 & No & No & 1968 & 934 & -- & 619 & 1.67 & 0.607 & 0.551 & 1070 \\
\hline 05531300 & 1 & No & No & 1969 & 982 & -- & 651 & 1.46 & 0.626 & 0.528 & 1100 \\
\hline 05531300 & 1 & No & No & 1970 & 808 & -- & 536 & 1.34 & 0.646 & 0.709 & 906 \\
\hline 05531300 & 1 & No & No & 1971 & 964 & -- & 639 & 0.364 & 0.658 & 0.575 & 1050 \\
\hline 05531300 & 1 & No & No & 1972 & 2230 & -- & 1479 & 1.26 & 0.67 & 0.0541 & 2280 \\
\hline
\end{tabular}




\begin{tabular}{|c|c|c|c|c|c|c|c|c|c|c|c|}
\hline $\begin{array}{l}\text { U.S. } \\
\text { Geological } \\
\text { Survey } \\
\text { streamgage } \\
\text { number }\end{array}$ & $\begin{array}{l}\text { Segment } \\
\text { number }\end{array}$ & $\begin{array}{l}\text { Streamgage used in } \\
\text { regression analyses } \\
\text { (non-redundant) }\end{array}$ & $\begin{array}{c}\text { Discharge value } \\
\text { used in } \\
\text { adjustment } \\
\text { regression }\end{array}$ & $\begin{array}{l}\text { Water } \\
\text { year }\end{array}$ & $\begin{array}{c}\text { Observed annual } \\
\text { maximum peak } \\
\text { discharge }\left(\mathrm{ft}^{3} / \mathrm{s}\right)\end{array}$ & $\begin{array}{l}\text { NWIS peak } \\
\text { code }\end{array}$ & $\begin{array}{l}\text { Observed annual } \\
\text { maximum peak } \\
\text { discharge with } \\
\text { segment intercept } \\
\text { value subtracted } \\
\left(\mathrm{ft}^{3} / \mathrm{s}\right)\end{array}$ & $\begin{array}{l}\text { Observed } \\
\text { precipitation } \\
\text { (inches) }\end{array}$ & Urban fraction & $\begin{array}{l}\text { Exceedance } \\
\text { probability }\end{array}$ & $\begin{array}{c}\text { Urban-adjusted } \\
\text { annual maximum } \\
\text { peak discharge } \\
\left(\mathrm{ft}^{3} / \mathrm{s}\right)\end{array}$ \\
\hline 05531300 & 1 & No & No & 1973 & 729 & -- & 483 & 1.24 & 0.682 & 0.8 & 795 \\
\hline 05531300 & 1 & No & No & 1974 & 1030 & -- & 683 & 1.04 & 0.694 & 0.553 & 1070 \\
\hline 05531300 & 1 & No & No & 1975 & 944 & -- & 626 & 0.965 & 0.706 & 0.641 & 976 \\
\hline 05531300 & 1 & No & No & 1976 & 1100 & -- & 730 & 1.07 & 0.718 & 0.519 & 1110 \\
\hline 05531300 & 0 & No & No & 1977 & 606 & -- & 411 & 1.18 & 0.73 & 0.905 & 648 \\
\hline 05531300 & 0 & No & No & 1978 & 914 & -- & 633 & 1.34 & 0.742 & 0.669 & 948 \\
\hline 05531300 & 0 & No & No & 1979 & 1520 & -- & 1077 & 0.731 & 0.754 & 0.206 & 1550 \\
\hline 05531300 & 0 & No & No & 1980 & 703 & -- & 509 & 0.764 & 0.766 & 0.833 & 750 \\
\hline 05531300 & 2 & No & No & 1989 & 1580 & $C$ & 1170 & 1.61 & 0.793 & 0.183 & 1630 \\
\hline 05531300 & 2 & No & No & 1990 & 1530 & C & 1133 & 1.59 & 0.796 & 0.2 & 1570 \\
\hline 05531300 & 2 & No & No & 1991 & 1270 & C & 940 & 1.96 & 0.797 & 0.358 & 1310 \\
\hline 05531300 & 2 & No & No & 1992 & 645 & $C, E$ & 478 & 0.984 & 0.798 & 0.889 & 674 \\
\hline 05531300 & 2 & No & No & 1993 & 1130 & C & 837 & 0.693 & 0.8 & 0.478 & 1160 \\
\hline 05531300 & 2 & No & No & 1994 & 1500 & C & 1111 & 2.48 & 0.801 & 0.216 & 1530 \\
\hline 05531300 & 2 & No & No & 1995 & 1390 & C & 1029 & 1.93 & 0.802 & 0.272 & 1420 \\
\hline 05531300 & 2 & No & No & 1996 & 1250 & C & 926 & 0.839 & 0.804 & 0.383 & 1280 \\
\hline 05531300 & 2 & No & No & 1997 & 1460 & C & 1081 & 2.57 & 0.805 & 0.236 & 1490 \\
\hline 05531300 & 0 & No & No & 1998 & 1050 & C & 779 & 0.498 & 0.806 & 0.55 & 1080 \\
\hline 05531300 & 0 & No & No & 1999 & 1300 & C & 965 & 1.68 & 0.807 & 0.341 & 1330 \\
\hline 05531300 & 0 & No & No & 2000 & 1250 & C & 930 & 1.43 & 0.809 & 0.385 & 1280 \\
\hline 05531300 & 0 & No & No & 2001 & 1110 & C & 827 & 2.34 & 0.811 & 0.502 & 1130 \\
\hline 05531300 & 0 & No & No & 2002 & 1520 & C & 1134 & 3 & 0.814 & 0.21 & 1550 \\
\hline 05531300 & 3 & No & No & 2003 & 1170 & $C$ & 874 & 1.35 & 0.816 & 0.454 & 1190 \\
\hline 05531300 & 3 & No & No & 2004 & 1070 & C & 799 & 1.1 & 0.818 & 0.54 & 1090 \\
\hline 05531300 & 3 & No & No & 2005 & 1040 & $C, E$ & 777 & 0.866 & 0.821 & 0.568 & 1050 \\
\hline 05531300 & 3 & No & No & 2006 & 798 & C & 596 & 0.512 & 0.823 & 0.789 & 809 \\
\hline 05531300 & 3 & No & No & 2007 & 1310 & C & 979 & 1.27 & 0.826 & 0.347 & 1320 \\
\hline 05531300 & 3 & No & No & 2008 & 1940 & C & 1449 & 3.46 & 0.828 & 0.0982 & 1950 \\
\hline 05531300 & 3 & No & No & 2009 & 1430 & C & 1068 & 0.974 & 0.831 & 0.264 & 1430 \\
\hline 05531380 & 1 & No & No & 1948 & 1560 & 7 & 1059 & 1.91 & 0.247 & 0.0512 & 2600 \\
\hline 05531380 & 1 & No & No & 1950 & 1250 & 7 & 849 & 1.23 & 0.269 & 0.109 & 2110 \\
\hline 05531380 & 1 & No & No & 1955 & 1380 & 7 & 937 & 3.45 & 0.384 & 0.112 & 2100 \\
\hline 05531380 & 1 & No & No & 1957 & 1010 & 7 & 686 & 2.74 & 0.43 & 0.304 & 1540 \\
\hline 05531380 & 1 & No & No & 1960 & 1240 & -- & 842 & 0.375 & 0.499 & 0.209 & 1720 \\
\hline 05531380 & 1 & No & No & 1961 & 911 & -- & 619 & 1.5 & 0.517 & 0.466 & 1310 \\
\hline
\end{tabular}




\begin{tabular}{|c|c|c|c|c|c|c|c|c|c|c|c|}
\hline $\begin{array}{c}\text { U.S. } \\
\text { Geological } \\
\text { Survey } \\
\text { streamgage } \\
\text { number } \\
\end{array}$ & $\begin{array}{c}\text { Segment } \\
\text { number }\end{array}$ & $\begin{array}{l}\text { Streamgage used in } \\
\text { regression analyses } \\
\text { (non-redundant) }\end{array}$ & $\begin{array}{c}\text { Discharge value } \\
\text { used in } \\
\text { adjustment } \\
\text { regression }\end{array}$ & $\begin{array}{l}\text { Water } \\
\text { year }\end{array}$ & $\begin{array}{c}\text { Observed annual } \\
\text { maximum peak } \\
\text { discharge }\left(\mathrm{ft}^{3} / \mathrm{s}\right)\end{array}$ & $\begin{array}{c}\text { NWIS peak } \\
\text { code }\end{array}$ & $\begin{array}{c}\text { Observed annual } \\
\text { maximum peak } \\
\text { discharge with } \\
\text { segment intercept } \\
\text { value subtracted } \\
\left(\mathrm{ft}^{3} / \mathrm{s}\right) \\
\end{array}$ & $\begin{array}{c}\text { Observed } \\
\text { precipitation } \\
\text { (inches) }\end{array}$ & Urban fraction & $\begin{array}{l}\text { Exceedance } \\
\text { probability }\end{array}$ & $\begin{array}{c}\text { Urban-adjusted } \\
\text { annual maximum } \\
\text { peak discharge } \\
\left(\mathrm{ft}^{3} / \mathrm{s}\right) \\
\end{array}$ \\
\hline 05531380 & 1 & No & No & 1962 & 1040 & -- & 706 & 0.251 & 0.535 & 0.369 & 1450 \\
\hline 05531380 & 1 & No & No & 1963 & 582 & -- & 395 & 1.26 & 0.553 & 0.813 & 872 \\
\hline 05531380 & 1 & No & No & 1964 & 562 & -- & 382 & 0.747 & 0.571 & 0.84 & 832 \\
\hline 05531380 & 1 & No & No & 1965 & 689 & -- & 468 & 0.16 & 0.589 & 0.749 & 962 \\
\hline 05531380 & 1 & No & No & 1966 & 1210 & -- & 822 & 1.29 & 0.607 & 0.299 & 1540 \\
\hline 05531380 & 1 & No & No & 1967 & 1200 & -- & 815 & 1.41 & 0.625 & 0.324 & 1510 \\
\hline 05531380 & 1 & No & No & 1968 & 770 & -- & 523 & 1.65 & 0.643 & 0.722 & 997 \\
\hline 05531380 & 1 & No & No & 1969 & 766 & -- & 520 & 1.43 & 0.661 & 0.741 & 973 \\
\hline 05531380 & 1 & No & No & 1970 & 1120 & -- & 760 & 1.46 & 0.679 & 0.443 & 1350 \\
\hline 05531380 & 1 & No & No & 1971 & 740 & -- & 502 & 0.36 & 0.69 & 0.784 & 913 \\
\hline 05531380 & 1 & No & No & 1972 & 1790 & -- & 1215 & 1.29 & 0.701 & 0.125 & 2050 \\
\hline 05531380 & 1 & No & No & 1973 & 1040 & -- & 706 & 1.25 & 0.711 & 0.541 & 1210 \\
\hline 05531380 & 1 & No & No & 1974 & 1080 & -- & 733 & 1.05 & 0.722 & 0.519 & 1240 \\
\hline 05531380 & 1 & No & No & 1975 & 1330 & -- & 903 & 0.977 & 0.733 & 0.33 & 1500 \\
\hline 05531380 & 1 & No & No & 1976 & 1430 & -- & 971 & 1.06 & 0.743 & 0.273 & 1580 \\
\hline 05531500 & 1 & Yes & Yes & 1946 & 775 & -- & 425 & 0.785 & 0.222 & 0.501 & 1420 \\
\hline 05531500 & 1 & Yes & Yes & 1947 & 1000 & -- & 549 & 2.15 & 0.233 & 0.323 & 1700 \\
\hline 05531500 & 1 & Yes & Yes & 1948 & 1920 & -- & 1053 & 1.92 & 0.243 & 0.0517 & 2900 \\
\hline 05531500 & 1 & Yes & Yes & 1949 & 836 & -- & 459 & 0.725 & 0.254 & 0.471 & 1460 \\
\hline 05531500 & 1 & Yes & Yes & 1950 & 1360 & -- & 746 & 1.23 & 0.265 & 0.162 & 2100 \\
\hline 05531500 & 1 & Yes & Yes & 1951 & 1200 & 2 & 658 & 0.346 & 0.287 & 0.236 & 1850 \\
\hline 05531500 & 1 & Yes & Yes & 1952 & 925 & $\mathrm{D}$ & 507 & 0.725 & 0.309 & 0.44 & 1510 \\
\hline 05531500 & 1 & Yes & Yes & 1953 & 506 & $\mathrm{E}$ & 278 & 1.14 & 0.331 & 0.832 & 947 \\
\hline 05531500 & 1 & Yes & Yes & 1954 & 1140 & -- & 625 & 1.47 & 0.353 & 0.316 & 1700 \\
\hline 05531500 & 1 & Yes & Yes & 1955 & 1710 & -- & 938 & 3.45 & 0.375 & 0.108 & 2370 \\
\hline 05531500 & 1 & Yes & Yes & 1956 & 624 & -- & 342 & 0.831 & 0.397 & 0.781 & 1030 \\
\hline 05531500 & 1 & Yes & Yes & 1957 & 1020 & -- & 559 & 2.69 & 0.419 & 0.459 & 1480 \\
\hline 05531500 & 1 & Yes & Yes & 1958 & 778 & -- & 427 & 1.45 & 0.441 & 0.689 & 1160 \\
\hline 05531500 & 1 & Yes & Yes & 1959 & 1200 & 2 & 658 & 0.379 & 0.463 & 0.365 & 1630 \\
\hline 05531500 & 1 & Yes & Yes & 1960 & 1220 & -- & 669 & 0.371 & 0.485 & 0.371 & 1620 \\
\hline 05531500 & 1 & Yes & Yes & 1961 & 1430 & -- & 784 & 1.97 & 0.503 & 0.258 & 1810 \\
\hline 05531500 & 1 & Yes & Yes & 1962 & 940 & $\mathrm{E}$ & 516 & 0.257 & 0.521 & 0.621 & 1250 \\
\hline 05531500 & 1 & Yes & Yes & 1963 & 585 & $\mathrm{E}$ & 321 & 1.26 & 0.539 & 0.889 & 849 \\
\hline 05531500 & 1 & Yes & Yes & 1964 & 650 & $\mathrm{E}$ & 357 & 0.749 & 0.557 & 0.86 & 899 \\
\hline 05531500 & 1 & Yes & Yes & 1965 & 700 & 2 & 384 & 0.129 & 0.575 & 0.839 & 933 \\
\hline
\end{tabular}




\begin{tabular}{|c|c|c|c|c|c|c|c|c|c|c|c|}
\hline $\begin{array}{l}\text { U.S. } \\
\text { Geological } \\
\text { Survey } \\
\text { streamgage } \\
\text { number }\end{array}$ & $\begin{array}{l}\text { Segment } \\
\text { number }\end{array}$ & $\begin{array}{l}\text { Streamgage used in } \\
\text { regression analyses } \\
\text { (non-redundant) }\end{array}$ & $\begin{array}{l}\text { Discharge value } \\
\text { used in } \\
\text { adjustment } \\
\text { regression }\end{array}$ & $\begin{array}{l}\text { Water } \\
\text { year }\end{array}$ & $\begin{array}{l}\text { Observed annual } \\
\text { maximum peak } \\
\text { discharge }\left(\mathrm{ft}^{3} / \mathrm{s}\right)\end{array}$ & $\begin{array}{l}\text { NWIS peak } \\
\text { code }\end{array}$ & $\begin{array}{l}\text { Observed annual } \\
\text { maximum peak } \\
\text { discharge with } \\
\text { segment intercept } \\
\text { value subtracted } \\
\left(\mathrm{ft}^{3} / \mathrm{s}\right)\end{array}$ & $\begin{array}{l}\text { Observed } \\
\text { precipitation } \\
\text { (inches) }\end{array}$ & Urban fraction & $\begin{array}{l}\text { Exceedance } \\
\text { probability }\end{array}$ & $\begin{array}{c}\text { Urban-adjusted } \\
\text { annual maximum } \\
\text { peak discharge } \\
\left(\mathrm{ft}^{3} / \mathrm{s}\right)\end{array}$ \\
\hline 05531500 & 1 & Yes & Yes & 1966 & 1460 & -- & 801 & 1.3 & 0.593 & 0.31 & 1710 \\
\hline 05531500 & 1 & Yes & Yes & 1967 & 1350 & -- & 741 & 1.4 & 0.61 & 0.4 & 1580 \\
\hline 05531500 & 1 & Yes & Yes & 1968 & 1220 & -- & 669 & 1.63 & 0.628 & 0.506 & 1410 \\
\hline 05531500 & 1 & Yes & Yes & 1969 & 1040 & -- & 570 & 0.943 & 0.646 & 0.659 & 1200 \\
\hline 05531500 & 1 & Yes & Yes & 1970 & 1110 & -- & 609 & 1.43 & 0.664 & 0.622 & 1250 \\
\hline 05531500 & 1 & Yes & Yes & 1971 & 739 & $\mathrm{D}, \mathrm{E}$ & 405 & 0.355 & 0.675 & 0.88 & 868 \\
\hline 05531500 & 1 & Yes & Yes & 1972 & 1790 & -- & 982 & 1.31 & 0.686 & 0.225 & 1890 \\
\hline 05531500 & 1 & Yes & Yes & 1973 & 1280 & -- & 702 & 0.811 & 0.697 & 0.532 & 1370 \\
\hline 05531500 & 1 & Yes & Yes & 1974 & 1160 & -- & 636 & 1.05 & 0.708 & 0.629 & 1240 \\
\hline 05531500 & 1 & Yes & Yes & 1975 & 1600 & -- & 878 & 0.992 & 0.719 & 0.346 & 1650 \\
\hline 05531500 & 1 & Yes & Yes & 1976 & 1460 & D & 801 & 1.05 & 0.731 & 0.447 & 1500 \\
\hline 05531500 & 0 & Yes & No & 1977 & 1000 & $E$ & 541 & 1.18 & 0.742 & 0.781 & 1030 \\
\hline 05531500 & 2 & Yes & Yes & 1978 & 1150 & $E$ & 613 & 1.3 & 0.753 & 0.708 & 1140 \\
\hline 05531500 & 2 & Yes & Yes & 1979 & 1930 & -- & 1030 & 0.765 & 0.764 & 0.243 & 1840 \\
\hline 05531500 & 2 & Yes & Yes & 1980 & 1370 & $\mathrm{E}$ & 731 & 0.712 & 0.775 & 0.575 & 1310 \\
\hline 05531500 & 2 & Yes & Yes & 1981 & 1450 & $\mathrm{E}$ & 773 & 1.79 & 0.778 & 0.528 & 1380 \\
\hline 05531500 & 2 & Yes & Yes & 1982 & 1330 & -- & 709 & 0.36 & 0.781 & 0.608 & 1270 \\
\hline 05531500 & 2 & Yes & Yes & 1983 & 2070 & -- & 1104 & 1.63 & 0.784 & 0.209 & 1930 \\
\hline 05531500 & 2 & Yes & Yes & 1984 & 1040 & -- & 555 & 0.552 & 0.787 & 0.803 & 995 \\
\hline 05531500 & 2 & Yes & Yes & 1985 & 1850 & -- & 987 & 1.32 & 0.789 & 0.297 & 1730 \\
\hline 05531500 & 0 & Yes & No & 1986 & 1330 & -- & 715 & 0.836 & 0.792 & 0.614 & 1260 \\
\hline 05531500 & 3 & Yes & Yes & 1987 & 3540 & -- & 1915 & 2.49 & 0.795 & 0.0324 & 3290 \\
\hline 05531500 & 3 & Yes & Yes & 1988 & 1180 & -- & 638 & 0.84 & 0.798 & 0.72 & 1120 \\
\hline 05531500 & 3 & Yes & Yes & 1989 & 1360 & -- & 736 & 1.45 & 0.801 & 0.596 & 1280 \\
\hline 05531500 & 3 & Yes & Yes & 1990 & 2320 & -- & 1255 & 1.82 & 0.804 & 0.155 & 2150 \\
\hline 05531500 & 3 & Yes & Yes & 1991 & 1760 & -- & 952 & 2.01 & 0.805 & 0.354 & 1640 \\
\hline 05531500 & 3 & Yes & Yes & 1992 & 1140 & E & 617 & 0.971 & 0.807 & 0.752 & 1070 \\
\hline 05531500 & 3 & Yes & Yes & 1993 & 1160 & C & 628 & 0.652 & 0.808 & 0.741 & 1090 \\
\hline 05531500 & 3 & Yes & Yes & 1994 & 1520 & C & 822 & 2.21 & 0.809 & 0.505 & 1420 \\
\hline 05531500 & 3 & Yes & Yes & 1995 & 1470 & C & 795 & 0.197 & 0.81 & 0.536 & 1370 \\
\hline 05531500 & 3 & Yes & Yes & 1996 & 1650 & C & 893 & 0.751 & 0.812 & 0.429 & 1530 \\
\hline 05531500 & 3 & Yes & Yes & 1997 & 2510 & C & 1358 & 2.55 & 0.813 & 0.122 & 2310 \\
\hline 05531500 & 0 & Yes & No & 1998 & 1470 & C & 811 & 0.64 & 0.814 & 0.522 & 1390 \\
\hline 05531500 & 0 & Yes & No & 1999 & 1550 & C & 872 & 1.47 & 0.816 & 0.456 & 1490 \\
\hline 05531500 & 0 & Yes & No & 2000 & 1520 & C & 872 & 1.44 & 0.817 & 0.457 & 1490 \\
\hline
\end{tabular}




\begin{tabular}{|c|c|c|c|c|c|c|c|c|c|c|c|}
\hline $\begin{array}{l}\text { U.S. } \\
\text { Geological } \\
\text { Survey } \\
\text { streamgage } \\
\text { number }\end{array}$ & $\begin{array}{l}\text { Segment } \\
\text { number }\end{array}$ & $\begin{array}{l}\text { Streamgage used in } \\
\text { regression analyses } \\
\text { (non-redundant) }\end{array}$ & $\begin{array}{c}\text { Discharge value } \\
\text { used in } \\
\text { adjustment } \\
\text { regression }\end{array}$ & $\begin{array}{l}\text { Water } \\
\text { year }\end{array}$ & $\begin{array}{c}\text { Observed annual } \\
\text { maximum peak } \\
\text { discharge }\left(\mathrm{ft}^{3} / \mathrm{s}\right)\end{array}$ & $\begin{array}{l}\text { NWIS peak } \\
\text { code }\end{array}$ & $\begin{array}{l}\text { Observed annual } \\
\text { maximum peak } \\
\text { discharge with } \\
\text { segment intercept } \\
\text { value subtracted } \\
\left(\mathrm{ft}^{3} / \mathrm{s}\right)\end{array}$ & $\begin{array}{l}\text { Observed } \\
\text { precipitation } \\
\text { (inches) }\end{array}$ & Urban fraction & $\begin{array}{l}\text { Exceedance } \\
\text { probability }\end{array}$ & $\begin{array}{c}\text { Urban-adjusted } \\
\text { annual maximum } \\
\text { peak discharge } \\
\left(\mathrm{ft}^{3} / \mathrm{s}\right)\end{array}$ \\
\hline 05531500 & 0 & Yes & No & 2001 & 1280 & C & 749 & 1.1 & 0.82 & 0.6 & 1280 \\
\hline 05531500 & 0 & Yes & No & 2002 & 2080 & C & 1241 & 2.53 & 0.824 & 0.169 & 2090 \\
\hline 05531500 & 4 & Yes & Yes & 2003 & 1220 & C & 742 & 1.38 & 0.827 & 0.616 & 1250 \\
\hline 05531500 & 4 & Yes & Yes & 2004 & 1110 & $C, E$ & 675 & 1.36 & 0.83 & 0.709 & 1140 \\
\hline 05531500 & 4 & Yes & Yes & 2005 & 1390 & $C, E$ & 845 & 1.02 & 0.834 & 0.505 & 1420 \\
\hline 05531500 & 4 & Yes & Yes & 2006 & 945 & $\mathrm{C}, \mathrm{E}$ & 575 & 0.536 & 0.837 & 0.822 & 963 \\
\hline 05531500 & 4 & Yes & Yes & 2007 & 1920 & C & 1168 & 1.63 & 0.841 & 0.208 & 1940 \\
\hline 05531500 & 4 & Yes & Yes & 2008 & 2890 & C & 1758 & 3.34 & 0.844 & 0.0484 & 2910 \\
\hline 05531500 & 4 & Yes & Yes & 2009 & 2080 & C & 1265 & 0.824 & 0.847 & 0.17 & 2090 \\
\hline 05531800 & 1 & No & No & 1961 & 389 & -- & 946 & 1.7 & 0.627 & 0.212 & 509 \\
\hline 05531800 & 1 & No & No & 1962 & 334 & -- & 812 & 0.226 & 0.641 & 0.342 & 442 \\
\hline 05531800 & 1 & No & No & 1963 & 303 & -- & 737 & 1.31 & 0.655 & 0.447 & 402 \\
\hline 05531800 & 1 & No & No & 1964 & 316 & -- & 769 & 1.09 & 0.669 & 0.423 & 412 \\
\hline 05531800 & 1 & No & No & 1965 & 311 & -- & 756 & 0.583 & 0.683 & 0.451 & 400 \\
\hline 05531800 & 1 & No & No & 1966 & 449 & -- & 1092 & 2.07 & 0.697 & 0.173 & 548 \\
\hline 05531800 & 1 & No & No & 1967 & 342 & -- & 832 & 0.946 & 0.711 & 0.391 & 424 \\
\hline 05531800 & 1 & No & No & 1968 & 388 & -- & 944 & 2.39 & 0.725 & 0.281 & 467 \\
\hline 05531800 & 1 & No & No & 1969 & 367 & -- & 893 & 1.37 & 0.739 & 0.349 & 439 \\
\hline 05531800 & 1 & No & No & 1970 & 406 & -- & 987 & 2.76 & 0.753 & 0.266 & 474 \\
\hline 05531800 & 1 & No & No & 1971 & 335 & -- & 815 & 0.376 & 0.758 & 0.46 & 397 \\
\hline 05531800 & 1 & No & No & 1972 & 343 & -- & 834 & 1.57 & 0.764 & 0.444 & 403 \\
\hline 05531800 & 1 & No & No & 1973 & 307 & -- & 747 & 0.498 & 0.77 & 0.551 & 361 \\
\hline 05531800 & 1 & No & No & 1975 & 239 & -- & 581 & 1.5 & 0.781 & 0.769 & 284 \\
\hline 05531800 & 1 & No & No & 1976 & 177 & -- & 430 & 1.78 & 0.787 & 0.921 & 217 \\
\hline 05531800 & 0 & No & No & 1977 & 233 & -- & NA & 1.61 & 0.792 & NA & NA \\
\hline 05531800 & 0 & No & No & 1978 & 236 & -- & NA & 1.27 & 0.798 & NA & NA \\
\hline 05531800 & 0 & No & No & 1979 & 271 & -- & NA & 0.841 & 0.804 & NA & NA \\
\hline 05531800 & 0 & No & No & 1980 & 216 & -- & NA & 0.836 & 0.809 & NA & NA \\
\hline 05532000 & 1 & Yes & Yes & 1951 & 295 & -- & 616 & 0.344 & 0.601 & 0.55 & 369 \\
\hline 05532000 & 1 & Yes & Yes & 1952 & 200 & -- & 418 & 0.829 & 0.626 & 0.834 & 265 \\
\hline 05532000 & 1 & Yes & Yes & 1953 & 57 & -- & 119 & 0.666 & 0.651 & 1 & 94.2 \\
\hline 05532000 & 1 & Yes & Yes & 1954 & 548 & -- & 1145 & 1.66 & 0.677 & 0.143 & 591 \\
\hline 05532000 & 1 & Yes & Yes & 1955 & 598 & -- & 1249 & 3.37 & 0.702 & 0.113 & 629 \\
\hline 05532000 & 1 & Yes & Yes & 1956 & 228 & -- & 476 & 0.389 & 0.727 & 0.84 & 263 \\
\hline 05532000 & 1 & Yes & Yes & 1957 & 560 & -- & 1170 & 2.15 & 0.753 & 0.165 & 564 \\
\hline
\end{tabular}




\begin{tabular}{|c|c|c|c|c|c|c|c|c|c|c|c|}
\hline $\begin{array}{c}\text { U.S. } \\
\text { Geological } \\
\text { Survey } \\
\text { streamgage } \\
\text { number }\end{array}$ & $\begin{array}{l}\text { Segment } \\
\text { number }\end{array}$ & $\begin{array}{l}\text { Streamgage used in } \\
\text { regression analyses } \\
\text { (non-redundant) }\end{array}$ & $\begin{array}{l}\text { Discharge value } \\
\text { used in } \\
\text { adjustment } \\
\text { regression }\end{array}$ & $\begin{array}{l}\text { Water } \\
\text { year }\end{array}$ & $\begin{array}{l}\text { Observed annual } \\
\text { maximum peak } \\
\text { discharge }\left(\mathrm{ft}^{3} / \mathrm{s}\right)\end{array}$ & $\begin{array}{l}\text { NWIS peak } \\
\text { code }\end{array}$ & $\begin{array}{l}\text { Observed annual } \\
\text { maximum peak } \\
\text { discharge with } \\
\text { segment intercept } \\
\text { value subtracted } \\
\left(\mathrm{ft}^{3} / \mathrm{s}\right)\end{array}$ & $\begin{array}{l}\text { Observed } \\
\text { precipitation } \\
\text { (inches) }\end{array}$ & Urban fraction & $\begin{array}{l}\text { Exceedance } \\
\text { probability }\end{array}$ & $\begin{array}{c}\text { Urban-adjusted } \\
\text { annual maximum } \\
\text { peak discharge } \\
\left(\mathrm{ft}^{3} / \mathrm{s}\right)\end{array}$ \\
\hline 05532000 & 1 & Yes & Yes & 1958 & 405 & -- & 846 & 1.45 & 0.778 & 0.445 & 411 \\
\hline 05532000 & 1 & Yes & Yes & 1959 & 273 & D & 570 & 0.67 & 0.803 & 0.8 & 280 \\
\hline 05532000 & 1 & Yes & Yes & 1960 & 295 & -- & 616 & 0.77 & 0.828 & 0.771 & 291 \\
\hline 05532000 & 1 & Yes & Yes & 1961 & 588 & -- & 1228 & 1.76 & 0.835 & 0.179 & 550 \\
\hline 05532000 & 1 & Yes & Yes & 1962 & 260 & -- & 543 & 1.73 & 0.841 & 0.856 & 256 \\
\hline 05532000 & 1 & Yes & Yes & 1963 & 325 & -- & 679 & 1.38 & 0.847 & 0.721 & 311 \\
\hline 05532000 & 1 & Yes & Yes & 1964 & 284 & -- & 593 & 0.909 & 0.854 & 0.817 & 273 \\
\hline 05532000 & 1 & Yes & Yes & 1965 & 434 & -- & 907 & 0.386 & 0.86 & 0.466 & 403 \\
\hline 05532000 & 1 & Yes & Yes & 1966 & 499 & -- & 1042 & 1.68 & 0.867 & 0.321 & 457 \\
\hline 05532000 & 1 & Yes & Yes & 1967 & 264 & -- & 552 & 1.02 & 0.873 & 0.874 & 249 \\
\hline 05532000 & 1 & Yes & Yes & 1968 & 584 & -- & 1220 & 1.72 & 0.879 & 0.203 & 524 \\
\hline 05532000 & 1 & Yes & Yes & 1969 & 553 & -- & 1155 & 0.839 & 0.886 & 0.244 & 494 \\
\hline 05532000 & 1 & Yes & Yes & 1970 & 449 & D & 938 & 1.79 & 0.892 & 0.467 & 402 \\
\hline 05532000 & 1 & Yes & Yes & 1971 & 336 & -- & 702 & 0.903 & 0.894 & 0.739 & 304 \\
\hline 05532000 & 1 & Yes & Yes & 1972 & 706 & -- & 1475 & 0.93 & 0.896 & 0.118 & 623 \\
\hline 05532000 & 1 & Yes & Yes & 1973 & 509 & -- & 1063 & 0.762 & 0.898 & 0.336 & 451 \\
\hline 05532000 & 1 & Yes & Yes & 1974 & 428 & -- & 894 & 0.72 & 0.899 & 0.521 & 381 \\
\hline 05532000 & 1 & Yes & Yes & 1975 & 598 & -- & 1249 & 1.26 & 0.901 & 0.201 & 526 \\
\hline 05532000 & 1 & Yes & Yes & 1976 & 454 & D & 948 & 0.963 & 0.903 & 0.469 & 402 \\
\hline 05532000 & 1 & Yes & Yes & 1977 & 359 & $\mathrm{E}$ & 750 & 1.42 & 0.905 & 0.701 & 319 \\
\hline 05532000 & 1 & Yes & Yes & 1978 & 607 & -- & 1268 & 0.999 & 0.907 & 0.196 & 531 \\
\hline 05532000 & 1 & Yes & Yes & 1979 & 591 & -- & 1235 & 0.547 & 0.909 & 0.214 & 516 \\
\hline 05532000 & 1 & Yes & Yes & 1980 & 662 & -- & 1383 & 0.546 & 0.911 & 0.156 & 576 \\
\hline 05532000 & 1 & Yes & Yes & 1981 & 548 & -- & 1145 & 0.835 & 0.911 & 0.272 & 478 \\
\hline 05532000 & 1 & Yes & Yes & 1982 & 839 & -- & 1753 & 0.757 & 0.911 & 0.0617 & 729 \\
\hline 05532000 & 1 & Yes & Yes & 1983 & 639 & -- & 1335 & 1.98 & 0.912 & 0.174 & 556 \\
\hline 05532000 & 1 & Yes & Yes & 1984 & 464 & -- & 969 & 1.5 & 0.912 & 0.457 & 407 \\
\hline 05532000 & 1 & Yes & Yes & 1985 & 556 & -- & 1162 & 1.35 & 0.912 & 0.26 & 485 \\
\hline 05532000 & 1 & Yes & Yes & 1986 & 391 & -- & 817 & 0.866 & 0.913 & 0.621 & 343 \\
\hline 05532000 & 1 & Yes & Yes & 1987 & 1120 & -- & 2340 & 3.33 & 0.913 & 0.0218 & 968 \\
\hline 05532000 & 1 & Yes & Yes & 1988 & 370 & -- & 773 & 0.59 & 0.913 & 0.68 & 325 \\
\hline 05532000 & 1 & Yes & Yes & 1989 & 541 & -- & 1130 & 2.02 & 0.913 & 0.285 & 471 \\
\hline 05532000 & 1 & Yes & Yes & 1990 & 895 & -- & 1870 & 3.29 & 0.914 & 0.0477 & 775 \\
\hline 05532000 & 1 & Yes & Yes & 1991 & 675 & -- & 1410 & 2.87 & 0.914 & 0.148 & 586 \\
\hline 05532000 & 0 & Yes & No & 1992 & 351 & C & 801 & 1.59 & 0.914 & 0.644 & 336 \\
\hline
\end{tabular}




\begin{tabular}{|c|c|c|c|c|c|c|c|c|c|c|c|}
\hline $\begin{array}{c}\text { U.S. } \\
\text { Geological } \\
\text { Survey } \\
\text { streamgage } \\
\text { number } \\
\end{array}$ & $\begin{array}{c}\text { Segment } \\
\text { number }\end{array}$ & $\begin{array}{l}\text { Streamgage used in } \\
\text { regression analyses } \\
\text { (non-redundant) }\end{array}$ & $\begin{array}{c}\text { Discharge value } \\
\text { used in } \\
\text { adjustment } \\
\text { regression }\end{array}$ & $\begin{array}{c}\text { Water } \\
\text { year }\end{array}$ & $\begin{array}{c}\text { Observed annual } \\
\text { maximum peak } \\
\text { discharge }\left(\mathrm{ft}^{3} / \mathrm{s}\right)\end{array}$ & $\begin{array}{c}\text { NWIS peak } \\
\text { code }\end{array}$ & $\begin{array}{l}\text { Observed annual } \\
\text { maximum peak } \\
\text { discharge with } \\
\text { segment intercept } \\
\text { value subtracted } \\
\left(\mathrm{ft}^{3} / \mathrm{s}\right)\end{array}$ & $\begin{array}{l}\text { Observed } \\
\text { precipitation } \\
\text { (inches) }\end{array}$ & Urban fraction & $\begin{array}{l}\text { Exceedance } \\
\text { probability }\end{array}$ & $\begin{array}{c}\text { Urban-adjusted } \\
\text { annual maximum } \\
\text { peak discharge } \\
\left(\mathrm{ft}^{3} / \mathrm{s}\right)\end{array}$ \\
\hline 05532000 & 2 & Yes & Yes & 1993 & 517 & C & 1288 & 1.82 & 0.914 & 0.192 & 536 \\
\hline 05532000 & 2 & Yes & Yes & 1994 & 425 & $C$ & 1059 & 2.01 & 0.915 & 0.363 & 442 \\
\hline 05532000 & 2 & Yes & Yes & 1995 & 273 & $\mathrm{C}, \mathrm{E}$ & 680 & 2.44 & 0.915 & 0.782 & 287 \\
\hline 05532000 & 2 & Yes & Yes & 1996 & 400 & $C$ & 997 & 2.14 & 0.915 & 0.432 & 416 \\
\hline 05532000 & 2 & Yes & Yes & 1997 & 753 & C & 1877 & 3.13 & 0.916 & 0.0475 & 777 \\
\hline 05532000 & 2 & Yes & Yes & 1998 & 466 & C & 1161 & 1.05 & 0.916 & 0.263 & 483 \\
\hline 05532000 & 2 & Yes & Yes & 1999 & 385 & C & 959 & 1.43 & 0.916 & 0.472 & 401 \\
\hline 05532000 & 2 & Yes & Yes & 2000 & 477 & C & 1189 & 2.25 & 0.917 & 0.244 & 494 \\
\hline 05532000 & 2 & Yes & Yes & 2001 & 379 & C & 945 & 1.09 & 0.92 & 0.492 & 393 \\
\hline 05532000 & 2 & Yes & Yes & 2002 & 652 & C & 1625 & 3.13 & 0.924 & 0.0878 & 669 \\
\hline 05532000 & 2 & Yes & Yes & 2003 & 567 & C & 1413 & 2.34 & 0.927 & 0.153 & 580 \\
\hline 05532000 & 2 & Yes & Yes & 2004 & 516 & C & 1286 & 1.67 & 0.931 & 0.2 & 526 \\
\hline 05532000 & 2 & Yes & Yes & 2005 & 377 & C & 940 & 1.44 & 0.934 & 0.512 & 385 \\
\hline 05532000 & 2 & Yes & Yes & 2006 & 343 & C & 855 & 0.864 & 0.938 & 0.603 & 349 \\
\hline 05532000 & 2 & Yes & Yes & 2007 & 777 & C & 1936 & 2.03 & 0.942 & 0.0461 & 784 \\
\hline 05532000 & 2 & Yes & Yes & 2008 & 808 & C & 2014 & 5.3 & 0.945 & 0.0414 & 813 \\
\hline 05532000 & 2 & Yes & Yes & 2009 & 727 & C & 1812 & 2.08 & 0.949 & 0.0613 & 729 \\
\hline 05532500 & 1 & No & No & 1940 & 2660 & -- & 330 & 0.401 & 0.145 & 0.623 & 4220 \\
\hline 05532500 & 1 & No & No & 1941 & 3580 & -- & 444 & 0.191 & 0.153 & 0.411 & 5360 \\
\hline 05532500 & 1 & No & No & 1942 & 3320 & -- & 412 & 0.729 & 0.16 & 0.473 & 5010 \\
\hline 05532500 & 1 & No & No & 1943 & 3820 & -- & 474 & 0.981 & 0.167 & 0.377 & 5570 \\
\hline 05532500 & 1 & No & No & 1944 & 4480 & -- & 556 & 1.1 & 0.175 & 0.275 & 6250 \\
\hline 05532500 & 1 & No & No & 1945 & 3670 & -- & 456 & 1.36 & 0.182 & 0.416 & 5330 \\
\hline 05532500 & 1 & No & No & 1946 & 3370 & -- & 418 & 0.545 & 0.19 & 0.486 & 4930 \\
\hline 05532500 & 1 & No & No & 1947 & 3780 & -- & 469 & 1.84 & 0.197 & 0.406 & 5390 \\
\hline 05532500 & 1 & No & No & 1948 & 6510 & -- & 808 & 1.84 & 0.204 & 0.105 & 8500 \\
\hline 05532500 & 1 & No & No & 1949 & 1890 & -- & 235 & 1.39 & 0.212 & 0.832 & 3070 \\
\hline 05532500 & 1 & No & No & 1950 & 6340 & D & 787 & 1.32 & 0.219 & 0.122 & 8190 \\
\hline 05532500 & 1 & No & No & 1951 & 3100 & -- & 385 & 0.191 & 0.232 & 0.589 & 4400 \\
\hline 05532500 & 1 & No & No & 1952 & 3620 & -- & 449 & 0.797 & 0.245 & 0.479 & 4970 \\
\hline 05532500 & 1 & No & No & 1953 & 1470 & -- & 183 & 0.624 & 0.257 & 0.918 & 2450 \\
\hline 05532500 & 1 & No & No & 1954 & 3980 & -- & 494 & 1.43 & 0.27 & 0.428 & 5270 \\
\hline 05532500 & 1 & No & No & 1955 & 6340 & -- & 787 & 2.89 & 0.283 & 0.147 & 7750 \\
\hline 05532500 & 1 & No & No & 1956 & 2460 & -- & 305 & 1.23 & 0.296 & 0.771 & 3440 \\
\hline 05532500 & 1 & No & No & 1957 & 5950 & -- & 739 & 1.85 & 0.308 & 0.184 & 7130 \\
\hline
\end{tabular}




\begin{tabular}{|c|c|c|c|c|c|c|c|c|c|c|c|}
\hline $\begin{array}{c}\text { U.S. } \\
\text { Geological } \\
\text { Survey } \\
\text { streamgage } \\
\text { number }\end{array}$ & $\begin{array}{l}\text { Segment } \\
\text { number }\end{array}$ & $\begin{array}{l}\text { Streamgage used in } \\
\text { regression analyses } \\
\text { (non-redundant) }\end{array}$ & $\begin{array}{l}\text { Discharge value } \\
\text { used in } \\
\text { adjustment } \\
\text { regression }\end{array}$ & $\begin{array}{c}\text { Water } \\
\text { year }\end{array}$ & $\begin{array}{l}\text { Observed annual } \\
\text { maximum peak } \\
\text { discharge }\left(\mathrm{ft}^{3} / \mathrm{s}\right)\end{array}$ & $\begin{array}{l}\text { NWIS peak } \\
\text { code }\end{array}$ & $\begin{array}{l}\text { Observed annual } \\
\text { maximum peak } \\
\text { discharge with } \\
\text { segment intercept } \\
\text { value subtracted } \\
\left(\mathrm{ft}^{3} / \mathrm{s}\right)\end{array}$ & $\begin{array}{l}\text { Observed } \\
\text { precipitation } \\
\text { (inches) }\end{array}$ & Urban fraction & $\begin{array}{l}\text { Exceedance } \\
\text { probability }\end{array}$ & $\begin{array}{c}\text { Urban-adjusted } \\
\text { annual maximum } \\
\text { peak discharge } \\
\left(\mathrm{ft}^{3} / \mathrm{s}\right)\end{array}$ \\
\hline 05532500 & 1 & No & No & 1958 & 2100 & -- & 261 & 1.24 & 0.321 & 0.849 & 2950 \\
\hline 05532500 & 1 & No & No & 1959 & 1680 & -- & 209 & 1.18 & 0.334 & 0.916 & 2460 \\
\hline 05532500 & 1 & No & No & 1960 & 5500 & -- & 683 & 0.0655 & 0.347 & 0.249 & 6440 \\
\hline 05532500 & 1 & No & No & 1961 & 4690 & -- & 582 & 1.95 & 0.355 & 0.373 & 5600 \\
\hline 05532500 & 1 & No & No & 1962 & 4230 & -- & 525 & 0.251 & 0.364 & 0.461 & 5080 \\
\hline 05532500 & 1 & No & No & 1963 & 2410 & -- & 299 & 1.29 & 0.373 & 0.826 & 3110 \\
\hline 05532500 & 1 & No & No & 1964 & 2500 & -- & 310 & 0.761 & 0.382 & 0.816 & 3170 \\
\hline 05532500 & 1 & No & No & 1965 & 3200 & -- & 397 & 0.311 & 0.391 & 0.695 & 3860 \\
\hline 05532500 & 1 & No & No & 1966 & 5210 & -- & 647 & 1.22 & 0.4 & 0.326 & 5880 \\
\hline 05532500 & 1 & No & No & 1967 & 4600 & -- & 571 & 1.48 & 0.409 & 0.433 & 5240 \\
\hline 05532500 & 1 & No & No & 1968 & 3600 & -- & 447 & 1.12 & 0.417 & 0.636 & 4150 \\
\hline 05532500 & 1 & No & No & 1969 & 4380 & -- & 544 & 0.871 & 0.426 & 0.489 & 4920 \\
\hline 05532500 & 1 & No & No & 1970 & 4890 & -- & 607 & 1.48 & 0.435 & 0.407 & 5390 \\
\hline 05532500 & 1 & No & No & 1971 & 3380 & -- & 420 & 0.436 & 0.443 & 0.702 & 3830 \\
\hline 05532500 & 1 & No & No & 1972 & 5460 & -- & 678 & 1.13 & 0.45 & 0.329 & 5860 \\
\hline 05532500 & 1 & No & No & 1973 & 4330 & -- & 538 & 1.35 & 0.457 & 0.527 & 4710 \\
\hline 05532500 & 1 & No & No & 1974 & 4290 & -- & 533 & 0.587 & 0.464 & 0.542 & 4640 \\
\hline 05532500 & 1 & No & No & 1975 & 4630 & -- & 575 & 0.794 & 0.472 & 0.485 & 4940 \\
\hline 05532500 & 1 & No & No & 1976 & 4830 & -- & 600 & 1.13 & 0.479 & 0.457 & 5100 \\
\hline 05532500 & 0 & No & No & 1977 & 2170 & -- & 283 & 1.11 & 0.486 & 0.902 & 2590 \\
\hline 05532500 & 2 & No & No & 1978 & 3380 & -- & 463 & 1.02 & 0.494 & 0.676 & 3950 \\
\hline 05532500 & 2 & No & No & 1979 & 5870 & -- & 805 & 0.776 & 0.501 & 0.239 & 6530 \\
\hline 05532500 & 2 & No & No & 1980 & 2960 & -- & 406 & 0.676 & 0.508 & 0.769 & 3450 \\
\hline 05532500 & 2 & No & No & 1981 & 3160 & -- & 433 & 1.65 & 0.512 & 0.736 & 3640 \\
\hline 05532500 & 2 & No & No & 1982 & 3840 & -- & 526 & 1.86 & 0.517 & 0.602 & 4330 \\
\hline 05532500 & 2 & No & No & 1983 & 6130 & -- & 840 & 1.57 & 0.521 & 0.223 & 6680 \\
\hline 05532500 & 2 & No & No & 1984 & 3250 & -- & 445 & 0.489 & 0.525 & 0.729 & 3680 \\
\hline 05532500 & 2 & No & No & 1985 & 6360 & -- & 872 & 1.18 & 0.529 & 0.205 & 6870 \\
\hline 05532500 & 2 & No & No & 1986 & 4180 & -- & 573 & 0.702 & 0.533 & 0.546 & 4610 \\
\hline 05532500 & 2 & No & No & 1987 & 9770 & -- & 1339 & 2.62 & 0.537 & 0.0519 & 10400 \\
\hline 05532500 & 2 & No & No & 1988 & 3250 & -- & 445 & 0.704 & 0.541 & 0.742 & 3610 \\
\hline 05532500 & 2 & No & No & 1989 & 3720 & -- & 510 & 1.27 & 0.545 & 0.652 & 4070 \\
\hline 05532500 & 2 & No & No & 1990 & 5950 & -- & 816 & 1.71 & 0.55 & 0.262 & 6330 \\
\hline 05532500 & 2 & No & No & 1991 & 5270 & -- & 722 & 1.7 & 0.553 & 0.367 & 5630 \\
\hline 05532500 & 2 & No & No & 1992 & 2760 & -- & 378 & 0.792 & 0.557 & 0.835 & 3050 \\
\hline
\end{tabular}




\begin{tabular}{|c|c|c|c|c|c|c|c|c|c|c|c|}
\hline $\begin{array}{c}\text { U.S. } \\
\text { Geological } \\
\text { Survey } \\
\text { streamgage } \\
\text { number }\end{array}$ & $\begin{array}{l}\text { Segment } \\
\text { number }\end{array}$ & $\begin{array}{l}\text { Streamgage used in } \\
\text { regression analyses } \\
\text { (non-redundant) }\end{array}$ & $\begin{array}{l}\text { Discharge value } \\
\text { used in } \\
\text { adjustment } \\
\text { regression }\end{array}$ & $\begin{array}{l}\text { Water } \\
\text { year }\end{array}$ & $\begin{array}{l}\text { Observed annual } \\
\text { maximum peak } \\
\text { discharge }\left(\mathrm{ft}^{3} / \mathrm{s}\right)\end{array}$ & $\begin{array}{l}\text { NWIS peak } \\
\text { code }\end{array}$ & $\begin{array}{l}\text { Observed annual } \\
\text { maximum peak } \\
\text { discharge with } \\
\text { segment intercept } \\
\text { value subtracted } \\
\left(\mathrm{ft}^{3} / \mathrm{s}\right)\end{array}$ & $\begin{array}{l}\text { Observed } \\
\text { precipitation } \\
\text { (inches) }\end{array}$ & Urban fraction & $\begin{array}{l}\text { Exceedance } \\
\text { probability }\end{array}$ & $\begin{array}{c}\text { Urban-adjusted } \\
\text { annual maximum } \\
\text { peak discharge } \\
\left(\mathrm{ft}^{3} / \mathrm{s}\right)\end{array}$ \\
\hline 05532500 & 2 & No & No & 1993 & 4690 & C & 643 & 0.975 & 0.56 & 0.475 & 5000 \\
\hline 05532500 & 2 & No & No & 1994 & 4880 & $\mathrm{C}$ & 669 & 0.694 & 0.564 & 0.444 & 5180 \\
\hline 05532500 & 2 & No & No & 1995 & 4720 & C & 647 & 1.12 & 0.567 & 0.476 & 5000 \\
\hline 05532500 & 2 & No & No & 1996 & 5270 & C & 722 & 0.799 & 0.571 & 0.383 & 5530 \\
\hline 05532500 & 2 & No & No & 1997 & 6990 & C & 958 & 2.26 & 0.574 & 0.18 & 7230 \\
\hline 05532500 & 0 & No & No & 1998 & 4450 & C & 624 & 1.37 & 0.578 & 0.518 & 4770 \\
\hline 05532500 & 3 & No & No & 1999 & 5680 & C & 814 & 1.12 & 0.581 & 0.287 & 6130 \\
\hline 05532500 & 3 & No & No & 2000 & 4660 & C & 667 & 1.68 & 0.585 & 0.466 & 5050 \\
\hline 05532500 & 3 & No & No & 2001 & 4040 & C & 579 & 1.76 & 0.592 & 0.595 & 4360 \\
\hline 05532500 & 3 & No & No & 2002 & 6050 & C & 867 & 1.51 & 0.6 & 0.253 & 6400 \\
\hline 05532500 & 3 & No & No & 2003 & 3840 & C & 550 & 1.03 & 0.607 & 0.65 & 4080 \\
\hline 05532500 & 3 & No & No & 2004 & 4780 & C & 685 & 0.74 & 0.614 & 0.472 & 5020 \\
\hline 05532500 & 3 & No & No & 2005 & 3930 & C & 563 & 0.621 & 0.622 & 0.646 & 4110 \\
\hline 05532500 & 3 & No & No & 2006 & 2550 & C & 365 & 0.891 & 0.629 & 0.892 & 2670 \\
\hline 05532500 & 3 & No & No & 2007 & 5790 & C & 829 & 1.32 & 0.636 & 0.318 & 5920 \\
\hline 05532500 & 3 & No & No & 2008 & 9560 & C & 1369 & 3.19 & 0.644 & 0.0663 & 9680 \\
\hline 05532500 & 3 & No & No & 2009 & 6110 & C & 875 & 0.875 & 0.651 & 0.284 & 6150 \\
\hline 05533000 & 1 & Yes & Yes & 1951 & 540 & -- & 631 & 1.45 & 0.495 & 0.429 & 870 \\
\hline 05533000 & 1 & Yes & Yes & 1952 & 398 & -- & 465 & 0.978 & 0.52 & 0.697 & 663 \\
\hline 05533000 & 1 & Yes & Yes & 1953 & 306 & -- & 358 & 1.27 & 0.545 & 0.851 & 534 \\
\hline 05533000 & 1 & Yes & Yes & 1954 & 770 & -- & 900 & 1.68 & 0.57 & 0.209 & 1080 \\
\hline 05533000 & 1 & Yes & Yes & 1955 & 1300 & -- & 1520 & 3.43 & 0.595 & 0.041 & 1720 \\
\hline 05533000 & 1 & Yes & Yes & 1956 & 285 & -- & 333 & 0.803 & 0.62 & 0.915 & 472 \\
\hline 05533000 & 1 & Yes & Yes & 1957 & 1350 & -- & 1578 & 2.21 & 0.645 & 0.0413 & 1710 \\
\hline 05533000 & 1 & Yes & Yes & 1958 & 388 & $E$ & 454 & 1.51 & 0.67 & 0.824 & 559 \\
\hline 05533000 & 1 & Yes & Yes & 1959 & 1550 & -- & 1812 & 1.24 & 0.695 & 0.0295 & 1870 \\
\hline 05533000 & 1 & Yes & Yes & 1960 & 800 & -- & 935 & 1.93 & 0.72 & 0.285 & 987 \\
\hline 05533000 & 1 & Yes & Yes & 1961 & 2680 & -- & 3133 & 1.77 & 0.73 & 0.00569 & 3090 \\
\hline 05533000 & 1 & Yes & Yes & 1962 & 344 & $E$ & 402 & 0.286 & 0.74 & 0.918 & 470 \\
\hline 05533000 & 1 & Yes & Yes & 1963 & 367 & -- & 429 & 1.17 & 0.749 & 0.902 & 488 \\
\hline 05533000 & 1 & Yes & Yes & 1964 & 375 & -- & 438 & 0.777 & 0.759 & 0.9 & 491 \\
\hline 05533000 & 1 & Yes & Yes & 1965 & 576 & -- & 673 & 0.527 & 0.769 & 0.644 & 699 \\
\hline 05533000 & 1 & Yes & Yes & 1966 & 975 & -- & 1140 & 1.41 & 0.779 & 0.189 & 1120 \\
\hline 05533000 & 1 & Yes & Yes & 1967 & 816 & D & 954 & 1.16 & 0.789 & 0.333 & 945 \\
\hline 05533000 & 1 & Yes & Yes & 1968 & 648 & -- & 758 & 1.4 & 0.799 & 0.568 & 755 \\
\hline
\end{tabular}




\begin{tabular}{|c|c|c|c|c|c|c|c|c|c|c|c|}
\hline $\begin{array}{c}\text { U.S. } \\
\text { Geological } \\
\text { Survey } \\
\text { streamgage } \\
\text { number }\end{array}$ & $\begin{array}{l}\text { Segment } \\
\text { number }\end{array}$ & $\begin{array}{l}\text { Streamgage used in } \\
\text { regression analyses } \\
\text { (non-redundant) }\end{array}$ & $\begin{array}{c}\text { Discharge value } \\
\text { used in } \\
\text { adjustment } \\
\text { regression }\end{array}$ & $\begin{array}{c}\text { Water } \\
\text { year }\end{array}$ & $\begin{array}{l}\text { Observed annual } \\
\text { maximum peak } \\
\text { discharge }\left(\mathrm{ft}^{3} / \mathrm{s}\right)\end{array}$ & $\begin{array}{c}\text { NWIS peak } \\
\text { code }\end{array}$ & $\begin{array}{c}\text { Observed annual } \\
\text { maximum peak } \\
\text { discharge with } \\
\text { segment intercept } \\
\text { value subtracted } \\
\left(\mathrm{ft}^{3} / \mathrm{s}\right)\end{array}$ & $\begin{array}{c}\text { Observed } \\
\text { precipitation } \\
\text { (inches) }\end{array}$ & Urban fraction & $\begin{array}{l}\text { Exceedance } \\
\text { probability }\end{array}$ & $\begin{array}{c}\text { Urban-adjusted } \\
\text { annual maximum } \\
\text { peak discharge } \\
\left(\mathrm{ft}^{3} / \mathrm{s}\right)\end{array}$ \\
\hline 05533000 & 1 & Yes & Yes & 1969 & 803 & \begin{tabular}{l|}
- \\
-
\end{tabular} & 939 & 0.883 & 0.809 & 0.374 & 915 \\
\hline 05533000 & 1 & Yes & Yes & 1970 & 400 & \begin{tabular}{l|}
-- \\
\end{tabular} & 468 & 0.955 & 0.819 & 0.91 & 479 \\
\hline 05533000 & 1 & Yes & Yes & 1971 & 188 & $E$ & 220 & 0.302 & 0.825 & 1 & 239 \\
\hline 05533000 & 1 & Yes & Yes & 1972 & 830 & -- & 970 & 1.29 & 0.831 & 0.363 & 923 \\
\hline 05533000 & 1 & Yes & Yes & 1973 & 786 & -- & 919 & 0.851 & 0.837 & 0.428 & 871 \\
\hline 05533000 & 1 & Yes & Yes & 1974 & 696 & \begin{tabular}{l|}
- \\
\end{tabular} & 814 & 0.56 & 0.843 & 0.549 & 770 \\
\hline 05533000 & 1 & Yes & Yes & 1975 & 1630 & -- & 1906 & 1.15 & 0.849 & 0.0385 & 1740 \\
\hline 05533000 & 1 & Yes & Yes & 1976 & 2230 & $\mathrm{D}$ & 2607 & 0.558 & 0.856 & 0.015 & 2360 \\
\hline 05533000 & 1 & Yes & Yes & 1977 & 560 & -- & 655 & 1.23 & 0.862 & 0.759 & 614 \\
\hline 05533000 & 1 & Yes & Yes & 1978 & 575 & -- & 672 & 0.774 & 0.868 & 0.747 & 624 \\
\hline 05533000 & 1 & Yes & Yes & 1979 & 967 & -- & 1130 & 0.968 & 0.874 & 0.251 & 1020 \\
\hline 05533000 & 1 & Yes & Yes & 1980 & 439 & -- & 513 & 0.594 & 0.88 & 0.912 & 477 \\
\hline 05533000 & 1 & Yes & Yes & 1981 & 823 & -- & 962 & 1.93 & 0.882 & 0.431 & 869 \\
\hline 05533000 & 1 & Yes & Yes & 1982 & 826 & -- & 966 & 0.316 & 0.884 & 0.43 & 870 \\
\hline 05533000 & 1 & Yes & Yes & 1983 & 1960 & -- & 2291 & 1.51 & 0.886 & 0.0218 & 2030 \\
\hline 05533000 & 1 & Yes & Yes & 1984 & 670 & $\mathrm{D}$ & 783 & 0.593 & 0.889 & 0.635 & 705 \\
\hline 05533000 & 1 & Yes & Yes & 1985 & 1390 & -- & 1625 & 1.24 & 0.891 & 0.0771 & 1440 \\
\hline 05533000 & 1 & Yes & Yes & 1986 & 636 & -- & 743 & 0.686 & 0.893 & 0.695 & 667 \\
\hline 05533000 & 1 & Yes & Yes & 1987 & 939 & \begin{tabular}{l|l}
-- & \\
\end{tabular} & 1098 & 2.06 & 0.895 & 0.295 & 974 \\
\hline 05533000 & 1 & Yes & Yes & 1988 & 759 & $\mathrm{E}$ & 887 & 0.783 & 0.897 & 0.526 & 789 \\
\hline 05533000 & 1 & Yes & Yes & 1989 & 708 & $E$ & 828 & 1.97 & 0.899 & 0.592 & 735 \\
\hline 05533000 & 1 & Yes & Yes & 1990 & 1910 & -- & 2233 & 2.71 & 0.902 & 0.0254 & 1960 \\
\hline 05533000 & 1 & Yes & Yes & 1991 & 1470 & -- & 1718 & 2.64 & 0.903 & 0.0649 & 1510 \\
\hline 05533000 & 1 & Yes & Yes & 1992 & 1100 & $C, E$ & 1286 & 2.5 & 0.904 & 0.188 & 1130 \\
\hline 05533000 & 1 & Yes & Yes & 1993 & 791 & C & 925 & 2.61 & 0.905 & 0.496 & 815 \\
\hline 05533000 & 1 & Yes & Yes & 1994 & 569 & $c$ & 665 & 1.19 & 0.906 & 0.79 & 589 \\
\hline 05533000 & 1 & Yes & Yes & 1995 & 567 & $\mathrm{C}, \mathrm{E}$ & 663 & 0.967 & 0.907 & 0.793 & 586 \\
\hline 05533000 & 1 & Yes & Yes & 1996 & 2300 & C & 2689 & 3.61 & 0.909 & 0.0154 & 2340 \\
\hline 05533000 & 1 & Yes & Yes & 1997 & 1540 & C & 1800 & 2.67 & 0.91 & 0.0544 & 1570 \\
\hline 05533000 & 1 & Yes & Yes & 1998 & 1160 & 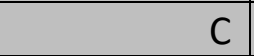 & 1356 & 2.31 & 0.911 & 0.166 & 1180 \\
\hline 05533000 & 1 & Yes & Yes & 1999 & 860 & C & 1005 & 1.17 & 0.912 & 0.42 & 879 \\
\hline 05533000 & 1 & Yes & Yes & 2000 & 633 & C & 740 & 1.77 & 0.913 & 0.719 & 648 \\
\hline 05533000 & 1 & Yes & Yes & 2001 & 745 & $C$ & 871 & 2.23 & 0.915 & 0.562 & 759 \\
\hline 05533000 & 1 & Yes & Yes & 2002 & 962 & $C$ & 1125 & 1.98 & 0.917 & 0.293 & 976 \\
\hline 05533000 & 1 & Yes & Yes & 2003 & 600 & C & 701 & 1.14 & 0.919 & 0.763 & 610 \\
\hline
\end{tabular}




\begin{tabular}{|c|c|c|c|c|c|c|c|c|c|c|c|}
\hline $\begin{array}{c}\text { U.S. } \\
\text { Geological } \\
\text { Survey } \\
\text { streamgage } \\
\text { number } \\
\end{array}$ & $\begin{array}{c}\text { Segment } \\
\text { number }\end{array}$ & $\begin{array}{l}\text { Streamgage used in } \\
\text { regression analyses } \\
\text { (non-redundant) }\end{array}$ & $\begin{array}{c}\text { Discharge value } \\
\text { used in } \\
\text { adjustment } \\
\text { regression } \\
\end{array}$ & $\begin{array}{c}\text { Water } \\
\text { year }\end{array}$ & $\begin{array}{c}\text { Observed annual } \\
\text { maximum peak } \\
\text { discharge }\left(\mathrm{ft}^{3} / \mathrm{s}\right)\end{array}$ & $\begin{array}{c}\text { NWIS peak } \\
\text { code }\end{array}$ & $\begin{array}{c}\text { Observed annual } \\
\text { maximum peak } \\
\text { discharge with } \\
\text { segment intercept } \\
\text { value subtracted } \\
\left(\mathrm{ft}^{3} / \mathrm{s}\right)\end{array}$ & $\begin{array}{c}\text { Observed } \\
\text { precipitation } \\
\text { (inches) }\end{array}$ & Urban fraction & $\begin{array}{l}\text { Exceedance } \\
\text { probability }\end{array}$ & $\begin{array}{c}\text { Urban-adjusted } \\
\text { annual maximum } \\
\text { peak discharge } \\
\left(\mathrm{ft}^{3} / \mathrm{s}\right) \\
\end{array}$ \\
\hline 05533000 & 1 & Yes & Yes & 2004 & 790 & C & 924 & 2.35 & 0.921 & 0.514 & 800 \\
\hline 05533000 & 1 & Yes & Yes & 2005 & 692 & $\mathrm{C}, \mathrm{E}$ & 809 & 1.1 & 0.922 & 0.644 & 700 \\
\hline 05533000 & 1 & Yes & Yes & 2006 & 559 & C & 653 & 1.67 & 0.924 & 0.817 & 565 \\
\hline 05533000 & 1 & Yes & Yes & 2007 & 1490 & C & 1742 & 1.85 & 0.926 & 0.0666 & 1500 \\
\hline 05533000 & 1 & Yes & Yes & 2008 & 1240 & C & 1450 & 3.06 & 0.928 & 0.141 & 1240 \\
\hline 05533000 & 1 & Yes & Yes & 2009 & 1330 & C & 1555 & 1.28 & 0.93 & 0.108 & 1330 \\
\hline 05533200 & 1 & Yes & Yes & 1961 & 200 & -- & 855 & 2.2 & 0.6 & 0.263 & 292 \\
\hline 05533200 & 1 & Yes & Yes & 1962 & 178 & -- & 761 & 0.42 & 0.626 & 0.389 & 262 \\
\hline 05533200 & 1 & Yes & Yes & 1963 & 49 & -- & 210 & 1.4 & 0.653 & 0.981 & 97.3 \\
\hline 05533200 & 1 & Yes & No & 1964 & 48 & $4, B$ & 205 & 0.42 & 0.679 & 0.984 & 91.3 \\
\hline 05533200 & 1 & Yes & Yes & 1965 & 219 & -- & 936 & 0.98 & 0.706 & 0.272 & 289 \\
\hline 05533200 & 1 & Yes & Yes & 1966 & 254 & -- & 1086 & 4.05 & 0.732 & 0.191 & 323 \\
\hline 05533200 & 1 & Yes & Yes & 1967 & 225 & -- & 962 & 1.18 & 0.759 & 0.293 & 283 \\
\hline 05533200 & 1 & Yes & Yes & 1968 & 257 & -- & 1099 & 3.07 & 0.785 & 0.213 & 311 \\
\hline 05533200 & 1 & Yes & Yes & 1969 & 202 & -- & 864 & 1.74 & 0.812 & 0.46 & 246 \\
\hline 05533200 & 1 & Yes & Yes & 1970 & 166 & -- & 710 & 1.23 & 0.838 & 0.673 & 199 \\
\hline 05533200 & 1 & Yes & Yes & 1971 & 168 & -- & 718 & 0.56 & 0.85 & 0.676 & 199 \\
\hline 05533200 & 1 & Yes & Yes & 1972 & 256 & -- & 1095 & 1.45 & 0.863 & 0.269 & 290 \\
\hline 05533200 & 1 & Yes & Yes & 1973 & 300 & -- & 1283 & 0.6 & 0.875 & 0.176 & 333 \\
\hline 05533200 & 1 & Yes & Yes & 1974 & 282 & -- & 1206 & 1.61 & 0.887 & 0.216 & 310 \\
\hline 05533200 & 1 & Yes & Yes & 1975 & 286 & -- & 1223 & 2.3 & 0.899 & 0.214 & 311 \\
\hline 05533200 & 1 & Yes & Yes & 1976 & 315 & -- & 1347 & 0.96 & 0.912 & 0.169 & 338 \\
\hline 05533200 & 1 & Yes & Yes & 1977 & 289 & -- & 1236 & 1.36 & 0.924 & 0.223 & 307 \\
\hline 05533200 & 1 & Yes & Yes & 1979 & 276 & -- & 1180 & 1.14 & 0.948 & 0.277 & 287 \\
\hline 05533300 & 1 & Yes & Yes & 1962 & 60 & -- & 619 & 0.42 & 0.306 & 0.289 & 116 \\
\hline 05533300 & 1 & Yes & Yes & 1963 & 20 & 2 & 206 & 1.4 & 0.344 & 0.922 & 55.8 \\
\hline 05533300 & 1 & Yes & Yes & 1964 & 35 & -- & 361 & 1.51 & 0.382 & 0.745 & 74.4 \\
\hline 05533300 & 1 & Yes & Yes & 1965 & 55 & -- & 567 & 0.98 & 0.421 & 0.449 & 100 \\
\hline 05533300 & 1 & Yes & Yes & 1966 & 151 & -- & 1558 & 4.12 & 0.459 & 0.0259 & 225 \\
\hline 05533300 & 1 & Yes & Yes & 1967 & 109 & -- & 1124 & 1.18 & 0.497 & 0.084 & 166 \\
\hline 05533300 & 1 & Yes & Yes & 1968 & 48 & -- & 495 & 3.07 & 0.536 & 0.665 & 80.9 \\
\hline 05533300 & 1 & Yes & Yes & 1969 & 73 & -- & 753 & 1.74 & 0.574 & 0.348 & 110 \\
\hline 05533300 & 1 & Yes & Yes & 1970 & 95 & -- & 980 & 1.3 & 0.612 & 0.186 & 132 \\
\hline 05533300 & 1 & Yes & Yes & 1971 & 70 & -- & 722 & 0.56 & 0.627 & 0.438 & 102 \\
\hline 05533300 & 1 & Yes & Yes & 1972 & 87 & -- & 897 & 3.3 & 0.642 & 0.257 & 119 \\
\hline
\end{tabular}




\begin{tabular}{|c|c|c|c|c|c|c|c|c|c|c|c|}
\hline $\begin{array}{l}\text { U.S. } \\
\text { Geological } \\
\text { Survey } \\
\text { streamgage } \\
\text { number }\end{array}$ & $\begin{array}{l}\text { Segment } \\
\text { number }\end{array}$ & $\begin{array}{l}\text { Streamgage used in } \\
\text { regression analyses } \\
\text { (non-redundant) }\end{array}$ & $\begin{array}{c}\text { Discharge value } \\
\text { used in } \\
\text { adjustment } \\
\text { regression }\end{array}$ & $\begin{array}{l}\text { Water } \\
\text { year }\end{array}$ & $\begin{array}{l}\text { Observed annual } \\
\text { maximum peak } \\
\text { discharge }\left(\mathrm{ft}^{3} / \mathrm{s}\right)\end{array}$ & $\begin{array}{l}\text { NWIS peak } \\
\text { code }\end{array}$ & $\begin{array}{l}\text { Observed annual } \\
\text { maximum peak } \\
\text { discharge with } \\
\text { segment intercept } \\
\text { value subtracted } \\
\left(\mathrm{ft}^{3} / \mathrm{s}\right)\end{array}$ & $\begin{array}{l}\text { Observed } \\
\text { precipitation } \\
\text { (inches) }\end{array}$ & Urban fraction & $\begin{array}{l}\text { Exceedance } \\
\text { probability }\end{array}$ & $\begin{array}{c}\text { Urban-adjusted } \\
\text { annual maximum } \\
\text { peak discharge } \\
\left(\mathrm{ft}^{3} / \mathrm{s}\right)\end{array}$ \\
\hline 05533300 & 1 & Yes & Yes & 1973 & 94 & -- & 970 & 2.17 & 0.656 & 0.214 & 126 \\
\hline 05533300 & 1 & Yes & Yes & 1974 & 102 & -- & 1052 & 1.61 & 0.671 & 0.179 & 134 \\
\hline 05533300 & 1 & Yes & Yes & 1975 & 146 & -- & 1506 & 2.3 & 0.686 & 0.0529 & 186 \\
\hline 05533300 & 1 & Yes & Yes & 1976 & 101 & -- & 1042 & 4.38 & 0.701 & 0.196 & 130 \\
\hline 05533400 & 1 & No & No & 1961 & 924 & -- & 1252 & 2.62 & 0.336 & 0.039 & 1340 \\
\hline 05533400 & 1 & No & No & 1962 & 195 & -- & 264 & 0.414 & 0.359 & 0.864 & 367 \\
\hline 05533400 & 1 & No & No & 1963 & 59 & -- & 80 & 1.38 & 0.382 & 1 & 148 \\
\hline 05533400 & 1 & No & No & 1964 & 156 & 4, B & 211 & 0.214 & 0.405 & 0.933 & 300 \\
\hline 05533400 & 1 & No & No & 1965 & 395 & $4, B$ & 535 & 0.214 & 0.428 & 0.504 & 593 \\
\hline 05533400 & 1 & No & No & 1966 & 984 & -- & 1333 & 3.99 & 0.451 & 0.0428 & 1300 \\
\hline 05533400 & 1 & No & No & 1967 & 725 & -- & 982 & 1.18 & 0.474 & 0.127 & 967 \\
\hline 05533400 & 1 & No & No & 1968 & 472 & -- & 639 & 2.98 & 0.497 & 0.42 & 649 \\
\hline 05533400 & 1 & No & No & 1969 & 541 & -- & 733 & 1.69 & 0.52 & 0.323 & 713 \\
\hline 05533400 & 1 & No & No & 1970 & 279 & -- & 378 & 1.2 & 0.543 & 0.827 & 394 \\
\hline 05533400 & 1 & No & No & 1971 & 385 & 4, B & 522 & 0.214 & 0.555 & 0.643 & 510 \\
\hline 05533400 & 1 & No & No & 1972 & 883 & -- & 1196 & 3.18 & 0.567 & 0.0841 & 1080 \\
\hline 05533400 & 1 & No & No & 1973 & 718 & -- & 973 & 0.793 & 0.58 & 0.176 & 872 \\
\hline 05533400 & 1 & No & No & 1974 & 654 & -- & 886 & 1.57 & 0.592 & 0.233 & 790 \\
\hline 05533400 & 1 & No & No & 1975 & 984 & -- & 1333 & 2.24 & 0.604 & 0.0648 & 1160 \\
\hline 05533400 & 1 & No & No & 1976 & 970 & -- & 1314 & 0.94 & 0.617 & 0.0702 & 1130 \\
\hline 05533400 & 1 & No & No & 1977 & 535 & -- & 725 & 2.42 & 0.629 & 0.437 & 638 \\
\hline 05533400 & 1 & No & No & 1978 & 835 & -- & 1131 & 1.96 & 0.642 & 0.133 & 956 \\
\hline 05533400 & 1 & No & No & 1979 & 916 & -- & 1241 & 1.1 & 0.654 & 0.0972 & 1040 \\
\hline 05533400 & 1 & No & No & 1986 & 494 & -- & 669 & 1.51 & 0.693 & 0.569 & 553 \\
\hline 05533400 & 1 & No & No & 1987 & 560 & -- & 759 & 3.73 & 0.697 & 0.463 & 621 \\
\hline 05533400 & 1 & No & No & 1988 & 396 & -- & 536 & 0.873 & 0.701 & 0.754 & 444 \\
\hline 05533400 & 1 & No & No & 1989 & 912 & -- & 1235 & 2.07 & 0.706 & 0.12 & 985 \\
\hline 05533400 & 1 & No & No & 1990 & 1730 & -- & 2344 & 3.15 & 0.71 & 0.0147 & 1840 \\
\hline 05533400 & 1 & No & No & 1991 & 1260 & -- & 1707 & 3.45 & 0.712 & 0.0384 & 1340 \\
\hline 05533400 & 1 & No & No & 1992 & 241 & $D, E$ & 326 & 0.214 & 0.715 & 0.949 & 276 \\
\hline 05533400 & 1 & No & No & 1993 & 1600 & -- & 2167 & 3.03 & 0.717 & 0.0178 & 1690 \\
\hline 05533400 & 1 & No & No & 1994 & 526 & -- & 713 & 1.6 & 0.719 & 0.541 & 570 \\
\hline 05533400 & 1 & No & No & 1995 & 456 & -- & 618 & 0.999 & 0.721 & 0.669 & 496 \\
\hline 05533400 & 1 & No & No & 1996 & 3070 & C & 4159 & 5.87 & 0.723 & 0 & 3210 \\
\hline 05533400 & 1 & No & No & 1997 & 1360 & C & 1842 & 2.8 & 0.726 & 0.0303 & 1430 \\
\hline
\end{tabular}




\begin{tabular}{|c|c|c|c|c|c|c|c|c|c|c|c|}
\hline $\begin{array}{l}\text { U.S. } \\
\text { Geological } \\
\text { Survey } \\
\text { streamgage } \\
\text { number }\end{array}$ & $\begin{array}{l}\text { Segment } \\
\text { number }\end{array}$ & $\begin{array}{l}\text { Streamgage used in } \\
\text { regression analyses } \\
\text { (non-redundant) }\end{array}$ & $\begin{array}{c}\text { Discharge value } \\
\text { used in } \\
\text { adjustment } \\
\text { regression }\end{array}$ & $\begin{array}{l}\text { Water } \\
\text { year }\end{array}$ & $\begin{array}{c}\text { Observed annual } \\
\text { maximum peak } \\
\text { discharge }\left(\mathrm{ft}^{3} / \mathrm{s}\right)\end{array}$ & $\begin{array}{l}\text { NWIS peak } \\
\text { code }\end{array}$ & $\begin{array}{l}\text { Observed annual } \\
\text { maximum peak } \\
\text { discharge with } \\
\text { segment intercept } \\
\text { value subtracted } \\
\left(\mathrm{ft}^{3} / \mathrm{s}\right)\end{array}$ & $\begin{array}{l}\text { Observed } \\
\text { precipitation } \\
\text { (inches) }\end{array}$ & Urban fraction & $\begin{array}{l}\text { Exceedance } \\
\text { probability }\end{array}$ & $\begin{array}{c}\text { Urban-adjusted } \\
\text { annual maximum } \\
\text { peak discharge } \\
\left(\mathrm{ft}^{3} / \mathrm{s}\right)\end{array}$ \\
\hline 05533400 & 1 & No & No & 1998 & 968 & C & 1311 & 3.94 & 0.728 & 0.102 & 1020 \\
\hline 05533400 & 1 & No & No & 1999 & 576 & C, D & 780 & 1.55 & 0.73 & 0.471 & 616 \\
\hline 05533400 & 1 & No & No & 2000 & 655 & C & 887 & 2.12 & 0.732 & 0.348 & 696 \\
\hline 05533400 & 1 & No & No & 2001 & 479 & $C, E$ & 649 & 0.773 & 0.738 & 0.644 & 510 \\
\hline 05533400 & 1 & No & No & 2002 & 1060 & C & 1436 & 2.71 & 0.744 & 0.0753 & 1100 \\
\hline 05533400 & 1 & No & No & 2003 & 387 & $\mathrm{C}, \mathrm{E}$ & 524 & 0.837 & 0.75 & 0.806 & 409 \\
\hline 05533400 & 1 & No & No & 2004 & 615 & C & 833 & 2.7 & 0.756 & 0.436 & 639 \\
\hline 05533400 & 1 & No & No & 2005 & 522 & C & 707 & 0.846 & 0.761 & 0.59 & 540 \\
\hline 05533400 & 1 & No & No & 2006 & 493 & $C, E$ & 668 & 2.04 & 0.767 & 0.649 & 507 \\
\hline 05533400 & 1 & No & No & 2007 & 821 & C & 1112 & 2.63 & 0.773 & 0.198 & 834 \\
\hline 05533400 & 1 & No & No & 2008 & 832 & C & 1127 & 3.56 & 0.779 & 0.194 & 841 \\
\hline 05533400 & 1 & No & No & 2009 & 1020 & C & 1382 & 2.06 & 0.784 & 0.101 & 1030 \\
\hline 05534300 & 1 & No & No & 1961 & 124 & -- & 266 & 1.14 & 0.0697 & 0.696 & 282 \\
\hline 05534300 & 1 & No & No & 1962 & 192 & -- & 411 & 0.211 & 0.0767 & 0.408 & 391 \\
\hline 05534300 & 1 & No & No & 1963 & 107 & -- & 229 & 1.44 & 0.0838 & 0.775 & 253 \\
\hline 05534300 & 1 & No & No & 1964 & 134 & -- & 287 & 0.833 & 0.0908 & 0.667 & 292 \\
\hline 05534300 & 1 & No & No & 1965 & 167 & -- & 358 & 0.178 & 0.0979 & 0.527 & 343 \\
\hline 05534300 & 1 & No & No & 1966 & 225 & -- & 482 & 0.587 & 0.105 & 0.324 & 428 \\
\hline 05534300 & 1 & No & No & 1967 & 240 & -- & 514 & 0.963 & 0.112 & 0.286 & 445 \\
\hline 05534300 & 1 & No & No & 1968 & 108 & 2 & 231 & 0.386 & 0.119 & 0.79 & 247 \\
\hline 05534300 & 1 & No & No & 1969 & 299 & -- & 640 & 2.81 & 0.126 & 0.172 & 524 \\
\hline 05534300 & 1 & No & No & 1970 & 290 & -- & 621 & 2.28 & 0.133 & 0.188 & 507 \\
\hline 05534300 & 1 & No & No & 1971 & 255 & -- & 546 & 0.311 & 0.143 & 0.267 & 455 \\
\hline 05534300 & 1 & No & No & 1972 & 295 & -- & 632 & 3.95 & 0.152 & 0.188 & 507 \\
\hline 05534300 & 1 & No & No & 1973 & 242 & -- & 518 & 1.59 & 0.162 & 0.313 & 432 \\
\hline 05534300 & 1 & No & No & 1974 & 230 & -- & 492 & 1.45 & 0.172 & 0.354 & 414 \\
\hline 05534300 & 1 & No & No & 1975 & 90 & 2 & 193 & 1.4 & 0.181 & 0.88 & 207 \\
\hline 05534300 & 1 & No & No & 1976 & 196 & -- & 420 & 2.21 & 0.191 & 0.485 & 360 \\
\hline 05534400 & 1 & No & No & 1960 & 321 & -- & 551 & 0.467 & 0.113 & 0.243 & 606 \\
\hline 05534400 & 1 & No & No & 1961 & 175 & -- & 300 & 1.2 & 0.122 & 0.663 & 385 \\
\hline 05534400 & 1 & No & No & 1962 & 250 & -- & 429 & 0.22 & 0.132 & 0.421 & 503 \\
\hline 05534400 & 1 & No & No & 1963 & 117 & -- & 201 & 1.37 & 0.141 & 0.85 & 291 \\
\hline 05534400 & 1 & No & No & 1964 & 174 & -- & 298 & 0.81 & 0.151 & 0.687 & 375 \\
\hline 05534400 & 1 & No & No & 1965 & 202 & -- & 347 & 0.538 & 0.16 & 0.605 & 414 \\
\hline 05534400 & 1 & No & No & 1966 & 269 & -- & 461 & 0.546 & 0.17 & 0.397 & 515 \\
\hline
\end{tabular}




\begin{tabular}{|c|c|c|c|c|c|c|c|c|c|c|c|}
\hline $\begin{array}{c}\text { U.S. } \\
\text { Geological } \\
\text { Survey } \\
\text { streamgage } \\
\text { number }\end{array}$ & $\begin{array}{l}\text { Segment } \\
\text { number }\end{array}$ & $\begin{array}{l}\text { Streamgage used in } \\
\text { regression analyses } \\
\text { (non-redundant) }\end{array}$ & $\begin{array}{l}\text { Discharge value } \\
\text { used in } \\
\text { adjustment } \\
\text { regression }\end{array}$ & $\begin{array}{l}\text { Water } \\
\text { year }\end{array}$ & $\begin{array}{l}\text { Observed annual } \\
\text { maximum peak } \\
\text { discharge }\left(\mathrm{ft}^{3} / \mathrm{s}\right)\end{array}$ & $\begin{array}{l}\text { NWIS peak } \\
\text { code }\end{array}$ & $\begin{array}{l}\text { Observed annual } \\
\text { maximum peak } \\
\text { discharge with } \\
\text { segment intercept } \\
\text { value subtracted } \\
\left(\mathrm{ft}^{3} / \mathrm{s}\right)\end{array}$ & $\begin{array}{l}\text { Observed } \\
\text { precipitation } \\
\text { (inches) }\end{array}$ & Urban fraction & $\begin{array}{l}\text { Exceedance } \\
\text { probability }\end{array}$ & $\begin{array}{c}\text { Urban-adjusted } \\
\text { annual maximum } \\
\text { peak discharge } \\
\left(\mathrm{ft}^{3} / \mathrm{s}\right)\end{array}$ \\
\hline 05534400 & 1 & No & No & 1967 & 355 & -- & 609 & 1.37 & 0.179 & 0.221 & 623 \\
\hline 05534400 & 1 & No & No & 1968 & 145 & 2 & 249 & 0.506 & 0.189 & 0.799 & 320 \\
\hline 05534400 & 1 & No & No & 1969 & 319 & -- & 547 & 2.44 & 0.198 & 0.301 & 569 \\
\hline 05534400 & 1 & No & No & 1970 & 278 & -- & 477 & 2.05 & 0.208 & 0.403 & 512 \\
\hline 05534400 & 1 & No & No & 1971 & 300 & 2 & 515 & 0.329 & 0.218 & 0.357 & 538 \\
\hline 05534400 & 1 & No & No & 1972 & 339 & -- & 582 & 3.17 & 0.228 & 0.28 & 582 \\
\hline 05534400 & 1 & No & No & 1973 & 327 & -- & 561 & 1.42 & 0.238 & 0.311 & 563 \\
\hline 05534400 & 1 & No & No & 1974 & 268 & -- & 460 & 1.22 & 0.248 & 0.465 & 480 \\
\hline 05534400 & 1 & No & No & 1975 & 156 & -- & 268 & 0.409 & 0.259 & 0.807 & 316 \\
\hline 05534400 & 1 & No & No & 1976 & 282 & -- & 484 & 1.91 & 0.269 & 0.443 & 492 \\
\hline 05534500 & 1 & Yes & Yes & 1953 & 79 & $\mathrm{E}$ & 137 & 0.742 & 0.144 & 0.932 & 252 \\
\hline 05534500 & 1 & Yes & Yes & 1954 & 247 & -- & 430 & 1.52 & 0.159 & 0.441 & 522 \\
\hline 05534500 & 1 & Yes & Yes & 1955 & 247 & -- & 430 & 1.01 & 0.174 & 0.453 & 515 \\
\hline 05534500 & 1 & Yes & Yes & 1956 & 207 & $\mathrm{E}$ & 360 & 1.24 & 0.189 & 0.602 & 442 \\
\hline 05534500 & 1 & Yes & Yes & 1957 & 284 & -- & 494 & 1.07 & 0.203 & 0.375 & 559 \\
\hline 05534500 & 1 & Yes & Yes & 1958 & 178 & $\mathrm{E}$ & 310 & 0.157 & 0.218 & 0.714 & 387 \\
\hline 05534500 & 1 & Yes & Yes & 1959 & 292 & -- & 508 & 1.53 & 0.233 & 0.377 & 557 \\
\hline 05534500 & 1 & Yes & Yes & 1960 & 284 & -- & 494 & 0.45 & 0.248 & 0.409 & 539 \\
\hline 05534500 & 1 & Yes & Yes & 1961 & 178 & -- & 310 & 1.02 & 0.26 & 0.743 & 374 \\
\hline 05534500 & 1 & Yes & Yes & 1962 & 268 & -- & 466 & 0.226 & 0.272 & 0.474 & 504 \\
\hline 05534500 & 1 & Yes & Yes & 1963 & 134 & $\mathrm{E}$ & 233 & 1.31 & 0.284 & 0.869 & 302 \\
\hline 05534500 & 1 & Yes & Yes & 1964 & 228 & -- & 397 & 0.808 & 0.296 & 0.62 & 432 \\
\hline 05534500 & 1 & Yes & Yes & 1965 & 208 & -- & 362 & 0.528 & 0.308 & 0.689 & 399 \\
\hline 05534500 & 1 & Yes & Yes & 1966 & 311 & -- & 541 & 0.517 & 0.321 & 0.4 & 544 \\
\hline 05534500 & 1 & Yes & Yes & 1967 & 386 & -- & 672 & 1.4 & 0.333 & 0.251 & 633 \\
\hline 05534500 & 1 & Yes & Yes & 1968 & 173 & $\mathrm{E}$ & 301 & 0.652 & 0.345 & 0.807 & 338 \\
\hline 05534500 & 1 & Yes & Yes & 1969 & 359 & -- & 625 & 2.2 & 0.357 & 0.32 & 589 \\
\hline 05534500 & 1 & Yes & Yes & 1970 & 268 & $\mathrm{D}$ & 466 & 1.9 & 0.369 & 0.562 & 459 \\
\hline 05534500 & 1 & Yes & Yes & 1971 & 341 & $E$ & 593 & 0.341 & 0.379 & 0.377 & 557 \\
\hline 05534500 & 1 & Yes & Yes & 1972 & 368 & $-{ }_{-}^{-}$ & 640 & 2.65 & 0.389 & 0.326 & 585 \\
\hline 05534500 & 1 & Yes & Yes & 1973 & 390 & $-{ }_{-1}$ & 679 & 1.3 & 0.399 & 0.289 & 607 \\
\hline 05534500 & 1 & Yes & Yes & 1974 & 362 & -- & 630 & 1.3 & 0.409 & 0.355 & 569 \\
\hline 05534500 & 1 & Yes & Yes & 1975 & 370 & 2 & 644 & 1.1 & 0.419 & 0.346 & 574 \\
\hline 05534500 & 1 & Yes & Yes & 1976 & 550 & -- & 957 & 1.71 & 0.429 & 0.121 & 804 \\
\hline 05534500 & 1 & Yes & Yes & 1977 & 58 & $\mathrm{E}$ & 101 & 1.09 & 0.439 & 1 & 139 \\
\hline
\end{tabular}




\begin{tabular}{|c|c|c|c|c|c|c|c|c|c|c|c|}
\hline $\begin{array}{l}\text { U.S. } \\
\text { Geological } \\
\text { Survey } \\
\text { streamgage } \\
\text { number }\end{array}$ & $\begin{array}{l}\text { Segment } \\
\text { number }\end{array}$ & $\begin{array}{l}\text { Streamgage used in } \\
\text { regression analyses } \\
\text { (non-redundant) }\end{array}$ & $\begin{array}{c}\text { Discharge value } \\
\text { used in } \\
\text { adjustment } \\
\text { regression }\end{array}$ & $\begin{array}{l}\text { Water } \\
\text { year }\end{array}$ & $\begin{array}{c}\text { Observed annual } \\
\text { maximum peak } \\
\text { discharge }\left(\mathrm{ft}^{3} / \mathrm{s}\right)\end{array}$ & $\begin{array}{l}\text { NWIS peak } \\
\text { code }\end{array}$ & $\begin{array}{l}\text { Observed annual } \\
\text { maximum peak } \\
\text { discharge with } \\
\text { segment intercept } \\
\text { value subtracted } \\
\left(\mathrm{ft}^{3} / \mathrm{s}\right)\end{array}$ & $\begin{array}{l}\text { Observed } \\
\text { precipitation } \\
\text { (inches) }\end{array}$ & Urban fraction & $\begin{array}{l}\text { Exceedance } \\
\text { probability }\end{array}$ & $\begin{array}{c}\text { Urban-adjusted } \\
\text { annual maximum } \\
\text { peak discharge } \\
\left(\mathrm{ft}^{3} / \mathrm{s}\right)\end{array}$ \\
\hline 05534500 & 1 & Yes & Yes & 1978 & 292 & -- & 508 & 1.67 & 0.449 & 0.566 & 457 \\
\hline 05534500 & 1 & Yes & Yes & 1979 & 358 & -- & 623 & 1.28 & 0.459 & 0.407 & 541 \\
\hline 05534500 & 1 & Yes & Yes & 1980 & 237 & -- & 412 & 0.671 & 0.469 & 0.732 & 379 \\
\hline 05534500 & 1 & Yes & Yes & 1981 & 348 & -- & 606 & 0.827 & 0.478 & 0.448 & 518 \\
\hline 05534500 & 1 & Yes & Yes & 1982 & 756 & -- & 1316 & 2.73 & 0.488 & 0.0483 & 1020 \\
\hline 05534500 & 1 & Yes & Yes & 1983 & 702 & -- & 1222 & 2.25 & 0.497 & 0.064 & 952 \\
\hline 05534500 & 1 & Yes & Yes & 1984 & 415 & $\mathrm{D}$ & 722 & 0.347 & 0.507 & 0.325 & 585 \\
\hline 05534500 & 1 & Yes & Yes & 1985 & 678 & -- & 1180 & 0.683 & 0.516 & 0.0745 & 908 \\
\hline 05534500 & 1 & Yes & Yes & 1986 & 465 & -- & 809 & 1.45 & 0.526 & 0.251 & 632 \\
\hline 05534500 & 1 & Yes & Yes & 1987 & 933 & -- & 1624 & 3.5 & 0.535 & 0.0277 & 1200 \\
\hline 05534500 & 1 & Yes & Yes & 1988 & 273 & $E$ & 475 & 0.713 & 0.545 & 0.704 & 393 \\
\hline 05534500 & 1 & Yes & Yes & 1989 & 210 & $E$ & 365 & 1.58 & 0.554 & 0.848 & 314 \\
\hline 05534500 & 1 & Yes & Yes & 1990 & 557 & -- & 969 & 1.76 & 0.564 & 0.171 & 722 \\
\hline 05534500 & 1 & Yes & Yes & 1991 & 462 & -- & 804 & 1.95 & 0.575 & 0.292 & 604 \\
\hline 05534500 & 0 & Yes & No & 1992 & 198 & $C, E$ & 340 & 2.25 & 0.586 & 0.894 & 286 \\
\hline 05534500 & 2 & Yes & Yes & 1993 & 469 & C & 793 & 1.33 & 0.597 & 0.323 & 585 \\
\hline 05534500 & 2 & Yes & Yes & 1994 & 590 & C & 997 & 2.18 & 0.608 & 0.177 & 714 \\
\hline 05534500 & 2 & Yes & Yes & 1995 & 561 & C & 948 & 1.54 & 0.619 & 0.206 & 673 \\
\hline 05534500 & 2 & Yes & Yes & 1996 & 559 & C & 945 & 1.57 & 0.63 & 0.215 & 665 \\
\hline 05534500 & 2 & Yes & Yes & 1997 & 630 & $C, E$ & 1065 & 2.64 & 0.641 & 0.161 & 738 \\
\hline 05534500 & 2 & Yes & Yes & 1998 & 239 & $C, E$ & 404 & 0.863 & 0.652 & 0.867 & 303 \\
\hline 05534500 & 2 & Yes & Yes & 1999 & 590 & C & 997 & 1.68 & 0.663 & 0.201 & 679 \\
\hline 05534500 & 2 & Yes & Yes & 2000 & 437 & $C$ & 738 & 1.7 & 0.675 & 0.465 & 510 \\
\hline 05534500 & 2 & Yes & Yes & 2001 & 537 & C & 907 & 2.85 & 0.688 & 0.284 & 608 \\
\hline 05534500 & 2 & Yes & Yes & 2002 & 787 & C & 1330 & 2.28 & 0.702 & 0.0888 & 869 \\
\hline 05534500 & 2 & Yes & Yes & 2003 & 309 & C & 522 & 0.702 & 0.716 & 0.782 & 352 \\
\hline 05534500 & 2 & Yes & Yes & 2004 & 386 & C & 652 & 0.902 & 0.73 & 0.63 & 426 \\
\hline 05534500 & 2 & Yes & Yes & 2005 & 377 & $C, E$ & 637 & 0.675 & 0.744 & 0.667 & 410 \\
\hline 05534500 & 2 & Yes & Yes & 2006 & 356 & $C$ & 602 & 0.789 & 0.758 & 0.726 & 382 \\
\hline 05534500 & 2 & Yes & Yes & 2007 & 513 & $C$ & 867 & 1.62 & 0.772 & 0.416 & 537 \\
\hline 05534500 & 2 & Yes & Yes & 2008 & 749 & $C$ & 1266 & 3.44 & 0.786 & 0.143 & 768 \\
\hline 05534500 & 2 & Yes & Yes & 2009 & 624 & C & 1054 & 0.803 & 0.8 & 0.25 & 632 \\
\hline 05534600 & 1 & No & No & 1960 & 358 & -- & 561 & 0.441 & 0.317 & 0.37 & 601 \\
\hline 05534600 & 1 & No & No & 1961 & 277 & -- & 434 & 1.26 & 0.327 & 0.581 & 482 \\
\hline 05534600 & 1 & No & No & 1962 & 307 & -- & 481 & 0.23 & 0.338 & 0.507 & 520 \\
\hline
\end{tabular}




\begin{tabular}{|c|c|c|c|c|c|c|c|c|c|c|c|}
\hline $\begin{array}{c}\text { U.S. } \\
\text { Geological } \\
\text { Survey } \\
\text { streamgage } \\
\text { number }\end{array}$ & $\begin{array}{l}\text { Segment } \\
\text { number }\end{array}$ & $\begin{array}{l}\text { Streamgage used in } \\
\text { regression analyses } \\
\text { (non-redundant) }\end{array}$ & $\begin{array}{l}\text { Discharge value } \\
\text { used in } \\
\text { adjustment } \\
\text { regression }\end{array}$ & $\begin{array}{l}\text { Water } \\
\text { year }\end{array}$ & $\begin{array}{l}\text { Observed annual } \\
\text { maximum peak } \\
\text { discharge }\left(\mathrm{ft}^{3} / \mathrm{s}\right)\end{array}$ & $\begin{array}{l}\text { NWIS peak } \\
\text { code }\end{array}$ & $\begin{array}{l}\text { Observed annual } \\
\text { maximum peak } \\
\text { discharge with } \\
\text { segment intercept } \\
\text { value subtracted } \\
\left(\mathrm{ft}^{3} / \mathrm{s}\right)\end{array}$ & $\begin{array}{l}\text { Observed } \\
\text { precipitation } \\
\text { (inches) }\end{array}$ & Urban fraction & $\begin{array}{l}\text { Exceedance } \\
\text { probability }\end{array}$ & $\begin{array}{c}\text { Urban-adjusted } \\
\text { annual maximum } \\
\text { peak discharge } \\
\left(\mathrm{ft}^{3} / \mathrm{s}\right)\end{array}$ \\
\hline 05534600 & 1 & No & No & 1963 & 204 & -- & 320 & 1.28 & 0.349 & 0.783 & 375 \\
\hline 05534600 & 1 & No & No & 1964 & 214 & -- & 335 & 0.802 & 0.359 & 0.767 & 384 \\
\hline 05534600 & 1 & No & No & 1965 & 273 & -- & 428 & 0.525 & 0.37 & 0.627 & 457 \\
\hline 05534600 & 1 & No & No & 1966 & 361 & -- & 566 & 0.5 & 0.381 & 0.416 & 573 \\
\hline 05534600 & 1 & No & No & 1967 & 470 & -- & 737 & 1.41 & 0.391 & 0.228 & 699 \\
\hline 05534600 & 1 & No & No & 1968 & 213 & -- & 334 & 0.734 & 0.402 & 0.796 & 367 \\
\hline 05534600 & 1 & No & No & 1969 & 424 & -- & 664 & 2.04 & 0.413 & 0.315 & 633 \\
\hline 05534600 & 1 & No & No & 1970 & 327 & -- & 512 & 1.8 & 0.423 & 0.535 & 505 \\
\hline 05534600 & 1 & No & No & 1971 & 430 & -- & 674 & 0.347 & 0.432 & 0.319 & 631 \\
\hline 05534600 & 1 & No & No & 1972 & 424 & -- & 664 & 2.32 & 0.44 & 0.338 & 620 \\
\hline 05534600 & 0 & No & No & 1973 & 473 & -- & 737 & 1.23 & 0.448 & 0.264 & 668 \\
\hline 05534600 & 2 & No & No & 1974 & 384 & -- & 595 & 1.25 & 0.457 & 0.443 & 558 \\
\hline 05534600 & 2 & No & No & 1975 & 399 & -- & 618 & 1.02 & 0.465 & 0.419 & 572 \\
\hline 05534600 & 2 & No & No & 1976 & 481 & -- & 745 & 1.3 & 0.474 & 0.274 & 661 \\
\hline 05534600 & 2 & No & No & 1978 & 307 & -- & 476 & 1.58 & 0.491 & 0.654 & 443 \\
\hline 05534600 & 2 & No & No & 1979 & 471 & -- & 730 & 0.949 & 0.499 & 0.309 & 636 \\
\hline 05534600 & 2 & No & No & 1980 & 232 & -- & 360 & 0.6 & 0.508 & 0.827 & 348 \\
\hline 05534900 & 1 & No & No & 1962 & 132 & -- & 456 & 0.21 & 0.376 & 0.587 & 223 \\
\hline 05534900 & 1 & No & No & 1963 & 73 & -- & 252 & 1.45 & 0.398 & 0.896 & 142 \\
\hline 05534900 & 1 & No & No & 1964 & 158 & -- & 546 & 3.07 & 0.42 & 0.48 & 249 \\
\hline 05534900 & 1 & No & No & 1965 & 158 & -- & 546 & 1.4 & 0.442 & 0.501 & 243 \\
\hline 05534900 & 1 & No & No & 1966 & 145 & -- & 501 & 0.589 & 0.464 & 0.593 & 221 \\
\hline 05534900 & 1 & No & No & 1967 & 244 & -- & 842 & 1.48 & 0.486 & 0.2 & 336 \\
\hline 05534900 & 1 & No & No & 1968 & 77 & -- & 266 & 1.04 & 0.508 & 0.926 & 131 \\
\hline 05534900 & 1 & No & No & 1969 & 374 & -- & 1291 & 2.82 & 0.53 & 0.0582 & 483 \\
\hline 05534900 & 1 & No & No & 1970 & 324 & -- & 1119 & 2.29 & 0.552 & 0.101 & 415 \\
\hline 05534900 & 1 & No & No & 1971 & 166 & -- & 573 & 0.309 & 0.56 & 0.572 & 226 \\
\hline 05534900 & 1 & No & No & 1972 & 350 & -- & 1208 & 3.99 & 0.568 & 0.081 & 441 \\
\hline 05534900 & 1 & No & No & 1973 & 280 & -- & 967 & 1.6 & 0.576 & 0.177 & 353 \\
\hline 05534900 & 1 & No & No & 1974 & 316 & -- & 1091 & 1.46 & 0.584 & 0.126 & 394 \\
\hline 05534900 & 1 & No & No & 1975 & 162 & -- & 559 & 1.41 & 0.592 & 0.623 & 214 \\
\hline 05534900 & 1 & No & No & 1976 & 395 & -- & 1364 & 2.22 & 0.6 & 0.0591 & 480 \\
\hline 05535000 & 1 & Yes & Yes & 1952 & 321 & -- & 914 & 2.3 & 0.211 & 0.0725 & 570 \\
\hline 05535000 & 1 & Yes & Yes & 1953 & 92 & $E$ & 262 & 0.802 & 0.225 & 0.797 & 218 \\
\hline 05535000 & 1 & Yes & Yes & 1954 & 257 & -- & 731 & 1.53 & 0.239 & 0.161 & 456 \\
\hline
\end{tabular}




\begin{tabular}{|c|c|c|c|c|c|c|c|c|c|c|c|}
\hline $\begin{array}{c}\text { U.S. } \\
\text { Geological } \\
\text { Survey } \\
\text { streamgage } \\
\text { number } \\
\end{array}$ & $\begin{array}{c}\text { Segment } \\
\text { number }\end{array}$ & $\begin{array}{l}\text { Streamgage used in } \\
\text { regression analyses } \\
\text { (non-redundant) }\end{array}$ & $\begin{array}{c}\text { Discharge value } \\
\text { used in } \\
\text { adjustment } \\
\text { regression }\end{array}$ & $\begin{array}{c}\text { Water } \\
\text { year }\end{array}$ & $\begin{array}{c}\text { Observed annual } \\
\text { maximum peak } \\
\text { discharge }\left(\mathrm{ft}^{3} / \mathrm{s}\right)\end{array}$ & $\begin{array}{c}\text { NWIS peak } \\
\text { code }\end{array}$ & $\begin{array}{c}\text { Observed annual } \\
\text { maximum peak } \\
\text { discharge with } \\
\text { segment intercept } \\
\text { value subtracted } \\
\left(\mathrm{ft}^{3} / \mathrm{s}\right)\end{array}$ & $\begin{array}{l}\text { Observed } \\
\text { precipitation } \\
\text { (inches) }\end{array}$ & Urban fraction & $\begin{array}{l}\text { Exceedance } \\
\text { probability }\end{array}$ & $\begin{array}{c}\text { Urban-adjusted } \\
\text { annual maximum } \\
\text { peak discharge } \\
\left(\mathrm{ft}^{3} / \mathrm{s}\right)\end{array}$ \\
\hline 05535000 & 1 & Yes & Yes & 1955 & 194 & -- & 552 & 0.673 & 0.252 & 0.333 & 364 \\
\hline 05535000 & 1 & Yes & Yes & 1956 & 155 & $E$ & 441 & 1.42 & 0.266 & 0.512 & 303 \\
\hline 05535000 & 1 & Yes & Yes & 1957 & 201 & $E$ & 572 & 1.19 & 0.28 & 0.327 & 365 \\
\hline 05535000 & 1 & Yes & Yes & 1958 & 98 & $E$ & 279 & 0.123 & 0.294 & 0.81 & 214 \\
\hline 05535000 & 1 & Yes & Yes & 1959 & 313 & $E$ & 891 & 1.8 & 0.308 & 0.104 & 517 \\
\hline 05535000 & 1 & Yes & Yes & 1960 & 250 & -- & 712 & 0.486 & 0.321 & 0.208 & 416 \\
\hline 05535000 & 1 & Yes & Yes & 1961 & 155 & -- & 441 & 1.15 & 0.341 & 0.58 & 283 \\
\hline 05535000 & 1 & Yes & Yes & 1962 & 194 & -- & 552 & 0.211 & 0.36 & 0.418 & 335 \\
\hline 05535000 & 1 & Yes & Yes & 1963 & 161 & $E$ & 458 & 1.44 & 0.379 & 0.585 & 282 \\
\hline 05535000 & 1 & Yes & Yes & 1964 & 151 & -- & 430 & 3.03 & 0.399 & 0.647 & 263 \\
\hline 05535000 & 1 & Yes & Yes & 1965 & 130 & -- & 370 & 0.684 & 0.418 & 0.756 & 232 \\
\hline 05535000 & 1 & Yes & Yes & 1966 & 176 & D & 501 & 0.583 & 0.437 & 0.567 & 287 \\
\hline 05535000 & 1 & Yes & Yes & 1967 & 295 & -- & 840 & 1.44 & 0.457 & 0.19 & 430 \\
\hline 05535000 & 1 & Yes & Yes & 1968 & 80 & $E$ & 228 & 0.38 & 0.476 & 0.94 & 157 \\
\hline 05535000 & 1 & Yes & Yes & 1969 & 315 & -- & 897 & 2.77 & 0.495 & 0.177 & 443 \\
\hline 05535000 & 1 & Yes & Yes & 1970 & 249 & -- & 709 & 2.26 & 0.515 & 0.348 & 358 \\
\hline 05535000 & 1 & Yes & Yes & 1971 & 223 & $\mathrm{E}$ & 635 & 0.312 & 0.522 & 0.449 & 325 \\
\hline 05535000 & 1 & Yes & Yes & 1972 & 367 & -- & 1045 & 1.13 & 0.529 & 0.123 & 497 \\
\hline 05535000 & 1 & Yes & Yes & 1973 & 302 & -- & 860 & 1.57 & 0.536 & 0.218 & 411 \\
\hline 05535000 & 1 & Yes & Yes & 1974 & 272 & -- & 774 & 1.42 & 0.544 & 0.297 & 374 \\
\hline 05535000 & 1 & Yes & Yes & 1975 & 185 & -- & 527 & 1.38 & 0.551 & 0.632 & 268 \\
\hline 05535000 & 1 & Yes & Yes & 1976 & 315 & -- & 897 & 2.18 & 0.558 & 0.205 & 419 \\
\hline 05535000 & 1 & Yes & Yes & 1977 & 70 & $E$ & 199 & 1.34 & 0.565 & 0.97 & 125 \\
\hline 05535000 & 1 & Yes & Yes & 1978 & 169 & -- & 481 & 1.98 & 0.573 & 0.719 & 244 \\
\hline 05535000 & 1 & Yes & Yes & 1979 & 274 & -- & 780 & 1.53 & 0.58 & 0.322 & 366 \\
\hline 05535000 & 1 & Yes & Yes & 1980 & 175 & -- & 498 & 0.775 & 0.587 & 0.708 & 247 \\
\hline 05535000 & 1 & Yes & Yes & 1981 & 201 & -- & 572 & 1.64 & 0.593 & 0.605 & 275 \\
\hline 05535000 & 1 & Yes & Yes & 1982 & 435 & -- & 1238 & 3.32 & 0.599 & 0.082 & 550 \\
\hline 05535000 & 1 & Yes & Yes & 1983 & 394 & -- & 1121 & 2.45 & 0.604 & 0.122 & 498 \\
\hline 05535000 & 1 & Yes & Yes & 1984 & 195 & -- & 555 & 0.482 & 0.61 & 0.646 & 264 \\
\hline 05535000 & 1 & Yes & Yes & 1985 & 265 & -- & 754 & 0.962 & 0.616 & 0.388 & 345 \\
\hline 05535000 & 1 & Yes & Yes & 1986 & 492 & -- & 1400 & 2.99 & 0.621 & 0.0572 & 606 \\
\hline 05535000 & 1 & Yes & Yes & 1987 & 259 & -- & 737 & 1.16 & 0.627 & 0.419 & 335 \\
\hline 05535000 & 1 & Yes & Yes & 1988 & 221 & -- & 629 & 0.81 & 0.633 & 0.564 & 288 \\
\hline 05535000 & 1 & Yes & Yes & 1989 & 102 & $E$ & 290 & 1.29 & 0.638 & 0.946 & 152 \\
\hline
\end{tabular}




\begin{tabular}{|c|c|c|c|c|c|c|c|c|c|c|c|}
\hline $\begin{array}{l}\text { U.S. } \\
\text { Geological } \\
\text { Survey } \\
\text { streamgage } \\
\text { number }\end{array}$ & $\begin{array}{l}\text { Segment } \\
\text { number }\end{array}$ & $\begin{array}{l}\text { Streamgage used in } \\
\text { regression analyses } \\
\text { (non-redundant) }\end{array}$ & $\begin{array}{l}\text { Discharge value } \\
\text { used in } \\
\text { adjustment } \\
\text { regression }\end{array}$ & $\begin{array}{l}\text { Water } \\
\text { year }\end{array}$ & $\begin{array}{l}\text { Observed annual } \\
\text { maximum peak } \\
\text { discharge }\left(\mathrm{ft}^{3} / \mathrm{s}\right)\end{array}$ & $\begin{array}{l}\text { NWIS peak } \\
\text { code }\end{array}$ & $\begin{array}{l}\text { Observed annual } \\
\text { maximum peak } \\
\text { discharge with } \\
\text { segment intercept } \\
\text { value subtracted } \\
\left(\mathrm{ft}^{3} / \mathrm{s}\right)\end{array}$ & $\begin{array}{l}\text { Observed } \\
\text { precipitation } \\
\text { (inches) }\end{array}$ & Urban fraction & $\begin{array}{l}\text { Exceedance } \\
\text { probability }\end{array}$ & $\begin{array}{c}\text { Urban-adjusted } \\
\text { annual maximum } \\
\text { peak discharge } \\
\left(\mathrm{ft}^{3} / \mathrm{s}\right)\end{array}$ \\
\hline 05535000 & 1 & Yes & Yes & 1990 & 272 & -- & 774 & 2.04 & 0.644 & 0.392 & 344 \\
\hline 05535000 & 1 & Yes & Yes & 1991 & 276 & -- & 786 & 1.82 & 0.653 & 0.387 & 345 \\
\hline 05535000 & 1 & Yes & Yes & 1992 & 138 & C & 393 & 2.72 & 0.661 & 0.884 & 187 \\
\hline 05535000 & 1 & Yes & Yes & 1993 & 294 & $C, D$ & 837 & 1.79 & 0.67 & 0.343 & 359 \\
\hline 05535000 & 1 & Yes & Yes & 1994 & 318 & C & 905 & 1.03 & 0.679 & 0.279 & 381 \\
\hline 05535000 & 1 & Yes & Yes & 1995 & 305 & C & 868 & 1.63 & 0.687 & 0.324 & 365 \\
\hline 05535000 & 1 & Yes & Yes & 1996 & 460 & C & 1309 & 1.9 & 0.696 & 0.092 & 534 \\
\hline 05535000 & 1 & Yes & Yes & 1997 & 496 & $\mathrm{C}, \mathrm{E}$ & 1412 & 2.52 & 0.705 & 0.0712 & 570 \\
\hline 05535000 & 1 & Yes & Yes & 1998 & 246 & $C$ & 700 & 2.07 & 0.713 & 0.551 & 292 \\
\hline 05535000 & 1 & Yes & Yes & 1999 & 472 & C & 1343 & 1.96 & 0.722 & 0.0912 & 536 \\
\hline 05535000 & 1 & Yes & Yes & 2000 & 429 & C & 1221 & 1.93 & 0.73 & 0.136 & 484 \\
\hline 05535000 & 1 & Yes & Yes & 2001 & 392 & C & 1116 & 2.95 & 0.744 & 0.184 & 438 \\
\hline 05535000 & 1 & Yes & Yes & 2002 & 580 & C & 1651 & 3.01 & 0.757 & 0.0473 & 634 \\
\hline 05535000 & 1 & Yes & Yes & 2003 & 238 & C & 677 & 0.757 & 0.77 & 0.639 & 266 \\
\hline 05535000 & 1 & Yes & Yes & 2004 & 267 & C & 760 & 0.967 & 0.783 & 0.549 & 292 \\
\hline 05535000 & 1 & Yes & Yes & 2005 & 208 & $\mathrm{C}, \mathrm{E}$ & 592 & 0.693 & 0.797 & 0.77 & 227 \\
\hline 05535000 & 1 & Yes & Yes & 2006 & 277 & C & 788 & 0.888 & 0.81 & 0.543 & 294 \\
\hline 05535000 & 1 & Yes & Yes & 2007 & 355 & C & 1010 & 1.17 & 0.823 & 0.307 & 370 \\
\hline 05535000 & 1 & Yes & Yes & 2008 & 464 & C & 1321 & 2.84 & 0.836 & 0.145 & 475 \\
\hline 05535000 & 1 & Yes & Yes & 2009 & 412 & C & 1173 & 0.76 & 0.85 & 0.211 & 417 \\
\hline 05535070 & 1 & No & No & 1967 & 410 & -- & 694 & 1.38 & 0.573 & 0.42 & 590 \\
\hline 05535070 & 1 & No & No & 1968 & 388 & $\mathrm{E}$ & 657 & 0.636 & 0.589 & 0.484 & 553 \\
\hline 05535070 & 1 & No & No & 1969 & 500 & -- & 847 & 2.18 & 0.606 & 0.275 & 673 \\
\hline 05535070 & 1 & No & No & 1970 & 368 & -- & 623 & 1.89 & 0.623 & 0.562 & 510 \\
\hline 05535070 & 1 & No & No & 1971 & 344 & -- & 583 & 0.339 & 0.629 & 0.625 & 478 \\
\hline 05535070 & 1 & No & No & 1972 & 570 & -- & 965 & 1.72 & 0.636 & 0.204 & 735 \\
\hline 05535070 & 1 & No & No & 1973 & 422 & -- & 715 & 0.685 & 0.642 & 0.462 & 566 \\
\hline 05535070 & 1 & No & No & 1974 & 440 & -- & 745 & 1.07 & 0.649 & 0.431 & 584 \\
\hline 05535070 & 1 & No & No & 1975 & 381 & -- & 645 & 0.599 & 0.655 & 0.564 & 509 \\
\hline 05535070 & 1 & No & No & 1976 & 512 & $\mathrm{D}$ & 867 & 1.7 & 0.661 & 0.299 & 655 \\
\hline 05535070 & 1 & No & No & 1977 & 164 & $E$ & 278 & 1.09 & 0.668 & 0.957 & 255 \\
\hline 05535070 & 1 & No & No & 1978 & 326 & -- & 552 & 1.65 & 0.674 & 0.712 & 437 \\
\hline 05535070 & 1 & No & No & 1979 & 388 & -- & 657 & 0.985 & 0.681 & 0.573 & 504 \\
\hline 05535070 & 1 & No & No & 1980 & 263 & -- & 445 & 0.916 & 0.687 & 0.845 & 360 \\
\hline 05535070 & 1 & No & No & 1981 & 351 & -- & 595 & 0.827 & 0.691 & 0.671 & 456 \\
\hline
\end{tabular}




\begin{tabular}{|c|c|c|c|c|c|c|c|c|c|c|c|}
\hline $\begin{array}{l}\text { U.S. } \\
\text { Geological } \\
\text { Survey } \\
\text { streamgage } \\
\text { number }\end{array}$ & $\begin{array}{l}\text { Segment } \\
\text { number }\end{array}$ & $\begin{array}{l}\text { Streamgage used in } \\
\text { regression analyses } \\
\text { (non-redundant) }\end{array}$ & $\begin{array}{c}\text { Discharge value } \\
\text { used in } \\
\text { adjustment } \\
\text { regression }\end{array}$ & $\begin{array}{l}\text { Water } \\
\text { year }\end{array}$ & $\begin{array}{c}\text { Observed annual } \\
\text { maximum peak } \\
\text { discharge }\left(\mathrm{ft}^{3} / \mathrm{s}\right)\end{array}$ & $\begin{array}{l}\text { NWIS peak } \\
\text { code }\end{array}$ & $\begin{array}{l}\text { Observed annual } \\
\text { maximum peak } \\
\text { discharge with } \\
\text { segment intercept } \\
\text { value subtracted } \\
\left(\mathrm{ft}^{3} / \mathrm{s}\right)\end{array}$ & $\begin{array}{l}\text { Observed } \\
\text { precipitation } \\
\text { (inches) }\end{array}$ & Urban fraction & $\begin{array}{l}\text { Exceedance } \\
\text { probability }\end{array}$ & $\begin{array}{c}\text { Urban-adjusted } \\
\text { annual maximum } \\
\text { peak discharge } \\
\left(\mathrm{ft}^{3} / \mathrm{s}\right)\end{array}$ \\
\hline 05535070 & 1 & No & No & 1982 & 716 & -- & 1213 & 2.51 & 0.695 & 0.124 & 869 \\
\hline 05535070 & 1 & No & No & 1983 & 724 & -- & 1226 & 2.58 & 0.699 & 0.121 & 875 \\
\hline 05535070 & 1 & No & No & 1984 & 381 & -- & 645 & 2.13 & 0.703 & 0.612 & 484 \\
\hline 05535070 & 1 & No & No & 1985 & 524 & -- & 888 & 0.825 & 0.707 & 0.321 & 643 \\
\hline 05535070 & 1 & No & No & 1986 & 584 & -- & 989 & 1.47 & 0.711 & 0.236 & 705 \\
\hline 05535070 & 1 & No & No & 1987 & 895 & -- & 1516 & 4.18 & 0.715 & 0.0569 & 1060 \\
\hline 05535070 & 1 & No & No & 1988 & 337 & -- & 571 & 0.505 & 0.719 & 0.728 & 428 \\
\hline 05535070 & 1 & No & No & 1989 & 298 & -- & 505 & 2 & 0.723 & 0.807 & 383 \\
\hline 05535070 & 1 & No & No & 1990 & 503 & -- & 852 & 1.46 & 0.727 & 0.385 & 610 \\
\hline 05535070 & 1 & No & No & 1991 & 477 & -- & 808 & 1.9 & 0.733 & 0.441 & 578 \\
\hline 05535070 & 1 & No & No & 1992 & 281 & $E$ & 476 & 1.83 & 0.738 & 0.848 & 358 \\
\hline 05535070 & 1 & No & No & 1993 & 372 & C & 630 & 0.777 & 0.744 & 0.677 & 454 \\
\hline 05535070 & 1 & No & No & 1994 & 665 & C & 1126 & 1.06 & 0.75 & 0.182 & 770 \\
\hline 05535070 & 1 & No & No & 1995 & 589 & C & 998 & 2 & 0.755 & 0.26 & 683 \\
\hline 05535070 & 1 & No & No & 1996 & 745 & C & 1262 & 1.62 & 0.761 & 0.134 & 851 \\
\hline 05535070 & 1 & No & No & 1997 & 894 & $C, E$ & 1514 & 2.79 & 0.767 & 0.0674 & 1010 \\
\hline 05535070 & 1 & No & No & 1998 & 300 & C & 508 & 0.999 & 0.773 & 0.839 & 363 \\
\hline 05535070 & 1 & No & No & 1999 & 880 & C & 1491 & 2.04 & 0.778 & 0.0735 & 986 \\
\hline 05535070 & 1 & No & No & 2000 & 634 & C & 1074 & 1.55 & 0.784 & 0.227 & 713 \\
\hline 05535070 & 1 & No & No & 2001 & 762 & C & 1291 & 2.69 & 0.796 & 0.138 & 844 \\
\hline 05535070 & 1 & No & No & 2002 & 1230 & C & 2083 & 3.06 & 0.809 & 0.0249 & 1330 \\
\hline 05535070 & 1 & No & No & 2003 & 454 & C & 769 & 0.865 & 0.821 & 0.577 & 502 \\
\hline 05535070 & 1 & No & No & 2004 & 536 & C & 908 & 1.13 & 0.833 & 0.436 & 581 \\
\hline 05535070 & 1 & No & No & 2005 & 553 & $\mathrm{C}, \mathrm{E}$ & 937 & 0.785 & 0.846 & 0.419 & 591 \\
\hline 05535070 & 1 & No & No & 2006 & 499 & C & 845 & 0.873 & 0.858 & 0.53 & 528 \\
\hline 05535070 & 1 & No & No & 2007 & 745 & C & 1262 & 1.75 & 0.871 & 0.182 & 771 \\
\hline 05535070 & 1 & No & No & 2008 & 1150 & C & 1948 & 2.98 & 0.883 & 0.0391 & 1170 \\
\hline 05535070 & 1 & No & No & 2009 & 857 & C & 1452 & 0.638 & 0.895 & 0.126 & 867 \\
\hline 05535150 & 1 & No & No & 1960 & 387 & -- & 654 & 0.436 & 0.471 & 0.377 & 565 \\
\hline 05535150 & 1 & No & No & 1961 & 320 & -- & 541 & 1.26 & 0.485 & 0.549 & 473 \\
\hline 05535150 & 1 & No & No & 1962 & 374 & -- & 632 & 0.233 & 0.499 & 0.431 & 536 \\
\hline 05535150 & 1 & No & No & 1963 & 267 & -- & 451 & 1.26 & 0.513 & 0.711 & 396 \\
\hline 05535150 & 1 & No & No & 1964 & 279 & -- & 471 & 0.793 & 0.527 & 0.694 & 405 \\
\hline 05535150 & 1 & No & No & 1965 & 285 & -- & 482 & 0.525 & 0.54 & 0.691 & 406 \\
\hline 05535150 & 1 & No & No & 1966 & 312 & -- & 527 & 1.15 & 0.554 & 0.634 & 432 \\
\hline
\end{tabular}




\begin{tabular}{|c|c|c|c|c|c|c|c|c|c|c|c|}
\hline $\begin{array}{l}\text { U.S. } \\
\text { Geological } \\
\text { Survey } \\
\text { streamgage } \\
\text { number }\end{array}$ & $\begin{array}{c}\text { Segment } \\
\text { number }\end{array}$ & $\begin{array}{l}\text { Streamgage used in } \\
\text { regression analyses } \\
\text { (non-redundant) }\end{array}$ & $\begin{array}{c}\text { Discharge value } \\
\text { used in } \\
\text { adjustment } \\
\text { regression }\end{array}$ & $\begin{array}{l}\text { Water } \\
\text { year }\end{array}$ & $\begin{array}{c}\text { Observed annual } \\
\text { maximum peak } \\
\text { discharge }\left(\mathrm{ft}^{3} / \mathrm{s}\right)\end{array}$ & $\begin{array}{l}\text { NWIS peak } \\
\text { code }\end{array}$ & $\begin{array}{l}\text { Observed annual } \\
\text { maximum peak } \\
\text { discharge with } \\
\text { segment intercept } \\
\text { value subtracted } \\
\left(\mathrm{ft}^{3} / \mathrm{s}\right)\end{array}$ & $\begin{array}{l}\text { Observed } \\
\text { precipitation } \\
\text { (inches) }\end{array}$ & Urban fraction & $\begin{array}{l}\text { Exceedance } \\
\text { probability }\end{array}$ & $\begin{array}{c}\text { Urban-adjusted } \\
\text { annual maximum } \\
\text { peak discharge } \\
\left(\mathrm{ft}^{3} / \mathrm{s}\right)\end{array}$ \\
\hline 05535150 & 1 & No & No & 1967 & 567 & 2 & 958 & 1.39 & 0.568 & 0.178 & 720 \\
\hline 05535150 & 1 & No & No & 1968 & 309 & -- & 522 & 0.766 & 0.582 & 0.668 & 416 \\
\hline 05535150 & 1 & No & No & 1969 & 450 & -- & 760 & 1.94 & 0.596 & 0.361 & 574 \\
\hline 05535150 & 0 & No & No & 1970 & 420 & -- & 712 & 1.73 & 0.61 & 0.434 & 535 \\
\hline 05535150 & 2 & No & No & 1971 & 404 & -- & 687 & 0.349 & 0.615 & 0.47 & 515 \\
\hline 05535150 & 2 & No & No & 1972 & 459 & -- & 780 & 2.13 & 0.62 & 0.36 & 574 \\
\hline 05535150 & 2 & No & No & 1973 & 385 & -- & 655 & 0.71 & 0.625 & 0.522 & 487 \\
\hline 05535150 & 2 & No & No & 1974 & 438 & -- & 745 & 1.23 & 0.63 & 0.413 & 546 \\
\hline 05535150 & 2 & No & No & 1975 & 399 & -- & 678 & 0.986 & 0.635 & 0.501 & 498 \\
\hline 05535150 & 2 & No & No & 1976 & 499 & -- & 848 & 1.51 & 0.64 & 0.3 & 606 \\
\hline 05535150 & 2 & No & No & 1978 & 303 & -- & 515 & 1.52 & 0.65 & 0.738 & 383 \\
\hline 05535150 & 2 & No & No & 1979 & 414 & -- & 704 & 1.18 & 0.655 & 0.488 & 505 \\
\hline 05535200 & 1 & No & No & 1960 & 786 & -- & 661 & 0.432 & 0.442 & 0.344 & 1160 \\
\hline 05535200 & 1 & No & No & 1961 & 688 & -- & 578 & 1.27 & 0.454 & 0.464 & 1030 \\
\hline 05535200 & 1 & No & No & 1962 & 674 & -- & 566 & 0.233 & 0.465 & 0.491 & 998 \\
\hline 05535200 & 1 & No & No & 1963 & 532 & -- & 447 & 1.25 & 0.477 & 0.687 & 807 \\
\hline 05535200 & 1 & No & No & 1964 & 532 & -- & 447 & 0.798 & 0.488 & 0.698 & 798 \\
\hline 05535200 & 1 & No & No & 1965 & 458 & -- & 385 & 0.521 & 0.5 & 0.791 & 700 \\
\hline 05535200 & 1 & No & No & 1966 & 616 & -- & 518 & 0.486 & 0.511 & 0.609 & 880 \\
\hline 05535200 & 1 & No & No & 1967 & 1070 & -- & 899 & 1.41 & 0.523 & 0.187 & 1400 \\
\hline 05535200 & 1 & No & No & 1968 & 519 & -- & 436 & 0.799 & 0.535 & 0.749 & 747 \\
\hline 05535200 & 1 & No & No & 1969 & 866 & -- & 728 & 1.91 & 0.546 & 0.353 & 1150 \\
\hline 05535200 & 0 & No & No & 1970 & 730 & 2 & NA & 1.72 & 0.558 & NA & NA \\
\hline 05535200 & 0 & No & No & 1971 & 608 & -- & NA & 0.351 & 0.564 & NA & NA \\
\hline 05535200 & 0 & No & No & 1972 & 998 & -- & NA & 2.06 & 0.57 & NA & NA \\
\hline 05535200 & 0 & No & No & 1973 & 678 & -- & NA & 1.18 & 0.576 & NA & NA \\
\hline 05535200 & 0 & No & No & 1974 & 755 & -- & NA & 1.22 & 0.582 & NA & NA \\
\hline 05535200 & 0 & No & No & 1975 & 704 & -- & NA & 0.967 & 0.588 & NA & NA \\
\hline 05535200 & 0 & No & No & 1976 & 1200 & -- & NA & 1.31 & 0.594 & NA & NA \\
\hline 05535200 & 0 & No & No & 1977 & 319 & -- & NA & 0.751 & 0.6 & NA & NA \\
\hline 05535300 & 1 & No & No & 1961 & 166 & -- & 388 & 1.38 & 0.242 & 0.593 & 357 \\
\hline 05535300 & 1 & No & No & 1962 & 128 & -- & 299 & 0.249 & 0.26 & 0.76 & 296 \\
\hline 05535300 & 1 & No & No & 1963 & 111 & -- & 259 & 1.13 & 0.277 & 0.829 & 266 \\
\hline 05535300 & 1 & No & No & 1964 & 162 & -- & 378 & 1.78 & 0.294 & 0.649 & 336 \\
\hline 05535300 & 1 & No & No & 1965 & 160 & -- & 374 & 0.504 & 0.311 & 0.67 & 328 \\
\hline
\end{tabular}




\begin{tabular}{|c|c|c|c|c|c|c|c|c|c|c|c|}
\hline $\begin{array}{l}\text { U.S. } \\
\text { Geological } \\
\text { Survey } \\
\text { streamgage } \\
\text { number }\end{array}$ & $\begin{array}{c}\text { Segment } \\
\text { number }\end{array}$ & $\begin{array}{l}\text { Streamgage used in } \\
\text { regression analyses } \\
\text { (non-redundant) }\end{array}$ & $\begin{array}{l}\text { Discharge value } \\
\text { used in } \\
\text { adjustment } \\
\text { regression }\end{array}$ & $\begin{array}{l}\text { Water } \\
\text { year }\end{array}$ & $\begin{array}{c}\text { Observed annual } \\
\text { maximum peak } \\
\text { discharge }\left(\mathrm{ft}^{3} / \mathrm{s}\right)\end{array}$ & $\begin{array}{l}\text { NWIS peak } \\
\text { code }\end{array}$ & $\begin{array}{l}\text { Observed annual } \\
\text { maximum peak } \\
\text { discharge with } \\
\text { segment intercept } \\
\text { value subtracted } \\
\left(\mathrm{ft}^{3} / \mathrm{s}\right)\end{array}$ & $\begin{array}{l}\text { Observed } \\
\text { precipitation } \\
\text { (inches) }\end{array}$ & Urban fraction & $\begin{array}{l}\text { Exceedance } \\
\text { probability }\end{array}$ & $\begin{array}{c}\text { Urban-adjusted } \\
\text { annual maximum } \\
\text { peak discharge } \\
\left(\mathrm{ft}^{3} / \mathrm{s}\right)\end{array}$ \\
\hline 05535300 & 1 & No & No & 1966 & 245 & -- & 572 & 0.417 & 0.328 & 0.364 & 448 \\
\hline 05535300 & 1 & No & No & 1967 & 325 & -- & 759 & 1.47 & 0.345 & 0.188 & 545 \\
\hline 05535300 & 1 & No & No & 1968 & 112 & -- & 262 & 1.15 & 0.363 & 0.87 & 249 \\
\hline 05535300 & 1 & No & No & 1969 & 301 & -- & 703 & 1.29 & 0.38 & 0.251 & 499 \\
\hline 05535300 & 1 & No & No & 1970 & 265 & -- & 619 & 1.34 & 0.397 & 0.359 & 450 \\
\hline 05535300 & 1 & No & No & 1971 & 193 & -- & 451 & 0.386 & 0.411 & 0.624 & 345 \\
\hline 05535300 & 1 & No & No & 1972 & 387 & -- & 904 & 0.693 & 0.424 & 0.144 & 599 \\
\hline 05535300 & 1 & No & No & 1973 & 299 & -- & 698 & 1.39 & 0.438 & 0.296 & 476 \\
\hline 05535300 & 1 & No & No & 1974 & 231 & -- & 539 & 0.524 & 0.451 & 0.519 & 384 \\
\hline 05535300 & 1 & No & No & 1975 & 178 & -- & 416 & 0.953 & 0.465 & 0.724 & 310 \\
\hline 05535300 & 1 & No & No & 1976 & 256 & -- & 598 & 0.976 & 0.478 & 0.459 & 409 \\
\hline 05535400 & 1 & No & No & 1961 & 324 & -- & 552 & 1.78 & 0.423 & 0.474 & 573 \\
\hline 05535400 & 1 & No & No & 1962 & 290 & -- & 494 & 0.248 & 0.44 & 0.582 & 514 \\
\hline 05535400 & 1 & No & No & 1963 & 247 & -- & 421 & 1.12 & 0.458 & 0.712 & 449 \\
\hline 05535400 & 1 & No & No & 1964 & 376 & -- & 640 & 1.79 & 0.476 & 0.4 & 616 \\
\hline 05535400 & 1 & No & No & 1965 & 208 & -- & 354 & 0.498 & 0.494 & 0.826 & 385 \\
\hline 05535400 & 1 & No & No & 1966 & 324 & -- & 552 & 1.32 & 0.512 & 0.557 & 526 \\
\hline 05535400 & 1 & No & No & 1967 & 571 & -- & 972 & 1.49 & 0.53 & 0.155 & 829 \\
\hline 05535400 & 1 & No & No & 1968 & 355 & -- & 605 & 1.17 & 0.548 & 0.515 & 550 \\
\hline 05535400 & 1 & No & No & 1969 & 418 & -- & 712 & 1.29 & 0.565 & 0.391 & 621 \\
\hline 05535400 & 1 & No & No & 1970 & 370 & -- & 630 & 1.33 & 0.583 & 0.514 & 550 \\
\hline 05535400 & 1 & No & No & 1971 & 272 & -- & 463 & 0.387 & 0.595 & 0.759 & 424 \\
\hline 05535400 & 1 & No & No & 1972 & 531 & -- & 904 & 0.69 & 0.607 & 0.229 & 725 \\
\hline 05535400 & 1 & No & No & 1973 & 477 & -- & 812 & 1.39 & 0.619 & 0.321 & 658 \\
\hline 05535400 & 1 & No & No & 1974 & 364 & -- & 620 & 1.04 & 0.631 & 0.575 & 517 \\
\hline 05535400 & 1 & No & No & 1975 & 367 & -- & 625 & 0.963 & 0.644 & 0.58 & 514 \\
\hline 05535400 & 1 & No & No & 1976 & 455 & -- & 775 & 0.96 & 0.656 & 0.403 & 615 \\
\hline 05535500 & 1 & Yes & Yes & 1953 & 86 & $\mathrm{E}$ & 123 & 0.651 & 0.251 & 0.961 & 197 \\
\hline 05535500 & 1 & Yes & Yes & 1954 & 411 & -- & 588 & 1.61 & 0.281 & 0.308 & 516 \\
\hline 05535500 & 1 & Yes & Yes & 1955 & 404 & -- & 578 & 0.786 & 0.311 & 0.343 & 500 \\
\hline 05535500 & 1 & Yes & Yes & 1956 & 199 & $E$ & 285 & 1.2 & 0.341 & 0.828 & 294 \\
\hline 05535500 & 1 & Yes & Yes & 1957 & 930 & -- & 1330 & 1.87 & 0.371 & 0.0351 & 956 \\
\hline 05535500 & 1 & Yes & Yes & 1958 & 159 & -- & 227 & 0.211 & 0.401 & 0.921 & 245 \\
\hline 05535500 & 1 & Yes & Yes & 1959 & 366 & -- & 523 & 1.07 & 0.431 & 0.524 & 419 \\
\hline 05535500 & 1 & Yes & Yes & 1960 & 275 & -- & 393 & 0.387 & 0.461 & 0.754 & 328 \\
\hline
\end{tabular}




\begin{tabular}{|c|c|c|c|c|c|c|c|c|c|c|c|}
\hline $\begin{array}{l}\text { U.S. } \\
\text { Geological } \\
\text { Survey } \\
\text { streamgage } \\
\text { number }\end{array}$ & $\begin{array}{l}\text { Segment } \\
\text { number }\end{array}$ & $\begin{array}{l}\text { Streamgage used in } \\
\text { regression analyses } \\
\text { (non-redundant) }\end{array}$ & $\begin{array}{c}\text { Discharge value } \\
\text { used in } \\
\text { adjustment } \\
\text { regression }\end{array}$ & $\begin{array}{l}\text { Water } \\
\text { year }\end{array}$ & $\begin{array}{l}\text { Observed annual } \\
\text { maximum peak } \\
\text { discharge }\left(\mathrm{ft}^{3} / \mathrm{s}\right)\end{array}$ & $\begin{array}{l}\text { NWIS peak } \\
\text { code }\end{array}$ & $\begin{array}{l}\text { Observed annual } \\
\text { maximum peak } \\
\text { discharge with } \\
\text { segment intercept } \\
\text { value subtracted } \\
\left(\mathrm{ft}^{3} / \mathrm{s}\right)\end{array}$ & $\begin{array}{l}\text { Observed } \\
\text { precipitation } \\
\text { (inches) }\end{array}$ & Urban fraction & $\begin{array}{l}\text { Exceedance } \\
\text { probability }\end{array}$ & $\begin{array}{c}\text { Urban-adjusted } \\
\text { annual maximum } \\
\text { peak discharge } \\
\left(\mathrm{ft}^{3} / \mathrm{s}\right)\end{array}$ \\
\hline 05535500 & 1 & Yes & Yes & 1961 & 502 & -- & 718 & 1.41 & 0.477 & 0.304 & 516 \\
\hline 05535500 & 1 & Yes & Yes & 1962 & 428 & -- & 612 & 0.246 & 0.493 & 0.453 & 451 \\
\hline 05535500 & 1 & Yes & Yes & 1963 & 350 & -- & 500 & 1.1 & 0.509 & 0.633 & 375 \\
\hline 05535500 & 1 & Yes & Yes & 1964 & 298 & -- & 426 & 1.82 & 0.525 & 0.755 & 327 \\
\hline 05535500 & 1 & Yes & Yes & 1965 & 285 & -- & 408 & 0.487 & 0.541 & 0.79 & 312 \\
\hline 05535500 & 1 & Yes & Yes & 1966 & 390 & $\mathrm{D}$ & 558 & 1.34 & 0.557 & 0.593 & 391 \\
\hline 05535500 & 1 & Yes & Yes & 1967 & 712 & -- & 1018 & 1.53 & 0.573 & 0.152 & 644 \\
\hline 05535500 & 1 & Yes & Yes & 1968 & 397 & -- & 568 & 1.23 & 0.59 & 0.608 & 384 \\
\hline 05535500 & 1 & Yes & Yes & 1969 & 634 & -- & 907 & 1.28 & 0.606 & 0.227 & 562 \\
\hline 05535500 & 1 & Yes & Yes & 1970 & 423 & -- & 605 & 1.32 & 0.622 & 0.587 & 393 \\
\hline 05535500 & 1 & Yes & Yes & 1971 & 358 & D & 512 & 0.389 & 0.635 & 0.729 & 338 \\
\hline 05535500 & 1 & Yes & Yes & 1972 & 680 & -- & 972 & 0.683 & 0.648 & 0.208 & 577 \\
\hline 05535500 & 1 & Yes & Yes & 1973 & 674 & -- & 964 & 1.39 & 0.662 & 0.222 & 566 \\
\hline 05535500 & 1 & Yes & Yes & 1974 & 557 & -- & 796 & 1.02 & 0.675 & 0.397 & 477 \\
\hline 05535500 & 1 & Yes & Yes & 1975 & 534 & D & 764 & 0.981 & 0.688 & 0.448 & 453 \\
\hline 05535500 & 1 & Yes & Yes & 1976 & 600 & -- & 858 & 0.931 & 0.702 & 0.35 & 496 \\
\hline 05535500 & 1 & Yes & Yes & 1977 & 175 & $\mathrm{E}$ & 250 & 1.18 & 0.715 & 0.976 & 173 \\
\hline 05535500 & 1 & Yes & Yes & 1978 & 569 & -- & 814 & 1.17 & 0.728 & 0.43 & 462 \\
\hline 05535500 & 1 & Yes & Yes & 1979 & 602 & -- & 861 & 0.87 & 0.741 & 0.39 & 479 \\
\hline 05535500 & 1 & Yes & Yes & 1980 & 582 & -- & 832 & 0.756 & 0.755 & 0.436 & 459 \\
\hline 05535500 & 1 & Yes & Yes & 1981 & 636 & -- & 909 & 0.934 & 0.759 & 0.351 & 495 \\
\hline 05535500 & 1 & Yes & Yes & 1982 & 1070 & -- & 1530 & 2.05 & 0.762 & 0.0641 & 807 \\
\hline 05535500 & 1 & Yes & Yes & 1983 & 744 & -- & 1064 & 1.51 & 0.766 & 0.222 & 566 \\
\hline 05535500 & 1 & Yes & Yes & 1984 & 646 & D & 924 & 1.28 & 0.77 & 0.347 & 497 \\
\hline 05535500 & 1 & Yes & Yes & 1985 & 666 & -- & 952 & 0.759 & 0.774 & 0.318 & 508 \\
\hline 05535500 & 1 & Yes & Yes & 1986 & 698 & -- & 998 & 0.789 & 0.778 & 0.278 & 528 \\
\hline 05535500 & 1 & Yes & Yes & 1987 & 1190 & -- & 1702 & 3 & 0.781 & 0.0459 & 879 \\
\hline 05535500 & 1 & Yes & Yes & 1988 & 346 & -- & 495 & 0.272 & 0.785 & 0.863 & 277 \\
\hline 05535500 & 1 & Yes & Yes & 1989 & 536 & -- & 766 & 1.68 & 0.789 & 0.547 & 409 \\
\hline 05535500 & 0 & Yes & No & 1990 & 719 & -- & 1142 & 2.33 & 0.793 & 0.195 & 591 \\
\hline 05535500 & 0 & Yes & No & 1991 & 446 & -- & 786 & 2.28 & 0.796 & 0.532 & 416 \\
\hline 05535500 & 0 & Yes & No & 1992 & 425 & $C, E$ & 832 & 2.06 & 0.8 & 0.484 & 438 \\
\hline 05535500 & 2 & Yes & Yes & 1993 & 418 & C & 908 & 0.536 & 0.803 & 0.403 & 474 \\
\hline 05535500 & 2 & Yes & Yes & 1994 & 767 & C & 1667 & 2.7 & 0.806 & 0.0524 & 845 \\
\hline 05535500 & 2 & Yes & Yes & 1995 & 390 & C & 847 & 1.39 & 0.81 & 0.477 & 441 \\
\hline
\end{tabular}




\begin{tabular}{|c|c|c|c|c|c|c|c|c|c|c|c|}
\hline $\begin{array}{l}\text { U.S. } \\
\text { Geological } \\
\text { Survey } \\
\text { streamgage } \\
\text { number }\end{array}$ & $\begin{array}{l}\text { Segment } \\
\text { number }\end{array}$ & $\begin{array}{l}\text { Streamgage used in } \\
\text { regression analyses } \\
\text { (non-redundant) }\end{array}$ & $\begin{array}{c}\text { Discharge value } \\
\text { used in } \\
\text { adjustment } \\
\text { regression }\end{array}$ & $\begin{array}{l}\text { Water } \\
\text { year }\end{array}$ & $\begin{array}{c}\text { Observed annual } \\
\text { maximum peak } \\
\text { discharge }\left(\mathrm{ft}^{3} / \mathrm{s}\right)\end{array}$ & $\begin{array}{l}\text { NWIS peak } \\
\text { code }\end{array}$ & $\begin{array}{l}\text { Observed annual } \\
\text { maximum peak } \\
\text { discharge with } \\
\text { segment intercept } \\
\text { value subtracted } \\
\left(\mathrm{ft}^{3} / \mathrm{s}\right)\end{array}$ & $\begin{array}{l}\text { Observed } \\
\text { precipitation } \\
\text { (inches) }\end{array}$ & Urban fraction & $\begin{array}{l}\text { Exceedance } \\
\text { probability }\end{array}$ & $\begin{array}{c}\text { Urban-adjusted } \\
\text { annual maximum } \\
\text { peak discharge } \\
\left(\mathrm{ft}^{3} / \mathrm{s}\right)\end{array}$ \\
\hline 05535500 & 2 & Yes & Yes & 1996 & 570 & C & 1239 & 0.564 & 0.813 & 0.165 & 629 \\
\hline 05535500 & 2 & Yes & Yes & 1997 & 507 & C & 1102 & 2.74 & 0.817 & 0.231 & 559 \\
\hline 05535500 & 2 & Yes & Yes & 1998 & 374 & $C, E$ & 813 & 1.54 & 0.82 & 0.526 & 419 \\
\hline 05535500 & 2 & Yes & Yes & 1999 & 522 & C & 1134 & 1.42 & 0.823 & 0.216 & 572 \\
\hline 05535500 & 2 & Yes & Yes & 2000 & 361 & C & 784 & 1.27 & 0.827 & 0.565 & 402 \\
\hline 05535500 & 2 & Yes & Yes & 2001 & 624 & C & 1356 & 2.88 & 0.836 & 0.133 & 673 \\
\hline 05535500 & 2 & Yes & Yes & 2002 & 848 & C & 1843 & 3.67 & 0.845 & 0.0424 & 902 \\
\hline 05535500 & 2 & Yes & Yes & 2003 & 369 & $\mathrm{C}, \mathrm{E}$ & 802 & 1.01 & 0.855 & 0.574 & 398 \\
\hline 05535500 & 2 & Yes & Yes & 2004 & 560 & C & 1217 & 1.42 & 0.864 & 0.196 & 590 \\
\hline 05535500 & 2 & Yes & Yes & 2005 & 300 & C & 652 & 0.535 & 0.873 & 0.773 & 319 \\
\hline 05535500 & 2 & Yes & Yes & 2006 & 308 & $C, E$ & 669 & 0.801 & 0.883 & 0.763 & 323 \\
\hline 05535500 & 2 & Yes & Yes & 2007 & 670 & C & 1456 & 2.81 & 0.892 & 0.123 & 687 \\
\hline 05535500 & 2 & Yes & Yes & 2008 & 637 & C & 1384 & 5.14 & 0.901 & 0.151 & 648 \\
\hline 05535500 & 2 & Yes & Yes & 2009 & 421 & C & 915 & 1.42 & 0.91 & 0.512 & 425 \\
\hline 05535700 & 1 & No & No & 1960 & 713 & -- & 785 & 0.33 & 0.455 & 0.227 & 1040 \\
\hline 05535700 & 1 & No & No & 1961 & 647 & -- & 712 & 1.57 & 0.474 & 0.309 & 952 \\
\hline 05535700 & 1 & No & No & 1962 & 491 & -- & 540 & 0.222 & 0.493 & 0.557 & 744 \\
\hline 05535700 & 1 & No & No & 1963 & 397 & -- & 437 & 0.944 & 0.511 & 0.73 & 616 \\
\hline 05535700 & 1 & No & No & 1964 & 432 & -- & 475 & 2.14 & 0.53 & 0.691 & 646 \\
\hline 05535700 & 1 & No & No & 1965 & 319 & -- & 351 & 0.384 & 0.549 & 0.862 & 500 \\
\hline 05535700 & 1 & No & No & 1966 & 678 & -- & 746 & 1.51 & 0.568 & 0.351 & 916 \\
\hline 05535700 & 1 & No & No & 1967 & 1140 & -- & 1255 & 1.93 & 0.587 & 0.0746 & 1450 \\
\hline 05535700 & 1 & No & No & 1968 & 527 & -- & 580 & 1.69 & 0.606 & 0.606 & 707 \\
\hline 05535700 & 1 & No & No & 1969 & 905 & -- & 996 & 1.3 & 0.624 & 0.184 & 1120 \\
\hline 05535700 & 1 & No & No & 1970 & 638 & -- & 702 & 1.3 & 0.643 & 0.478 & 809 \\
\hline 05535700 & 1 & No & No & 1971 & 472 & -- & 519 & 0.418 & 0.654 & 0.735 & 612 \\
\hline 05535700 & 1 & No & No & 1972 & 1000 & -- & 1101 & 2.11 & 0.664 & 0.155 & 1190 \\
\hline 05535700 & 1 & No & No & 1973 & 733 & -- & 807 & 0.712 & 0.674 & 0.383 & 890 \\
\hline 05535700 & 1 & No & No & 1974 & 666 & -- & 733 & 0.564 & 0.685 & 0.482 & 807 \\
\hline 05535700 & 1 & No & No & 1975 & 761 & -- & 838 & 1.16 & 0.695 & 0.368 & 902 \\
\hline 05535700 & 1 & No & No & 1976 & 937 & -- & 1031 & 1.41 & 0.706 & 0.205 & 1080 \\
\hline 05535700 & 1 & No & No & 1977 & 224 & -- & 247 & 1.17 & 0.716 & 0.978 & 297 \\
\hline 05535800 & 1 & No & No & 1960 & 1220 & 2 & 700 & 0.385 & 0.5 & 0.345 & 1320 \\
\hline 05535800 & 1 & No & No & 1961 & 1190 & -- & 683 & 1.4 & 0.512 & 0.378 & 1290 \\
\hline 05535800 & 1 & No & No & 1962 & 971 & -- & 557 & 0.227 & 0.524 & 0.561 & 1060 \\
\hline
\end{tabular}




\begin{tabular}{|c|c|c|c|c|c|c|c|c|c|c|c|}
\hline $\begin{array}{c}\text { U.S. } \\
\text { Geological } \\
\text { Survey } \\
\text { streamgage } \\
\text { number } \\
\end{array}$ & $\begin{array}{c}\text { Segment } \\
\text { number }\end{array}$ & $\begin{array}{l}\text { Streamgage used in } \\
\text { regression analyses } \\
\text { (non-redundant) }\end{array}$ & $\begin{array}{c}\text { Discharge value } \\
\text { used in } \\
\text { adjustment } \\
\text { regression }\end{array}$ & $\begin{array}{c}\text { Water } \\
\text { year }\end{array}$ & $\begin{array}{c}\text { Observed annual } \\
\text { maximum peak } \\
\text { discharge }\left(\mathrm{ft}^{3} / \mathrm{s}\right)\end{array}$ & $\begin{array}{c}\text { NWIS peak } \\
\text { code }\end{array}$ & $\begin{array}{c}\text { Observed annual } \\
\text { maximum peak } \\
\text { discharge with } \\
\text { segment intercept } \\
\text { value subtracted } \\
\left(\mathrm{ft}^{3} / \mathrm{s}\right)\end{array}$ & $\begin{array}{c}\text { Observed } \\
\text { precipitation } \\
\text { (inches) }\end{array}$ & Urban fraction & $\begin{array}{l}\text { Exceedance } \\
\text { probability }\end{array}$ & $\begin{array}{c}\text { Urban-adjusted } \\
\text { annual maximum } \\
\text { peak discharge } \\
\left(\mathrm{ft}^{3} / \mathrm{s}\right)\end{array}$ \\
\hline 05535800 & 1 & No & No & 1963 & 827 & -- & 474 & 1.12 & 0.536 & 0.698 & 917 \\
\hline 05535800 & 1 & No & No & 1964 & 763 & -- & 438 & 0.883 & 0.549 & 0.757 & 848 \\
\hline 05535800 & 1 & No & No & 1965 & 629 & -- & 361 & 0.166 & 0.561 & 0.857 & 716 \\
\hline 05535800 & 1 & No & No & 1966 & 1280 & -- & 734 & 1.31 & 0.573 & 0.371 & 1290 \\
\hline 05535800 & 1 & No & No & 1967 & 2130 & -- & 1222 & 1.66 & 0.585 & 0.0824 & 2040 \\
\hline 05535800 & 1 & No & No & 1968 & 793 & -- & 455 & 1.2 & 0.598 & 0.771 & 830 \\
\hline 05535800 & 1 & No & No & 1969 & 1350 & -- & 774 & 1.68 & 0.61 & 0.357 & 1310 \\
\hline 05535800 & 0 & No & No & 1970 & 1090 & -- & 659 & 1.55 & 0.622 & 0.514 & 1120 \\
\hline 05535800 & 0 & No & No & 1971 & 859 & -- & 547 & 0.379 & 0.628 & 0.676 & 939 \\
\hline 05535800 & 0 & No & No & 1972 & 1440 & -- & 966 & 1.45 & 0.634 & 0.203 & 1560 \\
\hline 05535800 & 0 & No & No & 1973 & 1040 & -- & 735 & 1.2 & 0.64 & 0.435 & 1210 \\
\hline 05535800 & 2 & No & No & 1974 & 1060 & -- & 789 & 1.09 & 0.647 & 0.376 & 1290 \\
\hline 05535800 & 2 & No & No & 1975 & 1080 & -- & 804 & 0.798 & 0.653 & 0.364 & 1300 \\
\hline 05535800 & 2 & No & No & 1976 & 1500 & $-{ }_{-1}$ & 1116 & 1.35 & 0.659 & 0.147 & 1750 \\
\hline 05535800 & 2 & No & No & 1977 & 661 & -- & 492 & 0.803 & 0.665 & 0.777 & 822 \\
\hline 05535800 & 2 & No & No & 1978 & 619 & -- & 461 & 1.34 & 0.671 & 0.817 & 770 \\
\hline 05535800 & 0 & No & No & 1979 & 1000 & -- & NA & 0.824 & 0.677 & NA & NA \\
\hline 05536000 & 1 & Yes & Yes & 1951 & 952 & -- & 549 & 1.68 & 0.351 & 0.414 & 1490 \\
\hline 05536000 & 1 & Yes & Yes & 1952 & 870 & -- & 502 & 0.981 & 0.371 & 0.503 & 1360 \\
\hline 05536000 & 1 & Yes & Yes & 1953 & 428 & $E$ & 247 & 0.756 & 0.39 & 0.898 & 790 \\
\hline 05536000 & 1 & Yes & Yes & 1954 & 1510 & -- & 871 & 1.52 & 0.41 & 0.154 & 2070 \\
\hline 05536000 & 1 & Yes & Yes & 1955 & 1700 & -- & 981 & 2.85 & 0.429 & 0.11 & 2270 \\
\hline 05536000 & 1 & Yes & Yes & 1956 & 1030 & -- & 594 & 1.26 & 0.449 & 0.436 & 1460 \\
\hline 05536000 & 1 & Yes & Yes & 1957 & 1850 & -- & 1068 & 2.13 & 0.468 & 0.0919 & 2380 \\
\hline 05536000 & 1 & Yes & Yes & 1958 & 647 & -- & 373 & 0.18 & 0.488 & 0.798 & 957 \\
\hline 05536000 & 1 & Yes & Yes & 1959 & 695 & -- & 401 & 1.14 & 0.507 & 0.774 & 992 \\
\hline 05536000 & 1 & Yes & Yes & 1960 & 1340 & -- & 773 & 0.382 & 0.527 & 0.285 & 1690 \\
\hline 05536000 & 1 & Yes & Yes & 1961 & 1290 & -- & 745 & 1.4 & 0.538 & 0.326 & 1620 \\
\hline 05536000 & 1 & Yes & Yes & 1962 & 1010 & -- & 583 & 0.228 & 0.55 & 0.548 & 1290 \\
\hline 05536000 & 1 & Yes & Yes & 1963 & 905 & $\mathrm{E}$ & 522 & 1.11 & 0.561 & 0.648 & 1160 \\
\hline 05536000 & 1 & Yes & Yes & 1964 & 810 & -- & 467 & 0.879 & 0.573 & 0.737 & 1050 \\
\hline 05536000 & 1 & Yes & Yes & 1965 & 658 & -- & 380 & 0.717 & 0.584 & 0.85 & 872 \\
\hline 05536000 & 1 & Yes & Yes & 1966 & 1360 & -- & 785 & 1.32 & 0.596 & 0.331 & 1610 \\
\hline 05536000 & 1 & Yes & Yes & 1967 & 2210 & -- & 1275 & 1.65 & 0.608 & 0.075 & 2500 \\
\hline 05536000 & 1 & Yes & Yes & 1968 & 858 & -- & 495 & 1.21 & 0.619 & 0.738 & 1050 \\
\hline
\end{tabular}




\begin{tabular}{|c|c|c|c|c|c|c|c|c|c|c|c|}
\hline $\begin{array}{c}\text { U.S. } \\
\text { Geological } \\
\text { Survey } \\
\text { streamgage } \\
\text { number }\end{array}$ & $\begin{array}{l}\text { Segment } \\
\text { number }\end{array}$ & $\begin{array}{l}\text { Streamgage used in } \\
\text { regression analyses } \\
\text { (non-redundant) }\end{array}$ & $\begin{array}{l}\text { Discharge value } \\
\text { used in } \\
\text { adjustment } \\
\text { regression }\end{array}$ & $\begin{array}{l}\text { Water } \\
\text { year }\end{array}$ & $\begin{array}{l}\text { Observed annual } \\
\text { maximum peak } \\
\text { discharge }\left(\mathrm{ft}^{3} / \mathrm{s}\right)\end{array}$ & $\begin{array}{l}\text { NWIS peak } \\
\text { code }\end{array}$ & $\begin{array}{l}\text { Observed annual } \\
\text { maximum peak } \\
\text { discharge with } \\
\text { segment intercept } \\
\text { value subtracted } \\
\left(\mathrm{ft}^{3} / \mathrm{s}\right)\end{array}$ & $\begin{array}{l}\text { Observed } \\
\text { precipitation } \\
\text { (inches) }\end{array}$ & Urban fraction & $\begin{array}{l}\text { Exceedance } \\
\text { probability }\end{array}$ & $\begin{array}{c}\text { Urban-adjusted } \\
\text { annual maximum } \\
\text { peak discharge } \\
\left(\mathrm{ft}^{3} / \mathrm{s}\right)\end{array}$ \\
\hline 05536000 & 1 & Yes & Yes & 1969 & 1300 & -- & 750 & 1.65 & 0.631 & 0.407 & 1500 \\
\hline 05536000 & 0 & Yes & No & 1970 & 1170 & -- & 688 & 1.52 & 0.642 & 0.495 & 1370 \\
\hline 05536000 & 0 & Yes & No & 1971 & 1070 & D & 642 & 0.378 & 0.648 & 0.561 & 1280 \\
\hline 05536000 & 0 & Yes & No & 1972 & 1440 & -- & 880 & 1.45 & 0.653 & 0.281 & 1690 \\
\hline 05536000 & 0 & Yes & No & 1973 & 1050 & -- & 654 & 1.21 & 0.659 & 0.555 & 1280 \\
\hline 05536000 & 2 & Yes & Yes & 1974 & 1070 & -- & 680 & 1.08 & 0.664 & 0.528 & 1320 \\
\hline 05536000 & 2 & Yes & Yes & 1975 & 1160 & -- & 737 & 0.845 & 0.67 & 0.462 & 1420 \\
\hline 05536000 & 2 & Yes & Yes & 1976 & 1550 & D & 985 & 1.36 & 0.675 & 0.216 & 1840 \\
\hline 05536000 & 2 & Yes & Yes & 1977 & 723 & $E$ & 459 & 0.811 & 0.681 & 0.826 & 913 \\
\hline 05536000 & 2 & Yes & Yes & 1978 & 1150 & -- & 731 & 1.03 & 0.686 & 0.486 & 1380 \\
\hline 05536000 & 0 & Yes & No & 1979 & 1150 & -- & 734 & 0.819 & 0.692 & 0.488 & 1380 \\
\hline 05536000 & 3 & Yes & Yes & 1980 & 712 & -- & 457 & 0.814 & 0.697 & 0.84 & 889 \\
\hline 05536000 & 3 & Yes & Yes & 1981 & 1160 & -- & 744 & 0.932 & 0.701 & 0.485 & 1390 \\
\hline 05536000 & 3 & Yes & Yes & 1982 & 1420 & -- & 911 & 2.57 & 0.704 & 0.294 & 1670 \\
\hline 05536000 & 3 & Yes & Yes & 1983 & 1480 & -- & 949 & 1.84 & 0.708 & 0.263 & 1720 \\
\hline 05536000 & 3 & Yes & Yes & 1984 & 1210 & -- & 776 & 1.77 & 0.711 & 0.456 & 1430 \\
\hline 05536000 & 3 & Yes & Yes & 1985 & 1350 & -- & 866 & 1.12 & 0.714 & 0.354 & 1580 \\
\hline 05536000 & 3 & Yes & Yes & 1986 & 1800 & -- & 1154 & 1.07 & 0.718 & 0.156 & 2060 \\
\hline 05536000 & 3 & Yes & Yes & 1987 & 2590 & -- & 1661 & 4.03 & 0.721 & 0.0427 & 2930 \\
\hline 05536000 & 3 & Yes & Yes & 1988 & 831 & $\mathrm{E}$ & 533 & 0.445 & 0.724 & 0.776 & 989 \\
\hline 05536000 & 3 & Yes & Yes & 1989 & 1780 & -- & 1141 & 2.2 & 0.728 & 0.166 & 2020 \\
\hline 05536000 & 3 & Yes & Yes & 1990 & 1600 & -- & 1026 & 1.84 & 0.731 & 0.224 & 1820 \\
\hline 05536000 & 3 & Yes & Yes & 1991 & 1360 & -- & 872 & 2.26 & 0.734 & 0.369 & 1560 \\
\hline 05536000 & 0 & Yes & No & 1992 & 893 & $C, E$ & 563 & 2.12 & 0.738 & 0.753 & 1020 \\
\hline 05536000 & 4 & Yes & Yes & 1993 & 1250 & C & 776 & 1 & 0.741 & 0.488 & 1380 \\
\hline 05536000 & 4 & Yes & Yes & 1994 & 1530 & C & 950 & 2.01 & 0.745 & 0.292 & 1670 \\
\hline 05536000 & 4 & Yes & Yes & 1995 & 1330 & $C, E$ & 826 & 1.32 & 0.748 & 0.437 & 1460 \\
\hline 05536000 & 4 & Yes & Yes & 1996 & 1220 & C & 757 & 0.805 & 0.752 & 0.52 & 1340 \\
\hline 05536000 & 4 & Yes & Yes & 1997 & 2260 & C & 1403 & 2.79 & 0.755 & 0.0866 & 2420 \\
\hline 05536000 & 4 & Yes & Yes & 1998 & 993 & C & 616 & 1.09 & 0.759 & 0.71 & 1090 \\
\hline 05536000 & 4 & Yes & Yes & 1999 & 1800 & C & 1117 & 1.31 & 0.762 & 0.191 & 1920 \\
\hline 05536000 & 4 & Yes & Yes & 2000 & 1290 & $C, E$ & 801 & 1.7 & 0.765 & 0.483 & 1390 \\
\hline 05536000 & 4 & Yes & Yes & 2001 & 1380 & C & 857 & 0.686 & 0.772 & 0.427 & 1470 \\
\hline 05536000 & 4 & Yes & Yes & 2002 & 1940 & C & 1204 & 3.13 & 0.779 & 0.163 & 2040 \\
\hline 05536000 & 4 & Yes & Yes & 2003 & 1130 & C & 702 & 1.36 & 0.785 & 0.623 & 1190 \\
\hline
\end{tabular}




\begin{tabular}{|c|c|c|c|c|c|c|c|c|c|c|c|}
\hline $\begin{array}{l}\text { U.S. } \\
\text { Geological } \\
\text { Survey } \\
\text { streamgage } \\
\text { number }\end{array}$ & $\begin{array}{c}\text { Segment } \\
\text { number }\end{array}$ & $\begin{array}{l}\text { Streamgage used in } \\
\text { regression analyses } \\
\text { (non-redundant) }\end{array}$ & $\begin{array}{c}\text { Discharge value } \\
\text { used in } \\
\text { adjustment } \\
\text { regression }\end{array}$ & $\begin{array}{l}\text { Water } \\
\text { year }\end{array}$ & $\begin{array}{c}\text { Observed annual } \\
\text { maximum peak } \\
\text { discharge }\left(\mathrm{ft}^{3} / \mathrm{s}\right)\end{array}$ & $\begin{array}{l}\text { NWIS peak } \\
\text { code }\end{array}$ & $\begin{array}{l}\text { Observed annual } \\
\text { maximum peak } \\
\text { discharge with } \\
\text { segment intercept } \\
\text { value subtracted } \\
\left(\mathrm{ft}^{3} / \mathrm{s}\right)\end{array}$ & $\begin{array}{l}\text { Observed } \\
\text { precipitation } \\
\text { (inches) }\end{array}$ & Urban fraction & $\begin{array}{l}\text { Exceedance } \\
\text { probability }\end{array}$ & $\begin{array}{c}\text { Urban-adjusted } \\
\text { annual maximum } \\
\text { peak discharge } \\
\left(\mathrm{ft}^{3} / \mathrm{s}\right)\end{array}$ \\
\hline 05536000 & 4 & Yes & Yes & 2004 & 1130 & $C, E$ & 702 & 1.2 & 0.792 & 0.631 & 1180 \\
\hline 05536000 & 4 & Yes & Yes & 2005 & 1210 & $C, E$ & 751 & 0.701 & 0.798 & 0.575 & 1260 \\
\hline 05536000 & 4 & Yes & Yes & 2006 & 965 & C & 599 & 0.753 & 0.805 & 0.769 & 999 \\
\hline 05536000 & 4 & Yes & Yes & 2007 & 1730 & C & 1074 & 1.46 & 0.812 & 0.245 & 1760 \\
\hline 05536000 & 4 & Yes & Yes & 2008 & 3340 & C & 2073 & 4.23 & 0.818 & 0.0261 & 3370 \\
\hline 05536000 & 4 & Yes & Yes & 2009 & 1980 & $C$ & 1229 & 2.14 & 0.825 & 0.174 & 1990 \\
\hline 05536105 & 1 & No & No & 1990 & 1890 & -- & 1188 & 1.97 & 0.746 & 0.155 & 2050 \\
\hline 05536105 & 1 & No & No & 1991 & 1540 & -- & 968 & 2.3 & 0.749 & 0.28 & 1680 \\
\hline 05536105 & 1 & No & No & 1992 & 965 & $C, D$ & 607 & 2.16 & 0.752 & 0.715 & 1070 \\
\hline 05536105 & 1 & No & No & 1993 & 1290 & C & 811 & 1.09 & 0.755 & 0.461 & 1410 \\
\hline 05536105 & 1 & No & No & 1994 & 1400 & C & 880 & 0.629 & 0.758 & 0.386 & 1520 \\
\hline 05536105 & 1 & No & No & 1995 & 1370 & $C, E$ & 861 & 1.23 & 0.761 & 0.411 & 1480 \\
\hline 05536105 & 1 & No & No & 1996 & 1280 & C & 805 & 0.856 & 0.764 & 0.478 & 1380 \\
\hline 05536105 & 1 & No & No & 1997 & 2360 & $\mathrm{C}$ & 1483 & 2.79 & 0.767 & 0.0723 & 2500 \\
\hline 05536105 & 1 & No & No & 1998 & 954 & $C, E$ & 600 & 1.16 & 0.77 & 0.739 & 1040 \\
\hline 05536105 & 1 & No & No & 2000 & 963 & $C, E$ & 605 & 1.07 & 0.776 & 0.737 & 1040 \\
\hline 05536105 & 1 & No & No & 2001 & 1620 & C & 1018 & 0.845 & 0.782 & 0.264 & 1710 \\
\hline 05536105 & 1 & No & No & 2002 & 1800 & C & 1131 & 3.18 & 0.788 & 0.197 & 1880 \\
\hline 05536105 & 1 & No & No & 2003 & 1130 & $C, E$ & 710 & 1.39 & 0.793 & 0.621 & 1190 \\
\hline 05536105 & 1 & No & No & 2004 & 1030 & $C, E$ & 647 & 1.12 & 0.799 & 0.711 & 1080 \\
\hline 05536105 & 1 & No & No & 2005 & 1200 & $C, E$ & 754 & 0.697 & 0.805 & 0.578 & 1240 \\
\hline 05536105 & 1 & No & No & 2006 & 1060 & C & 666 & 0.765 & 0.811 & 0.702 & 1090 \\
\hline 05536105 & 1 & No & No & 2007 & 2110 & C & 1326 & 1.55 & 0.817 & 0.135 & 2140 \\
\hline 05536105 & 1 & No & No & 2008 & 3580 & C & 2250 & 4.3 & 0.823 & 0.0195 & 3610 \\
\hline 05536105 & 1 & No & No & 2009 & 2420 & C & 1521 & 2.21 & 0.829 & 0.0811 & 2430 \\
\hline 05536178 & 1 & No & No & 1955 & 2480 & 7 & 876 & 3.37 & 0.0278 & 0.0529 & 3620 \\
\hline 05536178 & 1 & No & No & 1966 & 2080 & -- & 735 & 1.51 & 0.0561 & 0.0928 & 3020 \\
\hline 05536178 & 1 & No & No & 1967 & 991 & -- & 350 & 1.18 & 0.0586 & 0.509 & 1580 \\
\hline 05536178 & 1 & No & No & 1968 & 1390 & -- & 491 & 1.58 & 0.0611 & 0.284 & 2090 \\
\hline 05536178 & 1 & No & No & 1969 & 916 & -- & 324 & 0.496 & 0.0636 & 0.574 & 1460 \\
\hline 05536178 & 1 & No & No & 1970 & 1330 & -- & 470 & 1.49 & 0.0661 & 0.315 & 2010 \\
\hline 05536178 & 1 & No & No & 1971 & 430 & -- & 152 & 0.973 & 0.0736 & 0.896 & 796 \\
\hline 05536178 & 1 & No & No & 1972 & 816 & -- & 288 & 1.26 & 0.0812 & 0.658 & 1300 \\
\hline 05536178 & 1 & No & No & 1973 & 1860 & -- & 657 & 0.92 & 0.0887 & 0.147 & 2640 \\
\hline 05536178 & 1 & No & No & 1974 & 1380 & -- & 488 & 0.983 & 0.0963 & 0.31 & 2020 \\
\hline
\end{tabular}




\begin{tabular}{|c|c|c|c|c|c|c|c|c|c|c|c|}
\hline $\begin{array}{l}\text { U.S. } \\
\text { Geological } \\
\text { Survey } \\
\text { streamgage } \\
\text { number }\end{array}$ & $\begin{array}{l}\text { Segment } \\
\text { number }\end{array}$ & $\begin{array}{l}\text { Streamgage used in } \\
\text { regression analyses } \\
\text { (non-redundant) }\end{array}$ & $\begin{array}{l}\text { Discharge value } \\
\text { used in } \\
\text { adjustment } \\
\text { regression }\end{array}$ & $\begin{array}{l}\text { Water } \\
\text { year }\end{array}$ & $\begin{array}{l}\text { Observed annual } \\
\text { maximum peak } \\
\text { discharge }\left(\mathrm{ft}^{3} / \mathrm{s}\right)\end{array}$ & $\begin{array}{l}\text { NWIS peak } \\
\text { code }\end{array}$ & $\begin{array}{c}\text { Observed annual } \\
\text { maximum peak } \\
\text { discharge with } \\
\text { segment intercept } \\
\text { value subtracted } \\
\left(\mathrm{ft}^{3} / \mathrm{s}\right)\end{array}$ & $\begin{array}{l}\text { Observed } \\
\text { precipitation } \\
\text { (inches) }\end{array}$ & Urban fraction & $\begin{array}{l}\text { Exceedance } \\
\text { probability }\end{array}$ & $\begin{array}{c}\text { Urban-adjusted } \\
\text { annual maximum } \\
\text { peak discharge } \\
\left(\mathrm{ft}^{3} / \mathrm{s}\right)\end{array}$ \\
\hline 05536178 & 1 & No & No & 1975 & 1600 & -- & 565 & 1.34 & 0.104 & 0.222 & 2260 \\
\hline 05536178 & 1 & No & No & 1976 & 914 & -- & 323 & 1.57 & 0.111 & 0.613 & 1390 \\
\hline 05536178 & 1 & No & No & 1977 & 285 & -- & 101 & 0.722 & 0.119 & 0.957 & 545 \\
\hline 05536179 & 1 & No & No & 1990 & 927 & -- & 411 & 1.57 & 0.218 & 0.526 & 1240 \\
\hline 05536179 & 1 & No & No & 1991 & 3010 & -- & 1333 & 3.02 & 0.226 & 0.023 & 3650 \\
\hline 05536179 & 1 & No & No & 1992 & 631 & $\mathrm{E}$ & 279 & 0.532 & 0.234 & 0.775 & 868 \\
\hline 05536179 & 1 & No & No & 1993 & 1110 & -- & 492 & 1.85 & 0.242 & 0.408 & 1430 \\
\hline 05536179 & 1 & No & No & 1994 & 1590 & -- & 704 & 0.981 & 0.25 & 0.181 & 1960 \\
\hline 05536179 & 1 & No & No & 1995 & 721 & $E$ & 319 & 1.02 & 0.257 & 0.725 & 950 \\
\hline 05536179 & 1 & No & No & 1996 & 1420 & -- & 629 & 3.33 & 0.265 & 0.252 & 1740 \\
\hline 05536179 & 1 & No & No & 1997 & 1650 & -- & 731 & 2.06 & 0.273 & 0.175 & 1990 \\
\hline 05536179 & 1 & No & No & 1998 & 1230 & -- & 545 & 1.84 & 0.281 & 0.364 & 1510 \\
\hline 05536179 & 1 & No & No & 1999 & 1080 & -- & 478 & 0.607 & 0.289 & 0.469 & 1330 \\
\hline 05536179 & 1 & No & No & 2000 & 950 & -- & 421 & 1.29 & 0.297 & 0.578 & 1170 \\
\hline 05536179 & 1 & No & No & 2001 & 1120 & -- & 496 & 0.692 & 0.315 & 0.463 & 1340 \\
\hline 05536179 & 1 & No & No & 2002 & 1120 & -- & 496 & 1.68 & 0.332 & 0.478 & 1310 \\
\hline 05536179 & 1 & No & No & 2003 & 887 & -- & 393 & 1.24 & 0.35 & 0.669 & 1030 \\
\hline 05536179 & 1 & No & No & 2004 & 453 & -- & 201 & 0.88 & 0.368 & 0.932 & 543 \\
\hline 05536179 & 1 & No & No & 2005 & 1170 & -- & 518 & 1.17 & 0.386 & 0.491 & 1290 \\
\hline 05536179 & 1 & No & No & 2006 & 1430 & -- & 633 & 3.23 & 0.404 & 0.346 & 1540 \\
\hline 05536179 & 1 & No & No & 2007 & 2660 & -- & 1178 & 2.01 & 0.422 & 0.0585 & 2790 \\
\hline 05536179 & 1 & No & No & 2008 & 3110 & -- & 1377 & 4.33 & 0.44 & 0.0376 & 3200 \\
\hline 05536179 & 1 & No & No & 2009 & 1450 & -- & 642 & 1.17 & 0.457 & 0.381 & 1480 \\
\hline 05536190 & 1 & Yes & Yes & 1943 & 2280 & -- & 773 & 0.967 & 0.124 & 0.0957 & 3620 \\
\hline 05536190 & 1 & Yes & Yes & 1944 & 2420 & -- & 820 & 1.41 & 0.131 & 0.0814 & 3800 \\
\hline 05536190 & 1 & Yes & Yes & 1945 & 1270 & -- & 430 & 0.997 & 0.138 & 0.423 & 2190 \\
\hline 05536190 & 1 & Yes & Yes & 1946 & 780 & -- & 264 & 0.338 & 0.145 & 0.749 & 1480 \\
\hline 05536190 & 1 & Yes & Yes & 1947 & 2490 & -- & 844 & 2.89 & 0.152 & 0.0786 & 3830 \\
\hline 05536190 & 1 & Yes & Yes & 1948 & 1950 & -- & 661 & 1.04 & 0.159 & 0.172 & 3040 \\
\hline 05536190 & 1 & Yes & Yes & 1949 & 850 & -- & 288 & 0.919 & 0.166 & 0.717 & 1540 \\
\hline 05536190 & 1 & Yes & Yes & 1950 & 1570 & -- & 532 & 1.51 & 0.172 & 0.303 & 2510 \\
\hline 05536190 & 1 & Yes & Yes & 1951 & 1430 & -- & 485 & 2.24 & 0.183 & 0.373 & 2320 \\
\hline 05536190 & 1 & Yes & Yes & 1952 & 1190 & -- & 403 & 1.19 & 0.193 & 0.517 & 1970 \\
\hline 05536190 & 1 & Yes & Yes & 1953 & 960 & -- & 325 & 0.571 & 0.203 & 0.674 & 1630 \\
\hline 05536190 & 1 & Yes & Yes & 1954 & 1110 & -- & 376 & 1.51 & 0.214 & 0.591 & 1820 \\
\hline
\end{tabular}




\begin{tabular}{|c|c|c|c|c|c|c|c|c|c|c|c|}
\hline $\begin{array}{c}\text { U.S. } \\
\text { Geological } \\
\text { Survey } \\
\text { streamgage } \\
\text { number }\end{array}$ & $\begin{array}{l}\text { Segment } \\
\text { number }\end{array}$ & $\begin{array}{l}\text { Streamgage used in } \\
\text { regression analyses } \\
\text { (non-redundant) }\end{array}$ & $\begin{array}{l}\text { Discharge value } \\
\text { used in } \\
\text { adjustment } \\
\text { regression }\end{array}$ & $\begin{array}{l}\text { Water } \\
\text { year }\end{array}$ & $\begin{array}{c}\text { Observed annual } \\
\text { maximum peak } \\
\text { discharge }\left(\mathrm{ft}^{3} / \mathrm{s}\right)\end{array}$ & $\begin{array}{l}\text { NWIS peak } \\
\text { code }\end{array}$ & $\begin{array}{l}\text { Observed annual } \\
\text { maximum peak } \\
\text { discharge with } \\
\text { segment intercept } \\
\text { value subtracted } \\
\left(\mathrm{ft}^{3} / \mathrm{s}\right)\end{array}$ & $\begin{array}{l}\text { Observed } \\
\text { precipitation } \\
\text { (inches) }\end{array}$ & Urban fraction & $\begin{array}{l}\text { Exceedance } \\
\text { probability }\end{array}$ & $\begin{array}{c}\text { Urban-adjusted } \\
\text { annual maximum } \\
\text { peak discharge } \\
\left(\mathrm{ft}^{3} / \mathrm{s}\right)\end{array}$ \\
\hline 05536190 & 1 & Yes & Yes & 1955 & 2600 & -- & 881 & 3.41 & 0.224 & 0.0843 & 3760 \\
\hline 05536190 & 1 & Yes & Yes & 1956 & 1550 & -- & 525 & 0.778 & 0.234 & 0.355 & 2370 \\
\hline 05536190 & 1 & Yes & Yes & 1957 & 2060 & -- & 698 & 2.27 & 0.244 & 0.183 & 2970 \\
\hline 05536190 & 1 & Yes & Yes & 1958 & 960 & -- & 325 & 1.2 & 0.255 & 0.712 & 1550 \\
\hline 05536190 & 1 & Yes & Yes & 1959 & 2670 & -- & 905 & 1.37 & 0.265 & 0.0871 & 3730 \\
\hline 05536190 & 1 & Yes & Yes & 1960 & 855 & -- & 290 & 1.65 & 0.275 & 0.783 & 1390 \\
\hline 05536190 & 1 & Yes & Yes & 1961 & 960 & -- & 325 & 1.71 & 0.281 & 0.731 & 1510 \\
\hline 05536190 & 1 & Yes & Yes & 1962 & 715 & -- & 242 & 0.234 & 0.286 & 0.857 & 1190 \\
\hline 05536190 & 1 & Yes & Yes & 1963 & 885 & -- & 300 & 1.59 & 0.291 & 0.777 & 1400 \\
\hline 05536190 & 1 & Yes & Yes & 1964 & 465 & -- & 158 & 1.03 & 0.296 & 0.947 & 854 \\
\hline 05536190 & 1 & Yes & Yes & 1965 & 1160 & -- & 393 & 0.946 & 0.301 & 0.63 & 1730 \\
\hline 05536190 & 1 & Yes & Yes & 1966 & 2500 & -- & 847 & 1.7 & 0.307 & 0.125 & 3380 \\
\hline 05536190 & 1 & Yes & Yes & 1967 & 950 & -- & 322 & 1.2 & 0.312 & 0.757 & 1450 \\
\hline 05536190 & 1 & Yes & Yes & 1968 & 1250 & -- & 424 & 1.96 & 0.317 & 0.591 & 1810 \\
\hline 05536190 & 1 & Yes & Yes & 1969 & 720 & -- & 244 & 0.472 & 0.322 & 0.873 & 1150 \\
\hline 05536190 & 1 & Yes & Yes & 1970 & 1050 & -- & 356 & 1.47 & 0.328 & 0.714 & 1550 \\
\hline 05536190 & 1 & Yes & Yes & 1971 & 629 & -- & 213 & 0.962 & 0.338 & 0.913 & 1020 \\
\hline 05536190 & 1 & Yes & Yes & 1972 & 1250 & -- & 424 & 1.36 & 0.348 & 0.616 & 1760 \\
\hline 05536190 & 1 & Yes & Yes & 1973 & 1500 & -- & 508 & 0.841 & 0.358 & 0.481 & 2050 \\
\hline 05536190 & 1 & Yes & Yes & 1974 & 1540 & -- & 522 & 1.35 & 0.368 & 0.47 & 2080 \\
\hline 05536190 & 1 & Yes & Yes & 1975 & 1450 & -- & 491 & 1.31 & 0.378 & 0.527 & 1950 \\
\hline 05536190 & 1 & Yes & Yes & 1976 & 1270 & -- & 430 & 0.844 & 0.388 & 0.638 & 1710 \\
\hline 05536190 & 1 & Yes & Yes & 1977 & 1200 & -- & 407 & 1.11 & 0.398 & 0.686 & 1610 \\
\hline 05536190 & 1 & Yes & Yes & 1978 & 1120 & -- & 380 & 0.429 & 0.408 & 0.736 & 1500 \\
\hline 05536190 & 1 & Yes & Yes & 1979 & 1390 & -- & 471 & 0.783 & 0.418 & 0.6 & 1800 \\
\hline 05536190 & 1 & Yes & Yes & 1980 & 1380 & -- & 468 & 0.755 & 0.429 & 0.613 & 1770 \\
\hline 05536190 & 1 & Yes & No & 1981 & 1850 & 1,8 & 627 & 1.9 & 0.433 & 0.379 & 2300 \\
\hline 05536190 & 1 & Yes & Yes & 1982 & 1880 & -- & 637 & 1.24 & 0.438 & 0.37 & 2320 \\
\hline 05536190 & 1 & Yes & Yes & 1983 & 2110 & -- & 715 & 1.99 & 0.442 & 0.282 & 2560 \\
\hline 05536190 & 1 & Yes & Yes & 1984 & 1560 & -- & 529 & 0.69 & 0.447 & 0.531 & 1940 \\
\hline 05536190 & 1 & Yes & Yes & 1985 & 2400 & 2 & 813 & 0.891 & 0.451 & 0.202 & 2860 \\
\hline 05536190 & 1 & Yes & Yes & 1986 & 1700 & 2 & 576 & 1.12 & 0.456 & 0.469 & 2080 \\
\hline 05536190 & 1 & Yes & Yes & 1987 & 1640 & -- & 556 & 1.25 & 0.461 & 0.503 & 2000 \\
\hline 05536190 & 1 & Yes & Yes & 1988 & 1300 & -- & 441 & 0.76 & 0.465 & 0.688 & 1610 \\
\hline 05536190 & 1 & Yes & Yes & 1989 & 2100 & -- & 712 & 1.52 & 0.47 & 0.306 & 2490 \\
\hline
\end{tabular}




\begin{tabular}{|c|c|c|c|c|c|c|c|c|c|c|c|}
\hline $\begin{array}{c}\text { U.S. } \\
\text { Geological } \\
\text { Survey } \\
\text { streamgage } \\
\text { number }\end{array}$ & $\begin{array}{l}\text { Segment } \\
\text { number }\end{array}$ & $\begin{array}{l}\text { Streamgage used in } \\
\text { regression analyses } \\
\text { (non-redundant) }\end{array}$ & $\begin{array}{c}\text { Discharge value } \\
\text { used in } \\
\text { adjustment } \\
\text { regression }\end{array}$ & $\begin{array}{l}\text { Water } \\
\text { year }\end{array}$ & $\begin{array}{c}\text { Observed annual } \\
\text { maximum peak } \\
\text { discharge }\left(\mathrm{ft}^{3} / \mathrm{s}\right)\end{array}$ & $\begin{array}{l}\text { NWIS peak } \\
\text { code }\end{array}$ & $\begin{array}{l}\text { Observed annual } \\
\text { maximum peak } \\
\text { discharge with } \\
\text { segment intercept } \\
\text { value subtracted } \\
\left(\mathrm{ft}^{3} / \mathrm{s}\right)\end{array}$ & $\begin{array}{l}\text { Observed } \\
\text { precipitation } \\
\text { (inches) }\end{array}$ & Urban fraction & $\begin{array}{l}\text { Exceedance } \\
\text { probability }\end{array}$ & $\begin{array}{c}\text { Urban-adjusted } \\
\text { annual maximum } \\
\text { peak discharge } \\
\left(\mathrm{ft}^{3} / \mathrm{s}\right)\end{array}$ \\
\hline 05536190 & 1 & Yes & Yes & 1990 & 2170 & -- & 735 & 1.54 & 0.474 & 0.285 & 2550 \\
\hline 05536190 & 1 & Yes & Yes & 1991 & 3010 & -- & 1020 & 3.01 & 0.481 & 0.114 & 3470 \\
\hline 05536190 & 1 & Yes & Yes & 1992 & 1540 & -- & 522 & 2.65 & 0.488 & 0.581 & 1830 \\
\hline 05536190 & 1 & Yes & Yes & 1993 & 2360 & -- & 800 & 1.9 & 0.495 & 0.239 & 2710 \\
\hline 05536190 & 1 & Yes & Yes & 1994 & 2490 & -- & 844 & 0.908 & 0.502 & 0.209 & 2830 \\
\hline 05536190 & 1 & Yes & Yes & 1995 & 1590 & -- & 539 & 1.27 & 0.509 & 0.574 & 1850 \\
\hline 05536190 & 1 & Yes & Yes & 1996 & 2710 & -- & 918 & 3.48 & 0.515 & 0.174 & 3040 \\
\hline 05536190 & 1 & Yes & Yes & 1997 & 2700 & -- & 915 & 2.08 & 0.522 & 0.179 & 3010 \\
\hline 05536190 & 1 & Yes & Yes & 1998 & 2370 & -- & 803 & 1.29 & 0.529 & 0.259 & 2640 \\
\hline 05536190 & 1 & Yes & Yes & 1999 & 2470 & -- & 837 & 0.646 & 0.536 & 0.235 & 2720 \\
\hline 05536190 & 1 & Yes & No & 2000 & 1700 & 1,8 & 576 & 1.39 & 0.543 & 0.551 & 1900 \\
\hline 05536190 & 1 & Yes & No & 2001 & 1250 & 1,8 & 424 & 0.667 & 0.552 & 0.777 & 1400 \\
\hline 05536190 & 1 & Yes & No & 2002 & 2500 & 1,8 & 847 & 1.88 & 0.562 & 0.243 & 2690 \\
\hline 05536190 & 1 & Yes & Yes & 2003 & 2400 & -- & 813 & 1.24 & 0.572 & 0.28 & 2560 \\
\hline 05536190 & 1 & Yes & Yes & 2004 & 1440 & -- & 488 & 0.835 & 0.581 & 0.716 & 1550 \\
\hline 05536190 & 1 & Yes & Yes & 2005 & 2670 & -- & 905 & 1.13 & 0.591 & 0.219 & 2790 \\
\hline 05536190 & 1 & Yes & Yes & 2006 & 3260 & -- & 1105 & 1.23 & 0.6 & 0.127 & 3380 \\
\hline 05536190 & 1 & Yes & Yes & 2007 & 3060 & -- & 1037 & 1.89 & 0.61 & 0.16 & 3140 \\
\hline 05536190 & 1 & Yes & Yes & 2008 & 3840 & 2 & 1301 & 4.46 & 0.619 & 0.0729 & 3910 \\
\hline 05536190 & 1 & Yes & Yes & 2009 & 3160 & 2 & 1071 & 1.22 & 0.629 & 0.153 & 3190 \\
\hline 05536201 & 1 & Yes & No & 1955 & 475 & 7 & 762 & 4.9 & 0.198 & 0.128 & 672 \\
\hline 05536201 & 1 & Yes & No & 1957 & 1320 & 7 & 2117 & 2.54 & 0.212 & 0.00638 & 1710 \\
\hline 05536201 & 1 & Yes & Yes & 1962 & 116 & -- & 186 & 0.215 & 0.247 & 0.911 & 197 \\
\hline 05536201 & 1 & Yes & No & 1963 & 60 & 4, B & 96 & 0.215 & 0.255 & 0.98 & 116 \\
\hline 05536201 & 1 & Yes & Yes & 1964 & 134 & -- & 215 & 1.25 & 0.262 & 0.883 & 217 \\
\hline 05536201 & 1 & Yes & Yes & 1965 & 215 & -- & 345 & 1.1 & 0.269 & 0.689 & 315 \\
\hline 05536201 & 1 & Yes & Yes & 1966 & 502 & -- & 805 & 2.99 & 0.276 & 0.135 & 662 \\
\hline 05536201 & 1 & Yes & Yes & 1967 & 369 & -- & 592 & 1.46 & 0.284 & 0.305 & 497 \\
\hline 05536201 & 1 & Yes & Yes & 1968 & 1140 & -- & 1828 & 3.5 & 0.291 & 0.012 & 1410 \\
\hline 05536201 & 1 & Yes & Yes & 1969 & 139 & -- & 223 & 0.692 & 0.298 & 0.888 & 213 \\
\hline 05536201 & 1 & Yes & Yes & 1970 & 400 & -- & 641 & 1.52 & 0.306 & 0.265 & 523 \\
\hline 05536201 & 1 & Yes & Yes & 1971 & 440 & 2 & 706 & 1.37 & 0.31 & 0.207 & 567 \\
\hline 05536201 & 1 & Yes & Yes & 1972 & 218 & -- & 350 & 1.26 & 0.314 & 0.714 & 304 \\
\hline 05536201 & 1 & Yes & Yes & 1973 & 472 & -- & 757 & 1.24 & 0.318 & 0.178 & 601 \\
\hline 05536201 & 1 & Yes & Yes & 1974 & 1460 & -- & 2341 & 2.98 & 0.322 & 0.00582 & 1750 \\
\hline
\end{tabular}




\begin{tabular}{|c|c|c|c|c|c|c|c|c|c|c|c|}
\hline $\begin{array}{l}\text { U.S. } \\
\text { Geological } \\
\text { Survey } \\
\text { streamgage } \\
\text { number }\end{array}$ & $\begin{array}{l}\text { Segment } \\
\text { number }\end{array}$ & $\begin{array}{l}\text { Streamgage used in } \\
\text { regression analyses } \\
\text { (non-redundant) }\end{array}$ & $\begin{array}{c}\text { Discharge value } \\
\text { used in } \\
\text { adjustment } \\
\text { regression }\end{array}$ & $\begin{array}{l}\text { Water } \\
\text { year }\end{array}$ & $\begin{array}{l}\text { Observed annual } \\
\text { maximum peak } \\
\text { discharge }\left(\mathrm{ft}^{3} / \mathrm{s}\right)\end{array}$ & $\begin{array}{l}\text { NWIS peak } \\
\text { code }\end{array}$ & $\begin{array}{l}\text { Observed annual } \\
\text { maximum peak } \\
\text { discharge with } \\
\text { segment intercept } \\
\text { value subtracted } \\
\left(\mathrm{ft}^{3} / \mathrm{s}\right)\end{array}$ & $\begin{array}{l}\text { Observed } \\
\text { precipitation } \\
\text { (inches) }\end{array}$ & Urban fraction & $\begin{array}{l}\text { Exceedance } \\
\text { probability }\end{array}$ & $\begin{array}{c}\text { Urban-adjusted } \\
\text { annual maximum } \\
\text { peak discharge } \\
\left(\mathrm{ft}^{3} / \mathrm{s}\right)\end{array}$ \\
\hline 05536201 & 1 & Yes & Yes & 1975 & 300 & -- & 481 & 1.66 & 0.326 & 0.497 & 399 \\
\hline 05536201 & 1 & Yes & Yes & 1976 & 332 & -- & 532 & 2.2 & 0.33 & 0.421 & 435 \\
\hline 05536201 & 1 & Yes & Yes & 1977 & 191 & -- & 306 & 1.66 & 0.335 & 0.794 & 266 \\
\hline 05536201 & 1 & Yes & Yes & 1978 & 387 & -- & 621 & 1.35 & 0.339 & 0.311 & 494 \\
\hline 05536207 & 1 & Yes & Yes & 1962 & 155 & -- & 650 & 0.65 & 0.83 & 0.737 & 165 \\
\hline 05536207 & 1 & Yes & Yes & 1963 & 127 & -- & 532 & 2.3 & 0.833 & 0.861 & 136 \\
\hline 05536207 & 1 & Yes & Yes & 1964 & 276 & -- & 1157 & 1.82 & 0.835 & 0.21 & 288 \\
\hline 05536207 & 1 & Yes & Yes & 1965 & 274 & -- & 1148 & 1.8 & 0.837 & 0.217 & 286 \\
\hline 05536207 & 1 & Yes & Yes & 1966 & 406 & 2 & 1702 & 3.89 & 0.839 & 0.054 & 421 \\
\hline 05536207 & 1 & Yes & Yes & 1967 & 197 & -- & 826 & 1.55 & 0.842 & 0.534 & 206 \\
\hline 05536207 & 1 & Yes & Yes & 1968 & 1090 & -- & 4569 & 4.94 & 0.844 & 0 & 1120 \\
\hline 05536207 & 1 & Yes & Yes & 1969 & 312 & -- & 1308 & 1.96 & 0.846 & 0.154 & 322 \\
\hline 05536207 & 1 & Yes & Yes & 1970 & 238 & -- & 998 & 1.74 & 0.848 & 0.352 & 246 \\
\hline 05536207 & 1 & Yes & Yes & 1971 & 346 & -- & 1450 & 1.58 & 0.849 & 0.106 & 356 \\
\hline 05536207 & 1 & Yes & Yes & 1972 & 364 & -- & 1526 & 1.22 & 0.849 & 0.0865 & 375 \\
\hline 05536207 & 1 & Yes & Yes & 1973 & 244 & -- & 1023 & 1.43 & 0.849 & 0.324 & 252 \\
\hline 05536207 & 1 & Yes & Yes & 1974 & 758 & -- & 3177 & 4.24 & 0.85 & 0.00788 & 775 \\
\hline 05536207 & 1 & Yes & Yes & 1975 & 110 & -- & 461 & 1.26 & 0.85 & 0.93 & 116 \\
\hline 05536207 & 1 & Yes & Yes & 1976 & 179 & -- & 750 & 1.86 & 0.851 & 0.633 & 186 \\
\hline 05536207 & 1 & Yes & Yes & 1977 & 166 & -- & 696 & 2 & 0.851 & 0.706 & 172 \\
\hline 05536210 & 1 & No & No & 1965 & 684 & -- & 517 & 1.44 & 0.523 & 0.621 & 827 \\
\hline 05536210 & 1 & No & No & 1966 & 1520 & -- & 1148 & 3.14 & 0.528 & 0.086 & 1750 \\
\hline 05536210 & 1 & No & No & 1967 & 670 & -- & 506 & 1.48 & 0.534 & 0.647 & 802 \\
\hline 05536210 & 1 & No & No & 1968 & 2040 & -- & 1541 & 3.78 & 0.54 & 0.0338 & 2290 \\
\hline 05536210 & 1 & No & No & 1969 & 437 & -- & 330 & 1.19 & 0.546 & 0.883 & 539 \\
\hline 05536210 & 1 & No & No & 1970 & 886 & -- & 669 & 1.54 & 0.552 & 0.432 & 1020 \\
\hline 05536210 & 1 & No & No & 1971 & 950 & -- & 718 & 1.39 & 0.555 & 0.374 & 1090 \\
\hline 05536210 & 1 & No & No & 1972 & 714 & -- & 539 & 2.43 & 0.558 & 0.62 & 828 \\
\hline 05536210 & 1 & No & No & 1973 & 1330 & -- & 1005 & 1.27 & 0.561 & 0.153 & 1490 \\
\hline 05536210 & 1 & No & No & 1974 & 2220 & -- & 1677 & 3.21 & 0.564 & 0.0268 & 2440 \\
\hline 05536210 & 1 & No & No & 1975 & 667 & -- & 504 & 1.69 & 0.567 & 0.681 & 770 \\
\hline 05536210 & 1 & No & No & 1976 & 1040 & D & 786 & 1.69 & 0.57 & 0.306 & 1170 \\
\hline 05536210 & 1 & No & No & 1977 & 942 & -- & 712 & 1.71 & 0.573 & 0.399 & 1060 \\
\hline 05536210 & 1 & No & No & 1978 & 1070 & -- & 808 & 1.39 & 0.576 & 0.288 & 1190 \\
\hline 05536210 & 1 & No & No & 1979 & 1070 & -- & 808 & 0.356 & 0.579 & 0.29 & 1190 \\
\hline
\end{tabular}




\begin{tabular}{|c|c|c|c|c|c|c|c|c|c|c|c|}
\hline $\begin{array}{l}\text { U.S. } \\
\text { Geological } \\
\text { Survey } \\
\text { streamgage } \\
\text { number }\end{array}$ & $\begin{array}{l}\text { Segment } \\
\text { number }\end{array}$ & $\begin{array}{l}\text { Streamgage used in } \\
\text { regression analyses } \\
\text { (non-redundant) }\end{array}$ & $\begin{array}{l}\text { Discharge value } \\
\text { used in } \\
\text { adjustment } \\
\text { regression }\end{array}$ & $\begin{array}{l}\text { Water } \\
\text { year }\end{array}$ & $\begin{array}{l}\text { Observed annual } \\
\text { maximum peak } \\
\text { discharge }\left(\mathrm{ft}^{3} / \mathrm{s}\right)\end{array}$ & $\begin{array}{l}\text { NWIS peak } \\
\text { code }\end{array}$ & $\begin{array}{l}\text { Observed annual } \\
\text { maximum peak } \\
\text { discharge with } \\
\text { segment intercept } \\
\text { value subtracted } \\
\left(\mathrm{ft}^{3} / \mathrm{s}\right)\end{array}$ & $\begin{array}{l}\text { Observed } \\
\text { precipitation } \\
\text { (inches) }\end{array}$ & Urban fraction & $\begin{array}{l}\text { Exceedance } \\
\text { probability }\end{array}$ & $\begin{array}{c}\text { Urban-adjusted } \\
\text { annual maximum } \\
\text { peak discharge } \\
\left(\mathrm{ft}^{3} / \mathrm{s}\right)\end{array}$ \\
\hline 05536215 & 1 & No & No & 1950 & 780 & -- & 532 & 1.53 & 0.397 & 0.48 & 1090 \\
\hline 05536215 & 1 & No & No & 1951 & 831 & -- & 566 & 2.6 & 0.411 & 0.442 & 1140 \\
\hline 05536215 & 1 & No & No & 1952 & 780 & -- & 532 & 1.24 & 0.425 & 0.506 & 1060 \\
\hline 05536215 & 1 & No & No & 1953 & 848 & -- & 578 & 2.41 & 0.439 & 0.451 & 1130 \\
\hline 05536215 & 1 & No & No & 1954 & 729 & -- & 497 & 1.33 & 0.453 & 0.589 & 971 \\
\hline 05536215 & 1 & No & No & 1955 & 1180 & -- & 804 & 4.65 & 0.467 & 0.219 & 1470 \\
\hline 05536215 & 1 & No & No & 1956 & 830 & -- & 566 & 1.23 & 0.482 & 0.508 & 1060 \\
\hline 05536215 & 1 & No & No & 1957 & 2460 & -- & 1677 & 2.39 & 0.496 & 0.0218 & 2880 \\
\hline 05536215 & 1 & No & No & 1958 & 950 & -- & 648 & 1.71 & 0.51 & 0.421 & 1170 \\
\hline 05536215 & 1 & No & No & 1959 & 1060 & D & 723 & 1.57 & 0.524 & 0.34 & 1270 \\
\hline 05536215 & 1 & No & No & 1960 & 661 & -- & 451 & 1.05 & 0.538 & 0.732 & 813 \\
\hline 05536215 & 1 & No & No & 1961 & 1220 & -- & 832 & 2.38 & 0.544 & 0.245 & 1420 \\
\hline 05536215 & 1 & No & No & 1962 & 610 & -- & 416 & 0.705 & 0.55 & 0.786 & 746 \\
\hline 05536215 & 1 & No & No & 1963 & 528 & -- & 360 & 1.91 & 0.556 & 0.856 & 651 \\
\hline 05536215 & 1 & No & No & 1964 & 464 & -- & 316 & 1.22 & 0.562 & 0.904 & 577 \\
\hline 05536215 & 1 & No & No & 1965 & 756 & -- & 515 & 1.23 & 0.568 & 0.665 & 888 \\
\hline 05536215 & 1 & No & No & 1966 & 1690 & -- & 1152 & 2.72 & 0.574 & 0.098 & 1890 \\
\hline 05536215 & 1 & No & No & 1967 & 920 & -- & 627 & 1.4 & 0.58 & 0.515 & 1050 \\
\hline 05536215 & 1 & No & No & 1968 & 2600 & -- & 1772 & 3.15 & 0.586 & 0.0234 & 2840 \\
\hline 05536215 & 1 & No & No & 1969 & 636 & -- & 434 & 1.15 & 0.592 & 0.793 & 736 \\
\hline 05536215 & 1 & No & No & 1970 & 1080 & -- & 736 & 1.42 & 0.598 & 0.393 & 1210 \\
\hline 05536215 & 1 & No & No & 1971 & 1010 & -- & 688 & 1.28 & 0.601 & 0.454 & 1130 \\
\hline 05536215 & 1 & No & No & 1972 & 905 & -- & 617 & 2.23 & 0.603 & 0.551 & 1010 \\
\hline 05536215 & 1 & No & No & 1973 & 1570 & 2 & 1070 & 1.18 & 0.606 & 0.143 & 1710 \\
\hline 05536215 & 1 & No & No & 1974 & 2300 & -- & 1568 & 2.64 & 0.608 & 0.0384 & 2480 \\
\hline 05536215 & 1 & No & No & 1975 & 715 & -- & 487 & 1.14 & 0.611 & 0.741 & 803 \\
\hline 05536215 & 1 & No & No & 1976 & 1200 & D & 818 & 1.6 & 0.613 & 0.309 & 1310 \\
\hline 05536215 & 1 & No & No & 1977 & 1180 & -- & 804 & 1.54 & 0.616 & 0.327 & 1290 \\
\hline 05536215 & 1 & No & No & 1978 & 1190 & -- & 811 & 1.34 & 0.618 & 0.322 & 1290 \\
\hline 05536215 & 1 & No & No & 1979 & 1420 & -- & 968 & 0.464 & 0.621 & 0.196 & 1530 \\
\hline 05536215 & 1 & No & No & 1980 & 1180 & -- & 804 & 1.25 & 0.623 & 0.335 & 1280 \\
\hline 05536215 & 1 & No & No & 1981 & 2310 & -- & 1575 & 2.51 & 0.624 & 0.0395 & 2460 \\
\hline 05536215 & 1 & No & No & 1982 & 2120 & -- & 1445 & 3.97 & 0.624 & 0.0509 & 2260 \\
\hline 05536215 & 1 & No & No & 1983 & 2210 & -- & 1506 & 2.99 & 0.625 & 0.0452 & 2360 \\
\hline 05536215 & 1 & No & No & 1984 & 1530 & -- & 1043 & 1.42 & 0.625 & 0.164 & 1640 \\
\hline
\end{tabular}




\begin{tabular}{|c|c|c|c|c|c|c|c|c|c|c|c|}
\hline $\begin{array}{l}\text { U.S. } \\
\text { Geological } \\
\text { Survey } \\
\text { streamgage } \\
\text { number }\end{array}$ & $\begin{array}{l}\text { Segment } \\
\text { number }\end{array}$ & $\begin{array}{l}\text { Streamgage used in } \\
\text { regression analyses } \\
\text { (non-redundant) }\end{array}$ & $\begin{array}{l}\text { Discharge value } \\
\text { used in } \\
\text { adjustment } \\
\text { regression }\end{array}$ & $\begin{array}{l}\text { Water } \\
\text { year }\end{array}$ & $\begin{array}{c}\text { Observed annual } \\
\text { maximum peak } \\
\text { discharge }\left(\mathrm{ft}^{3} / \mathrm{s}\right)\end{array}$ & $\begin{array}{l}\text { NWIS peak } \\
\text { code }\end{array}$ & $\begin{array}{l}\text { Observed annual } \\
\text { maximum peak } \\
\text { discharge with } \\
\text { segment intercept } \\
\text { value subtracted } \\
\left(\mathrm{ft}^{3} / \mathrm{s}\right)\end{array}$ & $\begin{array}{l}\text { Observed } \\
\text { precipitation } \\
\text { (inches) }\end{array}$ & Urban fraction & $\begin{array}{l}\text { Exceedance } \\
\text { probability }\end{array}$ & $\begin{array}{c}\text { Urban-adjusted } \\
\text { annual maximum } \\
\text { peak discharge } \\
\left(\mathrm{ft}^{3} / \mathrm{s}\right)\end{array}$ \\
\hline 05536215 & 1 & No & No & 1985 & 957 & -- & 652 & 1.16 & 0.626 & 0.526 & 1040 \\
\hline 05536215 & 1 & No & No & 1986 & 1250 & $\mathrm{E}$ & 852 & 1.67 & 0.626 & 0.286 & 1350 \\
\hline 05536215 & 1 & No & No & 1987 & 1090 & -- & 743 & 1.46 & 0.627 & 0.412 & 1180 \\
\hline 05536215 & 1 & No & No & 1988 & 517 & $\mathrm{E}$ & 352 & 1.07 & 0.627 & 0.903 & 580 \\
\hline 05536215 & 1 & No & No & 1989 & 1710 & -- & 1166 & 2.42 & 0.628 & 0.114 & 1830 \\
\hline 05536215 & 1 & No & No & 1990 & 1130 & $\mathrm{E}$ & 770 & 1.57 & 0.628 & 0.381 & 1220 \\
\hline 05536215 & 1 & No & No & 1991 & 2120 & -- & 1445 & 2.03 & 0.629 & 0.0517 & 2250 \\
\hline 05536215 & 1 & No & No & 1992 & 908 & $\mathrm{C}, \mathrm{E}$ & 619 & 1.87 & 0.629 & 0.574 & 986 \\
\hline 05536215 & 1 & No & No & 1993 & 1470 & C & 1002 & 0.898 & 0.63 & 0.184 & 1570 \\
\hline 05536215 & 1 & No & No & 1994 & 1210 & C & 825 & 2.35 & 0.63 & 0.317 & 1300 \\
\hline 05536215 & 1 & No & No & 1995 & 763 & $C, E$ & 520 & 1.34 & 0.631 & 0.716 & 833 \\
\hline 05536215 & 1 & No & No & 1996 & 2700 & $2, \mathrm{C}$ & 1840 & 4.57 & 0.631 & 0.0232 & 2850 \\
\hline 05536215 & 1 & No & No & 1997 & 1370 & $C, E$ & 934 & 2.16 & 0.632 & 0.224 & 1460 \\
\hline 05536215 & 1 & No & No & 1998 & 1180 & C & 804 & 1.67 & 0.632 & 0.343 & 1270 \\
\hline 05536215 & 1 & No & No & 1999 & 1100 & C & 750 & 1.33 & 0.633 & 0.41 & 1180 \\
\hline 05536215 & 1 & No & No & 2000 & 1050 & C & 716 & 1.73 & 0.633 & 0.451 & 1130 \\
\hline 05536215 & 1 & No & No & 2001 & 692 & $C, E$ & 472 & 1.2 & 0.64 & 0.782 & 751 \\
\hline 05536215 & 1 & No & No & 2002 & 1290 & $C, E$ & 879 & 2.2 & 0.647 & 0.277 & 1360 \\
\hline 05536215 & 1 & No & No & 2003 & 1580 & $C, E$ & 1077 & 2.25 & 0.653 & 0.161 & 1650 \\
\hline 05536215 & 1 & No & No & 2004 & 867 & C & 591 & 1.85 & 0.66 & 0.644 & 910 \\
\hline 05536215 & 1 & No & No & 2005 & 1270 & C & 866 & 1.18 & 0.667 & 0.306 & 1310 \\
\hline 05536215 & 1 & No & No & 2006 & 2540 & C & 1731 & 2.39 & 0.674 & 0.0328 & 2600 \\
\hline 05536215 & 1 & No & No & 2007 & 1080 & C & 736 & 1.22 & 0.681 & 0.474 & 1100 \\
\hline 05536215 & 1 & No & No & 2008 & 2240 & C & 1527 & 3.68 & 0.687 & 0.0501 & 2270 \\
\hline 05536215 & 1 & No & No & 2009 & 817 & C & 557 & 0.646 & 0.694 & 0.723 & 824 \\
\hline 05536235 & 1 & Yes & Yes & 1948 & 663 & 5 & 630 & 1.94 & 0.142 & 0.185 & 1070 \\
\hline 05536235 & 1 & Yes & Yes & 1949 & 376 & $5, \mathrm{E}$ & 357 & 0.884 & 0.147 & 0.571 & 673 \\
\hline 05536235 & 1 & Yes & Yes & 1950 & 438 & 5 & 416 & 1.52 & 0.152 & 0.459 & 762 \\
\hline 05536235 & 1 & Yes & Yes & 1951 & 467 & 5 & 444 & 2.74 & 0.162 & 0.419 & 797 \\
\hline 05536235 & 1 & Yes & Yes & 1952 & 419 & 5 & 398 & 1.15 & 0.172 & 0.508 & 720 \\
\hline 05536235 & 1 & Yes & Yes & 1953 & 435 & 5 & 413 & 1.62 & 0.181 & 0.488 & 737 \\
\hline 05536235 & 1 & Yes & Yes & 1954 & 417 & 5 & 396 & 1.5 & 0.191 & 0.529 & 704 \\
\hline 05536235 & 1 & Yes & Yes & 1955 & 637 & 5 & 605 & 3.8 & 0.201 & 0.237 & 982 \\
\hline 05536235 & 1 & Yes & Yes & 1956 & 467 & 5 & 444 & 1.35 & 0.21 & 0.459 & 762 \\
\hline 05536235 & 1 & Yes & Yes & 1957 & 1380 & 5 & 1311 & 2.48 & 0.22 & 0.0241 & 1930 \\
\hline
\end{tabular}




\begin{tabular}{|c|c|c|c|c|c|c|c|c|c|c|c|}
\hline $\begin{array}{l}\text { U.S. } \\
\text { Geological } \\
\text { Survey } \\
\text { streamgage } \\
\text { number }\end{array}$ & $\begin{array}{l}\text { Segment } \\
\text { number }\end{array}$ & $\begin{array}{l}\text { Streamgage used in } \\
\text { regression analyses } \\
\text { (non-redundant) }\end{array}$ & $\begin{array}{c}\text { Discharge value } \\
\text { used in } \\
\text { adjustment } \\
\text { regression }\end{array}$ & $\begin{array}{l}\text { Water } \\
\text { year }\end{array}$ & $\begin{array}{l}\text { Observed annual } \\
\text { maximum peak } \\
\text { discharge }\left(\mathrm{ft}^{3} / \mathrm{s}\right)\end{array}$ & $\begin{array}{l}\text { NWIS peak } \\
\text { code }\end{array}$ & $\begin{array}{l}\text { Observed annual } \\
\text { maximum peak } \\
\text { discharge with } \\
\text { segment intercept } \\
\text { value subtracted } \\
\left(\mathrm{ft}^{3} / \mathrm{s}\right)\end{array}$ & $\begin{array}{l}\text { Observed } \\
\text { precipitation } \\
\text { (inches) }\end{array}$ & Urban fraction & $\begin{array}{l}\text { Exceedance } \\
\text { probability }\end{array}$ & $\begin{array}{c}\text { Urban-adjusted } \\
\text { annual maximum } \\
\text { peak discharge } \\
\left(\mathrm{ft}^{3} / \mathrm{s}\right)\end{array}$ \\
\hline 05536235 & 1 & Yes & Yes & 1958 & 471 & 5 & 448 & 1.56 & 0.23 & 0.469 & 754 \\
\hline 05536235 & 1 & Yes & Yes & 1959 & 850 & 5 & 808 & 1.38 & 0.239 & 0.119 & 1240 \\
\hline 05536235 & 1 & Yes & Yes & 1960 & 430 & 5 & 409 & 1.39 & 0.249 & 0.557 & 683 \\
\hline 05536235 & 1 & Yes & Yes & 1961 & 510 & 5 & 485 & 1.85 & 0.255 & 0.43 & 788 \\
\hline 05536235 & 1 & Yes & Yes & 1962 & 250 & $5, E$ & 238 & 0.231 & 0.261 & 0.852 & 439 \\
\hline 05536235 & 1 & Yes & Yes & 1963 & 149 & $5, E$ & 142 & 1.65 & 0.268 & 0.952 & 300 \\
\hline 05536235 & 1 & Yes & Yes & 1964 & 141 & $5, E$ & 134 & 1.1 & 0.274 & 0.957 & 284 \\
\hline 05536235 & 1 & Yes & Yes & 1965 & 443 & 5 & 421 & 0.851 & 0.28 & 0.562 & 680 \\
\hline 05536235 & 1 & Yes & Yes & 1966 & 898 & 5 & 853 & 1.93 & 0.286 & 0.114 & 1250 \\
\hline 05536235 & 1 & Yes & Yes & 1967 & 459 & 5 & 436 & 1.25 & 0.293 & 0.545 & 692 \\
\hline 05536235 & 1 & Yes & Yes & 1968 & 874 & 5 & 831 & 1.94 & 0.299 & 0.13 & 1210 \\
\hline 05536235 & 1 & Yes & Yes & 1969 & 558 & 5 & 530 & 0.6 & 0.305 & 0.403 & 812 \\
\hline 05536235 & 1 & Yes & Yes & 1970 & 447 & 5 & 425 & 1.38 & 0.311 & 0.584 & 664 \\
\hline 05536235 & 1 & Yes & Yes & 1971 & 289 & 5 & 275 & 0.245 & 0.317 & 0.829 & 463 \\
\hline 05536235 & 1 & Yes & Yes & 1972 & 342 & $5, E$ & 325 & 1.26 & 0.322 & 0.759 & 527 \\
\hline 05536235 & 1 & Yes & Yes & 1973 & 722 & 5 & 686 & 1.01 & 0.327 & 0.234 & 986 \\
\hline 05536235 & 1 & Yes & Yes & 1974 & 610 & 5 & 580 & 1.56 & 0.332 & 0.357 & 854 \\
\hline 05536235 & 1 & Yes & Yes & 1975 & 542 & 5 & 515 & 1.43 & 0.338 & 0.453 & 768 \\
\hline 05536235 & 1 & Yes & Yes & 1976 & 607 & 5 & 577 & 1.67 & 0.343 & 0.369 & 843 \\
\hline 05536235 & 1 & Yes & Yes & 1977 & 425 & $5, E$ & 404 & 1.24 & 0.348 & 0.648 & 612 \\
\hline 05536235 & 1 & Yes & Yes & 1978 & 454 & 5 & 431 & 0.417 & 0.353 & 0.608 & 645 \\
\hline 05536235 & 1 & Yes & Yes & 1979 & 533 & 5 & 507 & 0.676 & 0.358 & 0.485 & 741 \\
\hline 05536235 & 1 & Yes & Yes & 1980 & 616 & $5, E$ & 585 & 0.934 & 0.364 & 0.375 & 837 \\
\hline 05536235 & 1 & Yes & Yes & 1981 & 848 & 5 & 806 & 2.12 & 0.366 & 0.17 & 1110 \\
\hline 05536235 & 1 & Yes & Yes & 1982 & 704 & 5 & 669 & 0.564 & 0.368 & 0.278 & 933 \\
\hline 05536235 & 1 & Yes & Yes & 1983 & 848 & 5 & 806 & 2.39 & 0.37 & 0.172 & 1100 \\
\hline 05536235 & 1 & Yes & Yes & 1984 & 623 & 5 & 592 & 1.04 & 0.373 & 0.374 & 839 \\
\hline 05536235 & 1 & Yes & Yes & 1985 & 727 & 5 & 691 & 0.993 & 0.375 & 0.26 & 953 \\
\hline 05536235 & 1 & Yes & Yes & 1986 & 630 & 5 & 599 & 1.34 & 0.377 & 0.369 & 843 \\
\hline 05536235 & 1 & Yes & Yes & 1987 & 409 & 5 & 389 & 1.51 & 0.379 & 0.7 & 575 \\
\hline 05536235 & 1 & Yes & Yes & 1988 & 428 & 5 & 407 & 0.796 & 0.381 & 0.671 & 596 \\
\hline 05536235 & 1 & Yes & Yes & 1989 & 739 & $5, E$ & 702 & 1.82 & 0.384 & 0.254 & 959 \\
\hline 05536235 & 1 & Yes & Yes & 1990 & 446 & 5 & 424 & 0.891 & 0.386 & 0.647 & 614 \\
\hline 05536235 & 1 & Yes & Yes & 1991 & 809 & 5 & 769 & 2.41 & 0.39 & 0.2 & 1040 \\
\hline 05536235 & 1 & Yes & Yes & 1992 & 470 & $5, C$ & 447 & 0.417 & 0.394 & 0.617 & 638 \\
\hline
\end{tabular}




\begin{tabular}{|c|c|c|c|c|c|c|c|c|c|c|c|}
\hline $\begin{array}{c}\text { U.S. } \\
\text { Geological } \\
\text { Survey } \\
\text { streamgage } \\
\text { number } \\
\end{array}$ & $\begin{array}{c}\text { Segment } \\
\text { number }\end{array}$ & $\begin{array}{l}\text { Streamgage used in } \\
\text { regression analyses } \\
\text { (non-redundant) }\end{array}$ & $\begin{array}{c}\text { Discharge value } \\
\text { used in } \\
\text { adjustment } \\
\text { regression } \\
\end{array}$ & $\begin{array}{c}\text { Water } \\
\text { year }\end{array}$ & $\begin{array}{c}\text { Observed annual } \\
\text { maximum peak } \\
\text { discharge }\left(\mathrm{ft}^{3} / \mathrm{s}\right)\end{array}$ & $\begin{array}{c}\text { NWIS peak } \\
\text { code }\end{array}$ & $\begin{array}{c}\text { Observed annual } \\
\text { maximum peak } \\
\text { discharge with } \\
\text { segment intercept } \\
\text { value subtracted } \\
\left(\mathrm{ft}^{3} / \mathrm{s}\right) \\
\end{array}$ & $\begin{array}{c}\text { Observed } \\
\text { precipitation } \\
\text { (inches) }\end{array}$ & Urban fraction & $\begin{array}{l}\text { Exceedance } \\
\text { probability }\end{array}$ & $\begin{array}{c}\text { Urban-adjusted } \\
\text { annual maximum } \\
\text { peak discharge } \\
\left(\mathrm{ft}^{3} / \mathrm{s}\right)\end{array}$ \\
\hline 05536235 & 1 & Yes & Yes & 1993 & 830 & $5, C$ & 789 & 1.85 & 0.397 & 0.192 & 1050 \\
\hline 05536235 & 1 & Yes & Yes & 1994 & 707 & $5, C$ & 672 & 1.01 & 0.401 & 0.298 & 910 \\
\hline 05536235 & 1 & Yes & Yes & 1995 & 478 & $5, \mathrm{C}$ & 454 & 1.13 & 0.405 & 0.614 & 640 \\
\hline 05536235 & 1 & Yes & Yes & 1996 & 824 & $5, C$ & 783 & 3.56 & 0.409 & 0.2 & 1040 \\
\hline 05536235 & 1 & Yes & Yes & 1997 & 803 & $5, \mathrm{C}$ & 763 & 2.09 & 0.413 & 0.219 & 1010 \\
\hline 05536235 & 1 & Yes & Yes & 1998 & 632 & $5, C$ & 601 & 1.96 & 0.416 & 0.399 & 816 \\
\hline 05536235 & 1 & Yes & Yes & 1999 & 587 & $5, C$ & 558 & 0.617 & 0.42 & 0.463 & 760 \\
\hline 05536235 & 1 & Yes & Yes & 2000 & 678 & $5, C$ & 644 & 0.823 & 0.424 & 0.349 & 861 \\
\hline 05536235 & 1 & Yes & Yes & 2001 & 645 & $5, C$ & 613 & 0.635 & 0.447 & 0.41 & 806 \\
\hline 05536235 & 1 & Yes & Yes & 2002 & 755 & $5, \mathrm{C}$ & 718 & 1.57 & 0.47 & 0.299 & 908 \\
\hline 05536235 & 1 & Yes & Yes & 2003 & 597 & $5, C$ & 567 & 1.44 & 0.493 & 0.516 & 716 \\
\hline 05536235 & 1 & Yes & Yes & 2004 & 413 & $5, \mathrm{C}$ & 392 & 1.78 & 0.516 & 0.792 & 497 \\
\hline 05536235 & 1 & Yes & Yes & 2005 & 674 & $5, \mathrm{C}$ & 641 & 1.12 & 0.539 & 0.457 & 765 \\
\hline 05536235 & 0 & Yes & No & 2006 & 873 & $5, \mathrm{C}$ & NA & 1.67 & 0.562 & NA & NA \\
\hline 05536235 & 0 & Yes & No & 2007 & 1320 & $5, \mathrm{C}$ & NA & 1.55 & 0.585 & NA & NA \\
\hline 05536235 & 0 & Yes & No & 2008 & 1320 & $5, \mathrm{C}$ & NA & 3.78 & 0.608 & NA & NA \\
\hline 05536235 & 0 & Yes & No & 2009 & 509 & $5, \mathrm{C}$ & NA & 1.74 & 0.63 & NA & NA \\
\hline 05536238 & 1 & Yes & Yes & 1961 & 248 & -- & 639 & 1.62 & 0.0702 & 0.151 & 337 \\
\hline 05536238 & 1 & Yes & Yes & 1962 & 71 & -- & 183 & 0.246 & 0.0728 & 0.848 & 114 \\
\hline 05536238 & 1 & Yes & No & 1963 & 44 & 4, B & 113 & 0.246 & 0.0754 & 0.94 & 78 \\
\hline 05536238 & 1 & Yes & Yes & 1964 & 61 & -- & 157 & 0.867 & 0.078 & 0.89 & 101 \\
\hline 05536238 & 1 & Yes & Yes & 1965 & 132 & -- & 340 & 0.948 & 0.0806 & 0.55 & 191 \\
\hline 05536238 & 1 & Yes & Yes & 1966 & 201 & -- & 518 & 1.58 & 0.0832 & 0.264 & 275 \\
\hline 05536238 & 1 & Yes & Yes & 1967 & 100 & -- & 258 & 1.19 & 0.0858 & 0.723 & 150 \\
\hline 05536238 & 1 & Yes & Yes & 1968 & 372 & -- & 959 & 1.49 & 0.0885 & 0.0471 & 486 \\
\hline 05536238 & 1 & Yes & Yes & 1969 & 60 & -- & 155 & 0.492 & 0.0911 & 0.898 & 97.8 \\
\hline 05536238 & 1 & Yes & Yes & 1970 & 271 & -- & 699 & 1.45 & 0.0937 & 0.124 & 360 \\
\hline 05536238 & 1 & Yes & Yes & 1971 & 30 & -- & 77 & 1.03 & 0.109 & 0.975 & 54 \\
\hline 05536238 & 1 & Yes & Yes & 1972 & 75 & -- & 193 & 1.25 & 0.124 & 0.854 & 113 \\
\hline 05536238 & 1 & Yes & Yes & 1973 & 120 & -- & 309 & 1.29 & 0.139 & 0.657 & 165 \\
\hline 05536238 & 1 & Yes & Yes & 1974 & 299 & -- & 771 & 1.15 & 0.154 & 0.106 & 375 \\
\hline 05536238 & 1 & Yes & Yes & 1975 & 226 & -- & 583 & 1.13 & 0.169 & 0.242 & 283 \\
\hline 05536238 & 1 & Yes & Yes & 1976 & 150 & -- & 387 & 1.56 & 0.184 & 0.542 & 193 \\
\hline 05536238 & 1 & Yes & Yes & 1977 & 210 & -- & 541 & 0.752 & 0.199 & 0.309 & 259 \\
\hline 36238 & 1 & Yes & Yes & 1978 & 149 & -- & 384 & 1.17 & 0.214 & 0.575 & 186 \\
\hline
\end{tabular}




\begin{tabular}{|c|c|c|c|c|c|c|c|c|c|c|c|}
\hline $\begin{array}{c}\text { U.S. } \\
\text { Geological } \\
\text { Survey } \\
\text { streamgage } \\
\text { number } \\
\end{array}$ & $\begin{array}{c}\text { Segment } \\
\text { number }\end{array}$ & $\begin{array}{l}\text { Streamgage used in } \\
\text { regression analyses } \\
\text { (non-redundant) }\end{array}$ & $\begin{array}{c}\text { Discharge value } \\
\text { used in } \\
\text { adjustment } \\
\text { regression }\end{array}$ & $\begin{array}{c}\text { Water } \\
\text { year }\end{array}$ & $\begin{array}{c}\text { Observed annual } \\
\text { maximum peak } \\
\text { discharge }\left(\mathrm{ft}^{3} / \mathrm{s}\right)\end{array}$ & $\begin{array}{c}\text { NWIS peak } \\
\text { code }\end{array}$ & $\begin{array}{l}\text { Observed annual } \\
\text { maximum peak } \\
\text { discharge with } \\
\text { segment intercept } \\
\text { value subtracted } \\
\left(\mathrm{ft}^{3} / \mathrm{s}\right)\end{array}$ & $\begin{array}{l}\text { Observed } \\
\text { precipitation } \\
\text { (inches) }\end{array}$ & Urban fraction & $\begin{array}{l}\text { Exceedance } \\
\text { probability }\end{array}$ & $\begin{array}{c}\text { Urban-adjusted } \\
\text { annual maximum } \\
\text { peak discharge } \\
\left(\mathrm{ft}^{3} / \mathrm{s}\right)\end{array}$ \\
\hline 05536238 & 1 & Yes & Yes & 1979 & 573 & -- & 1477 & 0.767 & 0.229 & 0.0177 & 654 \\
\hline 05536255 & 1 & Yes & Yes & 1948 & 970 & -- & 925 & 1.87 & 0.124 & 0.0574 & 1590 \\
\hline 05536255 & 1 & Yes & Yes & 1949 & 465 & -- & 443 & 0.749 & 0.13 & 0.395 & 843 \\
\hline 05536255 & 1 & Yes & Yes & 1950 & 555 & -- & 529 & 1.19 & 0.136 & 0.283 & 961 \\
\hline 05536255 & 1 & Yes & Yes & 1951 & 525 & -- & 500 & 2.06 & 0.156 & 0.332 & 908 \\
\hline 05536255 & 1 & Yes & Yes & 1952 & 339 & -- & 323 & 0.957 & 0.175 & 0.658 & 621 \\
\hline 05536255 & 1 & Yes & Yes & 1953 & 810 & -- & 772 & 2.05 & 0.195 & 0.121 & 1280 \\
\hline 05536255 & 1 & Yes & Yes & 1954 & 450 & -- & 429 & 1.42 & 0.215 & 0.489 & 758 \\
\hline 05536255 & 1 & Yes & Yes & 1955 & 1380 & -- & 1315 & 4.25 & 0.235 & 0.0249 & 2000 \\
\hline 05536255 & 1 & Yes & Yes & 1956 & 510 & -- & 486 & 1.27 & 0.255 & 0.428 & 814 \\
\hline 05536255 & 1 & Yes & Yes & 1957 & 2000 & -- & 1906 & 2.4 & 0.275 & 0.0101 & 2750 \\
\hline 05536255 & 1 & Yes & Yes & 1958 & 311 & -- & 296 & 1.29 & 0.295 & 0.784 & 514 \\
\hline 05536255 & 1 & Yes & Yes & 1959 & 375 & $E$ & 357 & 1.48 & 0.315 & 0.702 & 587 \\
\hline 05536255 & 1 & Yes & Yes & 1960 & 318 & -- & 303 & 1.23 & 0.335 & 0.798 & 502 \\
\hline 05536255 & 1 & Yes & Yes & 1961 & 935 & -- & 891 & 2.12 & 0.348 & 0.119 & 1280 \\
\hline 05536255 & 1 & Yes & Yes & 1962 & 297 & -- & 283 & 0.231 & 0.361 & 0.84 & 459 \\
\hline 05536255 & 1 & Yes & Yes & 1963 & 187 & $\mathrm{E}$ & 178 & 1.8 & 0.375 & 0.949 & 311 \\
\hline 05536255 & 1 & Yes & Yes & 1964 & 251 & $\mathrm{E}$ & 239 & 1.03 & 0.388 & 0.905 & 388 \\
\hline 05536255 & 1 & Yes & Yes & 1965 & 405 & -- & 386 & 1.13 & 0.401 & 0.72 & 572 \\
\hline 05536255 & 1 & Yes & Yes & 1966 & 888 & -- & 846 & 2.33 & 0.415 & 0.169 & 1150 \\
\hline 05536255 & 1 & Yes & Yes & 1967 & 424 & -- & 404 & 1.33 & 0.428 & 0.713 & 578 \\
\hline 05536255 & 1 & Yes & Yes & 1968 & 1680 & -- & 1601 & 2.6 & 0.441 & 0.022 & 2070 \\
\hline 05536255 & 0 & Yes & No & 1969 & 432 & -- & 398 & 0.591 & 0.455 & 0.743 & 553 \\
\hline 05536255 & 2 & Yes & Yes & 1970 & 666 & -- & 592 & 1.31 & 0.468 & 0.458 & 787 \\
\hline 05536255 & 2 & Yes & Yes & 1971 & 376 & -- & 334 & 1.17 & 0.478 & 0.84 & 459 \\
\hline 05536255 & 2 & Yes & Yes & 1972 & 399 & -- & 354 & 1.24 & 0.488 & 0.822 & 479 \\
\hline 05536255 & 2 & Yes & Yes & 1973 & 820 & 2 & 728 & 1.09 & 0.498 & 0.31 & 927 \\
\hline 05536255 & 2 & Yes & Yes & 1974 & 1480 & -- & 1315 & 2.14 & 0.508 & 0.0507 & 1630 \\
\hline 05536255 & 2 & Yes & Yes & 1975 & 548 & -- & 487 & 1.52 & 0.518 & 0.661 & 619 \\
\hline 05536255 & 2 & Yes & Yes & 1976 & 586 & $D$ & 521 & 1.51 & 0.528 & 0.62 & 653 \\
\hline 05536255 & 2 & Yes & Yes & 1977 & 964 & -- & 856 & 1.4 & 0.538 & 0.222 & 1040 \\
\hline 05536255 & 2 & Yes & Yes & 1978 & 932 & -- & 828 & 1.28 & 0.548 & 0.249 & 999 \\
\hline 05536255 & 2 & Yes & Yes & 1979 & 730 & -- & 648 & 0.566 & 0.557 & 0.464 & 782 \\
\hline 05536255 & 2 & Yes & Yes & 1980 & 448 & -- & 398 & 1.34 & 0.567 & 0.819 & 481 \\
\hline 05536255 & 2 & Yes & Yes & 1981 & 1980 & -- & 1759 & 2.35 & 0.57 & 0.0229 & 2060 \\
\hline
\end{tabular}




\begin{tabular}{|c|c|c|c|c|c|c|c|c|c|c|c|}
\hline $\begin{array}{l}\text { U.S. } \\
\text { Geological } \\
\text { Survey } \\
\text { streamgage } \\
\text { number }\end{array}$ & $\begin{array}{l}\text { Segment } \\
\text { number }\end{array}$ & $\begin{array}{l}\text { Streamgage used in } \\
\text { regression analyses } \\
\text { (non-redundant) }\end{array}$ & $\begin{array}{c}\text { Discharge value } \\
\text { used in } \\
\text { adjustment } \\
\text { regression }\end{array}$ & $\begin{array}{l}\text { Water } \\
\text { year }\end{array}$ & $\begin{array}{l}\text { Observed annual } \\
\text { maximum peak } \\
\text { discharge }\left(\mathrm{ft}^{3} / \mathrm{s}\right)\end{array}$ & $\begin{array}{l}\text { NWIS peak } \\
\text { code }\end{array}$ & $\begin{array}{l}\text { Observed annual } \\
\text { maximum peak } \\
\text { discharge with } \\
\text { segment intercept } \\
\text { value subtracted } \\
\left(\mathrm{ft}^{3} / \mathrm{s}\right)\end{array}$ & $\begin{array}{l}\text { Observed } \\
\text { precipitation } \\
\text { (inches) }\end{array}$ & Urban fraction & $\begin{array}{l}\text { Exceedance } \\
\text { probability }\end{array}$ & $\begin{array}{c}\text { Urban-adjusted } \\
\text { annual maximum } \\
\text { peak discharge } \\
\left(\mathrm{ft}^{3} / \mathrm{s}\right)\end{array}$ \\
\hline 05536255 & 2 & Yes & Yes & 1982 & 2160 & -- & 1919 & 3.17 & 0.572 & 0.018 & 2240 \\
\hline 05536255 & 2 & Yes & Yes & 1983 & 1700 & -- & 1510 & 2.7 & 0.575 & 0.0397 & 1760 \\
\hline 05536255 & 2 & Yes & Yes & 1984 & 1020 & -- & 906 & 1.24 & 0.577 & 0.209 & 1060 \\
\hline 05536255 & 2 & Yes & Yes & 1985 & 816 & -- & 725 & 1.07 & 0.579 & 0.388 & 852 \\
\hline 05536255 & 2 & Yes & Yes & 1986 & 713 & -- & 633 & 1.49 & 0.582 & 0.508 & 744 \\
\hline 05536255 & 2 & Yes & Yes & 1987 & 711 & -- & 632 & 1.62 & 0.584 & 0.513 & 740 \\
\hline 05536255 & 2 & Yes & Yes & 1988 & 512 & -- & 455 & 0.423 & 0.586 & 0.763 & 535 \\
\hline 05536255 & 2 & Yes & Yes & 1989 & 870 & -- & 773 & 2.12 & 0.589 & 0.339 & 898 \\
\hline 05536255 & 2 & Yes & Yes & 1990 & 829 & -- & 736 & 2.12 & 0.591 & 0.385 & 854 \\
\hline 05536255 & 2 & Yes & Yes & 1991 & 2090 & -- & 1857 & 3.37 & 0.593 & 0.0198 & 2130 \\
\hline 05536255 & 2 & Yes & Yes & 1992 & 465 & C & 413 & 2.28 & 0.595 & 0.819 & 481 \\
\hline 05536255 & 2 & Yes & Yes & 1993 & 1070 & C & 951 & 2.5 & 0.596 & 0.193 & 1090 \\
\hline 05536255 & 2 & Yes & Yes & 1994 & 909 & $C, E$ & 807 & 1.78 & 0.598 & 0.307 & 929 \\
\hline 05536255 & 2 & Yes & Yes & 1995 & 575 & C & 511 & 1.14 & 0.6 & 0.702 & 589 \\
\hline 05536255 & 2 & Yes & Yes & 1996 & 2220 & C & 1972 & 5.24 & 0.601 & 0.0179 & 2250 \\
\hline 05536255 & 2 & Yes & Yes & 1997 & 1070 & $C, E$ & 951 & 2.25 & 0.603 & 0.196 & 1090 \\
\hline 05536255 & 2 & Yes & Yes & 1998 & 707 & C & 628 & 1.53 & 0.605 & 0.538 & 719 \\
\hline 05536255 & 2 & Yes & Yes & 1999 & 710 & C & 631 & 1.27 & 0.606 & 0.536 & 720 \\
\hline 05536255 & 2 & Yes & Yes & 2000 & 497 & C & 441 & 1.26 & 0.608 & 0.795 & 505 \\
\hline 05536255 & 2 & Yes & Yes & 2001 & 544 & C & 483 & 0.535 & 0.609 & 0.745 & 551 \\
\hline 05536255 & 2 & Yes & Yes & 2002 & 949 & C & 843 & 1.43 & 0.61 & 0.282 & 957 \\
\hline 05536255 & 2 & Yes & Yes & 2003 & 634 & C & 563 & 2.4 & 0.611 & 0.635 & 640 \\
\hline 05536255 & 2 & Yes & Yes & 2004 & 931 & C & 827 & 3.41 & 0.613 & 0.299 & 937 \\
\hline 05536255 & 2 & Yes & Yes & 2005 & 774 & $C, E$ & 688 & 1.02 & 0.614 & 0.468 & 779 \\
\hline 05536255 & 2 & Yes & Yes & 2006 & 2640 & C & 2345 & 3.02 & 0.615 & 0.012 & 2650 \\
\hline 05536255 & 2 & Yes & Yes & 2007 & 840 & C & 746 & 2.01 & 0.616 & 0.398 & 843 \\
\hline 05536255 & 2 & Yes & Yes & 2008 & 2020 & C & 1794 & 4.8 & 0.617 & 0.0245 & 2020 \\
\hline 05536255 & 2 & Yes & Yes & 2009 & 788 & C & 700 & 1.2 & 0.618 & 0.456 & 789 \\
\hline 05536265 & 1 & Yes & Yes & 1948 & 461 & 5 & 1081 & 1.12 & 0.0402 & 0.0285 & 321 \\
\hline 05536265 & 1 & Yes & Yes & 1949 & 371 & 5 & 870 & 0.766 & 0.0439 & 0.0564 & 266 \\
\hline 05536265 & 1 & Yes & Yes & 1950 & 301 & 5 & 706 & 1.17 & 0.0476 & 0.102 & 219 \\
\hline 05536265 & 1 & Yes & Yes & 1951 & 425 & 5 & 996 & 2.05 & 0.0626 & 0.0398 & 295 \\
\hline 05536265 & 1 & Yes & Yes & 1952 & 323 & 5 & 757 & 0.0211 & 0.0776 & 0.09 & 228 \\
\hline 05536265 & 1 & Yes & Yes & 1953 & 354 & 5 & 830 & 1.11 & 0.0925 & 0.0713 & 245 \\
\hline 05536265 & 1 & Yes & Yes & 1954 & 362 & 5 & 849 & 0.909 & 0.107 & 0.0697 & 247 \\
\hline
\end{tabular}




\begin{tabular}{|c|c|c|c|c|c|c|c|c|c|c|c|}
\hline $\begin{array}{c}\text { U.S. } \\
\text { Geological } \\
\text { Survey } \\
\text { streamgage } \\
\text { number }\end{array}$ & $\begin{array}{l}\text { Segment } \\
\text { number }\end{array}$ & $\begin{array}{l}\text { Streamgage used in } \\
\text { regression analyses } \\
\text { (non-redundant) }\end{array}$ & $\begin{array}{l}\text { Discharge value } \\
\text { used in } \\
\text { adjustment } \\
\text { regression }\end{array}$ & $\begin{array}{l}\text { Water } \\
\text { year }\end{array}$ & $\begin{array}{l}\text { Observed annual } \\
\text { maximum peak } \\
\text { discharge }\left(\mathrm{ft}^{3} / \mathrm{s}\right)\end{array}$ & $\begin{array}{l}\text { NWIS peak } \\
\text { code }\end{array}$ & $\begin{array}{l}\text { Observed annual } \\
\text { maximum peak } \\
\text { discharge with } \\
\text { segment intercept } \\
\text { value subtracted } \\
\left(\mathrm{ft}^{3} / \mathrm{s}\right)\end{array}$ & $\begin{array}{l}\text { Observed } \\
\text { precipitation } \\
\text { (inches) }\end{array}$ & Urban fraction & $\begin{array}{l}\text { Exceedance } \\
\text { probability }\end{array}$ & $\begin{array}{c}\text { Urban-adjusted } \\
\text { annual maximum } \\
\text { peak discharge } \\
\left(\mathrm{ft}^{3} / \mathrm{s}\right)\end{array}$ \\
\hline 05536265 & 1 & Yes & Yes & 1955 & 302 & 5 & 708 & 3.31 & 0.122 & 0.129 & 207 \\
\hline 05536265 & 1 & Yes & Yes & 1956 & 320 & 5 & 750 & 1.42 & 0.137 & 0.111 & 215 \\
\hline 05536265 & 1 & Yes & Yes & 1957 & 240 & $5, \mathrm{E}$ & 563 & 2.41 & 0.152 & 0.254 & 163 \\
\hline 05536265 & 1 & Yes & Yes & 1958 & 150 & $5, \mathrm{E}$ & 352 & 1.11 & 0.167 & 0.601 & 108 \\
\hline 05536265 & 1 & Yes & Yes & 1959 & 155 & 5 & 363 & 1.25 & 0.182 & 0.59 & 109 \\
\hline 05536265 & 1 & Yes & Yes & 1960 & 204 & 5 & 478 & 1.05 & 0.197 & 0.393 & 137 \\
\hline 05536265 & 1 & Yes & Yes & 1961 & 126 & $5, E$ & 295 & 1.5 & 0.204 & 0.73 & 89.7 \\
\hline 05536265 & 1 & Yes & Yes & 1962 & 77 & $5, E$ & 181 & 0.242 & 0.212 & 0.905 & 59.8 \\
\hline 05536265 & 1 & Yes & Yes & 1963 & 94 & $5, \mathrm{E}$ & 220 & 0.563 & 0.219 & 0.856 & 69.3 \\
\hline 05536265 & 1 & Yes & Yes & 1964 & 56 & $5, \mathrm{E}$ & 131 & 0.996 & 0.226 & 0.952 & 45.5 \\
\hline 05536265 & 1 & Yes & Yes & 1965 & 165 & 5 & 387 & 0.898 & 0.233 & 0.587 & 110 \\
\hline 05536265 & 1 & Yes & Yes & 1966 & 272 & 5 & 638 & 1.61 & 0.241 & 0.228 & 169 \\
\hline 05536265 & 1 & Yes & Yes & 1967 & 130 & $5, \mathrm{E}$ & 305 & 1.15 & 0.248 & 0.743 & 87.9 \\
\hline 05536265 & 1 & Yes & Yes & 1968 & 260 & 5 & 610 & 1.6 & 0.255 & 0.266 & 160 \\
\hline 05536265 & 1 & Yes & Yes & 1969 & 48 & $5, \mathrm{E}$ & 113 & 0.923 & 0.262 & 0.969 & 37.7 \\
\hline 05536265 & 1 & Yes & Yes & 1970 & 135 & $5, E$ & 316 & 1.48 & 0.269 & 0.738 & 88.7 \\
\hline 05536265 & 1 & Yes & Yes & 1971 & 96 & 5 & 225 & 0.931 & 0.278 & 0.877 & 65.8 \\
\hline 05536265 & 1 & Yes & Yes & 1972 & 105 & 5 & 246 & 1.07 & 0.286 & 0.852 & 70.1 \\
\hline 05536265 & 1 & Yes & Yes & 1973 & 238 & 5 & 558 & 0.892 & 0.294 & 0.356 & 144 \\
\hline 05536265 & 1 & Yes & Yes & 1974 & 228 & 5 & 534 & 1.31 & 0.302 & 0.394 & 137 \\
\hline 05536265 & 1 & Yes & Yes & 1975 & 199 & $5, \mathrm{E}$ & 467 & 1.31 & 0.31 & 0.507 & 120 \\
\hline 05536265 & 1 & Yes & Yes & 1976 & 202 & 5 & 474 & 1.55 & 0.318 & 0.502 & 121 \\
\hline 05536265 & 1 & Yes & Yes & 1977 & 188 & $5, \mathrm{E}$ & 441 & 1.02 & 0.327 & 0.568 & 112 \\
\hline 05536265 & 1 & Yes & Yes & 1978 & 138 & $5, \mathrm{E}$ & 324 & 1.16 & 0.335 & 0.769 & 83.9 \\
\hline 05536265 & 1 & Yes & Yes & 1979 & 222 & $5, \mathrm{E}$ & 520 & 0.801 & 0.343 & 0.45 & 129 \\
\hline 05536265 & 1 & Yes & Yes & 1980 & 176 & $5, E$ & 413 & 0.754 & 0.351 & 0.637 & 102 \\
\hline 05536265 & 1 & Yes & Yes & 1981 & 204 & 5 & 478 & 1.85 & 0.355 & 0.528 & 117 \\
\hline 05536265 & 1 & Yes & Yes & 1982 & 198 & 5 & 464 & 1.19 & 0.358 & 0.555 & 114 \\
\hline 05536265 & 1 & Yes & Yes & 1983 & 203 & 5 & 476 & 1.48 & 0.361 & 0.538 & 116 \\
\hline 05536265 & 1 & Yes & Yes & 1984 & 146 & 5 & 342 & 0.675 & 0.365 & 0.761 & 85.2 \\
\hline 05536265 & 1 & Yes & Yes & 1985 & 187 & 5 & 438 & 0.865 & 0.368 & 0.609 & 106 \\
\hline 05536265 & 1 & Yes & Yes & 1986 & 183 & $5, E$ & 429 & 1.08 & 0.372 & 0.627 & 104 \\
\hline 05536265 & 1 & Yes & Yes & 1987 & 136 & $5, \mathrm{E}$ & 319 & 1.17 & 0.375 & 0.8 & 78.9 \\
\hline 05536265 & 0 & Yes & No & 1988 & 109 & $5, \mathrm{E}$ & 371 & 0.393 & 0.379 & 0.727 & 90.2 \\
\hline 05536265 & 2 & Yes & Yes & 1989 & 142 & $5, E$ & 701 & 1.43 & 0.382 & 0.254 & 163 \\
\hline
\end{tabular}




\begin{tabular}{|c|c|c|c|c|c|c|c|c|c|c|c|}
\hline $\begin{array}{l}\text { U.S. } \\
\text { Geological } \\
\text { Survey } \\
\text { streamgage } \\
\text { number }\end{array}$ & $\begin{array}{c}\text { Segment } \\
\text { number }\end{array}$ & $\begin{array}{l}\text { Streamgage used in } \\
\text { regression analyses } \\
\text { (non-redundant) }\end{array}$ & $\begin{array}{c}\text { Discharge value } \\
\text { used in } \\
\text { adjustment } \\
\text { regression }\end{array}$ & $\begin{array}{l}\text { Water } \\
\text { year }\end{array}$ & $\begin{array}{c}\text { Observed annual } \\
\text { maximum peak } \\
\text { discharge }\left(\mathrm{ft}^{3} / \mathrm{s}\right)\end{array}$ & $\begin{array}{l}\text { NWIS peak } \\
\text { code }\end{array}$ & $\begin{array}{l}\text { Observed annual } \\
\text { maximum peak } \\
\text { discharge with } \\
\text { segment intercept } \\
\text { value subtracted } \\
\left(\mathrm{ft}^{3} / \mathrm{s}\right)\end{array}$ & $\begin{array}{l}\text { Observed } \\
\text { precipitation } \\
\text { (inches) }\end{array}$ & Urban fraction & $\begin{array}{l}\text { Exceedance } \\
\text { probability }\end{array}$ & $\begin{array}{c}\text { Urban-adjusted } \\
\text { annual maximum } \\
\text { peak discharge } \\
\left(\mathrm{ft}^{3} / \mathrm{s}\right)\end{array}$ \\
\hline 05536265 & 2 & Yes & Yes & 1990 & 129 & $5, \mathrm{E}$ & 637 & 2.68 & 0.385 & 0.327 & 149 \\
\hline 05536265 & 2 & Yes & Yes & 1991 & 197 & $5, \mathrm{E}$ & 973 & 3.83 & 0.389 & 0.0982 & 222 \\
\hline 05536265 & 2 & Yes & Yes & 1992 & 100 & $5, \mathrm{C}$ & 494 & 0.76 & 0.392 & 0.536 & 116 \\
\hline 05536265 & 2 & Yes & Yes & 1993 & 184 & $5, \mathrm{C}$ & 909 & 2 & 0.395 & 0.13 & 207 \\
\hline 05536265 & 2 & Yes & Yes & 1994 & 180 & $5, \mathrm{C}$ & 889 & 1.12 & 0.398 & 0.14 & 202 \\
\hline 05536265 & 2 & Yes & Yes & 1995 & 120 & $5, \mathrm{C}$ & 593 & 0.9 & 0.401 & 0.397 & 137 \\
\hline 05536265 & 2 & Yes & Yes & 1996 & 208 & $5, C$ & 1027 & 4.19 & 0.404 & 0.0862 & 231 \\
\hline 05536265 & 2 & Yes & Yes & 1997 & 168 & $5, \mathrm{C}$ & 830 & 2.06 & 0.407 & 0.174 & 187 \\
\hline 05536265 & 2 & Yes & Yes & 1998 & 114 & $5, \mathrm{C}$ & 563 & 1.19 & 0.41 & 0.446 & 129 \\
\hline 05536265 & 2 & Yes & Yes & 1999 & 150 & $5, \mathrm{C}$ & 741 & 0.81 & 0.413 & 0.238 & 167 \\
\hline 05536265 & 2 & Yes & Yes & 2000 & 169 & $5, \mathrm{C}$ & 835 & 2.02 & 0.416 & 0.175 & 187 \\
\hline 05536265 & 2 & Yes & Yes & 2001 & 119 & $5, \mathrm{C}$ & 588 & 0.8 & 0.427 & 0.426 & 132 \\
\hline 05536265 & 2 & Yes & Yes & 2002 & 116 & $5, \mathrm{C}$ & 573 & 2.56 & 0.438 & 0.457 & 127 \\
\hline 05536265 & 2 & Yes & Yes & 2003 & 169 & $5, \mathrm{C}$ & 835 & 1.74 & 0.448 & 0.189 & 181 \\
\hline 05536265 & 2 & Yes & Yes & 2004 & 100 & $5, \mathrm{C}$ & 494 & 2.2 & 0.459 & 0.599 & 108 \\
\hline 05536265 & 2 & Yes & Yes & 2005 & 127 & $5, \mathrm{C}$ & 627 & 1.95 & 0.47 & 0.411 & 135 \\
\hline 05536265 & 2 & Yes & Yes & 2006 & 202 & $5, \mathrm{C}$ & 997 & 5.56 & 0.48 & 0.123 & 210 \\
\hline 05536265 & 2 & Yes & Yes & 2007 & 123 & $5, \mathrm{C}$ & 607 & 2.69 & 0.491 & 0.457 & 127 \\
\hline 05536265 & 2 & Yes & Yes & 2008 & 219 & $5, C$ & 1081 & 5.35 & 0.502 & 0.0972 & 223 \\
\hline 05536265 & 2 & Yes & Yes & 2009 & 100 & $5, \mathrm{C}$ & 494 & 1.58 & 0.512 & 0.646 & 101 \\
\hline 05536270 & 1 & Yes & Yes & 1948 & 730 & 5 & 972 & 1.85 & 0.125 & 0.0491 & 1060 \\
\hline 05536270 & 1 & Yes & Yes & 1949 & 531 & 5 & 707 & 0.768 & 0.129 & 0.132 & 794 \\
\hline 05536270 & 1 & Yes & Yes & 1950 & 575 & 5 & 766 & 1.15 & 0.134 & 0.101 & 852 \\
\hline 05536270 & 1 & Yes & Yes & 1951 & 649 & 5 & 864 & 2.03 & 0.145 & 0.0722 & 943 \\
\hline 05536270 & 1 & Yes & Yes & 1952 & 435 & 5 & 579 & 0.0207 & 0.156 & 0.238 & 646 \\
\hline 05536270 & 1 & Yes & Yes & 1953 & 531 & 5 & 707 & 0.615 & 0.168 & 0.147 & 768 \\
\hline 05536270 & 1 & Yes & Yes & 1954 & 623 & 5 & 829 & 0.916 & 0.179 & 0.0899 & 884 \\
\hline 05536270 & 1 & Yes & Yes & 1955 & 692 & 5 & 921 & 3.32 & 0.19 & 0.0678 & 965 \\
\hline 05536270 & 1 & Yes & Yes & 1956 & 348 & 5 & 463 & 1.42 & 0.201 & 0.42 & 520 \\
\hline 05536270 & 1 & Yes & Yes & 1957 & 515 & $5, \mathrm{E}$ & 686 & 2.39 & 0.213 & 0.178 & 717 \\
\hline 05536270 & 1 & Yes & Yes & 1958 & 269 & 5 & 358 & 1.1 & 0.224 & 0.631 & 405 \\
\hline 05536270 & 1 & Yes & Yes & 1959 & 328 & 5 & 437 & 0.612 & 0.235 & 0.492 & 477 \\
\hline 05536270 & 1 & Yes & Yes & 1960 & 285 & $5, \mathrm{E}$ & 379 & 1.05 & 0.246 & 0.611 & 416 \\
\hline 05536270 & 1 & Yes & Yes & 1961 & 305 & 5 & 406 & 1.49 & 0.256 & 0.568 & 438 \\
\hline 05536270 & 1 & Yes & Yes & 1962 & 185 & $5, \mathrm{E}$ & 246 & 0.242 & 0.265 & 0.841 & 285 \\
\hline
\end{tabular}




\begin{tabular}{|c|c|c|c|c|c|c|c|c|c|c|c|}
\hline $\begin{array}{c}\text { U.S. } \\
\text { Geological } \\
\text { Survey } \\
\text { streamgage } \\
\text { number } \\
\end{array}$ & $\begin{array}{c}\text { Segment } \\
\text { number }\end{array}$ & $\begin{array}{l}\text { Streamgage used in } \\
\text { regression analyses } \\
\text { (non-redundant) }\end{array}$ & $\begin{array}{c}\text { Discharge value } \\
\text { used in } \\
\text { adjustment } \\
\text { regression }\end{array}$ & $\begin{array}{l}\text { Water } \\
\text { year }\end{array}$ & $\begin{array}{c}\text { Observed annual } \\
\text { maximum peak } \\
\text { discharge }\left(\mathrm{ft}^{3} / \mathrm{s}\right)\end{array}$ & $\begin{array}{c}\text { NWIS peak } \\
\text { code }\end{array}$ & $\begin{array}{c}\text { Observed annual } \\
\text { maximum peak } \\
\text { discharge with } \\
\text { segment intercept } \\
\text { value subtracted } \\
\left(\mathrm{ft}^{3} / \mathrm{s}\right)\end{array}$ & $\begin{array}{c}\text { Observed } \\
\text { precipitation } \\
\text { (inches) }\end{array}$ & Urban fraction & $\begin{array}{l}\text { Exceedance } \\
\text { probability }\end{array}$ & $\begin{array}{c}\text { Urban-adjusted } \\
\text { annual maximum } \\
\text { peak discharge } \\
\left(\mathrm{ft}^{3} / \mathrm{s}\right) \\
\end{array}$ \\
\hline 05536270 & 1 & Yes & Yes & 1963 & 295 & $5, E$ & 393 & 1.49 & 0.274 & 0.61 & 417 \\
\hline 05536270 & 1 & Yes & Yes & 1964 & 121 & $5, E$ & 161 & 0.991 & 0.283 & 0.941 & 199 \\
\hline 05536270 & 1 & Yes & Yes & 1965 & 234 & $5, E$ & 312 & 0.896 & 0.292 & 0.761 & 336 \\
\hline 05536270 & 1 & Yes & Yes & 1966 & 540 & 5 & 719 & 1.63 & 0.302 & 0.193 & 693 \\
\hline 05536270 & 1 & Yes & Yes & 1967 & 240 & 5 & 320 & 1.15 & 0.311 & 0.76 & 337 \\
\hline 05536270 & 1 & Yes & Yes & 1968 & 374 & 5 & 498 & 2.11 & 0.32 & 0.464 & 493 \\
\hline 05536270 & 1 & Yes & Yes & 1969 & 142 & $5, E$ & 189 & 0.945 & 0.329 & 0.931 & 213 \\
\hline 05536270 & 1 & Yes & Yes & 1970 & 244 & $5, \mathrm{E}$ & 325 & 1.47 & 0.338 & 0.769 & 331 \\
\hline 05536270 & 1 & Yes & Yes & 1971 & 220 & $5, \mathrm{E}$ & 293 & 0.924 & 0.35 & 0.822 & 299 \\
\hline 05536270 & 1 & Yes & Yes & 1972 & 266 & 5 & 354 & 1.09 & 0.361 & 0.741 & 348 \\
\hline 05536270 & 1 & Yes & Yes & 1973 & 390 & 5 & 519 & 0.887 & 0.373 & 0.478 & 486 \\
\hline 05536270 & 1 & Yes & Yes & 1974 & 328 & 5 & 437 & 1.3 & 0.384 & 0.625 & 409 \\
\hline 05536270 & 1 & Yes & Yes & 1975 & 340 & 5 & 453 & 1.12 & 0.396 & 0.609 & 417 \\
\hline 05536270 & 1 & Yes & Yes & 1976 & 497 & 5 & 662 & 1.56 & 0.407 & 0.314 & 586 \\
\hline 05536270 & 1 & Yes & Yes & 1977 & 238 & $5, \mathrm{E}$ & 317 & 1.02 & 0.418 & 0.828 & 294 \\
\hline 05536270 & 1 & Yes & Yes & 1978 & 249 & 5 & 331 & 1.16 & 0.43 & 0.816 & 302 \\
\hline 05536270 & 1 & Yes & Yes & 1979 & 505 & 5 & 672 & 0.803 & 0.441 & 0.329 & 576 \\
\hline 05536275 & 1 & No & No & 1947 & 4200 & -- & 1197 & 2.85 & 0.185 & 0.0298 & 5680 \\
\hline 05536275 & 1 & No & No & 1948 & 4040 & -- & 1151 & 2.07 & 0.192 & 0.0351 & 5460 \\
\hline 05536275 & 1 & No & No & 1949 & 1320 & -- & 376 & 0.815 & 0.2 & 0.578 & 2040 \\
\hline 05536275 & 1 & No & No & 1950 & 2010 & -- & 573 & 1.52 & 0.207 & 0.276 & 2880 \\
\hline 05536275 & 1 & No & No & 1951 & 2720 & -- & 775 & 2.36 & 0.22 & 0.129 & 3740 \\
\hline 05536275 & 1 & No & No & 1952 & 1570 & -- & 447 & 1.05 & 0.233 & 0.472 & 2290 \\
\hline 05536275 & 1 & No & No & 1953 & 2280 & -- & 650 & 1.74 & 0.246 & 0.22 & 3100 \\
\hline 05536275 & 1 & No & No & 1954 & 1890 & -- & 538 & 1.47 & 0.26 & 0.356 & 2620 \\
\hline 05536275 & 1 & No & No & 1955 & 3370 & -- & 960 & 3.95 & 0.273 & 0.0732 & 4370 \\
\hline 05536275 & 1 & No & No & 1956 & 2280 & -- & 650 & 1.32 & 0.286 & 0.244 & 3000 \\
\hline 05536275 & 1 & No & No & 1957 & 4700 & -- & 1339 & 2.41 & 0.299 & 0.0279 & 5790 \\
\hline 05536275 & 1 & No & No & 1958 & 1560 & -- & 444 & 1.33 & 0.312 & 0.548 & 2110 \\
\hline 05536275 & 1 & No & No & 1959 & 1780 & -- & 507 & 1.41 & 0.325 & 0.455 & 2340 \\
\hline 05536275 & 1 & No & No & 1960 & 1220 & D & 348 & 1.36 & 0.339 & 0.735 & 1660 \\
\hline 05536275 & 1 & No & No & 1961 & 2420 & -- & 689 & 1.93 & 0.347 & 0.244 & 3000 \\
\hline 05536275 & 1 & No & No & 1962 & 1100 & -- & 313 & 0.23 & 0.356 & 0.796 & 1490 \\
\hline 05536275 & 1 & No & No & 1963 & 975 & -- & 278 & 1.7 & 0.365 & 0.849 & 1330 \\
\hline 05536275 & 1 & No & No & 1964 & 621 & -- & 177 & 1.12 & 0.373 & 0.95 & 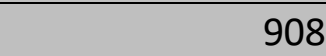 \\
\hline
\end{tabular}




\begin{tabular}{|c|c|c|c|c|c|c|c|c|c|c|c|}
\hline $\begin{array}{c}\text { U.S. } \\
\text { Geological } \\
\text { Survey } \\
\text { streamgage } \\
\text { number }\end{array}$ & $\begin{array}{l}\text { Segment } \\
\text { number }\end{array}$ & $\begin{array}{l}\text { Streamgage used in } \\
\text { regression analyses } \\
\text { (non-redundant) }\end{array}$ & $\begin{array}{l}\text { Discharge value } \\
\text { used in } \\
\text { adjustment } \\
\text { regression }\end{array}$ & $\begin{array}{l}\text { Water } \\
\text { year }\end{array}$ & $\begin{array}{l}\text { Observed annual } \\
\text { maximum peak } \\
\text { discharge }\left(\mathrm{ft}^{3} / \mathrm{s}\right)\end{array}$ & $\begin{array}{l}\text { NWIS peak } \\
\text { code }\end{array}$ & $\begin{array}{l}\text { Observed annual } \\
\text { maximum peak } \\
\text { discharge with } \\
\text { segment intercept } \\
\text { value subtracted } \\
\left(\mathrm{ft}^{3} / \mathrm{s}\right)\end{array}$ & $\begin{array}{l}\text { Observed } \\
\text { precipitation } \\
\text { (inches) }\end{array}$ & Urban fraction & $\begin{array}{l}\text { Exceedance } \\
\text { probability }\end{array}$ & $\begin{array}{c}\text { Urban-adjusted } \\
\text { annual maximum } \\
\text { peak discharge } \\
\left(\mathrm{ft}^{3} / \mathrm{s}\right)\end{array}$ \\
\hline 05536275 & 1 & No & No & 1965 & 1290 & -- & 368 & 1.05 & 0.382 & 0.735 & 1660 \\
\hline 05536275 & 1 & No & No & 1966 & 3240 & -- & 923 & 2.04 & 0.391 & 0.121 & 3810 \\
\hline 05536275 & 1 & No & No & 1967 & 1690 & -- & 481 & 1.27 & 0.399 & 0.564 & 2070 \\
\hline 05536275 & 1 & No & No & 1968 & 3420 & -- & 974 & 2.13 & 0.408 & 0.104 & 3950 \\
\hline 05536275 & 1 & No & No & 1969 & 1090 & -- & 311 & 0.68 & 0.417 & 0.835 & 1370 \\
\hline 05536275 & 1 & No & No & 1970 & 1830 & -- & 521 & 1.33 & 0.425 & 0.523 & 2170 \\
\hline 05536275 & 1 & No & No & 1971 & 1250 & -- & 356 & 1.1 & 0.432 & 0.784 & 1520 \\
\hline 05536275 & 1 & No & No & 1972 & 1410 & -- & 402 & 1.26 & 0.438 & 0.724 & 1680 \\
\hline 05536275 & 1 & No & No & 1973 & 3060 & -- & 872 & 1.03 & 0.445 & 0.168 & 3430 \\
\hline 05536275 & 1 & No & No & 1974 & 2970 & -- & 846 & 1.71 & 0.451 & 0.184 & 3310 \\
\hline 05536275 & 1 & No & No & 1975 & 1630 & -- & 464 & 1.45 & 0.457 & 0.642 & 1880 \\
\hline 05536275 & 1 & No & No & 1976 & 1940 & -- & 553 & 1.7 & 0.464 & 0.51 & 2200 \\
\hline 05536275 & 1 & No & No & 1977 & 1580 & -- & 450 & 1.28 & 0.47 & 0.676 & 1800 \\
\hline 05536275 & 1 & No & No & 1978 & 2020 & -- & 575 & 1.24 & 0.476 & 0.489 & 2250 \\
\hline 05536275 & 1 & No & No & 1979 & 2680 & -- & 764 & 0.641 & 0.483 & 0.263 & 2920 \\
\hline 05536275 & 1 & No & No & 1980 & 1440 & -- & 410 & 0.971 & 0.489 & 0.751 & 1620 \\
\hline 05536275 & 1 & No & No & 1981 & 4140 & -- & 1179 & 2.18 & 0.491 & 0.0701 & 4430 \\
\hline 05536275 & 1 & No & No & 1982 & 2370 & -- & 675 & 2.54 & 0.493 & 0.37 & 2580 \\
\hline 05536275 & 1 & No & No & 1983 & 3370 & -- & 960 & 2.46 & 0.494 & 0.146 & 3610 \\
\hline 05536275 & 1 & No & No & 1984 & 2060 & -- & 587 & 1.09 & 0.496 & 0.491 & 2250 \\
\hline 05536275 & 1 & No & No & 1985 & 2110 & -- & 601 & 1.01 & 0.497 & 0.472 & 2300 \\
\hline 05536275 & 1 & No & No & 1986 & 1630 & -- & 464 & 1.37 & 0.499 & 0.68 & 1790 \\
\hline 05536275 & 1 & No & No & 1987 & 1350 & -- & 385 & 1.98 & 0.501 & 0.792 & 1500 \\
\hline 05536275 & 0 & No & No & 1988 & 964 & -- & 279 & 0.715 & 0.502 & 0.912 & 1120 \\
\hline 05536275 & 2 & No & No & 1989 & 3000 & -- & 882 & 1.9 & 0.504 & 0.188 & 3290 \\
\hline 05536275 & 2 & No & No & 1990 & 1620 & -- & 476 & 1.51 & 0.506 & 0.667 & 1820 \\
\hline 05536275 & 2 & No & No & 1991 & 4210 & -- & 1238 & 2.87 & 0.508 & 0.0631 & 4570 \\
\hline 05536275 & 2 & No & No & 1992 & 1070 & C & 315 & 2.11 & 0.51 & 0.881 & 1230 \\
\hline 05536275 & 2 & No & No & 1993 & 2610 & C & 767 & 2.09 & 0.512 & 0.28 & 2860 \\
\hline 05536275 & 2 & No & No & 1994 & 1640 & C & 482 & 1.4 & 0.514 & 0.665 & 1830 \\
\hline 05536275 & 2 & No & No & 1995 & 1400 & C & 412 & 1.11 & 0.516 & 0.767 & 1570 \\
\hline 05536275 & 2 & No & No & 1996 & 4470 & C & 1314 & 4.28 & 0.518 & 0.0525 & 4810 \\
\hline 05536275 & 2 & No & No & 1997 & 3370 & C & 991 & 2.16 & 0.52 & 0.142 & 3640 \\
\hline 05536275 & 2 & No & No & 1998 & 2360 & C & 694 & 1.5 & 0.522 & 0.373 & 2570 \\
\hline 05536275 & 2 & No & No & 1999 & 2230 & C & 656 & 0.787 & 0.524 & 0.423 & 2430 \\
\hline
\end{tabular}




\begin{tabular}{|c|c|c|c|c|c|c|c|c|c|c|c|}
\hline $\begin{array}{c}\text { U.S. } \\
\text { Geological } \\
\text { Survey } \\
\text { streamgage } \\
\text { number } \\
\end{array}$ & $\begin{array}{c}\text { Segment } \\
\text { number }\end{array}$ & $\begin{array}{l}\text { Streamgage used in } \\
\text { regression analyses } \\
\text { (non-redundant) }\end{array}$ & $\begin{array}{c}\text { Discharge value } \\
\text { used in } \\
\text { adjustment } \\
\text { regression }\end{array}$ & $\begin{array}{c}\text { Water } \\
\text { year }\end{array}$ & $\begin{array}{c}\text { Observed annual } \\
\text { maximum peak } \\
\text { discharge }\left(\mathrm{ft}^{3} / \mathrm{s}\right)\end{array}$ & $\begin{array}{c}\text { NWIS peak } \\
\text { code }\end{array}$ & $\begin{array}{l}\text { Observed annual } \\
\text { maximum peak } \\
\text { discharge with } \\
\text { segment intercept } \\
\text { value subtracted } \\
\left(\mathrm{ft}^{3} / \mathrm{s}\right)\end{array}$ & $\begin{array}{c}\text { Observed } \\
\text { precipitation } \\
\text { (inches) }\end{array}$ & Urban fraction & $\begin{array}{l}\text { Exceedance } \\
\text { probability }\end{array}$ & $\begin{array}{c}\text { Urban-adjusted } \\
\text { annual maximum } \\
\text { peak discharge } \\
\left(\mathrm{ft}^{3} / \mathrm{s}\right)\end{array}$ \\
\hline 05536275 & 2 & No & No & 2000 & 1390 & C & 409 & 1.15 & 0.526 & 0.777 & 1540 \\
\hline 05536275 & 2 & No & No & 2001 & 1900 & $C$ & 559 & 0.636 & 0.533 & 0.568 & 2060 \\
\hline 05536275 & 2 & No & No & 2002 & 2920 & $\mathrm{C}$ & 859 & 1.46 & 0.541 & 0.222 & 3100 \\
\hline 05536275 & 2 & No & No & 2003 & 2730 & $C$ & 803 & 1.86 & 0.549 & 0.274 & 2880 \\
\hline 05536275 & 2 & No & No & 2004 & 2340 & C & 688 & 2.32 & 0.557 & 0.413 & 2460 \\
\hline 05536275 & 2 & No & No & 2005 & 3400 & C & 1000 & 1.12 & 0.564 & 0.157 & 3520 \\
\hline 05536275 & 2 & No & No & 2006 & 5540 & C & 1629 & 2.6 & 0.572 & 0.0302 & 5680 \\
\hline 05536275 & 0 & No & No & 2007 & 2820 & C & NA & 1.84 & 0.58 & NA & NA \\
\hline 05536275 & 0 & No & No & 2008 & 5860 & C & NA & 4.41 & 0.588 & NA & NA \\
\hline 05536275 & 0 & No & No & 2009 & 3490 & C & NA & 1.09 & 0.595 & NA & NA \\
\hline 05536290 & 1 & No & No & 1947 & 4760 & 5 & 1186 & 2.88 & 0.221 & 0.0343 & 6670 \\
\hline 05536290 & 1 & No & No & 1948 & 3810 & 5 & 949 & 1.04 & 0.23 & 0.0686 & 5440 \\
\hline 05536290 & 1 & No & No & 1949 & 1580 & 5 & 394 & 0.901 & 0.239 & 0.578 & 2500 \\
\hline 05536290 & 1 & No & No & 1950 & 2780 & 5 & 693 & 1.32 & 0.248 & 0.188 & 3990 \\
\hline 05536290 & 1 & No & No & 1951 & 3360 & 5 & 837 & 2.24 & 0.26 & 0.112 & 4730 \\
\hline 05536290 & 1 & No & No & 1952 & 2480 & 5 & 618 & 1 & 0.273 & 0.269 & 3550 \\
\hline 05536290 & 1 & No & No & 1953 & 2420 & 5 & 603 & 1.41 & 0.285 & 0.293 & 3440 \\
\hline 05536290 & 1 & No & No & 1954 & 2100 & 5 & 523 & 1.45 & 0.297 & 0.406 & 3030 \\
\hline 05536290 & 1 & No & No & 1955 & 4210 & 5 & 1049 & 3.71 & 0.31 & 0.0627 & 5600 \\
\hline 05536290 & 1 & No & No & 1956 & 2420 & 5 & 603 & 1.35 & 0.322 & 0.32 & 3340 \\
\hline 05536290 & 1 & No & No & 1957 & 4440 & 5 & 1106 & 2.24 & 0.334 & 0.0565 & 5760 \\
\hline 05536290 & 1 & No & No & 1958 & 1930 & 5 & 481 & 1.44 & 0.346 & 0.516 & 2680 \\
\hline 05536290 & 1 & No & No & 1959 & 2820 & 5 & 703 & 1.43 & 0.359 & 0.238 & 3680 \\
\hline 05536290 & 1 & No & No & 1960 & 1860 & 5 & 463 & 1.53 & 0.371 & 0.568 & 2530 \\
\hline 05536290 & 1 & No & No & 1961 & 2410 & 5 & 600 & 1.88 & 0.378 & 0.367 & 3170 \\
\hline 05536290 & 1 & No & No & 1962 & 1280 & 5 & 319 & 0.23 & 0.385 & 0.806 & 1810 \\
\hline 05536290 & 1 & No & No & 1963 & 1400 & 5 & 349 & 1.69 & 0.392 & 0.768 & 1940 \\
\hline 05536290 & 1 & No & No & 1964 & 995 & 5 & 248 & 1.08 & 0.399 & 0.901 & 1450 \\
\hline 05536290 & 1 & No & No & 1965 & 1710 & 5 & 426 & 0.964 & 0.406 & 0.66 & 2260 \\
\hline 05536290 & 1 & No & No & 1966 & 3490 & 5 & 870 & 1.76 & 0.413 & 0.156 & 4290 \\
\hline 05536290 & 1 & No & No & 1967 & 1890 & 5 & 471 & 1.24 & 0.42 & 0.601 & 2440 \\
\hline 05536290 & 1 & No & No & 1968 & 3060 & 5 & 762 & 1.85 & 0.427 & 0.228 & 3740 \\
\hline 05536290 & 1 & No & No & 1969 & 1340 & 5 & 334 & 0.505 & 0.434 & 0.815 & 1780 \\
\hline 05536290 & 1 & No & No & 1970 & 2340 & 5 & 583 & 1.39 & 0.441 & 0.445 & 2910 \\
\hline 05536290 & 1 & No & No & 1971 & 1520 & 5 & 379 & 1.02 & 0.448 & 0.764 & 1950 \\
\hline
\end{tabular}




\begin{tabular}{|c|c|c|c|c|c|c|c|c|c|c|c|}
\hline $\begin{array}{l}\text { U.S. } \\
\text { Geological } \\
\text { Survey } \\
\text { streamgage } \\
\text { number }\end{array}$ & $\begin{array}{l}\text { Segment } \\
\text { number }\end{array}$ & $\begin{array}{l}\text { Streamgage used in } \\
\text { regression analyses } \\
\text { (non-redundant) }\end{array}$ & $\begin{array}{c}\text { Discharge value } \\
\text { used in } \\
\text { adjustment } \\
\text { regression }\end{array}$ & $\begin{array}{l}\text { Water } \\
\text { year }\end{array}$ & $\begin{array}{l}\text { Observed annual } \\
\text { maximum peak } \\
\text { discharge }\left(\mathrm{ft}^{3} / \mathrm{s}\right)\end{array}$ & $\begin{array}{l}\text { NWIS peak } \\
\text { code }\end{array}$ & $\begin{array}{l}\text { Observed annual } \\
\text { maximum peak } \\
\text { discharge with } \\
\text { segment intercept } \\
\text { value subtracted } \\
\left(\mathrm{ft}^{3} / \mathrm{s}\right)\end{array}$ & $\begin{array}{l}\text { Observed } \\
\text { precipitation } \\
\text { (inches) }\end{array}$ & Urban fraction & $\begin{array}{l}\text { Exceedance } \\
\text { probability }\end{array}$ & $\begin{array}{c}\text { Urban-adjusted } \\
\text { annual maximum } \\
\text { peak discharge } \\
\left(\mathrm{ft}^{3} / \mathrm{s}\right)\end{array}$ \\
\hline 05536290 & 1 & No & No & 1972 & 1820 & 5 & 453 & 1.32 & 0.455 & 0.657 & 2270 \\
\hline 05536290 & 1 & No & No & 1973 & 3500 & 5 & 872 & 0.91 & 0.462 & 0.175 & 4120 \\
\hline 05536290 & 1 & No & No & 1974 & 2980 & 5 & 742 & 1.46 & 0.469 & 0.273 & 3520 \\
\hline 05536290 & 1 & No & No & 1975 & 2480 & 5 & 618 & 1.37 & 0.476 & 0.429 & 2960 \\
\hline 05536290 & 1 & No & No & 1976 & 2650 & 5 & 660 & 0.856 & 0.483 & 0.38 & 3130 \\
\hline 05536290 & 1 & No & No & 1977 & 2050 & 5 & 511 & 1.21 & 0.49 & 0.601 & 2440 \\
\hline 05536290 & 1 & No & No & 1978 & 2860 & 5 & 713 & 1.19 & 0.497 & 0.328 & 3310 \\
\hline 05536290 & 1 & No & No & 1979 & 3100 & 5 & 772 & 0.714 & 0.504 & 0.269 & 3540 \\
\hline 05536290 & 1 & No & No & 1980 & 2320 & 5 & 578 & 0.852 & 0.511 & 0.517 & 2680 \\
\hline 05536290 & 1 & No & No & 1981 & 4070 & 5 & 1014 & 2.03 & 0.513 & 0.129 & 4560 \\
\hline 05536290 & 1 & No & No & 1982 & 2790 & 5 & 695 & 0.507 & 0.515 & 0.365 & 3180 \\
\hline 05536290 & 1 & No & No & 1983 & 3520 & 5 & 877 & 2.2 & 0.518 & 0.196 & 3940 \\
\hline 05536290 & 1 & No & No & 1984 & 2550 & 5 & 635 & 0.927 & 0.52 & 0.447 & 2900 \\
\hline 05536290 & 1 & No & No & 1985 & 2980 & 5 & 742 & 0.942 & 0.522 & 0.315 & 3350 \\
\hline 05536290 & 1 & No & No & 1986 & 2510 & 5 & 625 & 1.23 & 0.525 & 0.464 & 2850 \\
\hline 05536290 & 1 & No & No & 1987 & 1950 & 5 & 486 & 1.32 & 0.527 & 0.672 & 2230 \\
\hline 05536290 & 1 & No & No & 1988 & 1470 & $5, C$ & 366 & 0.658 & 0.53 & 0.833 & 1710 \\
\hline 05536290 & 1 & No & No & 1989 & 3390 & $5, C$ & 845 & 1.69 & 0.532 & 0.227 & 3750 \\
\hline 05536290 & 1 & No & No & 1990 & 2070 & $5, C$ & 516 & 1.56 & 0.534 & 0.633 & 2340 \\
\hline 05536290 & 1 & No & No & 1991 & 4150 & $5, C$ & 1034 & 2.9 & 0.538 & 0.131 & 4550 \\
\hline 05536290 & 1 & No & No & 1992 & 1650 & $5, C$ & 411 & 2.43 & 0.541 & 0.785 & 1880 \\
\hline 05536290 & 1 & No & No & 1993 & 3020 & $5, C$ & 752 & 2.02 & 0.544 & 0.322 & 3320 \\
\hline 05536290 & 1 & No & No & 1994 & 2120 & $5, C$ & 528 & 0.946 & 0.547 & 0.626 & 2360 \\
\hline 05536290 & 1 & No & No & 1995 & 2010 & $5, C$ & 501 & 1.03 & 0.551 & 0.67 & 2240 \\
\hline 05536290 & 1 & No & No & 1996 & 3870 & $5, C$ & 964 & 3.83 & 0.554 & 0.169 & 4180 \\
\hline 05536290 & 1 & No & No & 1997 & 3540 & $5, C$ & 882 & 2.14 & 0.557 & 0.215 & 3820 \\
\hline 05536290 & 1 & No & No & 1998 & 2820 & $5, C$ & 703 & 1.41 & 0.561 & 0.398 & 3070 \\
\hline 05536290 & 1 & No & No & 1999 & 2630 & $5, C$ & 655 & 0.72 & 0.564 & 0.462 & 2860 \\
\hline 05536290 & 1 & No & No & 2000 & 2160 & $5, C$ & 538 & 1.29 & 0.567 & 0.63 & 2350 \\
\hline 05536290 & 1 & No & No & 2001 & 2410 & $5, C$ & 600 & 0.638 & 0.574 & 0.546 & 2590 \\
\hline 05536290 & 1 & No & No & 2002 & 3620 & $5, C$ & 902 & 1.69 & 0.581 & 0.215 & 3820 \\
\hline 05536290 & 1 & No & No & 2003 & 1610 & $5, C$ & 401 & 0.907 & 0.589 & 0.829 & 1730 \\
\hline 05536290 & 1 & No & No & 2004 & 1820 & $5, C$ & 453 & 2.12 & 0.596 & 0.771 & 1930 \\
\hline 05536290 & 1 & No & No & 2005 & 2310 & $5, C$ & 576 & 1.1 & 0.603 & 0.61 & 2410 \\
\hline 05536290 & 1 & No & No & 2006 & 2600 & $5, C$ & 648 & 2.67 & 0.61 & 0.516 & 2680 \\
\hline
\end{tabular}




\begin{tabular}{|c|c|c|c|c|c|c|c|c|c|c|c|}
\hline $\begin{array}{l}\text { U.S. } \\
\text { Geological } \\
\text { Survey } \\
\text { streamgage } \\
\text { number }\end{array}$ & $\begin{array}{l}\text { Segment } \\
\text { number }\end{array}$ & $\begin{array}{l}\text { Streamgage used in } \\
\text { regression analyses } \\
\text { (non-redundant) }\end{array}$ & $\begin{array}{c}\text { Discharge value } \\
\text { used in } \\
\text { adjustment } \\
\text { regression }\end{array}$ & $\begin{array}{l}\text { Water } \\
\text { year }\end{array}$ & $\begin{array}{c}\text { Observed annual } \\
\text { maximum peak } \\
\text { discharge }\left(\mathrm{ft}^{3} / \mathrm{s}\right)\end{array}$ & $\begin{array}{l}\text { NWIS peak } \\
\text { code }\end{array}$ & $\begin{array}{l}\text { Observed annual } \\
\text { maximum peak } \\
\text { discharge with } \\
\text { segment intercept } \\
\text { value subtracted } \\
\left(\mathrm{ft}^{3} / \mathrm{s}\right)\end{array}$ & $\begin{array}{l}\text { Observed } \\
\text { precipitation } \\
\text { (inches) }\end{array}$ & Urban fraction & $\begin{array}{l}\text { Exceedance } \\
\text { probability }\end{array}$ & $\begin{array}{c}\text { Urban-adjusted } \\
\text { annual maximum } \\
\text { peak discharge } \\
\left(\mathrm{ft}^{3} / \mathrm{s}\right)\end{array}$ \\
\hline 05536290 & 1 & No & No & 2007 & 2310 & $5, \mathrm{C}$ & 576 & 1.82 & 0.617 & 0.623 & 2370 \\
\hline 05536290 & 1 & No & No & 2008 & 3930 & $5, \mathrm{C}$ & 979 & 4.43 & 0.624 & 0.192 & 3980 \\
\hline 05536290 & 1 & No & No & 2009 & 2650 & $5, \mathrm{C}$ & 660 & 1.14 & 0.631 & 0.52 & 2670 \\
\hline 05536310 & 1 & Yes & No & 1955 & 389 & 7 & 998 & 3.51 & 0.577 & 0.163 & 503 \\
\hline 05536310 & 1 & Yes & No & 1957 & 467 & 7 & 1198 & 2.37 & 0.626 & 0.101 & 575 \\
\hline 05536310 & 1 & Yes & Yes & 1961 & 410 & -- & 1052 & 1.61 & 0.709 & 0.196 & 471 \\
\hline 05536310 & 1 & Yes & Yes & 1962 & 191 & -- & 490 & 0.249 & 0.717 & 0.818 & 232 \\
\hline 05536310 & 1 & Yes & Yes & 1963 & 259 & -- & 664 & 1.58 & 0.726 & 0.609 & 302 \\
\hline 05536310 & 1 & Yes & Yes & 1964 & 145 & -- & 372 & 1.03 & 0.734 & 0.933 & 179 \\
\hline 05536310 & 1 & Yes & Yes & 1965 & 306 & -- & 785 & 0.949 & 0.742 & 0.478 & 348 \\
\hline 05536310 & 1 & Yes & Yes & 1966 & 431 & -- & 1106 & 1.52 & 0.751 & 0.191 & 476 \\
\hline 05536310 & 1 & Yes & Yes & 1967 & 311 & -- & 798 & 1.19 & 0.759 & 0.48 & 347 \\
\hline 05536310 & 1 & Yes & Yes & 1968 & 502 & -- & 1288 & 1.5 & 0.767 & 0.127 & 545 \\
\hline 05536310 & 1 & Yes & Yes & 1969 & 210 & -- & 539 & 0.861 & 0.776 & 0.811 & 235 \\
\hline 05536310 & 1 & Yes & Yes & 1970 & 398 & -- & 1021 & 1.4 & 0.784 & 0.264 & 427 \\
\hline 05536310 & 1 & Yes & Yes & 1971 & 322 & -- & 826 & 0.269 & 0.79 & 0.48 & 347 \\
\hline 05536310 & 1 & Yes & Yes & 1972 & 265 & -- & 680 & 0.88 & 0.796 & 0.665 & 285 \\
\hline 05536310 & 1 & Yes & Yes & 1973 & 356 & -- & 913 & 0.927 & 0.802 & 0.397 & 377 \\
\hline 05536310 & 1 & Yes & Yes & 1974 & 385 & -- & 988 & 1.08 & 0.809 & 0.316 & 404 \\
\hline 05536310 & 0 & Yes & No & 1975 & 214 & -- & NA & 1.11 & 0.815 & NA & NA \\
\hline 05536310 & 0 & Yes & No & 1976 & 259 & -- & NA & 0.928 & 0.821 & NA & NA \\
\hline 05536335 & 1 & No & No & 1954 & 204 & -- & 545 & 1.65 & 0.27 & 0.355 & 379 \\
\hline 05536335 & 1 & No & No & 1955 & 481 & -- & 1285 & 3.27 & 0.294 & 0.0318 & 759 \\
\hline 05536335 & 1 & No & No & 1956 & 160 & -- & 427 & 1.37 & 0.319 & 0.585 & 300 \\
\hline 05536335 & 1 & No & No & 1957 & 479 & -- & 1279 & 2.41 & 0.343 & 0.037 & 732 \\
\hline 05536335 & 1 & No & No & 1958 & 145 & 2 & 387 & 1.4 & 0.368 & 0.693 & 267 \\
\hline 05536335 & 1 & No & No & 1959 & 195 & 2 & 521 & 1.26 & 0.392 & 0.493 & 330 \\
\hline 05536335 & 1 & No & No & 1960 & 180 & 2 & 481 & 0.0494 & 0.417 & 0.581 & 301 \\
\hline 05536335 & 1 & No & No & 1961 & 282 & -- & 753 & 1.45 & 0.428 & 0.237 & 427 \\
\hline 05536335 & 1 & No & No & 1962 & 191 & -- & 510 & 0.3 & 0.44 & 0.555 & 309 \\
\hline 05536335 & 1 & No & No & 1963 & 162 & -- & 433 & 1.51 & 0.452 & 0.689 & 268 \\
\hline 05536335 & 1 & No & No & 1964 & 65 & -- & 174 & 0.989 & 0.464 & 0.965 & 139 \\
\hline 05536335 & 1 & No & No & 1965 & 165 & -- & 441 & 0.89 & 0.476 & 0.697 & 266 \\
\hline 05536335 & 1 & No & No & 1966 & 332 & -- & 887 & 1.31 & 0.488 & 0.178 & 469 \\
\hline 05536335 & 1 & No & No & 1967 & 205 & -- & 548 & 1.15 & 0.499 & 0.552 & 310 \\
\hline
\end{tabular}




\begin{tabular}{|c|c|c|c|c|c|c|c|c|c|c|c|}
\hline $\begin{array}{l}\text { U.S. } \\
\text { Geological } \\
\text { Survey } \\
\text { streamgage } \\
\text { number }\end{array}$ & $\begin{array}{c}\text { Segment } \\
\text { number }\end{array}$ & $\begin{array}{l}\text { Streamgage used in } \\
\text { regression analyses } \\
\text { (non-redundant) }\end{array}$ & $\begin{array}{c}\text { Discharge value } \\
\text { used in } \\
\text { adjustment } \\
\text { regression }\end{array}$ & $\begin{array}{l}\text { Water } \\
\text { year }\end{array}$ & $\begin{array}{c}\text { Observed annual } \\
\text { maximum peak } \\
\text { discharge }\left(\mathrm{ft}^{3} / \mathrm{s}\right)\end{array}$ & $\begin{array}{l}\text { NWIS peak } \\
\text { code }\end{array}$ & $\begin{array}{l}\text { Observed annual } \\
\text { maximum peak } \\
\text { discharge with } \\
\text { segment intercept } \\
\text { value subtracted } \\
\left(\mathrm{ft}^{3} / \mathrm{s}\right)\end{array}$ & $\begin{array}{l}\text { Observed } \\
\text { precipitation } \\
\text { (inches) }\end{array}$ & Urban fraction & $\begin{array}{l}\text { Exceedance } \\
\text { probability }\end{array}$ & $\begin{array}{c}\text { Urban-adjusted } \\
\text { annual maximum } \\
\text { peak discharge } \\
\left(\mathrm{ft}^{3} / \mathrm{s}\right)\end{array}$ \\
\hline 05536335 & 1 & No & No & 1968 & 253 & -- & 676 & 1.46 & 0.511 & 0.386 & 368 \\
\hline 05536335 & 1 & No & No & 1969 & 166 & -- & 443 & 0.93 & 0.523 & 0.73 & 255 \\
\hline 05536335 & 1 & No & No & 1970 & 298 & -- & 796 & 1.1 & 0.535 & 0.27 & 410 \\
\hline 05536335 & 1 & No & No & 1971 & 189 & -- & 505 & 0.309 & 0.561 & 0.674 & 273 \\
\hline 05536335 & 1 & No & No & 1972 & 232 & -- & 620 & 1.23 & 0.587 & 0.532 & 317 \\
\hline 05536335 & 1 & No & No & 1973 & 580 & 2 & 1549 & 1.31 & 0.613 & 0.0404 & 712 \\
\hline 05536335 & 1 & No & No & 1974 & 234 & -- & 625 & 1.24 & 0.639 & 0.575 & 303 \\
\hline 05536335 & 1 & No & No & 1975 & 279 & -- & 745 & 0.665 & 0.665 & 0.447 & 347 \\
\hline 05536335 & 1 & No & No & 1976 & 201 & -- & 537 & 0.915 & 0.691 & 0.745 & 251 \\
\hline 05536335 & 1 & No & No & 1977 & 188 & -- & 502 & 1.4 & 0.717 & 0.805 & 229 \\
\hline 05536335 & 1 & No & No & 1978 & 280 & -- & 748 & 1.11 & 0.743 & 0.522 & 320 \\
\hline 05536335 & 1 & No & No & 1979 & 273 & -- & 729 & 0.847 & 0.769 & 0.571 & 304 \\
\hline 05536340 & 1 & Yes & Yes & 1951 & 375 & -- & 750 & 1.32 & 0.209 & 0.138 & 283 \\
\hline 05536340 & 1 & Yes & Yes & 1952 & 256 & $\mathrm{D}$ & 512 & 0.943 & 0.231 & 0.37 & 205 \\
\hline 05536340 & 1 & Yes & Yes & 1953 & 384 & -- & 768 & 1.14 & 0.253 & 0.145 & 279 \\
\hline 05536340 & 1 & Yes & Yes & 1954 & 248 & $\mathrm{E}$ & 496 & 1.65 & 0.275 & 0.428 & 193 \\
\hline 05536340 & 1 & Yes & Yes & 1955 & 569 & -- & 1138 & 3.27 & 0.296 & 0.0469 & 383 \\
\hline 05536340 & 1 & Yes & Yes & 1956 & 204 & $\mathrm{E}$ & 408 & 0.738 & 0.318 & 0.618 & 157 \\
\hline 05536340 & 1 & Yes & Yes & 1957 & 550 & -- & 1100 & 2.4 & 0.34 & 0.0585 & 360 \\
\hline 05536340 & 1 & Yes & Yes & 1958 & 168 & $\mathrm{E}$ & 336 & 1.4 & 0.362 & 0.767 & 130 \\
\hline 05536340 & 1 & Yes & Yes & 1959 & 228 & $\mathrm{E}$ & 456 & 1.26 & 0.383 & 0.593 & 162 \\
\hline 05536340 & 1 & Yes & Yes & 1960 & 210 & $\mathrm{E}$ & 420 & 0.0492 & 0.405 & 0.669 & 148 \\
\hline 05536340 & 1 & Yes & Yes & 1961 & 328 & $E$ & 656 & 1.45 & 0.415 & 0.328 & 213 \\
\hline 05536340 & 1 & Yes & Yes & 1962 & 210 & D & 420 & 0.3 & 0.426 & 0.686 & 145 \\
\hline 05536340 & 1 & Yes & Yes & 1963 & 226 & $E$ & 452 & 1.51 & 0.436 & 0.643 & 152 \\
\hline 05536340 & 1 & Yes & Yes & 1964 & 128 & $E$ & 256 & 0.493 & 0.446 & 0.911 & 97.5 \\
\hline 05536340 & 1 & Yes & Yes & 1965 & 192 & -- & 384 & 0.892 & 0.456 & 0.763 & 131 \\
\hline 05536340 & 1 & Yes & Yes & 1966 & 391 & $\mathrm{D}$ & 782 & 1.3 & 0.466 & 0.236 & 236 \\
\hline 05536340 & 1 & Yes & Yes & 1967 & 237 & -- & 474 & 1.15 & 0.477 & 0.644 & 152 \\
\hline 05536340 & 1 & Yes & Yes & 1968 & 314 & -- & 628 & 1.46 & 0.487 & 0.425 & 193 \\
\hline 05536340 & 1 & Yes & Yes & 1969 & 158 & -- & 316 & 0.925 & 0.497 & 0.874 & 107 \\
\hline 05536340 & 1 & Yes & Yes & 1970 & 296 & -- & 592 & 1.1 & 0.507 & 0.494 & 180 \\
\hline 05536340 & 1 & Yes & Yes & 1971 & 178 & -- & 356 & 0.308 & 0.528 & 0.843 & 114 \\
\hline 05536340 & 1 & Yes & Yes & 1972 & 271 & -- & 542 & 1.23 & 0.548 & 0.607 & 159 \\
\hline 05536340 & 1 & Yes & Yes & 1973 & 501 & -- & 1002 & 1.31 & 0.569 & 0.158 & 272 \\
\hline
\end{tabular}




\begin{tabular}{|c|c|c|c|c|c|c|c|c|c|c|c|}
\hline $\begin{array}{c}\text { U.S. } \\
\text { Geological } \\
\text { Survey } \\
\text { streamgage } \\
\text { number }\end{array}$ & $\begin{array}{l}\text { Segment } \\
\text { number }\end{array}$ & $\begin{array}{l}\text { Streamgage used in } \\
\text { regression analyses } \\
\text { (non-redundant) }\end{array}$ & $\begin{array}{l}\text { Discharge value } \\
\text { used in } \\
\text { adjustment } \\
\text { regression }\end{array}$ & $\begin{array}{l}\text { Water } \\
\text { year }\end{array}$ & $\begin{array}{c}\text { Observed annual } \\
\text { maximum peak } \\
\text { discharge }\left(\mathrm{ft}^{3} / \mathrm{s}\right)\end{array}$ & $\begin{array}{l}\text { NWIS peak } \\
\text { code }\end{array}$ & $\begin{array}{l}\text { Observed annual } \\
\text { maximum peak } \\
\text { discharge with } \\
\text { segment intercept } \\
\text { value subtracted } \\
\left(\mathrm{ft}^{3} / \mathrm{s}\right)\end{array}$ & $\begin{array}{l}\text { Observed } \\
\text { precipitation } \\
\text { (inches) }\end{array}$ & Urban fraction & $\begin{array}{l}\text { Exceedance } \\
\text { probability }\end{array}$ & $\begin{array}{c}\text { Urban-adjusted } \\
\text { annual maximum } \\
\text { peak discharge } \\
\left(\mathrm{ft}^{3} / \mathrm{s}\right)\end{array}$ \\
\hline 05536340 & 0 & Yes & No & 1974 & 210 & $5, C$ & 540 & 1.24 & 0.59 & 0.649 & 152 \\
\hline 05536340 & 2 & Yes & Yes & 1975 & 251 & $5, C$ & 829 & 0.665 & 0.61 & 0.295 & 220 \\
\hline 05536340 & 2 & Yes & Yes & 1976 & 201 & $5, \mathrm{C}$ & 664 & 1.05 & 0.631 & 0.516 & 176 \\
\hline 05536340 & 2 & Yes & Yes & 1977 & 166 & $5, C$ & 548 & 1.39 & 0.651 & 0.698 & 144 \\
\hline 05536340 & 2 & Yes & Yes & 1978 & 224 & $5, C$ & 740 & 0.822 & 0.672 & 0.46 & 187 \\
\hline 05536340 & 2 & Yes & Yes & 1979 & 253 & $5, C$ & 835 & 0.843 & 0.692 & 0.367 & 205 \\
\hline 05536340 & 2 & Yes & Yes & 1980 & 128 & $5, C$ & 423 & 1.04 & 0.713 & 0.885 & 105 \\
\hline 05536340 & 2 & Yes & Yes & 1981 & 350 & $5, C$ & 1156 & 1.91 & 0.717 & 0.156 & 274 \\
\hline 05536340 & 2 & Yes & Yes & 1982 & 192 & $5, C$ & 634 & 0.387 & 0.721 & 0.646 & 152 \\
\hline 05536340 & 2 & Yes & Yes & 1983 & 300 & $5, C$ & 991 & 1.42 & 0.725 & 0.244 & 234 \\
\hline 05536340 & 2 & Yes & Yes & 1984 & 244 & $5, \mathrm{C}$ & 806 & 0.745 & 0.73 & 0.441 & 190 \\
\hline 05536340 & 2 & Yes & Yes & 1985 & 222 & $5, C$ & 733 & 0.836 & 0.734 & 0.531 & 173 \\
\hline 05536340 & 2 & Yes & Yes & 1986 & 125 & $5, C$ & 413 & 0.844 & 0.738 & 0.908 & 98.5 \\
\hline 05536340 & 2 & Yes & Yes & 1987 & 267 & $5, C$ & 882 & 1.09 & 0.742 & 0.366 & 205 \\
\hline 05536340 & 0 & Yes & No & 1988 & 131 & $5, C$ & 502 & 0.606 & 0.747 & 0.827 & 118 \\
\hline 05536340 & 3 & Yes & Yes & 1989 & 305 & $5, C$ & 1356 & 1.3 & 0.751 & 0.0968 & 312 \\
\hline 05536340 & 3 & Yes & Yes & 1990 & 261 & $5, C$ & 1161 & 2.49 & 0.755 & 0.17 & 266 \\
\hline 05536340 & 3 & Yes & Yes & 1991 & 339 & $5, C$ & 1508 & 3.15 & 0.757 & 0.0664 & 345 \\
\hline 05536340 & 3 & Yes & Yes & 1992 & 183 & $5, C$ & 814 & 4.07 & 0.759 & 0.461 & 186 \\
\hline 05536340 & 3 & Yes & Yes & 1993 & 187 & $5, C$ & 832 & 1.06 & 0.761 & 0.443 & 190 \\
\hline 05536340 & 3 & Yes & Yes & 1994 & 152 & $5, C$ & 676 & 1.67 & 0.763 & 0.633 & 154 \\
\hline 05536340 & 3 & Yes & Yes & 1995 & 174 & $5, C$ & 774 & 1.14 & 0.765 & 0.514 & 176 \\
\hline 05536340 & 3 & Yes & Yes & 1996 & 382 & $5, C$ & 1699 & 3.48 & 0.767 & 0.0446 & 385 \\
\hline 05536340 & 3 & Yes & Yes & 1997 & 259 & $5, C$ & 1152 & 2.47 & 0.769 & 0.18 & 261 \\
\hline 05536340 & 3 & Yes & Yes & 1998 & 214 & $5, C$ & 952 & 1.57 & 0.771 & 0.315 & 215 \\
\hline 05536340 & 3 & Yes & Yes & 1999 & 197 & $5, C$ & 876 & 1.17 & 0.772 & 0.406 & 198 \\
\hline 05536340 & 3 & Yes & Yes & 2000 & 298 & $5, C$ & 1325 & 3.78 & 0.774 & 0.116 & 298 \\
\hline 05536340 & 3 & Yes & Yes & 2001 & 241 & $5, C$ & 1072 & 0.915 & 0.774 & 0.223 & 241 \\
\hline 05536340 & 3 & Yes & Yes & 2002 & 260 & $5, C$ & 1156 & 2.7 & 0.775 & 0.181 & 260 \\
\hline 05536340 & 3 & Yes & Yes & 2003 & 236 & $5, C$ & 1050 & 2.26 & 0.775 & 0.237 & 236 \\
\hline 05536340 & 3 & Yes & Yes & 2004 & 295 & $5, C$ & 1312 & 2.2 & 0.775 & 0.121 & 295 \\
\hline 05536340 & 3 & Yes & Yes & 2005 & 159 & $5, C$ & 707 & 0.925 & 0.775 & 0.604 & 159 \\
\hline 05536340 & 3 & Yes & Yes & 2006 & 217 & $5, C$ & 965 & 2.93 & 0.775 & 0.305 & 217 \\
\hline 05536340 & 3 & Yes & Yes & 2007 & 225 & $5, C$ & 1001 & 2.66 & 0.775 & 0.274 & 225 \\
\hline 05536340 & 3 & Yes & Yes & 2008 & 325 & $5, C$ & 1445 & 4.52 & 0.775 & 0.0825 & 325 \\
\hline
\end{tabular}




\begin{tabular}{|c|c|c|c|c|c|c|c|c|c|c|c|}
\hline $\begin{array}{c}\text { U.S. } \\
\text { Geological } \\
\text { Survey } \\
\text { streamgage } \\
\text { number }\end{array}$ & $\begin{array}{c}\text { Segment } \\
\text { number }\end{array}$ & $\begin{array}{l}\text { Streamgage used in } \\
\text { regression analyses } \\
\text { (non-redundant) }\end{array}$ & $\begin{array}{c}\text { Discharge value } \\
\text { used in } \\
\text { adjustment } \\
\text { regression }\end{array}$ & $\begin{array}{c}\text { Water } \\
\text { year }\end{array}$ & $\begin{array}{c}\text { Observed annual } \\
\text { maximum peak } \\
\text { discharge }\left(\mathrm{ft}^{3} / \mathrm{s}\right)\end{array}$ & $\begin{array}{c}\text { NWIS peak } \\
\text { code }\end{array}$ & $\begin{array}{c}\text { Observed annual } \\
\text { maximum peak } \\
\text { discharge with } \\
\text { segment intercept } \\
\text { value subtracted } \\
\left(\mathrm{ft}^{3} / \mathrm{s}\right)\end{array}$ & $\begin{array}{c}\text { Observed } \\
\text { precipitation } \\
\text { (inches) }\end{array}$ & Urban fraction & $\begin{array}{l}\text { Exceedance } \\
\text { probability }\end{array}$ & $\begin{array}{c}\text { Urban-adjusted } \\
\text { annual maximum } \\
\text { peak discharge } \\
\left(\mathrm{ft}^{3} / \mathrm{s}\right)\end{array}$ \\
\hline 05536340 & 3 & Yes & Yes & 2009 & 318 & $5, \mathrm{C}$ & 1414 & 1.76 & 0.775 & 0.09 & 318 \\
\hline 05536460 & 1 & No & No & 1961 & 386 & 2 & 468 & 1.43 & 0.21 & 0.418 & 674 \\
\hline 05536460 & 1 & No & No & 1962 & 261 & -- & 317 & 0.27 & 0.23 & 0.71 & 484 \\
\hline 05536460 & 1 & No & No & 1963 & 430 & -- & 522 & 1.51 & 0.25 & 0.371 & 709 \\
\hline 05536460 & 1 & No & No & 1964 & 109 & 4 & 132 & 0.983 & 0.27 & 0.958 & 252 \\
\hline 05536460 & 1 & No & No & 1965 & 337 & -- & 409 & 0.881 & 0.29 & 0.595 & 557 \\
\hline 05536460 & 1 & No & No & 1966 & 783 & -- & 950 & 1.38 & 0.31 & 0.0847 & 1130 \\
\hline 05536460 & 1 & No & No & 1967 & 488 & -- & 592 & 1.15 & 0.33 & 0.34 & 733 \\
\hline 05536460 & 1 & No & No & 1968 & 447 & -- & 542 & 1.45 & 0.35 & 0.423 & 671 \\
\hline 05536460 & 1 & No & No & 1969 & 210 & 2 & 255 & 0.419 & 0.37 & 0.881 & 357 \\
\hline 05536460 & 1 & No & No & 1970 & 999 & -- & 1212 & 1.09 & 0.39 & 0.0486 & 1320 \\
\hline 05536460 & 1 & No & No & 1971 & 197 & -- & 239 & 0.311 & 0.42 & 0.917 & 322 \\
\hline 05536460 & 1 & No & No & 1972 & 501 & -- & 608 & 1.23 & 0.449 & 0.418 & 675 \\
\hline 05536460 & 1 & No & No & 1973 & 715 & $-{ }_{-1}$ & 867 & 1.33 & 0.478 & 0.184 & 897 \\
\hline 05536460 & 1 & No & No & 1974 & 569 & -- & 690 & 1.2 & 0.508 & 0.364 & 714 \\
\hline 05536460 & 1 & No & No & 1975 & 575 & -- & 698 & 1.02 & 0.537 & 0.382 & 701 \\
\hline 05536460 & 1 & No & No & 1976 & 352 & -- & 427 & 0.924 & 0.566 & 0.783 & 434 \\
\hline 05536460 & 1 & No & No & 1977 & 266 & -- & 323 & 1.43 & 0.596 & 0.913 & 327 \\
\hline 05536460 & 1 & No & No & 1978 & 853 & 2 & 1035 & 1.09 & 0.625 & 0.167 & 934 \\
\hline 05536460 & 1 & No & No & 1979 & 260 & 2 & 315 & 0.863 & 0.654 & 0.938 & 293 \\
\hline 05536500 & 1 & Yes & Yes & 1951 & 505 & -- & 491 & 1.34 & 0.0462 & 0.274 & 889 \\
\hline 05536500 & 1 & Yes & Yes & 1952 & 405 & -- & 394 & 0.944 & 0.0615 & 0.426 & 746 \\
\hline 05536500 & 1 & Yes & Yes & 1953 & 530 & -- & 515 & 1.15 & 0.0768 & 0.263 & 901 \\
\hline 05536500 & 1 & Yes & Yes & 1954 & 367 & -- & 357 & 1.66 & 0.0922 & 0.523 & 667 \\
\hline 05536500 & 1 & Yes & Yes & 1955 & 1930 & -- & 1877 & 3.27 & 0.108 & 0.00744 & 2780 \\
\hline 05536500 & 1 & Yes & Yes & 1957 & 1730 & -- & 1683 & 2.37 & 0.138 & 0.0108 & 2450 \\
\hline 05536500 & 1 & Yes & Yes & 1958 & 227 & $\mathrm{E}$ & 221 & 0.974 & 0.154 & 0.826 & 436 \\
\hline 05536500 & 1 & Yes & Yes & 1959 & 455 & $\mathrm{E}$ & 443 & 1.26 & 0.169 & 0.427 & 745 \\
\hline 05536500 & 1 & Yes & Yes & 1960 & 395 & -- & 384 & 0.0504 & 0.184 & 0.548 & 650 \\
\hline 05536500 & 1 & Yes & Yes & 1961 & 453 & -- & 441 & 1.44 & 0.202 & 0.458 & 720 \\
\hline 05536500 & 1 & Yes & Yes & 1962 & 306 & -- & 298 & 0.268 & 0.219 & 0.736 & 511 \\
\hline 05536500 & 1 & Yes & Yes & 1963 & 484 & $\mathrm{E}$ & 471 & 1.52 & 0.237 & 0.437 & 737 \\
\hline 05536500 & 1 & Yes & Yes & 1964 & 110 & $\mathrm{E}$ & 107 & 0.982 & 0.254 & 0.972 & 225 \\
\hline 05536500 & 1 & Yes & Yes & 1965 & 391 & -- & 380 & 0.887 & 0.272 & 0.628 & 591 \\
\hline 05536500 & 1 & Yes & Yes & 1966 & 769 & $\mathrm{D}$ & 748 & 1.34 & 0.289 & 0.171 & 1040 \\
\hline
\end{tabular}




\begin{tabular}{|c|c|c|c|c|c|c|c|c|c|c|c|}
\hline $\begin{array}{c}\text { U.S. } \\
\text { Geological } \\
\text { Survey } \\
\text { streamgage } \\
\text { number }\end{array}$ & $\begin{array}{l}\text { Segment } \\
\text { number }\end{array}$ & $\begin{array}{l}\text { Streamgage used in } \\
\text { regression analyses } \\
\text { (non-redundant) }\end{array}$ & $\begin{array}{l}\text { Discharge value } \\
\text { used in } \\
\text { adjustment } \\
\text { regression }\end{array}$ & $\begin{array}{l}\text { Water } \\
\text { year }\end{array}$ & $\begin{array}{l}\text { Observed annual } \\
\text { maximum peak } \\
\text { discharge }\left(\mathrm{ft}^{3} / \mathrm{s}\right)\end{array}$ & $\begin{array}{l}\text { NWIS peak } \\
\text { code }\end{array}$ & $\begin{array}{c}\text { Observed annual } \\
\text { maximum peak } \\
\text { discharge with } \\
\text { segment intercept } \\
\text { value subtracted } \\
\left(\mathrm{ft}^{3} / \mathrm{s}\right)\end{array}$ & $\begin{array}{l}\text { Observed } \\
\text { precipitation } \\
\text { (inches) }\end{array}$ & Urban fraction & $\begin{array}{l}\text { Exceedance } \\
\text { probability }\end{array}$ & $\begin{array}{c}\text { Urban-adjusted } \\
\text { annual maximum } \\
\text { peak discharge } \\
\left(\mathrm{ft}^{3} / \mathrm{s}\right)\end{array}$ \\
\hline 05536500 & 1 & Yes & Yes & 1967 & 524 & $E$ & 510 & 1.15 & 0.307 & 0.435 & 739 \\
\hline 05536500 & 1 & Yes & Yes & 1968 & 495 & -- & 481 & 1.44 & 0.324 & 0.495 & 690 \\
\hline 05536500 & 1 & Yes & Yes & 1969 & 240 & $E$ & 233 & 0.426 & 0.342 & 0.893 & 369 \\
\hline 05536500 & 1 & Yes & Yes & 1970 & 1580 & -- & 1537 & 1.1 & 0.359 & 0.0199 & 1930 \\
\hline 05536500 & 1 & Yes & Yes & 1971 & 224 & $E$ & 218 & 0.309 & 0.382 & 0.924 & 333 \\
\hline 05536500 & 1 & Yes & Yes & 1972 & 491 & -- & 478 & 1.23 & 0.405 & 0.575 & 630 \\
\hline 05536500 & 1 & Yes & Yes & 1973 & 775 & -- & 754 & 1.32 & 0.427 & 0.236 & 933 \\
\hline 05536500 & 1 & Yes & Yes & 1974 & 670 & -- & 652 & 1.2 & 0.45 & 0.362 & 802 \\
\hline 05536500 & 1 & Yes & Yes & 1975 & 700 & -- & 681 & 1.02 & 0.473 & 0.345 & 817 \\
\hline 05536500 & 1 & Yes & Yes & 1976 & 456 & -- & 444 & 0.931 & 0.495 & 0.709 & 534 \\
\hline 05536500 & 1 & Yes & Yes & 1977 & 372 & -- & 362 & 1.42 & 0.518 & 0.831 & 430 \\
\hline 05536500 & 1 & Yes & Yes & 1978 & 1220 & -- & 1187 & 1.1 & 0.541 & 0.0792 & 1310 \\
\hline 05536500 & 1 & Yes & Yes & 1979 & 615 & -- & 598 & 1.03 & 0.564 & 0.539 & 657 \\
\hline 05536500 & 1 & Yes & Yes & 1980 & 270 & -- & 263 & 0.756 & 0.586 & 0.948 & 287 \\
\hline 05536500 & 1 & Yes & Yes & 1981 & 1560 & -- & 1517 & 1.93 & 0.589 & 0.0406 & 1600 \\
\hline 05536500 & 1 & Yes & Yes & 1982 & 713 & -- & 693 & 0.365 & 0.591 & 0.439 & 737 \\
\hline 05536500 & 1 & Yes & Yes & 1983 & 1310 & -- & 1274 & 1.81 & 0.594 & 0.0726 & 1340 \\
\hline 05536500 & 1 & Yes & Yes & 1984 & 708 & -- & 689 & 0.712 & 0.597 & 0.45 & 727 \\
\hline 05536500 & 1 & Yes & Yes & 1985 & 644 & -- & 626 & 0.824 & 0.599 & 0.535 & 660 \\
\hline 05536500 & 1 & Yes & Yes & 1986 & 490 & -- & 477 & 0.963 & 0.602 & 0.748 & 502 \\
\hline 05536500 & 1 & Yes & Yes & 1987 & 689 & -- & 670 & 1.05 & 0.604 & 0.481 & 702 \\
\hline 05536500 & 1 & Yes & Yes & 1988 & 361 & -- & 351 & 0.592 & 0.607 & 0.894 & 369 \\
\hline 05536500 & 1 & Yes & Yes & 1989 & 830 & -- & 807 & 1.27 & 0.61 & 0.318 & 840 \\
\hline 05536500 & 1 & Yes & Yes & 1990 & 758 & D & 737 & 2.28 & 0.612 & 0.405 & 765 \\
\hline 05536500 & 1 & Yes & Yes & 1991 & 1340 & -- & 1303 & 2.28 & 0.613 & 0.0713 & 1350 \\
\hline 05536500 & 1 & Yes & Yes & 1992 & 593 & C & 577 & 4.1 & 0.614 & 0.619 & 598 \\
\hline 05536500 & 1 & Yes & Yes & 1993 & 820 & C & 798 & 1.36 & 0.615 & 0.334 & 825 \\
\hline 05536500 & 1 & Yes & Yes & 1994 & 439 & $C, E$ & 427 & 0.51 & 0.616 & 0.817 & 442 \\
\hline 05536500 & 1 & Yes & Yes & 1995 & 564 & $C$ & 549 & 1.18 & 0.617 & 0.662 & 567 \\
\hline 05536500 & 1 & Yes & Yes & 1996 & 2010 & $C$ & 1955 & 3.38 & 0.618 & 0.0187 & 2020 \\
\hline 05536500 & 1 & Yes & Yes & 1997 & 1080 & $C$ & 1050 & 2.53 & 0.619 & 0.157 & 1080 \\
\hline 05536500 & 1 & Yes & Yes & 1998 & 604 & $C$ & 587 & 1.6 & 0.619 & 0.609 & 605 \\
\hline 05536500 & 1 & Yes & Yes & 1999 & 575 & $C$ & 559 & 0.847 & 0.62 & 0.65 & 576 \\
\hline 05536500 & 1 & Yes & Yes & 2000 & 582 & $C$ & 566 & 1.11 & 0.621 & 0.641 & 582 \\
\hline 05536500 & 1 & Yes & Yes & 2001 & 473 & $\mathrm{C}, \mathrm{E}$ & 460 & 0.767 & 0.621 & 0.782 & 473 \\
\hline
\end{tabular}




\begin{tabular}{|c|c|c|c|c|c|c|c|c|c|c|c|}
\hline $\begin{array}{c}\text { U.S. } \\
\text { Geological } \\
\text { Survey } \\
\text { streamgage } \\
\text { number } \\
\end{array}$ & $\begin{array}{c}\text { Segment } \\
\text { number }\end{array}$ & $\begin{array}{l}\text { Streamgage used in } \\
\text { regression analyses } \\
\text { (non-redundant) }\end{array}$ & $\begin{array}{c}\text { Discharge value } \\
\text { used in } \\
\text { adjustment } \\
\text { regression } \\
\end{array}$ & $\begin{array}{c}\text { Water } \\
\text { year }\end{array}$ & $\begin{array}{c}\text { Observed annual } \\
\text { maximum peak } \\
\text { discharge }\left(\mathrm{ft}^{3} / \mathrm{s}\right)\end{array}$ & $\begin{array}{c}\text { NWIS peak } \\
\text { code }\end{array}$ & $\begin{array}{c}\text { Observed annual } \\
\text { maximum peak } \\
\text { discharge with } \\
\text { segment intercept } \\
\text { value subtracted } \\
\left(\mathrm{ft}^{3} / \mathrm{s}\right) \\
\end{array}$ & $\begin{array}{c}\text { Observed } \\
\text { precipitation } \\
\text { (inches) }\end{array}$ & Urban fraction & $\begin{array}{l}\text { Exceedance } \\
\text { probability }\end{array}$ & $\begin{array}{c}\text { Urban-adjusted } \\
\text { annual maximum } \\
\text { peak discharge } \\
\left(\mathrm{ft}^{3} / \mathrm{s}\right) \\
\end{array}$ \\
\hline 05536500 & 1 & Yes & Yes & 2002 & 830 & C & 807 & 2.58 & 0.621 & 0.329 & 830 \\
\hline 05536500 & 1 & Yes & Yes & 2003 & 350 & $2, C$ & 340 & 0.505 & 0.621 & 0.91 & 350 \\
\hline 05536500 & 1 & Yes & Yes & 2004 & 690 & C & 671 & 1.94 & 0.621 & 0.497 & 690 \\
\hline 05536500 & 1 & Yes & Yes & 2005 & 712 & C & 693 & 0.922 & 0.621 & 0.469 & 712 \\
\hline 05536500 & 1 & Yes & Yes & 2006 & 1210 & C & 1177 & 1.94 & 0.621 & 0.107 & 1210 \\
\hline 05536500 & 1 & Yes & Yes & 2007 & 808 & C & 786 & 2.35 & 0.621 & 0.354 & 808 \\
\hline 05536500 & 1 & Yes & Yes & 2008 & 907 & C & 882 & 4.31 & 0.621 & 0.255 & 907 \\
\hline 05536500 & 1 & Yes & Yes & 2009 & 1190 & c & 1157 & 1.91 & 0.621 & 0.114 & 1190 \\
\hline 05536510 & 1 & Yes & Yes & 1961 & 260 & 2 & 762 & 1.43 & 0.631 & 0.393 & 293 \\
\hline 05536510 & 1 & Yes & Yes & 1962 & 166 & -- & 487 & 0.277 & 0.631 & 0.758 & 192 \\
\hline 05536510 & 1 & Yes & Yes & 1963 & 225 & -- & 660 & 1.54 & 0.632 & 0.522 & 255 \\
\hline 05536510 & 1 & Yes & Yes & 1964 & 133 & -- & 390 & 0.737 & 0.632 & 0.869 & 157 \\
\hline 05536510 & 1 & Yes & Yes & 1965 & 166 & -- & 487 & 0.899 & 0.633 & 0.759 & 192 \\
\hline 05536510 & 1 & Yes & Yes & 1966 & 222 & -- & 651 & 1.33 & 0.633 & 0.534 & 252 \\
\hline 05536510 & 1 & Yes & Yes & 1967 & 232 & -- & 680 & 1.15 & 0.633 & 0.497 & 263 \\
\hline 05536510 & 1 & Yes & Yes & 1968 & 273 & -- & 801 & 1.38 & 0.634 & 0.349 & 306 \\
\hline 05536510 & 1 & Yes & Yes & 1969 & 186 & -- & 545 & 0.42 & 0.634 & 0.684 & 213 \\
\hline 05536510 & 1 & Yes & Yes & 1970 & 423 & -- & 1240 & 1.12 & 0.635 & 0.0917 & 467 \\
\hline 05536510 & 1 & Yes & Yes & 1971 & 150 & -- & 440 & 0.933 & 0.644 & 0.822 & 172 \\
\hline 05536510 & 1 & Yes & Yes & 1972 & 221 & -- & 648 & 1.21 & 0.653 & 0.558 & 245 \\
\hline 05536510 & 1 & Yes & Yes & 1973 & 241 & -- & 707 & 1.31 & 0.663 & 0.492 & 264 \\
\hline 05536510 & 1 & Yes & Yes & 1974 & 377 & -- & 1106 & 1.14 & 0.672 & 0.157 & 403 \\
\hline 05536510 & 1 & Yes & Yes & 1975 & 196 & -- & 575 & 1.07 & 0.681 & 0.689 & 212 \\
\hline 05536510 & 1 & Yes & Yes & 1976 & 434 & -- & 1273 & 0.977 & 0.691 & 0.0999 & 455 \\
\hline 05536510 & 1 & Yes & Yes & 1977 & 244 & -- & 715 & 1.44 & 0.7 & 0.518 & 256 \\
\hline 05536510 & 1 & Yes & Yes & 1978 & 375 & -- & 1100 & 1.09 & 0.709 & 0.175 & 387 \\
\hline 05536510 & 1 & Yes & Yes & 1979 & 204 & -- & 598 & 0.853 & 0.719 & 0.695 & 210 \\
\hline 05536560 & 1 & Yes & Yes & 1962 & 137 & -- & 1514 & 0.283 & 0.972 & 0.14 & 382 \\
\hline 05536560 & 1 & Yes & Yes & 1963 & 67 & 2 & 740 & 1.61 & 0.973 & 0.776 & 187 \\
\hline 05536560 & 1 & Yes & Yes & 1964 & 35 & 2 & 387 & 0.783 & 0.974 & 0.983 & 98.4 \\
\hline 05536560 & 1 & Yes & Yes & 1965 & 56 & 2 & 619 & 0.496 & 0.975 & 0.895 & 156 \\
\hline 05536560 & 1 & Yes & Yes & 1966 & 143 & -- & 1580 & 1.59 & 0.976 & 0.121 & 397 \\
\hline 05536560 & 1 & Yes & Yes & 1967 & 91 & -- & 1006 & 1.18 & 0.977 & 0.493 & 253 \\
\hline 05536560 & 1 & Yes & Yes & 1968 & 117 & -- & 1293 & 1.51 & 0.978 & 0.227 & 324 \\
\hline 05536560 & 1 & Yes & Yes & 1969 & 79 & -- & 873 & 0.735 & 0.979 & 0.634 & 219 \\
\hline
\end{tabular}




\begin{tabular}{|c|c|c|c|c|c|c|c|c|c|c|c|}
\hline $\begin{array}{l}\text { U.S. } \\
\text { Geological } \\
\text { Survey } \\
\text { streamgage } \\
\text { number }\end{array}$ & $\begin{array}{l}\text { Segment } \\
\text { number }\end{array}$ & $\begin{array}{l}\text { Streamgage used in } \\
\text { regression analyses } \\
\text { (non-redundant) }\end{array}$ & $\begin{array}{c}\text { Discharge value } \\
\text { used in } \\
\text { adjustment } \\
\text { regression }\end{array}$ & $\begin{array}{l}\text { Water } \\
\text { year }\end{array}$ & $\begin{array}{c}\text { Observed annual } \\
\text { maximum peak } \\
\text { discharge }\left(\mathrm{ft}^{3} / \mathrm{s}\right)\end{array}$ & $\begin{array}{l}\text { NWIS peak } \\
\text { code }\end{array}$ & $\begin{array}{l}\text { Observed annual } \\
\text { maximum peak } \\
\text { discharge with } \\
\text { segment intercept } \\
\text { value subtracted } \\
\left(\mathrm{ft}^{3} / \mathrm{s}\right)\end{array}$ & $\begin{array}{l}\text { Observed } \\
\text { precipitation } \\
\text { (inches) }\end{array}$ & Urban fraction & $\begin{array}{l}\text { Exceedance } \\
\text { probability }\end{array}$ & $\begin{array}{c}\text { Urban-adjusted } \\
\text { annual maximum } \\
\text { peak discharge } \\
\left(\mathrm{ft}^{3} / \mathrm{s}\right)\end{array}$ \\
\hline 05536560 & 1 & Yes & Yes & 1970 & 183 & -- & 2022 & 1.23 & 0.981 & 0.0446 & 505 \\
\hline 05536560 & 0 & Yes & No & 1971 & 100 & -- & 858 & 0.452 & 0.981 & 0.656 & 215 \\
\hline 05536560 & 0 & Yes & No & 1972 & 104 & -- & 693 & 1.14 & 0.981 & 0.83 & 173 \\
\hline 05536560 & 0 & Yes & No & 1973 & 110 & -- & 569 & 1.27 & 0.982 & 0.932 & 142 \\
\hline 05536560 & 2 & Yes & Yes & 1974 & 166 & -- & 666 & 1.01 & 0.982 & 0.856 & 167 \\
\hline 05536560 & 2 & Yes & Yes & 1975 & 231 & -- & 927 & 1.36 & 0.982 & 0.575 & 231 \\
\hline 05536560 & 2 & Yes & Yes & 1976 & 408 & -- & 1637 & 0.983 & 0.983 & 0.106 & 409 \\
\hline 05536560 & 2 & Yes & Yes & 1977 & 234 & -- & 939 & 1.57 & 0.983 & 0.564 & 234 \\
\hline 05536560 & 2 & Yes & Yes & 1978 & 256 & -- & 1027 & 1.02 & 0.983 & 0.478 & 256 \\
\hline 05536560 & 2 & Yes & Yes & 1979 & 352 & -- & 1413 & 1.16 & 0.984 & 0.179 & 352 \\
\hline 05536560 & 2 & Yes & Yes & 1980 & 230 & -- & 923 & 1.02 & 0.984 & 0.581 & 230 \\
\hline 05536570 & 1 & No & No & 1962 & 520 & 2 & 1563 & 0.471 & 0.95 & 0.115 & 527 \\
\hline 05536570 & 1 & No & No & 1963 & 340 & -- & 1022 & 2.42 & 0.952 & 0.448 & 345 \\
\hline 05536570 & 1 & No & No & 1964 & 94 & -- & 283 & 1.14 & 0.954 & 1 & 96.5 \\
\hline 05536570 & 1 & No & No & 1965 & 212 & -- & 637 & 0.834 & 0.955 & 0.86 & 215 \\
\hline 05536570 & 1 & No & No & 1966 & 619 & -- & 1860 & 2.16 & 0.957 & 0.056 & 624 \\
\hline 05536570 & 1 & No & No & 1967 & 350 & -- & 1052 & 1.77 & 0.958 & 0.425 & 353 \\
\hline 05536570 & 1 & No & No & 1968 & 708 & -- & 2128 & 2.13 & 0.96 & 0.0359 & 711 \\
\hline 05536570 & 1 & No & No & 1969 & 301 & -- & 905 & 0.603 & 0.962 & 0.576 & 302 \\
\hline 05536570 & 1 & No & No & 1970 & 632 & -- & 1900 & 1.65 & 0.963 & 0.052 & 633 \\
\hline 05536570 & 0 & No & No & 1971 & 169 & -- & NA & 1.3 & 0.963 & NA & NA \\
\hline 05536570 & 0 & No & No & 1972 & 441 & -- & NA & 1.83 & 0.964 & NA & NA \\
\hline 05536570 & 0 & No & No & 1973 & 356 & -- & NA & 1.36 & 0.964 & NA & NA \\
\hline 05536570 & 0 & No & No & 1974 & 365 & -- & NA & 1.71 & 0.964 & NA & NA \\
\hline 05536570 & 0 & No & No & 1975 & 683 & -- & NA & 1.69 & 0.964 & NA & NA \\
\hline 05536570 & 0 & No & No & 1976 & 1570 & -- & NA & 0.97 & 0.964 & NA & NA \\
\hline 05536620 & 1 & No & No & 1961 & 208 & -- & 892 & 1.42 & 0.293 & 0.0984 & 270 \\
\hline 05536620 & 1 & No & No & 1962 & 101 & -- & 433 & 0.281 & 0.306 & 0.563 & 139 \\
\hline 05536620 & 1 & No & No & 1963 & 100 & -- & 429 & 1.53 & 0.319 & 0.583 & 136 \\
\hline 05536620 & 1 & No & No & 1964 & 54 & -- & 232 & 0.734 & 0.331 & 0.891 & 79.6 \\
\hline 05536620 & 1 & No & No & 1965 & 118 & -- & 506 & 0.884 & 0.344 & 0.473 & 154 \\
\hline 05536620 & 1 & No & No & 1966 & 330 & -- & 1415 & 1.42 & 0.357 & 0.0272 & 395 \\
\hline 05536620 & 1 & No & No & 1967 & 203 & -- & 870 & 1.15 & 0.37 & 0.138 & 247 \\
\hline 05536620 & 1 & No & No & 1968 & 134 & 2 & 574 & 1.42 & 0.382 & 0.405 & 167 \\
\hline 05536620 & 1 & No & No & 1969 & 69 & -- & 296 & 0.408 & 0.395 & 0.842 & 90.3 \\
\hline
\end{tabular}




\begin{tabular}{|c|c|c|c|c|c|c|c|c|c|c|c|}
\hline $\begin{array}{c}\text { U.S. } \\
\text { Geological } \\
\text { Survey } \\
\text { streamgage } \\
\text { number } \\
\end{array}$ & $\begin{array}{c}\text { Segment } \\
\text { number }\end{array}$ & $\begin{array}{l}\text { Streamgage used in } \\
\text { regression analyses } \\
\text { (non-redundant) }\end{array}$ & $\begin{array}{c}\text { Discharge value } \\
\text { used in } \\
\text { adjustment } \\
\text { regression }\end{array}$ & $\begin{array}{c}\text { Water } \\
\text { year }\end{array}$ & $\begin{array}{c}\text { Observed annual } \\
\text { maximum peak } \\
\text { discharge }\left(\mathrm{ft}^{3} / \mathrm{s}\right)\end{array}$ & $\begin{array}{c}\text { NWIS peak } \\
\text { code }\end{array}$ & $\begin{array}{l}\text { Observed annual } \\
\text { maximum peak } \\
\text { discharge with } \\
\text { segment intercept } \\
\text { value subtracted } \\
\left(\mathrm{ft}^{3} / \mathrm{s}\right)\end{array}$ & $\begin{array}{l}\text { Observed } \\
\text { precipitation } \\
\text { (inches) }\end{array}$ & Urban fraction & $\begin{array}{l}\text { Exceedance } \\
\text { probability }\end{array}$ & $\begin{array}{c}\text { Urban-adjusted } \\
\text { annual maximum } \\
\text { peak discharge } \\
\left(\mathrm{ft}^{3} / \mathrm{s}\right)\end{array}$ \\
\hline 05536620 & 1 & No & No & 1970 & 181 & -- & 776 & 1.09 & 0.408 & 0.205 & 214 \\
\hline 05536620 & 1 & No & No & 1971 & 38 & -- & 163 & 0.903 & 0.418 & 0.963 & 52 \\
\hline 05536620 & 1 & No & No & 1972 & 157 & -- & 673 & 1.21 & 0.429 & 0.318 & 184 \\
\hline 05536620 & 1 & No & No & 1973 & 163 & -- & 699 & 0.845 & 0.439 & 0.296 & 189 \\
\hline 05536620 & 1 & No & No & 1974 & 110 & -- & 472 & 1.14 & 0.45 & 0.625 & 129 \\
\hline 05536620 & 1 & No & No & 1975 & 122 & -- & 523 & 1.07 & 0.46 & 0.553 & 141 \\
\hline 05536620 & 1 & No & No & 1976 & 99 & -- & 424 & 0.889 & 0.471 & 0.716 & 114 \\
\hline 05536620 & 1 & No & No & 1977 & 80 & -- & 343 & 1.45 & 0.481 & 0.832 & 92.5 \\
\hline 05536630 & 1 & Yes & No & 1955 & 2050 & 7 & 5507 & 3.3 & 0.233 & 0 & 2610 \\
\hline 05536630 & 1 & Yes & Yes & 1961 & 174 & 2 & 467 & 1.42 & 0.324 & 0.518 & 237 \\
\hline 05536630 & 1 & Yes & Yes & 1962 & 117 & 2 & 314 & 0.282 & 0.336 & 0.782 & 166 \\
\hline 05536630 & 1 & Yes & Yes & 1963 & 109 & 2 & 293 & 1.53 & 0.347 & 0.82 & 154 \\
\hline 05536630 & 1 & Yes & Yes & 1964 & 61 & -- & 164 & 0.736 & 0.359 & 0.954 & 94.1 \\
\hline 05536630 & 1 & Yes & Yes & 1965 & 107 & 2 & 287 & 0.885 & 0.371 & 0.84 & 147 \\
\hline 05536630 & 1 & Yes & Yes & 1966 & 558 & -- & 1499 & 1.43 & 0.382 & 0.0237 & 659 \\
\hline 05536630 & 1 & Yes & Yes & 1967 & 251 & 2 & 674 & 1.15 & 0.394 & 0.29 & 307 \\
\hline 05536630 & 1 & Yes & Yes & 1968 & 130 & -- & 349 & 1.42 & 0.405 & 0.776 & 168 \\
\hline 05536630 & 1 & Yes & Yes & 1969 & 98 & 2 & 263 & 0.407 & 0.417 & 0.892 & 129 \\
\hline 05536630 & 1 & Yes & Yes & 1970 & 526 & -- & 1413 & 1.09 & 0.429 & 0.0334 & 603 \\
\hline 05536630 & 1 & Yes & Yes & 1971 & 59 & 2 & 159 & 0.902 & 0.437 & 0.968 & 80.3 \\
\hline 05536630 & 1 & Yes & Yes & 1972 & 188 & -- & 505 & 1.21 & 0.445 & 0.568 & 224 \\
\hline 05536630 & 1 & Yes & Yes & 1973 & 125 & -- & 336 & 1.34 & 0.453 & 0.825 & 153 \\
\hline 05536630 & 1 & Yes & Yes & 1974 & 411 & -- & 1104 & 1.14 & 0.462 & 0.0799 & 463 \\
\hline 05536630 & 1 & Yes & Yes & 1975 & 429 & -- & 1152 & 1.08 & 0.47 & 0.0711 & 480 \\
\hline 05536630 & 1 & Yes & Yes & 1976 & 309 & -- & 830 & 0.986 & 0.478 & 0.205 & 345 \\
\hline 05536630 & 1 & Yes & Yes & 1977 & 130 & -- & 349 & 1.45 & 0.486 & 0.827 & 152 \\
\hline 05536630 & 1 & Yes & Yes & 1978 & 325 & -- & 873 & 1.08 & 0.495 & 0.188 & 357 \\
\hline 05536630 & 1 & Yes & Yes & 1979 & 762 & -- & 2047 & 0.862 & 0.503 & 0.0138 & 817 \\
\hline 05537500 & 1 & Yes & Yes & 1951 & 390 & -- & 243 & 1.45 & 0.0399 & 0.724 & 639 \\
\hline 05537500 & 1 & Yes & Yes & 1952 & 1000 & -- & 622 & 1.25 & 0.0468 & 0.154 & 1260 \\
\hline 05537500 & 1 & Yes & Yes & 1953 & 495 & -- & 308 & 1.42 & 0.0537 & 0.602 & 742 \\
\hline 05537500 & 1 & Yes & Yes & 1954 & 658 & $E$ & 409 & 1.67 & 0.0607 & 0.399 & 916 \\
\hline 05537500 & 1 & Yes & Yes & 1955 & 3160 & -- & 1966 & 3.39 & 0.0676 & 0.00572 & 3330 \\
\hline 05537500 & 1 & Yes & Yes & 1956 & 348 & -- & 216 & 0.445 & 0.0745 & 0.792 & 578 \\
\hline 05537500 & 1 & Yes & Yes & 1957 & 2390 & -- & 1487 & 2.29 & 0.0815 & 0.0135 & 2560 \\
\hline
\end{tabular}




\begin{tabular}{|c|c|c|c|c|c|c|c|c|c|c|c|}
\hline $\begin{array}{c}\text { U.S. } \\
\text { Geological } \\
\text { Survey } \\
\text { streamgage } \\
\text { number }\end{array}$ & $\begin{array}{l}\text { Segment } \\
\text { number }\end{array}$ & $\begin{array}{l}\text { Streamgage used in } \\
\text { regression analyses } \\
\text { (non-redundant) }\end{array}$ & $\begin{array}{c}\text { Discharge value } \\
\text { used in } \\
\text { adjustment } \\
\text { regression }\end{array}$ & $\begin{array}{c}\text { Water } \\
\text { year }\end{array}$ & $\begin{array}{l}\text { Observed annual } \\
\text { maximum peak } \\
\text { discharge }\left(\mathrm{ft}^{3} / \mathrm{s}\right)\end{array}$ & $\begin{array}{c}\text { NWIS peak } \\
\text { code }\end{array}$ & $\begin{array}{c}\text { Observed annual } \\
\text { maximum peak } \\
\text { discharge with } \\
\text { segment intercept } \\
\text { value subtracted } \\
\left(\mathrm{ft}^{3} / \mathrm{s}\right)\end{array}$ & $\begin{array}{c}\text { Observed } \\
\text { precipitation } \\
\text { (inches) }\end{array}$ & Urban fraction & $\begin{array}{l}\text { Exceedance } \\
\text { probability }\end{array}$ & $\begin{array}{c}\text { Urban-adjusted } \\
\text { annual maximum } \\
\text { peak discharge } \\
\left(\mathrm{ft}^{3} / \mathrm{s}\right)\end{array}$ \\
\hline 05537500 & 1 & Yes & Yes & 1958 & 358 & $E$ & 223 & 1.47 & 0.0884 & 0.789 & 582 \\
\hline 05537500 & 1 & Yes & Yes & 1959 & 397 & $E$ & 247 & 1.38 & 0.0954 & 0.751 & 619 \\
\hline 05537500 & 1 & Yes & Yes & 1960 & 477 & -- & 297 & 1.86 & 0.102 & 0.656 & 693 \\
\hline 05537500 & 1 & Yes & Yes & 1961 & 1000 & -- & 622 & 0.607 & 0.116 & 0.18 & 1190 \\
\hline 05537500 & 1 & Yes & Yes & 1962 & 304 & \begin{tabular}{l|}
- \\
\end{tabular} & 189 & 0.296 & 0.129 & 0.864 & 507 \\
\hline 05537500 & 1 & Yes & Yes & 1963 & 348 & $E$ & 216 & 1.57 & 0.143 & 0.827 & 545 \\
\hline 05537500 & 0 & Yes & No & 1964 & 139 & $E$ & 108 & 0.996 & 0.157 & 0.957 & 354 \\
\hline 05537500 & 2 & Yes & Yes & 1965 & 263 & $\mathrm{E}$ & 256 & 0.89 & 0.17 & 0.776 & 594 \\
\hline 05537500 & 2 & Yes & Yes & 1966 & 1360 & 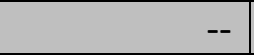 & 1325 & 1.68 & 0.184 & 0.0205 & 2150 \\
\hline 05537500 & 2 & Yes & Yes & 1967 & 616 & $E$ & 600 & 1.2 & 0.197 & 0.24 & 1080 \\
\hline 05537500 & 2 & Yes & Yes & 1968 & 400 & -- & 390 & 0.995 & 0.211 & 0.561 & 772 \\
\hline 05537500 & 2 & Yes & Yes & 1969 & 333 & -- & 324 & 0.926 & 0.225 & 0.692 & 665 \\
\hline 05537500 & 2 & Yes & Yes & 1970 & 1140 & -- & 1110 & 1.01 & 0.238 & 0.044 & 1800 \\
\hline 05537500 & 2 & Yes & Yes & 1971 & 217 & $E$ & 211 & 0.439 & 0.26 & 0.886 & 482 \\
\hline 05537500 & 2 & Yes & Yes & 1972 & 825 & -- & 804 & 1.16 & 0.282 & 0.138 & 1310 \\
\hline 05537500 & 2 & Yes & Yes & 1973 & 663 & -- & 646 & 0.826 & 0.304 & 0.259 & 1060 \\
\hline 05537500 & 2 & Yes & Yes & 1974 & 960 & -- & 935 & 1.06 & 0.326 & 0.0932 & 1450 \\
\hline 05537500 & 2 & Yes & Yes & 1975 & 1050 & -- & 1023 & 1.18 & 0.348 & 0.0736 & 1540 \\
\hline 05537500 & 2 & Yes & Yes & 1976 & 500 & \begin{tabular}{l|}
-- \\
\end{tabular} & 487 & 0.953 & 0.37 & 0.527 & 800 \\
\hline 05537500 & 2 & Yes & Yes & 1977 & 179 & $\mathrm{E}$ & 174 & 1.54 & 0.392 & 0.954 & 366 \\
\hline 05537500 & 2 & Yes & Yes & 1978 & 983 & -- & 958 & 1.01 & 0.414 & 0.114 & 1380 \\
\hline 05537500 & 2 & Yes & Yes & 1979 & 530 & 2 & 516 & 0.852 & 0.436 & 0.54 & 789 \\
\hline 05537500 & 2 & Yes & Yes & 1980 & 230 & $E$ & 224 & 0.867 & 0.458 & 0.938 & 405 \\
\hline 05537500 & 2 & Yes & Yes & 1981 & 1820 & -- & 1773 & 2.04 & 0.47 & 0.018 & 2310 \\
\hline 05537500 & 2 & Yes & Yes & 1982 & 795 & -- & 774 & 0.307 & 0.482 & 0.252 & 1070 \\
\hline 05537500 & 2 & Yes & Yes & 1983 & 740 & \begin{tabular}{l|l}
-- & \\
\end{tabular} & 721 & 1.56 & 0.494 & 0.315 & 995 \\
\hline 05537500 & 2 & Yes & Yes & 1984 & 567 & -- & 552 & 0.619 & 0.506 & 0.551 & 780 \\
\hline 05537500 & 2 & Yes & Yes & 1985 & 686 & -- & 668 & 1.34 & 0.518 & 0.402 & 915 \\
\hline 05537500 & 2 & Yes & Yes & 1986 & 365 & -- & 356 & 0.871 & 0.53 & 0.845 & 525 \\
\hline 05537500 & 2 & Yes & Yes & 1987 & 394 & $E$ & 384 & 2.16 & 0.542 & 0.82 & 552 \\
\hline 05537500 & 2 & Yes & Yes & 1988 & 302 & \begin{tabular}{|c|}
- \\
\end{tabular} & 294 & 0.358 & 0.554 & 0.92 & 441 \\
\hline 05537500 & 2 & Yes & Yes & 1989 & 521 & $E$ & 508 & 1.26 & 0.566 & 0.675 & 680 \\
\hline 05537500 & 2 & Yes & Yes & 1990 & 1200 & -- & 1169 & 2.44 & 0.578 & 0.0945 & 1450 \\
\hline 05537500 & 2 & Yes & Yes & 1991 & 1050 & -- & 1023 & 3.12 & 0.59 & 0.158 & 1260 \\
\hline 05537500 & 2 & Yes & Yes & 1992 & 707 & $E$ & 689 & 4.02 & 0.602 & 0.455 & 865 \\
\hline
\end{tabular}




\begin{tabular}{|c|c|c|c|c|c|c|c|c|c|c|c|}
\hline $\begin{array}{c}\text { U.S. } \\
\text { Geological } \\
\text { Survey } \\
\text { streamgage } \\
\text { number }\end{array}$ & $\begin{array}{l}\text { Segment } \\
\text { number }\end{array}$ & $\begin{array}{l}\text { Streamgage used in } \\
\text { regression analyses } \\
\text { (non-redundant) }\end{array}$ & $\begin{array}{c}\text { Discharge value } \\
\text { used in } \\
\text { adjustment } \\
\text { regression }\end{array}$ & $\begin{array}{c}\text { Water } \\
\text { year }\end{array}$ & $\begin{array}{c}\text { Observed annual } \\
\text { maximum peak } \\
\text { discharge }\left(\mathrm{ft}^{3} / \mathrm{s}\right)\end{array}$ & $\begin{array}{c}\text { NWIS peak } \\
\text { code }\end{array}$ & $\begin{array}{l}\text { Observed annual } \\
\text { maximum peak } \\
\text { discharge with } \\
\text { segment intercept } \\
\text { value subtracted } \\
\left(\mathrm{ft}^{3} / \mathrm{s}\right)\end{array}$ & $\begin{array}{c}\text { Observed } \\
\text { precipitation } \\
\text { (inches) }\end{array}$ & Urban fraction & $\begin{array}{l}\text { Exceedance } \\
\text { probability }\end{array}$ & $\begin{array}{c}\text { Urban-adjusted } \\
\text { annual maximum } \\
\text { peak discharge } \\
\left(\mathrm{ft}^{3} / \mathrm{s}\right)\end{array}$ \\
\hline 05537500 & 2 & Yes & Yes & 1993 & 942 & $\mathrm{C}$ & 918 & 1.03 & 0.614 & 0.224 & 1110 \\
\hline 05537500 & 2 & Yes & Yes & 1994 & 378 & $C, E$ & 368 & 0.747 & 0.626 & 0.887 & 482 \\
\hline 05537500 & 2 & Yes & Yes & 1995 & 519 & C & 506 & 1.17 & 0.638 & 0.74 & 627 \\
\hline 05537500 & 2 & Yes & Yes & 1996 & 5310 & C & 5173 & 4.73 & 0.65 & 0 & 5810 \\
\hline 05537500 & 2 & Yes & Yes & 1997 & 1670 & $\mathrm{C}$ & 1627 & 2.66 & 0.662 & 0.0393 & 1850 \\
\hline 05537500 & 2 & Yes & Yes & 1998 & 820 & $\mathrm{C}$ & 799 & 1.97 & 0.674 & 0.392 & 922 \\
\hline 05537500 & 2 & Yes & Yes & 1999 & 604 & C & 588 & 0.888 & 0.686 & 0.674 & 682 \\
\hline 05537500 & 2 & Yes & Yes & 2000 & 568 & C & 553 & 1.39 & 0.698 & 0.731 & 635 \\
\hline 05537500 & 2 & Yes & Yes & 2001 & 635 & C & 619 & 0.793 & 0.706 & 0.652 & $69 \varepsilon$ \\
\hline 05537500 & 2 & Yes & Yes & 2002 & 811 & C & 790 & 2.35 & 0.715 & 0.444 & $87 t$ \\
\hline 05537500 & 2 & Yes & Yes & 2003 & 1500 & $C, E$ & 1461 & 3.87 & 0.724 & 0.0672 & 1580 \\
\hline 05537500 & 2 & Yes & Yes & 2004 & 1090 & C & 1062 & 2.41 & 0.733 & 0.203 & 1150 \\
\hline 05537500 & 2 & Yes & Yes & 2005 & 668 & C & 651 & 0.938 & 0.742 & 0.645 & 704 \\
\hline 05537500 & 2 & Yes & Yes & 2006 & 305 & $\mathrm{C}$ & 297 & 1.72 & 0.75 & 0.966 & 326 \\
\hline 05537500 & 2 & Yes & Yes & 2007 & 859 & C & 837 & 2.37 & 0.759 & 0.436 & 884 \\
\hline 05537500 & 2 & Yes & Yes & 2008 & 1220 & C & 1188 & 3.7 & 0.768 & 0.165 & 1240 \\
\hline 05537500 & 2 & Yes & Yes & 2009 & 1250 & C & 1218 & 1.93 & 0.777 & 0.157 & 1260 \\
\hline 05538440 & 1 & Yes & No & 1955 & 112 & 7 & 1423 & 3.21 & 0.192 & 0.018 & 180 \\
\hline 05538440 & 1 & Yes & Yes & 1961 & 66 & -- & 839 & 1.42 & 0.296 & 0.125 & $10 t$ \\
\hline 05538440 & 1 & Yes & Yes & 1962 & 32 & 2 & 407 & 0.268 & 0.305 & 0.61 & 58.3 \\
\hline 05538440 & 1 & Yes & Yes & 1963 & 30 & 2 & 381 & 1.5 & 0.314 & 0.659 & 55.1 \\
\hline 05538440 & 1 & Yes & Yes & 1964 & 23 & -- & 292 & 0.926 & 0.323 & 0.807 & 45.2 \\
\hline 05538440 & 1 & Yes & Yes & 1965 & 23 & -- & 292 & 0.871 & 0.332 & 0.812 & $44 . \varepsilon$ \\
\hline 05538440 & 1 & Yes & Yes & 1966 & 78 & -- & 991 & 1.4 & 0.341 & 0.0803 & $11 \mathrm{~s}$ \\
\hline 05538440 & 1 & Yes & Yes & 1967 & 56 & -- & 712 & 1.14 & 0.35 & 0.225 & 87.3 \\
\hline 05538440 & 1 & Yes & Yes & 1968 & 37 & 2 & 470 & 1.47 & 0.359 & 0.545 & 62.4 \\
\hline 05538440 & 1 & Yes & Yes & 1969 & 39 & -- & 496 & 0.145 & 0.368 & 0.511 & 64.7 \\
\hline 05538440 & 1 & Yes & Yes & 1970 & 49 & -- & 623 & 1.08 & 0.377 & 0.338 & 77.3 \\
\hline 05538440 & 1 & Yes & Yes & 1971 & 15 & 2 & 191 & 0.931 & 0.39 & 0.944 & $31 . \varepsilon$ \\
\hline 05538440 & 1 & Yes & Yes & 1972 & 37 & -- & 470 & 1.23 & 0.404 & 0.588 & 59.7 \\
\hline 05538440 & 1 & Yes & Yes & 1973 & 62 & -- & 788 & 0.839 & 0.418 & 0.202 & 90.1 \\
\hline 05538440 & 1 & Yes & Yes & 1974 & 50 & -- & 635 & 1.21 & 0.432 & 0.367 & 75.1 \\
\hline 05538440 & 1 & Yes & Yes & 1975 & 46 & -- & 584 & 1.01 & 0.446 & 0.448 & 69.3 \\
\hline 05538440 & 1 & Yes & Yes & 1976 & 34 & -- & 432 & 0.904 & 0.459 & 0.697 & 53 \\
\hline 05538440 & 1 & Yes & Yes & 1977 & 38 & -- & 483 & 1.43 & 0.473 & 0.628 & 57.1 \\
\hline
\end{tabular}




\begin{tabular}{|c|c|c|c|c|c|c|c|c|c|c|c|}
\hline $\begin{array}{c}\text { U.S. } \\
\text { Geological } \\
\text { Survey } \\
\text { streamgage } \\
\text { number }\end{array}$ & $\begin{array}{l}\text { Segment } \\
\text { number }\end{array}$ & $\begin{array}{l}\text { Streamgage used in } \\
\text { regression analyses } \\
\text { (non-redundant) }\end{array}$ & $\begin{array}{l}\text { Discharge value } \\
\text { used in } \\
\text { adjustment } \\
\text { regression }\end{array}$ & $\begin{array}{l}\text { Water } \\
\text { year }\end{array}$ & $\begin{array}{l}\text { Observed annual } \\
\text { maximum peak } \\
\text { discharge }\left(\mathrm{ft}^{3} / \mathrm{s}\right)\end{array}$ & $\begin{array}{l}\text { NWIS peak } \\
\text { code }\end{array}$ & $\begin{array}{l}\text { Observed annual } \\
\text { maximum peak } \\
\text { discharge with } \\
\text { segment intercept } \\
\text { value subtracted } \\
\left(\mathrm{ft}^{3} / \mathrm{s}\right)\end{array}$ & $\begin{array}{l}\text { Observed } \\
\text { precipitation } \\
\text { (inches) }\end{array}$ & Urban fraction & $\begin{array}{l}\text { Exceedance } \\
\text { probability }\end{array}$ & $\begin{array}{c}\text { Urban-adjusted } \\
\text { annual maximum } \\
\text { peak discharge } \\
\left(\mathrm{ft}^{3} / \mathrm{s}\right)\end{array}$ \\
\hline 05539000 & 1 & Yes & No & 1942 & 9000 & 2,7 & 1488 & 0.0435 & 0.0789 & 0.0134 & 14000 \\
\hline 05539000 & 1 & Yes & Yes & 1945 & 2040 & -- & 337 & 0.829 & 0.0865 & 0.562 & 4000 \\
\hline 05539000 & 1 & Yes & Yes & 1946 & 1430 & -- & 236 & 0.379 & 0.0891 & 0.765 & 3080 \\
\hline 05539000 & 1 & Yes & Yes & 1947 & 10200 & -- & 1687 & 2.6 & 0.0916 & 0.00964 & 15500 \\
\hline 05539000 & 1 & Yes & Yes & 1948 & 4370 & -- & 723 & 1.84 & 0.0942 & 0.11 & 7390 \\
\hline 05539000 & 1 & Yes & Yes & 1949 & 2400 & -- & 397 & 0.767 & 0.0967 & 0.449 & 4540 \\
\hline 05539000 & 1 & Yes & Yes & 1950 & 3910 & -- & 647 & 1.2 & 0.0993 & 0.158 & 6640 \\
\hline 05539000 & 1 & Yes & Yes & 1951 & 2550 & D & 422 & 0.385 & 0.105 & 0.411 & 4730 \\
\hline 05539000 & 1 & Yes & Yes & 1952 & 3580 & -- & 592 & 1.27 & 0.11 & 0.198 & 6070 \\
\hline 05539000 & 1 & Yes & Yes & 1953 & 2350 & -- & 389 & 1.17 & 0.115 & 0.479 & 4380 \\
\hline 05539000 & 1 & Yes & Yes & 1954 & 3150 & -- & 521 & 1.74 & 0.121 & 0.283 & 5450 \\
\hline 05539000 & 1 & Yes & Yes & 1955 & 8130 & -- & 1344 & 3.24 & 0.126 & 0.0181 & 12300 \\
\hline 05539000 & 1 & Yes & Yes & 1956 & 2300 & -- & 380 & 0.779 & 0.132 & 0.509 & 4240 \\
\hline 05539000 & 1 & Yes & Yes & 1957 & 15200 & -- & 2514 & 2.95 & 0.137 & 0.00208 & 21900 \\
\hline 05539000 & 1 & Yes & Yes & 1958 & 1980 & -- & 327 & 1.21 & 0.142 & 0.626 & 3720 \\
\hline 05539000 & 1 & Yes & Yes & 1959 & 2870 & -- & 475 & 1.31 & 0.148 & 0.362 & 5000 \\
\hline 05539000 & 1 & Yes & Yes & 1960 & 1980 & -- & 327 & 0.0435 & 0.153 & 0.634 & 3680 \\
\hline 05539000 & 1 & Yes & Yes & 1961 & 4560 & $\mathrm{E}$ & 754 & 1.45 & 0.16 & 0.117 & 7280 \\
\hline 05539000 & 1 & Yes & Yes & 1962 & 2020 & -- & 334 & 0.527 & 0.166 & 0.631 & 3690 \\
\hline 05539000 & 1 & Yes & Yes & 1963 & 850 & $2, E$ & 141 & 0.415 & 0.173 & 0.935 & 2000 \\
\hline 05539000 & 1 & Yes & Yes & 1964 & 1180 & $\mathrm{E}$ & 195 & 1.02 & 0.18 & 0.876 & 2470 \\
\hline 05539000 & 1 & Yes & Yes & 1965 & 1650 & $\mathrm{E}$ & 273 & 0.883 & 0.186 & 0.758 & 3120 \\
\hline 05539000 & 1 & Yes & Yes & 1966 & 4680 & -- & 774 & 1.46 & 0.193 & 0.119 & 7250 \\
\hline 05539000 & 1 & Yes & Yes & 1967 & 2020 & $\mathrm{E}$ & 334 & 1.12 & 0.2 & 0.656 & 3580 \\
\hline 05539000 & 1 & Yes & Yes & 1968 & 2460 & -- & 407 & 1.99 & 0.206 & 0.522 & 4180 \\
\hline 05539000 & 1 & Yes & Yes & 1969 & 1220 & $\mathrm{E}$ & 202 & 0.849 & 0.213 & 0.88 & 2440 \\
\hline 05539000 & 1 & Yes & Yes & 1970 & 2670 & -- & 442 & 1.57 & 0.22 & 0.471 & 4430 \\
\hline 05539000 & 1 & Yes & Yes & 1971 & 1390 & $\mathrm{E}$ & 230 & 0.425 & 0.229 & 0.847 & 2630 \\
\hline 05539000 & 1 & Yes & Yes & 1972 & 1710 & -- & 283 & 1.33 & 0.239 & 0.772 & 3040 \\
\hline 05539000 & 1 & Yes & Yes & 1973 & 4080 & -- & 675 & 1.32 & 0.248 & 0.199 & 6080 \\
\hline 05539000 & 1 & Yes & Yes & 1974 & 5070 & -- & 838 & 1.14 & 0.258 & 0.11 & 7400 \\
\hline 05539000 & 1 & Yes & Yes & 1975 & 2680 & -- & 443 & 1.34 & 0.267 & 0.509 & 4240 \\
\hline 05539000 & 1 & Yes & Yes & 1976 & 3580 & D & 592 & 1.48 & 0.277 & 0.3 & 5340 \\
\hline 05539000 & 1 & Yes & Yes & 1977 & 1290 & $\mathrm{E}$ & 213 & 1.64 & 0.286 & 0.894 & 2350 \\
\hline 05539000 & 1 & Yes & Yes & 1978 & 3800 & E & 628 & 1.05 & 0.296 & 0.272 & 5510 \\
\hline
\end{tabular}




\begin{tabular}{|c|c|c|c|c|c|c|c|c|c|c|c|}
\hline $\begin{array}{l}\text { U.S. } \\
\text { Geological } \\
\text { Survey } \\
\text { streamgage } \\
\text { number }\end{array}$ & $\begin{array}{c}\text { Segment } \\
\text { number }\end{array}$ & $\begin{array}{l}\text { Streamgage used in } \\
\text { regression analyses } \\
\text { (non-redundant) }\end{array}$ & $\begin{array}{c}\text { Discharge value } \\
\text { used in } \\
\text { adjustment } \\
\text { regression }\end{array}$ & $\begin{array}{l}\text { Water } \\
\text { year }\end{array}$ & $\begin{array}{c}\text { Observed annual } \\
\text { maximum peak } \\
\text { discharge }\left(\mathrm{ft}^{3} / \mathrm{s}\right)\end{array}$ & $\begin{array}{l}\text { NWIS peak } \\
\text { code }\end{array}$ & $\begin{array}{l}\text { Observed annual } \\
\text { maximum peak } \\
\text { discharge with } \\
\text { segment intercept } \\
\text { value subtracted } \\
\left(\mathrm{ft}^{3} / \mathrm{s}\right)\end{array}$ & $\begin{array}{l}\text { Observed } \\
\text { precipitation } \\
\text { (inches) }\end{array}$ & Urban fraction & $\begin{array}{l}\text { Exceedance } \\
\text { probability }\end{array}$ & $\begin{array}{c}\text { Urban-adjusted } \\
\text { annual maximum } \\
\text { peak discharge } \\
\left(\mathrm{ft}^{3} / \mathrm{s}\right)\end{array}$ \\
\hline 05539000 & 1 & Yes & Yes & 1979 & 2750 & -- & 455 & 1.18 & 0.305 & 0.523 & 4180 \\
\hline 05539000 & 1 & Yes & Yes & 1980 & 1960 & $\mathrm{E}$ & 324 & 1.24 & 0.315 & 0.756 & 3130 \\
\hline 05539000 & 1 & Yes & Yes & 1981 & 17300 & $\mathrm{E}$ & 2861 & 2.27 & 0.322 & 0 & 21900 \\
\hline 05539000 & 1 & Yes & Yes & 1982 & 3620 & -- & 599 & 0.419 & 0.329 & 0.33 & 5170 \\
\hline 05539000 & 1 & Yes & Yes & 1983 & 8360 & -- & 1382 & 2.07 & 0.335 & 0.0277 & 10900 \\
\hline 05539000 & 1 & Yes & Yes & 1984 & 2670 & -- & 442 & 0.928 & 0.342 & 0.581 & 3920 \\
\hline 05539000 & 1 & Yes & Yes & 1985 & 3120 & -- & 516 & 1.28 & 0.349 & 0.462 & 4480 \\
\hline 05539000 & 1 & Yes & Yes & 1986 & 2320 & $E$ & 384 & 1.08 & 0.356 & 0.689 & 3440 \\
\hline 05539000 & 1 & Yes & Yes & 1987 & 1710 & $E$ & 283 & 1.11 & 0.363 & 0.841 & 2660 \\
\hline 05539000 & 1 & Yes & Yes & 1988 & 1090 & $E$ & 180 & 0.673 & 0.37 & 0.946 & 1870 \\
\hline 05539000 & 1 & Yes & Yes & 1989 & 4160 & $E$ & 688 & 1.29 & 0.377 & 0.264 & 5570 \\
\hline 05539000 & 1 & Yes & Yes & 1990 & 1950 & $\mathrm{E}$ & 322 & 2.01 & 0.383 & 0.8 & 2900 \\
\hline 05539000 & 1 & Yes & Yes & 1991 & 6220 & -- & 1029 & 3.16 & 0.395 & 0.0833 & 7980 \\
\hline 05539000 & 1 & Yes & Yes & 1992 & 1840 & $E$ & 304 & 3.59 & 0.406 & 0.837 & 2690 \\
\hline 05539000 & 1 & Yes & Yes & 1993 & 2540 & $C, E$ & 420 & 1.49 & 0.417 & 0.679 & 3480 \\
\hline 05539000 & 1 & Yes & Yes & 1994 & 5980 & $C, E$ & 989 & 1.54 & 0.428 & 0.106 & 7480 \\
\hline 05539000 & 1 & Yes & Yes & 1995 & 3780 & $C, E$ & 625 & 0.978 & 0.439 & 0.387 & 4860 \\
\hline 05539000 & 1 & Yes & Yes & 1996 & 8710 & C & 1440 & 4.74 & 0.45 & 0.0332 & 10400 \\
\hline 05539000 & 1 & Yes & Yes & 1997 & 5170 & $C, E$ & 855 & 2.3 & 0.462 & 0.184 & 6300 \\
\hline 05539000 & 1 & Yes & Yes & 1998 & 2990 & $C, E$ & 494 & 1.5 & 0.473 & 0.61 & 3790 \\
\hline 05539000 & 1 & Yes & Yes & 1999 & 2190 & $C, E$ & 362 & 1.7 & 0.484 & 0.81 & 2850 \\
\hline 05539000 & 1 & Yes & Yes & 2000 & 1980 & $C, E$ & 327 & 1.34 & 0.495 & 0.858 & 2570 \\
\hline 05539000 & 1 & Yes & Yes & 2001 & 2920 & $C, E$ & 483 & 0.535 & 0.513 & 0.663 & 3550 \\
\hline 05539000 & 1 & Yes & Yes & 2002 & 4400 & C & 728 & 2.18 & 0.531 & 0.34 & 5110 \\
\hline 05539000 & 1 & Yes & Yes & 2003 & 2640 & C & 437 & 1.06 & 0.549 & 0.759 & 3110 \\
\hline 05539000 & 1 & Yes & Yes & 2004 & 4630 & C & 766 & 1.52 & 0.567 & 0.327 & 5170 \\
\hline 05539000 & 1 & Yes & Yes & 2005 & 3290 & $C, E$ & 544 & 0.971 & 0.585 & 0.638 & 3660 \\
\hline 05539000 & 1 & Yes & Yes & 2006 & 3270 & C & 541 & 2.3 & 0.603 & 0.66 & 3570 \\
\hline 05539000 & 1 & Yes & Yes & 2007 & 4120 & C & 681 & 2.45 & 0.621 & 0.483 & 4380 \\
\hline 05539000 & 1 & Yes & Yes & 2008 & 7150 & C & 1182 & 4.15 & 0.639 & 0.112 & 7390 \\
\hline 05539000 & 0 & Yes & No & 2009 & 4920 & C & NA & 1.68 & 0.657 & NA & NA \\
\hline 05539870 & 1 & No & No & 1961 & 212 & -- & 475 & 0.945 & 0.387 & 0.563 & 402 \\
\hline 05539870 & 1 & No & No & 1962 & 138 & -- & 309 & 0.197 & 0.429 & 0.843 & 288 \\
\hline 05539870 & 1 & No & No & 1963 & 100 & -- & 224 & 0.874 & 0.471 & 0.941 & 228 \\
\hline 05539870 & 1 & No & No & 1964 & 147 & -- & 329 & 1.27 & 0.513 & 0.867 & 278 \\
\hline
\end{tabular}




\begin{tabular}{|c|c|c|c|c|c|c|c|c|c|c|c|}
\hline $\begin{array}{c}\text { U.S. } \\
\text { Geological } \\
\text { Survey } \\
\text { streamgage } \\
\text { number } \\
\end{array}$ & $\begin{array}{c}\text { Segment } \\
\text { number }\end{array}$ & $\begin{array}{l}\text { Streamgage used in } \\
\text { regression analyses } \\
\text { (non-redundant) }\end{array}$ & $\begin{array}{c}\text { Discharge value } \\
\text { used in } \\
\text { adjustment } \\
\text { regression } \\
\end{array}$ & $\begin{array}{c}\text { Water } \\
\text { year }\end{array}$ & $\begin{array}{c}\text { Observed annual } \\
\text { maximum peak } \\
\text { discharge }\left(\mathrm{ft}^{3} / \mathrm{s}\right)\end{array}$ & $\begin{array}{c}\text { NWIS peak } \\
\text { code }\end{array}$ & $\begin{array}{c}\text { Observed annual } \\
\text { maximum peak } \\
\text { discharge with } \\
\text { segment intercept } \\
\text { value subtracted } \\
\left(\mathrm{ft}^{3} / \mathrm{s}\right) \\
\end{array}$ & $\begin{array}{c}\text { Observed } \\
\text { precipitation } \\
\text { (inches) }\end{array}$ & Urban fraction & $\begin{array}{l}\text { Exceedance } \\
\text { probability }\end{array}$ & $\begin{array}{c}\text { Urban-adjusted } \\
\text { annual maximum } \\
\text { peak discharge } \\
\left(\mathrm{ft}^{3} / \mathrm{s}\right)\end{array}$ \\
\hline 05539870 & 1 & No & No & 1965 & 88 & -- & 197 & 0.0936 & 0.555 & 0.969 & 187 \\
\hline 05539870 & 1 & No & No & 1966 & 295 & -- & 661 & 0.931 & 0.598 & 0.487 & 435 \\
\hline 05539870 & 1 & No & No & 1967 & 542 & -- & 1214 & 0.866 & 0.64 & 0.1 & 714 \\
\hline 05539870 & 1 & No & No & 1968 & 535 & -- & 1199 & 1.13 & 0.682 & 0.124 & 680 \\
\hline 05539870 & 1 & No & No & 1969 & 238 & -- & 533 & 0.973 & 0.724 & 0.775 & 319 \\
\hline 05539870 & 1 & No & No & 1970 & 400 & -- & 896 & 1.07 & 0.766 & 0.375 & 484 \\
\hline 05539870 & 1 & No & No & 1971 & 395 & -- & 885 & 0.265 & 0.78 & 0.405 & 472 \\
\hline 05539870 & 1 & No & No & 1972 & 630 & -- & 1412 & 0.977 & 0.795 & 0.0967 & 722 \\
\hline 05539870 & 1 & No & No & 1973 & 390 & -- & 874 & 0.557 & 0.809 & 0.447 & 453 \\
\hline 05539870 & 1 & No & No & 1974 & 365 & -- & 818 & 0.447 & 0.823 & 0.524 & 419 \\
\hline 05539870 & 1 & No & No & 1975 & 480 & -- & 1075 & 0.599 & 0.838 & 0.264 & 533 \\
\hline 05539870 & 1 & No & No & 1976 & 418 & -- & 937 & 0.83 & 0.852 & 0.425 & 463 \\
\hline 05539870 & 0 & No & No & 1977 & 170 & -- & NA & 0.448 & 0.867 & NA & NA \\
\hline 05539870 & 0 & No & No & 1978 & 429 & -- & NA & 0.995 & 0.881 & NA & NA \\
\hline 05539870 & 0 & No & No & 1979 & 553 & -- & NA & 0.589 & 0.895 & NA & NA \\
\hline 05539890 & 1 & No & No & 1955 & 1620 & 7 & 2053 & 3.05 & 0.19 & 0.00674 & 2540 \\
\hline 05539890 & 1 & No & No & 1961 & 397 & -- & 503 & 1.31 & 0.29 & 0.431 & 717 \\
\hline 05539890 & 1 & No & No & 1962 & 306 & -- & 388 & 0.276 & 0.315 & 0.649 & 567 \\
\hline 05539890 & 1 & No & No & 1963 & 191 & 2 & 242 & 1.22 & 0.34 & 0.883 & 401 \\
\hline 05539890 & 1 & No & No & 1964 & 195 & -- & 247 & 1.75 & 0.365 & 0.888 & 396 \\
\hline 05539890 & 1 & No & No & 1965 & 209 & -- & 265 & 0.131 & 0.39 & 0.879 & 405 \\
\hline 05539890 & 1 & No & No & 1966 & 442 & -- & 560 & 1.3 & 0.415 & 0.455 & 699 \\
\hline 05539890 & 1 & No & No & 1967 & 1270 & -- & 1609 & 1.2 & 0.44 & 0.0215 & 1710 \\
\hline 05539890 & 1 & No & No & 1968 & 638 & -- & 808 & 1.56 & 0.465 & 0.214 & 900 \\
\hline 05539890 & 1 & No & No & 1969 & 375 & -- & 475 & 1.35 & 0.49 & 0.655 & 565 \\
\hline 05539890 & 1 & No & No & 1970 & 560 & -- & 710 & 1.49 & 0.515 & 0.348 & 778 \\
\hline 05539890 & 1 & No & No & 1971 & 494 & -- & 626 & 0.368 & 0.535 & 0.473 & 687 \\
\hline 05539890 & 1 & No & No & 1972 & 726 & -- & 920 & 1.39 & 0.554 & 0.19 & 939 \\
\hline 05539890 & 1 & No & No & 1973 & 482 & -- & 611 & 0.772 & 0.573 & 0.531 & 646 \\
\hline 05539890 & 1 & No & No & 1974 & 528 & -- & 669 & 0.62 & 0.593 & 0.471 & 688 \\
\hline 05539890 & 1 & No & No & 1975 & 487 & -- & 617 & 0.837 & 0.612 & 0.56 & 626 \\
\hline 05539890 & 1 & No & No & 1976 & 530 & -- & 672 & 1.15 & 0.631 & 0.506 & 664 \\
\hline 05539890 & 0 & No & No & 1977 & 269 & -- & NA & 0.624 & 0.65 & NA & NA \\
\hline 05539890 & 0 & No & No & 1978 & 808 & -- & NA & 1.38 & 0.67 & NA & NA \\
\hline 3989 & 0 & No & No & 1979 & 900 & -- & NA & $0 . \varepsilon$ & 0. & NA & NA \\
\hline
\end{tabular}




\begin{tabular}{|c|c|c|c|c|c|c|c|c|c|c|c|}
\hline $\begin{array}{c}\text { U.S. } \\
\text { Geological } \\
\text { Survey } \\
\text { streamgage } \\
\text { number } \\
\end{array}$ & $\begin{array}{c}\text { Segment } \\
\text { number }\end{array}$ & $\begin{array}{l}\text { Streamgage used in } \\
\text { regression analyses } \\
\text { (non-redundant) }\end{array}$ & $\begin{array}{c}\text { Discharge value } \\
\text { used in } \\
\text { adjustment } \\
\text { regression }\end{array}$ & $\begin{array}{c}\text { Water } \\
\text { year }\end{array}$ & $\begin{array}{c}\text { Observed annual } \\
\text { maximum peak } \\
\text { discharge }\left(\mathrm{ft}^{3} / \mathrm{s}\right)\end{array}$ & $\begin{array}{c}\text { NWIS peak } \\
\text { code }\end{array}$ & $\begin{array}{c}\text { Observed annual } \\
\text { maximum peak } \\
\text { discharge with } \\
\text { segment intercept } \\
\text { value subtracted } \\
\left(\mathrm{ft}^{3} / \mathrm{s}\right)\end{array}$ & $\begin{array}{l}\text { Observed } \\
\text { precipitation } \\
\text { (inches) }\end{array}$ & Urban fraction & $\begin{array}{l}\text { Exceedance } \\
\text { probability }\end{array}$ & $\begin{array}{c}\text { Urban-adjusted } \\
\text { annual maximum } \\
\text { peak discharge } \\
\left(\mathrm{ft}^{3} / \mathrm{s}\right)\end{array}$ \\
\hline 05539900 & 1 & Yes & Yes & 1961 & 450 & -- & 554 & 1.31 & 0.258 & 0.334 & 701 \\
\hline 05539900 & 1 & Yes & Yes & 1962 & 361 & -- & 444 & 0.277 & 0.28 & 0.518 & 580 \\
\hline 05539900 & 1 & Yes & Yes & 1963 & 217 & $E$ & 267 & 1.22 & 0.302 & 0.831 & 393 \\
\hline 05539900 & 1 & Yes & Yes & 1964 & 201 & -- & 247 & 1.74 & 0.324 & 0.869 & 366 \\
\hline 05539900 & 1 & Yes & Yes & 1965 & 250 & 2 & 308 & 0.131 & 0.345 & 0.798 & 416 \\
\hline 05539900 & 1 & Yes & Yes & 1966 & 537 & -- & 661 & 1.31 & 0.367 & 0.285 & 735 \\
\hline 05539900 & 1 & Yes & Yes & 1967 & 805 & -- & 991 & 1.19 & 0.389 & 0.0928 & 1030 \\
\hline 05539900 & 1 & Yes & Yes & 1968 & 340 & -- & 419 & 1.56 & 0.411 & 0.676 & 489 \\
\hline 05539900 & 1 & Yes & Yes & 1969 & 399 & -- & 491 & 1.34 & 0.432 & 0.579 & 545 \\
\hline 05539900 & 1 & Yes & Yes & 1970 & 521 & D & 641 & 1.48 & 0.454 & 0.379 & 671 \\
\hline 05539900 & 1 & Yes & Yes & 1971 & 333 & $\mathrm{E}$ & 410 & 0.367 & 0.477 & 0.742 & 452 \\
\hline 05539900 & 1 & Yes & Yes & 1972 & 715 & -- & 880 & 1.41 & 0.499 & 0.186 & 841 \\
\hline 05539900 & 1 & Yes & Yes & 1973 & 535 & -- & 659 & 0.77 & 0.521 & 0.417 & 646 \\
\hline 05539900 & 1 & Yes & Yes & 1974 & 522 & -- & 643 & 0.616 & 0.544 & 0.459 & 619 \\
\hline 05539900 & 1 & Yes & Yes & 1975 & 537 & -- & 661 & 0.844 & 0.566 & 0.456 & 621 \\
\hline 05539900 & 1 & Yes & Yes & 1976 & 557 & -- & 686 & 1.15 & 0.589 & 0.446 & 628 \\
\hline 05539900 & 0 & Yes & No & 1977 & 158 & $\mathrm{E}$ & 209 & 0.865 & 0.611 & 0.974 & 225 \\
\hline 05539900 & 2 & Yes & Yes & 1978 & 468 & -- & 663 & 0.929 & 0.633 & 0.519 & 581 \\
\hline 05539900 & 2 & Yes & Yes & 1979 & 779 & D & 1104 & 0.832 & 0.656 & 0.151 & 910 \\
\hline 05539900 & 2 & Yes & Yes & 1980 & 311 & -- & 441 & 0.687 & 0.678 & 0.844 & 383 \\
\hline 05539900 & 2 & Yes & Yes & 1981 & 483 & -- & 684 & 0.55 & 0.683 & 0.541 & 567 \\
\hline 05539900 & 2 & Yes & Yes & 1982 & 673 & -- & 954 & 0.343 & 0.688 & 0.245 & 767 \\
\hline 05539900 & 2 & Yes & Yes & 1983 & 984 & -- & 1394 & 1.74 & 0.693 & 0.0718 & 1100 \\
\hline 05539900 & 2 & Yes & Yes & 1984 & 392 & $E$ & 555 & 0.599 & 0.698 & 0.729 & 460 \\
\hline 05539900 & 2 & Yes & Yes & 1985 & 706 & -- & 1000 & 1.28 & 0.704 & 0.223 & 792 \\
\hline 05539900 & 2 & Yes & Yes & 1986 & 613 & $E$ & 869 & 0.804 & 0.709 & 0.345 & 692 \\
\hline 05539900 & 2 & Yes & Yes & 1987 & 958 & -- & 1358 & 2.24 & 0.714 & 0.0852 & 1060 \\
\hline 05539900 & 2 & Yes & Yes & 1988 & 377 & $E$ & 534 & 0.857 & 0.719 & 0.77 & 434 \\
\hline 05539900 & 2 & Yes & Yes & 1989 & 549 & $E$ & 778 & 1.21 & 0.724 & 0.467 & 614 \\
\hline 05539900 & 2 & Yes & Yes & 1990 & 571 & $E$ & 809 & 1.42 & 0.729 & 0.436 & 634 \\
\hline 05539900 & 2 & Yes & Yes & 1991 & 470 & -- & 666 & 1.7 & 0.732 & 0.614 & 524 \\
\hline 05539900 & 2 & Yes & Yes & 1992 & 278 & $E$ & 394 & 1.55 & 0.735 & 0.922 & 322 \\
\hline 05539900 & 2 & Yes & Yes & 1993 & 460 & C & 652 & 0.79 & 0.738 & 0.64 & 510 \\
\hline 05539900 & 2 & Yes & Yes & 1994 & 786 & C & 1114 & 5.06 & 0.742 & 0.184 & 849 \\
\hline 05539900 & 2 & Yes & Yes & 1995 & 643 & C & 911 & 2.13 & 0.745 & 0.334 & 698 \\
\hline
\end{tabular}




\begin{tabular}{|c|c|c|c|c|c|c|c|c|c|c|c|}
\hline $\begin{array}{l}\text { U.S. } \\
\text { Geological } \\
\text { Survey } \\
\text { streamgage } \\
\text { number }\end{array}$ & $\begin{array}{l}\text { Segment } \\
\text { number }\end{array}$ & $\begin{array}{l}\text { Streamgage used in } \\
\text { regression analyses } \\
\text { (non-redundant) }\end{array}$ & $\begin{array}{c}\text { Discharge value } \\
\text { used in } \\
\text { adjustment } \\
\text { regression }\end{array}$ & $\begin{array}{l}\text { Water } \\
\text { year }\end{array}$ & $\begin{array}{c}\text { Observed annual } \\
\text { maximum peak } \\
\text { discharge }\left(\mathrm{ft}^{3} / \mathrm{s}\right)\end{array}$ & $\begin{array}{l}\text { NWIS peak } \\
\text { code }\end{array}$ & $\begin{array}{l}\text { Observed annual } \\
\text { maximum peak } \\
\text { discharge with } \\
\text { segment intercept } \\
\text { value subtracted } \\
\left(\mathrm{ft}^{3} / \mathrm{s}\right)\end{array}$ & $\begin{array}{l}\text { Observed } \\
\text { precipitation } \\
\text { (inches) }\end{array}$ & Urban fraction & $\begin{array}{l}\text { Exceedance } \\
\text { probability }\end{array}$ & $\begin{array}{c}\text { Urban-adjusted } \\
\text { annual maximum } \\
\text { peak discharge } \\
\left(\mathrm{ft}^{3} / \mathrm{s}\right)\end{array}$ \\
\hline 05539900 & 2 & Yes & Yes & 1996 & 592 & C & 839 & 1.82 & 0.748 & 0.422 & 644 \\
\hline 05539900 & 2 & Yes & Yes & 1997 & 892 & $C, E$ & 1264 & 2.51 & 0.751 & 0.129 & 954 \\
\hline 05539900 & 2 & Yes & Yes & 1998 & 623 & $C, E$ & 883 & 1.2 & 0.754 & 0.378 & 672 \\
\hline 05539900 & 2 & Yes & Yes & 1999 & 666 & C & 944 & 1.42 & 0.757 & 0.31 & 713 \\
\hline 05539900 & 2 & Yes & Yes & 2000 & 517 & C & 733 & 1.4 & 0.761 & 0.558 & 556 \\
\hline 05539900 & 2 & Yes & Yes & 2001 & 825 & C & 1169 & 3.9 & 0.767 & 0.172 & 870 \\
\hline 05539900 & 2 & Yes & Yes & 2002 & 913 & C & 1294 & 3.79 & 0.774 & 0.127 & 957 \\
\hline 05539900 & 2 & Yes & Yes & 2003 & 565 & C & 801 & 2.27 & 0.78 & 0.499 & 594 \\
\hline 05539900 & 2 & Yes & Yes & 2004 & 538 & C & 762 & 1.4 & 0.786 & 0.549 & 562 \\
\hline 05539900 & 2 & Yes & Yes & 2005 & 495 & $C, E$ & 701 & 0.76 & 0.793 & 0.632 & 514 \\
\hline 05539900 & 2 & Yes & Yes & 2006 & 569 & C & 806 & 1.61 & 0.799 & 0.512 & 586 \\
\hline 05539900 & 2 & Yes & Yes & 2007 & 1040 & C & 1474 & 2.21 & 0.806 & 0.0851 & 1060 \\
\hline 05539900 & 2 & Yes & Yes & 2008 & 1840 & C & 2607 & 4.27 & 0.812 & 0.0137 & 1860 \\
\hline 05539900 & 2 & Yes & Yes & 2009 & 1080 & C & 1530 & 0.754 & 0.819 & 0.0759 & 1090 \\
\hline 05539950 & 1 & Yes & Yes & 1961 & 172 & -- & 478 & 1.41 & 0.197 & 0.393 & 379 \\
\hline 05539950 & 1 & Yes & Yes & 1962 & 107 & -- & 297 & 0.623 & 0.216 & 0.735 & 267 \\
\hline 05539950 & 1 & Yes & Yes & 1963 & 119 & -- & 330 & 1.36 & 0.235 & 0.689 & 281 \\
\hline 05539950 & 1 & Yes & Yes & 1964 & 101 & -- & 280 & 2.16 & 0.254 & 0.785 & 250 \\
\hline 05539950 & 1 & Yes & Yes & 1965 & 114 & -- & 317 & 0.791 & 0.273 & 0.74 & 266 \\
\hline 05539950 & 1 & Yes & Yes & 1966 & 440 & -- & 1222 & 1.49 & 0.292 & 0.0376 & 744 \\
\hline 05539950 & 1 & Yes & Yes & 1967 & 213 & -- & 591 & 1.31 & 0.311 & 0.326 & 403 \\
\hline 05539950 & 1 & Yes & Yes & 1968 & 224 & -- & 622 & 1.98 & 0.33 & 0.303 & 411 \\
\hline 05539950 & 1 & Yes & Yes & 1969 & 119 & -- & 330 & 1.35 & 0.349 & 0.768 & 256 \\
\hline 05539950 & 1 & Yes & Yes & 1970 & 148 & -- & 411 & 1.37 & 0.368 & 0.653 & 292 \\
\hline 05539950 & 1 & Yes & Yes & 1971 & 144 & -- & 400 & 0.335 & 0.404 & 0.702 & 278 \\
\hline 05539950 & 1 & Yes & Yes & 1972 & 888 & -- & 2466 & 2.11 & 0.439 & 0.00662 & 1260 \\
\hline 05539950 & 1 & Yes & Yes & 1973 & 165 & -- & 458 & 0.819 & 0.475 & 0.668 & 287 \\
\hline 05539950 & 1 & Yes & Yes & 1974 & 186 & -- & 517 & 0.584 & 0.51 & 0.61 & 305 \\
\hline 05539950 & 1 & Yes & Yes & 1975 & 170 & -- & 472 & 1.07 & 0.546 & 0.709 & 276 \\
\hline 05539950 & 1 & Yes & Yes & 1976 & 280 & -- & 778 & 1.12 & 0.582 & 0.327 & 401 \\
\hline 05539950 & 1 & Yes & Yes & 1977 & 141 & -- & 392 & 0.644 & 0.617 & 0.857 & 223 \\
\hline 05539950 & 1 & Yes & Yes & 1978 & 212 & -- & 589 & 0.835 & 0.653 & 0.639 & 296 \\
\hline 05539950 & 1 & Yes & Yes & 1979 & 412 & -- & 1144 & 0.989 & 0.688 & 0.148 & 516 \\
\hline 05540030 & 1 & No & No & 1955 & 1260 & 7 & 1036 & 3.47 & 0.232 & 0.0531 & 2130 \\
\hline 05540030 & 1 & No & No & 1961 & 758 & -- & 623 & 1.38 & 0.329 & 0.3 & 1270 \\
\hline
\end{tabular}




\begin{tabular}{|c|c|c|c|c|c|c|c|c|c|c|c|}
\hline $\begin{array}{c}\text { U.S. } \\
\text { Geological } \\
\text { Survey } \\
\text { streamgage } \\
\text { number } \\
\end{array}$ & $\begin{array}{c}\text { Segment } \\
\text { number }\end{array}$ & $\begin{array}{l}\text { Streamgage used in } \\
\text { regression analyses } \\
\text { (non-redundant) }\end{array}$ & $\begin{array}{c}\text { Discharge value } \\
\text { used in } \\
\text { adjustment } \\
\text { regression }\end{array}$ & $\begin{array}{l}\text { Water } \\
\text { year }\end{array}$ & $\begin{array}{c}\text { Observed annual } \\
\text { maximum peak } \\
\text { discharge }\left(\mathrm{ft}^{3} / \mathrm{s}\right)\end{array}$ & $\begin{array}{c}\text { NWIS peak } \\
\text { code }\end{array}$ & $\begin{array}{c}\text { Observed annual } \\
\text { maximum peak } \\
\text { discharge with } \\
\text { segment intercept } \\
\text { value subtracted } \\
\left(\mathrm{ft}^{3} / \mathrm{s}\right)\end{array}$ & $\begin{array}{c}\text { Observed } \\
\text { precipitation } \\
\text { (inches) }\end{array}$ & Urban fraction & $\begin{array}{l}\text { Exceedance } \\
\text { probability }\end{array}$ & $\begin{array}{c}\text { Urban-adjusted } \\
\text { annual maximum } \\
\text { peak discharge } \\
\left(\mathrm{ft}^{3} / \mathrm{s}\right) \\
\end{array}$ \\
\hline 05540030 & 1 & No & No & 1962 & 688 & -- & 565 & 0.285 & 0.345 & 0.387 & 1170 \\
\hline 05540030 & 1 & No & No & 1963 & 415 & 2 & 341 & 1.34 & 0.362 & 0.761 & 781 \\
\hline 05540030 & 1 & No & No & 1964 & 550 & 2 & 452 & 2.08 & 0.379 & 0.596 & 948 \\
\hline 05540030 & 1 & No & No & 1965 & 460 & 2 & 378 & 0.115 & 0.395 & 0.728 & 815 \\
\hline 05540030 & 1 & No & No & 1966 & 1020 & -- & 838 & 1.45 & 0.412 & 0.172 & 1520 \\
\hline 05540030 & 1 & No & No & 1967 & 1080 & -- & 888 & 1.28 & 0.429 & 0.154 & 1580 \\
\hline 05540030 & 1 & No & No & 1968 & 748 & -- & 615 & 1.91 & 0.445 & 0.406 & 1150 \\
\hline 05540030 & 1 & No & No & 1969 & 520 & 2 & 427 & 1.35 & 0.462 & 0.705 & 840 \\
\hline 05540030 & 1 & No & No & 1970 & 730 & -- & 600 & 1.42 & 0.479 & 0.456 & 1100 \\
\hline 05540030 & 1 & No & No & 1971 & 506 & 2 & 416 & 0.345 & 0.5 & 0.751 & 793 \\
\hline 05540030 & 1 & No & No & 1972 & 1670 & -- & 1373 & 1.99 & 0.521 & 0.0463 & 2200 \\
\hline 05540030 & 1 & No & No & 1973 & 869 & -- & 714 & 0.802 & 0.541 & 0.366 & 1200 \\
\hline 05540030 & 1 & No & No & 1974 & 740 & -- & 608 & 0.599 & 0.562 & 0.524 & 1020 \\
\hline 05540030 & 1 & No & No & 1975 & 651 & -- & 535 & 1.01 & 0.583 & 0.649 & 892 \\
\hline 05540030 & 1 & No & No & 1976 & 925 & -- & 760 & 1.15 & 0.604 & 0.369 & 1190 \\
\hline 05540030 & 1 & No & No & 1977 & 257 & -- & 211 & 1.34 & 0.625 & 0.975 & 399 \\
\hline 05540030 & 1 & No & No & 1978 & 584 & -- & 480 & 0.852 & 0.646 & 0.777 & 762 \\
\hline 05540030 & 1 & No & No & 1979 & 1220 & -- & 1003 & 0.281 & 0.667 & 0.199 & 1440 \\
\hline 05540060 & 1 & Yes & Yes & 1961 & 192 & -- & 313 & 1.3 & 0.189 & 0.687 & 255 \\
\hline 05540060 & 1 & Yes & Yes & 1962 & 175 & -- & 285 & 0.289 & 0.199 & 0.745 & 234 \\
\hline 05540060 & 1 & Yes & Yes & 1963 & 131 & -- & 214 & 1.22 & 0.208 & 0.862 & 182 \\
\hline 05540060 & 1 & Yes & Yes & 1964 & 637 & -- & 1038 & 1.65 & 0.218 & 0.0505 & 726 \\
\hline 05540060 & 1 & Yes & Yes & 1965 & 159 & -- & 259 & 0.134 & 0.228 & 0.803 & 209 \\
\hline 05540060 & 1 & Yes & Yes & 1966 & 356 & -- & 580 & 1.37 & 0.237 & 0.287 & 416 \\
\hline 05540060 & 1 & Yes & Yes & 1967 & 426 & -- & 694 & 1.17 & 0.247 & 0.186 & 485 \\
\hline 05540060 & 1 & Yes & Yes & 1968 & 203 & -- & 331 & 1.49 & 0.256 & 0.704 & 249 \\
\hline 05540060 & 1 & Yes & Yes & 1969 & 150 & -- & 245 & 0.1 & 0.266 & 0.844 & 191 \\
\hline 05540060 & 1 & Yes & Yes & 1970 & 200 & -- & 326 & 1.42 & 0.275 & 0.726 & 241 \\
\hline 05540060 & 1 & Yes & Yes & 1971 & 215 & -- & 350 & 0.358 & 0.28 & 0.687 & 255 \\
\hline 05540060 & 1 & Yes & Yes & 1972 & 278 & -- & 453 & 1.58 & 0.284 & 0.507 & 320 \\
\hline 05540060 & 1 & Yes & Yes & 1973 & 288 & -- & 469 & 0.753 & 0.289 & 0.483 & 329 \\
\hline 05540060 & 1 & Yes & Yes & 1974 & 282 & -- & 460 & 1.2 & 0.294 & 0.504 & 321 \\
\hline 05540060 & 1 & Yes & Yes & 1975 & 361 & -- & 588 & 0.901 & 0.298 & 0.32 & 400 \\
\hline 05540060 & 1 & Yes & Yes & 1976 & 389 & -- & 634 & 1.1 & 0.303 & 0.271 & 425 \\
\hline 05540060 & 1 & Yes & Yes & 1977 & 194 & -- & 316 & 1.15 & 0.307 & 0.763 & 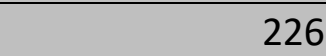 \\
\hline
\end{tabular}




\begin{tabular}{|c|c|c|c|c|c|c|c|c|c|c|c|}
\hline $\begin{array}{l}\text { U.S. } \\
\text { Geological } \\
\text { Survey } \\
\text { streamgage } \\
\text { number }\end{array}$ & $\begin{array}{l}\text { Segment } \\
\text { number }\end{array}$ & $\begin{array}{l}\text { Streamgage used in } \\
\text { regression analyses } \\
\text { (non-redundant) }\end{array}$ & $\begin{array}{c}\text { Discharge value } \\
\text { used in } \\
\text { adjustment } \\
\text { regression }\end{array}$ & $\begin{array}{l}\text { Water } \\
\text { year }\end{array}$ & $\begin{array}{c}\text { Observed annual } \\
\text { maximum peak } \\
\text { discharge }\left(\mathrm{ft}^{3} / \mathrm{s}\right)\end{array}$ & $\begin{array}{l}\text { NWIS peak } \\
\text { code }\end{array}$ & $\begin{array}{l}\text { Observed annual } \\
\text { maximum peak } \\
\text { discharge with } \\
\text { segment intercept } \\
\text { value subtracted } \\
\left(\mathrm{ft}^{3} / \mathrm{s}\right)\end{array}$ & $\begin{array}{l}\text { Observed } \\
\text { precipitation } \\
\text { (inches) }\end{array}$ & Urban fraction & $\begin{array}{l}\text { Exceedance } \\
\text { probability }\end{array}$ & $\begin{array}{c}\text { Urban-adjusted } \\
\text { annual maximum } \\
\text { peak discharge } \\
\left(\mathrm{ft}^{3} / \mathrm{s}\right)\end{array}$ \\
\hline 05540060 & 1 & Yes & Yes & 1978 & 407 & -- & 663 & 0.901 & 0.312 & 0.246 & 439 \\
\hline 05540060 & 1 & Yes & Yes & 1979 & 418 & -- & 681 & 0.878 & 0.316 & 0.232 & 448 \\
\hline 05540060 & 1 & Yes & Yes & 1980 & 267 & -- & 435 & 0.906 & 0.321 & 0.573 & 297 \\
\hline 05540060 & 1 & Yes & Yes & 1986 & 425 & -- & 693 & 0.832 & 0.331 & 0.231 & 449 \\
\hline 05540060 & 1 & Yes & Yes & 1987 & 573 & -- & 934 & 2.14 & 0.333 & 0.0953 & 599 \\
\hline 05540060 & 1 & Yes & Yes & 1988 & 181 & -- & 295 & 0.865 & 0.334 & 0.81 & 206 \\
\hline 05540060 & 1 & Yes & Yes & 1989 & 180 & -- & 293 & 2.01 & 0.336 & 0.813 & 205 \\
\hline 05540060 & 1 & Yes & Yes & 1990 & 326 & -- & 531 & 1.37 & 0.337 & 0.428 & 351 \\
\hline 05540060 & 1 & Yes & Yes & 1991 & 374 & -- & 610 & 0.95 & 0.343 & 0.328 & 396 \\
\hline 05540060 & 0 & Yes & No & 1992 & 134 & $E$ & 228 & 0.407 & 0.348 & 0.902 & 162 \\
\hline 05540060 & 0 & Yes & No & 1993 & 349 & -- & 619 & 1.56 & 0.354 & 0.324 & 398 \\
\hline 05540060 & 2 & Yes & Yes & 1994 & 369 & -- & 683 & 0.57 & 0.359 & 0.257 & 432 \\
\hline 05540060 & 2 & Yes & Yes & 1995 & 286 & -- & 530 & 0.972 & 0.365 & 0.455 & 340 \\
\hline 05540060 & 2 & Yes & Yes & 1996 & 1980 & -- & 3666 & 2.69 & 0.37 & 0 & 2190 \\
\hline 05540060 & 2 & Yes & Yes & 1997 & 579 & -- & 1072 & 2.23 & 0.376 & 0.0693 & 659 \\
\hline 05540060 & 2 & Yes & Yes & 1998 & 238 & -- & 441 & 1.88 & 0.381 & 0.616 & 281 \\
\hline 05540060 & 2 & Yes & Yes & 1999 & 241 & C & 446 & 1.4 & 0.387 & 0.612 & 283 \\
\hline 05540060 & 2 & Yes & Yes & 2000 & 225 & $C, E$ & 417 & 1.76 & 0.392 & 0.664 & 263 \\
\hline 05540060 & 2 & Yes & Yes & 2001 & 283 & C & 524 & 0.741 & 0.405 & 0.5 & 323 \\
\hline 05540060 & 2 & Yes & Yes & 2002 & 446 & C & 826 & 2.51 & 0.419 & 0.181 & 491 \\
\hline 05540060 & 2 & Yes & Yes & 2003 & 195 & C & 361 & 2.12 & 0.432 & 0.777 & 220 \\
\hline 05540060 & 2 & Yes & Yes & 2004 & 160 & C, D & 296 & 0.841 & 0.445 & 0.868 & 180 \\
\hline 05540060 & 2 & Yes & Yes & 2005 & 184 & $C, E$ & 341 & 0.741 & 0.458 & 0.821 & 201 \\
\hline 05540060 & 2 & Yes & Yes & 2006 & 149 & $\mathrm{C}, \mathrm{E}$ & 276 & 2.34 & 0.471 & 0.902 & 162 \\
\hline 05540060 & 2 & Yes & Yes & 2007 & 307 & C & 568 & 1.97 & 0.484 & 0.506 & 321 \\
\hline 05540060 & 2 & Yes & Yes & 2008 & 2100 & C & 3888 & 6.02 & 0.497 & 0 & 2140 \\
\hline 05540060 & 2 & Yes & Yes & 2009 & 448 & C & 829 & 1.54 & 0.51 & 0.225 & 454 \\
\hline 05540080 & 1 & Yes & Yes & 1961 & 158 & -- & 1043 & 1.7 & 0.741 & 0.219 & 201 \\
\hline 05540080 & 1 & Yes & Yes & 1962 & 70 & 2 & 462 & 0.303 & 0.749 & 0.87 & 101 \\
\hline 05540080 & 1 & Yes & Yes & 1963 & 103 & -- & 680 & 1.85 & 0.756 & 0.62 & 136 \\
\hline 05540080 & 1 & Yes & Yes & 1964 & 120 & -- & 792 & 3.63 & 0.764 & 0.491 & 155 \\
\hline 05540080 & 1 & Yes & Yes & 1965 & 147 & -- & 971 & 1.73 & 0.771 & 0.296 & 184 \\
\hline 05540080 & 1 & Yes & Yes & 1966 & 144 & -- & 951 & 1.97 & 0.779 & 0.325 & 180 \\
\hline 05540080 & 1 & Yes & Yes & 1967 & 166 & -- & 1096 & 1.64 & 0.786 & 0.215 & 202 \\
\hline 05540080 & 1 & Yes & Yes & 1968 & 222 & -- & 1466 & 3.49 & 0.794 & 0.0832 & 266 \\
\hline
\end{tabular}




\begin{tabular}{|c|c|c|c|c|c|c|c|c|c|c|c|}
\hline $\begin{array}{l}\text { U.S. } \\
\text { Geological } \\
\text { Survey } \\
\text { streamgage } \\
\text { number }\end{array}$ & $\begin{array}{l}\text { Segment } \\
\text { number }\end{array}$ & $\begin{array}{l}\text { Streamgage used in } \\
\text { regression analyses } \\
\text { (non-redundant) }\end{array}$ & $\begin{array}{c}\text { Discharge value } \\
\text { used in } \\
\text { adjustment } \\
\text { regression }\end{array}$ & $\begin{array}{c}\text { Water } \\
\text { year }\end{array}$ & $\begin{array}{c}\text { Observed annual } \\
\text { maximum peak } \\
\text { discharge }\left(\mathrm{ft}^{3} / \mathrm{s}\right)\end{array}$ & $\begin{array}{l}\text { NWIS peak } \\
\text { code }\end{array}$ & $\begin{array}{l}\text { Observed annual } \\
\text { maximum peak } \\
\text { discharge with } \\
\text { segment intercept } \\
\text { value subtracted } \\
\left(\mathrm{ft}^{3} / \mathrm{s}\right)\end{array}$ & $\begin{array}{l}\text { Observed } \\
\text { precipitation } \\
\text { (inches) }\end{array}$ & Urban fraction & $\begin{array}{l}\text { Exceedance } \\
\text { probability }\end{array}$ & $\begin{array}{c}\text { Urban-adjusted } \\
\text { annual maximum } \\
\text { peak discharge } \\
\left(\mathrm{ft}^{3} / \mathrm{s}\right)\end{array}$ \\
\hline 05540080 & 1 & Yes & Yes & 1969 & 177 & -- & 1169 & 1.48 & 0.801 & 0.187 & 212 \\
\hline 05540080 & 1 & Yes & Yes & 1970 & 152 & -- & 1004 & 1.27 & 0.809 & 0.299 & 183 \\
\hline 05540080 & 1 & Yes & Yes & 1971 & 147 & -- & 971 & 1.08 & 0.821 & 0.35 & 176 \\
\hline 05540080 & 1 & Yes & Yes & 1972 & 552 & -- & 3645 & 4.24 & 0.834 & 0.00366 & 617 \\
\hline 05540080 & 1 & Yes & Yes & 1973 & 152 & -- & 1004 & 1.76 & 0.846 & 0.342 & 177 \\
\hline 05540080 & 1 & Yes & Yes & 1974 & 113 & -- & 746 & 0.589 & 0.859 & 0.648 & 133 \\
\hline 05540080 & 1 & Yes & Yes & 1975 & 172 & -- & 1136 & 1.57 & 0.871 & 0.246 & 194 \\
\hline 05540080 & 1 & Yes & Yes & 1976 & 162 & -- & 1070 & 1.24 & 0.884 & 0.311 & 181 \\
\hline 05540080 & 1 & Yes & Yes & 1977 & 84 & -- & 555 & 2.29 & 0.896 & 0.889 & 97.7 \\
\hline 05540080 & 1 & Yes & Yes & 1978 & 232 & -- & 1532 & 2.01 & 0.909 & 0.106 & 251 \\
\hline 05540080 & 1 & Yes & Yes & 1979 & 178 & -- & 1175 & 1.43 & 0.921 & 0.257 & 191 \\
\hline 05540091 & 1 & No & No & 1992 & 118 & $C, E$ & 531 & 2.2 & 0.729 & 0.782 & 152 \\
\hline 05540091 & 1 & No & No & 1993 & 194 & C & 873 & 0.983 & 0.731 & 0.363 & 237 \\
\hline 05540091 & 1 & No & No & 1994 & 297 & C & 1337 & 2.7 & 0.732 & 0.096 & 352 \\
\hline 05540091 & 1 & No & No & 1995 & 213 & C & 959 & 1.58 & 0.734 & 0.276 & 256 \\
\hline 05540091 & 1 & No & No & 1996 & 393 & C & 1769 & 7.81 & 0.736 & 0.0363 & 457 \\
\hline 05540091 & 1 & No & No & 1997 & 310 & C & 1396 & 2.72 & 0.738 & 0.0831 & 365 \\
\hline 05540091 & 1 & No & No & 1998 & 179 & C & 806 & 1.12 & 0.74 & 0.451 & 219 \\
\hline 05540091 & 1 & No & No & 1999 & 203 & C & 914 & 0.892 & 0.742 & 0.327 & 244 \\
\hline 05540091 & 1 & No & No & 2000 & 282 & C & 1270 & 1.65 & 0.743 & 0.123 & 331 \\
\hline 05540091 & 1 & No & No & 2001 & 177 & C & 797 & 0.97 & 0.761 & 0.484 & 212 \\
\hline 05540091 & 1 & No & No & 2002 & 364 & C & 1639 & 3.57 & 0.779 & 0.0511 & 411 \\
\hline 05540091 & 1 & No & No & 2003 & 178 & C & 801 & 2.14 & 0.797 & 0.515 & 205 \\
\hline 05540091 & 1 & No & No & 2004 & 180 & C & 810 & 0.885 & 0.815 & 0.523 & 203 \\
\hline 05540091 & 1 & No & No & 2005 & 190 & $\mathrm{C}, \mathrm{E}$ & 855 & 1.47 & 0.832 & 0.492 & 210 \\
\hline 05540091 & 1 & No & No & 2006 & 201 & C & 905 & 2.47 & 0.85 & 0.457 & 217 \\
\hline 05540091 & 1 & No & No & 2007 & 333 & C & 1499 & 3.36 & 0.868 & 0.0988 & 349 \\
\hline 05540091 & 1 & No & No & 2008 & 391 & C & 1760 & 2.99 & 0.886 & 0.0551 & 403 \\
\hline 05540091 & 1 & No & No & 2009 & 372 & C & 1675 & 1.03 & 0.903 & 0.0716 & 378 \\
\hline 05540095 & 1 & No & No & 1969 & 612 & -- & 328 & 1.36 & 0.411 & 0.81 & 1010 \\
\hline 05540095 & 1 & No & No & 1970 & 990 & -- & 530 & 1.4 & 0.425 & 0.508 & 1470 \\
\hline 05540095 & 1 & No & No & 1971 & 641 & $E$ & 343 & 0.341 & 0.442 & 0.808 & 1010 \\
\hline 05540095 & 1 & No & No & 1972 & 1980 & -- & 1061 & 2.14 & 0.458 & 0.0911 & 2630 \\
\hline 05540095 & 1 & No & No & 1973 & 1200 & -- & 643 & 0.805 & 0.475 & 0.395 & 1660 \\
\hline 05540095 & 1 & No & No & 1974 & 1200 & -- & 643 & 0.596 & 0.491 & 0.41 & 1630 \\
\hline
\end{tabular}




\begin{tabular}{|c|c|c|c|c|c|c|c|c|c|c|c|}
\hline $\begin{array}{c}\text { U.S. } \\
\text { Geological } \\
\text { Survey } \\
\text { streamgage } \\
\text { number } \\
\end{array}$ & $\begin{array}{l}\text { Segment } \\
\text { number }\end{array}$ & $\begin{array}{l}\text { Streamgage used in } \\
\text { regression analyses } \\
\text { (non-redundant) }\end{array}$ & $\begin{array}{c}\text { Discharge value } \\
\text { used in } \\
\text { adjustment } \\
\text { regression }\end{array}$ & $\begin{array}{c}\text { Water } \\
\text { year }\end{array}$ & $\begin{array}{c}\text { Observed annual } \\
\text { maximum peak } \\
\text { discharge }\left(\mathrm{ft}^{3} / \mathrm{s}\right)\end{array}$ & $\begin{array}{c}\text { NWIS peak } \\
\text { code }\end{array}$ & $\begin{array}{c}\text { Observed annual } \\
\text { maximum peak } \\
\text { discharge with } \\
\text { segment intercept } \\
\text { value subtracted } \\
\left(\mathrm{ft}^{3} / \mathrm{s}\right)\end{array}$ & $\begin{array}{c}\text { Observed } \\
\text { precipitation } \\
\text { (inches) }\end{array}$ & Urban fraction & $\begin{array}{l}\text { Exceedance } \\
\text { probability }\end{array}$ & $\begin{array}{c}\text { Urban-adjusted } \\
\text { annual maximum } \\
\text { peak discharge } \\
\left(\mathrm{ft}^{3} / \mathrm{s}\right)\end{array}$ \\
\hline 05540095 & 1 & No & No & 1975 & 1080 & -- & 579 & 1.04 & 0.508 & 0.514 & 1460 \\
\hline 05540095 & 1 & No & No & 1976 & 1520 & -- & 814 & 1.15 & 0.525 & 0.246 & 1940 \\
\hline 05540095 & 1 & No & No & 1977 & 300 & $E$ & 161 & 0.911 & 0.541 & 0.982 & 487 \\
\hline 05540095 & 1 & No & No & 1978 & 916 & -- & 491 & 1.57 & 0.558 & 0.693 & 1200 \\
\hline 05540095 & 1 & No & No & 1979 & 1740 & $\mathrm{D}$ & 932 & 0.274 & 0.574 & 0.193 & 2100 \\
\hline 05540095 & 1 & No & No & 1980 & 761 & -- & 408 & 0.818 & 0.591 & 0.823 & 985 \\
\hline 05540095 & 1 & No & No & 1981 & 970 & -- & 520 & 1.89 & 0.596 & 0.685 & 1210 \\
\hline 05540095 & 1 & No & No & 1982 & 1210 & $E$ & 648 & 0.362 & 0.601 & 0.507 & 1470 \\
\hline 05540095 & 1 & No & No & 1983 & 2160 & -- & 1157 & 1.97 & 0.606 & 0.108 & 2520 \\
\hline 05540095 & 1 & No & No & 1984 & 1170 & $E$ & 627 & 0.683 & 0.611 & 0.545 & 1410 \\
\hline 05540095 & 1 & No & No & 1985 & 1620 & -- & 868 & 1.06 & 0.616 & 0.264 & 1890 \\
\hline 05540095 & 1 & No & No & 1986 & 1310 & $\mathrm{E}$ & 702 & 0.857 & 0.621 & 0.457 & 1560 \\
\hline 05540095 & 1 & No & No & 1987 & 3050 & -- & 1634 & 2.8 & 0.626 & 0.0351 & 3450 \\
\hline 05540095 & 1 & No & No & 1988 & 888 & $\mathrm{E}$ & 476 & 1.04 & 0.631 & 0.77 & 1080 \\
\hline 05540095 & 1 & No & No & 1989 & 750 & $E$ & 402 & 0.94 & 0.636 & 0.858 & 920 \\
\hline 05540095 & 1 & No & No & 1990 & 1520 & -- & 814 & 1.33 & 0.641 & 0.34 & 1750 \\
\hline 05540095 & 1 & No & No & 1991 & 1310 & -- & 702 & 1.59 & 0.645 & 0.48 & 1520 \\
\hline 05540095 & 1 & No & No & 1992 & 580 & $\mathrm{E}$ & 311 & 0.487 & 0.648 & 0.938 & 724 \\
\hline 05540095 & 1 & No & No & 1993 & 1010 & C & 541 & 0.755 & 0.652 & 0.707 & 1180 \\
\hline 05540095 & 1 & No & No & 1994 & 1590 & C & 852 & 3.02 & 0.655 & 0.311 & 1800 \\
\hline 05540095 & 1 & No & No & 1995 & 1360 & C & 729 & 1.5 & 0.659 & 0.461 & 1550 \\
\hline 05540095 & 1 & No & No & 1996 & 3470 & C & 1859 & 3.6 & 0.663 & 0.0246 & 3790 \\
\hline 05540095 & 1 & No & No & 1997 & 2640 & C & 1414 & 2.43 & 0.666 & 0.0633 & 2910 \\
\hline 05540095 & 1 & No & No & 1998 & 1500 & C & 804 & 1.35 & 0.67 & 0.382 & 1680 \\
\hline 05540095 & 1 & No & No & 1999 & 1630 & C & 873 & 0.787 & 0.673 & 0.304 & 1810 \\
\hline 05540095 & 1 & No & No & 2000 & 1590 & $\mathrm{C}, \mathrm{E}$ & 852 & 1.5 & 0.677 & 0.332 & 1760 \\
\hline 05540095 & 1 & No & No & 2001 & 1560 & $C$ & 836 & 0.644 & 0.687 & 0.361 & 1710 \\
\hline 05540095 & 1 & No & No & 2002 & 2130 & $C$ & 1141 & 2.13 & 0.697 & 0.153 & 2290 \\
\hline 05540095 & 1 & No & No & 2003 & 1460 & $C$ & 782 & 1.74 & 0.707 & 0.445 & 1580 \\
\hline 05540095 & 1 & No & No & 2004 & 1240 & $C, D$ & 664 & 1.04 & 0.717 & 0.6 & 1330 \\
\hline 05540095 & 1 & No & No & 2005 & 1100 & $C, E$ & 589 & 0.787 & 0.727 & 0.713 & 1170 \\
\hline 05540095 & 1 & No & No & 2006 & 1100 & $\mathrm{C}$ & 589 & 1.12 & 0.737 & 0.722 & 1160 \\
\hline 05540095 & 1 & No & No & 2007 & 1780 & $C$ & 954 & 1.33 & 0.747 & 0.29 & 1830 \\
\hline 05540095 & 1 & No & No & 2008 & 4930 & $C$ & 2641 & 4.63 & 0.757 & 0.0117 & 5000 \\
\hline 05540095 & 1 & No & No & 2009 & 2780 & C & 1489 & 1.08 & 0.767 & 0.0713 & 2800 \\
\hline
\end{tabular}




\begin{tabular}{|c|c|c|c|c|c|c|c|c|c|c|c|}
\hline $\begin{array}{l}\text { U.S. } \\
\text { Geological } \\
\text { Survey } \\
\text { streamgage } \\
\text { number }\end{array}$ & $\begin{array}{l}\text { Segment } \\
\text { number }\end{array}$ & $\begin{array}{l}\text { Streamgage used in } \\
\text { regression analyses } \\
\text { (non-redundant) }\end{array}$ & $\begin{array}{c}\text { Discharge value } \\
\text { used in } \\
\text { adjustment } \\
\text { regression }\end{array}$ & $\begin{array}{l}\text { Water } \\
\text { year }\end{array}$ & $\begin{array}{c}\text { Observed annual } \\
\text { maximum peak } \\
\text { discharge }\left(\mathrm{ft}^{3} / \mathrm{s}\right)\end{array}$ & $\begin{array}{l}\text { NWIS peak } \\
\text { code }\end{array}$ & $\begin{array}{l}\text { Observed annual } \\
\text { maximum peak } \\
\text { discharge with } \\
\text { segment intercept } \\
\text { value subtracted } \\
\left(\mathrm{ft}^{3} / \mathrm{s}\right)\end{array}$ & $\begin{array}{l}\text { Observed } \\
\text { precipitation } \\
\text { (inches) }\end{array}$ & Urban fraction & $\begin{array}{l}\text { Exceedance } \\
\text { probability }\end{array}$ & $\begin{array}{c}\text { Urban-adjusted } \\
\text { annual maximum } \\
\text { peak discharge } \\
\left(\mathrm{ft}^{3} / \mathrm{s}\right)\end{array}$ \\
\hline 05540110 & 1 & Yes & Yes & 1961 & 81 & -- & 381 & 1.35 & 0.131 & 0.508 & 121 \\
\hline 05540110 & 1 & Yes & Yes & 1962 & 71 & -- & 334 & 0.301 & 0.136 & 0.611 & 107 \\
\hline 05540110 & 1 & Yes & Yes & 1963 & 40 & -- & 188 & 1.31 & 0.141 & 0.871 & 66.7 \\
\hline 05540110 & 1 & Yes & Yes & 1964 & 214 & -- & 1006 & 1.8 & 0.146 & 0.0471 & 285 \\
\hline 05540110 & 1 & Yes & Yes & 1965 & 45 & -- & 211 & 0.135 & 0.152 & 0.838 & 72.3 \\
\hline 05540110 & 1 & Yes & Yes & 1966 & 102 & -- & 479 & 1.34 & 0.157 & 0.362 & 144 \\
\hline 05540110 & 1 & Yes & Yes & 1967 & 108 & -- & 508 & 1.35 & 0.162 & 0.327 & 151 \\
\hline 05540110 & 1 & Yes & Yes & 1968 & 60 & -- & 282 & 1.39 & 0.167 & 0.73 & 90.1 \\
\hline 05540110 & 1 & Yes & Yes & 1969 & 72 & -- & 338 & 0.587 & 0.172 & 0.628 & 104 \\
\hline 05540110 & 1 & Yes & Yes & 1970 & 66 & -- & 310 & 1.44 & 0.177 & 0.684 & 96.4 \\
\hline 05540110 & 1 & Yes & Yes & 1971 & 62 & 2 & 291 & 0.362 & 0.184 & 0.724 & 90.9 \\
\hline 05540110 & 1 & Yes & Yes & 1972 & 180 & -- & 846 & 2.18 & 0.191 & 0.0875 & 234 \\
\hline 05540110 & 1 & Yes & Yes & 1973 & 74 & -- & 348 & 1.36 & 0.198 & 0.63 & 104 \\
\hline 05540110 & 1 & Yes & Yes & 1974 & 93 & -- & 437 & 1.23 & 0.205 & 0.466 & 127 \\
\hline 05540110 & 1 & Yes & Yes & 1975 & 74 & -- & 348 & 1.02 & 0.212 & 0.64 & 103 \\
\hline 05540110 & 1 & Yes & Yes & 1976 & 134 & -- & 630 & 1.11 & 0.219 & 0.223 & 173 \\
\hline 05540110 & 1 & Yes & Yes & 1977 & 60 & -- & 282 & 1.26 & 0.226 & 0.766 & 84.5 \\
\hline 05540110 & 1 & Yes & Yes & 1978 & 187 & -- & 879 & 1.95 & 0.233 & 0.0872 & 235 \\
\hline 05540110 & 1 & Yes & Yes & 1979 & 110 & -- & 517 & 0.924 & 0.24 & 0.37 & 143 \\
\hline 05540130 & 1 & No & No & 1989 & 977 & C & 406 & 2.21 & 0.659 & 0.869 & 1200 \\
\hline 05540130 & 1 & No & No & 1990 & 2290 & C & 950 & 1.83 & 0.665 & 0.233 & 2600 \\
\hline 05540130 & 1 & No & No & 1991 & 3420 & C & 1419 & 1.41 & 0.669 & 0.063 & 3820 \\
\hline 05540130 & 1 & No & No & 1992 & 2020 & C, D & 838 & 1.53 & 0.672 & 0.343 & 2300 \\
\hline 05540130 & 1 & No & No & 1993 & 1530 & C & 635 & 1.69 & 0.675 & 0.598 & 1760 \\
\hline 05540130 & 1 & No & No & 1994 & 2320 & C & 963 & 3.02 & 0.679 & 0.233 & 2600 \\
\hline 05540130 & 1 & No & No & 1995 & 2150 & C & 892 & 1.55 & 0.682 & 0.293 & 2410 \\
\hline 05540130 & 1 & No & No & 1996 & 6620 & C & 2748 & 4.47 & 0.686 & 0.00859 & 7170 \\
\hline 05540130 & 1 & No & No & 1997 & 3260 & C & 1353 & 2.53 & 0.689 & 0.079 & 3590 \\
\hline 05540130 & 1 & No & No & 1998 & 2020 & C & 838 & 1.16 & 0.692 & 0.364 & 2250 \\
\hline 05540130 & 1 & No & No & 1999 & 1960 & C & 813 & 0.805 & 0.696 & 0.398 & 2180 \\
\hline 05540130 & 1 & No & No & 2000 & 2040 & $C, E$ & 847 & 1.47 & 0.699 & 0.361 & 2260 \\
\hline 05540130 & 1 & No & No & 2001 & 1910 & C & 793 & 0.716 & 0.709 & 0.435 & 2100 \\
\hline 05540130 & 1 & No & No & 2002 & 3090 & C & 1282 & 2.45 & 0.719 & 0.108 & 3320 \\
\hline 05540130 & 1 & No & No & 2003 & 1530 & C & 635 & 1.81 & 0.728 & 0.652 & 1660 \\
\hline 05540130 & 1 & No & No & 2004 & 1580 & $C, D$ & 656 & 1.22 & 0.738 & 0.634 & 1690 \\
\hline
\end{tabular}




\begin{tabular}{|c|c|c|c|c|c|c|c|c|c|c|c|}
\hline $\begin{array}{l}\text { U.S. } \\
\text { Geological } \\
\text { Survey } \\
\text { streamgage } \\
\text { number }\end{array}$ & $\begin{array}{l}\text { Segment } \\
\text { number }\end{array}$ & $\begin{array}{l}\text { Streamgage used in } \\
\text { regression analyses } \\
\text { (non-redundant) }\end{array}$ & $\begin{array}{c}\text { Discharge value } \\
\text { used in } \\
\text { adjustment } \\
\text { regression }\end{array}$ & $\begin{array}{l}\text { Water } \\
\text { year }\end{array}$ & $\begin{array}{c}\text { Observed annual } \\
\text { maximum peak } \\
\text { discharge }\left(\mathrm{ft}^{3} / \mathrm{s}\right)\end{array}$ & $\begin{array}{l}\text { NWIS peak } \\
\text { code }\end{array}$ & $\begin{array}{l}\text { Observed annual } \\
\text { maximum peak } \\
\text { discharge with } \\
\text { segment intercept } \\
\text { value subtracted } \\
\left(\mathrm{ft}^{3} / \mathrm{s}\right)\end{array}$ & $\begin{array}{l}\text { Observed } \\
\text { precipitation } \\
\text { (inches) }\end{array}$ & Urban fraction & $\begin{array}{l}\text { Exceedance } \\
\text { probability }\end{array}$ & $\begin{array}{c}\text { Urban-adjusted } \\
\text { annual maximum } \\
\text { peak discharge } \\
\left(\mathrm{ft}^{3} / \mathrm{s}\right)\end{array}$ \\
\hline 05540130 & 1 & No & No & 2005 & 1450 & $C, E$ & 602 & 0.919 & 0.748 & 0.717 & 1540 \\
\hline 05540130 & 1 & No & No & 2006 & 1110 & $C, E$ & 461 & 1.43 & 0.758 & 0.878 & 1180 \\
\hline 05540130 & 1 & No & No & 2007 & 1940 & C & 805 & 1.42 & 0.767 & 0.48 & 2000 \\
\hline 05540130 & 1 & No & No & 2008 & 4160 & C & 1727 & 4.21 & 0.777 & 0.0436 & 4230 \\
\hline 05540130 & 1 & No & No & 2009 & 2640 & C & 1096 & 1.05 & 0.787 & 0.216 & 2660 \\
\hline 05540140 & 1 & Yes & Yes & 1961 & 50 & -- & 560 & 1.88 & 0.312 & 0.367 & 102 \\
\hline 05540140 & 1 & Yes & Yes & 1962 & 38 & -- & 426 & 0.249 & 0.341 & 0.607 & 80.6 \\
\hline 05540140 & 1 & Yes & Yes & 1963 & 18 & -- & 202 & 1.15 & 0.37 & 0.932 & 50.9 \\
\hline 05540140 & 1 & Yes & Yes & 1964 & 14 & -- & 157 & 0.78 & 0.398 & 0.963 & 42.3 \\
\hline 05540140 & 1 & Yes & Yes & 1965 & 24 & -- & 269 & 0.0987 & 0.427 & 0.89 & 56.3 \\
\hline 05540140 & 1 & Yes & Yes & 1966 & 77 & -- & 863 & 1.37 & 0.456 & 0.177 & 126 \\
\hline 05540140 & 1 & Yes & Yes & 1967 & 68 & -- & 762 & 1.46 & 0.484 & 0.265 & 111 \\
\hline 05540140 & 1 & Yes & Yes & 1968 & 40 & -- & 448 & 1.25 & 0.513 & 0.715 & 72.3 \\
\hline 05540140 & 1 & Yes & Yes & 1969 & 40 & -- & 448 & 1.29 & 0.542 & 0.738 & 70.5 \\
\hline 05540140 & 1 & Yes & Yes & 1970 & 81 & -- & 908 & 1.28 & 0.57 & 0.204 & 119 \\
\hline 05540140 & 1 & Yes & Yes & 1971 & 28 & -- & 314 & 0.364 & 0.61 & 0.926 & 52.3 \\
\hline 05540140 & 1 & Yes & Yes & 1972 & 204 & -- & 2287 & 1.17 & 0.649 & 0.0139 & 261 \\
\hline 05540140 & 1 & Yes & Yes & 1973 & 67 & -- & 751 & 0.808 & 0.688 & 0.463 & 93 \\
\hline 05540140 & 1 & Yes & Yes & 1974 & 56 & -- & 628 & 1.05 & 0.727 & 0.661 & 76.4 \\
\hline 05540140 & 1 & Yes & Yes & 1975 & 105 & -- & 1177 & 1.02 & 0.766 & 0.168 & 128 \\
\hline 05540140 & 1 & Yes & Yes & 1976 & 81 & -- & 908 & 0.874 & 0.805 & 0.406 & 98.3 \\
\hline 05540140 & 1 & Yes & Yes & 1977 & 91 & -- & 1020 & 0.608 & 0.844 & 0.32 & 105 \\
\hline 05540140 & 1 & Yes & Yes & 1978 & 98 & -- & 1099 & 1.49 & 0.883 & 0.284 & 108 \\
\hline 05540140 & 1 & Yes & Yes & 1979 & 143 & -- & 1603 & 0.783 & 0.922 & 0.0924 & 151 \\
\hline 05540150 & 1 & No & No & 1961 & 207 & -- & 658 & 2.08 & 0.546 & 0.441 & 310 \\
\hline 05540150 & 1 & No & No & 1963 & 82 & -- & 261 & 1.34 & 0.582 & 0.948 & 148 \\
\hline 05540150 & 1 & No & No & 1964 & 64 & 2 & 204 & 0.815 & 0.6 & 0.974 & 119 \\
\hline 05540150 & 1 & No & No & 1965 & 107 & 2 & 340 & 0.113 & 0.619 & 0.909 & 171 \\
\hline 05540150 & 1 & No & No & 1966 & 468 & -- & 1488 & 1.45 & 0.637 & 0.048 & 591 \\
\hline 05540150 & 1 & No & No & 1967 & 303 & -- & 964 & 1.42 & 0.655 & 0.217 & 387 \\
\hline 05540150 & 1 & No & No & 1968 & 220 & -- & 700 & 1.87 & 0.673 & 0.511 & 289 \\
\hline 05540150 & 1 & No & No & 1969 & 227 & -- & 722 & 1.32 & 0.691 & 0.501 & 292 \\
\hline 05540150 & 1 & No & No & 1970 & 354 & -- & 1126 & 1.26 & 0.709 & 0.164 & 428 \\
\hline 05540150 & 1 & No & No & 1971 & 145 & 2 & 461 & 0.327 & 0.722 & 0.852 & 191 \\
\hline 05540150 & 1 & No & No & 1972 & 912 & -- & 2901 & 2.03 & 0.735 & 0.00809 & 1040 \\
\hline
\end{tabular}




\begin{tabular}{|c|c|c|c|c|c|c|c|c|c|c|c|}
\hline $\begin{array}{l}\text { U.S. } \\
\text { Geological } \\
\text { Survey } \\
\text { streamgage } \\
\text { number }\end{array}$ & $\begin{array}{l}\text { Segment } \\
\text { number }\end{array}$ & $\begin{array}{l}\text { Streamgage used in } \\
\text { regression analyses } \\
\text { (non-redundant) }\end{array}$ & $\begin{array}{c}\text { Discharge value } \\
\text { used in } \\
\text { adjustment } \\
\text { regression }\end{array}$ & $\begin{array}{l}\text { Water } \\
\text { year }\end{array}$ & $\begin{array}{c}\text { Observed annual } \\
\text { maximum peak } \\
\text { discharge }\left(\mathrm{ft}^{3} / \mathrm{s}\right)\end{array}$ & $\begin{array}{l}\text { NWIS peak } \\
\text { code }\end{array}$ & $\begin{array}{l}\text { Observed annual } \\
\text { maximum peak } \\
\text { discharge with } \\
\text { segment intercept } \\
\text { value subtracted } \\
\left(\mathrm{ft}^{3} / \mathrm{s}\right)\end{array}$ & $\begin{array}{l}\text { Observed } \\
\text { precipitation } \\
\text { (inches) }\end{array}$ & Urban fraction & $\begin{array}{l}\text { Exceedance } \\
\text { probability }\end{array}$ & $\begin{array}{c}\text { Urban-adjusted } \\
\text { annual maximum } \\
\text { peak discharge } \\
\left(\mathrm{ft}^{3} / \mathrm{s}\right)\end{array}$ \\
\hline 05540150 & 1 & No & No & 1973 & 278 & -- & 884 & 0.842 & 0.749 & 0.37 & 331 \\
\hline 05540150 & 1 & No & No & 1974 & 242 & -- & 770 & 0.533 & 0.762 & 0.516 & 287 \\
\hline 05540150 & 1 & No & No & 1975 & 303 & -- & 964 & 1.17 & 0.775 & 0.307 & 349 \\
\hline 05540150 & 1 & No & No & 1976 & 318 & -- & 1011 & 1.03 & 0.788 & 0.275 & 360 \\
\hline 05540150 & 1 & No & No & 1978 & 227 & -- & 722 & 1.53 & 0.815 & 0.629 & 255 \\
\hline 05540150 & 1 & No & No & 1979 & 360 & -- & 1145 & 0.978 & 0.828 & 0.213 & 390 \\
\hline 05540150 & 1 & No & No & 1980 & 218 & -- & 693 & 0.333 & 0.842 & 0.701 & 239 \\
\hline 05540160 & 1 & Yes & No & 1955 & 1590 & 7 & 2526 & 3.91 & 0.48 & 0.00675 & 2190 \\
\hline 05540160 & 1 & Yes & Yes & 1961 & 652 & -- & 1036 & 2.2 & 0.614 & 0.162 & 876 \\
\hline 05540160 & 1 & Yes & Yes & 1963 & 299 & -- & 475 & 1.38 & 0.648 & 0.784 & 438 \\
\hline 05540160 & 1 & Yes & Yes & 1964 & 227 & -- & 361 & 0.838 & 0.665 & 0.913 & 351 \\
\hline 05540160 & 1 & Yes & Yes & 1965 & 265 & -- & 421 & 1.2 & 0.681 & 0.867 & 385 \\
\hline 05540160 & 1 & Yes & Yes & 1966 & 993 & -- & 1578 & 1.58 & 0.698 & 0.047 & 1210 \\
\hline 05540160 & 1 & Yes & Yes & 1967 & 675 & -- & 1072 & 1.42 & 0.715 & 0.189 & 828 \\
\hline 05540160 & 1 & Yes & Yes & 1968 & 728 & -- & 1157 & 2.18 & 0.732 & 0.162 & 877 \\
\hline 05540160 & 1 & Yes & Yes & 1969 & 420 & -- & 667 & 1.34 & 0.749 & 0.63 & 523 \\
\hline 05540160 & 1 & Yes & Yes & 1970 & 725 & -- & 1152 & 1.26 & 0.765 & 0.178 & 848 \\
\hline 05540160 & 1 & Yes & Yes & 1971 & 321 & -- & 510 & 0.313 & 0.774 & 0.839 & 403 \\
\hline 05540160 & 1 & Yes & Yes & 1972 & 1720 & -- & 2732 & 2.47 & 0.783 & 0.0112 & 1920 \\
\hline 05540160 & 1 & Yes & Yes & 1973 & 786 & -- & 1249 & 0.859 & 0.791 & 0.152 & 897 \\
\hline 05540160 & 1 & Yes & Yes & 1974 & 530 & 2 & 842 & 0.545 & 0.8 & 0.472 & 616 \\
\hline 05540160 & 1 & Yes & Yes & 1975 & 661 & -- & 1050 & 1.24 & 0.808 & 0.26 & 747 \\
\hline 05540160 & 1 & Yes & Yes & 1976 & 575 & -- & 913 & 1.07 & 0.817 & 0.412 & 653 \\
\hline 05540160 & 1 & Yes & Yes & 1990 & 881 & C & 1400 & 1.38 & 0.872 & 0.133 & 933 \\
\hline 05540160 & 1 & Yes & Yes & 1991 & 747 & C & 1187 & 1.77 & 0.873 & 0.217 & 792 \\
\hline 05540160 & 1 & Yes & Yes & 1992 & 393 & C, D & 624 & 0.739 & 0.874 & 0.803 & 427 \\
\hline 05540160 & 1 & Yes & Yes & 1993 & 464 & C & 737 & 0.884 & 0.874 & 0.68 & 500 \\
\hline 05540160 & 1 & Yes & Yes & 1994 & 617 & C & 980 & 1.87 & 0.875 & 0.405 & 658 \\
\hline 05540160 & 1 & Yes & Yes & 1995 & 538 & C & 855 & 1.24 & 0.876 & 0.538 & 575 \\
\hline 05540160 & 1 & Yes & Yes & 1996 & 936 & C & 1487 & 3.9 & 0.876 & 0.106 & 987 \\
\hline 05540160 & 1 & Yes & Yes & 1997 & 925 & C & 1469 & 2.43 & 0.877 & 0.112 & 975 \\
\hline 05540160 & 1 & Yes & Yes & 1998 & 547 & C & 869 & 1.39 & 0.878 & 0.525 & 583 \\
\hline 05540160 & 1 & Yes & Yes & 1999 & 517 & C & 821 & 0.653 & 0.878 & 0.577 & 552 \\
\hline 05540160 & 1 & Yes & Yes & 2000 & 535 & C & 850 & 1.32 & 0.879 & 0.547 & 570 \\
\hline 05540160 & 1 & Yes & Yes & 2001 & 460 & C & 731 & 0.714 & 0.885 & 0.702 & 489 \\
\hline
\end{tabular}




\begin{tabular}{|c|c|c|c|c|c|c|c|c|c|c|c|}
\hline $\begin{array}{c}\text { U.S. } \\
\text { Geological } \\
\text { Survey } \\
\text { streamgage } \\
\text { number } \\
\end{array}$ & $\begin{array}{c}\text { Segment } \\
\text { number }\end{array}$ & $\begin{array}{l}\text { Streamgage used in } \\
\text { regression analyses } \\
\text { (non-redundant) }\end{array}$ & $\begin{array}{c}\text { Discharge value } \\
\text { used in } \\
\text { adjustment } \\
\text { regression }\end{array}$ & $\begin{array}{c}\text { Water } \\
\text { year }\end{array}$ & $\begin{array}{c}\text { Observed annual } \\
\text { maximum peak } \\
\text { discharge }\left(\mathrm{ft}^{3} / \mathrm{s}\right)\end{array}$ & $\begin{array}{c}\text { NWIS peak } \\
\text { code }\end{array}$ & $\begin{array}{c}\text { Observed annual } \\
\text { maximum peak } \\
\text { discharge with } \\
\text { segment intercept } \\
\text { value subtracted } \\
\left(\mathrm{ft}^{3} / \mathrm{s}\right) \\
\end{array}$ & $\begin{array}{c}\text { Observed } \\
\text { precipitation } \\
\text { (inches) }\end{array}$ & Urban fraction & $\begin{array}{l}\text { Exceedance } \\
\text { probability }\end{array}$ & $\begin{array}{c}\text { Urban-adjusted } \\
\text { annual maximum } \\
\text { peak discharge } \\
\left(\mathrm{ft}^{3} / \mathrm{s}\right) \\
\end{array}$ \\
\hline 05540160 & 1 & Yes & Yes & 2002 & 1040 & C & 1652 & 1.97 & 0.89 & 0.0722 & 1080 \\
\hline 05540160 & 1 & Yes & Yes & 2003 & 538 & C & 855 & 1.85 & 0.896 & 0.559 & 562 \\
\hline 05540160 & 1 & Yes & Yes & 2004 & 372 & C & 591 & 1.46 & 0.901 & 0.858 & 390 \\
\hline 05540160 & 1 & Yes & Yes & 2005 & 517 & $\mathrm{C}, \mathrm{E}$ & 821 & 1.42 & 0.907 & 0.608 & 534 \\
\hline 05540160 & 1 & Yes & Yes & 2006 & 386 & C & 613 & 2.26 & 0.912 & 0.846 & 398 \\
\hline 05540160 & 1 & Yes & Yes & 2007 & 840 & C & 1334 & 3.12 & 0.918 & 0.177 & 853 \\
\hline 05540160 & 1 & Yes & Yes & 2008 & 1190 & c & 1890 & 3.34 & 0.924 & 0.0473 & 1200 \\
\hline 05540160 & 1 & Yes & Yes & 2009 & 1040 & C & 1652 & 1.11 & 0.929 & 0.0835 & 1050 \\
\hline 05540190 & 1 & Yes & Yes & 1961 & 583 & -- & 1724 & 2 & 0.891 & 0.0614 & 624 \\
\hline 05540190 & 1 & Yes & Yes & 1962 & 139 & -- & 411 & 0.288 & 0.895 & 0.96 & 159 \\
\hline 05540190 & 1 & Yes & Yes & 1963 & 542 & -- & 1603 & 1.45 & 0.899 & 0.0846 & 577 \\
\hline 05540190 & 1 & Yes & Yes & 1964 & 123 & -- & 364 & 0.886 & 0.903 & 0.975 & 140 \\
\hline 05540190 & 1 & Yes & Yes & 1965 & 253 & -- & 748 & 0.843 & 0.907 & 0.705 & 273 \\
\hline 05540190 & 1 & Yes & Yes & 1966 & 497 & -- & 1470 & 1.78 & 0.911 & 0.127 & 524 \\
\hline 05540190 & 1 & Yes & Yes & 1967 & 314 & -- & 929 & 1.37 & 0.915 & 0.503 & 333 \\
\hline 05540190 & 1 & Yes & Yes & 1968 & 569 & -- & 1683 & 2.06 & 0.919 & 0.0739 & 594 \\
\hline 05540190 & 1 & Yes & Yes & 1969 & 247 & -- & 731 & 1.27 & 0.923 & 0.737 & 262 \\
\hline 05540190 & 1 & Yes & Yes & 1970 & 330 & -- & 976 & 1.22 & 0.927 & 0.467 & 345 \\
\hline 05540190 & 1 & Yes & Yes & 1971 & 267 & -- & 790 & 0.868 & 0.928 & 0.677 & 280 \\
\hline 05540190 & 1 & Yes & Yes & 1972 & 642 & -- & 1899 & 2.15 & 0.929 & 0.0473 & 664 \\
\hline 05540190 & 1 & Yes & Yes & 1973 & 448 & -- & 1325 & 0.856 & 0.93 & 0.186 & 464 \\
\hline 05540190 & 1 & Yes & Yes & 1974 & 344 & -- & 1017 & 0.983 & 0.931 & 0.429 & 358 \\
\hline 05540190 & 1 & Yes & Yes & 1975 & 620 & -- & 1834 & 1.32 & 0.932 & 0.0544 & 640 \\
\hline 05540190 & 1 & Yes & Yes & 1976 & 216 & -- & 639 & 0.964 & 0.933 & 0.838 & 227 \\
\hline 05540190 & 1 & Yes & Yes & 1977 & 213 & -- & 630 & 1.12 & 0.934 & 0.848 & 224 \\
\hline 05540195 & 1 & No & No & 1989 & 464 & C & 930 & 2.22 & 0.92 & 0.506 & 486 \\
\hline 05540195 & 1 & No & No & 1990 & 938 & C & 1881 & 1.75 & 0.92 & 0.0476 & 970 \\
\hline 05540195 & 1 & No & No & 1991 & 715 & C & 1434 & 1.71 & 0.92 & 0.143 & 742 \\
\hline 05540195 & 1 & No & No & 1992 & 560 & C, D & 1123 & 1.34 & 0.92 & 0.297 & 583 \\
\hline 05540195 & 1 & No & No & 1993 & 390 & C & 782 & 1.09 & 0.92 & 0.677 & 410 \\
\hline 05540195 & 1 & No & No & 1994 & 542 & c & 1087 & 1.57 & 0.92 & 0.337 & 565 \\
\hline 05540195 & 1 & No & No & 1995 & 322 & $\mathrm{C}, \mathrm{E}$ & 646 & 1.22 & 0.92 & 0.821 & 340 \\
\hline 05540195 & 1 & No & No & 1996 & 1280 & C & 2566 & 5.12 & 0.92 & 0.0175 & 1320 \\
\hline 05540195 & 1 & No & No & 1997 & 898 & C & 1800 & 2.55 & 0.92 & 0.0567 & 929 \\
\hline 05540195 & 1 & No & No & 1998 & 641 & C & 1285 & 1.83 & 0.92 & 0.196 & 665 \\
\hline
\end{tabular}




\begin{tabular}{|c|c|c|c|c|c|c|c|c|c|c|c|}
\hline $\begin{array}{l}\text { U.S. } \\
\text { Geological } \\
\text { Survey } \\
\text { streamgage } \\
\text { number }\end{array}$ & $\begin{array}{l}\text { Segment } \\
\text { number }\end{array}$ & $\begin{array}{l}\text { Streamgage used in } \\
\text { regression analyses } \\
\text { (non-redundant) }\end{array}$ & $\begin{array}{c}\text { Discharge value } \\
\text { used in } \\
\text { adjustment } \\
\text { regression }\end{array}$ & $\begin{array}{l}\text { Water } \\
\text { year }\end{array}$ & $\begin{array}{l}\text { Observed annual } \\
\text { maximum peak } \\
\text { discharge }\left(\mathrm{ft}^{3} / \mathrm{s}\right)\end{array}$ & $\begin{array}{l}\text { NWIS peak } \\
\text { code }\end{array}$ & $\begin{array}{l}\text { Observed annual } \\
\text { maximum peak } \\
\text { discharge with } \\
\text { segment intercept } \\
\text { value subtracted } \\
\left(\mathrm{ft}^{3} / \mathrm{s}\right)\end{array}$ & $\begin{array}{l}\text { Observed } \\
\text { precipitation } \\
\text { (inches) }\end{array}$ & Urban fraction & $\begin{array}{l}\text { Exceedance } \\
\text { probability }\end{array}$ & $\begin{array}{c}\text { Urban-adjusted } \\
\text { annual maximum } \\
\text { peak discharge } \\
\left(\mathrm{ft}^{3} / \mathrm{s}\right)\end{array}$ \\
\hline 05540195 & 1 & No & No & 1999 & 491 & C & 984 & 1.49 & 0.92 & 0.451 & 513 \\
\hline 05540195 & 1 & No & No & 2000 & 474 & C & 950 & 1.31 & 0.92 & 0.486 & 495 \\
\hline 05540195 & 1 & No & No & 2001 & 429 & C & 860 & 0.756 & 0.924 & 0.583 & 447 \\
\hline 05540195 & 1 & No & No & 2002 & 796 & C & 1596 & 2.02 & 0.928 & 0.0961 & 819 \\
\hline 05540195 & 1 & No & No & 2003 & 404 & C & 810 & 1.12 & 0.932 & 0.655 & 418 \\
\hline 05540195 & 1 & No & No & 2004 & 533 & C & 1069 & 1.52 & 0.936 & 0.381 & 547 \\
\hline 05540195 & 1 & No & No & 2005 & 467 & $C, E$ & 936 & 1.58 & 0.94 & 0.522 & 478 \\
\hline 05540195 & 1 & No & No & 2006 & 346 & C & 694 & 2.26 & 0.944 & 0.796 & 353 \\
\hline 05540195 & 1 & No & No & 2007 & 975 & C & 1955 & 3.27 & 0.948 & 0.0456 & 985 \\
\hline 05540195 & 1 & No & No & 2008 & 764 & C & 1532 & 2.87 & 0.952 & 0.125 & 770 \\
\hline 05540195 & 1 & No & No & 2009 & 886 & C & 1776 & 2.34 & 0.956 & 0.0681 & 889 \\
\hline 05540240 & 1 & Yes & Yes & 1961 & 532 & -- & 2513 & 1.93 & 0.557 & 0.00841 & 719 \\
\hline 05540240 & 1 & Yes & Yes & 1962 & 152 & -- & 718 & 0.292 & 0.584 & 0.402 & 233 \\
\hline 05540240 & 1 & Yes & Yes & 1963 & 441 & -- & 2084 & 1.42 & 0.612 & 0.0162 & 581 \\
\hline 05540240 & 1 & Yes & Yes & 1964 & 92 & -- & 435 & 3.18 & 0.639 & 0.824 & 148 \\
\hline 05540240 & 1 & Yes & Yes & 1965 & 351 & -- & 1658 & 1.46 & 0.666 & 0.0373 & 454 \\
\hline 05540240 & 1 & Yes & Yes & 1966 & 163 & -- & 770 & 3.03 & 0.694 & 0.446 & 223 \\
\hline 05540240 & 1 & Yes & Yes & 1967 & 177 & -- & 836 & 1.54 & 0.721 & 0.397 & 234 \\
\hline 05540240 & 1 & Yes & Yes & 1968 & 238 & -- & 1124 & 3.15 & 0.748 & 0.182 & 295 \\
\hline 05540240 & 1 & Yes & Yes & 1969 & 189 & -- & 893 & 1.17 & 0.776 & 0.39 & 235 \\
\hline 05540240 & 1 & Yes & Yes & 1970 & 128 & -- & 605 & 1.17 & 0.803 & 0.761 & 161 \\
\hline 05540240 & 1 & Yes & Yes & 1971 & 157 & -- & 742 & 0.209 & 0.816 & 0.604 & 190 \\
\hline 05540240 & 1 & Yes & Yes & 1972 & 465 & -- & 2197 & 3.09 & 0.829 & 0.0214 & 523 \\
\hline 05540240 & 1 & Yes & Yes & 1973 & 165 & -- & 780 & 0.912 & 0.841 & 0.586 & 194 \\
\hline 05540240 & 1 & Yes & Yes & 1974 & 204 & -- & 964 & 1.16 & 0.854 & 0.4 & 234 \\
\hline 05540240 & 1 & Yes & Yes & 1975 & 423 & -- & 1998 & 2 & 0.867 & 0.0344 & 464 \\
\hline 05540240 & 1 & Yes & Yes & 1976 & 112 & -- & 529 & 0.776 & 0.88 & 0.9 & 131 \\
\hline 05540240 & 1 & Yes & Yes & 1977 & 120 & -- & 567 & 1.53 & 0.893 & 0.875 & 137 \\
\hline 05540240 & 1 & Yes & Yes & 1978 & 149 & -- & 704 & 1.34 & 0.906 & 0.748 & 164 \\
\hline 05540240 & 1 & Yes & Yes & 1979 & 117 & -- & 553 & 1.2 & 0.919 & 0.907 & 129 \\
\hline 05540240 & 1 & Yes & Yes & 1980 & 112 & -- & 529 & 1.78 & 0.932 & 0.93 & 122 \\
\hline 05540250 & 0 & No & No & 1989 & 497 & C & NA & 1.63 & 0.789 & NA & NA \\
\hline 05540250 & 0 & No & No & 1990 & 1990 & C & NA & 2.11 & 0.792 & NA & NA \\
\hline 05540250 & 0 & No & No & 1991 & 1070 & C & NA & 1.56 & 0.793 & NA & NA \\
\hline 05540250 & 1 & No & No & 1992 & 615 & C, D & 518 & 1.6 & 0.793 & 0.845 & 689 \\
\hline
\end{tabular}




\begin{tabular}{|c|c|c|c|c|c|c|c|c|c|c|c|}
\hline $\begin{array}{c}\text { U.S. } \\
\text { Geological } \\
\text { Survey } \\
\text { streamgage } \\
\text { number }\end{array}$ & $\begin{array}{l}\text { Segment } \\
\text { number }\end{array}$ & $\begin{array}{l}\text { Streamgage used in } \\
\text { regression analyses } \\
\text { (non-redundant) }\end{array}$ & $\begin{array}{c}\text { Discharge value } \\
\text { used in } \\
\text { adjustment } \\
\text { regression }\end{array}$ & $\begin{array}{c}\text { Water } \\
\text { year }\end{array}$ & $\begin{array}{c}\text { Observed annual } \\
\text { maximum peak } \\
\text { discharge }\left(\mathrm{ft}^{3} / \mathrm{s}\right)\end{array}$ & $\begin{array}{c}\text { NWIS peak } \\
\text { code }\end{array}$ & $\begin{array}{l}\text { Observed annual } \\
\text { maximum peak } \\
\text { discharge with } \\
\text { segment intercept } \\
\text { value subtracted } \\
\left(\mathrm{ft}^{3} / \mathrm{s}\right)\end{array}$ & $\begin{array}{c}\text { Observed } \\
\text { precipitation } \\
\text { (inches) }\end{array}$ & Urban fraction & $\begin{array}{l}\text { Exceedance } \\
\text { probability }\end{array}$ & $\begin{array}{c}\text { Urban-adjusted } \\
\text { annual maximum } \\
\text { peak discharge } \\
\left(\mathrm{ft}^{3} / \mathrm{s}\right)\end{array}$ \\
\hline 05540250 & 1 & No & No & 1993 & 1100 & $\mathrm{C}$ & 927 & 1.82 & 0.794 & 0.371 & 1200 \\
\hline 05540250 & 1 & No & No & 1994 & 945 & $\mathrm{C}$ & 796 & 0.804 & 0.795 & 0.52 & 1030 \\
\hline 05540250 & 1 & No & No & 1995 & 858 & $C, D$ & 723 & 1.28 & 0.796 & 0.607 & $93 \varepsilon$ \\
\hline 05540250 & 1 & No & No & 1996 & 3980 & C & 3353 & 6.01 & 0.797 & 0.00508 & 4190 \\
\hline 05540250 & 1 & No & No & 1997 & 1910 & $\mathrm{C}$ & 1609 & 2.62 & 0.798 & 0.0593 & $204 C$ \\
\hline 05540250 & 1 & No & No & 1998 & 1090 & $\mathrm{C}$ & 918 & 1.28 & 0.799 & 0.387 & 1180 \\
\hline 05540250 & 1 & No & No & 1999 & 1200 & C & 1011 & 0.762 & 0.8 & 0.286 & 1290 \\
\hline 05540250 & 1 & No & No & 2000 & 938 & C & 790 & 1.29 & 0.801 & 0.532 & $102 C$ \\
\hline 05540250 & 1 & No & No & 2001 & 1070 & C & 901 & 0.82 & 0.808 & 0.416 & 1150 \\
\hline 05540250 & 1 & No & No & 2002 & 1680 & C & 1415 & 2.59 & 0.816 & 0.103 & 1770 \\
\hline 05540250 & 1 & No & No & 2003 & 668 & C & 563 & 1.11 & 0.823 & 0.822 & 716 \\
\hline 05540250 & 1 & No & No & 2004 & 989 & C & 833 & 1.26 & 0.83 & 0.514 & $104 C$ \\
\hline 05540250 & 1 & No & No & 2005 & 1380 & C & 1162 & 1.45 & 0.837 & 0.208 & 1430 \\
\hline 05540250 & 1 & No & No & 2006 & 573 & C & 483 & 2.36 & 0.844 & 0.913 & 600 \\
\hline 05540250 & 1 & No & No & 2007 & 1460 & C & 1230 & 3.08 & 0.851 & 0.186 & 1490 \\
\hline 05540250 & 1 & No & No & 2008 & 2410 & C & 2030 & 3.02 & 0.859 & 0.0316 & 2440 \\
\hline 05540250 & 1 & No & No & 2009 & 2140 & C & 1803 & 1.11 & 0.866 & 0.0474 & 2150 \\
\hline 05540275 & 1 & Yes & Yes & 1988 & 78 & -- & 263 & 1.11 & 0.481 & 0.918 & 115 \\
\hline 05540275 & 1 & Yes & Yes & 1989 & 80 & -- & 270 & 1.58 & 0.507 & 0.922 & 113 \\
\hline 05540275 & 1 & Yes & Yes & 1990 & 694 & D & 2343 & 1.81 & 0.534 & 0.00986 & 793 \\
\hline 05540275 & 1 & Yes & Yes & 1991 & 267 & -- & 901 & 1.48 & 0.545 & 0.195 & 314 \\
\hline 05540275 & 1 & Yes & Yes & 1992 & 134 & $E$ & 452 & 1.78 & 0.557 & 0.744 & 165 \\
\hline 05540275 & 1 & Yes & Yes & 1993 & 153 & -- & 516 & 1.72 & 0.568 & 0.663 & 18 \\
\hline 05540275 & 1 & Yes & Yes & 1994 & 147 & $\mathrm{E}$ & 496 & 0.712 & 0.579 & 0.704 & 175 \\
\hline 05540275 & 1 & Yes & Yes & 1995 & 107 & -- & 361 & 1.27 & 0.59 & 0.876 & 130 \\
\hline 05540275 & 1 & Yes & Yes & 1996 & 1750 & -- & 5907 & 3.67 & 0.602 & 0 & 1890 \\
\hline 05540275 & 1 & Yes & Yes & 1997 & 465 & -- & 1570 & 2.52 & 0.613 & 0.0388 & 507 \\
\hline 05540275 & 1 & Yes & Yes & 1998 & 391 & -- & 1320 & 1.25 & 0.624 & 0.0707 & 425 \\
\hline 05540275 & 1 & Yes & Yes & 1999 & 218 & -- & 736 & 0.599 & 0.635 & 0.429 & 235 \\
\hline 05540275 & 1 & Yes & Yes & 2000 & 178 & C & 601 & 1.49 & 0.647 & 0.616 & 192 \\
\hline 05540275 & 1 & Yes & Yes & 2001 & 208 & C & 702 & 0.694 & 0.654 & 0.489 & 224 \\
\hline 05540275 & 1 & Yes & Yes & 2002 & 538 & C & 1816 & 2.2 & 0.661 & 0.0267 & 563 \\
\hline 05540275 & 1 & Yes & Yes & 2003 & 129 & $\mathrm{C}$ & 435 & 0.902 & 0.668 & 0.843 & 135 \\
\hline 05540275 & 1 & Yes & Yes & 2004 & 164 & $\mathrm{C}$ & 554 & 1.74 & 0.675 & 0.712 & 173 \\
\hline 05540275 & 1 & Yes & Yes & 2005 & 195 & C & 658 & 1.19 & 0.683 & 0.574 & 203 \\
\hline
\end{tabular}




\begin{tabular}{|c|c|c|c|c|c|c|c|c|c|c|c|}
\hline $\begin{array}{c}\text { U.S. } \\
\text { Geological } \\
\text { Survey } \\
\text { streamgage } \\
\text { number }\end{array}$ & $\begin{array}{l}\text { Segment } \\
\text { number }\end{array}$ & $\begin{array}{l}\text { Streamgage used in } \\
\text { regression analyses } \\
\text { (non-redundant) }\end{array}$ & $\begin{array}{c}\text { Discharge value } \\
\text { used in } \\
\text { adjustment } \\
\text { regression }\end{array}$ & $\begin{array}{l}\text { Water } \\
\text { year }\end{array}$ & $\begin{array}{c}\text { Observed annual } \\
\text { maximum peak } \\
\text { discharge }\left(\mathrm{ft}^{3} / \mathrm{s}\right)\end{array}$ & $\begin{array}{l}\text { NWIS peak } \\
\text { code }\end{array}$ & $\begin{array}{c}\text { Observed annual } \\
\text { maximum peak } \\
\text { discharge with } \\
\text { segment intercept } \\
\text { value subtracted } \\
\left(\mathrm{ft}^{3} / \mathrm{s}\right)\end{array}$ & $\begin{array}{c}\text { Observed } \\
\text { precipitation } \\
\text { (inches) }\end{array}$ & Urban fraction & $\begin{array}{l}\text { Exceedance } \\
\text { probability }\end{array}$ & $\begin{array}{c}\text { Urban-adjusted } \\
\text { annual maximum } \\
\text { peak discharge } \\
\left(\mathrm{ft}^{3} / \mathrm{s}\right)\end{array}$ \\
\hline 05540275 & 1 & Yes & Yes & 2006 & 66 & $C, E$ & 223 & 1.62 & 0.69 & 0.981 & 70 \\
\hline 05540275 & 1 & Yes & Yes & 2007 & 188 & $C$ & 635 & 2.33 & 0.697 & 0.62 & 193 \\
\hline 05540275 & 1 & Yes & Yes & 2008 & 422 & $C$ & 1425 & 2.74 & 0.704 & 0.0691 & 427 \\
\hline 05540275 & 1 & Yes & Yes & 2009 & 473 & $C$ & 1597 & 1.01 & 0.711 & 0.0468 & 476 \\
\hline 05540500 & 1 & Yes & Yes & 1941 & 1730 & -- & 230 & 0.553 & 0.105 & 0.786 & 3280 \\
\hline 05540500 & 1 & Yes & Yes & 1942 & 4360 & -- & 578 & 1.13 & 0.109 & 0.211 & 6350 \\
\hline 05540500 & 1 & Yes & Yes & 1943 & 2640 & -- & 350 & 0.75 & 0.112 & 0.556 & 4380 \\
\hline 05540500 & 1 & Yes & Yes & 1944 & 2220 & -- & 295 & 1.46 & 0.116 & 0.67 & 3840 \\
\hline 05540500 & 1 & Yes & Yes & 1945 & 1820 & -- & 241 & 1.27 & 0.119 & 0.772 & 3360 \\
\hline 05540500 & 1 & Yes & Yes & 1946 & 3700 & -- & 491 & 0.394 & 0.122 & 0.323 & 5610 \\
\hline 05540500 & 1 & Yes & Yes & 1947 & 5200 & -- & 690 & 3.09 & 0.126 & 0.141 & 7330 \\
\hline 05540500 & 1 & Yes & Yes & 1948 & 11000 & -- & 1459 & 2.36 & 0.129 & 0.0154 & 13900 \\
\hline 05540500 & 1 & Yes & Yes & 1949 & 4500 & 2 & 597 & 0.761 & 0.133 & 0.206 & 6400 \\
\hline 05540500 & 1 & Yes & Yes & 1950 & 9070 & -- & 1203 & 1.29 & 0.136 & 0.0257 & 11700 \\
\hline 05540500 & 1 & Yes & Yes & 1951 & 4230 & D & 561 & 0.333 & 0.148 & 0.252 & 6040 \\
\hline 05540500 & 1 & Yes & Yes & 1952 & 2390 & -- & 317 & 1.31 & 0.159 & 0.657 & 3900 \\
\hline 05540500 & 1 & Yes & Yes & 1953 & 5100 & $\mathrm{E}$ & 677 & 1.61 & 0.171 & 0.167 & 6930 \\
\hline 05540500 & 1 & Yes & Yes & 1954 & 3830 & -- & 508 & 1.91 & 0.182 & 0.34 & 5500 \\
\hline 05540500 & 1 & Yes & Yes & 1955 & 12000 & -- & 1592 & 4.07 & 0.194 & 0.0141 & 14400 \\
\hline 05540500 & 1 & Yes & Yes & 1956 & 1880 & -- & 249 & 1.02 & 0.205 & 0.806 & 3170 \\
\hline 05540500 & 1 & Yes & Yes & 1957 & 6040 & -- & 801 & 3.18 & 0.217 & 0.113 & 7800 \\
\hline 05540500 & 1 & Yes & Yes & 1958 & 2880 & -- & 382 & 1.73 & 0.228 & 0.592 & 4220 \\
\hline 05540500 & 1 & Yes & Yes & 1959 & 4360 & -- & 578 & 1.37 & 0.24 & 0.291 & 5780 \\
\hline 05540500 & 1 & Yes & Yes & 1960 & 3460 & -- & 459 & 2.15 & 0.251 & 0.468 & 4810 \\
\hline 05540500 & 1 & Yes & Yes & 1961 & 4920 & -- & 653 & 1.47 & 0.264 & 0.228 & 6220 \\
\hline 05540500 & 1 & Yes & Yes & 1962 & 3960 & -- & 525 & 0.31 & 0.277 & 0.387 & 5240 \\
\hline 05540500 & 1 & Yes & Yes & 1963 & 1300 & $E$ & 172 & 1.47 & 0.29 & 0.934 & 2300 \\
\hline 05540500 & 1 & Yes & Yes & 1964 & 3330 & $E$ & 442 & 2.3 & 0.303 & 0.544 & 4430 \\
\hline 05540500 & 1 & Yes & Yes & 1965 & 1750 & 2 & 232 & 0.226 & 0.315 & 0.884 & 2720 \\
\hline 05540500 & 1 & Yes & Yes & 1966 & 6990 & -- & 927 & 1.78 & 0.328 & 0.0962 & 8130 \\
\hline 05540500 & 1 & Yes & Yes & 1967 & 3160 & -- & 419 & 1.36 & 0.341 & 0.618 & 4090 \\
\hline 05540500 & 1 & Yes & Yes & 1968 & 1760 & -- & 234 & 2.2 & 0.354 & 0.898 & 2620 \\
\hline 05540500 & 1 & Yes & Yes & 1969 & 1890 & -- & 251 & 0.975 & 0.367 & 0.884 & 2720 \\
\hline 05540500 & 1 & Yes & Yes & 1970 & 2800 & -- & 372 & 1.4 & 0.38 & 0.727 & 3590 \\
\hline 05540500 & 1 & Yes & Yes & 1971 & 1940 & -- & 257 & 0.298 & 0.393 & 0.888 & 2690 \\
\hline
\end{tabular}




\begin{tabular}{|c|c|c|c|c|c|c|c|c|c|c|c|}
\hline $\begin{array}{c}\text { U.S. } \\
\text { Geological } \\
\text { Survey } \\
\text { streamgage } \\
\text { number }\end{array}$ & $\begin{array}{l}\text { Segment } \\
\text { number }\end{array}$ & $\begin{array}{l}\text { Streamgage used in } \\
\text { regression analyses } \\
\text { (non-redundant) }\end{array}$ & $\begin{array}{l}\text { Discharge value } \\
\text { used in } \\
\text { adjustment } \\
\text { regression }\end{array}$ & $\begin{array}{l}\text { Water } \\
\text { year }\end{array}$ & $\begin{array}{l}\text { Observed annual } \\
\text { maximum peak } \\
\text { discharge }\left(\mathrm{ft}^{3} / \mathrm{s}\right)\end{array}$ & $\begin{array}{l}\text { NWIS peak } \\
\text { code }\end{array}$ & $\begin{array}{l}\text { Observed annual } \\
\text { maximum peak } \\
\text { discharge with } \\
\text { segment intercept } \\
\text { value subtracted } \\
\left(\mathrm{ft}^{3} / \mathrm{s}\right)\end{array}$ & $\begin{array}{l}\text { Observed } \\
\text { precipitation } \\
\text { (inches) }\end{array}$ & Urban fraction & $\begin{array}{l}\text { Exceedance } \\
\text { probability }\end{array}$ & $\begin{array}{c}\text { Urban-adjusted } \\
\text { annual maximum } \\
\text { peak discharge } \\
\left(\mathrm{ft}^{3} / \mathrm{s}\right)\end{array}$ \\
\hline 05540500 & 0 & Yes & No & 1972 & 6520 & -- & 977 & 2.52 & 0.407 & 0.103 & 7990 \\
\hline 05540500 & 2 & Yes & Yes & 1973 & 3110 & -- & 526 & 0.79 & 0.421 & 0.511 & 4590 \\
\hline 05540500 & 2 & Yes & Yes & 1974 & 5440 & -- & 920 & 1.08 & 0.435 & 0.14 & 7370 \\
\hline 05540500 & 2 & Yes & Yes & 1975 & 3840 & -- & 650 & 1.26 & 0.449 & 0.364 & 5370 \\
\hline 05540500 & 2 & Yes & Yes & 1976 & 4200 & -- & 711 & 1.03 & 0.463 & 0.301 & 5700 \\
\hline 05540500 & 2 & Yes & Yes & 1977 & 1080 & $\mathrm{E}$ & 183 & 1.53 & 0.477 & 0.963 & 1870 \\
\hline 05540500 & 2 & Yes & Yes & 1978 & 3450 & -- & 584 & 1.07 & 0.49 & 0.49 & 4700 \\
\hline 05540500 & 2 & Yes & Yes & 1979 & 6100 & -- & 1032 & 0.284 & 0.504 & 0.118 & 7740 \\
\hline 05540500 & 2 & Yes & Yes & 1980 & 2140 & -- & 362 & 0.887 & 0.518 & 0.831 & 3030 \\
\hline 05540500 & 2 & Yes & Yes & 1981 & 5050 & -- & 854 & 2.06 & 0.524 & 0.215 & 6350 \\
\hline 05540500 & 2 & Yes & Yes & 1982 & 4600 & -- & 778 & 0.359 & 0.529 & 0.283 & 5820 \\
\hline 05540500 & 2 & Yes & Yes & 1983 & 8030 & -- & 1359 & 2.18 & 0.535 & 0.0491 & 9770 \\
\hline 05540500 & 2 & Yes & Yes & 1984 & 3620 & -- & 612 & 0.703 & 0.541 & 0.497 & 4670 \\
\hline 05540500 & 2 & Yes & Yes & 1985 & 5760 & -- & 974 & 1.06 & 0.546 & 0.161 & 7060 \\
\hline 05540500 & 2 & Yes & Yes & 1986 & 2700 & -- & 457 & 0.909 & 0.552 & 0.734 & 3550 \\
\hline 05540500 & 2 & Yes & Yes & 1987 & 4780 & -- & 809 & 2.15 & 0.557 & 0.274 & 5870 \\
\hline 05540500 & 2 & Yes & Yes & 1988 & 3470 & D & 587 & 1.08 & 0.563 & 0.555 & 4380 \\
\hline 05540500 & 2 & Yes & Yes & 1989 & 2120 & $\mathrm{E}$ & 359 & 1.39 & 0.569 & 0.865 & 2840 \\
\hline 05540500 & 2 & Yes & Yes & 1990 & 6220 & -- & 1052 & 1.55 & 0.574 & 0.138 & 7420 \\
\hline 05540500 & 2 & Yes & Yes & 1991 & 4600 & -- & 778 & 1.64 & 0.581 & 0.325 & 5570 \\
\hline 05540500 & 2 & Yes & Yes & 1992 & 2580 & $\mathrm{E}$ & 436 & 1.33 & 0.587 & 0.786 & 3280 \\
\hline 05540500 & 2 & Yes & Yes & 1993 & 3610 & -- & 611 & 1.75 & 0.593 & 0.55 & 4400 \\
\hline 05540500 & 2 & Yes & Yes & 1994 & 3330 & C & 563 & 0.631 & 0.599 & 0.624 & 4060 \\
\hline 05540500 & 2 & Yes & Yes & 1995 & 2730 & C & 462 & 1.22 & 0.605 & 0.768 & 3380 \\
\hline 05540500 & 2 & Yes & Yes & 1996 & 17300 & C & 2927 & 4.51 & 0.612 & 0.00528 & 19300 \\
\hline 05540500 & 2 & Yes & Yes & 1997 & 7100 & C & 1201 & 2.48 & 0.618 & 0.0972 & 8120 \\
\hline 05540500 & 2 & Yes & Yes & 1998 & 3470 & C & 587 & 1.31 & 0.624 & 0.614 & 4100 \\
\hline 05540500 & 2 & Yes & Yes & 1999 & 4680 & C & 792 & 0.695 & 0.63 & 0.356 & 5400 \\
\hline 05540500 & 2 & Yes & Yes & 2000 & 3740 & C & 633 & 1.4 & 0.636 & 0.562 & 4340 \\
\hline 05540500 & 2 & Yes & Yes & 2001 & 4240 & C & 717 & 0.622 & 0.65 & 0.466 & 4830 \\
\hline 05540500 & 2 & Yes & Yes & 2002 & 5010 & C & 848 & 1.79 & 0.663 & 0.323 & 5570 \\
\hline 05540500 & 2 & Yes & Yes & 2003 & 2070 & C & 350 & 1.38 & 0.676 & 0.926 & 2410 \\
\hline 05540500 & 2 & Yes & Yes & 2004 & 3840 & C & 650 & 1.29 & 0.689 & 0.591 & 4210 \\
\hline 05540500 & 2 & Yes & Yes & 2005 & 3800 & $C, E$ & 643 & 1.1 & 0.702 & 0.614 & 4100 \\
\hline 05540500 & 2 & Yes & Yes & 2006 & 1870 & $C, E$ & 316 & 1.18 & 0.715 & 0.953 & 2060 \\
\hline
\end{tabular}




\begin{tabular}{|c|c|c|c|c|c|c|c|c|c|c|c|}
\hline $\begin{array}{c}\text { U.S. } \\
\text { Geological } \\
\text { Survey } \\
\text { streamgage } \\
\text { number }\end{array}$ & $\begin{array}{l}\text { Segment } \\
\text { number }\end{array}$ & $\begin{array}{l}\text { Streamgage used in } \\
\text { regression analyses } \\
\text { (non-redundant) }\end{array}$ & $\begin{array}{l}\text { Discharge value } \\
\text { used in } \\
\text { adjustment } \\
\text { regression }\end{array}$ & $\begin{array}{c}\text { Water } \\
\text { year }\end{array}$ & $\begin{array}{l}\text { Observed annual } \\
\text { maximum peak } \\
\text { discharge }\left(\mathrm{ft}^{3} / \mathrm{s}\right)\end{array}$ & $\begin{array}{l}\text { NWIS peak } \\
\text { code }\end{array}$ & $\begin{array}{l}\text { Observed annual } \\
\text { maximum peak } \\
\text { discharge with } \\
\text { segment intercept } \\
\text { value subtracted } \\
\left(\mathrm{ft}^{3} / \mathrm{s}\right)\end{array}$ & $\begin{array}{l}\text { Observed } \\
\text { precipitation } \\
\text { (inches) }\end{array}$ & Urban fraction & $\begin{array}{l}\text { Exceedance } \\
\text { probability }\end{array}$ & $\begin{array}{c}\text { Urban-adjusted } \\
\text { annual maximum } \\
\text { peak discharge } \\
\left(\mathrm{ft}^{3} / \mathrm{s}\right)\end{array}$ \\
\hline 05540500 & 2 & Yes & Yes & 2007 & 5310 & $\mathrm{C}$ & 898 & 1.62 & 0.728 & 0.331 & 5530 \\
\hline 05540500 & 2 & Yes & Yes & 2008 & 9440 & $C$ & 1597 & 3.46 & 0.741 & 0.0499 & 9650 \\
\hline 05540500 & 2 & Yes & Yes & 2009 & 7310 & $\mathrm{C}$ & 1237 & 0.986 & 0.754 & 0.14 & 7400 \\
\hline 05541750 & 1 & Yes & Yes & 1959 & 131 & -- & 415 & 0.663 & 0.00327 & 0.352 & 131 \\
\hline 05541750 & 1 & Yes & Yes & 1960 & 118 & -- & 374 & 0.0216 & 0.00327 & 0.418 & 118 \\
\hline 05541750 & 1 & Yes & Yes & 1961 & 59 & -- & 187 & 1.34 & 0.00327 & 0.811 & 59 \\
\hline 05541750 & 1 & Yes & Yes & 1962 & 110 & -- & 348 & 0.305 & 0.00327 & 0.468 & 110 \\
\hline 05541750 & 1 & Yes & Yes & 1963 & 90 & -- & 285 & 0.544 & 0.00327 & 0.612 & 90 \\
\hline 05541750 & 1 & Yes & Yes & 1964 & 19 & -- & 60 & 1.2 & 0.00327 & 0.977 & 19 \\
\hline 05541750 & 1 & Yes & Yes & 1965 & 72 & -- & 228 & 0.202 & 0.00327 & 0.731 & 72 \\
\hline 05541750 & 1 & Yes & Yes & 1966 & 119 & -- & 377 & 1.41 & 0.00327 & 0.413 & 119 \\
\hline 05541750 & 1 & Yes & Yes & 1967 & 100 & -- & 317 & 1.05 & 0.00327 & 0.537 & 100 \\
\hline 05541750 & 1 & Yes & Yes & 1968 & 163 & -- & 516 & 1.95 & 0.00327 & 0.219 & 163 \\
\hline 05541750 & 1 & Yes & Yes & 1969 & 44 & -- & 139 & 0.38 & 0.00327 & 0.891 & 44 \\
\hline 05541750 & 1 & Yes & Yes & 1970 & 132 & -- & 418 & 1.64 & 0.00327 & 0.347 & 132 \\
\hline 05541750 & 1 & Yes & Yes & 1971 & 48 & -- & 152 & 0.893 & 0.00327 & 0.873 & 48 \\
\hline 05541750 & 1 & Yes & Yes & 1972 & 108 & -- & 342 & 0.831 & 0.00327 & 0.48 & 108 \\
\hline 05541750 & 1 & Yes & Yes & 1973 & 77 & 2 & 244 & 0.977 & 0.00327 & 0.697 & 77 \\
\hline 05541750 & 1 & Yes & Yes & 1974 & 143 & -- & 453 & 1.04 & 0.00327 & 0.298 & 143 \\
\hline 05541750 & 1 & Yes & Yes & 1975 & 125 & -- & 396 & 0.647 & 0.00327 & 0.381 & 125 \\
\hline 05541750 & 1 & Yes & Yes & 1976 & 119 & -- & 377 & 0.549 & 0.00327 & 0.413 & 119 \\
\hline 05541750 & 1 & Yes & Yes & 1977 & 125 & -- & 396 & 1.4 & 0.00327 & 0.381 & 125 \\
\hline 05541750 & 1 & Yes & Yes & 1978 & 122 & -- & 387 & 0.864 & 0.00327 & 0.396 & 122 \\
\hline 05541750 & 1 & Yes & Yes & 1979 & 173 & -- & 548 & 1.25 & 0.00327 & 0.189 & 173 \\
\hline 05541750 & 1 & Yes & Yes & 1980 & 170 & -- & 539 & 0.556 & 0.00327 & 0.196 & 170 \\
\hline 05542000 & 1 & Yes & Yes & 1940 & 1090 & -- & 44 & 1.66 & 0.00936 & 0.989 & 1120 \\
\hline 05542000 & 1 & Yes & Yes & 1941 & 1820 & -- & 74 & 1.22 & 0.00947 & 0.964 & 1860 \\
\hline 05542000 & 1 & Yes & Yes & 1942 & 14000 & -- & 570 & 2.42 & 0.00959 & 0.175 & 14100 \\
\hline 05542000 & 1 & Yes & Yes & 1943 & 13600 & -- & 554 & 1.44 & 0.0097 & 0.187 & 13700 \\
\hline 05542000 & 1 & Yes & Yes & 1944 & 13700 & -- & 558 & 1.37 & 0.00982 & 0.184 & 13800 \\
\hline 05542000 & 1 & Yes & Yes & 1945 & 7900 & -- & 322 & 1.39 & 0.00994 & 0.531 & 8000 \\
\hline 05542000 & 1 & Yes & Yes & 1946 & 11600 & -- & 473 & 2.28 & 0.0101 & 0.277 & 11700 \\
\hline 05542000 & 1 & Yes & Yes & 1947 & 12500 & -- & 509 & 2.2 & 0.0102 & 0.231 & 12600 \\
\hline 05542000 & 1 & Yes & Yes & 1948 & 8120 & -- & 331 & 1.18 & 0.0103 & 0.51 & 8220 \\
\hline 05542000 & 1 & Yes & Yes & 1949 & 7340 & -- & 299 & 0.801 & 0.0104 & 0.587 & 7430 \\
\hline
\end{tabular}




\begin{tabular}{|c|c|c|c|c|c|c|c|c|c|c|c|}
\hline $\begin{array}{c}\text { U.S. } \\
\text { Geological } \\
\text { Survey } \\
\text { streamgage } \\
\text { number }\end{array}$ & $\begin{array}{l}\text { Segment } \\
\text { number }\end{array}$ & $\begin{array}{l}\text { Streamgage used in } \\
\text { regression analyses } \\
\text { (non-redundant) }\end{array}$ & $\begin{array}{l}\text { Discharge value } \\
\text { used in } \\
\text { adjustment } \\
\text { regression }\end{array}$ & $\begin{array}{l}\text { Water } \\
\text { year }\end{array}$ & $\begin{array}{c}\text { Observed annual } \\
\text { maximum peak } \\
\text { discharge }\left(\mathrm{ft}^{3} / \mathrm{s}\right)\end{array}$ & $\begin{array}{l}\text { NWIS peak } \\
\text { code }\end{array}$ & $\begin{array}{l}\text { Observed annual } \\
\text { maximum peak } \\
\text { discharge with } \\
\text { segment intercept } \\
\text { value subtracted } \\
\left(\mathrm{ft}^{3} / \mathrm{s}\right)\end{array}$ & $\begin{array}{l}\text { Observed } \\
\text { precipitation } \\
\text { (inches) }\end{array}$ & Urban fraction & $\begin{array}{l}\text { Exceedance } \\
\text { probability }\end{array}$ & $\begin{array}{c}\text { Urban-adjusted } \\
\text { annual maximum } \\
\text { peak discharge } \\
\left(\mathrm{ft}^{3} / \mathrm{s}\right)\end{array}$ \\
\hline 05542000 & 1 & Yes & Yes & 1950 & 17300 & -- & 705 & 1.67 & 0.0105 & 0.093 & 17500 \\
\hline 05542000 & 1 & Yes & Yes & 1951 & 11100 & D & 452 & 0.374 & 0.0107 & 0.304 & 11200 \\
\hline 05542000 & 1 & Yes & Yes & 1952 & 5880 & -- & 240 & 0.826 & 0.0109 & 0.712 & 5960 \\
\hline 05542000 & 1 & Yes & Yes & 1953 & 5180 & -- & 211 & 0.548 & 0.0111 & 0.77 & 5250 \\
\hline 05542000 & 1 & Yes & Yes & 1954 & 10300 & -- & 420 & 1.85 & 0.0113 & 0.35 & 10400 \\
\hline 05542000 & 1 & Yes & Yes & 1955 & 3760 & $\mathrm{E}$ & 153 & 0.759 & 0.0115 & 0.874 & 3810 \\
\hline 05542000 & 1 & Yes & Yes & 1956 & 2070 & $\mathrm{E}$ & 84 & 0.328 & 0.0117 & 0.956 & 2110 \\
\hline 05542000 & 1 & Yes & Yes & 1957 & 7800 & -- & 318 & 0.392 & 0.0119 & 0.542 & 7880 \\
\hline 05542000 & 1 & Yes & Yes & 1958 & 17600 & -- & 717 & 1.34 & 0.0121 & 0.0889 & 17700 \\
\hline 05542000 & 1 & Yes & Yes & 1959 & 8200 & -- & 334 & 1.2 & 0.0123 & 0.505 & 8280 \\
\hline 05542000 & 1 & Yes & Yes & 1960 & 5800 & -- & 236 & 0.963 & 0.0124 & 0.72 & 5860 \\
\hline 05542000 & 1 & Yes & Yes & 1961 & 5500 & $\mathrm{E}$ & 224 & 1.58 & 0.0126 & 0.746 & 5560 \\
\hline 05542000 & 1 & Yes & Yes & 1962 & 7010 & -- & 286 & 0.318 & 0.0127 & 0.618 & 7080 \\
\hline 05542000 & 1 & Yes & Yes & 1963 & 3680 & $E$ & 150 & 0.533 & 0.0128 & 0.879 & 3730 \\
\hline 05542000 & 1 & Yes & Yes & 1964 & 1200 & $\mathrm{E}$ & 49 & 1.11 & 0.0129 & 0.986 & 1220 \\
\hline 05542000 & 1 & Yes & Yes & 1965 & 9390 & -- & 383 & 1.12 & 0.013 & 0.41 & 9470 \\
\hline 05542000 & 1 & Yes & Yes & 1966 & 8650 & -- & 352 & 1.48 & 0.0131 & 0.467 & 8730 \\
\hline 05542000 & 1 & Yes & Yes & 1967 & 7710 & -- & 314 & 1.05 & 0.0132 & 0.552 & 7780 \\
\hline 05542000 & 1 & Yes & Yes & 1968 & 16800 & -- & 684 & 1.98 & 0.0134 & 0.102 & 16900 \\
\hline 05542000 & 1 & Yes & Yes & 1969 & 4470 & $\mathrm{E}$ & 182 & 1.17 & 0.0135 & 0.825 & 4520 \\
\hline 05542000 & 1 & Yes & Yes & 1970 & 16800 & $\mathrm{E}$ & 684 & 1.71 & 0.0136 & 0.102 & 16900 \\
\hline 05542000 & 1 & Yes & Yes & 1971 & 4750 & -- & 194 & 0.431 & 0.0138 & 0.804 & 4800 \\
\hline 05542000 & 1 & Yes & Yes & 1972 & 8000 & -- & 326 & 1.1 & 0.014 & 0.525 & 8060 \\
\hline 05542000 & 1 & Yes & Yes & 1973 & 12000 & -- & 489 & 0.738 & 0.0142 & 0.258 & 12100 \\
\hline 05542000 & 1 & Yes & Yes & 1974 & 9250 & -- & 377 & 1.08 & 0.0144 & 0.421 & 9320 \\
\hline 05542000 & 1 & Yes & Yes & 1975 & 7080 & -- & 288 & 1.14 & 0.0146 & 0.613 & 7130 \\
\hline 05542000 & 1 & Yes & Yes & 1976 & 8340 & -- & 340 & 0.926 & 0.0148 & 0.494 & 8400 \\
\hline 05542000 & 1 & Yes & Yes & 1977 & 4670 & -- & 190 & 1.47 & 0.015 & 0.81 & 4710 \\
\hline 05542000 & 1 & Yes & Yes & 1978 & 5560 & -- & 227 & 1.01 & 0.0152 & 0.743 & 5600 \\
\hline 05542000 & 1 & Yes & Yes & 1979 & 13300 & -- & 542 & 1.38 & 0.0154 & 0.198 & 13400 \\
\hline 05542000 & 1 & Yes & Yes & 1980 & 12200 & -- & 497 & 1.05 & 0.0156 & 0.249 & 12300 \\
\hline 05542000 & 1 & Yes & Yes & 1981 & 9970 & -- & 406 & 1.62 & 0.0157 & 0.373 & 10000 \\
\hline 05542000 & 1 & Yes & Yes & 1982 & 10200 & -- & 416 & 0.342 & 0.0158 & 0.359 & 10300 \\
\hline 05542000 & 1 & Yes & Yes & 1983 & 22400 & -- & 913 & 2.22 & 0.0159 & 0.0459 & 22500 \\
\hline 05542000 & 1 & Yes & Yes & 1984 & 16800 & -- & 684 & 0.626 & 0.016 & 0.103 & 16900 \\
\hline
\end{tabular}




\begin{tabular}{|c|c|c|c|c|c|c|c|c|c|c|c|}
\hline $\begin{array}{l}\text { U.S. } \\
\text { Geological } \\
\text { Survey } \\
\text { streamgage } \\
\text { number }\end{array}$ & $\begin{array}{l}\text { Segment } \\
\text { number }\end{array}$ & $\begin{array}{l}\text { Streamgage used in } \\
\text { regression analyses } \\
\text { (non-redundant) }\end{array}$ & $\begin{array}{c}\text { Discharge value } \\
\text { used in } \\
\text { adjustment } \\
\text { regression }\end{array}$ & $\begin{array}{l}\text { Water } \\
\text { year }\end{array}$ & $\begin{array}{c}\text { Observed annual } \\
\text { maximum peak } \\
\text { discharge }\left(\mathrm{ft}^{3} / \mathrm{s}\right)\end{array}$ & $\begin{array}{l}\text { NWIS peak } \\
\text { code }\end{array}$ & $\begin{array}{l}\text { Observed annual } \\
\text { maximum peak } \\
\text { discharge with } \\
\text { segment intercept } \\
\text { value subtracted } \\
\left(\mathrm{ft}^{3} / \mathrm{s}\right)\end{array}$ & $\begin{array}{l}\text { Observed } \\
\text { precipitation } \\
\text { (inches) }\end{array}$ & Urban fraction & $\begin{array}{l}\text { Exceedance } \\
\text { probability }\end{array}$ & $\begin{array}{c}\text { Urban-adjusted } \\
\text { annual maximum } \\
\text { peak discharge } \\
\left(\mathrm{ft}^{3} / \mathrm{s}\right)\end{array}$ \\
\hline 05542000 & 1 & Yes & Yes & 1985 & 18800 & -- & 766 & 0.778 & 0.0161 & 0.0736 & 18900 \\
\hline 05542000 & 1 & Yes & Yes & 1986 & 16800 & -- & 684 & 1.07 & 0.0162 & 0.103 & 16900 \\
\hline 05542000 & 1 & Yes & Yes & 1987 & 9310 & -- & 379 & 1.58 & 0.0163 & 0.418 & 9360 \\
\hline 05542000 & 1 & Yes & Yes & 1988 & 4610 & $\mathrm{E}$ & 188 & 0.468 & 0.0164 & 0.816 & 4640 \\
\hline 05542000 & 1 & Yes & Yes & 1989 & 11400 & -- & 464 & 2.14 & 0.0165 & 0.291 & 11500 \\
\hline 05542000 & 1 & Yes & Yes & 1990 & 10500 & -- & 428 & 0.572 & 0.0166 & 0.342 & 10500 \\
\hline 05542000 & 1 & Yes & Yes & 1991 & 20800 & -- & 847 & 1.41 & 0.0168 & 0.057 & 20900 \\
\hline 05542000 & 1 & Yes & Yes & 1992 & 3040 & $E$ & 124 & 0.731 & 0.017 & 0.917 & 3060 \\
\hline 05542000 & 1 & Yes & Yes & 1993 & 10700 & -- & 436 & 1.88 & 0.0171 & 0.331 & 10700 \\
\hline 05542000 & 1 & Yes & Yes & 1994 & 9150 & $E$ & 373 & 1.81 & 0.0173 & 0.431 & 9190 \\
\hline 05542000 & 1 & Yes & Yes & 1995 & 8140 & -- & 332 & 1.5 & 0.0175 & 0.515 & 8170 \\
\hline 05542000 & 1 & Yes & Yes & 1997 & 13500 & -- & 550 & 1.75 & 0.0179 & 0.193 & 13500 \\
\hline 05542000 & 1 & Yes & Yes & 1998 & 16600 & -- & 676 & 1.44 & 0.018 & 0.109 & 16600 \\
\hline 05542000 & 1 & Yes & Yes & 1999 & 10200 & -- & 416 & 1.36 & 0.0182 & 0.361 & 10200 \\
\hline 05542000 & 1 & Yes & Yes & 2000 & 3830 & $\mathrm{E}$ & 156 & 1.21 & 0.0184 & 0.872 & 3850 \\
\hline 05542000 & 1 & Yes & Yes & 2001 & 12600 & -- & 513 & 0.676 & 0.0187 & 0.231 & 12600 \\
\hline 05542000 & 1 & Yes & Yes & 2002 & 14100 & -- & 574 & 1.9 & 0.0189 & 0.175 & 14100 \\
\hline 05542000 & 1 & Yes & Yes & 2003 & 8220 & $\mathrm{E}$ & 335 & 1.73 & 0.0192 & 0.509 & 8240 \\
\hline 05542000 & 1 & Yes & Yes & 2004 & 8520 & $\mathrm{E}$ & 347 & 2.12 & 0.0195 & 0.483 & 8540 \\
\hline 05542000 & 1 & Yes & Yes & 2005 & 14300 & -- & 583 & 1.19 & 0.0197 & 0.17 & 14300 \\
\hline 05542000 & 1 & Yes & Yes & 2006 & 3170 & -- & 129 & 1.09 & 0.02 & 0.91 & 3180 \\
\hline 05542000 & 1 & Yes & Yes & 2007 & 12300 & -- & 501 & 2.05 & 0.0203 & 0.247 & 12300 \\
\hline 05542000 & 1 & Yes & Yes & 2008 & 18800 & -- & 766 & 2.52 & 0.0206 & 0.0742 & 18800 \\
\hline 05542000 & 1 & Yes & Yes & 2009 & 14000 & -- & 570 & 1.92 & 0.0208 & 0.179 & 14000 \\
\hline 05543830 & 1 & Yes & No & 1960 & 2500 & 7 & 1744 & 1.09 & 0.266 & 0.0129 & 3580 \\
\hline 05543830 & 1 & Yes & Yes & 1963 & 620 & -- & 432 & 0.44 & 0.293 & 0.553 & 1050 \\
\hline 05543830 & 1 & Yes & Yes & 1964 & 644 & -- & 449 & 1.29 & 0.303 & 0.531 & 1080 \\
\hline 05543830 & 1 & Yes & Yes & 1965 & 1240 & -- & 865 & 0.949 & 0.312 & 0.118 & 1860 \\
\hline 05543830 & 1 & Yes & Yes & 1966 & 1040 & -- & 725 & 0.72 & 0.321 & 0.197 & 1560 \\
\hline 05543830 & 1 & Yes & Yes & 1967 & 369 & -- & 257 & 1.57 & 0.331 & 0.859 & 685 \\
\hline 05543830 & 1 & Yes & Yes & 1968 & 542 & -- & 378 & 0.996 & 0.34 & 0.686 & 904 \\
\hline 05543830 & 1 & Yes & Yes & 1969 & 1220 & -- & 851 & 2.13 & 0.349 & 0.14 & 1770 \\
\hline 05543830 & 1 & Yes & Yes & 1970 & 660 & -- & 460 & 1.82 & 0.358 & 0.562 & 1040 \\
\hline 05543830 & 1 & Yes & Yes & 1971 & 660 & -- & 460 & 0.169 & 0.368 & 0.571 & 1030 \\
\hline 05543830 & 1 & Yes & Yes & 1972 & 1150 & -- & 802 & 2.88 & 0.378 & 0.177 & 1630 \\
\hline
\end{tabular}




\begin{tabular}{|c|c|c|c|c|c|c|c|c|c|c|c|}
\hline $\begin{array}{c}\text { U.S. } \\
\text { Geological } \\
\text { Survey } \\
\text { streamgage } \\
\text { number } \\
\end{array}$ & $\begin{array}{c}\text { Segment } \\
\text { number }\end{array}$ & $\begin{array}{l}\text { Streamgage used in } \\
\text { regression analyses } \\
\text { (non-redundant) }\end{array}$ & $\begin{array}{c}\text { Discharge value } \\
\text { used in } \\
\text { adjustment } \\
\text { regression }\end{array}$ & $\begin{array}{c}\text { Water } \\
\text { year }\end{array}$ & $\begin{array}{c}\text { Observed annual } \\
\text { maximum peak } \\
\text { discharge }\left(\mathrm{ft}^{3} / \mathrm{s}\right)\end{array}$ & $\begin{array}{c}\text { NWIS peak } \\
\text { code }\end{array}$ & $\begin{array}{c}\text { Observed annual } \\
\text { maximum peak } \\
\text { discharge with } \\
\text { segment intercept } \\
\text { value subtracted } \\
\left(\mathrm{ft}^{3} / \mathrm{s}\right)\end{array}$ & $\begin{array}{l}\text { Observed } \\
\text { precipitation } \\
\text { (inches) }\end{array}$ & Urban fraction & $\begin{array}{l}\text { Exceedance } \\
\text { probability }\end{array}$ & $\begin{array}{c}\text { Urban-adjusted } \\
\text { annual maximum } \\
\text { peak discharge } \\
\left(\mathrm{ft}^{3} / \mathrm{s}\right)\end{array}$ \\
\hline 05543830 & 1 & Yes & Yes & 1973 & 2260 & -- & 1576 & 3.21 & 0.387 & 0.0198 & 2990 \\
\hline 05543830 & 1 & Yes & Yes & 1974 & 1520 & -- & 1060 & 0.708 & 0.397 & 0.075 & 2090 \\
\hline 05543830 & 1 & Yes & Yes & 1975 & 1200 & -- & 837 & 0.74 & 0.406 & 0.17 & 1660 \\
\hline 05543830 & 1 & Yes & Yes & 1976 & 1080 & -- & 753 & 1.84 & 0.416 & 0.229 & 1490 \\
\hline 05543830 & 1 & Yes & Yes & 1977 & 379 & -- & 264 & 0.374 & 0.425 & 0.894 & 632 \\
\hline 05543830 & 1 & Yes & Yes & 1978 & 910 & -- & 635 & 1.91 & 0.435 & 0.37 & 1280 \\
\hline 05543830 & 1 & Yes & Yes & 1979 & 1270 & -- & 886 & 0.332 & 0.444 & 0.161 & 1690 \\
\hline 05543830 & 1 & Yes & Yes & 1980 & 569 & -- & 397 & 1.2 & 0.454 & 0.744 & 840 \\
\hline 05543830 & 1 & Yes & Yes & 1981 & 900 & -- & 628 & 0.756 & 0.458 & 0.4 & 1240 \\
\hline 05543830 & 1 & Yes & Yes & 1982 & 965 & -- & 673 & 1.47 & 0.462 & 0.346 & 1310 \\
\hline 05543830 & 1 & Yes & Yes & 1983 & 1060 & -- & 739 & 1.3 & 0.467 & 0.275 & 1410 \\
\hline 05543830 & 1 & Yes & Yes & 1984 & 562 & -- & 392 & 0.26 & 0.471 & 0.762 & 816 \\
\hline 05543830 & 1 & Yes & Yes & 1985 & 646 & -- & 451 & 0.423 & 0.475 & 0.68 & 911 \\
\hline 05543830 & 1 & Yes & Yes & 1986 & 955 & -- & 666 & 1.45 & 0.48 & 0.37 & 1280 \\
\hline 05543830 & 1 & Yes & Yes & 1987 & 653 & -- & 455 & 1.96 & 0.484 & 0.68 & 911 \\
\hline 05543830 & 1 & Yes & Yes & 1988 & 793 & -- & 553 & 0.168 & 0.488 & 0.533 & 1080 \\
\hline 05543830 & 1 & Yes & Yes & 1989 & 759 & -- & 529 & 0.394 & 0.492 & 0.574 & 1030 \\
\hline 05543830 & 1 & Yes & Yes & 1990 & 1210 & -- & 844 & 1.08 & 0.497 & 0.206 & 1540 \\
\hline 05543830 & 1 & Yes & Yes & 1991 & 755 & -- & 527 & 0.76 & 0.507 & 0.592 & 1010 \\
\hline 05543830 & 1 & Yes & Yes & 1992 & 479 & -- & 334 & 0.501 & 0.518 & 0.864 & 679 \\
\hline 05543830 & 1 & Yes & Yes & 1993 & 1520 & -- & 1060 & 2 & 0.529 & 0.116 & 1870 \\
\hline 05543830 & 1 & Yes & Yes & 1994 & 800 & -- & 558 & 1.77 & 0.539 & 0.575 & 1030 \\
\hline 05543830 & 1 & Yes & Yes & 1995 & 1070 & -- & 746 & 2.67 & 0.55 & 0.335 & 1320 \\
\hline 05543830 & 1 & Yes & Yes & 1996 & 967 & -- & 674 & 1.99 & 0.561 & 0.434 & 1200 \\
\hline 05543830 & 1 & Yes & Yes & 1997 & 1320 & -- & 921 & 3.51 & 0.572 & 0.197 & 1570 \\
\hline 05543830 & 1 & Yes & Yes & 1998 & 1460 & -- & 1018 & 1.94 & 0.582 & 0.156 & 1710 \\
\hline 05543830 & 1 & Yes & Yes & 1999 & 1010 & -- & 704 & 1.95 & 0.593 & 0.427 & 1210 \\
\hline 05543830 & 1 & Yes & Yes & 2000 & 1730 & -- & 1207 & 3.99 & 0.604 & 0.0916 & 1980 \\
\hline 05543830 & 1 & Yes & Yes & 2001 & 746 & -- & 520 & 2.27 & 0.619 & 0.706 & 886 \\
\hline 05543830 & 1 & Yes & Yes & 2002 & 963 & -- & 672 & 4.41 & 0.634 & 0.509 & 1100 \\
\hline 05543830 & 1 & Yes & Yes & 2003 & 497 & -- & 347 & 0.805 & 0.649 & 0.918 & 592 \\
\hline 05543830 & 1 & Yes & Yes & 2004 & 1300 & -- & 907 & 2.56 & 0.665 & 0.266 & 1420 \\
\hline 05543830 & 1 & Yes & Yes & 2005 & 542 & -- & 378 & 0.377 & 0.68 & 0.907 & 612 \\
\hline 05543830 & 1 & Yes & Yes & 2006 & 1200 & -- & 837 & 1.64 & 0.695 & 0.369 & 1280 \\
\hline 05543830 & 1 & Yes & Yes & 2007 & 815 & -- & 568 & 1.35 & 0.711 & 0.724 & 864 \\
\hline
\end{tabular}




\begin{tabular}{|c|c|c|c|c|c|c|c|c|c|c|c|}
\hline $\begin{array}{l}\text { U.S. } \\
\text { Geological } \\
\text { Survey } \\
\text { streamgage } \\
\text { number }\end{array}$ & $\begin{array}{l}\text { Segment } \\
\text { number }\end{array}$ & $\begin{array}{l}\text { Streamgage used in } \\
\text { regression analyses } \\
\text { (non-redundant) }\end{array}$ & $\begin{array}{l}\text { Discharge value } \\
\text { used in } \\
\text { adjustment } \\
\text { regression }\end{array}$ & $\begin{array}{l}\text { Water } \\
\text { year }\end{array}$ & $\begin{array}{l}\text { Observed annual } \\
\text { maximum peak } \\
\text { discharge }\left(\mathrm{ft}^{3} / \mathrm{s}\right)\end{array}$ & $\begin{array}{l}\text { NWIS peak } \\
\text { code }\end{array}$ & $\begin{array}{l}\text { Observed annual } \\
\text { maximum peak } \\
\text { discharge with } \\
\text { segment intercept } \\
\text { value subtracted } \\
\left(\mathrm{ft}^{3} / \mathrm{s}\right)\end{array}$ & $\begin{array}{l}\text { Observed } \\
\text { precipitation } \\
\text { (inches) }\end{array}$ & Urban fraction & $\begin{array}{l}\text { Exceedance } \\
\text { probability }\end{array}$ & $\begin{array}{c}\text { Urban-adjusted } \\
\text { annual maximum } \\
\text { peak discharge } \\
\left(\mathrm{ft}^{3} / \mathrm{s}\right)\end{array}$ \\
\hline 05543830 & 1 & Yes & Yes & 2008 & 2440 & -- & 1702 & 2.25 & 0.726 & 0.0401 & 2500 \\
\hline 05543830 & 1 & Yes & Yes & 2009 & 1910 & -- & 1332 & 2.77 & 0.741 & 0.1 & 1940 \\
\hline 05544200 & 1 & Yes & Yes & 1974 & 292 & -- & 536 & 0.568 & 0.0876 & 0.245 & 362 \\
\hline 05544200 & 1 & Yes & Yes & 1977 & 140 & -- & 257 & 0.332 & 0.103 & 0.735 & 183 \\
\hline 05544200 & 1 & Yes & Yes & 1978 & 235 & -- & 432 & 1.66 & 0.108 & 0.397 & 292 \\
\hline 05544200 & 1 & Yes & Yes & 1979 & 274 & -- & 503 & 1.08 & 0.113 & 0.3 & 334 \\
\hline 05544200 & 1 & Yes & Yes & 1980 & 184 & -- & 338 & 0.773 & 0.118 & 0.588 & 230 \\
\hline 05544200 & 1 & Yes & Yes & 1981 & 219 & -- & 402 & 0.428 & 0.122 & 0.459 & 270 \\
\hline 05544200 & 1 & Yes & Yes & 1982 & 225 & -- & 413 & 1.27 & 0.125 & 0.442 & 276 \\
\hline 05544200 & 1 & Yes & Yes & 1983 & 173 & -- & 318 & 1.34 & 0.129 & 0.635 & 215 \\
\hline 05544200 & 1 & Yes & Yes & 1984 & 145 & -- & 266 & 0.29 & 0.133 & 0.737 & 182 \\
\hline 05544200 & 1 & Yes & Yes & 1985 & 209 & -- & 384 & 0.203 & 0.137 & 0.506 & 254 \\
\hline 05544200 & 1 & Yes & Yes & 1986 & 278 & -- & 511 & 1.1 & 0.14 & 0.308 & 330 \\
\hline 05544200 & 1 & Yes & Yes & 1987 & 201 & -- & 369 & 0.0386 & 0.144 & 0.543 & 243 \\
\hline 05544200 & 1 & Yes & Yes & 1988 & 165 & -- & 303 & 0.155 & 0.148 & 0.676 & 201 \\
\hline 05544200 & 1 & Yes & Yes & 1989 & 240 & -- & 441 & 1.68 & 0.151 & 0.416 & 286 \\
\hline 05544200 & 1 & Yes & Yes & 1990 & 261 & -- & 479 & 0.638 & 0.155 & 0.361 & 308 \\
\hline 05544200 & 1 & Yes & Yes & 1991 & 193 & 5 & 355 & 0.892 & 0.165 & 0.593 & 229 \\
\hline 05544200 & 1 & Yes & Yes & 1992 & 204 & 5 & 375 & 1.15 & 0.175 & 0.559 & 239 \\
\hline 05544200 & 1 & Yes & Yes & 1993 & 268 & 5 & 492 & 1.66 & 0.185 & 0.364 & 306 \\
\hline 05544200 & 1 & Yes & Yes & 1994 & 199 & 5 & 366 & 0.918 & 0.194 & 0.596 & 228 \\
\hline 05544200 & 1 & Yes & Yes & 1995 & 210 & 5 & 386 & 1.58 & 0.204 & 0.562 & 237 \\
\hline 05544200 & 1 & Yes & Yes & 1996 & 224 & 5 & 412 & 1.66 & 0.214 & 0.52 & 250 \\
\hline 05544200 & 1 & Yes & Yes & 1997 & 270 & 5 & 496 & 1.61 & 0.224 & 0.388 & 296 \\
\hline 05544200 & 1 & Yes & Yes & 1998 & 130 & 5 & 239 & 0.549 & 0.234 & 0.837 & 145 \\
\hline 05544200 & 1 & Yes & Yes & 1999 & 236 & 5 & 434 & 1.81 & 0.244 & 0.505 & 255 \\
\hline 05544200 & 1 & Yes & Yes & 2000 & 200 & 5 & 367 & 2.09 & 0.253 & 0.636 & 214 \\
\hline 05544200 & 1 & Yes & Yes & 2001 & 200 & 5 & 367 & 0.015 & 0.259 & 0.641 & 213 \\
\hline 05544200 & 1 & Yes & Yes & 2002 & 172 & 5 & 316 & 1.06 & 0.265 & 0.736 & 183 \\
\hline 05544200 & 1 & Yes & Yes & 2003 & 170 & 5 & 312 & 0.861 & 0.271 & 0.746 & 179 \\
\hline 05544200 & 1 & Yes & Yes & 2004 & 240 & 5 & 441 & 1.89 & 0.277 & 0.522 & 250 \\
\hline 05544200 & 1 & Yes & Yes & 2005 & 241 & 5 & 443 & 0.938 & 0.283 & 0.524 & 249 \\
\hline 05544200 & 1 & Yes & Yes & 2006 & 238 & 5 & 437 & 1.05 & 0.288 & 0.539 & 244 \\
\hline 05544200 & 1 & Yes & Yes & 2007 & 317 & 5 & 582 & 1.69 & 0.294 & 0.325 & 323 \\
\hline 05544200 & 1 & Yes & Yes & 2008 & 364 & 5 & 669 & 1.54 & 0.3 & 0.234 & 368 \\
\hline
\end{tabular}




\begin{tabular}{|c|c|c|c|c|c|c|c|c|c|c|c|}
\hline $\begin{array}{l}\text { U.S. } \\
\text { Geological } \\
\text { Survey } \\
\text { streamgage } \\
\text { number }\end{array}$ & $\begin{array}{l}\text { Segment } \\
\text { number }\end{array}$ & $\begin{array}{l}\text { Streamgage used in } \\
\text { regression analyses } \\
\text { (non-redundant) }\end{array}$ & $\begin{array}{l}\text { Discharge value } \\
\text { used in } \\
\text { adjustment } \\
\text { regression }\end{array}$ & $\begin{array}{l}\text { Water } \\
\text { year }\end{array}$ & $\begin{array}{l}\text { Observed annual } \\
\text { maximum peak } \\
\text { discharge }\left(\mathrm{ft}^{3} / \mathrm{s}\right)\end{array}$ & $\begin{array}{l}\text { NWIS peak } \\
\text { code }\end{array}$ & $\begin{array}{l}\text { Observed annual } \\
\text { maximum peak } \\
\text { discharge with } \\
\text { segment intercept } \\
\text { value subtracted } \\
\left(\mathrm{ft}^{3} / \mathrm{s}\right)\end{array}$ & $\begin{array}{l}\text { Observed } \\
\text { precipitation } \\
\text { (inches) }\end{array}$ & Urban fraction & $\begin{array}{l}\text { Exceedance } \\
\text { probability }\end{array}$ & $\begin{array}{c}\text { Urban-adjusted } \\
\text { annual maximum } \\
\text { peak discharge } \\
\left(\mathrm{ft}^{3} / \mathrm{s}\right)\end{array}$ \\
\hline 05544200 & 1 & Yes & Yes & 2009 & 271 & 5 & 498 & 1.3 & 0.306 & 0.452 & 273 \\
\hline 05544300 & 1 & Yes & Yes & 1960 & 36 & -- & 411 & 0.991 & 0.0534 & 0.39 & 41.9 \\
\hline 05544300 & 1 & Yes & Yes & 1961 & 13 & -- & 149 & 0.392 & 0.0537 & 0.894 & 16.2 \\
\hline 05544300 & 1 & Yes & Yes & 1962 & 14 & -- & 160 & 0.0835 & 0.054 & 0.879 & 17.3 \\
\hline 05544300 & 1 & Yes & No & 1963 & 10 & 4, B & 114 & 0.0835 & 0.0543 & 0.935 & 12.7 \\
\hline 05544300 & 1 & Yes & Yes & 1964 & 26 & -- & 297 & 0.0835 & 0.0546 & 0.623 & 30.7 \\
\hline 05544300 & 1 & Yes & Yes & 1965 & 14 & -- & 160 & 1.24 & 0.0549 & 0.879 & 17.3 \\
\hline 05544300 & 1 & Yes & Yes & 1966 & 24 & -- & 274 & 0.725 & 0.0552 & 0.668 & 28.5 \\
\hline 05544300 & 1 & Yes & Yes & 1967 & 30 & -- & 343 & 0.466 & 0.0555 & 0.522 & 35.2 \\
\hline 05544300 & 1 & Yes & Yes & 1968 & 48 & -- & 549 & 0.874 & 0.0558 & 0.213 & 54.8 \\
\hline 05544300 & 1 & Yes & Yes & 1969 & 66 & -- & 754 & 2.24 & 0.0561 & 0.086 & 74.8 \\
\hline 05544300 & 1 & Yes & No & 1970 & 10 & $4, B$ & 114 & 0.0835 & 0.0564 & 0.935 & 12.7 \\
\hline 05544300 & 1 & Yes & Yes & 1971 & 30 & -- & 343 & 0.868 & 0.0618 & 0.528 & 34.9 \\
\hline 05544300 & 1 & Yes & Yes & 1973 & 31 & -- & 354 & 3.04 & 0.0727 & 0.512 & 35.6 \\
\hline 05544300 & 1 & Yes & Yes & 1974 & 50 & -- & 571 & 0.622 & 0.0782 & 0.201 & 55.8 \\
\hline 05544300 & 1 & Yes & Yes & 1975 & 44 & -- & 503 & 0.358 & 0.0836 & 0.283 & 49.2 \\
\hline 05544300 & 1 & Yes & Yes & 1976 & 56 & -- & 640 & 1.75 & 0.0891 & 0.158 & 61.8 \\
\hline 05544300 & 1 & Yes & Yes & 1977 & 11 & -- & 126 & 1.82 & 0.0946 & 0.932 & 13.1 \\
\hline 05544300 & 1 & Yes & Yes & 1978 & 58 & -- & 663 & 1.19 & 0.1 & 0.148 & 63.3 \\
\hline 05544300 & 1 & Yes & Yes & 1979 & 60 & -- & 686 & 0.33 & 0.105 & 0.136 & 65.2 \\
\hline 05544300 & 1 & Yes & Yes & 1980 & 58 & -- & 663 & 0.949 & 0.111 & 0.152 & 62.7 \\
\hline 05544300 & 1 & Yes & Yes & 1981 & 10 & -- & 114 & 1.62 & 0.112 & 0.946 & 11.6 \\
\hline 05545100 & 1 & Yes & Yes & 1962 & 125 & -- & 307 & 0.00118 & 0.0214 & 0.577 & 128 \\
\hline 05545100 & 1 & Yes & Yes & 1963 & 55 & -- & 135 & 0.551 & 0.0215 & 0.903 & 56.8 \\
\hline 05545100 & 1 & Yes & Yes & 1964 & 37 & -- & 91 & 0.637 & 0.0216 & 0.952 & 38.4 \\
\hline 05545100 & 1 & Yes & Yes & 1965 & 56 & -- & 137 & 1.16 & 0.0217 & 0.9 & 57.8 \\
\hline 05545100 & 1 & Yes & Yes & 1966 & 145 & -- & 356 & 0.824 & 0.0218 & 0.467 & 148 \\
\hline 05545100 & 1 & Yes & Yes & 1967 & 122 & -- & 299 & 2.31 & 0.022 & 0.597 & 125 \\
\hline 05545100 & 1 & Yes & Yes & 1968 & 73 & -- & 179 & 1.25 & 0.0221 & 0.833 & 75.1 \\
\hline 05545100 & 1 & Yes & Yes & 1969 & 125 & -- & 307 & 2 & 0.0222 & 0.578 & 128 \\
\hline 05545100 & 1 & Yes & Yes & 1970 & 95 & -- & 233 & 1.21 & 0.0223 & 0.733 & 97.4 \\
\hline 05545100 & 1 & Yes & Yes & 1971 & 150 & -- & 368 & 0.762 & 0.0225 & 0.444 & 153 \\
\hline 05545100 & 1 & Yes & Yes & 1972 & 225 & -- & 552 & 1.77 & 0.0227 & 0.193 & 229 \\
\hline 05545100 & 1 & Yes & Yes & 1973 & 900 & -- & 2209 & 1.94 & 0.0229 & 0.00305 & 912 \\
\hline 05545100 & 1 & Yes & Yes & 1974 & 315 & -- & 773 & 0.658 & 0.0231 & 0.073 & 320 \\
\hline
\end{tabular}




\begin{tabular}{|c|c|c|c|c|c|c|c|c|c|c|c|}
\hline $\begin{array}{c}\text { U.S. } \\
\text { Geological } \\
\text { Survey } \\
\text { streamgage } \\
\text { number }\end{array}$ & $\begin{array}{l}\text { Segment } \\
\text { number }\end{array}$ & $\begin{array}{l}\text { Streamgage used in } \\
\text { regression analyses } \\
\text { (non-redundant) }\end{array}$ & $\begin{array}{l}\text { Discharge value } \\
\text { used in } \\
\text { adjustment } \\
\text { regression }\end{array}$ & $\begin{array}{l}\text { Water } \\
\text { year }\end{array}$ & $\begin{array}{l}\text { Observed annual } \\
\text { maximum peak } \\
\text { discharge }\left(\mathrm{ft}^{3} / \mathrm{s}\right)\end{array}$ & $\begin{array}{l}\text { NWIS peak } \\
\text { code }\end{array}$ & $\begin{array}{l}\text { Observed annual } \\
\text { maximum peak } \\
\text { discharge with } \\
\text { segment intercept } \\
\text { value subtracted } \\
\left(\mathrm{ft}^{3} / \mathrm{s}\right)\end{array}$ & $\begin{array}{l}\text { Observed } \\
\text { precipitation } \\
\text { (inches) }\end{array}$ & Urban fraction & $\begin{array}{l}\text { Exceedance } \\
\text { probability }\end{array}$ & $\begin{array}{c}\text { Urban-adjusted } \\
\text { annual maximum } \\
\text { peak discharge } \\
\left(\mathrm{ft}^{3} / \mathrm{s}\right)\end{array}$ \\
\hline 05545100 & 1 & Yes & Yes & 1975 & 87 & -- & 214 & 0.414 & 0.0233 & 0.771 & 89.2 \\
\hline 05545100 & 1 & Yes & Yes & 1976 & 190 & -- & 466 & 1.67 & 0.0235 & 0.293 & 194 \\
\hline 05545100 & 1 & Yes & Yes & 1977 & 65 & -- & 160 & 1.1 & 0.0236 & 0.868 & 66.8 \\
\hline 05545100 & 1 & Yes & Yes & 1978 & 165 & -- & 405 & 1.89 & 0.0238 & 0.38 & 168 \\
\hline 05545100 & 1 & Yes & Yes & 1979 & 185 & -- & 454 & 0.234 & 0.024 & 0.31 & 188 \\
\hline 05545100 & 1 & Yes & Yes & 1980 & 92 & -- & 226 & 0.847 & 0.0242 & 0.75 & 94.2 \\
\hline 05545100 & 1 & Yes & Yes & 1981 & 170 & -- & 417 & 0.77 & 0.0245 & 0.362 & 173 \\
\hline 05545100 & 1 & Yes & Yes & 1982 & 295 & -- & 724 & 1.41 & 0.0248 & 0.0893 & 300 \\
\hline 05545100 & 1 & Yes & Yes & 1983 & 220 & -- & 540 & 1.79 & 0.0251 & 0.205 & 224 \\
\hline 05545100 & 1 & Yes & Yes & 1984 & 100 & -- & 245 & 0.606 & 0.0254 & 0.709 & 102 \\
\hline 05545100 & 1 & Yes & Yes & 1985 & 160 & -- & 393 & 0.388 & 0.0258 & 0.401 & 163 \\
\hline 05545100 & 1 & Yes & Yes & 1986 & 190 & -- & 466 & 0.222 & 0.0261 & 0.294 & 193 \\
\hline 05545100 & 1 & Yes & Yes & 1987 & 95 & -- & 233 & 1.37 & 0.0264 & 0.735 & 96.9 \\
\hline 05545100 & 1 & Yes & Yes & 1988 & 145 & -- & 356 & 0.189 & 0.0267 & 0.471 & 148 \\
\hline 05545100 & 1 & Yes & Yes & 1989 & 65 & -- & 160 & 2.55 & 0.027 & 0.869 & 66.5 \\
\hline 05545100 & 1 & Yes & Yes & 1990 & 75 & -- & 184 & 1.28 & 0.0273 & 0.828 & 76.6 \\
\hline 05545100 & 1 & Yes & Yes & 1991 & 75 & -- & 184 & 0.757 & 0.0284 & 0.828 & 76.5 \\
\hline 05545100 & 1 & Yes & Yes & 1992 & 45 & -- & 110 & 1.18 & 0.0295 & 0.934 & 46 \\
\hline 05545100 & 1 & Yes & Yes & 1993 & 235 & -- & 577 & 1.13 & 0.0307 & 0.178 & 238 \\
\hline 05545100 & 1 & Yes & Yes & 1994 & 185 & -- & 454 & 1.05 & 0.0318 & 0.314 & 187 \\
\hline 05545100 & 1 & Yes & Yes & 1995 & 83 & -- & 204 & 1.49 & 0.0329 & 0.794 & 84 \\
\hline 05545100 & 1 & Yes & Yes & 1996 & 215 & -- & 528 & 1.42 & 0.034 & 0.224 & 217 \\
\hline 05545100 & 1 & Yes & Yes & 1997 & 134 & -- & 329 & 1.72 & 0.0351 & 0.536 & 135 \\
\hline 05545100 & 1 & Yes & Yes & 1998 & 117 & -- & 287 & 0.785 & 0.0363 & 0.63 & 118 \\
\hline 05545100 & 1 & Yes & Yes & 1999 & 235 & -- & 577 & 2.13 & 0.0374 & 0.181 & 236 \\
\hline 05545100 & 1 & Yes & Yes & 2000 & 153 & -- & 376 & 1.17 & 0.0385 & 0.442 & 154 \\
\hline 05545100 & 1 & Yes & Yes & 2001 & 163 & -- & 400 & 2.01 & 0.0389 & 0.398 & 164 \\
\hline 05545100 & 1 & Yes & Yes & 2002 & 110 & -- & 270 & 0.886 & 0.0392 & 0.666 & 110 \\
\hline 05545100 & 1 & Yes & Yes & 2003 & 42 & -- & 103 & 0.908 & 0.0396 & 0.943 & 42.2 \\
\hline 05545100 & 1 & Yes & Yes & 2004 & 208 & -- & 511 & 1.14 & 0.04 & 0.247 & 208 \\
\hline 05545100 & 1 & Yes & Yes & 2005 & 110 & -- & 270 & 0.48 & 0.0403 & 0.667 & 110 \\
\hline 05545100 & 1 & Yes & Yes & 2006 & 344 & -- & 844 & 1.02 & 0.0407 & 0.0609 & 344 \\
\hline 05545100 & 1 & Yes & Yes & 2007 & 336 & -- & 825 & 1.37 & 0.0411 & 0.0649 & 336 \\
\hline 05545100 & 1 & Yes & Yes & 2008 & 277 & -- & 680 & 0.416 & 0.0415 & 0.115 & 277 \\
\hline 05545100 & 1 & Yes & Yes & 2009 & 277 & -- & 680 & 0.78 & 0.0418 & 0.115 & 277 \\
\hline
\end{tabular}




\begin{tabular}{|c|c|c|c|c|c|c|c|c|c|c|c|}
\hline $\begin{array}{l}\text { U.S. } \\
\text { Geological } \\
\text { Survey } \\
\text { streamgage } \\
\text { number }\end{array}$ & $\begin{array}{l}\text { Segment } \\
\text { number }\end{array}$ & $\begin{array}{l}\text { Streamgage used in } \\
\text { regression analyses } \\
\text { (non-redundant) }\end{array}$ & $\begin{array}{c}\text { Discharge value } \\
\text { used in } \\
\text { adjustment } \\
\text { regression }\end{array}$ & $\begin{array}{l}\text { Water } \\
\text { year }\end{array}$ & $\begin{array}{l}\text { Observed annual } \\
\text { maximum peak } \\
\text { discharge }\left(\mathrm{ft}^{3} / \mathrm{s}\right)\end{array}$ & $\begin{array}{l}\text { NWIS peak } \\
\text { code }\end{array}$ & $\begin{array}{l}\text { Observed annual } \\
\text { maximum peak } \\
\text { discharge with } \\
\text { segment intercept } \\
\text { value subtracted } \\
\left(\mathrm{ft}^{3} / \mathrm{s}\right)\end{array}$ & $\begin{array}{l}\text { Observed } \\
\text { precipitation } \\
\text { (inches) }\end{array}$ & Urban fraction & $\begin{array}{l}\text { Exceedance } \\
\text { probability }\end{array}$ & $\begin{array}{c}\text { Urban-adjusted } \\
\text { annual maximum } \\
\text { peak discharge } \\
\left(\mathrm{ft}^{3} / \mathrm{s}\right)\end{array}$ \\
\hline 05545200 & 1 & Yes & No & 1958 & 40 & -- & 156 & 0.199 & 0.00492 & 0.867 & 42.1 \\
\hline 05545200 & 1 & Yes & No & 1959 & 100 & -- & 390 & 0.199 & 0.00492 & 0.392 & 104 \\
\hline 05545200 & 1 & Yes & Yes & 1960 & 235 & -- & 916 & 2.14 & 0.00492 & 0.0444 & 242 \\
\hline 05545200 & 1 & Yes & Yes & 1961 & 80 & -- & 312 & 2.72 & 0.00508 & 0.551 & 83.2 \\
\hline 05545200 & 1 & Yes & Yes & 1962 & 57 & -- & 222 & 0.199 & 0.00525 & 0.746 & 59.6 \\
\hline 05545200 & 1 & Yes & Yes & 1963 & 50 & -- & 195 & 0.575 & 0.00541 & 0.797 & 52.3 \\
\hline 05545200 & 1 & Yes & Yes & 1964 & 50 & -- & 195 & 1.65 & 0.00557 & 0.797 & 52.3 \\
\hline 05545200 & 1 & Yes & Yes & 1965 & 70 & -- & 273 & 1.47 & 0.00574 & 0.638 & 72.9 \\
\hline 05545200 & 1 & Yes & Yes & 1966 & 225 & -- & 877 & 1.81 & 0.0059 & 0.0499 & 231 \\
\hline 05545200 & 1 & Yes & Yes & 1967 & 215 & -- & 838 & 1.97 & 0.00607 & 0.0574 & 221 \\
\hline 05545200 & 1 & Yes & Yes & 1968 & 58 & -- & 226 & 2.71 & 0.00623 & 0.738 & 60.5 \\
\hline 05545200 & 1 & Yes & Yes & 1969 & 167 & -- & 651 & 2.64 & 0.00639 & 0.12 & 172 \\
\hline 05545200 & 1 & Yes & Yes & 1970 & 35 & -- & 136 & 1.73 & 0.00656 & 0.897 & 36.8 \\
\hline 05545200 & 1 & Yes & Yes & 1971 & 100 & -- & 390 & 0.819 & 0.00934 & 0.395 & 103 \\
\hline 05545200 & 1 & Yes & Yes & 1972 & 155 & -- & 604 & 0.592 & 0.0121 & 0.152 & 159 \\
\hline 05545200 & 1 & Yes & Yes & 1973 & 290 & -- & 1130 & 1.9 & 0.0149 & 0.0227 & 296 \\
\hline 05545200 & 1 & Yes & Yes & 1974 & 75 & -- & 292 & 0.598 & 0.0177 & 0.608 & 76.9 \\
\hline 05545200 & 1 & Yes & Yes & 1975 & 105 & -- & 409 & 0.199 & 0.0205 & 0.372 & 107 \\
\hline 05545200 & 1 & Yes & Yes & 1976 & 65 & -- & 253 & 1.08 & 0.0233 & 0.691 & 66.3 \\
\hline 05545200 & 1 & Yes & Yes & 1977 & 70 & -- & 273 & 1.37 & 0.0261 & 0.652 & 71.1 \\
\hline 05545200 & 1 & Yes & Yes & 1978 & 110 & -- & 429 & 2.37 & 0.0289 & 0.349 & 111 \\
\hline 05545200 & 1 & Yes & Yes & 1979 & 66 & -- & 257 & 0.199 & 0.0316 & 0.688 & 66.6 \\
\hline 05545200 & 1 & Yes & Yes & 1980 & 87 & -- & 339 & 1.12 & 0.0344 & 0.513 & 87.5 \\
\hline 05545200 & 1 & Yes & Yes & 1981 & 95 & -- & 370 & 0.759 & 0.0344 & 0.45 & 95.5 \\
\hline 05545200 & 1 & Yes & Yes & 1982 & 205 & -- & 799 & 1.73 & 0.0344 & 0.0693 & 206 \\
\hline 05545200 & 1 & Yes & Yes & 1983 & 170 & -- & 662 & 2.13 & 0.0344 & 0.123 & 171 \\
\hline 05545200 & 1 & Yes & Yes & 1984 & 85 & -- & 331 & 2.98 & 0.0344 & 0.53 & 85.5 \\
\hline 05545200 & 1 & Yes & Yes & 1985 & 85 & -- & 331 & 0.282 & 0.0344 & 0.53 & 85.5 \\
\hline 05545200 & 1 & Yes & Yes & 1986 & 185 & -- & 721 & 1.51 & 0.0344 & 0.0927 & 186 \\
\hline 05545200 & 1 & Yes & Yes & 1987 & 80 & -- & 312 & 1.67 & 0.0344 & 0.577 & 80.5 \\
\hline 05545200 & 1 & Yes & Yes & 1988 & 80 & -- & 312 & 0.84 & 0.0344 & 0.577 & 80.5 \\
\hline 05545200 & 1 & Yes & Yes & 1989 & 97 & -- & 378 & 2.78 & 0.0344 & 0.435 & 97.5 \\
\hline 05545200 & 1 & Yes & Yes & 1990 & 101 & -- & 394 & 1.42 & 0.0344 & 0.406 & 102 \\
\hline 05545200 & 1 & Yes & Yes & 1991 & 58 & -- & 226 & 1.46 & 0.0344 & 0.755 & 58.4 \\
\hline 05545200 & 1 & Yes & Yes & 1992 & 35 & -- & 136 & 0.977 & 0.0344 & 0.905 & 35.3 \\
\hline
\end{tabular}




\begin{tabular}{|c|c|c|c|c|c|c|c|c|c|c|c|}
\hline $\begin{array}{l}\text { U.S. } \\
\text { Geological } \\
\text { Survey } \\
\text { streamgage } \\
\text { number }\end{array}$ & $\begin{array}{l}\text { Segment } \\
\text { number }\end{array}$ & $\begin{array}{l}\text { Streamgage used in } \\
\text { regression analyses } \\
\text { (non-redundant) }\end{array}$ & $\begin{array}{c}\text { Discharge value } \\
\text { used in } \\
\text { adjustment } \\
\text { regression }\end{array}$ & $\begin{array}{l}\text { Water } \\
\text { year }\end{array}$ & $\begin{array}{c}\text { Observed annual } \\
\text { maximum peak } \\
\text { discharge }\left(\mathrm{ft}^{3} / \mathrm{s}\right)\end{array}$ & $\begin{array}{l}\text { NWIS peak } \\
\text { code }\end{array}$ & $\begin{array}{l}\text { Observed annual } \\
\text { maximum peak } \\
\text { discharge with } \\
\text { segment intercept } \\
\text { value subtracted } \\
\left(\mathrm{ft}^{3} / \mathrm{s}\right)\end{array}$ & $\begin{array}{l}\text { Observed } \\
\text { precipitation } \\
\text { (inches) }\end{array}$ & Urban fraction & $\begin{array}{l}\text { Exceedance } \\
\text { probability }\end{array}$ & $\begin{array}{c}\text { Urban-adjusted } \\
\text { annual maximum } \\
\text { peak discharge } \\
\left(\mathrm{ft}^{3} / \mathrm{s}\right)\end{array}$ \\
\hline 05545200 & 1 & Yes & Yes & 1993 & 120 & -- & 468 & 1.47 & 0.0344 & 0.298 & 121 \\
\hline 05545200 & 1 & Yes & Yes & 1994 & 130 & -- & 506 & 1.37 & 0.0344 & 0.249 & 131 \\
\hline 05545200 & 1 & Yes & Yes & 1995 & 40 & -- & 156 & 2.08 & 0.0344 & 0.878 & 40.3 \\
\hline 05545200 & 1 & Yes & Yes & 1996 & 182 & -- & 709 & 1.55 & 0.0344 & 0.097 & 183 \\
\hline 05545200 & 1 & Yes & Yes & 1997 & 72 & -- & 281 & 1.54 & 0.0344 & 0.642 & 72.4 \\
\hline 05545200 & 1 & Yes & Yes & 1998 & 24 & -- & 94 & 1.02 & 0.0344 & 0.952 & 24.2 \\
\hline 05545200 & 1 & Yes & Yes & 1999 & 354 & -- & 1379 & 2.39 & 0.0344 & 0.0148 & 355 \\
\hline 05545200 & 1 & Yes & Yes & 2000 & 150 & -- & 584 & 1.49 & 0.0344 & 0.174 & 151 \\
\hline 05545200 & 1 & Yes & Yes & 2001 & 140 & 2 & 545 & 2.82 & 0.0349 & 0.204 & 141 \\
\hline 05545200 & 1 & Yes & Yes & 2002 & 24 & -- & 94 & 0.781 & 0.0354 & 0.952 & 24.2 \\
\hline 05545200 & 1 & Yes & Yes & 2004 & 310 & -- & 1208 & 0.378 & 0.0364 & 0.0193 & 311 \\
\hline 05545200 & 1 & Yes & Yes & 2005 & 52 & -- & 203 & 0.525 & 0.0369 & 0.798 & 52.2 \\
\hline 05545200 & 1 & Yes & Yes & 2006 & 61 & -- & 238 & 1.22 & 0.0374 & 0.733 & 61.2 \\
\hline 05545200 & 1 & Yes & Yes & 2007 & 141 & -- & 549 & 1.83 & 0.0379 & 0.201 & 141 \\
\hline 05545200 & 1 & Yes & Yes & 2008 & 230 & -- & 896 & 0.524 & 0.0384 & 0.0506 & 230 \\
\hline 05545200 & 1 & Yes & Yes & 2009 & 14 & -- & 55 & 0.938 & 0.0389 & 0.984 & 14 \\
\hline 05545300 & 1 & Yes & No & 1959 & 800 & -- & 383 & 0.000673 & 0.13 & 0.502 & 952 \\
\hline 05545300 & 1 & Yes & Yes & 1960 & 1900 & -- & 910 & 1.07 & 0.133 & 0.0613 & 2170 \\
\hline 05545300 & 1 & Yes & Yes & 1961 & 480 & -- & 230 & 0.331 & 0.134 & 0.8 & 589 \\
\hline 05545300 & 1 & Yes & Yes & 1962 & 790 & -- & 379 & 0.000673 & 0.135 & 0.516 & 936 \\
\hline 05545300 & 1 & Yes & Yes & 1963 & 420 & -- & 201 & 0.549 & 0.136 & 0.847 & 520 \\
\hline 05545300 & 1 & Yes & Yes & 1964 & 350 & -- & 168 & 0.104 & 0.138 & 0.896 & 440 \\
\hline 05545300 & 1 & Yes & Yes & 1965 & 560 & -- & 268 & 0.131 & 0.139 & 0.737 & 676 \\
\hline 05545300 & 1 & Yes & Yes & 1966 & 1850 & -- & 887 & 1.58 & 0.14 & 0.0669 & 2100 \\
\hline 05545300 & 1 & Yes & Yes & 1967 & 1690 & -- & 810 & 1.7 & 0.141 & 0.0872 & 1920 \\
\hline 05545300 & 1 & Yes & Yes & 1968 & 467 & -- & 224 & 2.33 & 0.142 & 0.815 & 569 \\
\hline 05545300 & 1 & Yes & Yes & 1969 & 1960 & -- & 939 & 2.17 & 0.143 & 0.0574 & 2210 \\
\hline 05545300 & 1 & Yes & Yes & 1970 & 500 & -- & 240 & 1.26 & 0.145 & 0.789 & 604 \\
\hline 05545300 & 1 & Yes & Yes & 1971 & 1140 & -- & 546 & 0.712 & 0.148 & 0.27 & 1300 \\
\hline 05545300 & 1 & Yes & Yes & 1972 & 1140 & -- & 546 & 0.842 & 0.151 & 0.272 & 1300 \\
\hline 05545300 & 1 & Yes & Yes & 1973 & 1470 & -- & 704 & 1.88 & 0.154 & 0.143 & 1660 \\
\hline 05545300 & 1 & Yes & Yes & 1974 & 1080 & -- & 518 & 0.598 & 0.157 & 0.311 & 1230 \\
\hline 05545300 & 1 & Yes & Yes & 1975 & 798 & -- & 382 & 0.00873 & 0.16 & 0.529 & 920 \\
\hline 05545300 & 1 & Yes & Yes & 1976 & 1140 & -- & 546 & 0.838 & 0.163 & 0.279 & 1290 \\
\hline 05545300 & 1 & Yes & Yes & 1977 & 258 & -- & 124 & 0.88 & 0.166 & 0.948 & 319 \\
\hline
\end{tabular}




\begin{tabular}{|c|c|c|c|c|c|c|c|c|c|c|c|}
\hline $\begin{array}{l}\text { U.S. } \\
\text { Geological } \\
\text { Survey } \\
\text { streamgage } \\
\text { number }\end{array}$ & $\begin{array}{l}\text { Segment } \\
\text { number }\end{array}$ & $\begin{array}{l}\text { Streamgage used in } \\
\text { regression analyses } \\
\text { (non-redundant) }\end{array}$ & $\begin{array}{c}\text { Discharge value } \\
\text { used in } \\
\text { adjustment } \\
\text { regression }\end{array}$ & $\begin{array}{l}\text { Water } \\
\text { year }\end{array}$ & $\begin{array}{l}\text { Observed annual } \\
\text { maximum peak } \\
\text { discharge }\left(\mathrm{ft}^{3} / \mathrm{s}\right)\end{array}$ & $\begin{array}{l}\text { NWIS peak } \\
\text { code }\end{array}$ & $\begin{array}{l}\text { Observed annual } \\
\text { maximum peak } \\
\text { discharge with } \\
\text { segment intercept } \\
\text { value subtracted } \\
\left(\mathrm{ft}^{3} / \mathrm{s}\right)\end{array}$ & $\begin{array}{l}\text { Observed } \\
\text { precipitation } \\
\text { (inches) }\end{array}$ & Urban fraction & $\begin{array}{l}\text { Exceedance } \\
\text { probability }\end{array}$ & $\begin{array}{c}\text { Urban-adjusted } \\
\text { annual maximum } \\
\text { peak discharge } \\
\left(\mathrm{ft}^{3} / \mathrm{s}\right)\end{array}$ \\
\hline 05545300 & 1 & Yes & Yes & 1978 & 722 & -- & 346 & 2.48 & 0.169 & 0.612 & 828 \\
\hline 05545300 & 1 & Yes & Yes & 1979 & 771 & -- & 369 & 0.226 & 0.172 & 0.567 & 879 \\
\hline 05545300 & 1 & Yes & Yes & 1980 & 380 & -- & 182 & 0.198 & 0.175 & 0.891 & 450 \\
\hline 05545300 & 1 & Yes & Yes & 1981 & 492 & -- & 236 & 0.981 & 0.176 & 0.813 & 572 \\
\hline 05545300 & 1 & Yes & Yes & 1982 & 1110 & -- & 532 & 3.07 & 0.177 & 0.306 & 1240 \\
\hline 05545750 & 1 & Yes & Yes & 1940 & 3150 & -- & 401 & 1.02 & 0.0505 & 0.406 & 3780 \\
\hline 05545750 & 1 & Yes & Yes & 1941 & 2130 & -- & 271 & 0.00333 & 0.0524 & 0.673 & 2660 \\
\hline 05545750 & 1 & Yes & Yes & 1942 & 1610 & -- & 205 & 0.835 & 0.0543 & 0.803 & 2100 \\
\hline 05545750 & 1 & Yes & Yes & 1943 & 5700 & -- & 725 & 1.34 & 0.0561 & 0.0963 & 6440 \\
\hline 05545750 & 1 & Yes & Yes & 1944 & 3100 & -- & 394 & 1.15 & 0.058 & 0.423 & 3700 \\
\hline 05545750 & 1 & Yes & Yes & 1945 & 2130 & -- & 271 & 1.52 & 0.0599 & 0.678 & 2640 \\
\hline 05545750 & 1 & Yes & Yes & 1946 & 4170 & -- & 530 & 0.732 & 0.0617 & 0.237 & 4760 \\
\hline 05545750 & 1 & Yes & Yes & 1947 & 2070 & -- & 263 & 1.08 & 0.0636 & 0.697 & 2570 \\
\hline 05545750 & 1 & Yes & Yes & 1948 & 5000 & -- & 636 & 1.67 & 0.0654 & 0.152 & 5630 \\
\hline 05545750 & 1 & Yes & Yes & 1949 & 2400 & -- & 305 & 0.14 & 0.0673 & 0.616 & 2910 \\
\hline 05545750 & 1 & Yes & Yes & 1950 & 2400 & -- & 305 & 0.406 & 0.0692 & 0.617 & 2900 \\
\hline 05545750 & 1 & Yes & Yes & 1951 & 3660 & -- & 465 & 0.278 & 0.0735 & 0.325 & 4220 \\
\hline 05545750 & 1 & Yes & Yes & 1952 & 4010 & -- & 510 & 0.582 & 0.0778 & 0.27 & 4540 \\
\hline 05545750 & 1 & Yes & Yes & 1953 & 1780 & -- & 226 & 0.0754 & 0.0822 & 0.779 & 2210 \\
\hline 05545750 & 1 & Yes & Yes & 1954 & 2050 & -- & 261 & 1.85 & 0.0865 & 0.717 & 2480 \\
\hline 05545750 & 1 & Yes & Yes & 1955 & 1810 & -- & 230 & 1.13 & 0.0909 & 0.777 & 2220 \\
\hline 05545750 & 1 & Yes & Yes & 1956 & 1680 & -- & 214 & 0.661 & 0.0952 & 0.808 & 2070 \\
\hline 05545750 & 1 & Yes & Yes & 1957 & 1350 & -- & 172 & 1.45 & 0.0995 & 0.878 & 1710 \\
\hline 05545750 & 1 & Yes & Yes & 1958 & 1010 & -- & 128 & 0.0766 & 0.104 & 0.932 & 1340 \\
\hline 05545750 & 1 & Yes & Yes & 1959 & 3010 & -- & 383 & 0.00769 & 0.108 & 0.484 & 3420 \\
\hline 05545750 & 1 & Yes & Yes & 1960 & 7520 & -- & 956 & 0.974 & 0.113 & 0.0499 & 7970 \\
\hline 05545750 & 1 & Yes & Yes & 1961 & 2220 & -- & 282 & 0.345 & 0.116 & 0.694 & 2580 \\
\hline 05545750 & 1 & Yes & Yes & 1962 & 4060 & -- & 516 & $2.42 \mathrm{E}-05$ & 0.119 & 0.288 & 4430 \\
\hline 05545750 & 1 & Yes & Yes & 1963 & 1290 & -- & 164 & 0.11 & 0.122 & 0.896 & 1600 \\
\hline 05545750 & 1 & Yes & Yes & 1964 & 1260 & -- & 160 & 0.756 & 0.125 & 0.902 & 1560 \\
\hline 05545750 & 1 & Yes & Yes & 1965 & 2880 & -- & 366 & 0.43 & 0.129 & 0.535 & 3220 \\
\hline 05545750 & 1 & Yes & Yes & 1966 & 3310 & -- & 421 & 0.602 & 0.132 & 0.434 & 3650 \\
\hline 05545750 & 1 & Yes & Yes & 1967 & 2380 & -- & 303 & 0.965 & 0.135 & 0.667 & 2690 \\
\hline 05545750 & 1 & Yes & Yes & 1968 & 1990 & -- & 253 & 0.461 & 0.138 & 0.763 & 2290 \\
\hline 05545750 & 1 & Yes & Yes & 1969 & 2260 & -- & 287 & 0.965 & 0.141 & 0.702 & 2550 \\
\hline
\end{tabular}




\begin{tabular}{|c|c|c|c|c|c|c|c|c|c|c|c|}
\hline $\begin{array}{l}\text { U.S. } \\
\text { Geological } \\
\text { Survey } \\
\text { streamgage } \\
\text { number }\end{array}$ & $\begin{array}{l}\text { Segment } \\
\text { number }\end{array}$ & $\begin{array}{l}\text { Streamgage used in } \\
\text { regression analyses } \\
\text { (non-redundant) }\end{array}$ & $\begin{array}{c}\text { Discharge value } \\
\text { used in } \\
\text { adjustment } \\
\text { regression }\end{array}$ & $\begin{array}{l}\text { Water } \\
\text { year }\end{array}$ & $\begin{array}{l}\text { Observed annual } \\
\text { maximum peak } \\
\text { discharge }\left(\mathrm{ft}^{3} / \mathrm{s}\right)\end{array}$ & $\begin{array}{l}\text { NWIS peak } \\
\text { code }\end{array}$ & $\begin{array}{l}\text { Observed annual } \\
\text { maximum peak } \\
\text { discharge with } \\
\text { segment intercept } \\
\text { value subtracted } \\
\left(\mathrm{ft}^{3} / \mathrm{s}\right)\end{array}$ & $\begin{array}{l}\text { Observed } \\
\text { precipitation } \\
\text { (inches) }\end{array}$ & Urban fraction & $\begin{array}{l}\text { Exceedance } \\
\text { probability }\end{array}$ & $\begin{array}{c}\text { Urban-adjusted } \\
\text { annual maximum } \\
\text { peak discharge } \\
\left(\mathrm{ft}^{3} / \mathrm{s}\right)\end{array}$ \\
\hline 05545750 & 1 & Yes & Yes & 1970 & 1840 & -- & 234 & 1.41 & 0.145 & 0.799 & 2120 \\
\hline 05545750 & 1 & Yes & Yes & 1971 & 3010 & 9 & 383 & 0.105 & 0.15 & 0.52 & 3280 \\
\hline 05545750 & 1 & Yes & Yes & 1972 & 3280 & -- & 417 & 0.729 & 0.156 & 0.46 & 3530 \\
\hline 05545750 & 1 & Yes & Yes & 1973 & 6530 & -- & 830 & 2.27 & 0.161 & 0.0852 & 6700 \\
\hline 05545750 & 1 & Yes & Yes & 1974 & 3950 & -- & 502 & 0.279 & 0.167 & 0.337 & 4140 \\
\hline 05545750 & 1 & Yes & Yes & 1975 & 2840 & -- & 361 & 0.566 & 0.172 & 0.585 & 3030 \\
\hline 05545750 & 1 & Yes & Yes & 1976 & 3700 & -- & 471 & 1.31 & 0.178 & 0.39 & 3860 \\
\hline 05545750 & 0 & Yes & No & 1977 & 1120 & -- & 154 & 0.831 & 0.183 & 0.926 & 1390 \\
\hline 05545750 & 2 & Yes & Yes & 1978 & 2340 & -- & 346 & 1.11 & 0.189 & 0.626 & 2860 \\
\hline 05545750 & 2 & Yes & Yes & 1979 & 5010 & -- & 741 & 0.518 & 0.194 & 0.138 & 5830 \\
\hline 05545750 & 2 & Yes & Yes & 1980 & 1650 & -- & 244 & 1.04 & 0.2 & 0.812 & 2050 \\
\hline 05545750 & 2 & Yes & Yes & 1981 & 2230 & -- & 330 & 1.21 & 0.202 & 0.665 & 2700 \\
\hline 05545750 & 2 & Yes & Yes & 1982 & 3510 & -- & 519 & 1.5 & 0.205 & 0.342 & 4120 \\
\hline 05545750 & 2 & Yes & Yes & 1983 & 4020 & -- & 595 & 1.36 & 0.208 & 0.252 & 4650 \\
\hline 05545750 & 2 & Yes & Yes & 1984 & 2400 & -- & 355 & 0.206 & 0.211 & 0.626 & 2860 \\
\hline 05545750 & 2 & Yes & Yes & 1985 & 3220 & -- & 476 & 0.822 & 0.213 & 0.409 & 3770 \\
\hline 05545750 & 2 & Yes & Yes & 1986 & 3600 & -- & 532 & 0.234 & 0.216 & 0.332 & 4170 \\
\hline 05545750 & 2 & Yes & Yes & 1987 & 3810 & -- & 564 & 1.23 & 0.219 & 0.295 & 4380 \\
\hline 05545750 & 2 & Yes & Yes & 1988 & 2390 & -- & 354 & 0.149 & 0.222 & 0.637 & 2810 \\
\hline 05545750 & 2 & Yes & Yes & 1989 & 1850 & -- & 274 & 2 & 0.224 & 0.778 & 2210 \\
\hline 05545750 & 2 & Yes & Yes & 1990 & 2770 & -- & 410 & 0.385 & 0.227 & 0.535 & 3220 \\
\hline 05545750 & 2 & Yes & Yes & 1991 & 2810 & -- & 416 & 1.02 & 0.233 & 0.53 & 3240 \\
\hline 05545750 & 2 & Yes & Yes & 1992 & 1900 & -- & 281 & 0.437 & 0.24 & 0.776 & 2230 \\
\hline 05545750 & 2 & Yes & Yes & 1993 & 5060 & -- & 748 & 1.73 & 0.246 & 0.154 & 5610 \\
\hline 05545750 & 2 & Yes & Yes & 1994 & 3600 & 2,9 & 532 & 1.06 & 0.252 & 0.358 & 4030 \\
\hline 05545750 & 2 & Yes & Yes & 1995 & 1860 & -- & 275 & 1.29 & 0.258 & 0.796 & 2130 \\
\hline 05545750 & 2 & Yes & Yes & 1996 & 2920 & -- & 432 & 1.43 & 0.265 & 0.527 & 3250 \\
\hline 05545750 & 2 & Yes & Yes & 1997 & 3260 & -- & 482 & 1.49 & 0.271 & 0.447 & 3590 \\
\hline 05545750 & 2 & Yes & Yes & 1998 & 1830 & -- & 271 & 0.551 & 0.277 & 0.813 & 2050 \\
\hline 05545750 & 2 & Yes & Yes & 1999 & 4670 & -- & 691 & 1.38 & 0.283 & 0.204 & 5010 \\
\hline 05545750 & 2 & Yes & Yes & 2000 & 3410 & -- & 504 & 1.42 & 0.29 & 0.428 & 3680 \\
\hline 05545750 & 2 & Yes & Yes & 2001 & 2990 & -- & 442 & 2.17 & 0.297 & 0.537 & 3210 \\
\hline 05545750 & 2 & Yes & Yes & 2002 & 2480 & -- & 367 & 1.84 & 0.303 & 0.676 & 2650 \\
\hline 05545750 & 2 & Yes & Yes & 2003 & 1420 & -- & 210 & 0.769 & 0.31 & 0.906 & 1530 \\
\hline 05545750 & 2 & Yes & Yes & 2004 & 4510 & -- & 667 & 2.08 & 0.317 & 0.246 & 4700 \\
\hline
\end{tabular}




\begin{tabular}{|c|c|c|c|c|c|c|c|c|c|c|c|}
\hline $\begin{array}{l}\text { U.S. } \\
\text { Geological } \\
\text { Survey } \\
\text { streamgage } \\
\text { number }\end{array}$ & $\begin{array}{l}\text { Segment } \\
\text { number }\end{array}$ & $\begin{array}{l}\text { Streamgage used in } \\
\text { regression analyses } \\
\text { (non-redundant) }\end{array}$ & $\begin{array}{c}\text { Discharge value } \\
\text { used in } \\
\text { adjustment } \\
\text { regression }\end{array}$ & $\begin{array}{l}\text { Water } \\
\text { year }\end{array}$ & $\begin{array}{l}\text { Observed annual } \\
\text { maximum peak } \\
\text { discharge }\left(\mathrm{ft}^{3} / \mathrm{s}\right)\end{array}$ & $\begin{array}{l}\text { NWIS peak } \\
\text { code }\end{array}$ & $\begin{array}{l}\text { Observed annual } \\
\text { maximum peak } \\
\text { discharge with } \\
\text { segment intercept } \\
\text { value subtracted } \\
\left(\mathrm{ft}^{3} / \mathrm{s}\right)\end{array}$ & $\begin{array}{l}\text { Observed } \\
\text { precipitation } \\
\text { (inches) }\end{array}$ & Urban fraction & $\begin{array}{l}\text { Exceedance } \\
\text { probability }\end{array}$ & $\begin{array}{c}\text { Urban-adjusted } \\
\text { annual maximum } \\
\text { peak discharge } \\
\left(\mathrm{ft}^{3} / \mathrm{s}\right)\end{array}$ \\
\hline 05545750 & 2 & Yes & Yes & 2005 & 2260 & 2,9 & 334 & 0.542 & 0.324 & 0.747 & 2360 \\
\hline 05545750 & 2 & Yes & Yes & 2006 & 2980 & -- & 441 & 1.18 & 0.331 & 0.572 & 3080 \\
\hline 05545750 & 2 & Yes & Yes & 2007 & 4880 & -- & 722 & 1.14 & 0.338 & 0.209 & 4980 \\
\hline 05545750 & 2 & Yes & Yes & 2008 & 5960 & -- & 882 & 1.23 & 0.345 & 0.123 & 6040 \\
\hline 05545750 & 2 & Yes & Yes & 2009 & 3600 & -- & 532 & 1.41 & 0.352 & 0.44 & 3630 \\
\hline 05547755 & 1 & Yes & Yes & 1990 & 153 & -- & 337 & 0.548 & 0.0661 & 0.546 & 228 \\
\hline 05547755 & 1 & Yes & Yes & 1991 & 168 & -- & 370 & 0.898 & 0.0789 & 0.486 & 245 \\
\hline 05547755 & 1 & Yes & Yes & 1992 & 89 & $\mathrm{E}$ & 196 & 0.592 & 0.0917 & 0.836 & 141 \\
\hline 05547755 & 1 & Yes & Yes & 1993 & 312 & -- & 686 & 0.812 & 0.105 & 0.135 & 415 \\
\hline 05547755 & 1 & Yes & Yes & 1994 & 225 & -- & 495 & 0.605 & 0.117 & 0.314 & 305 \\
\hline 05547755 & 1 & Yes & Yes & 1995 & 142 & -- & 312 & 1.01 & 0.13 & 0.646 & 200 \\
\hline 05547755 & 1 & Yes & Yes & 1996 & 299 & D & 658 & 0.886 & 0.143 & 0.168 & 385 \\
\hline 05547755 & 1 & Yes & Yes & 1997 & 232 & -- & 510 & 2.08 & 0.156 & 0.319 & 303 \\
\hline 05547755 & 1 & Yes & Yes & 1998 & 149 & $E$ & 328 & 0.495 & 0.169 & 0.644 & 201 \\
\hline 05547755 & 1 & Yes & Yes & 1999 & 187 & -- & 411 & 1.02 & 0.181 & 0.492 & 244 \\
\hline 05547755 & 1 & Yes & Yes & 2000 & 200 & -- & 440 & 1.92 & 0.194 & 0.452 & 256 \\
\hline 05547755 & 1 & Yes & Yes & 2001 & 265 & -- & 583 & 0.745 & 0.217 & 0.271 & 323 \\
\hline 05547755 & 1 & Yes & Yes & 2002 & 236 & -- & 519 & 1.84 & 0.239 & 0.367 & 285 \\
\hline 05547755 & 1 & Yes & Yes & 2003 & 86 & $C, E$ & 189 & 0.676 & 0.261 & 0.912 & 111 \\
\hline 05547755 & 1 & Yes & Yes & 2004 & 196 & C & 431 & 2.12 & 0.284 & 0.546 & 228 \\
\hline 05547755 & 1 & Yes & Yes & 2005 & 102 & $C, E$ & 224 & 0.638 & 0.306 & 0.889 & 121 \\
\hline 05548150 & 1 & Yes & Yes & 1962 & 112 & -- & 189 & 0.0032 & 0.0335 & 0.821 & 117 \\
\hline 05548150 & 1 & Yes & No & 1963 & 60 & 4, B & 101 & 0.0032 & 0.0338 & 0.944 & 63.3 \\
\hline 05548150 & 1 & Yes & No & 1964 & 60 & $4, B$ & 101 & 0.0032 & 0.0341 & 0.944 & 63.3 \\
\hline 05548150 & 1 & Yes & Yes & 1965 & 120 & -- & 203 & 0.0931 & 0.0344 & 0.797 & 125 \\
\hline 05548150 & 1 & Yes & Yes & 1966 & 175 & -- & 296 & 0.502 & 0.0346 & 0.612 & 181 \\
\hline 05548150 & 1 & Yes & Yes & 1967 & 255 & -- & 431 & 0.84 & 0.0349 & 0.349 & 263 \\
\hline 05548150 & 1 & Yes & No & 1968 & 60 & $4, B$ & 101 & 0.0032 & 0.0352 & 0.944 & 63.1 \\
\hline 05548150 & 1 & Yes & Yes & 1969 & 187 & -- & 316 & 0.951 & 0.0355 & 0.567 & 193 \\
\hline 05548150 & 1 & Yes & Yes & 1970 & 182 & -- & 308 & 1.02 & 0.0358 & 0.588 & 188 \\
\hline 05548150 & 1 & Yes & Yes & 1971 & 315 & -- & 532 & 0.499 & 0.0362 & 0.219 & 323 \\
\hline 05548150 & 1 & Yes & Yes & 1972 & 196 & -- & 331 & 0.831 & 0.0366 & 0.532 & 202 \\
\hline 05548150 & 1 & Yes & Yes & 1973 & 240 & -- & 406 & 0.746 & 0.037 & 0.388 & 247 \\
\hline 05548150 & 1 & Yes & Yes & 1974 & 222 & -- & 375 & 0.543 & 0.0374 & 0.442 & 229 \\
\hline 05548150 & 1 & Yes & Yes & 1975 & 180 & -- & 304 & 0.294 & 0.0378 & 0.598 & 186 \\
\hline
\end{tabular}




\begin{tabular}{|c|c|c|c|c|c|c|c|c|c|c|c|}
\hline $\begin{array}{l}\text { U.S. } \\
\text { Geological } \\
\text { Survey } \\
\text { streamgage } \\
\text { number }\end{array}$ & $\begin{array}{l}\text { Segment } \\
\text { number }\end{array}$ & $\begin{array}{l}\text { Streamgage used in } \\
\text { regression analyses } \\
\text { (non-redundant) }\end{array}$ & $\begin{array}{c}\text { Discharge value } \\
\text { used in } \\
\text { adjustment } \\
\text { regression }\end{array}$ & $\begin{array}{l}\text { Water } \\
\text { year }\end{array}$ & $\begin{array}{l}\text { Observed annual } \\
\text { maximum peak } \\
\text { discharge }\left(\mathrm{ft}^{3} / \mathrm{s}\right)\end{array}$ & $\begin{array}{l}\text { NWIS peak } \\
\text { code }\end{array}$ & $\begin{array}{l}\text { Observed annual } \\
\text { maximum peak } \\
\text { discharge with } \\
\text { segment intercept } \\
\text { value subtracted } \\
\left(\mathrm{ft}^{3} / \mathrm{s}\right)\end{array}$ & $\begin{array}{l}\text { Observed } \\
\text { precipitation } \\
\text { (inches) }\end{array}$ & Urban fraction & $\begin{array}{l}\text { Exceedance } \\
\text { probability }\end{array}$ & $\begin{array}{c}\text { Urban-adjusted } \\
\text { annual maximum } \\
\text { peak discharge } \\
\left(\mathrm{ft}^{3} / \mathrm{s}\right)\end{array}$ \\
\hline 05548150 & 1 & Yes & Yes & 1976 & 202 & -- & 341 & 0.885 & 0.0382 & 0.51 & 208 \\
\hline 05548150 & 1 & Yes & No & 1977 & 60 & $4, B$ & 101 & 0.0032 & 0.0386 & 0.945 & 62.8 \\
\hline 05548150 & 1 & Yes & Yes & 1978 & 164 & -- & 277 & 1.31 & 0.039 & 0.651 & 169 \\
\hline 05548150 & 1 & Yes & Yes & 1979 & 200 & -- & 338 & 1.21 & 0.0394 & 0.519 & 206 \\
\hline 05548150 & 1 & Yes & Yes & 1980 & 135 & -- & 228 & 0.631 & 0.0398 & 0.754 & 139 \\
\hline 05548150 & 1 & Yes & Yes & 1981 & 110 & -- & 186 & 0.759 & 0.0401 & 0.83 & 114 \\
\hline 05548150 & 1 & Yes & Yes & 1982 & 260 & -- & 439 & 0.324 & 0.0403 & 0.34 & 266 \\
\hline 05548150 & 1 & Yes & Yes & 1983 & 328 & -- & 554 & 1.45 & 0.0406 & 0.198 & 335 \\
\hline 05548150 & 1 & Yes & Yes & 1984 & 220 & -- & 372 & 1.13 & 0.0408 & 0.451 & 226 \\
\hline 05548150 & 1 & Yes & Yes & 1985 & 105 & -- & 177 & 0.512 & 0.0411 & 0.844 & 109 \\
\hline 05548150 & 1 & Yes & Yes & 1986 & 375 & -- & 634 & 0.176 & 0.0413 & 0.144 & 383 \\
\hline 05548150 & 1 & Yes & Yes & 1987 & 110 & -- & 186 & 0.822 & 0.0415 & 0.831 & 114 \\
\hline 05548150 & 1 & Yes & Yes & 1988 & 130 & -- & 220 & 0.598 & 0.0418 & 0.769 & 134 \\
\hline 05548150 & 1 & Yes & Yes & 1989 & 136 & -- & 230 & 1.84 & 0.042 & 0.752 & 140 \\
\hline 05548150 & 1 & Yes & Yes & 1990 & 160 & -- & 270 & 0.532 & 0.0423 & 0.667 & 164 \\
\hline 05548150 & 1 & Yes & Yes & 1991 & 180 & -- & 304 & 0.927 & 0.0427 & 0.602 & 185 \\
\hline 05548150 & 1 & Yes & Yes & 1992 & 85 & -- & 144 & 0.64 & 0.043 & 0.898 & 87.8 \\
\hline 05548150 & 1 & Yes & Yes & 1993 & 350 & -- & 592 & 1.27 & 0.0434 & 0.173 & 357 \\
\hline 05548150 & 1 & Yes & Yes & 1994 & 350 & 2 & 592 & 0.723 & 0.0438 & 0.173 & 357 \\
\hline 05548150 & 1 & Yes & Yes & 1995 & 85 & -- & 144 & 1.01 & 0.0442 & 0.898 & 87.7 \\
\hline 05548150 & 1 & Yes & Yes & 1996 & 204 & -- & 345 & 0.907 & 0.0446 & 0.508 & 209 \\
\hline 05548150 & 1 & Yes & Yes & 1997 & 295 & -- & 499 & 1.85 & 0.045 & 0.265 & 301 \\
\hline 05548150 & 1 & Yes & Yes & 1998 & 333 & -- & 563 & 0.82 & 0.0454 & 0.194 & 339 \\
\hline 05548150 & 1 & Yes & Yes & 1999 & 517 & -- & 874 & 1.27 & 0.0458 & 0.0559 & 525 \\
\hline 05548150 & 1 & Yes & Yes & 2000 & 563 & -- & 952 & 4.51 & 0.0462 & 0.0438 & 571 \\
\hline 05548150 & 1 & Yes & Yes & 2001 & 355 & -- & 600 & 2.37 & 0.048 & 0.169 & 360 \\
\hline 05548150 & 1 & Yes & Yes & 2002 & 237 & -- & 401 & 1.63 & 0.0498 & 0.405 & 241 \\
\hline 05548150 & 1 & Yes & Yes & 2003 & 39 & -- & 66 & 1.82 & 0.0516 & 0.978 & 40 \\
\hline 05548150 & 1 & Yes & Yes & 2004 & 238 & -- & 402 & 2.53 & 0.0534 & 0.405 & 241 \\
\hline 05548150 & 1 & Yes & Yes & 2005 & 302 & -- & 510 & 0.68 & 0.0552 & 0.256 & 305 \\
\hline 05548150 & 1 & Yes & Yes & 2006 & 92 & -- & 155 & 1.52 & 0.057 & 0.886 & 93 \\
\hline 05548150 & 1 & Yes & Yes & 2007 & 285 & -- & 482 & 2.12 & 0.0588 & 0.294 & 287 \\
\hline 05548150 & 1 & Yes & Yes & 2008 & 250 & -- & 423 & 0.51 & 0.0605 & 0.378 & 251 \\
\hline 05548150 & 1 & Yes & Yes & 2009 & 314 & -- & 531 & 0.622 & 0.0623 & 0.236 & 315 \\
\hline 05548280 & 1 & Yes & No & 1966 & 1810 & 7 & 587 & 0.513 & 0.084 & 0.192 & 2120 \\
\hline
\end{tabular}




\begin{tabular}{|c|c|c|c|c|c|c|c|c|c|c|c|}
\hline $\begin{array}{c}\text { U.S. } \\
\text { Geological } \\
\text { Survey } \\
\text { streamgage } \\
\text { number }\end{array}$ & $\begin{array}{l}\text { Segment } \\
\text { number }\end{array}$ & $\begin{array}{l}\text { Streamgage used in } \\
\text { regression analyses } \\
\text { (non-redundant) }\end{array}$ & $\begin{array}{c}\text { Discharge value } \\
\text { used in } \\
\text { adjustment } \\
\text { regression }\end{array}$ & $\begin{array}{c}\text { Water } \\
\text { year }\end{array}$ & $\begin{array}{l}\text { Observed annual } \\
\text { maximum peak } \\
\text { discharge }\left(\mathrm{ft}^{3} / \mathrm{s}\right)\end{array}$ & $\begin{array}{c}\text { NWIS peak } \\
\text { code }\end{array}$ & $\begin{array}{c}\text { Observed annual } \\
\text { maximum peak } \\
\text { discharge with } \\
\text { segment intercept } \\
\text { value subtracted } \\
\left(\mathrm{ft}^{3} / \mathrm{s}\right)\end{array}$ & $\begin{array}{c}\text { Observed } \\
\text { precipitation } \\
\text { (inches) }\end{array}$ & Urban fraction & $\begin{array}{l}\text { Exceedance } \\
\text { probability }\end{array}$ & $\begin{array}{c}\text { Urban-adjusted } \\
\text { annual maximum } \\
\text { peak discharge } \\
\left(\mathrm{ft}^{3} / \mathrm{s}\right)\end{array}$ \\
\hline 05548280 & 1 & Yes & Yes & 1967 & 2120 & \begin{tabular}{l|l}
- & \\
\end{tabular} & 687 & 1.52 & 0.0853 & 0.127 & 2470 \\
\hline 05548280 & 1 & Yes & Yes & 1968 & 267 & $E$ & 87 & 0.679 & 0.0865 & 0.964 & 366 \\
\hline 05548280 & 1 & Yes & Yes & 1969 & 931 & -- & 302 & 0.981 & 0.0878 & 0.636 & 1130 \\
\hline 05548280 & 1 & Yes & Yes & 1970 & 799 & -- & 259 & 0.981 & 0.0891 & 0.722 & 983 \\
\hline 05548280 & 1 & Yes & Yes & 1971 & 2430 & D & 788 & 0.496 & 0.0914 & 0.0827 & 2810 \\
\hline 05548280 & 1 & Yes & Yes & 1972 & 1360 & $\mathrm{D}$ & 441 & 0.793 & 0.0937 & 0.373 & 1610 \\
\hline 05548280 & 1 & Yes & Yes & 1973 & 1610 & -- & 522 & 0.742 & 0.0961 & 0.267 & 1880 \\
\hline 05548280 & 1 & Yes & Yes & 1974 & 1990 & -- & 645 & 1.02 & 0.0984 & 0.158 & 2290 \\
\hline 05548280 & 1 & Yes & Yes & 1975 & 1040 & -- & 337 & 0.0916 & 0.101 & 0.575 & 1240 \\
\hline 05548280 & 1 & Yes & Yes & 1976 & 2120 & -- & 687 & 1.29 & 0.103 & 0.134 & 2430 \\
\hline 05548280 & 1 & Yes & Yes & 1977 & 362 & D & 117 & 0.833 & 0.105 & 0.942 & 469 \\
\hline 05548280 & 1 & Yes & Yes & 1978 & 1020 & -- & 331 & 1.36 & 0.108 & 0.596 & 1210 \\
\hline 05548280 & 1 & Yes & Yes & 1979 & 1820 & -- & 590 & 0.291 & 0.11 & 0.2 & 2080 \\
\hline 05548280 & 1 & Yes & Yes & 1980 & 648 & -- & 210 & 0.763 & 0.112 & 0.823 & 788 \\
\hline 05548280 & 1 & Yes & Yes & 1981 & 578 & -- & 187 & 0.759 & 0.114 & 0.859 & 708 \\
\hline 05548280 & 1 & Yes & Yes & 1982 & 1800 & 2 & 584 & 0.33 & 0.115 & 0.209 & 2050 \\
\hline 05548280 & 1 & Yes & Yes & 1983 & 1820 & -- & 590 & 1.51 & 0.117 & 0.204 & 2070 \\
\hline 05548280 & 1 & Yes & Yes & 1984 & 1280 & $\mathrm{E}$ & 415 & 0.576 & 0.119 & 0.434 & 1480 \\
\hline 05548280 & 1 & Yes & Yes & 1985 & 962 & \begin{tabular}{l|l}
-- & \\
\end{tabular} & 312 & 0.505 & 0.12 & 0.639 & 1130 \\
\hline 05548280 & 1 & Yes & Yes & 1986 & 2910 & -- & 944 & 1.35 & 0.122 & 0.0535 & 3260 \\
\hline 05548280 & 1 & Yes & Yes & 1988 & 1270 & -- & 412 & 0.207 & 0.125 & 0.444 & 1460 \\
\hline 05548280 & 1 & Yes & Yes & 1989 & 603 & -- & 196 & 1.88 & 0.126 & 0.852 & 724 \\
\hline 05548280 & 1 & Yes & Yes & 1990 & 866 & \begin{tabular}{l|l}
-- & \\
\end{tabular} & 281 & 0.422 & 0.128 & 0.705 & 1010 \\
\hline 05548280 & 1 & Yes & Yes & 1991 & 1180 & -- & 383 & 0.95 & 0.13 & 0.503 & 1350 \\
\hline 05548280 & 1 & Yes & Yes & 1992 & 571 & $E$ & 185 & 0.686 & 0.133 & 0.872 & 683 \\
\hline 05548280 & 1 & Yes & Yes & 1993 & 2230 & \begin{tabular}{l|l}
-- & \\
\end{tabular} & 723 & 1.48 & 0.135 & 0.125 & 2480 \\
\hline 05548280 & 1 & Yes & Yes & 1994 & 2830 & -- & 918 & 0.835 & 0.138 & 0.0607 & 3130 \\
\hline 05548280 & 1 & Yes & Yes & 1995 & 715 & $\mathrm{E}$ & 232 & 1.14 & 0.141 & 0.801 & 833 \\
\hline 05548280 & 1 & Yes & Yes & 1996 & 1200 & -- & 389 & 1.1 & 0.143 & 0.501 & 1360 \\
\hline 05548280 & 1 & Yes & Yes & 1997 & 1770 & \begin{tabular}{l|}
- \\
\end{tabular} & 574 & 1.85 & 0.146 & 0.237 & 1960 \\
\hline 05548280 & 1 & Yes & Yes & 1998 & 758 & -- & 246 & 1.13 & 0.148 & 0.781 & 872 \\
\hline 05548280 & 1 & Yes & Yes & 1999 & 2870 & -- & 931 & 1.31 & 0.151 & 0.0601 & 3140 \\
\hline 05548280 & 1 & Yes & Yes & 2000 & 2860 & -- & 927 & 2.63 & 0.154 & 0.0611 & 3120 \\
\hline 05548280 & 1 & Yes & Yes & 2001 & 1200 & -- & 389 & 0.716 & 0.164 & 0.519 & 1330 \\
\hline 05548280 & 1 & Yes & Yes & 2002 & 893 & -- & 290 & 1.69 & 0.174 & 0.72 & 988 \\
\hline
\end{tabular}




\begin{tabular}{|c|c|c|c|c|c|c|c|c|c|c|c|}
\hline $\begin{array}{c}\text { U.S. } \\
\text { Geological } \\
\text { Survey } \\
\text { streamgage } \\
\text { number } \\
\end{array}$ & $\begin{array}{c}\text { Segment } \\
\text { number }\end{array}$ & $\begin{array}{l}\text { Streamgage used in } \\
\text { regression analyses } \\
\text { (non-redundant) }\end{array}$ & $\begin{array}{c}\text { Discharge value } \\
\text { used in } \\
\text { adjustment } \\
\text { regression }\end{array}$ & $\begin{array}{c}\text { Water } \\
\text { year }\end{array}$ & $\begin{array}{c}\text { Observed annual } \\
\text { maximum peak } \\
\text { discharge }\left(\mathrm{ft}^{3} / \mathrm{s}\right)\end{array}$ & $\begin{array}{c}\text { NWIS peak } \\
\text { code }\end{array}$ & $\begin{array}{l}\text { Observed annual } \\
\text { maximum peak } \\
\text { discharge with } \\
\text { segment intercept } \\
\text { value subtracted } \\
\left(\mathrm{ft}^{3} / \mathrm{s}\right)\end{array}$ & $\begin{array}{l}\text { Observed } \\
\text { precipitation } \\
\text { (inches) }\end{array}$ & Urban fraction & $\begin{array}{l}\text { Exceedance } \\
\text { probability }\end{array}$ & $\begin{array}{c}\text { Urban-adjusted } \\
\text { annual maximum } \\
\text { peak discharge } \\
\left(\mathrm{ft}^{3} / \mathrm{s}\right)\end{array}$ \\
\hline 05548280 & 1 & Yes & Yes & 2003 & 230 & $E$ & 75 & 0.759 & 0.184 & 0.984 & 266 \\
\hline 05548280 & 1 & Yes & Yes & 2004 & 1270 & -- & 412 & 2.03 & 0.194 & 0.501 & 1360 \\
\hline 05548280 & 1 & Yes & Yes & 2005 & 868 & -- & 281 & 0.718 & 0.203 & 0.755 & 926 \\
\hline 05548280 & 1 & Yes & Yes & 2006 & 537 & -- & 174 & 1.55 & 0.213 & 0.913 & 573 \\
\hline 05548280 & 1 & Yes & Yes & 2007 & 1740 & -- & 564 & 1.89 & 0.223 & 0.297 & 1790 \\
\hline 05548280 & 1 & Yes & Yes & 2008 & 1590 & -- & 516 & 2.12 & 0.233 & 0.367 & 1620 \\
\hline 05548280 & 1 & Yes & Yes & 2009 & 1410 & -- & 457 & 0.701 & 0.243 & 0.465 & 1430 \\
\hline 05549000 & 1 & Yes & Yes & 1949 & 246 & -- & 668 & 0.314 & 0.000715 & 0.107 & 386 \\
\hline 05549000 & 1 & Yes & Yes & 1950 & 190 & -- & 516 & 1.41 & 0.00074 & 0.218 & 304 \\
\hline 05549000 & 1 & Yes & Yes & 1951 & 118 & $E$ & 320 & 2.08 & 0.00106 & 0.527 & 205 \\
\hline 05549000 & 1 & Yes & Yes & 1952 & 186 & -- & 505 & 0.502 & 0.00138 & 0.231 & 298 \\
\hline 05549000 & 1 & Yes & Yes & 1953 & 52 & $\mathrm{E}$ & 141 & 0.651 & 0.0017 & 0.888 & 108 \\
\hline 05549000 & 1 & Yes & Yes & 1954 & 167 & $E$ & 454 & 1.42 & 0.00202 & 0.297 & 273 \\
\hline 05549000 & 1 & Yes & Yes & 1955 & 187 & -- & 508 & 0.323 & 0.00234 & 0.228 & 299 \\
\hline 05549000 & 1 & Yes & Yes & 1956 & 59 & $\mathrm{E}$ & 160 & 0.414 & 0.00266 & 0.858 & 118 \\
\hline 05549000 & 1 & Yes & Yes & 1957 & 90 & $\mathrm{E}$ & 244 & 0.778 & 0.00298 & 0.696 & 164 \\
\hline 05549000 & 1 & Yes & Yes & 1958 & 34 & $\mathrm{E}$ & 92 & 0.756 & 0.0033 & 0.948 & 78.8 \\
\hline 05549000 & 1 & Yes & Yes & 1959 & 137 & $\mathrm{E}$ & 372 & 0.433 & 0.00363 & 0.422 & 233 \\
\hline 05549000 & 1 & Yes & Yes & 1960 & 149 & -- & 405 & 0.544 & 0.00395 & 0.368 & 249 \\
\hline 05549000 & 1 & Yes & Yes & 1961 & 64 & $\mathrm{E}$ & 174 & 0.979 & 0.00588 & 0.836 & 125 \\
\hline 05549000 & 1 & Yes & Yes & 1962 & 88 & $\mathrm{E}$ & 239 & 0.274 & 0.00781 & 0.711 & 160 \\
\hline 05549000 & 1 & Yes & Yes & 1963 & 228 & $3, \mathrm{E}$ & 619 & 1.28 & 0.00974 & 0.141 & 357 \\
\hline 05549000 & 1 & Yes & Yes & 1964 & 81 & $E$ & 220 & 1.56 & 0.0117 & 0.754 & 150 \\
\hline 05549000 & 0 & Yes & No & 1965 & 175 & 2 & 465 & 0.119 & 0.0136 & 0.289 & 276 \\
\hline 05549000 & 2 & Yes & Yes & 1966 & 178 & D & 462 & 0.51 & 0.0155 & 0.293 & 274 \\
\hline 05549000 & 2 & Yes & Yes & 1967 & 112 & $E$ & 291 & 1.3 & 0.0175 & 0.611 & 186 \\
\hline 05549000 & 2 & Yes & Yes & 1968 & 45 & $\mathrm{E}$ & 117 & 1.29 & 0.0194 & 0.926 & 92.1 \\
\hline 05549000 & 2 & Yes & Yes & 1969 & 80 & 2 & 208 & 1.36 & 0.0213 & 0.781 & 141 \\
\hline 05549000 & 2 & Yes & Yes & 1970 & 276 & -- & 717 & 1.56 & 0.0232 & 0.0916 & 404 \\
\hline 05549000 & 2 & Yes & Yes & 1971 & 220 & $E$ & 571 & 0.429 & 0.0272 & 0.181 & 325 \\
\hline 05549000 & 2 & Yes & Yes & 1972 & 223 & -- & 579 & 1.04 & 0.0311 & 0.177 & 328 \\
\hline 05549000 & 2 & Yes & Yes & 1973 & 162 & -- & 421 & 1.56 & 0.035 & 0.364 & 251 \\
\hline 05549000 & 2 & Yes & Yes & 1974 & 252 & -- & 654 & 1.14 & 0.0389 & 0.13 & 366 \\
\hline 05549000 & 2 & Yes & Yes & 1975 & 106 & -- & 275 & 0.848 & 0.0428 & 0.658 & 173 \\
\hline 05549000 & 2 & Yes & Yes & 1976 & 258 & -- & 670 & 1.46 & 0.0468 & 0.123 & 372 \\
\hline
\end{tabular}




\begin{tabular}{|c|c|c|c|c|c|c|c|c|c|c|c|}
\hline $\begin{array}{l}\text { U.S. } \\
\text { Geological } \\
\text { Survey } \\
\text { streamgage } \\
\text { number }\end{array}$ & $\begin{array}{l}\text { Segment } \\
\text { number }\end{array}$ & $\begin{array}{l}\text { Streamgage used in } \\
\text { regression analyses } \\
\text { (non-redundant) }\end{array}$ & $\begin{array}{c}\text { Discharge value } \\
\text { used in } \\
\text { adjustment } \\
\text { regression }\end{array}$ & $\begin{array}{l}\text { Water } \\
\text { year }\end{array}$ & $\begin{array}{l}\text { Observed annual } \\
\text { maximum peak } \\
\text { discharge }\left(\mathrm{ft}^{3} / \mathrm{s}\right)\end{array}$ & $\begin{array}{l}\text { NWIS peak } \\
\text { code }\end{array}$ & $\begin{array}{l}\text { Observed annual } \\
\text { maximum peak } \\
\text { discharge with } \\
\text { segment intercept } \\
\text { value subtracted } \\
\left(\mathrm{ft}^{3} / \mathrm{s}\right)\end{array}$ & $\begin{array}{l}\text { Observed } \\
\text { precipitation } \\
\text { (inches) }\end{array}$ & Urban fraction & $\begin{array}{l}\text { Exceedance } \\
\text { probability }\end{array}$ & $\begin{array}{c}\text { Urban-adjusted } \\
\text { annual maximum } \\
\text { peak discharge } \\
\left(\mathrm{ft}^{3} / \mathrm{s}\right)\end{array}$ \\
\hline 05549000 & 2 & Yes & Yes & 1977 & 42 & $E$ & 109 & 0.866 & 0.0507 & 0.939 & 83.8 \\
\hline 05549000 & 2 & Yes & Yes & 1978 & 209 & -- & 543 & 1.52 & 0.0546 & 0.218 & 303 \\
\hline 05549000 & 2 & Yes & Yes & 1979 & 145 & -- & 377 & 0.295 & 0.0585 & 0.456 & 223 \\
\hline 05549000 & 2 & Yes & Yes & 1980 & 61 & $\mathrm{E}$ & 158 & 0.884 & 0.0625 & 0.884 & 110 \\
\hline 05549000 & 2 & Yes & Yes & 1981 & 91 & $\mathrm{E}$ & 236 & 1.46 & 0.0663 & 0.754 & 150 \\
\hline 05549000 & 2 & Yes & Yes & 1982 & 196 & -- & 509 & 0.291 & 0.0701 & 0.267 & 284 \\
\hline 05549000 & 2 & Yes & Yes & 1983 & 190 & -- & 493 & 1.61 & 0.0739 & 0.289 & 276 \\
\hline 05549000 & 2 & Yes & Yes & 1984 & 128 & -- & 332 & 0.626 & 0.0778 & 0.566 & 196 \\
\hline 05549000 & 2 & Yes & Yes & 1985 & 116 & -- & 301 & 0.531 & 0.0816 & 0.633 & 179 \\
\hline 05549000 & 2 & Yes & Yes & 1986 & 345 & -- & 896 & 1.47 & 0.0854 & 0.0572 & 472 \\
\hline 05549000 & 2 & Yes & Yes & 1987 & 113 & -- & 293 & 0.679 & 0.0893 & 0.653 & 174 \\
\hline 05549000 & 2 & Yes & Yes & 1988 & 115 & -- & 299 & 0.913 & 0.0931 & 0.646 & 176 \\
\hline 05549000 & 2 & Yes & No & 1989 & 95 & $4, B$ & 247 & 0.119 & 0.0969 & 0.752 & 150 \\
\hline 05549000 & 2 & Yes & Yes & 1990 & 56 & -- & 145 & 0.432 & 0.101 & 0.912 & 98.1 \\
\hline 05549000 & 2 & Yes & Yes & 1991 & 162 & -- & 421 & 0.881 & 0.113 & 0.42 & 234 \\
\hline 05549000 & 2 & Yes & Yes & 1992 & 50 & -- & 130 & 0.967 & 0.125 & 0.934 & 86.9 \\
\hline 05549700 & 1 & Yes & No & 1960 & 378 & 7 & 1745 & 0.513 & 0.0211 & 0.00767 & 513 \\
\hline 05549700 & 1 & Yes & Yes & 1962 & 172 & -- & 794 & 0.262 & 0.0235 & 0.0687 & 251 \\
\hline 05549700 & 1 & Yes & Yes & 1963 & 20 & -- & 92 & 1.27 & 0.0247 & 0.952 & 43.4 \\
\hline 05549700 & 1 & Yes & Yes & 1964 & 24 & -- & 111 & 0.914 & 0.0258 & 0.933 & 49.6 \\
\hline 05549700 & 1 & Yes & Yes & 1965 & 114 & -- & 526 & 0.0625 & 0.027 & 0.221 & 171 \\
\hline 05549700 & 1 & Yes & Yes & 1966 & 111 & -- & 512 & 0.481 & 0.0282 & 0.238 & 167 \\
\hline 05549700 & 1 & Yes & Yes & 1967 & 45 & -- & 208 & 1.29 & 0.0293 & 0.785 & 79.3 \\
\hline 05549700 & 1 & Yes & Yes & 1968 & 47 & -- & 217 & 1.24 & 0.0305 & 0.769 & 82 \\
\hline 05549700 & 1 & Yes & Yes & 1969 & 98 & -- & 452 & 1.34 & 0.0317 & 0.317 & 150 \\
\hline 05549700 & 1 & Yes & Yes & 1970 & 37 & -- & 171 & 1.52 & 0.0329 & 0.852 & 67.8 \\
\hline 05549700 & 1 & Yes & Yes & 1971 & 263 & -- & 1214 & 0.423 & 0.0349 & 0.0191 & 361 \\
\hline 05549700 & 1 & Yes & Yes & 1972 & 75 & -- & 346 & 0.999 & 0.037 & 0.499 & 120 \\
\hline 05549700 & 1 & Yes & Yes & 1973 & 82 & -- & 379 & 1.46 & 0.0391 & 0.437 & 129 \\
\hline 05549700 & 1 & Yes & Yes & 1974 & 57 & -- & 263 & 1.11 & 0.0412 & 0.682 & 94.4 \\
\hline 05549700 & 1 & Yes & Yes & 1975 & 33 & -- & 152 & 0.814 & 0.0432 & 0.886 & 61.5 \\
\hline 05549700 & 1 & Yes & Yes & 1976 & 220 & -- & 1016 & 1.58 & 0.0453 & 0.0358 & 307 \\
\hline 05549850 & 1 & Yes & No & 1960 & 492 & 7 & 995 & 0.481 & 0.239 & 0.0614 & 799 \\
\hline 05549850 & 1 & Yes & Yes & 1962 & 239 & -- & 483 & 0.266 & 0.259 & 0.436 & 431 \\
\hline 05549850 & 1 & Yes & Yes & 1963 & 170 & -- & 344 & 0.288 & 0.27 & 0.691 & 326 \\
\hline
\end{tabular}




\begin{tabular}{|c|c|c|c|c|c|c|c|c|c|c|c|}
\hline $\begin{array}{l}\text { U.S. } \\
\text { Geological } \\
\text { Survey } \\
\text { streamgage } \\
\text { number }\end{array}$ & $\begin{array}{l}\text { Segment } \\
\text { number }\end{array}$ & $\begin{array}{l}\text { Streamgage used in } \\
\text { regression analyses } \\
\text { (non-redundant) }\end{array}$ & $\begin{array}{c}\text { Discharge value } \\
\text { used in } \\
\text { adjustment } \\
\text { regression }\end{array}$ & $\begin{array}{l}\text { Water } \\
\text { year }\end{array}$ & $\begin{array}{c}\text { Observed annual } \\
\text { maximum peak } \\
\text { discharge }\left(\mathrm{ft}^{3} / \mathrm{s}\right)\end{array}$ & $\begin{array}{l}\text { NWIS peak } \\
\text { code }\end{array}$ & $\begin{array}{l}\text { Observed annual } \\
\text { maximum peak } \\
\text { discharge with } \\
\text { segment intercept } \\
\text { value subtracted } \\
\left(\mathrm{ft}^{3} / \mathrm{s}\right)\end{array}$ & $\begin{array}{l}\text { Observed } \\
\text { precipitation } \\
\text { (inches) }\end{array}$ & Urban fraction & $\begin{array}{l}\text { Exceedance } \\
\text { probability }\end{array}$ & $\begin{array}{c}\text { Urban-adjusted } \\
\text { annual maximum } \\
\text { peak discharge } \\
\left(\mathrm{ft}^{3} / \mathrm{s}\right)\end{array}$ \\
\hline 05549850 & 1 & Yes & Yes & 1964 & 175 & -- & 354 & 3.17 & 0.28 & 0.681 & 330 \\
\hline 05549850 & 1 & Yes & Yes & 1965 & 188 & -- & 380 & 0.0205 & 0.29 & 0.643 & 345 \\
\hline 05549850 & 1 & Yes & Yes & 1966 & 260 & -- & 526 & 0.532 & 0.3 & 0.405 & 445 \\
\hline 05549850 & 1 & Yes & Yes & 1967 & 233 & -- & 471 & 1.24 & 0.311 & 0.499 & 403 \\
\hline 05549850 & 1 & Yes & Yes & 1968 & 224 & -- & 453 & 3.2 & 0.321 & 0.54 & 385 \\
\hline 05549850 & 1 & Yes & Yes & 1969 & 286 & -- & 578 & 2.09 & 0.331 & 0.358 & 467 \\
\hline 05549850 & 1 & Yes & Yes & 1970 & 287 & -- & 580 & 2.43 & 0.341 & 0.364 & 464 \\
\hline 05549850 & 1 & Yes & Yes & 1971 & 356 & -- & 720 & 0.395 & 0.353 & 0.22 & 542 \\
\hline 05549850 & 1 & Yes & Yes & 1972 & 238 & -- & 481 & 0.704 & 0.365 & 0.532 & 389 \\
\hline 05549850 & 1 & Yes & Yes & 1973 & 274 & -- & 554 & 1.81 & 0.377 & 0.43 & 434 \\
\hline 05549850 & 1 & Yes & Yes & 1974 & 233 & -- & 471 & 1.06 & 0.389 & 0.572 & 373 \\
\hline 05549850 & 1 & Yes & Yes & 1975 & 221 & -- & 447 & 1.51 & 0.401 & 0.622 & 353 \\
\hline 05549850 & 1 & Yes & Yes & 1976 & 268 & -- & 542 & 1.68 & 0.413 & 0.479 & 412 \\
\hline 05549850 & 1 & Yes & Yes & 1990 & 419 & -- & 847 & 2.21 & 0.582 & 0.257 & 517 \\
\hline 05549850 & 1 & Yes & Yes & 1991 & 305 & -- & 617 & 0.295 & 0.588 & 0.537 & 387 \\
\hline 05549850 & 1 & Yes & Yes & 1992 & 132 & $\mathrm{E}$ & 267 & 0.447 & 0.594 & 0.947 & 190 \\
\hline 05549850 & 1 & Yes & Yes & 1993 & 305 & -- & 617 & 0.847 & 0.599 & 0.548 & 383 \\
\hline 05549850 & 1 & Yes & Yes & 1994 & 552 & $\mathrm{E}$ & 1116 & 0.608 & 0.605 & 0.124 & 658 \\
\hline 05549850 & 1 & Yes & Yes & 1995 & 451 & -- & 912 & 1.86 & 0.611 & 0.226 & 539 \\
\hline 05549850 & 1 & Yes & Yes & 1996 & 690 & -- & 1396 & 1.98 & 0.616 & 0.0571 & 807 \\
\hline 05549900 & 1 & Yes & Yes & 1956 & 4 & -- & 277 & 0.364 & 0.77 & 0.977 & 6.34 \\
\hline 05549900 & 1 & Yes & Yes & 1957 & 31 & -- & 2148 & 2.16 & 0.815 & 0.0225 & 35.8 \\
\hline 05549900 & 1 & Yes & No & 1958 & 3 & $4, B$ & 208 & 0.00585 & 0.86 & 1 & 4.11 \\
\hline 05549900 & 1 & Yes & Yes & 1959 & 22 & -- & 1525 & 0.757 & 0.905 & 0.107 & 24 \\
\hline 05549900 & 1 & Yes & Yes & 1960 & 13 & 2 & 901 & 0.543 & 0.95 & 0.567 & 13.8 \\
\hline 05549900 & 1 & Yes & Yes & 1961 & 8 & 2 & 554 & 0.821 & 0.95 & 0.926 & 8.69 \\
\hline 05549900 & 1 & Yes & Yes & 1962 & 5 & 2 & 346 & 0.351 & 0.95 & 0.986 & 5.55 \\
\hline 05549900 & 1 & Yes & No & 1963 & 3 & 4, B & 208 & 0.00585 & 0.95 & 1 & 3.36 \\
\hline 05549900 & 1 & Yes & Yes & 1964 & 23 & -- & 1594 & 1.65 & 0.95 & 0.105 & 24.1 \\
\hline 05549900 & 1 & Yes & Yes & 1965 & 22 & -- & 1525 & 0.843 & 0.95 & 0.127 & 23 \\
\hline 05549900 & 1 & Yes & Yes & 1966 & 10 & 2 & 693 & 0.358 & 0.95 & 0.803 & 10.7 \\
\hline 05549900 & 1 & Yes & Yes & 1967 & 6 & -- & 416 & 0.00585 & 0.95 & 0.971 & 6.61 \\
\hline 05549900 & 1 & Yes & Yes & 1968 & 25 & -- & 1732 & 1.22 & 0.95 & 0.0733 & 26.1 \\
\hline 05549900 & 1 & Yes & Yes & 1969 & 23 & -- & 1594 & 0.793 & 0.95 & 0.105 & 24.1 \\
\hline 05549900 & 1 & Yes & Yes & 1970 & 45 & -- & 3118 & 0.762 & 0.95 & 0.011 & 46.7 \\
\hline
\end{tabular}




\begin{tabular}{|c|c|c|c|c|c|c|c|c|c|c|c|}
\hline $\begin{array}{l}\text { U.S. } \\
\text { Geological } \\
\text { Survey } \\
\text { streamgage } \\
\text { number }\end{array}$ & $\begin{array}{l}\text { Segment } \\
\text { number }\end{array}$ & $\begin{array}{l}\text { Streamgage used in } \\
\text { regression analyses } \\
\text { (non-redundant) }\end{array}$ & $\begin{array}{c}\text { Discharge value } \\
\text { used in } \\
\text { adjustment } \\
\text { regression }\end{array}$ & $\begin{array}{l}\text { Water } \\
\text { year }\end{array}$ & $\begin{array}{l}\text { Observed annual } \\
\text { maximum peak } \\
\text { discharge }\left(\mathrm{ft}^{3} / \mathrm{s}\right)\end{array}$ & $\begin{array}{l}\text { NWIS peak } \\
\text { code }\end{array}$ & $\begin{array}{l}\text { Observed annual } \\
\text { maximum peak } \\
\text { discharge with } \\
\text { segment intercept } \\
\text { value subtracted } \\
\left(\mathrm{ft}^{3} / \mathrm{s}\right)\end{array}$ & $\begin{array}{l}\text { Observed } \\
\text { precipitation } \\
\text { (inches) }\end{array}$ & Urban fraction & $\begin{array}{l}\text { Exceedance } \\
\text { probability }\end{array}$ & $\begin{array}{c}\text { Urban-adjusted } \\
\text { annual maximum } \\
\text { peak discharge } \\
\left(\mathrm{ft}^{3} / \mathrm{s}\right)\end{array}$ \\
\hline 05549900 & 1 & Yes & Yes & 1971 & 26 & -- & 1802 & 0.905 & 0.95 & 0.063 & 27.2 \\
\hline 05549900 & 1 & Yes & Yes & 1972 & 59 & -- & 4089 & 1.06 & 0.95 & 0.0027 & 61 \\
\hline 05549900 & 1 & Yes & Yes & 1973 & 5 & -- & 346 & 0.716 & 0.95 & 0.986 & 5.55 \\
\hline 05549900 & 1 & Yes & No & 1974 & 3 & $4, B$ & 208 & 0.00585 & 0.95 & 1 & 3.36 \\
\hline 05549900 & 1 & Yes & Yes & 1975 & 9 & -- & 624 & 0.806 & 0.95 & 0.869 & 9.69 \\
\hline 05549900 & 1 & Yes & Yes & 1977 & 3 & -- & 208 & 0.21 & 0.95 & 1 & 3.36 \\
\hline 05549900 & 1 & Yes & Yes & 1978 & 28 & -- & 1940 & 2.04 & 0.95 & 0.0467 & 29.2 \\
\hline 05549900 & 1 & Yes & Yes & 1979 & 3.4 & -- & 236 & 1.16 & 0.95 & 1 & 3.81 \\
\hline 05550300 & 1 & Yes & Yes & 1962 & 303 & -- & 285 & 0.264 & 0.0684 & 0.656 & 424 \\
\hline 05550300 & 1 & Yes & Yes & 1963 & 74 & -- & 70 & 1.42 & 0.0716 & 0.977 & 129 \\
\hline 05550300 & 1 & Yes & Yes & 1965 & 368 & -- & 346 & 0.711 & 0.078 & 0.535 & 500 \\
\hline 05550300 & 1 & Yes & Yes & 1966 & 392 & -- & 368 & 0.938 & 0.0812 & 0.49 & 528 \\
\hline 05550300 & 1 & Yes & Yes & 1967 & 333 & -- & 313 & 2.94 & 0.0844 & 0.613 & 454 \\
\hline 05550300 & 1 & Yes & Yes & 1968 & 251 & -- & 236 & 3.33 & 0.0876 & 0.765 & 353 \\
\hline 05550300 & 1 & Yes & Yes & 1969 & 318 & -- & 299 & 0.109 & 0.0908 & 0.644 & 432 \\
\hline 05550300 & 1 & Yes & Yes & 1970 & 306 & -- & 287 & 2.15 & 0.094 & 0.668 & 417 \\
\hline 05550300 & 1 & Yes & Yes & 1971 & 358 & -- & 336 & 0.346 & 0.098 & 0.574 & 478 \\
\hline 05550300 & 1 & Yes & Yes & 1972 & 454 & -- & 427 & 1.39 & 0.102 & 0.401 & 592 \\
\hline 05550300 & 1 & Yes & Yes & 1973 & 488 & -- & 458 & 2.14 & 0.106 & 0.357 & 629 \\
\hline 05550300 & 1 & Yes & Yes & 1974 & 311 & -- & 292 & 1.13 & 0.11 & 0.67 & 415 \\
\hline 05550300 & 1 & Yes & Yes & 1975 & 261 & -- & 245 & 1.52 & 0.114 & 0.764 & 355 \\
\hline 05550300 & 1 & Yes & Yes & 1976 & 324 & -- & 304 & 1.64 & 0.118 & 0.652 & 427 \\
\hline 05550300 & 1 & Yes & Yes & 1978 & 398 & -- & 374 & 2.39 & 0.126 & 0.517 & 511 \\
\hline 05550300 & 1 & Yes & Yes & 1979 & 432 & -- & 406 & 1.6 & 0.13 & 0.46 & 550 \\
\hline 05550300 & 1 & Yes & Yes & 1980 & 208 & -- & 195 & 0.374 & 0.134 & 0.855 & 283 \\
\hline 05550300 & 1 & Yes & Yes & 1998 & 221 & $C, E$ & 208 & 0.627 & 0.204 & 0.869 & 273 \\
\hline 05550300 & 1 & Yes & Yes & 1999 & 953 & C & 895 & 1.09 & 0.208 & 0.0759 & 1080 \\
\hline 05550300 & 1 & Yes & Yes & 2000 & 735 & C & 691 & 1.02 & 0.211 & 0.174 & 834 \\
\hline 05550300 & 1 & Yes & Yes & 2001 & 602 & C & 566 & 0.67 & 0.225 & 0.296 & 681 \\
\hline 05550300 & 1 & Yes & Yes & 2002 & 1650 & C & 1550 & 1.99 & 0.239 & 0.0163 & 1790 \\
\hline 05550300 & 1 & Yes & Yes & 2003 & 778 & C & 731 & 0.917 & 0.252 & 0.166 & 850 \\
\hline 05550300 & 1 & Yes & Yes & 2004 & 795 & C & 747 & 1.5 & 0.266 & 0.163 & 858 \\
\hline 05550300 & 1 & Yes & Yes & 2005 & 432 & $C, E$ & 406 & 0.768 & 0.28 & 0.591 & 468 \\
\hline 05550300 & 1 & Yes & Yes & 2006 & 595 & C & 559 & 0.299 & 0.293 & 0.354 & 630 \\
\hline 05550300 & 1 & Yes & Yes & 2007 & 1430 & C & 1344 & 2.2 & 0.307 & 0.0282 & 1480 \\
\hline
\end{tabular}




\begin{tabular}{|c|c|c|c|c|c|c|c|c|c|c|c|}
\hline $\begin{array}{c}\text { U.S. } \\
\text { Geological } \\
\text { Survey } \\
\text { streamgage } \\
\text { number } \\
\end{array}$ & $\begin{array}{l}\text { Segment } \\
\text { number }\end{array}$ & $\begin{array}{l}\text { Streamgage used in } \\
\text { regression analyses } \\
\text { (non-redundant) }\end{array}$ & $\begin{array}{c}\text { Discharge value } \\
\text { used in } \\
\text { adjustment } \\
\text { regression }\end{array}$ & $\begin{array}{c}\text { Water } \\
\text { year }\end{array}$ & $\begin{array}{c}\text { Observed annual } \\
\text { maximum peak } \\
\text { discharge }\left(\mathrm{ft}^{3} / \mathrm{s}\right)\end{array}$ & $\begin{array}{c}\text { NWIS peak } \\
\text { code }\end{array}$ & $\begin{array}{c}\text { Observed annual } \\
\text { maximum peak } \\
\text { discharge with } \\
\text { segment intercept } \\
\text { value subtracted } \\
\left(\mathrm{ft}^{3} / \mathrm{s}\right)\end{array}$ & $\begin{array}{c}\text { Observed } \\
\text { precipitation } \\
\text { (inches) }\end{array}$ & Urban fraction & $\begin{array}{l}\text { Exceedance } \\
\text { probability }\end{array}$ & $\begin{array}{c}\text { Urban-adjusted } \\
\text { annual maximum } \\
\text { peak discharge } \\
\left(\mathrm{ft}^{3} / \mathrm{s}\right)\end{array}$ \\
\hline 05550300 & 1 & Yes & Yes & 2008 & 1250 & $\mathrm{C}$ & 1174 & 3.78 & 0.321 & 0.0454 & 1280 \\
\hline 05550300 & 1 & Yes & Yes & 2009 & 886 & $C$ & 832 & 0.699 & 0.335 & 0.143 & 897 \\
\hline 05550430 & 1 & Yes & Yes & 1961 & 72 & -- & 475 & 1.27 & 0.178 & 0.383 & 99.8 \\
\hline 05550430 & 1 & Yes & Yes & 1962 & 88 & -- & 581 & 0.269 & 0.193 & 0.259 & 117 \\
\hline 05550430 & 1 & Yes & Yes & 1963 & 41 & -- & 270 & 2.01 & 0.208 & 0.774 & 59 \\
\hline 05550430 & 1 & Yes & Yes & 1964 & 22 & -- & 145 & 3.86 & 0.224 & 0.941 & 34.6 \\
\hline 05550430 & 1 & Yes & Yes & 1965 & 61 & -- & 402 & 0.579 & 0.239 & 0.561 & 80.8 \\
\hline 05550430 & 1 & Yes & Yes & 1966 & 92 & -- & 607 & 0.559 & 0.254 & 0.269 & 115 \\
\hline 05550430 & 1 & Yes & Yes & 1967 & 275 & -- & 1814 & 1.23 & 0.27 & 0.0117 & 321 \\
\hline 05550430 & 1 & Yes & Yes & 1968 & 71 & 2 & 468 & 4.14 & 0.285 & 0.482 & 88.6 \\
\hline 05550430 & 1 & Yes & Yes & 1969 & 88 & -- & 581 & 2.46 & 0.3 & 0.332 & 106 \\
\hline 05550430 & 1 & Yes & Yes & 1970 & 80 & -- & 528 & 2.87 & 0.316 & 0.415 & 96 \\
\hline 05550430 & 1 & Yes & Yes & 1971 & 76 & -- & 501 & 0.24 & 0.331 & 0.468 & 90.1 \\
\hline 05550430 & 1 & Yes & Yes & 1972 & 96 & -- & 633 & 1.75 & 0.346 & 0.301 & 110 \\
\hline 05550430 & 1 & Yes & Yes & 1973 & 164 & -- & 1082 & 2.2 & 0.361 & 0.0651 & 183 \\
\hline 05550430 & 1 & Yes & Yes & 1974 & 106 & -- & 699 & 1.13 & 0.376 & 0.252 & 118 \\
\hline 05550430 & 1 & Yes & Yes & 1975 & 86 & -- & 567 & 1.85 & 0.391 & 0.423 & 95.2 \\
\hline 05550430 & 1 & Yes & Yes & 1976 & 114 & -- & 752 & 1.89 & 0.406 & 0.224 & 123 \\
\hline 05550430 & 1 & Yes & Yes & 1977 & 63 & -- & 416 & 1.6 & 0.421 & 0.69 & 68.1 \\
\hline 05550450 & 1 & No & No & 1961 & 200 & -- & 411 & 1.28 & 0.133 & 0.452 & 292 \\
\hline 05550450 & 1 & No & No & 1962 & 275 & -- & 565 & 0.27 & 0.146 & 0.247 & 373 \\
\hline 05550450 & 1 & No & No & 1963 & 118 & -- & 243 & 1.74 & 0.159 & 0.792 & 185 \\
\hline 05550450 & 1 & No & No & 1964 & 146 & -- & 300 & 3.15 & 0.172 & 0.7 & 216 \\
\hline 05550450 & 1 & No & No & 1965 & 200 & -- & 411 & 0.579 & 0.185 & 0.495 & 277 \\
\hline 05550450 & 1 & No & No & 1966 & 263 & -- & 541 & 0.533 & 0.198 & 0.309 & 345 \\
\hline 05550450 & 1 & No & No & 1967 & 410 & -- & 843 & 1.21 & 0.211 & 0.0933 & 510 \\
\hline 05550450 & 1 & No & No & 1968 & 217 & 2 & 446 & 3.27 & 0.224 & 0.467 & 287 \\
\hline 05550450 & 0 & No & No & 1969 & 137 & -- & 297 & 2.08 & 0.237 & 0.75 & 200 \\
\hline 05550450 & 2 & No & No & 1970 & 190 & -- & 434 & 2.4 & 0.25 & 0.51 & 273 \\
\hline 05550450 & 2 & No & No & 1971 & 179 & -- & 409 & 0.235 & 0.27 & 0.576 & 253 \\
\hline 05550450 & 2 & No & No & 1972 & 290 & -- & 662 & 0.662 & 0.289 & 0.234 & 380 \\
\hline 05550450 & 2 & No & No & 1973 & 374 & -- & 854 & 1.83 & 0.309 & 0.122 & 475 \\
\hline 05550450 & 2 & No & No & 1974 & 254 & -- & 580 & 0.957 & 0.329 & 0.354 & 327 \\
\hline 05550450 & 2 & No & No & 1975 & 128 & -- & 292 & 0.643 & 0.348 & 0.822 & 174 \\
\hline 05550450 & 2 & No & No & 1976 & 297 & -- & 678 & 1.64 & 0.368 & 0.268 & 362 \\
\hline
\end{tabular}




\begin{tabular}{|c|c|c|c|c|c|c|c|c|c|c|c|}
\hline $\begin{array}{l}\text { U.S. } \\
\text { Geological } \\
\text { Survey } \\
\text { streamgage } \\
\text { number }\end{array}$ & $\begin{array}{l}\text { Segment } \\
\text { number }\end{array}$ & $\begin{array}{l}\text { Streamgage used in } \\
\text { regression analyses } \\
\text { (non-redundant) }\end{array}$ & $\begin{array}{c}\text { Discharge value } \\
\text { used in } \\
\text { adjustment } \\
\text { regression }\end{array}$ & $\begin{array}{l}\text { Water } \\
\text { year }\end{array}$ & $\begin{array}{c}\text { Observed annual } \\
\text { maximum peak } \\
\text { discharge }\left(\mathrm{ft}^{3} / \mathrm{s}\right)\end{array}$ & $\begin{array}{l}\text { NWIS peak } \\
\text { code }\end{array}$ & $\begin{array}{l}\text { Observed annual } \\
\text { maximum peak } \\
\text { discharge with } \\
\text { segment intercept } \\
\text { value subtracted } \\
\left(\mathrm{ft}^{3} / \mathrm{s}\right)\end{array}$ & $\begin{array}{l}\text { Observed } \\
\text { precipitation } \\
\text { (inches) }\end{array}$ & Urban fraction & $\begin{array}{l}\text { Exceedance } \\
\text { probability }\end{array}$ & $\begin{array}{c}\text { Urban-adjusted } \\
\text { annual maximum } \\
\text { peak discharge } \\
\left(\mathrm{ft}^{3} / \mathrm{s}\right)\end{array}$ \\
\hline 05550450 & 2 & No & No & 1977 & 111 & -- & 254 & 1.35 & 0.388 & 0.89 & 147 \\
\hline 05550470 & 1 & Yes & Yes & 1961 & 97 & -- & 401 & 1.3 & 0.389 & 0.688 & 151 \\
\hline 05550470 & 1 & Yes & Yes & 1962 & 75 & -- & 310 & 0.274 & 0.433 & 0.845 & 123 \\
\hline 05550470 & 1 & Yes & Yes & 1963 & 72 & -- & 297 & 1.21 & 0.476 & 0.883 & 115 \\
\hline 05550470 & 1 & Yes & Yes & 1964 & 167 & -- & 690 & 1.76 & 0.519 & 0.375 & 206 \\
\hline 05550470 & 1 & Yes & Yes & 1965 & 128 & -- & 529 & 0.577 & 0.562 & 0.639 & 159 \\
\hline 05550470 & 1 & Yes & Yes & 1966 & 164 & -- & 677 & 1.29 & 0.605 & 0.473 & 188 \\
\hline 05550470 & 1 & Yes & Yes & 1967 & 565 & -- & 2334 & 1.19 & 0.648 & 0.0131 & 549 \\
\hline 05550470 & 1 & Yes & Yes & 1968 & 144 & -- & 595 & 1.57 & 0.691 & 0.671 & 154 \\
\hline 05550470 & 1 & Yes & Yes & 1969 & 173 & -- & 715 & 0.808 & 0.735 & 0.554 & 173 \\
\hline 05550470 & 1 & Yes & Yes & 1970 & 226 & -- & 934 & 1.5 & 0.778 & 0.344 & 211 \\
\hline 05550470 & 1 & Yes & Yes & 1971 & 201 & -- & 830 & 0.226 & 0.791 & 0.477 & 187 \\
\hline 05550470 & 1 & Yes & Yes & 1972 & 255 & -- & 1053 & 1.36 & 0.805 & 0.254 & 229 \\
\hline 05550470 & 0 & Yes & No & 1973 & 248 & -- & 1115 & 1.35 & 0.818 & 0.224 & 239 \\
\hline 05550470 & 0 & Yes & No & 1974 & 156 & -- & 764 & 0.624 & 0.832 & 0.594 & 166 \\
\hline 05550470 & 2 & Yes & Yes & 1975 & 158 & -- & 843 & 0.824 & 0.845 & 0.52 & 179 \\
\hline 05550470 & 2 & Yes & Yes & 1976 & 116 & -- & 619 & 1.3 & 0.859 & 0.796 & 133 \\
\hline 05550470 & 2 & Yes & Yes & 1977 & 102 & -- & 544 & 0.868 & 0.872 & 0.881 & 116 \\
\hline 05550470 & 2 & Yes & Yes & 1978 & 168 & -- & 896 & 1.39 & 0.886 & 0.505 & 182 \\
\hline 05550470 & 2 & Yes & Yes & 1979 & 346 & -- & 1845 & 0.316 & 0.899 & 0.0479 & 363 \\
\hline 05550500 & 1 & Yes & Yes & 1952 & 303 & -- & 366 & 0.458 & 0.0753 & 0.489 & 529 \\
\hline 05550500 & 1 & Yes & Yes & 1953 & 282 & $\mathrm{E}$ & 341 & 1.08 & 0.0838 & 0.551 & 494 \\
\hline 05550500 & 1 & Yes & Yes & 1954 & 310 & -- & 375 & 1.22 & 0.0922 & 0.486 & 531 \\
\hline 05550500 & 1 & Yes & Yes & 1955 & 291 & -- & 352 & 2.97 & 0.101 & 0.542 & 499 \\
\hline 05550500 & 1 & Yes & Yes & 1956 & 175 & -- & 212 & 0.728 & 0.109 & 0.819 & 333 \\
\hline 05550500 & 1 & Yes & Yes & 1957 & 286 & -- & 346 & 2.1 & 0.117 & 0.57 & 483 \\
\hline 05550500 & 1 & Yes & Yes & 1958 & 210 & -- & 254 & 0.223 & 0.126 & 0.755 & 376 \\
\hline 05550500 & 1 & Yes & Yes & 1959 & 258 & -- & 312 & 0.402 & 0.134 & 0.649 & 437 \\
\hline 05550500 & 1 & Yes & Yes & 1960 & 340 & -- & 411 & 0.466 & 0.143 & 0.46 & 548 \\
\hline 05550500 & 1 & Yes & Yes & 1961 & 327 & -- & 396 & 1.3 & 0.162 & 0.505 & 520 \\
\hline 05550500 & 1 & Yes & Yes & 1962 & 512 & -- & 619 & 0.272 & 0.18 & 0.211 & 741 \\
\hline 05550500 & 1 & Yes & Yes & 1963 & 171 & $E$ & 207 & 1.46 & 0.199 & 0.868 & 297 \\
\hline 05550500 & 1 & Yes & Yes & 1964 & 263 & -- & 318 & 2.43 & 0.218 & 0.699 & 409 \\
\hline 05550500 & 1 & Yes & Yes & 1965 & 350 & 2 & 423 & 0.0915 & 0.237 & 0.518 & 512 \\
\hline 05550500 & 1 & Yes & Yes & 1966 & 476 & -- & 576 & 0.509 & 0.255 & 0.305 & 656 \\
\hline
\end{tabular}




\begin{tabular}{|c|c|c|c|c|c|c|c|c|c|c|c|}
\hline $\begin{array}{l}\text { U.S. } \\
\text { Geological } \\
\text { Survey } \\
\text { streamgage } \\
\text { number }\end{array}$ & $\begin{array}{l}\text { Segment } \\
\text { number }\end{array}$ & $\begin{array}{l}\text { Streamgage used in } \\
\text { regression analyses } \\
\text { (non-redundant) }\end{array}$ & $\begin{array}{c}\text { Discharge value } \\
\text { used in } \\
\text { adjustment } \\
\text { regression }\end{array}$ & $\begin{array}{l}\text { Water } \\
\text { year }\end{array}$ & $\begin{array}{l}\text { Observed annual } \\
\text { maximum peak } \\
\text { discharge }\left(\mathrm{ft}^{3} / \mathrm{s}\right)\end{array}$ & $\begin{array}{l}\text { NWIS peak } \\
\text { code }\end{array}$ & $\begin{array}{l}\text { Observed annual } \\
\text { maximum peak } \\
\text { discharge with } \\
\text { segment intercept } \\
\text { value subtracted } \\
\left(\mathrm{ft}^{3} / \mathrm{s}\right)\end{array}$ & $\begin{array}{l}\text { Observed } \\
\text { precipitation } \\
\text { (inches) }\end{array}$ & Urban fraction & $\begin{array}{l}\text { Exceedance } \\
\text { probability }\end{array}$ & $\begin{array}{c}\text { Urban-adjusted } \\
\text { annual maximum } \\
\text { peak discharge } \\
\left(\mathrm{ft}^{3} / \mathrm{s}\right)\end{array}$ \\
\hline 05550500 & 1 & Yes & Yes & 1967 & 797 & -- & 964 & 1.2 & 0.274 & 0.0727 & 1040 \\
\hline 05550500 & 1 & Yes & Yes & 1968 & 298 & -- & 360 & 2.4 & 0.293 & 0.679 & 420 \\
\hline 05550500 & 1 & Yes & Yes & 1969 & 247 & -- & 299 & 1.71 & 0.312 & 0.791 & 351 \\
\hline 05550500 & 1 & Yes & Yes & 1970 & 560 & -- & 677 & 1.94 & 0.33 & 0.244 & 708 \\
\hline 05550500 & 1 & Yes & Yes & 1971 & 296 & $\mathrm{E}$ & 358 & 0.374 & 0.344 & 0.722 & 396 \\
\hline 05550500 & 1 & Yes & Yes & 1972 & 294 & -- & 356 & 0.653 & 0.358 & 0.736 & 387 \\
\hline 05550500 & 1 & Yes & Yes & 1973 & 896 & -- & 1084 & 1.46 & 0.372 & 0.0666 & 1070 \\
\hline 05550500 & 1 & Yes & Yes & 1974 & 552 & -- & 668 & 1.05 & 0.386 & 0.292 & 666 \\
\hline 05550500 & 1 & Yes & Yes & 1975 & 488 & $\mathrm{D}$ & 590 & 1.12 & 0.4 & 0.399 & 589 \\
\hline 05550500 & 1 & Yes & Yes & 1976 & 490 & D & 593 & 1.39 & 0.414 & 0.408 & 583 \\
\hline 05550500 & 1 & Yes & Yes & 1977 & 117 & $E$ & 142 & 1.1 & 0.428 & 0.976 & 157 \\
\hline 05550500 & 1 & Yes & Yes & 1978 & 571 & $E$ & 691 & 1.65 & 0.442 & 0.308 & 653 \\
\hline 05550500 & 1 & Yes & Yes & 1979 & 765 & -- & 925 & 0.292 & 0.456 & 0.146 & 853 \\
\hline 05550500 & 1 & Yes & Yes & 1980 & 370 & $2, E$ & 448 & 1.02 & 0.47 & 0.68 & 421 \\
\hline 05550500 & 1 & Yes & Yes & 1981 & 304 & $\mathrm{E}$ & 368 & 0.566 & 0.477 & 0.798 & 347 \\
\hline 05550500 & 1 & Yes & Yes & 1982 & 537 & $\mathrm{E}$ & 650 & 0.49 & 0.485 & 0.396 & 591 \\
\hline 05550500 & 1 & Yes & Yes & 1983 & 761 & -- & 920 & 1.84 & 0.493 & 0.164 & 820 \\
\hline 05550500 & 1 & Yes & Yes & 1984 & 394 & -- & 477 & 0.723 & 0.501 & 0.662 & 431 \\
\hline 05550500 & 1 & Yes & Yes & 1985 & 633 & -- & 766 & 1.48 & 0.508 & 0.279 & 675 \\
\hline 05550500 & 1 & Yes & Yes & 1986 & 719 & -- & 870 & 0.815 & 0.516 & 0.199 & 759 \\
\hline 05550500 & 1 & Yes & Yes & 1987 & 625 & -- & 756 & 1.78 & 0.524 & 0.3 & 657 \\
\hline 05550500 & 1 & Yes & Yes & 1988 & 441 & -- & 533 & 1 & 0.532 & 0.605 & 463 \\
\hline 05550500 & 1 & Yes & Yes & 1989 & 700 & -- & 847 & 1.91 & 0.54 & 0.23 & 723 \\
\hline 05550500 & 1 & Yes & Yes & 1990 & 669 & -- & 809 & 1.76 & 0.547 & 0.266 & 687 \\
\hline 05550500 & 1 & Yes & Yes & 1991 & 399 & -- & 483 & 1.64 & 0.55 & 0.698 & 411 \\
\hline 05550500 & 1 & Yes & Yes & 1992 & 212 & $\mathrm{E}$ & 256 & 0.381 & 0.553 & 0.942 & 220 \\
\hline 05550500 & 1 & Yes & Yes & 1993 & 691 & C & 836 & 0.906 & 0.555 & 0.248 & 704 \\
\hline 05550500 & 1 & Yes & Yes & 1994 & 779 & C & 942 & 0.616 & 0.558 & 0.181 & 791 \\
\hline 05550500 & 1 & Yes & Yes & 1995 & 580 & C & 702 & 2.36 & 0.561 & 0.4 & 588 \\
\hline 05550500 & 1 & Yes & Yes & 1996 & 437 & C & 529 & 1.76 & 0.564 & 0.641 & 443 \\
\hline 05550500 & 1 & Yes & Yes & 1997 & 1180 & C & 1427 & 2.76 & 0.566 & 0.046 & 1190 \\
\hline 05550500 & 1 & Yes & Yes & 1998 & 400 & $\mathrm{C}, \mathrm{E}$ & 484 & 1.55 & 0.569 & 0.712 & 403 \\
\hline 05550500 & 1 & Yes & Yes & 1999 & 882 & C & 1067 & 1.86 & 0.572 & 0.131 & 884 \\
\hline 05550500 & 1 & Yes & Yes & 2000 & 555 & $\mathrm{C}, \mathrm{E}$ & 671 & 1.36 & 0.574 & 0.451 & 555 \\
\hline 05550500 & 1 & Yes & Yes & 2001 & 600 & $C$ & 726 & 0.734 & 0.574 & 0.382 & 600 \\
\hline
\end{tabular}




\begin{tabular}{|c|c|c|c|c|c|c|c|c|c|c|c|}
\hline $\begin{array}{l}\text { U.S. } \\
\text { Geological } \\
\text { Survey } \\
\text { streamgage } \\
\text { number }\end{array}$ & $\begin{array}{l}\text { Segment } \\
\text { number }\end{array}$ & $\begin{array}{l}\text { Streamgage used in } \\
\text { regression analyses } \\
\text { (non-redundant) }\end{array}$ & $\begin{array}{c}\text { Discharge value } \\
\text { used in } \\
\text { adjustment } \\
\text { regression }\end{array}$ & $\begin{array}{l}\text { Water } \\
\text { year }\end{array}$ & $\begin{array}{c}\text { Observed annual } \\
\text { maximum peak } \\
\text { discharge }\left(\mathrm{ft}^{3} / \mathrm{s}\right)\end{array}$ & $\begin{array}{l}\text { NWIS peak } \\
\text { code }\end{array}$ & $\begin{array}{l}\text { Observed annual } \\
\text { maximum peak } \\
\text { discharge with } \\
\text { segment intercept } \\
\text { value subtracted } \\
\left(\mathrm{ft}^{3} / \mathrm{s}\right)\end{array}$ & $\begin{array}{l}\text { Observed } \\
\text { precipitation } \\
\text { (inches) }\end{array}$ & Urban fraction & $\begin{array}{l}\text { Exceedance } \\
\text { probability }\end{array}$ & $\begin{array}{c}\text { Urban-adjusted } \\
\text { annual maximum } \\
\text { peak discharge } \\
\left(\mathrm{ft}^{3} / \mathrm{s}\right)\end{array}$ \\
\hline 05550500 & 1 & Yes & Yes & 2002 & 637 & C & 770 & 3.95 & 0.574 & 0.328 & 637 \\
\hline 05550500 & 1 & Yes & Yes & 2003 & 552 & $\mathrm{C}$ & 668 & 0.955 & 0.574 & 0.455 & 552 \\
\hline 05550500 & 1 & Yes & Yes & 2004 & 367 & C & 444 & 1.46 & 0.574 & 0.768 & 367 \\
\hline 05550500 & 1 & Yes & Yes & 2005 & 407 & $C, E$ & 492 & 0.823 & 0.574 & 0.705 & 407 \\
\hline 05550500 & 1 & Yes & Yes & 2006 & 461 & C & 558 & 1.32 & 0.574 & 0.608 & 461 \\
\hline 05550500 & 1 & Yes & Yes & 2007 & 1120 & C & 1355 & 2.89 & 0.574 & 0.0559 & 1120 \\
\hline 05550500 & 1 & Yes & Yes & 2008 & 1560 & C & 1887 & 3.7 & 0.574 & 0.0187 & 1560 \\
\hline 05550500 & 1 & Yes & Yes & 2009 & 1170 & C & 1415 & 0.935 & 0.574 & 0.048 & 1170 \\
\hline 05551030 & 1 & Yes & Yes & 1962 & 125 & -- & 199 & 0.282 & 0.0701 & 0.822 & 245 \\
\hline 05551030 & 1 & Yes & Yes & 1963 & 73 & 2 & 116 & 1.22 & 0.0765 & 0.937 & 166 \\
\hline 05551030 & 1 & Yes & Yes & 1964 & 56 & 2 & 89 & 0.675 & 0.083 & 0.961 & 136 \\
\hline 05551030 & 1 & Yes & Yes & 1965 & 105 & 2 & 167 & 0.132 & 0.0894 & 0.881 & 212 \\
\hline 05551030 & 1 & Yes & Yes & 1966 & 201 & -- & 320 & 0.472 & 0.0959 & 0.609 & 344 \\
\hline 05551030 & 1 & Yes & Yes & 1967 & 687 & -- & 1093 & 1.18 & 0.102 & 0.0326 & 989 \\
\hline 05551030 & 1 & Yes & Yes & 1968 & 226 & -- & 360 & 1.53 & 0.109 & 0.532 & 375 \\
\hline 05551030 & 1 & Yes & Yes & 1969 & 158 & 2 & 251 & 1.31 & 0.115 & 0.754 & 280 \\
\hline 05551030 & 1 & Yes & Yes & 1970 & 292 & -- & 465 & 1.45 & 0.122 & 0.359 & 460 \\
\hline 05551030 & 1 & Yes & Yes & 1971 & 408 & -- & 649 & 0.362 & 0.133 & 0.169 & 602 \\
\hline 05551030 & 1 & Yes & Yes & 1972 & 671 & -- & 1068 & 0.626 & 0.144 & 0.0394 & 941 \\
\hline 05551030 & 1 & Yes & Yes & 1973 & 221 & -- & 352 & 1.36 & 0.155 & 0.591 & 351 \\
\hline 05551030 & 1 & Yes & Yes & 1974 & 408 & -- & 649 & 1.17 & 0.166 & 0.182 & 585 \\
\hline 05551030 & 1 & Yes & Yes & 1975 & 325 & -- & 517 & 0.0347 & 0.177 & 0.325 & 478 \\
\hline 05551030 & 1 & Yes & Yes & 1976 & 275 & -- & 438 & 1.13 & 0.188 & 0.452 & 412 \\
\hline 05551030 & 1 & Yes & Yes & 1978 & 156 & -- & 248 & 1.37 & 0.211 & 0.811 & 250 \\
\hline 05551030 & 1 & Yes & Yes & 1979 & 352 & -- & 560 & 0.76 & 0.222 & 0.301 & 492 \\
\hline 05551030 & 0 & Yes & No & 2003 & 100 & C & NA & 1.74 & 0.493 & NA & NA \\
\hline 05551030 & 0 & Yes & No & 2004 & 111 & C & NA & 1.28 & 0.502 & NA & NA \\
\hline 05551030 & 0 & Yes & No & 2005 & 62 & C & NA & 0.583 & 0.511 & NA & NA \\
\hline 05551030 & 0 & Yes & No & 2006 & 77 & C & NA & 0.717 & 0.52 & NA & NA \\
\hline 05551050 & 1 & Yes & No & 1957 & 890 & 7 & 3640 & 2.27 & 0.0815 & 0 & 1310 \\
\hline 05551050 & 1 & Yes & Yes & 1962 & 48 & -- & 196 & 0.289 & 0.107 & 0.842 & 105 \\
\hline 05551050 & 1 & Yes & Yes & 1963 & 33 & -- & 135 & 1.22 & 0.112 & 0.928 & 82.5 \\
\hline 05551050 & 1 & Yes & Yes & 1964 & 25 & -- & 102 & 0.683 & 0.118 & 0.956 & 67.7 \\
\hline 05551050 & 1 & Yes & Yes & 1965 & 43 & 2 & 176 & 0.134 & 0.124 & 0.881 & 96.5 \\
\hline 05551050 & 1 & Yes & Yes & 1966 & 115 & -- & 470 & 1.37 & 0.129 & 0.356 & 200 \\
\hline
\end{tabular}




\begin{tabular}{|c|c|c|c|c|c|c|c|c|c|c|c|}
\hline $\begin{array}{l}\text { U.S. } \\
\text { Geological } \\
\text { Survey } \\
\text { streamgage } \\
\text { number }\end{array}$ & $\begin{array}{l}\text { Segment } \\
\text { number }\end{array}$ & $\begin{array}{l}\text { Streamgage used in } \\
\text { regression analyses } \\
\text { (non-redundant) }\end{array}$ & $\begin{array}{c}\text { Discharge value } \\
\text { used in } \\
\text { adjustment } \\
\text { regression }\end{array}$ & $\begin{array}{l}\text { Water } \\
\text { year }\end{array}$ & $\begin{array}{c}\text { Observed annual } \\
\text { maximum peak } \\
\text { discharge }\left(\mathrm{ft}^{3} / \mathrm{s}\right)\end{array}$ & $\begin{array}{l}\text { NWIS peak } \\
\text { code }\end{array}$ & $\begin{array}{l}\text { Observed annual } \\
\text { maximum peak } \\
\text { discharge with } \\
\text { segment intercept } \\
\text { value subtracted } \\
\left(\mathrm{ft}^{3} / \mathrm{s}\right)\end{array}$ & $\begin{array}{l}\text { Observed } \\
\text { precipitation } \\
\text { (inches) }\end{array}$ & Urban fraction & $\begin{array}{l}\text { Exceedance } \\
\text { probability }\end{array}$ & $\begin{array}{c}\text { Urban-adjusted } \\
\text { annual maximum } \\
\text { peak discharge } \\
\left(\mathrm{ft}^{3} / \mathrm{s}\right)\end{array}$ \\
\hline 05551050 & 1 & Yes & Yes & 1967 & 617 & -- & 2523 & 1.17 & 0.135 & 0 & 876 \\
\hline 05551050 & 1 & Yes & Yes & 1968 & 85 & -- & 348 & 1.49 & 0.141 & 0.587 & 155 \\
\hline 05551050 & 1 & Yes & Yes & 1969 & 69 & -- & 282 & 1.27 & 0.146 & 0.715 & 132 \\
\hline 05551050 & 1 & Yes & Yes & 1970 & 214 & -- & 875 & 1.42 & 0.152 & 0.071 & 334 \\
\hline 05551050 & 1 & Yes & Yes & 1971 & 122 & -- & 499 & 0.357 & 0.155 & 0.334 & 205 \\
\hline 05551050 & 1 & Yes & Yes & 1972 & 97 & -- & 397 & 0.562 & 0.158 & 0.499 & 170 \\
\hline 05551050 & 1 & Yes & Yes & 1973 & 106 & -- & 434 & 1.37 & 0.161 & 0.436 & 183 \\
\hline 05551050 & 1 & Yes & Yes & 1974 & 102 & -- & 417 & 1.2 & 0.164 & 0.467 & 177 \\
\hline 05551050 & 1 & Yes & Yes & 1975 & 88 & -- & 360 & 0.0356 & 0.167 & 0.583 & 156 \\
\hline 05551050 & 1 & Yes & Yes & 1976 & 142 & -- & 581 & 1.1 & 0.169 & 0.244 & 228 \\
\hline 05551050 & 1 & Yes & Yes & 1977 & 35 & -- & 143 & 0.854 & 0.172 & 0.932 & 80.1 \\
\hline 05551050 & 1 & Yes & Yes & 1978 & 78 & -- & 319 & 1.36 & 0.175 & 0.665 & 140 \\
\hline 05551050 & 1 & Yes & Yes & 1979 & 194 & -- & 793 & 0.332 & 0.178 & 0.103 & 300 \\
\hline 05551060 & 1 & No & No & 1957 & 204 & 7 & 626 & 2.27 & 0.108 & 0.174 & 380 \\
\hline 05551060 & 1 & No & No & 1962 & 84 & -- & 258 & 0.289 & 0.138 & 0.756 & 194 \\
\hline 05551060 & 1 & No & No & 1963 & 54 & -- & 166 & 1.22 & 0.145 & 0.901 & 147 \\
\hline 05551060 & 1 & No & No & 1964 & 42 & -- & 129 & 0.683 & 0.151 & 0.94 & 126 \\
\hline 05551060 & 1 & No & No & 1965 & 75 & -- & 230 & 0.134 & 0.158 & 0.812 & 177 \\
\hline 05551060 & 1 & No & No & 1966 & 210 & -- & 645 & 1.37 & 0.165 & 0.185 & 372 \\
\hline 05551060 & 1 & No & No & 1967 & 954 & -- & 2928 & 1.17 & 0.172 & 0 & 1430 \\
\hline 05551060 & 1 & No & No & 1968 & 150 & -- & 460 & 1.49 & 0.179 & 0.406 & 288 \\
\hline 05551060 & 1 & No & No & 1969 & 124 & -- & 381 & 1.27 & 0.186 & 0.556 & 245 \\
\hline 05551060 & 1 & No & No & 1970 & 264 & -- & 810 & 1.42 & 0.193 & 0.0996 & 449 \\
\hline 05551060 & 1 & No & No & 1971 & 157 & -- & 482 & 0.357 & 0.2 & 0.39 & 293 \\
\hline 05551060 & 1 & No & No & 1972 & 152 & -- & 467 & 0.562 & 0.208 & 0.42 & 284 \\
\hline 05551060 & 1 & No & No & 1973 & 134 & -- & 411 & 1.37 & 0.216 & 0.522 & 254 \\
\hline 05551060 & 1 & No & No & 1974 & 98 & -- & 301 & 1.2 & 0.223 & 0.734 & 200 \\
\hline 05551060 & 1 & No & No & 1975 & 152 & -- & 467 & 0.0356 & 0.231 & 0.439 & 278 \\
\hline 05551060 & 1 & No & No & 1976 & 142 & -- & 436 & 1.1 & 0.239 & 0.497 & 261 \\
\hline 05551060 & 1 & No & No & 1977 & 47 & -- & 144 & 0.633 & 0.247 & 0.947 & 122 \\
\hline 05551060 & 1 & No & No & 1978 & 101 & -- & 310 & 1.36 & 0.254 & 0.739 & 199 \\
\hline 05551060 & 1 & No & No & 1979 & 149 & -- & 457 & 0.712 & 0.262 & 0.48 & 266 \\
\hline 05551200 & 1 & Yes & Yes & 1961 & 425 & -- & 218 & 1.53 & 0.0421 & 0.772 & 929 \\
\hline 05551200 & 1 & Yes & Yes & 1962 & 855 & -- & 439 & 0.277 & 0.0476 & 0.345 & 1570 \\
\hline 05551200 & 1 & Yes & Yes & 1963 & 360 & $E$ & 185 & 1.17 & 0.0531 & 0.837 & 819 \\
\hline
\end{tabular}




\begin{tabular}{|c|c|c|c|c|c|c|c|c|c|c|c|}
\hline $\begin{array}{c}\text { U.S. } \\
\text { Geological } \\
\text { Survey } \\
\text { streamgage } \\
\text { number } \\
\end{array}$ & $\begin{array}{c}\text { Segment } \\
\text { number }\end{array}$ & $\begin{array}{l}\text { Streamgage used in } \\
\text { regression analyses } \\
\text { (non-redundant) }\end{array}$ & $\begin{array}{c}\text { Discharge value } \\
\text { used in } \\
\text { adjustment } \\
\text { regression }\end{array}$ & $\begin{array}{c}\text { Water } \\
\text { year }\end{array}$ & $\begin{array}{c}\text { Observed annual } \\
\text { maximum peak } \\
\text { discharge }\left(\mathrm{ft}^{3} / \mathrm{s}\right)\end{array}$ & $\begin{array}{c}\text { NWIS peak } \\
\text { code }\end{array}$ & $\begin{array}{c}\text { Observed annual } \\
\text { maximum peak } \\
\text { discharge with } \\
\text { segment intercept } \\
\text { value subtracted } \\
\left(\mathrm{ft}^{3} / \mathrm{s}\right)\end{array}$ & $\begin{array}{c}\text { Observed } \\
\text { precipitation } \\
\text { (inches) }\end{array}$ & Urban fraction & $\begin{array}{l}\text { Exceedance } \\
\text { probability }\end{array}$ & $\begin{array}{c}\text { Urban-adjusted } \\
\text { annual maximum } \\
\text { peak discharge } \\
\left(\mathrm{ft}^{3} / \mathrm{s}\right)\end{array}$ \\
\hline 05551200 & 1 & Yes & Yes & 1964 & 195 & $E$ & 100 & 0.746 & 0.0586 & 0.95 & 549 \\
\hline 05551200 & 1 & Yes & Yes & 1965 & 531 & -- & 273 & 0.114 & 0.0641 & 0.677 & 1070 \\
\hline 05551200 & 1 & Yes & Yes & 1966 & 1700 & $\mathrm{D}$ & 874 & 0.742 & 0.0696 & 0.0592 & 2770 \\
\hline 05551200 & 1 & Yes & Yes & 1967 & 852 & -- & 438 & 1.55 & 0.0751 & 0.365 & 1540 \\
\hline 05551200 & 1 & Yes & Yes & 1968 & 1590 & -- & 817 & 2.48 & 0.0806 & 0.0721 & 2590 \\
\hline 05551200 & 1 & Yes & Yes & 1969 & 810 & -- & 416 & 0.108 & 0.0861 & 0.406 & 1460 \\
\hline 05551200 & 1 & Yes & Yes & 1970 & 1700 & -- & 874 & 1.85 & 0.0916 & 0.0624 & 2720 \\
\hline 05551200 & 1 & Yes & Yes & 1971 & 1970 & D & 1013 & 0.32 & 0.104 & 0.0419 & 3060 \\
\hline 05551200 & 1 & Yes & Yes & 1972 & 950 & -- & 488 & 1.62 & 0.116 & 0.322 & 1610 \\
\hline 05551200 & 1 & Yes & Yes & 1973 & 1850 & -- & 951 & 1.66 & 0.129 & 0.0532 & 2850 \\
\hline 05551200 & 1 & Yes & Yes & 1974 & 968 & -- & 498 & 0.881 & 0.141 & 0.327 & 1610 \\
\hline 05551200 & 1 & Yes & Yes & 1975 & 496 & -- & 255 & 1.17 & 0.154 & 0.769 & 934 \\
\hline 05551200 & 1 & Yes & Yes & 1976 & 864 & -- & 444 & 1.42 & 0.166 & 0.422 & 1440 \\
\hline 05551200 & 1 & Yes & Yes & 1977 & 156 & $E$ & 80 & 1.21 & 0.179 & 0.981 & 396 \\
\hline 05551200 & 1 & Yes & Yes & 1978 & 1270 & -- & 653 & 1.94 & 0.191 & 0.19 & 1920 \\
\hline 05551200 & 1 & Yes & Yes & 1979 & 1380 & D & 709 & 0.307 & 0.203 & 0.16 & 2050 \\
\hline 05551200 & 1 & Yes & Yes & 1980 & 646 & -- & 332 & 0.367 & 0.216 & 0.671 & 1080 \\
\hline 05551200 & 1 & Yes & Yes & 1981 & 967 & -- & 497 & 2.01 & 0.226 & 0.387 & 1500 \\
\hline 05551200 & 1 & Yes & Yes & 1982 & 925 & -- & 475 & 0.341 & 0.235 & 0.428 & 1430 \\
\hline 05551200 & 1 & Yes & Yes & 1983 & 1570 & -- & 807 & 1.41 & 0.245 & 0.121 & 2240 \\
\hline 05551200 & 1 & Yes & Yes & 1984 & 1090 & -- & 560 & 0.618 & 0.255 & 0.324 & 1610 \\
\hline 05551200 & 1 & Yes & Yes & 1985 & 1040 & -- & 535 & 1.27 & 0.265 & 0.365 & 1540 \\
\hline 05551200 & 1 & Yes & Yes & 1986 & 893 & -- & 459 & 0.995 & 0.275 & 0.488 & 1340 \\
\hline 05551200 & 1 & Yes & Yes & 1987 & 1180 & -- & 607 & 2.13 & 0.284 & 0.289 & 1670 \\
\hline 05551200 & 1 & Yes & Yes & 1988 & 922 & $E$ & 474 & 0.523 & 0.294 & 0.481 & 1350 \\
\hline 05551200 & 1 & Yes & Yes & 1989 & 407 & $E$ & 209 & 1.12 & 0.304 & 0.905 & 686 \\
\hline 05551200 & 1 & Yes & Yes & 1990 & 438 & $E$ & 225 & 1.22 & 0.314 & 0.891 & 717 \\
\hline 05551200 & 1 & Yes & Yes & 1991 & 759 & -- & 390 & 0.854 & 0.325 & 0.653 & 1100 \\
\hline 05551200 & 1 & Yes & Yes & 1992 & 193 & $E$ & 99 & 0.521 & 0.336 & 0.985 & 359 \\
\hline 05551200 & 1 & Yes & Yes & 1993 & 1320 & -- & 678 & 0.951 & 0.348 & 0.254 & 1740 \\
\hline 05551200 & 1 & Yes & Yes & 1994 & 1670 & -- & 858 & 0.629 & 0.359 & 0.14 & 2150 \\
\hline 05551200 & 1 & Yes & Yes & 1995 & 993 & -- & 510 & 1.04 & 0.371 & 0.49 & 1330 \\
\hline 05551200 & 1 & Yes & Yes & 1996 & 1990 & -- & 1023 & 2.61 & 0.382 & 0.0817 & 2490 \\
\hline 05551200 & 1 & Yes & Yes & 1997 & 2580 & -- & 1326 & 2.17 & 0.393 & 0.0376 & 3150 \\
\hline 05551200 & 1 & Yes & Yes & 1998 & 556 & $E$ & 286 & 1.13 & 0.405 & 0.86 & 778 \\
\hline
\end{tabular}




\begin{tabular}{|c|c|c|c|c|c|c|c|c|c|c|c|}
\hline $\begin{array}{c}\text { U.S. } \\
\text { Geological } \\
\text { Survey } \\
\text { streamgage } \\
\text { number } \\
\end{array}$ & $\begin{array}{c}\text { Segment } \\
\text { number }\end{array}$ & $\begin{array}{l}\text { Streamgage used in } \\
\text { regression analyses } \\
\text { (non-redundant) }\end{array}$ & $\begin{array}{c}\text { Discharge value } \\
\text { used in } \\
\text { adjustment } \\
\text { regression }\end{array}$ & $\begin{array}{c}\text { Water } \\
\text { year }\end{array}$ & $\begin{array}{c}\text { Observed annual } \\
\text { maximum peak } \\
\text { discharge }\left(\mathrm{ft}^{3} / \mathrm{s}\right)\end{array}$ & $\begin{array}{c}\text { NWIS peak } \\
\text { code }\end{array}$ & $\begin{array}{c}\text { Observed annual } \\
\text { maximum peak } \\
\text { discharge with } \\
\text { segment intercept } \\
\text { value subtracted } \\
\left(\mathrm{ft}^{3} / \mathrm{s}\right)\end{array}$ & $\begin{array}{l}\text { Observed } \\
\text { precipitation } \\
\text { (inches) }\end{array}$ & Urban fraction & $\begin{array}{l}\text { Exceedance } \\
\text { probability }\end{array}$ & $\begin{array}{c}\text { Urban-adjusted } \\
\text { annual maximum } \\
\text { peak discharge } \\
\left(\mathrm{ft}^{3} / \mathrm{s}\right)\end{array}$ \\
\hline 05551200 & 1 & Yes & Yes & 1999 & 1340 & -- & 689 & 0.965 & 0.416 & 0.29 & 1670 \\
\hline 05551200 & 1 & Yes & Yes & 2000 & 345 & $C, E$ & 177 & 0.991 & 0.427 & 0.958 & 506 \\
\hline 05551200 & 1 & Yes & Yes & 2001 & 781 & $C$ & 401 & 0.665 & 0.448 & 0.732 & 991 \\
\hline 05551200 & 1 & Yes & Yes & 2002 & 1430 & $C$ & 735 & 1.43 & 0.469 & 0.281 & 1690 \\
\hline 05551200 & 1 & Yes & Yes & 2003 & 807 & $\mathrm{C}, \mathrm{E}$ & 415 & 1.36 & 0.49 & 0.745 & 973 \\
\hline 05551200 & 1 & Yes & Yes & 2004 & 1160 & C & 596 & 1.68 & 0.51 & 0.491 & 1330 \\
\hline 05551200 & 1 & Yes & Yes & 2005 & 541 & C & 278 & 0.769 & 0.531 & 0.925 & 643 \\
\hline 05551200 & 1 & Yes & Yes & 2006 & 355 & $\mathrm{C}, \mathrm{E}$ & 182 & 1.1 & 0.552 & 0.975 & 419 \\
\hline 05551200 & 1 & Yes & Yes & 2007 & 2360 & C & 1213 & 3.04 & 0.573 & 0.0812 & 2490 \\
\hline 05551200 & 1 & Yes & Yes & 2008 & 2980 & C & 1532 & 4.41 & 0.594 & 0.0398 & 3080 \\
\hline 05551200 & 1 & Yes & Yes & 2009 & 2530 & C & 1300 & 0.83 & 0.614 & 0.072 & 2580 \\
\hline 05551330 & 1 & Yes & Yes & 1998 & 746 & $C, E$ & 1083 & 0.675 & 0.427 & 0.0765 & 896 \\
\hline 05551330 & 1 & Yes & Yes & 1999 & 1080 & C & 1568 & 1.1 & 0.441 & 0.0239 & 1250 \\
\hline 05551330 & 1 & Yes & Yes & 2000 & 191 & $C, E$ & 277 & 1.5 & 0.455 & 0.894 & 252 \\
\hline 05551330 & 1 & Yes & Yes & 2001 & 530 & C & 769 & 0.686 & 0.472 & 0.25 & 619 \\
\hline 05551330 & 1 & Yes & Yes & 2002 & 406 & C & 589 & 1.37 & 0.49 & 0.482 & 476 \\
\hline 05551330 & 1 & Yes & Yes & 2003 & 272 & C & 395 & 2.33 & 0.508 & 0.783 & 322 \\
\hline 05551330 & 1 & Yes & Yes & 2004 & 331 & C & 480 & 2.18 & 0.526 & 0.679 & 378 \\
\hline 05551330 & 1 & Yes & Yes & 2005 & 181 & C & 263 & 0.816 & 0.544 & 0.936 & 211 \\
\hline 05551330 & 1 & Yes & Yes & 2006 & 114 & $C, E$ & 165 & 1.55 & 0.562 & 0.983 & 132 \\
\hline 05551330 & 1 & Yes & Yes & 2007 & 676 & C & 981 & 3.16 & 0.58 & 0.172 & 710 \\
\hline 05551330 & 1 & Yes & Yes & 2008 & 4510 & C & 6547 & 4.12 & 0.598 & 0 & 4620 \\
\hline 05551330 & 1 & Yes & Yes & 2009 & 665 & C & 965 & 0.749 & 0.616 & 0.195 & 676 \\
\hline 05551520 & 1 & Yes & Yes & 1961 & 158 & -- & 555 & 1.7 & 0.0912 & 0.226 & 222 \\
\hline 05551520 & 1 & Yes & Yes & 1962 & 125 & -- & 439 & 0.379 & 0.0961 & 0.378 & 182 \\
\hline 05551520 & 1 & Yes & Yes & 1963 & 58 & -- & 204 & 1.88 & 0.101 & 0.828 & 94.6 \\
\hline 05551520 & 1 & Yes & Yes & 1964 & 84 & -- & 295 & 2.7 & 0.106 & 0.662 & 127 \\
\hline 05551520 & 1 & Yes & Yes & 1965 & 65 & -- & 228 & 0.139 & 0.111 & 0.791 & 103 \\
\hline 05551520 & 1 & Yes & Yes & 1966 & 262 & -- & 920 & 2.3 & 0.116 & 0.057 & 349 \\
\hline 05551520 & 1 & Yes & Yes & 1967 & 224 & -- & 787 & 2.51 & 0.121 & 0.0902 & 301 \\
\hline 05551520 & 1 & Yes & Yes & 1968 & 88 & -- & 309 & 1.32 & 0.126 & 0.649 & 130 \\
\hline 05551520 & 1 & Yes & Yes & 1969 & 171 & -- & 600 & 1.7 & 0.131 & 0.201 & 231 \\
\hline 05551520 & 1 & Yes & Yes & 1970 & 84 & -- & 295 & 1.56 & 0.136 & 0.683 & 124 \\
\hline 05551520 & 1 & Yes & Yes & 1971 & 80 & -- & 281 & 0.384 & 0.137 & 0.712 & 118 \\
\hline 05551520 & 1 & Yes & Yes & 1972 & 224 & -- & 787 & 6.07 & 0.139 & 0.0944 & 296 \\
\hline
\end{tabular}




\begin{tabular}{|c|c|c|c|c|c|c|c|c|c|c|c|}
\hline $\begin{array}{l}\text { U.S. } \\
\text { Geological } \\
\text { Survey } \\
\text { streamgage } \\
\text { number }\end{array}$ & $\begin{array}{l}\text { Segment } \\
\text { number }\end{array}$ & $\begin{array}{l}\text { Streamgage used in } \\
\text { regression analyses } \\
\text { (non-redundant) }\end{array}$ & $\begin{array}{c}\text { Discharge value } \\
\text { used in } \\
\text { adjustment } \\
\text { regression }\end{array}$ & $\begin{array}{l}\text { Water } \\
\text { year }\end{array}$ & $\begin{array}{c}\text { Observed annual } \\
\text { maximum peak } \\
\text { discharge }\left(\mathrm{ft}^{3} / \mathrm{s}\right)\end{array}$ & $\begin{array}{l}\text { NWIS peak } \\
\text { code }\end{array}$ & $\begin{array}{l}\text { Observed annual } \\
\text { maximum peak } \\
\text { discharge with } \\
\text { segment intercept } \\
\text { value subtracted } \\
\left(\mathrm{ft}^{3} / \mathrm{s}\right)\end{array}$ & $\begin{array}{l}\text { Observed } \\
\text { precipitation } \\
\text { (inches) }\end{array}$ & Urban fraction & $\begin{array}{l}\text { Exceedance } \\
\text { probability }\end{array}$ & $\begin{array}{c}\text { Urban-adjusted } \\
\text { annual maximum } \\
\text { peak discharge } \\
\left(\mathrm{ft}^{3} / \mathrm{s}\right)\end{array}$ \\
\hline 05551520 & 1 & Yes & Yes & 1973 & 92 & -- & 323 & 1.21 & 0.14 & 0.633 & 133 \\
\hline 05551520 & 1 & Yes & Yes & 1974 & 216 & -- & 759 & 1.4 & 0.141 & 0.108 & 286 \\
\hline 05551520 & 1 & Yes & Yes & 1975 & 188 & -- & 660 & 1.79 & 0.143 & 0.166 & 250 \\
\hline 05551520 & 1 & Yes & Yes & 1976 & 118 & -- & 414 & 1.16 & 0.144 & 0.456 & 166 \\
\hline 05551520 & 1 & Yes & Yes & 1977 & 40 & -- & 140 & 1.49 & 0.146 & 0.929 & 66.8 \\
\hline 05551520 & 1 & Yes & Yes & 1978 & 402 & -- & 1412 & 5.21 & 0.147 & 0.017 & 505 \\
\hline 05551520 & 1 & Yes & Yes & 1979 & 227 & -- & 797 & 0.544 & 0.148 & 0.0932 & 298 \\
\hline 05551530 & 1 & No & No & 1961 & 436 & -- & 474 & 1.76 & 0.223 & 0.42 & 773 \\
\hline 05551530 & 1 & No & No & 1962 & 270 & -- & 294 & 0.393 & 0.231 & 0.752 & 529 \\
\hline 05551530 & 1 & No & No & 1963 & 507 & -- & 551 & 1.98 & 0.239 & 0.324 & 854 \\
\hline 05551530 & 1 & No & No & 1964 & 433 & -- & 471 & 2.87 & 0.248 & 0.446 & 752 \\
\hline 05551530 & 1 & No & No & 1965 & 375 & -- & 408 & 0.14 & 0.256 & 0.566 & 661 \\
\hline 05551530 & 1 & No & No & 1966 & 658 & -- & 715 & 2.48 & 0.265 & 0.18 & 1030 \\
\hline 05551530 & 1 & No & No & 1967 & 694 & -- & 755 & 2.72 & 0.273 & 0.161 & 1080 \\
\hline 05551530 & 1 & No & No & 1968 & 478 & -- & 520 & 1.39 & 0.282 & 0.398 & 791 \\
\hline 05551530 & 1 & No & No & 1969 & 377 & -- & 410 & 2.13 & 0.29 & 0.592 & 643 \\
\hline 05551530 & 1 & No & No & 1970 & 575 & -- & 625 & 1.59 & 0.299 & 0.278 & 897 \\
\hline 05551530 & 1 & No & No & 1971 & 486 & -- & 528 & 0.163 & 0.306 & 0.406 & 785 \\
\hline 05551530 & 1 & No & No & 1972 & 742 & -- & 807 & 6.79 & 0.313 & 0.148 & 1110 \\
\hline 05551530 & 1 & No & No & 1973 & 511 & -- & 556 & 1.35 & 0.32 & 0.38 & 807 \\
\hline 05551530 & 1 & No & No & 1974 & 647 & -- & 704 & 1.43 & 0.327 & 0.219 & 964 \\
\hline 05551530 & 1 & No & No & 1975 & 596 & -- & 648 & 1.93 & 0.335 & 0.277 & 897 \\
\hline 05551530 & 1 & No & No & 1976 & 592 & -- & 644 & 0.00507 & 0.342 & 0.287 & 887 \\
\hline 05551530 & 1 & No & No & 1977 & 316 & -- & 344 & 0.658 & 0.349 & 0.749 & 530 \\
\hline 05551530 & 1 & No & No & 1978 & 744 & -- & 809 & 5.82 & 0.356 & 0.165 & 1070 \\
\hline 05551530 & 1 & No & No & 1979 & 696 & -- & 757 & 0.527 & 0.363 & 0.196 & 998 \\
\hline 05551620 & 1 & No & No & 1961 & 384 & -- & 313 & 1.22 & 0.0412 & 0.58 & 507 \\
\hline 05551620 & 1 & No & No & 1962 & 466 & -- & 380 & 0.3 & 0.0423 & 0.438 & 606 \\
\hline 05551620 & 1 & No & No & 1963 & 388 & -- & 316 & 1.25 & 0.0435 & 0.574 & 511 \\
\hline 05551620 & 1 & No & No & 1965 & 536 & -- & 437 & 0.161 & 0.0458 & 0.348 & 686 \\
\hline 05551620 & 1 & No & No & 1966 & 550 & -- & 448 & 1.38 & 0.0469 & 0.333 & 701 \\
\hline 05551620 & 1 & No & No & 1967 & 499 & -- & 406 & 1.07 & 0.0481 & 0.395 & 642 \\
\hline 05551620 & 1 & No & No & 1969 & 306 & -- & 249 & 0.875 & 0.0504 & 0.717 & 410 \\
\hline 05551620 & 1 & No & No & 1970 & 558 & -- & 454 & 1.46 & 0.0515 & 0.326 & 707 \\
\hline 05551620 & 1 & No & No & 1971 & 311 & -- & 253 & 0.376 & 0.0553 & 0.712 & 413 \\
\hline
\end{tabular}




\begin{tabular}{|c|c|c|c|c|c|c|c|c|c|c|c|}
\hline $\begin{array}{l}\text { U.S. } \\
\text { Geological } \\
\text { Survey } \\
\text { streamgage } \\
\text { number }\end{array}$ & $\begin{array}{l}\text { Segment } \\
\text { number }\end{array}$ & $\begin{array}{l}\text { Streamgage used in } \\
\text { regression analyses } \\
\text { (non-redundant) }\end{array}$ & $\begin{array}{c}\text { Discharge value } \\
\text { used in } \\
\text { adjustment } \\
\text { regression }\end{array}$ & $\begin{array}{l}\text { Water } \\
\text { year }\end{array}$ & $\begin{array}{c}\text { Observed annual } \\
\text { maximum peak } \\
\text { discharge }\left(\mathrm{ft}^{3} / \mathrm{s}\right)\end{array}$ & $\begin{array}{l}\text { NWIS peak } \\
\text { code }\end{array}$ & $\begin{array}{l}\text { Observed annual } \\
\text { maximum peak } \\
\text { discharge with } \\
\text { segment intercept } \\
\text { value subtracted } \\
\left(\mathrm{ft}^{3} / \mathrm{s}\right)\end{array}$ & $\begin{array}{l}\text { Observed } \\
\text { precipitation } \\
\text { (inches) }\end{array}$ & Urban fraction & $\begin{array}{l}\text { Exceedance } \\
\text { probability }\end{array}$ & $\begin{array}{c}\text { Urban-adjusted } \\
\text { annual maximum } \\
\text { peak discharge } \\
\left(\mathrm{ft}^{3} / \mathrm{s}\right)\end{array}$ \\
\hline 05551620 & 1 & No & No & 1972 & 352 & -- & 287 & 1.08 & 0.0591 & 0.646 & 460 \\
\hline 05551620 & 1 & No & No & 1973 & 302 & -- & 246 & 1.47 & 0.0629 & 0.732 & 400 \\
\hline 05551620 & 1 & No & No & 1974 & 640 & -- & 521 & 1.3 & 0.0666 & 0.25 & 788 \\
\hline 05551620 & 1 & No & No & 1975 & 345 & -- & 281 & 0.841 & 0.0704 & 0.665 & 446 \\
\hline 05551620 & 1 & No & No & 1976 & 403 & -- & 328 & 1.1 & 0.0742 & 0.572 & 512 \\
\hline 05551620 & 1 & No & No & 1977 & 196 & -- & 160 & 1.19 & 0.0779 & 0.887 & 268 \\
\hline 05551620 & 1 & No & No & 1978 & 576 & -- & 469 & 1.4 & 0.0817 & 0.326 & 708 \\
\hline 05551620 & 1 & No & No & 1979 & 511 & -- & 416 & 0.349 & 0.0855 & 0.406 & 632 \\
\hline 05551650 & 1 & Yes & Yes & 1961 & 44 & -- & 246 & 1.22 & 0 & 0.691 & 49.3 \\
\hline 05551650 & 1 & Yes & Yes & 1962 & 48 & -- & 268 & 0.299 & 0 & 0.643 & 53.6 \\
\hline 05551650 & 1 & Yes & Yes & 1963 & 25 & -- & 140 & 0.404 & 0 & 0.89 & 28.8 \\
\hline 05551650 & 1 & Yes & No & 1964 & 11 & 4, B & 62 & 0.109 & 0 & 0.976 & 13.2 \\
\hline 05551650 & 1 & Yes & Yes & 1965 & 32 & -- & 179 & 0.133 & 0 & 0.825 & 36.4 \\
\hline 05551650 & 1 & Yes & Yes & 1966 & 54 & -- & 302 & 1.37 & 0 & 0.571 & 60 \\
\hline 05551650 & 1 & Yes & Yes & 1967 & 146 & -- & 817 & 1.08 & 0 & 0.0608 & 158 \\
\hline 05551650 & 1 & Yes & Yes & 1968 & 14 & -- & 78 & 1.36 & 0 & 0.96 & 16.6 \\
\hline 05551650 & 1 & Yes & Yes & 1969 & 20 & -- & 112 & 0.109 & 0 & 0.928 & 23.3 \\
\hline 05551650 & 1 & Yes & Yes & 1970 & 346 & -- & 1935 & 1.46 & 0 & 0.005 & 369 \\
\hline 05551650 & 1 & Yes & Yes & 1971 & 19 & -- & 106 & 0.377 & 0.000196 & 0.933 & 22.2 \\
\hline 05551650 & 1 & Yes & Yes & 1972 & 43 & -- & 240 & 0.931 & 0.000393 & 0.703 & 48.2 \\
\hline 05551650 & 1 & Yes & Yes & 1973 & 73 & -- & 408 & 1.47 & 0.00059 & 0.36 & 80.4 \\
\hline 05551650 & 1 & Yes & Yes & 1974 & 316 & -- & 1767 & 1.3 & 0.000786 & 0.00705 & 337 \\
\hline 05551650 & 1 & Yes & Yes & 1975 & 76 & -- & 425 & 0.835 & 0.000983 & 0.336 & 83.5 \\
\hline 05551650 & 1 & Yes & Yes & 1976 & 66 & -- & 369 & 1.11 & 0.00118 & 0.426 & 72.9 \\
\hline 05551675 & 1 & No & No & 1998 & 603 & C & 567 & 0.863 & 0.219 & 0.291 & 736 \\
\hline 05551675 & 1 & No & No & 1999 & 1040 & C & 978 & 1.13 & 0.225 & 0.0625 & 1230 \\
\hline 05551675 & 1 & No & No & 2000 & 278 & C & 261 & 1.58 & 0.231 & 0.802 & 359 \\
\hline 05551675 & 1 & No & No & 2001 & 544 & C & 511 & 0.664 & 0.25 & 0.386 & 652 \\
\hline 05551675 & 1 & No & No & 2002 & 608 & C & 572 & 1.55 & 0.269 & 0.32 & 709 \\
\hline 05551675 & 1 & No & No & 2003 & 344 & $\mathrm{C}, \mathrm{E}$ & 323 & 1.01 & 0.288 & 0.739 & 407 \\
\hline 05551675 & 1 & No & No & 2004 & 425 & $C, E$ & 400 & 1.61 & 0.307 & 0.623 & 485 \\
\hline 05551675 & 1 & No & No & 2005 & 255 & $\mathrm{C}, \mathrm{E}$ & 240 & 0.746 & 0.325 & 0.879 & 294 \\
\hline 05551675 & 1 & No & No & 2006 & 143 & $C, E$ & 134 & 0.902 & 0.344 & 0.967 & 165 \\
\hline 05551675 & 1 & No & No & 2007 & 754 & $7, C$ & 709 & 0.513 & 0.363 & 0.236 & 796 \\
\hline 05551675 & 1 & No & No & 2008 & 1740 & C & 1636 & 4.12 & 0.382 & 0.0183 & 1790 \\
\hline
\end{tabular}




\begin{tabular}{|c|c|c|c|c|c|c|c|c|c|c|c|}
\hline $\begin{array}{c}\text { U.S. } \\
\text { Geological } \\
\text { Survey } \\
\text { streamgage } \\
\text { number }\end{array}$ & $\begin{array}{l}\text { Segment } \\
\text { number }\end{array}$ & $\begin{array}{l}\text { Streamgage used in } \\
\text { regression analyses } \\
\text { (non-redundant) }\end{array}$ & $\begin{array}{l}\text { Discharge value } \\
\text { used in } \\
\text { adjustment } \\
\text { regression }\end{array}$ & $\begin{array}{l}\text { Water } \\
\text { year }\end{array}$ & $\begin{array}{l}\text { Observed annual } \\
\text { maximum peak } \\
\text { discharge }\left(\mathrm{ft}^{3} / \mathrm{s}\right)\end{array}$ & $\begin{array}{l}\text { NWIS peak } \\
\text { code }\end{array}$ & $\begin{array}{l}\text { Observed annual } \\
\text { maximum peak } \\
\text { discharge with } \\
\text { segment intercept } \\
\text { value subtracted } \\
\left(\mathrm{ft}^{3} / \mathrm{s}\right)\end{array}$ & $\begin{array}{l}\text { Observed } \\
\text { precipitation } \\
\text { (inches) }\end{array}$ & Urban fraction & $\begin{array}{l}\text { Exceedance } \\
\text { probability }\end{array}$ & $\begin{array}{c}\text { Urban-adjusted } \\
\text { annual maximum } \\
\text { peak discharge } \\
\left(\mathrm{ft}^{3} / \mathrm{s}\right)\end{array}$ \\
\hline 05551675 & 1 & No & No & 2009 & 821 & C & 772 & 0.902 & 0.401 & 0.204 & 836 \\
\hline 05551700 & 1 & Yes & Yes & 1961 & 455 & -- & 277 & 1.36 & 0.0694 & 0.672 & 700 \\
\hline 05551700 & 1 & Yes & Yes & 1962 & 1000 & -- & 609 & 0.328 & 0.0727 & 0.172 & 1380 \\
\hline 05551700 & 1 & Yes & Yes & 1963 & 472 & -- & 288 & 1.45 & 0.076 & 0.656 & 717 \\
\hline 05551700 & 1 & Yes & Yes & 1964 & 159 & -- & 97 & 1.02 & 0.0793 & 0.955 & 298 \\
\hline 05551700 & 1 & Yes & Yes & 1965 & 605 & 2 & 369 & 0.136 & 0.0826 & 0.491 & 886 \\
\hline 05551700 & 1 & Yes & Yes & 1966 & 906 & D & 552 & 1.5 & 0.0859 & 0.226 & 1250 \\
\hline 05551700 & 1 & Yes & Yes & 1967 & 618 & -- & 377 & 1.2 & 0.0892 & 0.481 & 897 \\
\hline 05551700 & 1 & Yes & Yes & 1968 & 289 & -- & 176 & 1.15 & 0.0925 & 0.869 & 471 \\
\hline 05551700 & 1 & Yes & Yes & 1969 & 578 & -- & 352 & 0.1 & 0.0958 & 0.537 & 839 \\
\hline 05551700 & 1 & Yes & Yes & 1970 & 1300 & -- & 792 & 1.47 & 0.0991 & 0.0831 & 1740 \\
\hline 05551700 & 1 & Yes & Yes & 1971 & 686 & $D, E$ & 418 & 0.375 & 0.103 & 0.417 & 971 \\
\hline 05551700 & 1 & Yes & Yes & 1972 & 546 & -- & 333 & 1.05 & 0.108 & 0.592 & 788 \\
\hline 05551700 & 1 & Yes & Yes & 1973 & 619 & -- & 377 & 0.625 & 0.112 & 0.498 & 878 \\
\hline 05551700 & 1 & Yes & Yes & 1974 & 1320 & -- & 804 & 1.36 & 0.117 & 0.0831 & 1730 \\
\hline 05551700 & 1 & Yes & Yes & 1975 & 483 & -- & 294 & 1.14 & 0.121 & 0.674 & 698 \\
\hline 05551700 & 1 & Yes & Yes & 1976 & 637 & -- & 388 & 1.1 & 0.125 & 0.488 & 889 \\
\hline 05551700 & 1 & Yes & Yes & 1977 & 172 & $\mathrm{E}$ & 105 & 1.21 & 0.13 & 0.956 & 295 \\
\hline 05551700 & 1 & Yes & Yes & 1978 & 1030 & -- & 628 & 2.54 & 0.134 & 0.184 & 1350 \\
\hline 05551700 & 1 & Yes & Yes & 1979 & 1250 & -- & 762 & 0.386 & 0.138 & 0.105 & 1620 \\
\hline 05551700 & 1 & Yes & Yes & 1980 & 210 & $\mathrm{E}$ & 128 & 0.672 & 0.143 & 0.94 & 342 \\
\hline 05551700 & 1 & Yes & Yes & 1981 & 1140 & -- & 695 & 2.15 & 0.145 & 0.145 & 1470 \\
\hline 05551700 & 1 & Yes & Yes & 1982 & 766 & -- & 467 & 0.347 & 0.146 & 0.373 & 1030 \\
\hline 05551700 & 1 & Yes & Yes & 1983 & 2060 & -- & 1255 & 2.61 & 0.148 & 0.0228 & 2550 \\
\hline 05551700 & 1 & Yes & Yes & 1984 & 765 & -- & 466 & 0.799 & 0.15 & 0.376 & 1020 \\
\hline 05551700 & 1 & Yes & Yes & 1985 & 1290 & -- & 786 & 1.58 & 0.152 & 0.0978 & 1650 \\
\hline 05551700 & 1 & Yes & Yes & 1986 & 797 & -- & 486 & 0.914 & 0.154 & 0.351 & 1060 \\
\hline 05551700 & 1 & Yes & Yes & 1987 & 973 & -- & 593 & 2.12 & 0.156 & 0.223 & 1250 \\
\hline 05551700 & 1 & Yes & Yes & 1988 & 432 & -- & 263 & 1.04 & 0.157 & 0.758 & 611 \\
\hline 05551700 & 1 & Yes & Yes & 1989 & 188 & -- & 115 & 1.01 & 0.159 & 0.953 & 304 \\
\hline 05551700 & 1 & Yes & Yes & 1990 & 544 & -- & 332 & 1.63 & 0.161 & 0.632 & 743 \\
\hline 05551700 & 1 & Yes & Yes & 1991 & 1360 & -- & 829 & 0.845 & 0.167 & 0.0872 & 1710 \\
\hline 05551700 & 1 & Yes & Yes & 1992 & 172 & $\mathrm{E}$ & 105 & 0.507 & 0.173 & 0.962 & 275 \\
\hline 05551700 & 1 & Yes & Yes & 1993 & 724 & -- & 441 & 0.786 & 0.179 & 0.438 & 946 \\
\hline 05551700 & 1 & Yes & Yes & 1994 & 1070 & -- & 652 & 0.752 & 0.185 & 0.188 & 1340 \\
\hline
\end{tabular}




\begin{tabular}{|c|c|c|c|c|c|c|c|c|c|c|c|}
\hline $\begin{array}{l}\text { U.S. } \\
\text { Geological } \\
\text { Survey } \\
\text { streamgage } \\
\text { number }\end{array}$ & $\begin{array}{l}\text { Segment } \\
\text { number }\end{array}$ & $\begin{array}{l}\text { Streamgage used in } \\
\text { regression analyses } \\
\text { (non-redundant) }\end{array}$ & $\begin{array}{l}\text { Discharge value } \\
\text { used in } \\
\text { adjustment } \\
\text { regression }\end{array}$ & $\begin{array}{l}\text { Water } \\
\text { year }\end{array}$ & $\begin{array}{c}\text { Observed annual } \\
\text { maximum peak } \\
\text { discharge }\left(\mathrm{ft}^{3} / \mathrm{s}\right)\end{array}$ & $\begin{array}{l}\text { NWIS peak } \\
\text { code }\end{array}$ & $\begin{array}{l}\text { Observed annual } \\
\text { maximum peak } \\
\text { discharge with } \\
\text { segment intercept } \\
\text { value subtracted } \\
\left(\mathrm{ft}^{3} / \mathrm{s}\right)\end{array}$ & $\begin{array}{l}\text { Observed } \\
\text { precipitation } \\
\text { (inches) }\end{array}$ & Urban fraction & $\begin{array}{l}\text { Exceedance } \\
\text { probability }\end{array}$ & $\begin{array}{c}\text { Urban-adjusted } \\
\text { annual maximum } \\
\text { peak discharge } \\
\left(\mathrm{ft}^{3} / \mathrm{s}\right)\end{array}$ \\
\hline 05551700 & 1 & Yes & Yes & 1995 & 516 & -- & 314 & 1.22 & 0.191 & 0.686 & 686 \\
\hline 05551700 & 1 & Yes & Yes & 1996 & 5510 & -- & 3358 & 2.73 & 0.197 & 0 & 6410 \\
\hline 05551700 & 1 & Yes & Yes & 1997 & 2040 & -- & 1243 & 2.48 & 0.203 & 0.0277 & 2430 \\
\hline 05551700 & 1 & Yes & Yes & 1998 & 507 & -- & 309 & 1.01 & 0.209 & 0.709 & 663 \\
\hline 05551700 & 1 & Yes & Yes & 1999 & 959 & C & 584 & 1.14 & 0.215 & 0.269 & 1180 \\
\hline 05551700 & 1 & Yes & Yes & 2000 & 297 & $C, E$ & 181 & 1.54 & 0.221 & 0.908 & 410 \\
\hline 05551700 & 1 & Yes & Yes & 2001 & 609 & C & 371 & 0.65 & 0.242 & 0.621 & 755 \\
\hline 05551700 & 1 & Yes & Yes & 2002 & 562 & C & 342 & 1.54 & 0.262 & 0.687 & 686 \\
\hline 05551700 & 1 & Yes & Yes & 2003 & 313 & C & 191 & 0.99 & 0.282 & 0.917 & 394 \\
\hline 05551700 & 1 & Yes & Yes & 2004 & 1130 & C & 689 & 1.97 & 0.302 & 0.217 & 1270 \\
\hline 05551700 & 1 & Yes & Yes & 2005 & 403 & C & 246 & 0.781 & 0.323 & 0.871 & 468 \\
\hline 05551700 & 1 & Yes & Yes & 2006 & 164 & $C, E$ & 100 & 0.911 & 0.343 & 0.985 & 194 \\
\hline 05551700 & 1 & Yes & Yes & 2007 & 1070 & C & 652 & 0.553 & 0.363 & 0.292 & 1140 \\
\hline 05551700 & 1 & Yes & Yes & 2008 & 2130 & C & 1298 & 3.71 & 0.383 & 0.0392 & 2200 \\
\hline 05551700 & 1 & Yes & Yes & 2009 & 1270 & C & 774 & 0.911 & 0.403 & 0.205 & 1290 \\
\hline 05551800 & 1 & Yes & Yes & 1961 & 36 & -- & 216 & 1.3 & 0.13 & 0.822 & 75.2 \\
\hline 05551800 & 1 & Yes & Yes & 1962 & 29 & -- & 174 & 0.318 & 0.132 & 0.886 & 64.8 \\
\hline 05551800 & 1 & Yes & Yes & 1963 & 17 & -- & 102 & 0.725 & 0.135 & 0.959 & 45.1 \\
\hline 05551800 & 1 & Yes & Yes & 1964 & 11 & -- & 66 & 0.996 & 0.137 & 0.985 & 32.7 \\
\hline 05551800 & 1 & Yes & Yes & 1965 & 218 & -- & 1308 & 0.256 & 0.14 & 0.0194 & 324 \\
\hline 05551800 & 1 & Yes & Yes & 1966 & 96 & -- & 576 & 1.5 & 0.142 & 0.233 & 157 \\
\hline 05551800 & 1 & Yes & Yes & 1967 & 36 & -- & 216 & 1.14 & 0.145 & 0.829 & 74.1 \\
\hline 05551800 & 1 & Yes & Yes & 1968 & 14 & -- & 84 & 1.3 & 0.147 & 0.974 & 38.7 \\
\hline 05551800 & 1 & Yes & Yes & 1969 & 46 & -- & 276 & 0.994 & 0.15 & 0.73 & 88.1 \\
\hline 05551800 & 1 & Yes & Yes & 1970 & 242 & -- & 1452 & 1.53 & 0.153 & 0.0162 & 354 \\
\hline 05551800 & 1 & Yes & Yes & 1971 & 57 & -- & 342 & 0.375 & 0.157 & 0.611 & 103 \\
\hline 05551800 & 1 & Yes & Yes & 1972 & 192 & -- & 1152 & 1.5 & 0.161 & 0.032 & 287 \\
\hline 05551800 & 1 & Yes & Yes & 1973 & 56 & -- & 336 & 1.43 & 0.165 & 0.627 & 101 \\
\hline 05551800 & 1 & Yes & Yes & 1974 & 147 & -- & 882 & 0.594 & 0.169 & 0.0724 & 226 \\
\hline 05551800 & 1 & Yes & Yes & 1975 & 304 & -- & 1823 & 0.879 & 0.174 & 0.00927 & 431 \\
\hline 05551800 & 1 & Yes & Yes & 1976 & 64 & -- & 384 & 0.99 & 0.178 & 0.543 & 111 \\
\hline 05551800 & 1 & Yes & Yes & 1977 & 11 & -- & 66 & 1.39 & 0.182 & 0.988 & 30.3 \\
\hline 05551800 & 1 & Yes & Yes & 1978 & 320 & -- & 1919 & 1.65 & 0.186 & 0.00828 & 448 \\
\hline 05551800 & 1 & Yes & Yes & 1980 & 125 & -- & 750 & 0.638 & 0.195 & 0.133 & 191 \\
\hline 05551900 & 1 & Yes & Yes & 1965 & 680 & -- & 330 & 0.0791 & 0.00395 & 0.507 & 701 \\
\hline
\end{tabular}




\begin{tabular}{|c|c|c|c|c|c|c|c|c|c|c|c|}
\hline $\begin{array}{c}\text { U.S. } \\
\text { Geological } \\
\text { Survey } \\
\text { streamgage } \\
\text { number }\end{array}$ & $\begin{array}{l}\text { Segment } \\
\text { number }\end{array}$ & $\begin{array}{l}\text { Streamgage used in } \\
\text { regression analyses } \\
\text { (non-redundant) }\end{array}$ & $\begin{array}{c}\text { Discharge value } \\
\text { used in } \\
\text { adjustment } \\
\text { regression }\end{array}$ & $\begin{array}{l}\text { Water } \\
\text { year }\end{array}$ & $\begin{array}{l}\text { Observed annual } \\
\text { maximum peak } \\
\text { discharge }\left(\mathrm{ft}^{3} / \mathrm{s}\right)\end{array}$ & $\begin{array}{l}\text { NWIS peak } \\
\text { code }\end{array}$ & $\begin{array}{l}\text { Observed annual } \\
\text { maximum peak } \\
\text { discharge with } \\
\text { segment intercept } \\
\text { value subtracted } \\
\left(\mathrm{ft}^{3} / \mathrm{s}\right)\end{array}$ & $\begin{array}{l}\text { Observed } \\
\text { precipitation } \\
\text { (inches) }\end{array}$ & Urban fraction & $\begin{array}{l}\text { Exceedance } \\
\text { probability }\end{array}$ & $\begin{array}{c}\text { Urban-adjusted } \\
\text { annual maximum } \\
\text { peak discharge } \\
\left(\mathrm{ft}^{3} / \mathrm{s}\right)\end{array}$ \\
\hline 05551900 & 1 & Yes & Yes & 1966 & 795 & -- & 386 & 0.481 & 0.00408 & 0.397 & 818 \\
\hline 05551900 & 1 & Yes & Yes & 1967 & 668 & -- & 324 & 1.11 & 0.00421 & 0.52 & 688 \\
\hline 05551900 & 1 & Yes & Yes & 1968 & 243 & 2 & 118 & 1.33 & 0.00434 & 0.921 & 254 \\
\hline 05551900 & 1 & Yes & Yes & 1969 & 542 & -- & 263 & 0.11 & 0.00447 & 0.657 & 559 \\
\hline 05551900 & 1 & Yes & Yes & 1970 & 550 & -- & 267 & 1.46 & 0.0046 & 0.649 & 567 \\
\hline 05551900 & 1 & Yes & Yes & 1971 & 498 & -- & 242 & 0.378 & 0.00565 & 0.704 & 514 \\
\hline 05551900 & 1 & Yes & Yes & 1972 & 1410 & -- & 684 & 1.08 & 0.00671 & 0.0997 & 1440 \\
\hline 05551900 & 1 & Yes & Yes & 1973 & 744 & -- & 361 & 1.48 & 0.00776 & 0.446 & 763 \\
\hline 05551900 & 1 & Yes & Yes & 1974 & 1580 & -- & 767 & 1.33 & 0.00881 & 0.0723 & 1610 \\
\hline 05551900 & 1 & Yes & Yes & 1975 & 398 & -- & 193 & 0.844 & 0.00986 & 0.802 & 409 \\
\hline 05551900 & 1 & Yes & Yes & 1976 & 458 & -- & 222 & 1.09 & 0.0109 & 0.749 & 470 \\
\hline 05551900 & 1 & Yes & Yes & 1977 & 382 & -- & 185 & 0.141 & 0.012 & 0.818 & 392 \\
\hline 05551900 & 1 & Yes & Yes & 1978 & 713 & -- & 346 & 1.39 & 0.013 & 0.48 & 727 \\
\hline 05551900 & 1 & Yes & Yes & 1979 & 889 & -- & 432 & 0.353 & 0.0141 & 0.335 & 904 \\
\hline 05551930 & 1 & Yes & Yes & 1965 & 334 & -- & 347 & 0.0688 & 0.0442 & 0.503 & 366 \\
\hline 05551930 & 1 & Yes & Yes & 1966 & 398 & -- & 413 & 0.477 & 0.0443 & 0.381 & 434 \\
\hline 05551930 & 1 & Yes & Yes & 1967 & 306 & -- & 318 & 1.11 & 0.0444 & 0.571 & 336 \\
\hline 05551930 & 1 & Yes & Yes & 1968 & 155 & -- & 161 & 1.34 & 0.0446 & 0.874 & 175 \\
\hline 05551930 & 1 & Yes & Yes & 1969 & 314 & -- & 326 & 0.109 & 0.0447 & 0.551 & 344 \\
\hline 05551930 & 1 & Yes & Yes & 1970 & 354 & -- & 368 & 1.44 & 0.0449 & 0.462 & 387 \\
\hline 05551930 & 1 & Yes & Yes & 1971 & 297 & -- & 308 & 0.374 & 0.0458 & 0.595 & 326 \\
\hline 05551930 & 1 & Yes & Yes & 1972 & 563 & -- & 585 & 1.07 & 0.0468 & 0.179 & 606 \\
\hline 05551930 & 1 & Yes & Yes & 1973 & 334 & -- & 347 & 1.47 & 0.0478 & 0.507 & 365 \\
\hline 05551930 & 1 & Yes & Yes & 1974 & 694 & -- & 721 & 1.32 & 0.0488 & 0.0961 & 744 \\
\hline 05551930 & 1 & Yes & Yes & 1975 & 187 & -- & 194 & 0.855 & 0.0497 & 0.82 & 208 \\
\hline 05551930 & 1 & Yes & Yes & 1976 & 291 & -- & 302 & 1.09 & 0.0507 & 0.611 & 318 \\
\hline 05551930 & 1 & Yes & Yes & 1977 & 141 & -- & 146 & 0.834 & 0.0517 & 0.897 & 158 \\
\hline 05551930 & 1 & Yes & Yes & 1978 & 525 & -- & 545 & 1.39 & 0.0527 & 0.214 & 562 \\
\hline 05551930 & 1 & Yes & Yes & 1979 & 402 & -- & 418 & 0.354 & 0.0536 & 0.381 & 434 \\
\hline 05551930 & 1 & Yes & Yes & 1980 & 180 & -- & 187 & 0.913 & 0.0546 & 0.834 & 199 \\
\hline
\end{tabular}




\section{APPENDIX 7}

Table 8. Quantile regression coefficients from temporal analysis of 117 streamgages in northeastern Illinois and adjacent states, as a function of annual exceedance probability.

\begin{tabular}{|c|c|c|c|c|c|c|c|c|c|c|}
\hline \multirow{2}{*}{$\begin{array}{c}\text { Annual } \\
\text { exceedance } \\
\text { probability }\end{array}$} & \multicolumn{3}{|c|}{ Quantile regression coefficients } & \multicolumn{3}{|c|}{ Mean bootstrapped coefficients } & \multicolumn{3}{|c|}{$\begin{array}{l}\text { Standard error of bootstrapped } \\
\text { coefficients }\end{array}$} & \multirow{2}{*}{$\begin{array}{c}\text { Fitted } \\
\text { urban } \\
\text { fraction } \\
\text { coefficients }\end{array}$} \\
\hline & Intercept & $\begin{array}{c}\text { Urban } \\
\text { fraction }\end{array}$ & Precipitation & Intercept & $\begin{array}{l}\text { Urban } \\
\text { fraction }\end{array}$ & Precipitation & Intercept & $\begin{array}{l}\text { Urban } \\
\text { fraction }\end{array}$ & Precipitation & \\
\hline 0.990 & 1.6168 & 0.9125 & 0.1092 & 1.6017 & 0.9407 & 0.1107 & 0.0863 & 0.0864 & 0.0249 & 0.9288 \\
\hline 0.980 & 1.7265 & 0.8509 & 0.1114 & 1.7277 & 0.8676 & 0.1056 & 0.0832 & 0.0829 & 0.0182 & 0.8833 \\
\hline 0.950 & 1.9029 & 0.7730 & 0.1039 & 1.9067 & 0.7784 & 0.0997 & 0.0763 & 0.0752 & 0.0152 & 0.7790 \\
\hline 0.925 & 2.0056 & 0.7146 & 0.0979 & 2.0021 & 0.7238 & 0.0966 & 0.0759 & 0.0758 & 0.0113 & 0.7199 \\
\hline 0.900 & 2.0585 & 0.6760 & 0.1008 & 2.0590 & 0.6815 & 0.0993 & 0.0716 & 0.0724 & .0089 & 0.6778 \\
\hline 0.875 & 2.1073 & 0.6357 & 0.1054 & 2.1045 & 0.6461 & 0.1030 & 0.0720 & 0.0707 & .0084 & 0.6471 \\
\hline 0.825 & 2.1747 & 0.5978 & 0.1050 & 2.1715 & 0.6026 & 0.1045 & 0.0709 & 0.0696 & 0.0089 & 0.6049 \\
\hline 0.800 & 2.2041 & 0.5836 & 0.1019 & 2.1993 & 0.5888 & 0.1042 & 0.0718 & 0.0711 & 0.0098 & 0.5890 \\
\hline 0.750 & 2.2513 & 0.5557 & 0.1065 & 2.2490 & 0.5590 & 0.1063 & 0.0715 & 0.0716 & 0.0095 & 0.5618 \\
\hline 0.700 & 2.2904 & 0.5425 & 0.1059 & 2.2879 & 0.5439 & 0.1062 & 0.0711 & 0.0712 & 0.0079 & 0.5387 \\
\hline 0.600 & 2.3645 & 0.4967 & 0.1052 & 2.3606 & 0.5010 & 0.1053 & 0.0692 & 0.0702 & 0.0089 & 0.5055 \\
\hline 0.500 & 2.4129 & 0.4866 & 0.1065 & 2.4109 & 0.4905 & 0.1063 & 0.0692 & 0.0751 & 0.0075 & 0.4872 \\
\hline 0.400 & 2.4700 & 0.4679 & 0.1055 & 2.4668 & 0.4696 & 0.1063 & 0.0687 & 0.0740 & 0.0065 & 0.4679 \\
\hline 0.300 & 2.5281 & 0.4275 & 0.1098 & 2.5267 & 0.4297 & 0.1096 & 0.0682 & 0.0732 & 0.0068 & 0.4359 \\
\hline 0.250 & 2.5569 & 0.4152 & 0.1125 & 2.5557 & 0.4171 & 0.1121 & 0.0687 & 0.0771 & 0.0086 & 0.4187 \\
\hline 0.200 & 2.5867 & 0.4114 & 0.1152 & 2.5856 & 0.4128 & 0.1141 & 0.0674 & 0.0797 & 0.0081 & 0.4053 \\
\hline 0.100 & 2.6810 & 0.3907 & 0.1169 & 2.6798 & 0.3945 & 0.1148 & 0.0651 & 0.0776 & 0.0085 & 0.3912 \\
\hline 0.075 & 2.7109 & 0.3859 & 0.1208 & 2.7125 & 0.3807 & 0.1184 & 0.0646 & 0.0782 & 0.0101 & 0.3837 \\
\hline 0.050 & 2.7649 & 0.3582 & 0.1225 & 2.7577 & 0.3544 & 0.1278 & 0.0669 & 0.0861 & 0.0148 & 0.3683 \\
\hline 0.040 & 2.7671 & 0.3693 & 0.1429 & 2.7727 & 0.3523 & 0.1386 & 0.0671 & 0.0850 & 0.0154 & 0.3588 \\
\hline 0.030 & 2.7893 & 0.3455 & 0.1527 & 2.7904 & 0.3505 & 0.1481 & 0.0669 & 0.0794 & 0.0129 & 0.3466 \\
\hline 0.020 & 2.8290 & 0.3382 & 0.1495 & 2.8216 & 0.3468 & 0.1550 & 0.0667 & 0.0861 & 0.0201 & 0.3313 \\
\hline 0.010 & 2.9361 & 0.3245 & 0.1666 & 2.9281 & 0.3326 & 0.1570 & 0.0759 & 0.1340 & 0.0283 & 0.3123 \\
\hline 0.005 & 3.0352 & 0.3172 & 0.1440 & 3.0217 & 0.3008 & 0.1507 & 0.0735 & 0.1119 & 0.0188 & 0.3012 \\
\hline 0.002 & 3.1086 & 0.3003 & 0.1312 & 3.1149 & 0.2721 & 0.1365 & 0.0845 & 0.1151 & 0.0282 & .2939 \\
\hline
\end{tabular}




\section{APPENDIX 8}

Table 13. Components of variance of prediction for the selected spatial regression equations in northeastern Illinois.

[ $X$, basin characteristics matrix; $\Lambda$, generalized least squares (GLS) covariance matrix; $\mathrm{mi}^{2}$, square miles; NLCD_22_23_24, sum of fractions of 2011 National Land Cover Database (NLCD) classes 22, 23, and 24; DrainageClass1a, sum of fractions of Soil Survey Geographic (SSURGO) database fractions "very poorly drained" and "unknown (likely water)"; DEM_1_0_P, basin elevation range divided by basin perimeter]

\begin{tabular}{|c|c|c|c|c|c|c|c|}
\hline \multirow[b]{2}{*}{$\begin{array}{l}\text { Exceedance } \\
\text { probability }\end{array}$} & \multirow{2}{*}{$\begin{array}{c}\text { Model } \\
\text { error } \\
\text { variance, } v^{2}\end{array}$} & & \multicolumn{5}{|c|}{$\left(X^{t} \Lambda^{-1} X\right)^{-1}$ matrices } \\
\hline & & & Intercept & $\begin{array}{c}\log _{10}(\text { Drainage } \\
\left.\text { area }\left(\mathrm{mi}^{2}\right)\right)\end{array}$ & $N L C D \_22 \_23 \_24^{(1 / 2)}$ & DrainageClass1a ${ }^{(1 / 2)}$ & $\begin{array}{c}\log _{10}\left(D E M \_1{ }_{1} 0 \_P\right. \\
(\text { feet per mile }))\end{array}$ \\
\hline \multirow[t]{5}{*}{0.5} & 0.0341 & Intercept & $1.7748 \mathrm{E}-02$ & $-4.8488 E-03$ & $-4.3213 \mathrm{E}-03$ & $-2.3209 E-03$ & $-1.1554 \mathrm{E}-02$ \\
\hline & & $\begin{array}{c}\log _{10}(\text { Drainage area } \\
\left.\left(\mathrm{mi}^{2}\right)\right)\end{array}$ & $-4.8488 \mathrm{E}-03$ & $1.8558 \mathrm{E}-03$ & $8.0193 \mathrm{E}-04$ & $-1.4525 \mathrm{E}-03$ & $3.3657 \mathrm{E}-03$ \\
\hline & & $N L C D$ 22_23_24 $4^{(1 / 2)}$ & $-4.3213 E-03$ & 8.0193E-04 & $4.8550 \mathrm{E}-03$ & $-1.0945 \mathrm{E}-03$ & $1.2048 \mathrm{E}-03$ \\
\hline & & DrainageClass $1 a^{(1 / 2)}$ & $-2.3209 \mathrm{E}-03$ & $-1.4525 \mathrm{E}-03$ & $-1.0945 \mathrm{E}-03$ & $2.2544 \mathrm{E}-02$ & $-7.9265 \mathrm{E}-04$ \\
\hline & & $\begin{array}{l}\log _{10}\left(D E M \_1{ }_{1} 0 \_P\right. \\
(\text { feet per mile }))\end{array}$ & $-1.1554 \mathrm{E}-02$ & 3.3657E-03 & $1.2048 \mathrm{E}-03$ & $-7.9265 \mathrm{E}-04$ & $1.0157 \mathrm{E}-02$ \\
\hline \multirow[t]{5}{*}{0.2} & 0.0354 & Intercept & $1.8866 \mathrm{E}-02$ & $-5.1542 E-03$ & $-4.5317 E-03$ & $-2.5357 \mathrm{E}-03$ & $-1.2385 E-02$ \\
\hline & & $\begin{array}{c}\log _{10}(\text { Drainage area } \\
\left.\left(\mathrm{mi}^{2}\right)\right)\end{array}$ & $-5.1542 \mathrm{E}-03$ & $1.9885 \mathrm{E}-03$ & $7.8898 \mathrm{E}-04$ & $-1.5811 \mathrm{E}-03$ & $3.5946 \mathrm{E}-03$ \\
\hline & & NLCD_22_23_24 $4^{(1 / 2)}$ & $-4.5317 E-03$ & $7.8898 \mathrm{E}-04$ & $5.4322 \mathrm{E}-03$ & $-1.0841 \mathrm{E}-03$ & $1.3331 \mathrm{E}-03$ \\
\hline & & DrainageClass $1 a^{(1 / 2)}$ & $-2.5357 \mathrm{E}-03$ & $-1.5811 E-03$ & $-1.0841 E-03$ & $2.4300 \mathrm{E}-02$ & $-7.9253 \mathrm{E}-04$ \\
\hline & & $\begin{array}{c}\log _{10}\left(D E M \_1{ }_{1} 0 \_P\right. \\
(\text { feet per mile }))\end{array}$ & $-1.2385 \mathrm{E}-02$ & $3.5946 \mathrm{E}-03$ & $1.3331 \mathrm{E}-03$ & $-7.9253 \mathrm{E}-04$ & $1.0930 \mathrm{E}-02$ \\
\hline \multirow[t]{5}{*}{0.1} & 0.0387 & Intercept & $2.0648 \mathrm{E}-02$ & $-5.6268 \mathrm{E}-03$ & $-4.9823 \mathrm{E}-03$ & $-2.8672 \mathrm{E}-03$ & $-1.3589 E-02$ \\
\hline & & $\begin{array}{c}\log _{10}(\text { Drainage area } \\
\left.\left(\mathrm{mi}^{2}\right)\right)\end{array}$ & $-5.6268 \mathrm{E}-03$ & $2.1796 \mathrm{E}-03$ & 8.1797E-04 & $-1.7460 \mathrm{E}-03$ & $3.9348 \mathrm{E}-03$ \\
\hline & & $N L C D$ 22_23_24 $4^{(1 / 2)}$ & $-4.9823 E-03$ & 8.1797E-04 & $6.2746 \mathrm{E}-03$ & $-1.0692 \mathrm{E}-03$ & $1.4835 \mathrm{E}-03$ \\
\hline & & DrainageClass $1 a^{(1 / 2)}$ & $-2.8672 \mathrm{E}-03$ & $-1.7460 \mathrm{E}-03$ & $-1.0692 \mathrm{E}-03$ & $2.6792 \mathrm{E}-02$ & $-8.2944 \mathrm{E}-04$ \\
\hline & & $\begin{array}{c}\log _{10}\left(D E M \_1 \_0 \_P\right. \\
(\text { feet per mile }))\end{array}$ & $-1.3589 \mathrm{E}-02$ & $3.9348 \mathrm{E}-03$ & $1.4835 \mathrm{E}-03$ & $-8.2944 \mathrm{E}-04$ & $1.2037 \mathrm{E}-02$ \\
\hline \multirow[t]{4}{*}{0.04} & 0.0432 & Intercept & $2.4241 \mathrm{E}-02$ & $-6.5853 \mathrm{E}-03$ & $-5.9149 \mathrm{E}-03$ & $-3.4644 \mathrm{E}-03$ & $-1.6015 \mathrm{E}-02$ \\
\hline & & $\begin{array}{c}\log _{10}(\text { Drainage area } \\
\left.\left(\mathrm{mi}^{2}\right)\right)\end{array}$ & $-6.5853 \mathrm{E}-03$ & $2.5560 \mathrm{E}-03$ & $9.1721 \mathrm{E}-04$ & $-2.0573 \mathrm{E}-03$ & $4.6231 \mathrm{E}-03$ \\
\hline & & NLCD_22_23_24(1/2) & $-5.9149 \mathrm{E}-03$ & $9.1721 \mathrm{E}-04$ & $7.7961 \mathrm{E}-03$ & $-1.1086 \mathrm{E}-03$ & $1.7924 \mathrm{E}-03$ \\
\hline & & DrainageClass $1 a^{(1 / 2)}$ & $-3.4644 \mathrm{E}-03$ & $-2.0573 E-03$ & $-1.1086 \mathrm{E}-03$ & $3.1587 \mathrm{E}-02$ & $-9.4557 \mathrm{E}-04$ \\
\hline
\end{tabular}




\begin{tabular}{|c|c|c|c|c|c|c|c|}
\hline \multirow[b]{2}{*}{$\begin{array}{c}\text { Exceedance } \\
\text { probability }\end{array}$} & \multirow{2}{*}{$\begin{array}{c}\text { Model } \\
\text { error } \\
\text { variance, } v^{2}\end{array}$} & & \multicolumn{5}{|c|}{$\left(X^{t} \Lambda^{-1} X\right)^{-1}$ matrices } \\
\hline & & & Intercept & $\begin{array}{c}\log _{10}(\text { Drainage } \\
\left.\text { area }\left(\mathrm{mi}^{2}\right)\right)\end{array}$ & $N L C D \_22 \_23 \_24^{(1 / 2)}$ & DrainageClass1a $a^{(1 / 2)}$ & $\begin{array}{c}\log _{10}\left(D E M \_1 \_0 \_P\right. \\
(\text { feet per mile) })\end{array}$ \\
\hline & & $\begin{array}{l}\log _{10}\left(D E M \_1{ }_{1} 0 \_P\right. \\
(\text { feet per mile }))\end{array}$ & $-1.6015 E-02$ & $4.6231 \mathrm{E}-03$ & $1.7924 \mathrm{E}-03$ & $-9.4557 \mathrm{E}-04$ & $1.4229 \mathrm{E}-02$ \\
\hline \multirow[t]{5}{*}{0.02} & 0.0476 & Intercept & $2.7554 \mathrm{E}-02$ & $-7.4709 \mathrm{E}-03$ & $-6.7834 \mathrm{E}-03$ & $-3.9893 \mathrm{E}-03$ & $-1.8258 \mathrm{E}-02$ \\
\hline & & $\begin{array}{c}\log _{10}(\text { Drainage area } \\
\left.\left(\mathrm{mi}^{2}\right)\right)\end{array}$ & $-7.4709 \mathrm{E}-03$ & $2.8990 \mathrm{E}-03$ & $1.0231 \mathrm{E}-03$ & $-2.3346 \mathrm{E}-03$ & $5.2590 \mathrm{E}-03$ \\
\hline & & NLCD_22_23_24 $4^{(1 / 2)}$ & $-6.7834 \mathrm{E}-03$ & $1.0231 \mathrm{E}-03$ & $9.1415 \mathrm{E}-03$ & $-1.1653 \mathrm{E}-03$ & $2.0867 \mathrm{E}-03$ \\
\hline & & DrainageClass $1 a^{(1 / 2)}$ & $-3.9893 \mathrm{E}-03$ & $-2.3346 \mathrm{E}-03$ & $-1.1653 \mathrm{E}-03$ & 3.5905E-02 & $-1.0685 \mathrm{E}-03$ \\
\hline & & $\begin{array}{c}\log _{10}\left(D E M \_1 \_0 \_P\right. \\
(\text { feet per mile }))\end{array}$ & $-1.8258 \mathrm{E}-02$ & $5.2590 \mathrm{E}-03$ & $2.0867 \mathrm{E}-03$ & $-1.0685 \mathrm{E}-03$ & 1.6244E-02 \\
\hline \multirow[t]{5}{*}{0.01} & 0.0527 & Intercept & $3.1003 \mathrm{E}-02$ & $-8.3921 E-03$ & $-7.6988 \mathrm{E}-03$ & $-4.5311 \mathrm{E}-03$ & $-2.0598 \mathrm{E}-02$ \\
\hline & & $\begin{array}{c}\log _{10}(\text { Drainage area } \\
\left.\left(\mathrm{mi}^{2}\right)\right)\end{array}$ & $-8.3921 \mathrm{E}-03$ & $3.2522 \mathrm{E}-03$ & 1.1394E-03 & $-2.6149 \mathrm{E}-03$ & $5.9206 \mathrm{E}-03$ \\
\hline & & NLCD_22_23_24 $4^{(1 / 2)}$ & $-7.6988 \mathrm{E}-03$ & 1.1394E-03 & $1.0542 \mathrm{E}-02$ & $-1.2200 \mathrm{E}-03$ & $2.4035 \mathrm{E}-03$ \\
\hline & & DrainageClass $1 a^{(1 / 2)}$ & $-4.5311 E-03$ & $-2.6149 \mathrm{E}-03$ & $-1.2200 \mathrm{E}-03$ & 4.0324E-02 & $-1.2044 \mathrm{E}-03$ \\
\hline & & $\begin{array}{c}\log _{10}\left(D E M \_1 \_0 \_P\right. \\
\text { (feet per mile) })\end{array}$ & $-2.0598 \mathrm{E}-02$ & $5.9206 \mathrm{E}-03$ & $2.4035 \mathrm{E}-03$ & $-1.2044 \mathrm{E}-03$ & $1.8346 \mathrm{E}-02$ \\
\hline \multirow[t]{5}{*}{0.005} & 0.0575 & Intercept & $3.4885 \mathrm{E}-02$ & $-9.4316 \mathrm{E}-03$ & $-8.7290 \mathrm{E}-03$ & $-5.1224 \mathrm{E}-03$ & $-2.3242 \mathrm{E}-02$ \\
\hline & & $\begin{array}{c}\log _{10}(\text { Drainage area } \\
\left.\left(\mathrm{mi}^{2}\right)\right)\end{array}$ & $-9.4316 \mathrm{E}-03$ & $3.6476 \mathrm{E}-03$ & $1.2786 \mathrm{E}-03$ & $-2.9248 \mathrm{E}-03$ & $6.6678 \mathrm{E}-03$ \\
\hline & & $N L C D \_22 \_23{ }^{2} 24^{(1 / 2)}$ & $-8.7290 \mathrm{E}-03$ & $1.2786 \mathrm{E}-03$ & $1.2073 \mathrm{E}-02$ & $-1.2960 \mathrm{E}-03$ & $2.7674 \mathrm{E}-03$ \\
\hline & & DrainageClass1a $a^{(1 / 2)}$ & $-5.1224 \mathrm{E}-03$ & $-2.9248 \mathrm{E}-03$ & $-1.2960 \mathrm{E}-03$ & 4.5232E-02 & $-1.3663 \mathrm{E}-03$ \\
\hline & & $\begin{array}{c}\log _{10}\left(D E M \_1 \_0 \_P\right. \\
(\text { feet per mile }))\end{array}$ & $-2.3242 \mathrm{E}-02$ & $6.6678 \mathrm{E}-03$ & $2.7674 \mathrm{E}-03$ & $-1.3663 \mathrm{E}-03$ & $2.0711 \mathrm{E}-02$ \\
\hline \multirow[t]{5}{*}{0.002} & 0.0644 & Intercept & $4.0052 E-02$ & $-1.0814 \mathrm{E}-02$ & $-1.0114 \mathrm{E}-02$ & $-5.9035 \mathrm{E}-03$ & $-2.6770 \mathrm{E}-02$ \\
\hline & & $\begin{array}{c}\log _{10}(\text { Drainage area } \\
\left.\left(\mathrm{mi}^{2}\right)\right)\end{array}$ & $-1.0814 \mathrm{E}-02$ & 4.1676E-03 & $1.4716 \mathrm{E}-03$ & $-3.3236 \mathrm{E}-03$ & 7.6621E-03 \\
\hline & & NLCD_22_23_24 $4^{(1 / 2)}$ & $-1.0114 \mathrm{E}-02$ & $1.4716 \mathrm{E}-03$ & $1.4116 \mathrm{E}-02$ & $-1.3865 \mathrm{E}-03$ & $3.2677 \mathrm{E}-03$ \\
\hline & & DrainageClass1a ${ }^{(1 / 2)}$ & $-5.9035 \mathrm{E}-03$ & $-3.3236 \mathrm{E}-03$ & $-1.3865 \mathrm{E}-03$ & 5.1639E-02 & $-1.5932 \mathrm{E}-03$ \\
\hline & & $\begin{array}{l}\log _{10}\left(D E M \_1 D_{-} 0 \_P\right. \\
(\text { feet per mile) })\end{array}$ & $-2.6770 \mathrm{E}-02$ & $7.6621 \mathrm{E}-03$ & $3.2677 \mathrm{E}-03$ & $-1.5932 \mathrm{E}-03$ & $2.3871 \mathrm{E}-02$ \\
\hline
\end{tabular}




\section{APPENDIX 9}

Table 1-1. Skew statistics at U.S. Geological Survey streamgages used in the development of the regional skew model in this study in northeastern Illinois.

[USGS, U.S. Geological Survey; MSE, mean-square error; NLCD_22_23_24, urbanization variable that is the sum of fractions of National Land Cover Dataset 2011 classes 22 (developed, low intensity), 23 (developed, medium intensity), 24 (developed, high intensity); $\mathrm{mi}^{2}$, square miles]

\begin{tabular}{|c|c|c|c|c|c|c|c|c|c|}
\hline Index \# & $\begin{array}{c}\text { USGS } \\
\text { streamgage } \\
\text { number }\end{array}$ & $\begin{array}{l}\text { Pseudo } \\
\text { record } \\
\text { length, } \\
\text { in years }\end{array}$ & 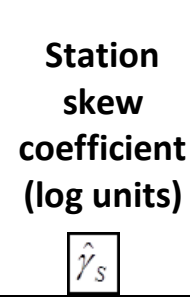 & 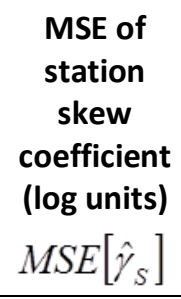 & $\begin{array}{c}\text { NLCD_22_23_24 } \\
\text { (fraction) }\end{array}$ & $\begin{array}{c}\text { Regional } \\
\text { skew } \\
\text { coefficient, } \\
\text { URBAN } \\
\text { model } \\
\text { (log units) }\end{array}$ & $\begin{array}{l}\text { Drainage } \\
\text { area } \\
\left(\mathrm{mi}^{2}\right)\end{array}$ & $\begin{array}{l}\text { Basin } \\
\text { centroid } \\
\text { latitude } \\
\text { (decimal } \\
\text { degrees) } \\
\end{array}$ & $\begin{array}{c}\text { Basin } \\
\text { centroid } \\
\text { longitude } \\
\text { (decimal } \\
\text { degrees) }\end{array}$ \\
\hline 1 & 04087300 & 17 & 0.86 & 0.37 & 0.342 & 0.178 & 1.50 & 42.4901 & -87.8452 \\
\hline 2 & 04087400 & 15 & 0.92 & 0.43 & 0.411 & 0.232 & 5.07 & 42.4695 & -87.8553 \\
\hline 3 & 05438250 & 30 & 0.51 & 0.21 & 0.070 & -0.133 & 85.12 & 42.1051 & -88.5673 \\
\hline 4 & 05438300 & 20 & -0.33 & 0.28 & 0.059 & -0.154 & 0.81 & 42.4627 & -88.5880 \\
\hline 5 & 05438500 & 70 & -0.92 & 0.15 & 0.091 & -0.097 & 536.74 & 42.2736 & -88.5997 \\
\hline 6 & 05438850 & 25 & -0.67 & 0.26 & 0.028 & -0.227 & 1.52 & 41.8513 & -88.9042 \\
\hline 7 & 05439000 & 30 & 0.75 & 0.24 & 0.076 & -0.123 & 77.41 & 41.8618 & -88.8311 \\
\hline 8 & 05439500 & 70 & -0.28 & 0.09 & 0.084 & -0.108 & 387.01 & 41.9869 & -88.7341 \\
\hline 9 & 05439550 & 18 & -0.26 & 0.29 & 0.009 & -0.300 & 1.78 & 42.1760 & -88.9284 \\
\hline 10 & 05440500 & 41 & -1.28 & 0.31 & 0.038 & -0.201 & 117.64 & 42.0004 & -88.9839 \\
\hline 11 & 05446950 & 16 & 0.50 & 0.35 & 0.040 & -0.195 & 0.66 & 41.7659 & -89.3352 \\
\hline 12 & 05447000 & 42 & -0.19 & 0.13 & 0.026 & -0.232 & 198.69 & 41.7540 & -89.1402 \\
\hline 13 & 05447500 & 70 & -0.42 & 0.10 & 0.025 & -0.236 & 999.57 & 41.5422 & -89.6641 \\
\hline 14 & 05526150 & 25 & -0.05 & 0.20 & 0.002 & -0.345 & 0.16 & 41.1934 & -87.9442 \\
\hline 15 & 05526500 & 26 & 1.77 & 0.61 & 0.023 & -0.244 & 13.00 & 41.1951 & -88.0511 \\
\hline 16 & 05527050 & 17 & 1.65 & 0.70 & 0.074 & -0.126 & 0.83 & 41.4448 & -87.8314 \\
\hline 17 & 05527800 & 50 & -0.42 & 0.13 & 0.120 & -0.053 & 123.65 & 42.5925 & -88.0017 \\
\hline 18 & 05527900 & 17 & -0.02 & 0.29 & 0.070 & -0.134 & 20.79 & 42.5011 & -88.0316 \\
\hline 19 & 05528150 & 17 & 0.08 & 0.29 & 0.395 & 0.220 & 10.92 & 42.2324 & -88.0451 \\
\hline 20 & 05528170 & 16 & 0.15 & 0.31 & 0.466 & 0.272 & 2.58 & 42.2549 & -88.0210 \\
\hline 21 & 05528360 & 16 & 0.69 & 0.37 & 0.669 & 0.403 & 2.83 & 42.1831 & -87.9694 \\
\hline 22 & 05528440 & 16 & 0.61 & 0.36 & 0.806 & 0.481 & 1.66 & 42.1888 & -88.0709 \\
\hline 23 & 05528500 & 57 & -0.58 & 0.13 & 0.586 & 0.352 & 19.64 & 42.1619 & -88.0246 \\
\hline 24 & 05529500 & 57 & 0.04 & 0.09 & 0.841 & 0.500 & 7.83 & 42.1128 & -87.9641 \\
\hline 25 & 05530000 & 59 & -0.41 & 0.12 & 0.904 & 0.532 & 12.73 & 42.0714 & -87.9719 \\
\hline 26 & 05530400 & 19 & -0.27 & 0.28 & 0.696 & 0.419 & 2.05 & 42.0350 & -87.9810 \\
\hline 27 & 05530480 & 21 & 0.26 & 0.25 & 0.901 & 0.531 & 17.92 & 42.0019 & -87.9461 \\
\hline 28 & 05530700 & 22 & 0.00 & 0.22 & 0.936 & 0.549 & 11.15 & 41.9417 & -87.9085 \\
\hline
\end{tabular}




\begin{tabular}{|c|c|c|c|c|c|c|c|c|c|}
\hline Index\# & $\begin{array}{c}\text { USGS } \\
\text { streamgage } \\
\text { number }\end{array}$ & $\begin{array}{l}\text { Pseudo } \\
\text { record } \\
\text { length, } \\
\text { in years }\end{array}$ & $\begin{array}{c}\text { Station } \\
\text { skew } \\
\text { coefficient } \\
\text { (log units) } \\
\hat{\gamma}_{S}\end{array}$ & $\begin{array}{c}\text { MSE of } \\
\text { station } \\
\text { skew } \\
\text { coefficient } \\
\text { (log units) } \\
M S E\left[\hat{\gamma}_{S}\right]\end{array}$ & $\begin{array}{c}\text { NLCD_22_23_24 } \\
\text { (fraction) }\end{array}$ & $\begin{array}{c}\text { Regional } \\
\text { skew } \\
\text { coefficient, } \\
\text { URBAN } \\
\text { model } \\
\text { (log units) }\end{array}$ & $\begin{array}{c}\text { Drainage } \\
\text { area } \\
\left(\mathrm{mi}^{2}\right)\end{array}$ & $\begin{array}{l}\text { Basin } \\
\text { centroid } \\
\text { latitude } \\
\text { (decimal } \\
\text { degrees) }\end{array}$ & $\begin{array}{c}\text { Basin } \\
\text { centroid } \\
\text { longitud } \\
\text { (decimal } \\
\text { degrees) }\end{array}$ \\
\hline 29 & 05530940 & 19 & 0.54 & 0.31 & 0.628 & 0.378 & 5.71 & 42.1138 & -88.0925 \\
\hline 30 & 05531080 & 19 & 1.90 & 0.79 & 0.812 & 0.484 & 6.17 & 41.9685 & -88.0878 \\
\hline 31 & 05531100 & 19 & 1.03 & 0.39 & 0.847 & 0.503 & 4.20 & 41.9900 & -88.0624 \\
\hline 32 & 05531500 & 64 & 0.47 & 0.11 & 0.760 & 0.456 & 116.25 & 41.9787 & -88.0209 \\
\hline 33 & 05532000 & 59 & -0.01 & 0.09 & 0.893 & 0.527 & 16.19 & 41.9126 & -87.9214 \\
\hline 34 & 05533000 & 59 & 0.26 & 0.10 & 0.849 & 0.504 & 16.82 & 41.7799 & -87.9263 \\
\hline 35 & 05533200 & 19 & 0.25 & 0.28 & 0.909 & 0.535 & 2.35 & 41.7494 & -87.9705 \\
\hline 36 & 05533300 & 15 & -0.24 & 0.34 & 0.675 & 0.407 & 3.11 & 41.7386 & -88.0032 \\
\hline 37 & 05534500 & 57 & -0.18 & 0.10 & 0.452 & 0.262 & 19.70 & 42.2481 & -87.8813 \\
\hline 38 & 05535000 & 58 & -0.23 & 0.10 & 0.552 & 0.330 & 12.63 & 42.2958 & -87.8717 \\
\hline 39 & 05535500 & 57 & -0.14 & 0.10 & 0.609 & 0.367 & 11.64 & 42.1757 & -87.8728 \\
\hline 40 & 05536000 & 59 & 0.33 & 0.11 & 0.639 & 0.386 & 99.34 & 42.1656 & -87.8330 \\
\hline 41 & 05536201 & 19 & 0.31 & 0.28 & 0.319 & 0.158 & 5.82 & 41.4471 & -87.7078 \\
\hline 42 & 05536207 & 16 & 0.86 & 0.39 & 0.836 & 0.497 & 3.93 & 41.4849 & -87.6904 \\
\hline 43 & 05536235 & 58 & 0.34 & 0.11 & 0.382 & 0.210 & 23.36 & 41.4523 & -87.6336 \\
\hline 44 & 05536238 & 19 & -0.22 & 0.28 & 0.528 & 0.315 & 1.76 & 41.4996 & -87.7682 \\
\hline 45 & 05536255 & 62 & 0.53 & 0.12 & 0.674 & 0.407 & 23.21 & 41.5099 & -87.7211 \\
\hline 46 & 05536265 & 62 & -0.35 & 0.11 & 0.413 & 0.234 & 8.02 & 41.4876 & -87.5551 \\
\hline 47 & 05536270 & 32 & 0.03 & 0.16 & 0.541 & 0.324 & 16.83 & 41.5118 & -87.5473 \\
\hline 48 & 05536340 & 59 & -0.06 & 0.09 & 0.831 & 0.494 & 13.62 & 41.5843 & -87.7878 \\
\hline 49 & 05536500 & 58 & 0.40 & 0.12 & 0.639 & 0.386 & 11.27 & 41.6162 & -87.8110 \\
\hline 50 & 05536510 & 19 & 0.48 & 0.30 & 0.717 & 0.431 & 1.60 & 41.6522 & -87.8058 \\
\hline 51 & 05536560 & 19 & -0.12 & 0.27 & 0.979 & 0.570 & 5.54 & 41.7403 & -87.7766 \\
\hline 52 & 05536630 & 19 & 0.90 & 0.36 & 0.601 & 0.362 & 10.52 & 41.6479 & -87.8558 \\
\hline 53 & 05537500 & 59 & 0.77 & 0.15 & 0.467 & 0.273 & 21.11 & 41.6361 & -87.9396 \\
\hline 54 & 05538440 & 18 & 0.52 & 0.32 & 0.624 & 0.376 & 1.54 & 41.6114 & -87.8903 \\
\hline 55 & 05539000 & 64 & 0.95 & 0.16 & 0.516 & 0.307 & 108.21 & 41.5345 & -87.8861 \\
\hline 56 & 05539900 & 49 & 0.28 & 0.12 & 0.716 & 0.431 & 27.95 & 41.9736 & -88.1515 \\
\hline 57 & 05539950 & 19 & 1.91 & 0.79 & 0.903 & 0.532 & 9.11 & 41.9251 & -88.1183 \\
\hline 58 & 05540060 & 44 & 1.64 & 0.42 & 0.531 & 0.317 & 18.63 & 41.8856 & -88.2377 \\
\hline 59 & 05540080 & 19 & 1.32 & 0.51 & 0.876 & 0.518 & 2.07 & 41.8567 & -88.1020 \\
\hline 60 & 05540110 & 19 & 0.65 & 0.32 & 0.278 & 0.121 & 3.69 & 41.8338 & -88.2219 \\
\hline 61 & 05540140 & 19 & 0.32 & 0.29 & 0.924 & 0.543 & 2.42 & 41.9417 & -88.0736 \\
\hline 62 & 05540160 & 36 & 0.57 & 0.19 & 0.795 & 0.475 & 25.21 & 41.8876 & -88.0491 \\
\hline 63 & 05540190 & 17 & -0.32 & 0.31 & 0.808 & 0.482 & 8.82 & 41.7919 & -88.0002 \\
\hline
\end{tabular}




\begin{tabular}{|c|c|c|c|c|c|c|c|c|c|}
\hline Index\# & $\begin{array}{c}\text { USGS } \\
\text { streamgage } \\
\text { number }\end{array}$ & $\begin{array}{l}\text { Pseudo } \\
\text { record } \\
\text { length, } \\
\text { in years }\end{array}$ & 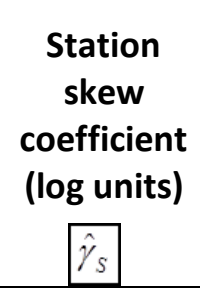 & $\begin{array}{c}\text { MSE of } \\
\text { station } \\
\text { skew } \\
\text { coefficient } \\
\text { (log units) } \\
M S E\left[\hat{\gamma}_{S}\right]\end{array}$ & $\begin{array}{c}\text { NLCD_22_23_24 } \\
\text { (fraction) }\end{array}$ & $\begin{array}{c}\text { Regional } \\
\text { skew } \\
\text { coefficient, } \\
\text { URBAN } \\
\text { model } \\
\text { (log units) }\end{array}$ & $\begin{array}{c}\text { Drainage } \\
\text { area } \\
\left(\mathrm{mi}^{2}\right)\end{array}$ & $\begin{array}{l}\text { Basin } \\
\text { centroid } \\
\text { latitude } \\
\text { (decimal } \\
\text { degrees) }\end{array}$ & $\begin{array}{c}\text { Basin } \\
\text { centroid } \\
\text { longitude } \\
\text { (decimal } \\
\text { degrees) }\end{array}$ \\
\hline 64 & 05540240 & 20 & 0.69 & 0.31 & 0.844 & 0.501 & 6.57 & 41.7670 & -88.0272 \\
\hline 65 & 05540275 & 22 & 0.87 & 0.31 & 0.614 & 0.370 & 9.89 & 41.7467 & -88.1887 \\
\hline 66 & 05540500 & 69 & 0.35 & 0.10 & 0.646 & 0.390 & 323.19 & 41.7774 & -88.1421 \\
\hline 67 & 05541750 & 22 & -0.05 & 0.23 & 0.010 & -0.294 & 4.74 & 41.1321 & -88.3521 \\
\hline 68 & 05542000 & 70 & -0.44 & 0.10 & 0.038 & -0.200 & 451.80 & 41.0978 & -88.3730 \\
\hline 69 & 05547755 & 16 & -0.64 & 0.37 & 0.237 & 0.082 & 16.37 & 42.2991 & -88.0787 \\
\hline 70 & 05548280 & 44 & -0.51 & 0.16 & 0.079 & -0.118 & 195.14 & 42.4499 & -88.3942 \\
\hline 71 & 05549000 & 44 & -0.31 & 0.14 & 0.056 & -0.161 & 15.65 & 42.3126 & -88.3616 \\
\hline 72 & 05549700 & 15 & 0.35 & 0.34 & 0.192 & 0.036 & 10.24 & 42.2907 & -88.1423 \\
\hline 73 & 05549850 & 31 & 1.07 & 0.27 & 0.375 & 0.204 & 35.57 & 42.1758 & -88.1350 \\
\hline 74 & 05549900 & 24 & -0.43 & 0.26 & 0.945 & 0.553 & 0.08 & 42.1985 & -88.2670 \\
\hline 75 & 05550300 & 43 & 0.57 & 0.15 & 0.257 & 0.101 & 39.04 & 42.0687 & -88.4027 \\
\hline 76 & 05550430 & 17 & 0.26 & 0.31 & 0.403 & 0.226 & 2.57 & 42.0866 & -88.1113 \\
\hline 77 & 05550470 & 19 & 1.43 & 0.56 & 0.879 & 0.520 & 5.15 & 42.0179 & -88.1727 \\
\hline 78 & 05550500 & 58 & 0.40 & 0.12 & 0.562 & 0.337 & 35.09 & 42.0556 & -88.1627 \\
\hline 79 & 05551030 & 17 & 0.01 & 0.28 & 0.416 & 0.235 & 15.28 & 41.9795 & -88.2304 \\
\hline 80 & 05551050 & 18 & 1.24 & 0.53 & 0.356 & 0.189 & 7.37 & 41.9397 & -88.2407 \\
\hline 81 & 05551200 & 49 & -0.59 & 0.15 & 0.293 & 0.135 & 51.80 & 41.9879 & -88.3924 \\
\hline 82 & 05551520 & 19 & -0.02 & 0.26 & 0.360 & 0.192 & 5.19 & 41.8291 & -88.2638 \\
\hline 83 & 05551650 & 16 & 0.51 & 0.36 & 0.018 & -0.260 & 1.95 & 41.8568 & -88.4135 \\
\hline 84 & 05551700 & 49 & 0.05 & 0.11 & 0.267 & 0.112 & 68.19 & 41.8015 & -88.4185 \\
\hline 85 & 05551800 & 19 & 0.03 & 0.25 & 0.093 & -0.095 & 0.45 & 41.6058 & -88.4699 \\
\hline 86 & 05551900 & 15 & 0.19 & 0.34 & 0.042 & -0.191 & 32.72 & 41.8396 & -88.5821 \\
\hline 87 & 05551930 & 16 & -0.19 & 0.32 & 0.065 & -0.142 & 22.75 & 41.8316 & -88.5204 \\
\hline 88 & 04093000 & 63 & -0.02 & 0.08 & 0.342 & 0.177 & 124.13 & 41.4570 & -87.3261 \\
\hline 89 & 04094000 & 65 & 0.38 & 0.10 & 0.104 & -0.078 & 66.01 & 41.5991 & -86.9707 \\
\hline 90 & 04094500 & 47 & 0.07 & 0.11 & 0.260 & 0.104 & 75.24 & 41.5078 & -87.0989 \\
\hline 91 & 05517500 & 61 & -0.07 & 0.09 & 0.040 & -0.196 & 1351.30 & 41.4402 & -86.4887 \\
\hline 92 & 05517890 & 36 & -0.53 & 0.18 & 0.041 & -0.193 & 30.62 & 41.4019 & -87.1220 \\
\hline 93 & 05519000 & 53 & 0.44 & 0.13 & 0.103 & -0.079 & 122.84 & 41.3160 & -87.3381 \\
\hline 94 & 05519500 & 24 & -0.52 & 0.25 & 0.142 & -0.024 & 55.05 & 41.3521 & -87.4975 \\
\hline 95 & 05536190 & 67 & -0.17 & 0.09 & 0.419 & 0.238 & 69.28 & 41.4626 & -87.5270 \\
\hline 96 & 04087204 & 46 & 0.51 & 0.15 & 0.559 & 0.335 & 24.93 & 42.8998 & -87.9147 \\
\hline 97 & 04087220 & 46 & 0.73 & 0.17 & 0.519 & 0.309 & 49.28 & 42.9366 & -88.0358 \\
\hline 98 & 04087230 & 32 & -0.80 & 0.23 & 0.031 & -0.218 & 4.01 & 42.7764 & -88.0403 \\
\hline
\end{tabular}




\begin{tabular}{|c|c|c|c|c|c|c|c|c|c|}
\hline Index\# & $\begin{array}{c}\text { USGS } \\
\text { streamgage } \\
\text { number }\end{array}$ & $\begin{array}{l}\text { Pseudo } \\
\text { record } \\
\text { length, } \\
\text { in years }\end{array}$ & 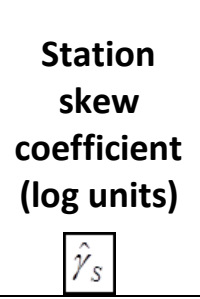 & 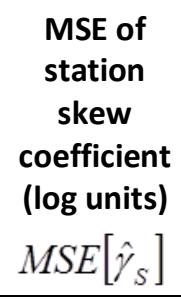 & $\begin{array}{c}\text { NLCD_22_23_24 } \\
\text { (fraction) }\end{array}$ & $\begin{array}{c}\text { Regional } \\
\text { skew } \\
\text { coefficient, } \\
\text { URBAN } \\
\text { model } \\
\text { (log units) }\end{array}$ & $\begin{array}{c}\text { Drainage } \\
\text { area } \\
\left(\mathrm{mi}^{2}\right)\end{array}$ & $\begin{array}{l}\text { Basin } \\
\text { centroid } \\
\text { latitude } \\
\text { (decimal } \\
\text { degrees) }\end{array}$ & $\begin{array}{c}\text { Basin } \\
\text { centroid } \\
\text { longitude } \\
\text { (decimal } \\
\text { degrees) }\end{array}$ \\
\hline 99 & 04087233 & 46 & -0.54 & 0.15 & 0.062 & -0.149 & 55.20 & 42.7452 & -88.0133 \\
\hline 100 & 04087250 & 50 & -0.65 & 0.15 & 0.417 & 0.236 & 7.24 & 42.5876 & -87.9111 \\
\hline 101 & 04087257 & 38 & -0.56 & 0.18 & 0.295 & 0.136 & 38.57 & 42.6604 & -87.8943 \\
\hline 102 & 05438283 & 17 & -0.34 & 0.32 & 0.046 & -0.183 & 9.26 & 42.5387 & -88.6475 \\
\hline 103 & 05543830 & 48 & 0.28 & 0.13 & 0.339 & 0.174 & 127.74 & 43.0773 & -88.2062 \\
\hline 104 & 05544200 & 34 & -0.29 & 0.17 & 0.056 & -0.161 & 72.09 & 42.8550 & -88.4424 \\
\hline 105 & 05544300 & 21 & -0.63 & 0.28 & 0.066 & -0.140 & 1.31 & 42.8376 & -88.3262 \\
\hline 106 & 05545100 & 48 & 0.20 & 0.12 & 0.085 & -0.108 & 6.19 & 42.6824 & -88.4912 \\
\hline 107 & 05545200 & 51 & -0.30 & & 0.017 & -0.263 & 2.36 & 42.6943 & -88.3774 \\
\hline 108 & 05545300 & 24 & -0.02 & 0.21 & 0.085 & -0.107 & 106.37 & 42.6101 & -88.4265 \\
\hline 109 & 05545750 & 70 & 0.01 & 0.08 & 0.130 & -0.040 & 804.31 & 42.8271 & -88.3187 \\
\hline 110 & 05548150 & 48 & -0.60 & 0.15 & 0.034 & -0.212 & 13.75 & 42.4981 & -88.4256 \\
\hline
\end{tabular}




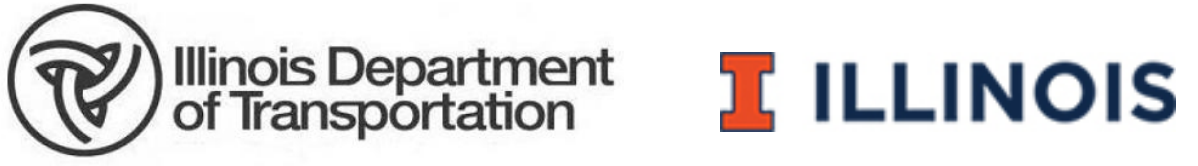

